\title{
Untersuchung zu mittelalterlichen und frühneuzeitlichen Messern
}

dargestellt am Beispiel von archä ologischen Funden vornehmlich aus dem weiteren Küstenbereich von $\mathrm{N}$ ord- und $\mathrm{O}$ stsee bis zur $\mathrm{M}$ ittelgebirg szone

Dissertation zur Erlangung des philosophischen Doktorgrades am Fachbereich Historisch-Philologische Wissenschaften der Georg-August-Universität zu Göttingen

vorgelegt von:

aus:
Gerhard Folke Wulf Holtmann

Münster

Göttingen 1993 
- II -

Berichterstatter:

Privatdozent Dr. H.-G. Stephan

Mitberichterstatter:

Prof. Dr. R. Brednich

Tag der mündlichen Prüfung:

09.02.1994 


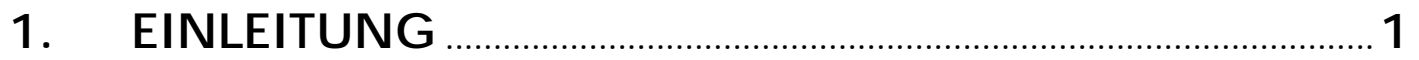

1.1. Der geographische Raum ....................................................................... 4

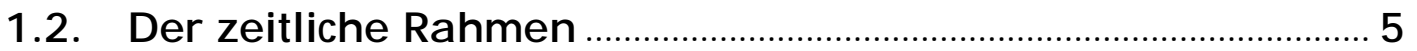

2. DIE ARCHÄOLOGISCHEN QUELLEN ...................................... 7

2.1. Die Fundorte................................................................................................... 7

2.2. Die Funde

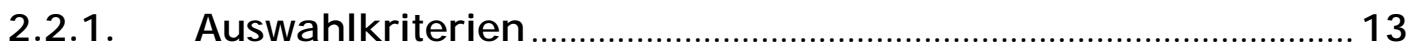

2.2.2. Fundverteilung innerhalb der Fundortgruppen.......................... 14

2.2.3. Die zeitliche Fundverteilung ……................................................. 16

2.2.4. Erhaltungszustand und Rekonstruktionsmöglichkeiten ............ 24

2.2.5. Kriterien der statistischen Auswertung........................................ 27

2.2.6. Abschließende Anmerkungen zur Quellenkritik und zum

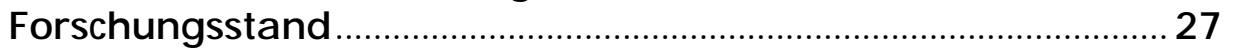

3. DAS KLASSIFIZIERUNGSMODELL_........................................31

3.1. Klassifizierungsmodelle in der Ur- und Frühgeschichte.........34

3.2. Klassifizierungsmodelle in der Mittelalter- und Neuzeitarchäologie Fragestellung und Probleme zur Sachgutbearbeitung..

3.3. Bestandteile des Messers...................................................................38

3.4. Die Verschlüsselung ……………………………............................39

3.5. Die Codebezeichnungen des Schlüssels..........................................42

3.5.1. Die Griffkonstruktionen................................................................. 42 
3.5.2. Die Klingenformen ...................................................................... 53

3.6. Kritische Anmerkungen zum Klassifizierungsmodell ..............59

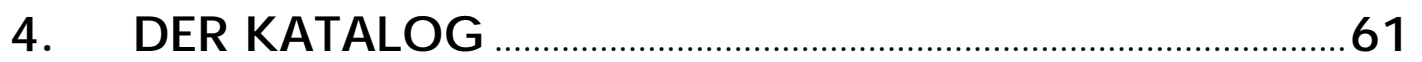

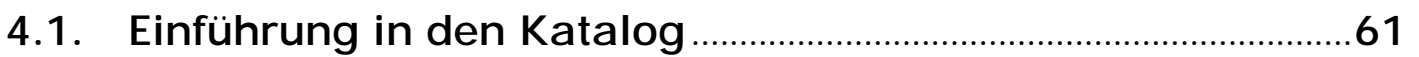

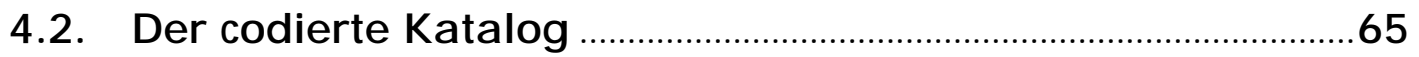

5. AUSWERTUNG UND ERGEBNISSE .................................104

5.1. Statistischer Teil...................................................................................... 104

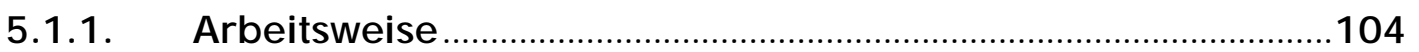

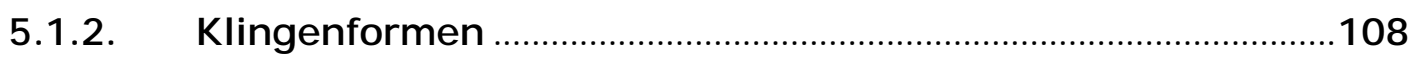

5.1.2.1. Die Verteilung der Klingenformen Ib (Auswertungsgruppe 1).......................110

5.1.2.2. Die Verteilung der Klingenformen IIb (A usw ertungsgruppe 2).......................118

5.1.2.3. Die Verteilung der Klingenformen IIIb (A uswertungsgruppe 3)......................123

5.1.2.4. Die Verteilung der Klingenformen IIlb (A uswertungsgruppe 4) ......................133

5.1.2.5. Die Verteilung der Klingenformen IIlb (A uswertungsgruppe 6)......................133

5.1.2.6. Die Verteilung der Klingenformen IIlb (A uswertungsgruppe 5).....................136

5.1.2.7. Die Verteilung der Klingenformen IIlb (A uswertungsgruppe 7).......................145

5.1.2.8. Abschließende Bemerkungen zu den Verteilungstendenzen

5.1.2.9. Die Verteilung der Klingenformen Ille (Auswertungsg ruppe 8) .......................151

5.1.2.10. Die Verteilung der Klingenformen Ille (Auswertungsg ruppe 9) .......................155

5.1.2.11. Die Verteilung der Klingenformen Ille (Auswertungsgruppe10).....................157

5.1.2.12. Abschließende Bemerkungen zu den Verteilungstendenzen der Klingenformen Ille .................................................................................158

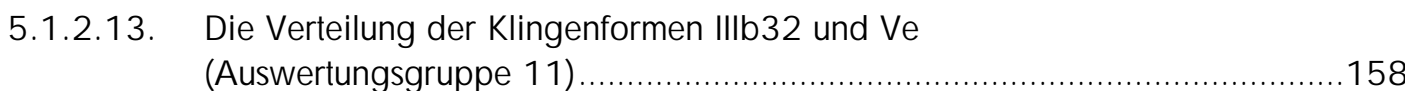

5.1.2.14. Die Verteilung der Klingenformen IIId 1 und IVi

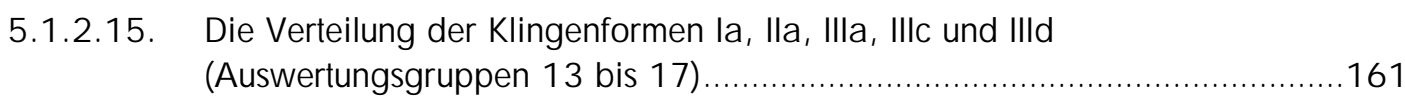

5.1.2.16. Die Verteilung der Sonderformen IV

5.1.2.17. Die Verteilung der Sonderformen $V$ (Klingenformen Va bis Ve) .....................170

5.1.2.18. Zusammenfassung der Ergebnisse zur Verteilung der hä ufiger a uftretenden Klingenformen ..... 
5.1.3. Die Klingenlängen...................................................................176

5.1.4. Die Griffkonstruktionen..........................................................192

5.1.4.1. Die zeitliche Verteilung von $\mathrm{G}$ riffangelmessern und $\mathrm{G}$ riffzungenmessern......192

5.1.4.2. Die zeitliche Verteilung der einzelnen $G$ riffangelkonstruktionen ..................208

5.1.4.2.1. Griffangelmesser mit und ohne Zusatzsicherungen ...............................209

5.1.4.2.2. Unter- und oberständige Griffe .........................................................219

5.1.4.2.3. Griffangelabsatzmöglichkeiten und die Anhebung des Griffs

5.1.4.2.4. Die einzelnen Griffsicherungskonstruktionen ........................................228

5.1.4.3. Die zeitliche Verteilung der einzelnen G riffzungenkonstruktionen................235

5.1.4.3.1. G riffzungenmesser mit und ohne Zusatzsicherungen ............................235

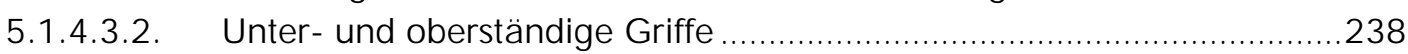

5.1.4.3.3. Die Anhebung des G riffs gegenüber der Schneide ...............................238

5.1.4.3.4. Die Griffsicherungskonstruktionen B5 bis B8 ....................................246

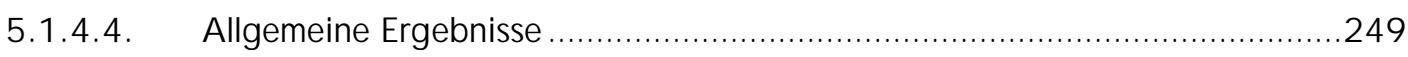

5.2. Beschreibender Teil ............................................................................. 250

5.2.1. Arbeitsweise..............................................................................250

5.2.2. Frühe Griffzungenkonstruktionen ...........................................250

5.2.3. Die Entwidklung der Griffkonstruktionen vom 14. bis 17. Jahrhundert am Beispiel der Niederlande............266

5.2.4. Die Griffiverzierungen ...............................................................273

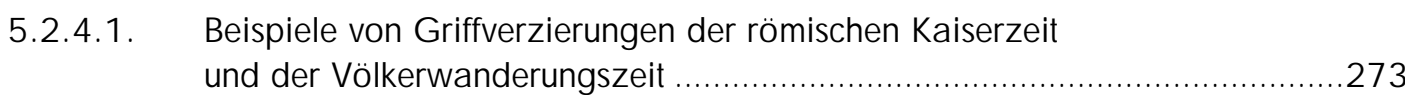

5.2.4.2. Griffverzierungen an $\mathrm{G}$ riffen aus organischen Materialien

5.2.4.2.1. Anthropomorph verzierte mittelalterliche Messergriffe

5.2.4.2.1.1 Beschreibung aller erfaßten G riffe ........................................................ 295

5.2.4.2.1.2 Zur historischen Einordnung der anthropomorph

5.2.4.2.1.3 Zur Frage der Herkunft, möglicher Vorbilder

und der Bildinhalte ................................................................. 330

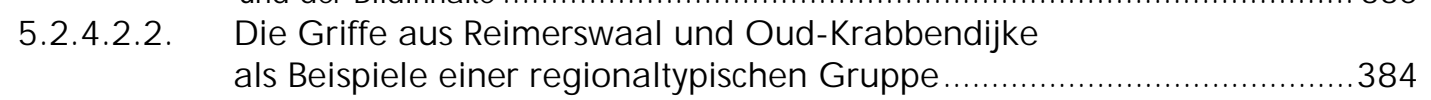

5.2.4.2.3. Die Verbreitung von Verzierungselementen - Beispiele............................388

5.2.4.3. Verzierungen an $\mathrm{G}$ riffen aus M etall und die Verwendung von
Metallapplikationen vom 9. bis zum 17. Jahrhundert...................................

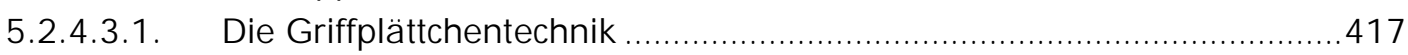

5.2.4.3.1.1 Die erfaßten G riffplättchenmesser............................................................... 418

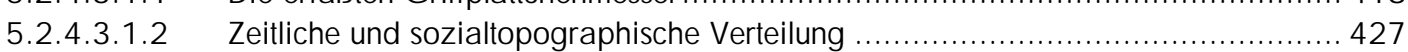

5.2.4.3.1.3 Zur typologischen und regionalen Differenzierung von $G$ riffplättchenmessern ........ 429

5.2.5. Klingenverzierungen .............................................................443 


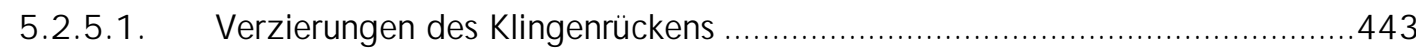

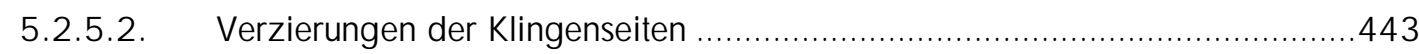

5.2.6. Die Klingenmarken ......................................................................451

5.2.7. Analyse fundortspezifischer Unterschiede von Messerklingen ...........................................................................459

6. MESSERFORMEN VOR 800 N. CHR..................................481

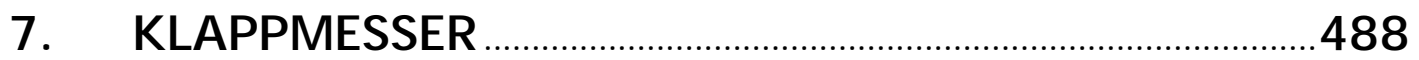

8. PRODUKTION UND GEBRAUCH.....................................493

8.1. Zur Herstellung von Messerklingen ...............................................493

8.1.1. Anmerkungen zu Herstellungsverfahren

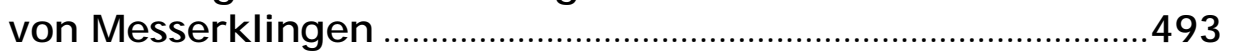

8.1.2. Eisenerzförderung und Eisenhandel...........................................505

8.1.3. Produktion und Handel mit Eisen- und Stahlwaren .................517

8.2. Zur Funktion von Messern ...............................................................538

8.2.1. Funktion und Funktionswandel aus der Sicht der historischen Forschung - Beispiele ..............................541

8.2.2. Beispiele von Messern in Bildquellen ........................................551

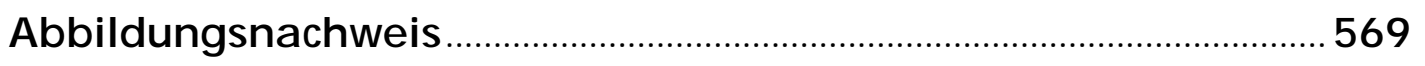

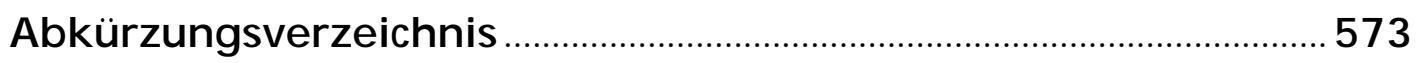

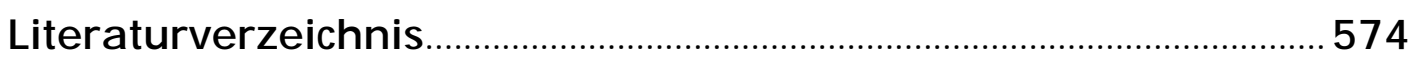




\section{Vorwort}

Die vorliegende Untersuchung zu mittelalterlichen und frühneuzeitlichen Messern ist aus einem Forschungsansatz entstanden, der sich aus zahlreichen Gesprächen mit in- und ausländischen Kolleginnen und Kollegen ergab und zwei Ziele verfolgt:

1. Die wissenschaftliche Bewältigung der umfangreichen Fundkomplexe, die in den letzten Jahrzehnten vor allem im Bereich der Stadtkernarchäologie angewachsen sind, und

2. die systematische Aufarbeitung und Vorlage der einzelnen Fundgruppen, um sie in ihrem jeweiligen historischen Umfeld unter Miteinbeziehung weiterer historischer Quellen diskutieren zu können.

Die Aufnahme des im Katalogteil erfaßten Fundmaterials wurde bis auf wenige Ausnahmen 1987 abgeschlossen.

Fundobjekte, die nach 1987 berücksichtigt wurden, sind in der Regel im beschreibenden Teil der Auswertung besprochen worden.

Die Fundbestände aus der Bundesrepublik Deutschland (alte Bundesländer) wurden vom Verfasser 1989 im Rahmen einer Magisterarbeit an der Westfälischen Wilhelms-Universität Münster vorgelegt.

Während der Entstehungszeit dieser Untersuchung erfuhr der Verfasser zahlreiche Anregungen und Hinweise, Unterstützung bei der Aufnahme des Fundmaterials und großes Entgegenkommen bei der Genehmigung zur Bearbeitung und Veröffentlichung bisher unpublizierter Fundbestände.

$\mathrm{Zu}$ besonderem Dank verpflichtet ist er Frau Kuratorin A. Ruempol und Frau A. van Dongen (Museum Boymans-van Beuningen, Rotterdam), Herrn Dr. J.M. Baart und Herrn A. Lagerweij (Historisches Museum Amsterdam, Archäologischer Dienst), Frau G. Faerden und Herrn A.E. Christensen (Museum für Nationale Altertümer der Universität Oslo), Herrn Dr. L. Redin (Staatliches Historisches Museum Stockholm), Herrn Prof. Dr. N.-K. Liebgott (Dänisches Nationalmuseum Kopenhagen), Herrn Museumsinspektor M. Andersen (Roskilde Museum, Roskilde), Frau M. Cinthio (Museum Kulturen, Lund), Herrn A. Falk M.A. (Amt für Vor- und Frühgeschichte, Lübeck), Herrn Prof. Dr. J. Bracker (Museum für Hamburgische Geschichte, Hamburg), Herrn Prof. H. Rötting (Niedersächsisches Institut für Denkmalpflege, Braunschweig),

Herrn Dr. G. Krause (Kultur- und Stadthistorisches Museum Duisburg), Herrn Dr. E. Schumacher (ehem. Archäologisches Museum Altenessen, Essen), Herrn Dr. R. Blank (Museum Hohenlimburg), Herrn Dr. J. Feldkamp und Herrn Dr. Wagener (Museum Burg Altena), Herrn F. Hallensleben (Altena) und Herrn Dr. S. Schütte (Amt für Bodendenkmalpflege, Köln). 
Weitere Vermittlungen von Fundbeständen, kritische Anregungen und Hinweise begleiteten die mehrjährige Arbeit in zahlreichen Gesprächen und Diskussionen.

Besonders gedankt sei an dieser Stelle Herrn Dr. habil. H.-G. Stephan (Universität Göttingen), Herrn Prof. Dr. L. Leciejewicz (Universität Breslau), Herrn Prof. Dr. W. Janssen (Universität Würzburg) und Herrn Prof. Dr. G. Fehring (Amt für Vorund Frühgeschichte Lübeck). Gleicher Dank gebührt Herrn Dr. D. Gaimster (London), Herrn Dr. P. Glüsing (Münster), Frau Dr. Z. Kurnatowska (Posen), Herrn Dr. V. Nekuda (Brünn), Herrn Dr. H.-J. Vogt (Dresden) und Herrn A. Zeischka (Oberhausen).

Herr A. Zeischka (Oberhausen) und Herr M. Pohlmann (Werdohl) fertigten einen Großteil der Zeichnungen an und gaben wertvolle Hilfe bei der Ausführung des Layout der Abbildungen.

Den größten Dank schuldet der Verfasser Herrn Th. Hinz (Münster), ohne dessen Erfahrung und Hilfe bei der computergestützten Auswertung die Untersuchung in der vorliegenden Form nicht durchführbar gewesen wäre. 


\section{Einleitung}

Mit der vorliegenden Arbeit wird zum ersten Mal eine überregionale Teilbestandsaufnahme von Messern aus archäologischen Quellen erstellt. Die im Titel enthaltene Zeitspanne von 800 bis 1650 nach Christus umfaßt in etwa den zeitlichen Rahmen der Mittelalter- und Neuzeitarchäologie, deren Publikationen ein Großteil der im Katalogteil enthaltenen Messer entnommen wurden.

Die Arbeit berücksichtigt hauptsächlich drei Schwerpunkte: Die Verschlüsselung von Angaben zu Formen und Materialien von Messern in ein System, dessen Terminologie eine Speicherung und statistische Auswertung in der Datenverarbeitung ermöglicht (Klassifizierungsmodell), eine repäsentative Bestandsaufnahme von ausgegrabenen Messern, die in ihrer räumlichen und zeitlichen Verteilung in etwa den derzeitigen Forschungsstand wiedergibt (Katalog) sowie eine historische Fragestellungen berücksichtigende Analyse des vorgelegten Bestandes, die einen statistischen und einen beschreibenden Teil enthält.

Ausgewertet wurden 1.300 Messer, die zu einem größeren Teil in Norwegen, Schweden, Dänemark, den Niederlanden, in Deutschland und Polen, im Baltikum und im nordwestlichen Rußland ausgegraben und publiziert worden sind. $\mathrm{Zu}$ einem geringeren Teil sind bisher unpublizierte Messer berücksichtigt worden. Vollständigkeit zu erreichen war weder möglich noch das Ziel dieser Arbeit. Für jede Region wurde der Grad an Quantität angestrebt, der unter Berücksichtigung arbeits- und quellenkritischer Überlegungen erforderlich war, um einen Querschnitt des vorhandenen Fundbestandes zu erfassen.

Bei der Bearbeitung des ausgewählten Fundbestandes stellten sich zwei grundsätzliche Probleme, bei deren Behandlung aus Gründen der Übersichtlichkeit und praktischen Handhabung der vorliegenden Untersuchung auf eine Berücksichtigung historischer Gegebenheiten verzichtet wurde.

Zum einen war es aus quellenkritischen Gründen bis auf wenige Ausnahmen nicht möglich, zeitgleiche Bestände aus den verschiedenen Regionen des Untersuchungsraums miteinander zu vergleichen. Des weiteren wurden bei der Einteilung des Untersuchungsraums die zum Zeitpunkt der Materialaufnahme und statistischen Auswertung des ausgewählten Bestandes gültigen Staatsgrenzen zugrunde gelegt, kulturräumliche, ethnische oder historische Grenzen wurden lediglich im zweiten, beschreibenden Teil der Auswertung berücksichtigt.

Die folgenden Begründungen betreffen weitgehend den Zustand vor 1987. In diesem Jahr wurde die Materialaufnahme abgeschlossen und die statistische Auswertung durchgeführt.

Die politischen Veränderungen nach 1989, deren Abschluß auch 1993 noch nicht endgültig zu sein scheint, sowie die wirtschaftlichen Einbrüche der letzten Jahre 
haben zu Einschränkungen und Schwerpunktveränderungen im kulturellen Bereich geführt, die hier nicht mehr berücksichtigt werden können.

Die quellenkritischen Einschränkungen bei der Analyse historisch vergleichbarer Fundbestände liegen vor allem in den verschiedenen Forschungsschwerpunkten der einzelnen Länder begründet. Neben den zumindest bis 1989 bestehenden Unterschieden zwischen den ost- und westeuropäischen Staaten in der Gewichtung und Bearbeitung von Gegenständen der materiellen Kultur, die vor allem historische Sachgüter aus alltäglichen Lebensbereichen umfaßt, bestehen auch im Westen in den einzelnen Ländern unterschiedliche Forschungsschwerpunkte. Diese sind nicht nur thematischer, sondern auch chronologischer Art.

Der Grund für diese Unterschiede liegt unter anderem in der verschiedenen Richtung und in den unterschiedlichen Trägerschaften der jeweiligen Forschung, deren Einfluß und Intention unterschiedliche Forschungsschwerpunkte und Ergebnisse gezeitigt haben.

Zwei Beispiele mögen dies verdeutlichen:

In den Niederlanden ist das Verhältnis von Industrie und Wirtschaft zum kulturellen Leben lange Zeit von zahlreichen Stiftungen, Schenkungen und Förderungen von Forschungsprojekten geprägt gewesen. Im kulturgeschichtlichen Zusammenhang sei hier das Museum Beumans-van Beuningen in Rotterdam genannt. Der Stolz der Förderer und Finanziers galt vor allem den Zeiten, in denen Handel und Wirtschaft die nördlichen Niederlande zur Hochblüte brachten. In die gleiche Zeit fällt - bedingt durch den rasch gewonnenen Reichtum des städtischen Bürgertums die Darstellung der Lebensumstände dieses Reichtums in der Malerei, die teilweise auch die niedrigeren sozialen Schichten umfaßt. Somit ist im Bereich der Mittelalter- und Neuzeitarchäologie in den Niederlanden neben dem "Goldenen Zeitalter" vor allem das 15. und 16. Jahrhundert behandelt worden. Das eigentliche Mittelalter ist im Vergleich hierzu stark unterrepräsentiert, lediglich die Alltagskultur des Spätmittelalters hat in den letzten zehn Jahren in Forschung und Repräsentation an Bedeutung gewonnen ${ }^{1}$.

Im Gegensatz hierzu setzte die Forschung in der ehemaligen DDR und in Polen andere Schwerpunkte. Nach dem Ende des zweiten Weltkrieges waren es in den beiden Staaten unter anderem zwei Ziele, die die vom Staat getragene archäologische Forschung, soweit sie das Mittelalter betraf, beherrschten: die

\footnotetext{
Eine Ausnahme bildet wie fast überall die niederländische Keramikforschung (vgl. z.B. die Grabungen von Brunssum-Schinveldt).

Zur Präsentation spätmittelalterlicher und frühneuzeitlicher Sachkultur aus niederländischen archäologischen Quellen vgl. u.a. J. Baart u.a., Opgravingen in Amsterdam, Amsterdam 1977; Thuis in de late middeleeuwen, Ausstellungskatalog mit Texten der gleichnamigen Ausstellung im Provinciaal Overijssels Museum Zwolle, Zwolle 1980; aus der Reihe "De eeuw van de Beeldenstorm" des Museums Boymans-van Beuningen: J.P. Ter Molen, A.P.E. Ruempol und A.G.A. van Dongen, Huisraad van een molenaars weduwe, Rotterdam 1987; ebenfalls in das Mittelalter hineinreichende Untersuchungen betreffen unter anderem die Publikationen des Verkehrsministeriums und des "Rijksdienst voor de Ijsselmeerpolders/ Museum voor Scheepsarcheologie" (Ketelhaven) über Schiffswrackfunde
} 
Slawenforschung und in Polen die Erforschung des Ursprungs des polnischen Staates. Vor allem in der Slawenforschung lag ein Schwerpunkt auf der Darstellung und Analyse der materiellen Kultur als einer wichtigen Quelle zur Erfassung der ökonomischen Grundlagen und Gesellschaftsformen der slawischen Bevölkerung ${ }^{2}$. Das Ergebnis war unter anderem eine durch Ausgrabungen zahlreicher slawischer Fundstätten erreichte breite Materialbasis. Sie betrifft vor allem die Zeit des 9. bis 12. Jahrhunderts und liegt in einem erheblich größeren Umfang als ähnliche in den westlichen Staaten publizierte Fundbestände vor. In der ehemaligen DDR betraf diese Forschung vor allem offene Siedlungen und Burgwälle, in Polen auch die wichtigsten Städte, bedingt durch das Ziel, die frühen Herrschaftszentren während der Entstehung und Festigung des polnischen Staates seit dem 10. Jahrhundert zu erfassen $^{3}$. Die weitere Aufarbeitung der Sachkultur des 13. und 14. Jahrhunderts erfolgte vor allem durch Stadtkerngrabungen, so unter anderem in Magdeburg, Erfurt, Leipzig, Danzig, Posen, Breslau und Oppeln, wobei in Polen für Städte wie Danzig mehrere Monographien erschienen sind ${ }^{4}$.

Diese vor allem die Zeit des 9. bis 12. Jahrhunderts betreffenden Forschungsschwerpunkte in der Mittelalter- und Neuzeitarchäologie legten das Gewicht auf die Frühgeschichte. Das Ergebnis war ein starker Gegensatz zum Beispiel zu den Niederlanden:

Wo im Westen die Sachkulturforschung auf archäologischer Basis im 14., vor allem im 15. Jahrhundert auf breiterer Basis vorliegt, dünnt sie im Osten im 15. Jahrhundert aus. So können, obwohl in beiden Ländern die Stadtkernarchäologie einen breiten Raum einnimmt, vor allem die nachmittelalterlichen Bestände aus den Niederlanden mit denen Polens kaum verglichen werden.

Probleme dieser Art wirkten sich bei der Auswertung für viele Vergleichskriterien und Untersuchungsräume nachteilig aus.

Die oben geschilderten unterschiedlichen Forschungsschwerpunkte haben wesentlich dazu beigetragen, daß bei der Auswahl räumlicher Gliederungsmöglichkeiten für die statistische Auswertung modernen Staatsgrenzen der Vorzug gegeben wurde.

Eine chronologische Abfolge der statistischen Analysen hätte alle wesentlichen Veränderungen der im Untersuchungsraum anfallenden Kultur- und Wirtschafts-

2 Vgl. unter anderem das von S. Epperlein 1965 ins Deutsche übersetzte Werk von W. Hensel, Die Slawen im frühen Mittelalter - Ihre materielle Kultur, Warschau 1956, dt. Ausgabe Berlin 1965; ders., Anfänge der Städte bei den Ost- und Westslawen, dt. Ausgabe Bautzen 1967 von P. Nowotny; J. Herrmann, Die Slawen in Deutschland, Berlin 1970; ders., Wikinger und Slawen - Zur Frühgeschichte der Ostseevölker, Neumünster-Berlin 1982; ders., Welt der Slawen, München-Leipzig 1986

3 Vgl. zusammenfassend W. Blaszczyk, Die Anfänge der polnischen Städte im Lichte der Bodenforschung, Ausstellungsführer mit Texten und weiterführenden Literaturangaben, Posen 1974, dt. Ausgabe anläßlich der gleichnamigen Ausstellung im Museum für Vor- und Frühgeschichte - Staatliche Museen Preußischer Kulturbesitz in Berlin

z.B. die für Posen und Danzig erschienenen Reihen "Poznan we wczesnym šredniowieczu" ab I-1959 (Breslau-Warschau) und "Gdansk wczesnošredniowieczny" ab I-1959 (Danzig) 
räume von der Karolingerzeit bis zur Späthanse bzw. bis zum 30-jährigen Krieg berücksichtigen müssen. Das publiziert angetroffene Fundmaterial lag aber auch nach der Aufnahme unpublizierter Fundbestände nicht in der erforderlichen Dichte vor, um regional und überregional vergleichbare Mengen vor dem Hintergrund der wichtigsten historischen Räume und Entwicklungen behandeln zu können. Dieses wurde an Einzelbeispielen im beschreibenden Teil der Auswertung durchgeführt.

Die im folgenden Kapitel beschriebene absolute Verteilung der mit dem hier vorgelegten Bestand zur Verfügung stehenden Fundmengen nach Jahrhunderten und Herkunftsländern zeigt eine chronologische und regionale Inhomogenität des betreffenden Fundmaterials, die sich auch durch die seit 1987 publizierten Grabungen nicht ausgleichen läßt.

Aus diesen Gründen und unter Berücksichtigung des sich über weite Strecken auf bereits vorhandene Literatur stützenden Inhalts der Untersuchung erschien eine Einteilung des Untersuchungsraums in die Länder, in denen die benutzten Publikationen erschienen waren, sinnvoller und nachvollziehbarer als in historische Räume.

Da die neueste politische Entwicklung erst nach Abschluß der Materialaufnahme und der statistischen Auswertung stattgefunden hat, wurden die 1987 aktuellen Bezeichnungen der in Frage kommenden Staaten beibehalten, zum Beispiel "ehemalige DDR", "ehemalige UdSSR" usw. Dies betrifft auch die Zitate der Literatur, die in diesen Staaten erschienen ist. Nach 1987 erschienene Forschungsergebnisse wurden nur dann berücksichtigt, wenn sie für das Thema dieser Untersuchung relevant waren und dem Autor zur Kenntnis gelangten.

\subsection{Der geographische Raum}

Der Bearbeitungsraum umfaßt in etwa die Anrainerstaaten der Nord- und Ostsee ohne den insularen Westen und Finnland.

Die im Katalog erfaßten Bestände an Messern wurden Publikationen und öffentlichen Einrichtungen folgender Staaten entnommen: den drei skandinavischen Staaten Norwegen, Schweden und Dänemark, den Niederlanden, der Bundesrepublik Deutschland, der ehemaligen Deutschen Demokratischen Republik und der ehemaligen Volksrepublik Polen. Aus der ehemaligen UdSSR wurden nur geringe Bestände aufgenommen, sie entstammen dem westlichen Teil der ehemaligen Russischen SFSR (dem Bereich der ehemaligen Novgoroder Slovenen) sowie der ehemaligen Estnischen, Lettischen und Weißrussischen SSR.

Geographisch umfaßt dieser Raum die östliche Nordseeküste, die westliche, südliche und südöstliche Ostseeküste, das norddeutsche Tiefland, den Baltischen Landrücken und das tiefere Vorland sowie den nördlichen Teil der Mittelgebirge vom Rheinischen Schiefergebirge bis zu den Oberläufen von Weichsel und San. Die wichtigsten Flüsse, die die nördlichen Mittelgebirge mit den jeweiligen Küstenregionen verbinden, sind Rhein, Weser, Elbe, Oder, Warthe und Weichsel, die Verbindungsflüsse des Baltischen Landrückens zur Ostsee neben Narew-Weichsel 
der Njemen (Memel), die Düna (oder westliche Dwina), die Narwa mit dem Pskover und dem Peipussee sowie der Volchov mit Lowat-Unterlauf, Ilmensee, Newa und Ladogasee (Karte Abb. 1).

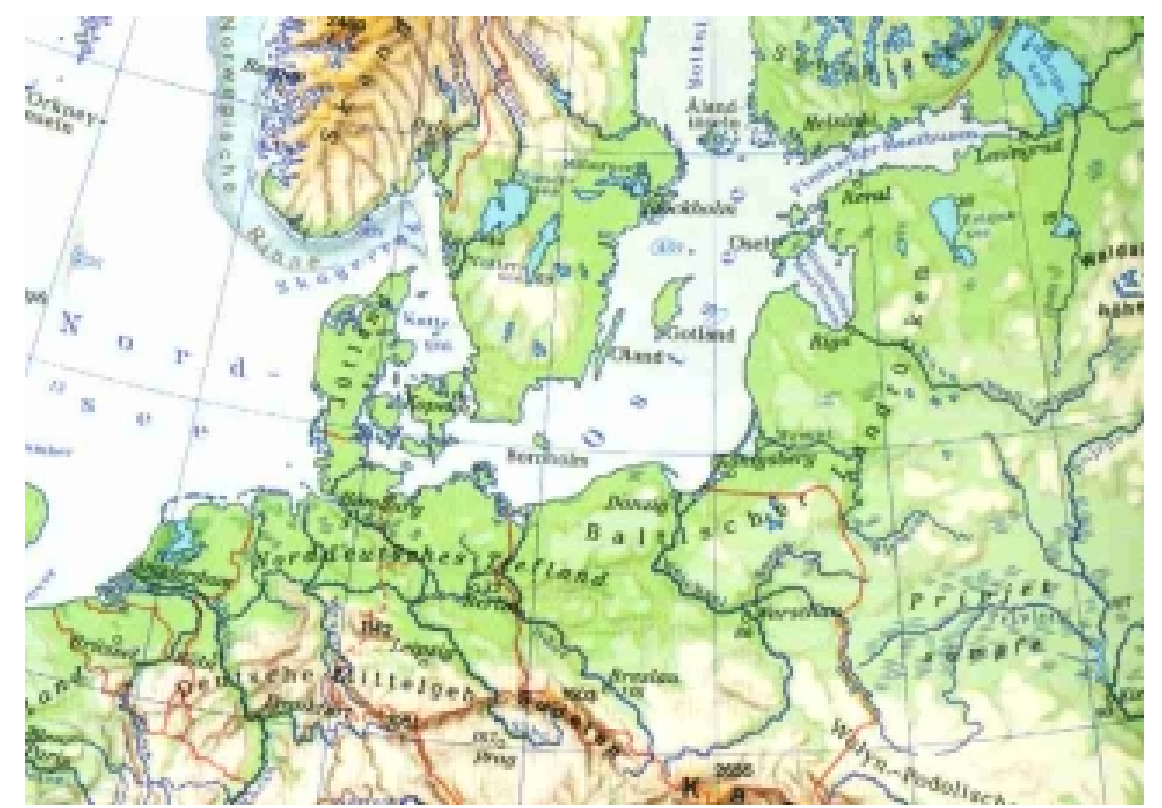

Abb. 1 Der geographische Raum

(Ausschnitt aus Diercke Weltatlas, Karte S. 72-73)

Eine Intensivierung der Aufnahme weiterer Bestände aus den zahlreichen Grabungen in der ehemaligen UdSSR sowie die Ausdehnung des Bearbeitungsraumes nach Westen vor allem auf die Britischen Inseln wäre in vieler Hinsicht wünschenswert gewesen. Dem Autor standen aber weder die Zeit noch Publikationen in der Menge zur Verfügung, wie sie für den oben angeführten Bereich vorhanden waren.

\subsection{Der zeitliche Rahmen}

Die im Titel angegebenen Grenzen des zeitlichen Rahmens - 800 bis 1650 n. Chr. bilden lediglich Eckdaten, die zwar im allgemeinen bindend waren, in Einzelfällen jedoch unter- oder überschritten wurden. Das galt besonders bei Fundorten, deren Besiedlungsdauer vor 800 begann oder über 1650 hinausging, wie zum Beispiel für Dorestad (Niederlande, 7./8. bis 9. Jahrhundert) oder Oslo-Christiania (Norwegen, 1625-1750). Einige Fundorte liegen in ihren Besiedlungsphasen insgesamt vor 800 . Sie wurden aufgenommen, wenn innerhalb des ergrabenen Fundbestandes eine gröBere Anzahl oder für die folgenden Zeitabschnitte wichtige Messer vorhanden waren.

Auf Messer der späten römischen Kaiserzeit und der Völkerwanderungszeit wurde in einem eigenen Kapitel eingegangen, um die wichtigsten Formen, die vor dem Mittelalter bereits vorhanden waren, vorzustellen ${ }^{5}$. 
Eine gleichmäßige zeitliche Verteilung konnte nicht erreicht werden. Das gilt nicht nur für den gesamten Bearbeitungsraum, sondern auch für jedes einzelne Land. Die Bestände des 8. bis 10. Jahrhunderts sind in der Regel sowohl in der Zahl der Fundorte als auch in der absoluten Anzahl der Messer geringer als diejenigen des 11. bis 14. Jahrhunderts, Messer des 15. bis 17. Jahrhunderts verteilen sich in den verschiedenen Untersuchungsländern sehr unterschiedlich ${ }^{6}$.

Der Zeitraum von 800 bis 1650 n. Chr. umfaßt in etwa den Bearbeitungszeitraum der Mittelalter- und Neuzeitarchäologie, wobei der Endpunkt 1650 nicht als obere Grenze derselben verstanden werden soll. Er wurde gewählt, weil die auf archäologischer Quellenbasis beruhende Materialgrundlage aufgrund fehlender Publikationen für die zweite Hälfte des 17. und des 18. Jahrhunderts so schmal ist, daß vergleichbare Mengen für diesen Zeitraum nicht mehr zur Verfügung standen. Die obertägig in Museen und Sammlungen verwahrten Bestände dieses Zeitraums sind dagegen erheblich. Die Auswahlkriterien von Sammlern oder die Bereitschaft späterer Besitzer, diese Bestände $\mathrm{zu}$ verwahren, sind aber von völlig anderen Gesichtspunkten abhängig und unterliegen nicht mehr dem Zufallsprinzip vieler archäologisch erfaßter Gegenstände. Die Bearbeitung jüngerer Messer unterblieb somit schon aus quellenkritischen Gründen. 


\section{Die archäologischen Quellen}

\subsection{Die Fundorte}

Die Verteilung der Funde innerhalb der verschiedenen Fundortgattungen gründet sich auf die Angaben der jeweiligen Befundbeschreibungen in den benutzten Publikationen. Diese wurden zum größten Teil auch in den Übersichtskarten angegeben (Verteilung der Fundorte im Untersuchungsraum auf einer Übersichts- und einer Zusatzkarte zum Baltikum).

Zwischen mittelalterlichen Städten und frühstädtischen Siedlungen des 8. bis 11. Jahrhunderts wurde in den beiden Übersichtskarten nicht unterschieden, da zum Beispiel in Polen diese beiden Gruppen in der Regel geographisch zusammenfallen, so in Wolin, Danzig oder Breslau. In den Niederlanden, der Bundesrepublik und Schweden waren mit Dorestad, Haithabu und Birka nur drei frühstädtische Handelsknotenpunkte betroffen, deren Fundmaterial aus Siedlungsfunden und Funden aus Gräberfeldern besteht. Hier waren die einzelnen fundortspezifischen Kriterien nicht so ausreichend mit Fundmaterial zu belegen, daß ein sinnvoller Vergleich möglich gewesen wäre.

Bei den Funden aus Burgen wurde differenzierter verfahren. Bei mehreren, vor allem polnischen Stadtkerngrabungen wird in der Befundbeschreibung unterschieden zwischen Burg und Vorburgsiedlung oder Stadt. Alle Messer, die zwar aus Stadtkerngrabungen stammen, deren genauer Befund aber eine Burg oder burgähnliche Anlage betrifft, wurden der Gruppe der ansonsten in Einzellage befindlichen Burgen zugerechnet. Dasselbe gilt für Burgen mit zugehörigen Siedlungen.

Für fundortspezifische Fragestellungen wurde das Material nach folgenden Kriterien unterschieden:

Städte Funde aus befestigten Städten zumeist des 12. bis 17. Jahrhunderts

Frühstädtische Sied- Funde aus geschlossenen, zum Teil befestigten Siedlungen lungen mit nachweisbarem überregionalen Handel und handwerklich spezialisierter Produktion zwischen dem 8. und 11. Jahrhundert

Burgen Funde aus befestigten Herrschafts- oder Verwaltungszentren mit oder ohne zugehörige Siedlungen des gesamten Bearbeitungszeitraums 
offene Siedlungen Funde aus unbefestigten Siedlungen auf zumeist landwirtschaftlicher Lebensgrundlage (Funde aus Dorfkirchen fallen ebenfalls in diese Kategorie)

Klöster

Gräberfelder

Schiffe

Streufunde
Funde aus Klosteranlagen (keine Dorfkirchenfunde)

Funde aus Gräberfeldern bzw. Friedhöfen (Die Funde aus Gräberfeldern und Friedhöfen wurden nicht spezifisch untersucht, da sie eine positive Auswahl darstellen und als Gruppe hier eine zu geringe Anzahl darstellen, um untereinander vergleichbar zu sein.)

Funde aus Schiffswracks

Zufällig geborgene Streu- oder Lesefunde ohne primären Befundzusammenhang, oft in sekundärer Lagerung (Flußfunde, Funde aus Ausbaggerungen von Seen, hochgepflügte Einzelfunde etc.), Streufunde wurden nur in einigen Einzeldarstellungen berücksichtigt.

Die in der Ur- und Frühgeschichte sonst übliche Einteilung nach Hort-, Siedlungsund Grabfunden war unergiebig, da es sich - mit Ausnahme der positiven Auslese aus Gräberfeldern und Friedhöfen- ausschließlich um Siedlungsfunde mit den bekannten Graden an Zufälligkeit des Verlusts handelt.

Diese differenziertere Fundortklassifizierung ist als Unterscheidungsgrundlage nur für einige wenige, auf diese Unterschiede abzielende Analysen benutzt worden.

Für die Übersichtskarten war eine unterteilende Kennzeichnung nicht immer möglich, weil oft Burgen, Siedlungen und Städte auf einen geographischen Punkt zusammenfielen. So wurden zum Beispiel Kalisch, Breslau und Oppeln als Städte gekennzeichnet, auch wenn einige Messer aus den jeweiligen befestigten Burgen als Keimzellen der späteren Städte stammen.

Bis auf Norwegen wurden alle identifizierbaren Fundorte markiert. Eine Zusatzkarte für Norwegen wurde nicht angelegt, da bis auf Oslo, Tonsberg und Trondheim alle norwegischen Funde nicht mitausgewertet wurden. Das lag einerseits an der geringen Menge, die sich im Gebiet Norwegens vergleichslos verteilt, vor allem aber an der Tatsache, daß fast alle norwegischen Messer außerhalb dieser drei Städte weder Datierungshinweise noch Befundbeschreibungen aufweisen - es handelt sich in der Regel um Streu- oder Lesefunde.

Die Verteilung der Fundorte im Untersuchungsraum ist nur teilweise flächendeckend. Vor allem in Skandinavien konzentrieren sich die Funde auf Orte in Dänemark und den äußersten Süden Schwedens (Schonen), welches während des überwiegenden Teiles des Bearbeitungszeitraums zum Königreich Dänemark gehörte. 
Im Nordwesten erweisen sich der Norden der Niederlande sowie das nördliche und westliche Niedersachsen als nahezu fundleere Räume. In Polen häufen sich die Fundorte im Bereich des ehemaligen Kujawien, Groß- und Kleinpolens und Krakaus zwischen Warthe, Netze und Weichsel, außerhalb dieser Grenzen dünnen sie vor allem nach Süden und Osten stark aus, allerdings liegen zwei der wichtigsten Stadtkerngrabungen in Orten an der Oder, Breslau und Oppeln.

Das Baltikum weist insgesamt nur zwölf Fundorte auf, die sich auf Gebiete der baltischen und ostslawischen Stämme und später auf den Staat des Deutschen Ordens, des Fürstentums Novgorod sowie des Fürstentums Litauen verteilen.

Auch das Verhältnis der verschiedenen Fundortarten untereinander ist insgesamt und in den einzelnen Ländern unterschiedlich:

Skandinavien 13 Städte, 3 Burgen, 1 offene Siedlung, 1 frühstädtische Siedlung (=1 Gräberfeld)

Niederlande 8 Städte, 2 Burgen, 2 offene Siedlungen (ohne Gorkum und Noordeinde), 1 frühstädtische Siedlung und 2 Schiffswracks

Bundesrepublik 16 Städte, 28 Burgen, 11 offene Siedlungen, Deutschland 3 Gräberfelder, 2 Klöster, 1 frühstädtische Siedlung und vor 1989

1 Schiffswrack

ehemalige Deutsche 8 Städte, 22 Burgen, 8 offene Siedlungen, 3 Gräberfelder Demokratische Re- und 1 befestigtes Herrschaftszentrum mit Handwerkersiedpublik lung und Verbindung zum Fernhandel (Lieps)

ehemalige Volksre- 21 Städte (mit 5 Burgen in späteren Städten: Kolberg, Ujspublik Polen cie, Gliecz, Ciechanow, Kalisch), 21 Burgen (ohne die unter "Städte" aufgeführten), 11 offene Siedlungen (darunter mit Rokietnica Tuliglowy 1 befestigte, als frühstädtisch bezeichnete Siedlung) ${ }^{7}, 24$ Gräberfelder und Friedhöfe, 1 Kloster (Hohensalza) und 4 frühstädtische Siedlungen aus der Zeit des 9. und 10. Jahrhunderts (Stettin, Wolin, Danzig und Breslau; die Funde aus Oppeln werden in das 10. bis 12. Jahrhundert datiert, alle 5 Städte sind bereits in den oben genannten enthalten $)^{8}$

ehemalige UdSSR 5 Städte, 1 Burg, 1 offene Siedlung mit Burg, 5 Gräberfelder

\section{Liste der Fundorte}

Die Liste der Fundorte enthält die Ortsnamen, unter denen die einzelnen Funde publiziert worden sind. Kreis- und Bezirksnamen sind im Katalog aufgeführt wor-

\footnotetext{
7 s. Katalogteil Polen, Kat. Nr. 374-376

8 s. Katalogteil Polen, Kat. Nr. 1, 12, 14-16, 38, 39, 310, 311, 330, 337, 338, 341
} 
den, soweit dies $\mathrm{zu}$ einer leichteren geographischen Einordnung notwendig erschien.

Die Fundorte sind nach den einzelnen Ländern geordnet und von 1 bis 236 durchnumeriert worden, die Zahlen entsprechen denen auf den beiden Übersichtskarten.

Die am Ende eingefügte große Übersichtskarte umfaßt die Fundorte der Niederlande, Südskandinaviens, der beiden ehemaligen deutschen Staaten und Polens sowie einige angrenzende Fundorte der ehemaligen Sowjetunion; die zweite Übersichtskarte bezeichnet die Lage aller baltischen und russischen Fundorte.

Die Signaturen sind denen in der allgemeinen Kartographie gebräuchlichen angepaßt worden, die Legende befindet sich auf der ersten Übersichtskarte.

\section{Liste der Fundorte}

$\begin{array}{ll}\text { Niederlande } \\ 1 & \text { Domburg } \\ 2 & \text { Vlissingen } \\ 3 & \text { Reimerswaal } \\ 4 & \text { Krabbendijke } \\ 5 & \text { Sluis } \\ 6 & \text { Den Haag } \\ 7 & \text { Leiden } \\ 8 & \text { Amsterdam } \\ 9 & \text { Noordeinde } \\ 10 & \text { Delft } \\ 11 & \text { Rotterdam } \\ 12 & \text { Zwijndrecht } \\ 13 & \text { Dordrecht } \\ 14 & \text { Gorinchen } \\ 15 & \text { Nederhemert } \\ 16 & \text { Tiel } \\ 17 & \text { Nijmegen } \\ 18 & \text { Utrecht } \\ 19 & \text { Nieuwegein } \\ 20 & \text { Dorestad } \\ 21 & \text { Voorst } \\ 22 & \text { Deventer } \\ 23 & \text { Zwolle } \\ 24 & \text { Ketelhaven } \\ 25 & \text { Lelystad } \\ & \\ \end{array}$

\section{Bundesrepublik Deutschland (alte Bundesländer)}

26 Praest-Blouswardt

27 Ahaus

28 Billerbeck

29 Coesfeld

30 Nottuln

31 Senden

32 Albersloh

33 Burg Kakesbeck

34 Burg Lüdinghausen

35 Haltern-Hullern

36 Holten

37 Duisburg

38 Burg Uda (Oedt)

39 Hs. Meer (Motte bei -)

40 Morken

41 Husterknupp

42 Köln

43 Burg Berge (Altenberge)

44 Isenburg (Essen)

45 Burg Isenberg (Hattingen)

46 Raffenburg u. Rücklenburg

47 Burg Altena

48 Emden

49 Bremen

50 Minden

51 Barntrup

52 Alt-Schieder

53 Höxter u. Tom Roden

54 Wildburg

55 Altenfels (Brilon) 


$\begin{array}{ll}56 & \text { Hünenkeller (Korbach) } \\ 57 & \text { Burg Rödersen (Wolfhagen) } \\ 58 & \text { Burg Schartenberg (Kassel) } \\ 59 & \text { Büraburg (Fritzlar) } \\ 60 & \text { Burg Wartenberg } \\ 61 & \text { Göttingen } \\ 62 & \text { Bengerode (Fredesloh) } \\ 63 & \text { Oldendorp (Einbeck) } \\ 64 & \text { Dörhai (Winzenburg) } \\ 65 & \text { Goslar } \\ 66 & \text { Schulenrode (Harzburg) } \\ 67 & \text { Woltwiesche (Peine) } \\ 68 & \text { Braunschweig } \\ 69 & \text { Kloster Wienhausen } \\ 70 & \text { Celle } \\ 71 & \text { Bunkenburg (Ahlden) } \\ 72 & \text { Növenthien } \\ 73 & \text { Fliessau-Spranz } \\ 74 & \text { Winsen/Luhe } \\ 75 & \text { Hamburg-Wilstorf } \\ 76 & \text { Hamburg-Sinstorf } \\ 77 & \text { Hamburg } \\ 78 & \text { Itzehoe } \\ 79 & \text { Slaw. Burg Lübeck } \\ 80 & \text { Lübeck } \\ 81 & \text { Bosau } \\ 82 & \text { Groß-Schlichtenberg u. } \\ & \text { Hochborre } \\ 83 & \text { Kiel } \\ 84 & \text { Haithabu } \\ 85 & \text { Haneburg (Westerohrstedt) } \\ & \\ \text { Dänemark, Schweden, } \\ \text { Norwegen }\end{array}$

$\begin{array}{ll}86 & \text { Ribe } \\ 87 & \text { Kolding } \\ 88 & \text { Århus } \\ 89 & \text { Randers } \\ 90 & \text { Store Valby } \\ 91 & \text { Roskilde } \\ 92 & \text { Burg Naesholm } \\ 93 & \text { Rørvig } \\ 94 & \text { København-Nørrevold } \\ 95 & \text { Dragør } \\ 96 & \text { Burg Skanör } \\ 97 & \text { Malmö }\end{array}$

$$
\begin{array}{ll}
98 & \text { Lund } \\
99 & \text { Tønsberg } \\
100 & \text { Oslo } \\
101 & \text { Burg Aranaes } \\
102 & \text { Birka }
\end{array}
$$

\section{Bundesrepublik Deutschland (neue Bundesländer, Berlin)}

103 Glasbach (Rennsteig)

104 Burg Manebach (Ilmenau)

105 Neideck (Arnstadt)

106 Gommerstedt

107 Erfurt

108 Hohenrode (Harz)

109 Camburg (Saale)

110 Kretzschau

111 Wiprechtsburg (Groitzsch)

112 Halle (Saale)

113 Leipzig

114 Beerwalde

115 Meißen

116 Brohna

117 Cottbus

118 Steinkirchen (Lübben)

119 Frankfurt (Oder)

120 Burg Köpenick (Ostberlin)

121 Spandau (Westberlin)

122 Göritz (Rädel)

123 Küsel (Burg)

124 Hildagsburg (Wolmirstedt)

125 Burg Wildberg

126 Tornow-Sechzehneichen

127 Mecklenburg

128 Schwerin

129 Trenntseesiedlung

130 Lübow-Levetzov (Wismar)

131 Burg Sternberg

132 Cambs

133 Burg Groß-Raden

134 Baggerfunde (Recknitz)

135 Marlow

136 Behren-Lübchin

137 Behren-Lübchin

138 Demmin (Peenefund)

139 Sanzkow

140 Anklam 


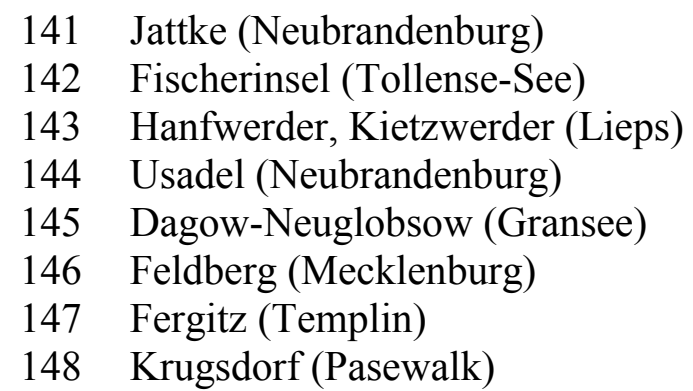

\section{Republik Polen (ehemalige Volksrep. Polen)}

149 Wolin

150 Kamień Pom. (Kammin)

151 Gardziec

152 Szczecin

153 Kunow

154 Stargard

155 Zantok (Zantoch)

156 Kołobrzeg (Kolberg)

157 Bardy

158 Cewlina

159 Radacz

160 Gdańsk (Danzig)

161 Gorzedziej

162 Wegry (Debia Góra)

163 Wegry (Debia Góra)

164 Klasztorek

165 Kałdus

166 Ujscie (Usch)

167 Pomarzanki

168 Wagrowiec

169 Komorowo

170 Gwiazdowo

171 Poznań (Posen)

172 Góra

173 Srem (Schrimm)

174 Witów

175 Wszembórz (Wembusch)

176 Giecz

177 Biale Piatkowo

178 Gniezno (Gnesen)

179 Biskupin

180 Niestronno

181 Inowroclaw (Hohensalza)

182 Kruszwica (Kruschnitz, Gräberfeld)
183 Kruszwica (Kruschnitz, Stadt)

184 Brzesc Kujawski

185 Kóscielna Wies

186 Kalisz (Kalisch)

187 Piwonice

188 Wrocław (Breslau)

189 Niemcza Slaska (Nimptsch)

190 Opole (Oppeln)

191 Bytom (Beuthen)

192 Strzemieszyce WLK.

193 Piekáry

194 Giebultów

195 Kraków (Krakau)

196 Raciborowice

197 Wieliczka

198 Łapczyca

199 Demblin

200 Pałeczznica

201 Szczaworyz

202 Samborzec

203 Rokietnica-Tuliglowy

204 Lublin

205 Kijany-Kolonia

206 Konskie

207 Stobnica-Trzymorgi

208 Rozprza

209 Psary

210 Zbrojewsko

211 Debina

212 Sieradz

213 Lutomiersk

214 Poddebice

215 Tum (Łeczyca)

216 Gostynin

217 Grodnia

218 Lewiczyn

219 Warszawa

(Warschau)-Wilanów

220 Błonie

221 Nasielsk

222 Ciechanów

223 Bazar Nowy

224 Rownina Dolna 
Rußland, Estland, Lettland,
Litauen (ehemalige UdSSR)

225 Grodno

226 Podros (Wolkowysk)

227 Novogrudok

228 Ryga (Riga)

229 Kente (Ogre)

230 Madi (Viljandi)
231 Pskov

232 Tamme (Kohtla-Järve)

233 Valjala Rahu (Kingisepp)

234 Gorodec (Luga)

235 Novgorod

236 Porzezce (Disna)

237 Talinn (Reval)

\subsection{Die Funde}

Die Grundlage dieser Arbeit sind ausschließlich Messer, die im weitesten Sinne durch archäologische Aufnahme erfaßt worden sind. Sie entstammen planmäßigen Grabungen, Notbergungen oder sind Streu- und Zufallsfunde aus Baugruben oder landwirtschaftlicher Bodenbearbeitung.

Gefunden wurden sie unter anderem in Altstadtkernen, in noch bestehenden oder wüstgefallenen Burgen und offenen Siedlungen sowie auf Friedhöfen und Gräberfeldern. Einige wenige entstammen Schiffswracks, andere wurden bei Flußausbaggerungen geborgen.

Die Messer verteilen sich sehr unterschiedlich auf die einzelnen Fundortgruppen. In den benutzten Publikationen aus Skandinavien und den Niederlanden übertrifft die Zahl der Funde aus Altstadtgrabungen zum Beispiel die der in Burgen und ländlichen Siedlungen gefundenen erheblich, in der dem Autor aus der ehemaligen DDR zur Verfügung stehenden Literatur ist das Verhältnis umgekehrt.

Dementsprechend unterschiedlich ist auch die Aussagekraft der den verschiedenen Arten von Fundorten entstammenden Messer. Ein oder mehrere Messer aus einem Schiffswrack sind in der Regel als geschlossener und gut datierbarer Fund zu betrachten, da die Schiffstypen auch in Details bekannt sind. Die Wracks aus den Poldern der ehemaligen Zuider Zee in den Niederlanden enthalten oft Münzen, über die die absolute Datierung noch enger gefaßt werden kann. Ein Flußfund entspricht eher einem Streufund, Aussagen sind hier oft nur aus dem Wissen über das Fundobjekt selbst zu treffen.

\subsubsection{Auswahlkriterien}

Bei der Erstellung des Kataloges wurde grundsätzlich versucht, wenigstens für bestimmte Zeitabschnitte und regional begrenzte Räume repräsentative Querschnitte vorzulegen.

Eine Auswahl aus den dem Autor zur Verfügung stehenden Publikationen wurde nur in seltenen Fällen getroffen, da zu Beginn weder Formen noch Konstruktionen als speziell oder selten beurteilbar waren. 
Darüber hinaus mußte die Auswahl berücksichtigt werden, die bereits in der benutzten Literatur vorgegeben war. In der Regel wechselten sich Grabungsberichte mit umfassenden oder nur sporadisch angelegten Fundkatalogen ab. Ob ein - oder alle - Messer aus einer Grabung ausführlich publiziert oder nur im Text erwähnt wurden, war in das Belieben eines jeden der zahlreichen Autoren gestellt. Somit stellt ein besonders reich verziertes Messer aus einer Siedlungsgrabung, welches den hundert anderen, unverzierten Messern bei der Publikation vorgezogen wurde, eine ebenso positive Auslese dar wie die Messerbeigaben reicher Grabausstattungen.

Die qualitativen Unterschiede in den Auswahlprinzipien der Bearbeiter waren oft derart gravierend, daß von einer aufgrund des Zufallsprinzips beim Verlustiggehen der Messer in Siedlungsgrabungen entstandenen Auswahl oft nicht ausgegangen werden kann. Das gilt auch für große Stadtkerngrabungen: eine ausdrücklich als repräsentativ bezeichnete Auswahl an Messern war auch hier die Ausnahme.

Demgegenüber stehen Siedlungsgrabungen, deren Fundmaterial in Typen und Formen nahezu komplett abgebildet wurde, wie die Grabungen in der Siedlungskammer der Lieps (ehemalige DDR) oder in Gommerstedt (ehemalige DDR), die bei der Auswertung auch für Mikroanalysen zur Verfügung standen.

Bei Gäberfeldern, den einzigen Fundorten mit Fundmaterial, welches zum Zeitpunkt der Niederlegung eine bewußte Auswahl darstellte, stellen sich durch unterschiedliche Publikationsintensität ähnliche Probleme. Neben Plätzen wie Birka oder Dorestad, deren Material als repräsentativer Querschnitt veröffentlicht worden ist, stehen Gräberfelder mit ganzen zwei Messern, die als typische Vertreter eines in Zahlen nicht genannten weitaus größeren Bestandes fungieren (zum Beispiel Lutomiersk, Polen, Kat. Nr. 270 und 271).

Der Autor war in der Regel nur bei der Aufnahme von unpublizierten Messern zu einer Auswahl gezwungen. Dabei handelt es sich in den seltenen Fällen, in denen das Material komplett zur Verfügung stand, bei der Auswahl um einen der Zusammensetzung des Bestandes entsprechenden Querschnitt. In anderen Fällen, in denen nur Teile von Sammlungen oder Beständen bearbeitet werden konnten, wurde das Material vollständig aufgenommen.

Voraussetzung für eine Aufnahme in den Katalog war ein Mindestmaß an Erhaltung und Informationen zum Fundort.

\subsubsection{Fundverteilung innerhalb der Fundortgruppen}

Von den im Katalog erfaßten 1.300 Messern konnten 1.273 den oben genannten Fundortgruppen zugeordnet werden. Davon entfallen 580 Messer auf Städte oder frühstädtische Siedlungen, 358 auf Burgen und burgähnliche Herrschaftszentren, 172 auf offene Siedlungen, 134 auf Gräberfelder, 13 auf Schiffswracks und 5 auf Klöster, 19 Messer (ohne die norwegischen) sind Streufunde. In den einzelnen Un- 
tersuchungsländern verschieben sich die Proportionen zwischen dem Fundaufkommen und der Art des Fundortes erheblich:

Skandinavien

Niederlande

Bundesrepublik

Deutschland

(alte Bundesländer)

Bundesrepublik

Deutschland

(neue Bundesländer)

ehemalige

Volksrepublik Polen

ehemalige UdSSR
Auf 13 skandinavische Städte entfallen 181 Messer, aus dem publizierten Bestand von Birka wurden 26 Messer ausgewählt.

25 Messer entstammen drei Burgen, 28 Messer sind einer Siedlungsgrabung entnommen worden.

Auf 8 niederländische Städte entfallen 72 Messer, 14 Messer wurden aus Dorestad übernommen.

Von 2 Burgen stammen 4 Messer, 4 weitere wurden in Schiffswracks gefunden, 35 Messer entstammen 5 offenen Siedlungen

In der Bundesrepublik verkehrt sich das Verhältnis zwischen Beständen aus Städten und Burgen:

Aus 16 Städten kommen hier 56 Messer, 10 Messer wurden Publikationen über Haithabu entnommen. 28 publizierte und unpublizierte Burggrabungen ergaben einen Bestand von 107 Messern, 11 offenen Siedlungen entstammen 15 Messer, 9 einem Schiffswrack, 6 Messer 3 Gräberfeldern und 2 Messer stammen aus 2 Klöstern. Ein Messer war als Streufund anzusehen.

In der ehemalige DDR wurden nur 17 Messer aus 8 Städten übernommen, dagegen stammen 112 Messer aus 22 Burgen sowie 23 Messer aus einem befestigten Herrschafts- und Kultzentrum.

46 Messer wurden in 8 offenen Siedlungen geborgen, 4 Messer in 3 Gräberfeldern. 18 Messer sind als Flußfunde keinem Fundort zuzuordnen gewesen.

In Polen fallen auf 17 Städte 143 Messer, davon stammen 3 aus einem Kloster.

Auf 26 Burgen kommen 84 Messer, 10 offenen und einer befestigten Siedlung entstammen 31 Messer.

Aus 24 Gräberfeldern wurden 110 Messer übernommen.

Von 12 ehemals sowjetischen Fundorten entfallen 52 Messer auf 5 Städte, 3 auf eine Burg, 18 auf eine befestigte Siedlung und 12 auf 5 Gräberfelder 


\subsubsection{Die zeitliche Fundverteilung}

Die Datierungsgrundlagen der im Katalog erfaßten Messer wurden bis auf die unpublizierten Bestände den Angaben in den jeweiligen Publikationen entnommen.

Ähnlich wie bei den Auswahlkriterien der veröffentlichten Messer in der Literatur waren auch der Spanne der Datierungshinweise keine Grenzen gesetzt:

Sie reichte von nicht datierten Messern über die Angaben wie "mittelalterlich oder neuzeitlich" bis zu einer aus stratigraphischen Befunden ermittelten Genauigkeit von 10 bis 25 Jahren eines Jahrhunderts; sie enthielt kunsthistorisch begründete Ansätze wie "um 1300" oder "spätgotisch", historisch belegbare Zeitrahmen vor allem bei Burggrabungen oder Vergleichsangaben mit Hinweisen auf ähnliche, besser datierte Funde.

Ein Großteil der Messer war, zumeist aufgrund keramischer Beifunde, zeitlich im Rahmen eines oder zweier Jahrhunderte eingeordnet worden.

Diesem Umstand mußte bei der statistischen Auswertung Rechnung getragen werden. Deshalb wurden für jede Fragestellung zwei Auswertungen vorgenommen:

Die erste enthält Messer mit Datierungsansätzen wie "13. Jahrhundert", "um 1320", "zweites Drittel 13. Jahrhundert", die zweite umfaßt alle Messer, die in einen zeitlichen Rahmen von 2 Jahrhunderten fielen, also zusätzlich zu erstgenannten zum Beispiel auch Messer des 13. bis 14. Jahrhunderts.

Im Vergleich beider Auswertungsdiagramme zeigte sich, daß fast ausnahmslos die Tendenzen der enger gefaßten Auswertung durch die zweite bestätigt wurden.

Mit diesem Verfahren wurde ein weiteres Problem, welches sich aus den oft willkürlich anmutenden Auswahlverfahren der einzelnen Autoren ergab, vermindert:

Bezogen auf die absoluten Zahlen war oft zu beobachten, daß sich einzelne, enger datierte Fundbestände aus befund- und ergebnisreichen Grabungen unverhältnismäßig stark gegenüber ähnlich genau datierten älteren oder jüngeren Einzelfunden bemerkbar machten. Durch die Hinzunahme der in der Regel umfangreicheren Bestände mit weiter gefaßten Datierungsansätzen konnte das Verhältnis in der Regel ausgeglichen werden.

Auf die Verteilungstendenzen der abgefragten Kriterien hatte dieser Umstand keine Auswirkungen.

In den folgenden Diagrammen wird die zeitliche Verteilung aller für die statistische Auswertung benutzten Messer in den einzelnen Ländern vorgestellt.

Sie zeigen neben den Schwerpunkten der jeweiligen nationalen Forschungen auch die Unterschiede bei den beiden Auswertungsgruppen der auf ein bzw. auf zwei Jahrhunderte datierten Funde. Bezogen auf Messer als Fundobjekte verdeutlichen sie besser als jede beschreibende Quellenkritik die Defizite, die sich aufgrund unterschiedlicher Forschungsansätze, uneinheitlicher Grabungspraxis und selektiver Auswahl bei der Publikation in einem Zeitraum von fast 60 Jahren archäologischer Forschung im nördlichen Teil Europas ergeben haben. 
Gleichzeitig zeigen sie aber auch, welche Mengen eines einzigen Fundgegenstandes der weiteren Forschung bereits zur Verfügung stehen, vor allem unter Berücksichtigung der Tatsache, daß das hier vorgestellte Material lediglich einen Ausschnitt von weit weniger als $30 \%$ des tatsächlich vorhandenen Bestandes an ausgegrabenen Messern darstellt ${ }^{9}$.
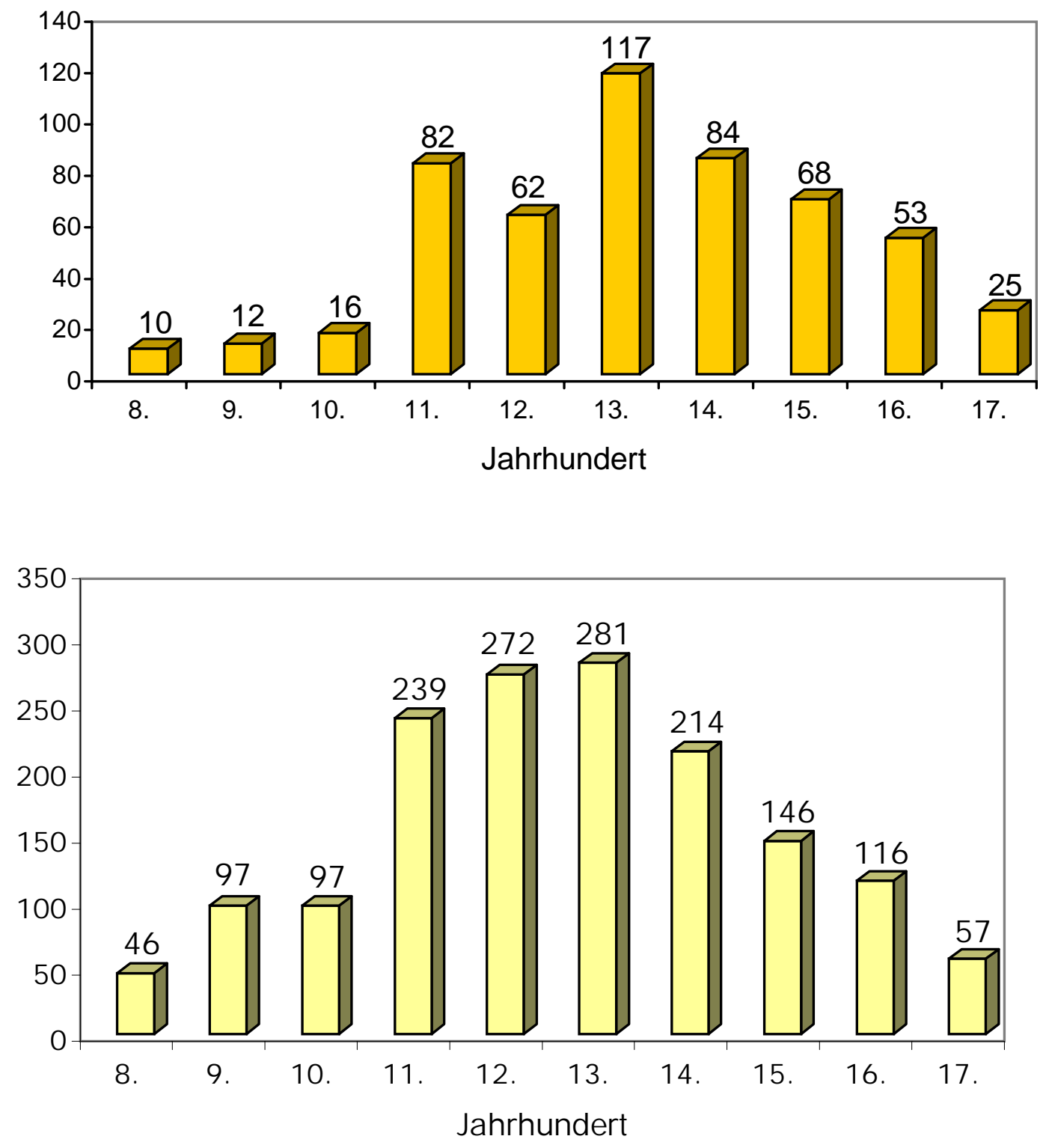

Abb. 2 Die zeitliche Verteilung der ausgewerteten Messer im gesamten Untersuchungsraum: oben: enger datierter Bestand; unten: grob datierter Bestand

\footnotetext{
9 Eine Einschätzung von ca. 10 bis 15\% ergab sich aus dem Vergleich mit den Beständen der Stadtarchäologien in Lund, Lübeck, Amsterdam, Duisburg und Höxter, des Museums Boymans-van Beuningen in Rotterdam, den publizierten Londoner Messern und den Angaben, die Prof. Dr. L. Leciejewicz dem Autor über polnische Fundbestände machte.
} 

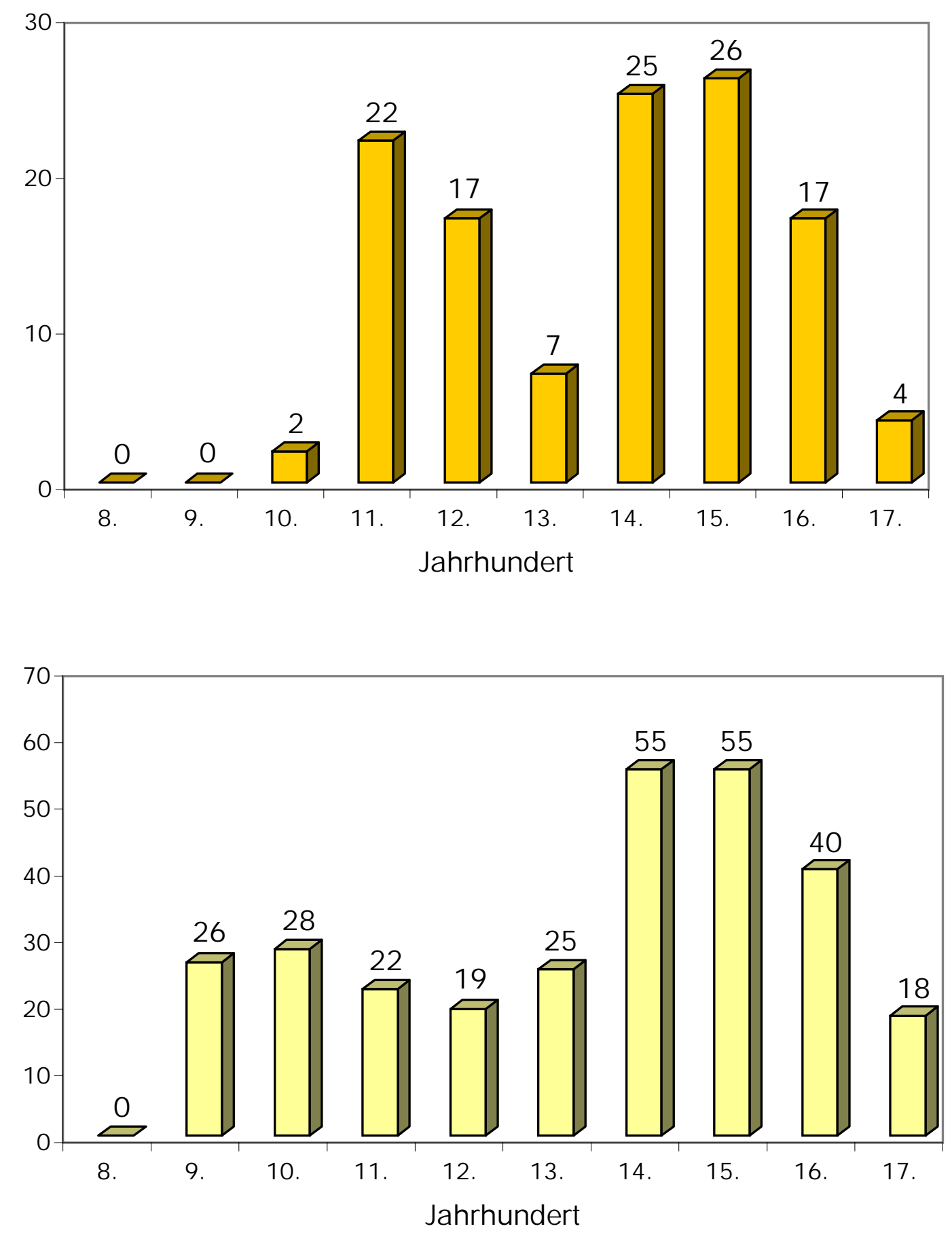

$A b b .3$ Die zeitliche Verteilung der ausgewerteten Messer in Skandinavien: oben: enger datierter Bestand; unten: grob datierter Bestand 

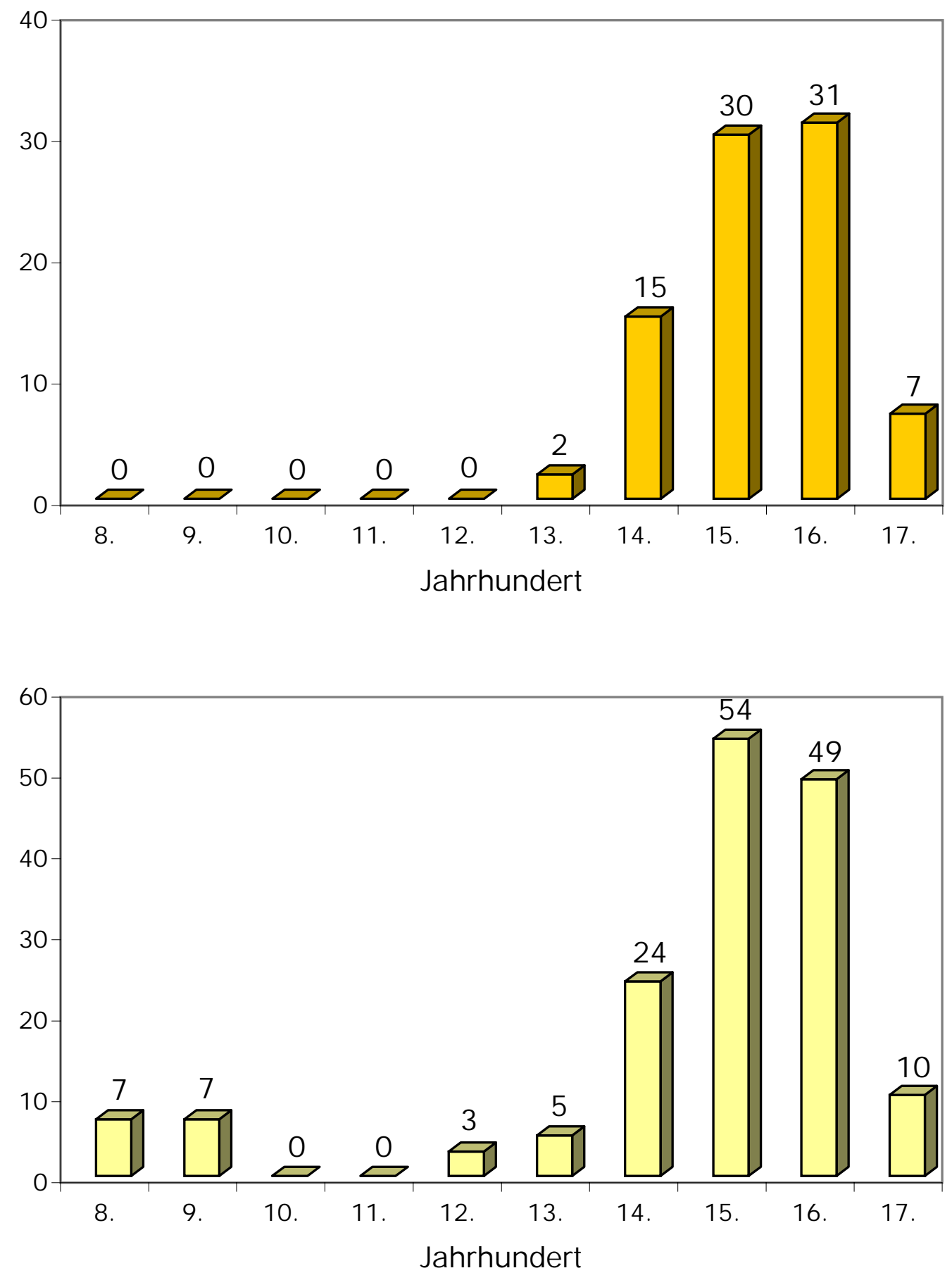

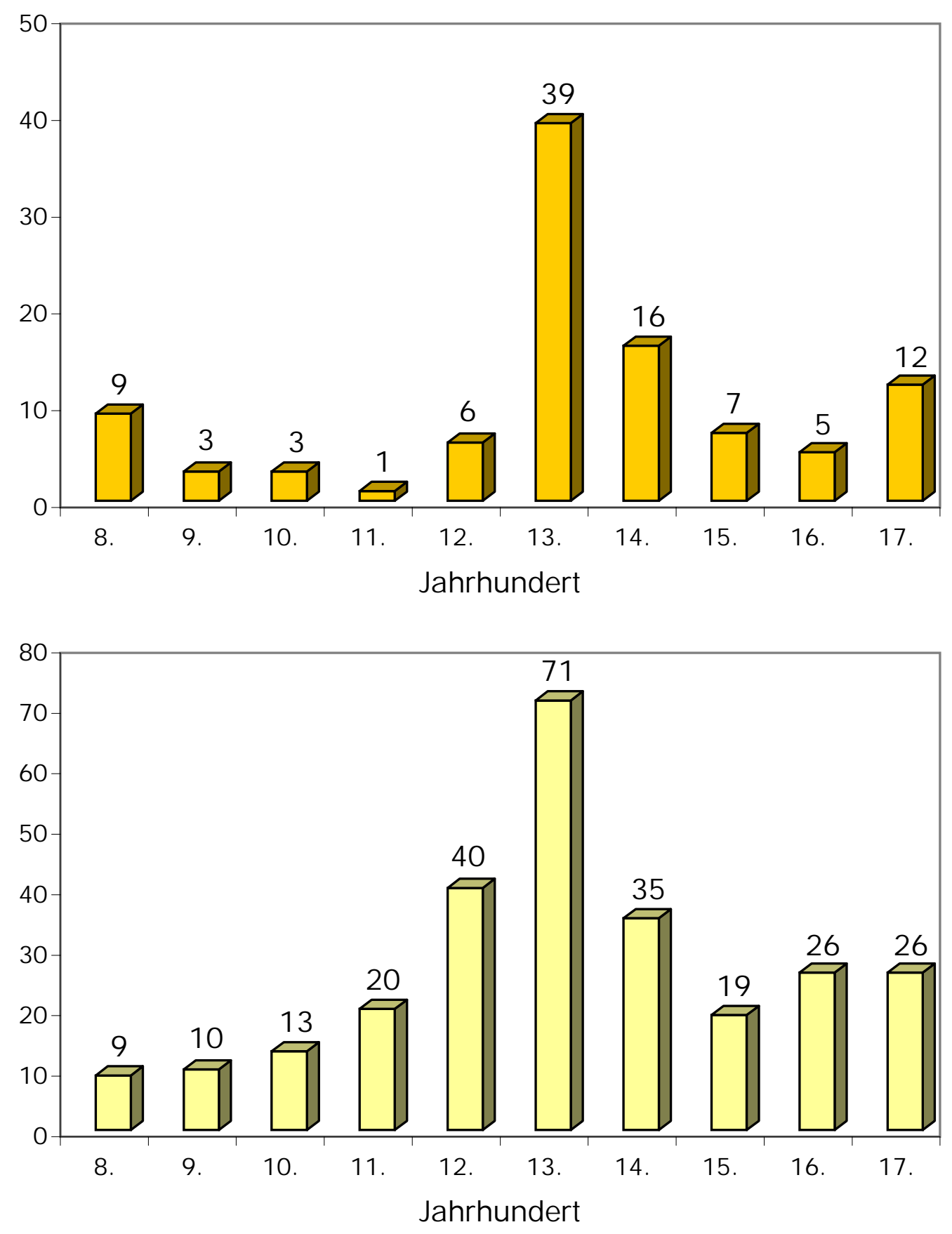

Abb. 5 Die zeitliche Verteilung der ausgewerteten Messer in Nordwestdeutschland: oben: enger datierter Bestand; unten: grob datierter Bestand 

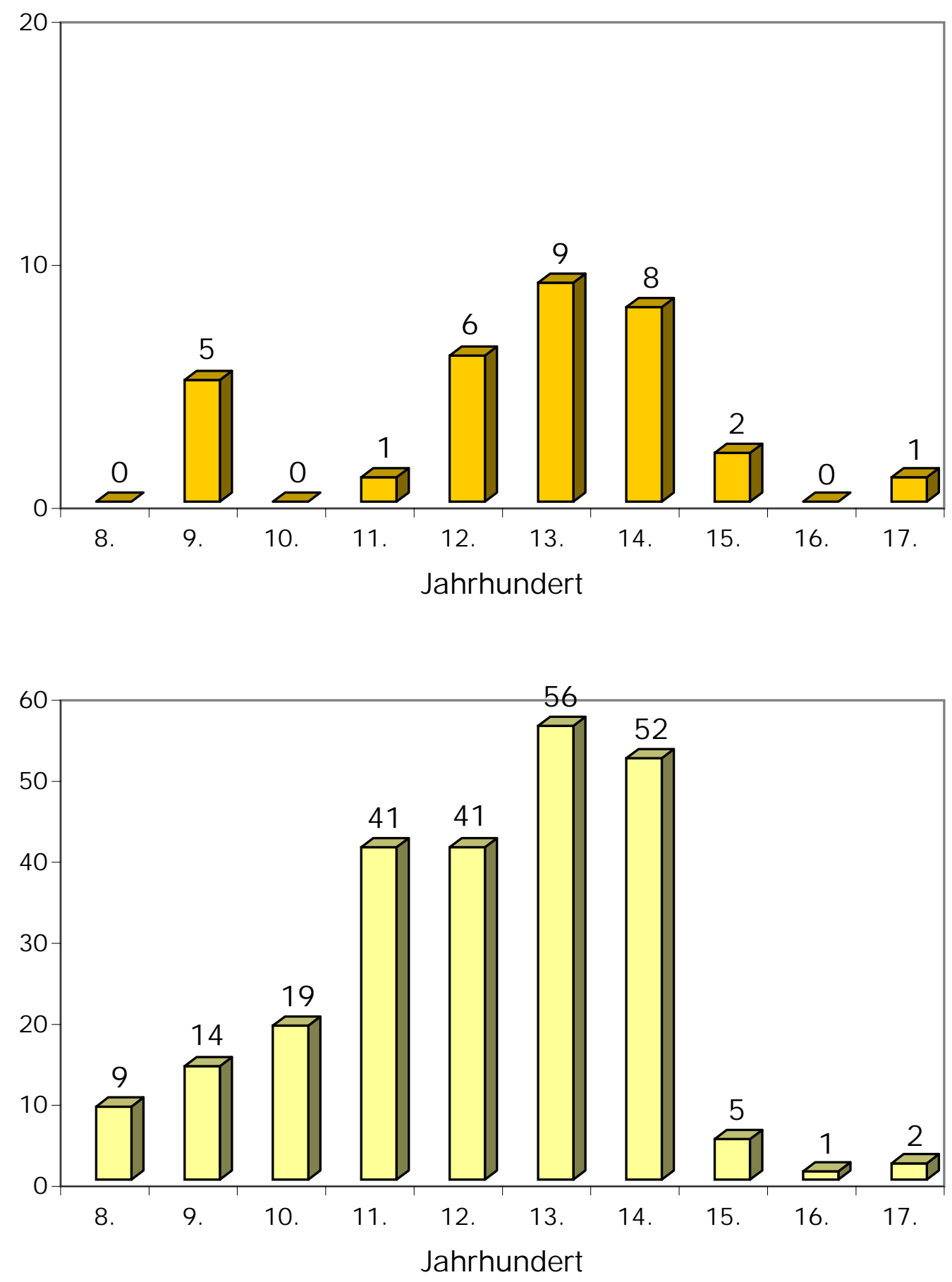

Abb. 6 Die zeitliche Verteilung der ausgewerteten Messer in Ostdeutschland: oben: enger datierter Bestand; unten: grob datierter Bestand 

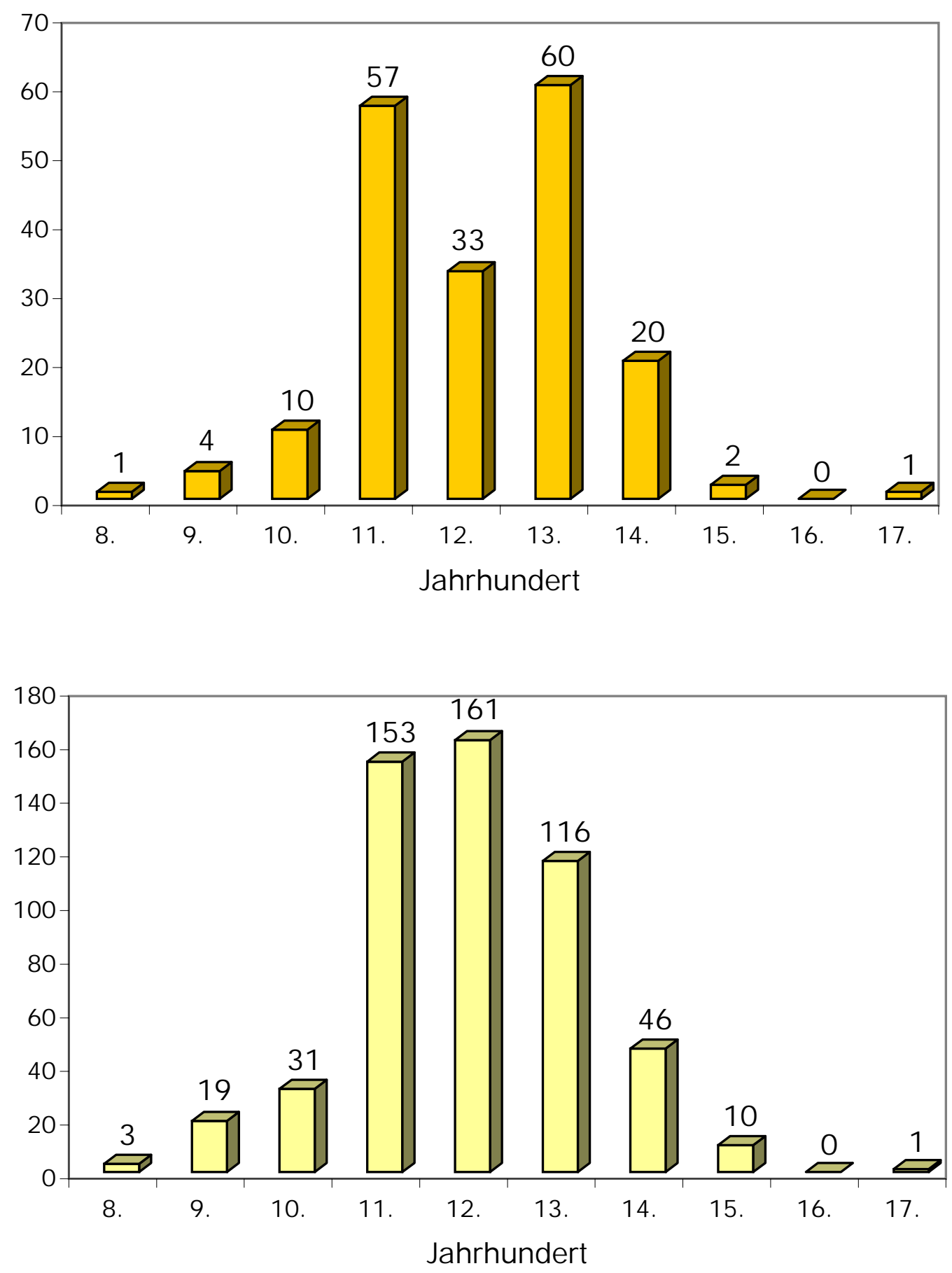

Abb. 7 Die zeitliche Verteilung der ausgewerteten Messer in Polen und Litauen: oben: enger datierter Bestand; unten: grob datierter Bestand 

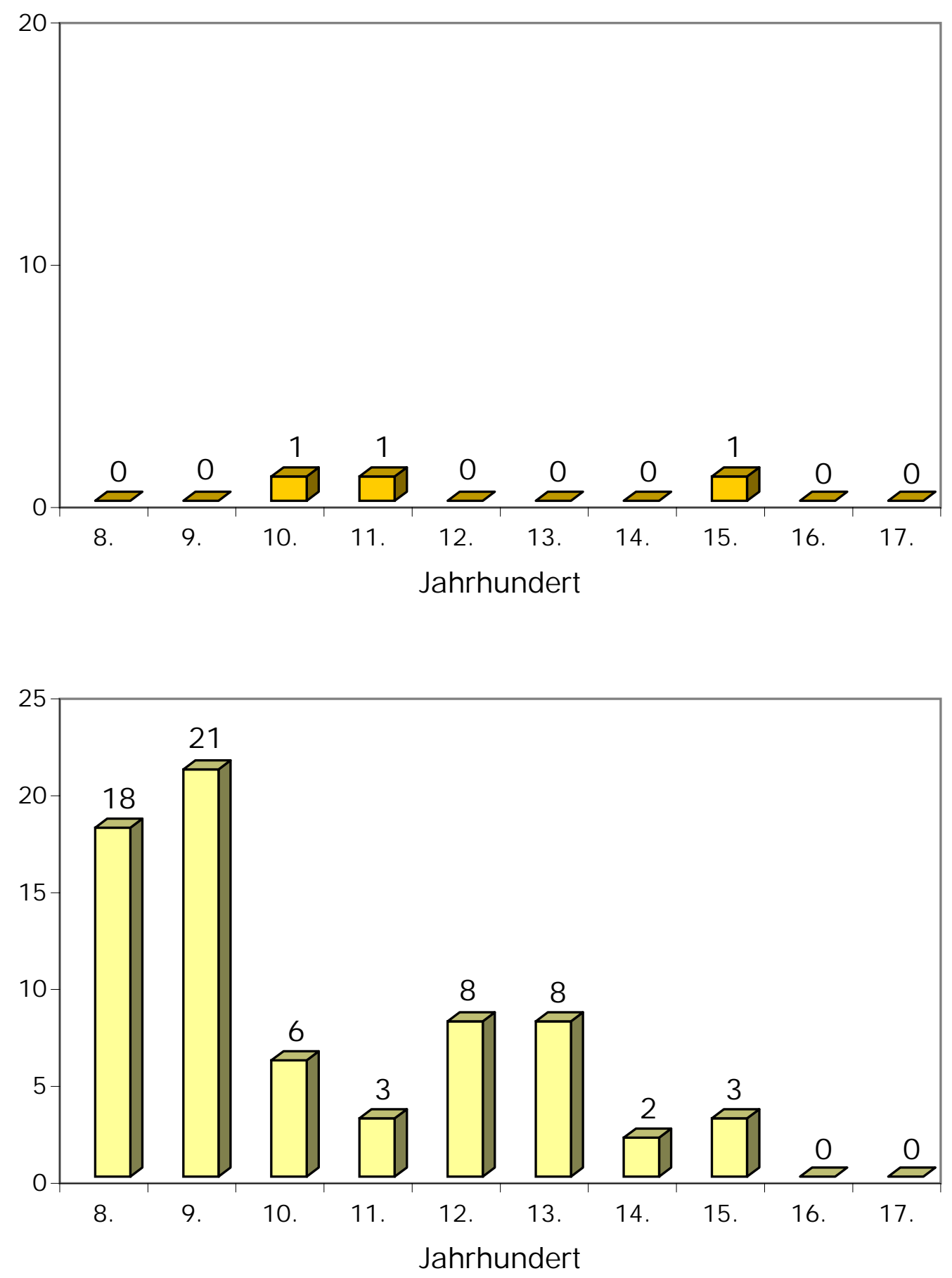

Abb. 8 Die zeitliche Verteilung der ausgewerteten Messer in der ehemaligen Sowjetunion: oben: enger datierter Bestand; unten: grob datierter Bestand 
In der alten Bundesrepublik liegt die Masse der Funde im 12., 13. und 14. Jahrhundert, vorher und nachher verteilen sie sich jeweils in etwa proportional, wobei die Funde aus der Büraburg bei Fritzlar (8. Jahrhundert) und die des Hamburger Elbwracks (um 1600) das Gesamtbild beeinflussen.

Bei den Messern aus Fundorten der ehemaligen DDR liegt der Schwerpunkt ebenfalls im 13. und 14. Jahrhundert, zum 12. tritt hier gleichstark noch das 11. Jahrhundert hinzu. Der Zeitraum vom 8. bis 10. Jahrhundert ist ähnlich vertreten. Im Gegensatz zur Bundesrepublik fehlt das 15. bis 17. Jahrhundert fast ganz, da die Forschungsschwerpunkte hier wie auch in Polen, im Baltikum und im nordwestrussichen Bearbeitungsraum diese Zeit archäologisch nur noch am Rande berücksichtigten.

Die Messer der übrigen ehemaligen sowjetischen Fundorte sind in ihrer geringen Zahl für eine Aussage nicht mehr geeignet, es fehlt aber auch hier das 16. und 17. Jahrhundert.

Ein völlig anderes Bild ergibt sich bei den beiden verbleibenden Regionen:

In den Niederlanden und in Skandinavien liegen die Schwerpunkte im 14. bis 17. Jahrhundert. Während in den Niederlanden aber die Zeit vor dem 14. Jahrhundert bis auf Dorestad nur sporadisch vertreten ist, kann sie in Skandinavien mit vor allem im 11. und 12. Jahrhundert enger datierten Funden besser belegt werden.

Für den gesamten Untersuchungsraum wurden von den 1.319 erfaßten Messern 1.070 ausgewertet, die Gruppe der auf ein Jahrhundert datierten Bestände umfaßt 530 Messer. Die übrigen waren zeitlich zu ungenau eingeordnet worden.

\subsubsection{Erhaltungszustand und Rekonstruktionsmöglichkei- ten}

Ein weiterer wesentlicher Faktor der Quellenkritik besteht in der geringen Anzahl vollständig erhaltener Messer.

Um das Material in eine kulturräumliche Gliederung oder in wirtschafts- und sozialgeschichtliche Zusammenhänge einbinden zu können, hätte es einer zeitlich und fundortspezifisch differenzierter angelegten Basis bedurft, wenigstens aber vergleichbarer Erhaltungszustände innerhalb der 1.300 im Katalog erfaßten Messer.

Erhalten sind aber bei den meisten Messern lediglich eine oft verschliffene Klinge und Reste der Griffkonstruktion.

Die Klinge eines Messers ist fast nie so erhalten, wie sie am Ende ihrer Fertigung ausgesehen hat, in nicht wenigen Fällen nicht einmal so, wie sie am Ende ihrer primären oder sekundären Funktionen durch Verlust oder als Abfall in die Erde gekommen ist. Unter bestimmten Voraussetzungen erschien es aber möglich, eine verschliffene und durch Korrosion zusätzlich zerfressene Klinge zu rekonstruieren. 
Der Verschliff einer Klinge betrifft in der Regel die Schneide. Das hängt neben der Funktion eines Messers auch von der Herstellungstechnik der Klinge ab. Die Schneidenpartie einer Klinge ist oft aus weicherem schleifbarem Eisen hergestellt worden als die sprödere Rükkenpartie ${ }^{10}$. Wie auch immer das Messer produziert worden ist, das wesentlichste Merkmal eines Verschliffs ist der Verlust an Länge der Klinge. Die Breite einer Klinge ist durch das Heft, den Absatzbereich von Griff und Klinge, festgelegt; der Heftbereich wird während der verschiedenen Verschliffstadien aber nur selten so in Mitleidenschaft gezogen, daß der Absatz zur Griffunterkante mit angeschliffen wird. Die Klingenoberkante und der Absatz zur Griffoberkante sind vom Verschliff einer Klinge so weit ausgeschlossen, wie die Klingenlänge insgesamt berührt ist. Es gibt demnach zwei feststehende Faktoren, die von einem Verschliff an der Klinge unberührt bleiben: die Höhe des Absatzes von der Griffoberkante zur Klingenoberkante und der Richtungsverlauf der Klingenoberkante (Abb. 9a).

Deutlich wird dies anhand von Beispielen stark verschliffener Messer aus Novgorod und Birka (Abb. 9b); selbst bei Messern, deren Klingen nur noch in Absätzen vorhanden sind, ist die obere Absatzkante unberührt geblieben. Fast immer ist auch der Richtungsverlauf der Klingenoberkante noch zu erkennen.

Um eine vertretbare Rekonstruktion zu erreichen, stellt sich allerdings die Frage nach der unteren Partie der Klinge. Diese ist bei starkem Verschliff nur dann noch zu rekonstruieren, wenn auch die untere Absatzkante am Heft unberührt geblieben ist.
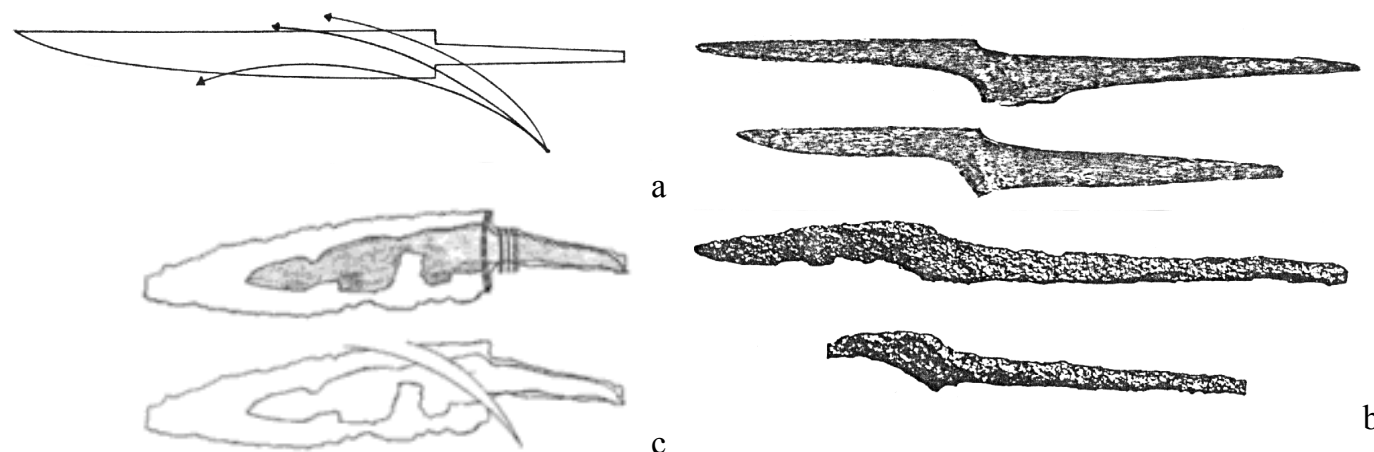

Abb. $9 a-c$

Verschliffstadien $(a-b)$ und Rekonstruktionsmöglichkeiten (c)

(Beispiele aus Arzichowskij/Koltschin (II) 1959, Abb. 41.2 und 3, aus Arbmann

1940, Tafel 182.9 und 12 sowie Kat. Nr. 161 un 162, BRD)

Da dieses jedoch nicht immer der Fall war, wurde versucht, die Relationen der Höhe der unteren und oberen Absatzkanten am Heft bezüglich der Größe, vor allem der Klingenlängen, festzustellen. Anhand zweier Beispiele von der Burg Altena und der Rücklenburg sei dies kurz erläutert.

Beide Messer gehören dem gleichen Typ an: der Griff ist plättchenversehen, die Klinge ist von gleicher Form und steht griffunterständig. Die Form ist unter ande- 
rem bekannt aus dem Kloster Tom Roden, aus Duisburg, von der Isenburg und der Raffenburg. Der wichtigste Unterschied zwischen den beiden Messern ist ihre Größe. Es zeigt sich deutlich, daß die Absatzkanten - korrespondierend zur Klingenlänge - bei dem Altenaer Messer niedriger sind als bei dem Exemplar von der Rücklenburg. Es scheint daher bedingt möglich zu sein, von der erhaltenen oberen Absatzkante am Heft auf die untere Kante - und damit auch bedingt auf die Klingenlänge schließen zu können, da der Richtungsverlauf einer Kante (der Klingenoberkante) ja bekannt ist (Abb. 9c).

Dieser Rekonstruktionsversuch ist in der Regel nur dann möglich, wenn der Grundtyp des verschliffenen Messers bekannt ist; in anderen Fällen muß eine derartige Rekonstruktion von Klingenform und -länge Hypothese bleiben.

Grundsätzlich ist eine Rekonstruktion je eher möglich, desto besser der zu rekonstruierende Teil erhalten ist. Daher wurde versucht, verschiedene Verschliffstadien und Erhaltungsmerkmale zu definieren. Unterschieden wurde nach folgenden Fragen:

1. Sind die Grundform und Länge der Klinge noch erhalten?

2. Sind die Grundform und Länge der Klinge noch zu erkennen?

3. Ist die Grundform der Klinge noch zu erkennen?

4. Ist die Klinge zum größten Teil vergangen?

Aus den Antworten auf diese vier Fragen ergaben sich vier Beurteilungskriterien und vier Verschliffstadien:

1. Die Schneide ist nur angeschliffen, der größte Teil der Klinge ist erhalten.

2. Die Schneide ist verschliffen, der Verlauf des Rückens und die Spitze sind noch erhalten.

3. Die Klinge ist stark verschliffen, erhalten ist nur noch der größte Teil des Rückenverlaufs.

4. Die Klinge ist bis auf einen kleinen Teil völlig verschliffen.

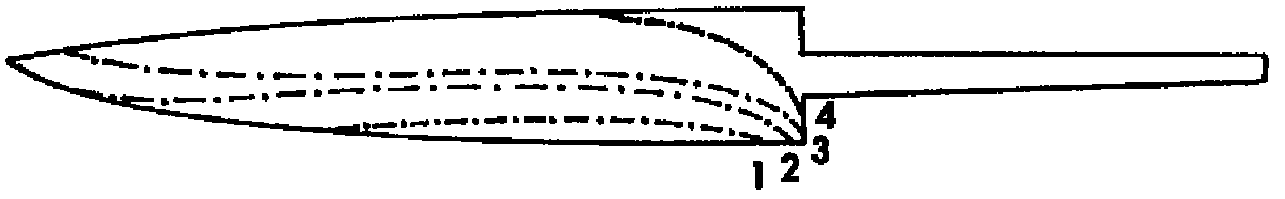

Dazu kommen Korrosionsschäden wie Ausbrüche in Rücken und Schneiden, abgebrochene Spitzen u.ä.

Für die Ansprache der Klingenform und die daraus resultierende Kennzeichnung über die Terminologie des Klassifizierungsmodells (Kodierung) wurden Messerklingen der Verschliffstadien 1 und 2 regelmäßig rekonstruiert, Klingen des Stadiums 3 nur in Kenntnis des Grundtyps, der sich vor allem bei umfangreicheren Beständen oft aus besser erhaltenen Belegen vom selben Fundort ergab. Klingen des Verschliffstadiums 4 wurden nicht rekonstruiert: 


\subsubsection{Kriterien der statistischen Auswertung}

Erkenntnisse über die Produktionstechniken und Metallzusammensetzungen sind zwar vorhanden, sie beschränken sich aber auf Bestände weniger Fundorte.

Vergleiche zwischen Messern verschiedener sozialer Herkunft sind ebenfalls kaum möglich, da für die betreffenden Fundortarten keine ausreichend vergleichbaren Mengen zur Verfügung standen. Nur in Einzelfällen konnten zeitgleiche Bestände aus verschiedenen sozialen Umfeldern miteinander verglichen werden ${ }^{11}$.

Die statistische Auswertung des im Katalog erfaßten Materials fußt auf derartigen Überlegungen. Sie bezieht sich in erster Linie auf Daten, die den Untersuchungsgegenstand selbst betreffen, seine Form, Konstruktion und Materialien, und berücksichtigt nur in seltenen Fällen sein gesellschaftliches Umfeld.

Für weitergehende Fragen ist es erforderlich, zeitlich wie räumlich repräsentative Mengen an Fundmaterial aufzuarbeiten, um vergleichbare Bestände zu erhalten.

Das in diesem Kapitel zusammengefaßte Material ist keine Bestandsaufnahme, sondern ein Ausschnitt, dem neben der zukünftigen Erfassung weiterer Quellengruppen die Aufarbeitung und Publizierung bereits untersuchter Produktionsstätten und der zahlreichen in Magazinen verwahrten Fundbestände folgen muß ${ }^{12}$.

\subsubsection{Abschließende Anmerkungen zur Quellenkritik und zum Forschungsstand}

Die bisher angesprochenen quellenkritischen Aspekte zur Materialbasis der vorliegenden Untersuchung korrespondieren mit einer von W. Janssen getroffenen Feststellung, die er 1986 im Zusammenhang mit der Erforschung des städtischen Handwerks nach einer deutlich formulierten Kritik am allgemeinen Zustand der deutschen Stadtkernarchäologie traf:

"Wir subsummieren diese ausschnitthaften Erkenntnismöglichkeiten der Archäologie des Mittelalters ... unter dem Sammelbegriff der erhaltungsbedingten ... und der publikationsbedingten Ausschnitthaftigkeit der archäologischen Quellen"13.

Eine über weite Strecken auf Literatur angewiesene, regional und zeitlich übergreifende Untersuchung wie die vorliegende kann die Defizite, die sie vorfindet, nicht ausgleichen. Sie kann lediglich auf sie hinweisen und einige spezifische Möglichkeiten zu ihrer Beseitigung formulieren.

\footnotetext{
$11 \quad$ Vgl. Kapitel 6, Abschnitt 2.7

12 Bereits an dieser Stelle sei auf eine 1987 erschienene Monographie über Messer und Messerscheiden aus der Londoner Innenstadt verwiesen: J. Cowgill u.a., Knives and scabbards, Medieval Finds from Excavations in London 1, London 1987

13 In leichter formaler Abänderung übernommen aus dem 1986 erschienenen Sammelband "Zur Lebensweise in der Stadt um 1200": W. Janssen 1986, S. 307-308
} 
Die Bearbeitung der vor allem durch die zahlreichen Stadtkerngrabungen erbrachten Fundbestände, die zum größten Teil noch unpubliziert in den Magazinen liegen, kann nur vor Ort durchgeführt werden. Dementsprechend sind die zu dieser Untersuchung mitberücksichtigten bisher unpublizierten Messer vor allem unter dem Aspekt aufgenommen worden, nach Durchsicht der Literatur erkennbare Lücken aufzufüllen oder zu bestimmten Fragestellungen repräsentative Mengen von Belegen über den bisher publizierten Bestand hinaus zu erhalten.

Der Entschluß, die Untersuchung zu mittelalterlichen und frühneuzeitlichen Messern überregional anzulegen, entsprang neben den im Vorwort genannten Anstößen auch der Tatsache, daß dieser Gegenstand von archäologischer Seite bisher wenig Beachtung gefunden hat.

Mittelalterliche und frühneuzeitliche Messer nehmen im Fundmaterial, gemessen an der Zahl der Gefäßkeramik oder anderer eiserner Objekte wie Nägel und Beschlagfragmente zwar einen weitaus geringeren Teil ein, liegen aber mittlerweile sicher in mehreren zehntausend Exemplaren in den Magazinen der europäischen Stadtkernarchäologien vor.

Im Gegensatz zu Gürtelschnallen, Riemenzungen, Beleuchtungsgeräten, Dreifußtöpfen (Grapen) oder anderen Alltagsgütern, deren Formenspektrum weniger umfangreich und deren Entwicklungsstufen im wesentlichen bekannt sind, liegen zeit-und raumübergreifende Studien zur Vielfalt von Messerformen, Funktionen und Herstellungstechniken bisher noch nicht vor.

Dazu beigetragen haben nicht nur die bisher beschriebenen quellenimmanenten Probleme, die eine Beschäftigung mit Messern mit sich bringt.

Die breite Spanne von Klingenformen, für deren gesicherte Ansprache bis heute jede Basis fehlt, läßt sich in regional begrenzten Ausschnitten nicht näher untersuchen. Deutlich zeigen dies Arbeiten wie die von Baart (u.a.) und Cowgill (u.a.) zu den in Amsterdam und London ausgegrabenen Messern ${ }^{14}$.

Kaum ein Gegenstand erscheint derart verschwommen im chronologischen Raster der Mittelalter- und Neuzeitarchäologie wie das Messer. Waffen, Schmuck und anderes persönliches Zubehör, selbst Hufeisen und Zaumzeug erfreuten sich sowohl in der Kunstgeschichte wie in der Archäologie größerer Beliebtheit, so daß sie in Fundkatalogen seit langem veröffentlicht und bearbeitet wurden. Messer wurden zumeist erst dann interessant, wenn ihre Griffe erhalten und verziert waren ${ }^{15}$.

Dieses ist um so erstaunlicher, wenn man sich die Bedeutung des Messers im alltäglichen mittelalterlichen Leben vor Augen führt. Das Messer gehörte während des gesamten Untersuchungszeitraumes und darüber hinaus zum Minimum persönlicher Ausrüstung (Benediktsregel, Grabausstattungen u.a.) beider Geschlechter. Sein Auftreten im Fundgut ist relativ häufig, seine Funktionen im $14 \quad$ Baart (u.a.) 1977

Cowgill (u.a.) 1987

15 Vgl. beispielhaft Billberg 1982, S. 32-34

Knorr 1971, S. 121-145

Leciejewicz 1974 
Auftreten im Fundgut ist relativ häufig, seine Funktionen im Alltag waren elementar und vielseitig. Seine Bedeutung erhellt nicht zuletzt durch den Umstand, daß es auf vielen Bildzeugnissen von der Karolingerzeit bis zur Malerei des 17. Jahrhunderts in seiner Anwendung und als Staffage festgehalten wurde.

Für diese Diskrepanz gibt es fund- wie gattungsspezifische Gründe. Der Erhaltungszustand der Messer vor allem des Mittelalters ist gegenüber dem von z.B. Waffen aus ersichtlichen Gründen erheblich schlechter: letztere waren ungleich höherer Belastung unterworfen, in der Regel aus besserem Stahl gefertigt und erhielten sich auch in feuchten Böden besser. Bei Messern war oft bestenfalls die Schneide gestählt, sie wurden im täglichen Gebrauch häufig benutzt, bis die Schneide verschliffen war. Dementsprechend sind viele geborgene Messer an der Schneide zerfressen, oft ist nur noch der Klingenansatz und ein Teil des Griffs vorhanden. Dazu kommt der Umstand, daß sich die Variationsbreite der Klingen aufgrund fehlender Informationen zur Funktionsspanne kaum erschließt. Lediglich Spezialmesser wie Werkzeugmesser von Sattlern und Fleischern, Rebmesser, Vorlegemesser bei Tisch oder Schnitzmesser fielen wegen ihrer Formen sofort auf und konnten teilweise klassifiziert und zugeordnet werden ${ }^{16}$. Diese Unzulänglichkeit des Materials mag einer der Gründe dafür sein, daß in den meisten publizierten Fundkatalogen die Forscher den Messern nur wenige lapidare Sätze widmen ${ }^{17}$. Die wenigen Arbeiten, die bereits zu Problemen der Einordnung ergrabener Messer Stellung nehmen, sind weder auf alle Messer anwendbar, noch reichen sie aus, aus den Fundkomplexen oder Sonderformen, für die sie erarbeitet wurden, weitergehende Analysen bezüglich ihrer Funktion oder ihres chronologischen Stellenwertes vorzunehmen $^{18}$.

Aus diesen Gründen ergab sich der Wunsch, Messer, in ihrer Vielfalt zu erfassen und zu untergliedern, um einen Einstieg in die Erforschung dieses für den Menschen zum ständigen Begleiter gewordenen Instrumentes zu ermöglichen.

16 Bereits 1959 stellte B.A. Koltschin neben einer Auswahl der in Novgorod geborgenen Messer auch Untersuchungen zur Funktion und Technologie der Messer an und unterschied "Universalmesser des bäuerlichen Haushalts" von "Messern zur Holzbearbeitung" sowie von "Schustermessern" und "Chirurgenmessern". Auch stellte er die typische Abfolge verschiedener Verschliffstadien dar: Koltschin 1959, v.a. S. 52-58 und Abb. 41-43

17 Eine Zusammenfassung in englischer Sprache liegt vor: Thompson 1967, S. 73-78, v.a. Abb. 74

3 Beispiele mögen hier stellvertretend einen Eindruck von der Art der Behandlung geben, die die Messer seitens der Forschung sehr oft erfahren,

1: "Diese Messerform kommt als einfaches Hausgerät während des gesamten Mittelalters vor und läßt keine differenziertere zeitliche Einordnung zu."

in: E. Plümer, Die Wüstung Oldendorp bei Einbeck, Einbeck 1978, S. 211

2: "... die Formen sind seit spätmerowingisch-karolingischer Zeit geläufig und leben bis in das hohe Mittelalter fort."

in: W. Janssen, M. Müller-Wille, Das Fundmaterial der Grabungen aus der Niederungsburg bei Haus Meer, in: Rheinische Ausgrabungen I, Köln 1968, S. 74 (Zitat dort nach L. Berger, Die Ausgrabungen am Petersberg in Basel, Basel 1963, S. $60 \mathrm{ff}$ )

3: "Für die Datierung sind die Messer nicht geeignet, da sie sich in fast unveränderter Form über Jahrhunderte gehalten haben und die einzelnen Formen lange nebeneinander vorkommen."

18 in: W. Timpel, Gomm Vgl. u.a. Knorr 1971

Schoknecht 1969 Drescher 1975 
Die in der statistischen Auswertung und im beschreibenden Teil vorgestellten und diskutierten Ergebnisse ließen sich nur teilweise in Verbindung mit Forschungsergebnissen historischer Nachbardisziplinen diskutieren, wie etwa das Aufkommen von Klingenmarken, der Beginn der serienmäßigen Verarbeitung von Buntmetallen am Griff, einzelne Verzierungsarten oder herausragende Befunde wie das Wittenberger Schiffswrack, dessen Messerladung im Zusammenhang mit wirtschaftshistorischen Fragestellungen diskutiert wird ${ }^{19}$.

Zahlreiche Fragestellungen, die sich aus den in der Auswertung ermittelten Daten ergaben, konnten jedoch nicht näher historisch eingebunden werden.

Das betrifft zunächst Ergebnisse, die sich am Objekt selbst manifestieren, wie Veränderungen der Klingenform oder der Klingenlänge. Letztere weisen wie auch einige Beobachtungen zur Entwicklung der Grifformen auf Forschungslücken, auf die jeweils an geeigneter Stelle hingewiesen wird.

In der Regel ergab sich die Unmöglichkeit nachweisbarer historischer Einbindungen vieler Aspekte und Daten wie in der Analyse fundortspezifischer Unterschiede von Messerklingen aus den fehlenden Begleitinformationen zum ausgewerteten Fundgut. Wesentliche Daten zur sozial- und wirtschaftshistorischen Umgebung, in der die Messer angetroffen worden waren, standen dem Autor nicht zur Verfügung, weil sie entweder nicht beobachtet oder nicht angegeben worden waren.

So konnten soziale Analysen, Fragen zu Messern als Ware in regionalem und überregionalem Handel und die Bandbreite der Funktionen vom Mehrzweckmesser bis zum Vorschneidemesser nur beispielhaft behandelt werden, wobei in allen Fällen Quellen und Erkenntnisse aus Nachbardisziplinen mitberücksichtigt wurden.

Eine schwerwiegende Forschungslücke ergab sich nach Durchsicht der Beiträge zu Produktionstechniken von Messerklingen.

Die publizierten Ergebnisse ${ }^{20}$ zeigen, welche Erkenntnismöglichkeiten in einer seriellen metallurgischen Objektanalyse verborgen liegen. Aussagen zur Technikund Wirtschaftsgeschichte (unter anderem aus Danzig und Novgorod), die teilweise Rückschlüsse auf wirtschaftsplitische und versorgungstechnische Mechanismen vor dem Einsetzen ausreichend vorhandener historischer Quellen zuließen, wurden beispielhaft zum Zweck der Verdeutlichung dieser Möglichkeiten vorgestellt.

Metallurgische Analysen gehören aber nach wie vor $\mathrm{zu}$ den Ausnahmeerscheinungen, so daß auf eine vergleichende Untersuchung zur Verbreitung der verschiedenen Produktionstechniken verzichtet werden mußte.

19 Vgl. Kapitel 6, Abschnitt 2.6 und Kapitel 9, Abschnitt 1.3
20 Vgl. Kapitel 9, Abschnitt 1 


\section{Das Klassifizierungsmodell}

Um die umfangreichen Fundmengen, die vor allem durch die europäischen Stadtkerngrabungen der letzten 40 Jahre geborgen worden sind, einer weitergehenden wissenschaftlichen Bearbeitung zuzuführen, wurden von den Trägern größerer Institutionen ähnlich den Umstrukturierungen in Museumsmagazinen und Archiven datenverarbeitungsgerechte Verschlüsselungen entwickelt, um die Aufnahme und Magazinierung von Funden zu beschleunigen, Kapazitäten zu erhöhen und einen sicheren und schnellen Zugriff auf das Material bei der wissenschaftlichen Weiterverarbeitung zu ermöglichen.

Mit dem vorliegenden Modell wird eine Verschlüsselung von Daten vorgestellt, die die Formen und Konstruktionen von Messerklingen und -griffen, die jeweils außer den immer vorhandenen Ausgangswerkstoffen Eisen und Stahl verwendeten zusätzlichen Materialien (Markeneinlagen, Verzierungseinlagen, Werkstoffe der Hilzen oder Griffplatten, der Niete und weiterer Applikationen), die Klingenlängen und zusätzliche Informationen wie Verzierungen und Marken umfassen.

Die bereits erwähnten, nicht erfaßten Daten wie weitere Maße (Stärke des Klingenrückens/-nackens), Breite (Höhe) der Klinge oder Informationen (Werkstoffe der Klinge und ihre Produktionstechnik) können, wenn zukünftige Analysen und Angaben eine ausreichende Datenmenge geliefert haben, problemlos hinzugefügt werden.

Computergestützte Fundkarteien und wissenschaftliche Auswertung sind in größeren Museen und Instituten heute keine Seltenheit mehr. Für die große Zahl kleinerer Institutionen sind sie dagegen nach wie vor oft unrealisierbar oder, da ein allgemein gültiges und benutzbares Verbundnetz mit zentralen Datenbanken fehlt, wenig relevant.

Das im folgenden beschriebene Klassifizierungsmodell ist daher sowohl manuell als auch mit Hilfe der Datenverarbeitung nutzbar.

Es enthält neben dem beschreibenden Teil eine graphische Darstellung der einzelnen, kodierten Klingenformen und Griffkonstruktionen, mit dessen Hilfe fast alle bisher bekannten Messerformen und -arten benannt werden können.

Eine Möglichkeit, auf bereits bestehende, allgemeine funktionale Differenzierungsmöglichkeiten als Kriterien zur Auswahl zurückzugreifen, war nicht vorhanden. So mußte der Rahmen so weit gefaßt werden, daß alle Messer erfaßt wurden, die in jeder erdenklichen Situation zum "Essen", d.h. zur Nahrungsbeschaffung, -zerkleinerung und -aufnahme gedient haben könnten. Das wiederum heißt, daß praktisch alle Messer Aufnahme fanden, bis auf klar zu erkennende Werkzeugmesser wie Sattler- oder Rebmesser. 
Das Modell enthält im ersten Teil die Formen und Arten von Griffkonstruktionen, darunter auch die Möglichkeiten der Angelausrichtung (Griffober- oder unterständigkeit).

Die Summe dieser Formen ergab sich aus den im Katalog erfaßten und darüber hinaus durchgesehenen Messerbeständen sowie aus logischen Erweiterungen von Konstruktionsmöglichkeiten, die sich zum Beispiel aus Erhaltungsgründen nicht belegen ließen.

Grundsätzlich ergaben sich bei der Aufstellung die beiden bekannten Griffbefestigungsarten der Angel und Zunge, die sich nach Abschluß der Materialaufnahme mit ihren Erweiterungen und Übergangsformen herausarbeiten ließen.

Die meist aus organischem Material bestehenden Vollgriffe (Hilzen) oder Griffschalen sind die am besten zu Verzierugszwecken geeigneten Bestandteile des Messers und unterlagen in ihrer Gestaltung auch den jeweils geltenden Kunst- und Moderichtungen. Damit ist der Griff des Messers eine wesentliche Datierungsgrundlage, zum Teil auch geeignet zu Funktionsbestimmungen und zur Einordnung in ein soziales Umfeld. Da sich jedoch nur selten die organischen Bestandteile des Messers erhalten haben, und unter diesen nur ein - wenn auch großer - Teil Verzierungen aufwies, relativiert sich die Aussagekraft der Griffe im archäologischen Bereich erheblich.

Im Gegensatz zum Klassifizierungsmodell der Klingen gab es für das Modell der Griffkonstruktionen bereits wissenschaftliche Erkenntnisse wie die Vorzeitigkeit der Griffangelkonstruktion und das Verbreitungsgebiet der plättchenverzierten Griffe, die sich im groben bestätigen, im Detail differenzieren und erweitern lieBen $^{21}$.

Der zweite Teil des Klassifizierungsmodells enthält die Klingenformen. Diese wurden im Gegensatz zu den Griffkonstruktionen unabhängig vom Fundmaterial unter allgemeinen typologischen und funktionstechnischen Vorgaben entwickelt und in Formengruppen unterteilt.

Schon bei der ersten Beschäftigung mit der Formenvielfalt der Klingen wurde deutlich, daß sich kaum bestimmte Formenentwicklungen chronologisch erfassen ließen oder einzelne Formen begrenzt auf regionale Räume $\mathrm{zu}$ beobachten waren. Es schien dagegen nicht nur auf den ersten Blick, als verstreue sich diese Vielfalt in den geographischen und zeitlichen Untersuchungsräumen.

Ursachen für diese Diffusität konnten aus dem Material selbst nicht ermittelt werden. Eine fiktiv angenommene Ordnung, die dieser in sich stark differenzierten Masse zugrunde liegen mochte, entzog sich auch nach stichprobenartigen Vergleichen mit Bildquellen noch völlig. Eine Gegenüberstellung von archäologischen Funden und zeitgenössischen Darstellungen am Schluß dieser Untersuchung ergab u.a., daß bestimmte Messerformen aus Bildquellen im Fundgut gar nicht, sehr viele

21 Eine einführende Zusammenfassung gibt der Artikel "Messer" in Baart u.a. 1977

S. 325-337. Zu den plättchenverzierten Griffen vgl. Knorr 1971 
Formen ergrabener Bestände ihrerseits auf zeitgenössischen Abbildungen nicht nachzuweisen waren.

Der formalen Diffusität folgt die Unsicherheit bei demVersuch, den einzelnen Messerformen Funktionen zuzuordnen. Diese Versuche geben sich in der Fachliteratur in der Regel als vage und kaum überprüfbare Verallgemeinerungen oder als ausschnitthafte Detailbeschreibungen aufgrund selektiv herangezogener Quellen zu erkennen ${ }^{22}$.

Die beobachtete grundsätzliche Unsicherheit bei der Funktionsbestimmung von Messern führte unter anderem zur Verwendung des Begriffs "Mehrzweckmesser" durch den Verfasser, der als fiktiver Arbeitsbegriff zu verstehen ist.

Er stützte sich auf die Tatsache, daß bis heute viele Messer multifunktional verwendet werden; zahlreiche bildliche Quellen weisen Messer oft als an der Seite getragene Instrumente aus, deren Funktionsbreite vom Willen des Besitzers abhing. Das gilt für Messer aus Gräberfeldern der Völkerwanderungszeit ebenso wie für Jagdbestecke des 17. Jahrhunderts ${ }^{23}$. Es wurde schon während der Materialaufnahme deutlich, daß diese die Hauptmasse des erfaßten Bestandes ausmachten: Messer, die trotz verschiedenster Formen weder einer bestimmten Funktion zuzuordnen waren, noch aufgrund ihrer Form zeitlich enger festgelegt werden konnten. So konnte eine bestimmte Messerform von zwei Menschen durchaus unterschiedlich benutzt werden; genauso konnten umgekehrt verschiedene Formen bei unterschiedlichen Besitzern den gleichen Funktionsanforderungen unterliegen ${ }^{24}$.

Dieser Vielzweckcharakter und seine Abhängigkeit von individuellen Bedürfnissen, die sich oft dem Zugriff wissenschaftlicher Analyse über faßbare Zivilisationsformen und standardisierbare Funktionszuweisungen entziehen, gewannen durch stichprobenhafte Vergleiche mit Bildquellen an Wahrscheinlichkeit. Dies bewog den Verfasser, ein Modell der Klingenformen zu erarbeiten, welches sich vor allem aus der Formenvielfalt und nicht - wie es in der volkskundlichen Sachkulturforschung bei wesentlich besserer Quellenlage möglich ist - aus funktionalen oder wie bei Typologien in der Ur- und Frühgeschichte üblich - aus chronologischen Erkenntnissen ergeben sollte, da erstere die einzig sichere Ausgangsbasis darstellte. Das Modell sollte in Bezug auf Funktionszuweisungen, die sich aus weiterführenden Untersuchungen ergeben können, breit angelegt sein und lediglich den zwei Grundanforderungen eines Messers, dem Stechen und Schneiden genügen, und mindestens alle im Katalog erfaßten Formen beinhalten, also auch die späteren rei-

\footnotetext{
22 Vgl. beispielhaft: Schiedlausky 1956

23 Grundsätzlich gilt für jedes Messer, auch wenn sein Funktionsrahmen - wie z.B. Jagdmesser - eingrenzbar erscheint, daß es für weitere Zwecke benutzt worden sein kann. Zur Frage der Funktionszuweisung vgl. Kapitel 9 
nen Tafelmesser, wie sie aus zahlreichen Sammlungen und bildlichen Darstellungen des 16. und 17. Jahrhunderts bekannt $\operatorname{sind}^{25}$.

Diese allgemeinen Anforderungen bilden den Rahmen der aufgelisteten Klingenformen und begrenzen die Formenbreite des Modells. Die wohl berechtigte Vorgabe, daß jede anzunehmende Variabilität von Klingenformen die Grundfunktionen nicht beeinträchtigen wird, läßt es wahrscheinlich erscheinen, daß vor allem funktionale und herstellungstechnische Gründe als formgebende Faktoren angenommen werden können. Unter dieser Prämisse bleibt der Raum für modische Veränderungen der Klingenformen begrenzt.

Es wurden daher fiktive Grundformen als Ausgangsformen von weiteren Differenzierungen angenommen. Diese Grundformen können daher keinesfalls chronologisch an den Anfang einer Entwicklung gestellt werden, sie dienten lediglich als Leitformen zur Entwicklung des Modells, um unter dem Aspekt der Nahrungsaufnahme das Material zu klassifizieren. Damit war von Anfang an der Versuch, das Fundmaterial inhaltlich zu gliedern, abgekoppelt von der Erstellung des Klassifizierungsmodells der Klingenformen.

Zusätzlich zu diesen Überlegungen erwuchs aus der Beschäftigung mit der Formenund Größenvielfalt ein weiteres Problem. Messern mit verschiedenen Klingenformen, aber fast gleichen Klingenmaßen, die durchaus einer gleichen oder gleichartigen Funktion unterlegen haben mochten, konnten in einigen Fällen am selben Fundort und zeitgleich andere Messer gegenübergestellt werden, die zwar gleiche Klingenformen aufweisen, sich aber in ihren Klingenmaßen erheblich unterscheiden und daher wahrscheinlich verschiedenen Funktionen gedient hatten.

Dieses Phänomen mahnte von Anfang an zur Vorsicht, in wieweit im nachhinein den einzelnen Formen auch differenzierte Funktionen würden zugeordnet werden können.

\subsection{Klassifizierungsmodelle in der Ur- und Frühge- schichte}

Das Aufstellen von Klassifizierungsmodellen ist in der Archäologie eine der wesentlichsten Methoden zur Erfassung des ergrabenen Bestandes an Sachgütern. Da begleitende Quellengruppen wie Bildzeugnisse nur sehr begrenzt vorhanden sind und schriftliche Überlieferungen bis zum Einsetzen der römischen Expansion nach Norden fast völlig fehlen, ist die Forschung angewiesen auf ein möglichst weitgehendes Befragen von Funden und Befunden. Die Klassifizierung des Sachguts ermöglicht Aussagen über Entwicklungsreihen der einzelnen Gerätschaften und

\footnotetext{
25 Vgl. beispielhaft: Schiedlausky 1956

Masterpieces of Cutlery and the Art of Eating, Ausstellungskatalog der gleichnamigen Ausstellung im Victoria and Albert Museum London, London 1979

P.W. Meister (u.a.), Messer, Löffel, Gabel, Museum für Kunsthandwerk Frankfurt, Frankfurt/Main 1965 (u.a. Slg. Bodo Glaub)
} 
Gegenstände. Sie schafft Übersichtlichkeit innerhalb einer Fundgruppe, ermöglicht zusammen mit den Ergebnissen aus Befunden und Fundzusammensetzungen eine klare Differenzierung und Bestimmung der einzelnen Gegenstände und führt über Vergleiche mit anderen Fundgruppen zur Möglichkeit, über die einzelnen Fundgruppen hinaus allgemeiner gefaßte kulturelle Gliederungen vorzunehmen.

Das Aufstellen von Klassifizierungsmodellen erfolgt oft durch ein Wechselspiel deduktiver und induktiver Arbeitsweise. Nicht immer sind Funde aus ihrer Lage heraus sicher zu datieren. So geben die fest datierbaren Exemplare einer Fundgattung ein Gerüst, in das unsichere und stratigraphisch oder aus Befunden nicht näher datierbare Funde eingegliedert werden.

Dabei spielen Überlegungen deduktiver Art eine wichtige Rolle: ausgehend von der Vorgabe, daß in der Entwicklungsgeschichte fast aller Gebrauchsgüter Regelhaftigkeiten zu beobachten sind, denen Menschen bei der Herstellung von Produkten lange unterworfen zu sein schienen, kann ein Gegenstand auf Grund seiner Form unter Umständen auch dann näher chronologisch eingeordnet werden, wenn er nicht aus einem geschlossenen Fundzusammenhang stammt.

Die Entwicklung dieser "Gesetzmäßigkeiten", die auf obiger, zunächst als "Hilfskonstruktion" anzusehender Überlegung beruht, hat richtungsweisend der Schwede Oscar Montelius exemplarisch an der Chronologie der nordischen Bronzezeit vorgenommen und mit typologischen Reihungen, die sich einerseits aus dem Material, andererseits aus bestimmten "Entwicklungsgesetzen" ergaben, zur stratigraphischen Methode eine weitere Datierungsmethode eingeführt ${ }^{26}$.

Diese Gesetzmäßigkeiten enthalten zwei Erkenntnisse, die für die weitere Fragestellung wichtig sind:

Zum einen legte Montelius dar, daß der Beginn einer typologischen Reihe vielfach in Abhängigkeit zur vorangegangenen Entwicklung steht, so zum Beispiel bei bronzenen Gürteldosen, deren älteste Vertreter die hölzernen Vorgänger nachahmen $^{27}$.

Im weiteren Verlauf der Entwicklung eines Typs wurde während der Loslösung aus dieser Abhängigkeit ein langsamer Formenwandel beobachtet, der vom Material abhängig ist und von seiner funktionalen Bestimmung beeinflußt wird, so bei der Entwicklung vom Bronzedolch zum Griffangelschwert ${ }^{28}$. Fremdeinflüsse und neues Dekorempfinden können dem neuen Typ ein spezifisches Gepräge geben.

26 Hoops, Reallexikon (Stichworte Typologie und Chronolgie)

Typologie, Katalog zur Ausstellung im Regionalmuseum Xanten 1977,

Red.: D. Soechting, Xanten 1977, S. 5-13

O. Montelius, Die typologische Methode, Stockholm 1903

H.J. Eggers, Einführung in die Vorgeschichte, München 1959, S. 98-101

G. Tromnau, Oscar Montelius und die typologische Methode, Informationsblatt des Helms-Museums Nr.

22, Hamburgisches Museum für Vor- und Frühgeschichte, Juni 1976

28

H.J. Eggers 1959, S. 99

H.J. Eggers 1959, S. 98 


\subsection{Klassifizierungsmodelle in der Mittelalter- und Neu- zeitarchäologie Fragestellung und Probleme zur Sachgutbearbeitung}

Die Übernahme von Klassifizierungsmodellen für die Erforschung des ergrabenen Sachguts von Kulturträgern, die sich seit dem Ende des 1. Jahrtausends nach Christi Geburt mehr und mehr durch Schrift-, Architektur- und Bildquellen mitteilen, ist zunächst aufgrund der gattungsgleichen Quelle nicht weiter verwunderlich. Doch ergeben sich aus der veränderten Quellensituation erhebliche Konsequenzen, vor allem bezüglich der verbesserten Kontrollmöglichkeiten.

Die ergrabenen Sachgüter galten in der Erforschung des Mittelalters lange Zeit als Zusatzinformation für ein bereits bestehendes Forschungssystem, in dem die Schriftquellen für die Geschichtsforschung, die Zeugnisse der bildenden Kunst für die Kunstgeschichte die Hauptquellengattungen bildeten.

Für die Mittelalter- und Neuzeitarchäologie wurden die schriftlichen und bildlichen Zeugnisse ihrerseits zu wertvollen flankierenden Quellen. Sie treten jedoch erst seit dem ausgehenden Mittelalter in der Anzahl und in der Genauigkeit auf, daß sie Erkenntnisse zum Alltagsleben auch mittlerer und unterer sozialer Schichten in ähnlicher Dichte beitragen können wie die großen Fundmengen der Stadtkerngrabungen, die bereits für das Früh- und Hochmittelalter einsetzen.

Aufgrund des Vorrangs, den die Forschung lange Zeit besonders auffälligen Funden zukommen ließ, lassen viel schlichte, in der Regel völlig unverzierte Alltagsgegenstände zeitlich oft nur sehr ungenau einordnen. Bei der Keramik zum Beispiel ergaben sich derartige Schwierigkeiten bei der Chronologie langlebiger Formen. Die exklusiven Gefäße der Siegburger Meister, besonders verzierte, mit Auflagen versehene und datierte Krüge aus Frechen, Raeren oder dem Westerwald waren beliebte Sammel- und Studienobjekte, während das Steinzeuggeschirr des Alltags im 15. und 16. Jahrhundert lange nach Bildquellen datiert wurde, Irdenware des Früh- und Hochmittelalters (die sogenannten "Bombentöpfe") als "mittelalterlich" oder "gotisch" bezeichnet wurden. Es fehlten sowohl systematische Grabungen in den wichtigen Töpferzentren als auch hinreichend ausgewertete Befunde und Funde derartigen Alltagsgeschirrs bei Siedlungsgrabungen.

Die Untersuchungen der "Scherbenhügel" in Siegburg, die Erfassung münzdatierter Gefäße und die Kartierung von Steinzeugtöpfereien im Rheinland und in Westfalen, vor allem aber auch Ausgrabungen wie die in den Töpferzentren Coppengrave und Duingen führten zu Erkenntnissen, die zum Beispiel neue Datierungsansätze bestimmter Formen des rheinischen Steinzeugs notwendig machten, das lange als 
relativ sicher datierbares Material auch für die chronologische Einordnung unsicherer Stratigraphien und Funde gedient hatte ${ }^{29}$.

Die Vielfalt der Quellengruppen und der gestiegene Umfang des geborgenen Sachguts entheben nicht der Notwendigkeit einer Aufarbeitung jeder Fundgruppe, welche nach Methoden wie denen der Ur- und Frühgeschichte bewerkstelligt werden muß. Die Idee von Oscar Montelius, möglichst mehrere Methoden zur Datierung einer Fundgruppe zu entwickeln, wird aufgrund der vielseitigeren Quellensituation in der Mittelalter- und Neuzeitarchäologie nicht überflüssig, sondern gewinnt gerade wegen der zusätzlich vorhandenen Kontrollen an Möglichkeiten zur Verwirklichung.

Die Menge der Funde und die Parallelität vieler Befunde machen es möglich, regional auftretende Forschungslücken zu schließen und Quellendefizite auszugleichen. Regional begrenzte Forschungsschwerpunkte können, wenn sie auf einer funktionierenden Bodendenkmalpflege beruhen, bereits aus der Beobachtung der archäologischen Quellen weitreichende Erkenntnisse zum historischen Umfeld gewinnen, da diese in den hoch- und spätmittelalterlichen Städten in einer Dichte und Komplexität auftreten, die der prähistorischen Forschung in der Regel nicht zur Verfügung steht.

Darüber hinaus liefern bildliche und schriftliche Quellen zusätzliche Informationen zur Chronologie, Hinweise auf die Funktionen der einzelnen Fundgegenstände und Erkenntnisse über ihre sozial und wirtschaftsgeschichtliche Einordnung.

Diese auf interdisziplinärer Arbeit beruhende Forschungsweise ist in der Mittelalter- und Neuzeitarchäologie seit langem bekannt, selbstverständlich ist sie jedoch noch nicht ${ }^{30}$.

Um den vielfältigen Kontroll- und Vergleichsmöglichkeiten dieser Disziplin gerecht zu werden, empfiehlt es sich, einzelne Funde und Fundgruppen nicht nur im Kontext und von Beginn an mit eventuell vorhandenen Schrift- und Bildquellen zu bearbeiten, sondern auch zu versuchen, sie in ein separates Raster aus entsprechenden, überregional vorliegenden Fundbeständen einzuordnen.

Nach einer derartigen Analyse, die sich im Rahmen etwa der prähistorischen und volkskundlichen Sachgutforschung bewegt, können die Ergebnisse mit Resultaten verglichen werden, die sich auf den oder die gleichen Gegenstände beziehen, aber aus regional begrenzter, interdisziplinärer Quellenauswertung gewonnen worden sind. Erst dieser abschließende Vergleich ermöglicht Ergebnisse, die - für mehrere

29 Vgl. beispielhaft H.G. Stephan, The development of production of medieval stoneware in Germany, in:

30

P. Davey, R. Hodges, Ceramics and Trade, Sheffield 1983, S. 95-120

Vgl. beispielhaft:

J.G.N. Renaud, Archeologie als derde dimensie in de middeleeuwse geschiedenis, in: Westerheem XX 5-6 1971 zum 10-jährigen Bestehen der AWN, S. 265-284

H. Jankuhn, Umrisse einer Archäologie des Mittelalters, in: Zeitschrift für Archäologie des Mittelalters 1-1973, S. 9-19

H. Bockmann, F.A. Dreier, W. Hübener, G. Wiegelmann, Überlegungen zu Möglichkeiten und Grenzen einer interdisziplinären Zusammenarbeit von Geschichte, Kunstgeschichte, Archäologie und Volkskunde, in: Lübecker Schriften zur Archäologie und Kulturgeschichte 4-1980, S. 220-228 
Gebrauchsgerätetypen entsprechend entwickelt - zur Erfassung von kulturellen Entwicklungsprozessen und kulturräumlichen Gliederungsmodellen führen können $^{31}$.

Zwar ist der ergrabene Bestand an Sachgütern des Mittelalters und der frühen Neuzeit mittlerweile bedeutend, die Auswertung dieser großen Fundmenge befindet sich jedoch erst in den Anfängen. So wichtig es für die einzelnen Regionen auch ist, Fundkomplexe zeitlich und räumlich begrenzt aufzuarbeiten, um den Befundveröffentlichungen möglichst adäquate und umfassende Materialvorlagen an die Seite zu stellen, so notwendig sind zeitlich und räumlich übergreifende Analysen einzelner Gegenstände und Gegenstandsgruppen sowohl für die Regionalforschung als auch für überregionale Schwerpunktforschungen.

\subsection{Bestandteile des Messers}

Das Messer besteht hauptsächlich aus drei Bestandteilen: der Klinge, der Angel bzw. Zunge und dem aus meist organischem Material bestehenden Griff.

Man unterscheidet Messer zunächst nach ihrer Griffkonstruktion in Griffangelmesser und Griffzungenmesser ${ }^{32}$ (Abb. 10).

Bei ersteren wird ein beispielsweise hölzerner Vollgriff auf den aus der Klinge auslaufenden Dorn gesteckt, bei letzteren werden zwei Griffplatten auf jede Seite der als flaches, hochkant zur Klinge stehendes und mehrfach durchlochtes Bandeisen gearbeiteten Griffzunge aufgelegt und mit mehreren Nieten befestigt.

Bei der Angelkonstruktion wird ein Weiterrutschen des Vollgriffs auf die Klinge meist aufgrund einer Stoßkante am Heft verhindert, die dadurch entsteht, daß man die Angel zum Klingenrücken und zur Schneide hin absetzt. Bei der Zungenkonstruktion verhindert die Querverbindung der Niete ein Vorrutschen. Weitere Zusatzbefestigungen können sich am Heft oder am hinteren Griffende befinden. Die Klinge besteht technologisch in der Regel aus zwei Bestandteilen: der weicher geschmiedeten Rückenpartie und der daran angeschweißten, härteren gestählten Schneide ${ }^{33}$.

\footnotetext{
31

Vgl. beispielhaft:

W. Dexel, Das Hausgerät Mitteleuropas, Braunschweig-Berlin 1962

M. Hasse, Neues Hausgerät, neue Häuser, neue Kleider - Eine Betrachtung der städtischen Kultur im 13. und 14. Jahrhundert..., in: Zeitschrift für Archäologie des Mittelalters 7-1979, S. 7-83

T. Dexel, Gebrauchsgerätetypen, Braunschweig 1980

M. Hasse, Die Bedeutung des metallenen Hausgerätes für die Bürger des 13. und 14. Jahrhunderts, in:

Lübecker Schriften zur Archäologie und Kulturgeschichte 4-1980, S. 133-138

Die angeführten Autoren stehen in ihrer Arbeitsweise der bisherigen Forschung nahe. Sie betrachten die Gegenstände von vorneherein im Kontext als bekannt vorausgesetzter kulturgeschichtlicher Entwicklungsstufen. Obwohl ihre Ergebnisse oft pauschal erscheinen und nur selten konsequent hinterfragt worden sind, bleibt die Notwendigkeit derartig übergreifender Versuche bestehen. Es fehlen vor allem Materialanalysen, die Aufarbeitung des einzelnen Gegenstandes, seiner Entwicklung und Verbreitung, um derartig weitgreifende Themen auf eine solide Materialbasis stellen zu können.

Baart u.a. 1977, S. 325

Vgl. Kapitel 9, Abschnitt 1
} 

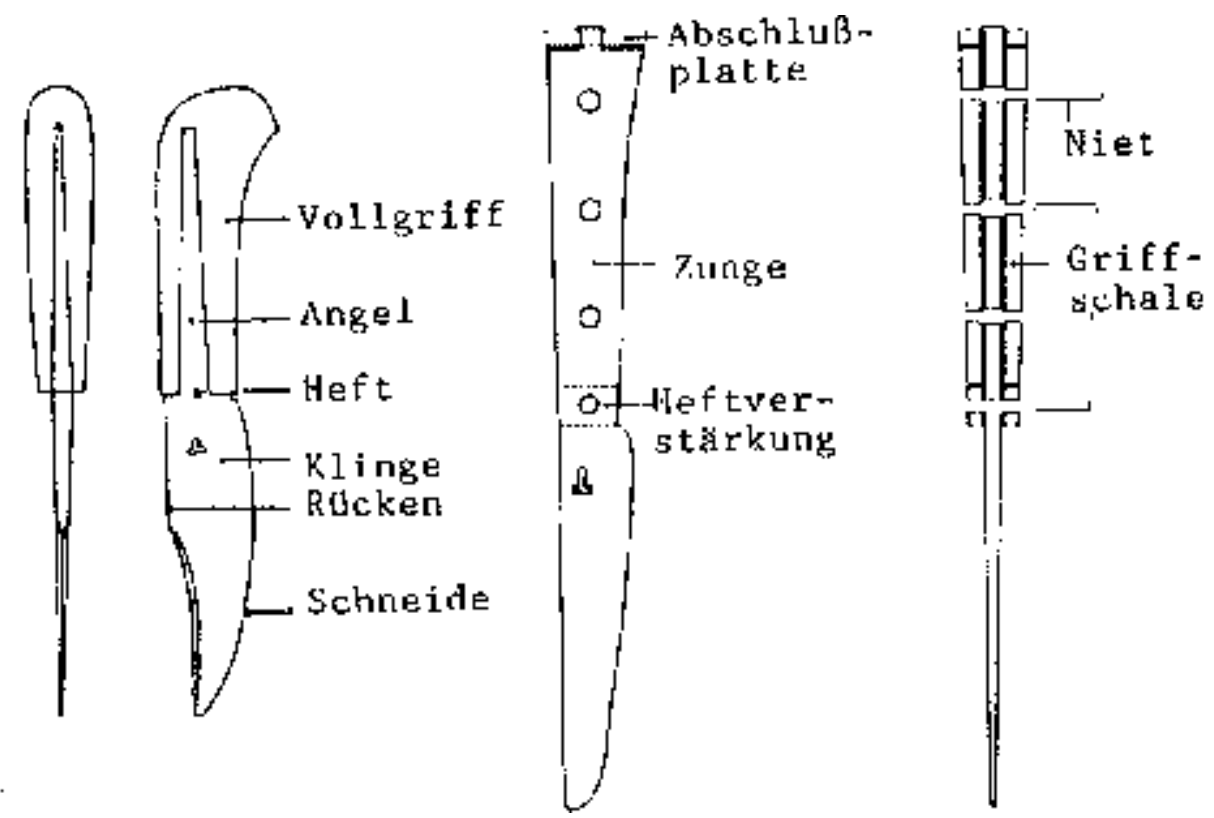

Abb. 10:

Bestandteile des Messers und die beiden grundsätzlichen Griffkonstruktionen (aus Baart u.a., Opgravingen in Amsterdam, Amsterdam 1977, Abb. 116, S. 326)

\subsection{Die Verschlüsselung}

Für die Verschlüsselung zur Speicherfähigkeit und Auswertbarkeit der einzelnen Messerbestandteile und weiterer Kriterien, die in der statistischen Auswertung relevant sind, wurden diese in Codes aus Zahlen- und Buchstabenkombinationen übersetzt.

Die einzelnen Codes des Schlüssels werden im folgenden detailliert aufgeführt und beschrieben. Auf einem Faltblatt am Ende dieser Beschreibung sind sie vollständig mit aufgeführt.

Zum besseren Verständnis der Verschlüsselung wird an Idealbeispielen ihre Anwendung erläutert:

Die Verschlüsselung umfaßt im vorderen Teil den Code für die Klingenform, nach einem Trennsymbol den Code für die Griffkonstruktion.

Im Anschluß daran folgt die Benennung der für den Griff verwandten Materialien: der Grundbuchstabe bezeichnet das Material des auf der Angel oder der Zunge befestigten Vollgriffs (Hilze) oder der Griffplatten. Die verschiedenen Buchstabensymbole sind in einer separaten Tabelle aufgelistet (Abb. 12). Sie enthält auch die Bezeichnungen zusätzlich verwendeter Materialien wie Bunt- oder Edelmetalle, zum Beispiel von Applikationen oder Griffplättchen, die im Code der Griffmaterialien dem Grundbuchstaben als Hochziffer zugeordnet erscheinen. 
Bei der Verwendung mehrerer zusätzlicher Materialien (zum Beispiel zu Verzierungszwecken) bilden diese Hochziffern eine Reihe.

Die Summe aus dieser Reihe und dem Grundbuchstaben ergibt die Summe aller zum Aufbau des Griffs benutzten organischen und nichtorganischen Materialien mit Ausnahme des immer aus Eisen bestehenden Kerns, der Angel oder Zunge. Nach dem Code der Griffmaterialien folgt die in Klammern gesetzte Angabe der Klingenlänge, zum Beispiel (14) $=14 \mathrm{~cm}$.

Bei erkennbaren Zierelementen am Griff, zum Beispiel bei Gravuren oder Schnitzereien, wird dem gesamten Code die Buchstabenkombination GV (= Griffverzierung) am Ende beigefügt.

Für den Fall einer Klingenverzierung erscheint an dieser Stelle oder zusätzlich die Kombination KV (= Klingenverzierung).

Am Schluß der Codereihe wird auf Zeichen, Marken oder ähnliche Identifikationssymbole in der Messerklinge verwiesen, in diesen Fällen steht dort der Sammelbegriff "Marke".

Dieses Verfahren zur Erfassung eines bestimmten Grifftyps sei an einem Beispiel kurz erläutert:

$$
\text { ... / A 2.3.3 } \mathrm{H}_{1}{ }^{\text {(B) } \ldots \mathrm{GV}}
$$

Die Übersetzung dieser Verschlüsselung sähe wie folgt aus:

Griffangelkonstruktion mit einer beidseitig abgesetzten Angel, aufgestecktem hölzernen und verziertem Vollgriff und zusätzlicher Endabsicherung am hinteren Griffende, zum Beispiel einem als Knauf oder Balluster gestaltetem Abschluß aus Buntmetall. Der Begriff GV wird jeweils im Katalog genauer erläutert.

Die Konstruktion des direkt an die Klinge geschmiedeten Volleisengriffs wird im Code durch einen Pfeil zwischen Griffkonstruktionsform und -material gekennzeichnet:

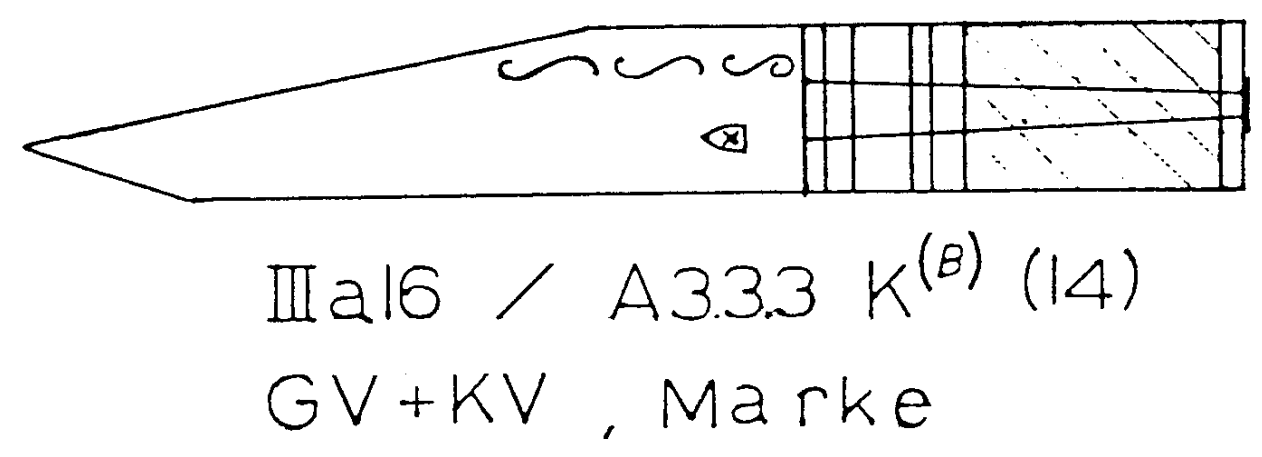
$\ldots / \mathrm{A} 1 \rightarrow \mathrm{E}_{1} \ldots$

Abb. 11: $\quad$ Schematisierte Darstellung einer Messerverschlüsselung

Die Verschlüsselung eines vollständig erhaltenen Idealmessers faßt das Verfahren noch einmal zusammen:

Bei diesem Idealmesser handelt es sich um ein Griffangelmesser mit der Klingenform IIIa16 und einem auf die Angel geschobenen Vollgriff, der in einzelne 
Knochensegmente unterteilt worden ist. Diese Segmente sind durch zwischengeschobene Buntmetallplättchen voneinander getrennt worden, den Abschluß bildet ein stärkeres Griffplättchen.

Diese Griffplättchenkonstruktion A.3 wird, da sie mehrere Lagen von Plättchen enthält, als Griffverzierung geführt.

Die Klinge ist durch Gravuren verziert und mit einer Marke versehen worden. Die Länge der Klinge beträgt $14 \mathrm{~cm}$.

\section{Materialien}

Holz

Horn (Geweih)

Knochen, Zahn (Bein)

Eisen

Buntmetalle (v.a. Messing)

Edelmetalle (v.a. Silber)

Glas (Glasfluß), Email, Edelsteine,

Wachs (Meerschaum, Harz, Bern-

stein etc.)

Leder

Blei

\section{Code}

H 1

$\mathrm{H} 2$

$\mathrm{K}$

E 1

B

E 2

E 3

$\mathrm{L}$

$\mathrm{P}$

Abb. 12: $\quad$ Die bei der Griffgestaltung verwendeten Materialien

Am Ende des Schlüssels zu den Griffkonstruktionen befindet sich die Bezeichnung "Klappmesser". Sie fällt unter die Formen der Gruppe B, da Klappmesser (in der Literatur auch als Faltmesser bezeichnet) ausnahmslos Griffzungenkonstruktionen besitzen. Die scharnierartige Vorrichtung zum Einklappen der Klinge befindet sich im vom eigentlichen Griff leicht abgesetzten Heft. Formale Differenzierungen wurden nicht vorgenommen, da die Menge der erfaßten Klappmesser zu gering war (Abb. 16b).

Das Prinzip, die Klinge nicht feststehend aus dem Griff auslaufen zu lassen, sondern sie am Heft mit einer scharnierartigen Klappvorrichtung zu versehen, um sie vor oder in den Griff zu klappen, ist für den gesamten hier behandelten Zeitraum nachweisbar. Für eine Klassifizierung oder eine weitergehende Analyse zur regionalen Verbreitung bestimmter Typen war das Material nicht aussagekräftig genug; es sollte zunächst genügen, die erfaßten Klappmesser im Katalog vorzustellen und einige generelle Aspekte zu dieser Gruppe anzusprechen ${ }^{34}$. 


\subsection{Die Codebezeichnungen des Schlüssels}

\subsubsection{Die Griffkonstruktionen}

Aus der Gesamtheit der im Katalog erfaßten Griffkonstruktionen ergaben sich sechs verschiedene Arten der Griffangelkonstruktion sowie neun verschiedene Arten der Griffzungenkonstruktion. Sie werden im folgenden in ihren Charakteristika beschrieben.

\section{Die Griffangelkonstruktionen}

Die Konstruktionsformen A.1-A.6 umfassen alle im bearbeiteten Fundgut vertetenen Griffangelkonstruktionen. Gemeinsam ist ihnen die Angel, eine dornartige, am Heft oft abgesetzte und nach hinten aus der Klinge auslaufende Verlängerung, auf die der eigentliche Vollgriff, welcher z.B. aus einem Röhrenknochen, einem Stück Geweih oder aus einem längs durchbohrten Holzschaft bestehen konnte, von hinten aufgesetzt und manchmal auch ganz durchgeschoben wurde:

bei letzterer Befestigungsart wurde das Angelende entweder umgebogen oder bildete als Platte oder Kopf den Griffabschluß.

Oft treten am Heft und am Angelende die Ansatzstellen verstärkende, zum Teil zwingenartige Zusatzhalterungen auf, wie Abschlußkonstruktionen aus Metallscheiben, massiven Metallkörpern oder am Heft angebrachten Metallhülsen (Abb. 13). 


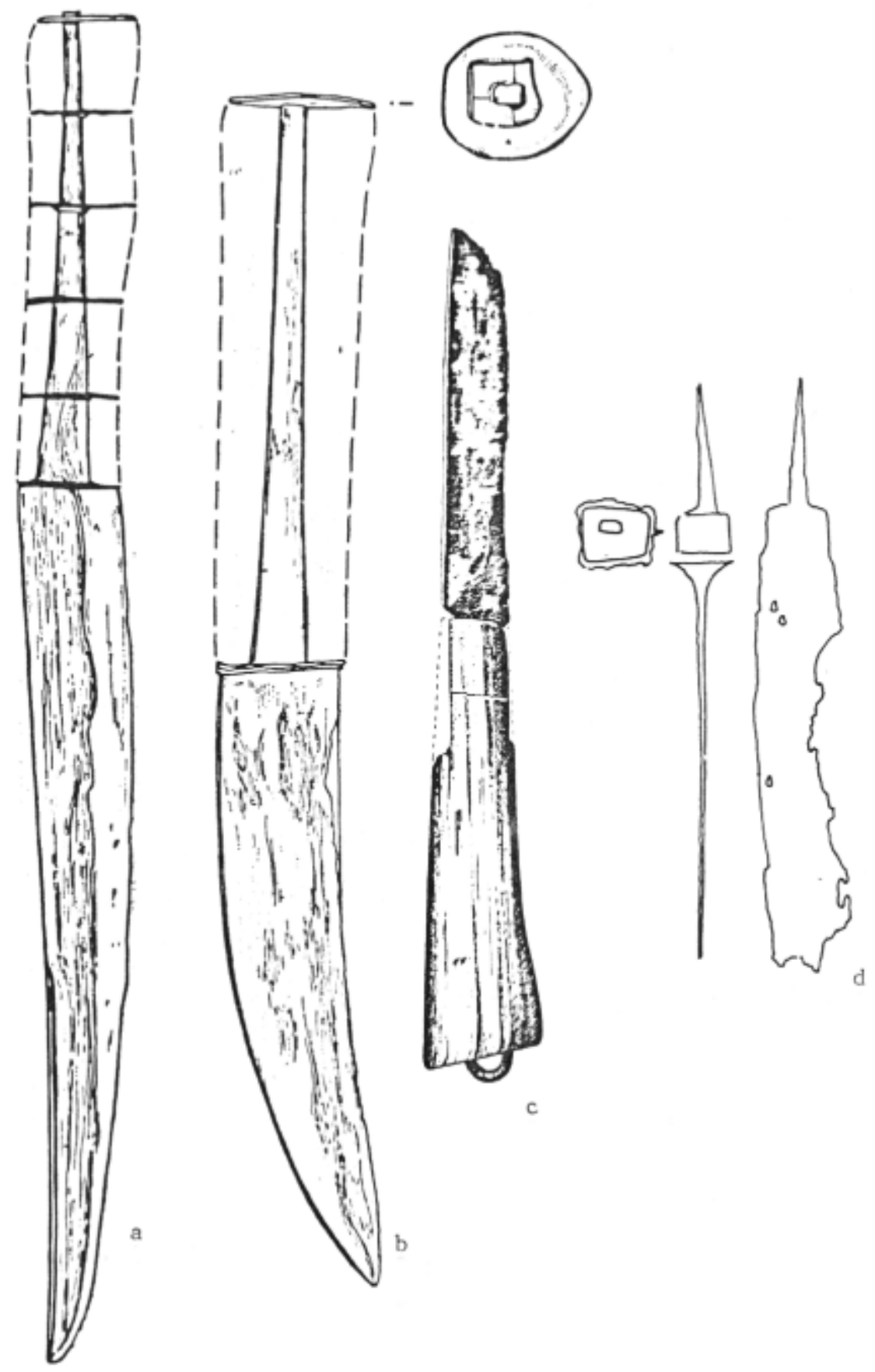




\section{Die Konstruktion A1 (Abb. 14)}

Unter diese Bezeichnung fallen Griffangelkonstruktionen ohne irgendeine zusätzliche Befestigungsart. Die Gruppen A1.0 bis A1.3 umfassen neun Möglichkeiten, die Angel am Ansatz zum Heft abzusetzen, bei ihrer Leitform fällt der Absatz weg: die Angel springt hier direkt aus dem Heft. Die Gruppen A1.4 und A1.5 beinhalten sechs Absetzungsmöglichkeiten für den Fall, daß die Angel schräg nach oben bzw. nach unten verläuft.

Für die Gruppen der Konstruktionsform A1 gilt, daß der jeweilige Vollgriff aus einem organischen Material besteht, da er nachgiebig sein muß, um fest auf der Angel zu verbleiben, ohne zusätzlichen Halt von hinten zu bekommen.

Für die sehr seltenen Fälle, in denen die Angel direkt zu einem eisernen Vollgriff ausgeschmiedet worden ist, wurde keine separate Bezeichnung gewählt, da technologisch für die Endfertigung des Griffs ebenfalls nur ein Schritt erforderlich ist. In diesen Fällen wird die Konstruktion aus der Schreibweise der Verschlüsselung ersichtlich: Ein aufgeschobener Holzvollgriff erscheint als Code: - / A 1. $\mathrm{H}_{1}(-)$, ein direkt an die Klinge geschmiedeter Volleisengriff erhält zwischen Konstruktion und Material einen Pfeil: - / A 1. $E_{1}(-)$.

\section{Die Konstruktion A2 (Abb. 15)}

Dieser Code bezeichnet die gleiche Konstruktion wie die des Codes A 1. Sie ist hier allerdings versehen mit einer zusätzlichen Halterung am Griffangelende, indem diese entweder umgebogen oder als Platte oder verdickter Kopf gearbeitet worden ist.

Eine zweite Halterungsmöglichkeit dieser Konstruktionsform ist eine Heftverstärkung in Form einer bereits am Messerrohling ausgeschmiedeten Verdickung am Angelansatz oder eines vor dem organischen Griff auf die Angel gesteckten Griffplättchens als Griffabschluß zum Heft. Diese Art der Heftgestaltung tritt auch zusammen mit den Zusatzhalterungen am Griffende auf, wobei zum Beispiel oft ein Griffplättchen am Heft und zusätzlich eines am Griffende befestigt worden ist.

\section{Die Konstruktion A3 (Abb. 16a)}

Der Code A3 beinhaltet eine Sonderkonstruktion, die in der "Plättchentechnik" verzierten Messergriffe ${ }^{35}$.

Die Griffangel wird immer mit mindestens jeweils einem am Heft und am Griffende auf die Angel gesteckten Griffplättchen versehen, die den Griff beidseitig abschließen. Im Fall der Plättchenverzierung ist der Vollgriff zusätzlich in einzelne 
Segmente unterteilt, die jeweils von Griffplättchen am Heft und am Angelende eingerahmt werden.

Die Plättchen können seltener aus Silberblech, häufiger aus Kupferoder Messingblech bestehen, die Segmente des Vollgriffs bestehen oft neben dem ursprünglichen Material des segmentierten Vollgriffs wie Wurzelholz aus zusätzlich zwischengesetzten Leder-, Horn- oder Bleisegmenten. Den Angelabschluß bilden verschiedene Arten von Nietknöpfen oder kleinere Abschlußscheibchen, sehr selten sind Beispiele mit massiveren Metallabschlüssen. Ursprünglich sollte die allgemeine Verwendung von Griffplättchen in einem eigenen Code zusammengefaßt werden. Davon wurde vor allem wegen der unterschiedlichen Funktionen ihrer Verwendung Abstand genommen:

Im Code A2 ist die zwingenartige Anbringung metallener Griffplättchen eine reine Befestigungsart und somit eine unter mehreren Arten, den auf die Angel gesteckten, aus organischem Material bestehenden Vollgriff zu stabilisieren.

Im Code A3 wird diese Befestigungsart durch die Segmentierung des Griffs und Einschub weiterer Plättchen ausgeweitet und so zusätzlich zu einer besonderen Art, den Griff zu verzieren. So ist diese Konstruktionsform eigentlich eine Sonderform des Codes A2. Sie wurde getrennt, da die Verbreitung dieser Verzierungsart relativ einheitlich und auch zeitlich einzugrenzen ist. Zwei größere Publikationen haben diese Technik in ihrem Erscheinungsbild und ihrer geographischen Verbreitung bereits bearbeitet, so daß eine gesonderte Behandlung dieser Griffgestaltung nahe $\operatorname{lag}^{36}$.

\section{Die Konstruktion A4 (Abb. 16b)}

Unter diesem Code sind Griffangelkonstruktionen zusammengefaßt worden, bei denen das vordere, an das Heft stoßende Vollgriffende von einem Metallblech ummantelt ist. Diese Befestigungsart kann als breiter Ring gearbeitet sein, der nachträglich um das vordere Vollgriffende gelegt wurde; sie kann aber auch aus einer zylindrischen oder konischen Hülse bestehen, die vor dem Aufstecken des Vollgriffs auf die Angel gesetzt worden ist. Eine zusätzliche Befestigung am hinteren Griffende in der Art der Konstruktion des Codes A2 kann zusätzlich auftreten, sie ist für diese Befestigungsart aber nicht regelmäßig zu beobachten.

Ob ein am Heft um den an die Klinge stoßenden Vollgriff gelegter Ring, Draht oder eine Manschette als Reparatur eines vorne eingerissenen Griffs oder als serienmäBige Zusatzsicherung anzusehen ist, war oft nicht einwandfrei festzustellen.

36 Vgl. vor allem Knorr 1971

Drescher 1975 


\section{Die Konstruktion A5 (Abb. 16c)}

Für diesen Code gibt es nur wenige Belegexemplare. Zwischen dem eigentlichen Dorn und dem hinteren Klingenende befindet sich am Heft ein im Schnitt mehreckiger oder zylindrischer Metallkörper, welcher zum Beispiel Plättchen oder Hülsen am Heft ersetzt und bereits den vorderen Teil des Griffs darstellt. Lediglich im hinteren Teil besteht der Griff noch aus einem auf die Angel geschobenen organischen Vollgriff.

\section{Die Konstruktion A6 (Abb. 16d)}

Der Code A6 bezeichnet eine äußerst selten beobachtete Konstruktionsform. Sie bildet typologisch die Übergangsform von der Griffangel- zur Griffzungenkonstruktion:

Bei meist breiter und kurzer, seltener auch längerer Angel sind im Anschlußbereich der Klinge an die Angel ein oder zwei Nietlöcher vorhanden. Leider hat sich bei keinem der vorhandenen Exemplare der Vollgriff erhalten, so daß sich über die Vernietung am Heft nur Mutmaßungen anstellen lassen. Denkbar ist jedoch, daß der Vollgriff im vorderen Bereich das Heft umfaßte wie zwei Griffschalen, indem er vorne eingeschnitten worden war.

Die Konstruktionsform A6 ist typologisch in ihrer Gesamterscheinung noch eine Angelkonstruktion mit zusätzlicher Heftverstärkung. Exemplare des 12. bis 13. Jahrhunderts liegen aber noch so früh, daß chronologisch von einer zur Griffzungenkonstruktion führenden Übergangsform nur bedingt gesprochen werden $\mathrm{kann}^{37}$. 

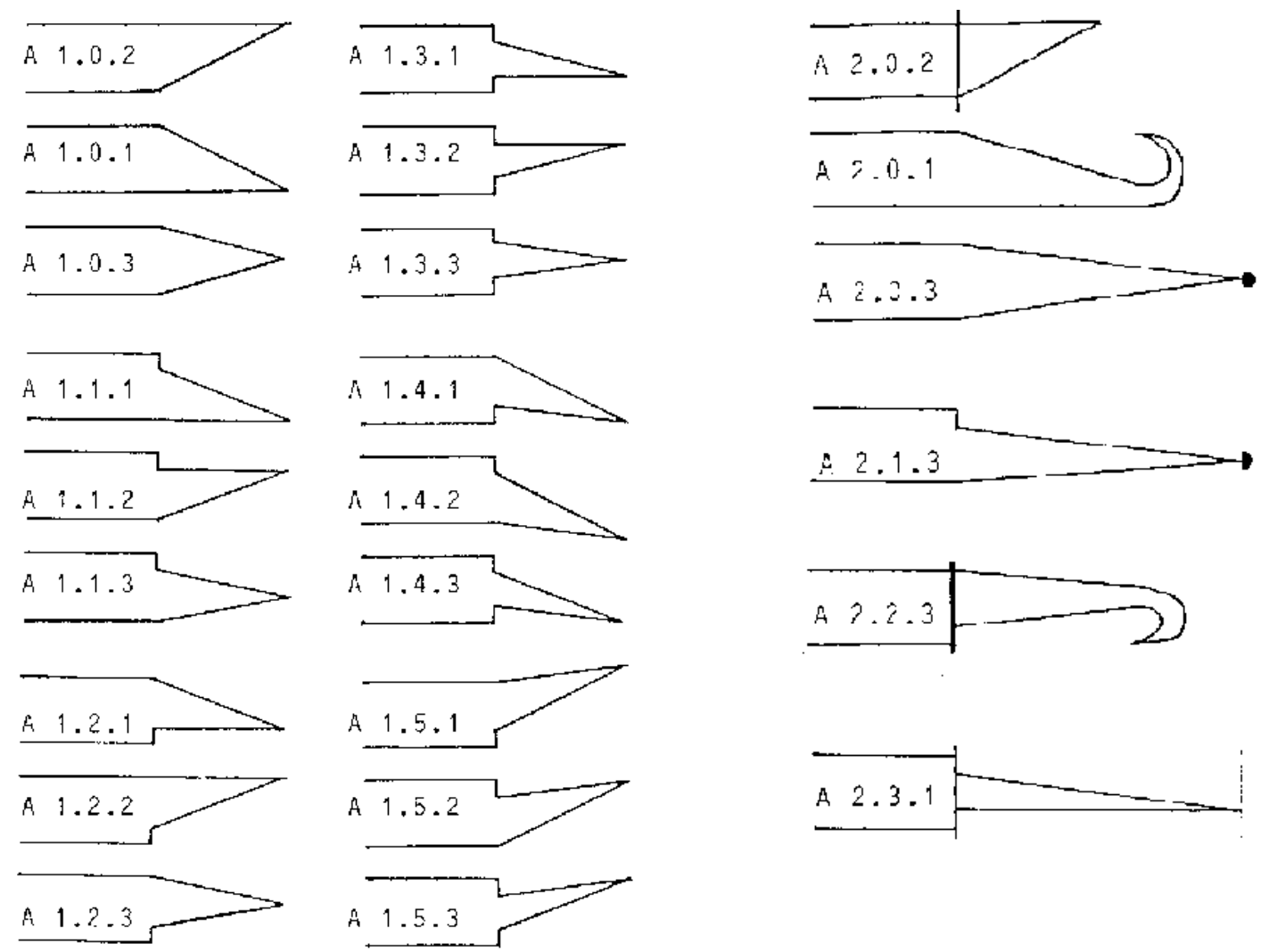

Abb. 14

Die Griffangelkonstruktion Al

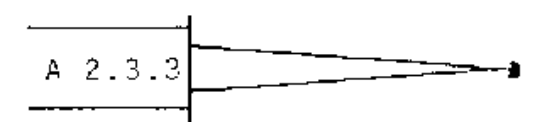

Abb. 15 Die Griffangelkonstuktion A2 

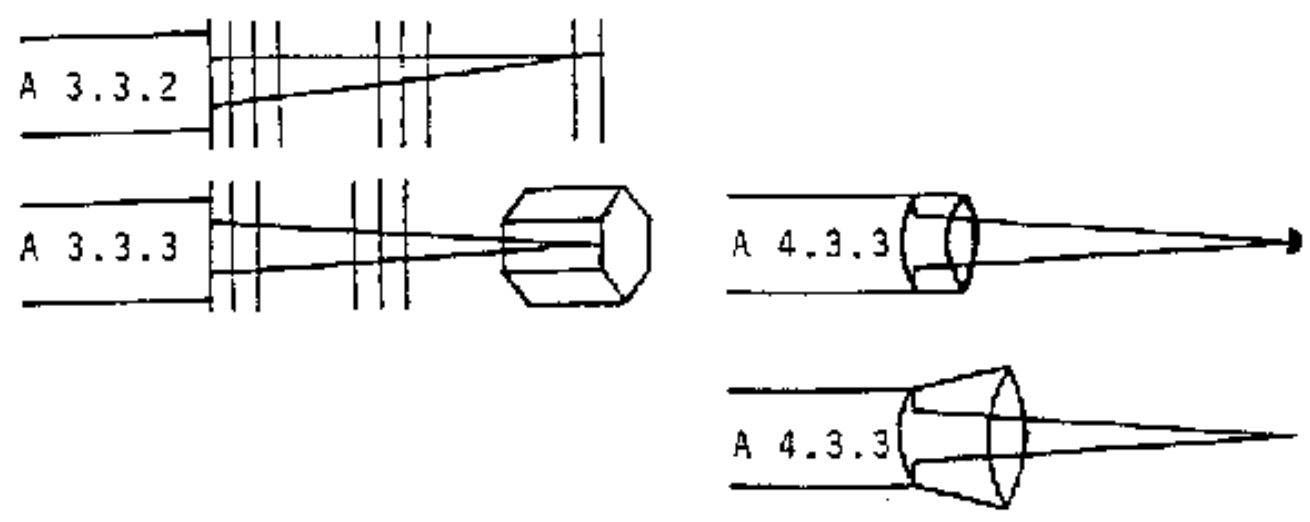

a

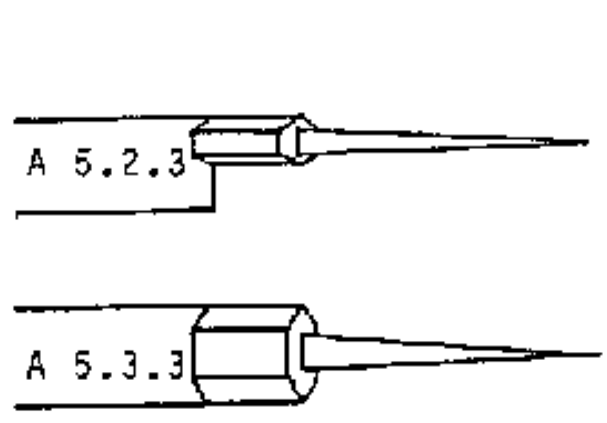

c $b$
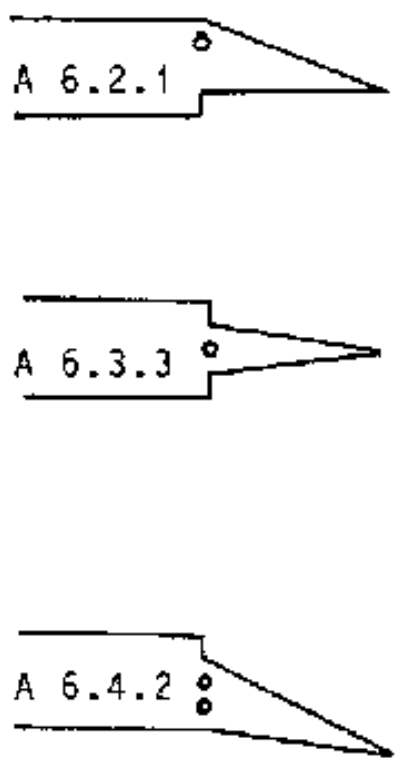

$a$

Abb. 16
a: Die Griffangelkonstruktion A 3
b: Die Griffangelkonstruktion A 4
c: Die Griffangelkonstruktion A 5
d: Die Griffangelkonstruktion A 6 


\section{Die Griffzungenkonstruktionen}

Das Prinzip der Griffzungenkonstruktion beruht auf der verbesserten Haltbarkeit und Festigkeit des Griffs: auf ein flaches Bandeisen, welches in der Regel zur Schneide hin abgesetzt ist, ansonsten aber in der Stärke der Klinge hochkant nach hinten weiterverläuft, werden beidseitig zwei aus meist organischem Material bestehende Griffschalen mit mehreren querverlaufenden Nieten befestigt. Diese Art der Griffkonstruktion ist die jüngere Grifform ${ }^{38}$. Insgesamt wurden im bearbeiteten Material neun verschiedene Arten von Griffzungenkonstruktionen beobachtet, wobei die Konstruktion mit der Codebezeichnung B5 nur aufgrund ihrer Verbindung mit der Konstruktionsform B6 in die Kategorie Griffzungenkonstruktionen mit eingeordnet wurde.

\section{Die Konstruktionsform B1 (Abb. 17a)}

Dieser Code bezeichnet die Grundform der Griffzungenkonstruktion ohne jede weitere Zusatzsicherung. Die Form der Zunge ist rechtekkig, zumeist läuft der Klingenrücken ansatzlos in den Griffzungenrücken über.

\section{Die Konstruktionsform B2 (Abb. 17b)}

Ähnlich wie bei der entsprechenden Angelkonstruktion ist auch hier der Griff mit einer zusätzlichen, oft zwingenartigen Griffsicherung am Heft und/oder am hinteren Zungenende versehen worden. Das Prinzip der Zwinge kann sich durch eine zusätzlich am Heft angebrachte Verstärkung, zum Beispiel in Form zweier Messingplatten, die an den Seiten des Heftes durch Niete befestigt sind, deutlich dokumentieren. Die hintere Abschlußgestaltung kann sich in mannigfaltiger Art zeigen, so in aus Buntmetall oder Edelmetall bestehenden gegossenen Aufsätzen auch zoomorphen Charakters, in aus Edel- oder Buntmetallblechen bestehenden, gravierten Manschetten und Hülsen oder in über die Zunge nach unten herausragenden und dort verbreiterten Griffschalenenden.

\section{Die Konstruktionsform B3 (Abb. 17c)}

Der Code B3 bezeichnet die Form einer trapezoid nach hinten verbreiterten Griffzunge, wobei eine beidseitige Trapezoidität nicht Voraussetzung ist. So ist die häufigste Vertreterin dieser Art die Form B3.2 ohne Verbreiterung der Oberkante. Der Einfall der unteren Zungenkante zum Heft verhindert das Abrutschen der Finger auf die Schneide, ohne daß es noch eines zusätzlichen Heftschutzes bedarf.

\footnotetext{
38 s. Kapitel 6, Abschnitt 2.3, in dem die Ergebnisse der Auswertung zu dieser Frage
} zusammengefaßt behandelt werden. 
Die Konstruktionsform B4 (Abb. 17d)

Unter diesen Code fallen die Formen von B3, die zusätzlich mit einer zwingenartigen hinteren Abschlußverstärkung versehen sind, ähnlich den Konstruktionen der Form B2. Eine zusätzliche Heftverstärkung ist auch hier nicht zwingend. Die hintere Abschlußverstärkung kann beispielsweise aus metallenen Aufsätzen oder aus hinten überstehenden Griffschalenenden bestehen.

\section{Die Konstruktionsformen B5 und B6 (Abb. 17e)}

Der Code B5 bezeichnet strenggenommen eine eigenständige Art der Griffkonstruktion: sie besteht aus einer direkt am Heft ansitzenden Hülse, die einen Vollgriff ohne dornartige Angel oder Zunge aufnimmt. Diese konisch zum Heft hin zulaufende Hülse kann als Zusatzsicherung einen Nagel oder einen Niet als Querverbindung enthalten. Diese Art der Griffzungenkonstruktion ist für Messer selten; sie ist eher bei haus- und landwirtschaftlichen Geräten oder Werkzeugen zu beobachten.

Bei den Beispielen der Konstruktionsform B6 stellen die metallene Hülse in der Art der Form B5 oder auch Metallringe eine zusätzliche Heftverstärkung dar, sie umfassen im vorderen Bereich die Zunge, die ansonsten das Prinzip der Griffschalenvernietung beibehält. Eine zusätzliche hintere Zungenendabsicherung kann hinzutreten. 

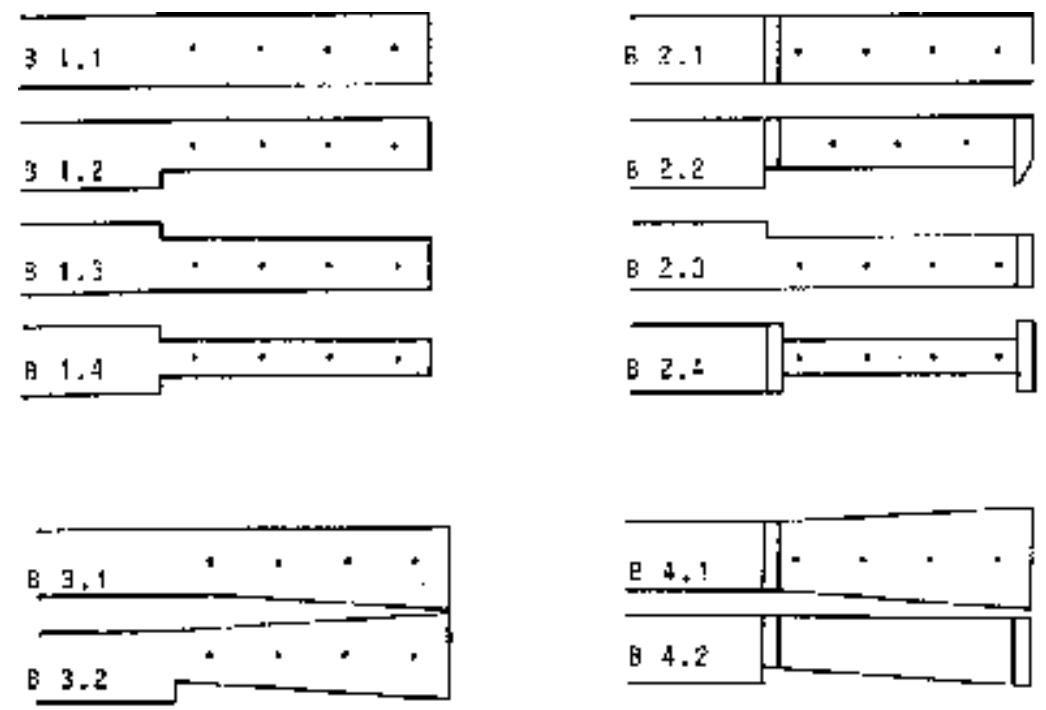

B 3.3

B 4.9

e 3.4

34,4
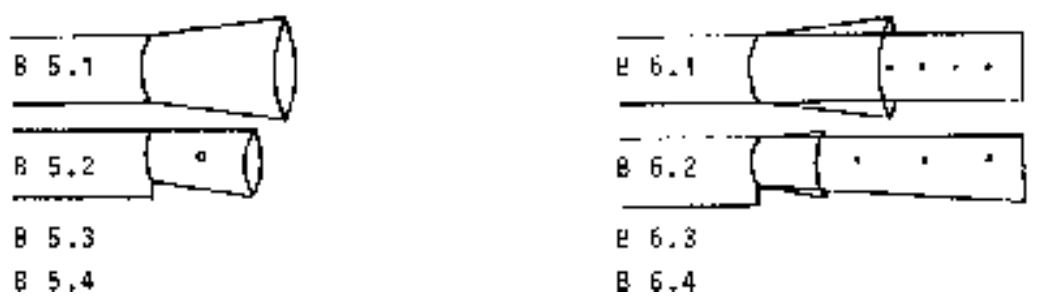

$A b b .17$

Die Griffangelkonstruktion B1 bis B 6

\section{Die Konstruktionsform B7 (Abb. 18a)}

Dieser Code bezeichnet eine Sonderform, bei der das Prinzip der Griffschalenvernietung auf einem flachen Bandeisen beibehalten wird. Die Griffzunge wird aber gegenüber der Klinge um eine Vierteldrehung versetzt, so daß jetzt das Flacheisen nicht mehr hochkant, sondern waagerecht vor der Klinge steht und die Griffschalen - von oben nach unten vernietet - sich auf und unter der Zunge befinden. Diese Form ist äußerst selten beobachtet worden. Es scheint, als trete sie zumeist in Kombination mit vollmetallenen, ausgeschmiedeten Partien ähnlich denen der Formen A5 und (seltener) A3 auf.

Bei einem Absatz des Griffs zur Schneide ist auch das Volleisenstück am Heft zur Schneide abgesetzt, die waagerecht zur Klinge stehende Zunge läuft dann nur aus diesem Griffteil heraus. 


\section{Die Konstruktionsform B8 (Abb. 18b)}

Die Form B8 stellt das Pendant zur Griffangelform A5 dar. Auch hier besteht der vordere Teil des Griffs aus einem zylindrischen oder mehreckigen Vollmetallstück, welches am Heft ansitzt. Aus diesem Schaft springt nach hinten die eigentliche, von Griffschalen ummantelte Zunge. Wie die Form A5 ist auch B8 äußerst selten aufgetreten.

\section{Die Konstruktionsform B9 (Abb. 18c)}

Eine weitere äußerst seltene Griffzungenform bezeichnet der Code B9. Hier handelt es sich um die gleiche Art der ohne Zusatzbefestigungen auftretenden Zungenkonstruktion wie bei der Form B1. Nur läuft hier die Zunge nach hinten konisch zu. Typologisch scheint diese Zungenform das Anschlußglied an die Konstruktionsform A6 zu sein, da die Kanten der Zunge ähnlich konisch zulaufen, die längslaufende Vernietung aber schon an eine Griffzunge erinnert. Die Griffzunge der Form B9 kann hinten spitz oder abgeschnitten enden.

Am Anfang des codierten Kataloges (Kapitel 5, Abschnitt 2) sind alle hier behandelten Konstruktionsformen noch einmal aufgeführt und graphisch dargestellt worden (Codierungstafeln 4 und 5).
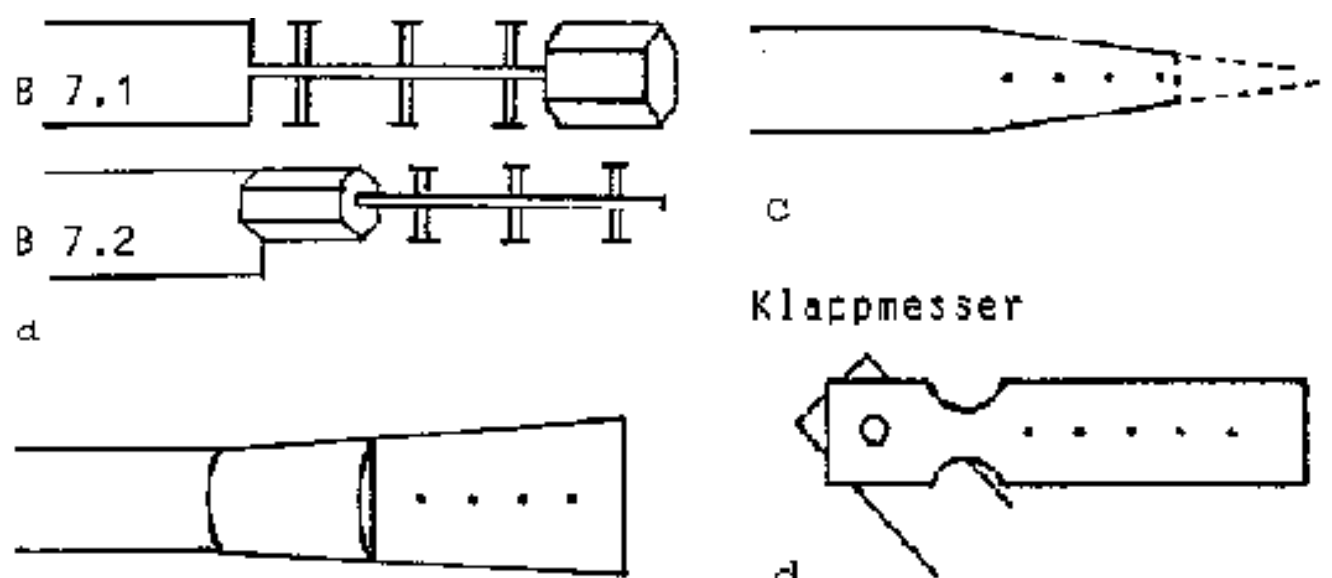

b

\section{Klappmesser}

d

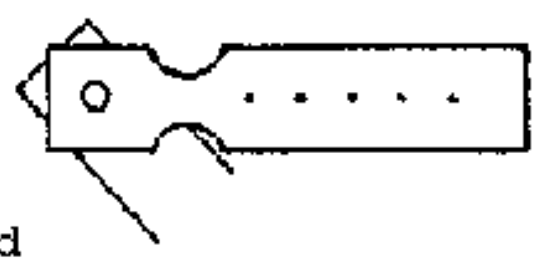

Abb. 18

a: Die Griffzungenkonstruktion B 7

b: Die Griffzungenkonstruktion $B 8$

c: Die Griffzungenkonstruktion B9

$d$ : Schematisierte Klappkonstruktion 


\subsubsection{Die Klingenformen}

Im Gegensatz zu der vom erfaßten Fundmaterial ausgehenden Klassifizierung der Griffkonstruktionen ist das Klassifizierungsmodell der Klingenformen deduktiv erstellt worden.

Unter der bereits erwähnten Voraussetzung, daß für ein Messer, welches auch zum Essen, das heißt zum Schneiden und Spießen bzw. Stechen benutzt werden soll, eine spitze und eine Schneidekante erforderlich sind, ergeben sich ausgehend von einem länglichen Rechteck als geometrischer Grundfigur drei Möglichkeiten der Abwandlung:

Zur Erreichung der Spitze verläuft entweder die Unterkante schräg nach oben zur Oberkante (Ia), oder die Oberkante fällt schräg nach unten auf die Unterkante (IIa), oder beide Kanten fallen im gleichen Winkel nach vorn zur Spitze zusammen (IIIa) (Abb. 19a).
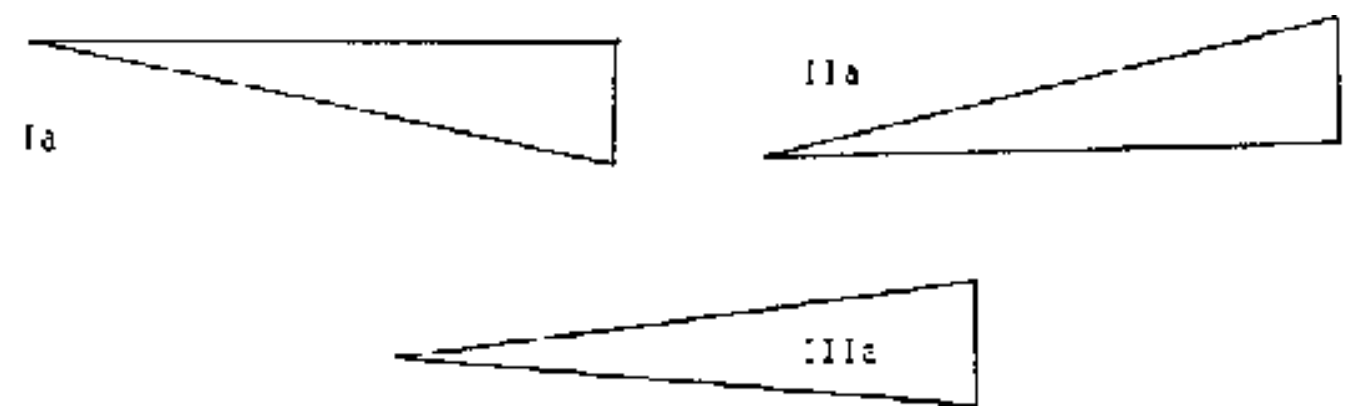

Abb. $19 a$

Die drei grundsätzlichen Möglichkeiten zur Ausformung einer Spitze

Bei einer Schärfung der Unterkante entstehen so die drei Grundformen des Messers mit geradem Rücken und einfallender Schneide, mit einfallendem Rücken und gerader Schneide und mit einfallendem Rücken und einfallender Schneide. Aus letzterer ergibt sich bei Anschliff beider Seiten die gleichseitige Grundform des Dolchs.

Die jeweils zwei Abwandlungsarten der Formen Ia und IIa: Ib, Ic sowie IIb, IIc sowie die vier aus der Form IIIa abgewandelten Formen IIIb, IIIc, IIId und IIIe ergeben sich aus der Veränderung der jeweils abgeschrägten Kanten in konvexe und konkave Bögen. Somit entstehen für die Struktur des Klassifizierungsmodells elf Leitformen (Abb. 19b). 

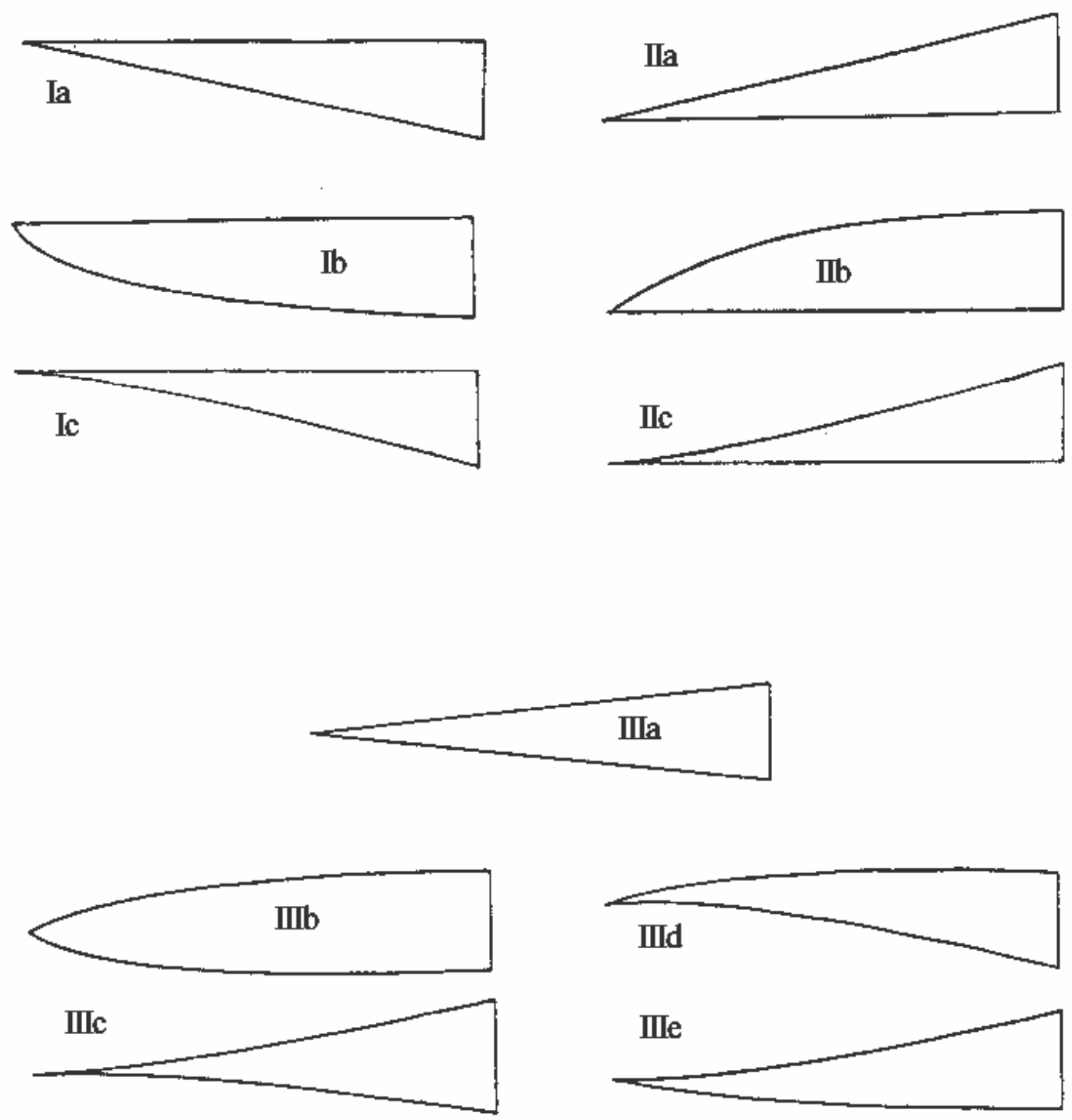

Abb. $19 b$

Die elf Leitformen des Klingenmodells

Die weitere Untergliederung des Modells in seine Haupt- und Einzelformen erfolgte durch Erfassung von Proportionalitätsverschiebungen und unter Berücksichtigung der verschiedenen Neigungsmöglichkeiten des Rückens und der Schneide.

Zunächst wird die Untergliederung der elf Leitformen in 88 Hauptformen beschrieben.

Das Prinzip dieser Unterteilung besteht aus den unterschiedlichen Möglichkeiten, die Längen des Rückens und der Schneide vor ihrer Abschrägung zur Spitze zu verändern. Um nicht Gefahr zu laufen, am Schluß des Modells weit mehr errechnete als tatsächlich vorhandene Formen zu erhalten, wurde nur eine Möglichkeit der Proportionalitätsveränderung durchgeführt: 
bei grundsätzlicher Beibehaltung der jeweils abgeschrägten Kante des Messers wurde die Klinge in ihrer Länge mittig geteilt. Sodann wurde die in der Leitform noch die ganze Kante erfassende Schrägung verkleinert, in dem die in der Leitform abgeschrägte Kante (bei der Form Ia also die Schneide) parallel zur anderen, geraden Kante über die Mitte der Klinge hinaus bzw. nur bis kurz vor die Mitte der Klinge vorgezogen wurde, um erst nach (so bei der Hauptform Ia1) bzw. vor der Mitte (so bei der Hauptform Ia2) zur Spitze einzufallen. Dieses Prinzip wiederholt sich bei allen anderen Leitformen.

So treten zu den Leitformen Ia-c und Ila-c jeweils zwei Hauptformen hinzu (Ia1, Ia2, Ib1 usw.).

Bei den Leitformen IIIa bis IIIe treten aufgrund der beidseitigen Abschrägung der Kanten zunächst jeweils vier Hauptformen hinzu. Im Fall der Leitform IIIb sind dies die Hauptformen IIIb1, bis IIIb4. Wegen der verschiedenen Möglichkeiten, die einzelnen Abschrägungen gerade oder gebogen zu halten, kommen auf jede dieser Leitformen noch einmal jeweils 14 Hauptformen. Im Fall der Leitform IIIb sind dies die Hauptformen IIIb13-18 und IIIb29-36 ${ }^{39}$ (Abb. 20).

Die Hauptform IIIb29 durchbricht die eigentliche Vorgabe des beidseitigen Einfalls von Schneide und Rücken der Leitform IIIa. Ausgegangen wurde für diese und die folgenden Hauptformen IIIb30 bis IIIb32 von der Einzelform IIIb29.1, bei der der Rücken gerade abgeschrägt zur Spitze verläuft, die Schneide bis über die Mitte gebogen, dann gerade zur Spitze hochsteigt.

Diese Form ergab sich aus einer Weiterentwicklung der Hauptformen IIIb13 und IIIb16. Da im Modell außer bei gebogenem Rücken in Hauptformen wenigstens der erste Teil des Rückens nach dem Heftansatz rechtwinklig zum Heft steht, wurde in diesem Fall der Rücken gerade gestellt und die so veränderte Einzelform an den Anfang einer Gruppe gestellt (Abb. 21a).

39 Diese Formen ergeben sich daraus, daß die Möglichkeit der Proportionalitätsverschiebung der Seiten bei den Formen IIIb, IIIc, IIId und IIIe durch die Tatsache der beidseitigen Krümmung um zwei grundsätzliche Varianten erweitert werden kann. Es sind dies die Möglichkeiten, eine Seite ganz und die zweite Seite zum Teil gebogen zu halten. So fällt bei der Form IIIb13 der Rücken ganz, die Schneide nur in ihrem letzten Viertel zur Spitze ein. Weiterhin besteht die Möglichkeit, daß bei beidseitiger Abschrägung die Kanten der einen Seite beide gerade verlaufen, während eine Kante der anderen Seite gerade, die zweite gebogen verläuft, so daß faktisch nur ein Teil einer Seite gebogen erscheint (bei der Form IIIb16 fällt der Rücken noch vor der Klingenmitte gerade zur Spitze ein, während sich die gerade Schneide nach der Klingenmitte hochbiegt). Die direkten Vorbilder dieser Formen waren neben den Hauptformen IIIa1IIIa4 die Einzelformen Ia1.1 und IIa1.2 als zwei zueinander spiegelverkehrte Ausführungen einer Form mit einer insgesamt abgeschrägten und einer zum Teil abgeschrägten Kante. Alle weiteren Möglichkeiten wiederholen sich in Haupt- und Einzelformen der Leitformen Ia und IIa, Ib und IIb.

Die zwischen den Hauptformen IIIb4 (IIIc4 usw.) und IIIb13 (IIIc13 usw.), IIIb18 (IIIc18 usw.) und IIIb29 (IIIc29 usw.) auftretenden Unterbrechungen betreffen Formen, die zwar aufgrund weitergehender Variationsmöglichkeiten entwickelt wurden, für die in dieser Untersuchung behandelten Messer aber unzutreffend waren und deshalb auch nicht im Codeverzeichnis erscheinen. Sie durchbrachen durchweg die an den Anfang gestellten funktionalen und formalen Einschränkungen und ließen sich im Fundgut nicht eindeutig belegen. 

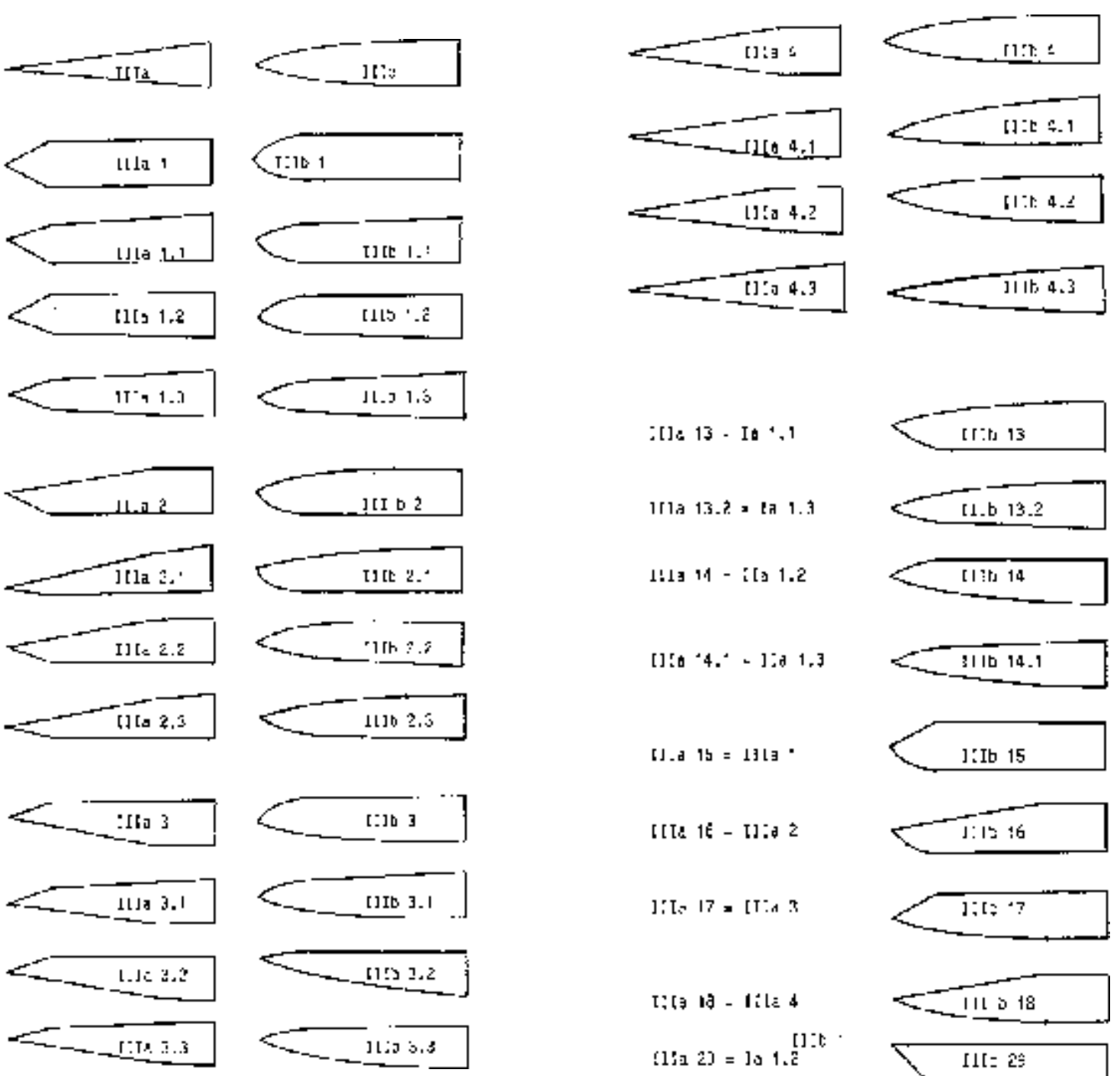

$[1]: 13-I d+1$

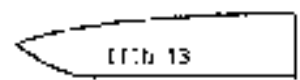

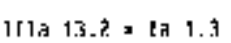

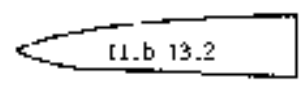

$1 ! 1314-\left[\begin{array}{lll}3 & 1.2\end{array}\right.$

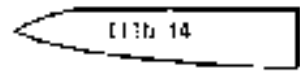

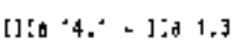

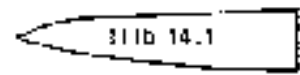

[ ] d $14=1113^{\circ}$

] Ib 15

[L] 1E- I]:d 2

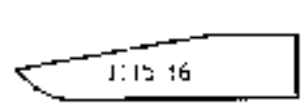

] T. $17=[1: 4: 7$

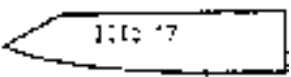

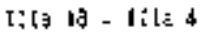

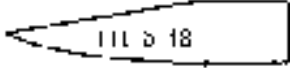

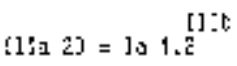

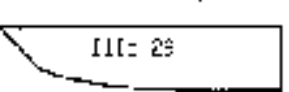

Abb. 20

Die Haupt- und Einzelformen der Leitformen III a und III $b$
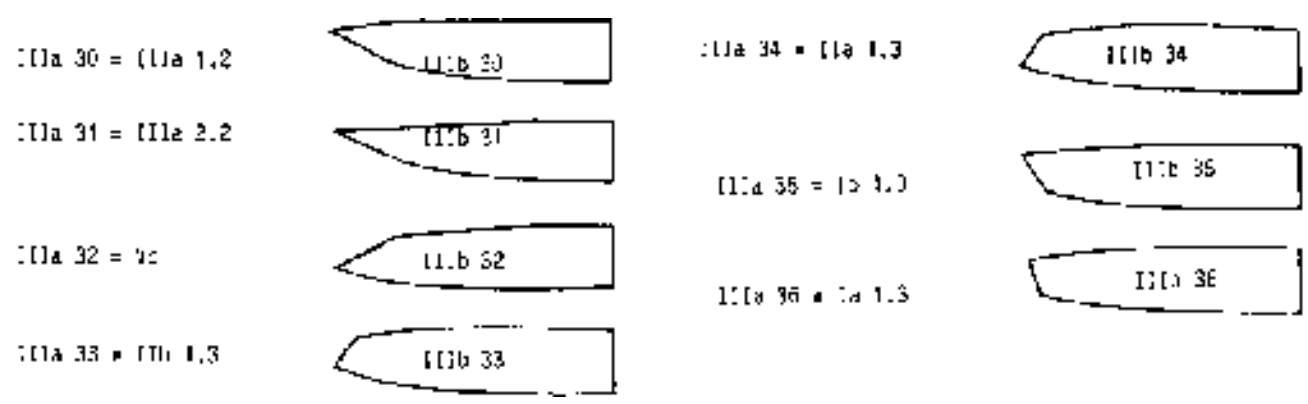

Abb. 20

Fortsetzung 


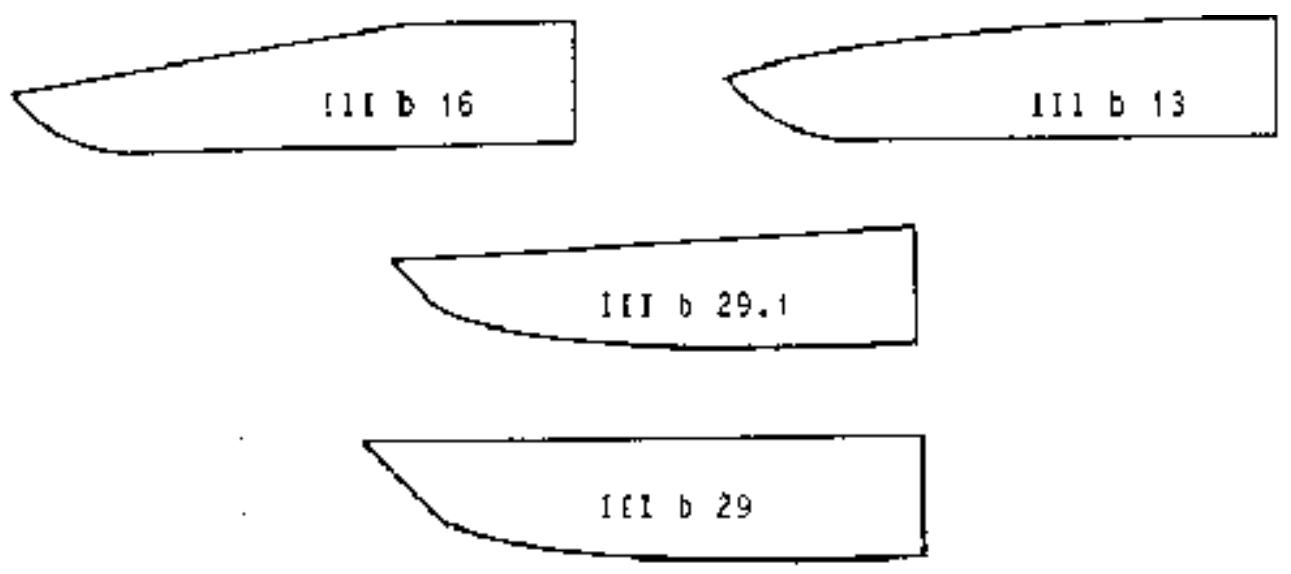

Die Lintwicklung der lionn UI b 29
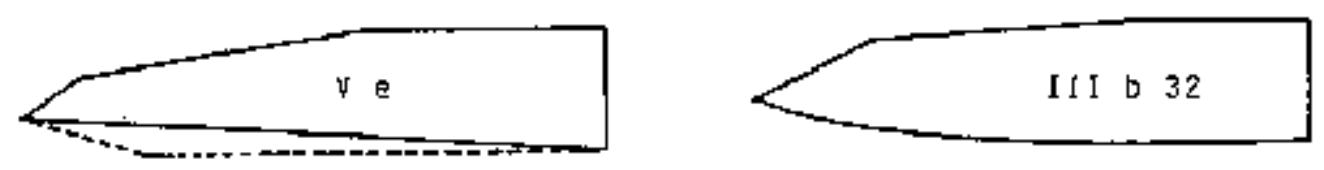

[Die [iotmen Ve und ItI h 32 - Lintersehicale

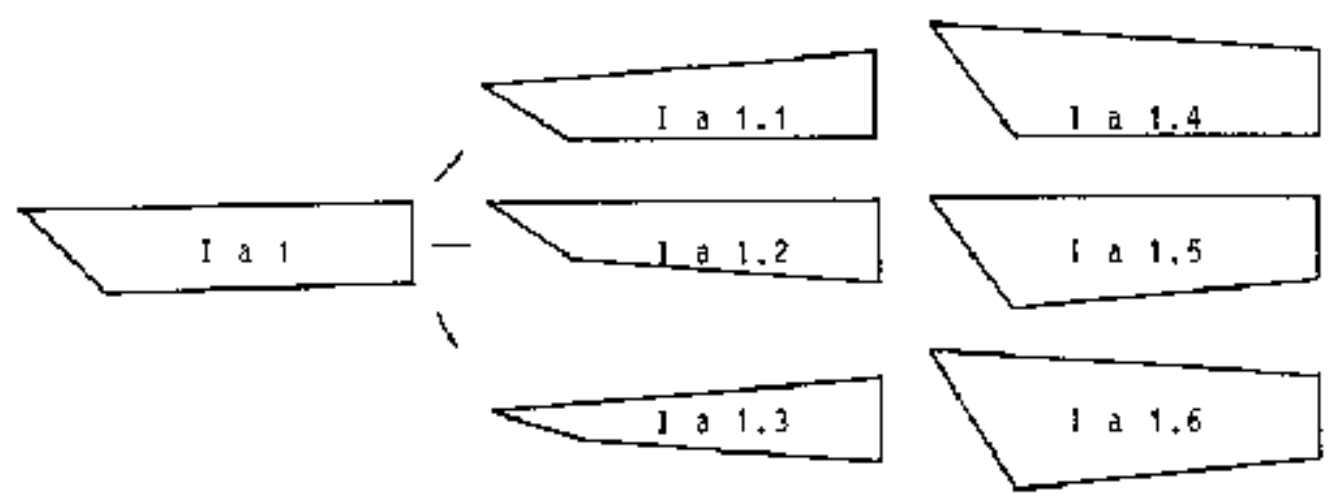

Die Feinumtergliederung der Finzelformen

Abb. 21 
Bei der Hauptform IIIb32 verläuft die Schneide wieder durchgängig gebogen vom Heftansatz zur Spitze. Durch die Richtungsänderung des letzten, gerade abgeschrägten Teiles der Schneide entsteht eine zweifache Abschrägung des Rückens, die sich bei gerade verlaufender Schneide auch in der Sonderform Ve findet (Abb. 21b).

Bei den Hauptformen IIIc29-IIIc32, IIId29-IIId32 und IIIe29-IIIe32 wurde das Prinzip der Leitform IIIa, die beidseitige Abschrägung, nicht mehr weiterentwickelt, da die so entstehenden Formen sich nur wiederholt hätten ${ }^{40}$.

In den Codierungstafeln 1 bis 3 am Anfang von Kapitel 5, Abschnitt 2 (Katalog) wurde nur ein Teil der Hauptformen graphisch dargestellt. Das war vor allem deshalb unproblematisch, da die Entwicklung der Hauptformen aus den elf Leitformen dem selben Schema folgt. Exemplarisch wurden unter anderem alle Hauptformen der Leitformen IaIc, IIa-IIc und IIIb abgebildet.

Die letzte Art der Untergliederung erfolgte durch die Einzelformen. Zu jeder Hauptform ergaben sich sechs Einzelformen, die sich durch unterschiedliche Neigung der beiden Kanten (Rücken und Schneide) zur Spitze voneinander unterscheiden. Die Formen Ia1.1-Ia1.6 mögen dies verdeutlichen (Abb. 21c).

Bei der Form Ia1.1 ällt der Rücken vom Heft zur Spitze unter einem Winkel von $90^{\circ}$ ein, während die Schneide genau in einem $90^{\circ}$-Winkel zum Heft steht.

Die Form Ia1.2 kehrt dieses Verhältnis um. Bei der Form Ia1.3 fallen Rücken und Schneide beide vom Heft zur Spitze unter einem Winkel von $90^{\circ}$ ein.

Die Form Ia1.4 läßt bei waagerechter Schneide den Rücken in einem Winkel von über $90^{\circ}$ vom Heft aufsteigen, die Form Ia1.5 ist die Umkehrung dieser Form, so daß hier die Schneide in einem Winkel von über $90^{\circ}$ nach unten wegfällt und der Rücken waagerecht zum Heft steht.

Die drei letzten Formen waren im erfaßten Fundmaterial nur gering vertreten, sie widersprachen in ihrer möglichen Handhabung vor allem dann der Funktion als Eßund Tafelmesser, wenn zusätzlich zu dieser Form die Klinge extrem breit und dicknackig war. ${ }^{41}$.

$40 \quad$ Nach der bereits beschriebenen Art der Proportionalitätsveränderung wurde die Vorgabe variiert, daß bei jeweils einer Abschrägung des Rückens und der Schneide je zwei der vier entstehenden Kanten gerade, zwei gebogen verlaufen. Bis auf die Formen IIId29, IIIe29, IIId30 und IIIe30 kommen die Haupt- und Einzelformen dieser Serie im Fundgut kaum vor. Die Hauptformen IIIb33-IIIb36, IIId33-IIId36 und IIIe33-IIIe36 wiederholen bereits bekannte Formen, wobei sie im Gegensatz zu den letzteren abgestumpfte Spitzen haben, die ihrerseits gebogen oder gerade verlaufen können.

Formen, bei denen der Rücken nach kaum einem Drittel oder schon nach wenigen Zentimetern meist gebogen zur Spitze einfällt, büßen ihre Grundform als spitzes Schneidemesser allerdings auch dann nicht ein, wenn der erste Teil des Rückens vor dem Einbiegen zunächst aufsteigt, wie z.B. bei der Form IIIe4.4. Bei den Formen Xxx.4-Xxx.6 mußte daher in jedem Fall bei der Anlage des Katalogs neu entschieden werden, ob die Gesamtform der Klinge der Funktion als Küchen-, Tafel- oder Mehrzweckmesser noch gerecht wurde oder nicht. Dabei wurde, um die Subjektivität dieser Entscheidung etwas auszugleichen, nach dem Prinzip verfahren, lieber ein strittiges Exemplar zuviel aufzunehmen, als ein möglicherweise auch nur selten zum Essen benutztes Mehrzweckmesser zuwenig. 
Bei den Formen, die sich durch mindestens eine insgesamt gebogene Kante ausweisen, fallen eine oder alle Möglichkeiten der Feinuntergliederung weg.

Auch die Einzelformen sind in der Codeübersicht nicht mehr alle abgebildet worden, da sich das Prinzip ständig wiederholt.

Mit den Codebezeichnungen IVa-IVj und Va-Vg wurden am Schluß der Codierungstafeln die Klingenformen aufgeführt, die sich aus dem bearbeiteten Fundmaterial ergaben, ohne vom Klassifizierungsmodell der Klingenformen erfaßt zu werden (Codierungstafel 4). Es handelt sich dabei um Formen, die von den funktionalen Vorgaben des Modells abweichen (IV) oder die bisherigen Kriterien weiter kombinieren (V).

\subsection{Kritische Anmerkungen zum Klassifizierungsmodell}

Die Klassifizierungsmodelle der Griffkonstruktionen und Klingenformen wurden vorrangig für die Anwendung in der Datenverarbeitung entwickelt. Die statistische Auswertung ausgewählter Kriterien, die für jedes Messer in den Codes der Verschlüsselung enthalten sind, hat ihre Verwendbarkeit nachgewiesen.

Bezüglich einer bereits in den Modellen enthaltenen historischen Aussagefähigkeit sind sie grundsätzlich unterschiedlich zu bewerten:

Das Modell der Griffkonstruktionen ist induktiv erarbeitet worden und stellt daher bezüglich seiner Anwendung auf historische Fragestellungen die wissenschaftliche Anwendung vor keine gravierenden Probleme.

Es ist listenartig angelegt und als Reihung der verschiedenen Möglichkeiten unbegrenzt ausbaufähig. Die Konstruktionsvarianten, die sich zwar auch aus einer gewissen Gesetzmäßigkeit, aber doch vor allem aufgrund erfaßter Einzelformen ergaben, wurden mit aufgenommen, so daß sich innerhalb des Modells eine in sich geschlossene Abfolge von Formen ergibt.

Diese kann durchaus als Grundlage für eine Typologie der Griffkonstruktionen bestimmter räumlicher oder zeitlicher Abschnitte dienen, wie sie innerhalb dieser Arbeit am Beispiel niederländischer Messer des 14. bis 17. Jahrhunderts beispielhaft belegt worden ist.

Bei der Entwicklung des Modells der Klingenformen hatten jedoch Aspekte bezüglich der Verwendbarkeit in der Datenverarbeitung Vorrang, da für eine Einarbeitung chronologischer Daten jede wissenschaftliche Grundlage in Form bisheriger Forschungsergebnisse fehlte.

Das angewandte Ordnungsprinzip geht von den grundsätzlich möglichen Grundformen aus und führt über festgelegte Veränderungen am Verlauf der Klingenkanten zu verschiedenen Einzelformen, die sich schrittweise und in Abhängigkeit von den jeweiligen Grundformen ergeben.

Dieses Verfahren entsprach der Gesamtabsicht, im Katalog alle Messertypen zusammenzufassen, die zwar regelmäßig, aber nicht unbedingt ausschließlich zum Essen benutzt worden waren. 
So folgt das Modell der Klingenformen einem formalen Entwicklungsschema. Vorgegebene Gesetzmäßigkeiten waren das Prinzip der Funktionalität und das Entwicklungsprinzip von einfachen zu differenzierteren Formen. Dieses Experiment erwies sich nach seiner Erstellung als durchaus fähig, die Menge der aufgenommenen Messer in ihrer Formenbreite abzudecken. Sieben Sonderformen waren durch Kombination bereits im Modell vorhandener Formen bedingt, zehn Sonderformen nicht integrierbar. Sie wurden als induktiv ermittelte Formen an das Modell angehängt. Möglichkeiten der Erweiterung des Modells bestehen durchaus, sei es durch zusätzliche Vorgaben bei der Erstellung der Leitformen oder auch durch weitergehende Variierungsmöglichkeiten bei den Haupt- und Einzelformen. Da aber innerhalb des Modells ein sehr großer Teil von Formen durch ergrabene Exemplare nicht abzudecken war, die Formenbreite des Modells die des ergrabenen Bestandes also schon übersteigt, sollte eine Veränderung oder Ausweitung des Modells zweckmäßigerweise erst dann erfolgen, wenn auch die viel größeren Mengen an geborgenen Messern aus den Magazinen von Instituten und Museen bearbeitet worden sind. 


\section{Der Katalog}

\subsection{Einführung in den Katalog}

Der zweibändige Katalogteil beinhaltet 1.300 Messer, die jeweils abgebildet und auf der gegenüberliegenden Seite beschrieben worden sind. Sie stammen mit einigen Ausnahmen aus der Zeit von 750/800 n. Chr. bis 1650 n. Chr.

Der Katalog ist nach den einzelnen Untersuchungsländern geordnet: Skandinavien (277 Messer), die Niederlande (135 Messer), die Bundesrepublik Deutschland ohne die neuen Bundesländer (206 Messer), die ehemalige DDR (221 Messer), Polen (376 Messer) und der nordwestliche Teil der ehemaligen UdSSR (85 Messer).

Das bezogen auf die absoluten Zahlen angetroffene Ost-West-Gefälle im Bereich der publizierten Sachkultur des Mittelalters machte es zusätzlich zu den bereits angesprochenen Beweggründen erforderlich, für die west- und nordeuropäischen Länder zum bereits veröffentlichten Bestand unpublizierte Gruppen aus Museen mitaufzunehmen, um in etwa ein Gleichgewicht herzustellen ${ }^{42}$.

Die unveröffentlichten Bestände machen etwas mehr als ein Drittel der Gesamtzahl der aus diesen Ländern erfaßten Messer aus. Die Bestände der übrigen Länder sind durchweg der Literatur entnommen worden.

Der Textteil ist listenförmig angelegt und in drei Sparten aufgeteilt worden, die vor der ersten Sparte befindliche Numerierung erfolgte für jedes Land aus Gründen der Übersichtlichkeit separat.

In der ersten Textsparte befinden sich alle Angaben zum Fundort, und zwar in Abfolge Name und Bezirk oder Kreis des geographischen Ortes, dann die Bezeichnung des eigentlichen Fundplatzes (wie Straßenoder Grundstücksname, Name des Stadtviertels o.ä.), danach Angaben zur Stratigraphie und - seltener Kurzerklärungen zur historischen Einordnung des Fundplatzes. Alle Angaben wurden den jeweiligen Publikationen entnommen und mit Kurzzitaten (Seitenangaben) versehen. Angaben aus sekundär herangezogenen Publikationen wurden mit erweiterten Kurzzitaten (Autor, Erscheinungsjahr und Seitenangaben) versehen. Bei einigen wenigen Fundplätzen wurde ein Abriß der gesamten historischen Entwicklung mit Angaben zu den ökonomischen und sozialen Grundlagen der Bewohner verfaßt.

\footnotetext{
Von 135 niederländischen Messern sind 65 unveröffentlicht. Sie stammen fast alle aus dem Rotterdamer Museum Boymans-van Beuningen.

Von 277 skandinavischen Messern sind 101 Messer unveröffentlicht. Sie wurden vom Museum der Universität Oslo (Oldsaksamling), vom Museum Kulturen in Lund, vom städt. Museum Roskilde und vom dänischen Nationalmuseum in Kopenhagen zur Verfügung gestellt. Die 60 unveröffentlichten Messer aus der alten Bundesrepublik entstammen neben Museen auch Sammlungen und Archiven.
} 
Generell war eine einheitliche Beschreibungsgrundlage für alle Fundplätze wegen der unterschiedlichen Publikationsstandards nicht zu erreichen. Die Extrema reichten von Schwierigkeiten, den Fundort überhaupt festzustellen, bis zu Versuchen der Autoren, das von ihnen publizierte Fundgut bestimmten Personen zuzuordnen. Somit richtet sich die Genauigkeit der Angaben zum Fundort auch nach den individuellen Publikationen, denen die Messer entnommen wurden.

Die zweite Textsparte beinhaltet Angaben zur Zeitstellung der jeweiligen Messer. Diese Angaben korrespondieren zum Teil mit stratigraphischen Angaben in der ersten Sparte.

Bei der Frage der Datierung ergaben sich die größten Probleme aufgrund der Unterschiedlichkeit der Publikationsweisen. In einigen Fällen waren überhaupt keine oder nur sehr grobe Zeitrahmen angegeben worden, so daß - wie für viele der unpublizierten Messer - Datierungsvorschläge aufgrund von Vergleichen mit enger datierbaren Messern gemacht werden mußten. In nicht wenigen Fällen waren Messer und anderes Sachgut zur Illustration der Grabungsberichte ohne Angaben zur Größe und zur Zeitstellung angefügt worden. Sehr oft entsprechen die im Katalog angegebenen Zeitstellungen auch der Gesamtbesiedlungsdauer oder einer bestimmten Besiedlungsphase des jeweiligen Fundplatzes. In einigen sehr günstigen Fällen, die fast ausschließlich osteuropäischen Publikationen betreffen, war die gesamte stratigraphische Auswertung listenförmig mitveröffentlicht worden, so daß die hier geborgenen Messer genauer zu datieren waren.

Die Zeitangaben in der zweiten Sparte geben demnach oft nur einen Anhaltspunkt über die Zeit der Verwendung bzw. des Verlustes eines Messers. Angaben über den Zeitpunkt der Herstellung sind davon fast nie betroffen.

In der dritten Textsparte befindet sich eine Kurzbeschreibung zu jedem Messer. Die Abkürzungen A, G, K und T bedeuten Art des Messers (Griffzungenmesser, Griffangelmesser, selten Klappmesser), Angaben zum Griff, Angaben zur Klinge und die Typbezeichnung ${ }^{43}$.

Die Angaben in dieser Sparte sind vom Erhaltungsgrad des jeweiligen Messers abhängig. Im günstigsten Fall enthält die Kurzbeschreibung Erläuterungen zur Art und zum Material des Griffs oder zu seiner Verarbeitung, weiterhin Angaben über Verzierungen sowie eine exakte Beschreibung der Klingenform.

Die Angaben zur Form und zum Material unter G (Griff) und K (Klinge) betreffen grundsätzlich den jetzigen Zustand eines Messers; sie wurden bei den unpublizierten Messern vom Autor am Material selbst aufgenommen oder ihm von den jeweiligen Museen und Institutionen auf Anfrage zur Verfügung gestellt. Bei den publizierten Messern wurden die Angaben aus der Literatur entnommen, soweit sie dort aufgeführt waren. Angaben zu einer rekonstruierten Klingen- oder Grifform 
sind mit dem Hinweis "rek." am Ende der Verschlüsselung kenntlich gemacht worden.

Die Breite der Klinge, die Dicke des Klingenrückens und die Länge des Griffs konnten aus mehreren Gründen nicht berücksichtigt werden.

Zunächst war nur ein kleiner Teil des publizierten Fundgutes in allen Ansichten abgebildet worden, so daß zum Beispiel eine Erfassung der Dicke des Rückens (in der Literatur auch oft als Klingen-"nacken" bezeichnet) nicht möglich war. Die Breite (Höhe) der Klinge war nur bei vollständig erhaltenen Klingen zu ermitteln. Um nicht noch zusätzlich hypothetische Daten in die Auswertung einzubringen, wurde von einer Aufnahme dieses Maßes in die Verschlüsselung der Typbezeichnung ebenfalls Abstand genommen.

Die Länge und Breite des Griffs war nur bei erhaltenen Griffen zu ermitteln. Die Griffangel war in der Regel kürzer als der auf diese gesteckte Vollgriff (Hilze). Insgesamt war für die Nichtaufnahme der Griffmaße die Überlegung ausschlaggebend, daß sie sich zumeist an die Maße der menschlichen Hand mit einer Länge zwischen 7 und $12 \mathrm{~cm}$ anlehnen.

Weiterhin wurde erfaßt, ob die Klinge zum Griff ober- oder unterständig steht. Dieses Kriterium ist bereits früher schon Gegenstand von Untersuchungen gewesen ${ }^{44}$. Ohne allerdings von diesem Faktor Funktionen abzuleiten, wurde lediglich versucht, das Kriterium selbst zu definieren. Griffober- oder -unterständigkeit wurde erst dann erkannt, wenn der Richtungsverlauf der Klinge erkennbar von dem des Griffs abwich. Nicht erforderlich für dieses Kriterium war, daß die Klingenspitze über oder unter die Messerlängsachse ragen mußte. Schwache Ober- oder Unterständigkeit der Klinge lag dann vor, wenn die Abweichung der Richtungsverläufe von Griff und Klinge nur mit auf die Abbildung projizierten Hilfslinien festzustellen war (Abb. 22).

Für jedes Land wurde ein eigenes Literaturverzeichnis geschaffen, um die Übersichtlichkeit in der Menge der benutzten Literatur $\mathrm{zu}$ bewahren und um die Handhabung des Katalogs nicht unnötig zu erschweren. Dort wird die Numerierung des Katalogs wiederholt; unter jeder Nummer ist die für dieses Messer benutzte Literatur zitiert worden. Gleichzeitig wurden die Abbildungsnachweise und die $\mathrm{Zi}$ tate der Textstellen, die sich in der Literatur explizit mit diesem Messer beschäftigen, aufgeführt. Die im Katalogtext zusätzlich verwandte Literatur, die dort als Kurzzitat kenntlich gemacht worden ist, wurde im Anschluß an dieses Verzeichnis separat aufgeführt.

Im Anschluß daran befindet sich ein Abkürzungsverzeichnis, welches die benutzten Zeitschriften, Reihen u.a. aufführt. 
Die im Katalogtext benutzten Abkürzungen sind allgemein gebräuchlich und ergeben sich aus dem Zusammenhang.

Da der Katalogtext listenförmig angelegt worden ist, wurde auf eine ständige Wiederholung gleicher Fundorte, Fundplätze, stratigraphischer Angaben oder Datierungsangaben sowie deren Nachweise durch die Angaben von Seitenzahlen verzichtet. An ihrer Stelle erscheint die Abkürzung s.o. (= siehe oben). Sie bezieht sich grundsätzlich auf die Zeile der darüberstehenden Katalognummer.
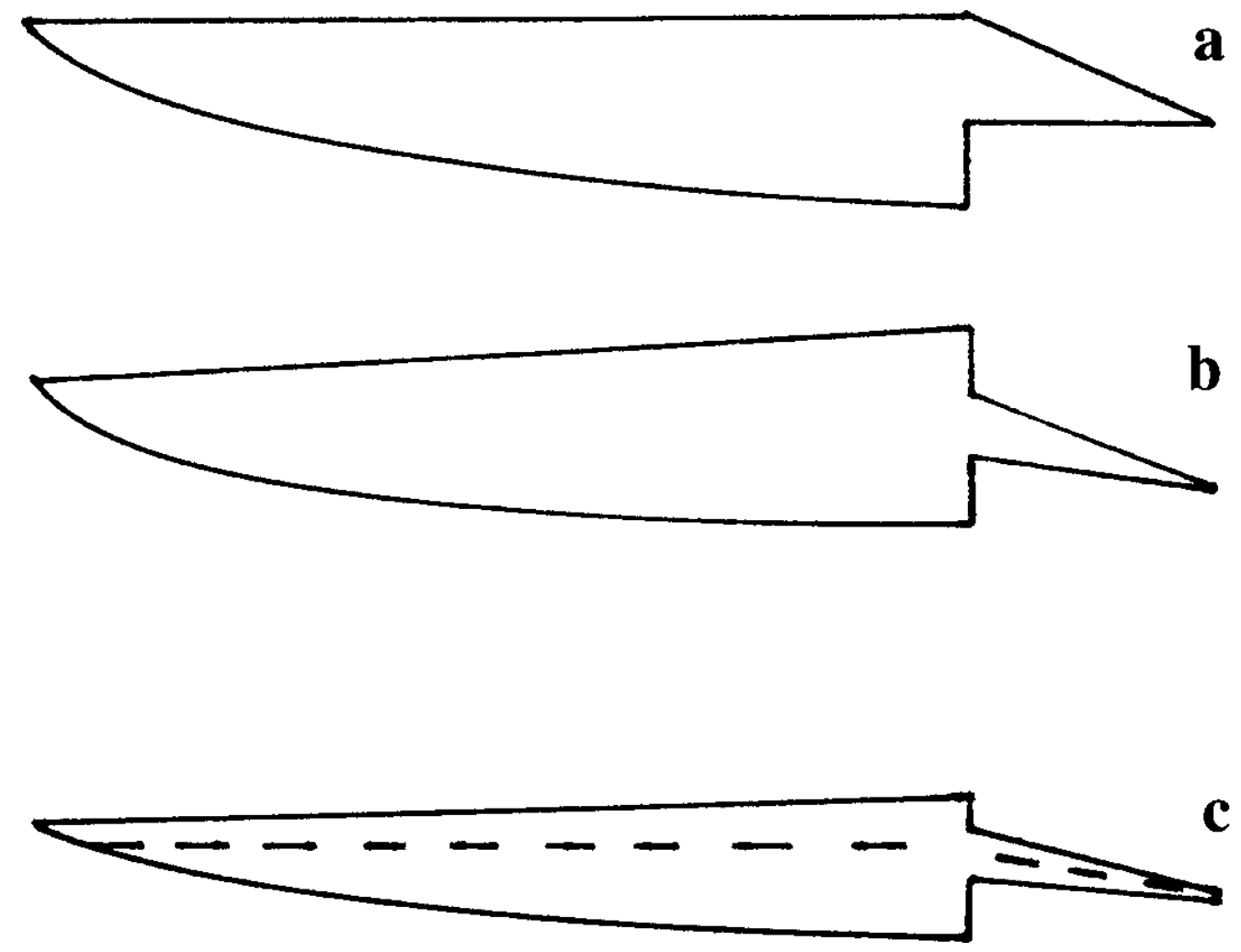

Abb. 22

a: Nicht griffunterständig; $b$ : Griffunterständig

c: Schwach griffunterständig 


\section{Die häufigsten Abkürzungen im Katalogtext}

o.n.A. ohne nähere Angaben
o.A. ohne Angaben

u.A. unter Abbildung

T.u.Abb. Text unter Abbildung

s. siehe

s.o. siehe oben

S. Seite

Abb. Abbildung

u.a. unter anderem, und andere (nachgestellt)

vgl. Anm. vergleiche Anmerkung

v.a. vor allem

ma. mittelalterlich

nztl. neuzeitlich

a.a.O. am angegebenen Ort
A. Art des Messers
G. Griff (Beschreibung)
K. Klinge (Beschreibung)
T. Verschlüsselung für die computergestützte statistische Auswertung

\subsection{Der codierte Katalog}

Die folgenden sechs Codierungstafeln umfassen alle Formen des Klassifizierungsmodells, nach denen das im Katalog zusammengefaßte Fundmaterial codiert, gespeichert und ausgewertet worden ist.

Im Anschluß an die Übersichtstafeln der codierten Formen und Konstruktionen wird der Bestand des Katalogs in seiner gespeicherten Form aufgeführt.

Er enthält Angaben zur Katalognummer, zur Klingenform, zur Griffkonstruktion, zu Griffmaterialien, zur Klingenlänge, zu Klingen- und Griffverzierungen und zur Zeitstellung und ist nach den einzelnen Untersuchungsländern geordnet worden. 

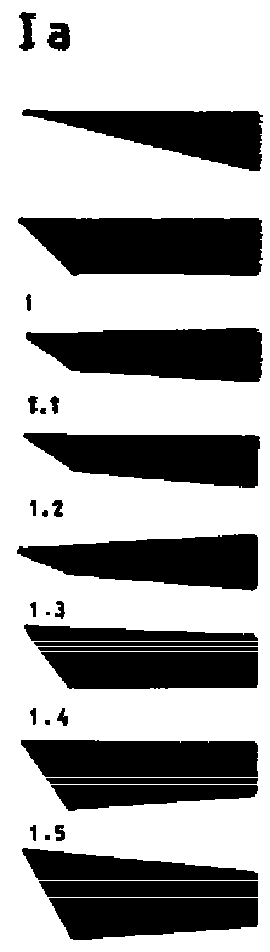

1.6

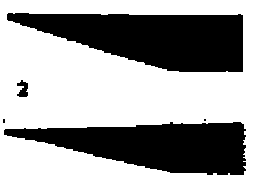

2.t

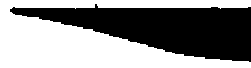

2,2

2,3
I b
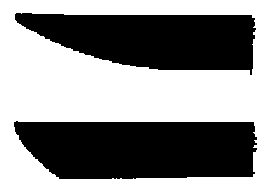

1

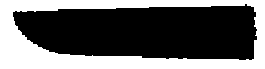

1.1

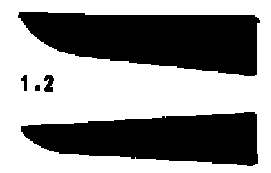

1.3

1.4

1.5

1.6

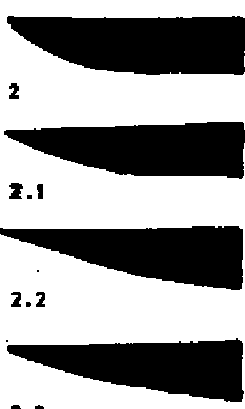

2.3
Ic

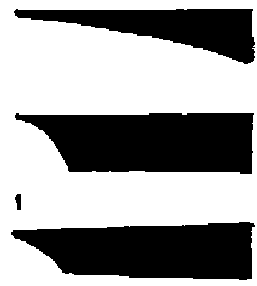

1,1

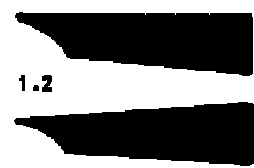

1.3

1.4

1.5

1.6

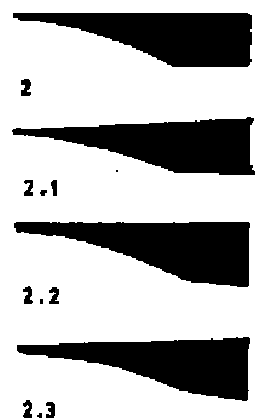

Codierungstafel 1 
II a
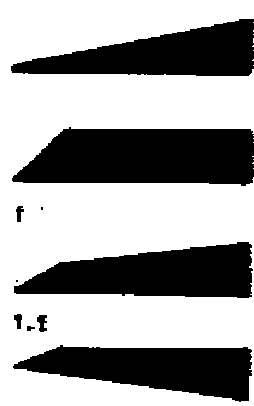

1.2

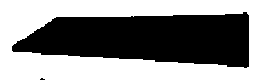

1.3

1.4

1.5

1.6

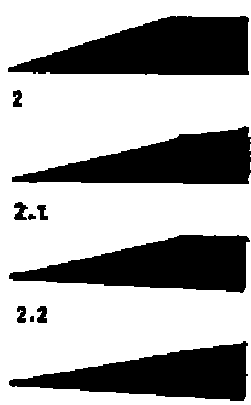

2.3
II b
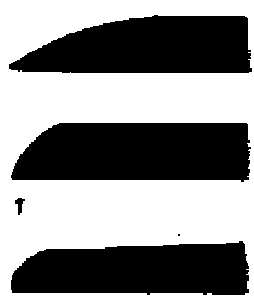

$\mathbf{f - 1}$

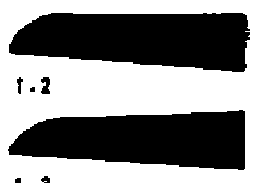

1,3

1.4

1.5

1.6

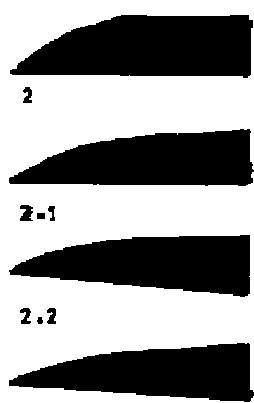

2.3
II C

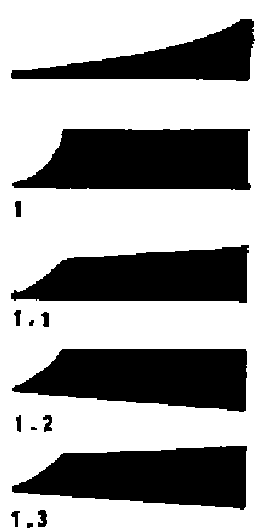

1.4

$1 \cdot 5$

1.6

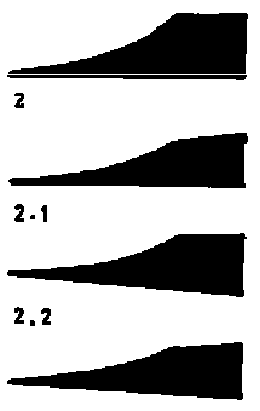

2.3

Codierungstafel 2 
$-68-$
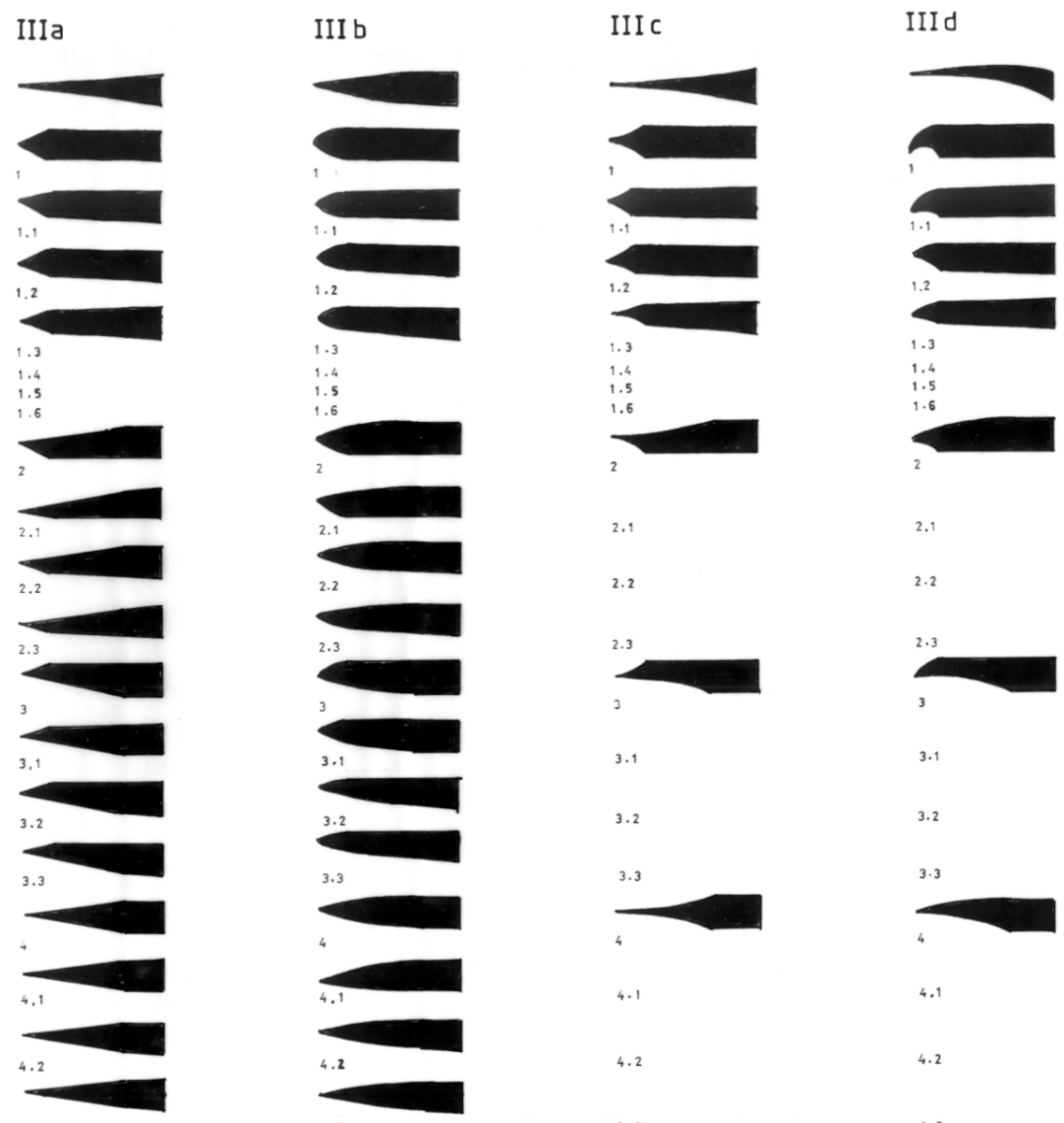

III e
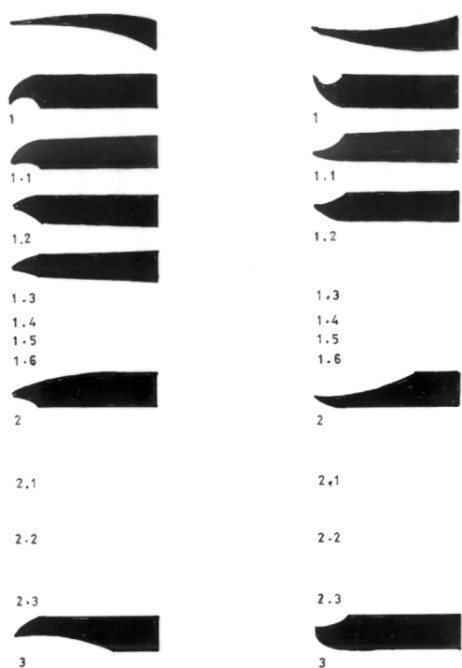

4.3
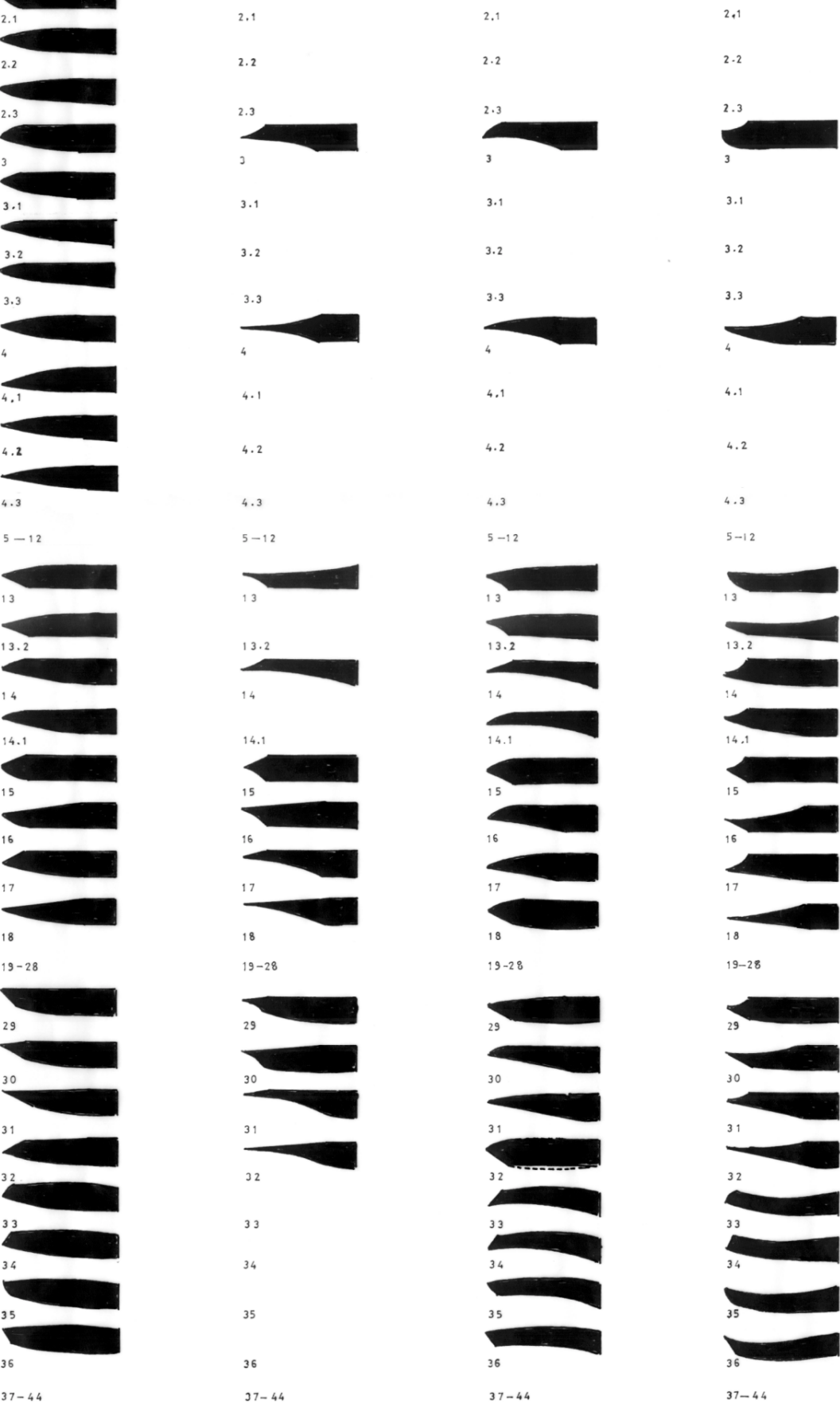

4.1

$5-12$
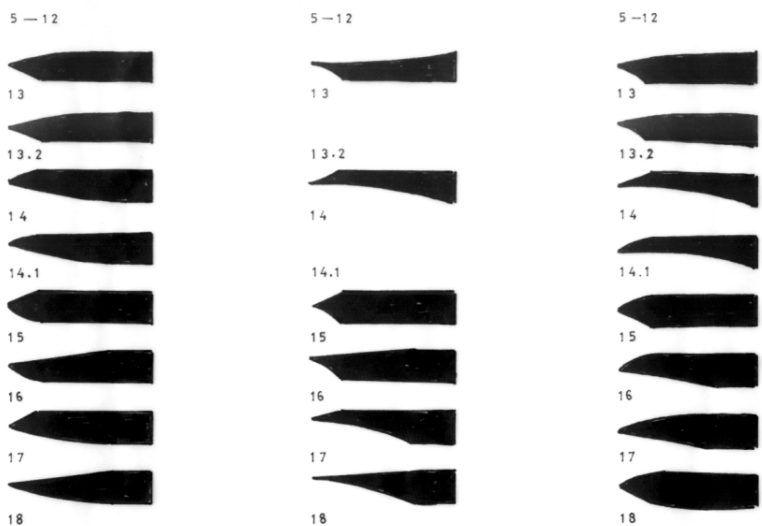

$9-28$

:9-28

4.1
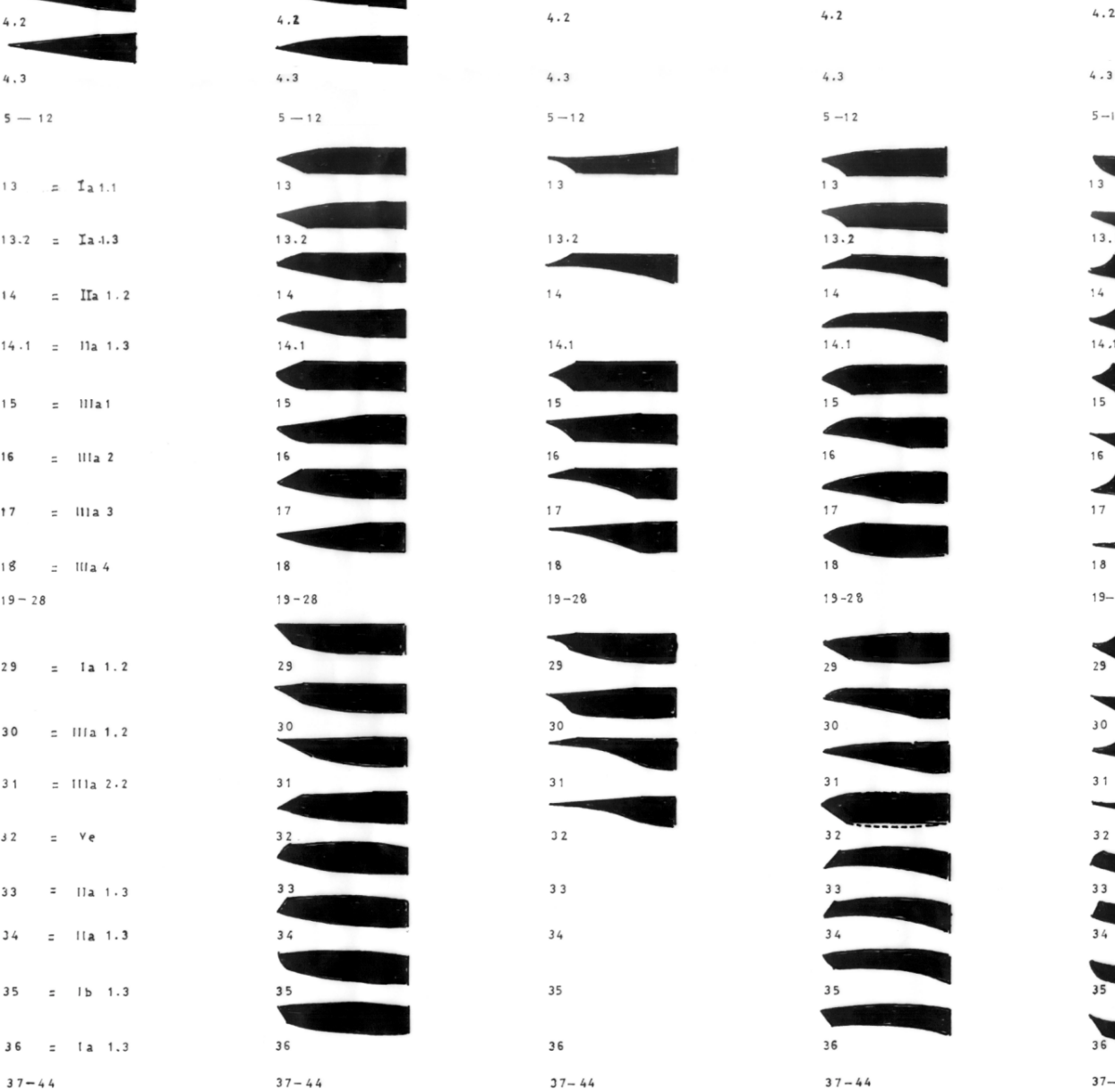


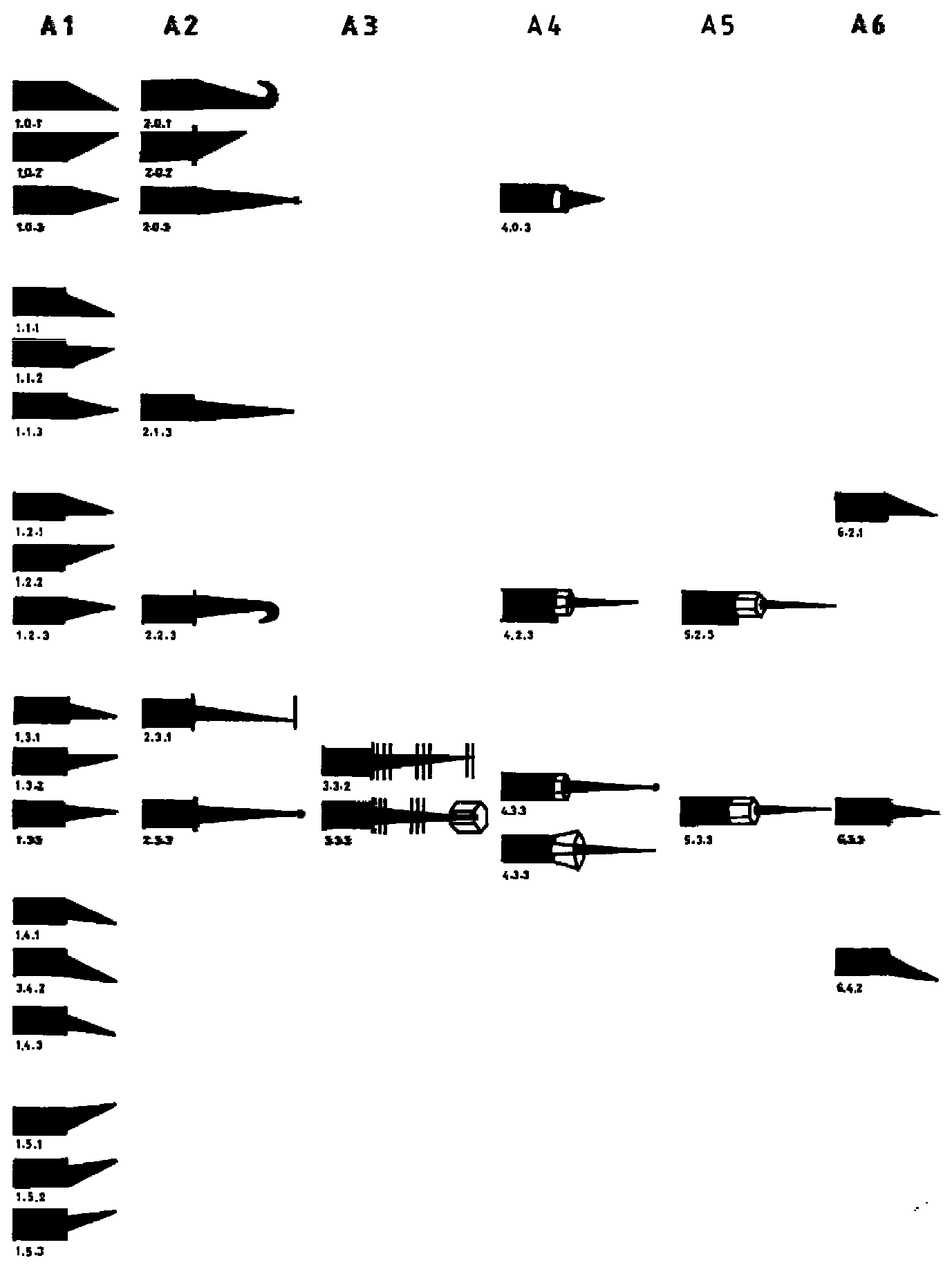

Codierungstafel 4 
-70 -

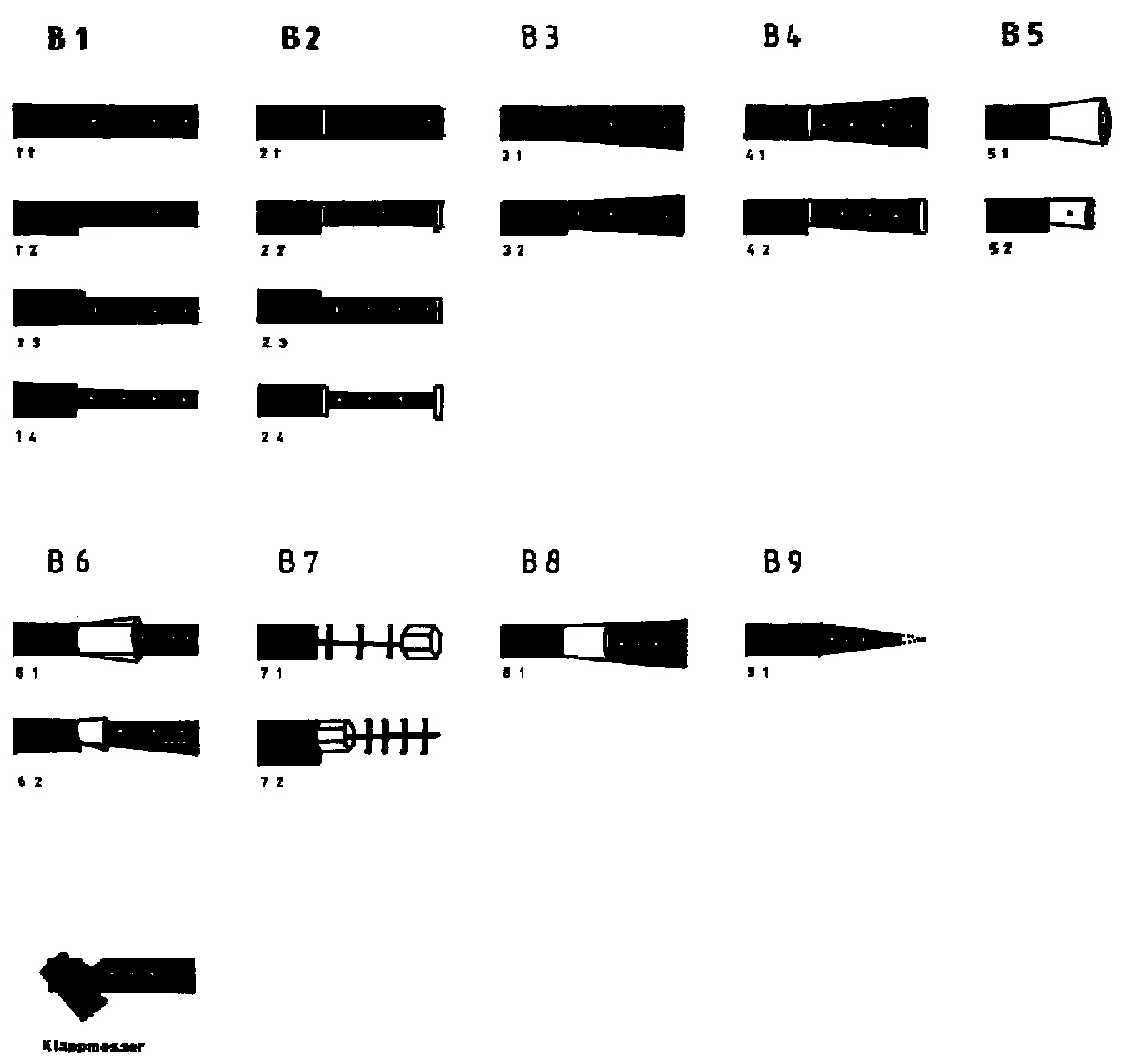

Codierungstafel 5 


\section{Sonderformen IV+V}

IV
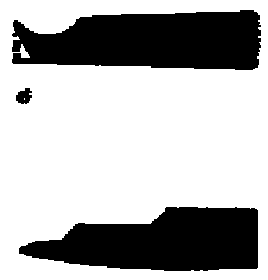

5

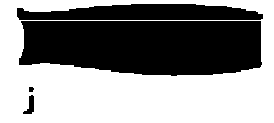

V

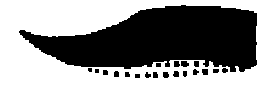

2

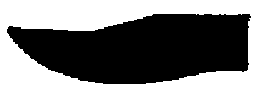

d

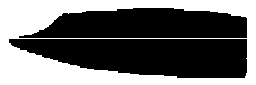

곡
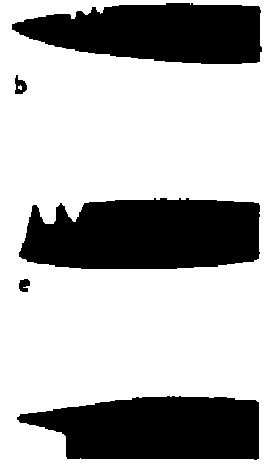

h
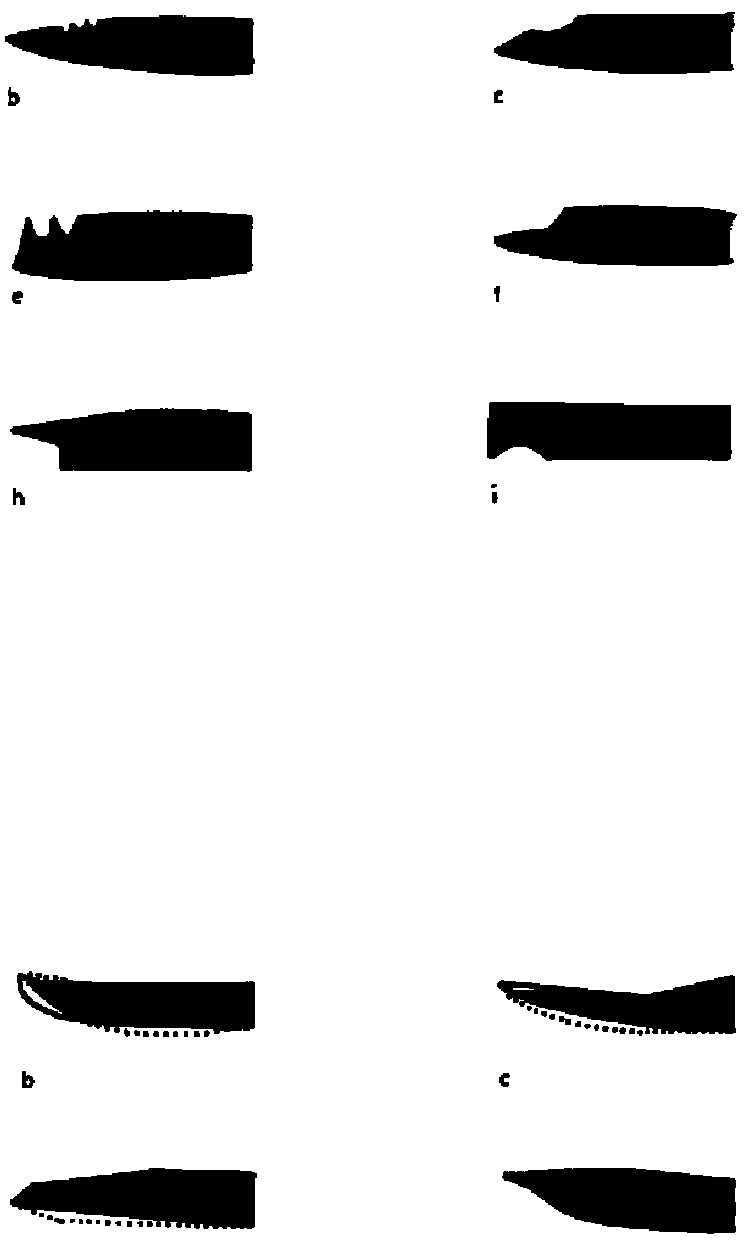
- 72 - 


\section{Der codierte Katalog}

\begin{tabular}{|c|c|c|c|c|c|c|c|}
\hline \multicolumn{8}{|c|}{ Niederlande } \\
\hline $11 \mathrm{C}$ & $3 \mathrm{~B}$ & A143 & $\mathrm{H} 1$ & & 11,40 & 1250 & -1300 \\
\hline $21 \mathrm{C}$ & 3B141 & A133 & & & 11,40 & 1300 & -1400 \\
\hline $31 \mathrm{C}$ & $3 \mathrm{~B}$ & A143 & & & 10,60 & 1300 & -1400 \\
\hline $4 \mathrm{AC}$ & $4 \mathrm{~F} ?$ & A143 & & & 14,00 & & \\
\hline $51 \mathrm{C}$ & 3B4 & A131 & & & 13,00 & & \\
\hline $61 \mathrm{C}$ & 3E29 & B32 & & & 25,00 & 1450 & -1550 \\
\hline $7 \mathrm{1C}$ & $3 \mathrm{~B}$ & B41 & H1 & B & 9,50 & 1500 & -1550 \\
\hline $81 \mathrm{C}$ & $3 \mathrm{~B}$ & B42 & $\mathrm{H} 1$ & & 12,00 & 1450 & -1550 \\
\hline $8 \mathrm{~A} 1 \mathrm{C}$ & & B42 & $\mathrm{H} 1$ & $\mathrm{~B}$ ? & & 1450 & -1550 \\
\hline $9 \mathrm{1C}$ & 2B1 & A221 & $\mathrm{H} 1$ & B & 11,50 & 1500 & -1550 \\
\hline $101 \mathrm{C}$ & $4 \mathrm{~F}$ & B41 & $\mathrm{K}$ & $\mathrm{B}$ & 11,50 & 1500 & -1550 \\
\hline $11 \mathrm{1B}$ & 3B14 & A231 & E3 & B E1 & 10,80 & 1450 & -1550 \\
\hline $121 \mathrm{~B}$ & $2 \mathrm{~B}$ & A133 & & & 17,00 & 1100 & -1300 \\
\hline $131 \mathrm{~B}$ & 3E4 & A133 & & & 11,00 & 650 & -900 \\
\hline $141 \mathrm{~B}$ & $5 \mathrm{~A}$ & A131 & & & 10,00 & 650 & -900 \\
\hline $151 \mathrm{~B}$ & $3 \mathrm{~B}$ & A132 & & & 11,40 & 650 & -900 \\
\hline $161 \mathrm{~B}$ & $4 \mathrm{~F}$ & A133 & & & 8,60 & 650 & -900 \\
\hline $17 \mathrm{1B}$ & 3E4 4 & A152 & & & 7,60 & 650 & -900 \\
\hline $18 \mathrm{1B}$ & $3 \mathrm{~A}$ & A152 & H1 & & 6,80 & 650 & -900 \\
\hline $191 \mathrm{~B}$ & $2 \mathrm{~B}$ & A131 & & & & 700 & -900 \\
\hline $201 \mathrm{~B}$ & $3 \mathrm{E} 24$ & A133 & & & & 700 & -900 \\
\hline $21 \mathrm{1B}$ & 3E1 4 & A133 & & & & 700 & -900 \\
\hline $22 \mathrm{1B}$ & $3 \mathrm{~B} 154$ & A133 & & & & 700 & -900 \\
\hline $231 \mathrm{~B}$ & $2 \mathrm{C} 13$ & A131 & & & & 700 & -900 \\
\hline $24 \mathrm{1B}$ & 3E1 & A133 & & & & 700 & -900 \\
\hline $251 \mathrm{~B}$ & & & $\mathrm{~K}$ & & & 650 & -900 \\
\hline $261 \mathrm{~B}$ & & A1? & $\mathrm{K}$ & & & 650 & -900 \\
\hline $271 \mathrm{~B}$ & $2 \mathrm{~B}$ & A243 & & & & 1200 & -1300 \\
\hline $281 \mathrm{~B}$ & & B32 & $\mathrm{K}$ & & & 1350 & -1500 \\
\hline $291 \mathrm{~B}$ & & $\mathrm{~A} 1 ?$ & $\mathrm{~K}$ & & & 1400 & -1500 \\
\hline $301 \mathrm{~B}$ & $3 \mathrm{~B} 153$ & B42 & $\mathrm{K}$ & B & 8,50 & 1400 & -1500 \\
\hline $31 \mathrm{1B}$ & $3 \mathrm{~B} 13$ & A233 & H1 & & 11,30 & 1300 & -1400 \\
\hline $32 \mathrm{1B}$ & $4 \mathrm{~F}$ & A23? & H1 & & 12,30 & 1300 & -1350 \\
\hline $331 \mathrm{~B}$ & 3E301 & B12 & $\mathrm{H} 1$ & B & 10,60 & 1400 & \\
\hline $341 \mathrm{~B}$ & $3 \mathrm{~B} 165$ & B42 & $\mathrm{H} 1$ & B E1 & 8,20 & 1500 & -1600 \\
\hline $351 \mathrm{~B}$ & $3 \mathrm{~B}$ & B42 & $\mathrm{K}$ & E3E1 & 10,60 & 1350 & -1400 \\
\hline $361 \mathrm{~B}$ & $3 \mathrm{~B} 13$ & B42 & $\mathrm{H} 2$ & B & & 1400 & -1450 \\
\hline $37 \mathrm{1B}$ & $3 \mathrm{~B} 33$ & A4? & $\mathrm{H} 1$ & E1 & & 1300 & -1350 \\
\hline $381 \mathrm{~B}$ & $3 \mathrm{~B} 162$ & A 233 & $\mathrm{~K}$ & & 14,80 & 1300 & -1350 \\
\hline
\end{tabular}




\begin{tabular}{|c|c|c|c|c|c|c|c|}
\hline 39 1B & 3B14 & A243 & B & & 7,00 & 1450 & -1500 \\
\hline 40 1B & 3B14 & B42 & $\mathrm{K}$ & & 7,40 & 1300 & -1500 \\
\hline 41 1B & 2B0 2 & B42 & $\mathrm{K}$ & B & 7,10 & 1450 & -1500 \\
\hline 42 1B & 2B1 3 & B12 & E1 & $?$ & 5,60 & 1450 & -1500 \\
\hline 43 1B & 3B141 & B42 & H1 & B E1 & 10,10 & 1400 & \\
\hline 44 1B & 3B141 & B32 & H1 & & 9,50 & 1500 & -1550 \\
\hline 45 1B & 3B & B42 & & E2 & 9,60 & 1500 & -1550 \\
\hline 46 1B & 3B & A223 & E2 & & 14,80 & 1550 & -1600 \\
\hline 47 1B & 3B & A223 & E2 & & 14,30 & 1550 & -1600 \\
\hline 48 1B & 2B1 1 & A123 & H1 & & 11,10 & 1600 & \\
\hline 49 1B & & A2 & B & E3 & & 1600 & -1650 \\
\hline $501 \mathrm{~B}$ & 3B141 & B41 & & E1 & 8,90 & 1450 & -1500 \\
\hline $511 \mathrm{~B}$ & 3B141 & B42 & & B & 9,50 & 1500 & -1550 \\
\hline 52 1B & & A2? & $\mathrm{B}$ & E3 & & 1600 & -1700 \\
\hline 53 1B & 3B & B42 & & B & 8,80 & 1500 & -1550 \\
\hline 54 1B & $3 \mathrm{~B}$ & B42 & H1 & E1 & 9,60 & 1500 & -1550 \\
\hline 55 1B & & A633 & & & 12,50 & 1100 & -1300 \\
\hline $561 \mathrm{~B}$ & $3 \mathrm{~B}$ & A133 & & & 18,75 & 1100 & -1300 \\
\hline 57 1B & 2B1 4 & B42 & $\mathrm{K}$ & & 7,80 & 1550 & -1600 \\
\hline 58 1B & & A1? & K & & & 1300 & -1350 \\
\hline 59 1B & $5 \mathrm{D}$ & A1? & H1 & & 6,75 & 1300 & -1350 \\
\hline 60 1B & 4D0 3 & A1? & H1 & & 12,00 & 1300 & -1350 \\
\hline $61 \mathrm{1B}$ & $3 \mathrm{~B} 13$ & A2? & H1 & & 12,00 & 1300 & -1350 \\
\hline 62 1B & 3B141 & A4? & H1 & & 8,60 & 1300 & -1400 \\
\hline 63 1B & 1B0 1 & B42 & H1 & E1 & 11,50 & 1300 & -1350 \\
\hline 64 1B & 1B0 1 & B42 & H1 & & 11,00 & 1350 & -1450 \\
\hline 65 1B & 3E14 & B42 & H1 & E1 & 11,60 & 1350 & -1450 \\
\hline 66 1B & 3B & B42 & $\mathrm{H} 2$ & & 11,30 & 1450 & -1500 \\
\hline 67 1B & $3 \mathrm{~B}$ & B42 & H1 & E1 & 8,00 & 1450 & -1500 \\
\hline 68 1B & 3B141 & A223 & H1 & B E1 & 9,20 & 1450 & -1500 \\
\hline 69 1B & 3B141 & A233 & H1 & & 9,20 & 1450 & -1500 \\
\hline 70 1B & 3B & B42 & H1 & B E1 & 12,00 & 1450 & -1550 \\
\hline 71 1B & & B42 & B & & & 1450 & -1500 \\
\hline 72 1B & & B42 & B & & & 1450 & -1550 \\
\hline 73 1B & & B42 & & B & & 1450 & -1500 \\
\hline 74 1B & & B42 & & B & & 1450 & -1550 \\
\hline 75 1B & & B42 & & B & & 1450 & -1550 \\
\hline 76 1B & & B42 & & B & & 1450 & -1550 \\
\hline 77 1B & $3 \mathrm{~B}$ & B42 & H1 & B & 7,70 & 1450 & -1550 \\
\hline 78 1B & & B82 & E1? & & 7,50 & 1450 & -1550 \\
\hline 79 1B & & A2? & B & & & 1450 & -1550 \\
\hline 80 1B & $3 \mathrm{~B}$ & B41 & H1 & B & 6,00 & 1500 & -1550 \\
\hline 81 1B & & A522 & B H1 & & & 1500 & -1550 \\
\hline 82 1B & $3 \mathrm{~B} 155$ & A523 & E1B & & 9,20 & 1550 & \\
\hline 83 1B & & A223 & $\mathrm{B}$ & & 10,20 & 1550 & -1600 \\
\hline 84 1B & & A22? & B & & & 1550 & -1600 \\
\hline 85 1B & 3B141 & A223 & B & & 11,40 & 1550 & -1600 \\
\hline $861 \mathrm{~B}$ & 3B & A122 & E1 & & 10,50 & 1500 & -1600 \\
\hline 87 1B & $3 \mathrm{~B}$ & B82 & E1B & & 6,50 & 1550 & -1600 \\
\hline 88 1B & $3 \mathrm{~B} 33$ & B42 & $\mathrm{H} 2$ & B & 13,30 & 1550 & -1650 \\
\hline
\end{tabular}




\begin{tabular}{|c|c|c|c|c|c|c|c|}
\hline 89 1B & & $\mathrm{A} 2 ?$ & B & & & 1550 & -1650 \\
\hline $901 \mathrm{~B}$ & & A22? & B & E3 & & 1550 & -1650 \\
\hline $911 \mathrm{~B}$ & 2B1 & A22? & $\mathrm{K}$ & & 15,80 & 1600 & -1650 \\
\hline $921 \mathrm{~B}$ & & A22? & E2 & & & 1600 & -1700 \\
\hline $93 \mathrm{1B}$ & 3B141 & A22? & B & E3 & 7,80 & 1550 & -1600 \\
\hline $941 \mathrm{~A}$ & 3B171 & A423 & H1 & E2 & 12,50 & 1600 & \\
\hline $951 \mathrm{~A}$ & & $\mathrm{~A} 1 ?$ & $\mathrm{~K}$ & & & 1300 & -1400 \\
\hline $961 \mathrm{~A}$ & & B42 & $\mathrm{K}$ & & & 1300 & -1500 \\
\hline $971 \mathrm{~A}$ & & B32 & $\mathrm{K}$ & & & 1400 & -1500 \\
\hline $981 \mathrm{~A}$ & & B32 & $\mathrm{K}$ & & & 1400 & -1500 \\
\hline $991 \mathrm{~A}$ & & B42 & $\mathrm{K}$ & & & 1400 & -1500 \\
\hline $1001 \mathrm{~A}$ & & B42 & $\mathrm{K}$ & & & 1400 & -1500 \\
\hline $1011 \mathrm{~A}$ & & B42 & $\mathrm{K}$ & & & 1400 & -1500 \\
\hline $1021 \mathrm{~A}$ & & B42 & $\mathrm{K}$ & & & 1400 & -1550 \\
\hline $1031 \mathrm{~A}$ & & B42 & $\mathrm{K}$ & & & 1400 & -1500 \\
\hline $1041 \mathrm{~A}$ & & B42 & $\mathrm{K}$ & & & 1400 & -1500 \\
\hline $1051 \mathrm{~A}$ & & B32 & $\mathrm{K}$ & & & 1400 & -1500 \\
\hline $1061 \mathrm{~A}$ & & B42 & $\mathrm{K}$ & & & 1400 & -1500 \\
\hline $1071 \mathrm{~A}$ & & B32 & $\mathrm{K}$ & & & 1400 & -1500 \\
\hline $1081 \mathrm{~A}$ & & B42 & $\mathrm{K}$ & & & 1450 & -1600 \\
\hline $1091 \mathrm{~A}$ & & B31 & $\mathrm{K}$ & & & 1300 & -1400 \\
\hline $1101 \mathrm{~A}$ & & B1? & $\mathrm{K}$ & & & & \\
\hline $1111 \mathrm{~A}$ & & B31 & $\mathrm{K}$ & & & 1300 & -1500 \\
\hline $1121 \mathrm{~A}$ & & A1? & $\mathrm{K}$ & & & 1300 & -1500 \\
\hline $1131 \mathrm{~A}$ & & B42 & $\mathrm{K}$ & & & 1400 & -1500 \\
\hline $1141 \mathrm{~A}$ & & B42 & K & & & 1400 & -1500 \\
\hline $1151 \mathrm{~A}$ & & B42 & $\mathrm{K}$ & & & 1400 & -1500 \\
\hline $1161 \mathrm{~A}$ & & B42 & $\mathrm{K}$ & & & 1400 & -1500 \\
\hline $1171 \mathrm{~A}$ & & B32 & $\mathrm{K}$ & & & 1300 & -1500 \\
\hline $1181 \mathrm{~A}$ & & B42 & $\mathrm{K}$ & & & 1300 & -1500 \\
\hline $1191 \mathrm{~A}$ & & B32 & $\mathrm{H} 2$ & & & 1400 & -1550 \\
\hline $1201 \mathrm{~A}$ & 3B141 & B41 & & B & 4,70 & 1500 & -1550 \\
\hline $1211 \mathrm{~A}$ & & B31 & $\mathrm{K}$ & E3E1 & & & \\
\hline $1221 \mathrm{~A}$ & & B32? & $\mathrm{K}$ & E3 & & & \\
\hline $1231 \mathrm{~A}$ & & B32? & $\mathrm{K}$ & E3 & & & \\
\hline $1241 \mathrm{~A}$ & $2 \mathrm{~B} 21$ & A523 & K E1 & & 6,20 & 1500 & -1600 \\
\hline $1251 \mathrm{~A}$ & $3 \mathrm{~B}$ & A133 & & & 8,50 & 700 & -900 \\
\hline $1261 \mathrm{~A}$ & & B42 & K & B & & 1500 & -1550 \\
\hline $1271 \mathrm{~B}$ & 2B0 2 & B42 & H1 & E1 & 11,40 & 1450 & -1500 \\
\hline $1281 \mathrm{~B}$ & & A? & $\mathrm{K}$ & & & 1500 & \\
\hline $1291 \mathrm{~B}$ & $3 \mathrm{~B} 33$ & B42 & H1 & B & 9,20 & 1500 & \\
\hline $1301 \mathrm{~B}$ & $3 \mathrm{~B}$ & B41 & H1 & B & 7,50 & 1500 & \\
\hline $131 \mathrm{1B}$ & 3E144 & B41 & $\mathrm{H} 1$ & B & & 1500 & \\
\hline $132 \mathrm{1B}$ & $3 \mathrm{~B}$ & A123 & B & & 7,80 & 1500 & -1550 \\
\hline $1331 \mathrm{~B}$ & & B22 & B & & & 1500 & -1550 \\
\hline $1341 \mathrm{~B}$ & & B42 & B & K9 & 3,50 & 1500 & -1550 \\
\hline $1351 \mathrm{~B}$ & & A223 & & E3 & & 1600 & \\
\hline
\end{tabular}




\section{Bundesrepublik Deutschland (alte Bundesländer)}

\begin{tabular}{|c|c|c|c|c|c|c|c|}
\hline 1 & $2 \mathrm{E} 1$ & 1B1 & A141 & & & 15,00 & $800-900$ \\
\hline 2 & 2E1 & 1B1 & A133 & & & 12,00 & $800-1100$ \\
\hline 3 & 2E1 & 3B1 1 & A112 & & & 08,80 & $800-1100$ \\
\hline 4 & $2 \mathrm{E} 1$ & 1B0 1 & A113 & $\mathrm{H} 1$ & & 05,25 & $800-1100$ \\
\hline 5 & $2 \mathrm{E} 1$ & 3E146 & & & & 04,80 & $800-1100$ \\
\hline 6 & 2E1 & 3B3 1 & A133 & & & 05,50 & $800-1100$ \\
\hline 7 & $2 \mathrm{E} 1$ & & A122 & & & & $800-1100$ \\
\hline 8 & 2E1 & 1B0 1 & A153 & & & 08,50 & $900-1000$ \\
\hline 9 & $2 \mathrm{E} 1$ & $3 \mathrm{~B} 154$ & A153 & & & 09,00 & $900-1000$ \\
\hline 10 & 2E1 & 3B164 & A133 & & & 08,80 & $900-1000$ \\
\hline 11 & $2 \mathrm{E} 1$ & $3 \mathrm{~B}$ & A141 & & & 10,50 & $1100-1300$ \\
\hline 12 & 2E1 & & B11 & $\mathrm{K}$ & B & & $1150-1300$ \\
\hline 13 & $2 \mathrm{E} 1$ & 1B0 1 & A131 & & & 26,40 & \\
\hline 14 & 2E1 & $5 \mathrm{C}$ & A243 & & & 14,00 & $1200-1400$ \\
\hline 15 & 2E1 & 3B13 & A131 & & & 10,00 & $1200-1400$ \\
\hline 16 & $2 \mathrm{E} 1$ & 2B & A131 & & & 08,00 & $1200-1400$ \\
\hline 17 & 2E1 & 1B1? & A133 & & & 09,50 & $1350-1500$ \\
\hline 18 & 2E1 & 3B141 & B7 & & & 14,00 & $1000-1150$ \\
\hline 19 & $2 \mathrm{E} 1$ & $3 \mathrm{~B} ?$ & A103 & & & 05,40 & $1000-1150$ \\
\hline 20 & 2E1 & 3B & A203 & & & 05,00 & $1000-1150$ \\
\hline 21 & 2E1 & 2B1 3 & A133 & & & 09,20 & $1000-1150$ \\
\hline 22 & $2 \mathrm{E} 1$ & 1B1 3 & A133 & & & 09,00 & $1000-1150$ \\
\hline 23 & $2 \mathrm{E} 1$ & $3 \mathrm{~B} ?$ & A133 & & & 11,20 & $1000-1150$ \\
\hline 24 & 2E1 & $3 \mathrm{E} 24$ & A103 & & & 06,50 & $1000-1150$ \\
\hline 25 & 2E1 & 3B & A123 & & & 06,80 & $1000-1150$ \\
\hline 26 & 2E1 & 1B1 & A153 & & & 07,00 & $1000-1150$ \\
\hline 27 & $2 \mathrm{E} 1$ & $3 \mathrm{E} 1$ & A123 & & & 08,60 & $1000-1150$ \\
\hline 28 & $2 \mathrm{E} 1$ & 3E2 & A113 & & & 07,60 & $1000-1150$ \\
\hline 29 & $2 \mathrm{E} 1$ & & A153 & $\mathrm{K}$ & & & $1250-1300$ \\
\hline $29 \mathrm{~A}$ & $2 \mathrm{E} 1$ & & A1 & $\mathrm{K}$ & & & $1250-1300$ \\
\hline 30 & $2 \mathrm{E} 1$ & & $\mathrm{~A} 1 ?$ & $\mathrm{~K}$ & & & $1200-1400$ \\
\hline 31 & 2E1 & & B1 & $\mathrm{K}$ & E1 & & \\
\hline 32 & $2 \mathrm{E} 1$ & 1B0 1 & A143 & & & 11,20 & $1300-1400$ \\
\hline 33 & 2E1 & $3 \mathrm{E}$ & A331 & H1 & B & 20,70 & $1300-1500$ \\
\hline 34 & 2E1 & $3 \mathrm{~B} ?$ & B42 & $\mathrm{K}$ & & 06,00 & $1500-1550$ \\
\hline 35 & $2 \mathrm{E} 1$ & & B31 & $\mathrm{K}$ & B & & \\
\hline 36 & 2E1 & 3B & A233 & H1 & B & 10,40 & $1450-1500$ \\
\hline 37 & 2E1 & 3E14 & B12 & & & 10,50 & \\
\hline 38 & $2 \mathrm{E} 1$ & & B42 & H1B & & & $1500-1600$ \\
\hline 39 & 2E1 & 3B141 & B42 & H1B & & 10,50 & $1500-1600$ \\
\hline 40 & 2E1 & 3E4 4 & A133 & & & 12,15 & $1300-1400$ \\
\hline 41 & $2 \mathrm{E} 1$ & $3 \mathrm{E} ?$ & A621 & & & 12,00 & $1300-1400$ \\
\hline 42 & $2 \mathrm{E} 1$ & 3B3 1 & A133 & & & 11,50 & $1200-1300$ \\
\hline 43 & 2E1 & $3 \mathrm{E} 1$ & A233 & & & 16,50 & $1300-1400$ \\
\hline 44 & $2 \mathrm{E} 1$ & & A453 & $\mathrm{H} 1$ & & 11,00 & 1000 \\
\hline 45 & 2E1 & 3E3 5 & A133 & & & 12,20 & $850-950$ \\
\hline
\end{tabular}




\begin{tabular}{|c|c|c|c|c|c|c|c|}
\hline 46 & $2 \mathrm{E} 1$ & 5E0 4 & A253 & H1 & & 07,80 & $1150-1200$ \\
\hline 47 & 2E1 & 3 E141 & A231 & & & 13,80 & $1200-1400$ \\
\hline 48 & $2 \mathrm{E} 1$ & & A13? & $\mathrm{K}$ & & & $1250-1400$ \\
\hline 49 & 2E1 & & $\mathrm{A} 1 ?$ & $\mathrm{~K}$ & & & \\
\hline 50 & $2 \mathrm{E} 1$ & $3 \mathrm{~B}$ & B42 & H1 & B & 11,00 & $1500-1550$ \\
\hline $50 \mathrm{~A}$ & 2E1 & & A243 & & & & $1150-1200$ \\
\hline 51 & 2E1 & 3 B3 2 & A523 & E1H2 & B & 13,00 & 1600 \\
\hline 52 & 2E1 & 3B1 4 & B32 & H1 & B & 14,00 & 1600 \\
\hline 53 & $2 \mathrm{E} 1$ & $3 \mathrm{~B} 14$ & B32 & $\mathrm{H} 1$ & B & 14,00 & 1600 \\
\hline 54 & $2 \mathrm{E} 1$ & 3B14 & B32 & & & 09,80 & 1600 \\
\hline 55 & 2E1 & 3B14 & B32 & & & 15,50 & 1600 \\
\hline 56 & 2E1 & 3B14 & B32 & & & 12,80 & 1600 \\
\hline 57 & 2E1 & 3B14 & B32 & & B & 14,50 & 1600 \\
\hline 58 & 2E1 & 3B14 & B32 & & & 15,30 & 1600 \\
\hline 59 & 2E1 & 3B14 & B32 & & B & 15,50 & 1600 \\
\hline 60 & 2E1 & 3B & A133 & & & 10,10 & $1300-1400$ \\
\hline 61 & 2E1 & 1B & A122 & & & 07,80 & $1100-1400$ \\
\hline 62 & 2E1 & $3 \mathrm{D}$ & A231 & & B & 14,50 & $1300-1400$ \\
\hline 63 & 2E2 & & A42 & $\mathrm{K}$ & & & $1600-1700$ \\
\hline 64 & $2 \mathrm{E} 2$ & $3 \mathrm{~B} 3$ & A233 & B & E2 & & $1600-1650$ \\
\hline 65 & $2 \mathrm{E} 2$ & 2B1 & A421 & $\mathrm{K}$ & & & $1600-1650$ \\
\hline 66 & $2 \mathrm{E} 3$ & & A13 & $\mathrm{H} 2$ & & & $950-1050$ \\
\hline 67 & $2 \mathrm{E} 3$ & & A23 & $\mathrm{H} 2$ & & & $900-1400$ \\
\hline 68 & $2 \mathrm{~F}$ & $3 \mathrm{~B} 13$ & A143 & & & 11,60 & $900-1100$ \\
\hline 69 & $2 \mathrm{~F}$ & 1B0 1 & A233 & & & 08,00 & $900-1100$ \\
\hline 70 & $2 \mathrm{~F}$ & $3 \mathrm{E} 1$ & $\mathrm{~A} 4 ?$ & $\mathrm{H} 1$ & E1? & & $1100-1300$ \\
\hline 71 & $2 \mathrm{~F}$ & $3 \mathrm{~B} 1$ & A433 & H1 & E1? & & $1100-1300$ \\
\hline 72 & $2 \mathrm{D}$ & 3B34 & & & & 11,10 & $1200-1400$ \\
\hline 73 & 2D & 3B0 3 & B31? & H1 & B & & $1300-1400$ \\
\hline 74 & $2 \mathrm{D}$ & 3B181 & A443 & K E2 & & 11,10 & 1300 \\
\hline 75 & 2D & & A343 & & B L & & $1250-1350$ \\
\hline $75 \mathrm{~A}$ & $2 \mathrm{D}$ & 1B1 1 & A333 & $\mathrm{K}$ & E3 L & 08,00 & $1250-1300$ \\
\hline 76 & $2 \mathrm{D}$ & $5 \mathrm{~A}$ & A123 & & & 09,00 & $800-850$ \\
\hline 77 & $2 \mathrm{D}$ & 3B16 & A133 & & & 09,00 & $800-850$ \\
\hline 78 & $2 \mathrm{D}$ & 3B14 & B42 & $\mathrm{K}$ & B & & $1400-1500$ \\
\hline 79 & $2 \mathrm{C}$ & 1B0 1 & A633 & & & 12,00 & $1100-1150$ \\
\hline 80 & $2 \mathrm{C}$ & $2 \mathrm{~B}$ & A143 & & & 10,30 & $1100-1150$ \\
\hline 81 & $2 \mathrm{C}$ & $5 \mathrm{E}$ & A143 & & & 13,00 & $1100-1200$ \\
\hline 82 & $2 \mathrm{C}$ & $3 \mathrm{~B}$ & $\mathrm{~A} 633$ & & & 13,00 & $800-1300$ \\
\hline 83 & $2 \mathrm{C}$ & 1B1 & A133 & & & 11,60 & $800-1300$ \\
\hline 84 & $2 \mathrm{C}$ & 3D35 & A133 & & & 09,60 & $800-1300$ \\
\hline 85 & $2 \mathrm{C}$ & $3 \mathrm{~B}$ & A243 & & B & 11,20 & $800-1300$ \\
\hline 86 & $2 \mathrm{C}$ & & B42 & $\mathrm{H} 2$ & & & $1500-1700$ \\
\hline 87 & $2 \mathrm{C}$ & & B42 & $\mathrm{H} 2$ & & & $1500-1700$ \\
\hline 88 & $2 \mathrm{C}$ & & B42 & $\mathrm{H} 2$ & & & $1500-1700$ \\
\hline 89 & $2 \mathrm{C}$ & 3B141 & A543 & E1? & & 10,90 & $1500-1700$ \\
\hline 90 & $2 \mathrm{C}$ & 3B141 & A523 & E1? & & 09,90 & $1500-1700$ \\
\hline 91 & $2 \mathrm{C}$ & 3B141 & A523 & E1? & & 07,80 & $1500-1700$ \\
\hline 92 & $2 \mathrm{C}$ & $3 \mathrm{~B}$ & B22 & H1B & & 08,50 & $1450-1500$ \\
\hline
\end{tabular}




\begin{tabular}{|c|c|c|c|c|c|c|c|}
\hline 93 & $2 \mathrm{C}$ & $3 \mathrm{E}$ & B43 & K & & 12,60 & $1450-1500$ \\
\hline 94 & $2 \mathrm{C}$ & & B22 & $\mathrm{K}$ & & & $1500-1700$ \\
\hline 95 & $2 \mathrm{C}$ & $2 \mathrm{~A} 14$ & B32 & $\mathrm{K}$ & & 08,00 & $1500-1700$ \\
\hline 96 & $2 \mathrm{C}$ & 1B1 & A131 & & & 10,40 & $1000-1400$ \\
\hline 97 & $2 \mathrm{C}$ & $3 \mathrm{~B} 163$ & A131 & & & 08,40 & $1150-1400$ \\
\hline 98 & $2 \mathrm{C}$ & $2 \mathrm{~A} 14$ & A141 & & & 08,00 & $1100-1300$ \\
\hline 99 & $2 \mathrm{C}$ & 2B1 & A222 & & & 09,40 & $1100-1300$ \\
\hline 100 & $2 \mathrm{C}$ & 3B34 & A433 & & & 09,00 & $1100-1300$ \\
\hline 101 & $2 \mathrm{C}$ & 3E13 & A143 & & & 11,50 & $1300-1400$ \\
\hline 102 & $2 \mathrm{C}$ & 3B141 & & & & 10,20 & $1300-1400$ \\
\hline 103 & $2 \mathrm{C}$ & $3 \mathrm{~B}$ & A243 & & B & 10,00 & $1200-1350$ \\
\hline 104 & $2 \mathrm{C}$ & $3 \mathrm{E} 4$ & A131 & & & 09,50 & $1200-1400$ \\
\hline 105 & $2 \mathrm{C}$ & $4 \mathrm{~F}$ & B32 & & & 09,10 & $1200-1400$ \\
\hline 106 & $2 \mathrm{C}$ & & A333 & & B ? & & $1150-1350$ \\
\hline 107 & $2 \mathrm{C}$ & 1B0 1 & A133 & & & 11,00 & $1150-1200$ \\
\hline 108 & $2 \mathrm{C}$ & & B1? & $\mathrm{K}$ & & & 1200 \\
\hline 109 & $2 \mathrm{C}$ & 3B & B42 & $\mathrm{K}$ & & 11,00 & $1300-1500$ \\
\hline 110 & $2 \mathrm{C}$ & 1B & B22 & $\mathrm{K}$ & & 07,50 & 1300 \\
\hline 111 & $2 \mathrm{C}$ & 1B0 1 & B12 & H1 & & 03,20 & 1300 \\
\hline 112 & $2 \mathrm{C}$ & $3 \mathrm{~B}$ & B92 & & & 08,50 & 1300 \\
\hline 113 & $2 \mathrm{C}$ & & B22 & $\mathrm{K}$ & & & 1300 \\
\hline 114 & $2 \mathrm{C}$ & & B6? & H1 & B & & 1300 \\
\hline 115 & $2 \mathrm{C}$ & & B1? & $\mathrm{K}$ & B & & $1500-1550$ \\
\hline 116 & $2 \mathrm{~A}$ & 1B & B32 & H1 & B & 10,80 & $1300-1500$ \\
\hline 117 & $2 \mathrm{~A}$ & 1B0 1 & B32 & $\mathrm{K}$ & E2 & 10,00 & $1300-1500$ \\
\hline 118 & $2 \mathrm{~A}$ & 1B0 1 & A443 & H1 & E1 & 10,20 & $1300-1400$ \\
\hline 119 & $2 \mathrm{~A}$ & & B42 & & & & $1500-1650$ \\
\hline 120 & $2 \mathrm{~A}$ & & B42 & $\mathrm{K}$ & & & $1550-1650$ \\
\hline 121 & $2 \mathrm{~A}$ & 2B1 4 & B42 & $\mathrm{K}$ & & 08,80 & $1550-1650$ \\
\hline 122 & $2 \mathrm{~A}$ & 2B1 4 & B42 & $\mathrm{H} 2$ & & & $1550-1650$ \\
\hline 123 & $2 \mathrm{~A}$ & $2 \mathrm{~A} 24$ & A431 & H1 & E1 & 11,00 & $1100-1300$ \\
\hline 124 & $2 \mathrm{~A}$ & & B42 & $\mathrm{K}$ & & & \\
\hline 125 & $2 \mathrm{~A}$ & 3B14 & B42 & & B & 09,70 & $1400-1500$ \\
\hline 126 & $2 \mathrm{~A}$ & $3 \mathrm{~B}$ & B32 & $\mathrm{K}$ & & 04,50 & $1500-1650$ \\
\hline 127 & $2 \mathrm{~A}$ & 2B1 6 & B41 & $\mathrm{K}$ & E1 & 07,00 & $1550-1650$ \\
\hline 128 & $2 \mathrm{~A}$ & & B32 & $\mathrm{K}$ & & & $1450-1550$ \\
\hline 129 & $2 \mathrm{~A}$ & & B42 & H1 & B & & $1450-1550$ \\
\hline 130 & $2 \mathrm{~A}$ & 3B & B82 & $\mathrm{H}$ & E1 & 11,30 & $1450-1550$ \\
\hline 131 & $2 \mathrm{~A}$ & 3B174 & A133 & & & 07,40 & $700-1100$ \\
\hline 132 & $2 \mathrm{~B}$ & 3D34 & A133 & & & 12,60 & $800-1000$ \\
\hline 133 & $2 \mathrm{~A}$ & & B42 & $\mathrm{K}$ & E1 & & \\
\hline 134 & $2 \mathrm{~B}$ & & $\mathrm{~A} 1 ?$ & $\mathrm{~K}$ & & & $1200-1300$ \\
\hline 135 & $2 \mathrm{~B}$ & & A132 & & & 02,90 & $1200-1300$ \\
\hline 136 & $2 \mathrm{~B}$ & & A133 & & & & $1200-1300$ \\
\hline 137 & $2 \mathrm{~B}$ & 3B34 & B11? & & & 12,00 & $1200-1300$ \\
\hline 138 & $2 \mathrm{~B}$ & $1 \mathrm{~B} 04$ & & H1 & & & $1200-1300$ \\
\hline 139 & $2 \mathrm{~B}$ & 3E1 & A143 & & & 07,70 & $1200-1300$ \\
\hline 140 & $2 \mathrm{~B}$ & & A343 & & B ? & 03,70 & $1200-1300$ \\
\hline 141 & $2 \mathrm{~B}$ & $3 \mathrm{E} 2$ & A433 & & B & 07,20 & $1200-1300$ \\
\hline
\end{tabular}




\begin{tabular}{|c|c|c|c|c|c|c|c|}
\hline 142 & $2 \mathrm{~B}$ & $5 \mathrm{~A}$ & $\mathrm{~B}$ ? & & B & 16,00 & $1200-1300$ \\
\hline 143 & $2 \mathrm{~B}$ & & B12 & $\mathrm{K}$ & & & $1200-1300$ \\
\hline 144 & $2 \mathrm{~B}$ & 3E1 & A131 & & & 16,20 & $1200-1250$ \\
\hline 145 & $2 \mathrm{~B}$ & $3 \mathrm{~B} 31$ & A643 & & & 18,00 & $1200-1250$ \\
\hline 146 & $2 \mathrm{~B}$ & 3B13 & A643 & & & 16,20 & $1200-1250$ \\
\hline 147 & $2 \mathrm{~B}$ & $3 \mathrm{~B}$ & A123 & & & 13,80 & $1200-1250$ \\
\hline 148 & $2 \mathrm{~B}$ & $3 \mathrm{~B}$ & A651 & & & 10,80 & $1200-1250$ \\
\hline 149 & $2 \mathrm{~B}$ & 3B13 & A643 & & & 12,00 & $1200-1250$ \\
\hline 150 & $2 \mathrm{~B}$ & & B1? & $\mathrm{K}$ & & & $1200-1250$ \\
\hline 151 & $2 \mathrm{~B}$ & $3 \mathrm{E} 2$ & A243 & & B & 10,00 & $1100-1300$ \\
\hline 152 & $2 \mathrm{~B}$ & $4 \mathrm{~A}$ & A143 & & & 09,80 & $1250-1300$ \\
\hline 153 & $2 \mathrm{~B}$ & $4 \mathrm{~A}$ & A143 & & & 10,20 & $1100-1300$ \\
\hline 154 & $2 \mathrm{~B}$ & $5 \mathrm{C}$ & A223 & & B & 08,00 & $1250-1300$ \\
\hline 155 & $2 \mathrm{~B}$ & $3 \mathrm{~B}$ & A343 & & B ? & 10,10 & $1250-1300$ \\
\hline 156 & $2 \mathrm{~B}$ & 3B & A143 & & & 13,40 & $1100-1300$ \\
\hline 157 & $2 \mathrm{~B}$ & $3 \mathrm{~B}$ & A343 & & B ? & 07,70 & $1100-1300$ \\
\hline 158 & $2 \mathrm{~B}$ & $3 \mathrm{~B}$ & A143 & & & 07,30 & $1250-1300$ \\
\hline 159 & $2 \mathrm{~B}$ & $3 \mathrm{~B}$ & A143 & & & 10,80 & $1100-1300$ \\
\hline 160 & $2 \mathrm{~B}$ & 1B0 1 & A143 & & & 07,30 & $1100-1300$ \\
\hline 161 & $2 \mathrm{~B}$ & 3B & A243 & & B & 11,50 & $1200-1250$ \\
\hline 162 & $2 \mathrm{~B}$ & 3B & A343 & & B K & 07,60 & $1200-1300$ \\
\hline 163 & $2 \mathrm{~B}$ & $3 \mathrm{E} 33$ & A233 & $\mathrm{K}$ & & 20,10 & $1400-1500$ \\
\hline 164 & $2 \mathrm{~B}$ & 3B17 & A133 & & & 10,80 & $800-1000$ \\
\hline 165 & $2 \mathrm{~B}$ & 3E3 4 & A133 & & & 10,00 & $800-1000$ \\
\hline 166 & $2 \mathrm{~B}$ & $2 \mathrm{~B}$ & A133 & & & 10,50 & $800-1000$ \\
\hline 167 & $2 \mathrm{~B}$ & $2 \mathrm{~A} 1$ & B12 & & & 06,50 & $1400-1500$ \\
\hline 168 & $2 \mathrm{~B}$ & $3 \mathrm{~B}$ & A243 & & & 11,00 & $1250-1300$ \\
\hline $168 \mathrm{~A}$ & $2 \mathrm{~B}$ & 1B1 2 & B42 & H1 & B & 09,20 & $1450-1550$ \\
\hline $168 \mathrm{~B}$ & $2 \mathrm{~B}$ & & B42 & H1 & B & & $1450-1550$ \\
\hline $168 \mathrm{C}$ & $2 \mathrm{~B}$ & 1B1 1 & B32 & & & & $1450-1550$ \\
\hline 169 & $2 \mathrm{~B}$ & $3 \mathrm{~B} 33$ & A131 & & & 11,40 & $1200-1400$ \\
\hline 170 & $2 \mathrm{~B}$ & 4D & A133 & & & 09,50 & $1000-1150$ \\
\hline 171 & $2 \mathrm{~B}$ & $3 \mathrm{~B} 154$ & & $\mathrm{~K}$ & & 11,50 & $1300-1700$ \\
\hline 172 & $2 \mathrm{~B}$ & & $\mathrm{~A} 2 ?$ & $\mathrm{~K}$ & & & $1200-1400$ \\
\hline 173 & $2 \mathrm{~B}$ & 3B34 & B22 & $\mathrm{K}$ & B & 08,00 & $1400-1550$ \\
\hline 174 & $2 \mathrm{~B}$ & 3E1 4 & A133 & & & 12,00 & $1000-1200$ \\
\hline 175 & $2 \mathrm{~B}$ & $3 \mathrm{E} 14$ & A133 & & & 12,00 & $1000-1200$ \\
\hline 176 & $2 \mathrm{~B}$ & 5E0 4 & A131 & & & 15,00 & $1000-1200$ \\
\hline 177 & $2 \mathrm{~B}$ & 3E1 & A133 & & & 18,00 & $1000-1200$ \\
\hline 178 & $2 \mathrm{~B}$ & 3B144 & A133 & & & 12,00 & $1250-1300$ \\
\hline 179 & $2 \mathrm{~B}$ & & $\mathrm{~A} 2 ?$ & $\mathrm{~K}$ & & & $1000-1250$ \\
\hline 180 & $2 \mathrm{~B}$ & 1B2 1 & A143 & & & 14,60 & $1250-1400$ \\
\hline 181 & $2 \mathrm{C}$ & $1 \mathrm{~A} 1$ & A143 & & & 09,30 & $1050-1300$ \\
\hline 182 & $2 \mathrm{C}$ & $3 \mathrm{~B}$ & A133 & & & 12,00 & $1050-1300$ \\
\hline 183 & $2 \mathrm{C}$ & 1B1 3 & A133 & & & 08,00 & $1150-1250$ \\
\hline 184 & $2 \mathrm{C}$ & $3 \mathrm{E} 12$ & A14? & & & 10,00 & $1150-1250$ \\
\hline 185 & $2 \mathrm{C}$ & 3B15 & A642 & & & 09,60 & $1150-1250$ \\
\hline 186 & $2 \mathrm{C}$ & $3 \mathrm{~B}$ & A133 & & & 07,00 & $850-1000$ \\
\hline 187 & $2 \mathrm{C}$ & 3B13 & A143 & & & 08,50 & $850-1000$ \\
\hline
\end{tabular}


-80 -

$\begin{array}{llll}188 & 2 \mathrm{G} & 3 \mathrm{~B} 16 & \mathrm{~A} 133 \\ 189 & 2 \mathrm{G} & 3 \mathrm{~B} 144 & \mathrm{~A} 132 \\ 190 & 2 \mathrm{G} & 3 \mathrm{~B} 141 & \mathrm{~A} 132 \\ 191 & 2 \mathrm{G} & 2 \mathrm{~B} 1 & \mathrm{~A} 133 \\ 192 & 2 \mathrm{G} & 3 \mathrm{~B} 4 & \mathrm{~A} 133 \\ 193 & 2 \mathrm{G} & 3 \mathrm{~B} 1 & \mathrm{~A} 133 \\ 194 & 2 \mathrm{G} & 2 \mathrm{~A} 1 & \\ 195 & 2 \mathrm{G} & 2 \mathrm{~B} & \mathrm{~A} 122 \\ 196 & 2 \mathrm{G} & 5 \mathrm{E} & \\ 197 & 2 \mathrm{G} & 3 \mathrm{~B} 16 & \mathrm{~A} 143 \\ 198 & 2 \mathrm{G} & 3 \mathrm{~B} 17 & \mathrm{~A} 143 \\ 199 & 2 \mathrm{G} & 1 \mathrm{~B} 01 & \mathrm{~A} 133 \\ 200 & 2 \mathrm{G} & 3 \mathrm{E} 1 & \mathrm{~A} 143 \\ 201 & 2 \mathrm{G} & 2 \mathrm{~B} 1 & \mathrm{~A} 143 \\ 202 & 2 \mathrm{G} & 4 \mathrm{H} & \mathrm{A} 143 \\ 203 & 2 \mathrm{G} & 2 \mathrm{~A} 1 & \mathrm{~A} 443 \\ 204 & 2 \mathrm{G} & 4 \mathrm{I} & \mathrm{A} 133 \\ 205 & 2 \mathrm{G} & 3 \mathrm{~B} & \mathrm{~A} 143\end{array}$

\begin{tabular}{|c|c|c|}
\hline & 11,00 & 700 \\
\hline & 10,40 & 700 \\
\hline & 09,00 & 700 \\
\hline & 08,40 & 700 \\
\hline & 07,80 & 700 \\
\hline & 08,00 & 700 \\
\hline & 10,00 & 700 \\
\hline & 06,40 & 700 \\
\hline & & 700 \\
\hline & 10,00 & $1200-1300$ \\
\hline & 13,20 & $1200-1300$ \\
\hline & 12,80 & $1200-1300$ \\
\hline & 12,40 & $1200-1300$ \\
\hline & 08,40 & $1200-1300$ \\
\hline & 09,60 & $1200-1300$ \\
\hline B ? & 08,80 & $1200-1300$ \\
\hline & 09,40 & $1200-1300$ \\
\hline & 08,00 & $1200-1300$ \\
\hline
\end{tabular}




\section{Bundesrepublik Deutschland (neue Bundesländer)}

\begin{tabular}{|c|c|c|c|c|c|c|c|}
\hline 1 & $3 \mathrm{~A} 1$ & 3B141 & & & & 07,60 & $600-700$ \\
\hline 2 & $3 \mathrm{~A} 1$ & 3B17 & A233 & H1 & & 09,60 & $800-900$ \\
\hline 3 & $3 \mathrm{~A} 1$ & 3B17 & & & & 16,00 & $800-900$ \\
\hline 4 & $3 \mathrm{~A} 1$ & 3B & A133 & & & 07,60 & $800-900$ \\
\hline 5 & $3 \mathrm{~A} 1$ & $3 \mathrm{~B} 17$ & A253 & & & 09,20 & $850-900$ \\
\hline 6 & $3 \mathrm{~A} 1$ & & A243 & & $\mathrm{B}$ & 05,40 & $1000-1050$ \\
\hline 7 & $3 \mathrm{~A} 1$ & 3B15 & A133 & & & 08,60 & $1000-1150$ \\
\hline 8 & $3 \mathrm{~A} 1$ & 3E14 & A133 & & & 07,40 & $1000-1150$ \\
\hline 9 & $3 \mathrm{~A} 1$ & 3B & A141 & & & 07,60 & $1150-1250$ \\
\hline 10 & $3 \mathrm{~A} 1$ & 3E4 4 & A133 & & & 07,60 & $800-1200$ \\
\hline 11 & $3 \mathrm{~A} 1$ & 3B & A632 & & & 10,00 & $1000-1200$ \\
\hline 12 & $3 \mathrm{~A} 1$ & $3 \mathrm{E} 1$ & A133 & & & 09,60 & $1000-1200$ \\
\hline 13 & $3 \mathrm{~A} 1$ & $3 \mathrm{E} 1$ & A133 & & & 08,60 & $1000-1200$ \\
\hline 14 & $3 \mathrm{~A} 1$ & 3B & A103 & & & 09,00 & $1000-1200$ \\
\hline 15 & $3 \mathrm{~A} 1$ & 3B & & & & 09,70 & $800-950$ \\
\hline 16 & $3 \mathrm{~A} 1$ & 3B & A343 & & B & 11,25 & $1200-1300$ \\
\hline 17 & $3 \mathrm{~A} 1$ & 3B & A103 & & & 14,20 & $1050-1200$ \\
\hline 18 & $3 \mathrm{~A} 1$ & $2 \mathrm{~B}$ & A143 & & & 08,00 & $1050-1200$ \\
\hline 19 & $3 \mathrm{~A} 1$ & $4 \mathrm{~F}$ & A133 & & & 09,00 & $1050-1200$ \\
\hline 20 & $3 \mathrm{~A} 1$ & 1B2 1 & A133 & & & 07,00 & $1050-1200$ \\
\hline 21 & $3 \mathrm{~A} 1$ & $3 \mathrm{C} 1$ & & & & 09,00 & $1050-1200$ \\
\hline 22 & $3 \mathrm{~A} 1$ & 1B0 1 & A133 & & & 06,40 & $600-800$ \\
\hline 23 & $3 \mathrm{~A} 1$ & 3E1 3 & A133 & & & 05,20 & $600-800$ \\
\hline 24 & $3 \mathrm{~A} 1$ & $4 \mathrm{~F}$ & A132 & & & 05,60 & $600-800$ \\
\hline 25 & $3 \mathrm{~A} 1$ & $4 \mathrm{~F}$ & A133 & & & 06,00 & $600-800$ \\
\hline 26 & $3 \mathrm{~A} 1$ & 3B & A133 & & & 06,40 & $600-800$ \\
\hline 27 & $3 \mathrm{~A} 1$ & $4 \mathrm{~F}$ & A133 & $\mathrm{H} 1$ & & 07,20 & $600-800$ \\
\hline 28 & $3 \mathrm{~A} 1$ & $3 \mathrm{E} 14$ & A133 & & & 10,00 & $600-800$ \\
\hline 29 & $3 \mathrm{~A} 1$ & 3E1 3 & A133 & $\mathrm{K}$ & & 05,80 & $600-800$ \\
\hline 30 & $3 \mathrm{~A} 1$ & 1B1 & A13? & $\mathrm{H} 1$ & & 09,70 & $800-1000$ \\
\hline 31 & $3 \mathrm{~A} 1$ & 3B13 & A23? & H1 & & 06,70 & $800-1000$ \\
\hline 32 & $3 \mathrm{~A} 1$ & 3B1 1 & A13? & H1 & & 07,90 & $800-1000$ \\
\hline 33 & $3 \mathrm{~A} 1$ & 3B1 1 & A23? & $\mathrm{H} 1$ & & 07,90 & $800-1000$ \\
\hline 34 & $3 \mathrm{~A} 1$ & 3E141 & A153 & & & 10,60 & \\
\hline 35 & $3 \mathrm{~A} 1$ & 3E2 1 & A343 & K B & & 12,25 & $1350-1400$ \\
\hline 36 & $3 \mathrm{~A} 2$ & 3B3 1 & A133 & & & 08,00 & $1000-1200$ \\
\hline 37 & $3 \mathrm{~A} 2$ & $5 \mathrm{~A}$ & A343 & & & 10,20 & $1100-1300$ \\
\hline 38 & $3 \mathrm{~A} 2$ & 3B33 & A143 & & & 16,00 & \\
\hline 39 & $3 \mathrm{~A} 2$ & $5 \mathrm{C}$ & A13? & & & 14,70 & \\
\hline 40 & $3 \mathrm{~A} 2$ & & A243 & & & & $1400-1600$ \\
\hline 41 & $3 \mathrm{~A} 2$ & 3B3 1 & A213 & & & 06,40 & $800-1000$ \\
\hline 42 & $3 \mathrm{~A} 2$ & 3B & A113 & & & 04,40 & $800-1000$ \\
\hline 43 & $3 \mathrm{~A} 2$ & 3B3 4 & A203 & & & 06,40 & $800-1000$ \\
\hline 44 & $3 \mathrm{~A} 2$ & $3 \mathrm{E} 14$ & A2 & $\mathrm{E} 2$ & & 08,30 & $950-1200$ \\
\hline 45 & $3 \mathrm{~A} 2$ & $3 \mathrm{E} 2$ & A231 & & & 10,00 & $950-1200$ \\
\hline 46 & $3 \mathrm{~A} 2$ & $3 \mathrm{E} 1$ & A132 & & & 09,50 & $950-1200$ \\
\hline
\end{tabular}




\begin{tabular}{|c|c|c|c|c|c|c|c|}
\hline 47 & $3 \mathrm{~A} 2$ & 1B0 1 & A132 & & & 06,50 & $950-1200$ \\
\hline 48 & $3 \mathrm{~A} 2$ & $3 B 184$ & A133 & & & 13,00 & $950-1200$ \\
\hline 49 & $3 \mathrm{~A} 2$ & 3B151 & A23? & H1 & & 10,50 & $1150-1250$ \\
\hline 50 & $3 \mathrm{~A} 2$ & 1B0 1 & A133 & & & 07,80 & $1000-1250$ \\
\hline 51 & $3 \mathrm{~A} 2$ & 3E4 1 & A343 & & B & 23,00 & $1200-1500$ \\
\hline 52 & $3 \mathrm{~A} 2$ & $3 \mathrm{E} 4$ & A131 & & & & $1100-1300$ \\
\hline 53 & $3 \mathrm{~A} 2$ & 3B3 & B22 & $\mathrm{K}$ & B & 09,20 & $900-1500$ \\
\hline 54 & $3 \mathrm{~A} 2$ & 1B2 & A133 & & & 26,00 & $900-1500$ \\
\hline 55 & $3 \mathrm{~A} 2$ & 2B2 & A133 & & & 26,40 & $900-1500$ \\
\hline 56 & $3 \mathrm{~A} 2$ & $5 \mathrm{~B} 01$ & B11 & & & 18,00 & $900-1500$ \\
\hline 57 & $3 \mathrm{~A} 2$ & $3 \mathrm{~B}$ & A133 & & & 09,00 & $900-1500$ \\
\hline 58 & $3 \mathrm{~A} 2$ & 3B3 1 & A143 & & & 10,00 & $900-1500$ \\
\hline 59 & $3 \mathrm{~A} 2$ & 1B1 3 & A233 & & B & 27,00 & $900-1500$ \\
\hline 60 & $3 \mathrm{~A} 2$ & 3B141 & A133 & & & 21,00 & $900-1500$ \\
\hline 61 & $3 \mathrm{~A} 2$ & 3B141 & A133 & & & 20,00 & $900-1500$ \\
\hline 62 & $3 \mathrm{~A} 2$ & 1B1 1 & A133 & & & 25,50 & $900-1500$ \\
\hline 63 & $3 \mathrm{~A} 2$ & 3B171 & A131 & & & 25,00 & $900-1500$ \\
\hline 64 & $3 \mathrm{~A} 2$ & 3B1 1 & A143 & & & 23,00 & $900-1500$ \\
\hline 65 & $3 \mathrm{~A} 2$ & 3B141 & A131 & & & 19,50 & $900-1500$ \\
\hline 66 & $3 \mathrm{~A} 2$ & 3B321 & A343 & $\mathrm{H} 1$ & E2 & 20,20 & $1200-1400$ \\
\hline 67 & $3 \mathrm{~A} 2$ & 3E301 & A343 & H1 & & 16,00 & $1200-1400$ \\
\hline 68 & $3 \mathrm{~A} 2$ & 3E141 & A343 & & B L & 18,00 & $1200-1400$ \\
\hline 69 & $3 \mathrm{~A} 2$ & 3B15 & A333 & & B & 17,25 & $1200-1400$ \\
\hline 70 & $3 \mathrm{~A} 2$ & $3 \mathrm{~B}$ & A343 & $\mathrm{H} 1$ & $\mathrm{H} 2 \mathrm{~L} \mathrm{~B}$ & 09,75 & $1200-1400$ \\
\hline 71 & $3 \mathrm{~A} 2$ & $3 \mathrm{~B}$ & A343 & H1 & B L & 13,50 & $1200-1400$ \\
\hline 72 & $3 \mathrm{~A} 2$ & $3 \mathrm{~B}$ & A343 & & $\mathrm{H} 2 \mathrm{~B}$ & 12,00 & $1200-1400$ \\
\hline 73 & $3 \mathrm{~A} 2$ & 3B17 & B31 & & & 09,00 & \\
\hline 74 & $3 \mathrm{~A} 2$ & $4 \mathrm{~F}$ & A133 & & & 08,80 & $950-1050$ \\
\hline 75 & $3 \mathrm{~A} 2$ & 1B2 1 & A103 & H1 & & 09,10 & $1000-1200$ \\
\hline 76 & $3 \mathrm{~A} 2$ & 3B181 & A233 & H1 & & 09,80 & $1000-1200$ \\
\hline 77 & $3 \mathrm{~A} 2$ & 1B1 3 & A133 & & & 11,50 & $1000-1250$ \\
\hline 78 & $3 \mathrm{~A} 2$ & 1B0 1 & A252 & & & 07,60 & $1000-1250$ \\
\hline 79 & $3 \mathrm{~A} 2$ & 1B0 1 & A143 & & & 07,80 & $1000-1250$ \\
\hline 80 & $3 \mathrm{~A} 2$ & $3 \mathrm{~B} 184$ & A133 & & & 12,20 & $1000-1200$ \\
\hline 81 & $3 \mathrm{~A} 2$ & 3B152 & A153 & & & 07,60 & $1000-1250$ \\
\hline 82 & $3 \mathrm{~A} 2$ & 3B181 & A113 & & & 08,30 & $1000-1200$ \\
\hline 83 & $3 \mathrm{~A} 2$ & $5 \mathrm{~A}$ & A113 & & & 09,00 & $1000-1200$ \\
\hline 84 & $3 \mathrm{~A} 2$ & 3E1 3 & A233 & & & 09,40 & $1000-1250$ \\
\hline 85 & $3 \mathrm{~A} 2$ & 3E1 & A133 & & & 09,00 & $1000-1250$ \\
\hline 86 & $3 \mathrm{~A} 2$ & 3E1 1 & A133 & & & 07,80 & $1000-1200$ \\
\hline 87 & $3 \mathrm{~A} 2$ & 3E4 1 & A133 & & & 05,00 & $1000-1200$ \\
\hline 88 & $3 \mathrm{~A} 2$ & 3B32 & A132 & & & 09,00 & $1000-1200$ \\
\hline 89 & $3 \mathrm{~A} 2$ & $3 \mathrm{~B}$ & A133 & & & 11,80 & $1000-1250$ \\
\hline 90 & $3 \mathrm{~A} 2$ & $3 \mathrm{~B} 23$ & A132 & & & 07,00 & $1000-1200$ \\
\hline 91 & $3 \mathrm{~A} 2$ & $3 \mathrm{~B}$ & A133 & & & 07,30 & $1000-1250$ \\
\hline 92 & $3 \mathrm{~A} 2$ & $3 \mathrm{~B}$ & A133 & & & 11,40 & $1000-1250$ \\
\hline 93 & $3 \mathrm{~A} 2$ & $2 \mathrm{~B}$ & A433 & H1 & E1 & 18,80 & $1200-1250$ \\
\hline 94 & $3 \mathrm{~A} 2$ & 3B13 & A132 & & & 16,80 & $1000-1200$ \\
\hline 95 & $3 \mathrm{~A} 2$ & $3 \mathrm{E}$ & 153 & & & 08,80 & $1000-1250$ \\
\hline
\end{tabular}




\begin{tabular}{|c|c|c|c|c|c|c|c|}
\hline 96 & $3 \mathrm{~A} 2$ & $3 \mathrm{~B} 25$ & A121 & & & 09,40 & $700-1200$ \\
\hline 97 & $3 \mathrm{~A} 2$ & & B31 & $\mathrm{H} 2$ & & 09,50 & $1200-1300$ \\
\hline 98 & $3 \mathrm{~A} 2$ & & A2? & $\mathrm{H} 2$ & & & $1000-1300$ \\
\hline 99 & $3 \mathrm{~A} 2$ & & A2? & $\mathrm{K}$ & & & $1000-1300$ \\
\hline 100 & $3 \mathrm{~A} 2$ & 1B2 1 & A621 & & & 14,30 & $1000-1300$ \\
\hline 101 & $3 \mathrm{~A} 2$ & 3E141 & A131 & & & 09,00 & $1000-1300$ \\
\hline 102 & $3 \mathrm{~A} 2$ & 3B1 3 & A133 & & & 10,40 & $1000-1300$ \\
\hline 103 & $3 \mathrm{~A} 2$ & 1B & A11? & & & 07,80 & $1000-1300$ \\
\hline 104 & $3 \mathrm{~A} 2$ & 1B5 & A131 & & & 09,00 & $1000-1300$ \\
\hline 105 & $3 \mathrm{~A} 2$ & 1B0 1 & A133 & & & 15,50 & $1000-1300$ \\
\hline 106 & $3 \mathrm{~A} 2$ & $3 \mathrm{E} 44$ & $\mathrm{~A} 233$ & & & 08,40 & $1000-1300$ \\
\hline 107 & $3 \mathrm{~A} 2$ & $5 \mathrm{~A}$ & A253 & & & 08,80 & $1000-1300$ \\
\hline 108 & $3 \mathrm{~A} 2$ & 3E141 & A23? & & E1 & 10,30 & $1000-1300$ \\
\hline 109 & $3 \mathrm{~A} 2$ & $3 \mathrm{E} 44$ & A13? & & & 09,60 & $1000-1300$ \\
\hline 110 & $3 \mathrm{~A} 2$ & $3 \mathrm{E}$ & A133 & & & 11,00 & $1100-1300$ \\
\hline 111 & $3 \mathrm{~A} 2$ & $5 \mathrm{~A}$ & A233 & & & 08,80 & $1000-1300$ \\
\hline 112 & $3 \mathrm{~A} 2$ & 3E2 4 & A131 & & & 07,40 & $1000-1300$ \\
\hline 113 & $3 \mathrm{~A} 2$ & $3 \mathrm{~B} 13$ & A122 & & & 11,40 & $1000-1300$ \\
\hline 114 & $3 \mathrm{~A} 2$ & 3B & A133 & H1 & & 09,00 & $1000-1300$ \\
\hline 115 & $3 \mathrm{~A} 2$ & 3B & A232 & & & 08,40 & $1000-1300$ \\
\hline 116 & $3 \mathrm{~A} 2$ & $3 \mathrm{~B}$ & A231 & & & 08,20 & $1000-1300$ \\
\hline 117 & $3 \mathrm{~A} 2$ & 3B & A152 & & & 08,00 & $1000-1300$ \\
\hline 118 & $3 \mathrm{~A} 2$ & 3B & A121 & & & 05,80 & $1000-1300$ \\
\hline 119 & $3 \mathrm{~A} 2$ & 3B141 & A142 & & & 07,00 & $1000-1300$ \\
\hline 120 & $3 \mathrm{~A} 2$ & $4 \mathrm{~F} 1$ & A133 & & & 07,00 & $1000-1300$ \\
\hline 121 & $3 \mathrm{~A} 2$ & $5 \mathrm{~A}$ & A111 & & & 08,20 & $1000-1300$ \\
\hline 122 & $3 \mathrm{~A} 2$ & & A222 & & B & 03,60 & $1000-1300$ \\
\hline 123 & $3 \mathrm{~A} 2$ & 1B0 1 & A131 & & & 08,20 & $1000-1300$ \\
\hline 124 & $3 \mathrm{~A} 2$ & 3E1 3 & A133 & & & 11,80 & $1000-1300$ \\
\hline 125 & $3 \mathrm{~A} 2$ & 3E2 4 & A133 & & & 09,40 & $900-1200$ \\
\hline 126 & $3 \mathrm{~A} 2$ & 3B14 & A2 & E1 & & 08,50 & $600-800$ \\
\hline 127 & $3 \mathrm{~A} 2$ & 1B2 1 & A113 & H1 & & 06,30 & $950-1200$ \\
\hline 128 & $3 \mathrm{~A} 2$ & 3B141 & A143 & $\mathrm{H} 1$ & & 09,00 & $950-1200$ \\
\hline 129 & $3 \mathrm{~A} 2$ & 1B0 1 & A131 & & & 09,00 & $950-1200$ \\
\hline 130 & $3 \mathrm{~B} 2$ & $3 \mathrm{~B} 2$ & B13 & & & 21,00 & $1200-1350$ \\
\hline 131 & $3 \mathrm{~B} 2$ & $3 \mathrm{~B} 18$ & A143 & & & 09,60 & $950-1100$ \\
\hline 132 & $3 \mathrm{~B} 2$ & 1B0 3 & A 121 & & & 06,00 & $950-1100$ \\
\hline 133 & $3 \mathrm{~B} 2$ & $3 \mathrm{~A} 23$ & A133 & & & 08,80 & $950-1100$ \\
\hline 134 & $3 \mathrm{~B} 2$ & 3B14 & A133 & & & 08,00 & $950-1100$ \\
\hline 135 & 3B2 & $3 \mathrm{E} 4$ & A133 & & & 08,00 & $950-1100$ \\
\hline 136 & $3 \mathrm{~B} 2$ & $3 \mathrm{~B} 25$ & A121 & & & 10,00 & $950-1100$ \\
\hline 137 & $3 \mathrm{~B} 2$ & 1B1 & A133 & & & 08,00 & $950-1100$ \\
\hline 138 & $3 \mathrm{~B} 2$ & 3B15 & A133 & & & 06,00 & $950-1100$ \\
\hline 139 & $3 \mathrm{~B} 2$ & $3 \mathrm{E} 3$ & A343 & & B & 13,50 & $1350-1450$ \\
\hline 140 & $3 \mathrm{~B} 2$ & $3 \mathrm{~B} 15$ & $\mathrm{~A} 2 ?$ & E1 & & 15,00 & $1000-1200$ \\
\hline 141 & 3B2 & 3B18 & A133 & & & 12,00 & $1300-1400$ \\
\hline 142 & $3 \mathrm{~B} 2$ & 1B0 1 & A143 & & & 15,00 & $1300-1400$ \\
\hline 143 & $3 \mathrm{~B} 2$ & & B32 & $\mathrm{K}$ & & & $1300-1400$ \\
\hline 144 & $3 \mathrm{~B} 2$ & & $\mathrm{~A} 4$ ? & $\mathrm{K}$ & & & $1200-1250$ \\
\hline
\end{tabular}




\begin{tabular}{|c|c|c|c|c|c|c|c|}
\hline 145 & $3 \mathrm{~B} 2$ & 1B0 1 & A133 & & & 07,30 & $1150-1200$ \\
\hline 146 & $3 \mathrm{~B} 2$ & 1B1 2 & A133 & & & 10,50 & $1150-1200$ \\
\hline 147 & $3 \mathrm{~B} 2$ & $3 \mathrm{~B} 132$ & A133 & & & 09,60 & $1150-1200$ \\
\hline 148 & $3 \mathrm{~B} 2$ & $4 \mathrm{~A} 05$ & A122 & & & 12,00 & $1150-1200$ \\
\hline 149 & $3 \mathrm{~B} 2$ & 3B18 & A133 & & & 09,60 & $1200-1250$ \\
\hline 150 & $3 \mathrm{~B} 2$ & 3E3 1 & A143 & & & 06,80 & $1000-1200$ \\
\hline 151 & $3 \mathrm{~B} 2$ & $3 \mathrm{~B} 15$ & A143 & & & 10,50 & $1000-1200$ \\
\hline 152 & 3B2 & 3B132 & A133 & & & 09,60 & $1000-1200$ \\
\hline 153 & $3 \mathrm{~B} 2$ & 3E1 3 & A133 & & & 13,50 & $1000-1200$ \\
\hline 154 & 3B2 & $4 \mathrm{~F}$ & A1 & & & 08,20 & $1000-1200$ \\
\hline 155 & 3B2 & $4 \mathrm{~F}$ & A143 & & & 09,30 & $1250-1300$ \\
\hline 156 & $3 \mathrm{~B} 2$ & $3 \mathrm{~B} 16$ & A123 & & & 10,80 & $1200-1250$ \\
\hline 157 & $3 \mathrm{~B} 1$ & $3 \mathrm{~B}$ & A 343 & & B & 09,75 & $1300-1500$ \\
\hline 158 & 3B1 & & A333 & & B & & 1300 \\
\hline 159 & 3B3 & $5 \mathrm{~B}$ & A331 & & B & 09,70 & $1350-1400$ \\
\hline 160 & $3 \mathrm{D}$ & & B11 & $\mathrm{H} 2$ & & & $1000-1200$ \\
\hline 161 & $3 \mathrm{D}$ & 5B0 1 & A133 & & & 14,80 & $1000-1150$ \\
\hline 162 & $3 \mathrm{C} 3$ & & $\mathrm{~B} 1 ?$ & K & & & $950-1100$ \\
\hline 163 & $3 \mathrm{D}$ & $5 \mathrm{C}$ & A133 & & & 11,00 & $850-950$ \\
\hline $163 \mathrm{~A}$ & $3 \mathrm{C}$ & $3 \mathrm{~B} 15$ & A132 & & & 10,60 & $950-1200$ \\
\hline $163 \mathrm{~B}$ & $3 \mathrm{C}$ & 3B154 & A453 & & & 11,40 & $950-1200$ \\
\hline $163 \mathrm{C}$ & $3 \mathrm{C}$ & $3 \mathrm{E} 16$ & A153 & & & 12,00 & $950-1200$ \\
\hline $163 \mathrm{D}$ & $3 \mathrm{C}$ & 2B0 1 & A153 & & & 10,00 & $950-1200$ \\
\hline $163 \mathrm{E}$ & $3 \mathrm{C}$ & 3B16 & A133 & & & 12,00 & $950-1200$ \\
\hline $163 \mathrm{~F}$ & $3 \mathrm{C}$ & $3 \mathrm{~B} 151$ & A143 & & & 10,60 & $950-1200$ \\
\hline $163 \mathrm{G}$ & $3 \mathrm{C}$ & 1B1 1 & A132 & & & 10,00 & $950-1200$ \\
\hline $163 \mathrm{H}$ & $3 \mathrm{C}$ & 3E1 4 & A133 & & & 12,00 & $950-1200$ \\
\hline $163 \mathrm{I}$ & $3 \mathrm{C}$ & $5 \mathrm{~A}$ & A133 & & & 08,70 & $950-1200$ \\
\hline $163 \mathrm{~J}$ & $3 \mathrm{C}$ & $5 \mathrm{~A}$ & A103 & & & 09,00 & $950-1200$ \\
\hline 164 & $3 \mathrm{C} 1$ & 3B15 & A133 & & & 10,20 & $1150-1400$ \\
\hline 165 & $3 \mathrm{C} 1$ & 3B3 1 & A121 & & & 10,90 & $1150-1400$ \\
\hline 166 & $3 \mathrm{C} 1$ & $2 \mathrm{~B}$ & A151 & & & 09,40 & $1150-1400$ \\
\hline 167 & $3 \mathrm{C} 1$ & $5 \mathrm{~A}$ & A121 & & & 06,80 & $1150-1400$ \\
\hline $167 \mathrm{~A}$ & $3 \mathrm{C} 1$ & 1B0 1 & A133 & & & 14,40 & $1150-1400$ \\
\hline 168 & $3 \mathrm{C} 1$ & & A1? & $\mathrm{H} 2$ & & & $1100-1200$ \\
\hline 169 & $3 \mathrm{C} 1$ & & $\mathrm{~A} 1 ?$ & K & & & $1100-1150$ \\
\hline 170 & $3 \mathrm{C} 1$ & $3 \mathrm{E} 41$ & A143 & & & 12,00 & $1200-1300$ \\
\hline 171 & $3 \mathrm{C} 1$ & 3E14 & A331 & & B & 09,30 & $1200-1300$ \\
\hline 172 & $3 \mathrm{C} 1$ & 1B & B42 & & & 18,80 & 1450 \\
\hline 173 & $3 \mathrm{C} 1$ & $2 \mathrm{~B}$ & B32 & H1 & & 18,00 & 1450 \\
\hline 174 & $3 \mathrm{C} 1$ & $5 \mathrm{~A}$ & A143 & & & 08,00 & $800-900$ \\
\hline 175 & $3 \mathrm{C} 3$ & $5 \mathrm{~A}$ & $\mathrm{~A} 1 ?$ & H1 & & 07,50 & $1250-1400$ \\
\hline 176 & $3 \mathrm{C} 2$ & & B12 & & & & $1200-1400$ \\
\hline 177 & $3 \mathrm{C} 2$ & 3B141 & B32 & H1 & B & 07,80 & $1200-1400$ \\
\hline 178 & $3 \mathrm{C} 2$ & 3B & A241 & & & 05,60 & $1200-1400$ \\
\hline 179 & $3 \mathrm{C} 2$ & 3B & A243 & & & 07,00 & $1200-1400$ \\
\hline 180 & $3 \mathrm{C} 2$ & $3 \mathrm{E}$ & A143 & & & 08,60 & $1250-1350$ \\
\hline 181 & $3 \mathrm{C} 2$ & 1B1 & & & & 07,00 & $1250-1350$ \\
\hline 182 & $3 \mathrm{C} 2$ & 3B3 3 & B32 & & & 09,60 & $1600-1750$ \\
\hline
\end{tabular}




\begin{tabular}{|c|c|c|c|c|c|c|c|}
\hline 183 & $3 \mathrm{C} 2$ & 1B0 1 & A131 & & & 11,80 & $1600-1650$ \\
\hline 184 & $3 \mathrm{C} 2$ & $4 \mathrm{~A}$ & A143 & & & 08,60 & $1050-1400$ \\
\hline 185 & $3 \mathrm{C} 2$ & $4 \mathrm{G}$ & & & & 08,00 & $1200-1400$ \\
\hline 186 & $3 \mathrm{C} 2$ & & B11 & & B & & $1200-1400$ \\
\hline 187 & $3 \mathrm{C} 2$ & 3B14 & A143 & & & 10,30 & $1200-1400$ \\
\hline 188 & $3 \mathrm{C} 2$ & & A243 & & B & 07,00 & $1200-1400$ \\
\hline 189 & $3 \mathrm{C} 2$ & $4 \mathrm{~A}$ & A143 & & & 08,00 & $1200-1400$ \\
\hline 190 & $3 \mathrm{C} 2$ & 3B1 4 & B12 & & & 11,20 & $1200-1400$ \\
\hline 191 & $3 \mathrm{C} 2$ & & B9 & & & & $1200-1400$ \\
\hline 192 & $3 \mathrm{C} 2$ & & B9 & & B & & $1200-1400$ \\
\hline 193 & $3 \mathrm{C} 2$ & $3 \mathrm{E} 4$ & B12 & & B & 08,20 & $1200-1400$ \\
\hline 194 & $3 \mathrm{C} 2$ & $2 \mathrm{~A} 1$ & & & & 08,40 & $1200-1400$ \\
\hline 195 & $3 \mathrm{C} 2$ & $4 \mathrm{~A}$ & A143 & & & 06,40 & $1200-1400$ \\
\hline 196 & $3 \mathrm{C} 2$ & $4 \mathrm{H}$ & B32 & & & 17,20 & $1200-1400$ \\
\hline 197 & $3 \mathrm{C} 2$ & & B9 & & B & & $1200-1400$ \\
\hline 198 & $3 \mathrm{C} 2$ & $3 B 342$ & A122 & & & 09,00 & $1200-1400$ \\
\hline 199 & $3 \mathrm{C} 2$ & $3 \mathrm{E}$ & A622 & & & 09,00 & $1200-1400$ \\
\hline 200 & $3 \mathrm{C} 2$ & & B8 & & & & $1200-1400$ \\
\hline 201 & $3 \mathrm{C} 2$ & 3B & B12 & & & 08,20 & $1050-1400$ \\
\hline 202 & $3 \mathrm{C} 2$ & $3 \mathrm{E} 4$ & A133 & & & 07,80 & $1200-1400$ \\
\hline 203 & $3 \mathrm{C} 2$ & $2 \mathrm{~A} 13$ & B12 & & & 07,60 & $1200-1400$ \\
\hline 204 & $3 \mathrm{C} 2$ & & B12 & & & & $1200-1400$ \\
\hline 205 & $3 \mathrm{C} 2$ & $4 \mathrm{~F}$ & B12 & & & 07,00 & $1200-1400$ \\
\hline 206 & $3 \mathrm{C} 2$ & 3B & B12 & & & 08,00 & $1200-1400$ \\
\hline 207 & $3 \mathrm{C} 2$ & $5 \mathrm{~A}$ & A143 & & & 08,50 & $1100-1400$ \\
\hline 208 & $3 \mathrm{C} 2$ & $5 \mathrm{~A}$ & A143 & & & 10,00 & $1100-1400$ \\
\hline 209 & $3 \mathrm{C} 2$ & 3B171 & B32 & $\mathrm{H} 1$ & & 07,00 & $1100-1400$ \\
\hline 210 & $3 \mathrm{C} 2$ & 1B1 5 & B42 & & & 11,50 & $1100-1400$ \\
\hline 211 & $3 \mathrm{C} 2$ & $2 \mathrm{C} 1$ & A131 & & & 07,60 & $1050-1400$ \\
\hline 212 & $3 \mathrm{C} 2$ & $4 \mathrm{~A}$ & A131 & & & 07,60 & $1050-1400$ \\
\hline 213 & $3 \mathrm{C} 2$ & 3B14 & A131 & & & 08,00 & $800-1050$ \\
\hline 214 & $3 \mathrm{C} 2$ & $3 \mathrm{E} 145$ & A133 & & & 15,00 & $1200-1400$ \\
\hline 215 & $3 \mathrm{C} 2$ & $4 \mathrm{~A}$ & A143 & & & 10,50 & $1200-1400$ \\
\hline 216 & $3 \mathrm{C} 2$ & 3E144 & A133 & & & 11,00 & $1200-1400$ \\
\hline 217 & $3 \mathrm{C} 2$ & $3 \mathrm{~B}$ & A143 & & & 10,30 & $1200-1400$ \\
\hline 218 & $3 \mathrm{C} 2$ & $4 \mathrm{~A}$ & A133 & & & 08,30 & $1200-1400$ \\
\hline 219 & $3 \mathrm{C} 2$ & $2 \mathrm{~B}$ & A133 & & & 08,60 & $1200-1400$ \\
\hline 220 & $3 \mathrm{C} 2$ & 3B34 & B32 & & & 08,10 & $1300-1400$ \\
\hline 221 & $3 \mathrm{C} 2$ & 3E4 1 & A343 & & B & 07,50 & $1300-1400$ \\
\hline
\end{tabular}




\section{Polen}

\begin{tabular}{|c|c|c|c|c|c|c|}
\hline 4A1 & $3 \mathrm{~B}$ & $\mathrm{~A} 2 ?$ & E1? & & 07,00 & $850-950$ \\
\hline $4 \mathrm{~A} 1$ & & A143 & $\mathrm{H} 1$ & & & $1000-1150$ \\
\hline 4A1 & $3 \mathrm{~B}$ & A143 & H1 & & 09,30 & $1000-1150$ \\
\hline 4A1 & 1B1 3 & A131 & & & 06,80 & $1000-1200$ \\
\hline $4 \mathrm{~A} 1$ & $2 \mathrm{~B} 02$ & $\mathrm{~A} 2 ?$ & $\mathrm{H} 1$ & & & $1150-1250$ \\
\hline $4 \mathrm{~A} 1$ & & $\mathrm{~A} 1 ?$ & $\mathrm{H} 2$ & & & $1200-1300$ \\
\hline 4A1 & 3E1 1 & A121 & & & 07,00 & $950-1100$ \\
\hline 4A1 & $2 \mathrm{~B} 02$ & A243 & & B? & 08,00 & $1300-1350$ \\
\hline 4A1 & $5 \mathrm{~B}$ & A233 & & & 09,50 & $1300-1350$ \\
\hline 4A1 & $5 \mathrm{C}$ & & & & 17,40 & $1250-1300$ \\
\hline 4A1 & $5 \mathrm{~A}$ & A221 & H1 & & 10,50 & $1000-1150$ \\
\hline 4A1 & & $\mathrm{A} 1 ?$ & H1 & & & $850-900$ \\
\hline 4A1 & $1 \mathrm{~B}$ & A133 & & & 06,00 & $1000-1050$ \\
\hline 4A1 & 1B0 1 & A133 & & & 06,00 & $900-1000$ \\
\hline 4A1 & $3 B$ & & & & 08,20 & $900-1000$ \\
\hline 4A1 & 3B141 & A133 & & & 08,00 & $900-1000$ \\
\hline 4A1 & $4 \mathrm{~F}$ & A131 & & & 09,50 & $1000-1100$ \\
\hline 4A1 & $3 \mathrm{E} 154$ & A131 & & & 10,00 & $1100-1200$ \\
\hline 4A1 & 3B181 & A122 & & & & $1000-1200$ \\
\hline 4A1 & $3 \mathrm{~B}$ & A131 & & & 09,00 & $700-1300$ \\
\hline 4A1 & 4D0 4 & A131 & & & 06,60 & $900-1200$ \\
\hline $4 \mathrm{~A} 1$ & $5 \mathrm{~A}$ & A131 & & & 07,00 & $900-1200$ \\
\hline 4A1 & 3B13 & A121 & & & & $850-950$ \\
\hline 4A1 & 3B1 & A233 & H1 & E1 & & $850-950$ \\
\hline 4A1 & $5 \mathrm{C}$ & A153 & & & & $1100-1200$ \\
\hline 4A1 & $5 \mathrm{C}$ & A141 & & & 08,70 & $850-950$ \\
\hline 4A1 & $3 \mathrm{E} 1$ & A133 & & & 06,25 & $850-950$ \\
\hline 4A1 & $3 \mathrm{~B}$ & A133 & & & 05,40 & $850-950$ \\
\hline $4 \mathrm{~A} 1$ & $3 \mathrm{~A} 4$ & A143 & H1 & & 08,20 & $1000-1100$ \\
\hline 4A1 & $5 \mathrm{~B}$ & A141 & H1 & & 12,00 & $1000-1050$ \\
\hline 4A1 & $5 \mathrm{~A} ?$ & A143 & & & 12,00 & $1000-1050$ \\
\hline 4A1 & $33 \mathrm{E}$ & A123 & & & 08,50 & $1000-1100$ \\
\hline 4A1 & 3B141 & A122 & H1 & & 09,00 & $1000-1100$ \\
\hline $4 \mathrm{~A} 1$ & 3B34 & $\mathrm{A} 1 ?$ & H1 & & 10,50 & $1000-1100$ \\
\hline $4 \mathrm{~A} 1$ & $3 \mathrm{~B}$ & A133 & & & 08,00 & $700-800$ \\
\hline 4A1 & $5 \mathrm{~A}$ & & & & 07,20 & $900-1000$ \\
\hline 4A1 & $5 \mathrm{~A}$ & & & & 06,20 & $900-1000$ \\
\hline $4 \mathrm{~A} 2$ & $3 \mathrm{E} 21$ & A141 & & & 08,00 & $950-1000$ \\
\hline $4 \mathrm{~A} 2$ & 1B1 1 & A131 & & & & $950-1000$ \\
\hline $4 \mathrm{~A} 2$ & 1B2 1 & A133 & & & 08,00 & $1000-1050$ \\
\hline $4 \mathrm{~A} 2$ & 1B2 1 & A121 & & & 09,70 & $1000-1050$ \\
\hline $4 \mathrm{~A} 2$ & 3B141 & A131 & & & 08,00 & $1000-1050$ \\
\hline $4 \mathrm{~A} 2$ & 3E1 1 & A133 & & & 07,00 & $1000-1050$ \\
\hline $4 \mathrm{~A} 2$ & 1B2 & A121 & & & 07,30 & 1050 \\
\hline $4 \mathrm{~A} 2$ & $3 \mathrm{~B}$ & A132 & & & 09,00 & $1050-1100$ \\
\hline $4 \mathrm{~A} 2$ & $3 \mathrm{~B}$ ? & A123 & & & 07,20 & $1050-1100$ \\
\hline
\end{tabular}




\begin{tabular}{|c|c|c|c|c|c|c|}
\hline 47 & $4 \mathrm{~A} 2$ & $3 \mathrm{E} 14$ & A132 & & 08,50 & $1050-1100$ \\
\hline 48 & $4 \mathrm{~A} 2$ & 3E1 3 & A122 & & 08,70 & $1050-1100$ \\
\hline 49 & $4 \mathrm{~A} 2$ & $5 \mathrm{~A}$ & A133 & & 08,20 & $1050-1100$ \\
\hline 50 & $4 \mathrm{~A} 2$ & 3E3 1 & A133 & & 14,30 & $1050-1100$ \\
\hline 51 & $4 \mathrm{~A} 2$ & 3A1 4 & A122 & & 06,80 & $1100-1150$ \\
\hline 52 & $4 \mathrm{~A} 2$ & 3E3 1 & A131 & & 09,00 & $1100-1150$ \\
\hline 53 & $4 \mathrm{~A} 2$ & 3D321 & A133 & & 06,90 & 1150 \\
\hline 54 & $4 \mathrm{~A} 2$ & 1B0 1 & A? & & 09,00 & 1150 \\
\hline 55 & $4 \mathrm{~A} 2$ & $5 \mathrm{C}$ & A143 & & 07,50 & $1150-1200$ \\
\hline 56 & $4 \mathrm{~A} 2$ & 1B1 1 & A141 & & 10,30 & $1150-1200$ \\
\hline 57 & $4 \mathrm{~A} 2$ & $3 \mathrm{~B}$ & A133 & & 08,50 & $1150-1200$ \\
\hline 58 & $4 \mathrm{~A} 2$ & 1B0 1 & A143 & & 07,00 & $1200-1250$ \\
\hline 59 & $4 \mathrm{~A} 2$ & 3E14 & A133 & & 06,50 & $1200-1250$ \\
\hline 60 & $4 \mathrm{~A} 2$ & 3E3 1 & A123 & & 08,50 & $1200-1250$ \\
\hline 61 & $4 \mathrm{~A} 2$ & 3B152 & A143 & & 10,50 & $1200-1250$ \\
\hline 62 & $4 \mathrm{~A} 2$ & 3E1 & A113 & & 07,00 & $1250-1300$ \\
\hline 63 & $4 \mathrm{~A} 2$ & $5 \mathrm{~A}$ & A133 & & 08,00 & $1250-1300$ \\
\hline 64 & $4 \mathrm{~A} 2$ & 3E1 2 & A131 & & 08,00 & 1300 \\
\hline 65 & $4 \mathrm{~A} 2$ & 1B1 1 & A143 & & 07,30 & 1300 \\
\hline 66 & $4 \mathrm{~A} 2$ & $2 \mathrm{C} 26$ & A141 & & 09,00 & $1200-1300$ \\
\hline 67 & $4 \mathrm{~A} 2$ & 3B34 & A603 & & 09,50 & $1200-1300$ \\
\hline 68 & $4 \mathrm{~A} 2$ & $3 \mathrm{~B} 173$ & A133 & & 11,00 & $1200-1300$ \\
\hline 69 & $4 \mathrm{~A} 2$ & 1B1 3 & A133 & & 12,00 & $1200-1300$ \\
\hline 70 & $4 \mathrm{~A} 2$ & $3 \mathrm{E} 13$ & A131 & & 14,00 & $1200-1300$ \\
\hline 71 & $4 \mathrm{~A} 2$ & 1B1 2 & A131 & & 13,50 & $1200-1300$ \\
\hline 72 & $4 \mathrm{~A} 2$ & 3B13 & B11 & $\mathrm{K}$ & & $1300-1350$ \\
\hline 73 & $4 \mathrm{~A} 2$ & $3 \mathrm{~B}$ & A131 & & & $1300-1350$ \\
\hline 74 & $4 \mathrm{~A} 2$ & 1B & A233 & & & $1300-1350$ \\
\hline 75 & $4 \mathrm{~A} 2$ & 3E1 1 & A133 & & & $1300-1350$ \\
\hline 76 & $4 \mathrm{~A} 2$ & & A1? & $\mathrm{K}$ & & $1300-1350$ \\
\hline 77 & $4 \mathrm{~A} 2$ & 1B0 1 & A143 & & 09,60 & $1250-1300$ \\
\hline 78 & $4 \mathrm{~A} 2$ & 3 & A133 & & 06,40 & $1300-1350$ \\
\hline 79 & $4 \mathrm{~A} 2$ & 3B13 & A132 & & 08,00 & $1300-1350$ \\
\hline 80 & $4 \mathrm{~A} 2$ & 1B1 & A133 & & 15,00 & $1300-1350$ \\
\hline 81 & $4 \mathrm{~A} 2$ & $5 \mathrm{~A}$ & A143 & & 08,00 & $1350-1400$ \\
\hline 82 & $4 \mathrm{~A} 2$ & 3B35 & A132 & & 09,20 & 1400 \\
\hline 83 & $4 \mathrm{~A} 2$ & 3B13 & A143 & & 14,00 & 1400 \\
\hline 84 & $4 \mathrm{~A} 2$ & 3B1 4 & A533 & & 10,00 & $1150-1400$ \\
\hline 85 & $4 \mathrm{~A} 2$ & 3E1 4 & A2 & E1 & 09,00 & $1000-1200$ \\
\hline 86 & $4 \mathrm{~A} 2$ & 1B1 & A133 & & 10,40 & $1000-1200$ \\
\hline 87 & $4 \mathrm{~A} 2$ & & A1? & $\mathrm{K}$ & & $1000-1100$ \\
\hline 88 & $4 \mathrm{~A} 2$ & 3B & A132 & & 09,00 & $1000-1100$ \\
\hline 89 & $4 \mathrm{~A} 2$ & 3B1 & A12? & & 08,30 & $1300-1400$ \\
\hline 90 & $4 \mathrm{~A} 2$ & $4 \mathrm{H}$ & A133 & & 17,00 & $1200-1400$ \\
\hline 91 & $4 \mathrm{~A} 2$ & 3B1 3 & A143 & & 10,00 & $1000-1200$ \\
\hline 92 & $4 \mathrm{~A} 2$ & $3 \mathrm{~B} 16$ & A133 & & 15,00 & $1000-1200$ \\
\hline 93 & $4 \mathrm{~A} 2$ & 1B1 5 & A103 & & 12,00 & $1000-1200$ \\
\hline 94 & $4 \mathrm{~A} 2$ & $5 \mathrm{C}$ & A131 & & 09,00 & $1000-1200$ \\
\hline 95 & $4 \mathrm{~A} 2$ & 1B2 1 & 141 & & 09,00 & $1000-1200$ \\
\hline
\end{tabular}


-88 -

\begin{tabular}{|c|c|c|c|c|c|c|c|}
\hline 96 & $4 \mathrm{~A} 2$ & $3 \mathrm{~B} 154$ & A133 & K & & 15,00 & $1000-1200$ \\
\hline 97 & $4 \mathrm{~A} 2$ & 1B1 1 & A133 & & & 09,50 & $1000-1200$ \\
\hline 98 & $4 \mathrm{~A} 2$ & 3B & A133 & & & 10,40 & $1000-1200$ \\
\hline 99 & $4 \mathrm{~A} 2$ & $3 \mathrm{~B}$ & A123 & & & 10,00 & $1000-1200$ \\
\hline 100 & $4 \mathrm{~A} 2$ & $5 \mathrm{~A}$ & A131 & & & 10,40 & $1000-1200$ \\
\hline 101 & $4 \mathrm{~A} 2$ & $1 \mathrm{~A} 11$ & A131 & & & 08,00 & $1000-1200$ \\
\hline 102 & $4 \mathrm{~A} 2$ & 3B15 & A133 & & & 07,00 & $1000-1200$ \\
\hline 103 & $4 \mathrm{~A} 2$ & $3 \mathrm{~B} 13$ & A133 & & & 09,20 & $1000-1200$ \\
\hline 104 & $4 \mathrm{~A} 2$ & $3 \mathrm{E} 1$ & A133 & & & 07,00 & $1000-1200$ \\
\hline 105 & 4A4 & $5 \mathrm{~A}$ & B2? & H1 & B?E2 & 08,50 & $1250-1450$ \\
\hline 106 & 4A4 & $1 \mathrm{~A} 1$ & A2? & H1 & & 12,50 & $1200-1400$ \\
\hline 107 & $4 \mathrm{~A} 4$ & $5 \mathrm{~A}$ & A2? & H1 & & 11,50 & $1250-1450$ \\
\hline 108 & $4 \mathrm{~A} 4$ & $1 \mathrm{~A} 11$ & A133 & H1 & & 11,60 & $1250-1450$ \\
\hline 109 & $4 \mathrm{~A} 3$ & $3 B 24$ & A143 & & & 11,60 & $1000-1050$ \\
\hline 110 & $4 \mathrm{~A} 3$ & $3 \mathrm{~B} 132$ & A143 & & & 17,20 & $1000-1150$ \\
\hline 111 & $4 \mathrm{~A} 3$ & 3B3 1 & A132 & & & 10,80 & $1000-1150$ \\
\hline 112 & $4 \mathrm{~A} 3$ & $3 \mathrm{~B} 13$ & A253 & & & 09,60 & $1000-1150$ \\
\hline 113 & $4 \mathrm{~A} 3$ & 3E2 3 & A1? & $\mathrm{K}$ ? & & 08,40 & $1000-1250$ \\
\hline 114 & $4 \mathrm{~A} 3$ & 3B & A141 & & & 06,40 & $1200-1300$ \\
\hline 115 & $4 \mathrm{~A} 3$ & $4 \mathrm{~A}$ & B11 & & & 12,00 & $1300-1400$ \\
\hline 116 & $4 \mathrm{~B}$ & $4 \mathrm{~F}$ & A132 & & & 09,30 & $900-1300$ \\
\hline 117 & $4 \mathrm{~B}$ & 3B35 & A133 & & & 14,40 & $900-1000$ \\
\hline 118 & $4 \mathrm{~B}$ & 3B3 1 & A233 & & & 08,00 & $850-1000$ \\
\hline 119 & $4 \mathrm{~B}$ & $3 \mathrm{E} 1$ & A133 & & & 12,00 & $850-1000$ \\
\hline 120 & $4 \mathrm{~B}$ & 3B3 1 & A131 & & & 08,00 & $850-1000$ \\
\hline 121 & $4 \mathrm{~B}$ & 3B3 1 & A133 & & & 07,60 & $850-1000$ \\
\hline 122 & 4B & 3B3 1 & A133 & & & 08,00 & $850-1000$ \\
\hline 123 & $4 \mathrm{~B}$ & 3B & A133 & & & 08,20 & $850-1000$ \\
\hline 124 & $4 \mathrm{~B}$ & $4 \mathrm{~F}$ & A123 & & & 05,80 & $850-1000$ \\
\hline 125 & $4 \mathrm{~B}$ & 3B3 1 & A133 & & & 06,00 & $850-1000$ \\
\hline 126 & $4 \mathrm{~B}$ & 1B2 1 & A133 & & & 06,00 & $850-1000$ \\
\hline 127 & $4 \mathrm{~B}$ & $3 \mathrm{E} 44$ & A133 & & & 07,70 & $900-1100$ \\
\hline 128 & $4 \mathrm{~B}$ & & $\mathrm{~A} 1 ?$ & $\mathrm{H} 1$ & & & $1000-1100$ \\
\hline 129 & $4 \mathrm{~B}$ & 3E144 & A133 & & & & $800-1100$ \\
\hline 130 & $4 \mathrm{~B}$ & & A1? & H1 & & & $1000-1050$ \\
\hline 131 & $4 \mathrm{~B}$ & 3E1 & A4? & H1 & ? & & $800-1050$ \\
\hline 132 & $4 \mathrm{~B}$ & $3 \mathrm{E} 16$ & A? & & & 17,50 & $900-1300$ \\
\hline 133 & $4 \mathrm{~B}$ & $3 \mathrm{~B} 25$ & A131 & & & 08,40 & $900-1300$ \\
\hline 134 & $4 \mathrm{~B}$ & $3 \mathrm{E} 1$ & & & & & $900-1300$ \\
\hline 135 & $4 \mathrm{~B}$ & $5 \mathrm{~A}$ & A122 & & & 10,50 & $900-1300$ \\
\hline 136 & $4 \mathrm{~B}$ & $5 \mathrm{~A}$ & A133 & & & 05,20 & $600-800$ \\
\hline 137 & $4 \mathrm{~B}$ & 3E4 4 & A143 & & & 06,40 & $600-800$ \\
\hline 138 & $4 \mathrm{~B}$ & $3 \mathrm{~B} 152$ & A133 & & & & $800-1200$ \\
\hline 139 & $4 \mathrm{~B}$ & 1B1 & A131 & & & 06,00 & $1000-1050$ \\
\hline 140 & $4 \mathrm{~B}$ & $3 \mathrm{E} 1$ & A121 & & & 10,30 & $1000-1050$ \\
\hline 141 & $4 \mathrm{~B}$ & 1B0 1 & A123 & & & 18,80 & $1100-1200$ \\
\hline 142 & 4B & $4 \mathrm{H} ?$ & A121 & & & 05,50 & $1100-1250$ \\
\hline 143 & $4 \mathrm{~B}$ & $3 \mathrm{E} 3$ & A131 & & & 09,00 & $1100-1250$ \\
\hline 144 & $4 \mathrm{~B}$ & $4 \mathrm{~F}$ & A122 & & & & $1100-1300$ \\
\hline
\end{tabular}




\begin{tabular}{|c|c|c|c|c|c|c|c|}
\hline 145 & $4 \mathrm{~B}$ & 1B2 3 & A143 & & & & $1100-1300$ \\
\hline 146 & $4 \mathrm{~B}$ & 3D15 & A143 & & & 07,00 & $1200-1300$ \\
\hline 147 & $4 \mathrm{~B}$ & $3 \mathrm{E} 1$ & $\mathrm{~A} 1 ?$ & & & 08,20 & $1200-1250$ \\
\hline 148 & $4 \mathrm{~B}$ & 3B & B11 & K & & 11,50 & $1250-1300$ \\
\hline 149 & $4 \mathrm{~B}$ & & A633 & & & 05,70 & $1250-1300$ \\
\hline 150 & 4B & 3B & A132 & & & 08,00 & 1250 \\
\hline 151 & $4 \mathrm{~B}$ & $3 \mathrm{~B} 36$ & A1 & & & 08,00 & 1250 \\
\hline 152 & $4 \mathrm{~B}$ & 1B1 1 & A111 & & & & $1250-1400$ \\
\hline 153 & 4B & 1B1 1 & A152 & & & & $1300-1400$ \\
\hline 154 & 4B & & B33 & & & & $1300-1500$ \\
\hline 155 & 4B & $5 \mathrm{D}$ & A131 & & & 08,50 & $1300-1500$ \\
\hline 156 & $4 \mathrm{~B}$ & $3 \mathrm{E} 44$ & A123 & & & 05,50 & $1300-1500$ \\
\hline 157 & $4 \mathrm{~B}$ & $5 \mathrm{D}$ & A133 & & & 08,00 & $1300-1500$ \\
\hline 158 & $4 \mathrm{~B}$ & 3E1 4 & A141 & & & 07,50 & $1300-1500$ \\
\hline 159 & 4B & $3 \mathrm{~B} 2$ & A133 & & & 07,30 & $1300-1500$ \\
\hline 160 & 4B & $3 \mathrm{~B} 132$ & & & & 09,50 & $1300-1500$ \\
\hline $160 \mathrm{~A}$ & 4B & & B32 & K & E1 & & $1300-1500$ \\
\hline 161 & $4 \mathrm{~B}$ & 3B141 & A523 & E1? & & 12,50 & $1600-1650$ \\
\hline 162 & 4B & $3 \mathrm{~B} 3$ & A133 & & & 08,00 & $950-1050$ \\
\hline 163 & $4 \mathrm{~B}$ & $3 \mathrm{~B} 33$ & A133 & & & 07,50 & $950-1050$ \\
\hline 164 & $4 \mathrm{~B}$ & $3 \mathrm{~B} 34$ & A123 & & & 09,50 & $950-1050$ \\
\hline 165 & 4B & $3 \mathrm{~B} 1$ & A233 & & & 10,00 & $1000-1300$ \\
\hline 166 & 4B & 3E1 4 & A131 & & & 11,80 & $900-1300$ \\
\hline 167 & $4 \mathrm{~B}$ & 3B1 3 & A143 & & & 08,00 & $1250-1300$ \\
\hline 168 & 4B & 1B & A143 & & & 08,50 & $1250-1300$ \\
\hline 169 & $4 \mathrm{~B}$ & $3 \mathrm{E} 13$ & A132 & & & 06,15 & $900-1300$ \\
\hline 170 & $4 \mathrm{~B}$ & $3 \mathrm{~B}$ & & & & 07,40 & $1250-1300$ \\
\hline 171 & 4B & 5B0 1 & A133 & & & 07,00 & $1250-1300$ \\
\hline 172 & 4B & $4 \mathrm{~A} ?$ & A1? & $\mathrm{H} 2$ & & 06,80 & $1300-1350$ \\
\hline 173 & 4B & $4 \mathrm{~A}$ & A243 & E1 & & 11,20 & $1300-1350$ \\
\hline 174 & $4 \mathrm{~B}$ & $2 \mathrm{~B}$ & A231 & & & 09,00 & $1300-1350$ \\
\hline 175 & $4 \mathrm{~B}$ & $3 \mathrm{~B} 162$ & A133 & & & 07,30 & $1000-1100$ \\
\hline 176 & $4 \mathrm{~B}$ & $3 \mathrm{~B}$ & A123 & & & 09,00 & $1250-1350$ \\
\hline 177 & 4B & & $\mathrm{A} 2 ?$ & $\mathrm{~K}$ ? & & & $1000-1150$ \\
\hline 178 & $4 \mathrm{~B}$ & 1B1 1 & A132 & & & 06,20 & $1000-1150$ \\
\hline 179 & 4D1 & $3 \mathrm{E} 16$ & A151 & & & 11,00 & $1100-1300$ \\
\hline 180 & 4D1 & & $\mathrm{B} 1 ?$ & K & & & $1200-1350$ \\
\hline 181 & 4D1 & $5 \mathrm{~A}$ & A123 & & & 08,00 & \\
\hline 182 & 4D1 & 1B2 1 & A132 & K & & 06,10 & $1050-1150$ \\
\hline 183 & 4D1 & 1B1 3 & A1? & & & 10,50 & $1050-1150$ \\
\hline 184 & $4 \mathrm{D} 1$ & 1B1 3 & A133 & & & 08,55 & $1050-1150$ \\
\hline 185 & 4D1 & & A141 & & & 15,00 & $1050-1150$ \\
\hline 186 & 4D1 & $4 \mathrm{~F}$ & A123 & & & 07,50 & $1050-1150$ \\
\hline 187 & 4D1 & 1B & A132 & & & 08,25 & $1050-1150$ \\
\hline 188 & 4D1 & 1B2 3 & A241 & H1 & & 06,75 & $1050-1150$ \\
\hline 189 & 4D1 & 3B & A131 & & & 08,25 & $1050-1150$ \\
\hline 190 & 4D1 & $3 \mathrm{~B}$ & A132 & & & 08,00 & $1050-1150$ \\
\hline 191 & 4D1 & $3 \mathrm{~B} 18$ & A143 & H1 & & 09,00 & $1050-1150$ \\
\hline 192 & 4D1 & $5 \mathrm{~A}$ & A123 & H1 & & 10,40 & $1050-1150$ \\
\hline
\end{tabular}




\begin{tabular}{|c|c|c|c|c|c|c|}
\hline 193 & 4D1 & $3 \mathrm{E}$ & A133 & & 11,00 & $1050-1150$ \\
\hline 194 & 4D1 & 1B2 1 & A143 & & 07,40 & $1050-1150$ \\
\hline 195 & 4D1 & 1B0 1 & A143 & & 08,40 & $1150-1250$ \\
\hline 196 & 4D1 & $1 \mathrm{~B} 11 ?$ & A133 & & 11,60 & $1150-1250$ \\
\hline 197 & 4D1 & 3B1 3 & A133 & & 07,70 & $1150-1250$ \\
\hline 198 & 4D1 & $5 \mathrm{~A}$ & A141 & & 07,60 & $1100-1300$ \\
\hline 199 & 4D1 & 1B1 1 & A143 & & 12,40 & $1100-1300$ \\
\hline 200 & 4D1 & $4 \mathrm{~A}$ & A132 & & 06,50 & $1200-1300$ \\
\hline 201 & 4D1 & $4 \mathrm{~A}$ & A131 & & 05,60 & $1200-1300$ \\
\hline 202 & 4D1 & $5 \mathrm{~A}$ & A143 & & 20,40 & $1200-1300$ \\
\hline 203 & 4D1 & 1B2 & A143 & & 12,40 & $1200-1300$ \\
\hline 204 & 4D1 & 3B & A131 & & 10,00 & $1200-1300$ \\
\hline 205 & 4D1 & 3B1 & A123 & & 11,20 & $1200-1300$ \\
\hline 206 & 4D1 & & B12 & & & $1200-1300$ \\
\hline 207 & 4D1 & $3 \mathrm{~B} 13$ & A141 & & 11,20 & $1200-1300$ \\
\hline 208 & 4D1 & $3 \mathrm{E}$ & A131 & & 09,00 & $1200-1300$ \\
\hline 209 & 4D1 & $3 \mathrm{~B} 13$ & A141 & & 08,00 & $1200-1300$ \\
\hline 210 & 4D1 & 1B2 1 & A141 & & & $1200-1300$ \\
\hline 211 & 4D1 & 1B2 1 & A143 & & 06,20 & $1200-1300$ \\
\hline 212 & 4D1 & 1B2 1 & A131 & & 11,00 & $1200-1300$ \\
\hline 213 & 4D1 & $3 \mathrm{~B} 155$ & A133 & & 14,40 & $1200-1300$ \\
\hline 214 & 4D1 & $1 \mathrm{~A} 1$ & A133 & & 14,00 & $1200-1300$ \\
\hline 215 & 4D1 & 1B2 1 & A143 & & 17,60 & $1200-1300$ \\
\hline 216 & 4D1 & 3B & A131 & & 14,40 & $1200-1300$ \\
\hline 217 & 4D1 & $5 \mathrm{C}$ & A & & 14,40 & $1200-1300$ \\
\hline 218 & 4D1 & $5 \mathrm{C}$ & & & 14,20 & $1200-1300$ \\
\hline 219 & 4D1 & $3 \mathrm{E} 1$ & A143 & & 09,50 & $1000-1300$ \\
\hline 220 & 4D1 & $2 \mathrm{~B} 1$ & A131 & & 07,50 & $1000-1200$ \\
\hline 221 & $4 \mathrm{D} 2$ & $3 \mathrm{~B} 13$ & A123 & & 07,50 & $1000-1300$ \\
\hline 222 & $4 \mathrm{D} 2$ & $5 \mathrm{C}$ & A233 & $\mathrm{B}$ ? & 12,60 & $1000-1200$ \\
\hline 223 & $4 \mathrm{D} 2$ & 1B2 & A143 & & 07,00 & $1100-1200$ \\
\hline 224 & $4 \mathrm{D} 2$ & 3E1 5 & A133 & & 09,00 & $1100-1200$ \\
\hline 225 & $4 \mathrm{D} 2$ & 1B2 1 & & & & $1100-1200$ \\
\hline 226 & $4 \mathrm{D} 2$ & 2B1 & B92 & & 09,00 & $1100-1200$ \\
\hline 227 & $4 \mathrm{D} 2$ & 3B171 & A153 & & 10,80 & $1100-1200$ \\
\hline 228 & $4 \mathrm{D} 2$ & $5 \mathrm{~A}$ & A131 & & 05,30 & $1100-1200$ \\
\hline 229 & $4 \mathrm{D} 2$ & $3 \mathrm{E} 44$ & A133 & & 03,70 & $1000-1200$ \\
\hline 230 & $4 \mathrm{D} 2$ & 5B & A133 & & 06,30 & $1000-1200$ \\
\hline 231 & $4 \mathrm{D} 2$ & 1B0 1 & A153 & & 08,00 & $1000-1200$ \\
\hline 232 & 4D2 & 1B1 1 & A133 & & 06,30 & $1000-1200$ \\
\hline 233 & $4 \mathrm{D} 2$ & 1B0 1 & A153 & & 09,30 & $1000-1200$ \\
\hline 234 & $4 \mathrm{D} 2$ & 1B1 & A123 & & 08,00 & $1100-1200$ \\
\hline 235 & $4 \mathrm{D} 2$ & 3B141 & A133 & & 06,20 & $1100-1200$ \\
\hline 236 & $4 \mathrm{D} 2$ & $3 \mathrm{~B} 13$ & A133 & & 05,00 & $1100-1200$ \\
\hline 237 & $4 \mathrm{D} 2$ & 3E1 4 & A133 & & 11,00 & $1000-1200$ \\
\hline 238 & 4D2 & $3 \mathrm{E} 1$ & A153 & & 06,50 & $1000-1200$ \\
\hline 239 & $4 \mathrm{D} 2$ & & A103 & & & $1000-1200$ \\
\hline 240 & $4 \mathrm{D} 2$ & $3 \mathrm{~B}$ & A133 & & 09,50 & $1000-1200$ \\
\hline 241 & $4 \mathrm{D} 2$ & $3 \mathrm{E} 3$ & A133 & & 10,80 & $1000-1200$ \\
\hline
\end{tabular}




\begin{tabular}{|c|c|c|c|c|c|c|}
\hline 242 & 4D2 & $3 \mathrm{E} 4$ & A133 & & 05,50 & $1000-1200$ \\
\hline 243 & 4D2 & $5 \mathrm{~A}$ & A121 & & 11,00 & $1000-1200$ \\
\hline 244 & 4D2 & $3 \mathrm{~B}$ & A143 & & 11,50 & $1000-1200$ \\
\hline 245 & 4D2 & $5 \mathrm{~B}$ & A133 & & 08,80 & 1050 \\
\hline 246 & 4D2 & 1B1 3 & A141 & & 06,60 & 1050 \\
\hline 247 & 4D2 & 3E1 4 & A13? & $\mathrm{H} 2$ & 10,80 & $1050-1100$ \\
\hline 248 & 4D2 & 1B1 3 & A132 & & 07,60 & $1050-1250$ \\
\hline 249 & 4D2 & 3B13 & A141 & & 09,40 & $1050-1150$ \\
\hline 250 & 4D2 & $2 \mathrm{~B} 2$ & A121 & & 10,20 & $1000-1100$ \\
\hline 251 & 4D2 & 1B2 & A132 & & 08,20 & $1050-1150$ \\
\hline 252 & 4D2 & 3E1 4 & A133 & & 12,70 & $1050-1150$ \\
\hline 253 & 4D2 & $3 \mathrm{E}$ & & & & $1050-1150$ \\
\hline 254 & 4D2 & $3 \mathrm{E}$ & A11? & & 10,00 & $1050-1150$ \\
\hline 255 & 4D2 & & A121 & & 07,00 & $1000-1100$ \\
\hline 256 & 4D2 & 3B141 & A133 & & 07,20 & $1050-1150$ \\
\hline 257 & 4D2 & 3E1 5 & A101 & & 09,00 & $1050-1150$ \\
\hline 258 & 4D2 & 3E1 6 & A103 & & 12,00 & $1050-1150$ \\
\hline 259 & 4D2 & 1B2 1 & A131 & & 08,00 & $1050-1150$ \\
\hline 260 & 4D2 & 1B5 1 & A121 & & 07,00 & $1050-1150$ \\
\hline 261 & 4D2 & 3E1 1 & A122 & & 07,00 & $1050-1150$ \\
\hline 262 & 4D2 & 3B15 & A143 & & 14,00 & $1050-1150$ \\
\hline 263 & 4D2 & $3 \mathrm{E} 26$ & A123 & & 13,00 & $1050-1150$ \\
\hline 264 & 4D2 & 1B2? & A132 & & 06,40 & $1050-1150$ \\
\hline 265 & 4D2 & $3 \mathrm{E} 2$ & A141 & & 12,20 & $1050-1150$ \\
\hline 266 & 4D2 & 3B & A132 & & 11,00 & $1050-1150$ \\
\hline 267 & 4D2 & $3 \mathrm{E}$ & A141 & & 12,70 & $1200-1350$ \\
\hline 268 & 4D2 & $3 \mathrm{E}$ & & & 10,80 & $1200-1350$ \\
\hline 269 & 4D2 & $3 \mathrm{~B} 33$ & A133 & & 14,00 & $1300-1350$ \\
\hline 270 & 4D2 & 3E1 4 & A133 & & 14,10 & $1000-1100$ \\
\hline 271 & 4D2 & 3B1 & A133 & & 11,80 & $1000-1100$ \\
\hline 272 & 4D2 & 1B2 1 & A103 & & 08,00 & $1100-1250$ \\
\hline 273 & 4D2 & $3 \mathrm{E} 16$ & A101 & & 08,00 & $1100-1250$ \\
\hline 274 & 4D2 & 1B2 1 & A133 & & 07,60 & $1100-1250$ \\
\hline 275 & 4D2 & 3E1 1 & A131 & & 11,50 & $1100-1250$ \\
\hline 276 & 4D2 & 3B141 & A132 & & 06,20 & $1100-1250$ \\
\hline 277 & 4D2 & $5 \mathrm{~A}$ & A101 & & 06,00 & $1100-1250$ \\
\hline 278 & 4D2 & 3B34 & A133 & & 12,40 & $1100-1250$ \\
\hline 279 & 4D2 & $3 \mathrm{E} 1$ & A141 & & 10,00 & $1100-1250$ \\
\hline 280 & 4D2 & & A1? & K & & $1100-1300$ \\
\hline 281 & 4D2 & & B3? & K & & $1100-1300$ \\
\hline 282 & 4D2 & 3E1 4 & A141 & & 10,50 & $1100-1300$ \\
\hline 283 & 4D2 & $3 \mathrm{~B} 155$ & A133 & & 11,70 & $1100-1300$ \\
\hline 284 & 4D2 & 3E1 6 & A152 & & 09,60 & $1100-1300$ \\
\hline 285 & 4D2 & 3B4 1 & A141 & & 11,20 & $1100-1300$ \\
\hline 286 & 4D2 & 3B13 & A241 & E1 & 06,60 & $1100-1300$ \\
\hline 287 & 4D2 & $5 \mathrm{~B}$ & A153 & & 10,00 & $1100-1300$ \\
\hline 288 & 4D2 & 2B0 2 & A131 & & 06,60 & $1100-1300$ \\
\hline 289 & 4D2 & $4 \mathrm{~F}$ & & & 08,80 & $1000-1100$ \\
\hline 290 & 4D2 & 1B2 1 & A133 & & 06,50 & $1000-1100$ \\
\hline
\end{tabular}




\begin{tabular}{|c|c|c|c|c|c|c|c|}
\hline 291 & $4 \mathrm{D} 2$ & 1B2 1 & A131 & & & 09,40 & $1000-1100$ \\
\hline 292 & 4D2 & 3B13 & A133 & & & 05,70 & $1000-1100$ \\
\hline 293 & $4 \mathrm{D} 2$ & $4 \mathrm{~F}$ & A122 & & & 10,00 & $1000-1100$ \\
\hline 294 & $4 \mathrm{D} 2$ & 1B2 1 & A133 & & & 08,20 & $1000-1100$ \\
\hline 295 & $4 \mathrm{D} 2$ & $3 \mathrm{E} 1$ & A133 & & & 10,50 & $1000-1100$ \\
\hline 296 & $4 \mathrm{D} 2$ & 1B & & & & 03,40 & $1000-1100$ \\
\hline 297 & 4D2 & 2B1 5 & & & & & $1000-1100$ \\
\hline 298 & 4D2 & $3 \mathrm{~B} 33$ & A112 & & & 10,40 & $1000-1100$ \\
\hline 299 & 4D2 & 3E1 1 & A131 & & & 11,00 & $1000-1200$ \\
\hline 300 & 4D2 & 1B1 1 & A133 & & & 09,00 & $1000-1200$ \\
\hline 301 & 4D2 & $3 \mathrm{~B} 13$ & B12 & & $?$ & 09,00 & $1000-1200$ \\
\hline 302 & $4 \mathrm{D} 2$ & 3E1 4 & A131 & & & 12,00 & $1000-1300$ \\
\hline 303 & 4D2 & $3 \mathrm{E} 1$ & A123 & & & 10,00 & $1050-1100$ \\
\hline 304 & 4D2 & $3 \mathrm{E} 1$ & A133 & & & 12,00 & $1050-1100$ \\
\hline 305 & $4 \mathrm{E}$ & 1B2 1 & & & & 12,80 & $900-1100$ \\
\hline 306 & $4 \mathrm{E}$ & 2B1 3 & B22 & & & 07,00 & $1100-1300$ \\
\hline 307 & $4 \mathrm{E}$ & $3 \mathrm{E} 1$ & B?2 & & & 08,40 & $1100-1300$ \\
\hline 308 & $4 \mathrm{E}$ & 3B141 & A131 & & & 13,70 & $1100-1300$ \\
\hline 309 & $4 \mathrm{E}$ & & B22 & & & 05,40 & $1100-1300$ \\
\hline 310 & $4 \mathrm{E}$ & $5 \mathrm{~A}$ & A123 & & & 09,00 & $900-1000$ \\
\hline 311 & $4 \mathrm{E}$ & $3 \mathrm{E} 1$ & A133 & & & 10,50 & $900-1000$ \\
\hline 312 & $4 \mathrm{E}$ & $5 \mathrm{~A}$ & A133 & & & 09,00 & $1000-1100$ \\
\hline 313 & $4 \mathrm{E}$ & $3 \mathrm{~B} 184$ & A133 & & & & $1000-1200$ \\
\hline 314 & $4 \mathrm{E}$ & 3B13 & A143 & & & & $1100-1300$ \\
\hline 315 & $4 \mathrm{E}$ & 2B1 1 & A343 & & $\mathrm{H} 2 \mathrm{~L}$ & 09,00 & $1100-1250$ \\
\hline 316 & $4 \mathrm{E}$ & 4F0 4 & A143 & & & 07,20 & $1200-1300$ \\
\hline 317 & $4 \mathrm{E}$ & 1B0 1 & A143 & & & 09,60 & $1200-1300$ \\
\hline 318 & $4 \mathrm{E}$ & 3B & A133 & & & 09,80 & $1200-1300$ \\
\hline 319 & $4 \mathrm{E}$ & 1B0 1 & & & & 09,00 & $1200-1300$ \\
\hline 320 & $4 \mathrm{E}$ & $4 \mathrm{~J}$ & A143 & & & 09,00 & $1200-1300$ \\
\hline 321 & $4 \mathrm{E}$ & 1B1 1 & & & & 09,60 & $1100-1300$ \\
\hline 322 & $4 \mathrm{E}$ & 1B1 1 & A141 & & & 10,00 & $1200-1300$ \\
\hline 323 & $4 \mathrm{E}$ & $4 \mathrm{E}$ & & & & 08,30 & $1200-1300$ \\
\hline 324 & $4 \mathrm{E}$ & 3B & A132 & & & 07,20 & $1200-1300$ \\
\hline 325 & $4 \mathrm{E}$ & $4 \mathrm{E}$ & A143 & & & 07,00 & $1200-1300$ \\
\hline 326 & $4 \mathrm{E}$ & $3 \mathrm{~B} 16$ & A131 & & & 10,50 & $1200-1300$ \\
\hline 327 & $4 \mathrm{E}$ & 1B0 1 & A131 & & & 09,00 & $1200-1300$ \\
\hline 328 & $4 \mathrm{E}$ & $3 \mathrm{~B} 132$ & A121 & & & 13,00 & $1200-1300$ \\
\hline 329 & $4 \mathrm{E}$ & 3B141 & A133 & & & 10,00 & $1000-1100$ \\
\hline 330 & $4 \mathrm{E}$ & & A1? & H1 & & & $900-1200$ \\
\hline 332 & $4 \mathrm{E}$ & & B1? & $\mathrm{K}$ & & & $1050-1100$ \\
\hline 333 & $4 \mathrm{E}$ & $3 \mathrm{~B} 2$ & A131 & & & 11,60 & $1050-1150$ \\
\hline 334 & $4 \mathrm{E}$ & $3 \mathrm{~B} 156$ & A103 & & & 10,00 & $1100-1200$ \\
\hline 335 & $4 \mathrm{E}$ & $3 \mathrm{~B}$ & A133 & & & 09,70 & $1050-1100$ \\
\hline 336 & $4 \mathrm{E}$ & 3B & A133 & & & 06,70 & $1100-1200$ \\
\hline 337 & $4 \mathrm{E}$ & & B12 & $\mathrm{K}$ & E1 & & $900-1200$ \\
\hline 338 & $4 \mathrm{E}$ & & B1? & $\mathrm{K}$ & & & $900-1200$ \\
\hline 339 & $4 \mathrm{E}$ & 1B1 3 & A1? & & & 08,20 & $1100-1200$ \\
\hline 340 & $4 \mathrm{E}$ & $5 \mathrm{C}$ & A233 & E1 & & 05,60 & 1150 \\
\hline
\end{tabular}




\begin{tabular}{|c|c|c|c|c|c|c|c|}
\hline 341 & $4 \mathrm{E}$ & $3 \mathrm{E} 26$ & A133 & & & 13,80 & $900-1200$ \\
\hline 342 & $4 \mathrm{E}$ & 1B1 3 & A133 & & & 13,00 & $1100-1350$ \\
\hline 343 & $4 \mathrm{E}$ & $3 \mathrm{~B} 175$ & A14? & $\mathrm{H} 2$ & & 09,30 & $1000-1200$ \\
\hline 344 & $4 \mathrm{E}$ & 1B0 1 & A143 & $\mathrm{H} 2$ & & 11,70 & $1100-1150$ \\
\hline 345 & $4 \mathrm{E}$ & 3B & A133 & & & 09,50 & $1100-1200$ \\
\hline 346 & $4 \mathrm{E}$ & 2B1 3 & A133 & & & 05,20 & $1000-1250$ \\
\hline 347 & $4 \mathrm{E}$ & $3 \mathrm{E} 2$ & A141 & & & 11,00 & $1100-1200$ \\
\hline 348 & $4 \mathrm{E}$ & 1B2 3 & A333 & $\mathrm{K}$ & B P & 12,00 & $1100-1300$ \\
\hline 349 & $4 \mathrm{E}$ & 3 E1 6 & A123 & & & & $1000-1100$ \\
\hline 350 & $4 \mathrm{E}$ & 3E1 3 & A151 & & & & $1000-1100$ \\
\hline 351 & $4 \mathrm{E}$ & 3E1 5 & A131 & & & & $1000-1100$ \\
\hline 352 & $4 \mathrm{C} 2$ & 3E4 1 & A133 & & & 11,40 & $1250-1350$ \\
\hline 353 & $4 \mathrm{C} 2$ & 3B14 & A131 & & & 14,50 & $1250-1350$ \\
\hline 354 & $4 \mathrm{C} 2$ & $4 \mathrm{~F}$ & A123 & & & 05,10 & $1250-1400$ \\
\hline 355 & $4 \mathrm{C} 2$ & 2B2 2 & A123 & & & 09,30 & $1200-1300$ \\
\hline 356 & $4 \mathrm{C} 2$ & 3E1 4 & A133 & & & 11,20 & $1100-1300$ \\
\hline 357 & $4 \mathrm{C} 2$ & $5 \mathrm{C}$ & A153 & & & 11,00 & $1000-1100$ \\
\hline 358 & $4 \mathrm{C} 2$ & 1B1 1 & A153 & & & 11,60 & $1000-1100$ \\
\hline 359 & $4 \mathrm{C} 2$ & $5 \mathrm{C}$ & A153 & & & 10,00 & $1000-1100$ \\
\hline 360 & $4 \mathrm{C} 2$ & $5 \mathrm{~B} ?$ & $\mathrm{~A} 1 ?$ & & & 10,80 & $1000-1100$ \\
\hline 361 & $4 \mathrm{C} 2$ & 1B0 1 & A123 & & & 07,00 & $1000-1300$ \\
\hline 362 & $4 \mathrm{C} 2$ & 3E4 4 & A122 & & & 07,00 & $1000-1250$ \\
\hline 363 & $4 \mathrm{C} 2$ & 1B0 2 & A13? & & & 14,00 & $1000-1250$ \\
\hline 364 & $4 \mathrm{C} 2$ & $5 \mathrm{~A} ?$ & A133 & & & & $900-1300$ \\
\hline 365 & $4 \mathrm{C} 2$ & $3 \mathrm{~B} 15$ & A132 & & & 08,00 & $600-850$ \\
\hline 366 & $4 \mathrm{C} 2$ & $3 \mathrm{~B} 173$ & A133 & & & 07,80 & $800-1100$ \\
\hline 367 & $4 \mathrm{E}$ & 1B2 1 & A143 & & & 04,60 & $1000-1200$ \\
\hline 368 & $4 \mathrm{E}$ & 3E1 6 & A233 & & & 09,80 & $1000-1200$ \\
\hline 369 & $4 \mathrm{E}$ & 1B2 1 & A140 & & & 07,80 & $1000-1200$ \\
\hline 370 & $4 \mathrm{E}$ & $3 \mathrm{~B} 36$ & A131 & & & 08,80 & $1000-1200$ \\
\hline 371 & $4 \mathrm{E}$ & 1A1 1 & A133 & & & 05,80 & $1000-1200$ \\
\hline 372 & $4 \mathrm{E}$ & $3 \mathrm{E} 16$ & A133 & & & 08,60 & $1000-1200$ \\
\hline 373 & $4 \mathrm{E}$ & 3B141 & A133 & & & 09,00 & $1000-1200$ \\
\hline 374 & $4 \mathrm{E}$ & 1B0 1 & A133 & & & & $800-900$ \\
\hline 375 & $4 \mathrm{E}$ & 2B1 3 & A133 & & & & $800-900$ \\
\hline 376 & $4 \mathrm{E}$ & $3 \mathrm{~B}$ & A132 & & & & $800-900$ \\
\hline 44 & $6 \mathrm{~A}$ & & A1? & $\mathrm{K}$ & & & $1200-1400$ \\
\hline 45 & $6 \mathrm{~A}$ & 1B1 1 & A14? & $\mathrm{K}$ & & 11,00 & $1200-1400$ \\
\hline $63 \mathrm{~A}$ & $4 \mathrm{~A} 2$ & & A1 & $\mathrm{K}$ & & & $1200-1300$ \\
\hline 67 & $6 \mathrm{C} 1$ & $3 \mathrm{~B} 155$ & A133 & & & 08,00 & $1100-1150$ \\
\hline 68 & $6 \mathrm{C} 1$ & 3B17 & A133 & & & 07,30 & $1100-1150$ \\
\hline 69 & $6 \mathrm{C} 1$ & $3 \mathrm{~B} 16$ & A1? & & & 12,00 & $1100-1150$ \\
\hline 70 & $6 \mathrm{C} 1$ & $5 \mathrm{C}$ & A132 & & & 04,60 & $1150-1200$ \\
\hline 71 & $6 \mathrm{C} 1$ & $3 \mathrm{~B}$ & A133 & & & 05,70 & $1150-1200$ \\
\hline 72 & $6 \mathrm{C} 1$ & $3 \mathrm{E} 1$ & A1? & & & 07,80 & $1150-1200$ \\
\hline 73 & $6 \mathrm{C} 1$ & $3 \mathrm{E} 1$ & A1? & & & 07,50 & $1150-1200$ \\
\hline 74 & $6 \mathrm{C} 1$ & & A13? & $\mathrm{K}$ & & & $1200-1300$ \\
\hline 75 & $6 \mathrm{C} 1$ & $3 \mathrm{~B} 17$ & A133 & & & 08,60 & $1000-1200$ \\
\hline 76 & $6 \mathrm{C} 1$ & $5 \mathrm{C}$ & A143 & & & 06,00 & $1000-1200$ \\
\hline
\end{tabular}


- 94 -

$\begin{array}{lllllll}77 & 6 \mathrm{C} 1 & 3 \mathrm{E} 1 & \mathrm{~A} 122 & & 06,00 & 1000-1200 \\ 78 & 6 \mathrm{C} 1 & 3 \mathrm{~B} 17 & \mathrm{~A} 13 ? & & 07,50 & 1000-1200 \\ 79 & 6 \mathrm{C} 1 & 1 \mathrm{~B} 21 & \mathrm{~A} 133 & & 10,50 & 1000-1200 \\ 80 & 6 \mathrm{C} 1 & 3 \mathrm{~B} 17 & \mathrm{~A} & & 07,00 & 1000-1200 \\ 81 & 6 \mathrm{C} 1 & 3 \mathrm{E} & & & 10,60 & 1000-1250 \\ 82 & 6 \mathrm{C} 1 & & \mathrm{~B} 1 ? & \mathrm{~K} & & 1100-1300 \\ 83 & 6 \mathrm{C} 1 & 3 \mathrm{~B} 184 & \mathrm{~A} 23 ? & \mathrm{~K} & 07,00 & 1200-1400 \\ 84 & 6 \mathrm{C} 1 & 5 \mathrm{~B} & \mathrm{~A} 133 & & 10,60 & 1200-1400 \\ 85 & 6 \mathrm{C} 1 & 3 \mathrm{~A} & \mathrm{~A} 133 & & 10,00 & 1200-1400\end{array}$




\section{Dänemark}

\begin{tabular}{|c|c|c|c|c|c|c|}
\hline $5 \mathrm{~A} 2$ & & A533 & K E2 & & & 1300 \\
\hline $5 \mathrm{~A} 2$ & $3 \mathrm{~B}$ & B42 & H1 & B & 10,10 & $1450-1500$ \\
\hline $5 \mathrm{~A} 2$ & 2B0 2 & B42 & H1 & B & 09,20 & $1450-1500$ \\
\hline $5 \mathrm{~A} 2$ & 3B191 & A133 & & & 07,50 & $900-1000$ \\
\hline $5 \mathrm{~A} 2$ & $3 \mathrm{~B} 123$ & A133 & & & 08,50 & $900-1250$ \\
\hline $5 \mathrm{~A} 2$ & 1B0 1 & A233 & & & 09,00 & $900-1250$ \\
\hline $5 \mathrm{~A} 2$ & 1B0 1 & A133 & & & 08,30 & $1200-1350$ \\
\hline $5 \mathrm{~A} 2$ & 2B1 1 & A433 & H1 & & 04,10 & $1200-1350$ \\
\hline $5 \mathrm{~A} 2$ & $3 \mathrm{~B}$ & A133 & & & 06,60 & $900-1000$ \\
\hline $5 \mathrm{~A} 2$ & $3 \mathrm{~B}$ & A133 & $\mathrm{H} 1$ & & 08,00 & $900-1250$ \\
\hline $5 \mathrm{~A} 2$ & $3 \mathrm{~B}$ & A233 & H1 & & 11,50 & $1200-1350$ \\
\hline $5 \mathrm{~A} 2$ & 1B13 & A133 & & & 10,00 & $1200-1350$ \\
\hline $5 \mathrm{~A} 2$ & 3B13 & A131 & & & 11,30 & $1200-1350$ \\
\hline $5 \mathrm{~A} 2$ & $5 \mathrm{D}$ & & & & 07,50 & $1300-1400$ \\
\hline $5 \mathrm{~A} 2$ & 3E33 & A143 & & & 11,30 & $1200-1350$ \\
\hline $5 \mathrm{~A} 2$ & $3 \mathrm{~B}$ & B2? & & B & 11,00 & $1450-1550$ \\
\hline $5 \mathrm{~A} 2$ & & A1? & $\mathrm{K}$ & & & $900-1250$ \\
\hline $5 \mathrm{~A} 2$ & & A1? & $\mathrm{H} 2$ & & & $900-1250$ \\
\hline $5 \mathrm{~A} 2$ & & $\mathrm{~A} 2$ & $\mathrm{~K}$ & & & $1200-1350$ \\
\hline $5 \mathrm{~A} 2$ & & A1? & K & & & $1200-1350$ \\
\hline $5 \mathrm{~A} 2$ & $3 \mathrm{~B}$ & A152 & & & 06,20 & \\
\hline $5 \mathrm{~A} 2$ & $3 \mathrm{~B}$ & B42 & $\mathrm{K}$ & & & $1500-1600$ \\
\hline $5 \mathrm{~A} 2$ & $3 \mathrm{~B}$ & B42 & B & & & $1550-1600$ \\
\hline $5 \mathrm{~A} 2$ & $3 \mathrm{E}$ & A343 & & B & 14,50 & 1300 \\
\hline $5 \mathrm{~A} 2$ & 1B0 1 & A131 & & & 11,25 & 1300 \\
\hline $5 \mathrm{~A} 2$ & $3 \mathrm{E}$ & A143 & & & 11,50 & 1300 \\
\hline $5 \mathrm{~A} 2$ & $3 \mathrm{E}$ & A133 & & & 14,25 & 1300 \\
\hline $5 \mathrm{~A} 2$ & 1B0 1 & A133 & & & 09,25 & 1300 \\
\hline $5 \mathrm{~A} 2$ & 1B0 1 & A233 & & B & 07,80 & 1300 \\
\hline $5 \mathrm{~A} 2$ & & A433 & H1 & $\mathrm{H} 2 ?$ & 08,40 & 1300 \\
\hline $5 \mathrm{~A} 2$ & & A23? & & $\mathrm{B}$ & & 1300 \\
\hline $5 \mathrm{~A} 2$ & & A33? & & B & & 1300 \\
\hline $5 \mathrm{~A} 2$ & & A33? & & B & & 1300 \\
\hline $5 \mathrm{~A} 2$ & & $\mathrm{~B}$ ? & $\mathrm{K}$ & & & 1300 \\
\hline $5 \mathrm{~A} 2$ & $3 \mathrm{~B}$ & B41 & & B & 07,00 & $1450-1550$ \\
\hline $5 \mathrm{~A} 2$ & 2B1 3 & B23? & $\mathrm{K}$ & E2 & 15,00 & $1200-1400$ \\
\hline $5 \mathrm{~A} 2$ & 1B0 1 & & & & 11,10 & $1000-1100$ \\
\hline $5 \mathrm{~A} 2$ & $3 \mathrm{~B}$ & A143 & & & 07,50 & 1300 \\
\hline $5 \mathrm{~A} 2$ & $3 \mathrm{E} 24$ & A143 & & & 13,30 & $1000-1300$ \\
\hline $5 \mathrm{~A} 2$ & $1 \mathrm{~B}$ & B23 & & & 09,00 & $1300-1400$ \\
\hline $5 \mathrm{~A} 2$ & $3 \mathrm{~B}$ & A133 & & & 06,00 & \\
\hline $5 \mathrm{~A} 2$ & $5 \mathrm{~B}$ & A133 & & & 09,50 & \\
\hline $5 \mathrm{~A} 2$ & $3 \mathrm{E} 14$ & A132 & & & 06,50 & \\
\hline $5 \mathrm{~A} 2$ & & A141 & & & & $1200-1300$ \\
\hline $5 \mathrm{~A} 2$ & $3 \mathrm{~B}$ & A133 & & & 06,70 & \\
\hline $5 \mathrm{~A} 2$ & $3 \mathrm{~B}$ & & & & 10,70 & \\
\hline
\end{tabular}




\begin{tabular}{|c|c|c|c|c|c|c|c|}
\hline 47 & $5 \mathrm{~A} 2$ & $1 \mathrm{~B} 13$ & & & & 14,10 & 1300 \\
\hline 48 & $5 \mathrm{~A} 2$ & & B3? & $\mathrm{KB}$ & & & $1450-1550$ \\
\hline 49 & $5 \mathrm{~A} 2$ & 1B & B32 & & & 07,00 & $1500-1600$ \\
\hline 50 & $5 \mathrm{~A} 2$ & & B12 & H1 & B & & $1500-1600$ \\
\hline 51 & $5 \mathrm{~A} 2$ & 3B & A153 & & & 08,20 & \\
\hline 52 & $5 \mathrm{~A} 2$ & & $\mathrm{~B} 4 ? 2$ & K & & & $1550-1650$ \\
\hline 53 & $5 \mathrm{~A} 2$ & 3B & B12 & & B & 10,40 & $1500-1700$ \\
\hline 54 & $5 \mathrm{~A} 2$ & & B42 & & & 05,10 & $1500-1700$ \\
\hline 55 & $5 \mathrm{~A} 2$ & 1B & B42 & & & 07,50 & $1600-1700$ \\
\hline 56 & $5 \mathrm{~A} 2$ & & A232 & & & & $1600-1700$ \\
\hline 57 & $5 \mathrm{~A} 2$ & 2B1 4 & B42 & $\mathrm{K}$ & & 09,00 & \\
\hline 58 & $5 \mathrm{~A} 2$ & 2B1 4 & B42 & $\mathrm{K}$ & & 06,80 & \\
\hline 59 & $5 \mathrm{~A} 2$ & 3B & A423 & K E1 & & 12,80 & \\
\hline 60 & $5 \mathrm{~A} 2$ & & A523 & E1? & & & $1600-1800$ \\
\hline 61 & $5 \mathrm{~A} 2$ & $3 \mathrm{~B} 35$ & A221 & & & 09,75 & \\
\hline 62 & $5 \mathrm{~A} 2$ & 3B & A233 & & & 11,00 & \\
\hline 63 & $5 \mathrm{~A} 2$ & 3 E2 6 & A133 & & & 07,50 & \\
\hline 64 & $5 \mathrm{~A} 2$ & 3B & A131 & & & 12,50 & \\
\hline 65 & $5 \mathrm{~A} 2$ & & A1? & $\mathrm{K}$ & & & $1200-1400$ \\
\hline 66 & $5 \mathrm{~A} 2$ & & $\mathrm{~A} 1 ?$ & $\mathrm{H} 2 ?$ & & & $1200-1400$ \\
\hline 67 & $5 \mathrm{~A} 2$ & & A1? & $\mathrm{K}$ & & & $1300-1400$ \\
\hline 68 & $5 \mathrm{~A} 2$ & & B1? & $\mathrm{K}$ & & & $1300-1400$ \\
\hline 69 & $5 \mathrm{~A} 2$ & $3 \mathrm{E}$ & B32 & $\mathrm{K}$ & E2 & 09,80 & $1350-1500$ \\
\hline 70 & $5 \mathrm{~A} 2$ & 3B14 & A222 & E1 & & 11,50 & $1500-1600$ \\
\hline 71 & $5 \mathrm{~A} 2$ & 3B141 & B22 & & & 09,50 & $1400-1500$ \\
\hline 72 & $5 \mathrm{~A} 2$ & $3 \mathrm{E}$ & B42 & $\mathrm{H} 1$ & $\mathrm{~B}$ & 11,70 & $1400-1500$ \\
\hline 73 & $5 \mathrm{~A} 2$ & 3B & B41 & & B E2 & & $1450-1550$ \\
\hline 74 & $5 \mathrm{~A} 2$ & 3B & B41 & & B E2 & & $1450-1550$ \\
\hline 75 & $5 \mathrm{~A} 2$ & 3E4 4 & A14? & $\mathrm{K}$ & & 09,50 & $1350-1450$ \\
\hline 76 & $5 \mathrm{~A} 2$ & 1B1 3 & A133 & & & 06,00 & $1350-1450$ \\
\hline 77 & $5 \mathrm{~A} 2$ & 5В0 3 & A133 & & & 09,00 & $1350-1450$ \\
\hline 78 & $5 \mathrm{~A} 2$ & 3B141 & A132 & & & 09,70 & $1350-1450$ \\
\hline 79 & $5 \mathrm{~A} 2$ & $3 \mathrm{~B} 33$ & A122 & E1 & & 07,80 & $1350-1450$ \\
\hline 80 & $5 \mathrm{~A} 2$ & 2B1 1 & A133 & & & 09,80 & $1350-1450$ \\
\hline 81 & $5 \mathrm{~A} 2$ & $4 \mathrm{~F}$ & B32 & H1? & & 05,00 & $1350-1450$ \\
\hline 82 & $5 \mathrm{~A} 2$ & & B21 & H1 & B & & $1350-1450$ \\
\hline 83 & $5 \mathrm{~A} 2$ & 3B & B41 & & B E2 & & $1400-1500$ \\
\hline 84 & $5 \mathrm{~A} 2$ & 2B1 2 & B11 & & & 11,00 & $1400-1500$ \\
\hline 85 & $5 \mathrm{~A} 2$ & 3B & B22 & & B & 11,50 & $1400-1500$ \\
\hline 86 & $5 \mathrm{~A} 2$ & & B22 & $\mathrm{H} 1$ & B & & $1350-1450$ \\
\hline 87 & $5 \mathrm{~A} 2$ & & B32 & $\mathrm{H} 2$ & & & $1350-1500$ \\
\hline 88 & $5 \mathrm{~A} 2$ & 3B & B42 & H1 & B & 06,50 & $1500-1550$ \\
\hline 89 & $5 \mathrm{~A} 2$ & $3 \mathrm{~B} 133$ & B42 & $\mathrm{H} 1$ & $\mathrm{~B}$ & 08,00 & $1550-1650$ \\
\hline 90 & $5 \mathrm{~A} 2$ & & A223 & H1 & & & $1550-1650$ \\
\hline 91 & $5 \mathrm{~A} 2$ & 3B & B42 & $\mathrm{H} 2$ & B & & $1500-1550$ \\
\hline
\end{tabular}




\section{Norwegen}

\begin{tabular}{|c|c|c|c|c|c|c|}
\hline $5 \mathrm{~B} 1$ & 1B0 1 & A13? & $\mathrm{K}$ & & 04,00 & $1300-1400$ \\
\hline $5 \mathrm{~B} 1$ & $3 \mathrm{~B}$ & A333 & B & B E3? & 10,20 & $1150-1200$ \\
\hline $5 \mathrm{~B} 1$ & $3 \mathrm{~B}$ & A132 & & & 07,20 & 1050 \\
\hline $5 \mathrm{~B} 1$ & $5 \mathrm{E}$ & A133 & & & 11,50 & 1100 \\
\hline $5 \mathrm{~B} 1$ & $3 \mathrm{~B}$ & A133 & & & 04,80 & 1100 \\
\hline $5 \mathrm{~B} 1$ & $3 \mathrm{~B}$ & A133 & & & 06,30 & 1100 \\
\hline $5 \mathrm{~B} 1$ & $3 \mathrm{E} 2$ & A133 & & & 08,00 & $1100-1200$ \\
\hline $5 \mathrm{~B} 1$ & 3E1 3 & A132 & & & 07,60 & $1150-1200$ \\
\hline $5 \mathrm{~B} 1$ & 3B174 & A132 & & & 07,00 & $1150-1200$ \\
\hline $5 \mathrm{~B} 1$ & $3 \mathrm{~B}$ & A133 & & & 08,20 & $1150-1200$ \\
\hline $5 \mathrm{~B} 1$ & $3 \mathrm{~B}$ & A133 & & & 10,00 & $1150-1200$ \\
\hline $5 \mathrm{~B} 1$ & $3 \mathrm{~B}$ & A533 & & & 07,80 & $1150-1200$ \\
\hline $5 \mathrm{~B} 1$ & 3 E33 & A153 & & & 05,50 & $1150-1200$ \\
\hline $5 \mathrm{~B} 1$ & 3E1 4 & A153 & & & 08,70 & $1150-1200$ \\
\hline $5 \mathrm{~B} 1$ & 3E2 4 & A133 & & & 11,00 & $1150-1200$ \\
\hline $5 \mathrm{~B} 1$ & 3E144 & A143 & & & 09,50 & $1150-1250$ \\
\hline $5 \mathrm{~B} 1$ & 4D & A131 & & & 08,70 & $1150-1250$ \\
\hline $5 \mathrm{~B} 1$ & & A13? & $\mathrm{H} 1$ & & 04,20 & $1200-1250$ \\
\hline $5 \mathrm{~B} 1$ & $3 \mathrm{~B}$ & A132 & & & 07,60 & $1300-1400$ \\
\hline $5 \mathrm{~B} 1$ & 3B34 & A153 & & & 13,50 & $1300-1400$ \\
\hline $5 \mathrm{~B} 1$ & 3B171 & A13? & & & 16,50 & $1400-1500$ \\
\hline $5 \mathrm{~B} 1$ & 3 & A133 & & & 05,80 & \\
\hline $5 \mathrm{~B} 1$ & 3B171 & A153 & & & 05,70 & $1500-1650$ \\
\hline $5 \mathrm{~B} 1$ & 3B13 & B12 & & & 09,40 & $1300-1450$ \\
\hline $5 \mathrm{~B} 1$ & & $\mathrm{~A} 1$ & $\mathrm{~K}$ & & & $1300-1400$ \\
\hline $5 \mathrm{~B} 1$ & & A1 & K & & & $1250-1350$ \\
\hline $5 \mathrm{~B} 1$ & & A2? & $\mathrm{K}$ & & & \\
\hline $5 \mathrm{~B} 1$ & $4 \mathrm{~F}$ & B32 & & & 17,40 & \\
\hline $5 \mathrm{~B} 1$ & $3 \mathrm{~B}$ & B82 & K H2 & & 10,90 & \\
\hline $5 \mathrm{~B} 1$ & & B32 & $\mathrm{K}$ & E2 & & $1500-1600$ \\
\hline $5 \mathrm{~B} 1$ & & B41 & & $\mathrm{B}$ & 07,00 & $1450-1550$ \\
\hline $5 \mathrm{~B} 1$ & $3 \mathrm{~B}$ & A223 & H1B & & 13,00 & \\
\hline $5 \mathrm{~B} 1$ & & B32 & & & & \\
\hline $5 \mathrm{~B} 1$ & $3 \mathrm{~B}$ & A13? & $\mathrm{K}$ & & 08,40 & \\
\hline $5 \mathrm{~B} 1$ & & A223 & $\mathrm{H} 2$ & & & $1600-1750$ \\
\hline $5 \mathrm{~B} 1$ & & A223 & $\mathrm{H} 2$ & & & $1600-1750$ \\
\hline $5 \mathrm{~B} 1$ & & A1 & $\mathrm{K}$ & & & $1600-1750$ \\
\hline $5 \mathrm{~B} 1$ & & B32 & $\mathrm{H} 2$ & & & $1650-1700$ \\
\hline $5 \mathrm{~B} 1$ & $3 \mathrm{~B} 1$ & B42 & & & & $1600-1750$ \\
\hline $5 \mathrm{~B} 1$ & & A223 & $\mathrm{H} 2$ & & 04,70 & $1600-1750$ \\
\hline $5 \mathrm{~B} 2$ & & A223 & B & & & $1600-1650$ \\
\hline $5 \mathrm{~B} 2$ & & $\mathrm{~A}$ ? & $\mathrm{K}$ & & & \\
\hline $5 \mathrm{~B} 2$ & $3 \mathrm{~B} ?$ & A153 & & & & \\
\hline $5 \mathrm{~B} 2$ & $4 \mathrm{~A}$ & A153 & & & & \\
\hline $5 \mathrm{~B} 2$ & $4 \mathrm{~A}$ & A153 & & & 10,50 & \\
\hline $5 \mathrm{~B} 2$ & $4 \mathrm{~A}$ & A153 & & & 17,00 & \\
\hline
\end{tabular}


- 98 -

\begin{tabular}{|c|c|c|c|c|c|}
\hline 47 & $5 \mathrm{~B} 2$ & $4 \mathrm{~A}$ & A132 & & 16,50 \\
\hline 48 & $5 \mathrm{~B} 2$ & $3 \mathrm{~B}$ & A133 & & 08,75 \\
\hline 49 & $5 \mathrm{~B} 2$ & 3E1 4 & A133 & & 11,60 \\
\hline 50 & $5 \mathrm{~B} 2$ & 3B16 & A523 & & 09,60 \\
\hline 51 & $5 \mathrm{~B} 2$ & 3E4 4 & A533 & & 15,10 \\
\hline 52 & $5 \mathrm{~B} 2$ & 3B14 & A13? & E1 & 18,25 \\
\hline 53 & $5 \mathrm{~B} 2$ & $3 \mathrm{~B} 141$ & B32 & E1? & 08,80 \\
\hline 54 & $5 \mathrm{~B} 2$ & 1B0 1 & A133 & & 16,30 \\
\hline 55 & $5 \mathrm{~B} 2$ & 3E4 4 & A533 & & 08,70 \\
\hline 56 & $5 \mathrm{~B} 2$ & 3E14 & A133 & & 08,60 \\
\hline 57 & $5 \mathrm{~B} 2$ & 3B141 & B72 & H1 & 11,50 \\
\hline
\end{tabular}




\section{Schweden}

\begin{tabular}{|c|c|c|c|c|c|c|}
\hline $5 \mathrm{C} 2$ & 1B2 1 & A23? & H1 & E2 & 07,50 & $800-1000$ \\
\hline $5 \mathrm{C} 2$ & $3 \mathrm{~B}$ & A43? & H1 & E2 & 06,40 & $800-1000$ \\
\hline $5 \mathrm{C} 2$ & & A1? & E1 & E2B & & $800-1000$ \\
\hline $5 \mathrm{C} 2$ & & & & & 07,80 & $800-1000$ \\
\hline $5 \mathrm{C} 2$ & $5 \mathrm{~A}$ & A243 & $\mathrm{H} 1$ & E2 & 08,50 & $800-1000$ \\
\hline $5 \mathrm{C} 2$ & $5 \mathrm{~A}$ & A243 & H1 & E2 & 07,50 & $800-1000$ \\
\hline $5 \mathrm{C} 2$ & 3B1 & A233 & H1 & E2 & 07,10 & $800-1000$ \\
\hline $5 \mathrm{C} 2$ & $3 \mathrm{~B}$ & A133 & H1 & & 08,20 & $800-1000$ \\
\hline $5 \mathrm{C} 2$ & $3 \mathrm{~B}$ & A243 & H1 & E2 & 05,90 & $800-1000$ \\
\hline $5 \mathrm{C} 2$ & 3B153 & A11? & & & 05,00 & $800-1000$ \\
\hline $5 \mathrm{C} 2$ & $3 \mathrm{~B}$ & A112 & & & 15,30 & $800-1000$ \\
\hline $5 \mathrm{C} 2$ & $3 \mathrm{~B}$ & A223 & & & 09,30 & $800-1000$ \\
\hline $5 \mathrm{C} 2$ & $3 \mathrm{~B}$ & A23? & H1 & E2 & 10,40 & $800-1000$ \\
\hline $5 \mathrm{C} 2$ & $3 \mathrm{~B}$ & A133 & & & 08,60 & $800-1000$ \\
\hline $5 \mathrm{C} 2$ & $3 \mathrm{~B}$ & A132 & & & 08,00 & $800-1000$ \\
\hline $5 \mathrm{C} 2$ & $3 \mathrm{~B}$ & A133 & & & 07,30 & $800-1000$ \\
\hline $5 \mathrm{C} 2$ & $3 \mathrm{~B}$ & A133 & & & 07,30 & $800-1000$ \\
\hline $5 \mathrm{C} 2$ & $3 \mathrm{~B}$ & A153 & & & 05,70 & $800-1000$ \\
\hline $5 \mathrm{C} 2$ & $3 \mathrm{~B}$ & A133 & & & 04,40 & $800-1000$ \\
\hline $5 \mathrm{C} 2$ & $3 \mathrm{~B}$ & A133 & & & 05,00 & $800-1000$ \\
\hline $5 \mathrm{C} 2$ & 1B2 1 & A133 & & & 07,30 & $800-1000$ \\
\hline $5 \mathrm{C} 2$ & 3B141 & A133 & & & 10,60 & $800-1000$ \\
\hline $5 \mathrm{C} 2$ & 3B141 & A133 & & & 09,30 & $800-1000$ \\
\hline $5 \mathrm{C} 2$ & 1B0 1 & A143 & & & 06,00 & $800-1000$ \\
\hline $5 \mathrm{C} 2$ & $2 \mathrm{~B}$ & A121 & & & 06,60 & $800-1000$ \\
\hline $5 \mathrm{C} 2$ & 3E3 1 & A133 & & & 09,60 & $800-1000$ \\
\hline $5 \mathrm{C} 1$ & 1B0 3 & A153 & & & 08,00 & $1100-1400$ \\
\hline $5 \mathrm{C} 1$ & $3 \mathrm{~B}$ & A121 & & & 06,20 & $1100-1400$ \\
\hline $5 \mathrm{C} 1$ & $5 \mathrm{C}$ & A133 & & & 13,70 & $1100-1400$ \\
\hline $5 \mathrm{C} 1$ & $3 \mathrm{E}$ & B12 & H1 & & 12,00 & $1100-1400$ \\
\hline $5 \mathrm{C} 1$ & $3 \mathrm{~B}$ & A243 & & & 15,40 & $1100-1400$ \\
\hline $5 \mathrm{C} 1$ & $3 \mathrm{~B}$ & A133 & & & 07,20 & $1100-1400$ \\
\hline $5 \mathrm{C} 1$ & $3 \mathrm{~B}$ & A133 & & & 08,40 & $1100-1400$ \\
\hline $5 \mathrm{C} 1$ & 3B332 & A132 & & & 09,00 & $1100-1400$ \\
\hline $5 \mathrm{C} 1$ & 3B34 & A133 & & & 08,00 & $1100-1400$ \\
\hline $5 \mathrm{C} 1$ & $3 \mathrm{~B}$ & A143 & & & 08,00 & $1100-1350$ \\
\hline $5 \mathrm{C} 1$ & $4 \mathrm{C}$ & A133 & & & 08,00 & $1100-1350$ \\
\hline $5 \mathrm{C} 1$ & 3B1 3 & B42 & H1 & B & 11,00 & $1100-1400$ \\
\hline $5 \mathrm{C} 1$ & 1B1 1 & B32 & H1 & & 08,20 & $1100-1400$ \\
\hline $5 \mathrm{C} 1$ & 3E4 1 & B32 & H1 & & 08,20 & $1100-1400$ \\
\hline $5 \mathrm{~A} 1$ & & A1? & $\mathrm{K}$ & & & $1300-1400$ \\
\hline $5 \mathrm{~A} 1$ & & A1? & B & & & \\
\hline $5 \mathrm{~A} 1$ & & A1? & $\mathrm{K}$ & & & $1500-1600$ \\
\hline $5 \mathrm{~A} 1$ & & A24? & K & E2 & & $1400-1500$ \\
\hline $5 \mathrm{~A} 1$ & $3 \mathrm{~B}$ & B42 & $\mathrm{H} 2$ & B E2 & 08,50 & $1450-1550$ \\
\hline $5 \mathrm{~A} 1$ & & A1? & $\mathrm{K}$ & & & $1200-1300$ \\
\hline
\end{tabular}




\begin{tabular}{|c|c|c|c|c|c|c|c|}
\hline 47 & $5 \mathrm{~A} 1$ & & A1? & $\mathrm{H} 2$ & & & $1000-1050$ \\
\hline 48 & $5 \mathrm{~A} 1$ & & A1? & $\mathrm{H} 2$ & & & $1000-1050$ \\
\hline 49 & $5 \mathrm{~A} 1$ & & A1? & K & & & $1100-1200$ \\
\hline 50 & $5 \mathrm{~A} 1$ & & A2? & H1 & E1 & & $1100-1150$ \\
\hline 51 & $5 \mathrm{~A} 1$ & & A2? & H1 & & & $1100-1200$ \\
\hline 52 & $5 \mathrm{~A} 1$ & 3E1 5 & A143 & & & 23,80 & $1000-1050$ \\
\hline 53 & $5 \mathrm{~A} 1$ & $3 \mathrm{E}$ & A1 & & & 12,50 & $1000-1050$ \\
\hline 54 & $5 \mathrm{~A} 1$ & 3E1 6 & A133 & & & 11,20 & $1000-1050$ \\
\hline 55 & $5 \mathrm{~A} 1$ & $3 \mathrm{E} 14$ & A133 & & & 09,60 & $1000-1050$ \\
\hline 56 & $5 \mathrm{~A} 1$ & 3B174 & A133 & & & 05,30 & $1000-1050$ \\
\hline 57 & $5 \mathrm{~A} 1$ & $3 \mathrm{~B}$ & A133 & & & 03,36 & $1000-1050$ \\
\hline 58 & $5 \mathrm{~A} 1$ & $3 \mathrm{~B}$ & A133 & & & 03,84 & $1000-1050$ \\
\hline 59 & $5 \mathrm{~A} 1$ & $3 \mathrm{~B}$ & A133 & & & 03,36 & $1000-1050$ \\
\hline 60 & $5 \mathrm{~A} 1$ & $3 \mathrm{~B}$ & A143 & & & 07,36 & $1000-1050$ \\
\hline 61 & $5 \mathrm{~A} 1$ & $3 \mathrm{~B}$ & A143 & & & 06,40 & $1000-1050$ \\
\hline 62 & $5 \mathrm{~A} 1$ & $4 \mathrm{~A}$ & A132 & & & 08,00 & $1150-1200$ \\
\hline 63 & $5 \mathrm{~A} 1$ & 1B0 1 & $\mathrm{~A} 2 ?$ & H1 & & 09,70 & $1200-1300$ \\
\hline 64 & $5 \mathrm{~A} 1$ & 2B1 3 & A133 & & & 08,60 & $1200-1300$ \\
\hline 65 & $5 \mathrm{~A} 1$ & 1B0 1 & A141 & E1 & & 07,20 & $1300-1400$ \\
\hline 66 & $5 \mathrm{~A} 1$ & $1 \mathrm{~A}$ & A221 & H1 & B & 05,40 & $1200-1300$ \\
\hline 67 & $5 \mathrm{~A} 1$ & $3 \mathrm{~B} 155$ & A243 & H1 & & 16,70 & $1000-1050$ \\
\hline 68 & $5 \mathrm{~A} 1$ & $3 \mathrm{~B}$ & A1? & H1 & & 06,60 & $1000-1050$ \\
\hline 69 & $5 \mathrm{~A} 1$ & 3E33 & A133 & & & 07,80 & $1000-1050$ \\
\hline 70 & $5 \mathrm{~A} 1$ & 3B151 & A143 & & & 14,00 & $1000-1050$ \\
\hline 71 & $5 \mathrm{~A} 1$ & 3B171 & A153 & & & 08,80 & $1000-1050$ \\
\hline 72 & $5 \mathrm{~A} 1$ & $3 \mathrm{E} 16$ & A133 & & & 10,40 & $1000-1050$ \\
\hline 73 & $5 \mathrm{~A} 1$ & $3 \mathrm{~B}$ & A133 & & & 05,60 & $1000-1050$ \\
\hline 74 & $5 \mathrm{~A} 1$ & $3 \mathrm{~B}$ & A133 & & & 04,60 & $1000-1050$ \\
\hline 75 & $5 \mathrm{~A} 1$ & $4 \mathrm{~F}$ & A243 & & & 09,50 & $1200-1300$ \\
\hline 76 & $5 \mathrm{~A} 1$ & $3 \mathrm{~B} 33$ & B92 & & & 11,50 & $1200-1400$ \\
\hline 77 & $5 \mathrm{~A} 1$ & 3B17 & B32 & & & 15,50 & \\
\hline 78 & $5 \mathrm{~A} 1$ & $3 \mathrm{~B}$ & B42 & $\mathrm{K}$ & B & 08,50 & $1500-1600$ \\
\hline 79 & $5 \mathrm{~A} 1$ & $3 \mathrm{~B}$ & B12 & & & 08,30 & $1200-1400$ \\
\hline 80 & $5 \mathrm{~A} 1$ & $3 \mathrm{~B}$ & B22? & & & 07,00 & $1300-1500$ \\
\hline 81 & $5 \mathrm{~A} 1$ & 3B13 & B12 & & & 08,00 & $1200-1400$ \\
\hline 82 & $5 \mathrm{~A} 1$ & $3 B$ & B12 & & & 10,00 & $1200-1400$ \\
\hline 83 & $5 \mathrm{~A} 1$ & 3B14 & $\mathrm{B} 42 ?$ & & & 10,50 & $1300-1500$ \\
\hline 84 & $5 \mathrm{~A} 1$ & 3B34 & B12? & $\mathrm{K}$ & & 04,50 & $1300-1400$ \\
\hline 85 & $5 \mathrm{~A} 1$ & 2B1 2 & B42 & & & 07,00 & $1400-1500$ \\
\hline 86 & $5 \mathrm{~A} 1$ & 2B0 2 & B22 & & & 07,30 & $1400-1500$ \\
\hline 87 & $5 \mathrm{~A} 1$ & 2B1 3 & B42 & & B & 09,50 & $1400-1500$ \\
\hline 88 & $5 \mathrm{~A} 1$ & 2B1 3 & B42 & & B & 10,00 & $1400-1500$ \\
\hline 89 & $5 \mathrm{~A} 1$ & 2B1 3 & B42 & $\mathrm{H} 1$ & B & 09,00 & $1400-1500$ \\
\hline 90 & $5 \mathrm{~A} 1$ & 2B1 3 & B42 & & $\mathrm{B}$ ? & 07,00 & $1400-1500$ \\
\hline 91 & $5 \mathrm{~A} 1$ & 2B1 3 & B32? & & & 08,80 & $1400-1500$ \\
\hline 92 & $5 \mathrm{~A} 1$ & 2B0 3 & B42 & $\mathrm{H} 2$ & $\mathrm{~B}$ ? & 08,70 & $1400-1500$ \\
\hline 93 & $5 \mathrm{~A} 1$ & 2B1 2 & B42 & $\mathrm{K}$ & $\mathrm{B}$ ? & 05,50 & $1400-1500$ \\
\hline 94 & $5 \mathrm{~A} 1$ & 2B1 3 & B42 & & B & 06,00 & $1400-1500$ \\
\hline 95 & $5 \mathrm{~A} 1$ & $3 \mathrm{~B}$ & B32 & & & 09,00 & $1400-1500$ \\
\hline
\end{tabular}




\begin{tabular}{|c|c|c|c|c|c|c|c|}
\hline 96 & $5 \mathrm{~A} 1$ & $3 \mathrm{~B}$ & B342 & & & 08,50 & $1450-1550$ \\
\hline 97 & $5 \mathrm{~A} 1$ & 3B & B32 & & & 07,50 & $1450-1550$ \\
\hline 98 & $5 \mathrm{~A} 1$ & 3B & B32 & & & 07,00 & $1400-1550$ \\
\hline 99 & $5 \mathrm{~A} 1$ & 1B0 1 & B42 & & B & 11,20 & $1400-1500$ \\
\hline 100 & $5 \mathrm{~A} 1$ & 3B & A523 & $\mathrm{H} 1$ & B & 11,80 & $1450-1500$ \\
\hline 101 & $5 \mathrm{~A} 1$ & 3E33 & B42 & & & 08,80 & $1400-1550$ \\
\hline 102 & $5 \mathrm{~A} 1$ & & B42 & & $\mathrm{B}$ ? & & $1400-1550$ \\
\hline 103 & $5 \mathrm{~A} 1$ & $4 \mathrm{H}$ & B32 & & & 09,50 & $1400-1500$ \\
\hline 104 & $5 \mathrm{~A} 1$ & $3 \mathrm{~B}$ & B22 & & B & 06,40 & $1400-1500$ \\
\hline 105 & $5 \mathrm{~A} 1$ & 2B1 3 & B42 & & B & 08,00 & $1400-1500$ \\
\hline 106 & $5 \mathrm{~A} 1$ & & B21 & & B & & $1450-1550$ \\
\hline 107 & $5 \mathrm{~A} 1$ & & B42 & & B & & $1400-1550$ \\
\hline 108 & $5 \mathrm{~A} 1$ & $3 \mathrm{~B}$ & B42 & & B & 07,00 & $1450-1500$ \\
\hline 109 & $5 \mathrm{~A} 1$ & & B42 & $\mathrm{K}$ & B & 06,50 & $1500-1600$ \\
\hline 110 & $5 \mathrm{~A} 1$ & 3B & B42 & $\mathrm{K}$ & B & 08,00 & $1500-1550$ \\
\hline 111 & $5 \mathrm{~A} 1$ & 3B & B42 & & B & 11,50 & $1500-1550$ \\
\hline 112 & $5 \mathrm{~A} 1$ & 3B141 & B22 & & B & 08,50 & $1500-1600$ \\
\hline 113 & $5 \mathrm{~A} 1$ & $3 \mathrm{~B}$ & B22 & $\mathrm{K}$ & B E2 & 09,00 & $1500-1600$ \\
\hline 114 & $5 \mathrm{~A} 1$ & & A523 & E1 & & & $1500-1600$ \\
\hline 115 & $5 \mathrm{~A} 1$ & 3B141 & B42 & $\mathrm{K}$ & B & 10,50 & $1550-1600$ \\
\hline 116 & $5 \mathrm{~A} 1$ & & B32 & $\mathrm{K}$ & & & $1500-1700$ \\
\hline 117 & $5 \mathrm{~A} 1$ & 3B & B42 & $\mathrm{H} 2 \mathrm{~K}$ & B & 08,00 & $1450-1550$ \\
\hline 118 & $5 \mathrm{~A} 1$ & & A4? & H1 & K & & $1200-1450$ \\
\hline 119 & $5 \mathrm{~A} 1$ & 3B171 & A343 & & K & 06,20 & $1200-1450$ \\
\hline 120 & $5 \mathrm{~A} 1$ & & $\mathrm{~A} 1 ?$ & H1 & & 07,00 & $1200-1450$ \\
\hline 121 & $5 \mathrm{~A} 1$ & & A33? & & K & & $1200-1450$ \\
\hline 122 & $5 \mathrm{~A} 1$ & & A33? & & B & & $1200-1450$ \\
\hline 123 & $5 \mathrm{~A} 1$ & & $\mathrm{~A} 2 ?$ & & E1 & & $1200-1450$ \\
\hline 124 & $5 \mathrm{~A} 1$ & & $\mathrm{~A}$ ? & $\mathrm{K}$ & & & $1200-1450$ \\
\hline 125 & $5 \mathrm{~A} 1$ & & B3? & $\mathrm{K}$ & B & & $1200-1450$ \\
\hline 126 & $5 \mathrm{~A} 1$ & & B3? & $\mathrm{K}$ & & & $1200-1450$ \\
\hline 127 & $5 \mathrm{~A} 1$ & & B32 & $\mathrm{K}$ & & & $1200-1450$ \\
\hline 128 & $5 \mathrm{~A} 1$ & & B4? & $\mathrm{K}$ & $\mathrm{B}$ ? & & $1200-1450$ \\
\hline 129 & $5 \mathrm{~A} 1$ & 1B & B33 & $\mathrm{H} 1$ & & 24,00 & $1200-1450$ \\
\hline
\end{tabular}




\section{ehemalige UdSSR}

$\begin{array}{rlll}1 & 6 \mathrm{~B} & 3 \mathrm{~B} 11 & \mathrm{~A} 13 ? \\ 2 & 6 \mathrm{~B} & 3 \mathrm{~B} 12 & \mathrm{~A} 13 ? \\ 3 & 6 \mathrm{~B} & 3 \mathrm{~B} 17 & \mathrm{~A} 133 \\ 4 & 6 \mathrm{~B} & 1 \mathrm{~B} 11 & \mathrm{~A} 132 \\ 5 & 6 \mathrm{~B} & 1 \mathrm{~B} 11 & \mathrm{~A} 131 \\ 6 & 6 \mathrm{~B} & 3 \mathrm{~B} 13 & \mathrm{~A} 13 ? \\ 7 & 6 \mathrm{~B} & 3 \mathrm{~B} 141 & \mathrm{~A} 13 ? \\ 8 & 6 \mathrm{~B} & 3 \mathrm{E} & \mathrm{A} 13 ? \\ 9 & 6 \mathrm{~B} & 1 \mathrm{~B} 13 & \mathrm{~A} 133 \\ 10 & 6 \mathrm{~B} & 3 \mathrm{~B} 141 & \mathrm{~A} 43 ? \\ 11 & 6 \mathrm{~B} & 3 \mathrm{~B} & \mathrm{~A} 131 \\ 12 & 6 \mathrm{~B} & 3 \mathrm{~B} & \mathrm{~A} 133 \\ 13 & 6 \mathrm{~B} & 1 \mathrm{~B} 23 & \mathrm{~A} 131 \\ 14 & 6 \mathrm{~B} & 5 \mathrm{~A} & \mathrm{~A} 143 \\ 15 & 6 \mathrm{~B} & 3 \mathrm{~B} & \mathrm{~A} 133 \\ 16 & 6 \mathrm{~B} & 3 \mathrm{~B} 13 & \mathrm{~A} 133 \\ 17 & 6 \mathrm{~B} & 1 \mathrm{~B} 23 & \mathrm{~A} 131 \\ 18 & 6 \mathrm{~B} & 3 \mathrm{~B} & \mathrm{~A} 13 ? \\ 19 & 6 \mathrm{~B} & 3 \mathrm{~B} 13 & \mathrm{~A} 13 ? \\ 20 & 6 \mathrm{~B} & 3 \mathrm{~B} 13 & \mathrm{~A} 14 ? \\ 21 & 6 \mathrm{~B} & 3 \mathrm{E} 132 & \mathrm{~A} 14 ? \\ 22 & 6 \mathrm{~B} & 1 \mathrm{~B} 13 & \mathrm{~A} 13 ? \\ 23 & 6 \mathrm{~B} & 3 \mathrm{~B} 16 & \mathrm{~A} 133 \\ 24 & 6 \mathrm{~B} & 5 \mathrm{~A} & \mathrm{~A} 233 \\ 25 & 6 \mathrm{~B} & 3 \mathrm{~B} 132 & \mathrm{~A} 143 \\ 26 & 6 \mathrm{~B} & 5 \mathrm{C} & \mathrm{A} 43 ? \\ 27 & 6 \mathrm{~B} & 3 \mathrm{E} & \mathrm{A} 133 \\ 28 & 6 \mathrm{~B} & 1 \mathrm{~B} 11 & \mathrm{~A} 13 ? \\ 29 & 6 \mathrm{~B} & 3 \mathrm{E} & \mathrm{A} 23 ? \\ 30 & 6 \mathrm{~B} & 3 \mathrm{E} 1 & \mathrm{~A} 133 \\ 31 & 6 \mathrm{~A} & 1 \mathrm{~B} 12 & \mathrm{~A} 102 \\ 32 & 6 \mathrm{~B} & 3 \mathrm{~B} 13 & \mathrm{~A} 133 \\ 33 & 6 \mathrm{~B} & 3 \mathrm{~B} 16 & \mathrm{~A} 133 \\ 34 & 6 \mathrm{~B} & 3 \mathrm{~B} 13 & \mathrm{~A} 133 \\ 35 & 6 \mathrm{~A} & 3 \mathrm{~B} 13 & \mathrm{~A} 133 \\ 36 & 6 \mathrm{~B} & 5 \mathrm{~A} & \mathrm{~A} 133 \\ 37 & 6 \mathrm{~B} & 3 \mathrm{~B} & \mathrm{~A} 233 \\ 38 & 6 \mathrm{~B} & 3 \mathrm{~B} 13 & \mathrm{~A} 13 ? \\ 39 & 6 \mathrm{~B} & & \mathrm{~A} 13 ? \\ 40 & 6 \mathrm{~B} & 1 \mathrm{~B} 21 & \mathrm{~A} 13 ? \\ 42 & 6 \mathrm{~B} & 1 \mathrm{~B} 21 & \mathrm{~A} 13 ? \\ & 6 \mathrm{~B} & 3 \mathrm{~B} 171 & \mathrm{~A} 153 \\ \text { 46 } & \text { 1B1 } 1 & \mathrm{~A} 523 \\ \mathrm{~A} 133 \\ \mathrm{~A} 133 \\ \end{array}$

$\mathrm{K}$

K

06,00

05,40

05,70

07,30

05,70

K

K

K

$\mathrm{H} 2$

H1

H1

H1

H1

K

K

H1

H1

10,70

12,50

12,60

10,50

09,20

H1

E2

11,50

16,20

15,40

H1

08,50

08,50

08,60

08,40

08,00

08,50

10,00

09,20

08,20

05,50

05,00

10,50

07,50

07,20

08,00

09,20
$900-1200$

$900-1200$

$900-1200$

$900-1200$

$1000-1100$

$1100-1300$

$1100-1300$

$1100-1300$

$1300-1500$

$1300-1500$

$900-1250$

$900-1250$

$800-1000$

$800-1000$

$800-1000$

$900-1250$

$1000-1300$

$1000-1300$

$1000-1500$

$1000-1500$

$1000-1500$

$1000-1500$

$1000-1500$

$1400-1500$

$700-850$

$700-850$

$700-850$ 


\begin{tabular}{|c|c|c|c|c|c|}
\hline 49 & $6 \mathrm{~A}$ & 1B2 & A133 & 12,00 & $700-850$ \\
\hline 50 & $6 \mathrm{~A}$ & 3B16 & A133 & 12,00 & $700-850$ \\
\hline 51 & $6 \mathrm{~A}$ & $3 \mathrm{E}$ & A153 & 13,50 & $700-850$ \\
\hline 52 & $6 \mathrm{~A}$ & 1B2 5 & A133 & 15,50 & $700-850$ \\
\hline 53 & $6 \mathrm{~A}$ & $5 \mathrm{C} 05$ & A132 & 17,00 & $700-850$ \\
\hline 54 & $6 \mathrm{~A}$ & $5 \mathrm{C}$ & A132 & 17,00 & $700-850$ \\
\hline 55 & $6 \mathrm{~A}$ & 3B174 & A13? & 11,50 & $700-850$ \\
\hline 56 & $6 \mathrm{~A}$ & $5 \mathrm{~A}$ & A233 & 12,00 & $700-850$ \\
\hline 57 & $6 \mathrm{~A}$ & $3 \mathrm{E} 132$ & A133 & 10,00 & $700-850$ \\
\hline 58 & $6 \mathrm{~A}$ & 3 E2 2 & A133 & 10,00 & $700-850$ \\
\hline 59 & $6 \mathrm{~A}$ & $5 \mathrm{C}$ & A122 & 10,50 & $700-850$ \\
\hline 60 & $6 \mathrm{~A}$ & 3B & A1 & 10,00 & $700-850$ \\
\hline 61 & $6 \mathrm{~A}$ & 1B5 1 & A141 & 12,50 & $700-850$ \\
\hline 62 & $6 \mathrm{~A}$ & 3B & A1 & 11,00 & $700-850$ \\
\hline 63 & $6 \mathrm{~A}$ & 3B & A1 & 12,50 & $700-850$ \\
\hline 64 & $6 \mathrm{C} 2$ & $3 \mathrm{E}$ & A112 & & $950-1000$ \\
\hline 65 & $6 \mathrm{C} 2$ & 3B & A123 & & $900-1100$ \\
\hline 66 & $6 \mathrm{C} 2$ & 1B2 & A133 & & $900-1100$ \\
\hline
\end{tabular}




\section{Auswertung und Ergebnisse}

\subsection{Statistischer Teil}

\subsubsection{Arbeitsweise}

Mit Hilfe eines datenverarbeitenden Gerätes wurden alle durch Codes verschlüsselten Daten mit für diesen Zweck entworfenen Programmen ausgewertet.

Der Gesamtbestand der statistischen Auswertung umfaßt für jedes Land separat und für den gesamten Untersuchungsraum die zeitliche Verteilung aller im Fundmaterial aufgetretenen Klingenformen, Griffkonstruktionen und Klingenlängen.

Für die Verarbeitung der Daten wurden aus dem Katalog für jedes auswertbare Messer die Katalognummer, die Zeitstellung und die Verschlüsselung als Datengerüst gespeichert.

Aus den hunderten von Einzelauswertungen, die teilweise auch lediglich zu Kontrollzwecken durchgeführt wurden, haben nur die aussagefähigen Analysen in die hier vorgelegte Untersuchung Eingang gefunden.

Dies gilt vor allem für die zahlreichen Fälle, in denen die absolute Zahl abgefragter Kriterien im Verhältnis zur jeweiligen Vergleichsmenge statistisch auswertbar war. Zur zeitlichen Verteilung der einzelnen Kriterien wurden in der Regel die Ergebnisse der beiden beschriebenen Zeitrahmen einander gegenübergestellt ${ }^{45}$. Grundsätzlich gilt auch hier, daß statistisch nicht repräsentative Mengen keine Berücksichtigung erfuhren.

In Ausnahmefällen fanden aber Analysen Aufnahme, die trotz geringer absoluter Zahl der Untersuchungsmenge statistisch abgesicherten Entwicklungstendenzen folgten und damit trotz fehlender Repräsentativität aussagekräftig erschienen.

Ein grundsätzliches Problem ergab sich aus der geringen Anzahl von Funden aus dem Gebiet der ehemaligen Sowjetunion. Für alle übrigen Untersuchungsländer war die erfaßte Materialbasis ausreichend, um zu den wichtigsten Fragestellungen Analysen durchführen zu können.

Von den 85 Messern ehemals sowjetischer Fundorte stammen allein 29 aus Novgorod, für die bereits weitreichende wissenschaftliche Vorarbeiten vorlagen.

Die übrigen 56 Messer verteilen sich auf 11 Fundorte, von denen 17 in der lettischen Burg und Siedlung Kente gefunden und in das 8. bis beginnende 9 . Jahrhundert datiert wurden, somit den zeitlichen Rahmen dieser Untersuchung durchbrechen. Für statistische Analysen war das Material mit zwei ersichtlichen 
Schwerpunkten, die jede gemeinsame Auswertung überproportional beeinflussen mußten, denkbar ungeeignet.

Eine ausschließlich historische Aspekte berücksichtigende Gliederung war aufgrund der geringen Fundortanzahl ebenfalls nur schwer durchzuführen.

So konnten zu keiner historischen Epoche Bestände baltischer und ostslawischer, später der Kiewer Rus oder deutscher, litauischer und russischer Fundorte einander annähernd adäquat gegenübergestellt werden.

Neben den Fundorten der Novgoroder Slowenen verteilt sich das Material aus der ehemaligen Sowjetunion auf verschiedenste Zeitabschnitte und ethnische Gruppen. Für die statistische Auswertung wurden aus den Grenzen der ehemaligen Sowjetunion zwei Messer aus Riga herausgenommen, die in das 13. bis 14. Jahrhundert datiert werden ${ }^{46}$ und mit hoher Wahrscheinlichkeit unter dem Einfluß der Hanse, der Riga 1282 beitrat, in das 1201 von Deutschen als Stadt gegründete Riga gelangten. Sie finden unter anderem in Polen einen Parallelfund vom Schloßberg in Stettin aus der gleichen Zeit ${ }^{47}$. Weiterhin betroffen sind sechs Messer des 11. bis 12. Jahrhunderts von einem masowischen oder masowisch beeinflußten Gräberfeld (Podros) in der Berührungszone von Masowien und Litauen ${ }^{48}$.

13 Messer aus den Städten Grodno und Novogrudok, die fast alle dem 12. bis beginnenden 14. Jahrhundert entstammen, wurden ebenfalls ausgesondert ${ }^{49}$.

$\mathrm{Zu}$ Beginn dieser Zeit war das Gebiet des oberen Njemen nordwestlich der Pripjetsümpfe Teil des russischen Teilfürstentums von Polozk; Grodno wurde noch am Ende des 12. Jahrhunderts von dem sich immer weiter nach Südosten ausdehnenden Litauen erobert, im 13. Jahrhundert folgten bereits die Gebiete um Novogrudok und der Zuflüsse des oberen Njemen. Neben den Landschaften Schamaiten (Zemaiten) am Unterlauf des Njemen und Aukstaiten am Verlauf der Wilija zählt dieses Gebiet (Schwarzreußen) zu dem sogenannten "eigentlichen Litauen", dem Kerngebiet des späteren Großfürstentums Litauen, welches seit 1386 bzw. 1401 mit Polen verbunden war ${ }^{50}$.

Demgegenüber stehen einige estnische und lettische Fundorte, die zum Teil so breit datiert waren, daß sie aus der Auswertung herausfielen (Valjala Rahu, Tamme, Madi) oder so früh datierten, daß sie mit dem Material der anderen Fundorte aus der Sowjetunion nicht mehr verglichen werden konnten (Kente).

Als zusammenhängend erwies sich lediglich noch das Gebiet der Novgoroder Slowenen mit den Fundorten Novgorod, Pskov und Gorodec (bei Luga).

Somit kamen für das bearbeitete Material des11. bis 14. Jahrhunderts auf dem Gebiet der nordwestlichen ehemaligen Sowjetunion drei Gliederungsräume in Frage: das Gebiet der baltischen Stammesverbände, das zum größten Teil im 13. Jahrhun-

\footnotetext{
46

s. Katalogteil ehemalige UdSSR, Kat. Nr. 44 und 4

47 s. Katalogteil Polen, Kat. Nr. 6

48 s. Katalogteil ehemalige UdSSR, Kat. Nr. 75-80

49 s. Katalogteil ehemalige UdSSR, Kat. Nr. 67-74 und 81-85

50 Rhode 1965, S. 118-119
} 
dert vom Deutschen Orden besetzt wurde, das Kerngebiet des späteren Großfürstentums Litauen und das russische Fürstentum Novgorod ${ }^{51}$.

Eigenständige Untersuchungsgebiete zu schaffen war für die beiden Räume Litauens und des Deutschen Ordens wegen der geringen Anzahl der Funde unmöglich, obwohl dies bei der Vielschichtigkeit der wirtschaftlichen und kulturellen Beziehungen beider Gebiete untereinander und mit den östlich angrenzenden russischen Fürstentümern, mit Polen und - was vor allem das Ordensgebiet angeht - mit Deutschland und Westeuropa - das historisch sinnvollste gewesen wäre.

Ein einzelnes großes Untersuchungsgebiet im Raum der sowjetischen Fundorte wäre wegen der räumlichen, vor allem aber zeitlichen Lücken nicht auszuwerten gewesen, abgesehen von der geringen Anzahl der Messer.

So wurden für die statistische Auswertung im Osten zwei Untersuchungsgebiete getrennt, von denen das eine Polen, Riga und die litauischen Städte Grodno und Novogrudok sowie das Gräberfeld von Podros, das zweite einen Teil des ehemalige Gebietes der Novgoroder Slowenen sowie zwei Fundorte des 8. bis 9. bzw. 10. bis 11. Jahrhunderts umfaßt, die wohl livische Burg und Siedlung Kente sowie das slawisch-skandinavische Gräberfeld von Porcecze ${ }^{52}$.

Im einzelnen wurden aus dem im Katalog erfaßten Bestand folgende Mengen aus den einzelnen Ländern ausgewertet, die zeitlich eng genug einzugrenzen waren:

Skandinavien: von 277 Messern wurden 207 ausgewertet

Niederlande: von 135 Messern wurden 121 ausgewertet

Bundesrepublik Deutschland (alte und neue Bundesländer): von 426 Messern wurden 316 ausgewertet

Polen (ohne die 21 Messer aus baltischen und litauischen Fundorten): von 376 Messern wurden 359 ausgewertet

ehemalige Sowjetunion: von 85 Messern wurden 61 ausgewertet

In wenigen Fällen wurden regional begrenzte Analysen durchgeführt, wobei bestimmte Kriterien abgefragt wurden, die sich oft auch aus den begrenzten Möglichkeiten des ergrabenen Bestandes einer Region ergaben.

In zwei Fällen war innerhalb einer regionalen Untersuchung der Vergleich mit Ergebnissen mikroanalytischer Arbeitsweise möglich. Sie betreffen das Gebiet der

51 Bogucka 1983, Karten S. 23 und S. 42 Donnert 1983, Karten S. 82 und S. 20 Grabois 1981, S. 377 und S. 507 Lebedev 1982, S. 235-238

Putzger - Historischer Weltatlas, Jubiläumsausgabe, Velhagen und Klasing 1963, Karten S. 40, S. 54-55, S. 57, S. 60

Rhode 1965 , S. 118-125

von Rimscha 1970, S. 65 ff, Abb. S. 21 und S. 66

Völker, Staaten und Kulturen - Ein Kartenwerk zur Geschichte, Westermann 1969, Karten S. 30, S. 32,

52 S. 34 , S. 35 , S. 38 , S. 39 , S. 42 , S. 44 , S. 46

52 Die Schreibweise lehnt sich an die des polnischen Berichtes an, s. H.C. Holubowiczowa, SlowianskoWareskie Cmentarzysko Kuranowe Kolo Porzecza W Pow. Dzisnienskim, in: Prz. Arch. Tom VI, 19371939, Poznan 1939 
späten Westslawen des 11. bis frühen 13. Jahrhunderts, innerhalb dessen sich in der Siedlungskammer "Lieps" ein weitreichend dokumentiertes Siedlungskonglomerat befand. Die dort ausgegrabenen Bestände an Messern sind mit denen der übrigen spätslawischen Fundorte auf dem Gebiet der ehemaligen nördlichen DDR sowohl chronologisch als auch im Umfang der vorkommenden Formen und in der absoluten Zahl vergleichbar ${ }^{53}$. Ähnlich günstige Voraussetzungen gab es nur noch für das 13. bis 14. Jahrhundert mit dem Fundbestand der Burg und Siedlung Gommerstedt in Thüringen.

Jede Einzelanalyse innerhalb der statistischen Auswertung wird durch eine Graphik veranschaulicht. Diese computererstellten Graphiken sind das eigentliche Gerüst der Auswertung.

Die Graphiken stellen nicht nur positiver Ergebnisse, sondern auch Mängel innerhalb des aufgenommenen Fundgutes dar; mehrere Fragestellungen zeitigten nur ausschnitthafte Ergebnisse. In der Regel werden die Resultate sowie quellenkritische Anmerkungen in den begleitenden Texten diskutiert und am Ende einer Auswertungsgruppe kurz zusammengefaßt.

Alle computererstellten Graphiken sind nach dem gleichen Schema aufgebaut:

Das linke Säulendiagramm veranschaulicht die chronologische Verteilung der absoluten Zahl ausgewerteter Messer. Das rechte Diagramm stellt die relative Verteilung derselben Kriterien zum Beispiel im Vergleich zu allen übrigen Messern dar. Beide Diagramme sind Säulen chronologisch nach Jahrhunderten angelegt und umfassen den Zeitraum vom 8. bis 17. Jahrhundert. In jeder Graphik sind die absoluten Zahlen der jeweils analysierten Messer und ihre prozentualen Anteile aufgeführt.

Mit der direkten Vergleichsmöglichkeit zwischen den Entwicklungstendenzen eines bestimmten Kriteriums und dem tatsächlichen absoluten Vorkommen desselben im abgefragten Komplex wird die Aussagekraft einer Graphik auch ohne die begleitenden Texte transparent. 


\subsubsection{Klingenformen}

Als nach Abschluß der Materialcodierung das Auswertungsprogramm getestet wurde, zeigte sich bald, daß eine Auswertung der zeitlichen Verteilung aller einzelnen Klingenformen wenig Aussicht auf Erfolg versprach.

Zum einen verteilte sich das aufgenommene Fundmaterial nicht gleichmäßig genug auf die 88 Hauptformen und ihre Einzelformen, zum anderen ergaben Vergleiche, daß Messer mit ähnlichen Klingenformen auch zeitlich ähnlich verbreitet gewesen waren.

Deshalb wurden für die statistische Auswertung Messerklingen mit ähnlichen Grundformen in Auswertungsgruppen zusammengefaßt.

Diese Gruppen sind mit neun der elf Leitformen, 48 Haupt- und Einzelformen sowie fünf Sonderformen eine formale Verdichtung des Klassifizierungsmodells, welche sich im Gegensatz zu letzterem auch am tatsächlich vorhandenen Klingenbestand orientiert. Gleichzeitig wurde mit diesem Vorgehen die Tatsache berücksichtigt, daß viele Klingen im Fundgut abgenutzt und korrosionsgeschädigt waren, so daß eine Zuweisung zu einander ähnelnden Klingenformen oft im Bereich des Möglichen lag.

Ausgewertet wurden 62 codierte Klingenformen, die in 17 Gruppen zusammengefaßt wurden. Die verschiedenen formalen Kriterien sind der Auflistung dieser Gruppen (Abb. 23) zu entnehmen. 

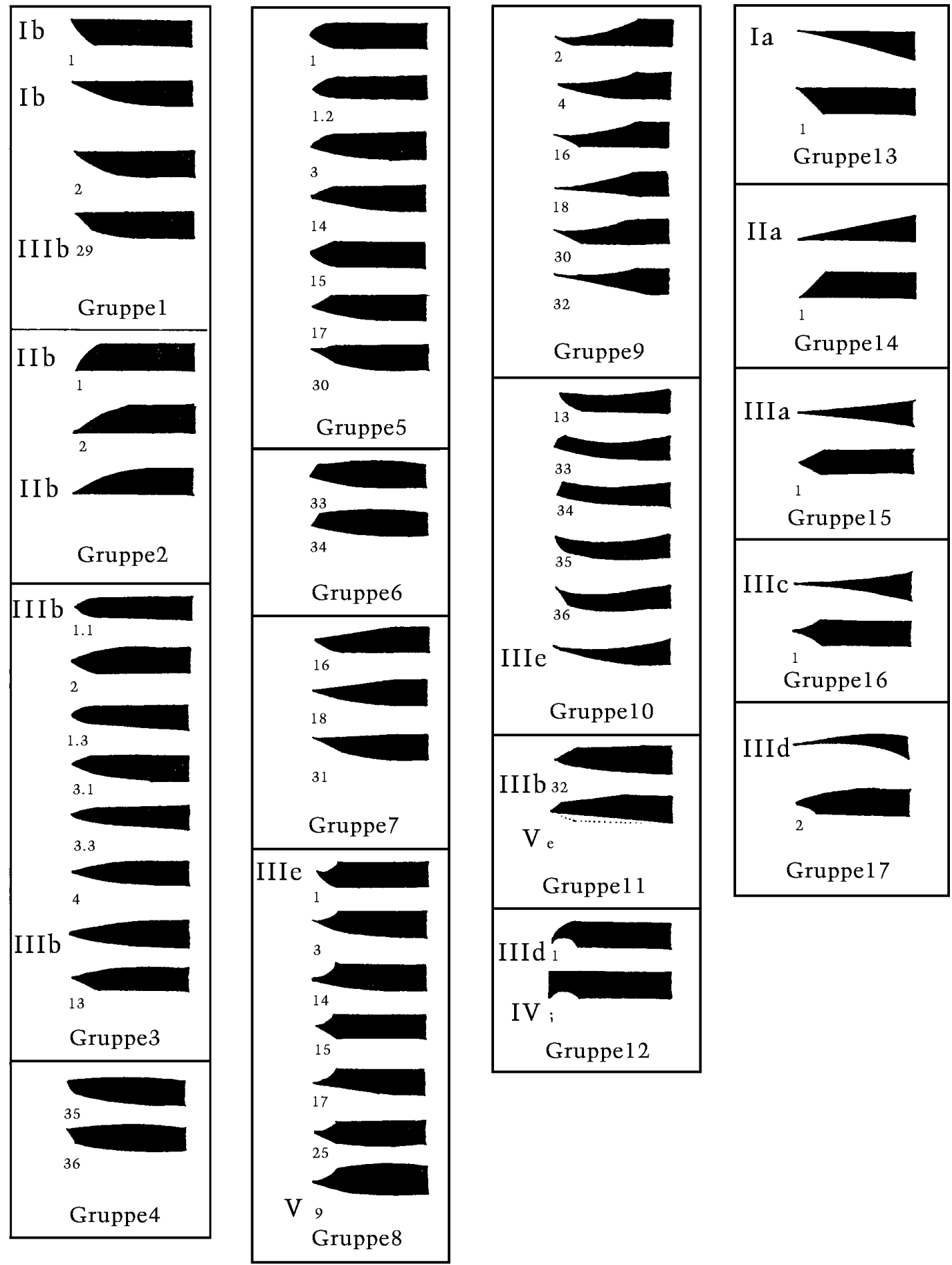

Abb. 23 


\subsubsection{Die Verteilung der Klingenformen Ib (Auswertungsgruppe 1)}

Die Auswertungsgruppe 1 umfaßt Messer mit geradem Rücken und ganz oder teilweise gebogener Schneide, im einzelnen die codierten Formen Ib1, Ib, Ib2 und IIIb29.

Im gesamten Untersuchungsgebiet entfallen 73 Messer auf diese Gruppe, deren Datierung im Rahmen eines Jahrhunderts liegt.

Die Form Ib1 ist vom 9. bis zum 15., die gesamte Gruppe vom 9. bis zum 17. Jahrhundert nachweisbar. Ihre Hauptverbreitungszeit reicht vom 9. bis zum 14. Jahrhundert (Abb. 24).

Der zeitlich weiter gefaßte Bestand dieser Gruppe umfaßt 194 Messer. Die zeitliche Begrenzung der Form Ib1 scheint sich mit einem Exemplar des 15. bis 16. Jahrhunderts zu bestätigen. Der Schwerpunkt aller vier Formen mit jeweils fast $20 \%$ liegt im 11. und 12. Jahrhundert, ebenso die Hauptverbreitungszeit der Form Ib1 (Abb. 25).

Es scheint bei der zeitlichen Gesamtverteilung und der Hauptverbreitungszeit von Messern mit geraden Rücken keine größere Rolle zu spielen, ob die Schneide ganz oder teilweise gebogen war. Wahrscheinlich muß bei der Verbreitung der Form Ib1 mit Verschliffstufen gerechnet werden, die nicht erkennbar waren, so daß die zeitliche Beschränkung auf das 9. bis 15./16. Jahrhundert hier nur eine Tendenz anzeigen kann.

Im Untersuchungsraum verteilt sich der Gesamtbestand dieser Messer unterschiedlich.

In Skandinavien sind Messer mit geraden Rücken vom 9. bis zum 17. Jahrhundert nachweisbar (Abb. 26).

Vom 9. bis 11. Jahrhundert erscheinen mit abfallender Tendenz nur Klingen mit weitgehend gebogener Schneide. Das Fehlen im 12. Jahrhundert darf aufgrund der fallenden absoluten Zahlen nicht überbewertet werden. 


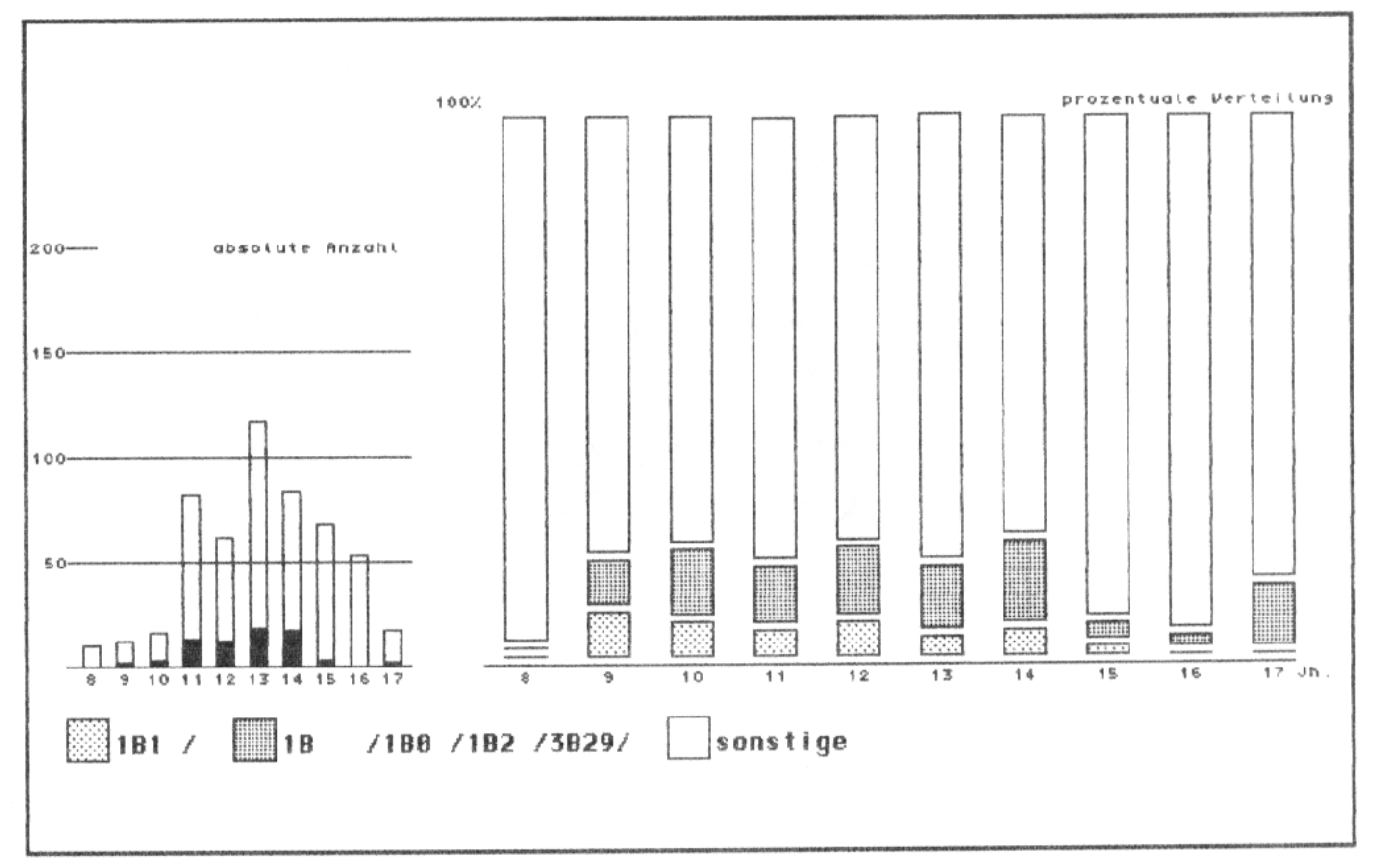

\begin{tabular}{|c|c|c|c|c|c|c|c|c|c|c|}
\hline 8. & 9. & 10. & 11. & 12. & 13. & 14. & 15. & 16. & 17. & Jh. \\
\hline$\emptyset$ & 1 & 1 & 4 & 4 & 4 & 4 & 1 & 0 & $\emptyset$ & Block 1 \\
\hline$\emptyset$ & 1 & 2 & 9 & 8 & 14 & 13 & 2 & 1 & 2 & Block 2 \\
\hline 10 & 10 & 13 & 69 & 50 & 99 & 67 & 65 & 52 & 15 & Block \\
\hline - & $\overline{12}$ & -16 & 82 & $\overline{6}$ & $\overline{117}$ & $\overline{84}$ & $\overline{6}$ & $-\overline{53}$ & $\overline{17}$ & Suntro \\
\hline
\end{tabular}

8. 9. 10. 11. 12. 13. 14. 15. 16. 17. Jh.

$\begin{array}{lllllllllll}0.0 & 8.3 & 6.2 & 4.9 & 6.5 & 3.4 & 4.8 & 1.5 & \emptyset . \emptyset & 0.0 & \text { Block } 1\end{array}$

$\begin{array}{lllllllllll}0.0 & 8.3 & 12.5 & 11.0 & 12.9 & 12.0 & 15.5 & 2.9 & 1.9 & 11.8 & \text { Block 2 }\end{array}$

$\begin{array}{lllllllllll}100 . \emptyset & 83.3 & 81.2 & 84.1 & 80.6 & 84.6 & 79.8 & 95.6 & 98.1 & 88.2 & \text { Block } 3\end{array}$

Abb. 24

Die zeitliche Verteilung von Klingenformen der Auswertungsgruppe 1 im gesamten Untersuchungsraum (enger datierter Bestand) 


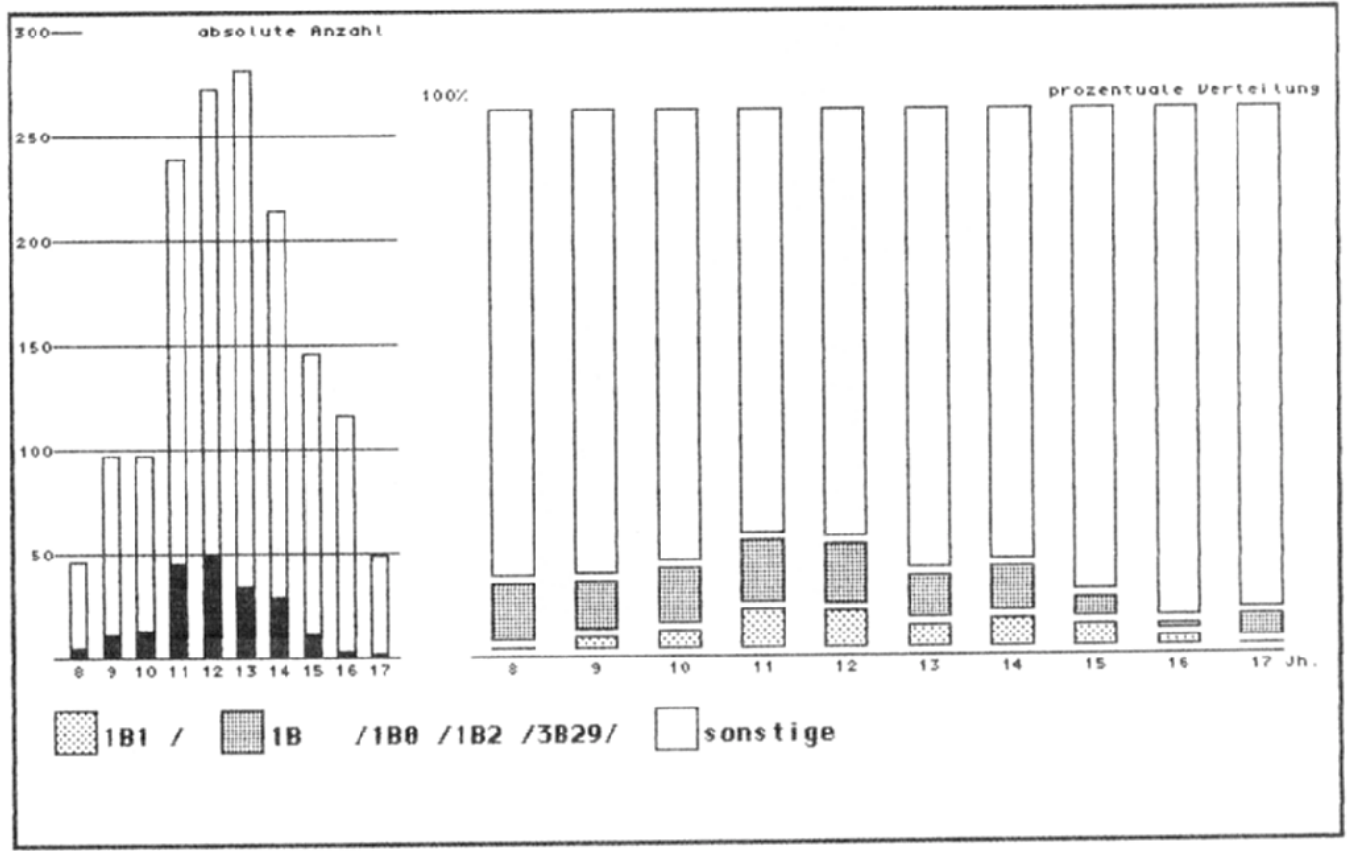

\begin{tabular}{|c|c|c|c|c|c|c|c|c|c|c|}
\hline 8. & 9. & 10. & 11. & 12. & 13. & 14. & 15. & 16. & 17. & Jh. \\
\hline 0 & 2 & 3 & 17 & 19 & 11 & 11 & 6 & 2 & 0 & Block 1 \\
\hline 5 & 9 & 10 & 28 & 30 & 23 & 18 & 5 & 1 & 2 & Block \\
\hline 41 & 86 & 84 & 194 & 223 & 247 & 185 & 135 & 113 & 47 & Block \\
\hline 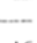 & $\cdots$ & $\overline{-}$ & $\bar{m}$ & - & - & - & $\overline{\text { sec }}$ & $\overline{116}$ & $\overline{49}$ & \\
\hline 46 & 97 & 97 & 239 & 272 & 281 & 214 & 146 & 116 & 49 & Sumine \\
\hline
\end{tabular}

8. 9. 10. $11.12 .13 .14 .415 .16 .17 . \quad$ Jh.

$\begin{array}{lllllllllll}0.0 & 2.1 & 3.1 & 7.1 & 7.0 & 3.9 & 5.1 & 4.1 & 1.7 & 0.0 & \text { Block } 1\end{array}$

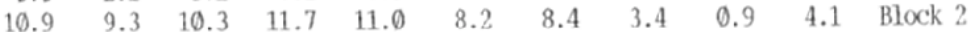

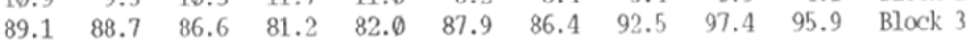

Abb. 25

Die zeitliche Verteilung von Klingenformen der Auswertungsgruppe 1 im gesamten Untersuchungsraum (grob datierter Bestand) 


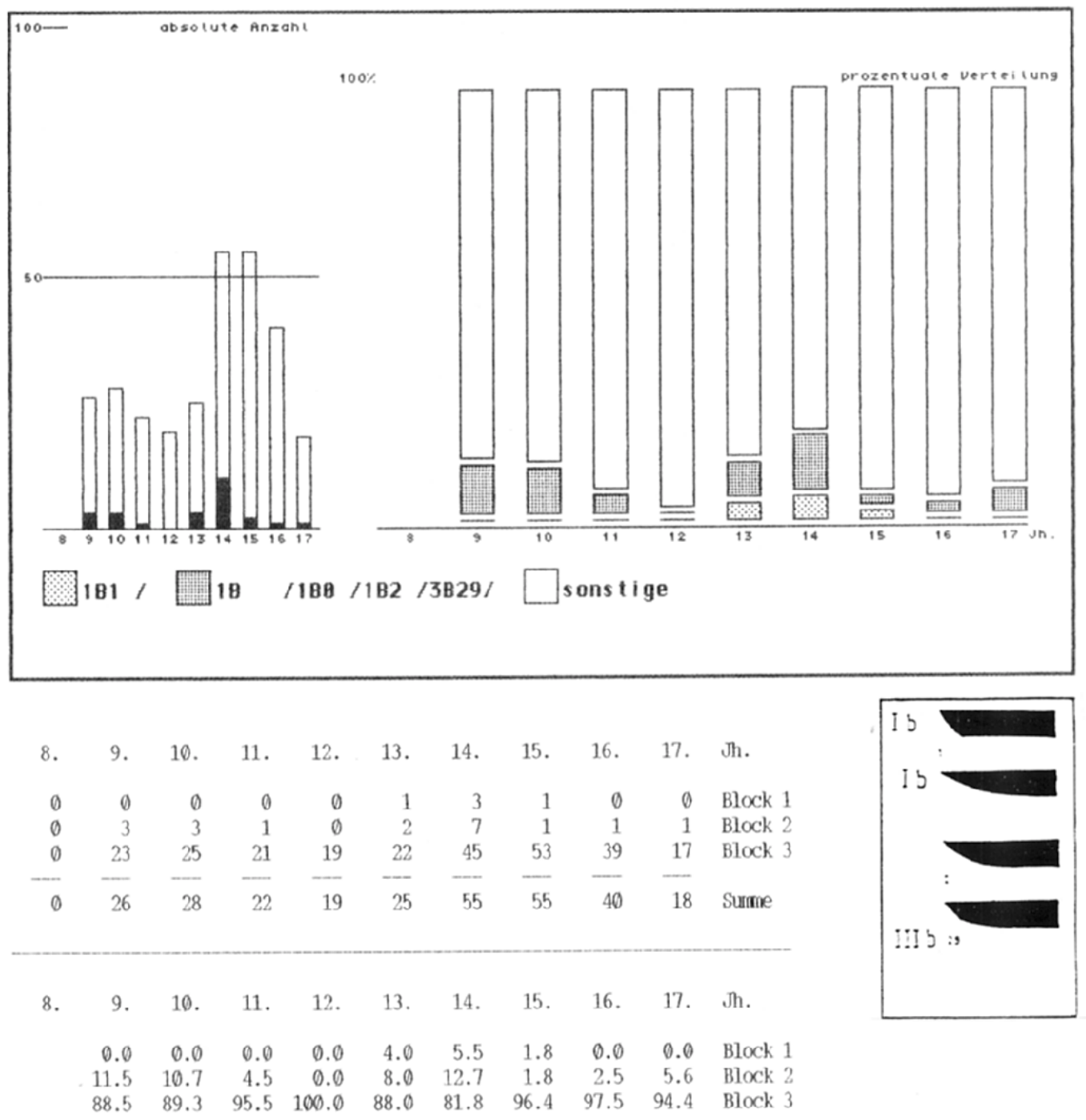

Abb. 26

Die zeitliche Verteilung von Klingenformen der Auswertungsgruppe 1 in Skandinavien (grob datierter Bestand) 
Die Form Ib1 begegnet erstmals im 13. Jahrhundert, in dem auch die übrigen Formen wieder einsetzen.

Im Gegensatz zur Gesamtverteilung liegt in Skandinavien die Hauptverbreitungszeit nicht im 11. bis 12 . Jahrhundert, sondern im 13., vor allem im 14. Jahrhundert mit über $18 \%$ aller beobachteten Klingenformen.

In der nördlichen Hälfte der Bundesrepublik (alte Bundesländer) und in der ehemaligen DDR liegen unterschiedliche Vergleichszeiträume vor:

Aussagefähig sind die Bestände in den alten Bundesländern vom 10./11. bis 17., in den neuen Bundesländern vom 10. bis 14. Jahrhundert (Abb. 27 und 28). Trotz verschiedener Prozentanteile sind die Verteilungstendenzen in beiden Untersuchungsräumen ähnlich, vor allem der Einbruch im 13. Jahrhundert ist auffällig.

Eine ethnisch begründete Bevorzugung dieser Formen, wie sie der in etwa regelmäßige Rückgang in den ehemals slawischen Siedlungsgebieten vom 10. bis 14. Jahrhundert nahe legen könnte, läßt sich im Vergleich mit den weiter westlich der Elbe gelegenen Räumen nicht belegen.

Der Rückgang von Messern mit geraden Rücken nach dem 15. Jahrhundert scheint sich dagegen zu bestätigen.

In Polen sind alle Klingenformen unter dem Code Ib im aussagekräftigen Zeitraum vom 10. bis 14. Jahrhundert aufgetreten. Ihre Hauptverbreitungszeit reicht vom 11. bis zum 13. Jahrhundert, im 12. Jahrhundert erreichen sie einen Anteil von fast 24\% aller Messer (Abb. 29). 


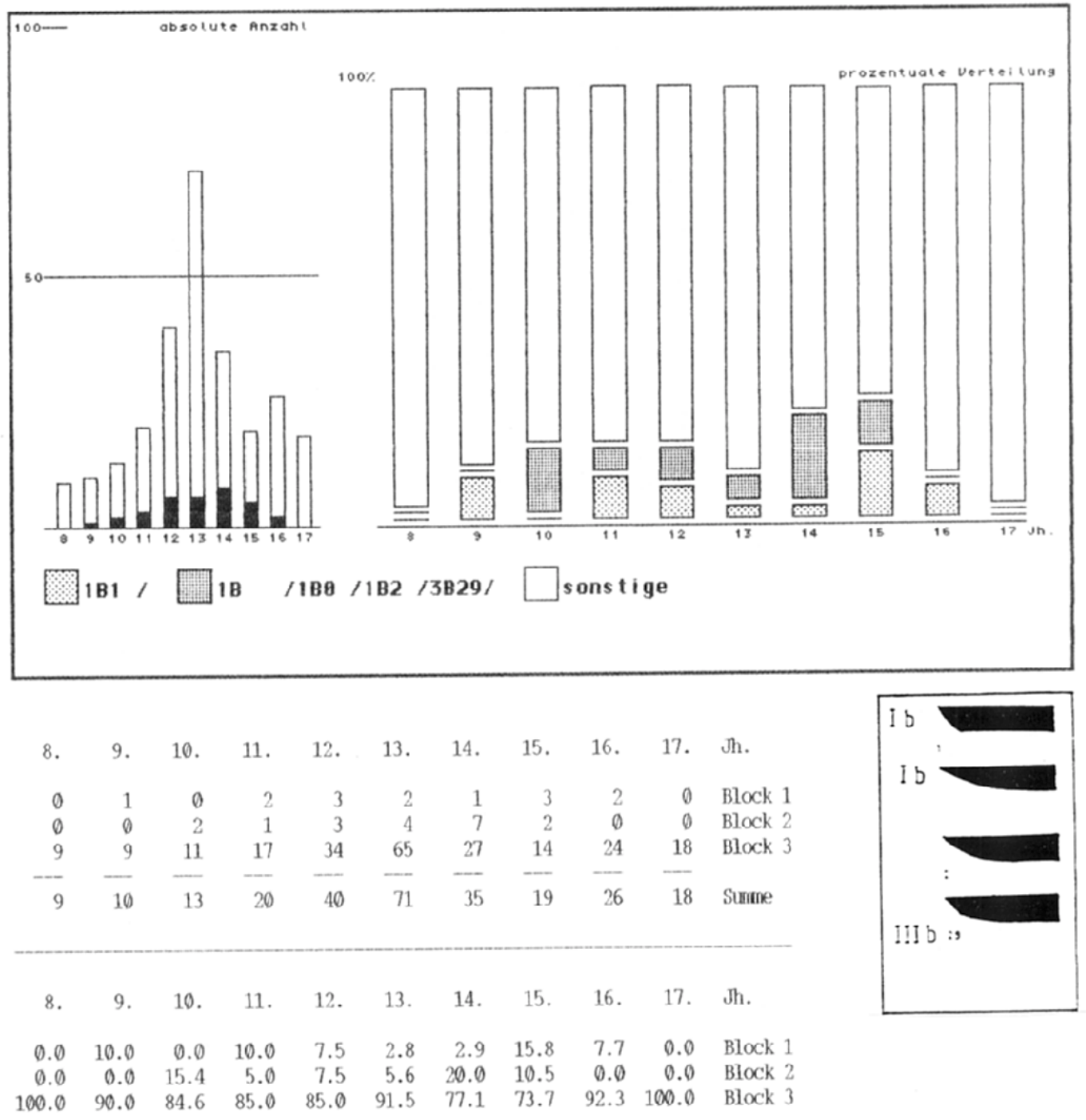

Abb. 27

Die zeitliche Verteilung von Klingenformen der Auswertungsgruppe 1 in Nordwestdeutschland (grob datierter Bestand) 


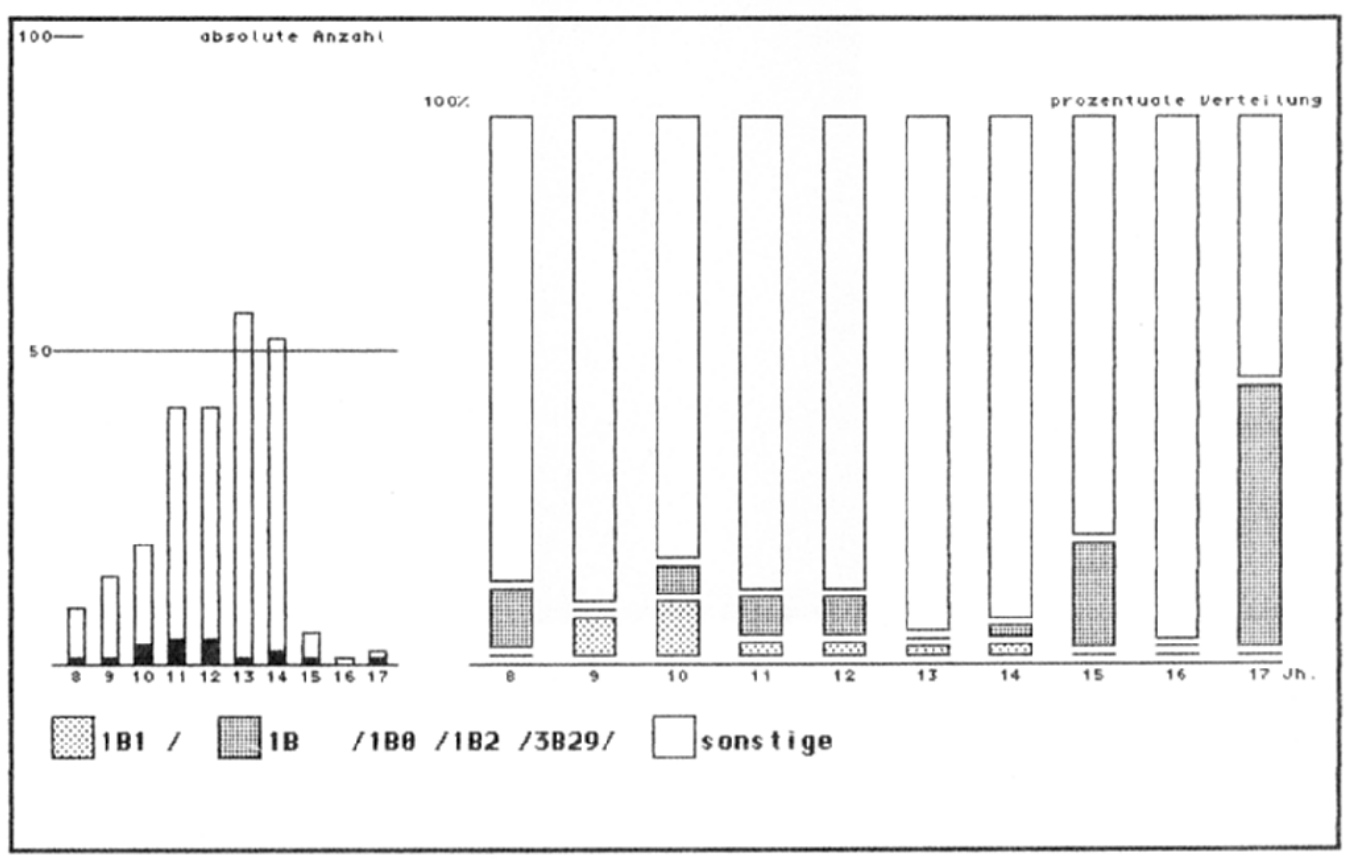

$\begin{array}{rrrrrrrrrrl}8 . & 9 . & 10 . & 11 . & 12 . & 13 . & 14 . & 15 . & 16 . & 17 . & \text { Jh. } \\ 0 & 1 & 2 & 1 & 1 & 1 & 1 & 0 & 0 & 0 & \text { Block 1 } \\ 1 & 0 & 1 & 3 & 3 & 0 & 1 & 1 & \emptyset & 1 & \text { Block 2 } \\ 8 & 13 & 16 & 37 & 37 & 55 & 50 & 4 & 1 & 1 & \text { Block 3 } \\ -9 & -14 & -19 & 41 & 41 & 56 & 52 & 5 & 1 & 2 & \text { Summe }\end{array}$

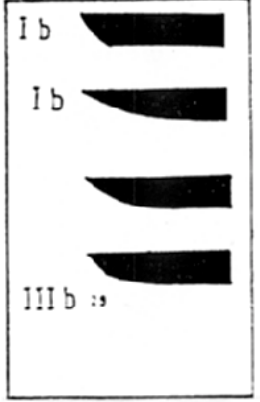

8. 9. 10. 11. 12. 13. 14. $15.16 .17 . \quad$ Jh.

$\begin{array}{lllllllllll}0.0 & 7.1 & 10.5 & 2.4 & 2.4 & 1.8 & 1.9 & 0.0 & 0.0 & 0.0 & \text { Block } 1\end{array}$

$\begin{array}{lllllllllll}11.1 & 0.0 & 5.3 & 7.3 & 7.3 & 0.0 & 1.9 & 20.0 & 0.0 & 50.0 & \text { Block 2 }\end{array}$

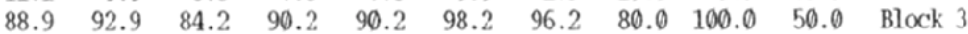

Abb. 28

Die zeitliche Verteilung von Klingenformen der Auswertungsgruppe 1 in Ostdeutschland (grob datierter Bestand) 


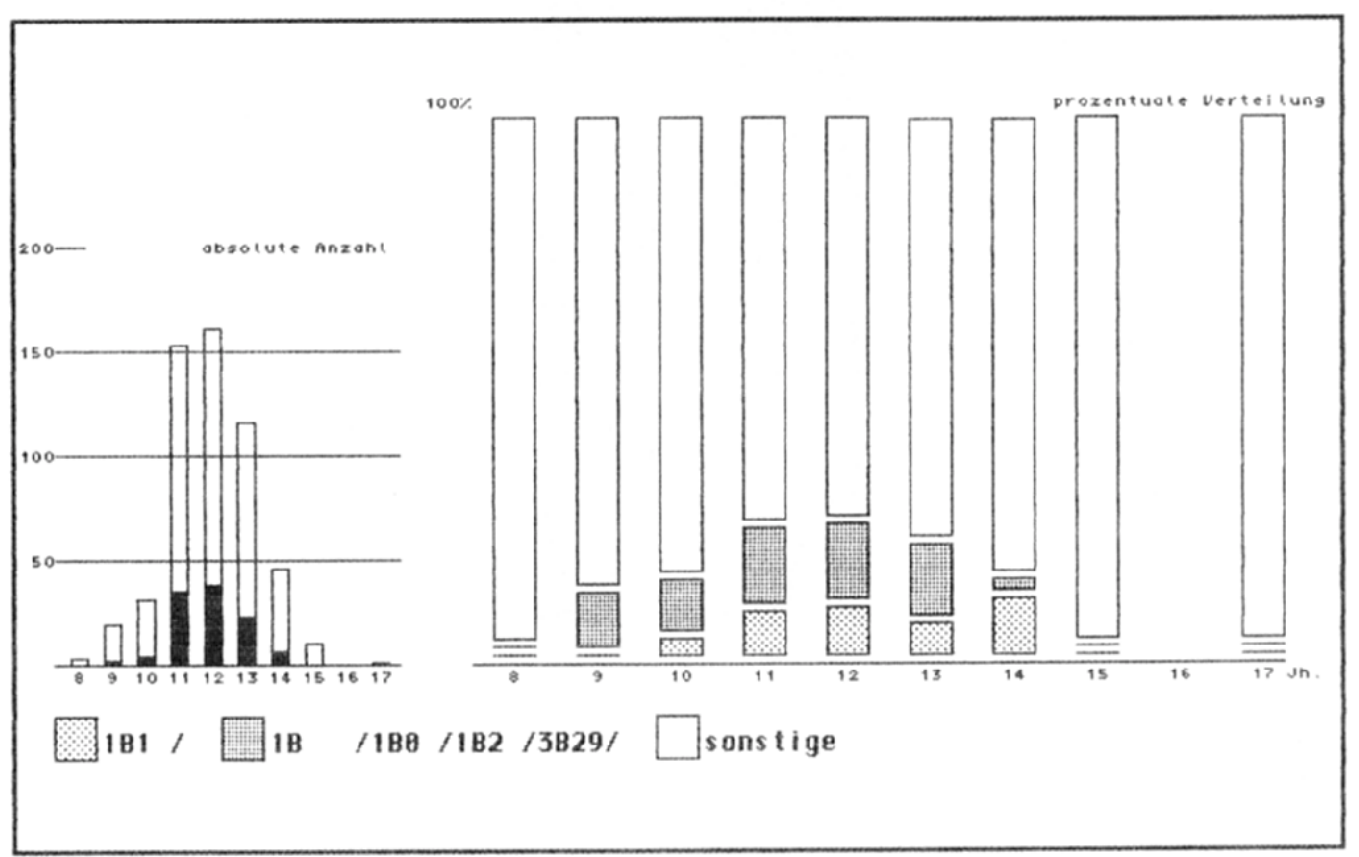

\begin{tabular}{|c|c|c|c|c|c|c|c|c|c|c|c|}
\hline 8. & 9. & 10. & 11. & 12. & 13. & 14. & 15. & 16. & 17. & $\mathrm{Jh}$. & \\
\hline$\emptyset$ & 0 & 1 & 13 & 15 & 7 & 5 & 0 & 0 & 0 & Block 1 & \\
\hline 0 & 2 & 3 & 22 & 23 & 16 & 1 & 0 & 0 & 0 & Block 2 & \\
\hline 3 & 17 & 27 & 118 & 123 & 93 & 40 & 10 & 0 & 1 & Block 3 & \\
\hline- & - & - & - & - & - & - & $-\cdots$ & - & - & & \\
\hline 3 & 19 & 31 & 153 & 161 & 116 & 46 & 10 & 0 & 1 & Sume & \\
\hline 8. & 9. & 10. & 11. & 12. & 13. & 14. & 15. & 16. & 17. & Jh. & \\
\hline 0.0 & 0.0 & 3.2 & 8.5 & 9.3 & 6.0 & 10.9 & 0.0 & & 0.0 & Block 1 & \\
\hline 0.0 & 10.5 & 9.7 & 14.4 & 14.3 & 13.8 & 2.2 & 0.0 & & 0.0 & Block 2 & \\
\hline 00.0 & 89.5 & 87.1 & 77.1 & 76.4 & 80.2 & 87.0 & 100.0 & & 100.0 & Block 3 & \\
\hline
\end{tabular}

Abb. 29

Die zeitliche Verteilung von Klingenformen der Auswertungsgruppe 1 in Polen (grob datierter Bestand) 


\subsubsection{Die Verteilung der Klingenformen IIb (Auswertungsgruppe 2)}

Nur 38 Klingen aus dem im Rahmen eines Jahrhunderts datierten Gesamtbestand von 526 Messern weisen gebogene Rücken und gerade Schneiden auf. Repräsentative Vergleichsmengen liegen für die Gruppe IIb vom 11. bis 16. Jahrhundert vor (Abb. 30).

In diesem Zeitraum erreichen sie kaum mehr als 5\% des Gesamtbestandes, durchbrochen von $25 \%$ im 15 . Jahrhundert.

Die gleiche Verteilung zeigt die Auswertung des zeitlich weiter gefaßten Bestandes (Abb. 31).

Nachweisbar im gesamten Untersuchungszeitraum, bleiben die Klingenformen jetzt bis zum 15. Jahrhundert unter 7\%, ihre Hauptverbreitungszeit liegt mit fast $13 \% \mathrm{im}$ 15. und noch über $10 \%$ im 17 . Jahrhundert.

Das Ansteigen im 15. Jahrhundert läßt sich mit dem Aufkommen nahezu gleichförmiger Griffzungenmesser in niederländischen, nordwestdeutschen und südskandinavischen Städten in Verbindung bringen, die aufgrund ihrer standardisierten Maße als Teile des frühneuzeitlichen Tafelbestecks angesprochen werden können (Abb. 32).

Dem entspricht das allgemein stärkere Vorkommen der Klingenformen des Codes IIb im 16. und 17. Jahrhundert, ein Zeitraum, in dem der größte Teil der geborgenen Messer auch durch Bildquellen belegt der Tafel zugeordnet werden kann.

Möglicherweise korrespondiert dieser Hinweis auf die Funktion mit einer allgemeinen funktionstechnischen Erfahrung, daß Klingen mit gerade verlaufender Schneide vor allem zum Zerschneiden und Zerteilen von Nahrungsmitteln tauglich sind.

Unterschiedliche zeitliche Verteilungen in den einzelnen Untersuchungsländern ließen sich auch aufgrund der geringen Anzahl nicht erkennen.

Die bereits angesprochene Konzentration von Messern mit den unter Ilb zusammengefaßten Klingenformen nach 1400, die auch in der Verbreitungskarte (Abb. 33) sichtbar wird, deckt einen Teil des frühneuzeitlichen niederländischskandinavischen Wirtschaftsraumes ab, dessen Anschluß auch über nordwestdeutsche Verkehrswege lief. Möglicherweise dokumentiert sich mit diesen ${ }^{54}$ und weiteren Tafelmessern des 15. bis 17. Jahrhunderts ein Teil des Handelsgutes, welches im spät- und nachhansischen Handel zwischen den Niederlanden und Südskandinavien direkt verhandelt wurde.

54 Vgl. neben den in Abb. 10 aufgeführten niederländischen Beispielen die Bestände aus Lund und Kolding 


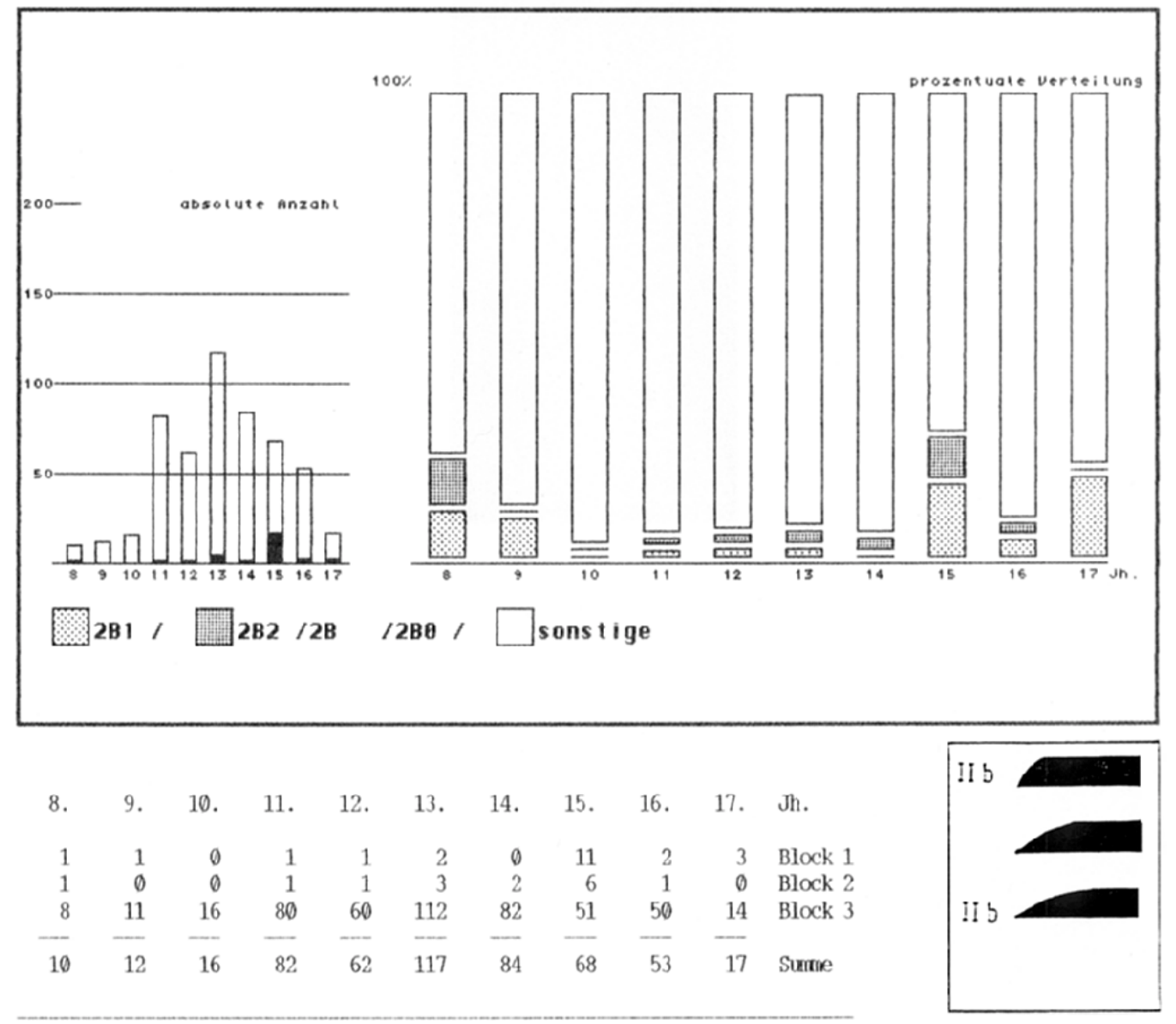

8. 9. 10. 11. 12. 13. 14. 15. 16. 17. Jh.

$\begin{array}{lllllllllll}10.0 & 8.3 & 0.0 & 1.2 & 1.6 & 1.7 & 0.0 & 16.2 & 3.8 & 17.6 & \text { Block 1 }\end{array}$

$\begin{array}{lllllllllll}10.0 & 0.0 & 0.0 & 1.2 & 1.6 & 2.6 & 2.4 & 8.8 & 1.9 & 0.0 & \text { Block 2 }\end{array}$

$\begin{array}{lllllllllll}80.0 & 91.7 & 100.0 & 97.6 & 96.8 & 95.7 & 97.6 & 75.0 & 94.3 & 82.4 & \text { Block } 3\end{array}$

Abb. 30

Die zeitliche Verteilung von Klingenformen der Auswertungsgruppe 2 im gesamten Untersuchungsraum (enger datierter Berstand) 


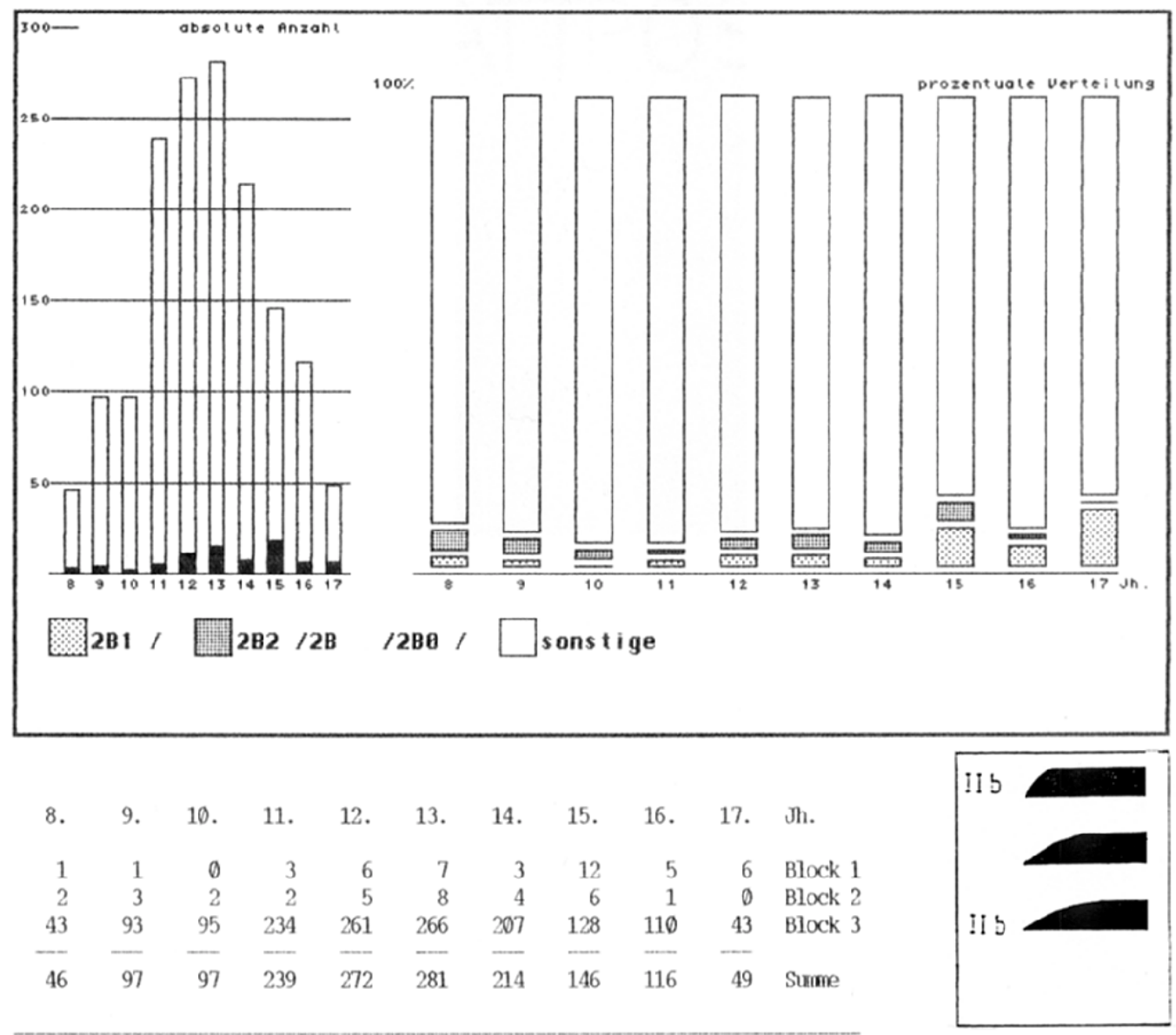

8. 9. 10. 11. 12. 13. 14. 15. 16. 17. Jh.

$\begin{array}{lllllllllll}2.2 & 1.0 & 0.0 & 1.3 & 2.2 & 2.5 & 1.4 & 8.2 & 4.3 & 12.2 & \text { Block 1 }\end{array}$

$\begin{array}{lllllllllll}4.3 & 3.1 & 2.1 & 0.8 & 1.8 & 2.8 & 1.9 & 4.1 & 0.9 & 0.0 & \text { Block 2 }\end{array}$

$\begin{array}{lllllllllll}93.5 & 95.9 & 97.9 & 97.9 & 96.0 & 94.7 & 96.7 & 87.7 & 94.8 & 87.8 & \text { Block 3 }\end{array}$

Abb. 31

Die zeitliche Verteilung von Klingenformen der Auswertungsgruppe 2 im gesamten Untersuchungsraum (grob datierter Berstand) 


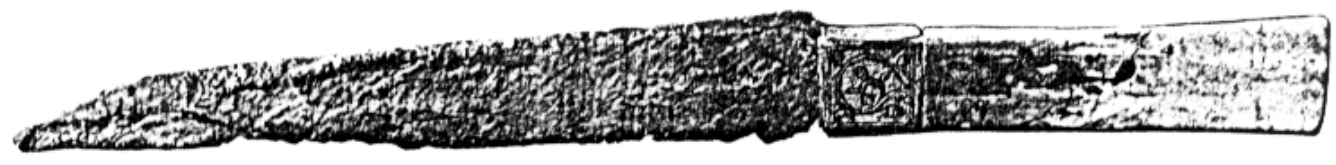

a

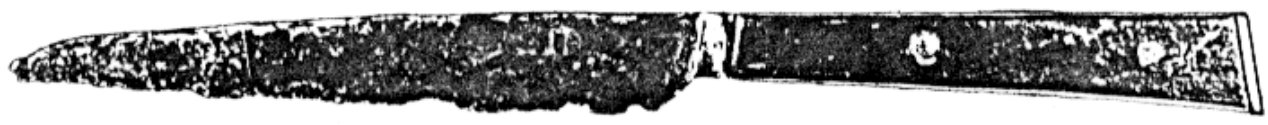

b

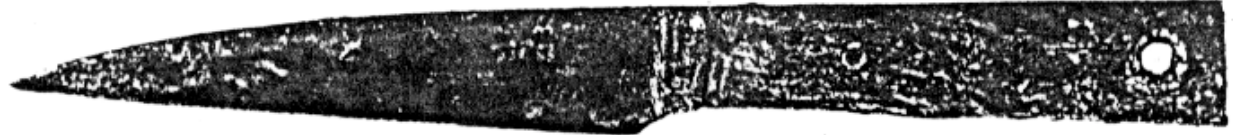

Abb. 32 Beispiele von Klingenformen der Auswertungsgruppe 2 (gebogener Rücken und gerade verlaufende Schneide)

$a:$

$b-c$ :
Einzelmesser des 13. Jahrhunderts (Store Valby)

Standardisierte Messer des 15. Jahrhunderts (Zwijndrecht, Gorkum) 
Verbreitungskarte 1

Blatt 1

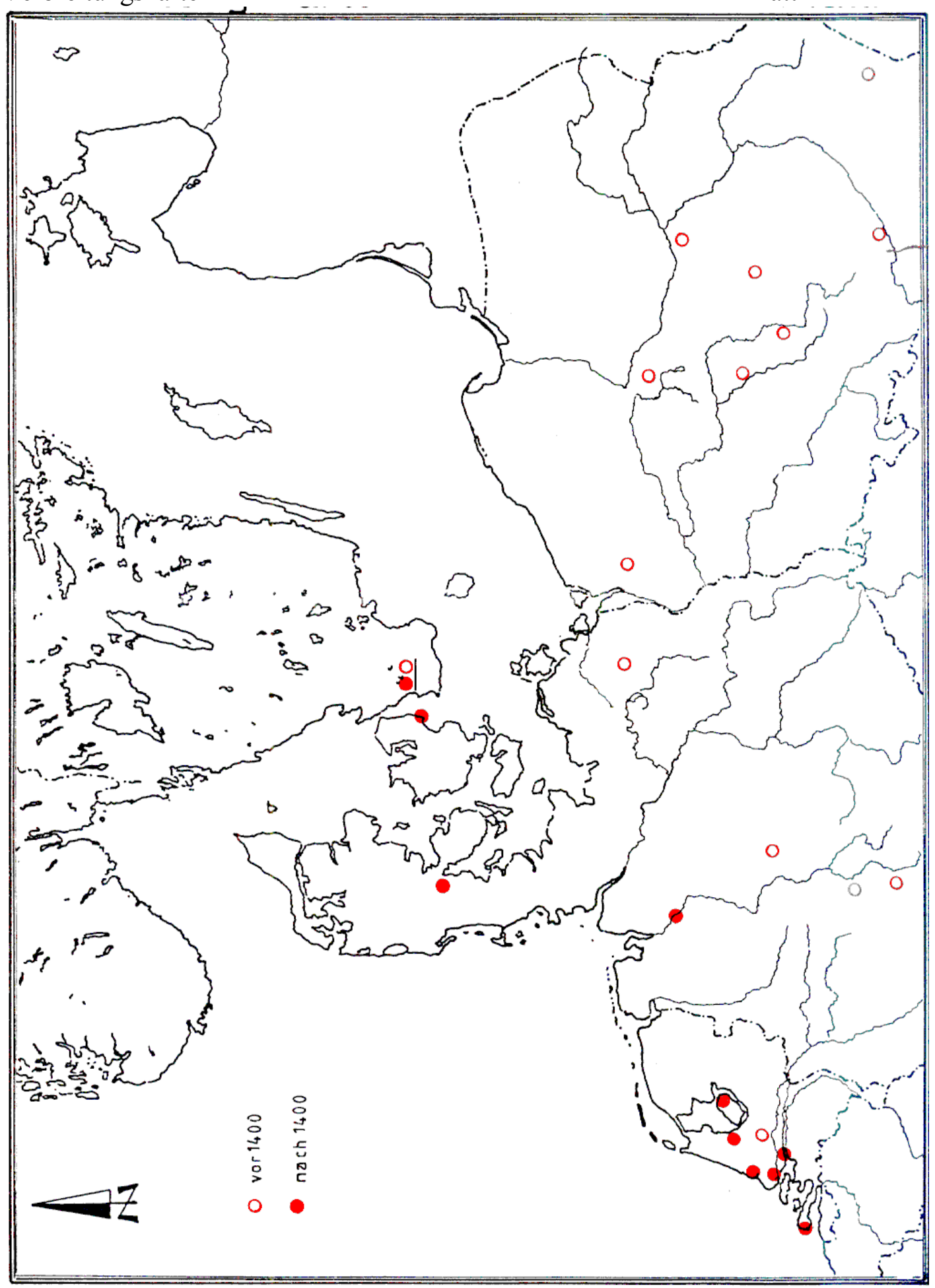

Abb 33

Die Verbreitung der Klingenform IIb vor und nach 1400 


\subsubsection{Die Verteilung der Klingenformen IIIb (Auswertungsgruppe 3)}

Messerklingen mit gebogenen bzw. gerade zur Spitze einfallenden Kanten, von denen in der Auswertung die Form IIIb13 getrennt wurde ${ }^{55}$, machen mit 103 Exemplaren fast $20 \%$ der 526 insgesamt vorhandenen, im Rahmen eines Jahrhunderts datierten Messer aus. Im Diagramm der Auswertung dieses Bestandes für den gesamten Untersuchungsraum ist die Gruppe lediglich im 17. Jahrhundert nicht angetroffen worden. In den übrigen Jahrhunderten liegt ihr prozentualer Anteil zwischen 10,7\% und 37,7\% (Abb. 34), wobei die Form IIIb13 nur vom 11. bis zum 15. Jahrhundert auftritt.

Einem leichten Anstieg zum 13. Jahrhundert folgt ein erneuter Anstieg zum 16. Jahrhundert, der von fast $40 \%$ auf das völlige Fehlen im 17. Jahrhundert abfällt.

Bereits an dieser Stelle sei auf die veränderte Entwicklung dieser Formengruppe hingewiesen, der diese vom 11. zum 14. Jahrhundert in Skandinavien unterliegt (Abb. 35).

Die Auswertung dieser Gruppe im zeitlich weiter gefaßten Rahmen zeigt mit unterschiedlichen Schwerpunkten die gleiche Tendenz (Abb. 36):

Insgesamt steigt sie vom 8. zum 9. und 10. Jahrhundert auf 38,2\% des Gesamtbestandes an, fällt zum 11. Jahrhundert auf $17,6 \%$ zurück und steigt bis zum 13. Jahrhundert wieder regelmäßig auf $20,4 \%$ an. Einem leichten Einbruch im 14. Jahrhundert folgt der bereits beobachtete Anstieg, hier auf 31,1\%, zum 16 . Jahrhundert und ein deutlicher Abfall auf 8,1\% im 17. Jahrhundert.

Vom 9. Jahrhundert an ist die Form IIIb13 zu beobachten, die ihre Hauptverbreitungszeit vom 9. bis 14. Jahrhundert zu haben scheint. Ihr allgemein geringes Aufkommen (maximal 6,2\% im 10. Jahrhundert) und unterschiedliche Schwankungen in den beiden Auswertungsdiagrammen zum gesamten Untersuchungsraum lassen lediglich den Schluß zu, daß sie nach dem 15. Jahrhundert nur noch eine sehr untergeordnete Rolle im Formenspektrum der Messerklingen eingenommen hat.

Zur Interpretation dieses Verteilungsbildes fehlt jede Information. Beobachten ließ sich im aufgenommenen Bestand eine Variabilität der Klingenbreiten (-höhen), die aber aus genannten Gründen der vorliegenden Materialbasis nicht ausgewertet worden sind.

Eine breitere Ausgangsbasis wird möglicherweise die Anzahl besser erhaltener Messer erhöhen können, so daß eine Hinzunahme dieses Maßes vielleicht Aufschluß über Differenzierungsmöglichkeiten in der Formengruppe gibt.

Feststellbar bleibt, daß Messerklingen dieser Formengruppe einen bedeutenden Anteil an Klingenformen im gesamten Untersuchungszeitraum innehatten. Daß es im

55 Klinge mit gebogenem Rücken und gerade verlaufender Schneide, die im letzten Drittel gerade zur Spitze hochbiegt 
Verlauf des frühen Hochmittelalters und am Ende des 16. Jahrhunderts Veränderungen gab, korrespondiert auch mit anderen untersuchten Kriterien ${ }^{56}$.

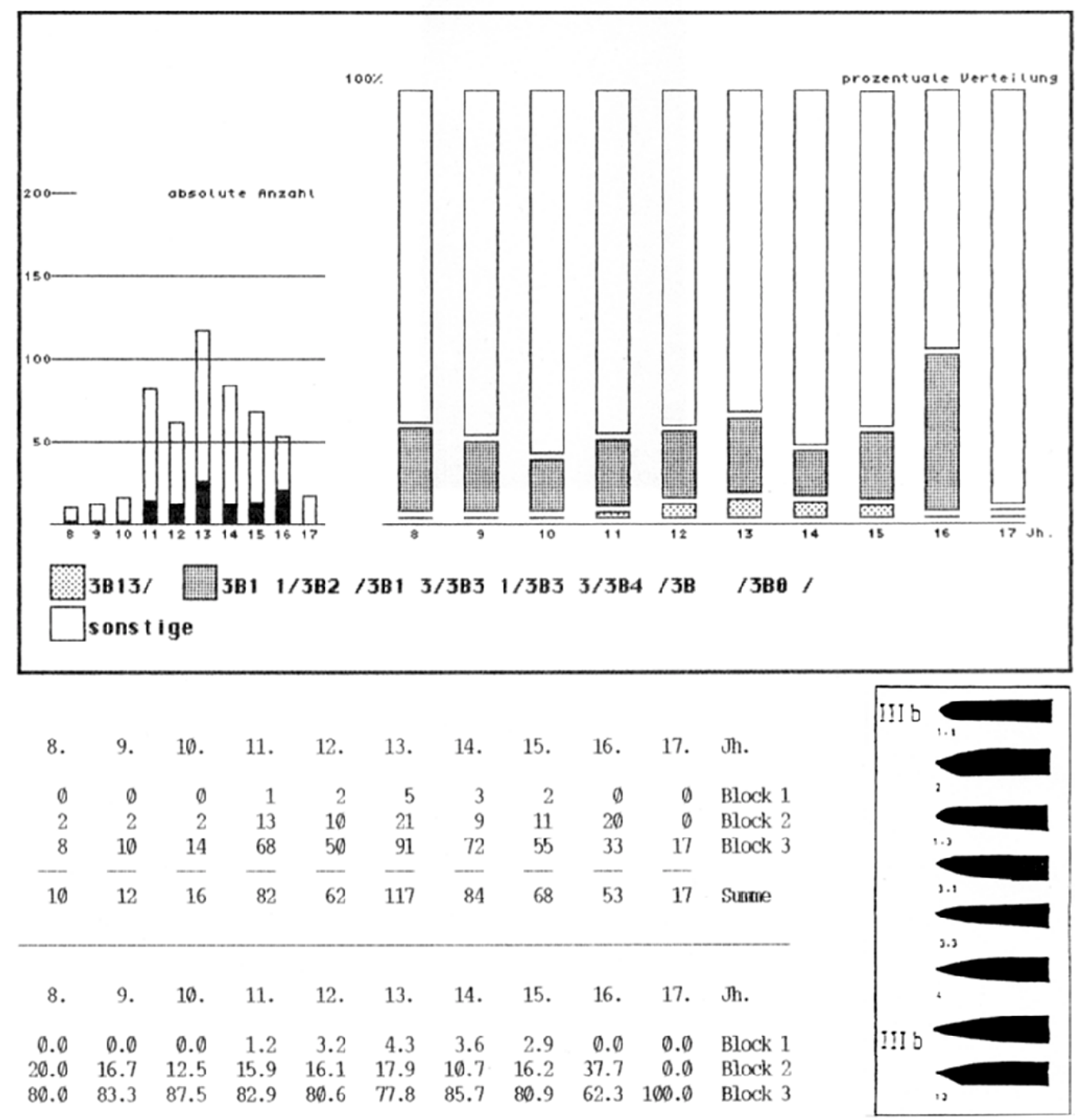

Abb. 34

Die zeitliche Verteilung von Klingenformen der Auswertungsgruppe 3 im gesamten Untersuchungsraum (enger datierter Berstand) 


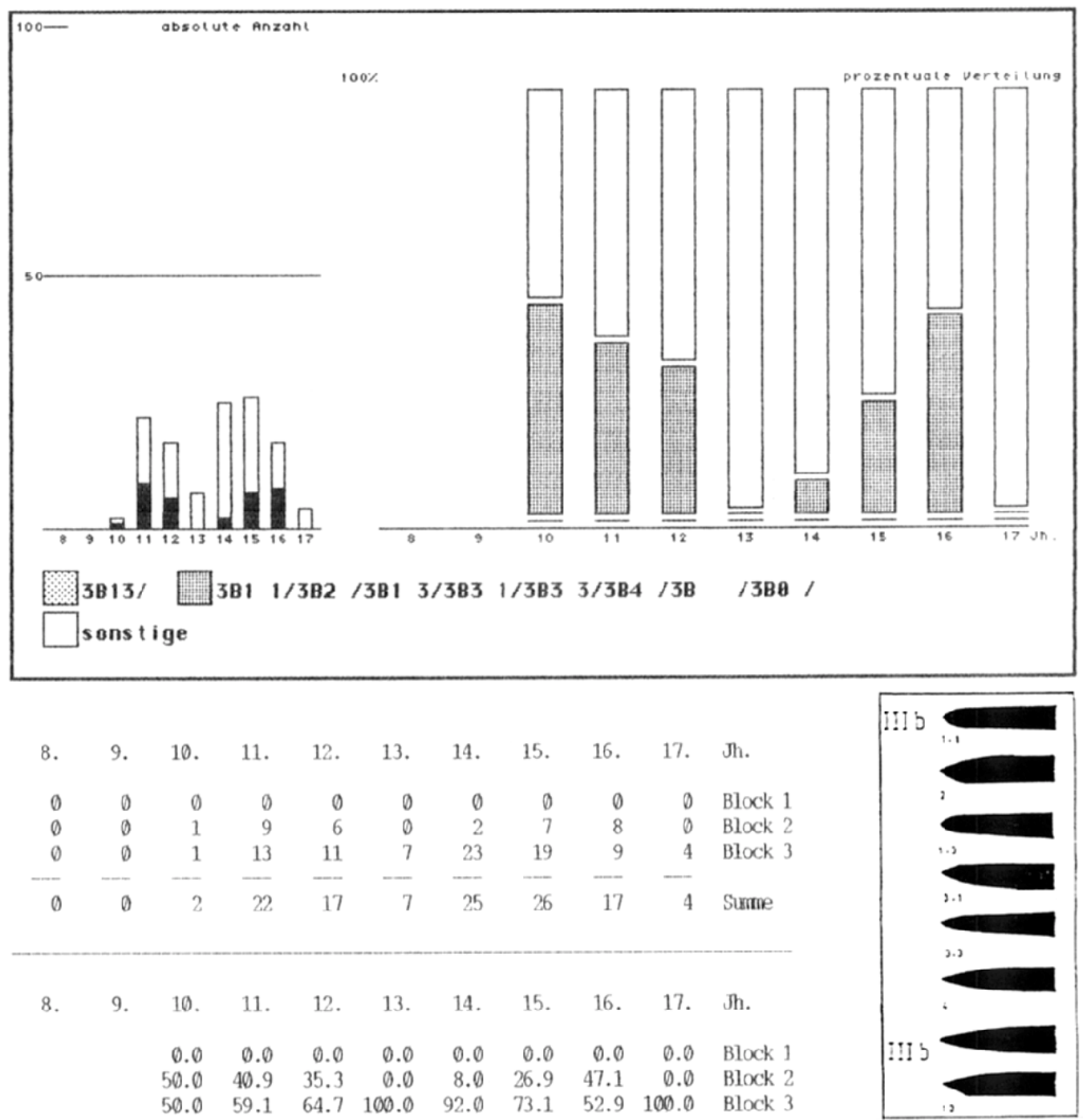

Abb. 35

Die zeitliche Verteilung von Klingenformen der Auswertungsgruppe 3 in Skandinavien (enger datierter Berstand) 


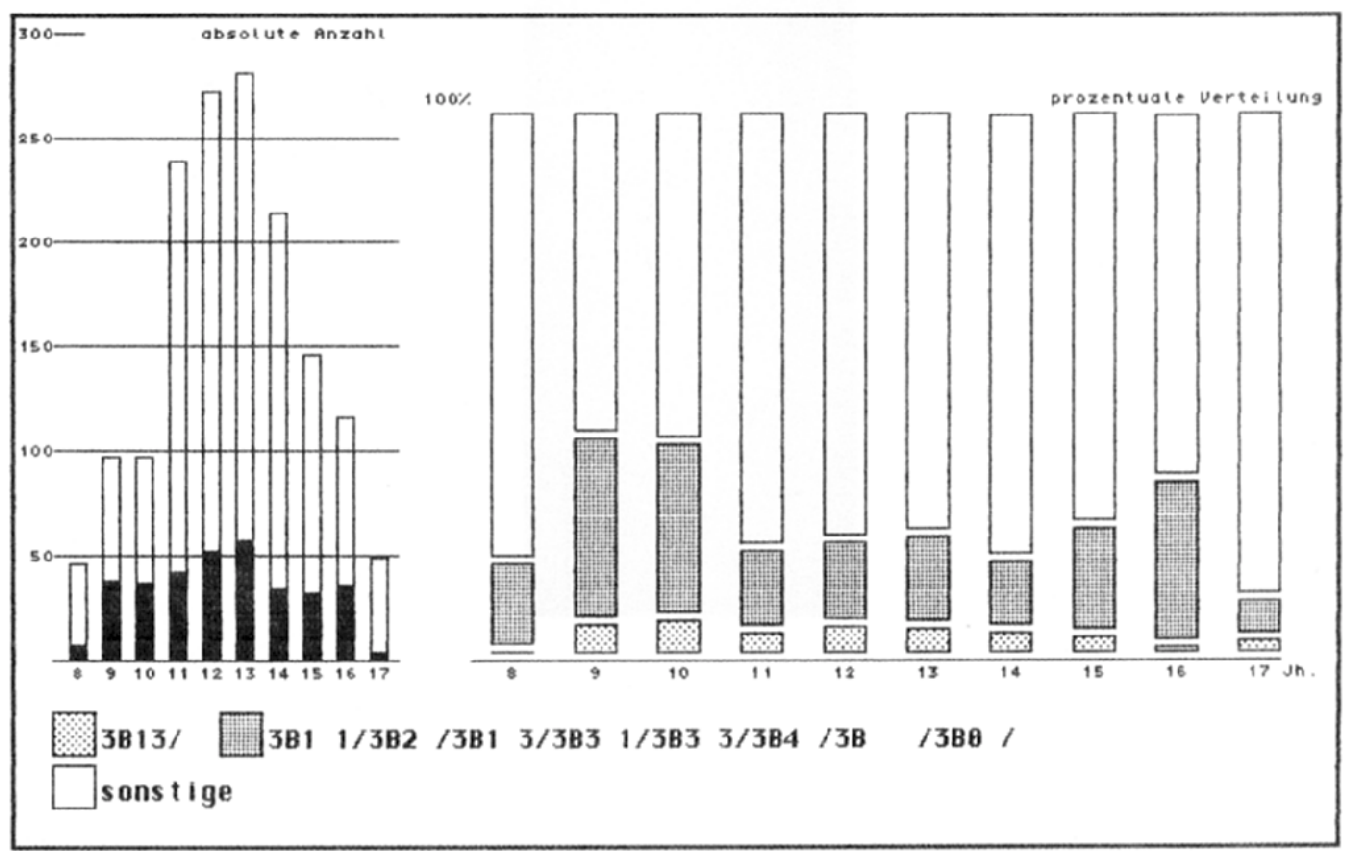

\begin{tabular}{|c|c|c|c|c|c|c|c|c|c|c|}
\hline 8. & 9. & 10. & 11. & 12. & 13. & 14. & 15. & 16. & 17. & $\mathrm{~h}$ \\
\hline 0 & 5 & 6 & 9 & 13 & 12 & 8 & 4 & 1 & 1 & Block 1 \\
\hline 7 & 33 & 31 & 33 & 39 & 45 & 26 & 28 & 35 & 3 & Block 2 \\
\hline 39 & 59 & 60 & 197 & 220 & 224 & 180 & 114 & 80 & 45 & Block 3 \\
\hline 46 & $\overline{07}$ & $\overline{07}$ & $\overline{33}$ & $\overline{\eta \eta}$ & $\bar{x}$ & $\bar{x}$ & $\overline{119}$ & $\overline{116}$ & $\overline{49}$ & \\
\hline 46 & 97 & 97 & 239 & 272 & 281 & 214 & 146 & 116 & 49 & Surme \\
\hline
\end{tabular}

8. 9. 10. 11. 12. 13. 14. 15. 16. 17. Jh.

$\begin{array}{rrrrrrrrrrr}0.0 & 5.2 & 6.2 & 3.8 & 4.8 & 4.3 & 3.7 & 2.7 & 0.9 & 2.0 & \text { Block 1 } \\ 15.2 & 34.0 & 32.0 & 13.8 & 14.3 & 16.0 & 12.1 & 19.2 & 30.2 & 6.1 & \text { Block 2 }\end{array}$

$\begin{array}{lllllllllll}84.8 & 60.8 & 61.9 & 82.4 & 80.9 & 79.7 & 84.1 & 78.1 & 69.0 & 91.8 & \text { Block } 3\end{array}$

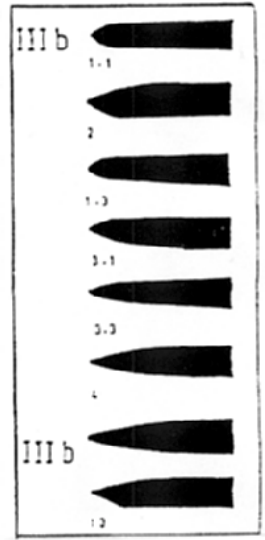

$A b b .36$

Die zeitliche Verteilung von Klingenformen der Auswertungsgruppe 3 im gesamten Untersuchungsraum (grob datierter Bestand) 
Interpretationsversuche hinsichtlich einer Gegenüberstellung der beobachteten Tendenzveränderungen mit allgemeinen historischen Epochengrenzen und technischen wie wirtschaftlichen Entwicklungen, die sich hinter dem Anstieg vom 14. zum 16. Jahrhundert verbergen könnten ${ }^{57}$, mögen als Denkanstoß tauglich erscheinen, für eine fundiertere Einbindung in historische Umfelder reichen diese tendenziellen Entwicklungsähnlichkeiten nicht aus.

Das gilt auch für die Unterschiede, die sich bei der Bestandsauswertung der einzelnen Untersuchungsländer ergaben.

Auch hier bleibt lediglich die Möglichkeit, diese festzustellen und ohne jeden Kommentar vorzulegen, als Belege für Informationsdefizite, die bisher nicht einmal bekannt waren.

In Skandinavien verläuft die tendenzielle Verteilungskurve vom 10. bis zum 14. Jahrhundert gleichmäßig abnehmend, ohne den Abfall zum 11. und erneuten Anstieg zum 13. Jahrhundert zu wiederholen, der im Bestand der nordwestdeutschen und teilweise auch der ostdeutschen und polnischen Messerfunde in Korrelation zum Bild der Gesamtauswertung zu beobachten ist (Abb. 37).

Dieser regional feststellbare Unterschied wird erhärtet durch die bereits angesprochene Verteilungstendenz im zeitlich enger gefaßten Bestand, diese Kurve bei einem erheblich geringeren Auswertungsbestand wiederholt.

Der bewertbare Zeitrahmen vom 14. bis 17. Jahrhundert zeigt in den Niederlanden eine etwas unterschiedliche Verteilung gegenüber dem gesamten Untersuchungsraum (Abb. 38).

Die getrennt vorgenommenen Untersuchungen der betreffenden Messer in den alten und neuen Bundesländern ergeben im vergleichbaren Zeitraum unterschiedliche Verteilungstendenzen im 13. Jahrhundert (Abb. 39 und 40).

Unterschiede zum Gesamterscheinungsbild zeigt der regelmäßige Abfall vom 15. zum 17. Jahrhundert im Bestand der nordwestlichen Bundesrepublik.

Im polnischen Messerbestand verläuft die Entwicklung ähnlich wie im ostdeutschen Bestand (Abb. 41). Auch hier fehlt der in Nordwestdeutschland beobachtete Anstieg vom 12. zum 13. Jahrhundert.

Die Form IIIb13 verteilt sich in den aussagefähigen Zeiträumen in jedem Untersuchungsland unterschiedlich. In einem Fall erreicht sie einen Wert von über $10 \%$ nordwestdeutschen Bestand des 10. Jahrhunderts. 


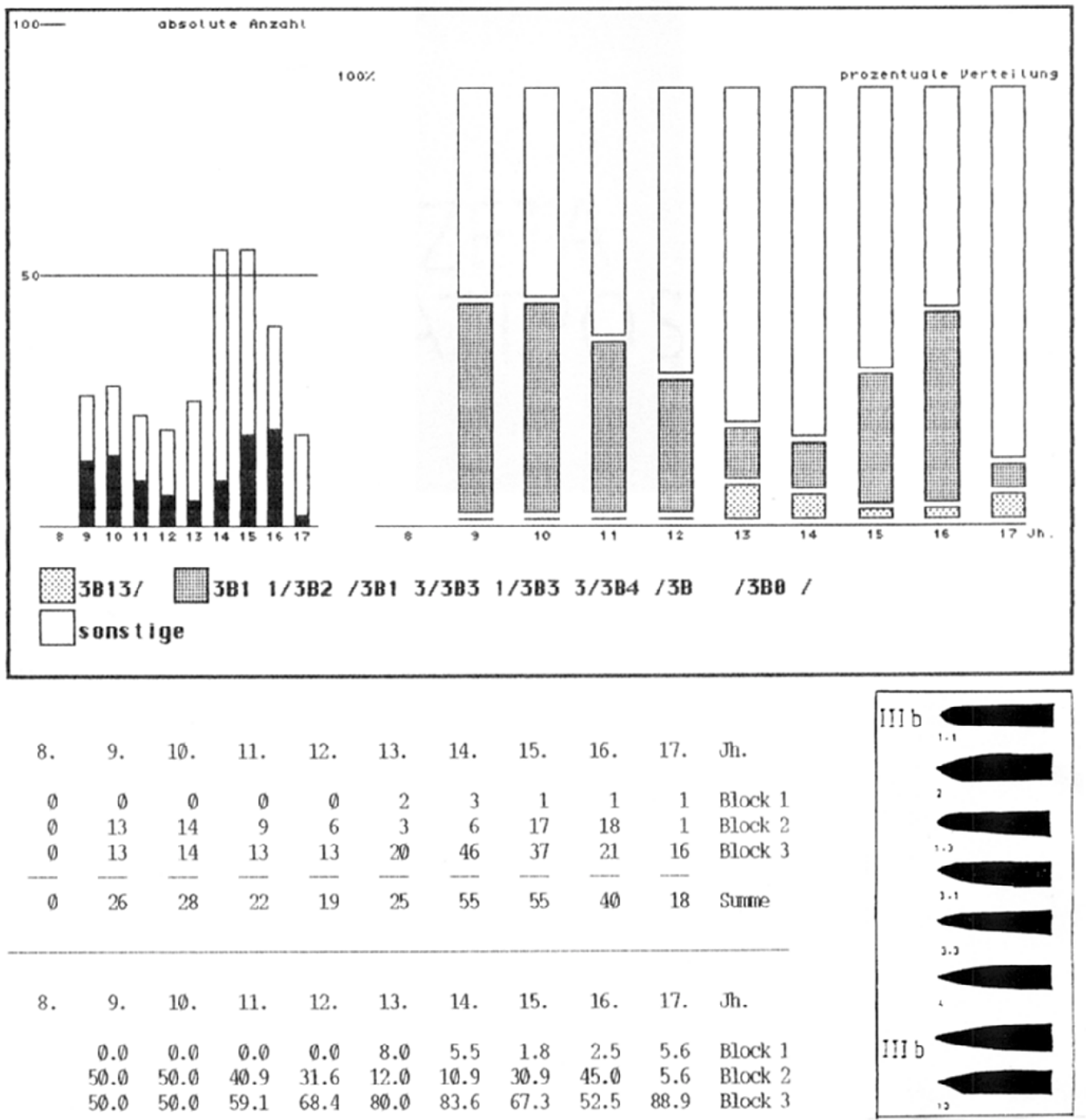

Abb. 37

Die zeitliche Verteilung von Klingenformen der Auswertungsgruppe 3 in Skandinavien (grob datierter Bestand) 


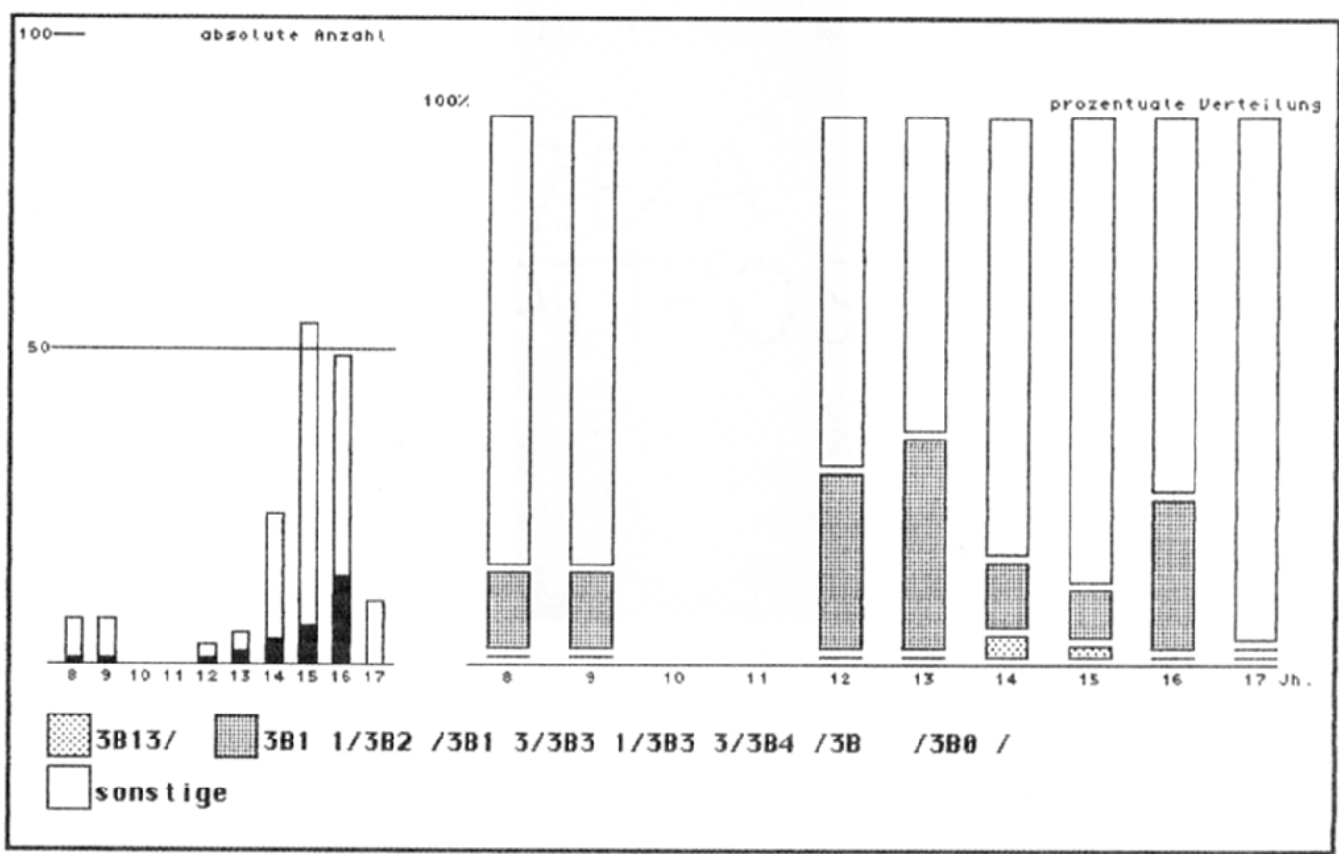

8. 9. 10. 11. 12. 13. 14. 15. 16. $17 . \quad$ Jh.

\begin{tabular}{rrrrrrrrrrl}
0 & 0 & 0 & 0 & 0 & 0 & 1 & 1 & 0 & 0 & Block 1 \\
1 & 1 & 0 & 0 & 1 & 2 & 3 & 5 & 14 & 0 & Block 2 \\
6 & 6 & 0 & 0 & 2 & 3 & 20 & 48 & 35 & 10 & Block 3 \\
\hline 7 & -7 & 0 & 0 & 3 & 5 & 24 & 54 & 49 & 10 & Surme
\end{tabular}

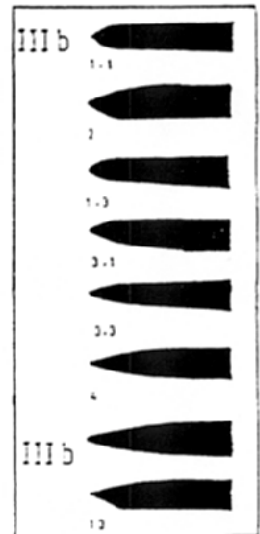

Abb. 38

Die zeitliche Verteilung von Klingenformen der Auswertungsgruppe 3 in den Niederlanden (grob datierter Bestand) 


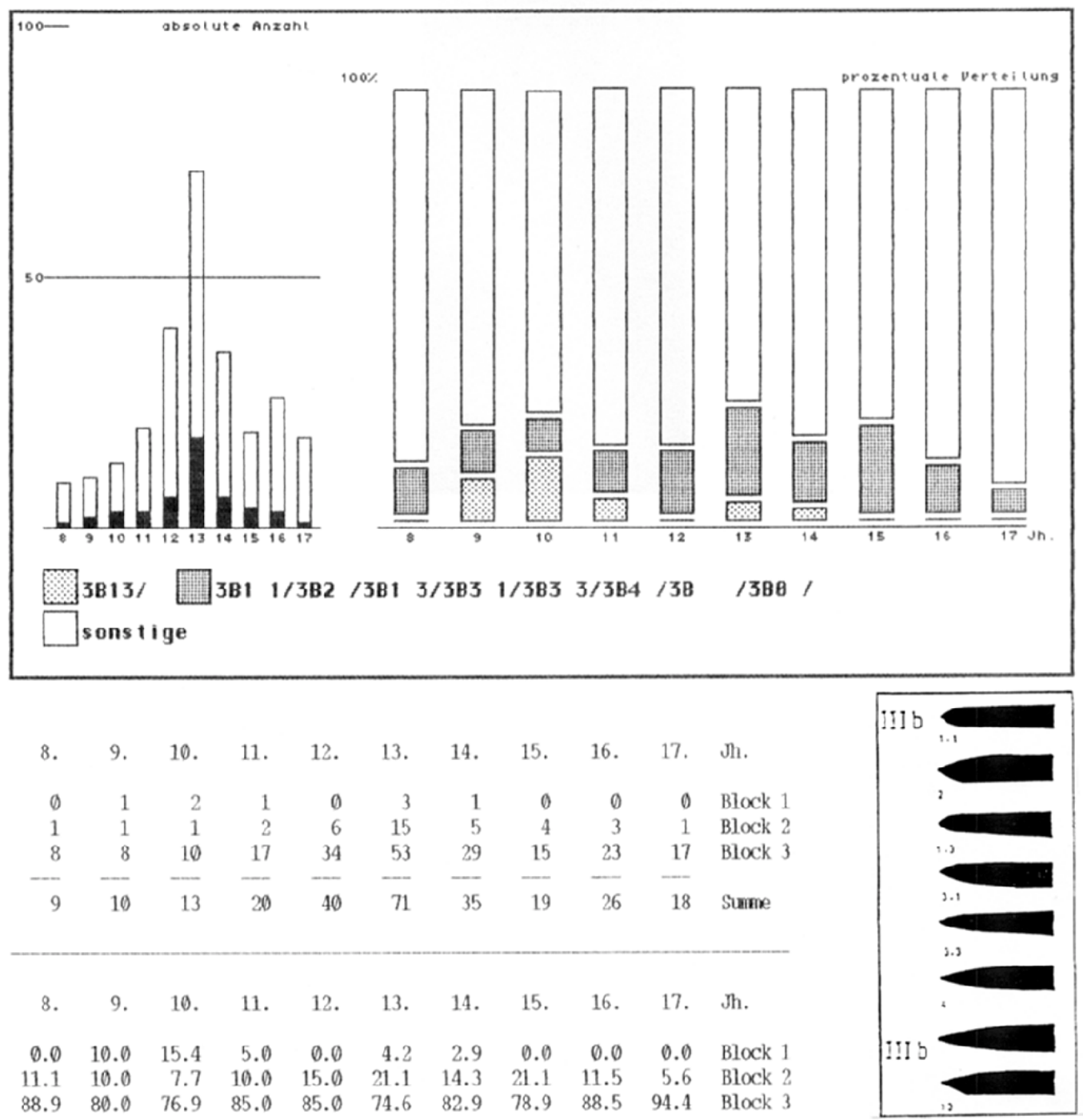

Abb. 39

Die zeitliche Verteilung von Klingenformen der Auswertungsgruppe 3 in Nordwestdeutschland (grob datierter Bestand) 


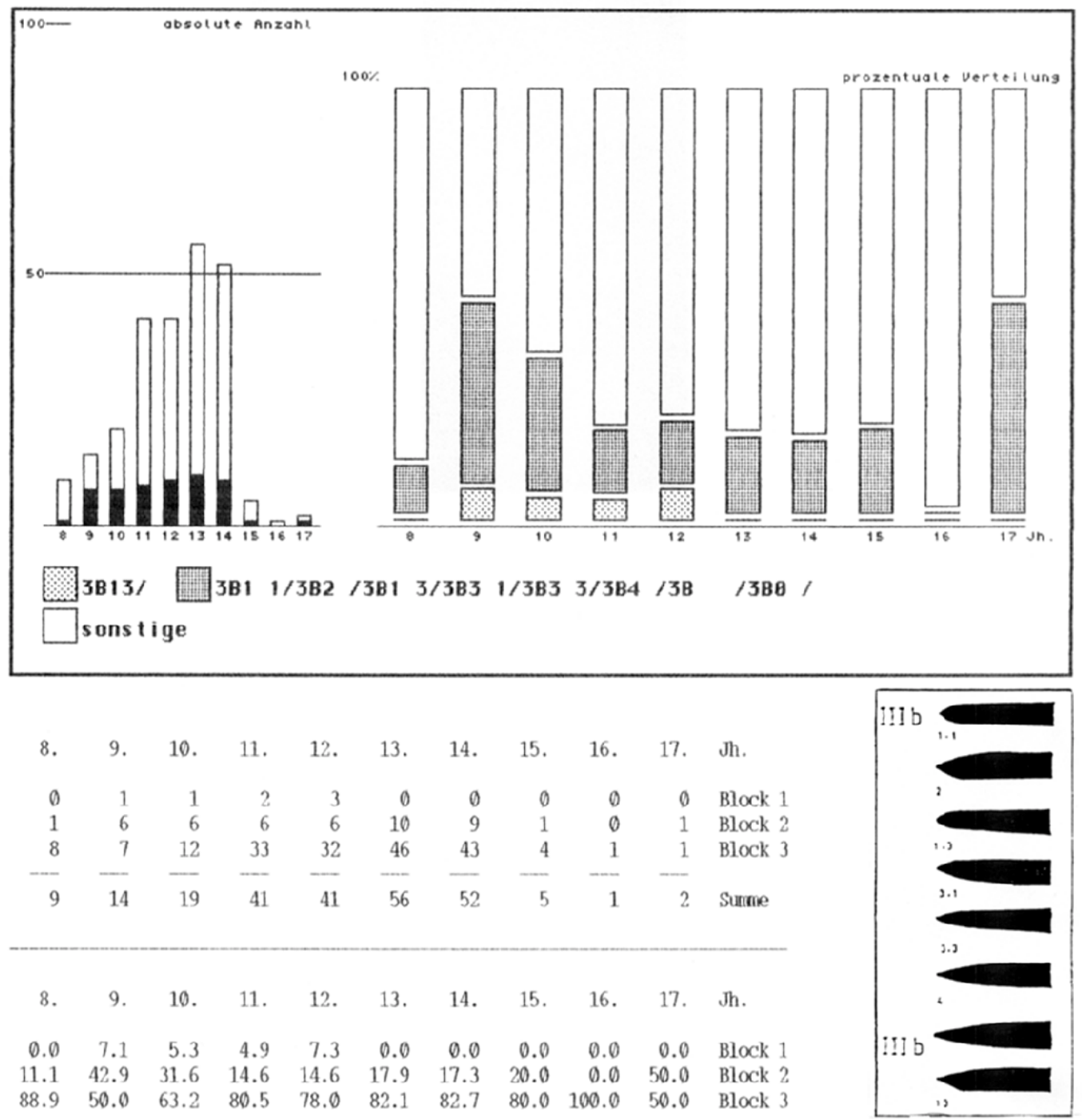

Abb. 40

Die zeitliche Verteilung von Klingenformen der Auswertungsgruppe 3 in Ostdeutschland (grob datierter Bestand) 


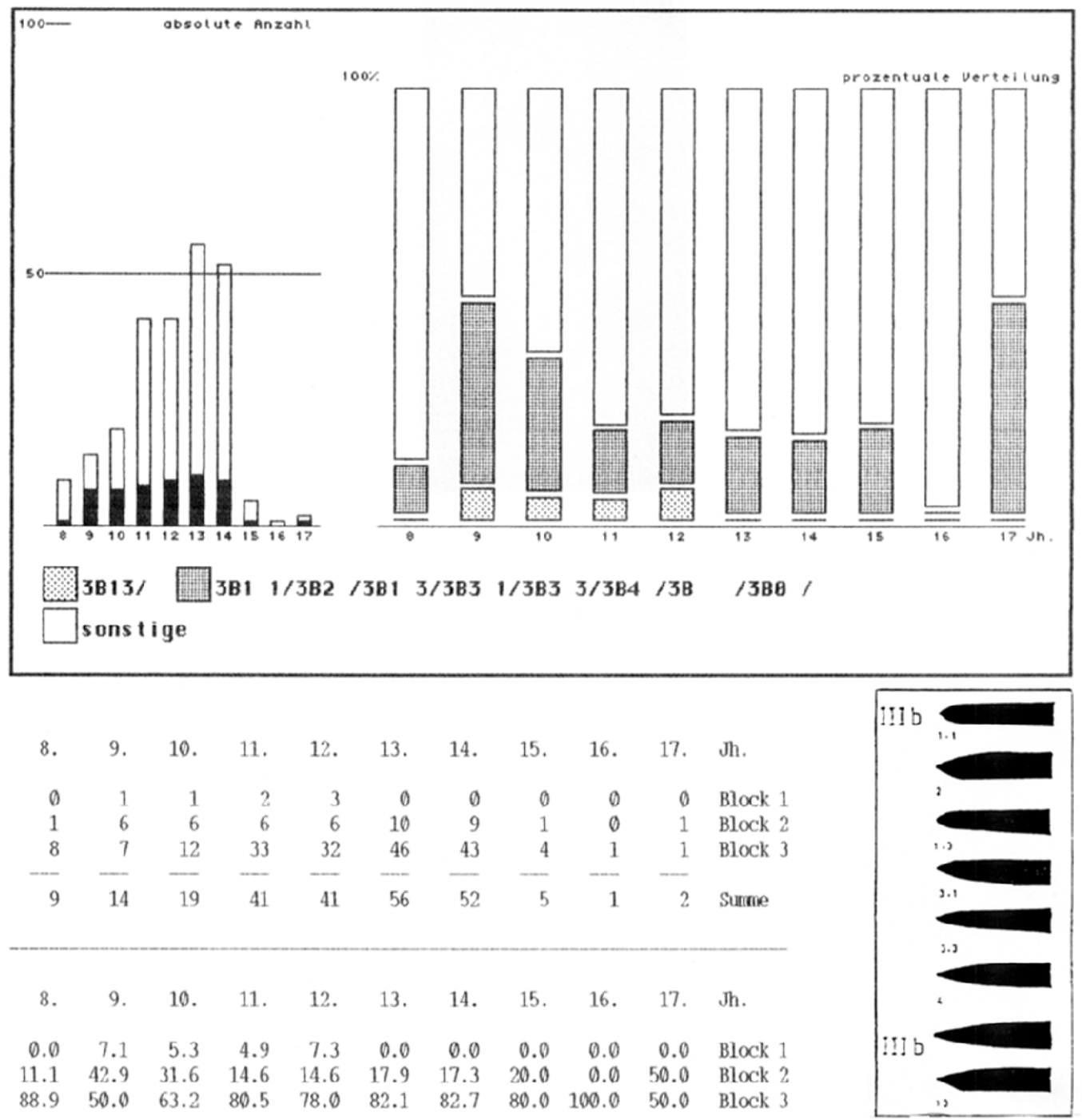

Abb. 41

Die zeitliche Verteilung von Klingenformen der Auswertungsgruppe 3 in Polen (grob datierter Bestand) 


\subsubsection{Die Verteilung der Klingenformen IIlb (Auswertungsgruppe 4)}

Messerklingen mit gebogenen Kanten und abgestumpften oder abgeschrägten Spitzen sind im ausgewerteten Messerbestand extrem selten aufgetreten.

In der Auswertung des zeitlich enger gefaßten Bestandes, die aufgrund der exakteren Datierungsgrundlage zur Kontrolle der übrigen Ergebnisse grundsätzliche Bedeutung hat, fielen von 526 Messern nur zwei Exemplare des 10. bzw. 15. Jahrhunderts in diese Gruppe. Aussagen zur Verteilung sind daher nicht möglich.

\subsubsection{Die Verteilung der Klingenformen IIIb (Auswertungsgruppe 6)}

Erstaunlicherweise ließen sich für diese Formen Verteilungstendenzen erkennen, obwohl der einzige Unterschied zu den Formen der Auswertungsgruppe 4 in der umgekehrten Abschrägung der Spitze liegt. Auch hier steht das Gesamtaufkommen der untersuchten Formen IIIb33 und IIIb34 in keinem Verhältnis zur Menge des Restbestandes. 10 Exemplare aus dem zeitlich enger gefaßten Auswertungsbestand belegen aber das sporadische Vorkommen dieser Formen vom 11. Bis 16. Jahrhundert, welches im 14. Jahrhundert einen Wert von $6 \%$ erreicht (Abb. 42).

Die Auswertung des zeitlich weiter gefaßten Bestandes von 30 Belegen bestätigt in etwa dieses Bild (Abb. 43):

Klingenformen der Auswertungsgruppe 6 sind vor dem 10. Jahrhundert nicht aufgetreten. Sie scheinen im 13. bis 14. Jahrhundert eine etwas größere Verbreitung erfahren zu haben, in den übrigen Jahrhunderten sind sie nur selten belegbar.

Weder zur Funktion dieser Klingenformen noch zu ihrer spätmittelalterlichen und neuzeitlichen Zunahme im Fundgut gibt es Informationen, die Interpretationsansätze ermöglichen. 


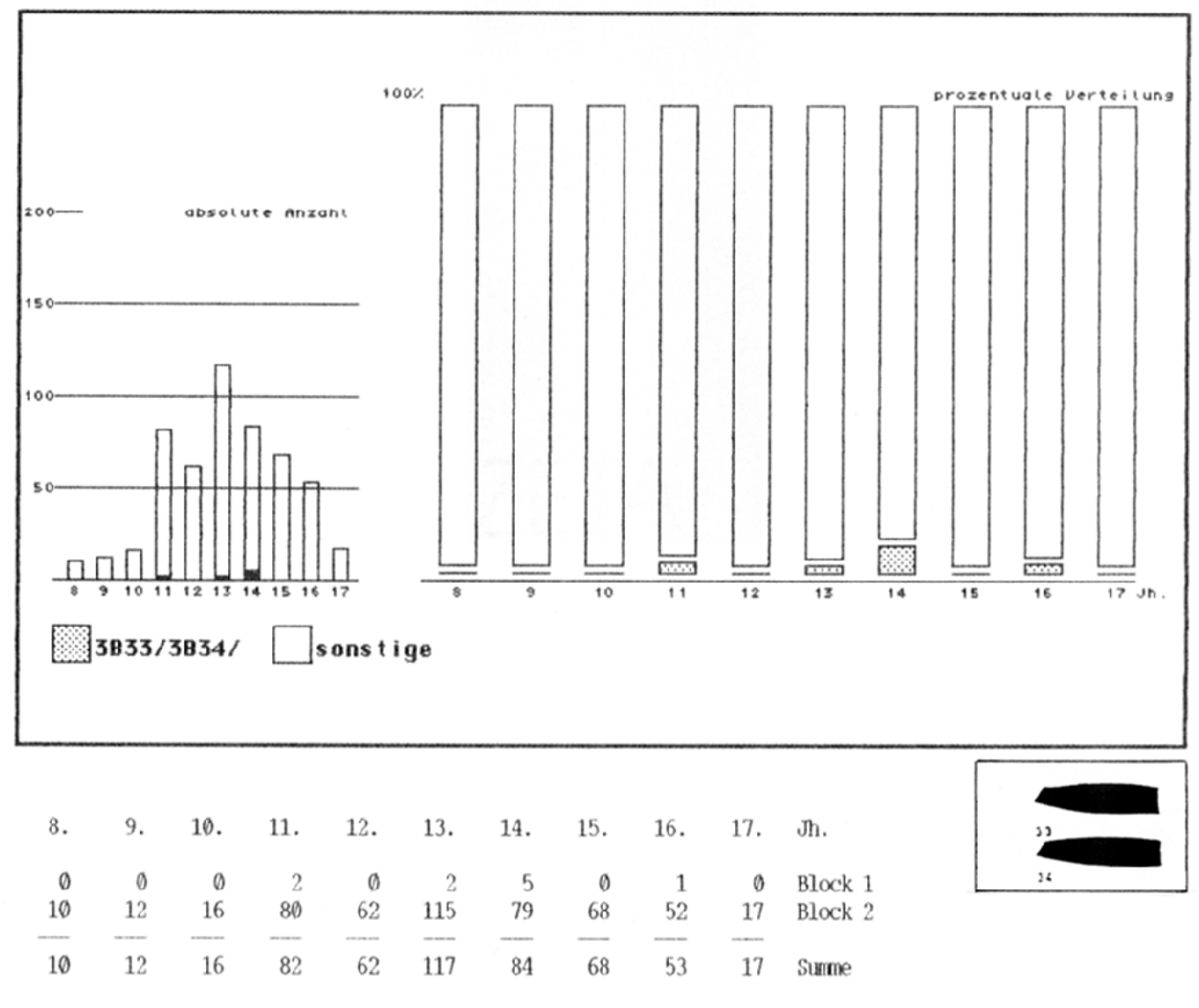

8. 9. 10. 11. 12. 13. 14. 15. 16. $17 . \mathrm{Jh}$.

$\begin{array}{lllllllllll}0.0 & 0.0 & 0.0 & 2.4 & 0.0 & 1.7 & 6.0 & 0.0 & 1.9 & 0.0 & \text { Block } 1\end{array}$ $\begin{array}{lllllllllll}100.0 & 100.0 & 100.0 & 97.6 & 100.0 & 98.3 & 94.0 & 100.0 & 98.1 & 100.0 & \text { Block } 2\end{array}$

$A b b .42$

Die zeitliche Verteilung von Klingenformen der Auswertungsgruppe 6 im gesamten Untersuchungsraum (enger datierter Berstand) 


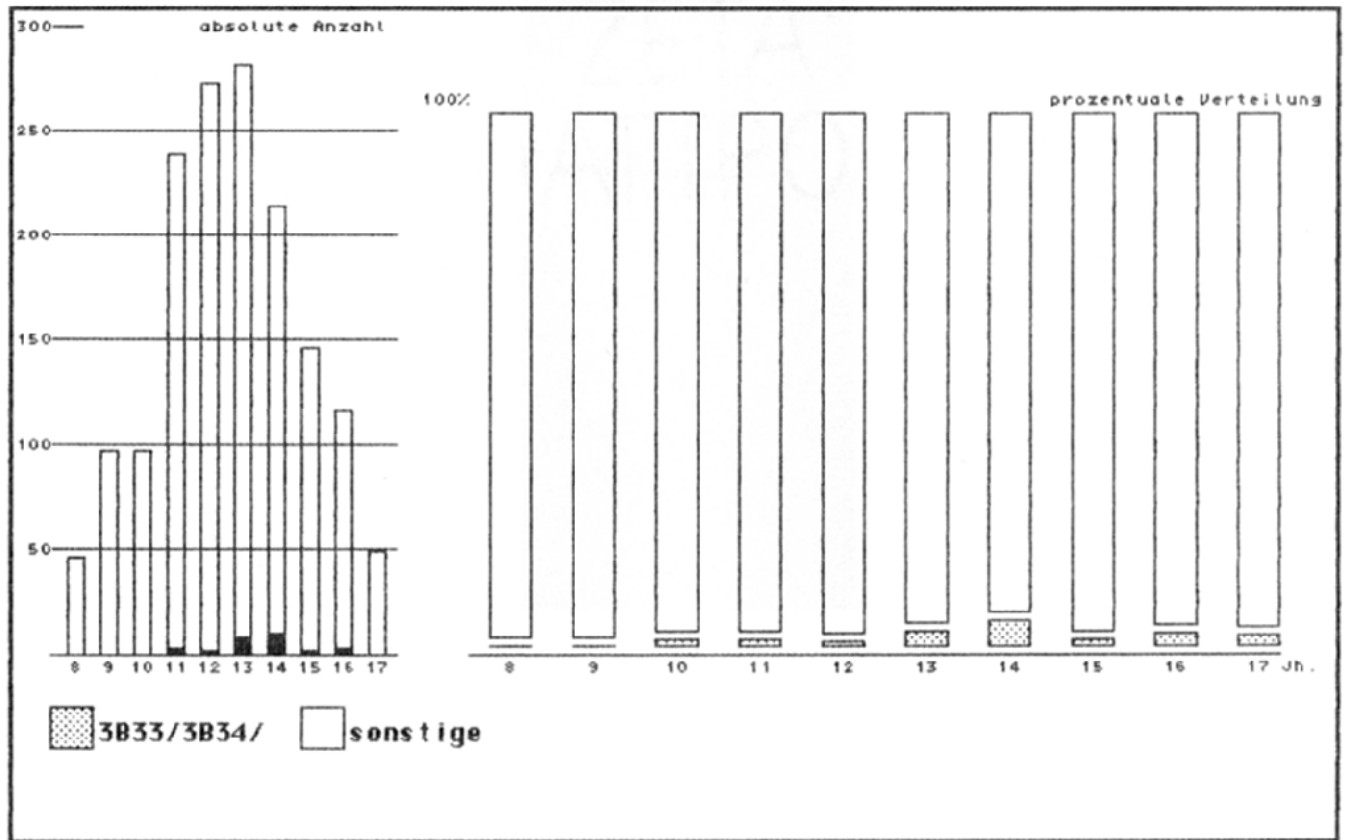

\begin{tabular}{rrrrrrrrrrl}
8. & 9. & 10. & 11. & 12. & 13. & 14. & 15. & 16. & 17. & Jh. \\
0 & 0 & 1 & 3 & 2 & 8 & 10 & 2 & 3 & 1 & Block 1 \\
46 & 97 & 96 & 236 & 270 & 273 & 204 & 144 & 113 & 48 & Block 2 \\
\hline 46 & $\frac{9}{97}$ & $\frac{1}{97}$ & $\frac{2}{23}$ & $\frac{272}{281}$ & $\frac{1}{214}$ & $\frac{146}{116}$ & $\frac{49}{49}$ & Sume
\end{tabular}

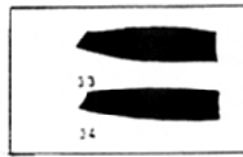

8. 9. 10. 11. 12. 13. 14. 15. 16. 17. Jh.

$\begin{array}{rrrrrrrrrrr}0.0 & 0.0 & 1.0 & 1.3 & 0.7 & 2.8 & 4.7 & 1.4 & 2.6 & 2.0 & \text { Block 1 }\end{array}$

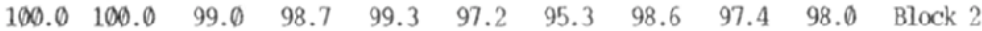

$A b b .43$

Die zeitliche Verteilung von Klingenformen der Auswertungsgruppe 6 im gesamten Untersuchungsraum (grob datierter Bestand) 


\subsubsection{Die Verteilung der Klingenformen IIIb (Auswertungsgruppe 5)}

In dieser Gruppe wurden Klingen mit den Codebezeichnungen IIIb1, IIIb1.2, IIIlb3 und IIIb14 sowie mit den Bezeichnungen IIIb15 und IIIb17 getrennt voneinander untersucht. Die Form IIIb30 ist nicht aufgetreten.

Die ersten vier Formen umfassen Klingen mit gerade verlaufendem Rücken, der vor der Spitze konvex gebogen einfällt. Die Schneide kann gerade oder gebogen verlaufen, der Gefahr einer möglichen Verfälschung der Ergebnisse durch Verschliffstadien wurde damit Rechnung getragen.

Bei den beiden letzten Formen biegt der Rücken nicht gebogen, sondern gerade zur Spitze ein.

Das Auswertungsdiagramm des zeitlich engeren Messerbestandes zeigt im aussagefähigen Zeitraum einen Anstieg dieser Formengruppe nach dem 14. Jahrhundert, die Hauptverbreitungszeit der Klingenformen IIIb15 und IIIb17 scheint vor dem 13. Jahrhundert zu liegen (Abb. 44), im 17. Jahrhundert steigt ihr Anteil wieder auf $5,9 \%$.

In der zweiten Graphik bestätigen sich diese Tendenzen (Abb. 45):

Die prozentuale Verteilung der gesamten Gruppe fällt vom 8. bis zum 14. Jahrhundert regelmäßig auf $5,2 \%$ ab und steigt bis zum 17. Jahrhundert wider auf $18,4 \%$ des gesamten Auswertungsbestandes an. Die Klingen des Elbfunds von Wittenbergen wurden wegen ihres Exportcharakters für Übersee nicht berücksichtigt.

Die Klingenformen IIIb15 und IIIb17 sind hauptsächlich vom 9. bis 12. und im 17. Jahrhundert mit Werten zwischen 4,1\% und 7,2\% verbreitet.

Weitere Differenzierungsmöglichkeiten zu den beiden grundsätzlichen Klingenformen sind im Gegensatz zu den in der Auswertungsgruppe 3 zusammengefaßten Formen weniger wahrscheinlich, da die Unterschiede hier den Schneidenverlauf betreffen.

Die Veranschaulichung der anteiligen Verteilung in den einzelnen Untersuchungsländern zeigt innerhalb der aussagefähigen Zeiträume ähnliche Tendenzen.

Unterschiede bestehen in Schwerpunktveränderungen, aber auch in einigen Tendenzabweichungen:

In Skandinavien setzt der beobachtete Rückgang erst nach dem 11. Jahrhundert ein, in welchem die Formen IIIb15 und IIIb17 über 18\% einnehmen. Im 13. Jahrhundert wurden die Formen der Auswertungsgruppe 5 nicht angetroffen (Abb. 46).

In den Niederlanden sind derartig geformte Klingen nach einem Beleg aus Dorestad erst seit dem 14. Jahrhundert nachgewiesen worden (Abb. 47). 


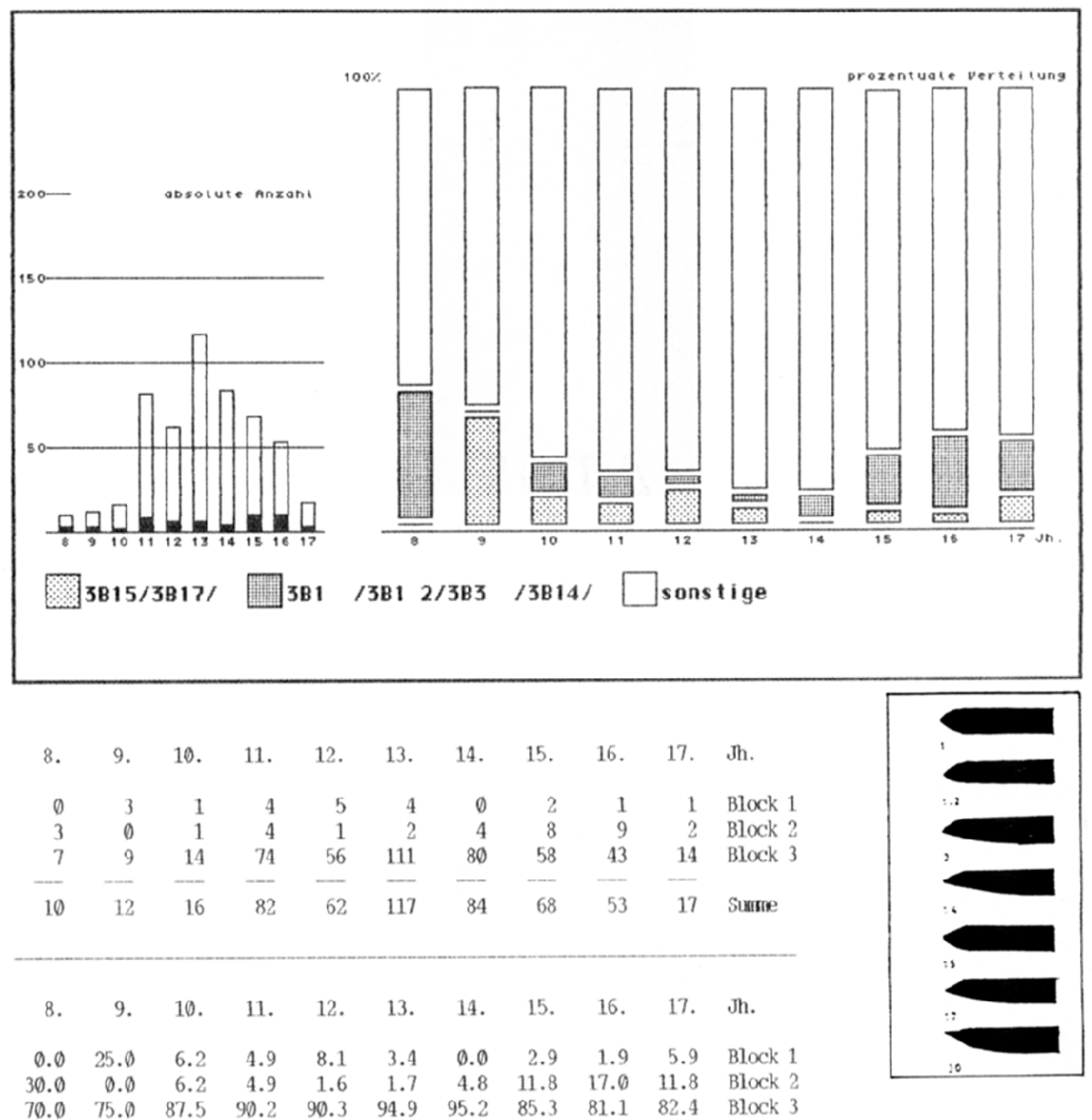

Abb. 44

Die zeitliche Verteilung von Klingenformen der Auswertungsgruppe 5 im gesamten Untersuchungsraum (enger datierter Berstand) 


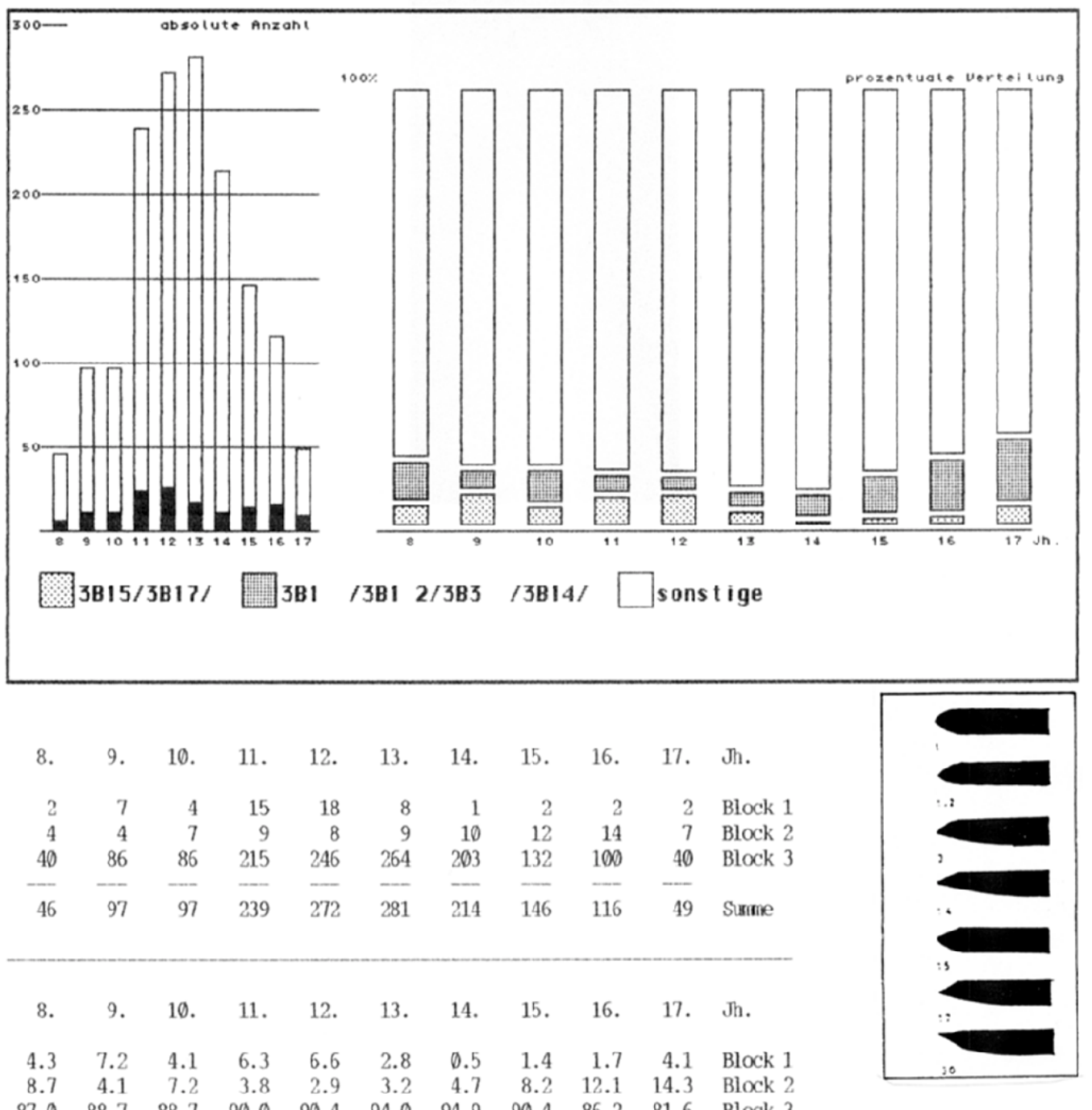

$87.0 \quad 88.7 \quad 88.7 \quad 90.0 \quad 99.4 \quad 94.0 \quad 94.9-90.4 \quad 86.2 \quad 81.6 \quad$ Block 3

Abb. 45

Die zeitliche Verteilung von Klingenformen der Auswertungsgruppe 5 im gesamten Untersuchungsraum (grob datierter Bestand) 
In der Bundesrepublik verteilen sich beide Gruppen mit unterschiedlichen Schwerpunkten vom 10. (9.) Jahrhundert abfallend bis zum 14. Jahrhundert.

Im Nordwesten scheint der Schwerpunkt der Formen IIIb15 und IIIb17 klar vor dem 11. Jahrhundert zu liegen (15,4\% im 10. Jahrhundert), nach dem 13. Jahrhundert tritt sie jedoch nicht mehr auf (Abb. 48). Die übrigen Formen steigen vom 14. bis zum 17. Jahrhundert auf $22,2 \%$ des Gesamtbestandes an (ebenfalls ohne die Messer des Wittenberger Elbfundes).

Im Osten der Bundesrepublik (neue Bundesländer) nimmt der Gesamtanteil der ausgewerteten Formen regelmäßig vom 9. bis 14. Jahrhundert ab, die Formen IIIb15 und IIIb17 sind hier auch nach dem 10. Jahrhundert mit Anteilen von bis zu 9,8\% beobachtet worden (Abb. 49).

In den Beständen aus den polnischen Fundorten wurde ein in etwa gleichbleibender Prozentsatz vom 10. bis zum 12. Jahrhundert festgestellt, der adäquat zur Verteilung im gesamten Untersuchungsraum und $\mathrm{zu}$ den beiden deutschen Untersuchungsgebieten zum 14. Jahrhundert abfällt (Abb. 50). 


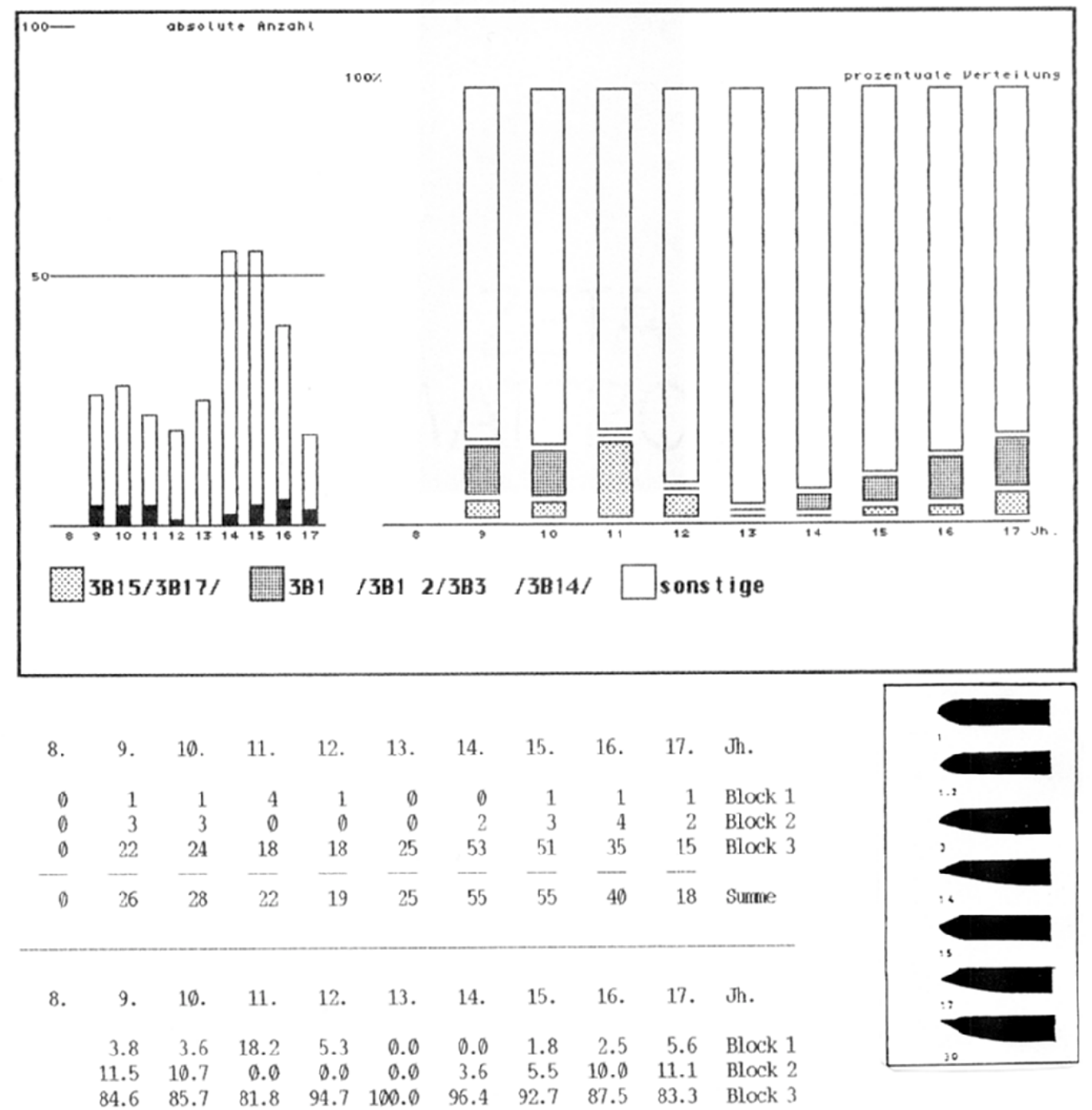

Abb. 46

Die zeitliche Verteilung von Klingenformen der Auswertungsgruppe 5 in Skandinavien (grob datierter Bestand) 


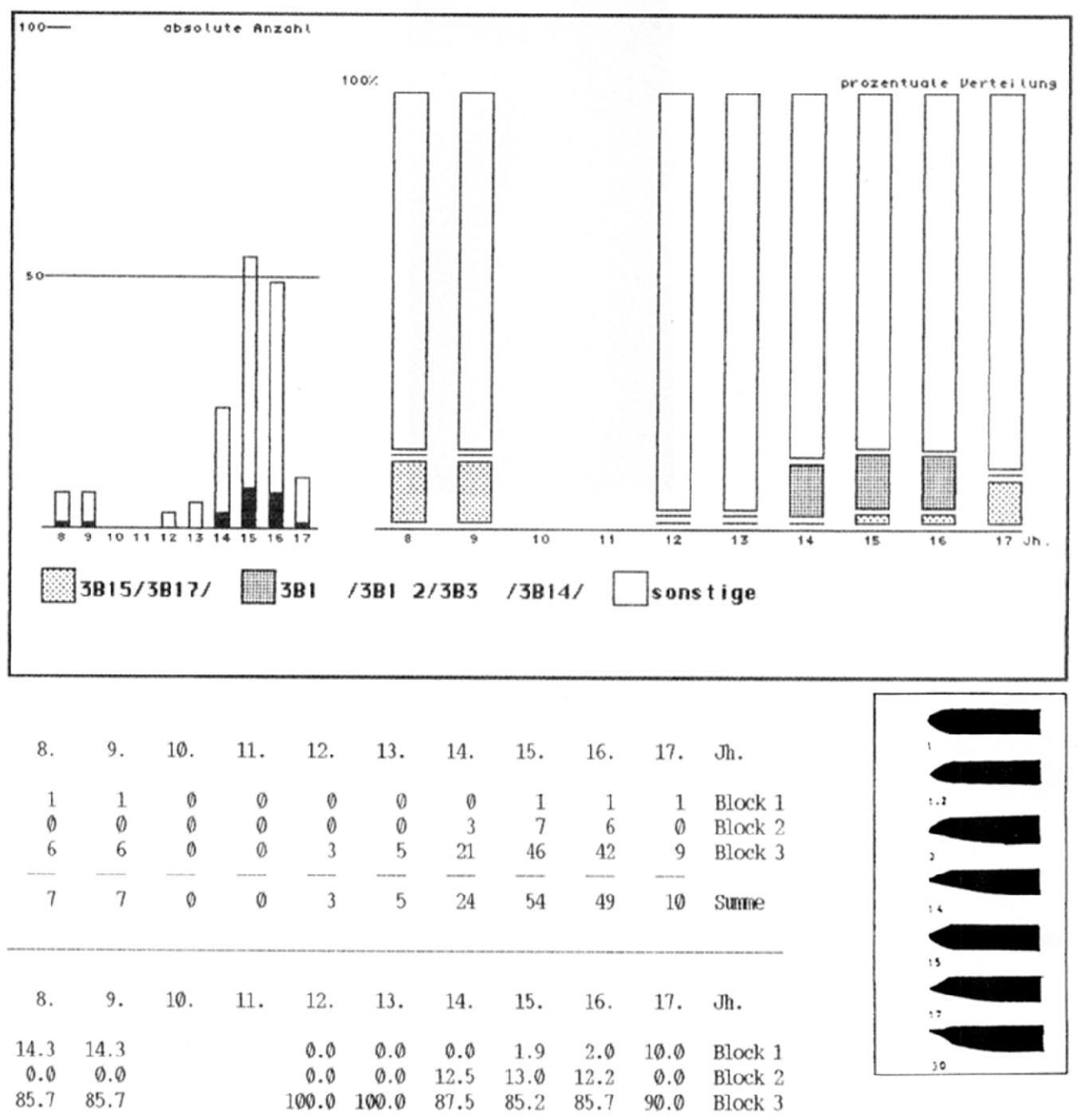

Abb. 47

Die zeitliche Verteilung von Klingenformen der Auswertungsgruppe 5 in den Niederlanden (grob datierter Bestand) 


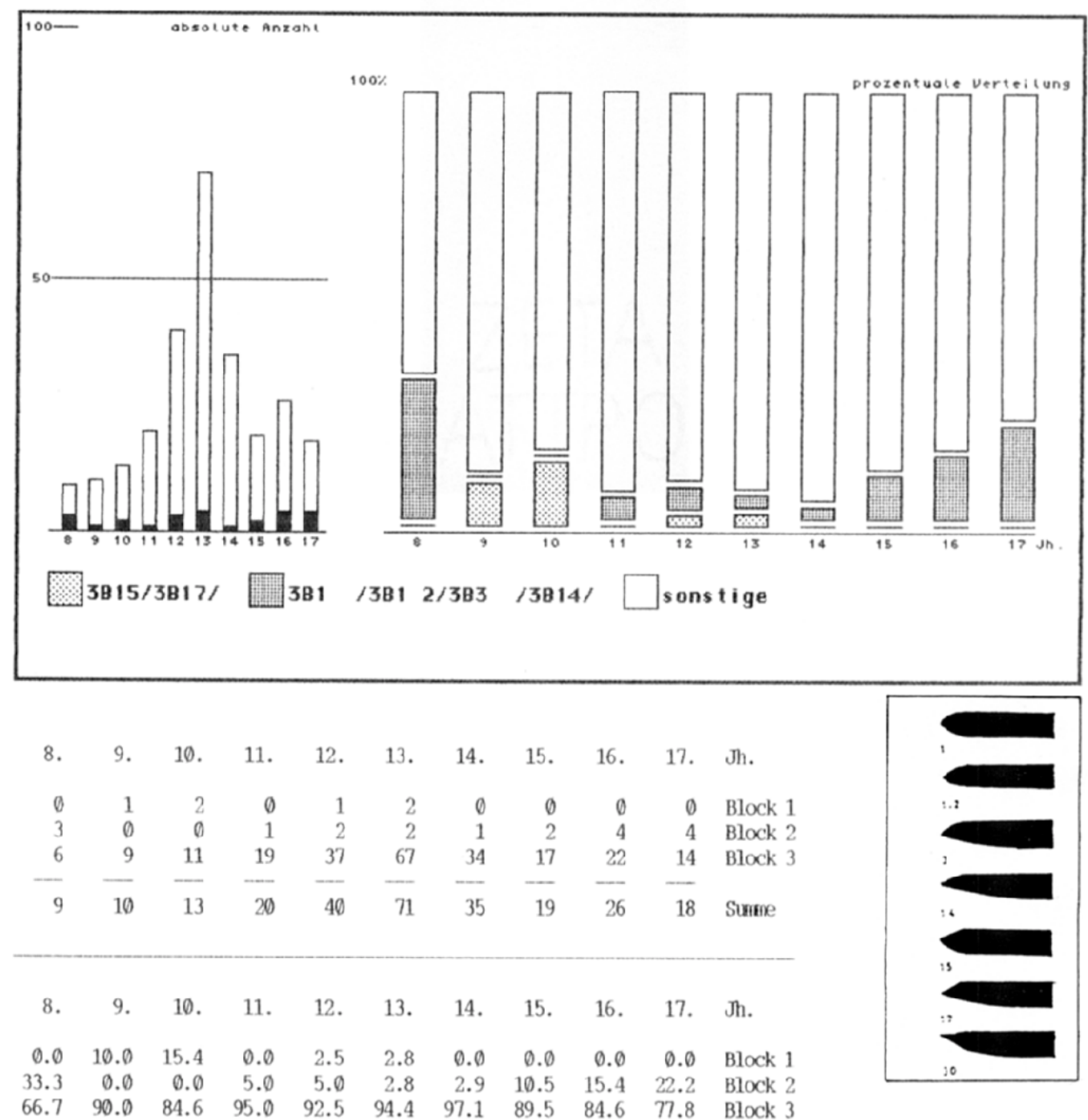

Abb. 48

Die zeitliche Verteilung von Klingenformen der Auswertungsgruppe 5 in Nordwestdeutschland (grob datierter Bestand) 


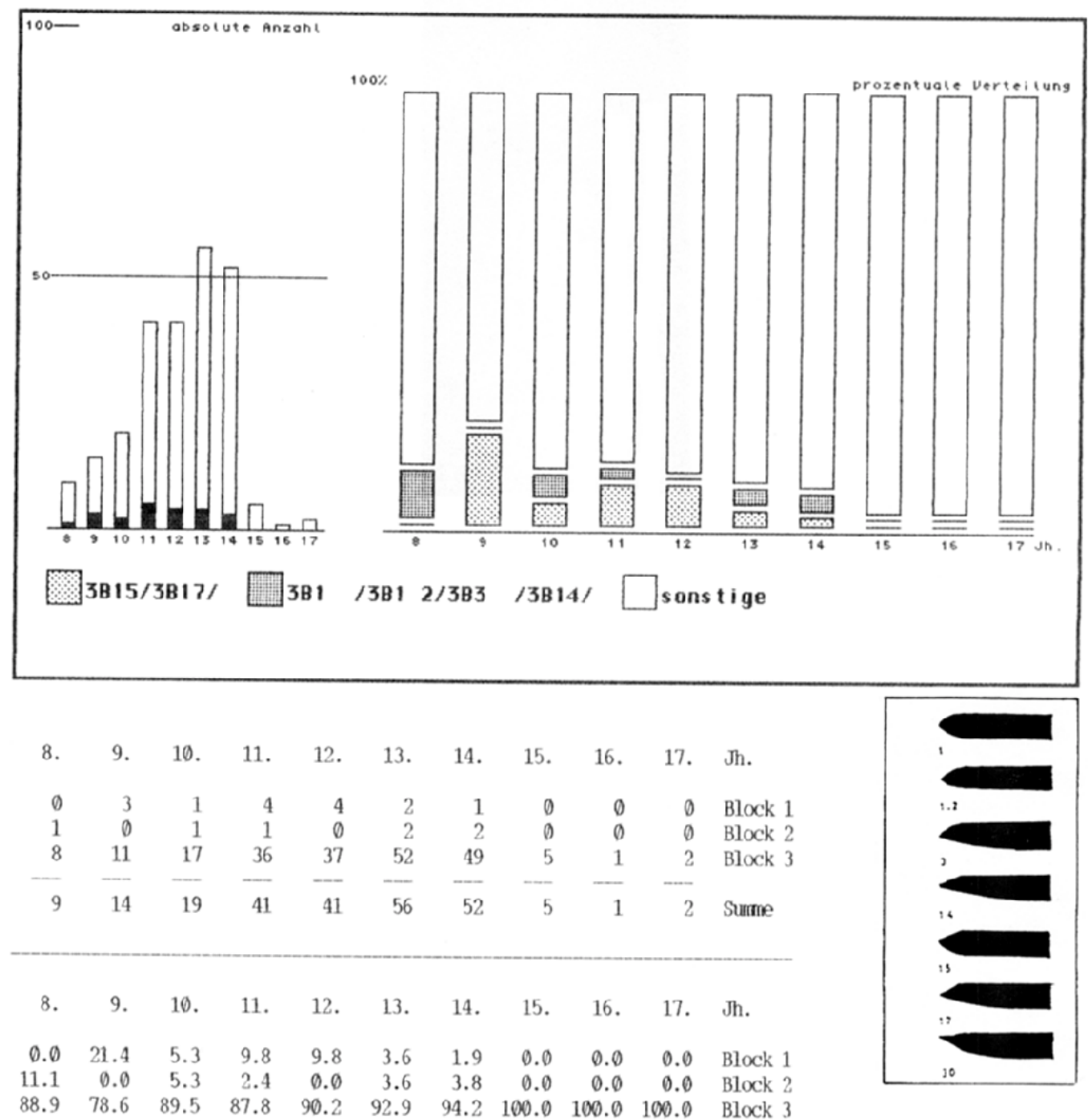

Abb. 49

Die zeitliche Verteilung von Klingenformen der Auswertungsgruppe 5 in Ostdeutschland (grob datierter Bestand) 


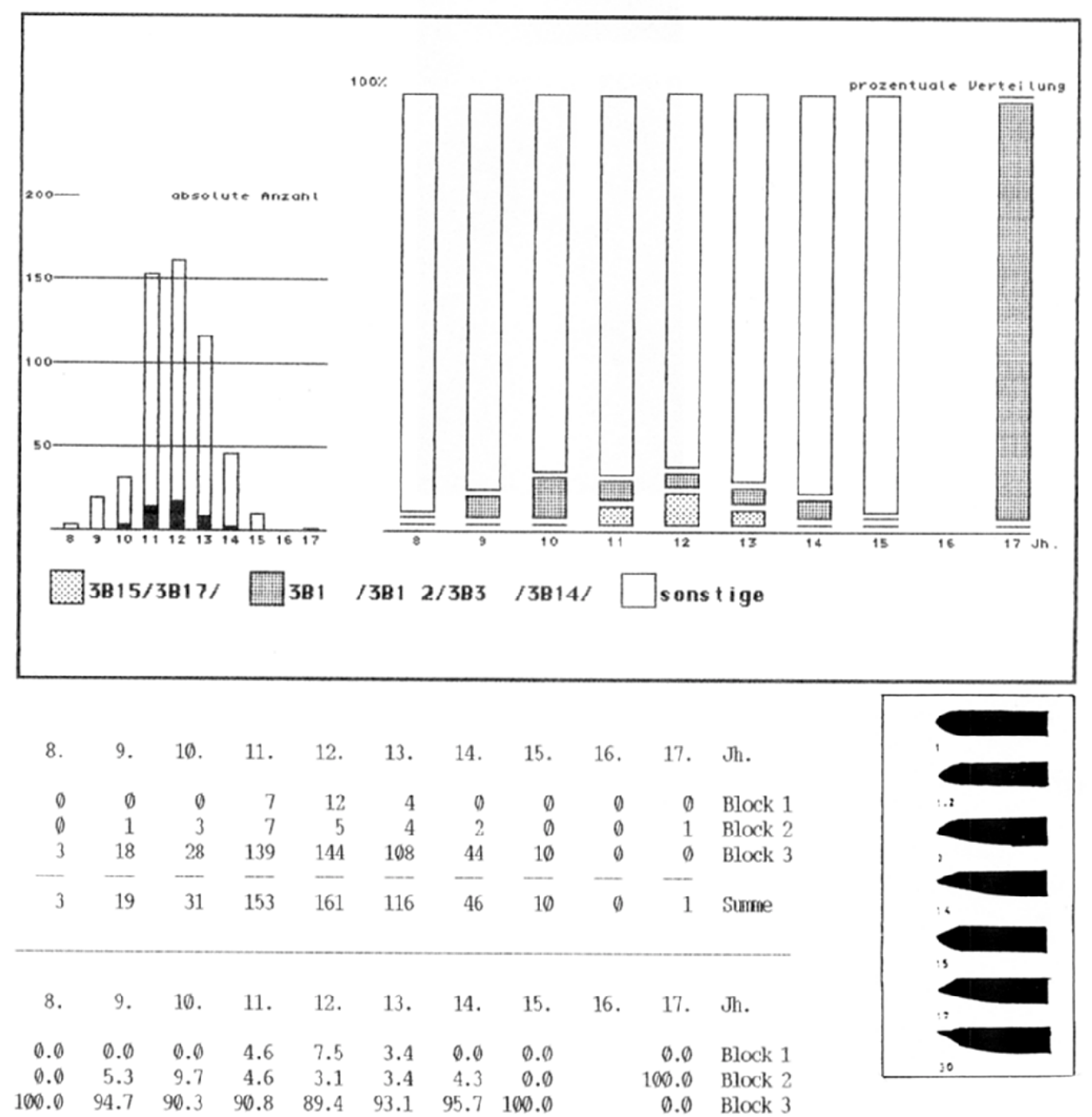

Abb. 50

Die zeitliche Verteilung von Klingenformen der Auswertungsgruppe 5 in Polen (grob datierter Bestand) 


\subsubsection{Die Verteilung der Klingenformen IIIb (Auswertungsgruppe 7)}

In dieser Gruppe wurde die Verteilung von Messern untersucht, deren Rücken über mehr als die Hälfte der Klingenlänge gerade abgeschrägt zur Spitze einfallen, wobei auch hier der Schneidenverlauf unberücksichtigt blieb (IIIb16 und IIIb18).

Die Form IIIb31 ist im bearbeiteten Bestand nicht aufgetreten.

Die in ihrem Erscheinungsbild auffälligen Klingenformen wurden nur sehr selten im bearbeiteten Messerbestand angetroffen. 14 Belege waren zeitlich enger zu begrenzen, 11 weitere fielen in die zweite Untersuchungsgruppe.

Die Auswertung des gesamten Bestandes dieser Formen zeigt eine leicht unregelmäßige, aber insgesamt beständig abnehmende Tendenz vom 8. zum 14. Jahrhundert (Abb. 51). Das vereinzelte Auftreten einer Klinge mit der Form IIlb16.5 im 16. Jahrhundert betrifft ein Messer aus Dordrecht (Kat. Nr. 34) und belegt die bereits mehrfach beobachtete Auf- oder Zunahme älterer Formen im 15. bis 17. Jahrhundert.

Die Graphik zur Verteilung im Rahmen des zeitlich enger begrenzten Bestandes zeigt neben dem Rückgang vom 8. zum 11. Jahrhundert einen Anstieg von 1,2\% im 11. auf 3,6\% im 14. Jahrhundert. Auch hier wird das Exemplar des 16. Jahrhunderts erfaßt (Abb. 52).

Die in dieser Gruppe behandelten Formen können formal mit vormittelalterlichen Saxklingen verglichen werden, die in der Literatur mehrfach als Vorbilder für die kleineren und zum Teil jüngeren Messerklingenformen angesehen worden sind.

Die hier beobachtete Tendenz mit Anteilen im 8. und 9. Jahrhundert, die später nicht mehr erreicht werden, belegt zumindest, daß es Klingenformen gab, die in der Karolingerzeit ihre Hauptverbreitungszeit hatten und im Mittelalter langsam ausliefen. 


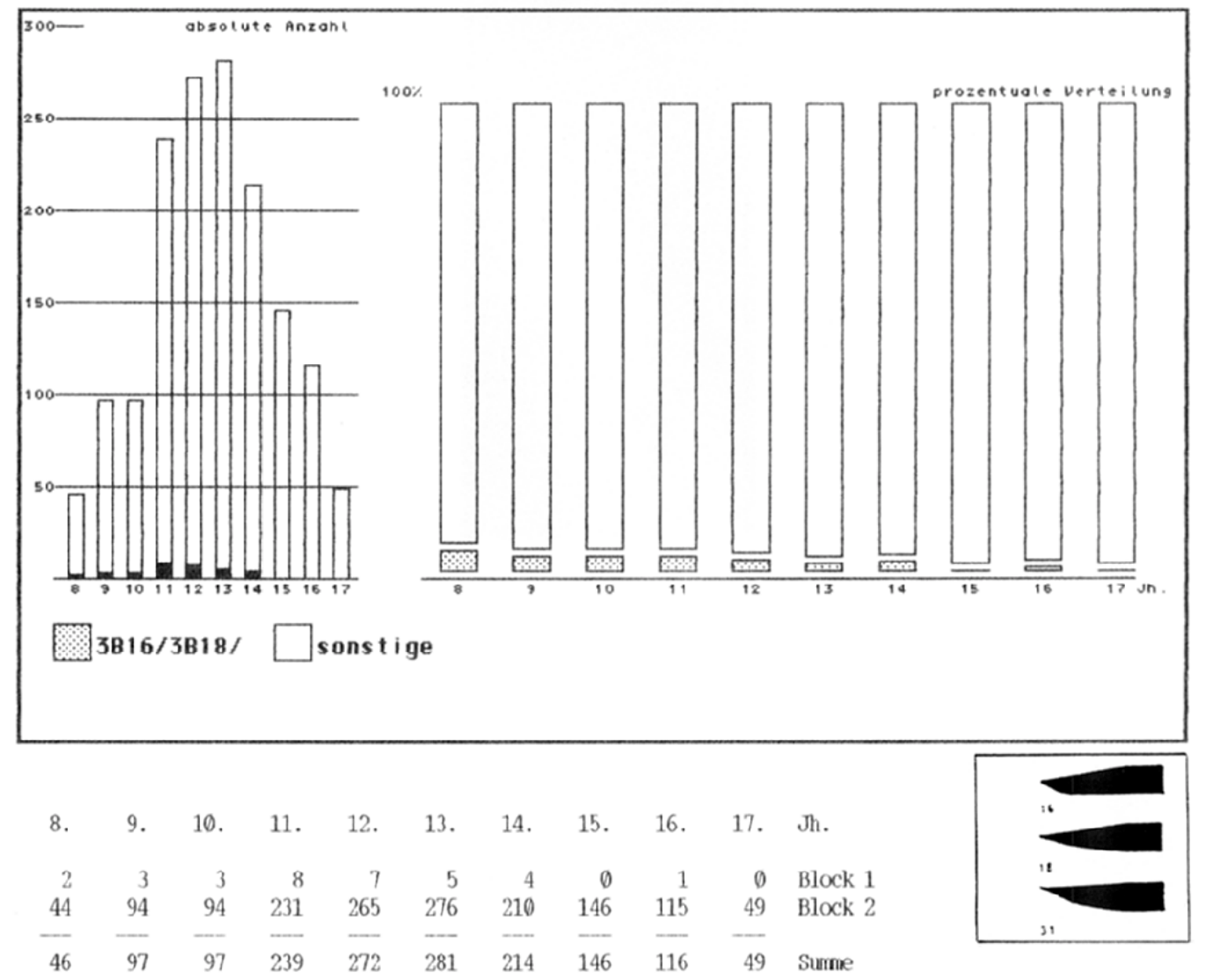

8. 9. 10. 11. 12. 13. 14. 15. 16. 17. Jh.

$\begin{array}{lllllllllll}4.3 & 3.1 & 3.1 & 3.3 & 2.6 & 1.8 & 1.9 & 0.0 & 0.9 & 0.0 & \text { Block 1 }\end{array}$

$\begin{array}{lllllllllll}95.7 & 96.9 & 96.9 & 96.7 & 97.4 & 98.2 & 98.1 & 100.0 & 99.1 & 100.0 & \text { Block } 2\end{array}$

$A b b .51$

Die zeitliche Verteilung von Klingenformen der Auswertungsgruppe 7 im gesamten Untersuchungsraum (grob datierter Bestand) 


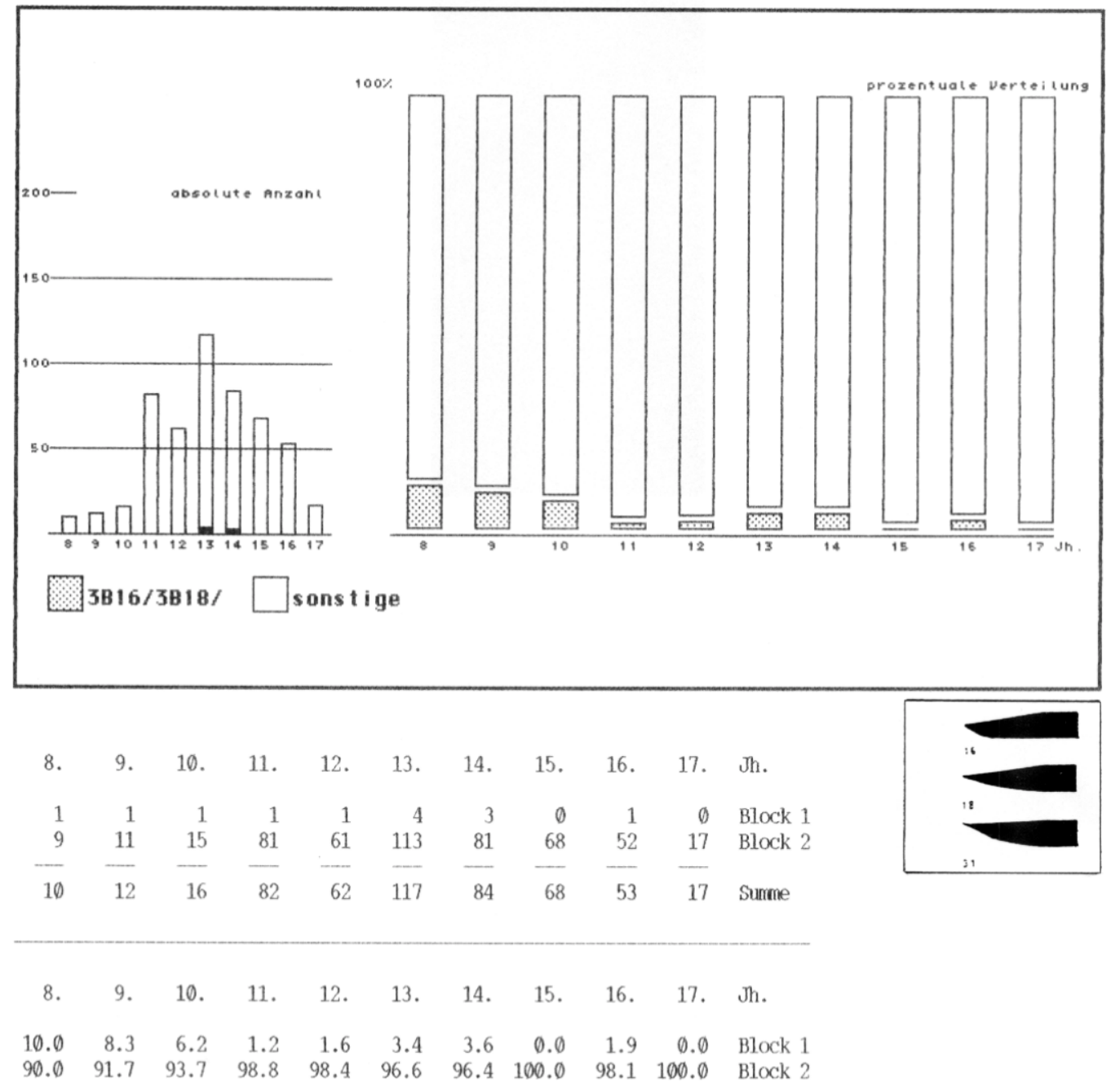

Abb. 52

Die zeitliche Verteilung von Klingenformen der Auswertungsgruppe 7 im gesamten Untersuchungsraum (enger datierter Berstand) 


\subsubsection{Abschließende Bemerkungen zu den Verteilungstendenzen der Klingenformen IIIb}

Die in den einzelnen Auswertungsgruppen zusammengefaßten Klingenformen verteilen sich sowohl quantitativ als auch chronologisch unterschiedlich im ausgewerteten Fundgut.

Gewisse Tendenzen und Abschnitte wiederholen sich dagegen, so daß von Zeitabschnitten gesprochen werden kann, die die Messer der Formengruppen IIIb unabhängig von formalen Unterschieden in Entwicklungsstufen zu gliedern scheint.

Die bisher behandelten Messer unter der Leitform IIIb machen insgesamt im Durchschnitt 38\% des gesamten untersuchten Messerbestandes aus.

Im zeitlich enger datierten Bestand nimmt ihr Gesamtanteil von 60\% im 8. auf $31,7 \%$ im 11. Jahrhundert regelmäßig ab und bleibt bis zum 15. Jahrhundert relativ konstant. Im 16. Jahrhundert steigt ihr Anteil noch einmal auf über $60 \%$ an und geht im 17. Jahrhundert auf 23.5\% wieder zurück (Abb. 53).

Die Auswertung .des umfangreicheren grob datierten Bestandes zeigt einen Anstieg vom 8. zum 9. Jahrhundert und stellt den Rückgang zum 11. Jahrhundert weniger gleichmäßig dar. Ansonsten sind die Tendenzen gleich (Abb. 54).

Signifikante Tendenzveränderungen wurden bei der Verteilung der Auswertungsgruppen, die die meisten Exemplare auf sich vereinigen, nach dem 10. (Gruppe 3) und nach dem 14. Jahrhundert (Gruppen 3 und 6) festgestellt, während sich bei den seltenen Formen ein unregelmäßiger Anstieg vor und ein unregelmäßiger Abfall nach dem 14. Jahrhundert (IIIb33, IIIb34) bzw. ein unregelmäßiger Rückgang vom 8. bis zum 14. Jahrhundert (IIIb16, IIIb18) ergab.

Davon unberührt bleibt die Möglichkeit, daß ältere und auch bereits vorher ausgelaufene Formen nach dem Mittelalter wieder aufgegriffen oder neu installiert wurden.

Die Tendenzveränderungen nach dem 10. und 14. Jahrhundert können sich unterschiedlich auswirken. Sie können Rückgang oder Zunahme beinhalten und sind, obwohl sie hier lediglich formale Veränderungen anzeigen, auch als Indikatoren für funktionale Veränderungen zu bewerten ${ }^{58}$.

Die sich daraus ergebenden Zeitabschnitte teilen den Untersuchungszeitraum in drei Teile:

In die Zeit vor dem 11. Jahrhundert, vom 11. bis zum 14. Jahrhundert und nach dem 14. Jahrhundert.

Die den Zeitabschnitt vor dem 11. Jahrhundert betreffenden Ergebnisse sind mit Zurückhaltung zu betrachten, da vor allem das 8. und 9. Jahrhundert im Verhältnis zu den nachfolgenden Jahrhunderten unterrepräsentiert sind. Dies betrifft vor allem beobachtete Tendenzveränderungen nach dem 8 . Jahrhundert, die zwar historisch 
wahrscheinlich, aber mit dem vorgelegten Material allein nicht ausreichend belegbar sind.

Im Hoch- und Spätmittelalter sind in der Regel unterschiedliche Entwicklungen zu beobachten, die sich weder im Vergleich der überregionalen Auswertungen zu den einzelnen Formengruppen noch im Vergleich der in den einzelnen Untersuchungsländern festgestellten Unterschiede zusammenfassen lassen.

In der frühen Neuzeit wurde in den meisten Fällen ein deutlicher Anstieg der untersuchten Formen festgestellt, der sich nicht immer bis in das 17. Jahrhundert fortsetzt und sich vereinzelt auch als kurzlebiges Aufgreifen älterer Formen zu dokumentieren scheint.

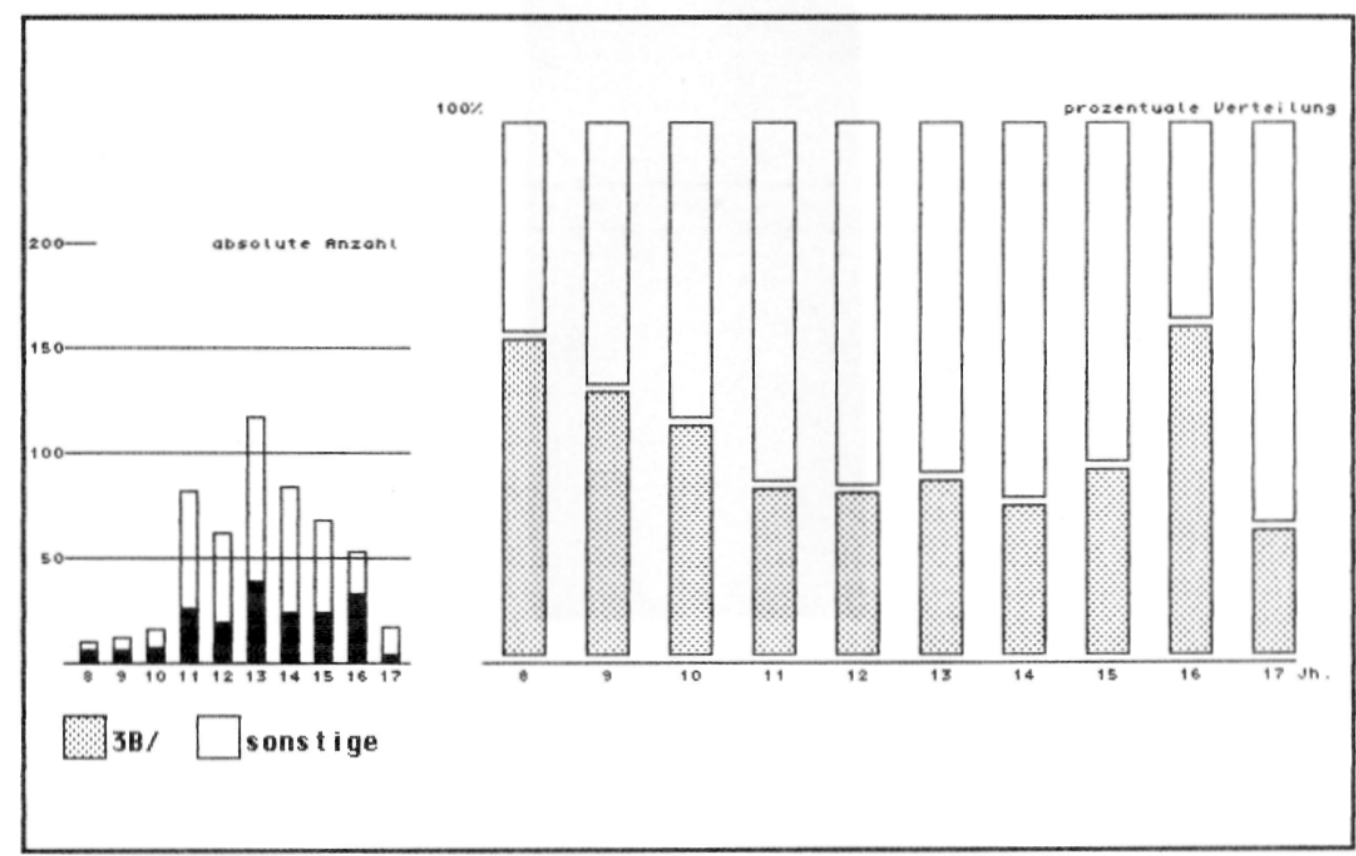

\begin{tabular}{|c|c|c|c|c|c|c|c|c|c|c|}
\hline 8. & 9. & 10. & 11. & 12. & 13. & 14. & 15. & 16. & 17. & $\mathrm{~h}$. \\
\hline 6 & 6 & 7 & 26 & 19 & 39 & 24 & 24 & 33 & 4 & Block 1 \\
\hline 4 & 6 & 9 & 56 & 43 & 78 & 60 & 44 & 20 & 13 & Block 2 \\
\hline - & - & - & - & -- & - & - & - & - & - & \\
\hline 10 & 12 & 16 & 82 & 62 & 117 & 84 & 68 & 53 & 17 & Summe \\
\hline
\end{tabular}

\footnotetext{
8. 9. 10. 11. 12. 13. 14. 15. 16. $17 . \quad \mathrm{Jh}$.

$\begin{array}{lllllllllll}60.0 & 50.0 & 43.8 & 31.7 & 30.6 & 33.3 & 28.6 & 35.3 & 62.3 & 23.5 & \text { Block 1 }\end{array}$

$\begin{array}{lllllllllll}40.0 & 50.0 & 56.2 & 68.3 & 69.4 & 66.7 & 71.4 & 64.7 & 37.7 & 76.5 & \text { Block } 2\end{array}$
} gesamten Untersuchungsraum (enger datierter Berstand) 


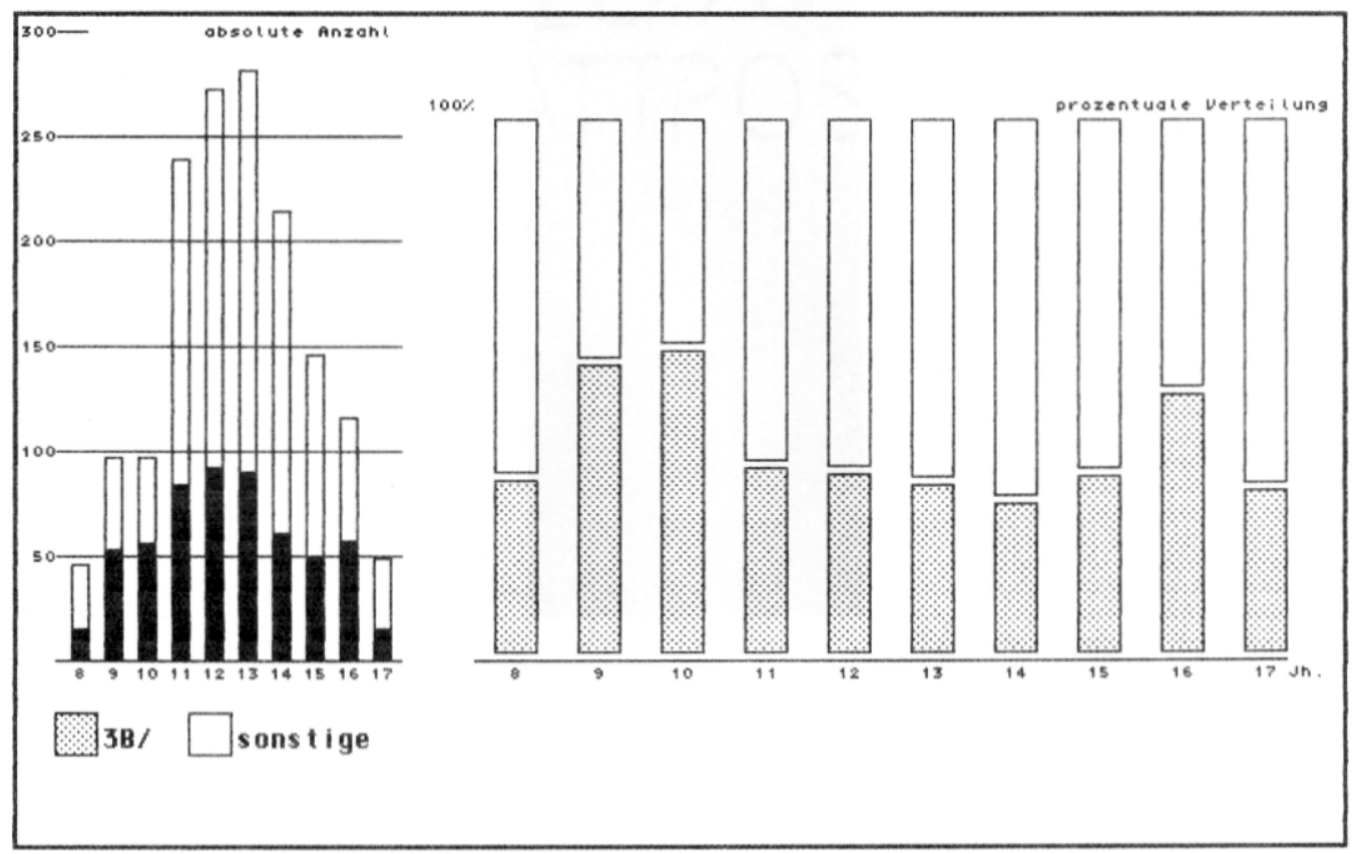

$\begin{array}{rrrrrrrrrrl}8 . & 9 . & 10 . & 11 . & 12 . & 13 . & 14 . & 15 . & 16 . & 17 . & \text { Jh. } \\ 15 & 53 & 56 & 84 & 92 & 90 & 61 & 49 & 57 & 15 & \text { Block 1 } \\ \frac{31}{46} & \frac{44}{97} & \frac{41}{97} & \frac{155}{239} & \frac{180}{272} & \frac{191}{281} & \frac{153}{214} & \frac{97}{146} & \frac{59}{116} & \frac{34}{49} & \text { Block 2 } \\ & & & \end{array}$

8. 9. 10. 11. 12. 13. 14. 15. 16. $17 . \quad \mathrm{h}$.

$\begin{array}{lllllllllll}32.6 & 54.6 & 57.7 & 35.1 & 33.8 & 32.0 & 28.5 & 33.6 & 49.1 & 30.6 & \text { Block } 1\end{array}$

$\begin{array}{lllllllllll}67.4 & 45.4 & 42.3 & 64.9 & 66.2 & 68.0 & 71.5 & 66.4 & 50.9 & 69.4 & \text { Block 2 }\end{array}$

Abb. 54

Die zeitliche Verteilung aller unter IIIb zusammengefassten Klingenformen im gesamten Untersuchungsraum (grob datierter Bestand) 


\subsubsection{Die Verteilung der Klingenformen Ille (Auswertungsgruppe 8)}

Die in der Auswertungsgruppe 8 zusammengefaßten Messer kennzeichnet ein konkav gebogener Einsprung des Rückens zur Spitze, der weniger als die Hälfte der Klingenlänge ausmacht. Die aufgrund dieses Kriteriums hier mitbehandelte Form $\mathrm{Vg}$ ist im Fundgut nicht beobachtet worden.

Die Auswertung des zeitlich enger gefaßten Bestandes ergab 38 Exemplare dieser Formengruppe, die sich zeitlich zwischen dem 10. und 14. Jahrhundert verteilen (Abb. 55).

Der Beleg des 16. Jahrhunderts betrifft ein niederländisches Messer, dessen Klinge stark ausgebrochen ist (Kat. Nr. 131); der Code zur Klingenform ist aufgrund eines Rekonstruktionsversuchs vergeben worden.

Auffällig ist die absolute Verteilung in den einzelnen Untersuchungsländern:

Von den 38 untersuchten Messern wurden 26 Messer in polnischen und litauischen Fundorten ausgegraben, weitere sechs Messer stammen aus Skandinavien. Fünf Messer wurden in der Bundesrepublik und ein unsicheres Exemplar (siehe oben) in den Niederlanden geborgen.

Der zweite, zeitlich weiter gefaßte Auswertungsbestand umfaßt 90 Messer, von denen wiederum 51 Messer aus dem polnisch-litauischen Raum stammen. 25 Messer wurden im Nordwesten und im Osten der Bundesrepublik geborgen, zehn Messer in Skandinavien und vier in den Niederlanden.

Die zeitliche Verteilung im gesamten Untersuchungsraum zeigt einen Anteil zwischen $7,2 \%$ und $10,9 \%$ in den ersten drei Jahrhunderten des Untersuchungszeitraums, der im 11. und 12. Jahrhundert auf 17,2\% bzw. 15,4\% ansteigt und vom 13. Jahrhundert an regelmäßig abnimmt (Abb. 56).

Das zusätzlich erscheinende Exemplar im 16. Jahrhundert ist ein messerförmiges Maß, welches die maximal erlaubte Länge von Messern und Kurzwehren innerhalb der Stadtmauern des niederländischen Deventer angab (Kat. Nr. 6) und in das 15. bis 16. Jahrhundert datiert wird.

Somit verringert sich ebenfalls die absolute Anzahl der im 15. Jahrhundert beobachteten Exemplare dieser Formengruppe.

Die beiden niederländischen Belege sind kein Beweis für den Anteil der hier behandelten Formen im 15. und 16. Jahrhundert, sie legen lediglich nahe, daß mit einer Fortdauer dieser Formen bis in das 16. Jahrhundert zumindest gerechnet werden muß.

Der Vergleich mit der Graphik des ersten Auswertungsbestandes erlaubt aber den Schluß, daß Klingen dieser Formengruppe nach dem 14. Jahrhundert äußerst selten anzutreffen sind. 
Die chronologische Verteilung der Messer in den einzelnen Untersuchungsländern zeigt Unterschiede, die mit den vorab behandelten quellenkritischen Anmerkungen allein nicht zu erklären sind.

In Skandinavien verteilt sich der entsprechende Bestand auf das 9. bis 13. Jahrhundert mit deutlichen Spitzen von über 15\% im 11. und 12. Jahrhundert. Im 13. Jahrhundert sinkt sein Anteil auf unter 5\%.

In den Niederlanden sind Messer dieser Formengruppe außer mit zwei Exemplaren des 8. bis 9. Jahrhunderts nur noch sicher im 14. bis 15. Jahrhundert aufgetreten.

Im Bearbeitungsbereich der Bundesrepublik verteilen sich die Belege mit unterschiedlichen Schwerpunkten vom 8. bis zum 15. Jahrhundert.

Im Bereich der alten Bundesländer reicht die Hauptverbreitungszeit vom 9. bis zum 12. Jahrhundert mit Anteilen bis zu 20\%, die im 13. und 14. Jahrhundert schrittweise auf unter $10 \%$ zurückgehen.

In der ehemaligen DDR sind derartige Klingenformen im 8. Jahrhundert sowie mit einem Anteil von über 10\% im 11. bis 12. Jahrhundert angetroffen worden, der auf unter $10 \%$ im 13. und 14. Jahrhundert zurückgeht. Ein Beleg wurde in das 14. bis 15. Jahrhundert datiert.

Die Hauptmenge der hier behandelten Messer wurde in Polen und in den angrenzenden litauischen Fundorten geborgen. Dort verteilen sie sich vom 9. bis zum 14. Jahrhundert mit Anteilen, die im 11. und 12. Jahrhundert über 17\% erreichen und erst mit 6,7\% im 14. Jahrhundert unter die Zehnprozentmarke fallen. Das Messer des 15. Jahrhunderts ist wie das niederländische Exemplar ein Rekonstruktionsversuch.

Im Bestand der übrigen ehemals sowjetischen Fundorte sind diese Formen nicht aufgetreten.

Aus dem Vergleich dieser Verteilungsanteile läßt sich schließen, daß der Rückgang nach dem 12. Jahrhundert, der sich in gleicher Stärke nach dem 13. Jahrhundert fortzusetzen scheint, regional unterschiedlich stattgefunden hat:

Im 12. Jahrhundert beträgt der Anteil dieser Klingenformen in allen Untersuchungsländern außer den Niederlanden über $15 \%$. Während er in Skandinavien danach auf $4 \%$ und im bearbeiteten deutschen Raum auf durchschnittlich 7,9\% abfällt, bleibt er im östlichen Bearbeitungsraum auf fast $13 \%$; erst im 14. Jahrhundert fällt er adäquat zu den westlich angrenzenden Räumemauclø,8\%\%eint der Rückgang im Norden eher und stärker eingesetzt zu haben, dort fehlen Belege für das 14. Jahrhundert.

Im Osten scheint diese Formengruppe am längsten Bestand gehabt zu haben und auch mit einem Ost-West-Gefälle geendet zu sein, da der Bestand aus den Fundorten der alten Bundesländer unter $6 \%$ liegt.

In den westlich angrenzenden Niederlanden haben Messer mit derartigen Klingenformen im Hauptverbreitungszeitraum keine Rolle gespielt. 


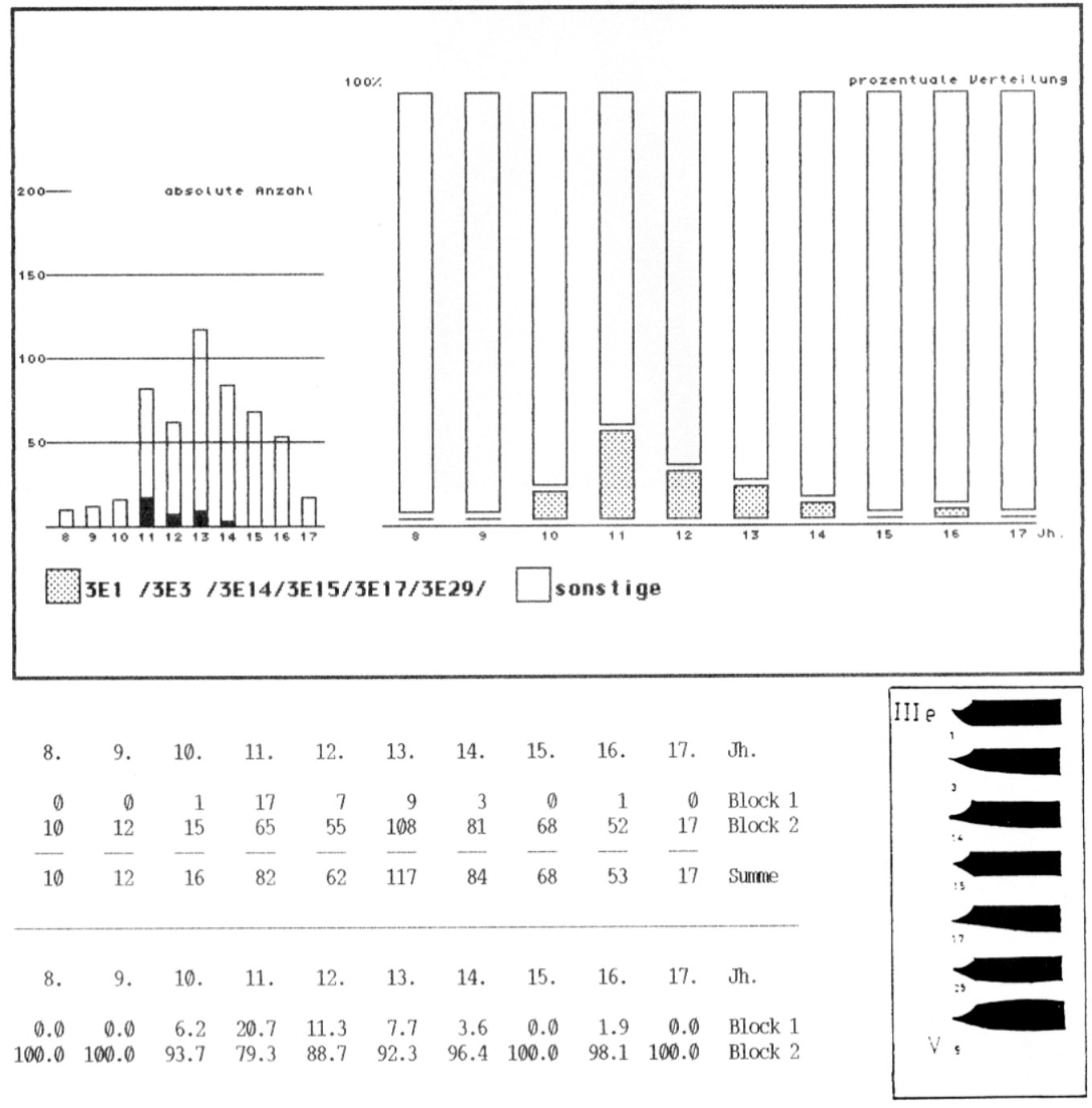

Abb. 55

Die zeitliche Verteilung von Klingenformen der Auswertungsgruppe 8 im gesamten Untersuchungsraum (enger datierter Berstand) 


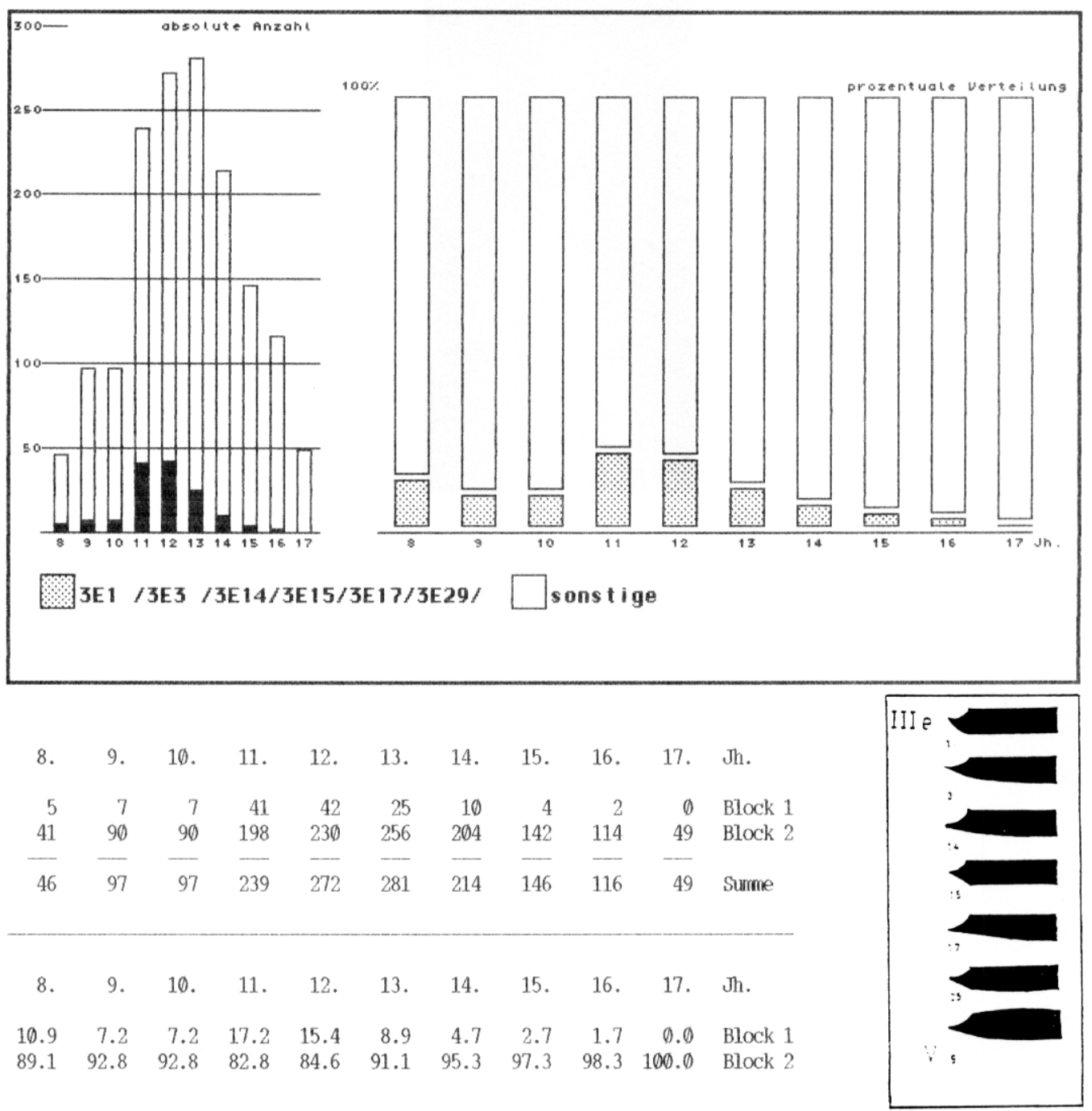

Abb. 56

Die zeitliche Verteilung von Klingenformen der Auswertungsgruppe 8 im gesamten Untersuchungsraum (grob datierter Bestand) 


\subsubsection{Die Verteilung der Klingenformen IIle (Auswertungsgruppe 9)}

Das Hauptmerkmal dieser Gruppe besteht aus einem konkav gebogenen Rücken, der nur im hinteren Bereich gerade verläuft.

Im Fundgut nachgewiesen wurden die Formen IIIe2 und IIIe4 sowie mit einem niederländischen Messer des 15. Jahrhunderts die Form IIIe30.

Im Bestand der zeitlich enger gefaßten Messer treten Belege für die Formen IIIe2 und IIIe4 mit 12 Exemplaren nur vereinzelt auf.

Im gesamten Auswertungsbestand verteilen sie sich mit leichten Schwankungen von 6,5\% im 8. Jahrhundert bis 4,7\% im 14. Jahrhundert in etwa linear (Abb. 57). Der Prozentanteil von 2,1\% im 15. Jahrhundert beruht neben dem bereits erwähnten niederländischen Messer auf zwei Belegen, die in das 14. bis 15 . Jahrhundert datiert wurden.

Aus der Auswertung der regionalen Fundbestände ergibt sich die Begrenzung der Anteile des 8. und 9. Jahrhunderts auf niederländische, polnische und lettische Fundorte.

Vom 10. bis 13. Jahrhundert sind diese Klingenformen in deutschen und polnischen Fundbeständen aufgetreten, in Skandinavien ließen sie sich nur im 12. Jahrhundert nachweisen.

Wie im vorangegangenen Bestand scheint auch in diesem Fall ein Auftreten im 15. Jahrhundert nur wahrscheinlich zu sein.

Im 14. Jahrhundert sind die Formen IIIe2 und IIIe4 dagegen allgemein verbreitet. 


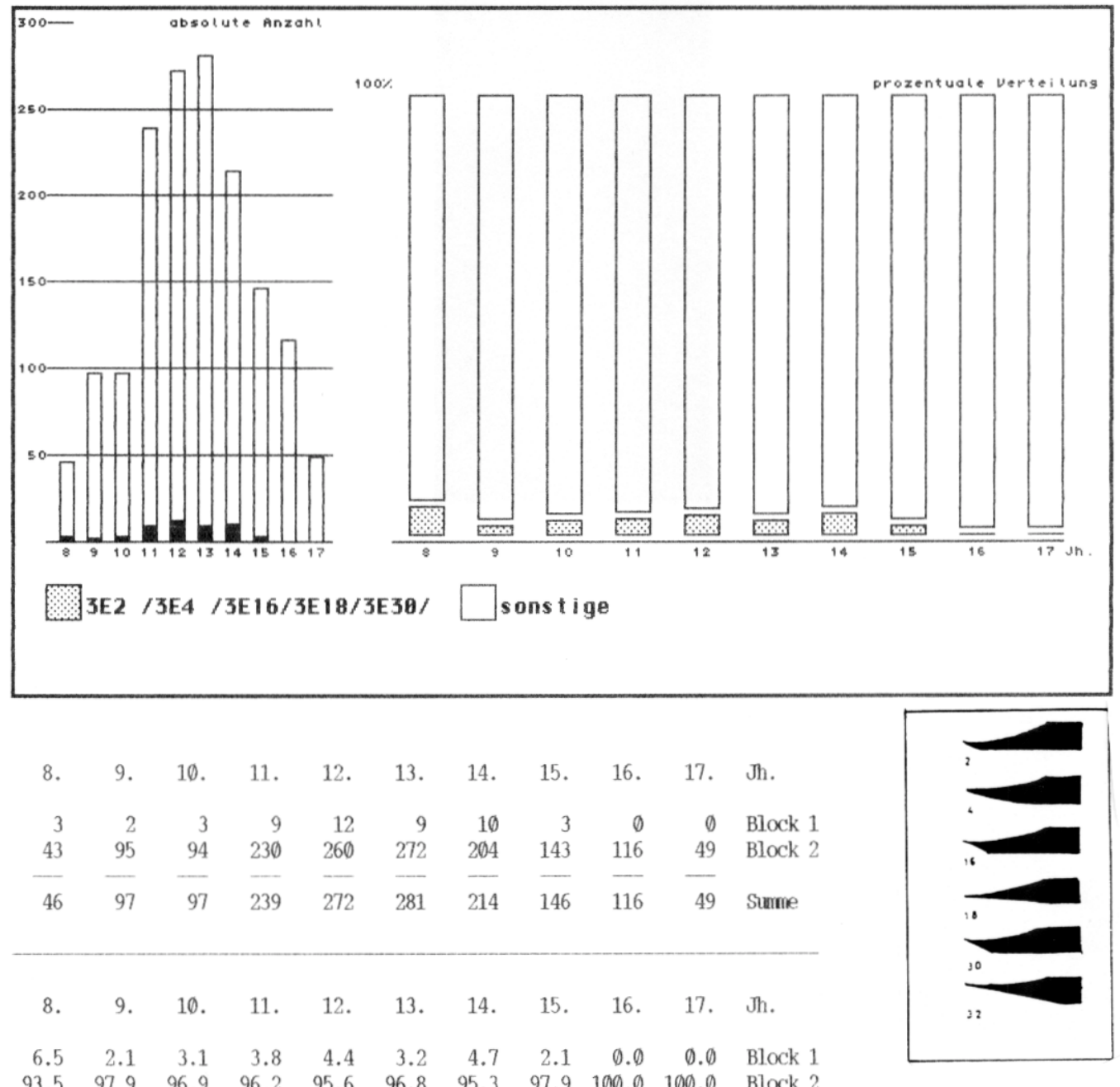

Abb. 57

Die zeitliche Verteilung von Klingenformen der Auswertungsgruppe 9 im gesamten Untersuchungsraum (grob datierter Bestand) 


\subsubsection{Die Verteilung der Klingenformen IIle (Auswertungsgruppe10)}

Die letzte Gruppe unter der Leitform IIIe besteht aus Messern, deren Rücken im gesamten Verlauf konkav gebogen sind. Nachgewiesen wurden im ausgewerteten Bestand die Formen IIIe, IIIe33 und IIIe13.

Am häufigsten unter den insgesamt 32 Messern dieser Gruppe ist die Form IIIe mit 24 Exemplaren vertreten. Sie wurde bis auf die Niederlande im gesamten Untersuchungsraum nachgewiesen.

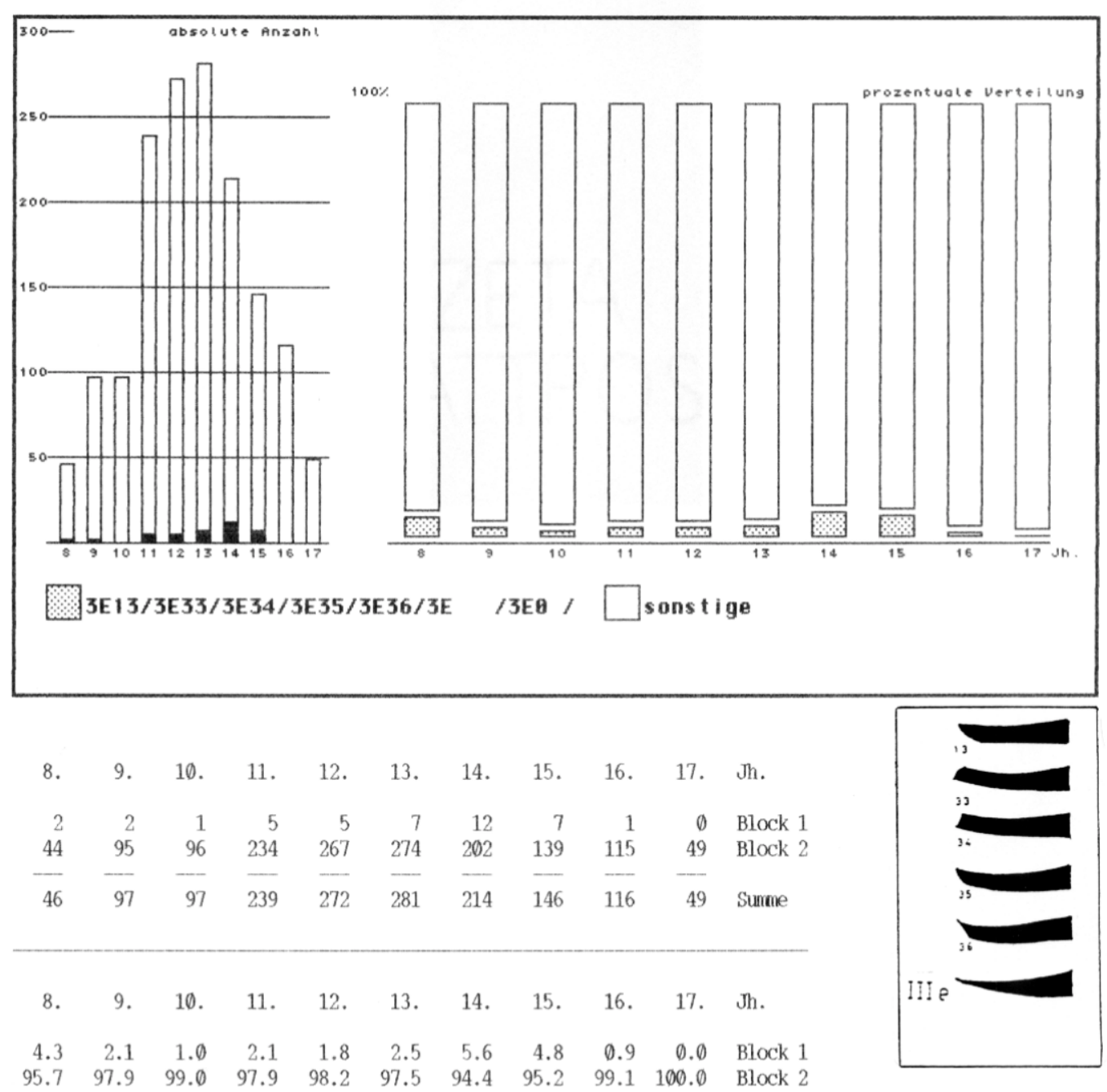


Die Formen IIIe13 und IIIe33 traten nur in Skandinavien, im westlichen Teil der Bundesrepublik und in der ehemaligen westlichen Sowjetunion auf. In den Niederlanden wurden Formen dieser Auswertungsgruppe nicht angetroffen.

Die zeitliche Verteilung umfaßt den Zeitraum vom 8. bis zum 15. Jahrhundert, das Exemplar des 16. Jahrhunderts, ein Messer aus Lund (Kat. Nr. 101, Codebezeichnung IIIe33), wird in das 15. bis 16. Jahrhundert datiert (Abb. 58).

Die prozentualen Anteile liegen mit steigender Tendenz zwischen 1\% im 10. Jahrhundert und 5,6\% im 14. Jahrhundert, im 15. Jahrhundert beträgt ihr Anteil noch 4,8\%. In Polen liegt der Schwerpunkt vom 11. bis 13., im östlichen Teil der Bundesrepublik vom 13. bis 14. Jahrhundert.

Die beiden frühen Exemplare des 8. bis 9. Jahrhunderts stammen aus Lettland (Kente, Kat. Nr. 51 und 57).

\subsubsection{Abschließende Bemerkungen zu den Verteilungstendenzen der Klingenformen Ille}

Bis auf einige Belege des 15. bzw. 15. bis 16. Jahrhunderts, die der Leitform IIIe nahestehen, sind Klingen mit konkav zur Spitze einfallenden Rückenabschnitten im ausgewerteten Messerbestand nach dem 14. Jahrhundert nicht eindeutig nachgewiesen worden, obwohl es Hinweise darauf gibt, daß sie in Einzelfällen möglicherweise bis in das 16. Jahrhundert vorkommen können.

Am häufigsten vertreten sind Klingen, deren konkaver Rückeneinfall erst kurz vor der Spitze beginnt. Ihre Hauptverbreitungszeit liegt im 11. und 12. Jahrhundert, zum Verbreitungsraum und den einzelnen Regressionsphasen wurden regionale Unterschiede festgestellt.

\subsubsection{Die Verteilung der Klingenformen IIIb32 und Ve (Auswertungsgruppe 11)}

Mit der Form IIIb32 und der Sonderform Ve wurden Messer mit einem zweifach abgeknickten Rückenverlauf erfaßt, deren hervorragendstes Beispiel ein Exemplar aus Hamburg aus dem letzten Viertel des 12. Jahrhunderts zu sein scheint (Kat. Nr.46).

Bis auf ein Klingenfragment von der Büraburg bei Fritzlar (Kat. Nr. 196) stammen sechs der sieben Belege aus dem 11. und 12. Jahrhundert.

Der Nachweis dieser Klingenformen gestaltete sich äußerst schwierig (Abb. 59).

Bei dem bereits erwähnten Hamburger Messer besteht die Möglichkeit, daß die Spitze abgebrochen ist.

Der Mittelknick im Rücken ist bei den Exemplaren vom Dörhai (Kat. Nr. 81) und aus Anklam (Kat. Nr. 66) nur schwach ausgeprägt. Die Messerklinge von der Nie- 
derungsburg bei Haus Meer (Kat. Nr. 176) ist vorne ausgebrochen, vom Belegexemplar von der Büraburg ist nur die Klingenspitze erhalten. Das Messer von der Fischerinsel (Neubrandenburg, Kat. Nr. 88) ist insgesamt schwach ausgeprägt, das Messer aus Oslo (Kat. Nr. 4), welches den doppelten Rückenknick am deutlichsten zeigt, ist gleichzeitig mit einem Absatz im Rückenverlauf eine Kombination mit der Form IVf.

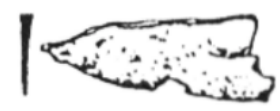

a

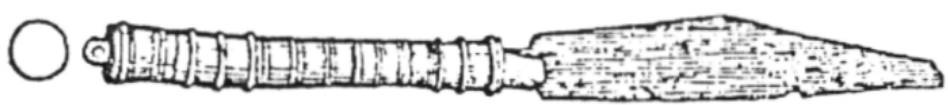

$\mathrm{b}$

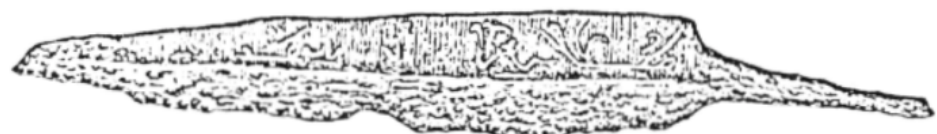

C

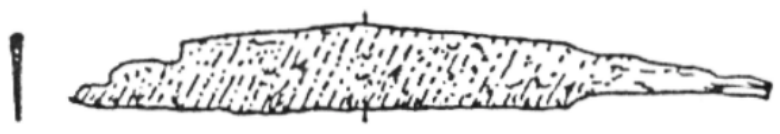

d

Abb. 59

Beispiele und Fragmente für die Klingenform Ve
a: Büraburg bei Fritzlar
b: Hamburg
c: Wall am Dörhai
d: Niederungsburg bei Haus Meer

Der unterschiedliche Schneidenverlauf der beiden Klingenformen wurde nicht berücksichtigt, die absolute zeitliche Verteilung der erfaßten Belege konzentriert sich auf das 11. bis 12. Jahrhundert (Abb. 60).

Messer mit doppelt abgeknicktem Rückenverlauf sind äußerst selten und anscheinend vor allem im Hochmittelalter anzutreffen. 


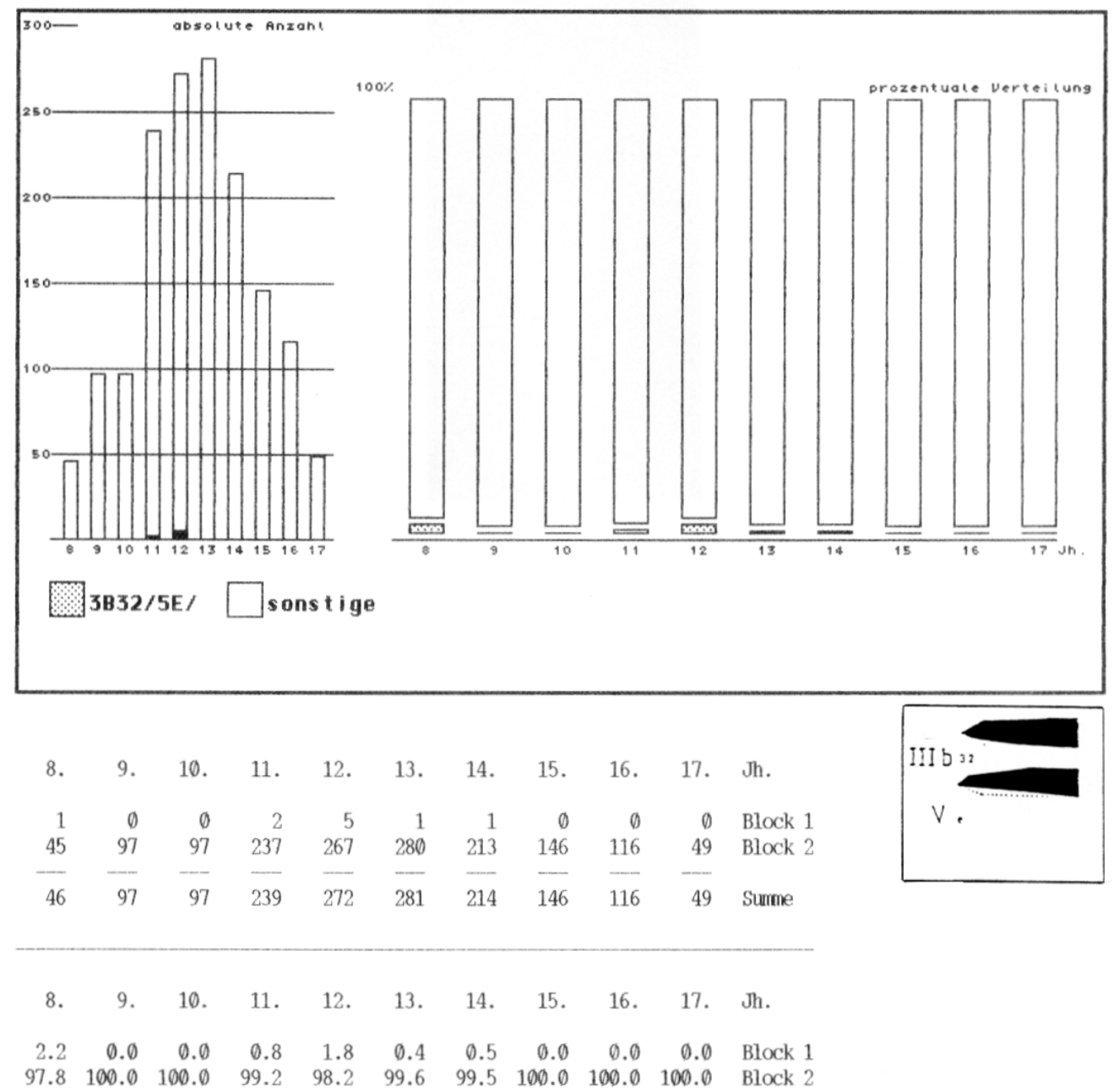

Abb. 60

Die zeitliche Verteilung von Klingenformen der Auswertungsgruppe 11 im gesamten Untersuchungsraum (grob datierter Bestand)

\subsubsection{Die Verteilung der Klingenformen IIId1 und IVi (Auswertungsgruppe 12)}

Die beiden Formen IIId1 und IVi konnten im gesamten Bestand von 1300 Messern nicht eindeutig nachgewiesen werden.

Ein einziges Messer mit der rekonstruierten Form IVi wurde in der Burg Wartenberg in Oberhessen gefunden (Kat. Nr. 204), die Schneide ist jedoch ausgebrochen, so daß eine formale Zuweisung zur Sonderform IVa ebenfalls möglich ist (Abb. 61).

Dieses Ergebnis ist um so erstaunlicher, als sich ähnliche Formen auf zahlreichen mittelalterlichen Darstellungen aus dem Bereich der Tafel nachweisen lassen. Bei 
beiden Formen weist die Spitze durch eine Einbuchtung im vorderen Schneidenabschnitt nach unten auf die Arbeitskante (Schneide) des Messers.

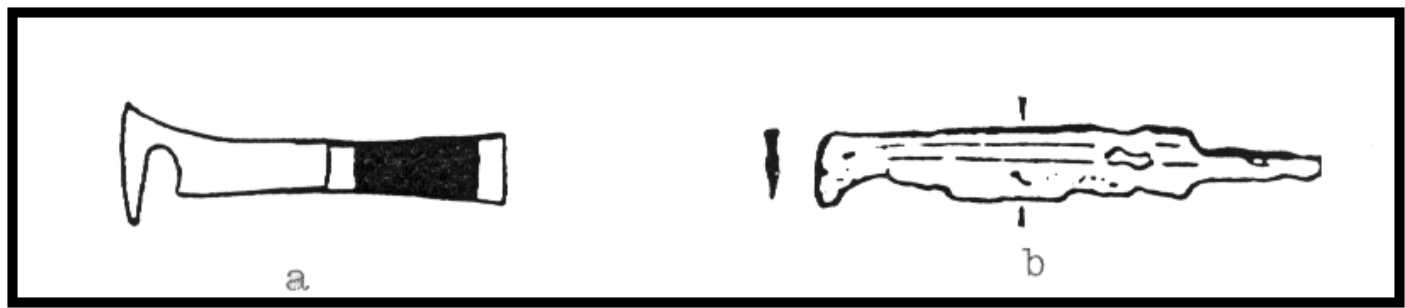

Abb. 61

Die Klingenform IV $i$

a: Hortus Deliciarum, um 1170

b: Burg Wartenberg, 13. Jahrhundert

\subsubsection{Die Verteilung der Klingenformen Ia, Ila, IIIa, IIIc und IIId (Auswertungsgruppen 13 bis 17)}

Die Merkmale der Leitformen Ia bis IIIa, grundsätzlich gerade verlaufende Kanten, waren im Fundgut wegen der bereits erwähnten Unsicherheiten zum Verlauf der Schneiden kaum exakt nachzuweisen.

In der Regel handelt es sich bei den entsprechenden Codezuweisungen um Rekonstruktionen.

Die beiden Leitformen IIIc und IIId kennzeichnen gegensätzlich konvex bzw. konkav verlaufende Klingenkanten.

Alle Leit- und zugehörigen Hauptformen wurden nur sehr selten angetroffen oder als Code nach Rekonstruktionsvorschlägen vergeben.

Neben einem Beleg aus Skandinavien (Abb. 62, Kindermesser aus Lund, Kat. Nr. 66) wurden in der Bundesrepublik (alte und neue Bundesländer) und in Polen insgesamt 21 Messer erfaßt, von denen elf im Rahmen eines Jahrhunderts datiert worden waren.

Außer einem Messer des 15. und einem des 16. bis 17. Jahrhunderts mit der Form Ia verteilen sich die Belege für diese Formen auf den Zeitraum vor dem15. Jahrhundert. 


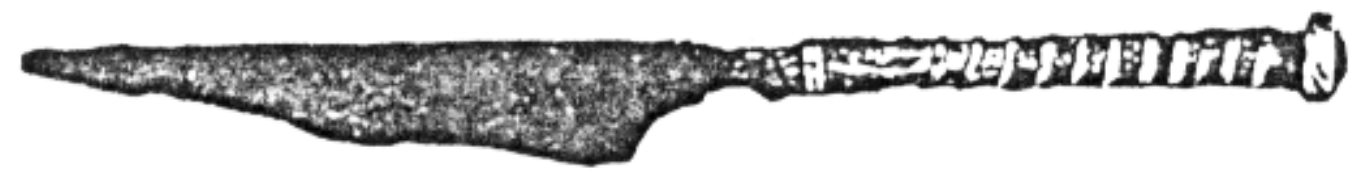

Im 8. bis 9. Jahrhundert tritt jeweils nur eine Form auf (IIa, IIId), im 10. und 11. Jahrhundert zwei bzw. drei Formen (IIIa, IIId bzw. Ia, IIIa und IIIc).

Die Hauptverbreitungszeit dieser nur schwer nachweisbaren Klingenformen scheint der Zeitraum vom 12. bis zum 14. Jahrhundert gewesen zu sein, in dem im 12. Jahrhundert alle fünf und im 13. und 14. Jahrhundert bis auf die Form IIIc die übrigen vier Formen angetroffen wurden (Abb. 63 und 64).

\subsubsection{Die Verteilung der Sonderformen IV (Klingenformen IVa, IVc bis IVj)}

Die hier zusammengefaßten Sonderformen sind mit ausgesprochen speziellen Merkmalen versehen, die Annahme, daß es sich bei den meisten der insgesamt 59 Belege und Messer mit stark eingegrenzten Funktionsbereichen handelt, liegt daher nahe. Besonders bei sechs Formen steht zu vermuten, daß es sich um spezielle Tafelmesser handelt, da einige von ihnen in diesem Funktionszusammenhang von mittelalterlichen Bildquellen bekannt sind. Es handelt sich um die Formen IVa, IVe, IVf, IVh, IVi und IVj (Abb. 65).

Der früheste Beleg der Form IVf stammt aus dem 7. Jahrhundert (Abb. 66a), der abgesetzte Klingenrücken ist mit einer Rille versehen.

Dieses Merkmal bildet möglicherweise die technologische Ursache für die Entstehung dieser Klingenform. Im Verlauf der folgenden Belege des 11. bis 12. und 12. bis 13. Jahrhunderts wird die Form zunächst beibehalten, obwohl die längs des Rückens verlaufende Rille bereits fehlt (Abb. 66b-c).

Bei ähnlicher Grundform zeigt das Beispiel aus Morken, datiert in das 13. Jahrhundert, den Knick im Rückenverlauf nur noch rudimentär (Abb. 66d). 


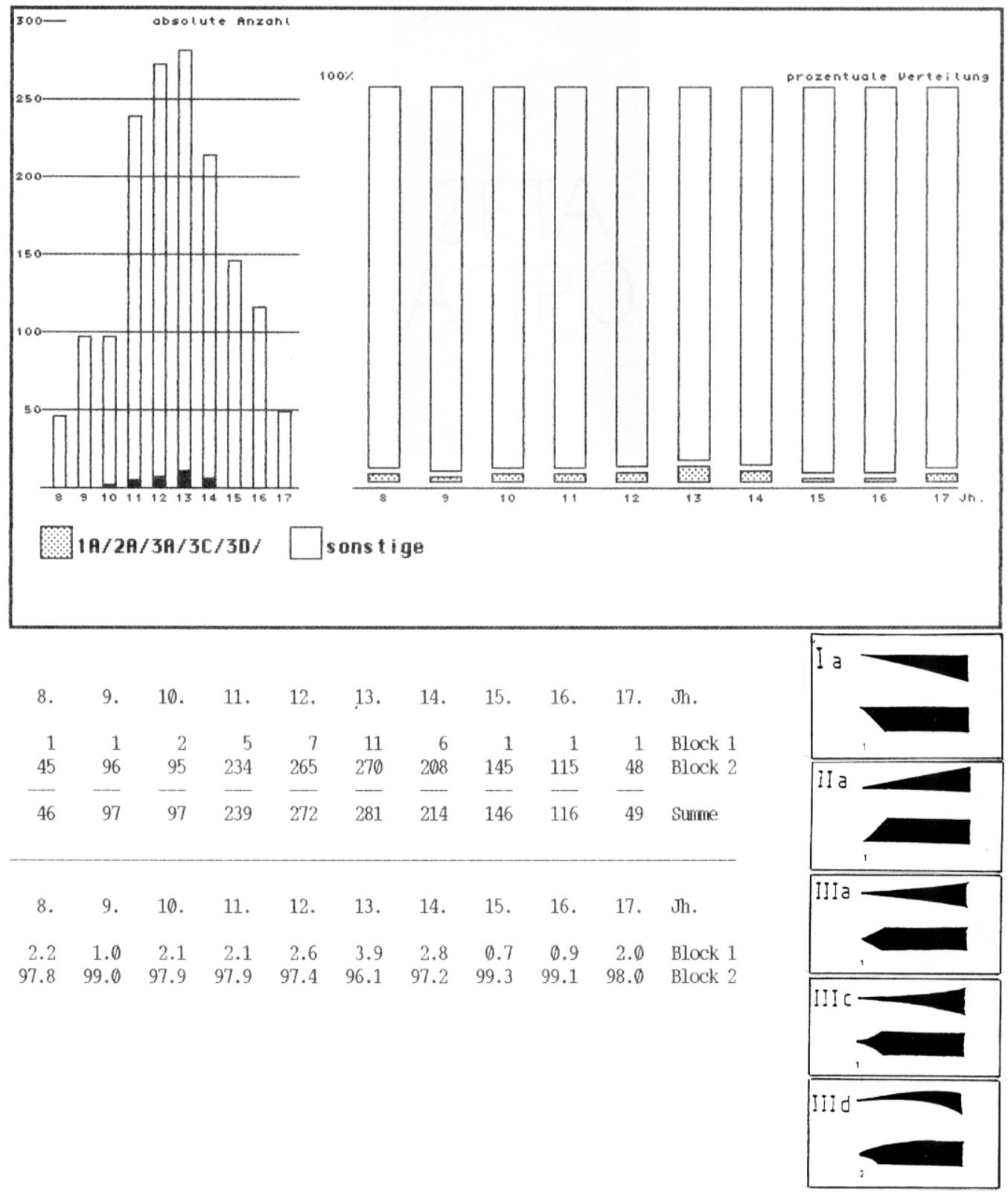

Abb. 63

Die zeitliche Verteilung von Klingenformen der Auswertungsgruppe 13 bis 17 im gesamten Untersuchungsraum (grob datierter Bestand) 


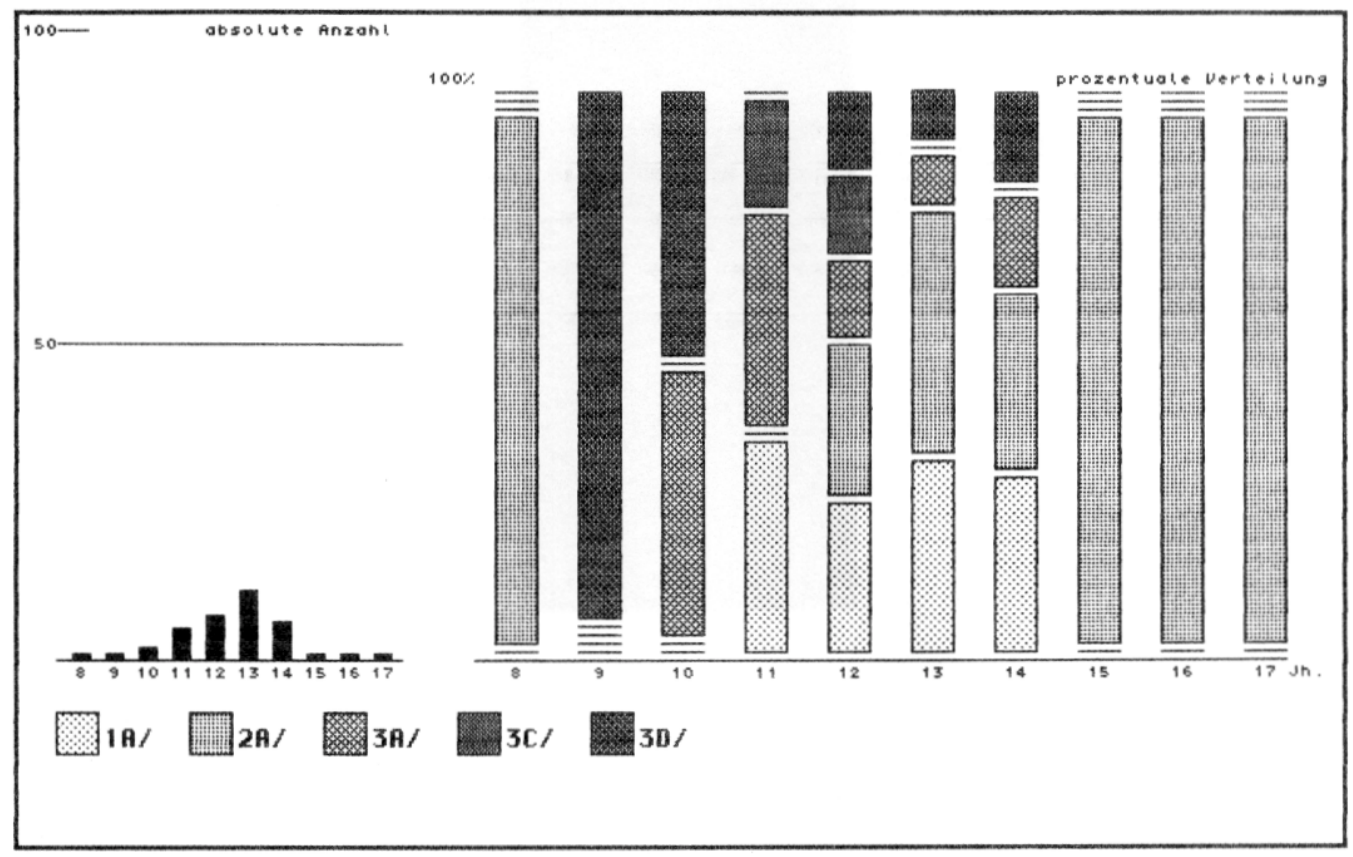

8. 9. 10. 11. 12. 13. 14. 15. 16. 17. Jh.

$\begin{array}{rrrrrrrrrrl}\emptyset & 0 & \emptyset & 2 & 2 & 4 & 2 & \emptyset & \emptyset & \emptyset & \text { Block 1 } \\ 1 & \emptyset & \emptyset & \emptyset & 2 & 5 & 2 & 1 & 1 & 1 & \text { Block 2 } \\ \emptyset & 0 & 1 & 2 & 1 & 1 & 1 & \emptyset & \emptyset & \emptyset & \text { Block 3 } \\ \emptyset & \emptyset & \emptyset & 1 & 1 & \emptyset & \emptyset & \emptyset & \emptyset & \emptyset & \text { Block 4 } \\ \emptyset & 1 & 1 & \emptyset & 1 & 1 & 1 & \emptyset & \emptyset & \emptyset & \text { Block 5 } \\ -1 & -1 & - & - & - & -1 & - & - & -- & - & \\ 1 & 1 & -11 & 6 & 1 & 1 & 1 & \text { Summe }\end{array}$

8. 9. 10. 11. 12. 13. 14. 15. 16. $17 . \quad$ Jh.

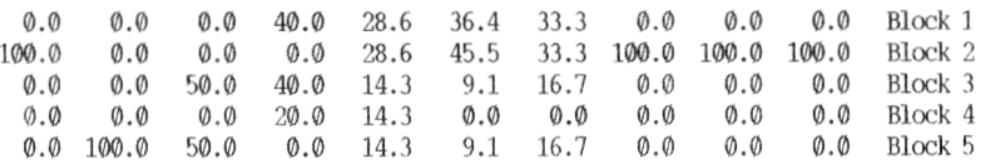

Abb. 64

Die anteilige Verteilung der Klingenformen Ia, Iia, IIIa, IIIc und IIId im Bestand der Auswertungsgruppen 13 bis 17 

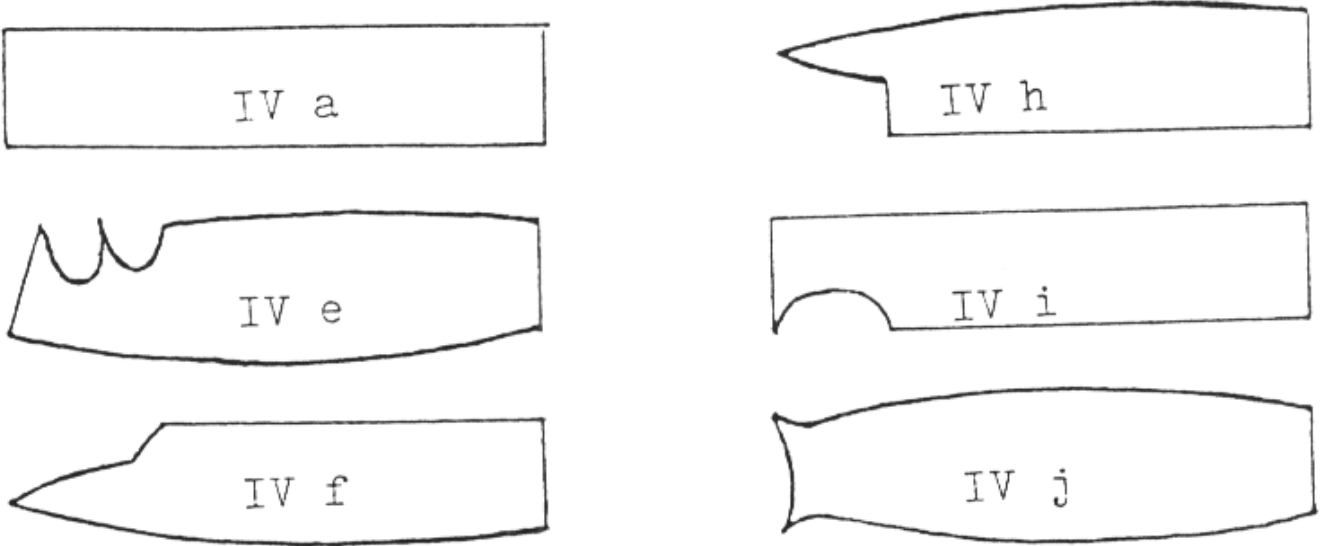

Abb. 65

Seit dieser Zeit scheint das Merkmal des Rückenabsatzes auch nicht mehr an die alte Grundform gebunden gewesen zu sein.

Das Messer aus Lund (Abb. 66e), dessen Datierung allerdings unsicher ist, zeigt die Rekonstruktion einer korrodierten Klinge mit einer dornartigen Klingenspitze, die in etwa die Form IVh (Abb. 65) umkehrt.

Weitere Variationsmöglichkeiten liegen mit zwei Messern des 14. und des 16. Jahrhunderts vor (Abb. 66f-g).

Die beiden Exemplare des 13. und 14. Jahrhunderts mögen aufgrund der besonderen Ausprägung ihrer Klingenformen am ehesten mit den erwähnten Bildquellen zum spätmittelalterlichen Tafelbesteck in Verbindung gebracht werden ${ }^{59}$.

Insgesamt wurden 30 Messer der Form IVf zugeordnet. 


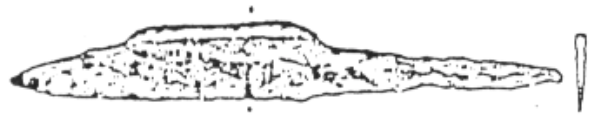

a.

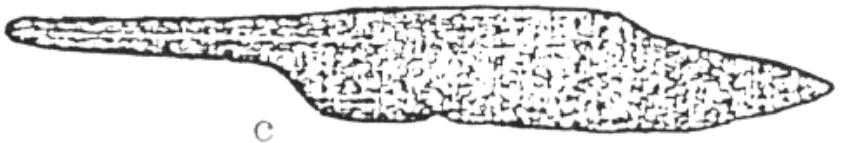

C

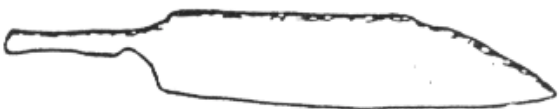

d
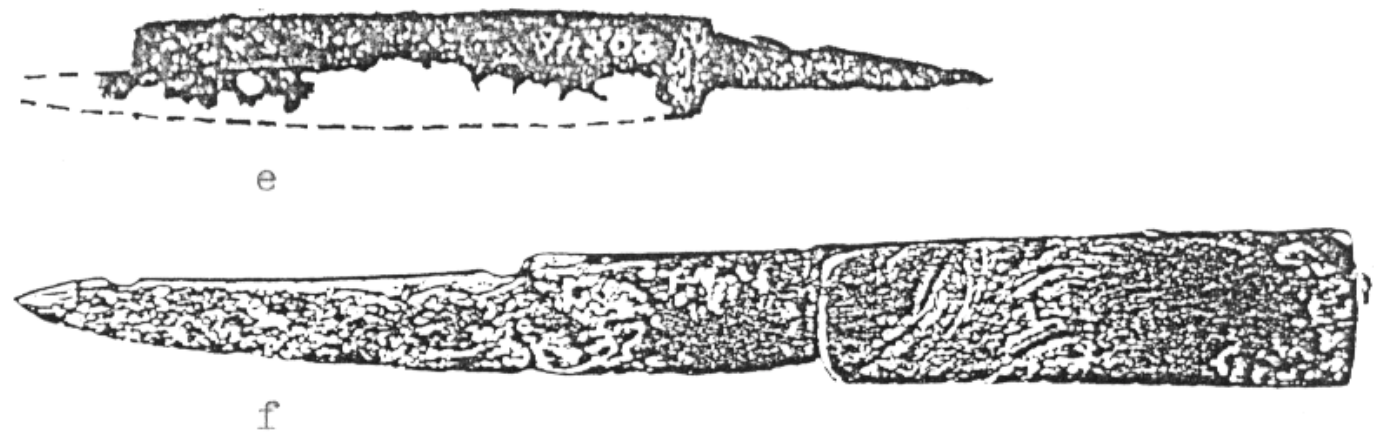

f

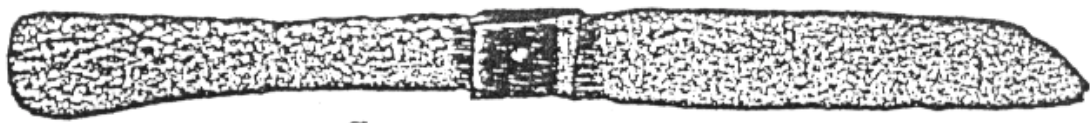

g

Abb. 66 Die verschiedenen Ausprägungen der Klingenform IV $f$

a: Sternberger Burg, 7. bis 8. Jahrhundert

b: Slawische Burg bei Lübeck, 11. bis 12. Jahrhundert

c: Posen, 12. bis 13. Jahrhundert

d: Morken, 13. Jahrhundert

e: Lund, 13. Jahrhundert

f: Dordrecht, 14. Jahrhundert

g: Ketelhaven, 16. Jahrhundert

Messer mit den Klingenformen IVa und IVh wurden zum größten Teil in Burgen gefunden, belegt durch neun Exemplare der Form IVa und drei Exemplare der Form IVh. Zwei Messer der Form IVa stammen aus einer ländlichen Siedlung mit einer Burg als Zentrum, zwei weitere aus einem Kloster; nur ein Messer dieser Form sowie eines der Form IVh (Lund) wurden bei einer Stadtkerngrabung geborgen. 
Diese 18 Messer stammen fast durchweg aus dem 12. bis 14. Jahrhundert, lediglich das Messer aus Lund wurde wegen seiner Griffkonstruktion in das 15. Jahrhundert datiert (Kat. Nr. 103).

Sehr viel seltener sind Messer der Formen IVe, IVi und IVj. Die Form IVi ist nur mit einem strittigen Exemplar des 13. Jahrhunderts vertreten (Burg Wartenberg, Kat. Nr. 204). Die einzigen sicheren Nachweise der Formen IVe und IVj wurden in Breslau gefunden, die drei Messer stammen ebenfalls aus dem 13. Jahrhundert (Kat. Nr. 320, 323, 325).

Beispiele für die verbleibenden Formen der Gruppe IV sind kaum angetroffen worden. Die Form IVb ist als zusätzliches Merkmal bei einem niederländischen Messer des 14. Jahrhunderts einmal angeklungen (Kat. Nr. 32).

Ein Messer aus der Burg Gommerstedt ist der einzig sichere Nachweis der Form IVg (Kat. Nr. 185).

Das Merkmal der Form IVc, der gebogene Einsprung im vorderen Rückenverlauf, ähnelt in seinen Ausprägungen oft der Form IVf, das Merkmal wurde nur einmal an einem Messer des 12. Jahrhunderts aus Novogrudok beobachtet, allerdings bei einem veränderten Restverlauf des Rückens (Kat. Nr. 67).

Auch die Form IVd, deren Spitze nach oben weist, ist im gesamten Bestand nur dreimal nachgewiesen worden, je einmal in den Niederlanden (14. Jahrhundert, Kat. Nr. 60), in der Bundesrepublik (11. bis 12. Jahrhundert, Kat. Nr. 170) und in Norwegen (12. bis 13. Jahrhundert, Kat. Nr. 17).

Bei der Auswertung wurde wegen des geringen Bestandes neben einer prozentualen Aufrechnung mit dem Gesamtbestand auch die Einzelverteilung innerhalb der Auswertungsgruppe erstellt.

Die Auswertungsgraphik weist die Zeit vom 11. bis 15. Jahrhundert als Verbreitungszeit der Formen IVa bis IVj aus, lediglich die Form IVf ist durchgängig vom 8. bis 16. Jahrhundert vertreten. die Hauptverbreitungszeit der Formengruppe IV ist das 11. bis 14. Jahrhundert, in denen pro Jahrhundert mehr als zwei dieser Formen nachweisbar auftreten. In diesem Zeitraum kommen die Formen IVa, IVc (12. bis Mitte 14. Jahrhundert, vgl. Katalogteil Schweden, Kat. Nr. 37), IVe, IVg, IVi und IVj vom 12. bis zum 14. Jahrhundert vor, die Formen IVd und IVh sind vom 11. bis 14. Jahrhundert vertreten (Abb. 67 und 68). 


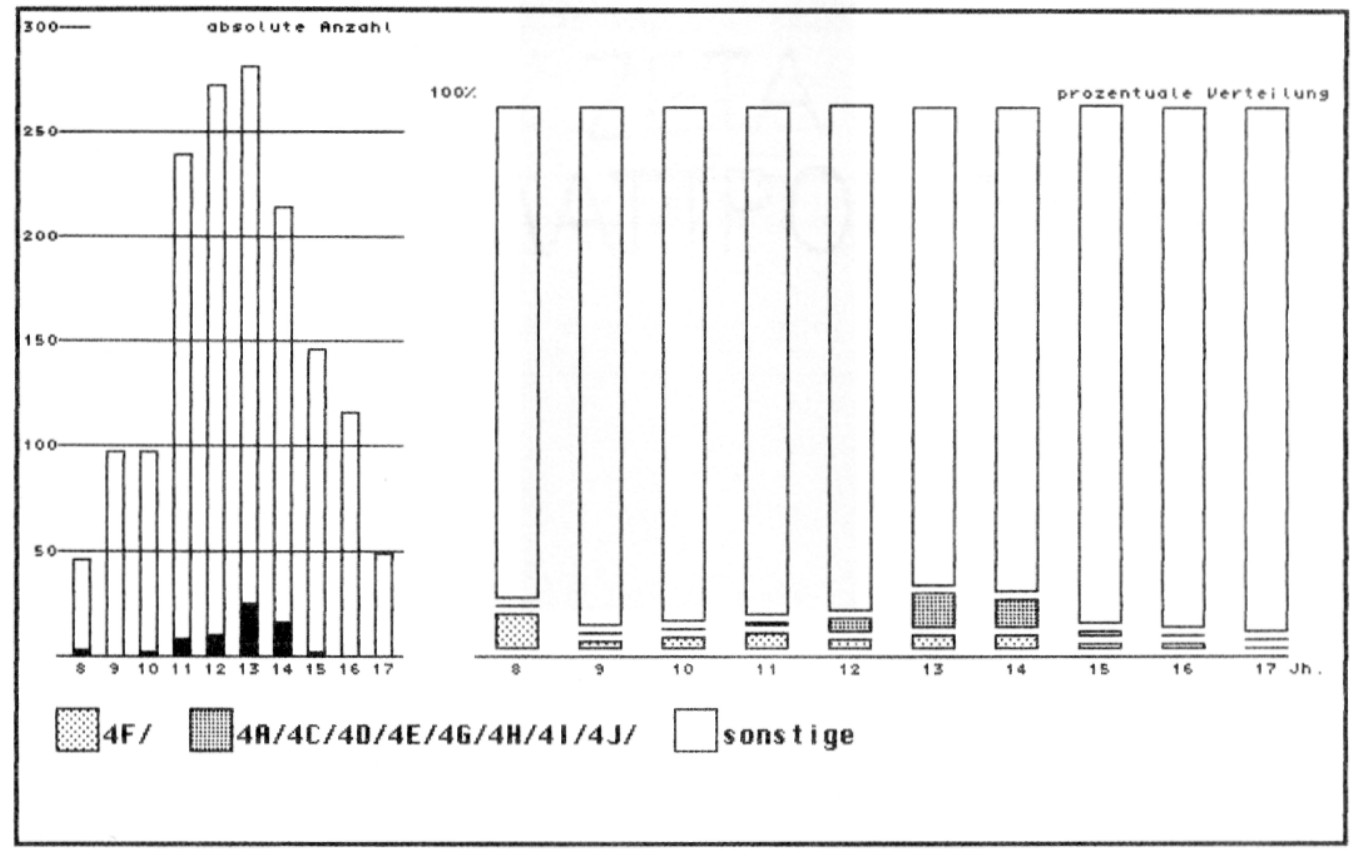

\begin{tabular}{|c|c|c|c|c|c|c|c|c|c|c|}
\hline 8. & 9. & 10. & 11. & 12. & 13. & 14. & 15. & 16. & 17. & Jh. \\
\hline 3 & 1 & 2 & 7 & 4 & 7 & 5 & 1 & 1 & $\emptyset$ & Block 1 \\
\hline$\emptyset$ & 0 & $\emptyset$ & 1 & 6 & 18 & 11 & 1 & 0 & $\emptyset$ & Block 2 \\
\hline 43 & 96 & 95 & 231 & 262 & 256 & 198 & 144 & 115 & 49 & Block 3 \\
\hline$\ldots$ & - & $\cdots$ & - & - & - & - & - & - & - & \\
\hline 46 & 97 & 97 & 239 & 272 & 281 & 214 & 146 & 116 & 49 & Surme \\
\hline
\end{tabular}

8. 9. 10. 11. 12. 13. 14. 15. 16. 17. Jh.

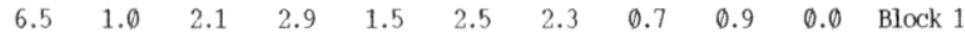

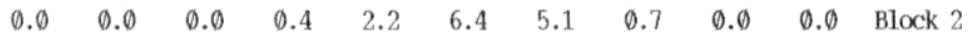

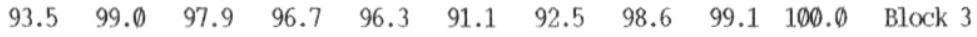

Abb. 67

Die zeitliche Verteilung von Klingenformen der Formengruppe IV

Sonderformen im gesamten Untersuchungsraum (grob datierter Bestand) 


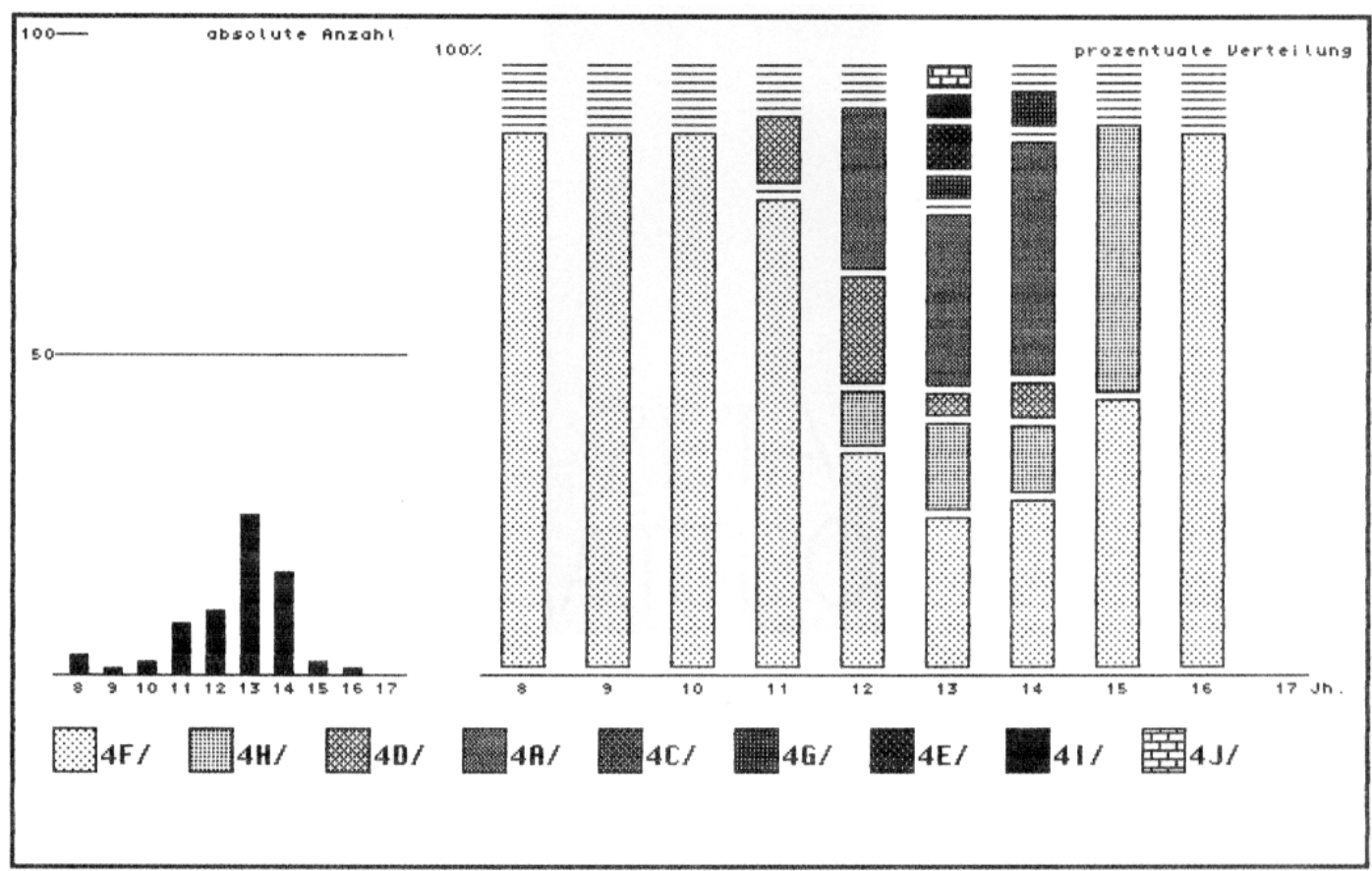

Abb. 68 Die anteiligen Verteilungen der Klingenformen IVa, IVc, IVd, IVe, IVf, IVg, IVh, IVi, IVj im Bestand der Formengruppe IV-Sonderfomen

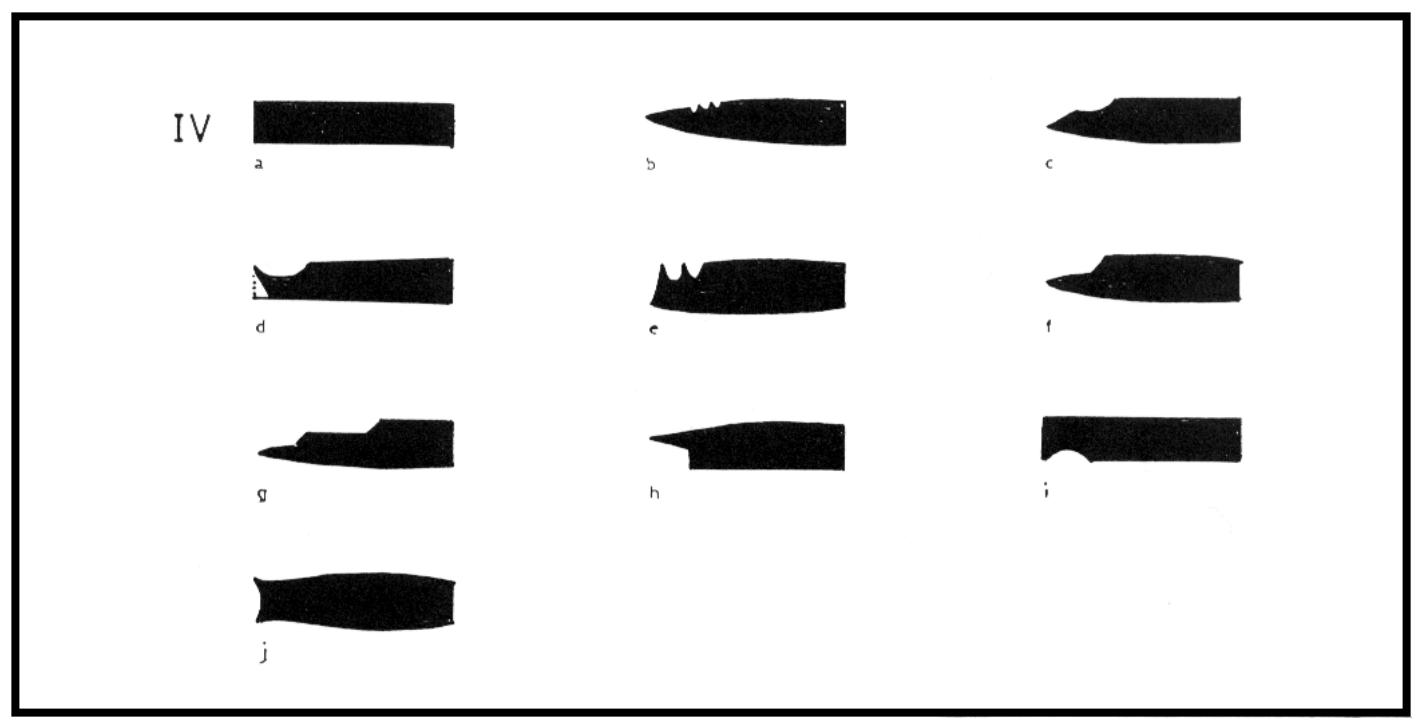

Abb. 68 (Fortsetzung) 


\subsubsection{Die Verteilung der Sonderformen V (Klingenformen Va bis Ve)}

Die im bearbeiteten Fundgut eheblich häufiger aufgetretenen Klingenformen der Gruppe V, in der Regel Kombinationen aus bereits im Klassifizierungsmodell enthaltenen Formkriterien, verteilen sich auf den Zeitraum vom 8. bis 15. Jahrhundert, zu einer späteren Zeitstellung ließen sich Belege dieser Formen nicht mehr nachweisen.

Die insgesamt 91 Messer belegen die fünf Formen Va bis Ve.

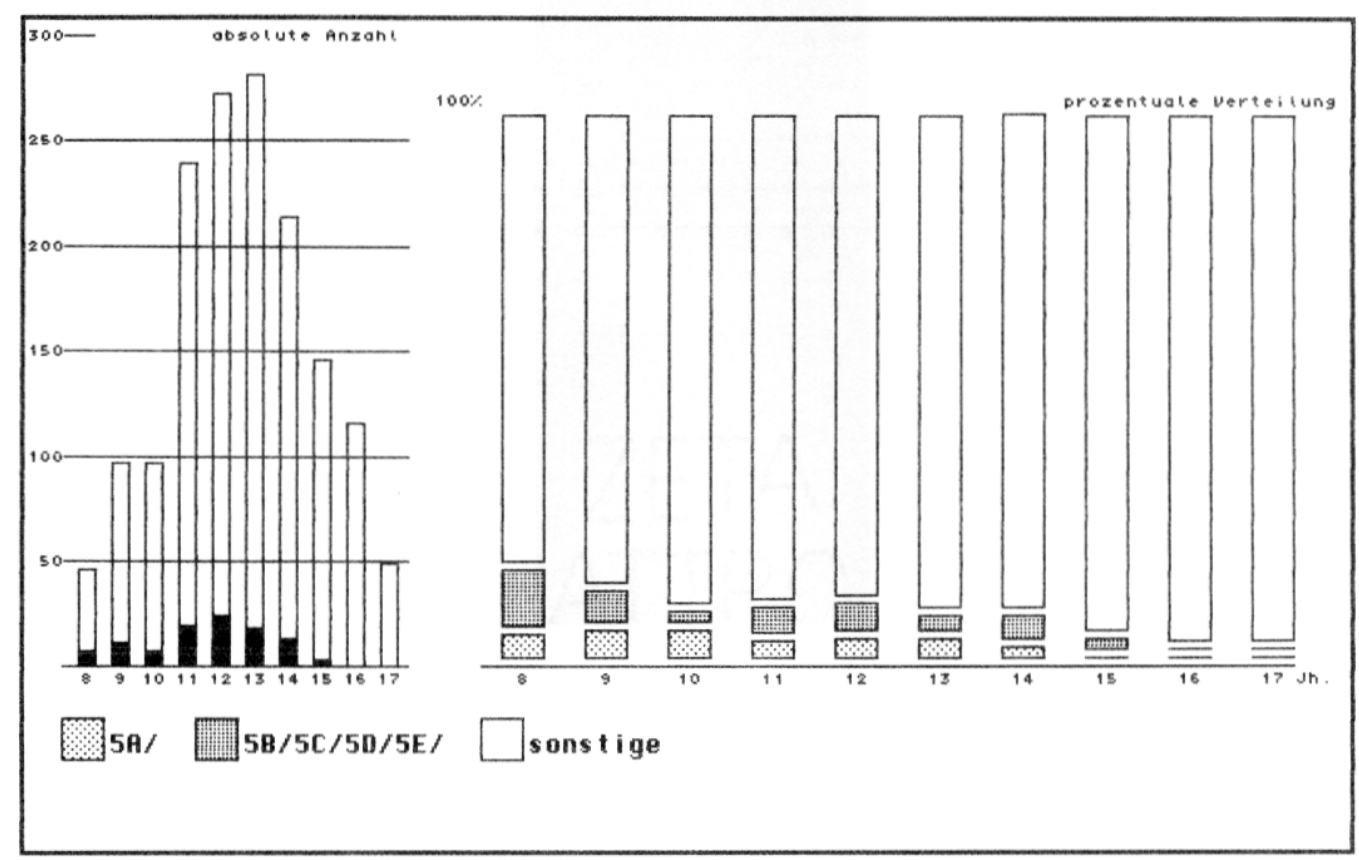

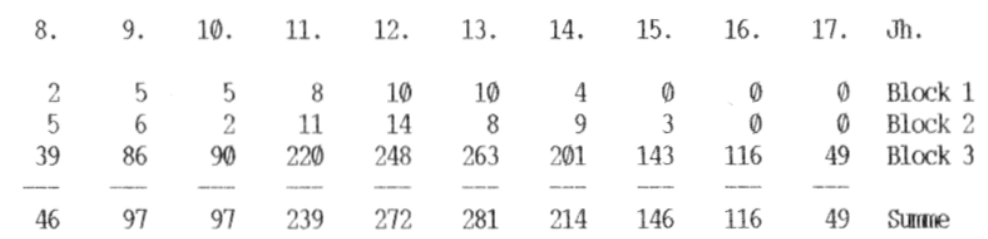

$\begin{array}{rrrrrrrrrrl}8 . & 9 . & 10 . & 11 . & 12 . & 13 . & 14 . & 15 . & 16 . & 17 . & \text { Jh. } \\ 4.3 & 5.2 & 5.2 & 3.3 & 3.7 & 3.6 & 1.9 & \emptyset . \emptyset & \emptyset .0 & \emptyset . \emptyset & \text { Block 1 } \\ 10.9 & 6.2 & 2.1 & 4.6 & 5.1 & 2.8 & 4.2 & 2.1 & \emptyset .0 & \emptyset . \emptyset & \text { Block 2 } \\ 84.8 & 88.7 & 92.8 & 92.1 & 91.2 & 93.6 & 93.9 & 97.9 & 100.0 & 100 . \emptyset & \text { Block 3 }\end{array}$

Abb. 69 Die anteilige Verteilung der Klingenformen Va, Vb, Vc, Vd und Ve imBestand der Formengruppe $V$ - Sonderformen 


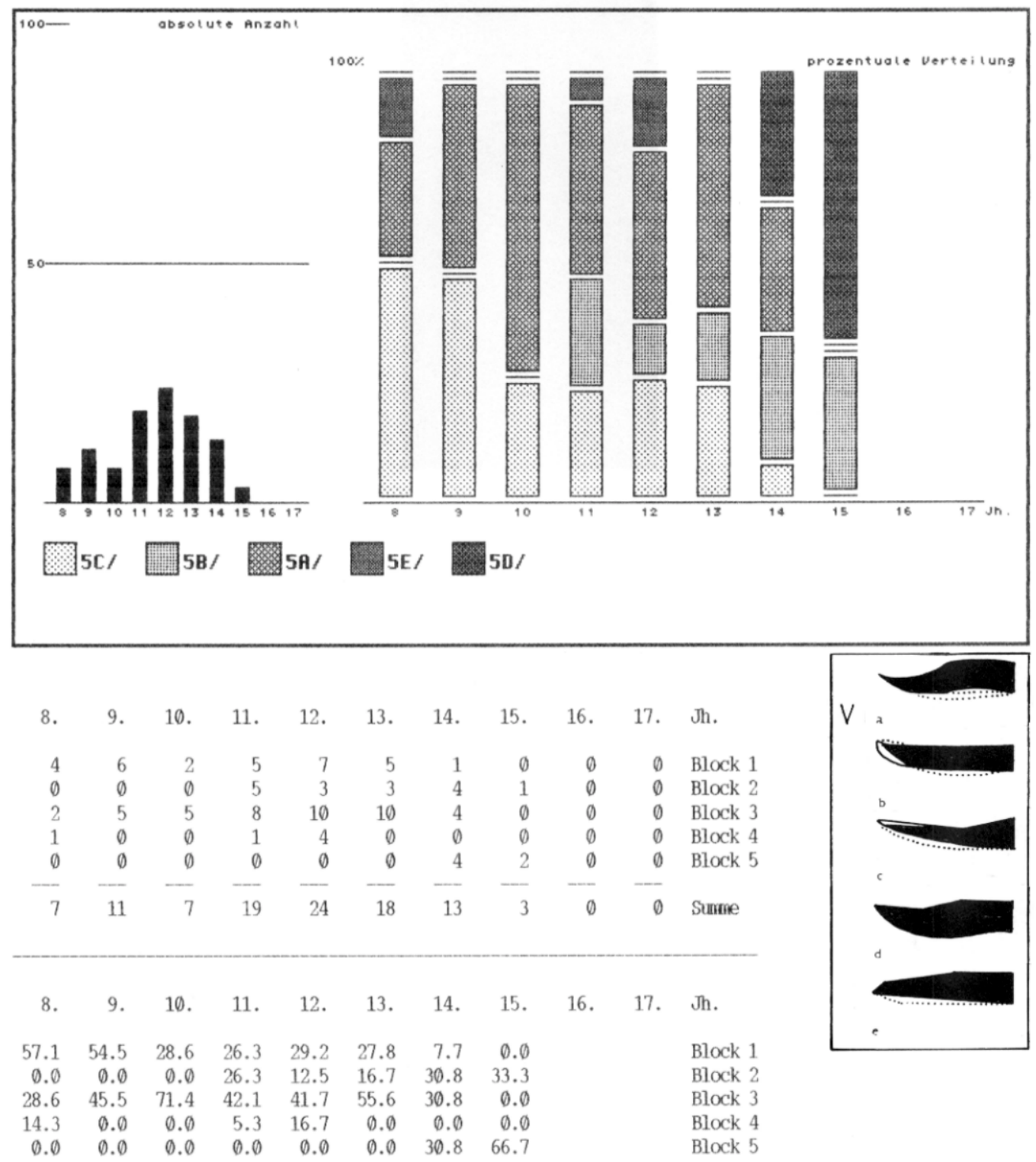

Abb. $70 \quad$ Die anteilige Verteilung der Klingenformen $V a, V b, V c$, Vdund Ve im Bestand der Formengruppe $V$ - Sonderformen

Die Form Va, eine Klinge mit s-förmig gebogenem Rücken, erscheint vom 8. bis zum 14. Jahrhundert, die Formen $\mathrm{Vb}$ und $\mathrm{Vc}$, Klingen mit hochgezogener Spitze bzw. mit abgeknicktem Rückenverlauf, laufen vom 7./8. Jahrhundert bis in das 15. Jahrhundert durch (Abb 69 und 70).

Die Form Vd, die ein abgetreppter Rückenverlauf kennzeichnet, ist mit nur drei Messern des 14. bis 15. Jahrhunderts vertreten; zwei Exemplare aus Polen (Kat. Nr. $155,157)$ sind aber nicht so gut erhalten, daß die Form Vd als gesichert nachweisbar gelten kann. Das dritte Messer aus Arhus ist zwar ebenfalls nicht vollständig erhalten, die Formzuweisung ist hier allerdings sehr wahrscheinlich (Kat. Nr. 14). 
Die Form Ve, eine Klinge mit doppelt abgeknicktem Rückenverlauf, wurde bereits im Zusammenhang mit der Klingenform IIIb32 behandelt. An dieser Stelle wurden nur die Messer mit möglicherweise gerade verlaufenden Schneiden erfaßt.

Die Formen Vf und Vg sind im bearbeiteten Fundbestand nicht nachgewiesen worden.

Die geographische Verteilung der Messer mit den Klingenformen $\mathrm{Va}, \mathrm{Vb}$ und $\mathrm{Vc}$ zeigt deutlich eine Häufung im Bereich der polnischen und russischen Fundorte (Abb. 71).

Von 33 Messern der Form Va stammen 20 Messer aus Polen, zwei aus dem Gebiet der nordwestlichen ehemaligen Sowjetunion, sechs aus der ehemaligen DDR, drei aus der Bundesrepublik (alte Bundesländer) und zwei aus Skandinavien.

Von den drei Messern aus den alten Bundesländern wurden zwei in westslawischem Kontext gefunden (Kat. Nr. 25, 76), das dritte Exemplar ist in seiner formalen Zuweisung strittig (Kat. Nr. 143). Auch die beiden skandinavischen Messer (Birka, Kat. Nr. 5, 6) sind unsichere Rekonstruktionsvorschläge von in Scheiden befindlichen Grabfunden. Von den sechs Messern aus der ehemaligen DDR wurden vier ebenfalls innerhalb westslawischer Siedlungskontexte geborgen (Kat. Nr. 37, 52, 83, 132), ein Messer wurde aus der Recknitz gebaggert, ein weiteres ist nur fragmentarisch erhalten, die Rekonstruktion ist ebenfalls unsicher (Kat. Nr. 174, 175).

Die Form Va ist demnach nur sporadisch und weitgehend ungesichert außerhalb des slawischen Kulturkreises aufgetreten. Mit hoher Wahrscheinlichkeit handelt es sich um eine Klingenform, deren Verbreitung bis zum 12. und 13. Jahrhundert sowohl zeitlich als auch räumlich mit den Ausdehnungs- und Regressionsphasen der slawischen Bevölkerung im Untersuchungsraum einherzugehen scheint.

Die Beispiele aus der Zeit des 13. bis 14. Jahrhunderts außerhalb Polens sind durchweg problematisch in ihrer formalen Zuweisung, vor allem die Proportionen der Klingen unterscheiden sich von den möglicherweise westslawischen Vorläufern. Am ehesten finden sich die auslaufenden Formen der Form Va in dieser Zeit im polnischen Bereich, vor allem - wie Messer aus Rownina Dolna und Danzig zeigen (Kat. Nr. 63, 81, 105, 107) - in deutsch-polnischen Berührungszonen.

Zwei Beispiele aus Kente und Novgorod zeigen, daß die Form Va auch im ostslawischen und baltischen Bereich anzunehmen ist (Kat. Nr. 14, 56). 
Verbreitungskarte 2

Blatt 1

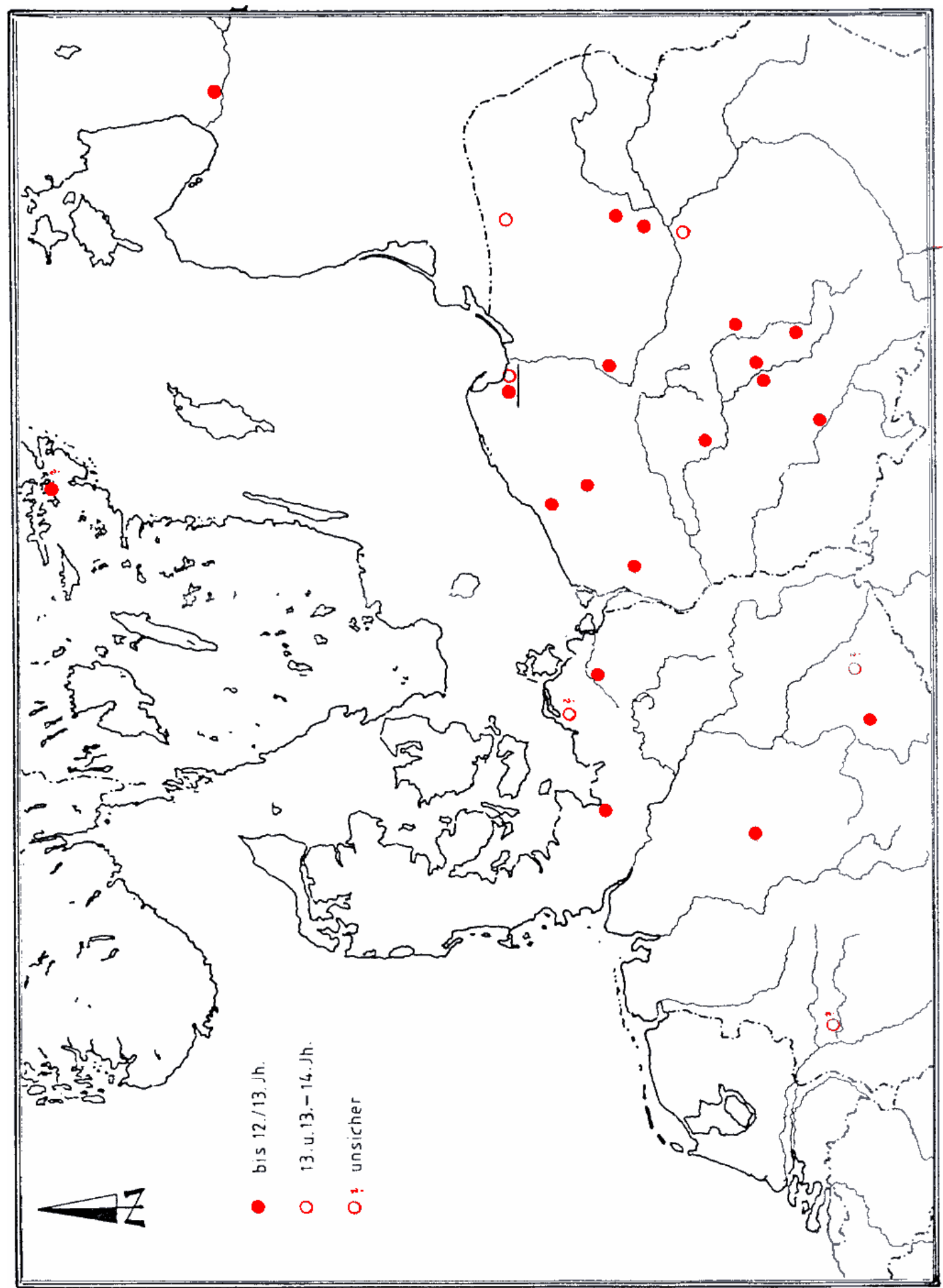

Abb. 71

Die Verbreitung der Klingenform Va vom 12. bis zum 14. Jahrhundert 
Die Formen $\mathrm{Vb}$ und $\mathrm{Vc}$ treten ebenfalls massiert in Polen und den angrenzenden nordwestrussischen Gebieten auf, dort wurden 14 von 20 Messern der Form Vb und 23 von 28 Messern der Form Vc gefunden.

Sie sind jedoch weder geographisch noch zeitlich dem westslawischen Kulturbereich allein zuzuordnen. Vor allem in Skandinavien wurden weitere Beispiele dieser Formen gefunden. Bis auf ein Messer des 12. Jahrhunderts aus Oslo (Kat. Nr. 15), einer Kombination aus den Formen Vb und IVf, sind es durchweg Messer des 14. bis 15. Jahrhunderts (Dänemark, Kat. Nr. 24, 26, 27, 69, 77). Ein Vergleich mit den Messern polnischer und nordwestrussischer Fundorte fällt aus den bereits angeführten quellenkritischen Gründen schwer, obwohl in diesem Fall ein unsicherer Beleg des 14. bis 15. Jahrhunderts aus Novgorod und ein weiteres Messer des 14. Jahrhunderts aus Stargard vorliegen (ehemalige UdSSR, Kat. Nr. 21, Polen, Kat. Nr. 9).

\subsubsection{Zusammenfassung der Ergebnisse zur Verteilung der häufiger auftretenden Klingenformen}

Die unter den jeweiligen Leitformen zusammengefaßten vier Klingenformen und ihre zahlreichen Variationsformen verteilen sich zeitlich, zum Teil auch räumlich unterschiedlich.

Messerklingen mit geradem Rücken und gebogener Schneide (Leitform Ib) nehmen vom 8. bis zum 12. Jahrhundert bis auf einen Umfang von fast $20 \%$ des Gesamtbestandes zu. Im Spätmittelalter geht ihre Anzahl leicht, im 15. und 16. Jahrhundert stark zurück, im 17. Jahrhundert wurde ein erneuter leichter Anstieg beobachtet.

Messerklingen mit gebogenem Rücken und gerader Schneide (Leitform Ilb) verteilen sich im Untersuchungsraum und -zeitraum nahezu entgegengesetzt. Ihr Bestand nimmt vom 8. bis zum 10. Jahrhundert ab. Im Verlauf des Hoch- und Spätmittelalters steigt der Bestand zum 13. Jahrhundert leicht an und fällt im 14. Jahrhundert wieder $\mathrm{ab}$.

Die Hauptverbreitungszeit dieser Klingenformen liegt im 15. und 17. Jahrhundert.

Schlanke Messerklingen mit teils zur Spitze abgeschrägten, teils gebogenen Kanten, die immer gebogen zur Spitze einfallen, scheinen mit fast 40\% im 9. und 10. Jahrhundert ihre erste Hauptverbreitung gefunden zu haben. Nach einem Rückgang auf unter $20 \%$ im 11. Jahrhundert wiederholt sich eine ähnliche Tendenz wie bei den Klingen unter der Leitform IIb. Der Anstieg nach dem 14. Jahrhundert setzt sich hier allerdings bis zum 16. Jahrhundert auf über 30\% fort, im 17. Jahrhundert fällt der Bestand auf unter 10\% zurück.

Regionale Unterschiede in den einzelnen Untersuchungsländern betreffen veränderte Tendenzen in einzelnen Zeitabschnitten: 
In Skandinavien verläuft die Entwicklung vom 10. bis zum 14. Jahrhundert nahezu gleichmäßig abnehmend, in Nordwestdeutschland geht der betreffende Bestand vom 15. bis zum 17. Jahrhundert gleichmäßig zurück.

Messerklingen mit gerade verlaufendem Rücken, der erst kurz vor der Spitze gebogen oder abgeschrägt zur Spitze einfällt, gehen allgemein vom 8. bis zum 14 . Jahrhundert leicht zurück.

Ihre Hauptverbreitungszeit setzt mit dem 15. Jahrhundert ein und erreicht im 17. Jahrhundert über $25 \%$ des Gesamtbestandes.

Auch hier wurden regional begrenzte Abweichungen festgestellt, die vor allem zeitliche Verschiebungen betreffen.

Messerklingen, deren Rückenverlauf kurz vor der Spitze konkav gebogen ist, sind vom 8. bis zum 14. Jahrhundert sicher nachgewiesen worden.

Mit 17,2\% und 15,4\% liegen ihre Höchstwerte im 11. und 12. Jahrhundert.

Die Regressionsphase seit dem 12. Jahrhundert hat regional unterschiedlich stattgefunden. Sie setzt im Norden des Untersuchungsraums eher und stärker ein, im Osten gehen diese Klingenformen erst nach dem 13. Jahrhundert zurück.

Messerklingen mit einem Rückenverlauf, der über zwei Drittel der Klingenlänge konkav gebogen ist, sind nur selten aufgetreten.

Sie sind sicher vom 8 . bis zum 15. Jahrhundert nachgewiesen worden, nur im 14. Jahrhundert wurden sie in allen Untersuchungsländern beobachtet.

Messerklingen, deren Rücken insgesamt konkav gebogen verläuft,treten sicher vom 8. bis zum 14. Jahrhundert auf. Sie sind ähnlich selten angetroffen worden wie die vorangegangenen Messer, in den niederländischen Fundbeständen konnten sie nicht nachgewiesen werden.

Im 15. Jahrhundert wurden Messerklingen mit konkaven Einsprüngen im Rücken vereinzelt angetroffen, regionale Begrenzungen ließen sich bisher nicht feststellen. 


\subsubsection{Die Klingenlängen}

Die Auswertung der Klingenlängen unterliegt in noch stärkerem Maße den unterschiedlichen Erhaltungszuständen der Messer als die Untersuchungen zu den Klingenformen.

In der Regel konnten die Maße stark korrodierter und abgebrochener Klingen nicht rekonstruiert werden, so daß zum einen die Anzahl der auswertbaren Maße niedriger ist als der Bestand ausgewerteter Messer, der sich auf Klingenformen und Griffkonstruktionen bezieht. Aus dem gleichen Grund finden die Minimalwerte, die neben den Maximalwerten, den Mittelwerten und den Standardabweichungen untersucht worden sind, in der Diskussion keine Berücksichtigung, da vor allem sie oft Verschliff oder den schlechten Erhaltungszustand einer Klinge repräsentieren.

Die Behandlung der Länge einer Messerklinge berührt zwei Themenbereiche:

Die Funktionsspanne des Messers und seine Abgrenzung zu Dolchmessern und Dolchen ${ }^{60}$.

Neben der Form einer Klinge und ihrer Breite und Nackenstärke ist das Längenmaß ein wichtiger Faktor, der Aufschluß über eine oder mehrere Funktionen eines Messers geben kann.

$\mathrm{Da}$ begleitende Informationen in den meisten Fällen fehlen, vorhandene Zusatzquellen wie bildliche Darstellungen nur in Auswahlmengen zur Verfügung stehen und die zahlreichen Schriftquellen zwar die Nahrungsmittel und Verhaltensweisen bei Tisch beschreiben, das seltener erwähnte Besteckinventar aber ohne die erwähnten Informationen kaum mit den vorhandenen Fundinventaren zu verbinden ist, kann an dieser Stelle lediglich nach Funktionsspannen und -veränderungen, nicht aber nach den einzelnen Funktionen direkt gefragt werden.

Mit dem Messermaß an einem Stadttor der niederländischen Stadt Deventer aus der zweiten Hälfte des 15. bis beginnenden 16. Jahrhunderts (Abb. 72a) liegt ein Hinweis für die maximal erlaubte Länge eines "Messers" $(40 \mathrm{~cm})$ innerhalb der Mauern einer frühneuzeitlichen Stadt vor, welches zunächst als Beleg für die tatsächlich durchgeführte Kontrolle im Rahmen der innerstädtischen Waffenbegrenzungen, verbote und weiterer Pazifizierungsmaßnahmen gelten darf.

Die Klingenlänge des Maßes beträgt $25 \mathrm{~cm}$. Sie umfaßt aus dem hier vorgelegten Bestand bis auf ein Dolchmesser (Abb. 72c) sowohl waffenartige Messer als auch Messer mit überlangen Klingen (persönliche Mehrzweck- oder Jagdmesser (?), sogenannte "Vorschneidemesser", Fleischermesser (?) oder Küchenmesser), deren Klingenlängen alle über $20 \mathrm{~cm}$ liegen (Abb. 72b, 72d, 72e-72i). 
Die Funktionszuweisung und damit eine Differenzierung dieser Messer ist bisher kaum möglich.

Bezeichnungen wie "Vorschneidemesser", die in der Literatur gerne dann vergeben werden, wenn das jeweilige Messer unter anderem aufgrund einer relativ überlangen Klinge (gemessen am Restbestand vorhandener oder ausgegrabener Messer) auffällt, sind kaum hinreichend zu belegen:

So besitzt das "Vorschneidemesser" aus Store Valby (Dänemark, Kat. Nr. 36), welches als klösterliches Geschenk an einen Bauernhof interpretiert wird, nur eine Klingenlänge von $15 \mathrm{~cm}$.

Hier machen die Klingenform und der verzierte Griff eine Zuweisung des Messers zu einer gehobenen Position an der Tafel wahrscheinlich, ohne daß es sich dabei zwangsläufig um ein Servier- oder Vorschneidemesser handeln muß.

Auch die Messer aus Lund (Abb. 72e) und von der Burg Altena (Abb. 72g) sind im Bereich der Nahrungsmittelverarbeitung anzusiedeln, da ihre Klingenformen und längen eine jagdliche oder multifunktionale Verwendung wenig wahrscheinlich machen.

Die Spanne zwischen $20 \mathrm{~cm}$ und 26,4 cm, die sich an den ausgewählten Beispielen dokumentiert, umfaßt neben diesen nur noch einige Dolch- bzw. Stechmesser, die ohne Datierungsansatz aus nordostdeutschen Flüssen gebaggert worden sind.

Für die Auswertung der Klingenlängen wurden die Waffen und überlangen Messer ausgeklammert, da sie im ausgewerteten Bestand nur sechs Belege umfassen und sich in der Regel deutlich von den übrigen Messern unterscheiden.

Zum Vergleich werden in der Auswertungsgraphik zum gesamten Untersuchungsraum die Werte mit den in Abbildung 72 vorgestellten Messern zusätzlich aufgeführt, repräsentative Veränderungen können daraus aber nicht abgeleitet werden (Abb. 73, untere Tabelle).

Die Verteilung des Gesamtbestandes an ausgewerteten Klingenmaßen zeigt zwei tendenzielle Veränderungen nach dem 10. und nach dem 16. Jahrhundert (Abb. 73, Diagramm und obere Tabelle).

Die Mittelwerte liegen im gesamten Untersuchungszeitraum deutlich unter $11 \mathrm{~cm}$.

Vom 8. zum 10. Jahrhundert ist eine rückläufige Entwicklung von 9,5 cm auf $8,2 \mathrm{~cm}$ zu beobachten, die, leicht versetzt, von einem Rückgang der Maximalwerte von $17 \mathrm{~cm}$ auf 15,3 cm begleitet wird. Auch die Standardabweichung geht in ihren oberen Extremwerten zurück und erreicht im 10. Jahrhundert ihren niedrigsten Wert. Mit diesem allgemeinen Rückgang aller betreffenden Maße und Werte deutet sich ein Wandel und eine Einschränkung des funktionalen Rahmens der untersuchten Messer an, der im 10. Jahrhundert seinen Abschluß gefunden hat. 


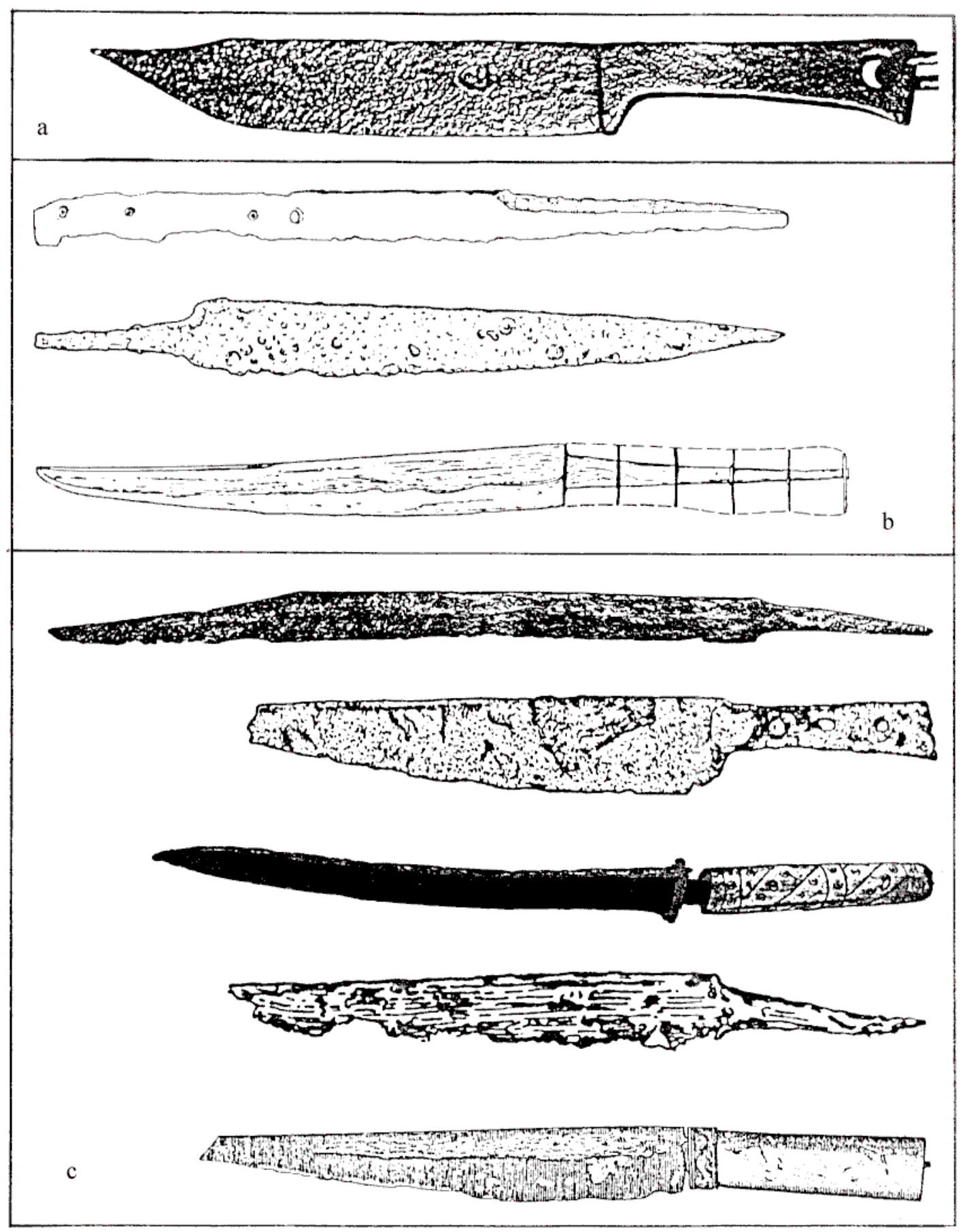

$A b b .72$

Messermaß (a), Dolch- und Stechmesser $(b-d)$ und "Küchen-" bzw. "Vorschneidemesser" (e-i) mit Klingenlängen über $20 \mathrm{~cm}$ 


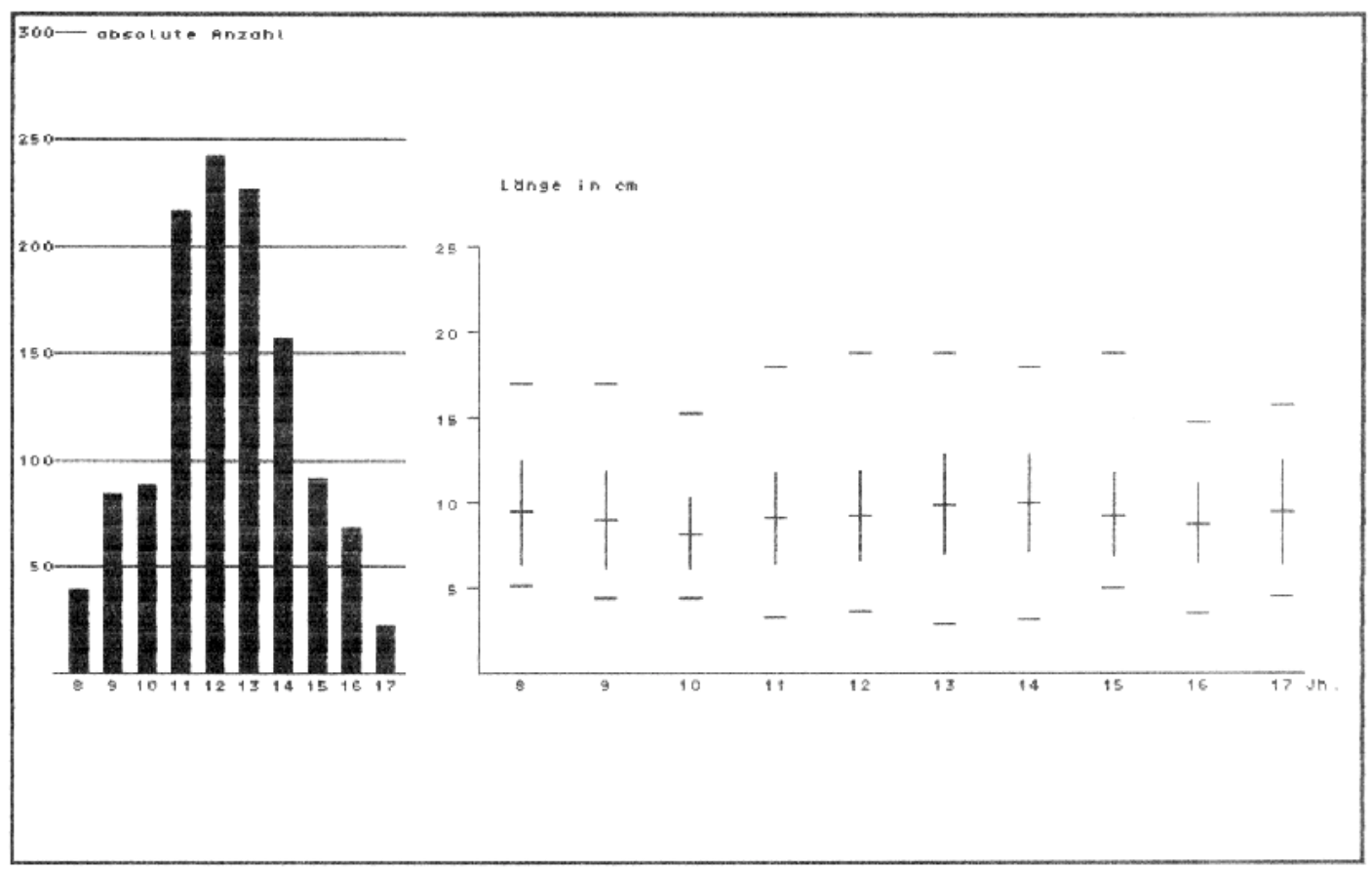

$\begin{array}{rrrrrrrrrrl}8 . & 9 . & 10 . & 11 . & 12 . & 13 . & 14 . & 15 . & 16 . & 17 . & \text { Jh. } \\ 39 & 84 & 88 & 217 & 242 & 227 & 157 & 91 & 68 & 22 & \text { Anzahl } \\ 5.20 & 4.40 & 4.40 & 3.36 & 3.70 & 2.90 & 3.20 & 5.00 & 3.50 & 4.50 & \text { Minimum } \\ 17.00 & 17.09 & 15.30 & 18.00 & 18.80 & 18.80 & 18.00 & 18.80 & 14.80 & 15.80 & \text { Maximum } \\ & & & & & & & & & & \\ 9.53 & 9.07 & 8.20 & 9.15 & 9.28 & 10.00 & 10.04 & 9.37 & 8.86 & 9.52 & \text { Mittelwert } \\ 3.05 & 2.82 & 2.06 & 2.65 & 2.63 & 2.95 & 2.84 & 2.46 & 2.28 & 3.08 & \text { Standardabweichung }\end{array}$

$\begin{array}{rrrrrrrrrrl}8 . & 9 . & 10 & 11 . & 12 . & 13 . & 14 . & 15 . & 16 . & 17 . & \text { Jh. } \\ 39 & 84 & 88 & 218 & 242 & 230 & 160 & 94 & 69 & 22 & \text { Anzahi } \\ 5.20 & 4.40 & 4.40 & 3.36 & 3.70 & 2.90 & 3.20 & 5.00 & 3.50 & 4.50 & \text { Minimani } \\ 17.00 & 17.00 & 15.30 & 23.80 & 18.80 & 21.00 & 21.00 & 25.90 & 25.00 & 15.80 & \text { Maxinim } \\ 9.53 & 9.07 & 8.20 & 9.21 & 9.28 & 10.14 & 10.24 & 9.77 & 9.10 & 9.52 & \text { Mittelwert } \\ 3.05 & 2.82 & 2.06 & 2.82 & 2.63 & 3.17 & 3.16 & 3.31 & 2.98 & 3.08 & \text { Standardabweichung }\end{array}$

Abb. 73 Die Entwicklung der Klingenlängen im gesamten Untersuchungsraum mit (untere Tabelle) und ohne Waffen und Messer mit Klingenlängen über $20 \mathrm{~cm}$ (Graphik und obere Tabelle) 
Welcher Art diese Veränderungen in funktionaler Hinsicht waren, ob sie zum Beispiel im Zusammenhang mit der Veränderung der Bewaffnung im Frühmittelalter standen oder auf Rohstoffknappheit ${ }^{61}$ zurückzuführen sind, läßt sich bisher nicht beantworten.

Das Hoch- und Spätmittelalter grenzt sich deutlich zu den vorangegangenen und nachfolgenden Jahrhunderten ab.

Die Mittelwerte nehmen in zwei Schüben zu: im 11. und 12. Jahrhundert auf über 9 $\mathrm{cm}$, im 13. und 14. Jahrhundert auf über $10 \mathrm{~cm}$. Auch die Standardabweichungen sowie ihre oberen Extremwerte nehmen in diesen Abschnitten $\mathrm{zu}$, die Maximalwerte der Klingenlängen steigen im 11. Jahrhundert auf $18 \mathrm{~cm}$, im 12 . und 13. Jahrhundert liegen sie bei $18,8 \mathrm{~cm}$.

Der Anstieg der Mittelwerte belegt eine Zunahme der Klingenlängen um durchschnittlich fast $2 \mathrm{~cm}$. Parallel zu den beiden zeitlichen Abschnitten, in denen diese Zunahme erfolgte, wächst die Zahl der Abweichungen von diesen Mittelwerten, während die Höchstwerte sprunghaft ansteigen und vom 11. bis 14. Jahrhundert in etwa gleich bleiben.

Damit erscheint das Hoch- und Spätmittelalter als ein Zeitraum, in dem erhebliche Größenunterschiede und eine zunehmende Menge an unterschiedlichen Klingenlängen auf eine wachsende Ausweitung des funktionalen Rahmens hinweisen. Diese Ausweitung berührt nachweislich im zweiten Abschnitt auch den Anwendungsraum von Kurzwaffen:

Ulrich Schoknecht hat anhand mecklenburgischer Nierendolche zwei Gruppen von Kurzwehren vorgestellt, deren Durchschnittslängen zwischen $42,5 \mathrm{~cm}$ und $30,9 \mathrm{~cm}$ bzw. 29,0 cm und 18,4 cm liegen; die Gruppe der Dolche mit den kürzeren Klingen reicht in ihren unteren Extremwerten von 15,6 cm bis 22,5 $\mathrm{cm}^{62}$.

Die Klingenlängen der Messer aus dem ausgewerteten Bestand liegen in ihren Maximalwerten alle unter $20 \mathrm{~cm}$, die Brührungszone zwischen den von Schoknecht vorgestellten Dolchen und den hier besprochenen Messern liegt zwischen 15,6 cm und 18,8 cm; letzterer Wert wird jeweils im 12. und 14. Jahrhundert erreicht.

Ohne die Diskussion um die Klingenformen von Messern, Dolchmessern und Dolchen läßt sich die Berührungszone von Werkzeug und Waffe nicht genau umreißen. Auch ohne die ausgeklammerten, durch Klingenform, -länge und (bei vollständiger Erhaltung) Heftgestaltung gekennzeichneten Dolchmesser, kleineren Nierendolche, "pooke" ${ }^{63}$ etc. fällt demnach ein Teil der behandelten Messer noch in die Längenzonen von Kurzwaffen. Auch ohne die formalen Kriterien einer dolchartigen Waffe zu erfüllen, mögen einige von ihnen dennoch "mißbraucht" worden sein.

Das belegt wiederum das Deventer Längenmaß (Abb. 72a), welches nach den von Schoknecht besprochenen Längengruppen und den hier ermittelten Maßen wahr-

\footnotetext{
61 Vgl. Kapitel 9, Abschnitt 1.2

62 Schoknecht 1980, S. 209 ff, sowie Abb. 8, S. 227

63 Vgl. die betreffenden Abschnitte, unter anderem die Diskussion um den Begriff "pook", in Kapitel 9, Abschnitt 1.3 und 2
} 
scheinlich nur die Gruppe der längeren Kurzwehren ausschloß. Die gewählte Form des Maßes entspricht aber nicht einer Waffe, sondern eher einem Werkzeug, als Stichwaffe wäre es mit der zu diesem Zeitpunkt bereits nicht mehr üblichen Klingenform trotz der Klingenlänge von fast $25 \mathrm{~cm}$ unbrauchbar gewesen.

Die Wahl gerade dieser Form eines Griffzungenmessers und die in zeitgenössischen niederländischen Abbildungen oft dargestellten "Bauernwaffen" ähnlicher Form scheinen aber zu belegen, daß die Grauzone zwischen Waffen und Messern erheblich war und allein durch Längenmaße nicht zu erhellen ist.

Die oft zitierte Auffassung, mittelalterliche Messer seien im Gegensatz zu neuzeitlichen Tafelmessern eher als Mehrzweckmesser geführt worden ${ }^{64}$, läßt sich mit dem vorliegenden Ergebnis nicht belegen.

Es ist im Gegenteil eher davon auszugehen, daß die Vielfalt von Messern mit verschiedenen und extrem auseinanderliegenden Klingenlängenmaßen (im 12. Jahrhundert beträgt der Maximalwert mehr als das Doppelte des Mittelwerts), sowie die bereits beschriebene Vielfalt an Klingenformen mit einer hohen Anzahl verschiedenartiger und $\mathrm{zu}$ begrenzten Funktionen benutzten Messern korrespondiert.

Der Beginn dieser Vielfalt im 11. Jahrhundert, der auch vor dem Hintergrund des Abklingens einiger Klingenformen nach dem 10. Jahrhundert zu betrachten ist, scheint eher die von Breivik und Christensen geäußerte Auffassung zu belegen, daß das an der Seite getragene Mehrzweckmesser, welches sich bis zur Aufgabe der Beigabensitte in Gräbern findet, nach $1000 \mathrm{n}$. Chr. außerhalb Skandinaviens in fast ganz Europa durch eine große Anzahl von Spezialmessern (alltäglicher Gebrauch, Küche, Werkstatt und Tafel) und Klappmessern ersetzt worden sei ${ }^{65}$.

Diese Auffassung muß aber ebenfalls differenziert werden, und zwar vor allem hinsichtlich der Tatsache, daß in völkerwanderungszeitlichen Gräbern unabhängig regionaler Faktoren, oft aber abhängig von der Reichhaltigkeit der Gesamtausstattung neben mehreren Waffen auch mehr als ein Messer auftritt. Diese unterscheiden sich durchaus voneinander und geben eher Aufschluß über die soziale Stellung ihres ehemalige Besitzers, der es sich leisten konnte, beispielsweise neben Spatha, Sax und mehreren Messern einen Satz goldgriffverzierter, der Tafel zugerechneter Messer zu besitzen ${ }^{66}$.

Daß diese Vielfalt vor dem Hochmittelalter selten und auf begüterte Personenkreise begrenzt war, während sie nach dem 10. Jahrhundert verbreiteter anzutreffen ist, begleitet von einer Zunahme von Spezialformen und Größenunterschieden, die nach dem 12. Jahrhundert noch einmal in Bewegung zu geraten scheinen, läßt sich als Ergebnis festhalten.

\footnotetext{
$64 \quad$ Vgl. Kapitel 9, Abschnitt 2.1

65 Breivik o.J. Christensen 1983

66 Vgl. Kapitel 6, Abschnitt 2.4.3 und Pirling 1986
} 
$\mathrm{Da}$ darunter auch weiterhin multifunktionale Messertypen wie das skandinavische Tollekniv auftreten, ist sehr wahrscheinlich, vor allem unter Berücksichtigung der trotz fortschreitender Verstädterung und landwirtschaftlicher Intensivierung im gesamten Mittelalter nicht geringen Lebensbereiche, in denen Menschen am Rande der Zivilisation lebten, vor allem im Bereich der Neukolonisation.

Auch ein gewisses Stadt-Land-Gefälle und die langwierigen Distanzüberwindungen (Wallfahrten, Handelsreisen u.ä.) werden zu berücksichtigen sein. In diesen Fällen werden die Betroffenen weiterreichende Anforderungen an ihre Waffen und Werkzeuge gestellt haben, die auch von den jeweiligen naturräumlichen Gegebenheiten abhingen.

Um derartige Differenzierungen, die Aufschluß über die in Betracht kommenden Funktionen geben könnten, durchführen zu können, wird es notwendig sein, neben den großen Fundmengen aus mittelalterlichen Städten auch die Bestände aus abseits gelegenen Klöstern, Dörfern in neubesiedelten Gegenden und Siedlungseinheiten in Streulage aufzuarbeiten, um festzustellen, ob sich hier formale und Größenunterschiede ergeben. Erste vergleichende Untersuchungen zu dieser Fragestellung werden im Anschluß an die Darstellungen zu Verzierungsarten und Klingenmarken vorgestellt ${ }^{67}$.

In den letzten drei Jahrhunderten des Untersuchungszeitraums verändern sich die untersuchten Werte erneut (Abb. 73).

Nach dem 14. Jahrhundert gehen die Mittelwerte um über einen Zentimeter zurück auf $8,8 \mathrm{~cm}$ im 16. Jahrhundert, zum 17. Jahrhundert steigt der Wert wieder auf 9,5 $\mathrm{cm}$ an.

Die Maximalwerte fallen erst nach dem 15. Jahrhundert um $4 \mathrm{~cm}$ auf $14,8 \mathrm{~cm}$ im 16. und $15,8 \mathrm{~cm}$ im 17. Jahrhundert.

Die Standardabweichung geht im 15. und 16. Jahrhundert ebenfalls leicht zurück, erreicht aber im 17. Jahrhundert ihren Höchstwert.

Somit teilt sich der Zeitraum der frühen Neuzeit in eine Regressionsphase, in der sich die mittelalterlichen Maße leicht versetzt vermindern. Auch die Anzahl der Abweichungen geht zurück, so daß im 16. Jahrhundert der Funktionsrahmen der bearbeiteten Messer erheblich eingeschränkt erscheint.

Die Zunahme von Abbildungen alltäglicher häuslicher Szenen und der Beginn von Genredarstellungen erlaubt vor allem seit dem 16. Jahrhundert, die Masse der angetroffenen Messer als Tafelmesser anzusprechen, die in fortschreitendem Maße einer standardisierten Produktion entstammen ${ }^{68}$. Dieses gilt vermehrt für den Bestand des 17. Jahrhunderts, der, das zeigt die Annäherung des Maximalwertes an den oberen Grenzwert der Standardabweichung, seine Ausdehnungsgrenze bezüglich der Klingenlänge zwischen 14,6 und 15,8 cm findet. Zwischen diesen Werten und dem

\footnotetext{
$67 \quad 67$ Vgl. Kapitel 6, Abschnitt 2.7

68 Vgl. Kapitel 9, Abschnitt 2
} 
Mittelwert von 9,5 cm befindet sich ein deutlich größerer Bestand an unterschiedlich langen Messerklingen als in den beiden Jahrhunderten davor.

Da sich die produktionstechnisch bedingte Standardisierung von Tafelmessern unabhängig von einer Zunahme von Gestaltungsmöglichkeiten im 17. Jahrhundert fortsetzt, belegt diese Zunahme einen erneuten Spezialisierungsprozeß, der durch das hier behandelte Material auf die Nahrungszubereitung (Küche) und Nahrungsaufnahme (Tafel) zu konzentrieren ist ${ }^{69}$.

Zusammenfassend lassen sich anhand der ausgewerteten Klingenlängen des Gesamtbestandes vier Entwicklungsphasen beobachten. Die Trennungslinien verlaufen nach dem 10., nach dem 14. und nach dem 16. Jahrhundert.

Einem Rückgang aller untersuchten Werte bis zum 10. Jahrhundert, begleitet von den bereits beschriebenen Veränderungen in den Mengen einiger Klingenformen, folgt vom 11. bis zum 14. Jahrhundert eine Phase der Zunahme dieser Werte.

Die zunehmende Vielfalt der Klingenformen, die während dieses Zeitraums ebenfalls festgestellt wurde, findet also ihre Entsprechung ebenfalls in einer Zunahme von Messern unterschiedlicher Klingenlängen sowie von Messern mit erheblich längeren Klingen. Der Einbruch im 15. und 16. Jahrhundert ist signifikant. Er betrifft eine Abnahme von Messern unterschiedlicher Klingenlänge sowie einen Rückgang des Maximal- und Mittelwertes und entspricht wiederum der Verringerung der Anzahl verschiedener Klingenformen im gleichen Zeitraum.

Der um 1450 exemplarisch am Beispiel der niederländischen Funde beobachtete sprunghafte Anstieg verschiedener Griffkonstruktionen, der nach 1550 wieder zurückgeht, ohne den gleichzeitig einsetzenden Gestaltungsschub im Bereich der Verzierungselemente zu berühren, führt im 17. Jahrhundert zu einer breit gefächerten Auswahl an spezialisierten, produktionstechnisch standardisierten Messern, deren durchschnittliche Länge zwar zunimmt, ohne jedoch die Maximalwerte des Mittelalters wieder zu erreichen.

Ihr äußerer Funktionsrahmen ist begrenzt auf den Bereich der Küche und Tafel.

Im Vergleich mit den Auswertungen der Klingenlängen in den einzelnen Untersuchungsländern zeigen sich deutliche Unterschiede, die mit einiger Wahrscheinlichkeit auch Traditionsgebiete und Innovationszentren voneinander trennen.

Wenn Christensen und Breivik das Tollekniv als vor- und frühgeschichtliches Universalmesser nach 1000 n. Chr. nur noch in Skandinavien antreffen, korrespondiert diese Auffassung mit dem Ergebnis der Auswertung von in Skandinavien ausgegrabenen Messern (Abb. 74).

69 Dieser Spezialisierungsprozeß ist bei fast allen Besteck- und Gefäßformen zwischen dem ausgehenden 16. und beginnenden 17. Jahrhundert zu beobachten (v.a. bei Hohlgläsern und Gefäßkeramik), er deutet sich ebenfalls mit einem deutlichen Bruch zwischen 1550 und 1600 bei der Entwicklung der Messergriffe an (vgl. Kapitel 6, Abschnitt 2.3) 
Bis zum 10. Jahrhundert liegt hier ein Bestand vor, der in unveränderter Weise eine erheblich geringere Durchschnittslänge bei vergleichsweise niedrigen Abweichungen aufweist. Nur wenige Messer erreichen die Maximallänge von 15,3 cm.

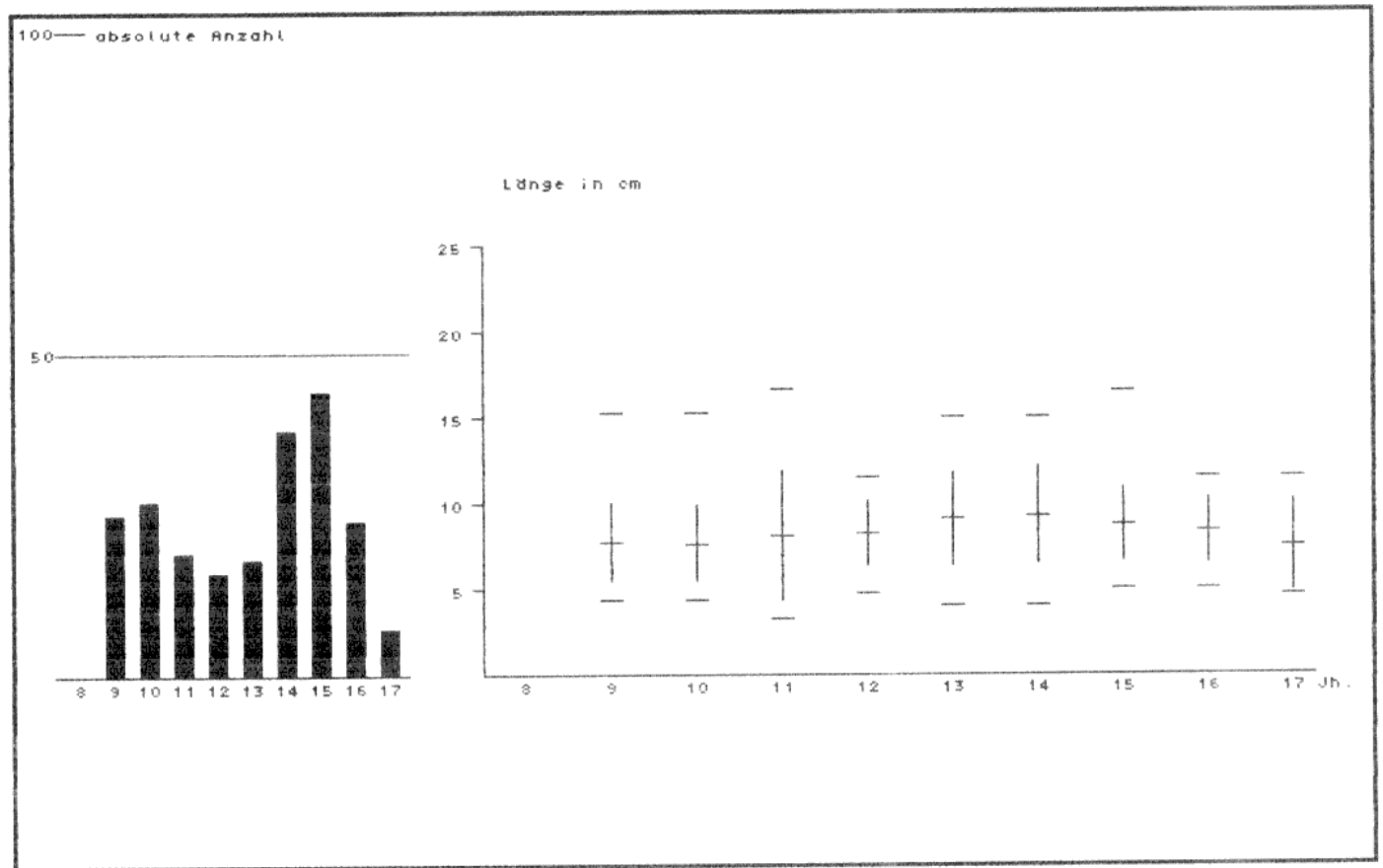

$\begin{array}{rrrrrrrrll}\text { 8. } 9 . & 10 . & 11 . & 12 . & 13 . & 14 . & 15 . & 16 . & 17 . & \text { Mh. } \\ 25 & 27 & 19 & 16 & 18 & 38 & 44 & 24 & 7 & \text { Anzahl } \\ 4.40 & 4.40 & 3.36 & 4.80 & 4.10 & 4.00 & 5.00 & 5.10 & 4.70 & \text { Minimum } \\ 15.30 & 15.30 & 16.70 & 11.50 & 15.00 & 15.00 & 16.50 & 11.50 & 11.50 & \text { Maximum } \\ & & & & & & & & & \\ 7.78 & 7.73 & 8.20 & 8.30 & 9.16 & 9.33 & 8.82 & 8.40 & 7.56 & \text { Mittelwert } \\ 2.27 & 2.19 & 3.73 & 1.87 & 2.70 & 2.80 & 2.08 & 1.85 & 2.63 & \text { Standardabweichung }\end{array}$


Im 11. Jahrhundert nimmt die Zahl der Abweichungen und die Maximallänge zu, ohne daß ein vorangegangener Einbruch, der auf eine grundlegende Veränderung des funktionalen Rahmens hinweisen könnte, stattgefunden hat.

Um zwei Jahrhunderte versetzt zum überregional ermittelten Allgemeinbild erscheint dieser Einbruch im 12. Jahrhundert, allerdings ohne daß sich der Durchschnittswert erheblich verändert.

Vom 13. bis zum 15. Jahrhundert erscheint eine ähnliche Entwicklung wie im gesamten Untersuchungsraum, der Durchschnittswert nimmt im 15. Jahrhundert nur leicht ab.

Diese Entwicklung setzt sich bis zum 17. Jahrhundert fort, dem bisherigen Ergebnis entsprechen aber der Rückgang der Maximalwerte und die Zunahme der Standardabweichung im 17. Jahrhundert.

Insgesamt erscheinen die Veränderungen in Skandinavien weniger durchgreifend und zeitlich stark versetzt. Die Mittelwerte steigen langsam vom 9. bis zum 14. Jahrhundert an und gehen danach bis zum 17. Jahrhundert kontinuierlich zurück. Ein formaler und funktionaler Wandel scheint sich nur zögernd vollzogen zu haben.

Der aussagefähige Zeitraum in den Niederlanden zeigt den Rückgang der durchschnittlichen Klingenlänge schärfer (Abb. 75).

Hervorzuheben ist der bereits im 14. Jahrhundert angetroffene Rückgang des Maximalwertes, der sich im 15. Jahrhundert fortsetzt.

Die Zunahme der Standardabweichung und der erneute Anstieg der Maximallänge erfolgen bereits im 16. Jahrhundert, dem im 17. Jahrhundert eine Anhebung des Durchschnittswertes, welche der allgemein beobachteten Entwicklung entspräche, $\mathrm{zu}$ folgen scheint.

In den Niederlanden setzen die Veränderungen, die im Gesamtbestand nacheinander im 15. und 16. Jahrhundert erfolgen, bereits im 14. Jahrhundert ein.

Im 16. Jahrhundert beginnen hier erneut Entwicklungen, die im übrigen Bestand erst einhundert Jahre später anzutreffen sind.

Die Auswertungen der Klingenmaße von Messern aus Fundorten der Bundesrepublik und Polens bestätigen im wesentlichen die Entwicklung, die sich in der Analyse des gesamten Bestandes dokumentiert.

Regionale Unterschiede, soweit sie nicht durch unterrepräsentierte Mengen verursacht wurden, betreffen Abweichungen in der Entwicklung der Maximallängen oder den Zeitraum vor dem 10. Jahrhundert.

So zeigt zum Beispiel die Entwicklung der Klingenlängen im Bestand aus dem Untersuchungsbereich Nordwestdeutschlands eine Tendenzabweichung vom 8. zum 9. Jahrhundert, etwas höhere Mittelwerte vor dem 15. Jahrhundert und einen signifikanten Rückgang der Maximallängen bereits im 15. Jahrhundert (Abb. 76). 


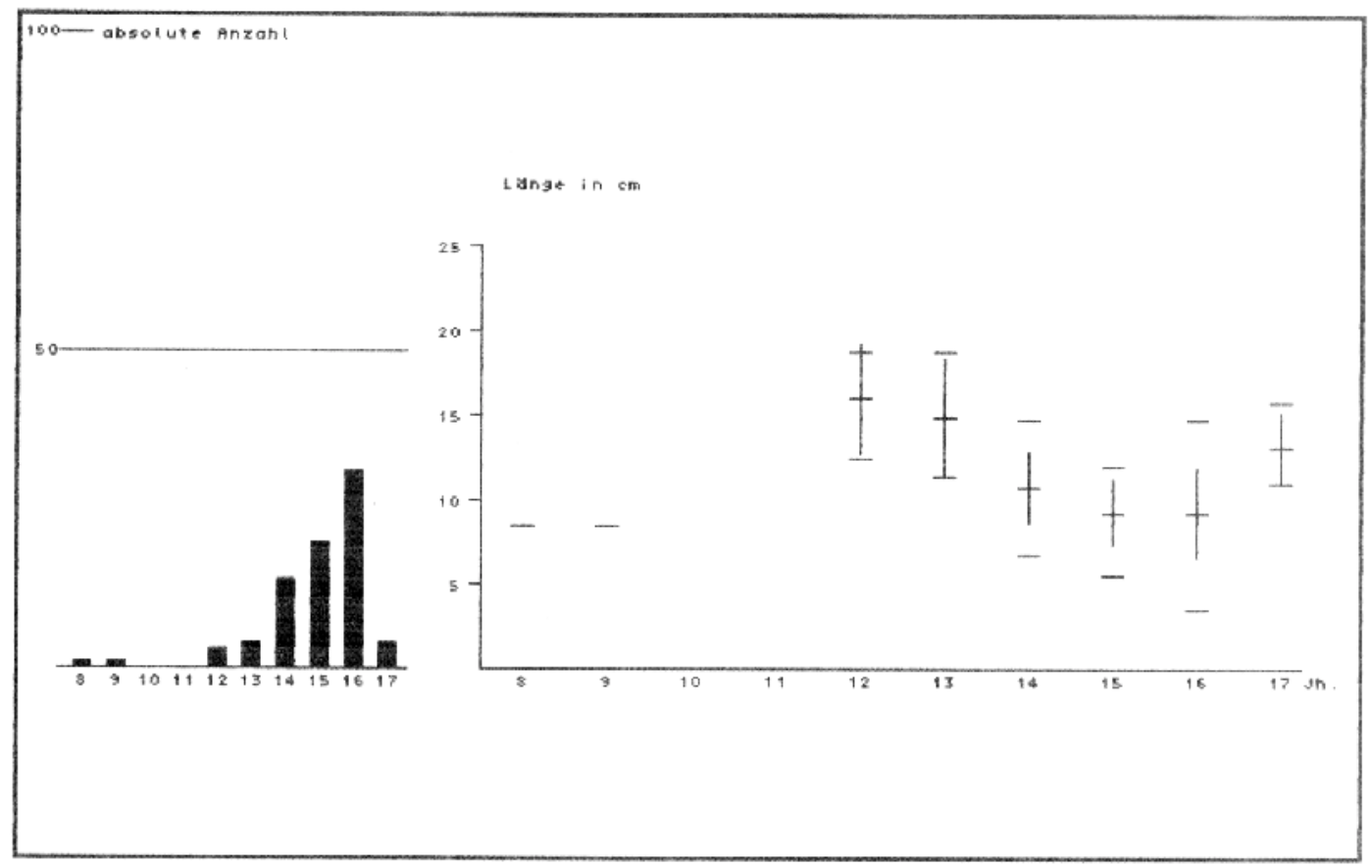

8. 9. 10. 11. 12. 13. 14. 15. 16. 17 . Th.

$\begin{array}{rr}1 & 1 \\ 8.50 & 8.50 \\ 8.50 & 8.50 \\ 8.50 & 8.50\end{array}$

$\begin{array}{rrrrrrl}3 & 4 & 14 & 20 & 31 & 4 & \text { Anzahl } \\ 12.50 & 11.40 & 6.75 & 5.60 & 3.50 & 11.10 & \text { Minimum } \\ 18.75 & 18.75 & 14.80 & 12.00 & 14.80 & 15.30 & \text { Maximm } \\ & & & & & & \\ 16.08 & 14.91 & 10.85 & 9.34 & 9.30 & 13.18 & \text { Mittelwert } \\ 3.22 & 3.52 & 2.97 & 1.94 & 2.61 & 1.97 & \text { Standandabweichung }\end{array}$

Abb. 75

Die Entwicklung der Klingenlängen in den Niederlanden 


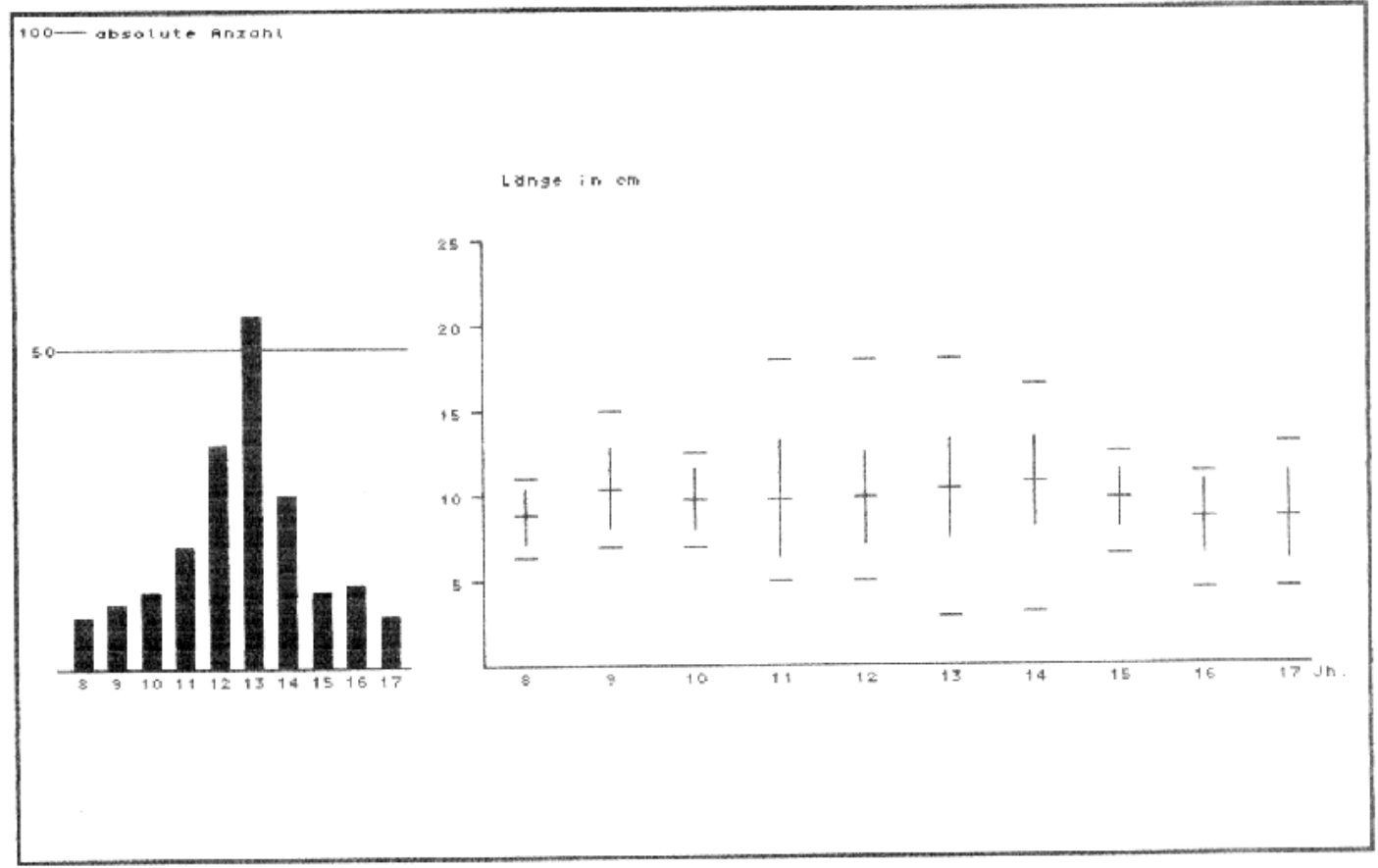

8. 9. 10. 11. 12. 13. 14. 15. 16. 17. Jh.

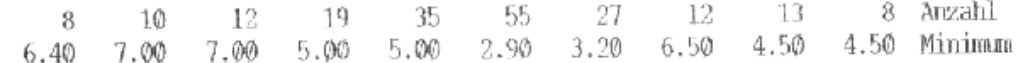

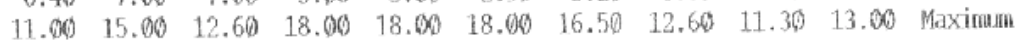

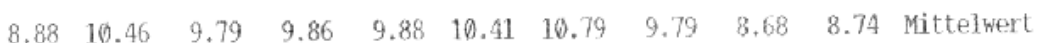

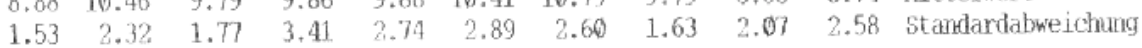

$A b b .76$

Die Entwicklung der Klingenlängen in Nordwestdeutschland 


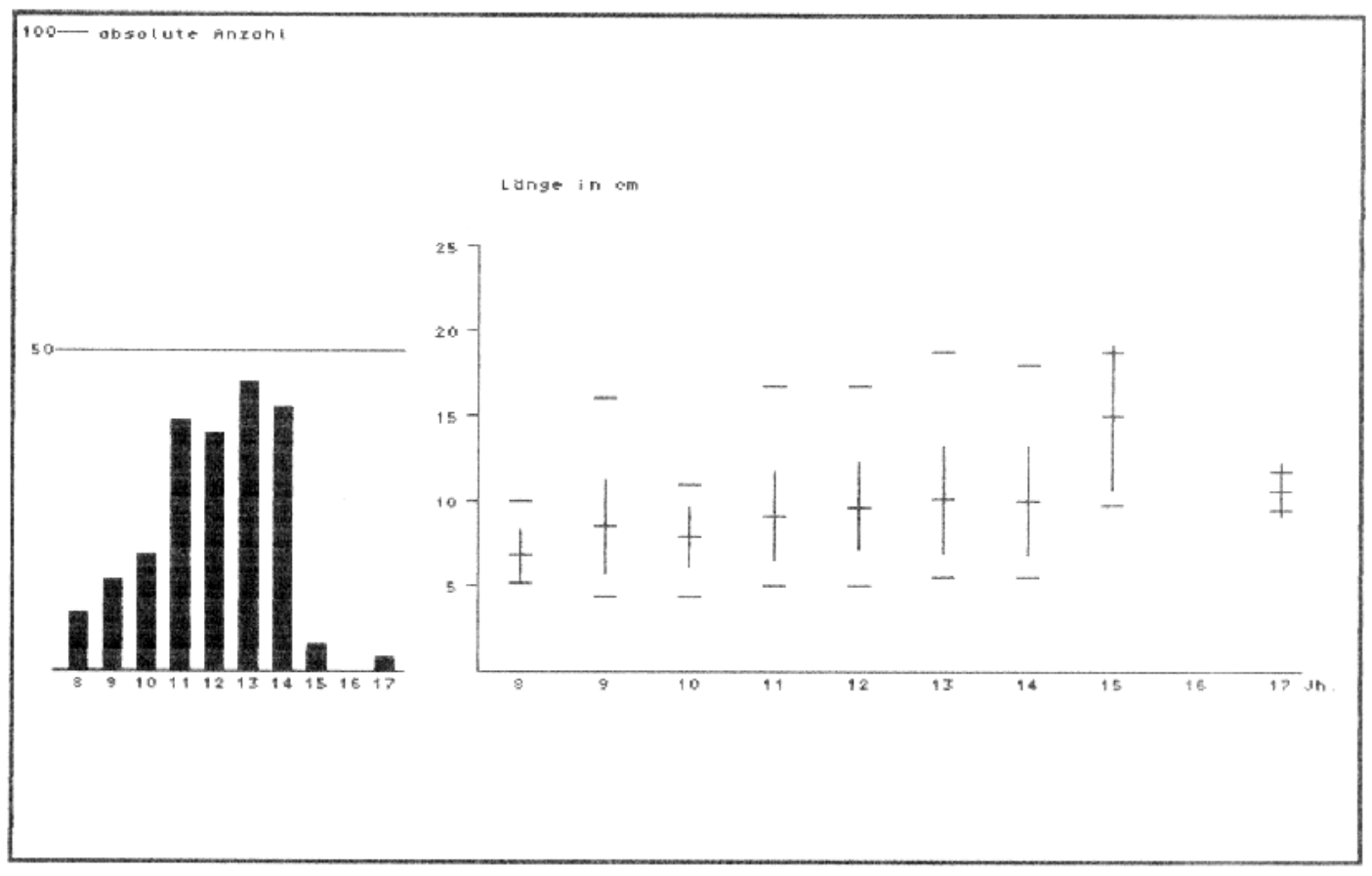

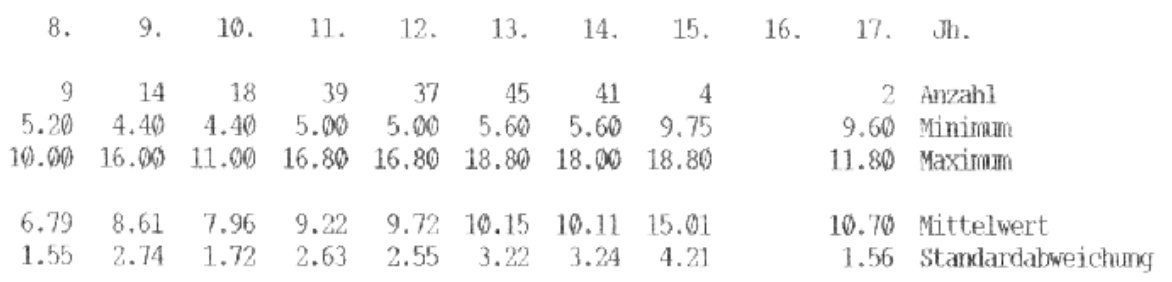

Abb. 77

Die Entwicklung der Klingenlängen in Ostdeutschland 
Ethnische Faktoren und historische Ereignisse scheinen die auffällige Veränderung vom 10. zum 11. Jahrhundert nicht beeinflußt zu haben.

Die Entwicklung der untersuchten Werte in den östlichen Bundesländern, deren Bestände vor dem 12. Jahrhundert vor allem westslawischen Siedlungskontexten entnommen worden sind, zeigt zwar absolut leicht unterschiedliche Maße, tendenziell entspricht sie jedoch auch in dem leichten Sprung nach dem 12. Jahrhundert dem Bild der Klingenwerte aus den nordwestlichen Bundesländern (Abb. 77).

Der Zeitraum vor dem 11. Jahrhundert konnte zwar in einigen Untersuchungsländern nur unvollständig oder unterrepräsentiert dargestellt werden, für das 9. und 10. Jahrhundert ergaben sich aber Hinweise auf regionale Unterschiede, die möglicherweise durch weiteres Fundmaterial verifiziert werden können.

\begin{tabular}{|lcc|}
\hline & 9. Jahrhundert & 10. Jahrhundert \\
Skandinavien & $7,78 \mathrm{~cm}$ & $7,73 \mathrm{~cm}$ \\
Polen & $7,46 \mathrm{~cm}$ & $8,11 \mathrm{~cm}$ \\
ehem. D D R & $8,61 \mathrm{~cm}$ & $7,96 \mathrm{~cm}$ \\
alte BRD & $10,46 \mathrm{~cm}$ & $9,79 \mathrm{~cm}$ \\
N iederlande & $10-12 \mathrm{~cm}$ & --- \\
\hline
\end{tabular}

Abb. 78 Die Mittelwerte der Klingenlängen im 9. und 10. Jahrhundert

Da das bearbeitete niederländische Fundmaterial in diesem Zeitraum nicht ausreichend aussagekräftig war, wurde der Durchschnittswert aller Messerklingen aus Dorestad (8. bis 9. Jahrhundert) mit 10 bis $12 \mathrm{~cm}^{70}$ mitberücksichtigt.

Die Übersicht zeigt bezüglich der Durchschnittslängen von Messerklingen ein Gefälle, welches vor allem im 9. Jahrhundert deutlich wird (Abb. 78):

In den Beständen aus niederländischen und nordwestdeutschen Fundorten liegen die Werte um bzw. über $10 \mathrm{~cm}$, in den fast ausschließlich westslawischen Beständen aus den östlichen Bundesländern unter $9 \mathrm{~cm}$. In den aus polnischen Fundorten stammenden Beständen liegt der Durchschnittswert unter $8 \mathrm{~cm}$, ebenso bei den skandinavischen Messern.

Im 10. Jahrhundert verändert sich die Reihenfolge leicht, da bei bis auf den in Polen gefundenen Messern alle Klingenlängen leicht zurückgehen.

Abgesehen von den skandinavischen Fundbeständen liegt somit eine Tendenz vor, die sich in der allgemeinen Entwicklung der Klingenlängen und der zeitlichen Verteilung der meisten Klingenformen so nicht nachweisen läßt.

70 Vgl. Katalogteil Niederlande, Kat. Nr. 19, Anmerkung 1 
Die Durchschnittslängen in den einzelnen Untersuchungsländern liegen auch nach dem 10. Jahrhundert in den östlichen Untersuchungsräumen und in Skandinavien unter denen der westlichen Gebiete.

Unabhängig von den verschiedenen Abweichungsmengen und den unterschiedlichen Maximalwerten scheint sich mit der Hauptmenge der durchschnittlichen Klingenlängen anzudeuten, daß im Nordwesten des Untersuchungsraumes die Klingen im gesamten Bearbeitungszeitraum länger waren als in Ostdeutschland, Polen und Skandinavien. Davon unberührt blieb eine tendenziell ähnliche Entwicklung von Rückgangs- und Anstiegsphasen, die sich in den niederländischen Fundbeständen eher dokumentieren, in Skandinavien dagegen erst mit zum Teil erheblicher zeitlicher Verzögerung einsetzen.

Die Auswertung der polnischen Messerfunde zeigt abschließend beispielhaft, daß regional unterschiedliche Tendenzen sich auch unabhängig von ethnischen Gemeinsamkeiten dokumentieren können (Abb. 79).

Wie bereits erwähnt, folgen die tendenziellen Entwicklungen der westslawischen Messer aus der ehemaligen DDR unter Einbeziehung der Maximalwerte den Werten der westlich benachbarten Gebiete. Der einzige deutliche Unterschied besteht in der unterschiedlichen Intensität des Anwachsens der Mittelwerte nach dem $10 \mathrm{Jahr}-$ hundert.

Im Gegensatz hierzu entwickeln sich die Maximal- und Mittelwerte in den polnischen Fundbeständen vom 9. zum 10. Jahrhundert mit steigender Tendenz, so daß sich trotz der Übereinstimmung vom 10. zum 11. Jahrhundert ein durchaus unterschiedliches Bild ergibt. Im 12. Jahrhundert liegt mit $18,8 \mathrm{~cm}$ der höchste, insgesamt für dieses Jahrhundert beobachtete Wert vor, während in den Niederlanden und Westdeutschland, in denen die Durchschnittslängen für die vergleichbaren Zeiträume deutlich höher angesiedelt sind, Maximallängen von 18,75 cm und 18,0 $\mathrm{cm}$ angetroffen wurden.

Abschließend läßt sich festhalten, daß sich ähnlich wie bei einigen Klingenformen die allgemein ermittelten Entwicklungsphasen in den einzelnen Untersuchungsländern zeitlich versetzt vollzogen haben.

Der Initialraum ist wahrscheinlich im westlichen Bearbeitungsgebiet anzusiedeln, im Norden haben sich diese Phasen erst mit starker zeitlicher Verzögerung durchgesetzt.

Die Basis, auf der sich vor allem nach dem 10. und 14. Jahrhundert Veränderungen ergeben haben, sind unterschiedliche Grundlängen, die im westlichen Bearbeitungsraum höher liegen als im östlichen.

Ob die beobachteten Entwicklungsphasen und allgemein längere Klingenmaße als Indikatoren für Innovationsschübe oder innovative 


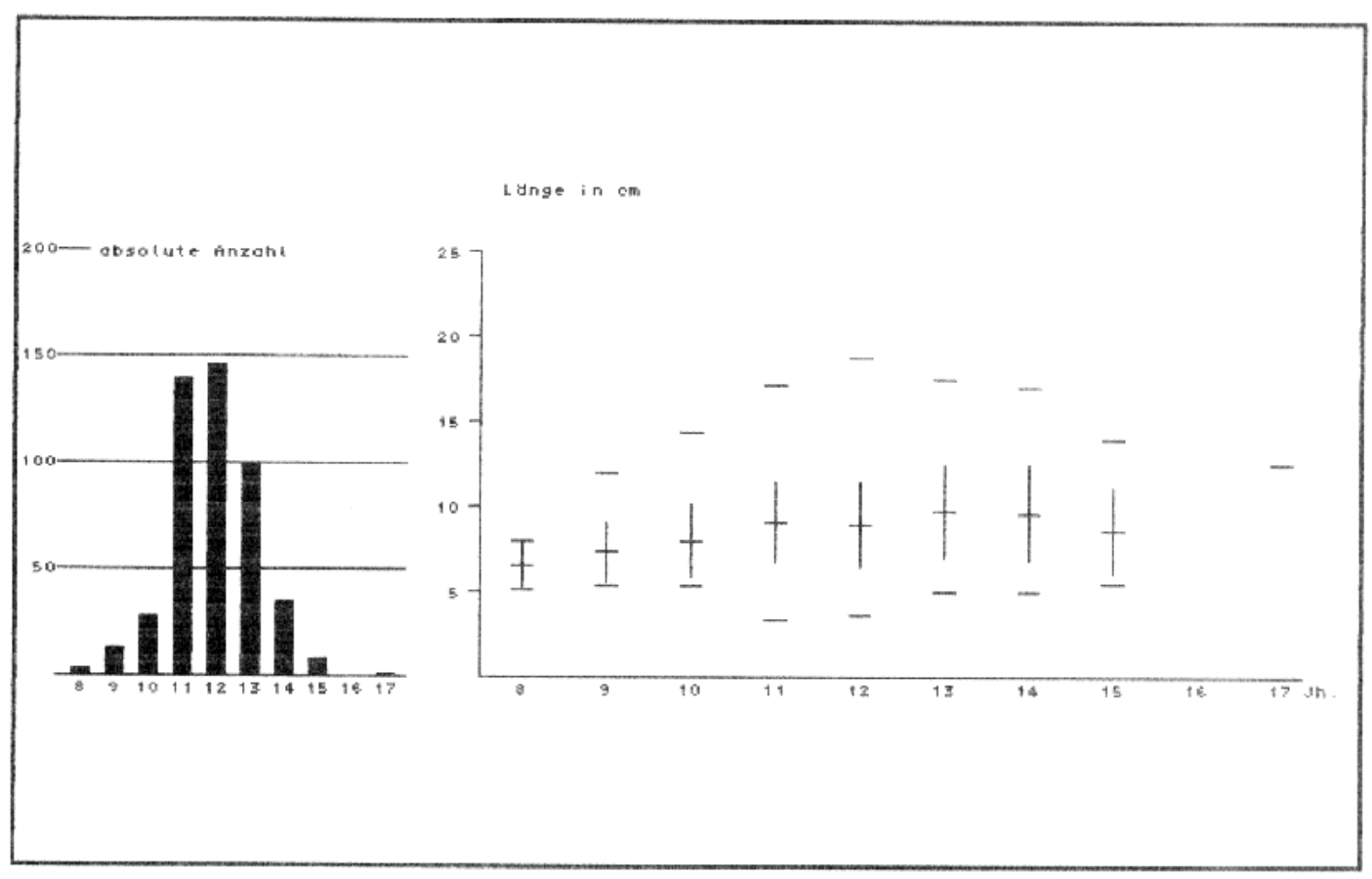

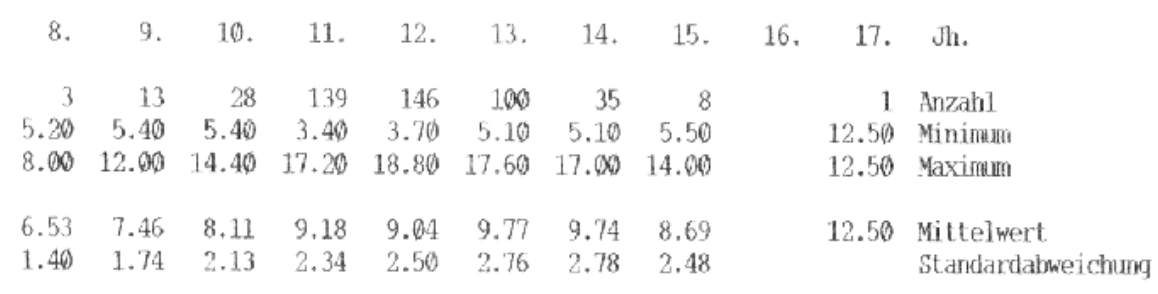

Abb. 79

Die Entwicklung der Klingenlängen in Polen

Räume zu bewerten sind, kann abschließend nicht beantwortet werden.

Sie belegen aber Veränderungen der Funktionsrahmen, die sich nicht gleichzeitig oder regional begrenzt, sondern mit einer faßbaren Richtung verbreitet haben.

Mit dieser Einschränkung erscheint der skandinavische Raum als dauerhaftes Reliktgebiet, der niederländische Raum zumindest seit dem 14. Jahrhundert als Innovationsgebiet. 


\subsubsection{Die Griffkonstruktionen}

Die statistischen Untersuchungen zur zeitlichen Verteilung der im bearbeiteten Fundgut angetroffenen Griffkonstruktionen folgen wie die vorangegangenen Untersuchungen $\mathrm{zu}$ den Klingenformen den im Klassifizierungsmodell vorgestellten Kriterien.

Die Auswertung der Griffkonstruktionen wird in drei Abschnitten vorgestellt:

Am Beginn steht die generelle Frage nach dem zeitlichen Einsetzen der Griffzungenkonstruktionen und die Behandlung von technologischen Übergangsformen.

Im zweiten Abschnitt werden die verschiedenen Griffangelkonstruktionen, am Schluß die Griffzungenkonstruktionen besprochen.

Wenn einzelne Konstruktionsformen im bearbeiteten Fundgut nur so vereinzelt aufgetreten sind, daß Aussagen über ihre Entstehung und Entwicklung nicht möglich sind, wird dieses im Text ohne graphische Darstellung vermerkt.

Auch für die im folgenden behandelten Fragestellungen spielt die Erhaltung der Fundobjekte eine nicht geringe Rolle.

Nach den verschiedenen organischen Materialien der Vollgriffe (Hilzen) und Griffschalen wurde aus diesem Grund nicht mehr gefragt. Betroffen waren aber auch die Griffendgestaltung und zusätzliche Applikationen an den Griffen.

Untersucht wurden in erster Linie technische Elemente wie Befestigungsarten und Zusatzbefestigungen, die bereits bei der Erstellung des Codesystems Eingang in das Klassifizierungsmodell gefunden haben.

Im beschreibenden Teil der Auswertung werden im Anschluß an die statistische Auswertung die in Frage kommenden Messer detailliert besprochen.

\subsubsection{Die zeitliche Verteilung von Griffangelmessern und Griffzungenmessern}

Die Befestigung von Griffschalen aus Holz oder Knochen durch mehrere Niete auf beiden Seiten der flach gehämmerten Griffzunge ist weder an Messer gebunden, noch ist sie technologisch eine neue Erfindung des Spätmittelalters ${ }^{71}$.

Im Zusammenhang der hier behandelten Fragen finden Griffbefestigungen an Waffen und Werkzeugen sowie vorkaiserzeitliche Messergriffkonstruktionen keine Berücksichtigung.

Der Einschluß nordalpiner kaiserzeitlicher Messer im anschließenden beschreibenden Teil erfolgte vor allem, um den Charakter einer Neuaufnahme sehr viel älterer 
Konstruktionsformen, den der beobachtete Beginn der Griffzunge im 10. Jahrhundert besitzt, deutlich zu machen.

Gleichzeitig wird damit die Frage nach einer möglichen Tradition, die auch im $\mathrm{Zu}-$ sammenhang mit Edelmetallgriffen an Angelmessern auftritt ${ }^{72}$, behandelt.

Die Auswertung des gesamten bearbeiteten Bestands zu den beiden grundsätzlichen Griffkonstruktionen Angel (Codebezeichnung A) und Zunge (Codebezeichnung B) zeigt im 10. Jahrhundert den frühestmöglichen Beginn der Griffzunge, die technologisch die aufwendigere Griffbefestigungsart darstellt, nach der Karolingerzeit an (Abb. 80).

Der Beleg des 10. Jahrhunderts betrifft eine verzierte Knochenschale, die auf dem slawischen Burgwall in Meißen gefunden wurde und in die zweite Hälfte des 10. bis in das 11. Jahrhundert datiert wurde.

Ihre Ansprache als Griffelement ist nicht unumstritten ${ }^{73}$.

Bis zum 12. Jahrhundert verbreitet sich diese Konstruktionsform nur langsam. Im 13. und 14. Jahrhundert wächst ihr Auftreten jeweils um mehr als das Doppelte.

Im15. und 16. Jahrhundert drängt die Griffzunge die ältere Griffangel auf weniger als ein Viertel des Gesamtbestandes zurück. Ein Jahrhundert später nimmt die Griffzunge etwas mehr, die Griffangel etwas weniger als die Hälfte dieses Bestandes ein.

Die Auswertung des zeitlich enger begrenzten Fundbestandes zeigt tendenziell die gleiche Entwicklung (Abb. 81).

Der Beginn der Griffzungenkonstruktion setzt hier im 11. Jahrhundert ein, der Sprung vom 14. zum 15. Jahrhundert ist noch ausgeprägter (von $21 \%$ auf $82,4 \%$ ).

Die Griffangelkonstruktion nimmt hier vom 15. (17,6\%) zum 17. Jahrhundert $(60 \%)$ stark zu.

Der erneute Beginn der Griffzungenkonstruktion läßt sich demnach mit hoher Wahrscheinlichkeit nach einem Abbruch seit der römischen Kaiserzeit im 11. Jahrhundert ansiedeln.

Ihre Hauptverbreitungszeit findet sie nach einer Expansionsphase, in der auch ihre wichtigsten Konstruktionsformen entwickelt worden $\operatorname{sind}^{74}$, im 15. und 16. Jahrhundert.

Zur genaueren Lokalisierung dieses Beginns wurden die zeitlich enger faßbaren Bestände der einzelnen Untersuchungsländer ausgewertet. 


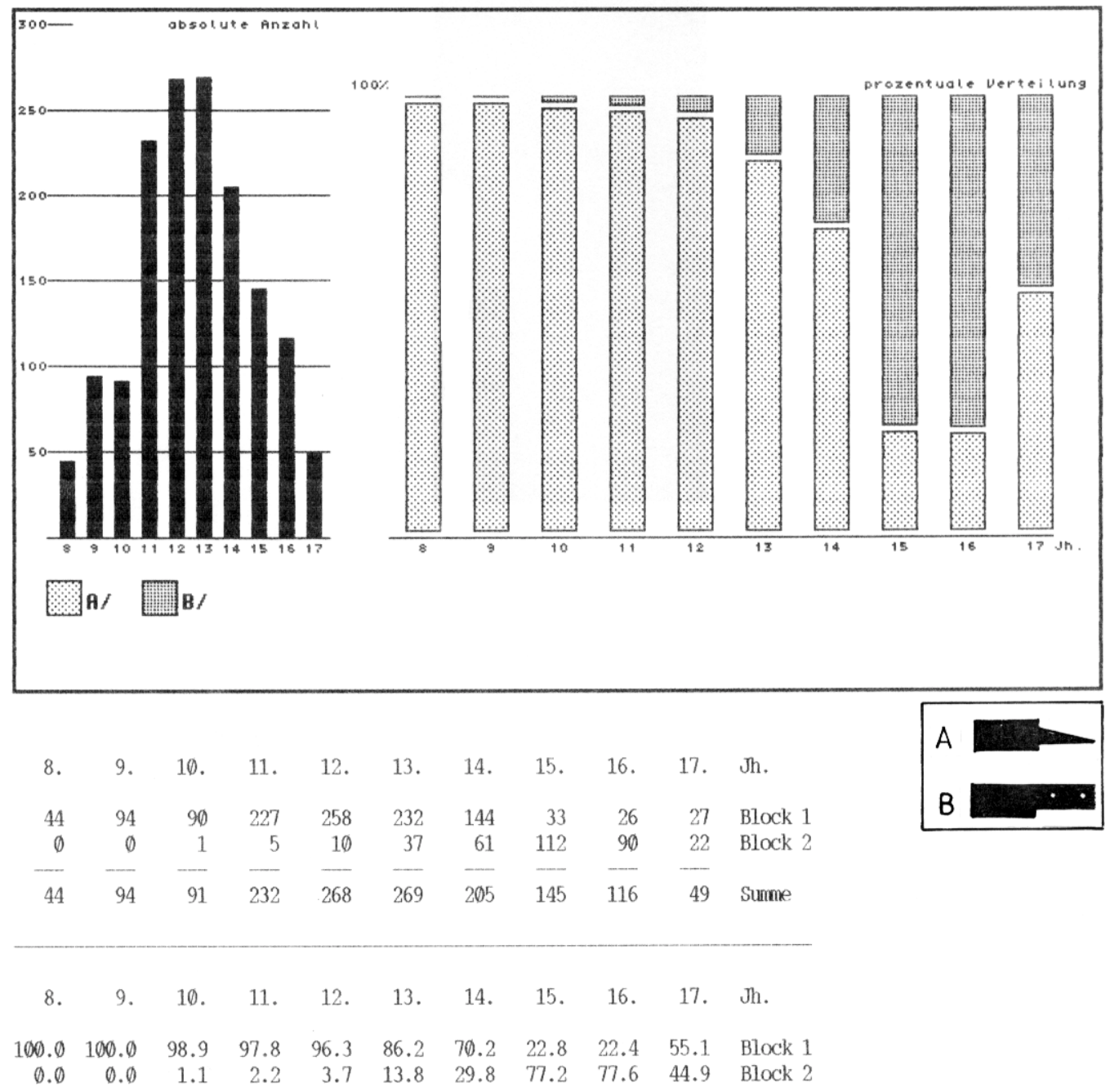

Abb. 80

Der Wiederbeginn der Griffzungenkonstruktion an Messergriffen nach der römischen Kaiserzeit im gesamten Untersuchungsraum (grob datierter Bestand) 


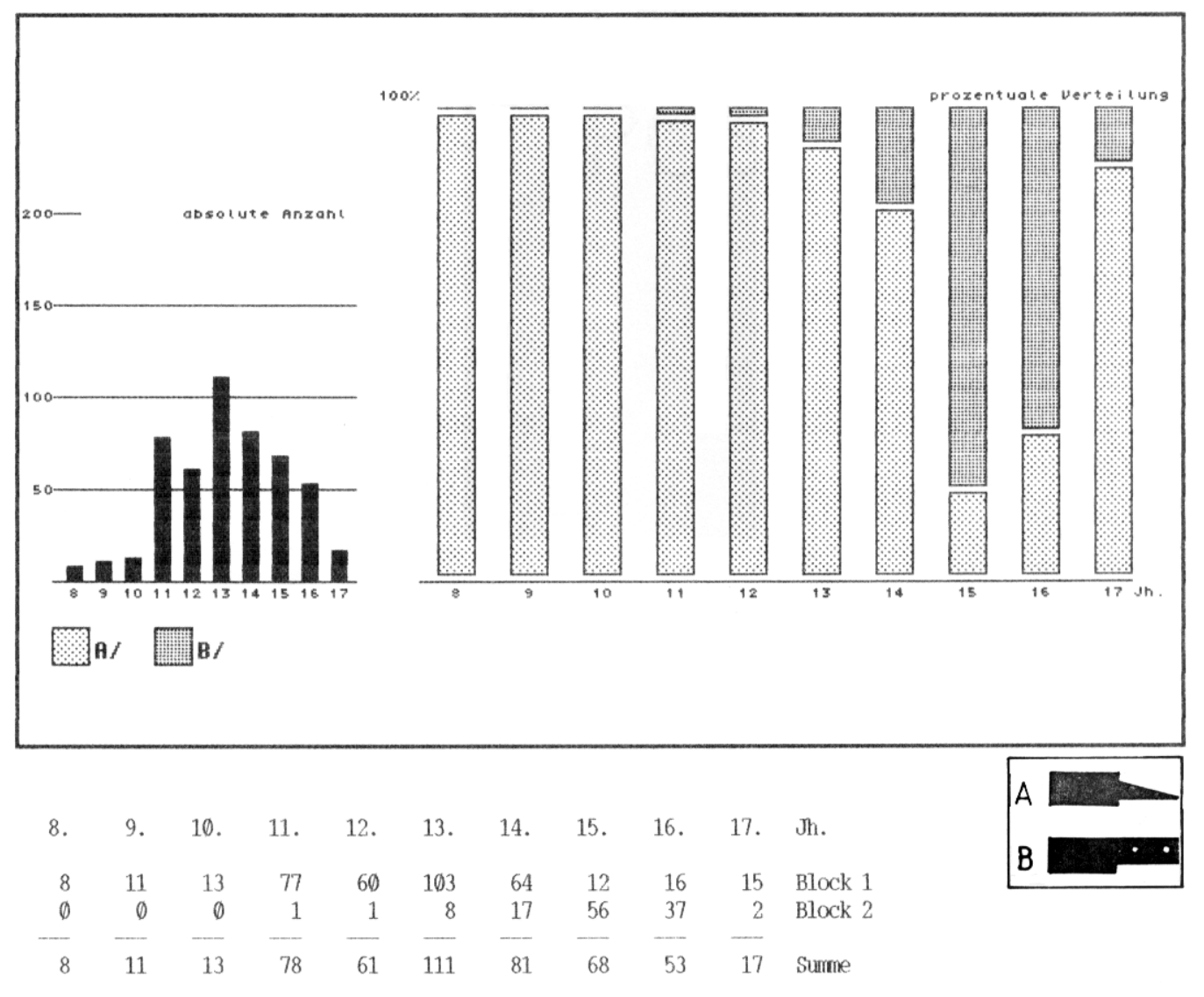

8. 9. 10. 11. 12. 13. 14. 15. 16. 17. Jh.

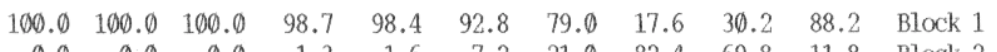

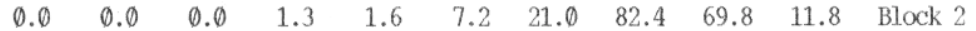

Abb. 81

Der Wiederbeginn der Griffkonstruktion an Messergriffen nach der römischen Kaiserzeit im gesamten Untersuchungsraum (enger datierter Berstand) 
In den bearbeiteten Fundbeständen aus Skandinavien setzen sicher nachweisbare Griffzungenmesser erst im 14. Jahrhundert ein (Abb. 82). Da auch im gesamten skandinavischen Fundmaterial nur vier Belege für Zungenkonstruktionen des 13. bis 14. Jahrhunderts angetroffen wurden, scheint sich die bereits an anderer Stelle beobachtete Reliktstellung dieses Raumes zu bestätigen. Die vier Griffzungenmesser des 13. bis 14. Jahrhunderts sind undatierte Altfunde aus Lund, die nach ähnlichen, stratigraphisch gesicherten Funden zeitlich eingeordnet wurden.

Der Prozentsatz von 17,4\% im 14. Jahrhundert sowie der außerordentlich scharfe Rückgang der Angelkonstruktionen auf 11,5\% im 15. Jahrhundert deutet trotz der geringen Anzahl der ausgewerteten Messer auf eine späte, dann aber plötzliche und sehr rasche Aufnahme dieser Konstruktionsform, die zumindest seit dem 11. Jahrhundert im nordalpinen Europa wieder bekannt war.

Im niederländischen Messerbestand wiederholt sich dieses Bild, der einzige Unterschied besteht in der sehr frühen Wiederaufnahme von Angelkonstruktionen, die im 16. Jahrhundert wieder über $40 \%$ einnehmen (Abb. 83).

Da auch in den bearbeiteten Beständen des 12. bis 13. Jahrhunderts keine Zungenkonstruktionen vorhanden sind, die im 14. Jahrhundert auftretenden Griffzungenmesser aber bereits entwickeltere Formen darstellen, wie sie im 15. und 16. Jahrhundert allgemein üblich sind (vgl. Kat. Nr. 63 und 65), scheint auch hier eine plötzliche Übernahme vorzuliegen, die wahrscheinlich von den holländischen Großstädten ausgegangen ist.

Dem frühen Anstieg der Angelkonstruktionen entspricht der starke Rückgang der Zungenkonstruktionen im 17. Jahrhundert, der in der Auswertungsgraphik aufgrund der geringen absoluten Zahl zu stark gezeichnet erscheint, sich aber im gesamten niederländischen Fundbestand mit einem Beleg nur unbedeutend relativiert.

In diesem Fall erscheinen vor allem die niederländischen Städte, in denen der Großteil der bearbeiteten Funde ausgegraben wurde, als ein Raum, in dem die Zungenkonstruktion zwar erst spät, aber vor allem im 15. Jahrhundert rasch aufgenommen und bis um 1550 technologisch und künstlerisch stark variiert worden ist. Dieser Gestaltungsschub erfaßte bereits im 16. Jahrhundert auch die Griffangelmesser, die hier nach 1600 bereits wieder die Masse der vor allem an der Tafel benutzten Messer ausmachen ${ }^{75}$.

Im entsprechenden Bestand des Bearbeitungsraums der alten Bundesländer erscheinen Griffzungenmesser sicher datiert seit dem 13. Jahrhundert, Griffschalen aus Knochen sind hier frühestens kurz vor oder um 1200 angetroffen worden (Kat. Nr. 12, 108, 150). Im zeitlich weiter gefaßten Bestand konnte nur ein früherer Nachweis erbracht werden, ein Griffzungenmesser des 11. bis beginnenden 12 . Jahrhunderts von der slawischen Burg in Lübeck (Kat. Nr. 18). 


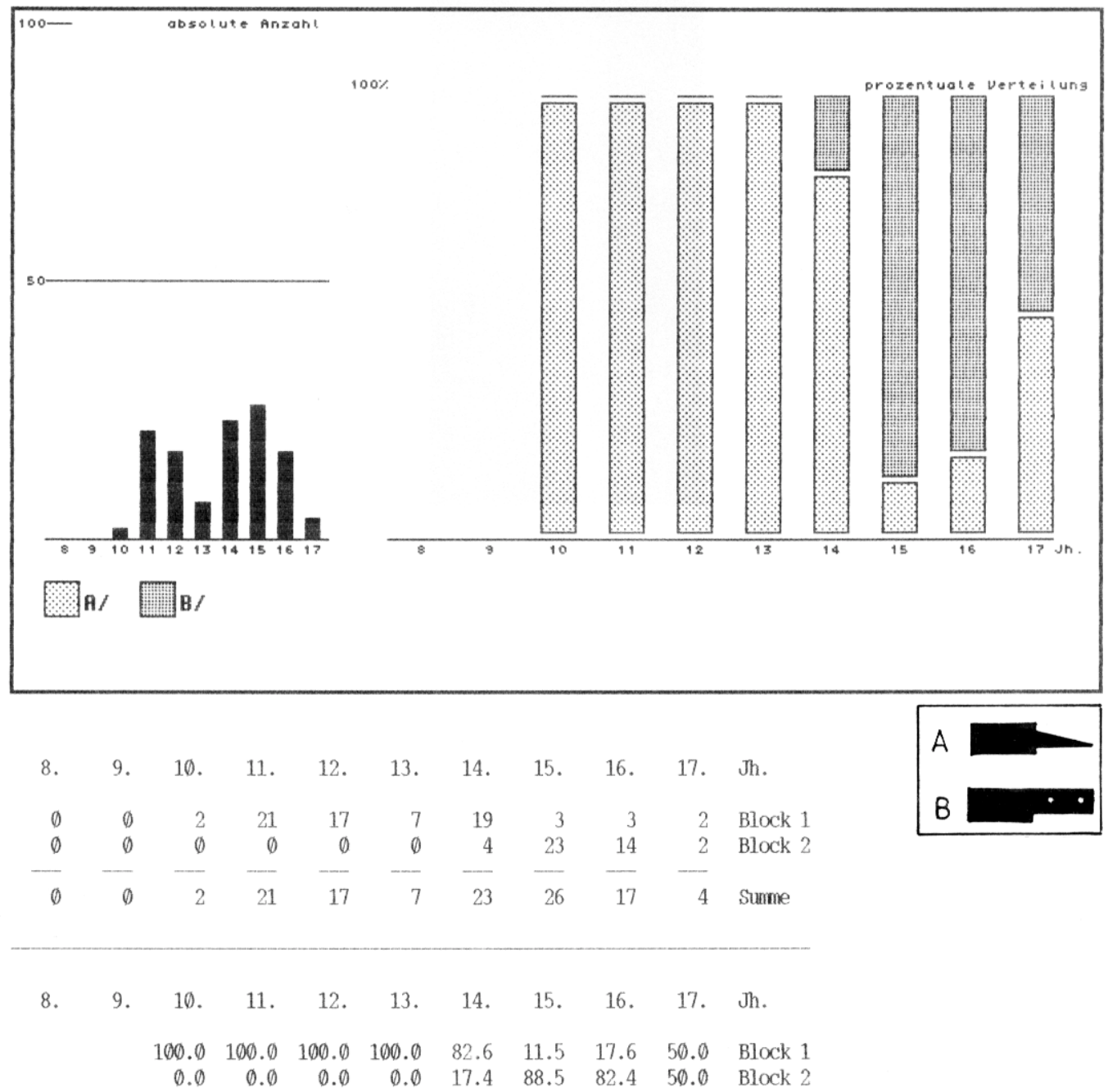

Abb. 82

Griffzungenmesser in Skandinavien (enger datierter Berstand) 


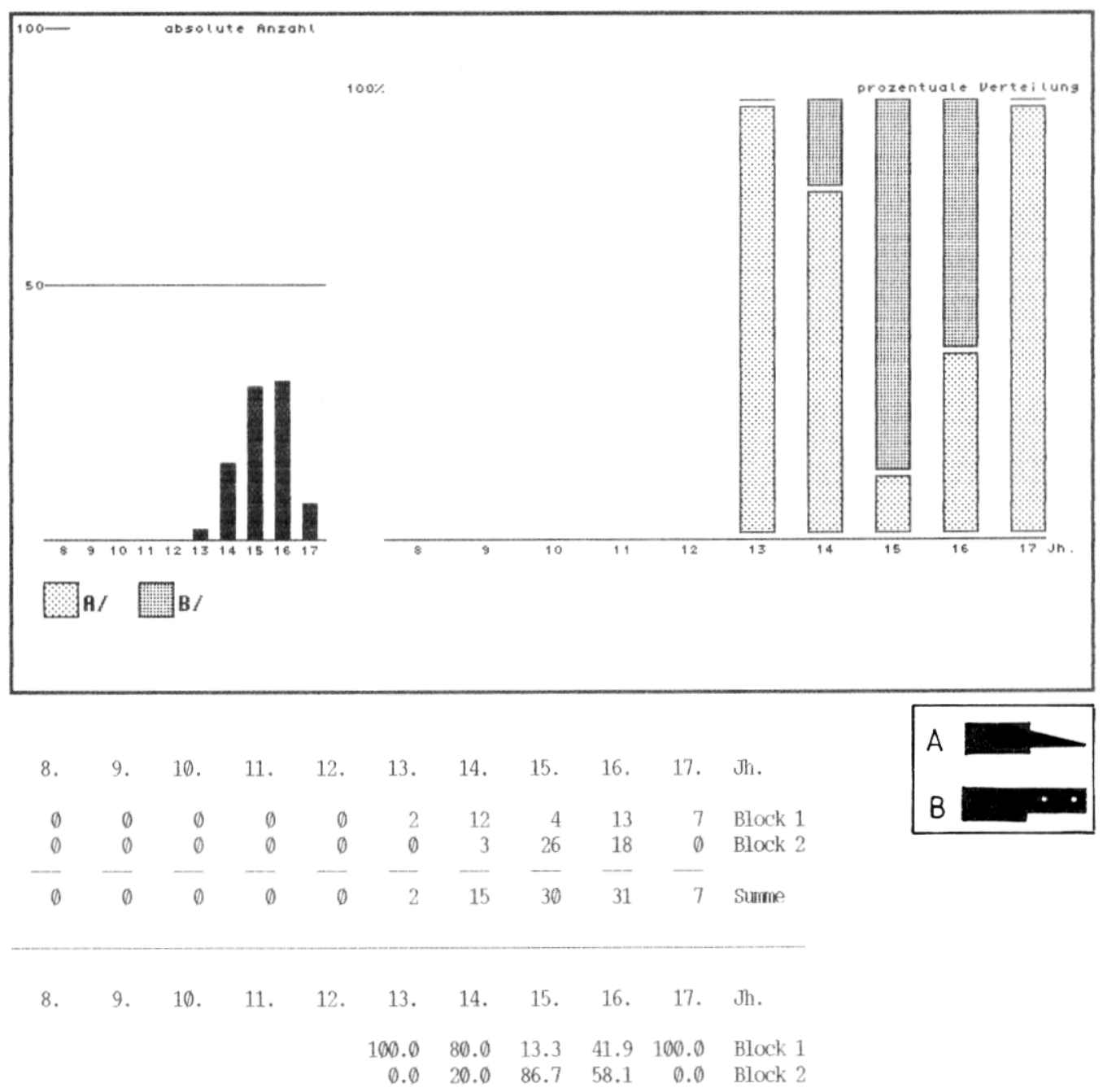

Abb. 83

Griffzungenmesser in den Niederlanden (enger datierter Berstand) 
Unter dem bereits erwähnten Vorbehalt der geringen Anzahl, die die allgemeinen Tendenzen teilweise überzeichnet, läßt sich anhand der zeitlichen Verteilung erkennen, daß im nördlichen und westlichen Deutschland Griffzungenmesser in regelmäßigen Wachstumsphasen vom 13. bis zum 16. Jahrhundert zunehmen, bis sie die Angelkonstruktionen fast völlig verdrängt haben (Abb. 84).

Außer den Zungenmessern des Wrackfundes von Wittenberge, die nicht mitberücksichtigt wurden (kurz vor 1600?), sind enger datierte Griffzungenmesser nach 1600 nicht aufgetreten.

Der zeitlich enger datierte Bestand aus den östlichen Bundesländern (ehemalige DDR) ist zu gering, um für eine Aussage herangezogen zu werden.

Im gesamten ostdeutschen Bestand erscheint neben einer unsicheren Knochenschale aus Meißen (Kat. Nr. 162) ein westslawisches Griffzungenmesser im 11. bis 12 . Jahrhundert (Kat. Nr. 160), im 13. Jahrhundert beginnt eine rasche Zunahme von Zungenkonstruktionen, die im 14. Jahrhundert über 30\%, und auch im enger datierten Bestand 25\% ausmachen (Abb. 85).

Der Zeitraum nach dem 14. Jahrhundert ist nicht mehr repräsentativ.

Die Auswertung des betreffenden Fundbestands aus Polen belegt den Beginn des Auftretens von Griffzungenmessern dort vom 11. bis zum 14. Jahrhundert (Abb. 86).

Die frühen Beispiele scheinen an politische und wirtschaftliche Zentren gebunden zu sein:

Aus dem ausgehenden 11. Jahrhundert (Schlußdatum 1102) stammt eine verzierte Griffschale, die in Oppeln ausgegraben wurde (Kat. Nr. 332). In Kalisch wurde ein Griffzungenmesser in Schichten des 12. Jahrhunderts angetroffen (Kat. Nr. 226). Zwei weitere Griffzungenmesser des 13. Jahrhunderts wurden in Posen und der Burg in Błonie gefunden.

Dazu treten eine verzierte Griffschale des 12. bis 13. Jahrhunderts aus Tum (Kat. Nr. 281) sowie zwei ebenfalls verzierte Schalen des 10. bis 12. Jahrhunderts aus Oppeln, die aufgrund ihrer weiten Datierungsspanne in der Auswertung keine Berücksichtigung gefunden haben.

Die Entwicklung nach dem 14. Jahrhundert ist anhand des aufgenommenen polnischen Fundmaterials nicht mehr zu verfolgen. 


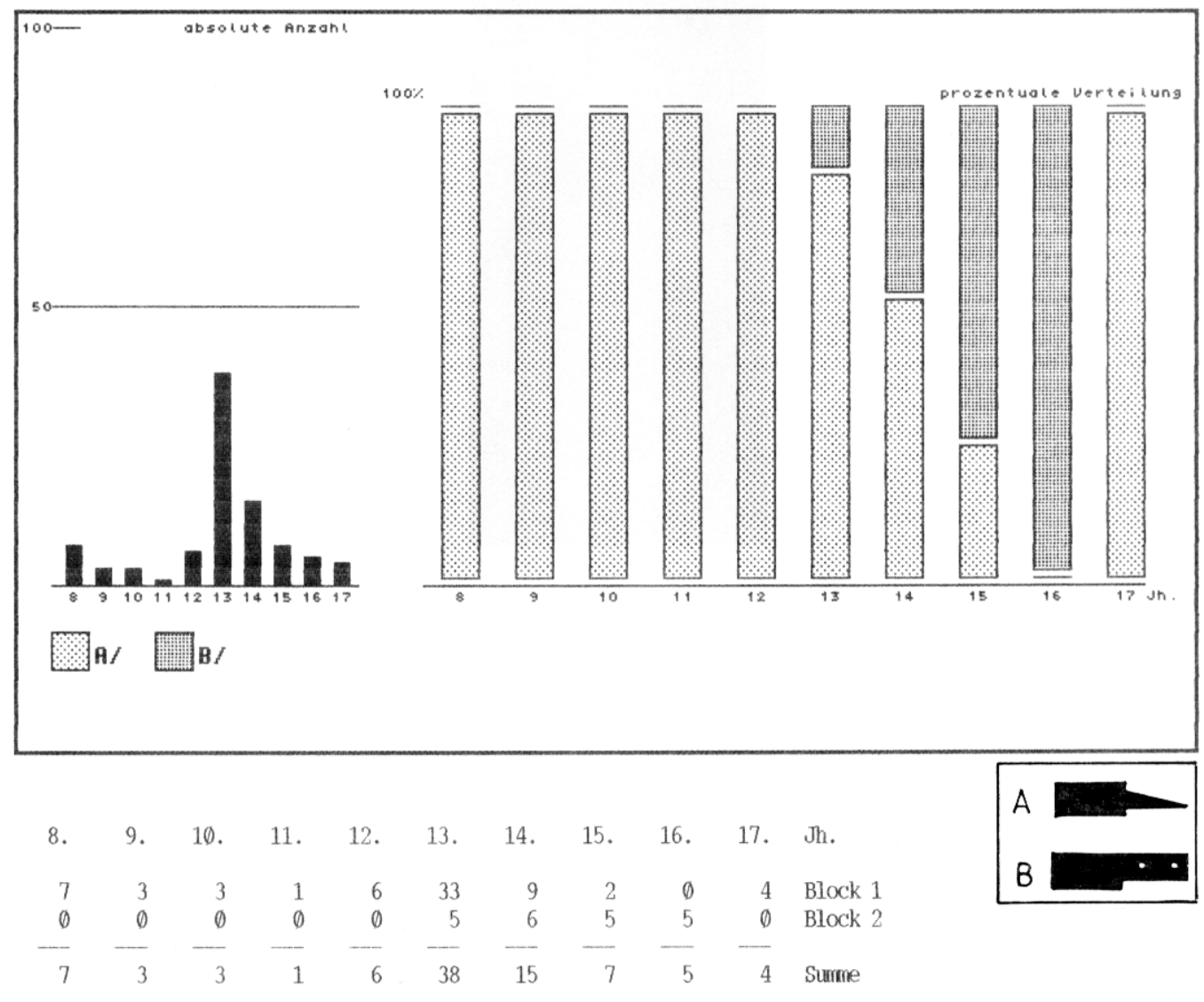

8. 9. 10. 11. $12 . \quad 13.14 .15 .416 .17 . \quad$ Jh.

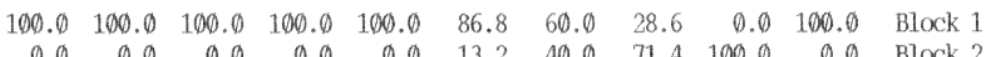

Abb. 84

Griffzungenmesser in Nordwestdeutschland (enger datierter Berstand) 


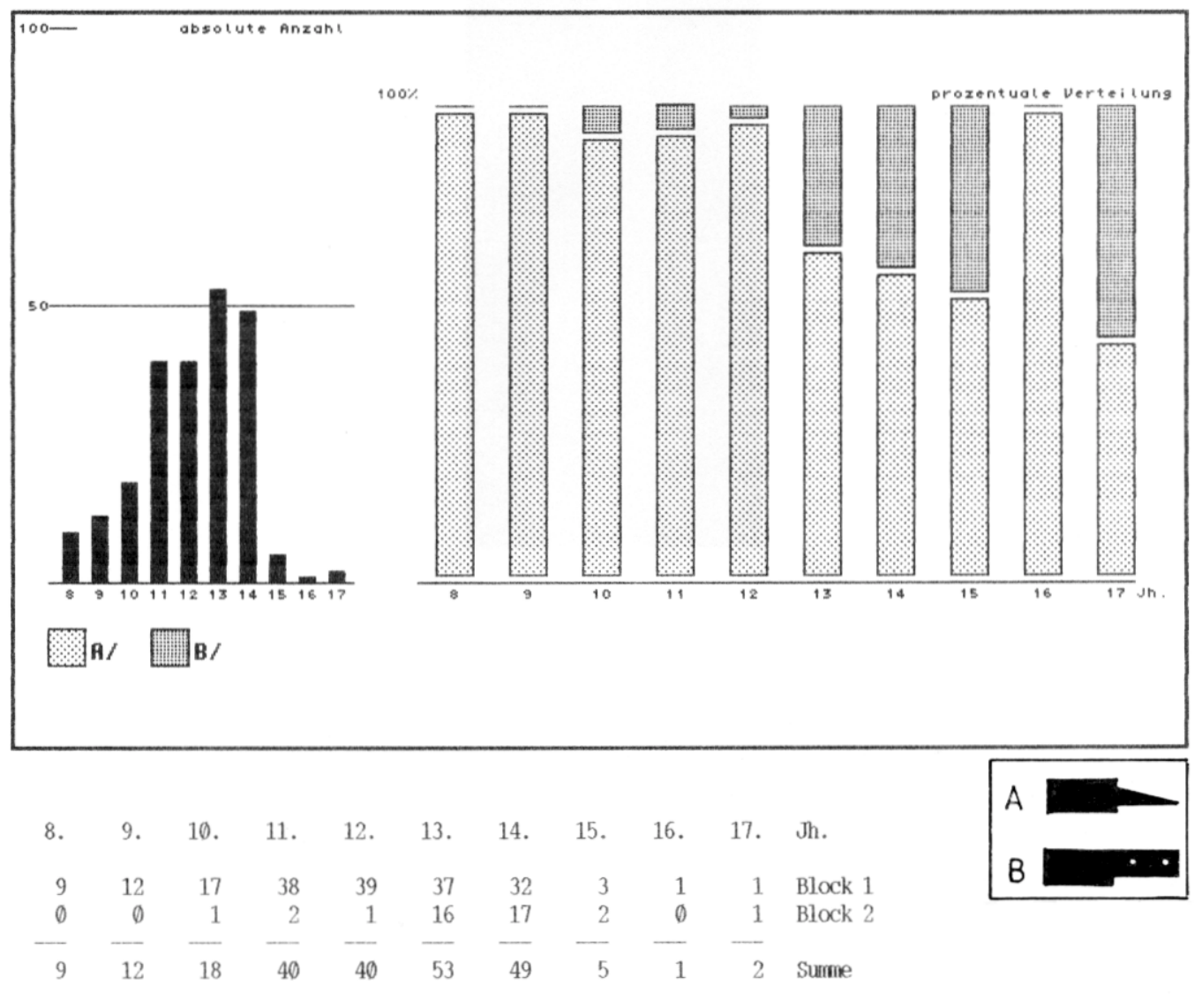

8. 9. 10. 11. 12. 13. 14. 15. 16. 17. Jh.

$\begin{array}{lllllllllll}100 . \emptyset & 100 . \emptyset & 94.4 & 95.0 & 97.5 & 69.8 & 65.3 & 60.0 & 100.0 & 50.0 & \text { Block } 1\end{array}$

$\begin{array}{lllllllllll}\emptyset .0 & 0 . \emptyset & 5.6 & 5.0 & 2.5 & 30.2 & 34.7 & 40.0 & 0.0 & 50.0 & \text { Block 2 }\end{array}$

Abb. 85

Griffzungenmesser in Ostdeuschland (grob datierter Bestand) 


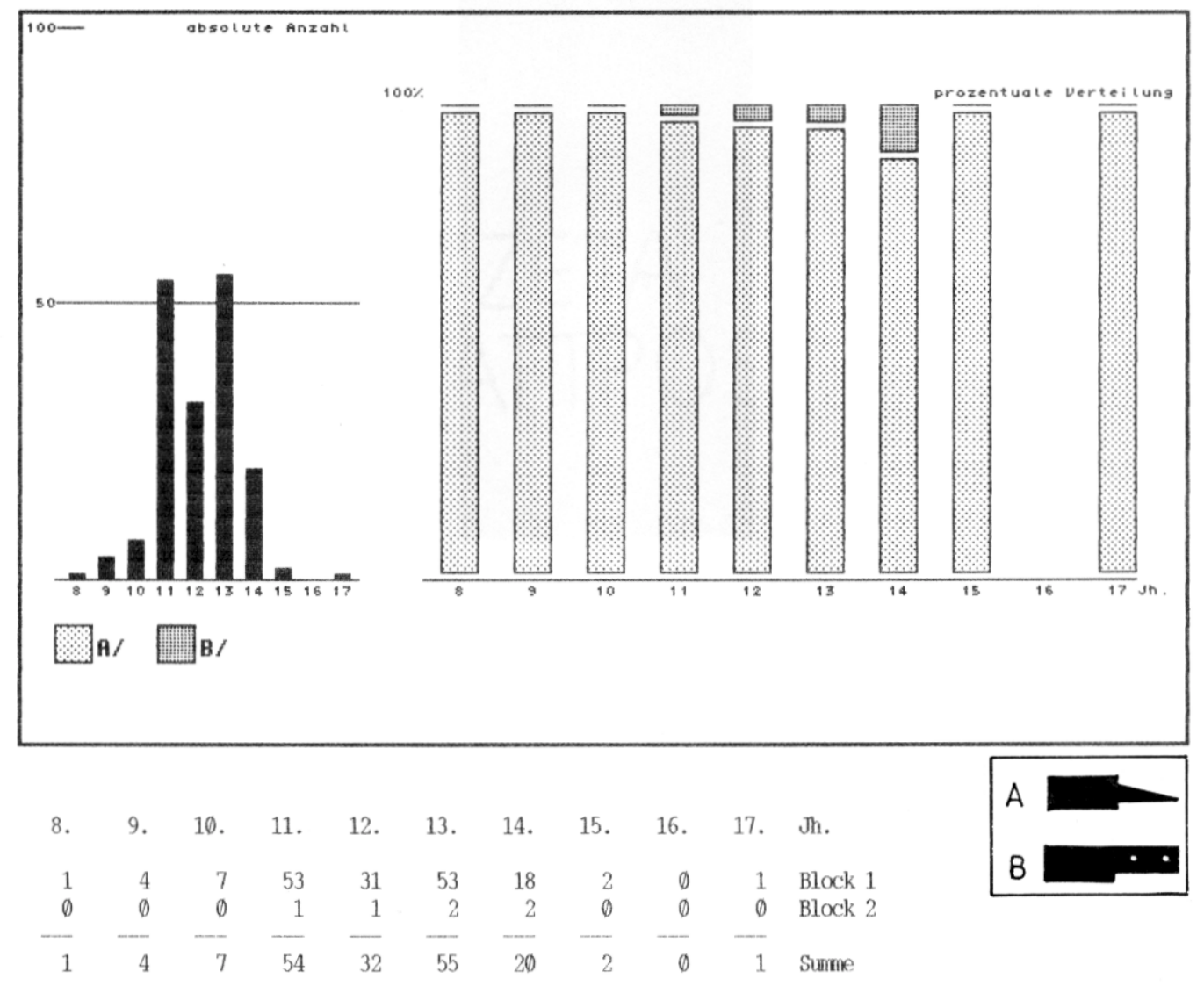

8. 9. 10. 11. 12. 13. 14. 15. 16. 17. Jh.

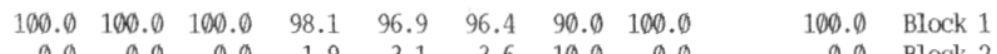

Abb. 86

Griffzungenmesser in Polen (enger datierter Berstand) 
Die in der Gesamtauswertung (Abb. 80) im 11. und 12. Jahrhundert auftretenden Belege für Griffzungenkonstruktionen stammen aus slawischen Siedlungsbefunden. Diese sowie die in Ostdeutschland und in Polen zusätzlich angetroffenen Beispiele des 10. bis 12. Jahrhunderts sind ein deutlicher Hinweis auf die Räume, in denen nach der Völkerwanderungszeit und der Zeit der ersten politischen Zentralisierungen Griffzungenmesser wieder in Gebrauch kommen. Ob die durchweg in slawischen Burgen und Hauptorten gefundenen Messer und Griffteile einer älteren Tradition entstammen, muß bezweifelt werden. In Stichproben wurden Inventare von frühslawischen Gräberfeldern daraufhin untersucht, ohne daß sich Belege für diese Ansicht finden ließen.

Weitgehend unberücksichtigt blieb der südslawische und südlich angrenzende Raum, so daß bisher nicht ausgeschlossen werden kann, daß in Südosteuropa und im Orient ältere Belege für Griffzungenkonstruktionen an Messern vorhanden sind. Im westlichen Teil des Untersuchungsgebietes wurden nach der römischen Kaiserzeit keine Griffzungenmesser vor dem 13. Jahrhundert angetroffen.

Der Gesamtbestand der hier bearbeiteten Messer enthielt mit den Formen A6 und B9 Konstruktionen, die technologisch den Eindruck von Zwischenstufen erwecken. Die Form A6, eine am Ansatz leicht verbreiterte Angel mit einem oder mehreren Nietlöchern, ist vom 11. bis zum 14. Jahrhundert aufgetreten. In der graphischen Darstellung erscheint sie sowohl gegenüber den Angelmessern als auch gegenüber den Zungenmessern abgesetzt (Abb. 87).

Im 13. Jahrhundert, in dem in den westlichen und nördlichen Teilen des Untersuchungsgebietes die ersten Griffzungenkonstruktionen auftreten, besitzt die Form A6 mit 3,4\% ihren höchsten Anteil.

In Nordwestdeutschland scheinen Messer mit der Griffkonstruktion A6 am häufigsten aufgetreten zu sein (Abb. 88).

Im 12. und 13. Jahrhundert erreichen sie Anteile von 5,0\% bzw. 7,2\%, im 14. Jahrhundert noch von 3\% des Gesamtbestandes.

In den Niederlanden konnte nur ein Beleg aus dem 12. bis 13. Jahrhundert aufgenommen werden, in Skandinavien wurde die Konstruktionsform nicht beobachtet. Im ostdeutschen Fundbestand wurden zwei Griffangelmesser mit Nietlöchern aus dem 11. bis 12. sowie aus dem 13. bis 14. Jahrhundert angetroffen, im polnischen Bestand traten zwei Belege des 13. Jahrhunderts auf. 


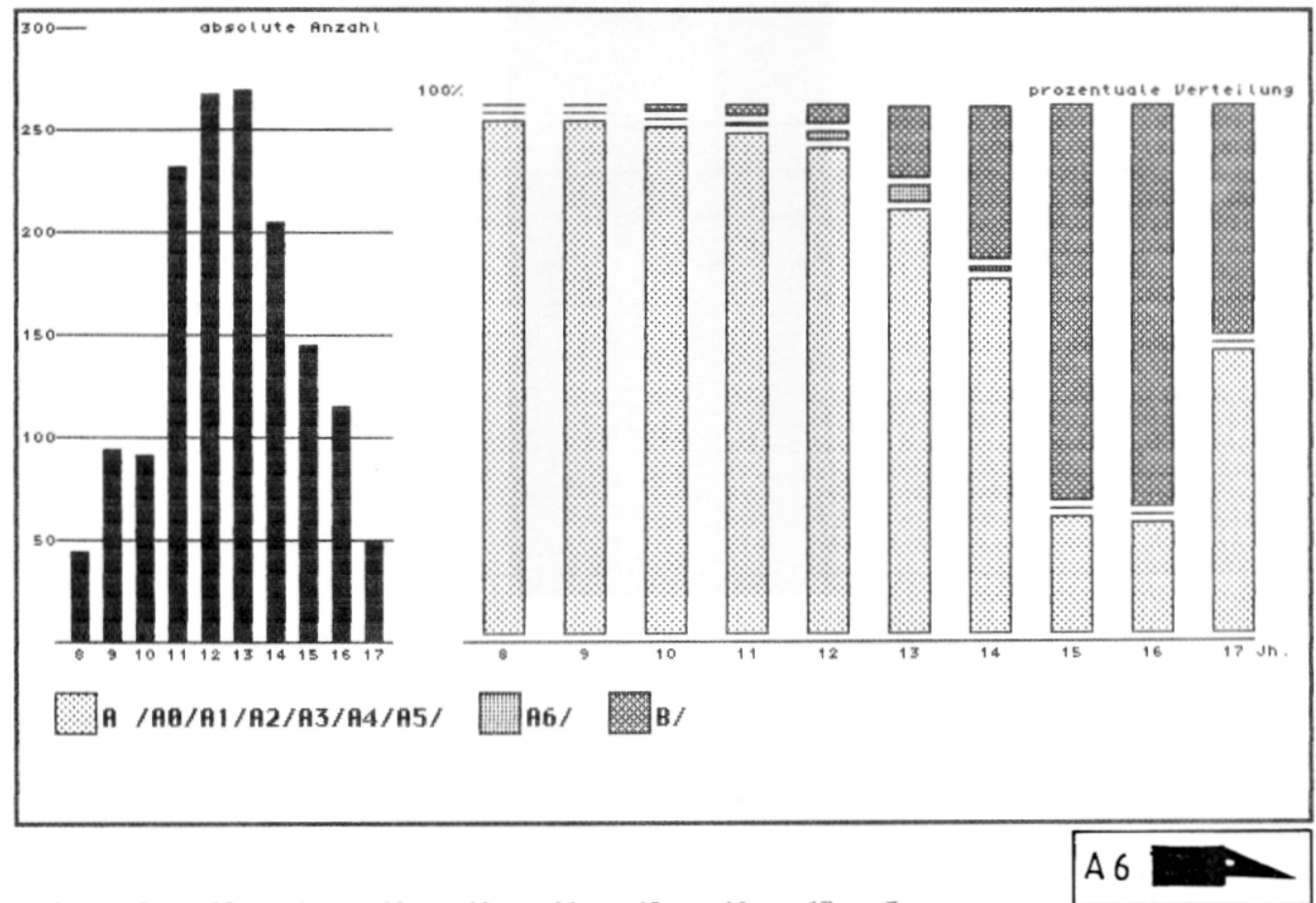

\begin{tabular}{|c|c|c|c|c|c|c|c|c|c|c|}
\hline 8. & 9. & 10. & 11. & 12. & 13. & 14. & 15. & 16. & 17. & $\mathrm{~J}$. \\
\hline 44 & 94 & 90 & 226 & 253 & 223 & 142 & 33 & 25 & 27 & Block 1 \\
\hline 0 & 0 & 0 & 1 & 4 & 9 & 2 & 0 & $\emptyset$ & 0 & Block 2 \\
\hline$\emptyset$ & 0 & 1 & 5 & 10 & 37 & 61 & 112 & 90 & 22 & Block 3 \\
\hline- & - & - & - & -- & - & - & -- & - & - & \\
\hline 44 & 94 & 91 & 232 & 267 & 269 & 205 & 145 & 115 & 49 & Summe \\
\hline
\end{tabular}

$\begin{array}{rrrrrrrrrrl}8 . & 9 . & 10 . & 11 . & 12 . & 13 . & 14 . & 15 . & 16 . & 17 . & \text { Jh. } \\ 100.0 & 100.0 & 98.9 & 97.4 & 94.8 & 82.9 & 69.3 & 22.8 & 21.7 & 55.1 & \text { Block 1 } \\ 0.0 & 0.0 & \emptyset .0 & 0.4 & 1.5 & 3.3 & 1.0 & 0.0 & \emptyset .0 & \emptyset . \emptyset & \text { Block 2 } \\ 0.0 & 0.0 & 1.1 & 2.2 & 3.7 & 13.8 & 29.8 & 77.2 & 78.3 & 44.9 & \text { Block 3 }\end{array}$

Abb. 87

Das Auftreten der Angelkonstruktionen A6 vom 11. bis zum 14. Jahrhundert im frühen Entwicklungszeitraum von Griffzungenmessern im gesamten Untersuchungsraum (grob datierter Bestand) 


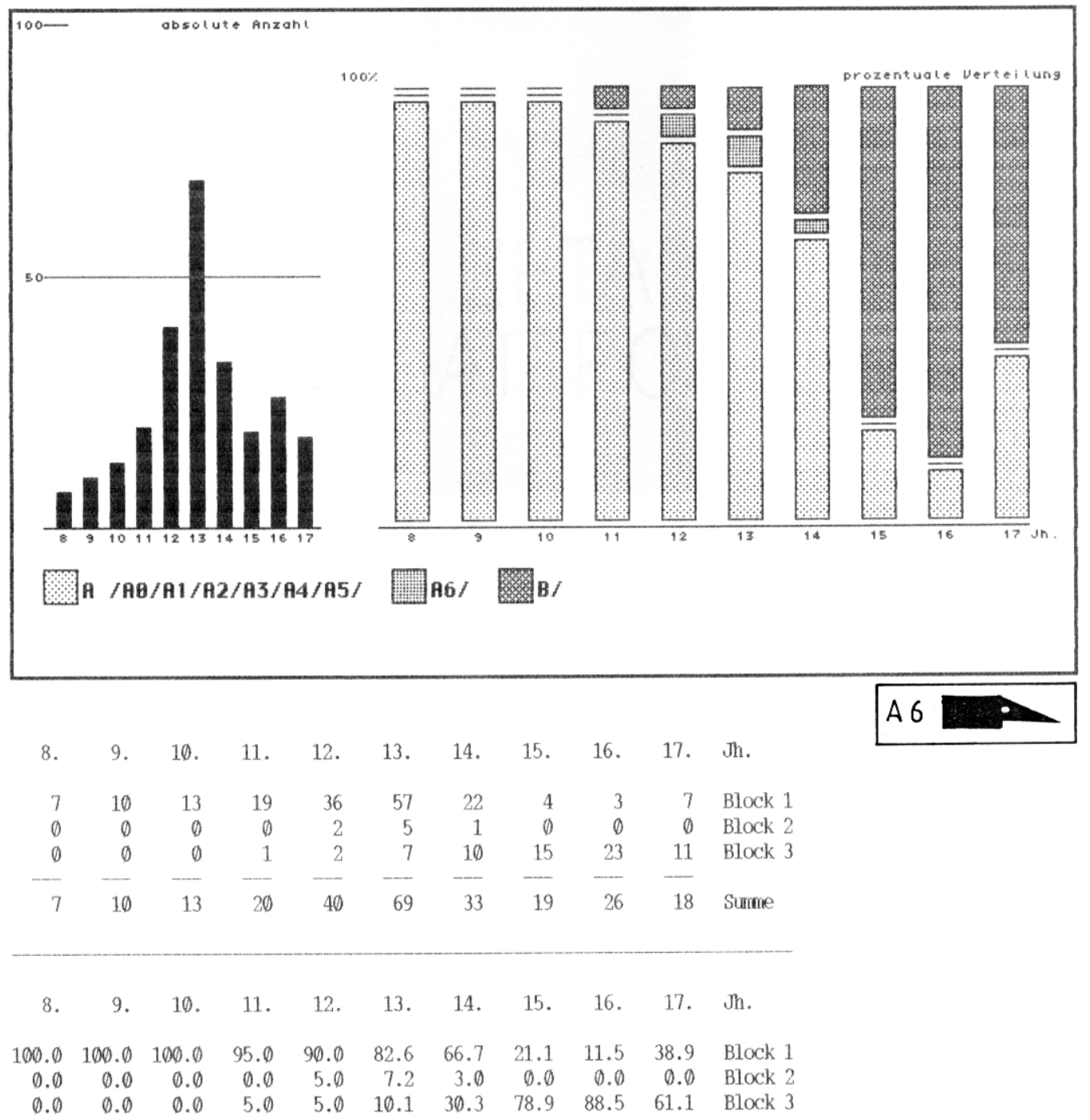

Abb. 88 Die Angelkonstruktion A6 in Nordwestdeutschland (grob datierter Bestand) 
In den östlich angrenzenden Bearbeitungsräumen wurden bis auf eine typologisch unsichere Knochenschale aus Grodno (12. bis 13. Jahrhundert) vor dem 14. Jahrhundert weder Griffzungenkonstruktionen noch Belege für die Konstruktionsform A6 angetroffen.

Die vorliegenden Ergebnisse legen nahe, das Aufkommen von Griffzungenmessern im slawischen Siedlungsraum im 10. bis 12. Jahrhundert zunächst unabhängig von den Entwicklungen des 12. bis 13. Jahrhunderts in den westlich angrenzenden Räumen zu betrachten.

Im westlichen Teil des Untersuchungsgebiets scheint mit der Griffkonstruktion A6 eine frühe Entwicklungsstufe vorzuliegen, die im 12. Jahrhundert entstanden ist und neben den ausgereiften Griffzungenkonstruktionen, die seit dem 13. Jahrhundert nachweisbar sind, bis zum 14. Jahrhundert Verwendung fand.

Die Möglichkeit, daß es sich bei diesen Messern um Klappmesser handelt, ist angesichts der auch beobachteten Doppelvernietung am Heft zwar unwahrscheinlich, aber nicht ganz auszuschließen ${ }^{76}$.

Der Vergleich mit der frühen Griffzungenkonstruktion B9 (12. bis 14. Jahrhundert) erlaubt aber selbst in diesem Fall, von technologischen Vorstufen zu sprechen (Abb. 89):

Mit einem Versatz von einem Jahrhundert geht die Form A6 parallel zur Zunahme der Form B9 zurück.

Der Zeitraum des 12. bis 14. Jahrhunderts ist aber nur begrenzt als Entwicklungszeitraum bis zum ausgereiften Griffzungenmesser, dessen Griff zur Schneide abgesetzt ist, zu bezeichnen.

Die Anzahl von insgesamt 19 Belegen für beide Formen läßt sie trotz ihrer Konzentration auf den nordwestdeutschen Raum nur bedingt als typologische Vorstufen erscheinen. Sicher ist jedoch, daß es zwischen der Angel- und der Zungenkonstruktion westlich des slawischen Kulturraums technologische Entwicklungsformen gab, die möglicherweise regional begrenzt blieben und vielleicht Klappmesser miteinschließen.

In den bearbeiteten slawischen Räumen gab es bereits mit, wahrscheinlich vor dem ersten Nachweis der Form A6 Griffzungenmesser, die besonders durch die Burgwallfunde aus Steinkirchen (Kat. Nr. 160) und Lübeck (Kat. Nr. 18), beide in das 11. bis 12. Jahrhundert datiert, belegt werden.

Ältere Vorstufen zu diesen Messern, die sich von den allgemein seit dem 13. Jahrhundert anzutreffenden Beispielen formal und technisch unterscheiden, sind nicht bekannt geworden.

76 Vgl. Kpitel 6, Abschnitt 2.2 und Kapitel 8, in denen auf diese Frage näher eingegangen wird. 


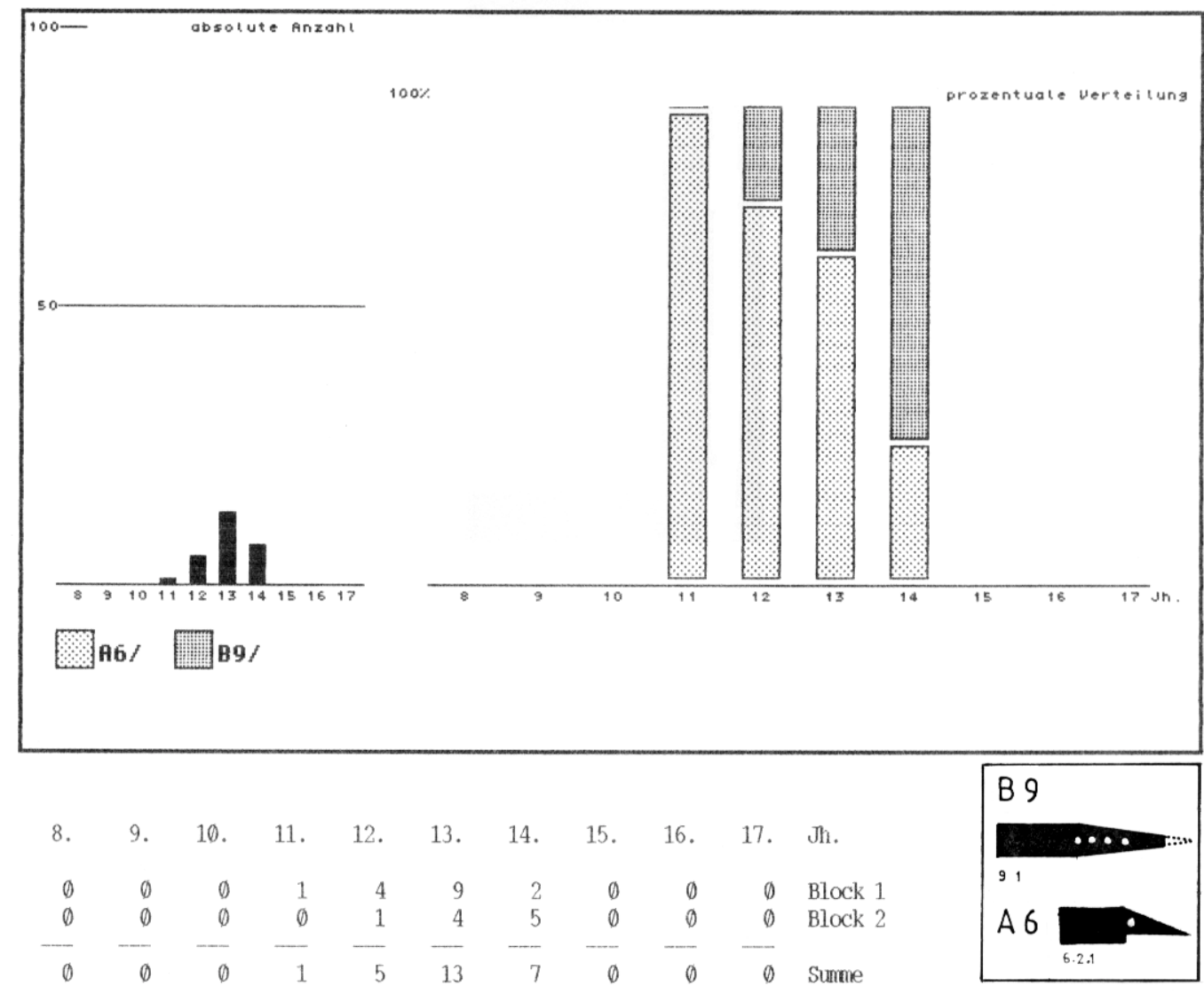

8. 9. 10. 11. 12. 13. 14. 15. 16. 17. Jh.

$\begin{array}{rrrrr}100 . \emptyset & 80.0 & 69.2 & 28.6 & \text { Block 1 } \\ 0 . \emptyset & 2 \emptyset .0 & 30.8 & 71.4 & \text { Block 2 }\end{array}$

Abb. 89

Das zeitlich versetzte Auftreten der Angelkonstruktion A6 und der frühen Zungenkonstruktion B9 im gesamten Untersuchungsraum (grob datierter Bestand) 
Die beiden einzigen Belege der Form A6 aus diesem Raum vor dem 13. Jahrhundert stammen von der Mecklenburg (Kat. Nr. 11) und von der befestigten Siedlung auf dem Hanfwerder (Kat. Nr. 100), beide werden in das ausgehende 10. bzw. beginnende 11. bis in das ausgehende 12. Jahrhundert datiert. Auch hier bleibt die Möglichkeit bestehen, sie als Klappmesser anzusprechen.

Die späteren Belege lassen sich aus historischen Gründen nicht mehr auf die slawische Bevölkerung beschränken.

Mit einer gewissen Wahrscheinlichkeit läßt sich abschließend vermuten, daß die slawische Vorgängerproduktion des 10. bis 12. Jahrhunderts keine Auswirkung auf die Neuentwicklung von Griffzungenmessern in den westlich angrenzenden Räumen hatte.

Ihr Einfluß auf die Entwicklung östlich der Elbe nach 1200 ist anhand der polnischen Belege aus Posen (Kat. Nr. 148), Danzig (Kat. Nr. 72) und Usch (Kat. Nr. 115) durchaus anzunehmen.

$\mathrm{Da}$ neben diesen auf wahrscheinlich ältere Traditionen zurückgehenden Formen möglicherweise durch den Zustrom deutscher Bürger und Siedler auch die von der Schneide nach oben abgesetzte Grifform Eingang fand, belegen die entsprechenden Funde aus Kalisch (Kat. Nr. 226), sicher aus Gommerstedt (Kat. Nr. 193, 203, 206 und 210) und Glasbach (Kat. Nr. 177) ${ }^{77}$.

\subsubsection{Die zeitliche Verteilung der einzelnen Griffangelkonstruktionen}

Grundsätzlich zu differenzieren sind Angelkonstruktionen mit einfachen oder zusätzlich gesicherten Griffen.

Die einfachen Griffangelmesser der Gruppe A1 besitzen lediglich einen auf die Angel geschlagenen röhrenförmigen Griff, meist aus organischem Material, ohne feststellbare Zusatzsicherungen.

Die Messergriffe der Gruppen A2 bis A4 sind auf verschiedene Arten zusätzlich gesichert, zum Beispiel mit Plättchen, Manschetten oder umgeschlagenen Angelenden.

Zur generellen Möglichkeit der Unterscheidung dieser beiden Gruppen sind folgende quellenkritische Aspekte zu berücksichtigen:

Viele Messer aus Siedlungsgrabungen besitzen bei ihrer Auffindung weder Griff noch Teile eventueller Zusatzsicherungen, während Messer aus Gräbern oder Depotfunden bei ihrer Niederlegung vollständig erhalten waren und in ihrer Art nach der Bergung zumindest in Teilen nachweisbar und daher in der Regel auch zu rekonstruieren sind.

77 Vgl. die entsprechenden Abbildungen in Kapitel 6, Abschnitt 2.2, in dem die einzelnen Belege früher Griffzungenmesser eingehend besprochen werden. 
Der Nachweis von Griffmaterialien und -konstruktionen ist darüberhinaus abhängig von der Bodenbeschaffenheit, in der die Funde angetroffen werden. So sind in der Regel Fundobjekte aus Metall oder organischen Substanzen in luftdicht abgeschlossenen Böden besser erhalten als in offenen und durchlässigen Böden.

Daher zeigen die in Brunnen, Abfallschächten oder von zahlreichen Siedlungsschichten und Fußböden überlagert geborgenen Messer aus Stadtkerngrabungen wie Amsterdam, London oder Lund eine oft vollständigere Erhaltung als Messer aus offenen, ländlichen Siedlungen.

Dieses muß bei den folgenden Analysen berücksichtigt werden, um zu vermeiden, nachweislose Zeitspannen überzuinterpretieren und das zweifellos anzunehmende Stadt-Land-Gefälle nicht zur ständigen Begründung für qualitative und quantitative Unterschiede werden zu lassen.

Für die vorangegangenen Untersuchungen waren diese Einschränkungen weniger relevant, da sich die grundsätzlichen Klingenformen eher durch Verschliff dem wissenschaftlichen Zugriff entziehen, Angel und Zunge dagegen fast immer eindeutig voneinander $\mathrm{zu}$ unterscheiden sind.

Relativierend läßt sich wiederum anführen, daß die Abhängigkeit von der Bodenbeschaffenheit alle Fundgattungen betrifft, also auch die Funde aus Gräberfeldern. Fast vollständig erhaltene Messer aus Burgen und ländlichen Siedlungen belegen zudem, daß die in Stadtkernen angetroffenen Befunde eher die Menge der besser erhaltenen Funde beeinflussen.

Die herausragenden Beispiele aus Gräberfeldern wie die Gold- und Silbergriffmesser aus Krefeld-Gellep oder Birka stellen eine positive Auslese dar. Sie repräsentieren als wertvolle Einzelstücke den Besitzstand der jeweiligen Verstorbenen und nicht, wie die zahlreichen übrigen Gebrauchsmesser, den technischen und künstlerischen Standard ihrer jeweiligen Epochen.

Für das gesamte Mittelalter gilt zudem, daß Zusatzsicherungen aus Bunt- oder Edelmetall wahrscheinlich in großer Zahl von verschliffenen oder unbrauchbaren Messern vor der Deponierung als Abfall abgelöst wurden.

\subsection{G riffangelmesser mit und ohne Zusatzsicherungen}

Die Auswertung des Gesamtbestandes zeigt eine vom 8. bis zum 17. Jahrhundert zunehmende Tendenz zusätzlich gesicherter Angelgriffe, die vom 9. und 10. Jahrhundert durchbrochen wird (Abb. 90).

Diese Jahrhunderte repräsentieren möglicherweise quantitativ den tatsächlich vorhandenen Bestand an zusätzlich gesicherten und mit Applikationen versehenen Griffangelmessern, da sie die erwähnten Grabfunde von skandinavischen und slawischen Gräberfeldern enthalten. Dieser Prozentsatz an zum Teil äußerst wertvollen Messern fehlt in allen darauf folgenden Jahrhunderten, in steigendem Maße begegnen sie als obertägig verwahrte Kunstobjekte seit dem 13. Jahrhundert in den Sammlungen von Kunstgewerbemuseen, seltener als Einzelfunde an heraus- 
ragenden Fundplätzen oder unter besonders günstigen Fundbedingungen wie das hochverzierte Messer aus dem Kloster Wienhausen (Kat. Nr. 74).

Somit hat die Zunahme derartiger Messergriffe im 9. und 10. Jahrhundert keine tendenzielle Bedeutung.

Zusätzlich gesicherte Angelgriffe gehören bis zum 11. Jahrhundert zu den Ausnahmen im Gesamtbestand.

Vom 12. bis zum 15. Jahrhundert wächst ihre Anzahl regelmäßig auf über $40 \%$ an.

Vom 15. zum 16. Jahrhundert verdoppelt sich ihr Bestand. Messer, deren Griffe einfach auf die Angel geschlagen wurden, sind seit dieser Zeit eine Randerscheinung.

Wie bereits mehrfach beobachtet, hat sich auch diese technische und materialbetreffende Veränderung nicht gleichzeitig im gesamten Untersuchungsraum durchgesetzt.

Die Auswertung des skandinavischen Fundbestandes zeigt, daß der Prozentsatz des 9. bis 10. Jahrhunderts, der die Grabfunde aus Birka enthält, hier in etwa den auch im gesamten Mittelalter anzunehmenden Anteil zusätzlich gesicherter Messergriffe umfaßt. Er liegt, abgesehen vom 11. Jahrhundert, immer über 20\% (Abb. 91). Die Zunahme auf über 50\% erfolgt im 16. Jahrhundert, zum 17. Jahrhundert vergrössert sich ihr prozentualer Anteil noch einmal auf über $70 \%$.

Im bearbeiteten niederländischen Fundbestand erfolgt der Einschnitt zur Majorität zusätzlich gesicherter Angelgriffe im 15. Jahrhundert, bereits im 14. Jahrhundert beträgt ihr Anteil aber schon 46\%. Ob die Zunahme seit dem Spätmittelalter regelmäßig stattgefunden hat, ist aufgrund der geringen Menge nicht zu entscheiden (Abb. 92).

Die Messer aus Dorestad (8.-9. Jahrhundert) weisen keine Griffsicherungen oder Applikationen auf, sie wurden in der Mehrzahl im Hafenbereich ausgegraben.

Im Bestand der nordwestlichen Bundesrepublik sind Angelgriffe mit Zusatzsicherungen seit dem 10. Jahrhundert nachweisbar. Sie setzen sich hier gegenüber den unbefestigten Griffen ebenfalls bereits im 15. Jahrhundert mehrheitlich durch (Abb. 93). Von den zehn nach 1500 datierten Messern waren alle zusätzlich gesichert.

Die Auswertung des bearbeiteten Bestandes aus den neuen Bundesländern (ehemalige DDR) belegt beispielhaft die bereits erwähnte Abhängigkeit der hier durchgeführten Untersuchungen von unterschiedlichen Forschungsschwerpunkten in den zugrunde liegenden regionalen Publikationen. 


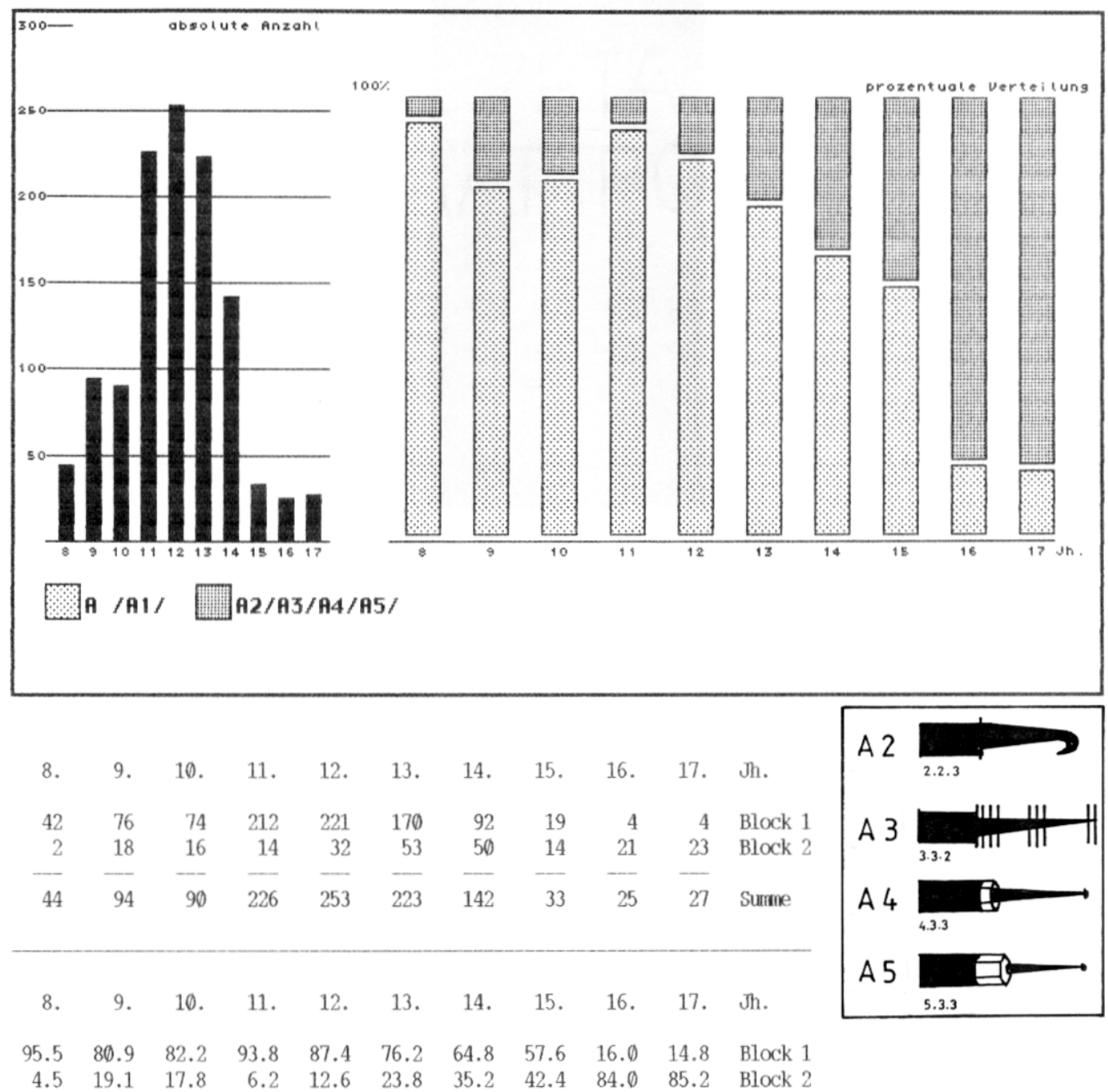

Abb. 90

Die zeitliche Verteilung von zusätzlich gesicherten Angelmessern gegenüber einfachen Angelmessern im gesamten Untersuchungsraum (grob datierter Bestand) 


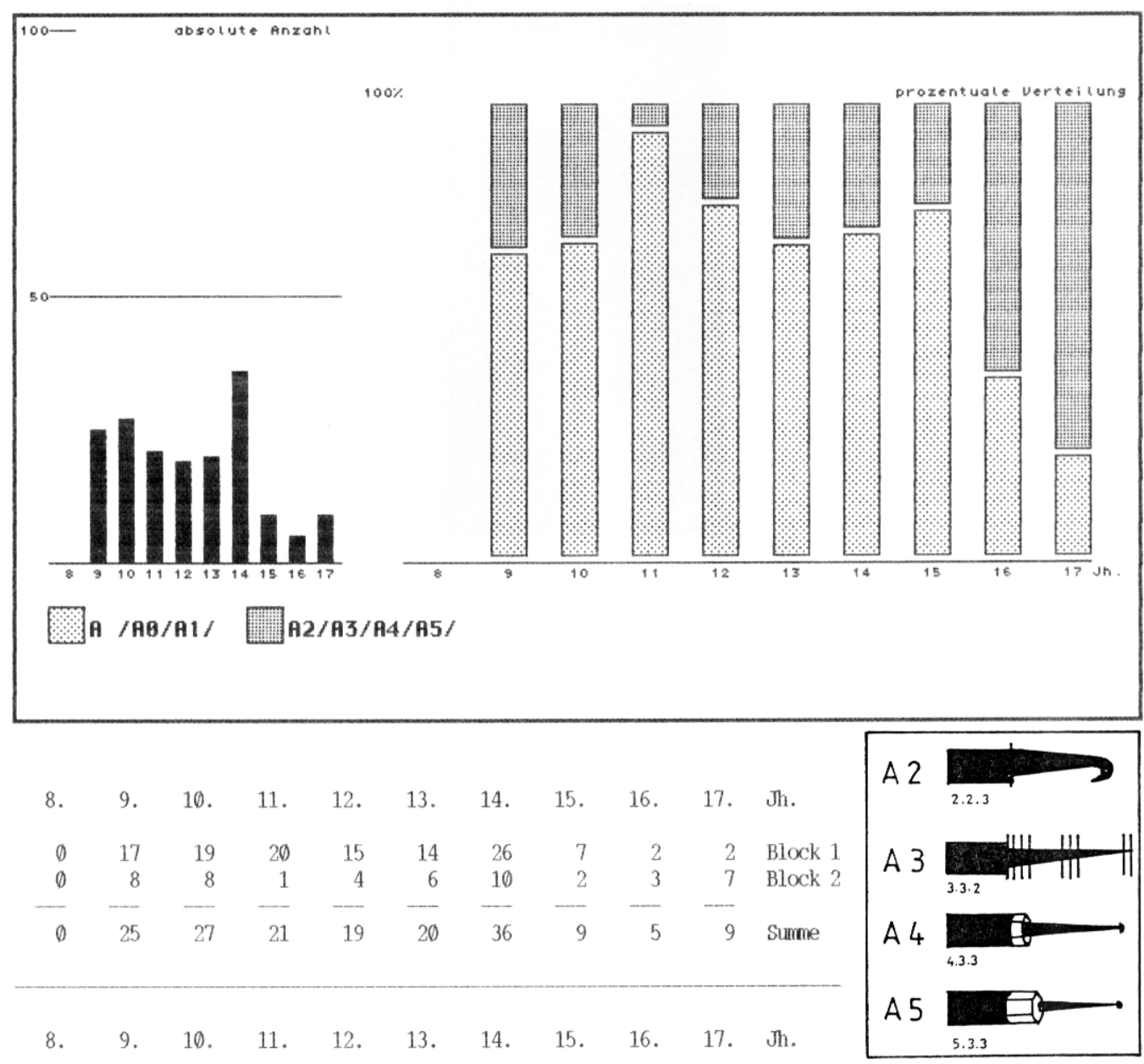

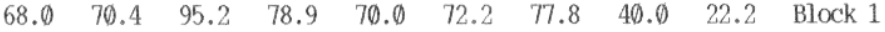

$\begin{array}{llllllllll}32.0 & 29.6 & 4.8 & 21.1 & 30.0 & 27.8 & 22.2 & 60.0 & 77.8 & \text { Block } 2\end{array}$ 


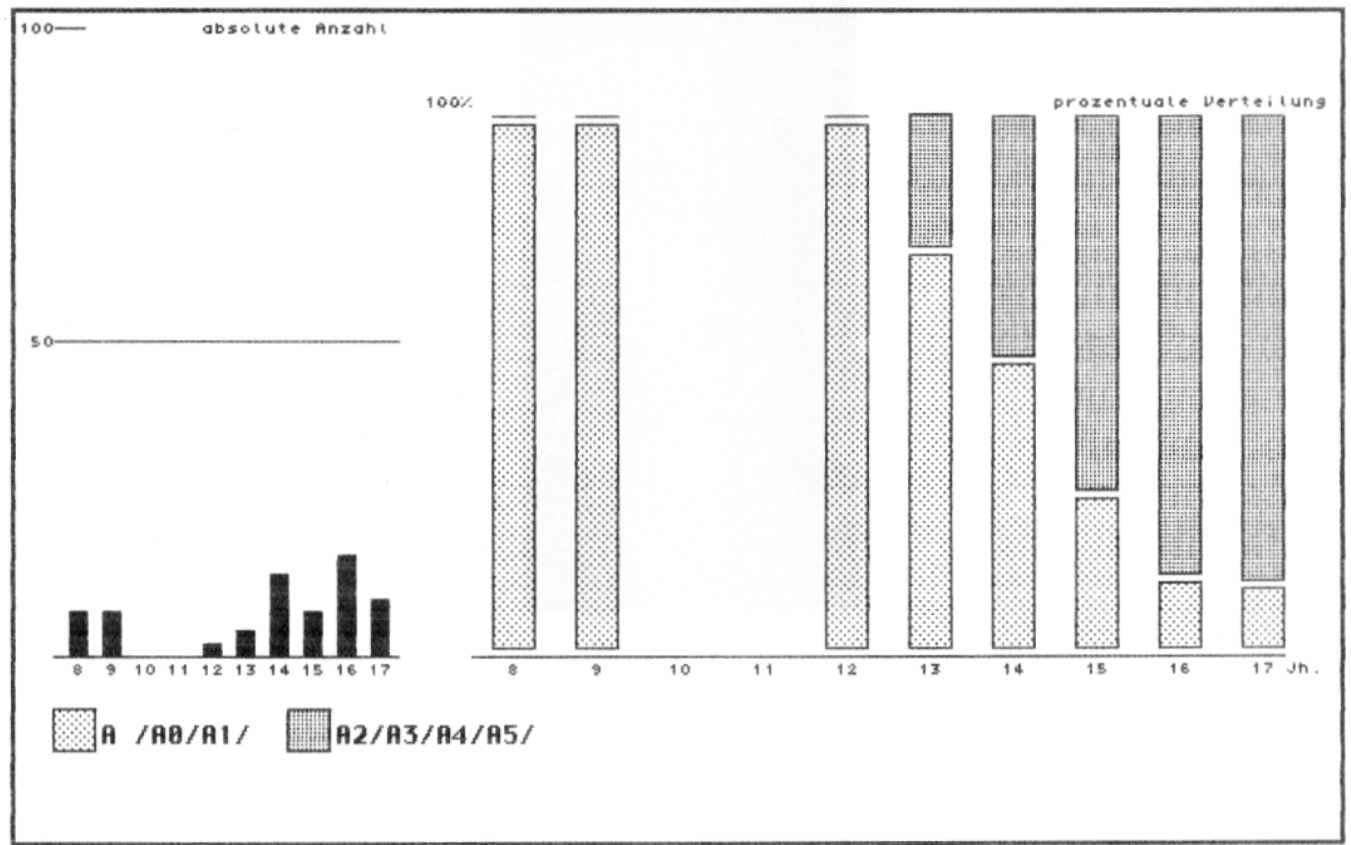

\begin{tabular}{rrrrrrrrrrl}
8. & 9. & 10. & 11. & 12. & 13. & 14. & 15. & 16. & 17. & Jh. \\
7 & 7 & 0 & 0 & 2 & 3 & 7 & 2 & 2 & 1 & Block 1 \\
0 & 0 & 0 & 0 & 0 & 1 & 6 & 5 & 14 & 8 & Block 2 \\
\hline 7 & $-\frac{1}{7}$ & 0 & 0 & 2 & 4 & 13 & 7 & -16 & 9 & Summe
\end{tabular}

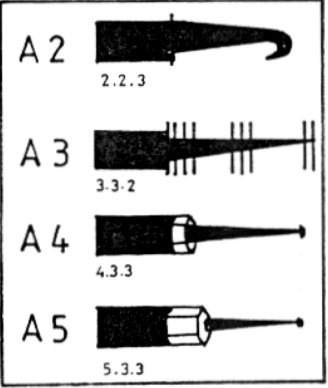

8. 9. 10. 11. 12. 13. 14. 15. 16. 17. Jh.

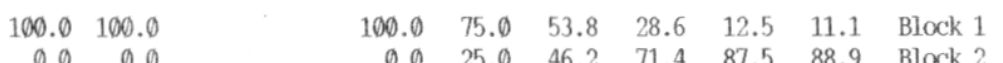

Abb. 92

Zusätzlich gesicherte Angelmesser in den Niederlanden (grob datierter Bestand) 


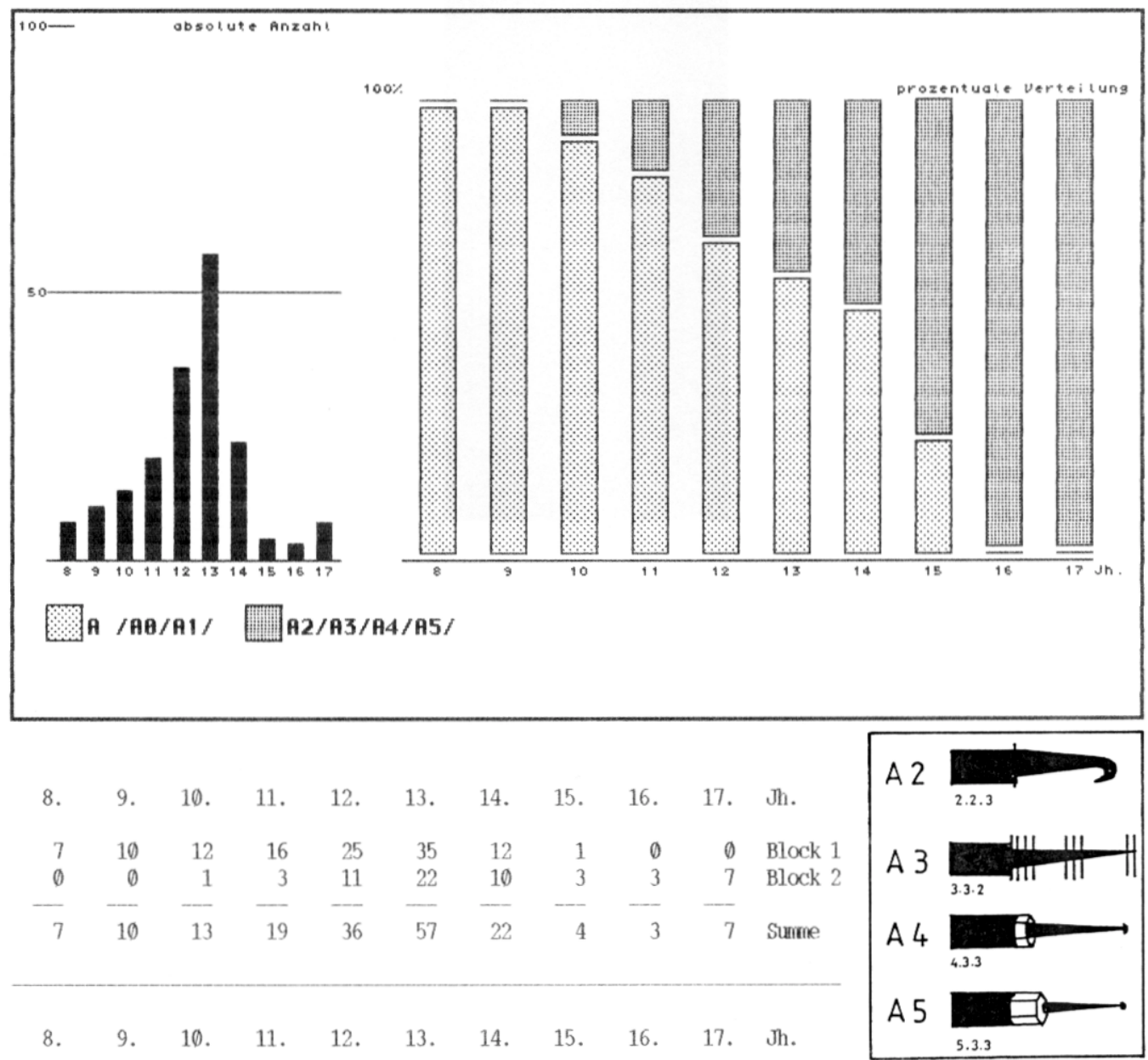

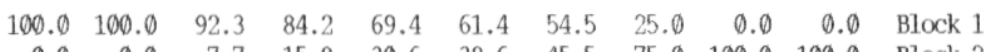

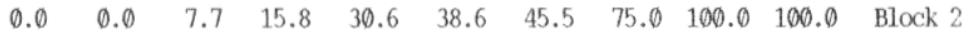

Abb. 93

Zusätzlich gesicherte Angelmesser in Nordwestdeutschland (grob datierter Bestand) 
Auffallend im aussagekräftigen Bereich sind vor allem die Anteile zusätzlich gesicherter Angelgriffe von 50\% im 9. und 13. Jahrhundert (Abb. 94).

Der Anteil im 10. Jahrhundert entspricht in etwa den im skandinavischen Bestand angetroffenen Werten des 9. bis 10. Jahrhunderts, auch der starke Rückgang im 11. und 12. Jahrhundert findet vergleichbare Tendenzen in den östlich angrenzenden Gebieten (Abb. 95) und in Skandinavien.

Im Gegensatz zu den genannten Räumen, in denen die bearbeiteten Funde aus zahlreichen Grabungen stammen, gibt im Bereich der ehemaligen DDR ein Umstand Ausschlag, der bisher kaum ins Gewicht fiel:

Die Messer des 9. bis 13. Jahrhunderts entstammen zu einem erheblichen Teil den veröffentlichten Beständen aus der Burgwallanlage in Groß-Raden (Sternberg) aus dem 9. bis 10. Jahrhundert und den entsprechenden Messern des 11. bis 13. Jahrhunderts aus der Siedlungskammer "Lieps" (Neubrandenburg).

Aus Groß-Raden wurden vor allem hervorragend erhaltene und verzierte Messer mit zum Teil umgeschlagenen Griffangeln veröffentlicht, also eine publikationsbedingte positive Auslese. Der Bestand aus den einzelnen Siedlungsarealen der Lieps wurde repräsentativ publiziert, auf 51 Messer kommen hier 13 Messer mit Zusatzsicherungen.

Die Messer der Formen A2 bis A4 machen in den Siedlungen der "Lieps", die um ein politisch-religiöses Zentrum angesiedelt waren, rund 26,5\% aus. Der gleiche Bestand erreicht für das 11. und 12. Jahrhundert in der Gesamtauswertung der ehemaligen DDR lediglich $8,1 \%$ bzw. 10,5\%, da die meisten Messer aus der Lieps aufgrund ihrer weitgefaßten Zeitstellung keine Berücksichtigung fanden.

Somit repräsentieren die Verteilungen des 9. bis 10. Jahrhunderts den zu vermutenden Gesamtbestand dieser Messer, möglicherweise mit einem leicht überproportionalen Anteil an herausragenden, auf vermögende Bevölkerungsteile beschränkten Beispielen.

Das Bild des 11. bis 12. Jahrhunderts belegt eher den alltäglichen Bestand der in der westslawischen Bevölkerung benutzten Messer, in dem der Bestandteil an zusätzlich gesicherten Griffen, der in Hauptorten des wirtschaftlichen und politischen Lebens häufiger anzutreffen ist, trotz einiger Belege von Burgwällen unterrepräsentiert erscheint.

Der in den Siedlungszentren der "Lieps" ermittelte Anteil von 26,5\% sowie die für die Bestände des 11. und 12. Jahrhunderts aus der ehemalige DDR vorliegenden Werte von $8,1 \%$ bzw. 10,5\% belegen als Rahmendaten jedenfalls, daß vom 9. bis zum 12. Jahrhundert unabhängig von möglichen Schwankungen mit durchschnittlichen Anteilen von mindestens 24\% und höchstens 32\% gerechnet werden kann. 


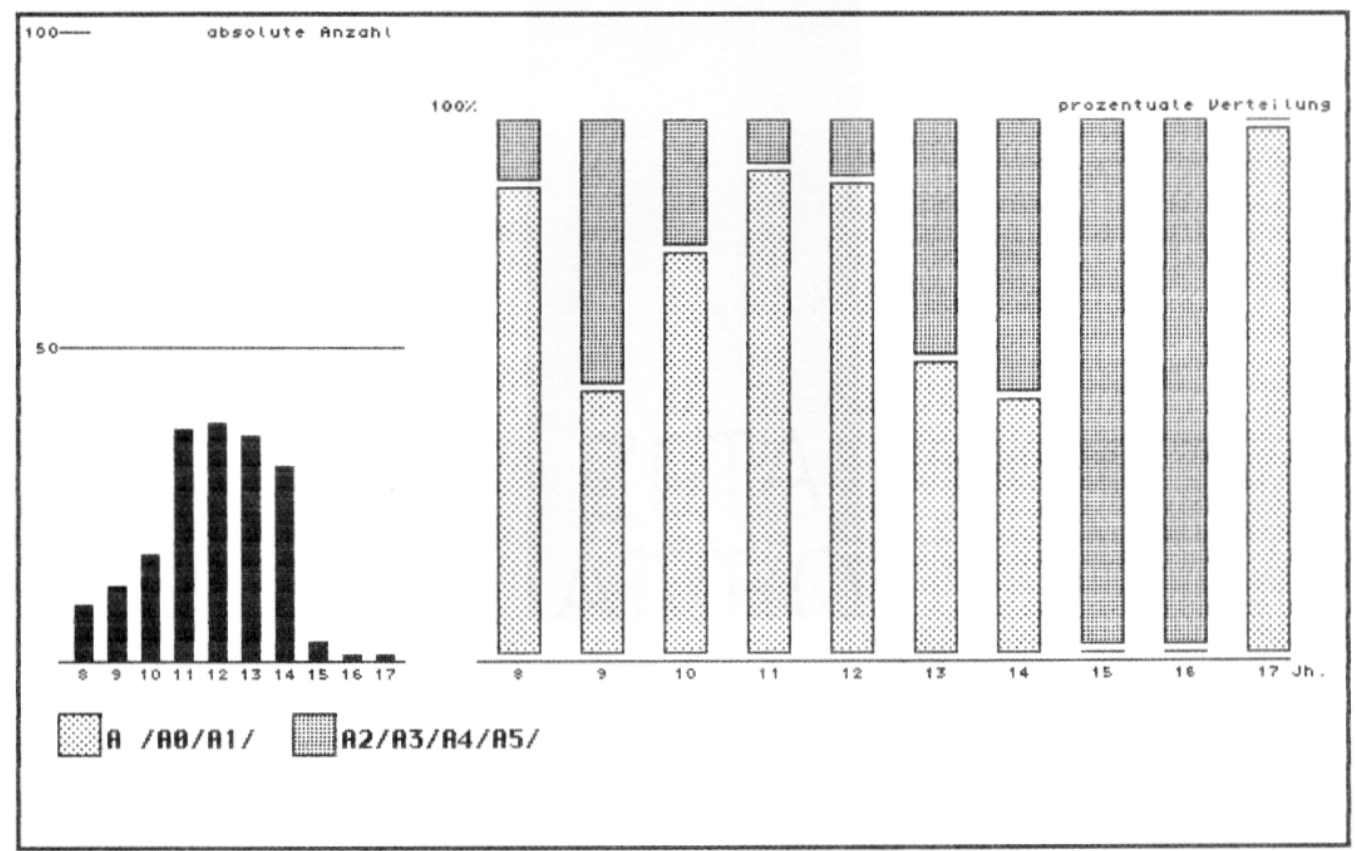

$$
\begin{array}{rrrrrrrrrrl}
\text { 8. } & 9 . & 10 . & 11 . & 12 . & 13 . & 14 . & 15 . & 16 . & 17 . & \text { Jh. } \\
8 & 6 & 13 & 34 & 34 & 20 & 15 & \emptyset & \emptyset & 1 & \text { Block 1 } \\
1 & 6 & 4 & 3 & 4 & 16 & 16 & 3 & 1 & \emptyset & \text { Block 2 } \\
\hline 9 & -12 & -17 & -37 & 38 & 36 & 31 & 3 & 1 & 1 & \text { Surme }
\end{array}
$$

8. 9. 10. 11. 12. 13. 14. 15. 16. 17. Jh.

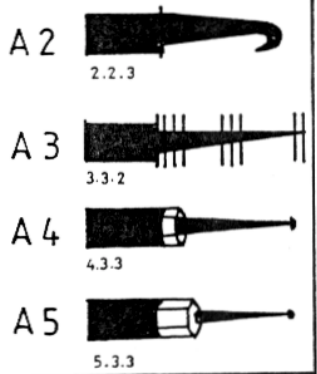

$\begin{array}{lllllllllll}88.9 & 50 . \emptyset & 76.5 & 91.9 & 89.5 & 55.6 & 48.4 & 0.0 & \emptyset . \emptyset & 100 . \emptyset & \text { Block } 1\end{array}$

$\begin{array}{lllllllllll}11.1 & 50.0 & 23.5 & 8.1 & 10.5 & 44.4 & 51.6 & 100.0 & 100.0 & 0.0 & \text { Block } 2\end{array}$ 
Der in Skandinavien und Ostdeutschland beobachtete Rückgang vom 9. zum 11. Jahrhundert ist somit in dieser Stärke sicher überzeichnet. Dem folgte im 13. Jahrhundert, in dem auf dem Gebiet der ehemaligen DDR nahezu überall die Ansiedlung westlicher Bevölkerungsgruppen und eine erneute Städtegründungsphase zu berücksichtigen sind, der Anstieg des Anteils zusätzlich befestigter Messergriffe auf über 40\%, im 14. Jahrhundert auf 50\% aller Griffangelmesser.

Im Vergleich zu den untersuchten Fundbeständen aus den Niederlanden und der nordwestlichen Bundesrepublik scheinen sich aber die bereits bei der Verteilung der Griffzungenkonstruktionen beobachteten Schwerpunkte zu bestätigen:

Der höhere Anteil von Griffangelmessern mit zusätzlichen Sicherungen zumeist am Griffende im slawischen Siedlungsgebiet ist mit den unterschiedlichen Publikationsschwerpunkten allein nicht zu erklären.

So besteht ein nicht geringer Anteil der im nordwestdeutschen Fundgut angetroffenen Zusatzbefestigungen des 10. bis 12. Jahrhunderts aus Messern, die ebenfalls in slawischen Siedlungskontexten angetroffen wurden (Burgwall von Alt-Lübeck, Kat. Nr. 18-28; Gräberfelder in Fliessau-Spranz und Növenthien, Kat. Nr. 68-71).

Mit einiger Wahrscheinlichkeit sind im alten Siedlungsbereich der Westslawen von ihnen verwendete Techniken und Konstruktionsformen auch nach dem 12. Jahrhundert weitergeführt worden.

In den nordwestdeutschen Beständen läßt sich der Anstieg im 13. Jahrhundert, der bedingt auch in den Niederlanden anzunehmen ist, im Fundgut dagegen als deutlicher Bruch zu den älteren Messern belegen. Vor allem die Messer von der Hattinger Isenburg und der Niederungsburg bei Haus Meer (Grevenbroich) zeigen im Vergleich mit den Messern der Essener Isenburg, der Raffenburg, der Rücklenburg und der Burg Altena deutlich die Unterschiede vor und nach dem beginnenden 13. Jahrhundert, unter anderem auch hinsichtlich der seit dem 13. Jahrhundert aufkommenden Griffplättchen ${ }^{78}$.

Die Verteilung der entsprechenden Messer in den bearbeiteten polnischen und litauischen Fundbeständen unterscheidet sich vor allem in den geringeren Anteilsmengen von dem übrigen Untersuchungsgebiet (Abb. 95).

Aber auch hier erscheint der in Skandinavien und im westslawischen Raum beobachtete Rückgang vom 9. zum 11. Jahrhundert, der demnach trotz der unterschiedlichen Quellenlage tendenziell für diese Räume als wahrscheinlich anzunehmen ist.

Insgesamt haben zusätzliche Sicherungen an Angelmessern im polnischen Bestand, der absolut betrachtet am besten repräsentiert ist, nur eine untergeordnete Rolle gespielt. Der Durchbruch der hier behandelten Konstruktionsformen scheint ähnlich wie in Skandinavien nicht vor dem 15. Jahrhundert stattgefunden zu haben. 


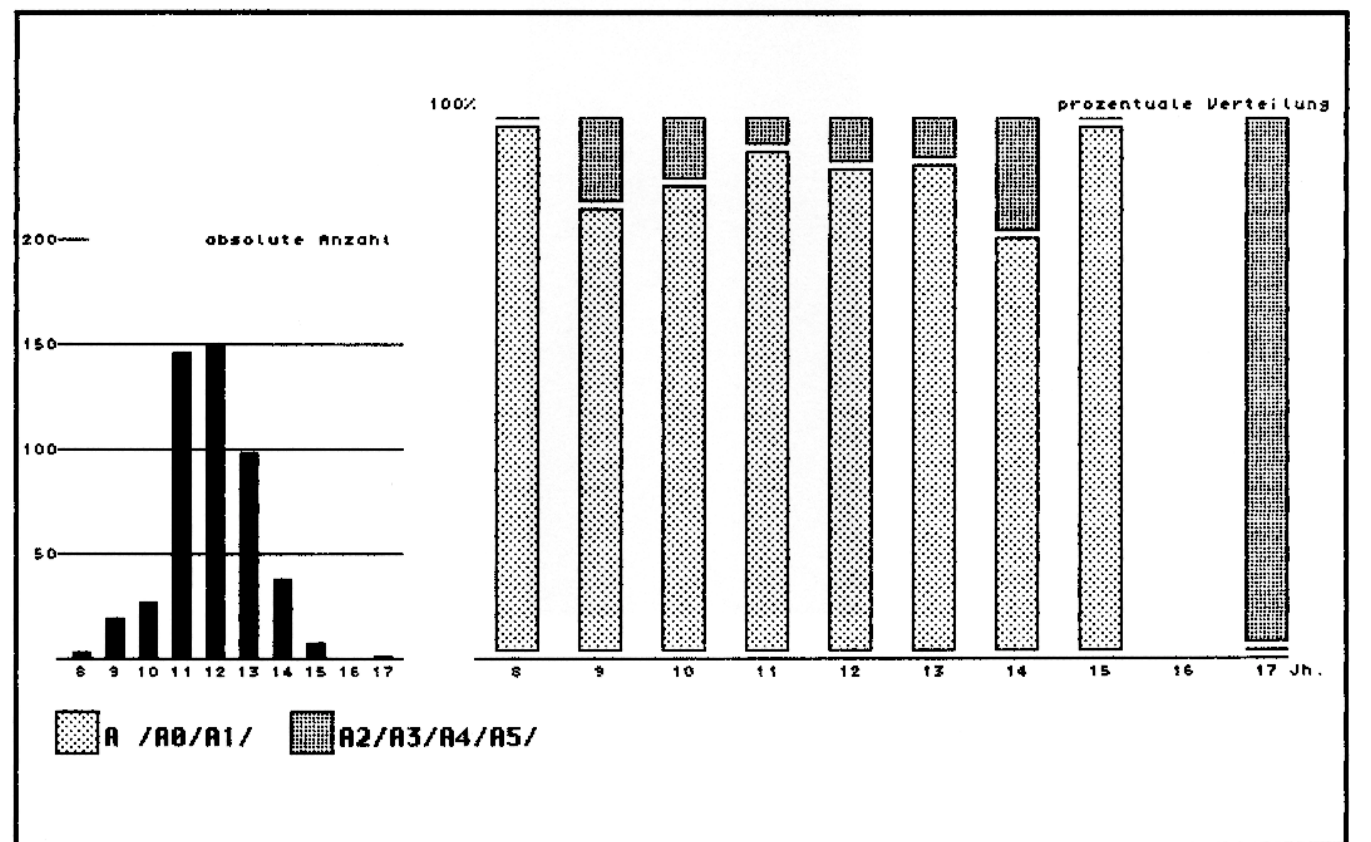

\begin{tabular}{rrrrrrrrrrl}
8. & 9. & 10. & 11. & 12. & 13. & 14. & 15. & 16. & 17. & Jh. \\
3 & 16 & 24 & 139 & 138 & 91 & 30 & 7 & $\emptyset$ & 0 & Block 1 \\
0 & 3 & 3 & 7 & 12 & 7 & 8 & 0 & $\emptyset$ & 1 & Block 2 \\
\hline 3 & -19 & -27 & 146 & $\frac{150}{9}$ & 98 & 38 & 7 & 0 & 1 & Summe
\end{tabular}

8. $\quad 9.10 .11 .12 .13 .14 .15 .16 .17 . \quad$ Jh.

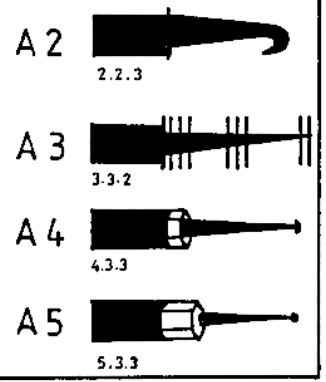

$\begin{array}{rrrrrrrrrr}100 . \emptyset & 84.2 & 88.9 & 95.2 & 92 . \emptyset & 92.9 & 78.9 & 100 . \emptyset & \emptyset . \emptyset & \text { Block 1 } \\ \emptyset . \emptyset & 15.8 & 11.1 & 4.8 & 8 . \emptyset & 7.1 & 21.1 & \emptyset . \emptyset & 100 . \emptyset & \text { Block 2 }\end{array}$ 
In den verbleibenden Untersuchungsräumen der nordwestlichen ehemaligen UdSSR sind Messer mit zusätzlich gesicherten Angelgriffen im 8., 9., 12. und 13. Jahrhundert nachzuweisen, das 14. bis 17. Jahrhundert läßt hier, ähnlich wie das 15. bis 17. Jahrhundert in den Beständen der ehemaligen DDR und Polens, keine verläßlichen Aussagen mehr zu.

Abschließend läßt sich festhalten, daß Angelkonstruktionen mit zusätzlichen Sicherungselementen im gesamten Untersuchungsgebiet und während des größten Teils der Untersuchungszeit bekannt waren. Möglicherweise waren sie in Zentren mit wirtschaftlicher und politisch-kultureller Kulmination stärker verbreitet als an anderen Orten. Es ist wahrscheinlich, daß sie im westlichen Bearbeitungsraum vor dem 12. Jahrhundert kaum jemals mehr als 30\% der Angelkonstruktionen ausgemacht haben. Wie die Bestände aus der Bundesrepublik und den Niederlanden zeigen, scheint es von Beginn ihres Auftretens an hier eine fortschreitende Zunahme gegeben zu haben.

In Skandinavien und im bearbeiteten slawischen Siedlungsraum waren zusätzlich gesicherte Griffangelmesser vor dem 12. Jahrhundert stärker verbreitet als im Westen. Vom 9. zum 11. Jahrhundert wurde ein allgemeiner Rückgang beobachtet, die folgenden Jahrhunderte zeigen jeweils unterschiedliche Entwicklungen und Anteile im Norden, in Ostdeutschland und in Polen.

Die Griffangelmesser des 15. bis 17. Jahrhunderts besaßen wohl zum größten Teil zusätzlich gesicherte Griffe, vor allem im Heftbereich. Als auffällige Vorläufer sind vor allem die griffplättchenversehenen Messer des 13. und 14. Jahrhundert anzusehen $^{79}$.

\subsection{Unter- und oberständige Griffe}

Das Phänomen der Griffunter- und -oberständigkeit hat wahrscheinlich funktionstechnische Gründe, ist in seinen Anwendungsbereichen aber bis jetzt kaum hinreichend bekannt.

Für die Feststellung der Griffunterständigkeit galten die im Zuge der eingangs erfolgten Anmerkungen $\mathrm{zu}$ den archäologischen Quellen bereits vorgestellten Kriterien $^{80}$. Untersucht wurde sie für alle Griffangelmesser.

Nachzuweisen ist die Griffunterständigkeit im gesamten Untersuchungsgebiet vom 8. bis zum 17. Jahrhundert. Vom 8. bis zum 13. Jahrhundert steigen ihre Anteile von $5 \%$ bis auf $45 \%$ an, danach fallen sie über noch $30 \%$ im 15 . Jahrhundert auf 4,5\% im 17. Jahrhundert ab. Die Hauptverbreitungszeit griffunterständiger Messer liegt im 13. und 14. Jahrhundert (Abb. 96).

\footnotetext{
79 Vgl. Kapitel 6, Abschnitt 2.4.3.1

80

Vgl. Kapitel 3, Abschnitt 2.5
} 


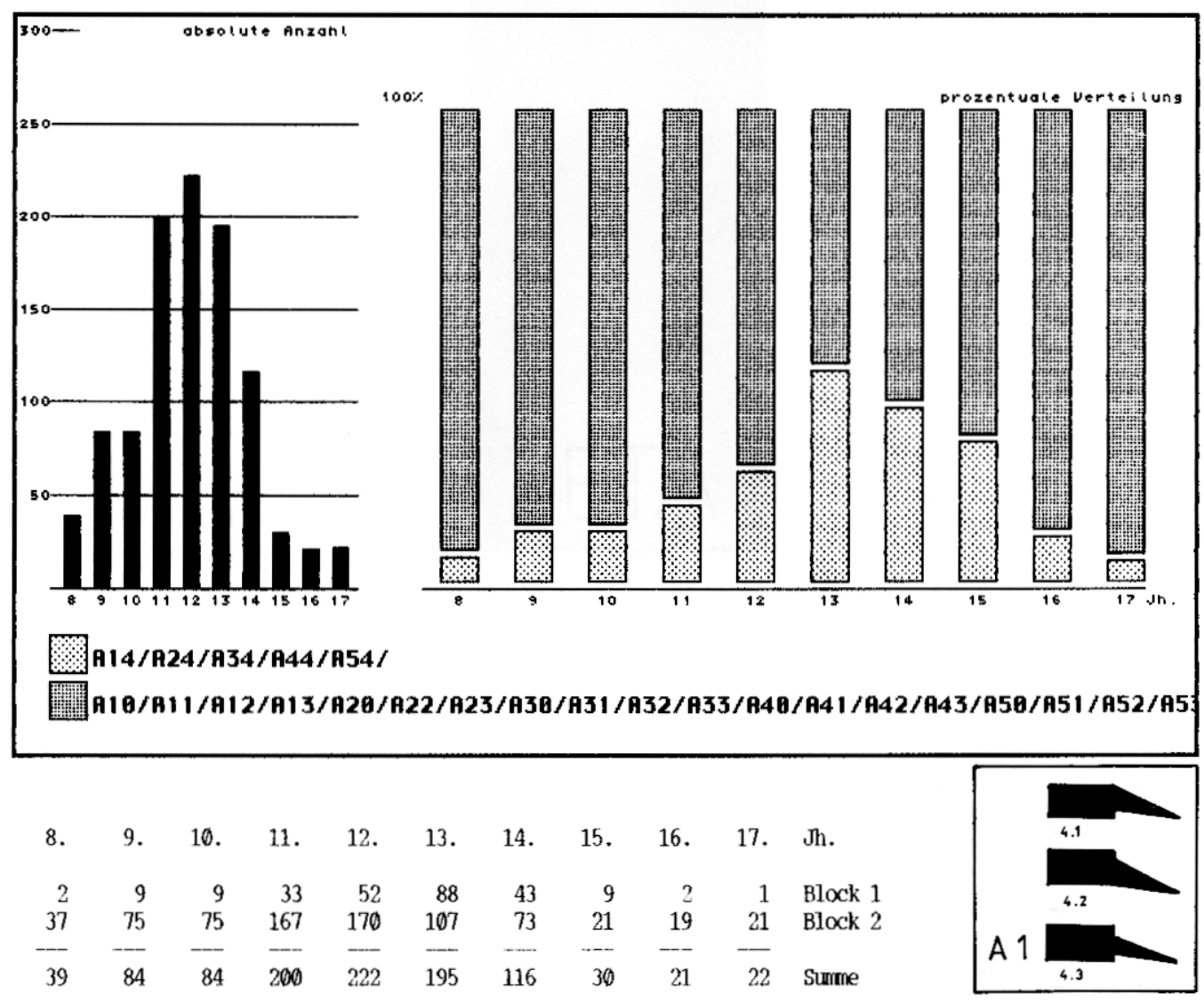

8. 9. 10. 11. $12.13 .14 .15 .16 .17 . \quad \mathrm{Jh}$.

$\begin{array}{lllllllllll}5.1 & 10.7 & 10.7 & 16.5 & 23.4 & 45.1 & 37.1 & 30.0 & 9.5 & 4.5 & \text { Block 1 }\end{array}$

$\begin{array}{lllllllllll}94.9 & 89.3 & 89.3 & 83.5 & 76.6 & 54.9 & 62.9 & 70 . \emptyset & 90.5 & 95.5 & \text { Block 2 }\end{array}$

Abb. 96

Griffunterständige Angelmesser im gesamten Untersuchungsraum (grob datierter Bestand) 
Gründe für das unterschiedlich starke Aufkommen können nicht genannt werden, es ist jedoch möglicherweise damit zu rechnen, daß sich mit der Griffunterständigkeit nicht nur eine feste Funktion verbindet, wie Koltschin 1959 anhand der Novgoroder Messer darlegte ${ }^{81}$, denn sie tritt auch an Angelmessern auf, die sich aufgrund ihrer Griffgestaltung und Klingenform deutlich als Tafel- bzw. Speisemesser zu erkennen geben.

Der Rückgang im 16. und 17. Jahrhundert folgt nicht der allgemeinen Entwicklung von Griffangelmessern, die nach 1550/1600 gegenüber den Griffzungenmessern wieder zunehmen.

Die Griffoberständigkeit zur Klinge ist äußerst selten aufgetreten. Im Vergleich zur Griffunterständigkeit von Angelmessern scheinen ihre prozentualen Anteile vom 8. bis zum 17. Jahrhundert in etwa linear zu verlaufen, ihre höchsten Anteile liegen mit 6,2\% und 7,1\% im 11. und 12. Jahrhundert (Abb. 97).

\subsection{G Giffangelabsatzmöglichkeiten und die Anhebung des Griffs gegenüber der Schneide}

Die verschiedenen Absatzmöglichkeiten der Angel oder des Griffs werden im Klassifizierungsmodell durch die Codebezeichnungen A1.0 (ohne Absatz), A1.1 (zum Rücken abgesetzt), A1.2 (zur Schneide abgesetzt) und A1.3 (mittige Angel) erfaßt. Diese vier Angelstellungen erscheinen auch in der Auswertungsgraphik getrennt (Abb. 98).

Da nicht zusätzlich gesicherte Angelmesser (Form A1) nach dem 15. Jahrhundert kaum noch anzutreffen sind, ist dieses Kriterium im 16. und 17. Jahrhundert nicht mehr relevant.

Die am häufigsten beobachtete Absatzart ist die des beidseitigen Absatzes der Angel zum Rücken und zur Schneide (A1.3), die im gesamten Untersuchungsgebiet vom 8 . bis 15 . Jahrhundert immer über $70 \%$ ausmacht.

Die Form A1.2 (eine von der Schneide abgesetzte Angel) ist durchgehend nachweisbar.

Griffangeln, die nur zum Rücken hin abgesetzt wurden (A1.1), sind nur vereinzelt vom 9. bis zum 14. Jahrhundert angetroffen worden. Auch Messer, deren Angeln ohne Absatz in die Klingenkanten übergehen (A1.0), treten in nur geringer Anzahl auf, sie scheinen auf das 11. bis 13. Jahrhundert beschränkt werden zu können.

Die gleiche Auswertung für den Bestand gesicherter und ungesicherter Angelmesser zeigt die gleichen Tendenzen (Abb. 99). 


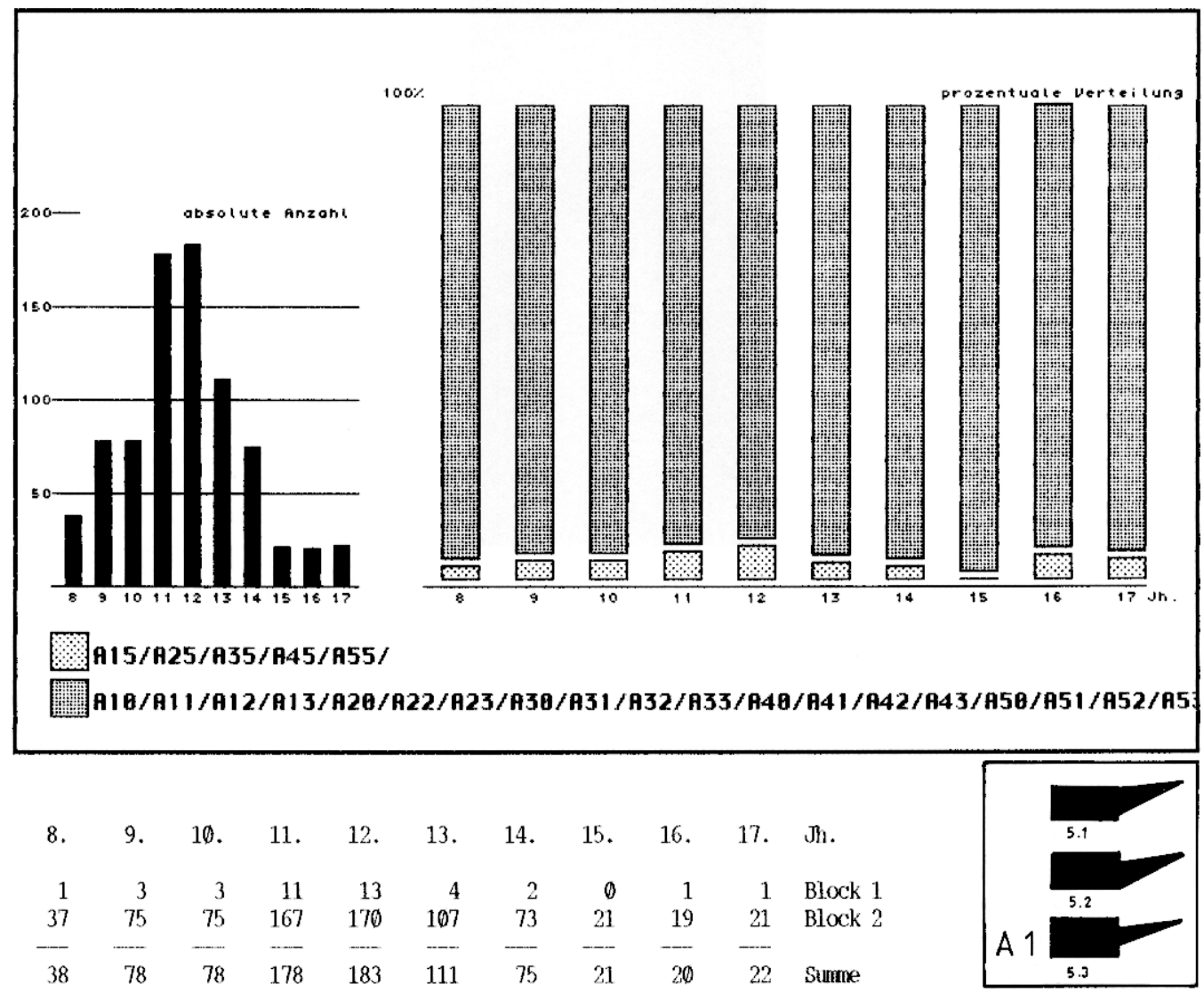

8. 9. 10. 11. 12. 13. 14. 15. 16. $17 . \quad$ Jh.

$\begin{array}{rrrrrrrrrrr}2.6 & 3.8 & 3.8 & 6.2 & 7.1 & 3.6 & 2.7 & 0.0 & 5.0 & 4.5 & \text { Block 1 } \\ 97.4 & 96.2 & 96.2 & 93.8 & 92.9 & 96.4 & 97.3 & 100 . \emptyset & 95 . \emptyset & 95.5 & \text { Block 2 }\end{array}$ 


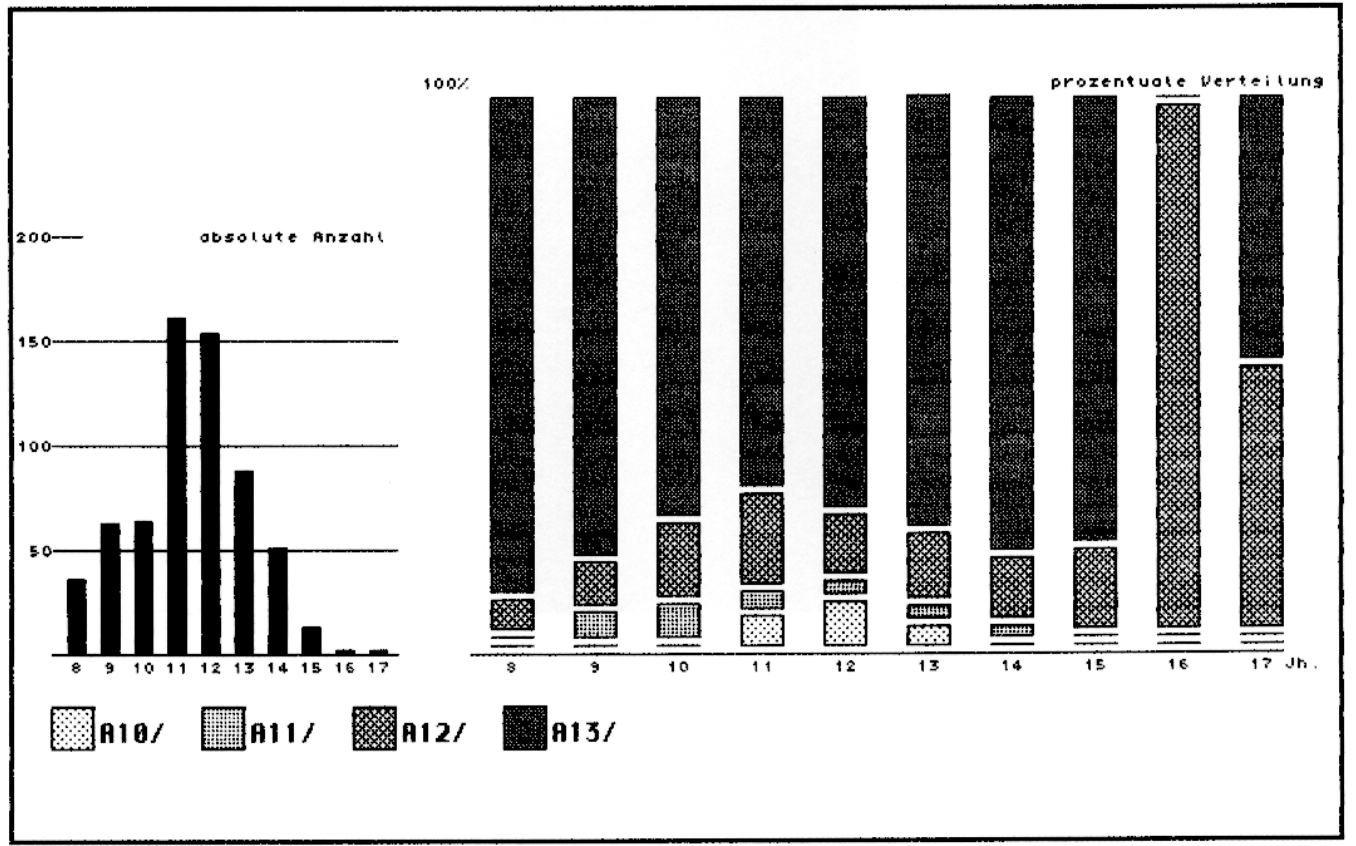

$$
\begin{array}{rrrrrrrrrrl}
8 . & 9 . & 10 . & 11 . & 12 . & 13 . & 14 . & 15 . & 16 . & 17 . & \text { Jh. } \\
\emptyset & 0 & 0 & 9 & 13 & 3 & 0 & 0 & \emptyset & \emptyset & \text { Block 1 } \\
0 & 3 & 4 & 5 & 4 & 2 & 1 & 0 & \emptyset & \emptyset & \text { Block 2 } \\
2 & 5 & 9 & 28 & 17 & 11 & 6 & 2 & 2 & 1 & \text { Block 3 } \\
34 & 55 & 51 & 119 & 120 & 72 & 44 & 11 & 0 & 1 & \text { Block 4 } \\
\hline 36 & -63 & 64 & 161 & 154 & 88 & 51 & 13 & 2 & 2 & \text { Surme }
\end{array}
$$

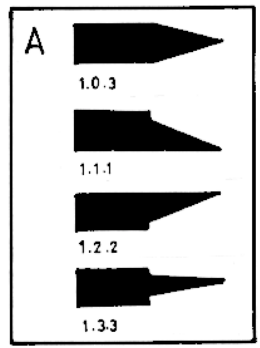

\begin{tabular}{rrrrrrrrrrl}
8. & 9. & 10. & 11. & 12. & 13. & 14. & \multicolumn{1}{c}{15.} & \multicolumn{1}{c}{16.} & 17. & Jh. \\
0.0 & 0.0 & 0.0 & 5.6 & 8.4 & 3.4 & $\emptyset . \emptyset$ & $\emptyset . \emptyset$ & $\emptyset . \emptyset$ & $\emptyset . \emptyset$ & Block 1 \\
0.0 & 4.8 & 6.2 & 3.1 & 2.6 & 2.3 & $2 . \emptyset$ & $\emptyset . \emptyset$ & $\emptyset . \emptyset$ & $\emptyset . \emptyset$ & Block 2 \\
5.6 & 7.9 & 14.1 & 17.4 & 11.0 & 12.5 & 11.8 & 15.4 & $100 . \emptyset$ & $5 \emptyset . \emptyset$ & Block 3 \\
94.4 & 87.3 & 79.7 & 73.9 & 77.9 & 81.8 & 86.3 & 84.6 & $\emptyset . \emptyset$ & $50 . \emptyset$ & Block 4
\end{tabular}

Abb. 98

Die unterschiedlichen Absatzmöglichkeiten von Griffangeln bei einfachen (ungesicherten) Angelmessern im gesamten Untersuchungsraum (grob datierter Bestand) 


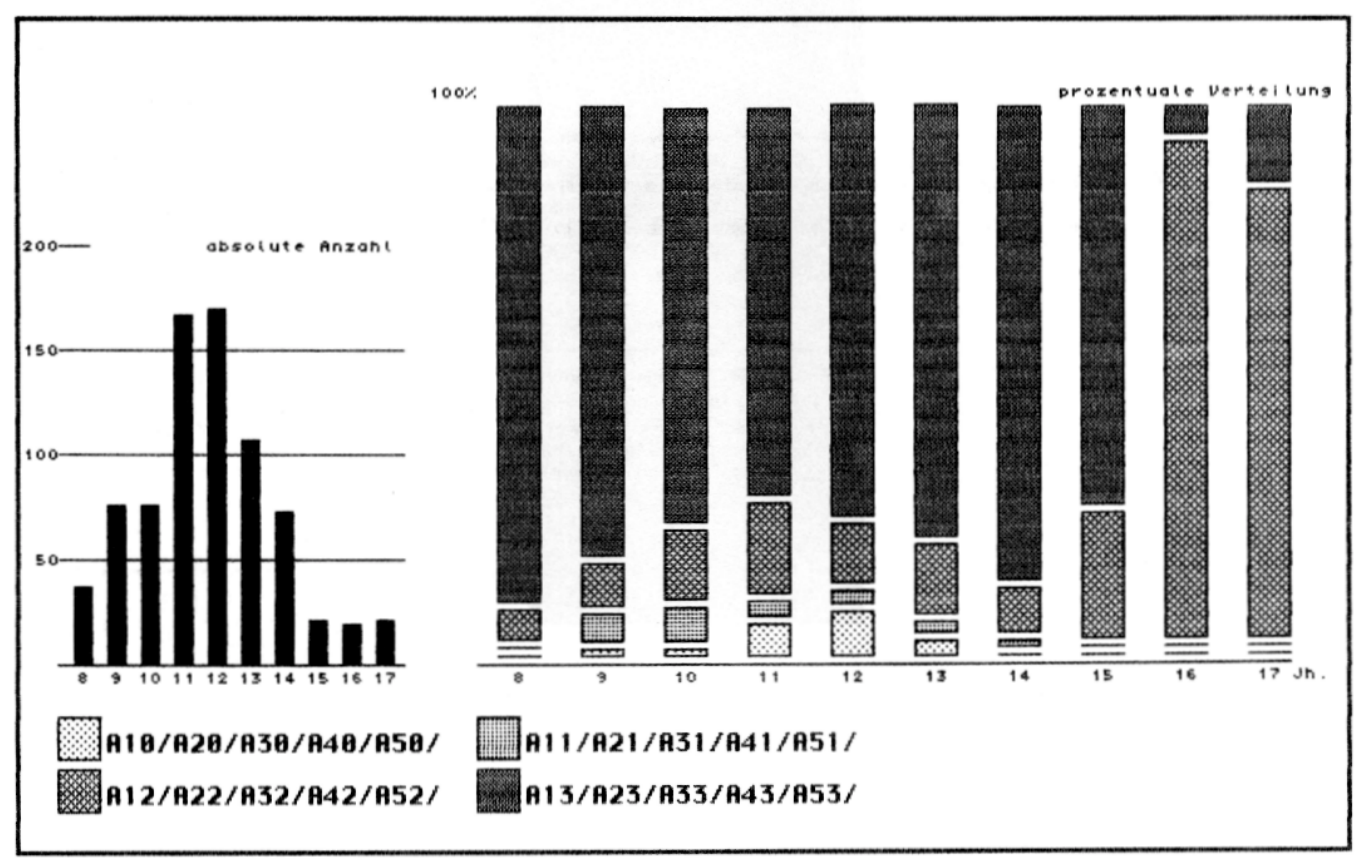

\begin{tabular}{rrrrrrrrrrl}
8. & 9. & 10. & 11. & 12. & 13. & 14. & 15. & 16. & 17. & Jh. \\
0 & 1 & 1 & 10 & 14 & 3 & 0 & 0 & 0 & 0 & Block 1 \\
0 & 4 & 5 & 5 & 4 & 2 & 1 & 0 & 0 & 0 & Block 2 \\
2 & 6 & 10 & 29 & 19 & 14 & 6 & 5 & 18 & 18 & Block 3 \\
35 & 65 & 60 & 123 & 133 & 88 & 66 & 16 & 1 & 3 & Block 4 \\
\hline 37 & -76 & 76 & 167 & 170 & 107 & 73 & 21 & 19 & 21 & Surme
\end{tabular}

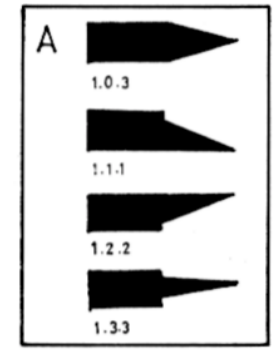

$\begin{array}{rrrrrrrrrrl}8 . & 9 . & 10 . & 11 . & 12 . & 13 . & 14 . & 15 . & 16 . & 17 . & \text { Jh. } \\ 0.0 & 1.3 & 1.3 & 6.0 & 8.2 & 2.8 & 0.0 & 0.0 & \emptyset .0 & \emptyset .0 & \text { Block 1 } \\ 0.0 & 5.3 & 6.6 & 3.0 & 2.4 & 1.9 & 1.4 & 0.0 & \emptyset .0 & \emptyset .0 & \text { Block 2 } \\ 5.4 & 7.9 & 13.2 & 17.4 & 11.2 & 13.1 & 8.2 & 23.8 & 94.7 & 85.7 & \text { Block 3 } \\ 94.6 & 85.5 & 78.9 & 73.7 & 78.2 & 82.2 & 90.4 & 76.2 & 5.3 & 14.3 & \text { Block 4 }\end{array}$

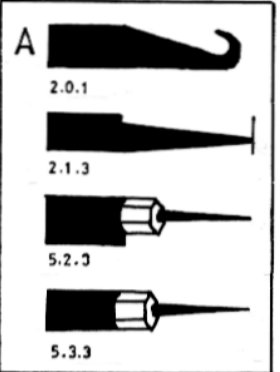

Abb. 99

Die unterschiedlichen Absatzmöglichkeiten von Griffangeln bei einfachen und zusätzlich gesicherten Angelmessern im gesamten Untersuchungsraum (grob datierter Bestand) 
Auch hier umfassen vom 8. bis zum 15. Jahrhundert mittig ansitzende Angeln immer einen Anteil von über $70 \%$.

Von der Schneide abgesetzte Angeln erscheinen jeweils zum 11. und 13. Jahrhundert leicht ansteigend, mit dem 15. Jahrhundert (jetzt bereits 23,8\%) beginnt eine erneute Zunahme, die im 16. und 17. Jahrhundert auf rund $90 \%$ aller beobachteten Griffangelmesser ansteigt. Grundsätzliche Unterschiede betreffen die Angelmesser vor und nach 1400:

Während bei den früheren Messern der Absatz in der Regel nur die Angel betrifft, bei aufgestecktem Griff also nicht mehr oder nur noch schwach sichtbar ist, erscheint bei den späteren Messern der ganze Griff zur Schneide hin klar abgesetzt (Abb. 100).

Der genaue Zeitpunkt, von dem an der Griff aus Sicherheitsgründen vollständig zur Schneide hin abgesetzt wurde, ist nur schwer zu bestimmen, da die Schneiden fast immer angeschliffen sind. Bei Messern mit gesicherten Heftbereichen (Heftmanschetten und -hülsen (A4) sowie Vollmetallstücken zwischen Klinge und Angel (A5)) wurde die Anhebung des Griffs ebenfalls erst nach dem 14. Jahrhundert beobachtet (Abb. 101). An einem griffplättchenversehenen Messer von der Burg Schartenberg (Nordhessen) aus dem 13. bis 14. Jahrhundert, welches dem Autor erst nach Abschluß des Katalogs zur Kenntnis gelangte, ist anhand der Griffplättchenmaße aber zu erkennen, daß bereits hier der ganze Griff zur sehr gut erhaltenen Schneide abgesetzt worden war (Abb. 100d).

Die Absetzung des Griffs zur Schneide, die bei Angelmessern nach dem 14. Jahrhundert regelmäßig zu beobachten ist, setzt somit wahrscheinlich schon im Spätmittelalter ein. Sie war nicht auf Griffplättchenmesser beschränkt, an denen sich lediglich anhand der Plättchenmaße die ursprüngliche Griffbreite zu erkennen gibt. Das zeigen Beispiele mit erhaltenen Vollgriffen aus Wienhausen (Kat. Nr. 74), Göttingen (Kat. Nr. 110) und Coesfeld (Kat. Nr. 118). Als regelhaftig ist die Griffanhebung in dieser Zeit aber noch nicht zu bezeichnen, da der übergangslose Verlauf der Schneide zur Griffunterkante ebenso belegbar ist (vgl. Dordrecht (Kat. Nr. 31-33), Lübeck (Kat. Nr. 32), Winsen (Kat. Nr. 62) und Minden (Kat. Nr. 75)). 


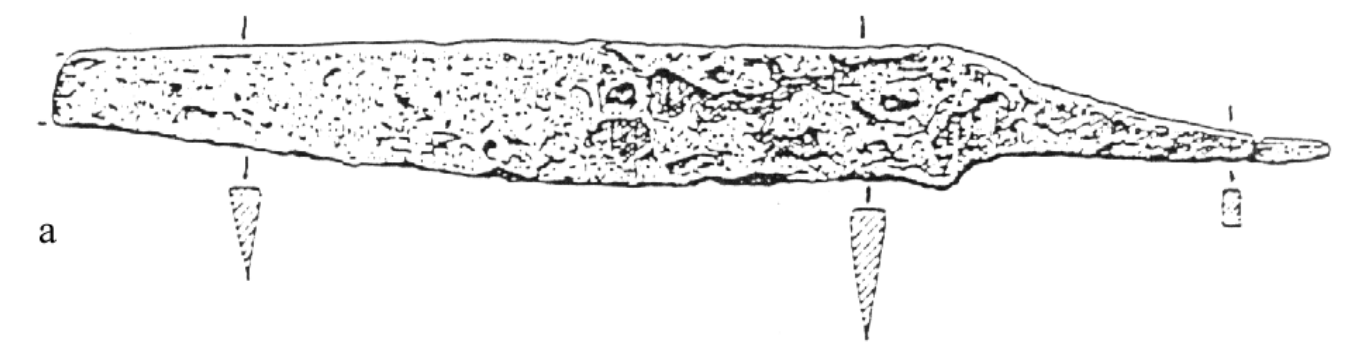

$\mathrm{b}$
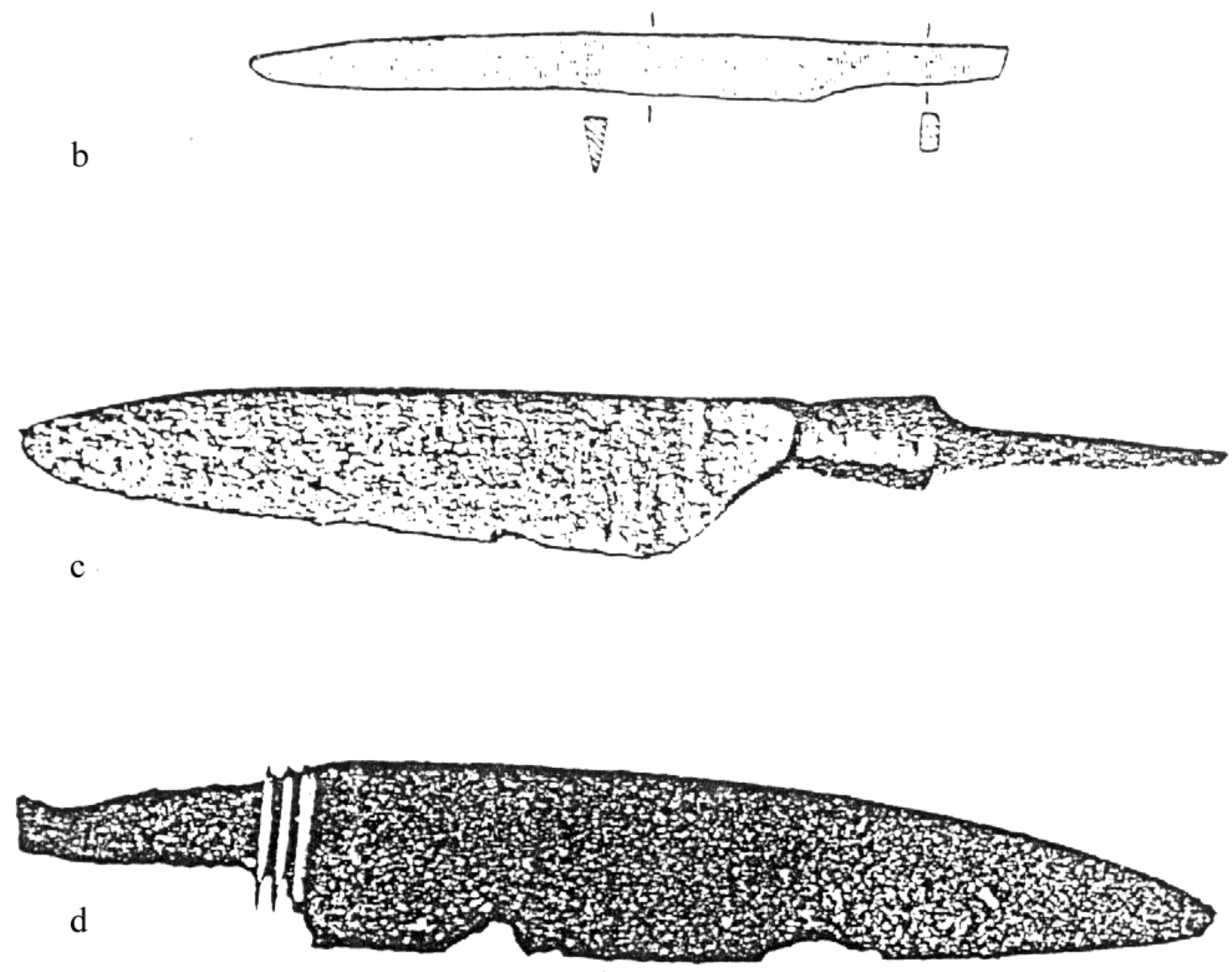

Abb. $100 \quad$ Beispiele für Griffangelmesser mit zur Schneide abgesetzter Angel (a-b) und abgesetztem Griff $(c-d)$

a: $\quad$ Recknitzfund, 12. - 13. Jahrhundert

b: $\quad$ Hanfwerder, 11. - 13. Jahrhundert

c: $\quad$ Barntrup, 16. - 17. Jahrhundert

d: $\quad$ Burg Schartenberg, 13. - 14. Jahrhundert (außer Katalog) 


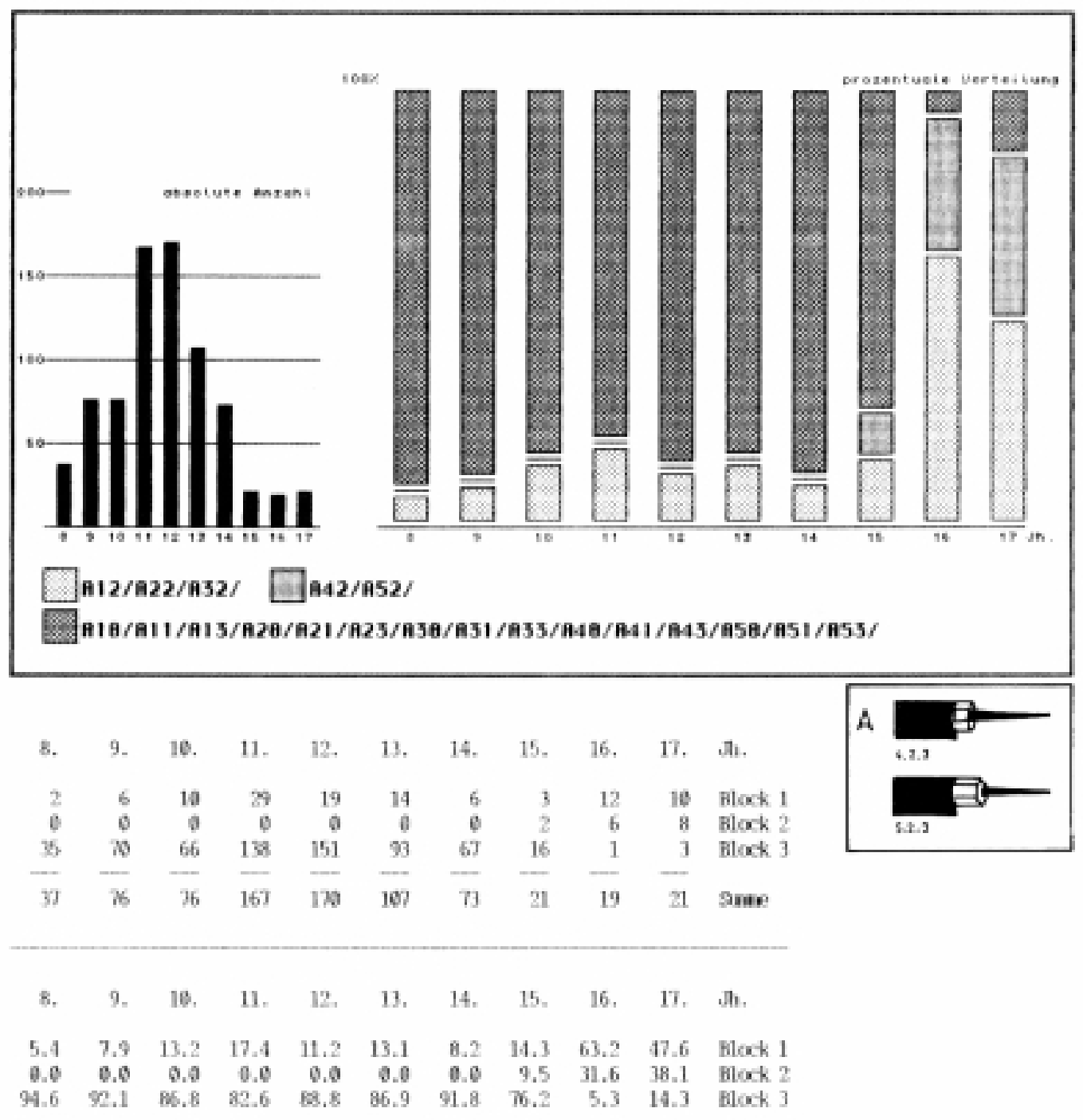

Abb. 101

Die Hauptverbreitungszeit von hochgesetzten Griffen am Beispiel der Angelkonstruktionen A42 und A52 im gesamten Untersuchungsraum (grob datierter Bestand) 


\subsection{Die einzelnen G riffsicherungskonstruktionen}

Die bereits im Klassifizierungsmodell erfaßten Zusatzsicherungen an Angelgriffen A2 bis A5 sowie die Übergangsform zur Zungenkonstruktion A6 treten in unterschiedlichen Zeiträumen auf (Abb. 102).

Im einzelnen verteilen sich die Konstruktionsformen gegenüber der im gesamten Bearbeitungszeitraum auftretenden Formengruppe A2 folgendermaßen:

Metallene Manschetten und Hülsen, die den Heftbereich des Griffs umschließen und damit vor allem die Aufspleißung und Risse hölzerner Vollgriffe (Hilzen) verhindern, wurden vom 9. bis 14. und im 17. Jahrhundert beobachtet (Abb. 103).

Das Fehlen dieses Konstruktionselements im 15. und 16. Jahrhundert findet möglicherweise seine Erklärung darin, daß in diesem Zeitabschnitt hauptsächlich Griffzungenmesser aufgetreten sind.

Wahrscheinlich ist mit Heftmanschetten und -hülsen bei Angelmessern im gesamten Bearbeitungszeitraum zu rechnen.

Die häufigsten Arten der zusätzlichen Griffsicherung sind in den Codebezeichnungen A2 und A3 zusammengefaßt worden, wobei die Konstruktionen der Gruppe A3 eine modische Erweiterung einer bereits in der Gruppe A2 erfaßten Befestigungsart darstellen, die sogenannte Griffplättchenverzierung ${ }^{82}$.

Die Auswertungsgraphik zeigt, daß die Verwendung von Griffplättchen zu Zierzwecken eine zeitlich begrenzte Erscheinung ist. Sie beginnt im 12. Jahrhundert, belegt im 13. und 14. Jahrhundert hohe prozentuale Anteile innerhalb der Messer der Formengruppe A2 und läuft im 15. Jahrhundert aus (Abb. 104).

Die übrigen Elemente zusätzlicher Sicherungen an Angelmessern sind im gesamten Untersuchungszeitraum nachzuweisen, sie umfassen am Ende umgebogene Angeln, Drahtumwicklungen am Heft, ausgeschmiedete oder angelötete Verdickungen des Angelendes sowie Heft- und Griffendplättchen.

Regionale Schwerpunkte konnten nur bei den Griffplättchen ausgemacht werden, allerdings verteilt sich die Mehrzahl dieser Konstruktionen auf Fundorte aus den Niederlanden, der Bundesrepublik, Dänemarks und Südschwedens, in den norwegischen, polnischen und nordwestrussischen Fundbeständen war ihre Anzahl geringer.

Die Form A5 umfaßt Messer, deren Heftbereich aus einem vor das hintere Klingenende gesetzten oder angeschmiedeten Vollmetallstück besteht.

Im bearbeiteten Fundbestand treten Beispiele dieser Konstruktionsform im 12. und vom 14. bis zum 17. Jahrhundert auf, von insgesamt 15 Belegen sind 10 Messer nach 1500 datiert worden (Abb. 105).

82

Vgl. Kapitel 6, Abschnitt 2.4.3.1, in dem der Anwendungsbereich der Griffplättchen beschrieben wird. 


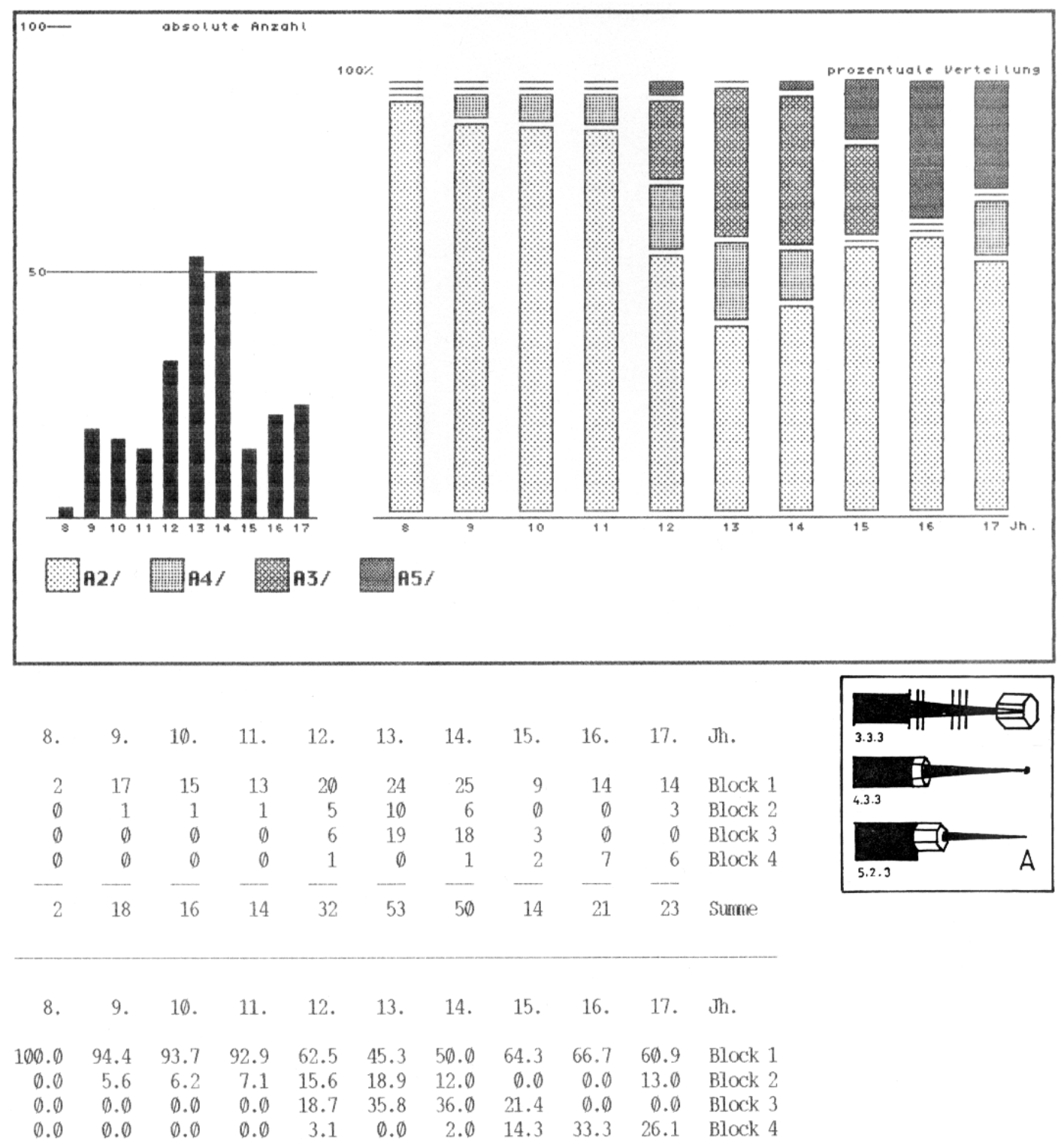

Abb. 102

Die zeitliche Verteilung der aufwendigeren Sicherungskonstruktionen A3, A4, und A5 im Vergleich zu einfacher gesicherten Angelmessern im gesamten Untersuchungsraum (grob datierter Bestand) 


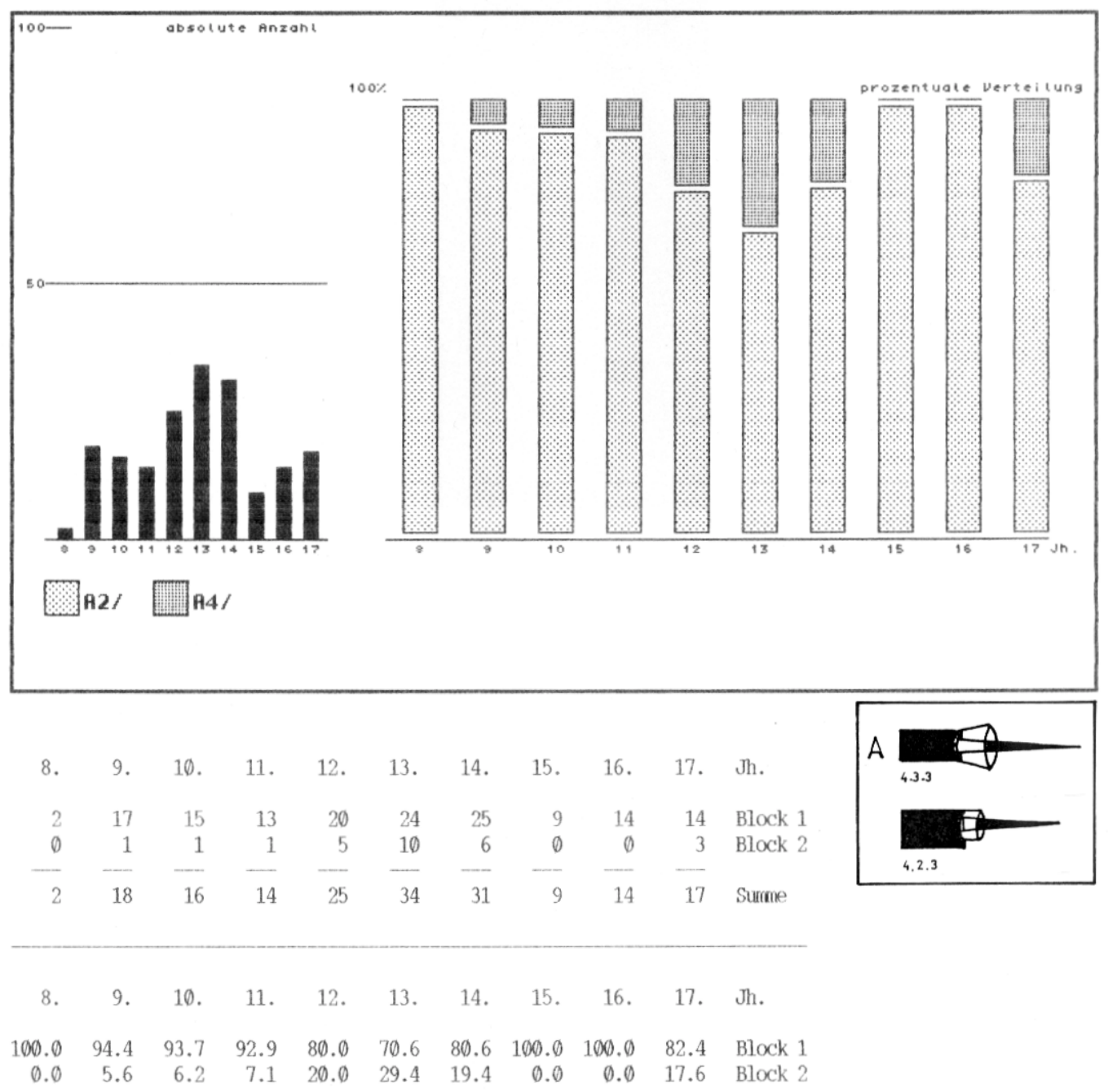

Abb. 103 Das Auftreten von Angelmessern mit Heftmanschetten oder Heftringen (A4) im gesamten Untersuchungsraum (grob datierter Bestand) 


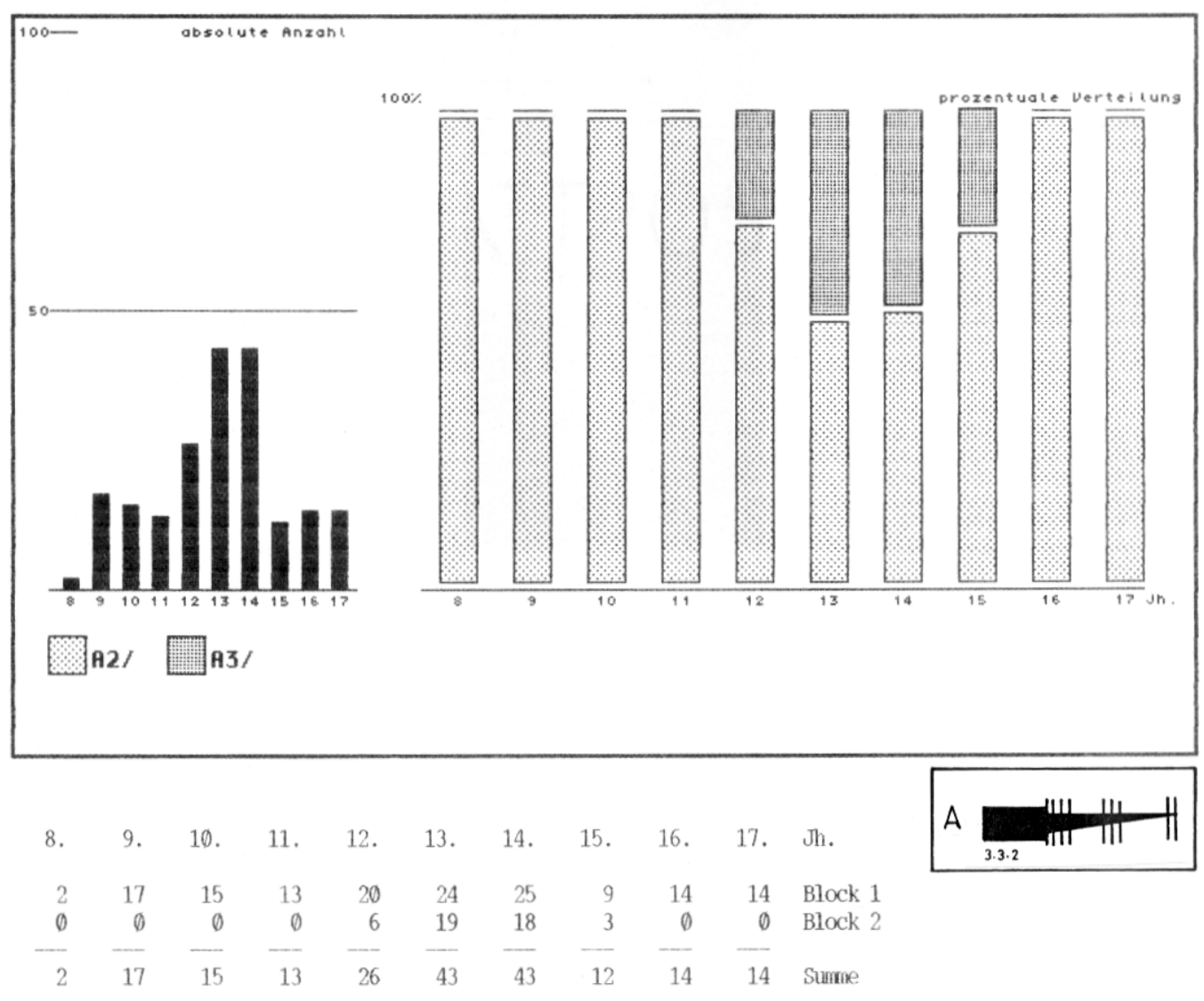

8. 9. 10. 11. 12. 13. 14. 15. 16. 17. Jh.

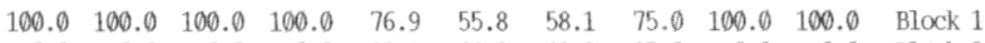

$\begin{array}{lllllllllll}\emptyset . \emptyset & 0 . \emptyset & \emptyset . \emptyset & 0 . \emptyset & 23.1 & 44.2 & 41.9 & 25 . \emptyset & 0.0 & 0 . \emptyset & \text { Block } 2\end{array}$

Abb. 104

Das Auftreten der Griffplättchenverzierung (A3) im gesamten Untersuchungsraum (grob datierter Bestand) 


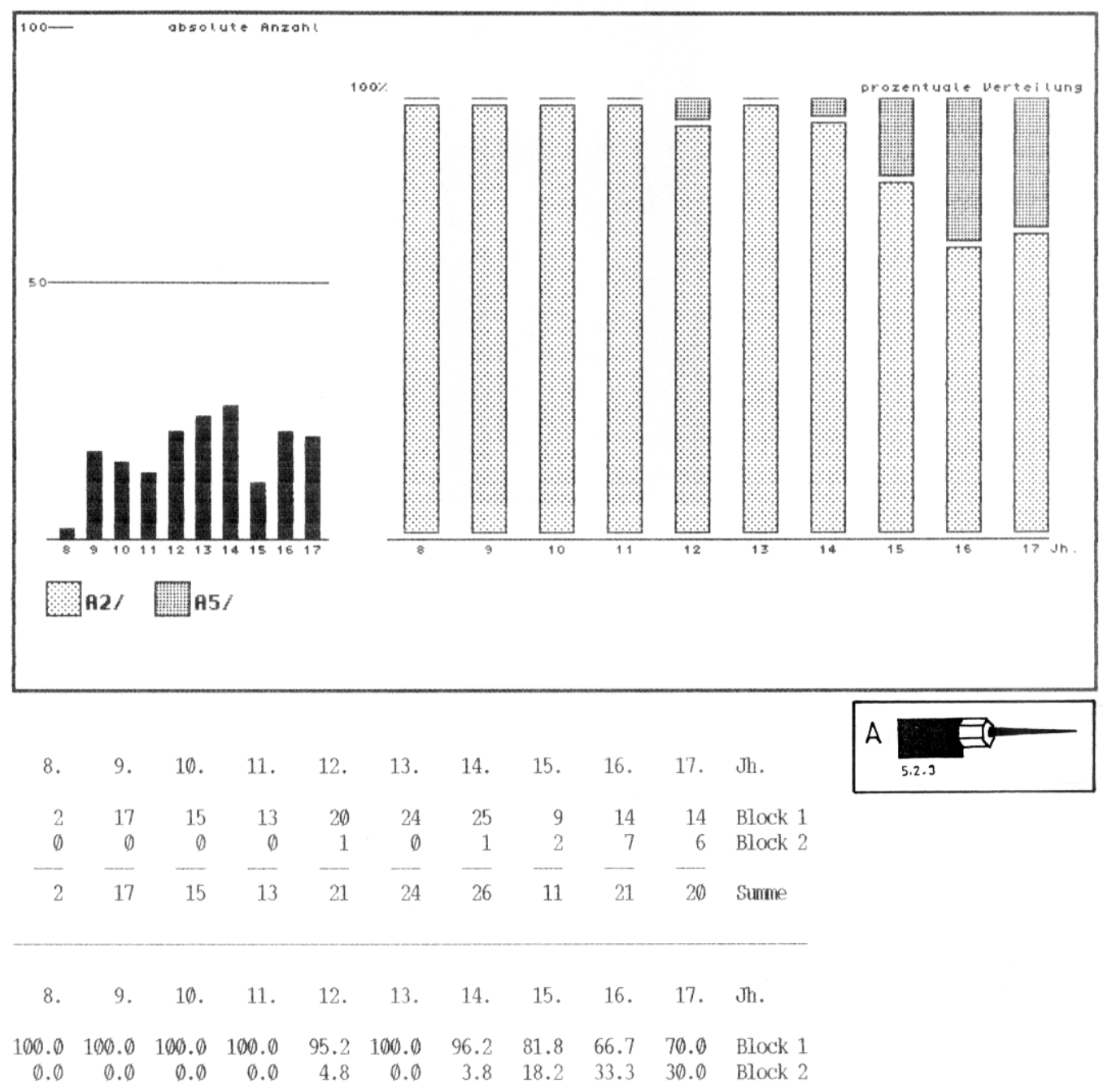

Abb. 105

Das Auftreten von Angelmessern mit Vollmetallsegmenten im vorderen Griffbereich (A5) im gesamten Untersuchungsraum (grob datierter Bestand) 
Die Exemplare des 15. bis 17. Jahrhunderts weisen bis auf ein griffunterständiges Messer alle gegenüber der Schneide angehobene Griffe auf, die die Vollmetallsegmente miteinschließen (Abb. 100c).

Die drei Messer des 12. bzw. 14. Jahrhunderts sind von unterschiedlicher Konstruktion.

Das erste ist ein griffplättchenverziertes Messer mit je einem länglich sechseckigen, gegossenen Körper aus Buntmetall am Heft und am Griffende. Es wurde in Oslo gefunden und stammt aus der Mitte bis zweiten Hälfte des 12. Jahrhunderts (Kat. Nr. 2). Ebenfalls aus Oslo stammt ein Messer des 12. Jahrhunderts mit einer aus einem eisernen Vollkörper bestehenden Trennzone zwischen Heft und Griffangel; ob dieser aufgesetzt oder direkt angeschmiedet worden ist, geht aus der Abbildung nicht hervor (Kat. Nr. 12).

Das dritte Exemplar, ein Messer mit einem vollplastisch ausgeschnitzten Beingriff aus Ribe, besitzt vor dem Griff einen silbernen oder mit Silberblech umfaßten mehreckigen Körper, dessen Felder verziert sind. Er dient gleichzeitig als Basis für den figuralen Griff. Das Messer wird in die Zeit um 1300 datiert (Kat. Nr. 1).

Die Vernietung von Angelgriffen (A6) wurde vom 11. bis zum 14. Jahrhundert angetroffen (Abb. 106).

Ihr Stellenwert zwischen Angel- und Zungenkonstruktionen wurde bereits im Zusammenhang mit dem Beginn der Griffzungenmesser beschrieben.

Zusammenfassend lassen sich zeitlich begrenzte Konstruktionselemente wie Griffplättchen von durchgehend beobachteten Befestigungsarten wie Heftmanschetten trennen.

Erstere umfassen neben Verzierungsarten Zusatzsicherungen, die vor allem in Zeiten technischer Innovationen auftreten (A3, A5, A6):

Die Angelvernietung tritt zumindest regional als Vorläufer der Zungenkonstruktion auf, Heftpartien aus Vollmetallstücken beginnen in etwa parallel zur Griffplättchenverwendung in einer Zeit, in der im westlichen Teil des Untersuchungsraums zusätzlich befestigte Griffe allgemein einsetzen.

Während die Griffplättchentechnik im 15. Jahrhundert ausläuft, erfährt das Vollmetallheft seine Blüte im 16. und 17. Jahrhundert.

Am Ende umgebogene Angeln, ausgeschmiedete Verdickungen sowie angelötete oder -gesetzte Griffendsicherungen, Drahtumwicklungen und das Heft umfassende Manschetten oder Hülsen (A2 und A4) sind in ihrem Auftreten lediglich abhängig vom Kenntnisstand der jeweils verwendeten Produktionstechnik und von der Verwendung des Griffangelprinzips. 


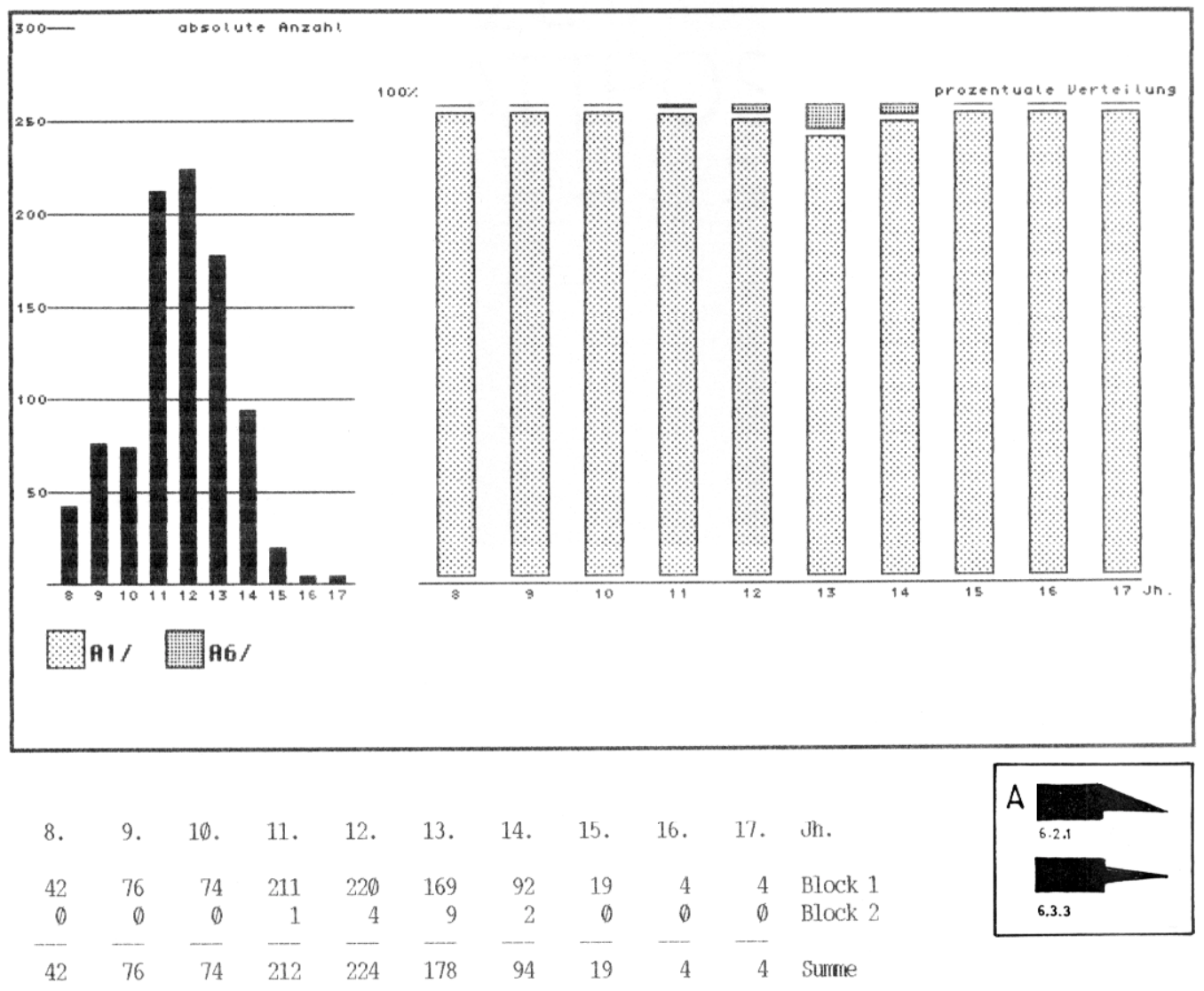

8. 9. 10. 11. 12. 13. 14. 15. 16. 17. Jh.

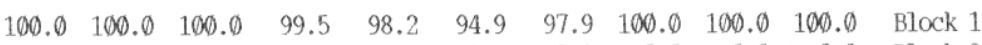

$\begin{array}{lllllllllll}0 . \emptyset & 0 . \emptyset & 0 . \emptyset & 0.5 & 1.8 & 5.1 & 2.1 & \emptyset . \emptyset & \emptyset . \emptyset & \emptyset . \emptyset & \text { Block } 2\end{array}$

Abb. 106

Das Auftreten von Angelmessern mit vernieteten Vollgriffen (A6) im gesamten Untersuchungsraum (grob datierter Bestand) 


\subsubsection{Die zeitliche Verteilung der einzelnen Griffzungenkonstruktionen}

\subsection{G Giffzungenmesser mit und ohne Zusatzsicherungen}

Unabhängig vom konstruktionsbedingten Umstand, daß mit der Verwendung von Nieten bereits ein zusätzliches Befestigungselement vorliegt, lassen sich auch Griffzungenmesser grundsätzlich unterscheiden nach einfachen und gesicherten Griffkonstruktionen.

Die häufigste Art der zusätzlichen Griffbefestigung besteht aus geschmiedeten Stoßkanten am Heft, die die vernieteten Griffschalen vorne begrenzen, und eisernen Abschlußplatten am Griffende, belegt vor allem durch drei Messer des 14. und beginnenden 15. Jahrhundert aus Amsterdam (Kat. Nr. 63 bis 65).

Die verschiedenen, vor allem im 15. und 16. Jahrhundert verwandten Techniken und Materialien zur zusätzlichen Sicherung oder Befestigung von Griffplatten werden am Beispiel niederländischer Funde exemplarisch beschrieben. ${ }^{83}$.

An dieser Stelle wird zunächst nach der allgemeinen zeitlichen Verteilung der beiden unterschiedenen Gruppen gefragt.

Zusatzbefestigungen an Griffzungenmessern treten bis auf eine Ausnahme, ein Messer der Form B7 aus der slawischen Burg in Alt-Lübeck (Kat. Nr. 18), nicht vor dem 12. Jahrhundert auf (Abb. 107).

Bis zum 16. Jahrhundert nehmen die Messer der Formen B2, B4 und B8 mit unterschiedlichen Schüben auf über $80 \%$ aller Griffzungenmesser zu.

$\mathrm{Da}$ in der Auswertungsgrafik im 17. Jahrhundert ihr Anteil wieder zugunsten unbefestigter Griffzungenmesser zurückgeht, bedarf des Hinweises auf die Messer des Wrackfundes von Wittenberge (Elbe, Kat. Nr. 52 bis 59). Diese acht Messer stellen einen Querschnitt durch die Messerladung eines Handelsschiffes dar, die mit hoher Wahrscheinlichkeit für den Export nach Übersee bestimmt war und erscheinen deshalb nicht in den übrigen Auswertungen.

Die Ladung besteht aus einer billig hergestellten Massenware. Man hatte sich nicht einmal die Mühe gemacht, die Form der Zunge der der Griffschalen anzugleichen, so daß am Ende Messer mit den Griffformen B1.2 und B9 mit Schalen der Form B3.2 beschlagen worden sind.

Daher erscheint im hier ausgewerteten Bestand des 17. Jahrhunderts der Anteil unbefestigter Zungenmesser überrepräsentiert. Es ist unklar, ob Messer dieser niedrigen Qualitätsstufe auf den europäischen Märkten überhaupt abzusetzen waren, nachgewiesen wurden sie im bearbeiteten Bestand nach 1500, ansonsten nicht mehr. Die gleiche Auswertung ohne diese Messer zeigt, daß auch im 17. Jahr- 
hundert Griffzungenmesser mit zusätzlichen Sicherungselementen die Regel waren (Abb. 108).

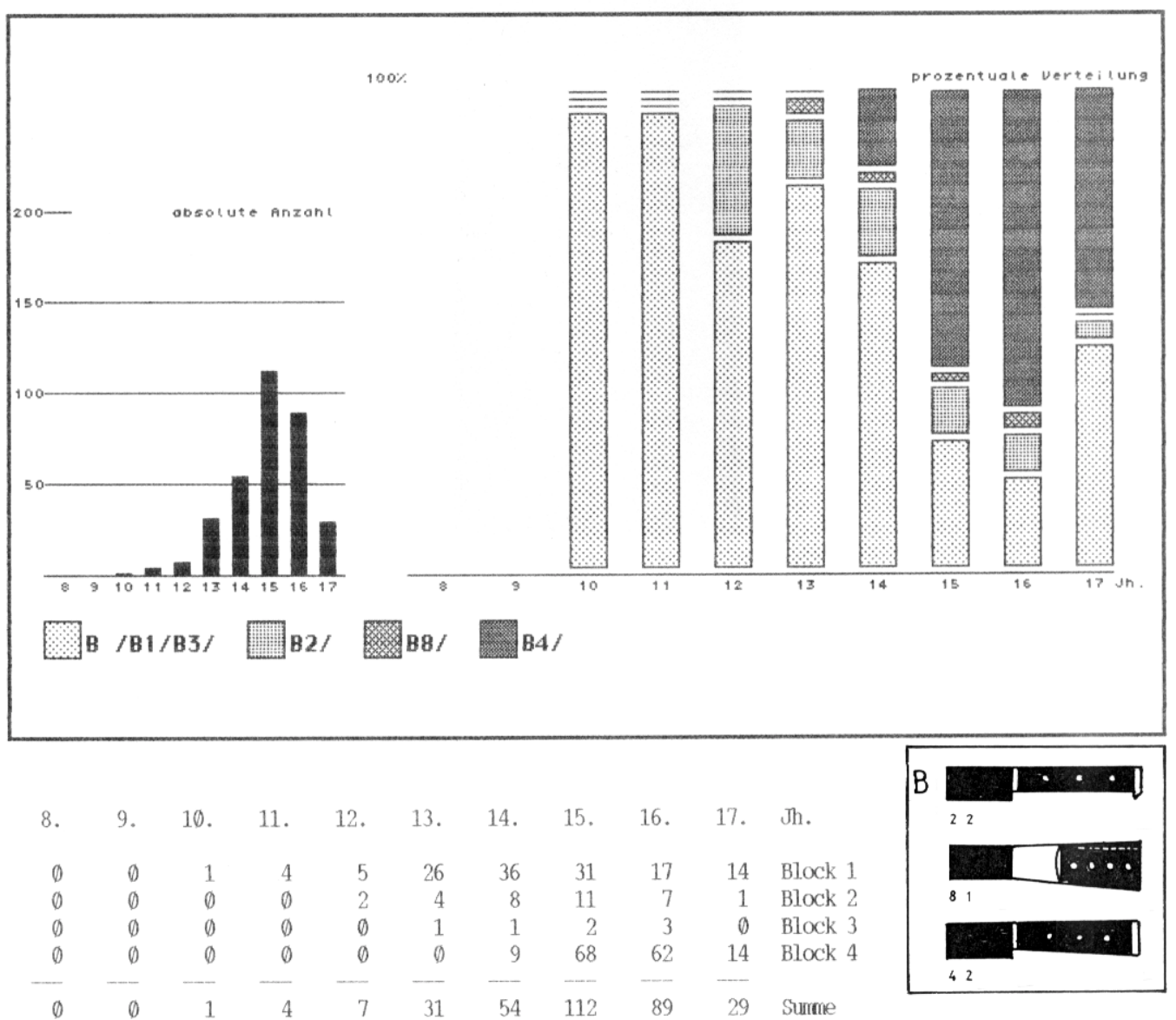

8. 9. 10. 11. 12. 13. 14. 15. 16. 17. Jh.

$\begin{array}{rrrrrrrrr}100 . \emptyset & 100 . \emptyset & 71.4 & 83.9 & 66.7 & 27.7 & 19.1 & 48.3 & \text { Block 1 } \\ 0.0 & 0.0 & 28.6 & 12.9 & 14.8 & 9.8 & 7.9 & 3.4 & \text { Block 2 } \\ 0.0 & 0 . \emptyset & \emptyset . \emptyset & 3.2 & 1.9 & 1.8 & 3.4 & \emptyset .0 & \text { Block 3 } \\ 0 . \emptyset & 0 . \emptyset & 0.0 & 0 . \emptyset & 16.7 & 60.7 & 69.7 & 48.3 & \text { Block 4 }\end{array}$




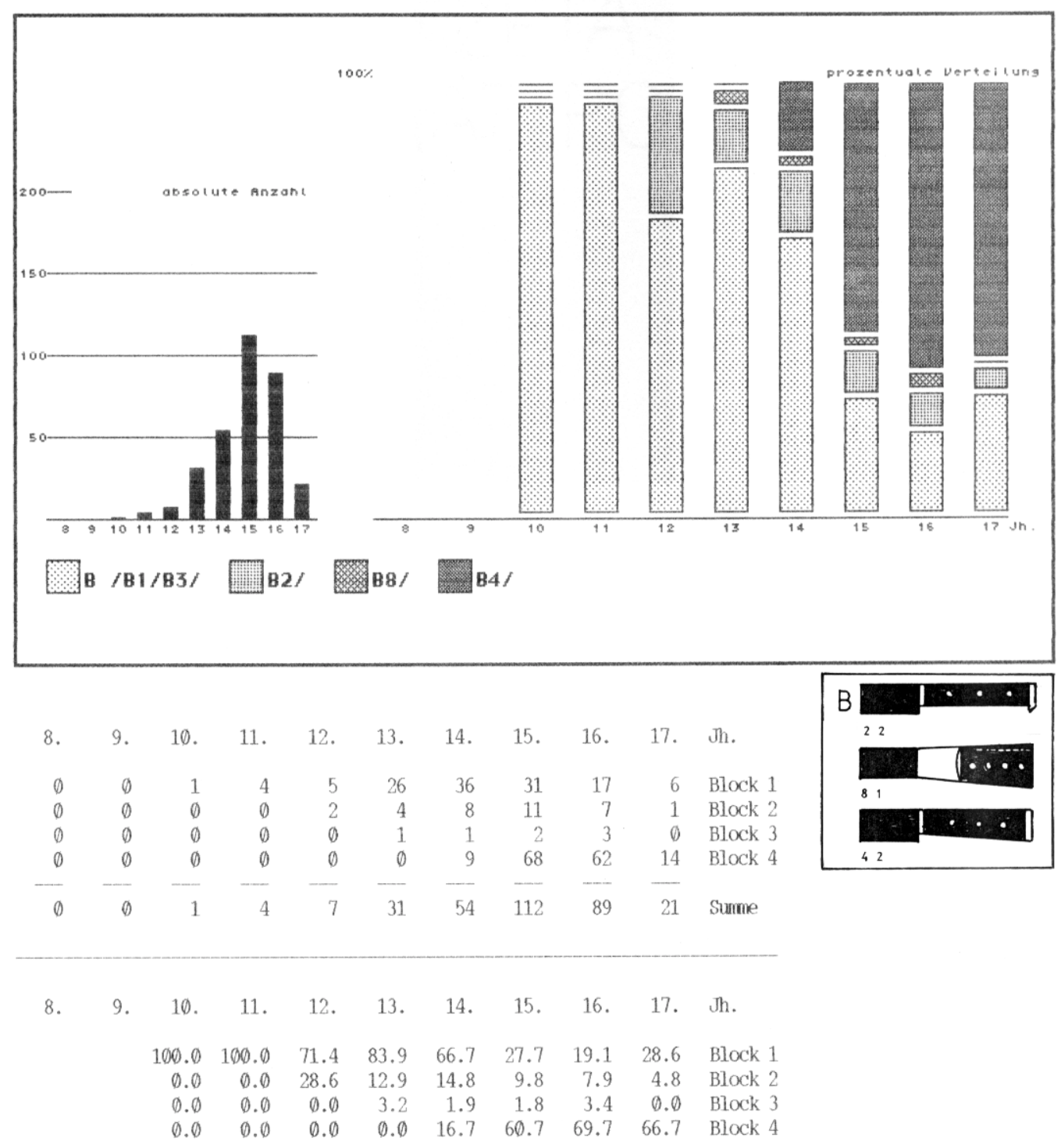

Abb. 108

Die zeitliche Verteilung von zusätzlich gesicherten Zungenmessern gegenüber einfachen Zungenmessern im gesamten Untersuchungsraum (grob datierter Bestand ohne das "Seegut" des Wrackfundes von Wittenbergen 


\subsection{Unter- und oberständige G riffe}

Die bei Griffangelmessern angetroffene Unter- und Oberständigkeit des Griffs zur Klinge wurde bei Griffzungenmessern nicht beobachtet. Die beiden in Gommerstedt und Manebach (Katalogteil ehemalige DDR, Kat. Nr. 190 und 220) gefundenen Messer aus dem 13. bis 14. Jahrhundert mit leicht unterständigen Zungen belegen eher die technische Unvollkommenheit einer frühen Entwicklungsstufe.

\subsection{Die Anhebung des G riffs gegenüber der Schneide}

Den beobachteten Möglichkeiten, die Angel in verschiedenen Ansätzen und unterschiedlichen Neigungen zur Klinge zu stellen, stehen nur vier nachgewiesene Gestaltungsmöglichkeiten bei Griffzungenmessern gegenüber:

Die Zungenkanten verlaufen entweder parallel (B1 und B2) oder zum Heft hin zusammen (B3 und B4), die gesamte Zunge läuft entweder ansatzlos in die Klinge über (B1.1, B2.1, B3.1 und B4.1) oder sie ist zur Schneide hin abgesetzt worden (B1.2, B2.2, B3.2 und B4.2).

Die jeweils letzteren Prinzipien verfolgen beide das Ziel, den Griff gegenüber der Schneide abzusetzen, indem sie entweder den ganzen Griff anheben (B1.2), ihn nach hinten verbreitern (B3.1) oder den bereits zum Heft einfallenden Griff zusätzlich gegenüber der Schneide anheben (B4.2).

Ansatzlos in die Klinge übergehende Griffzungen mit gerade verlaufenden Kanten ohne zusätzliche Sicherungen nehmen im Bestand der nicht verbreiterten Zungengriffe vom 11. bis zum 15. Jahrhundert regelmäßig ab (Abb. 109).

Die Form B1.1 ist insgesamt nur achtmal belegt worden. Das früheste und in der formalen Zuweisung sicherste Exemplar ist ein Messer aus Steinkirchen (Kreis Burg, Kat. Nr. 160) aus dem 11. bis 12. Jahrhundert. Ein weiteres, ebenfalls gut erhaltenes Beispiel des 14. Jahrhunderts stammt aus Usch (Polen, Kat. Nr. 115). Das jüngste Beispiel aus dem 15. Jahrhundert wurde in Dragör ausgegraben (Dänemark, Kat. Nr. 84).

Verglichen mit allen übrigen Griffzungenkonstruktionen erreichen die nicht verbreiterten Zungengriffe vom 11. bis zum 13. Jahrhundert Anteile über 60\%, bis zum 17. Jahrhundert gehen sie schrittweise auf $9 \%$ zurück (Abb. 110).

Messer der Form B1.2 sind mit 17 Exemplaren ebenfalls relativ selten angetroffen worden. Von ihnen wurden sechs Messer des 13. bis 14. Jahrhunderts in Gommerstedt (Thüringen, Kat. Nr. 190, 193, 203-206) gefunden. Ihre Hauptverbreitungszeit reicht mit $24 \%$ noch bis in das 14 . Jahrhundert, während die gleichen Messer mit zusätzlichen Befestigungselementen (B2.2) nur sporadisch auftreten und vom 13. bis zum 17. Jahrhundert regelmäßig zurückzugehen scheinen. 


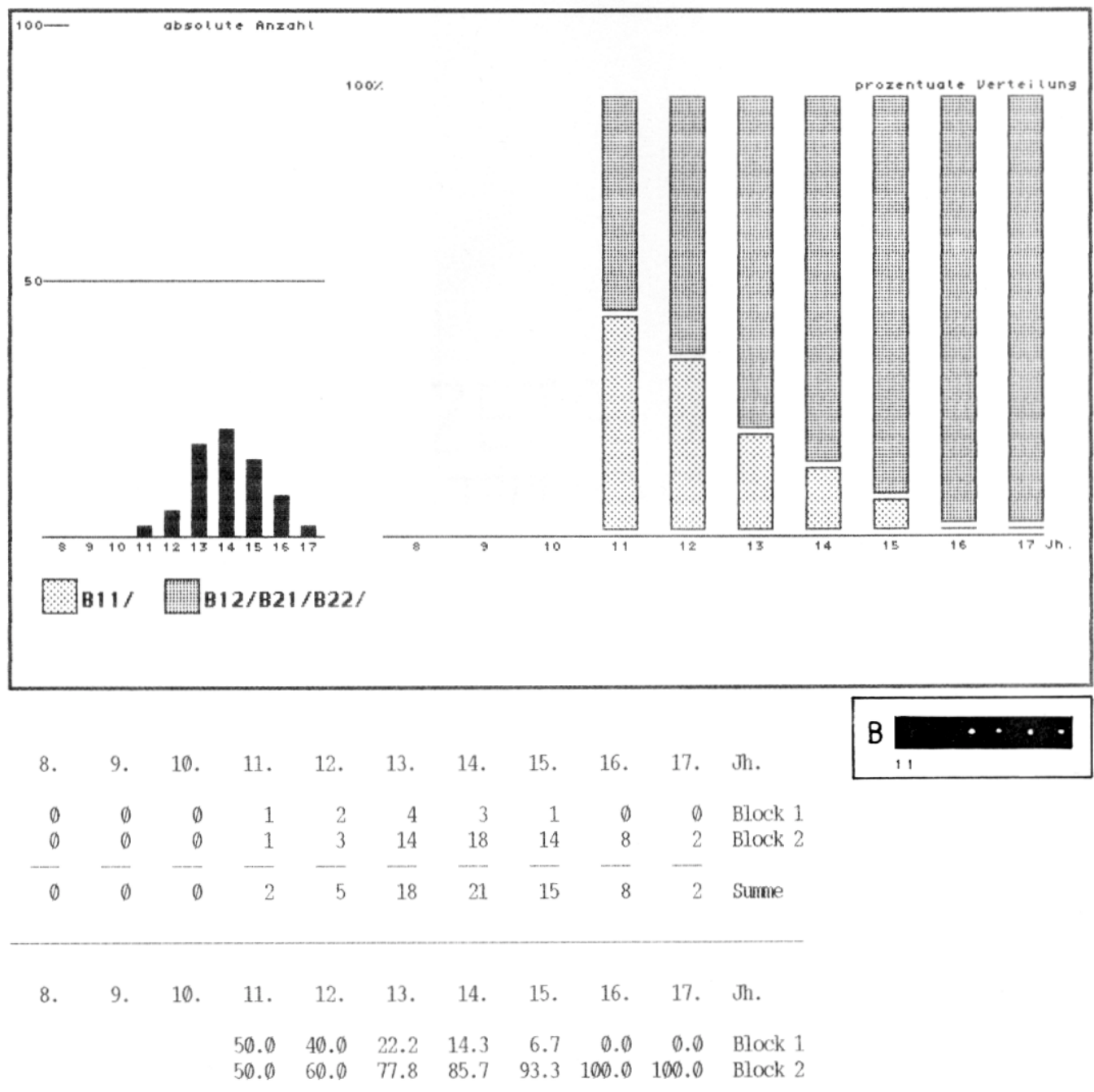

Abb. 109

Der Rückgang nicht zusätzlich gesicherter und nicht gegenüber der Schneide angehobener Zungengriffe (B11) im gesamten Untersuchungsraum (grob datierter Bestand) 


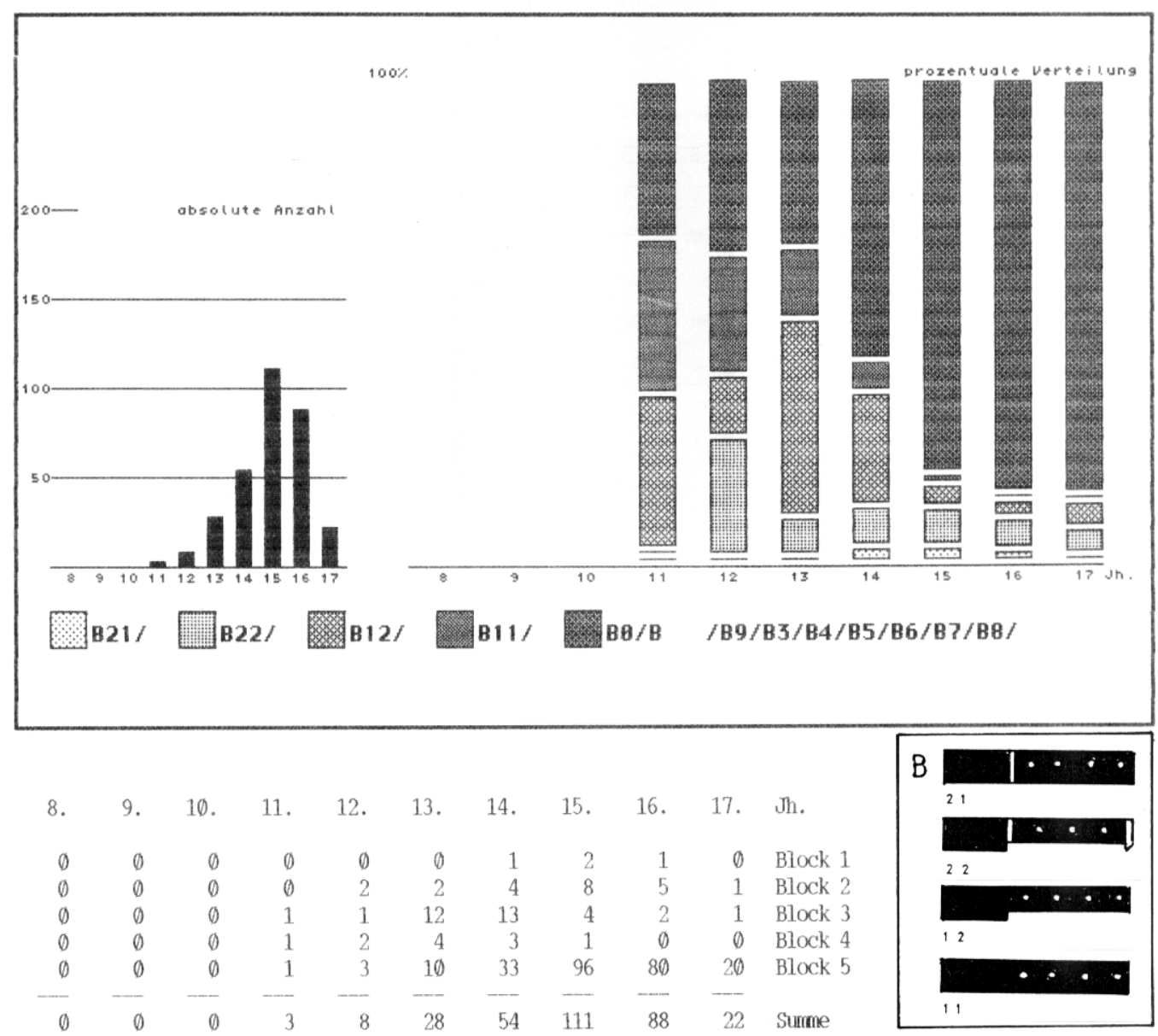

\begin{tabular}{rrrrrrrrl} 
8. 9. 10. 11. & 12. & 13. & 14. & \multicolumn{1}{c}{15.} & \multicolumn{1}{c}{16.} & \multicolumn{1}{l}{17.} & Jh. \\
& & & & & & & & \\
& $\emptyset . \emptyset$ & $\emptyset . \emptyset$ & $\emptyset . \emptyset$ & 1.9 & 1.8 & 1.1 & $\emptyset . \emptyset$ & Block 1 \\
$\emptyset . \emptyset$ & $25 . \emptyset$ & 7.1 & 7.4 & 7.2 & 5.7 & 4.5 & Block 2 \\
33.3 & 12.5 & 42.9 & 24.1 & 3.6 & 2.3 & 4.5 & Block 3 \\
& 33.3 & $25 . \emptyset$ & 14.3 & 5.6 & $\emptyset .9$ & $\emptyset . \emptyset$ & $\emptyset . \emptyset$ & Block 4 \\
& 33.3 & 37.5 & 35.7 & 61.1 & 86.5 & $9 \emptyset .9$ & $9 \emptyset .9$ & Block 5
\end{tabular}

Abb. 110

Der Rückgang nicht nach hinten verbreiterten Zungengriffen mit (2) und ohne (B1) zusätzliche Griffsicherungen im gesamten Untersuchungsraum (grob datierter Bestand) 
Nach hinten verbreiterte Zungengriffe ohne Absatzkante am Heft zur Schneide sind im Bestand der hinten verbreiterten Zungengriffe vom 13. bis zum 15. Jahrhundert aufgetreten (Abb. 111). Die regelmäßig abnehmende Tendenz ist hier trotz der auffallenden Ähnlichkeit mit der Entwicklung der Form B1.1 nur bedingt wahrscheinlich, da die Form B3.1 nur viermal nachgewiesen werden konnte.

Im Vergleich zu allen übrigen Griffzungenkonstruktionen nehmen die Anteile aller nach hinten verbreiterten Zungengriffe vom 13. bis zum 16. Jahrhundert von 11,7\% auf $83,9 \%$ zu und nehmen auch im 17. Jahrhundert (ohne Berücksichtigung der Wittenberger Schiffsladung) leicht zu (Abb. 112).

Von insgesamt 151 Belegexemplaren der Formengruppen B3 und B4 sind 125 Messer sicher nach 1400 datiert worden.

Die Anhebung des Griffs gegenüber der Schneide erfolgte demnach bei Griffzungenmessern stufenweise:

Vom 11. bis zum 15. Jahrhundert setzten sich bei nicht nach hinten verbreiterten Zungengriffen die Absatzkanten am Heft durch, während zeitlich versetzt die prozentualen Anteile dieser Griffe zugunsten der seit dem 13. Jahrhundert auftretenden, nach hinten verbreiterten Griffzungen bis zum 17. Jahrhundert auf unter $10 \%$ zurückgingen.

Im 16. und 17. Jahrhundert sind keine Zungengriffe mehr aufgetreten, die nicht in irgendeiner Form das Durchrutschen der Hand über das Heft hinaus zu verhindern suchen.

Zusätzliche Befestigungselemente an den Zungengriffen der Formen B1 und B3 treten jeweils mindestens ein Jahrhundert später auf als das Einsetzen der jeweiligen Konstruktionsform (Abb. 113 und 114).

Die Grifformen B2.1 (14. bis 16. Jahrhundert) und B4.1 (15. bis 17. Jahrhundert) unterscheiden sich nicht nur durch die vornehmlich im Heftbereich eingesetzten Stoßkanten oder vernieteten Heftplatten von den nicht zusätzlich befestigten Formen B1.1 und B3.1. Die Griffunterkante verläuft bei diesen späten Konstruktionsstufen auch nicht mehr in gerader Linie zur Schneide, sondern nimmt einen leicht s-förmig geschwungenen Verlauf, so daß im Heftbereich zwar keine Absatzkante, aber eine Kehle entsteht, die den Griff von der Schneide trennt.

Beispielhaft verdeutlichen dies drei Messer (B4.1) aus Zwolle (Kat. Nr. 7), Delft (Kat. Nr. 50) und Amsterdam (Kat. Nr. 80) gegenüber drei Messern aus Den Haag (Kat. Nr. 53) und Amsterdam (Kat. Nr. 67 und 70), bei denen im Kehlbereich ein Knick oder ein leichter Absatz (B4.2) erscheint. 


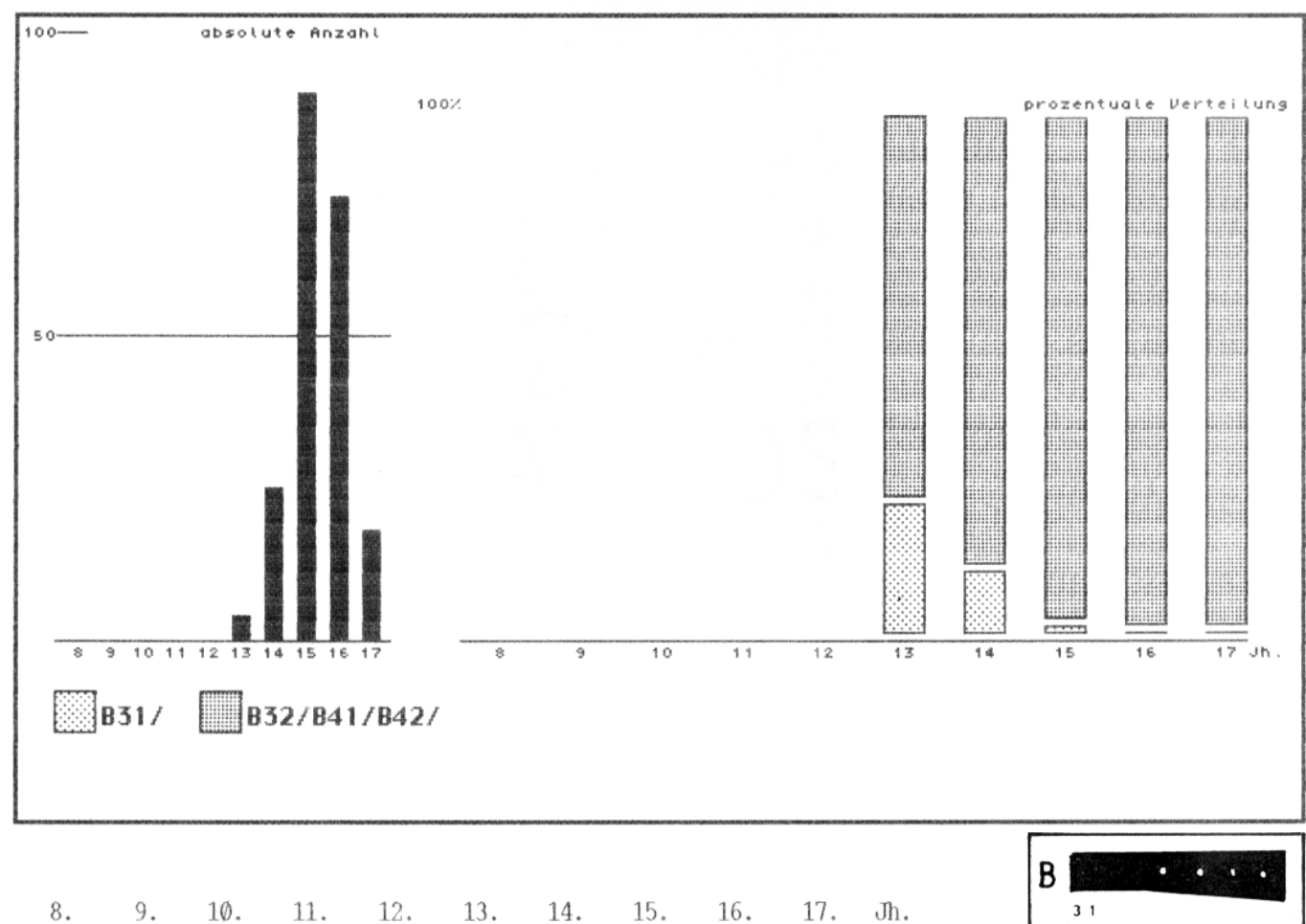

\begin{tabular}{|c|c|c|c|c|c|c|c|c|c|c|}
\hline 8. & 9. & 0. & 11. & 12. & 13. & 14. & 15. & 16. & 17. & $\mathrm{~J}$. \\
\hline () & 0 & 0 & $\emptyset$ & $\emptyset$ & 1 & 3 & 1 & 0 & $\emptyset$ & Block 1 \\
\hline$\emptyset$ & $\emptyset$ & 0 & $\emptyset$ & 0 & 3 & 22 & 89 & 73 & 18 & Block 2 \\
\hline 0 & $\theta$ & 0 & 0 & 0 & 4 & 25 & 90 & 73 & 18 & Surme \\
\hline
\end{tabular}

8. 9. 10. 11. 12. 13. 14. 15. 16. 17. Jh.

$\begin{array}{llllll}25.0 & 12 . \emptyset & 1.1 & 0 . \emptyset & \emptyset . \emptyset & \text { Block } 1\end{array}$

$\begin{array}{llllll}75 . \emptyset & 88.0 & 98.9 & 100 . \emptyset & 100 . \emptyset & \text { Block } 2\end{array}$

Abb. 111

Die Abnahme nicht zusätzlich gesicherter und nach hinten verbreiterter Zungengriffe ohne Absatz zur Schneide (B31) im gesamten Untersuchungsraum (grob datierter Bestand) 


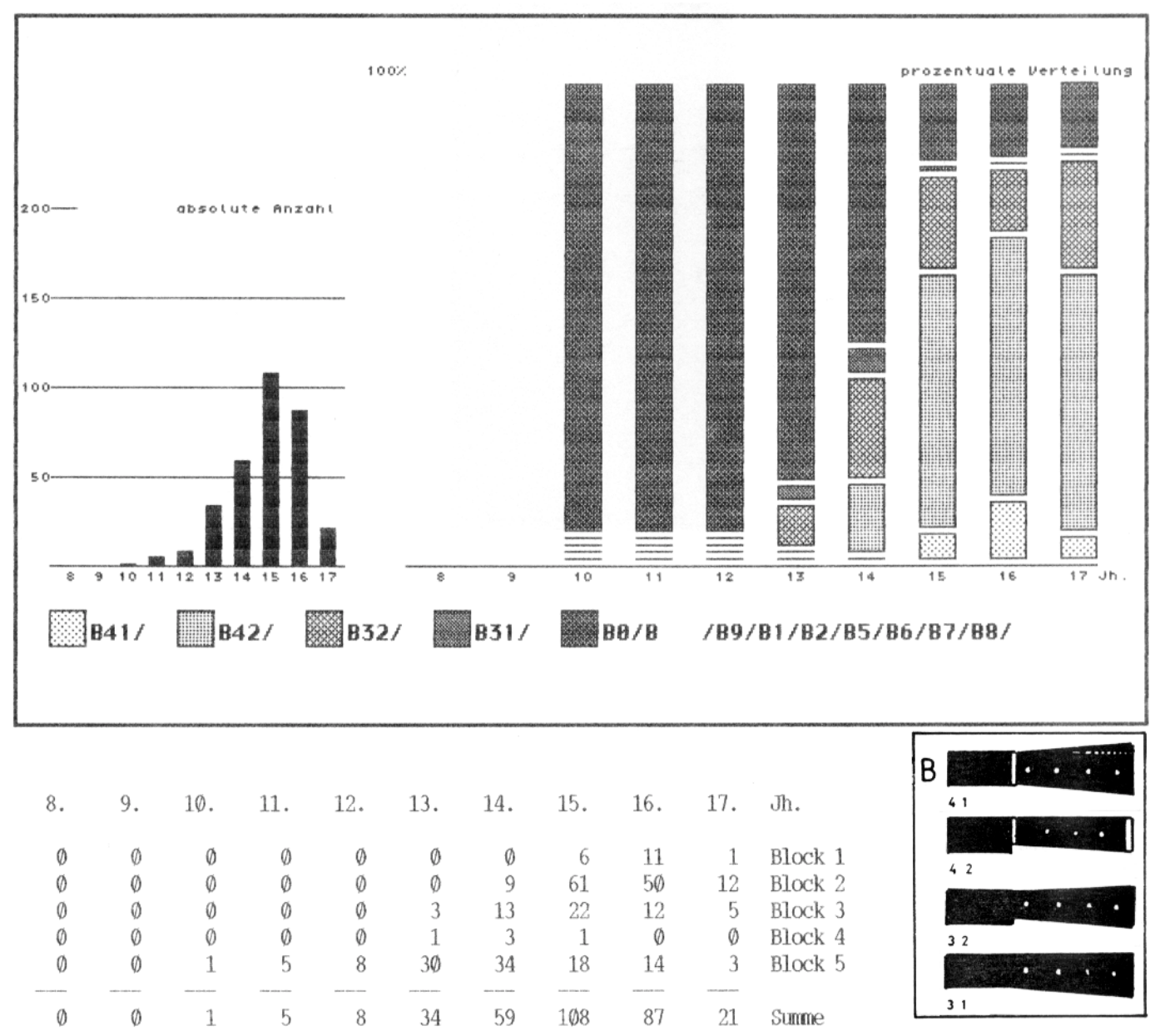

8. 9. 10. 11. 12. 13. 14. 15. 16. 17. Jh.

$\begin{array}{lllllllll}\emptyset . \emptyset & \emptyset . \emptyset & 0 . \emptyset & \emptyset . \emptyset & \emptyset . \emptyset & 5.6 & 12.6 & 4.8 & \text { Block 1 }\end{array}$

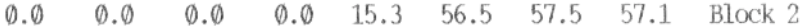

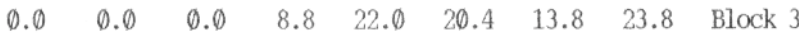

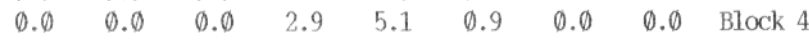

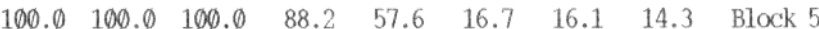

Abb. 112

Die Zunahme von nach hinten verbreiterten Zungengriffen mit (B4) und ohne (B3) zusätzliche Griffsicherungen im gesamten Untersuchungsraum (grob datierter Bestand) 


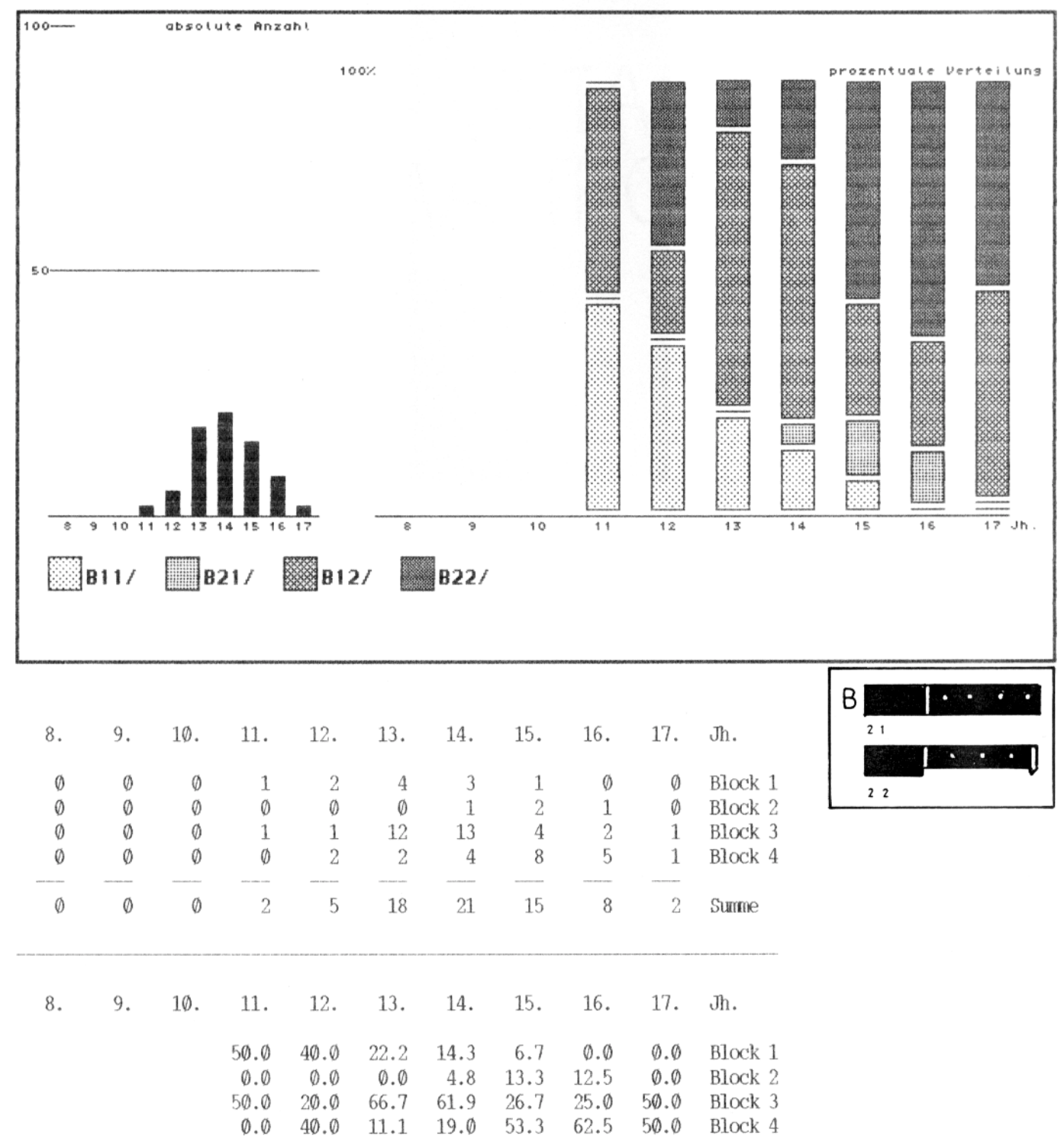

Abb. 113

Das Einsetzen von Zusatzsicherungen an Zungenmessern mit nicht nach hinten verbreiterten Griffen im gesamten Untersuchungsraum (B21 und B22, grob datierter Bestand) 


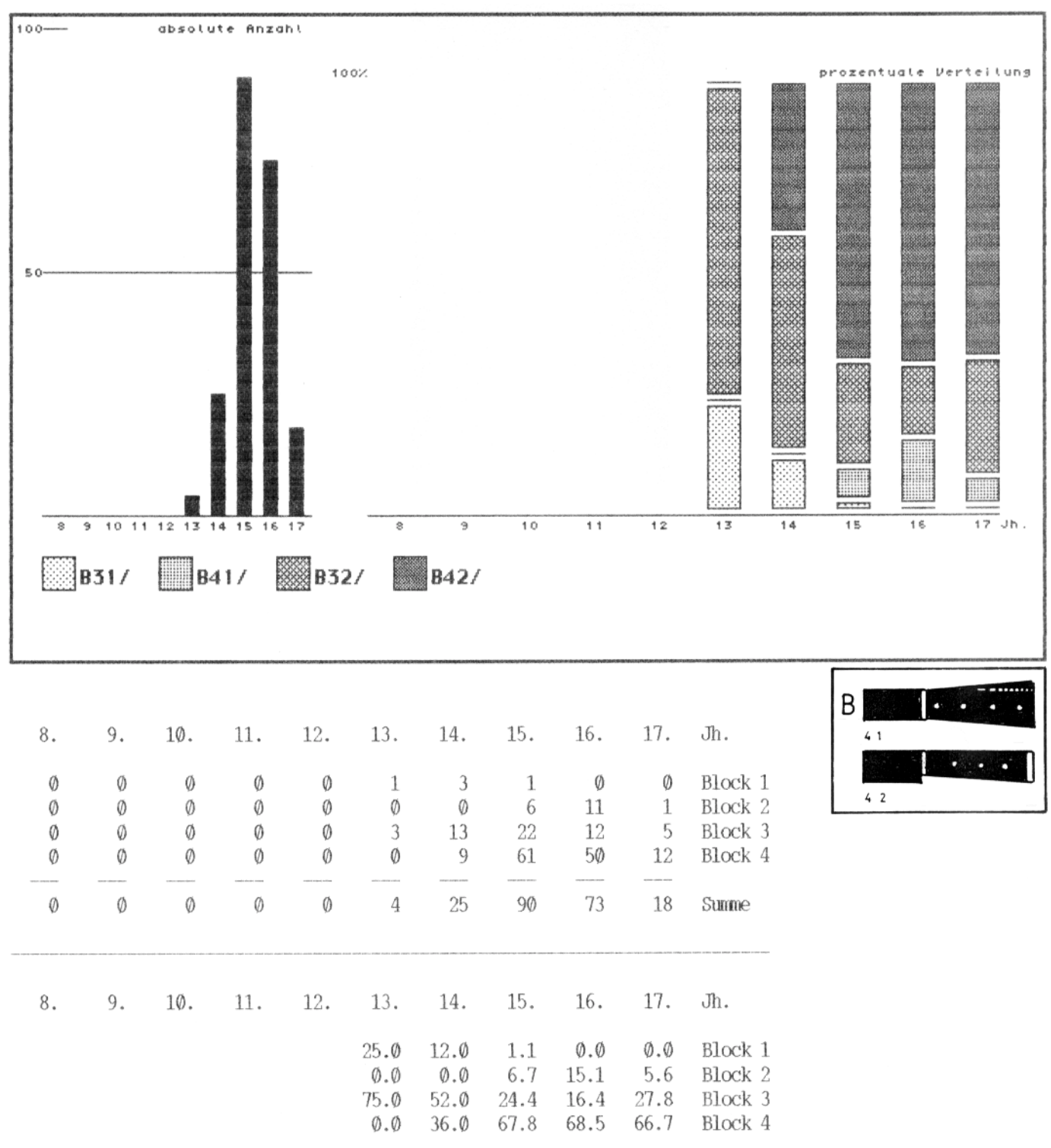

Abb. 114

Das Einsetzen von Zusatzsicherungen an Zungenmessern mit nach hinten verbreiterten Griffen im gesamten Untersuchungsraum (B41 und B42 grob datierter Bestand) 
Fast alle Messer dieser speziellen Konstruktionsform sind am Heft und Griffende mit oft verzierten Buntmetallschalen gesichert und besitzen lancettförmig gebogene Klingen, so daß sich mit dem zeitlich begrenzten Auftreten der Konstruktionsform B4.1 vor allem im 16. Jahrhundert möglicherweise eine hauptsächlich in niederländischen und südskandinavischen Fundorten aufgetretene Messerform verbinden läßt, die einen kleinen Teil des Bestandes der Konstruktionsform B4.2 4 miteinschlie $\beta t^{84}$.

Messer der Konstruktionsform B2 mit zusätzlichen Vorrichtungen zur sicheren Handhabung des Griffs (B2.2) sind vor 1400 zumindest äusserst selten aufgetreten. Zwei Exemplare des 12. bis 13. Jahrhunderts aus Lublin (Kat. Nr. 306, 309) sind bezüglich ihrer Datierung umstritten ${ }^{85}$. Bei den beiden übrigen Belegen aus Göttingen (Kat. Nr. 110, 113) betreffen die Sicherungsmerkmale ein Herunterziehen bzw. eine Vergrößerung der Griffschalenenden, durch die das Messer besser in der Hand liegt; die Griffenden sind in einem Fall zu einem Tierkopf ausgeschnitzt worden. Derartige Griffverbreiterungen sind auch von Griffangelmessern bekannt.

Technische Vorrichtungen, die als eiserne Stoßkanten oder zusätzlich montierte Metallapplikationen die Griffschalen vor allem im Heftbereich verstärken, erscheinen in der Regel erst nach 1400. Von den 125 Messern der Formengruppen B3 und B4 nach 1400 gehören 87 der Konstruktionsform B4.2 an, das heißt, daß ihre Griffe zusätzlich gesichert und zur Schneide hin abgesetzt worden sind. Die Sicherheitsvorrichtungen bestehen fast ausschließlich aus zusätzlich angebrachten Monturen aus Eisen- oder Buntmetallblechen, die im Heft vernietet sind, sowie zum Teil aus Griffendbeschlägen.

Nicht zusätzlich gesicherte und nicht zur Schneide abgesetzte Zungengriffe der Formen B1 und B3 laufen im 15. Jahrhundert aus, bei Messern mit angehobenen Griffen nehmen zusätzlich gesicherte Konstruktionsformen bis zum 16. Jahrhundert kontinuierlich zu und behalten wahrscheinlich auch im 17. Jahrhundert die höheren Anteile ${ }^{86}$.

\subsection{Die Griffsicherungskonstruktionen B5 bis B8}

Von den im Klassifizierungsmodell beschriebenen vier Konstruktionsformen B5 bis B8 konnte die Form B5 nicht nachgewiesen werden.

Die Konstruktion eines aus organischem Material bestehenden Griffs, der in einer Metallhülse oder -manschette nur durch einen Nagel oder Niet befestigt ist, betraf bei weiteren Nachforschungen ausschließlich Werkzeuge meist landwirtschaftlicher Anwendung.

\footnotetext{
84 Vgl. die Aufstellung niederländischer Griffzungenmesser des 14. bis 16. Jahrhunderts in Kapitel 6, Abschnitt 2.3

85 Vgl. Kapitel 6, Abschnitt 2.2

86 Die Verteilung von jeweils 50\% der Formen B1.2 und B2.2 im 17. Jahrhundert ist nicht aussagefähig (Abb. 113).
} 
Messer mit der Grifform B6 sind nur einmal aufgetreten.

Das Exemplar aus einer Knochenschnitzerwerkstatt aus Göttingen, datiert um 1300, weist am Heft eine Manschette auf, die nach der Beschreibung aus Buntmetallblech gefertigt wurde und die Griffschalen am Heft ummantelt (Kat. Nr. 114).

Die Form B7 mit waagerecht vor der Klinge liegender Zunge ist dreimal nachgewiesen worden.

Ein frühes Beispiel des 11. bis 12. Jahrhunderts aus der slawischen Burg bei Lübeck (Kat. Nr. 18) besitzt einen eisernen Vollgriff, der mittig eine Aussparung aufweist; diese wurde als liegende Zunge gestaltet (Abb. 115a).

Die beiden Exemplare des 16. bis 17. Jahrhunderts wurden in Trondheim (Kat. Nr. 57) und Amsterdam ${ }^{87}$ gefunden. Das Heft ist bei beiden Messern zu einem Vollkörper ausgeschmiedet worden, der auch als Stoßkante dient (Abb. 115b-c).

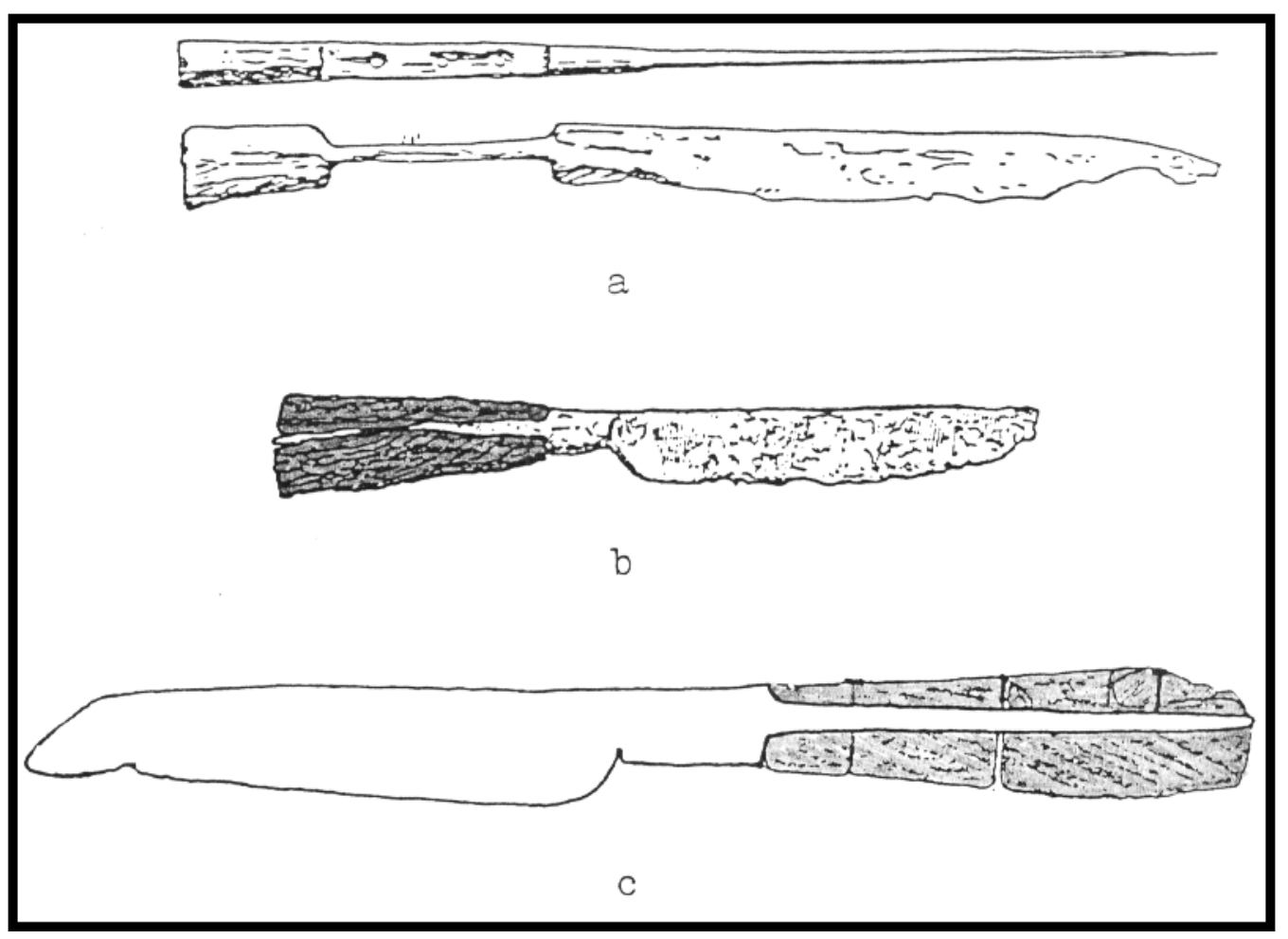

Abb. 115

Beispiel der Form B 7

a: Slawische Burg bei Lübeck, 11. bis 12. Jahrhundert

b: Trondheim, 16. bis 17. Jahrhundert

c: Amsterdam, Streufund

Die Konstruktionsform B8 konnte ebenfalls nur mit acht Messern belegt werden, die alle die für diese Form typische vollmetallene (eiserne) Übergangszone im

87 Das Messer aus Amsterdam gelangte dem Autor erst nach Abschluß des Kataloges zur Kenntnis. Verbleib: Privatbesitz 
Heftbereich aufweisen. Wie bei der Form B7 diente sie auch hier als Stoßkante für die vorderen Griffschalenenden. Diese Übergangszone nimmt im Gegensatz zu den

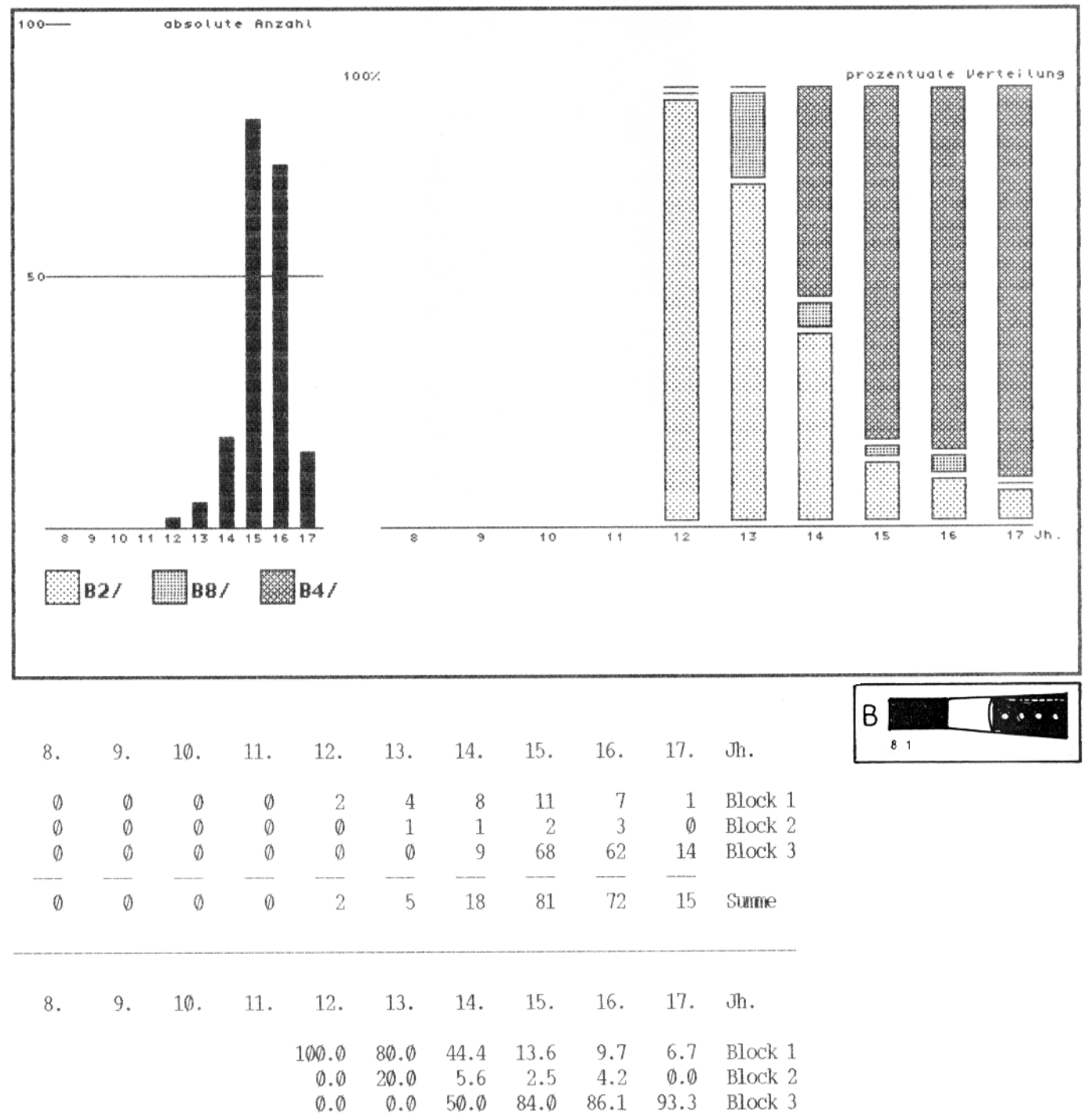

$A b b .116$

Das zeitliche Aufkommen der Zungenkonstruktion B8 zwischen Griffzungenmessern ohne (B2) und mit (B4) nach hinten verbreiterten Griffen im gesamten Untersuchungsraum (grob datierter Bestand) 
ausgeschmiedeten Stoßkanten der Formen B2 und B4 in der Regel ein bis zwei Drittel der Grifflänge als eisernes Vollgriffsegment ein.

Die zeitliche Verteilung vom 13. bis in das 16. Jahrhundert scheint trotz der geringen Anzahl von Belegen anzudeuten, daß diese Konstruktionsform möglicherweise älter ist als die Stoßkanten an Griffzungenmessern der Formen B2.2 und B4.2 (Abb. 116).

Ihre Parallelform findet sie in der Griffangelkonstruktion A5, die ebenfalls bereits früh und über einen längeren Zeitraum nachgewiesen werden konnte (Abb. 102: 12. und 14. bis 17. Jahrhundert).

\subsubsection{Allgemeine Ergebnisse}

Im Untersuchungszeitraum vom 9. bis 17. Jahrhundert sind zwei grundsätzliche Befestigungsarten des Griffs aufgetreten, die Angel- und die Zungenkonstruktion. Vom 9. bis zum 14. Jahrhundert dominiert die Angelkonstruktion im gesamten Untersuchungsraum. In dieser Zeit treten mehrere verschiedene Möglichkeiten auf, den auf die Angel aufgeschlagenen Vollgriff zusätzlich zu befestigen. Eine dieser Möglichkeiten, die Verwendung von Stich- und Abschlußplättchen am Heft und Griffende, enwickelt sich fast parallel zum Anwendungszeitraum der einfachen Befestigung $\mathrm{zu}$ einer modisch bedingten Verzierungsart, der Verwendung mehrerer Plättchenpakete und der Segmentierung des Vollgriffs. Die Hauptverbreitungszeit sowohl der Anwendung der einfachen Zusatzbefestigung als auch der Griffverzierung mit Griffplättchen liegt im 13. und 14. Jahrhundert, im 15. Jahrhundert ist sie - wenn auch nur sporadisch und regional begrenzt - noch anzutreffen.

Griffzungenkonstruktionen setzen verstärkt und allgemein verbreitet erst im 13. bis 14. Jahrhundert ein. Ihre zum Teil ausgereiften Vorläufer reichen bis in das 11 . Jahrhundert zurück, Griffzungenmesser vor 1200 scheinen auf die slawisch besiedelten Räume des Untersuchungsgebietes beschränkt zu sein.

Im 15. und 16. Jahrhundert dominiert das Griffzungenprinzip, belegbar aus quellenkritischen Gründen nur für den westlichen Teil des Untersuchungsgebietes.

Im 17. Jahrhundert ist für diesen Raum wieder eine stärkere Rückkehr zur Griffangelkonstruktion $\mathrm{zu}$ beobachten, sie hat in ihrer Intensität unterschiedlich stattgefunden.

Vom 15. bis 17. Jahrhundert machen Angel- und Zungenmesser eine gleichartige Entwicklung durch:

Die bereits im 13. und 14. Jahrhundert beobachtete Praxis, den Griff insgesamt zur Schneide abzusetzen, um ein Verrutschen der Hand auf die Klinge zu verhindern, wird im 15. und beginnenden 16. Jahrhundert variiert und erfährt im späten 16. und 17. Jahrhundert allgemein eine einheitlich klare Ausprägung, die unabhängig von Konstruktion und Verzierung vorhanden ist. 


\subsection{Beschreibender Teil}

\subsubsection{Arbeitsweise}

Im beschreibenden Teil der Auswertung werden anhand ausgewählter Beispiele Themen diskutiert, die im statistischen Teil nur angeschnitten werden konnten. Dazu treten Beschreibungen und katalogartige Erfassungen von Verzierungselementen und Klingenmarken sowie beispielhaft ein typologischer Überblick von Messern des 14. bis 17. Jahrhunderts und die Darstellung einer regionaltypischen Messergruppe.

Zwei besonders herausragende Gestaltungsarten spätmittelalterlicher Messergriffe werden darüber hinaus detailliert vorgestellt und in einem allgemeiner gefaßten kulturgeschichtlichen Zusammenhang diskutiert.

\subsubsection{Frühe Griffzungenkonstruktionen}

Die römische Zivilisation war zu Beginn der Kaiserzeit in der Organisation gewerblicher Produktion und in fast allen Techniken der Materialbearbeitung den von ihnen nördlich der Alpen unterworfenen Völkern weit überlegen.

Das Kulturgefälle zwischen den provinzialrömischen Gebieten und dem freien Germanien vor sowie in den römisch beeinflußten Gebieten nach dem Niedergang des römischen Kaiserreichs läßt sich an den Gütern der Massenproduktion, vor allem an Keramik- und Glasgefässen, nachweisen ${ }^{88}$. Viele Techniken, die von römischen Manufakturen beherrscht wurden, sind später erst wieder im Verlauf des Hochund Spätmittelalters nachweisbar, wobei eine Kontinuität über rund 800 Jahre in der Regel nicht anzunehmen ist.

Alle Analysen, die die Verbreitung der beiden hauptsächlichen Griffarten Angel und Zunge betreffen, zeigen übereinstimmend den wahrscheinlichen Beginn der Griffzungenkonstruktion seit dem 11. Jahrhundert, wobei der Auslöser der Entwicklung aus dem Osten zu kommen scheint.

Eine möglicherweise nur regional nachweisbare typologische Entwicklung von der Griffangel zur -zunge über die Formen A6, B9 und B1 am Ende des 12. und im 13. Jahrhundert und der Nachweis ausgereifter Griffzungenmesser vor dem 13. Jahrhundert mahnen zur Vorsicht bei der Suche nach Herkunft und Richtung dieser Entwicklung.

88 Vgl. beispielhaft das Fundgut aus dem Gräberfeld Krefeld-Gellep, in: Pirling 1975, S. 170-172; dieselbe 1986 
Die Zeit, in der Griffzungenmesser im Bearbeitungsraum nicht nachweisbar sind, umfaßt die gesamte Völkerwanderungszeit bis in das 10. Jahrhundert. Eine Kontinuität zwischen der römischen Kaiserzeit und dem Hochmittelalter ist daher eher unwahrscheinlich.

Um so erstaunlicher ist die Tatsache, daß im provinzialrömischen Bereich fast alle Grundtechniken und Verzierungsarten an Messergriffen vorkommen.

Von Anfang an brachten die römischen Truppen, Handwerker und Händler eine ausgefeilte, spezialisierte und standardisierte materielle Kultur in die Länder nördlich der Alpen, und hier finden sich auch bereits der Formenreichtum und die technologische Vielfalt von Messergriffkonstruktionen, die sich erst zu Beginn der Neuzeit wieder in ähnlichem Umfang belegen lassen.

Am Beispiel einiger ausgewählter Messer, die zum größten Teil aus spättiberischen und claudischen Militärstationen des Donauraumes stammen, wird ein Ausschnitt aus dieser Palette vorgestellt. Das Prinzip der Griffzungenkonstruktion ist mit und ohne Zusatzbefestigungen in den römischen Lagerbeständen vorhanden (Abb. 95c bis 95h), selbst Verzierungselemente wie Kreuzschraffur und Punktaugen, die in der gleichen Anordnung auf mittelalterlichen Messergriffen erscheinen, wurden verwandt (Abb. 117a und 117b).

Halterungsösen am Griffende sind an Griffangel- und Griffzungenmessern bekannt (Abb. 117h und 118c), technisch zu vergleichen etwa mit einem frühslawischen Messer des 7. bis 8. Jahrhunderts aus Feldberg (Mecklenburg) ${ }^{89}$.

Aus dem zeitgleichen Bestand des Legionslagers Augsburg-Oberhausen wurde unter anderem eine typlogische Abfolge A2-B2 anhand von Ringgriffmessern zusammengestellt, die ungeachtet der typologischen Stufen zeitlich nebeneinander vorhanden waren (Abb. 118b bis 118f). Dieses Beispiel mag die Schwierigkeiten, wenn nicht die Unmöglichkeit belegen, typlogischen Entwicklungen an Messergriffen eine chronologische Abfolge zugrunde zu legen, wie zum Beispiel bei der weiter unten geführten Diskussion um die Formen A6 und B9.

In diesem Zusammenhang sei auch auf die Möglichkeit hingewiesen, daß sich unter den zahlenmäßig bedeutenden Beständen, die als römische Funde aus Lagern, Stationen, Kastellen, villae rusticae und Städten der römischen Kaiserzeit nördlich der Alpen publiziert worden sind, weitaus jüngere Streufunde befinden können. Einen nachträglichen Nachweis zu führen, ist für Außenstehende kaum möglich. An das Ende der römischen Messer wurden aber zwei Griffe aus dem Donaukastell Aislingen gestellt, die aus dem Rahmen der übrigen, relativ einheitlichen Formen herausfallen (Abb. 118i und 118j). 

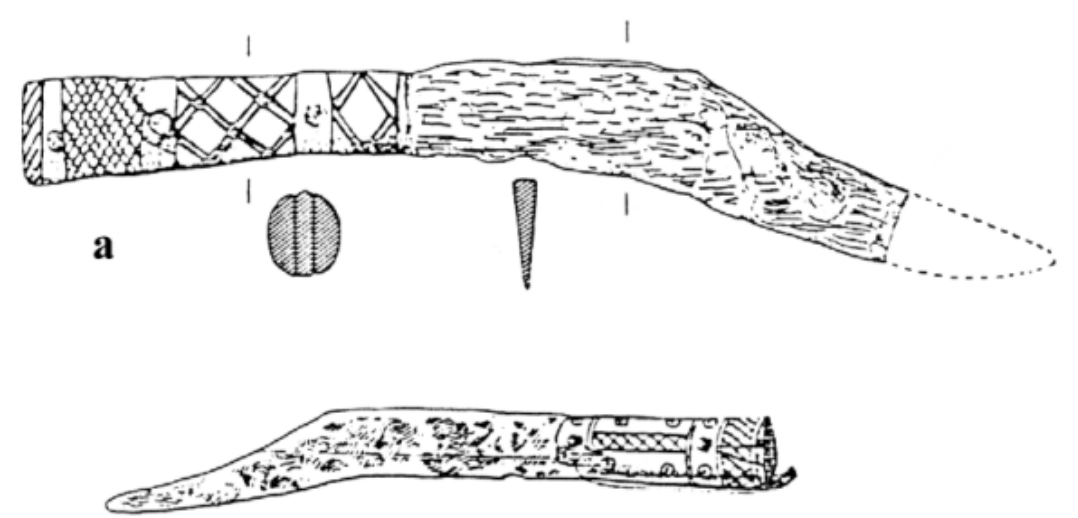

b

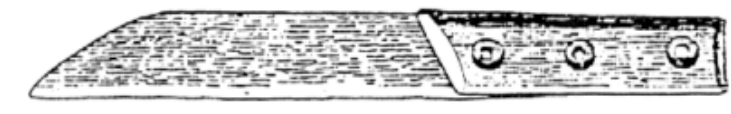

c

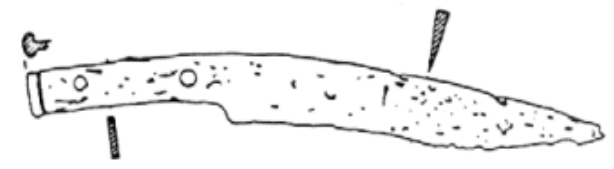

d
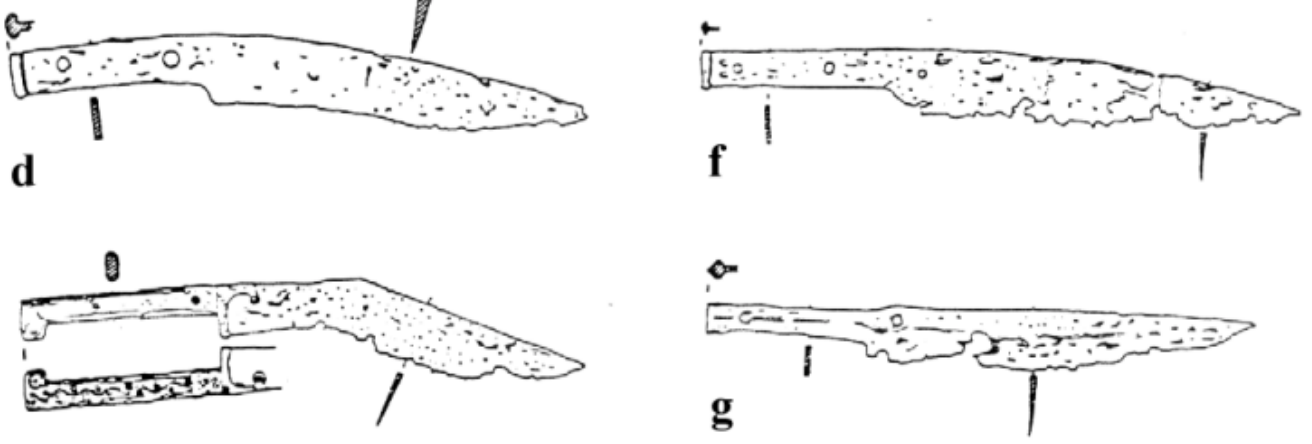

$\mathbf{e}$

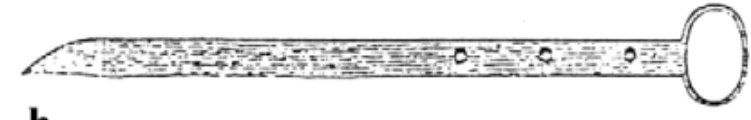

h

Abb. $117 \quad$ Griffzungenmesser der römischen Kaiserzeit $I^{90}$
a: $\quad$ Kastell Rißtissen;
b: Heddernheim;
c: $\quad$ Diemeser Ort;
d-g: Legionslager Augsburg-Oberhausen;
h: $\quad$ Diesmeser Ort 


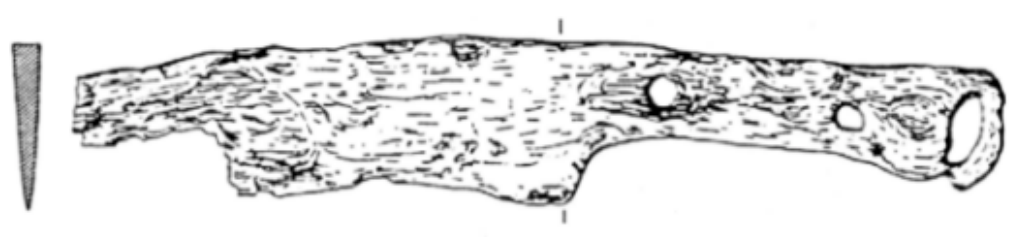

a

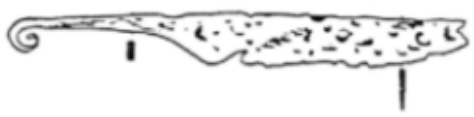

$\mathrm{b}$

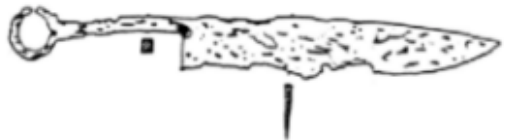

C

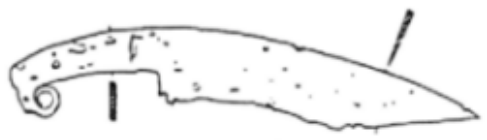

d
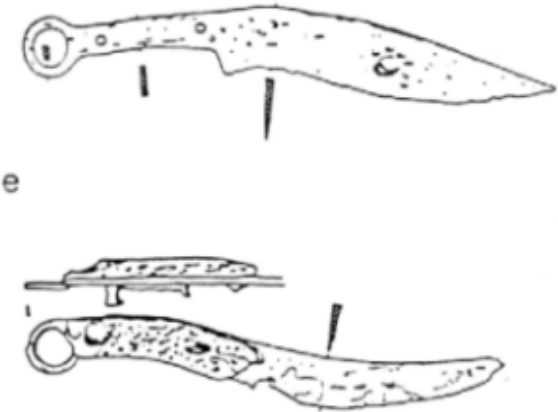

f

$A b b .118$

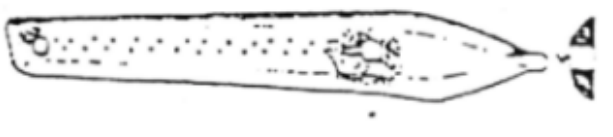

g

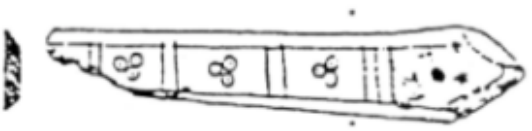

h

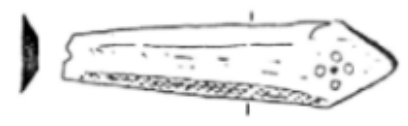

i

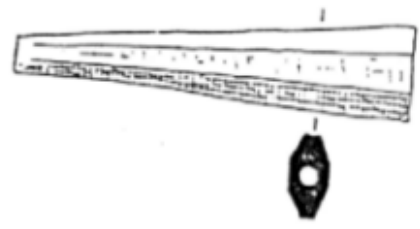

$j$

Griffzungenmesser der römischen Kaiserzeit $I^{91}$

a: Kastell Rißtissen:

b-f: $\quad$ Legionslager Augsburg-Oberhausen;

g-h: $\quad$ Burg in Skanör, A. 15. Jahrhundert;

i-j: $\quad$ Kastell Aislingen

90

a: Ulbert 1970, Abb. 272, Tafel 17

b-c, h: Lindenschmitt 1870 Abb. 1, 6 und 5, Tafel 4 aus Bd. II, Heft IV

91 d-g: Hübener 1973, Abb. 5, 6, 15 und 20, Tafel 20, Text S. 51-52

a: Ulbert 1970, Abb. 273, Tafel 17

b-f: Hübener 1973, Abb. 18, 3, 1, 4 und 2, Tafel 20

i-j: Ulbert 1959, Abb. 21 und 22, Tafel 26 
Vor allem das erste Beispiel, eine Knochenschale mit spitzem Abschluß und einer aus Kreisaugen bestehenden kreuzförmigen Verzierung, findet drei nahezu identische Parallelen aus Lübeck und von der Burg Skanör auf Schonen ${ }^{92}$. Die beiden Griffschalen aus Schonen stammen aus der Zeit vor 1425 (Abb. 118g und 118h). Aus den nachkaiserzeitlichen Jahrhunderten ist dem Autor im Bearbeitungsraum kein Beispiel eines Zungenmessers bekannt geworden.

\section{Griffzungenmesser vor 1200}

Die frühesten Beispiele von angeblichen Griffzungenmessern im Bearbeitungsraum nach der Völkerwanderungszeit bestehen aus Knochenschalen, deren Funktion als Griffschalen von Messern mehr als unsicher ist. Neben dem möglicherweise vorkarolingischen Fundstück aus Dorestad (Abb. 119a) sind noch zwei Knochenschalen zu erwähnen, von denen eine mit Sicherheit als Fragment eines Dreilagenkamms angesehen werden $\mathrm{mu}^{93}$ (Abb. 119c).

Das zweite Stück ist eine mit Wellenband und Punktaugen verzierte Knochenschale, die an einem Ende ausgebrochen ist. Nietlöcher sind nicht vorhanden, es besteht aber die Möglichkeit, daß die Schale für die endgültige Verwendung noch nicht fertig bearbeitet war, bevor sie verlorenging. Gegen eine Verwendung als Griffschale spricht die Abschrägung des erhaltenen Endes, die für Messer nicht üblich ist. Die Schale wurde auf dem Burgberg in Meißen gefunden und wird in die zweite Hälfte des 10. oder in das 11. Jahrhundert datiert ${ }^{94}$ (Abb. 119b).

a.
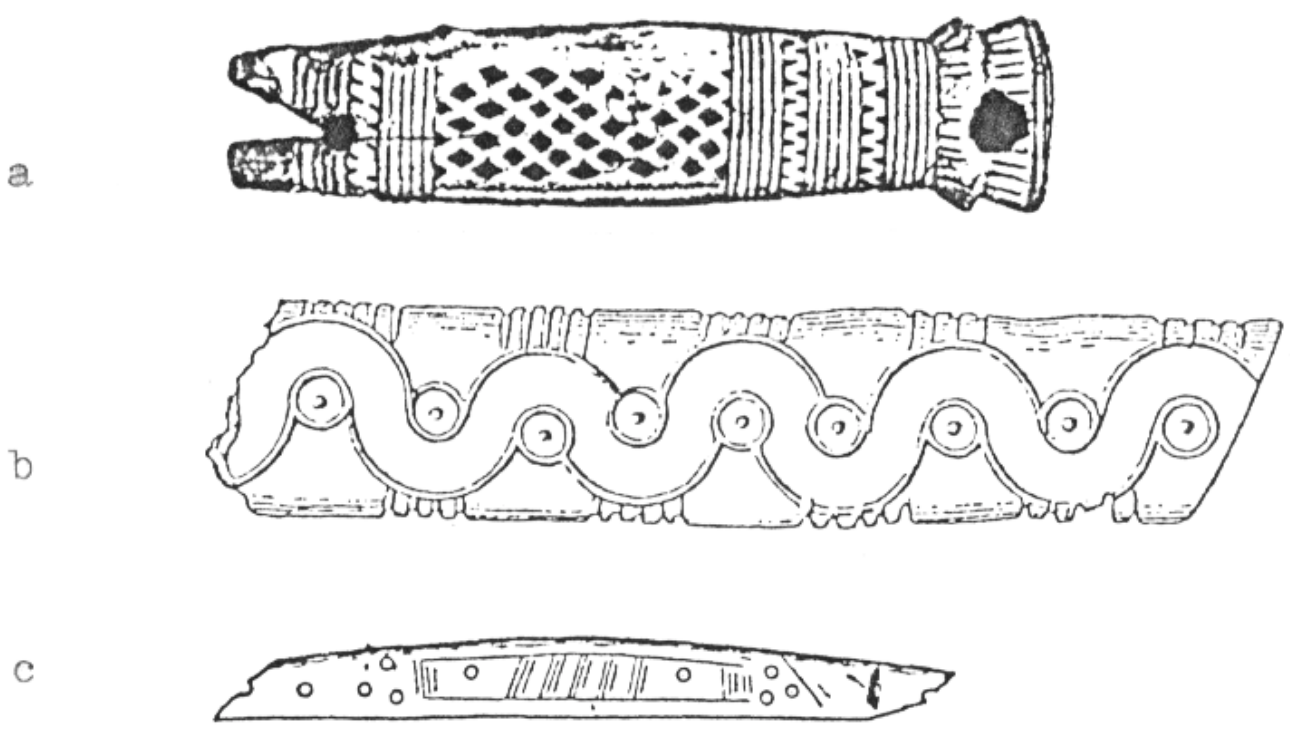

Abb. $119 \quad$ Knochenschalen außerhalb des Anwendungsbereichs als Messergriffschalen

\footnotetext{
92 Vgl. Katalogteil BRD, Kat. Nr. 35; Schweden, Kat. Nr. 125, 126; vgl. auch N.-K.Liebgott, Stakhaven, Kobenhavn 1979, S. 121: dort nennt Liebgott die Zeit um 1270 als den ältesten Nachweis für Griffzungenmesser.

Vgl. Katalogteil ehemalige DDR, Kat. Nr. 162
} 
Für die Zeit vor dem 13. Jahrhundert sind im gesamten Bestand von 1300 Messern nur fünf Beispiele vorhanden, die relativ sicher als Griffzungenmesser angesprochen werden können; sie stammen alle aus dem slawischen Kulturkreis (Abb. 120a, $120 \mathrm{~b}, 120 \mathrm{~d}, 120 \mathrm{e}, 120 \mathrm{~h})$. Das am weitesten westlich geborgene Exemplar aus Steinkirchen bei Lübben (ehemalige DDR) ist relativ gut erhalten, die vier polnischen Funde sind Griffschalen ${ }^{95}$.

Hinzu kommt ein ausgeprägtes Griffzungenmesser aus Rozprza (Polen), welches von den Ausgräbern in das 11. bis 12. Jahrhundert datiert wurde. Genaue stratigraphische Angaben wurden nicht gemacht, die Datierung bezieht sich auf eine den gesamten Grabungsabschnitt betreffende Besiedlungsphase (Abb 120j).

Es mag an dieser Stelle erlaubt sein, die Datierung in Frage zu stellen, auch angesichts der Tatsache, daß es bisher keine überregional angelegten Studien zur allgemeinen Entwicklungsgeschichte des Messers gibt, Vergleichsmöglichkeiten also 1966, im Jahre der Veröffentlichung der Grabungen in Rozprza, nicht vorlagen. Das Messer ist nach den Erkenntnissen der Auswertungen des Bestandes dieser Studie kaum vor das 13. bis 14. Jahrhundert zu datieren, da erst zu dieser Zeit derart ausgeprägte, im Heft zur Schneide abgesetzte Griffzungenmesser belegt sind.

Diese Zweifel gelten in noch stärkerem Maße für die beiden Messer aus Lublin (Abb. 120k und 1201), die in das 12. bis 13. Jahrhundert datiert worden sind. Diese beiden Beispiele weisen am Heft profilierte Heftverstärkungen auf, die ansonsten frühestens im 15. Jahrhundert nachzuweisen sind; die früheste Heftverstärkung an einem Griffzungenmesser überhaupt tritt bei einem Messer des 13. bis 14. Jahrhunderts aus Gommerstedt (ehemalige DDR) und bei einem Amsterdamer Messer des 14. Jahrhunderts auf (Abb. 123a und 123i).

Bisher nachweisbar sind Griffzungenmesser im 11. bis 12. Jahrhundert im slawischen Kulturraum, die frühesten sicheren Belege im westlich angrenzenden deutschen Kulturraum stammen frühestens aus der Zeit um 1200.

95 Unter ihnen befindet sich ein Messergriff, dessen heftseitiger Abschluß die einzige ähnliche Parallele zu der Griffgestaltung des umstrittenen Griffs aus Dorestad (Niederlande, Kat. Nr. 25) darstellt (Abb. 120h). Es kann daher nicht ausgeschlossen werden, daß dieser Griff nicht vorkarolingisch, sondern ein Beispiel einer Griffzungenkonstruktion in den Niederlanden für das 11. Jahrhundert ist. 

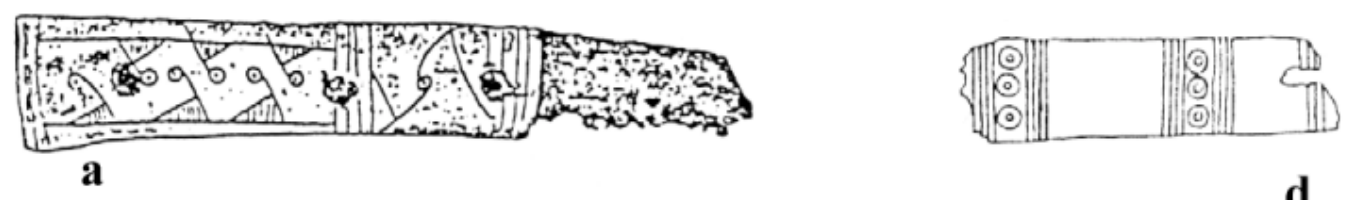

d
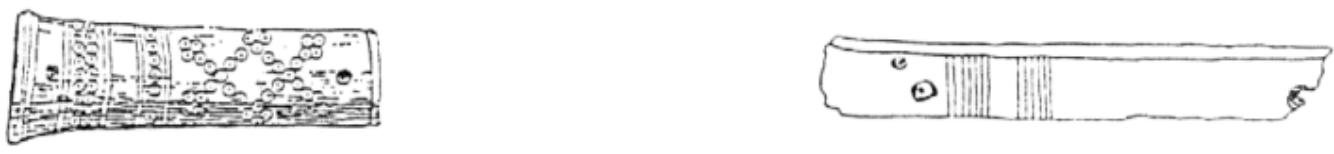

b
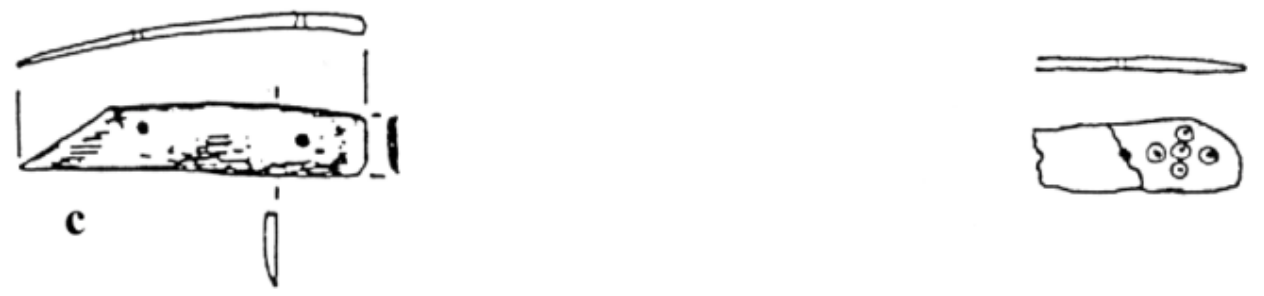

\section{f}

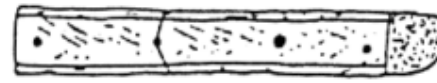

g

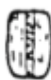

盟.

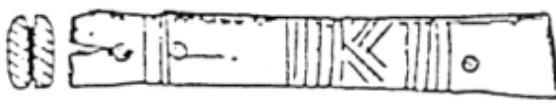

h

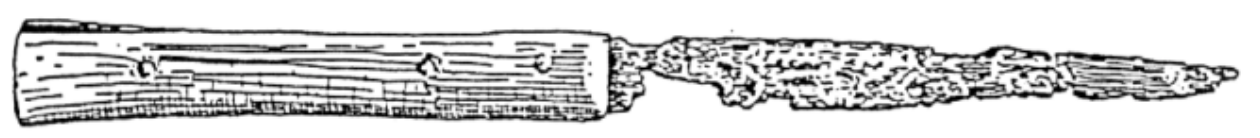

i
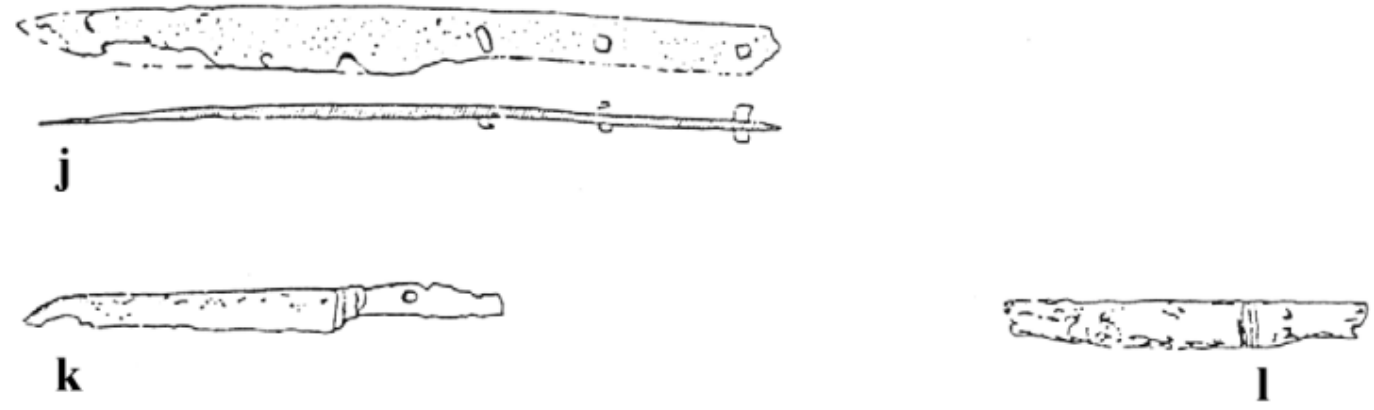

Abb. 120

Frühe Griffzungenmesser I

a: $\quad$ Steinkirchen, 11. - 12. Jh.; b: Tum, 12. - 13. Jh.; c: Göttingen, um 1200; d-e: Oppeln, 10. - 12. Jh.; f: Isenburg, E 12. - A.13. Jh.: g: Kiel, E12. - A.14. Jh.; h: Oppeln, E.11 - A.12. Jh.; i: Posen, 2.H.13. Jh.; j: Rozprza, 11. - 12. Jh.(?); k-l: Lublin, 12. - 13. Jh.(?) 


\section{Die Formen A6 und B9}

In diese Zeit der frühen Griffzungenmesser im mittleren und nördlichen Europa fällt der Nachweis einer bis jetzt lediglich konstruktionstechnisch als Vorläufer zu bezeichnenden Entwicklungsstufe, bezeichnet als Formen A6 und B9.

Technologisch lassen sich drei Stufen feststellen, die zeitlich in dieser Reihenfolge allerdings nicht regelmäßig aufgetreten sind, so daß von einer sicheren chronologischen Abfolge technischer Novationen zumindest derzeit noch nicht gesprochen werden kann:

1. Vollgriffe, die im Heftbereich mit Hilfe durch die Angel geschlagener Niete zusätzlich gesichert wurden

2. Griffschalen, die durch mehrere Niete auf einer zwar am Heftansatz breit ausgeschmiedeten, hinten aber noch wie eine Angel spitz zulaufenden Zunge befestigt wurden

3. Ausgebildete Griffzungen, deren Kanten entweder parallel verlaufen (in der Regel die ältere Form) oder deren Unterkante zum Griffende hin nach unten verläuft, so daß das Griffende breiter ist als das Heft (in der Regel die jüngere Form).

Der Grund für die Abfolge der ersten beiden Stufen liegt nicht nur in der zeitlichen Überschneidung von Messern der Formen A6, B9 und B1.1; er betrifft auch die Tatsache, daß die theoretisch angenommene Befestigungsart A6 mit keinem ausreichend erhaltenen Beispiel, sondern nur mit Fragmenten belegt werden kann.

In der Regel werden daher Messer der Form A6 in der Literatur als Klappmesser bezeichnet, obwohl sich die erhaltenen Klappmesser des Mittelalters durchaus in Form und Konstruktion von den Messern der Form A6 unterscheiden ${ }^{96}$.

Ein typisches Klappmesser mit abstehender Angel wurde unter anderem auf der Isenburg bei Hattingen (Ruhr) gefunden (Abb. 122e). 


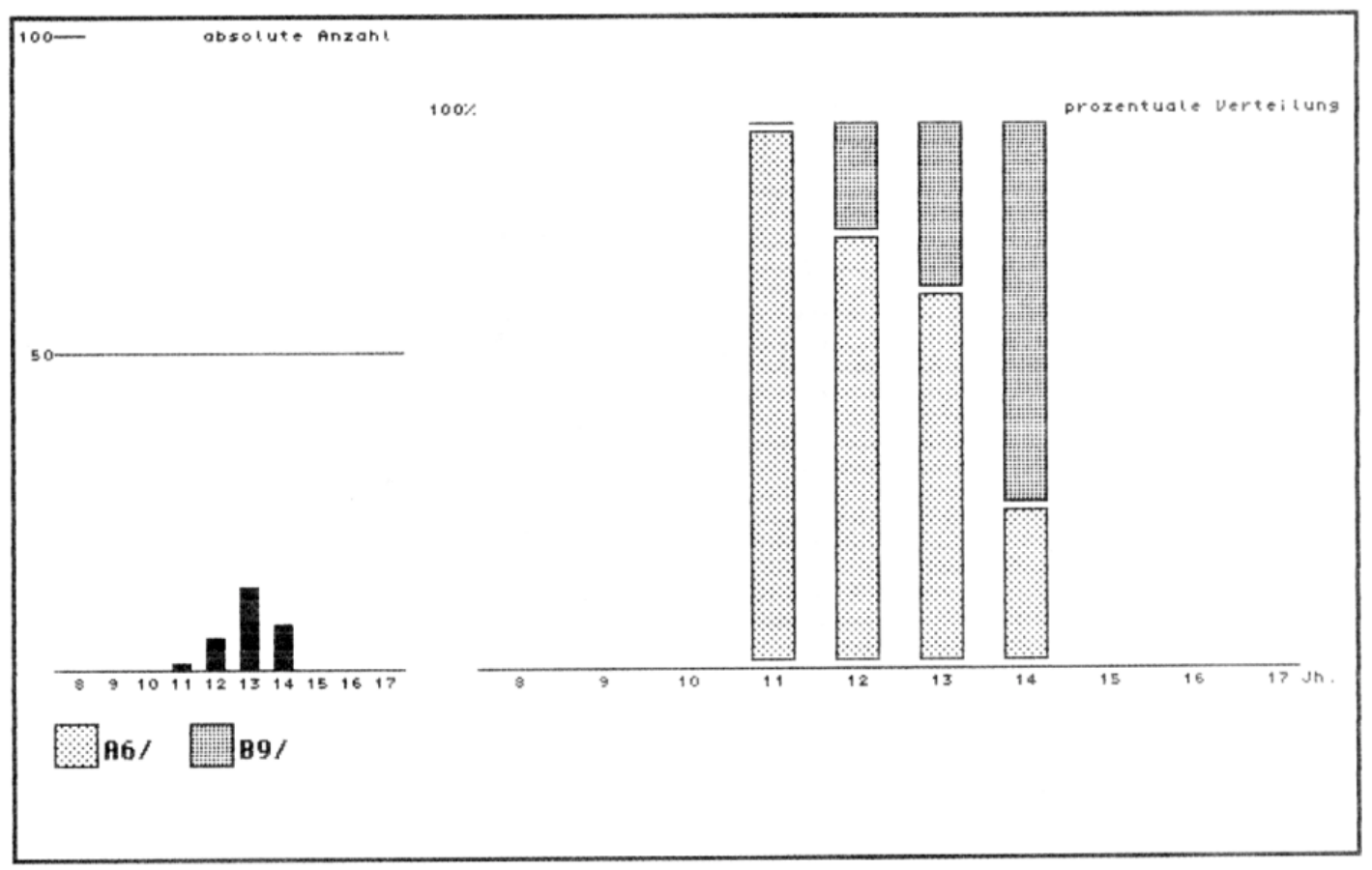

Abb. 121

Das Auftreten der Griffkonstruktionen A6 un B9 im gesamten Untersuchungsraum (grob datierter Bestand)

Klingenform, dornartig verkümmertes und schräg nach oben abstehendes Angelrelikt sowie ein Scharnierniet weisen es als ein solches aus, zu vergleichen etwa mit zeitgleichen Messern aus Novgorod ${ }^{97}$. Schon ein weiteres Messer aus der Burg Rödersen (12. Jahrhundert bis $1262^{98}$, Abb. 122f) zeigt Merkmale, die sich mit der Funktion als Klappmesser nicht mehr in Einklang bringen lassen: es besitzt zwei übereinander angeordnete Nietlöcher, der Angelstumpf weist zur Schneide, eine Hebelwirkung ist dadurch ebensowenig gegeben wie ein Scharniermechanismus. Ein Rekonstruktionsvorschlag für dieses Messer kann aufgrund fehlender Vergleichsmöglichkeiten nicht gemacht werden.

Auch die übrigen Beispiele der Form B6 (Abb. 122a bis 122d, 122g, 122h) fallen aus den bekannten Formen von Klappmessern heraus. Ob es sich bei ihnen um eine bisher nicht nachgewiesene Form von Klappmessern oder um zusätzlich durch Vernietung des Heftes gesicherte feststehende Angelmesser handelt, kann abschließend noch nicht beantwortet werden. Die Verbindung der Bezeichnung A6 mit letzterer Rekonstruktion ist daher als Vorschlag zu betrachten.

\footnotetext{
97 Koltschin 1959, Abb. 45, S. 58

98 Nach freundlicher Auskunft von Herrn Dr. H.-G. Stephan, Universität Göttingen, macht die Auswertung der Keramik von der Burg Rödersen eine Korrektur des bisherigen Anfangsdatums um 1180 erforderlich.
} 
Das zeitliche Aufkommen im 12., 11. bis 13., 12. bis 13. und 14. Jahrhundert deckt sich mit dem Auftreten der Form B9, einer frühen Art der Griffzungenkonstruktion im 12., 13. und 13. bis 14. Jahrhundert (Abb. 122i bis 122k).

Messergriffe mit den Formen A6 und B9 sind im Bearbeitungsraum vor allem im deutschsprachigen Raum, vereinzelt im westslawischen und polnischen Bereich aufgetreten und vom 12. bis 14. Jahrhundert mit steigender Tendenz nachweisbar (Abb. 121).

Regional begrenzt und seit dem 13. Jahrhundert neben der bereits technisch ausgereiften Zungenkonstruktion stehend, fällt es schwer, von einer allgemeinen typologischen Stufe zu sprechen. Möglicherweise wurde die Griffzunge in verschiedenen Räumen mit unterschiedlicher Geschwindigkeit gleichzeitig entwickelt, zum Teil typologisch über die Formen A6 und B9, zum Teil direkt als technische Innovation. 

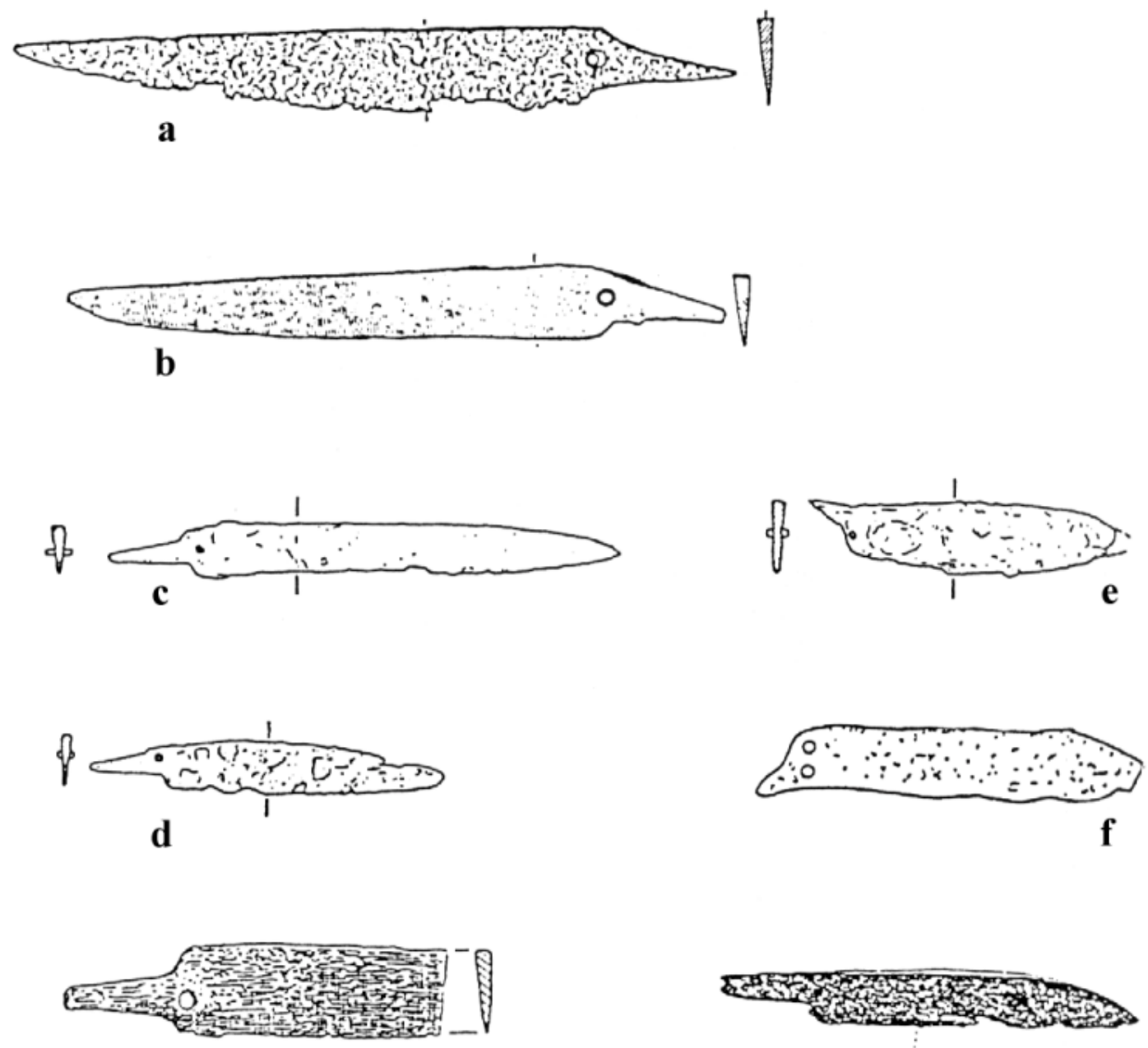

g

目

i

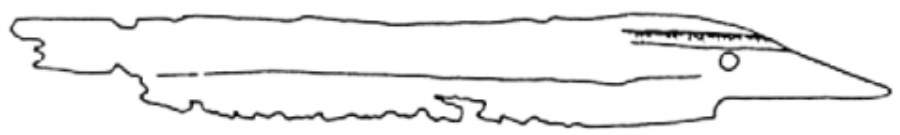

h

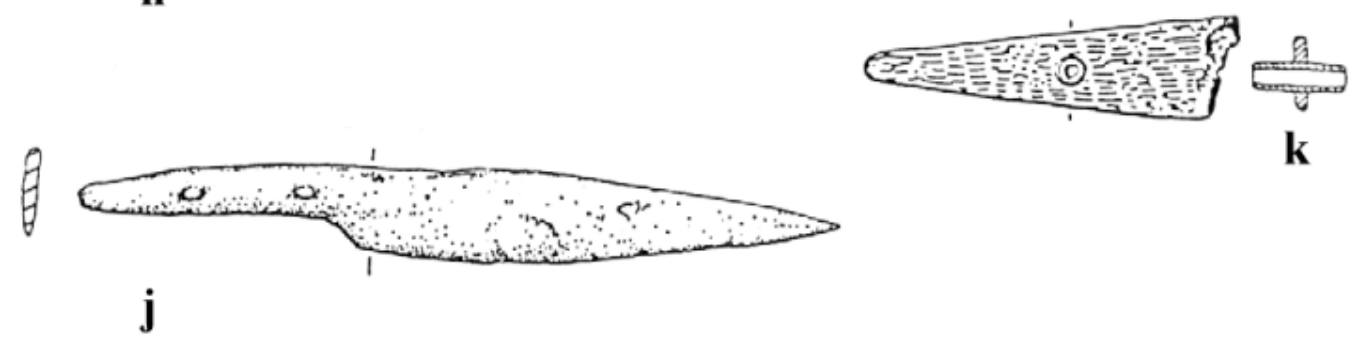

Abb. $122 \quad$ Beispiele der Fiormen A6 und B9

a: Dörhai-Winzenburg, 12. Jh.; b: Hanfwerder, 11. - 13. Jh.; c-e: Isenburg, E12. A.13. Jh.; f: Burg Rödersen, E.12. - 13. Jh.; g: Posen, 2.H.13. Jh.; h: Itzehoe, 14. Jh.; i: Kalisch, 12. Jh.; j: Göttingen, um 1300; k: Gommerstedt, 13. - 14. Jh. 


\section{Die Entwicklung vom 13. bis 15. Jahrhundert}

Das 13. Jahrhundert ist als der Zeitraum zu betrachten, in dem das entwickelte Griffzungenmesser im gesamten Untersuchungsraum nachweisbar ist (Abb. 81 und 87).

Funde aus Göttingen, von der Isenburg, aus Kiel und Posen zeigen verzierte und unverzierte Griffschalen und ein vollständig erhaltenes Messer der Form B1.1 (Abb. 120c, 120f, 120h und 120i).

Im 14. Jahrhundert ist von der übergangslos aus der Klinge hervorgehenden Zunge der Form B1.1 bis zu zusätzlich an Heft und Griffende gesicherten, zur Schneide abgesetzten Griffzungen der Form B4.2 (Abb. 123a, 123i und 123j) die gesamte Palette der konstruktionstechnischen Kriterien nachweisbar, ohne daß sie zeitlich genauer zu gliedern ist. Bis auf die Beispiele aus Gommerstedt und Glasbach (13. bis 14. Jahrhundert, Abb. 123a bis 123c) stammen alle Messer aus dem 14. Jahrhundert.

Da für das 13. Jahrhundert diese Vielfalt nicht nachzuweisen ist, darf angenommen werden, daß diese Entwicklung möglicherweise erst gegen Ende des 13. Jahrhunderts begonnen und sich dann sehr rasch im 14. Jahrhundert über den gesamten Raum ausgebreitet hat. Vergleicht man die in etwa zeitgleichen Messer aus Amsterdam, Göttingen und Danzig (Abb. 123f bis 123i), so liegt der Schluß nahe, daß diese Entwicklung von Westen nach Osten stattgefunden hat.

Dieser Schluß ist allerdings ebenfalls als Vorschlag zu betrachten, der nur durch erheblich dichter belegbares und besser datiertes Material zu erhärten ist.

Eine Besonderheit liegt im Fall eines Göttinger Messers aus der Zeit um 1300 vor (Abb. 123f). Die Griffzunge (-angel?) reicht nur zu einem Teil in den lediglich im vorderen Bereich aufgeschnittenen Vollgriff, sie ist mit nur einem Niet befestigt. Die beiden übrigen Niete stabilisieren den Griff und verhindern ein weitergehendes Aufreißen.

Ein Amsterdamer Exemplar aus der ersten Hälfte des 14. Jahrhunderts weist bereits alle wesentlichen Konstruktionselemente der Messer des 14. bis 15. und 15. Jahrhunderts auf (Abb. 123i). Vor allem die Griffendabsicherung, die bei dem Messer aus Göttingen noch in der Art der Vollgriffe zu Verzierungszwecken genutzt worden ist, weist den Westen als wahrscheinliches Ausgangszentrum dieser technischen Entwicklung aus.

Daß sich im 14. und beginnenden 15. Jahrhundert durchaus nicht überall diese neuartige Befestigungstechnik durchgesetzt hat, zeigt ein Blick auf weitere Belege dieser Zeit (Abb. 124).

Das Messer aus Dordrecht, nach Vergleich in die Zeit nach 1400 datiert, steht technologisch vor dem Messer aus Amsterdam (Abb. 124e und 123i). Auch die beiden Beispiele aus Duisburg (Abb. 124a) und Lübeck (Abb. 124b) sind noch als Vetreter 
der frühen technischen Stufe der Griffzungenkonstruktion zu betrachten. Schlüsse hinsichtlich der Richtung und der Zeit der Ausbreitung technischer Neuerungen können daraus nur bedingt gezogen werden, da die Datierungsansätze gerade dieser beiden Messer nicht eindeutig sind:

Das Duisburger Messer ist zwar stratigraphisch in das 15. Jahrhundert datiert worden ${ }^{99}$, die Form der Klinge und die Art der Griffzunge verweisen aber stark in das 13. bis 14. Jahrhundert, beispielhaft belegt durch die Messer aus Gommerstedt und Glasbach (ehemalige DDR). Es erscheint zwar unwahrscheinlich, aber nicht unmöglich, daß ein etwa 100 Jahre altes Messer in einem Keller im 15. Jahrhundert verlorenging oder dort mit Abraum deponiert wurde. 

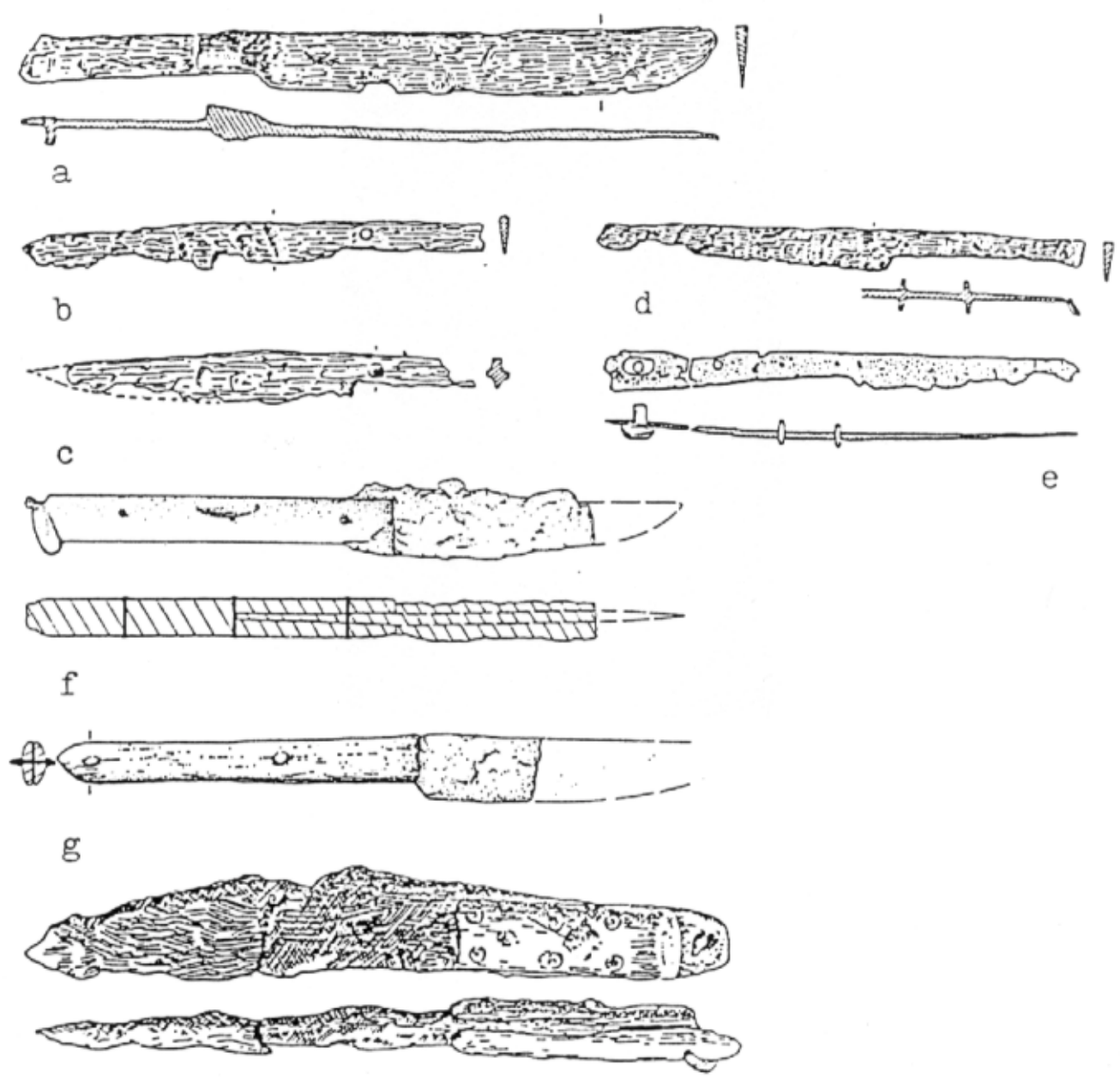

h

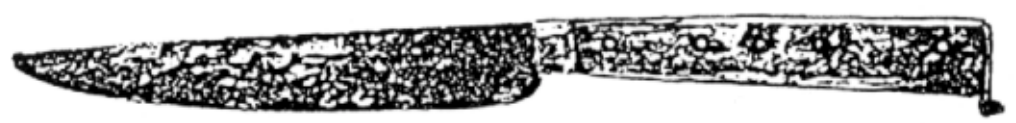

i

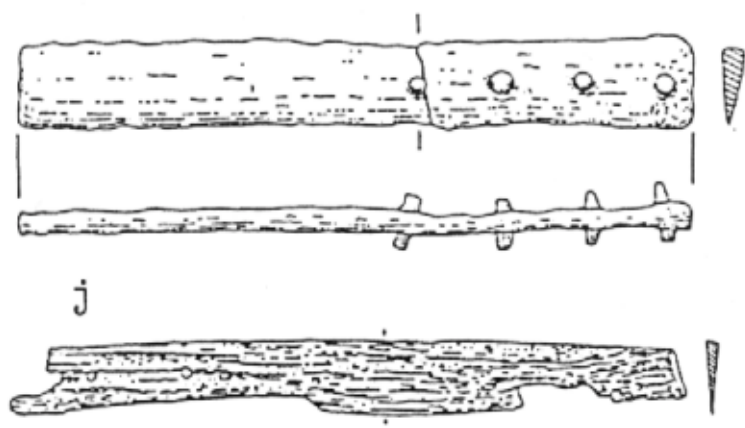

k

Abb. $123 \quad$ Frühe Griffzungenmesser II

a-d: Gommerstedt, 13. - 14. Jh.; e: Glasbach, 13. - 14. Jh.; f-g: Göttingen, um 1300; h: Danzig, A.14. Jh.; i: Amsterdam, 1.H. 14. Jh.; j: Usch, 14. Jh.; k: Manebach, 14. Jh. 
Das Messer aus Lübeck ist ein Altfund, der stilistisch in die Zeit um 1500 datiert worden ist ${ }^{100}$. Klingenverzierungen dieser Art sind im Fundgut bis in das 14. Jahrhundert belegbar, eine direkte Parallele ist nicht bekannt. Die Klingenverzierungen des 16. bis 17. Jahrhunderts betreffen in der Mehrzahl lediglich den an das Heft grenzenden Klingenbereich, auch die Art und die Plazierung der Verzierungselemente unterscheiden sich deutlich von diesem Stück ${ }^{101}$.

Der Hauptgrund für die Zweifel an einer Datierung dieses Messers in das beginnende 16. Jahrhundert besteht in der Klingenform und der Griffkonstruktion. Fast alle Zungenmesser des 16. Jahrhunderts weisen im Heftbereich Verstärkungen aus Buntmetallblechen auf; das für diese Zusatzbefestigung notwendige Nietloch im Heft fehlt bei dem Lübecker Messer.

Aus diesen Gründen mag das Messer eher in die Zeit vom 14. bis beginnenden 15. Jahrhundert eingeordnet werden; ein sicheres Belegexemplar ist es allerdings nicht.

Drei weitere Beispiele aus Ahaus, Lübeck und Posen (Abb. 124d, 124g, 124f) sind ebenfalls nicht genauer zu datieren. Der Lübecker Griff findet zwei vor 1425 datierte Parallelen von der Burg in Skanör ${ }^{102}$, Schonen. Er ist an den Kanten abgeschrägt worden, so daß ein rautenförmiger Querschnitt entstanden ist; in seiner Fläche ist er durch Silberstifte verziert worden. Die gleiche Abschrägung an den Kanten und Silbereinlagen weist das Messer aus Ahaus auf. Sein Griff verläuft ebenfalls nach hinten verbreitert, das Ende ist zu einer kronenförmigen Verzierung ausgeschnitzt worden. Parallelen zu diesem Messer finden sich in den Niederlanden, vor allem aus dem sogenannten Zeeuws Vlaanderen, wo an zwei Fundstellen derartige Griffe verstärkt aufgetreten sind ${ }^{103}$.

Eine Datierung dieser beiden Messer in das ausgehende 14. und beginnende 15. Jahrhundert mag daher angebracht erscheinen.

Das Messer aus Posen, ebenfalls nur grob in das 14. bis 15. Jahrhundert datiert, ist nicht genauer einzuordnen, da die Stellung zur Klinge nicht zu rekonstruieren ist (ähnlich wie das Dordrechter Messer, Abb. 124e).

Das letzte Beispiel dieser Messer aus der Übergangszeit von der ersten Etablierung der Zungenkonstruktionen bis zur endgültigen Durchsetzung im späten 15. und 16. Jahrhundert bildet ein Exemplar aus Oslo, stratigraphisch datiert in das Jahrhundert zwischen 1325 und 1425 (Abb. 124c).

\footnotetext{
100 Vgl. Katalogteil BRD, Kat. Nr. 37

101 Vgl. in diesem Kapitel den Abschnitt "Die Klingenverzierungen"

102 Vgl. Katalogteil Schweden, Kat. Nr. 125 und 126

103 Vgl. Katalogteil Niederlande, Kat. Nr. 95 ff.
} 


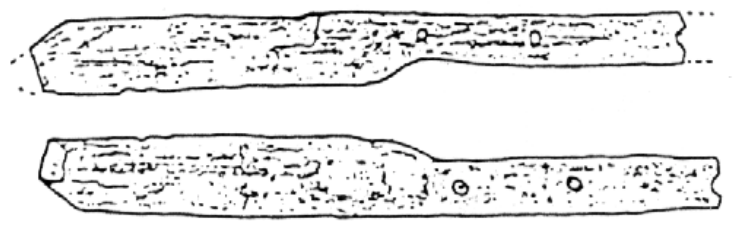

a

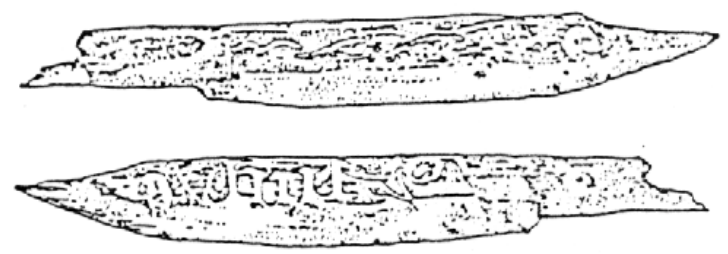

b

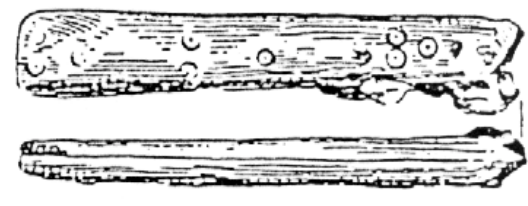

$\underline{1}$
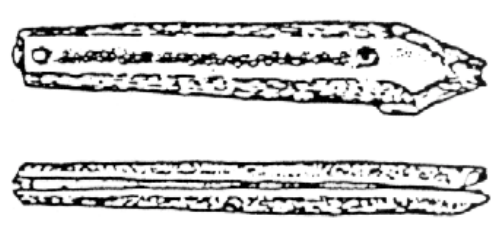

g
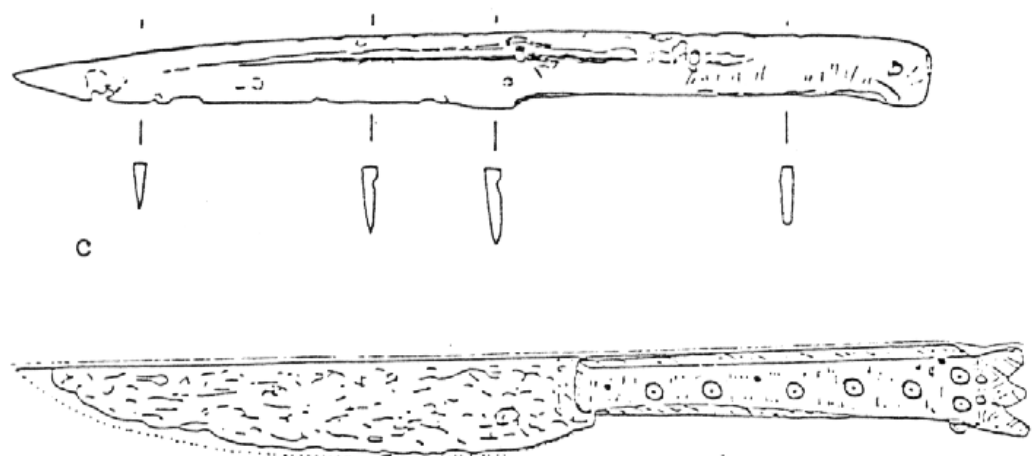

d

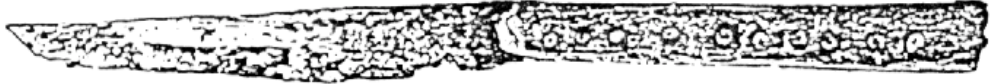

e

Abb. 124

Frühe Griffzungenmesser III

a: Duisburg, 15. Jh. (?); b: Lübeck, 14.-15. Jh. (?); c: Oslo, 2.V.14. - 1.V.15. Jh.; d: Ahaus, 14. - 15. Jh.; e: Dordrecht, um 1400; f: Posen, 14. - 15. Jh.; g: Lübeck, A.16. Jh. (?) 


\subsubsection{Die Entwicklung der Griffkonstruktionen vom 14. bis 17. Jahrhundert am Beispiel der Niederlande}

In den Niederlanden wurden für den Zeitraum vom 14. bis 17. Jahrhundert die Voraussetzungen für eine detaillierte Betrachtung der technischen Entwicklung bei den Messergriffen angetroffen.

Bisher sind die Konstruktionsmerkmale der Griffe nur grob erfaßt worden, für die meisten Messer ist eine genauere Untersuchung der verschiedenen Techniken aufgrund schlechter Erhaltungszustände oder fehlender Datierungen nur bedingt möglich.

Eine zusätzliche Heftverstärkung zum Beispiel kann aber auf sehr unterschiedliche Art und Weise angebracht werden, so daß sich für die Frage nach der zeitlichen Verbreitung der Formen A2 oder B4 durchaus Differenzierungen ergeben. Die Verstärkung kann aufgeschoben, ausgeschmiedet oder vernietet sein, sie kann im Heftbereich als Stoßkante vor oder als Manschette um den aus organischem Material bestehenden Griff gelegt worden sein.

Hervorragende Erhaltungsbedingungen und eine ungestörte, dichte Stratigraphie ergaben bei den Ausgrabungen in der Amsterdamer Altstadt eine Fülle gut erhaltener, zum Teil auf 25 Jahre und noch exakter zu datierender Funde, die das Gerüst für die Darstellung der Entwicklung der niederländischen Messer lieferte.

Aus dem Bestand der im Katalog erfaßten niederländischen Messer wurden 75 Exemplare ausgewählt, die zeitlich auf 25 bis 100 Jahre genau datierbar sind und deren Konstruktionstechniken erkennbar waren. Neben den zum Teil bereits publizierten Amsterdamer Funden sind sie vom Museum Boymans-van Beuningen in Rotterdam zur Verfügung gestellt worden ${ }^{104}$, diese stammen aus verschiedenen niederländischen Städten, darunter Dordrecht, Vlissingen, Rotterdam, Delft, Den Haag und Zwolle (eine Auswahl bieten die Abbildungen 125 und 126).

104 Baart u.a. 1970 

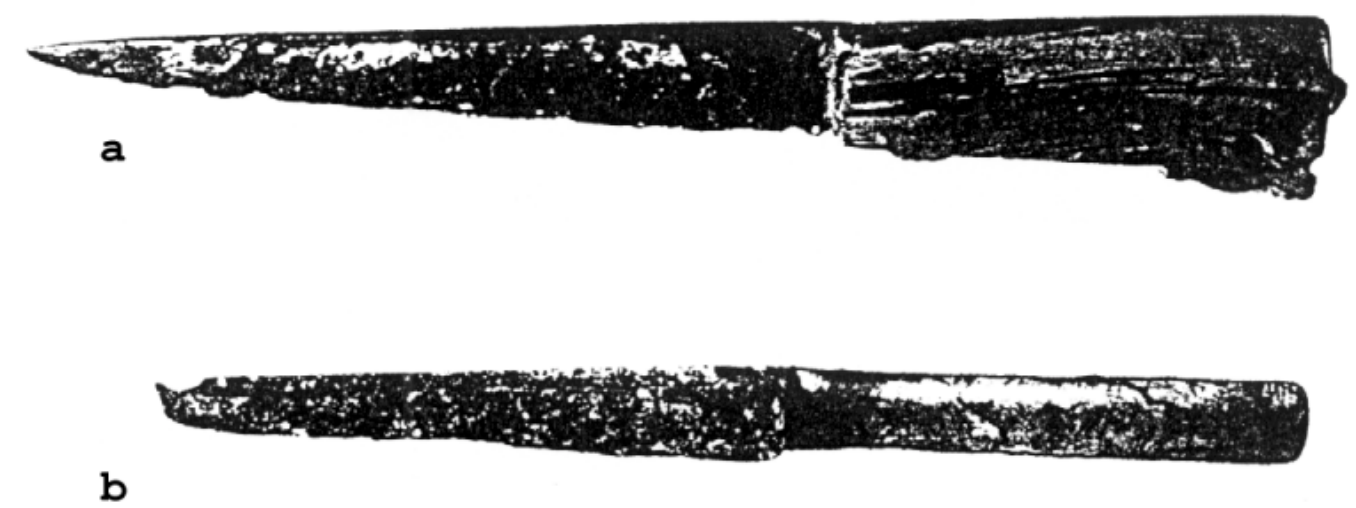

b

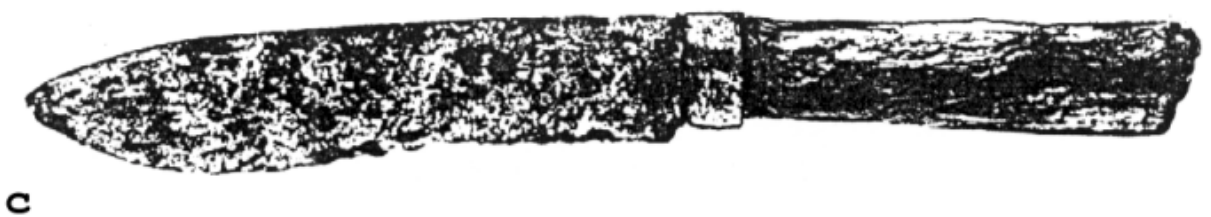

C

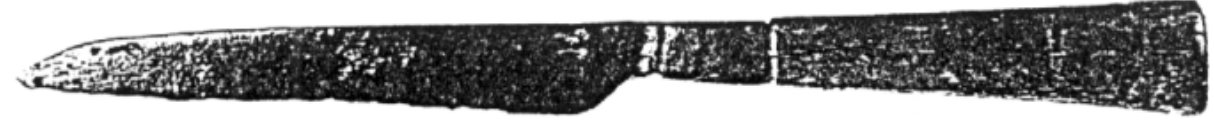

d
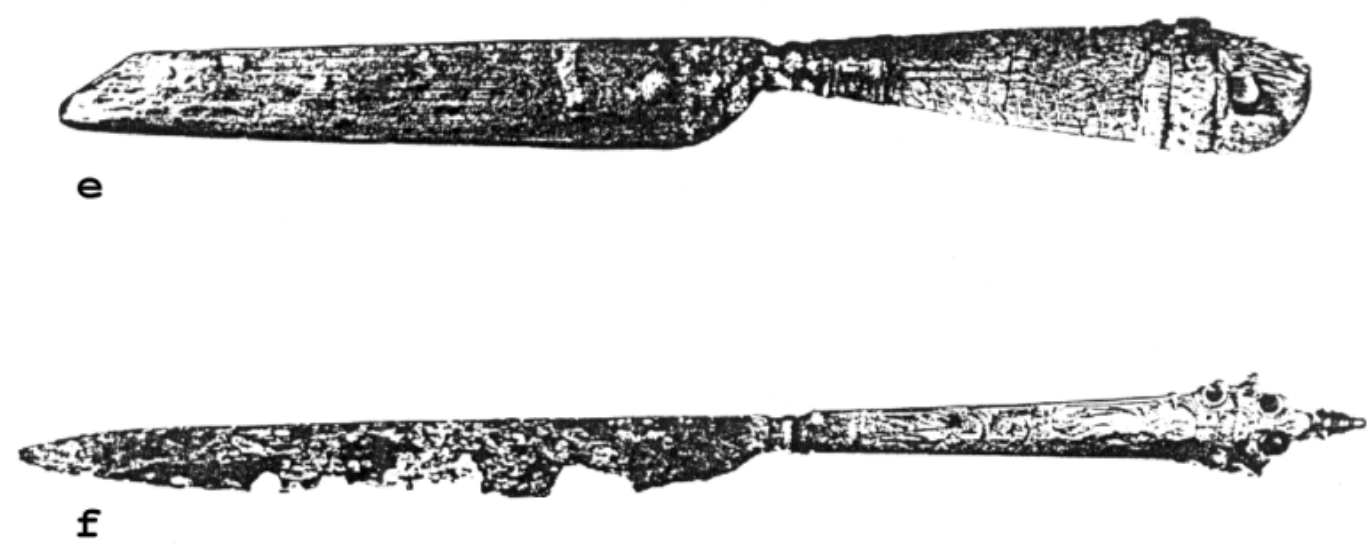

Abb. $125 \quad$ Niederländische Griffangelmesser
a: Dordrecht, 14. Jh.;
b: Amsterdam, 1.H. 14. Jh.;
c: Amsterdam, 2.-3. V. 14. Jh.,
d: Vlissingen 16. Jh.;
e: $\quad$ Noordeinde, 1596;
f: $\quad$ Rotterdam, um 1580 


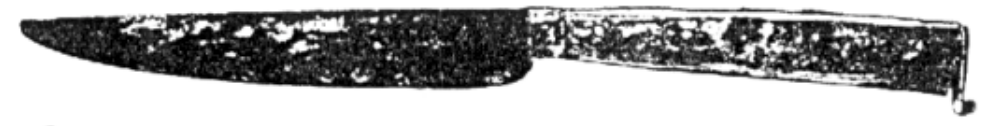

a

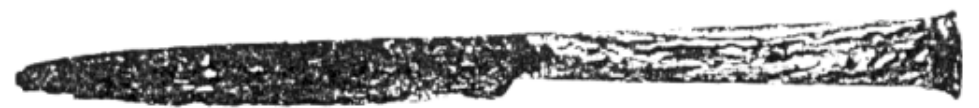

b
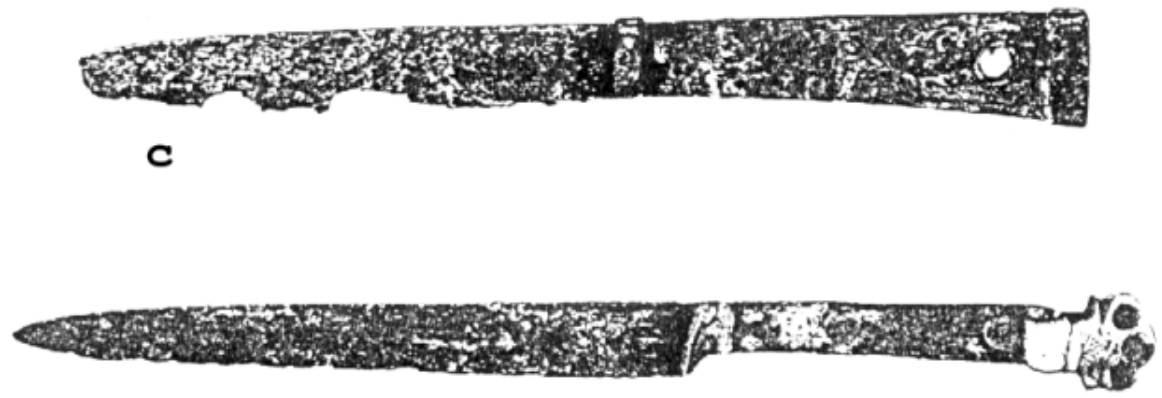

d

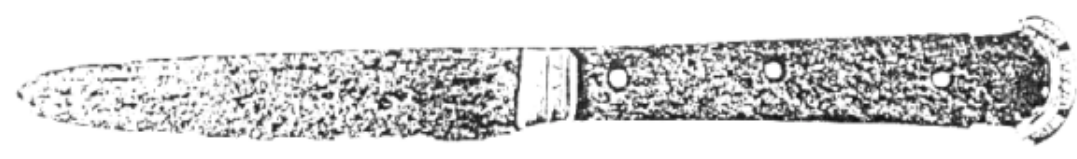

e

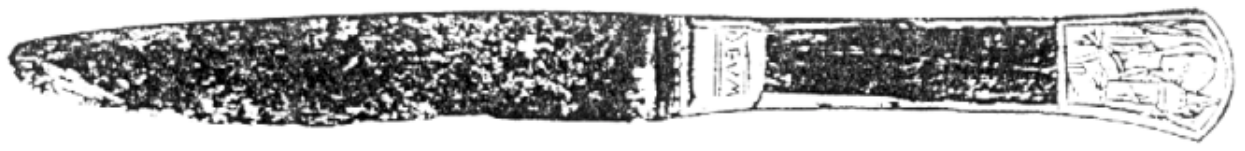

f

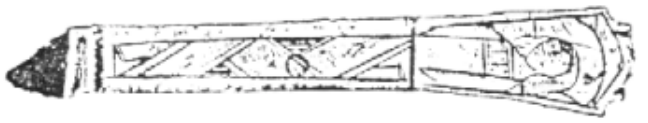

g

Abb. $126 \quad$ Niederländische Griffzungenmesser

a: Amserdam, 1.H. 14.Jh.; $\quad$ b: Amsterdam, 2.H. 15.Jh.;

c: Delft, 2.H. 15.Jh.; $\quad$ d: Delft, 1.H. 16. Jh..;

e: Den Haag, 1.V. 16. Jh.; f: Zwolle, 1.v. 16.Jh,;

g: Amsterdam, 2.H. 15.-1.V. 16. Jh. 
Aus diesem Bestand ergaben sich neun unterschiedliche Griffangelkonstruktionen und 14 verschiedene Griffzungenkonstruktionen sowie die Sonderform B8 (Abb. 127 und 128):

Neben unbefestigten Griffangelmessern (a) treten Messer auf, die am Heft einen Ring (b), eine schmale, angeschmiedete Stoßkante (c), eine breite, ausgeschmiedete Heftzone mit Stoßkante (d) sowie ein von Klinge und Griff abgesetztes, meist profiliert oder ballusterförmig ausgestaltetes Heft (e) aufweisen. Weiterhin wurden die Vollgriffe entweder mit Edelmetallblechen ummantelt (f) oder aus Bunt- bzw. Edelmetallen gegossen (g). Griffendbefestigungen treten als aufgesetzte Kappen auf (h) oder seltener als vollmetallene Endstücke, die hinter dem Vollgriff auf die Angel montiert wurden (i); kleine, auf das Angelende gesetzte Endplättchen wurden ebenfalls beobachtet. Griffzungenmesser wurden am Griff zum Teil technisch gleichartig befestigt ( $\mathrm{j}$ bis $\mathrm{n}, \mathrm{r}, \mathrm{s}$ und $\mathrm{u}$ ). Als spezifisch für Zungenkonstruktionen verwandte Zusatzbefestigungen sind Buntmetall- und Eisenplatten zu nennen, die am Heft (o und p) oder Griffende (v und w) beidseitig vernietet wurden. In einigen Fällen waren die gesamten Griffschalen in Buntmetall gegossen und vernietet worden, sie erinnern nur noch durch eingravierte, zonal gruppierte Verzierungen an die einfachere Version (q).

Ein weiteres, nur bei Zungenmessern auftretendes Element ist die Ausgestaltung der Griffenden durch gegossene, am Zungenende montierte Applikationen, die gleichzeitig als Griffendsicherung fungieren ( $\mathrm{t}$ ).

Seit dem 15. und 16. Jahrhundert sind diese Konstruktionselemente fast durchweg auch Träger von Verzierungen, teils bereits in Formen wie Dreipässen gegossen (Abb. 126d), teils mit Gravuren versehen (Abb. 126e bis 126g). Auch Griffendkappen oder ausgeschmiedete Heftverstärkungen sind zumindest mit Gurtgruppen verziert worden (Abb. 126b und 126e).

Vor dem 15. Jahrhundert sind verzierte Konstruktionselemente die Ausnahme, Verzierungen treten fast durchweg auf den Vollgriffen oder Griffschalen auf (Abb. 124d und 125a). Die Befestigungstechnik der Metallzonen am Heft und am Griffende ist oft nur durch Überprüfung am Original festzustellen, da zum Beispiel die Buntmetallplatten vor der Gravur poliert worden sind, so daß die Grenzen zwischen Plattenoberfläche und Nietkopf verschwimmen. In der Regel wurden eiserne Heftund Griffendkörper aufgeschmiedet oder angeschweißt, gegossene Körper aus Bunt- oder Edelmetallen vernietet (Abb. 126c und 126f).

Die Zwischenlagen in der Griffmitte bestanden aus organischen Materialien, seltener aus anorganischen Materialien wie Bergkristall ${ }^{105}$.

105 Vgl. Katalogteil Niederlande, Kat. Nr. 11 


\section{GRIFFKONSTRUKTIONEN IN DEN NIEDERLANDEN}

a Angelmesser ohne Zusatzmontierung (Vollgriff aus organischem Material)

b Angelmesser mit Ring am Heft (seltener konische Hülse)

c Angelmesser mit ausgeschmiedeter, schmaler Stoßkante am Heft

d Angelmesser mit ausgeschmiedeter, breiter Stoßkante (geschmiedetes Heft)

e Angelmesser mit ausgeschmiedetem Ballusterheft

f Angelmesser mit ummanteltem Vollgriff (metallisches Mantelblech)

g Angelmesser mit metallenem Vollgriff (meist gegossen)

$\mathrm{h}$ Angelmesser mit metallener Griffkappe

i Angelmesser mit metallenem Griffendstück (ausgeschmiedet, seltener gegossen)

j Zungenmesser ohne Zusatzmontierung (Griffschalen aus organischem Material)

$\mathrm{k} \quad$ Zungenmesser mit Ring am Heft

1 Zungenmesser mit ausgeschmiedeter, schmaler Stoßkante

m Zungenmesser mit ausgeschmiedeter, breiter Stoßkante (geschmiedetes Heft)

$\mathrm{n} \quad$ Zungenmesser mit ausgeschmiedetem Ballusterheft

o Zungenmesser mit vernieteten Buntmetallplatten am Heft

$\mathrm{p} \quad$ Zungenmesser mit vernieteten Eisenplatten am Heft

q Zungenmesser mit vernieteten Buntmetallschalen (gegossene Griffschalen)

$\mathrm{r} \quad$ Zungenmesser mit metallener Griffendplatte (vor das Griffende montiert)

s Zungenmesser mit metallener Griffendkappe

$\mathrm{t}$ Zungenmesser mit metallenen Griffendapplikationen (verschiedenartig montierte, nicht vernietete gegossene Appliken)

$\mathrm{u} \quad$ Zungenmesser mit metallenem Griffendstück (ausgeschmiedet, seltener gegossen)

$\mathrm{v} \quad$ Zungenmesser mit vernieteten Buntmetallplatten am Griffende

w Zungenmesser mit vernieteten Eisenplatten am Griffende

B8 siehe Modell der Griffkonstruktionen

Abb. 127 
Griffkonstruktionen in den Niederlanden II

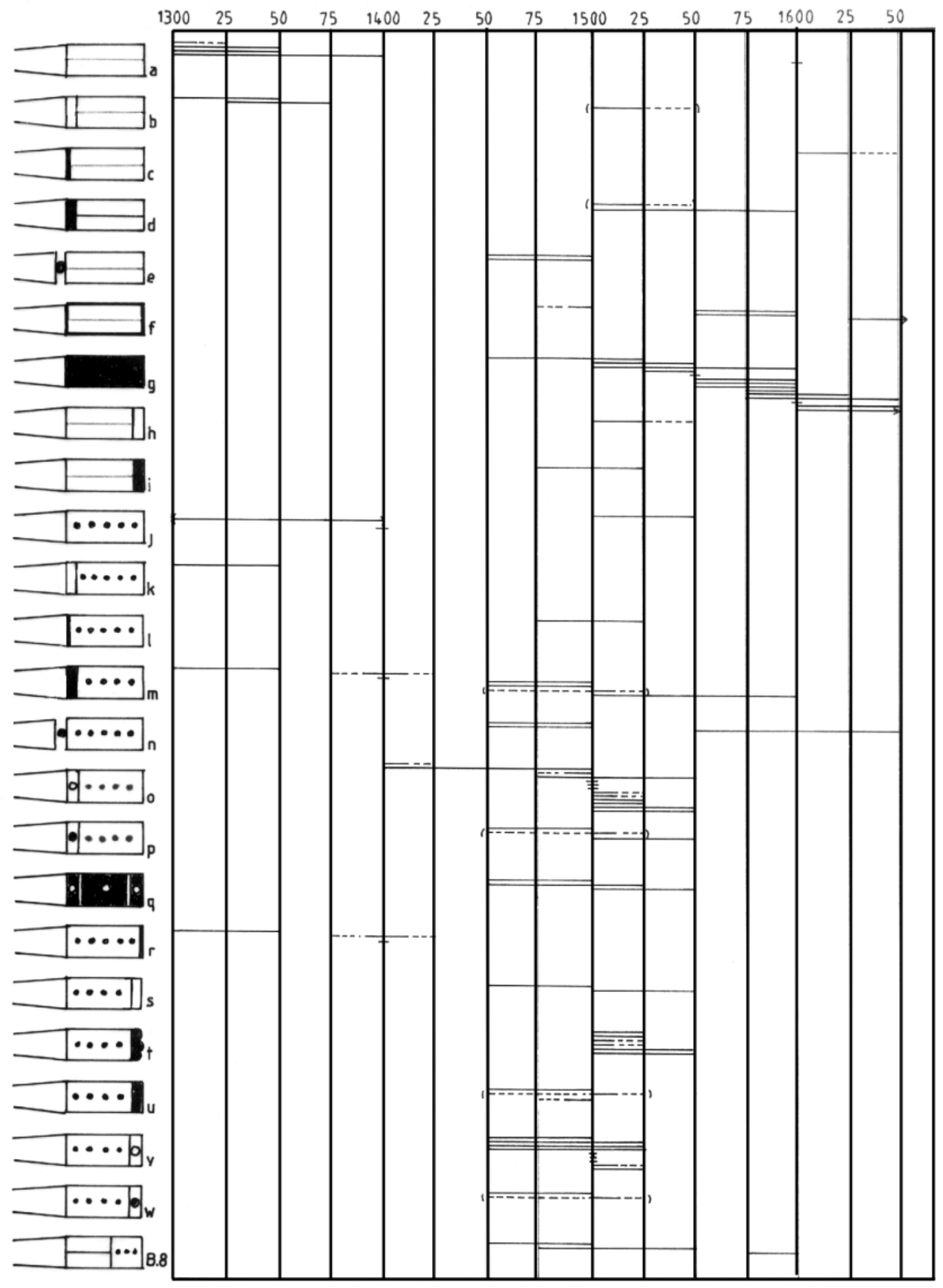




\section{Chronologie und Typologie}

Die Auswertung der zeitlichen Verteilung der neun Griffangel- und vierzehn Griffzungenkonstruktionen in den Niederlanden unterliegt der bereits beschriebenen Quellenkritik. So stehen von den ausgewählten 75 Messern 13 Messer vor 145062 Messern nach 1450 gegenüber.

Dennoch zeigt die Gegenüberstellung eine deutlich sichtbare Entwicklung (Abb. 128). In der Mitte des 15. Jahrhunderts liegt ein deutlicher Schnitt, der die Messer in zwei Gruppen trennt:

Vor 1450 sind zwei Griffangel- und fünf Griffzungenkonstruktionen nachzuweisen (a, b, j, k, m und r). Nach 1450 erhöht sich die Zahl auf mindestens acht Griffangel- und zwölf Griffzungenkonstruktionen sowie die Form B8, eine Angelkonstruktion, deren Griffende zu einer Zunge mit vernieteten Schalen gearbeitet worden ist ( $a,(b$ ?), $c$ bis j, 1 bis q, s bis w und B8).

Im 14. Jahrhundert treten neben unbefestigten Griffangel- und -zungenmessern die beiden Konstruktionen Heftringe (Manschetten) und bei Zungenmessern zusätzlich ausgeschmiedete Heftverstärkungen und Griffendplatten auf. Befestigungen wie Kupfer- oder Messingplättchen an Griffangelmessern, die vor allem in der nördlichen Hälfte Deutschlands im 13. und 14. Jahrhundert auftreten, kommen in den bearbeiteten Beständen aus den Niederlanden nicht vor.

Um 1400 treten zum ersten Mal vernietete Buntmetallplatten am Heft auf, sie bestehen aus dünnen Blechen, die oft den Klingenansatz mit umfassen. Seit der Mitte des 15. Jahrhunderts setzen gleichzeitig fast alle Konstruktionselemente ein, die in dem bearbeiteten Bestand aufgetreten sind. Heftringe und Griffendplatten, die vor das Ende von Griffzungenmessern gesetzt wurden ( $b$, $k$ und $r$ ), sind bis auf ein unsicheres Exemplar danach nicht mehr beobachtet worden.

Ebenfalls nachgewiesen wurde die bereits aus der Gesamtauswertung bekannte Abnahme von Zungenkonstruktionen zugunsten der Angelkonstruktionen im 17. Jahrhundert. In den Niederlanden zeigt sich dieser Wandel schnittartig um 1550. Die Mehrzahl der bearbeiteten niederländischen Angelmesser zwischen 1550 und 1650 weist gegossene vollmetallene oder von Metallblechen ummantelte Vollgriffe auf ( $\mathrm{f}$ und $\mathrm{g}$ ), die immer verziert sind.

Am Beispiel der Konstruktionstypen niederländischer Messergriffe zeigt sich, welche Erkenntnisse bei entsprechend sorgfältig durchgeführter und nicht nur punktuell, sondern breit angelegter Bodendenkmalpflege - in diesem Fall in der Stadtkernarchäologie - abseits der übergreifenden historisch-topographischen Fragestellungen zur Erforschung der Alltagsgeschichte zu gewinnen sind.

Es konnte nicht nur eine archäologisch abgesicherte Typologie erarbeitet werden, die innerhalb eines zeitlichen Rahmens von 350 Jahren 24 verschiedene Konstruktionsformen in ihrer chronologischen Abfolge umfaßt. Auch Hinweise zur Wirtschafts- und Technikgeschichte wie die explosionsartig einsetzende Massenverwendung von Messing um 1450 und die technischen Novationen bei den Angelkonstruktionen nur 100 Jahre später ergeben sich aus dieser Analyse und zeigen die Bedeutung, die eine sachgerechte Erfassung eines einzigen Alltagsgegenstandes erlangen kann. 


\subsubsection{Die Griffverzierungen}

\subsubsection{Beispiele von Griffverzierungen der römischen Kaiserzeit und der Völkerwanderungszeit}

Die im Bearbeitungszeitraum auftretenden grundsätzlichen Verzierungsarten an Messergriffen finden wie viele Konstruktionselemente ihre Parallelen im provinzialrömischen Bereich (Abb. 129), zum Teil darüber hinaus in der Völkerwanderungszeit.

Das vom 11. bis zum 16. Jahrhundert nachgewiesene Zirkelschlagornament (Punktoder Kreisauge) war in der römischen Kaiserzeit innerhalb und außerhalb des von den Römern besetzten Gebietes bekannt (Heddernheim, Abb. 117b; Rißtissen, Abb. 129a; Vimose, Abb. 129h).

Auch zonale Griffeinteilungen und flächendeckendes Dekor aus Kreuzschraffuren, Gurtpaaren und Netzwerk sind von kaiserzeitlichen Messergriffen bekannt (Abb. $117 \mathrm{a}, 117 \mathrm{~b}, 129 \mathrm{f})$.

Das ganze Ausmaß technischen Könnens und künstlerischer Gestaltung an römischen Messergriffen zeigt sich jedoch an anthropomorph und zoomorph ausgeschnitzten Griffen aus organischen Materialien, an gegossenen Vollgriffen und an der Verwendung von Edelmetallblechen (Abb. 129b bis 129e, 129i bis 1291).

Ein aus dem Rhein bei Diemeser Ort in der Nähe von Mainz stammendes römisches Messer mit Metallgriff ahmt mit seiner zonal angeordneten Griffgestaltung einen gedrechselten Holzgriff nach (Abb. 129f).

Die einfachen Verzierungselemente wie Kreuzschraffur oder Punktauge sind in der Völkerwanderungszeit genauso nachweisbar wie fast im gesamten Bearbeitungszeitraum dieser Studie.

Eine direkte Tradition ist kaum nachweisbar, sie belegen möglicherweise eher die Wahrscheinlichkeit, daß sich Techniken wie Verzierungselemente aus dem jeweiligen technischen Standard, Formempfinden und überregionalen Kontakten zu Räumen, deren Traditionen weiter zurückgehen können, auch nach langen Zeiten kultureller Umbrüche immer wieder ergeben können.

Im Fall der Verwendung von Mantelblechen aus Edelmetallen zeigt sich, daß der Beginn und das Ende einer technisch-künstlerischen Erscheinung in günstigen Fällen als Beispiel der Kontinuität eines kulturellen Elementes dienen kann, deren Ende aber durchaus nicht ein Wiederbeleben desselben ausschließen muß 


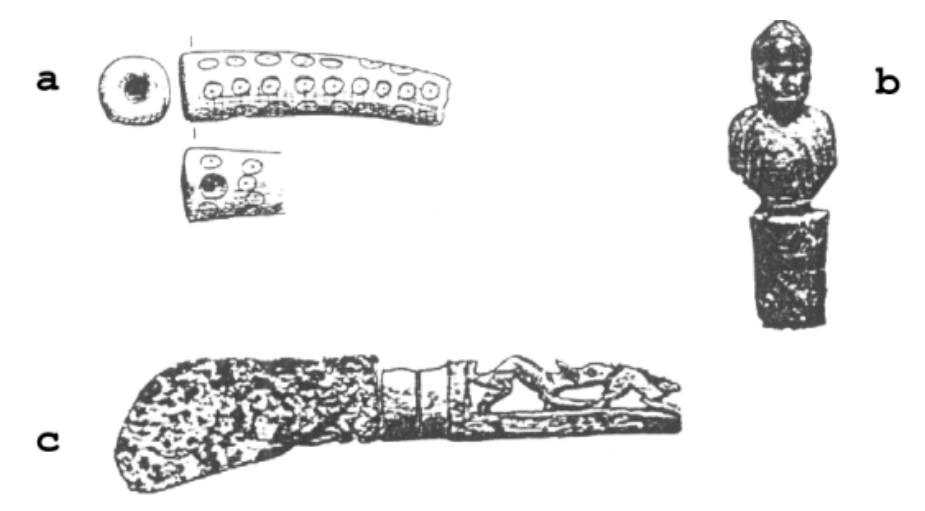

d 2

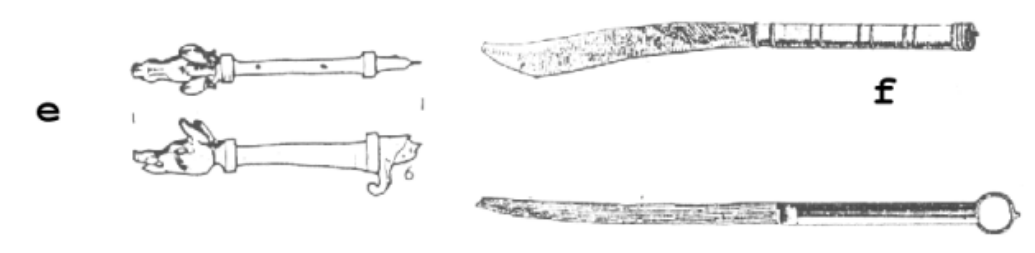

g

h

i
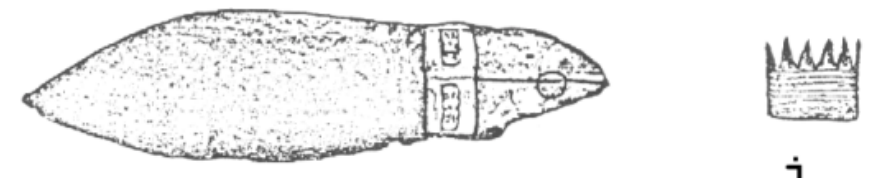

j

$\mathbf{k}$
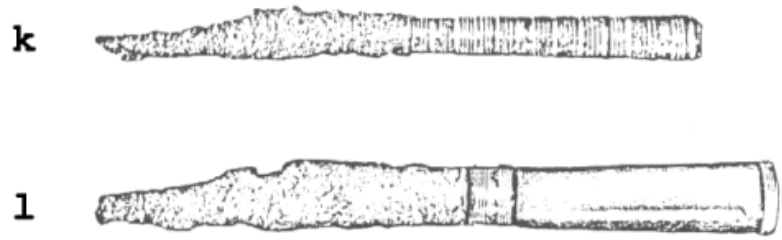

Abb. 129
Kaiserzeitliche Griffverzierung ${ }^{106}$.
a: Kastell Rißtissen; b Mainz; c: Regensburg; d: Rheinfund bei Diemeser Ort;
e: Legionslager Augsburg; f: Rheinfund wie d; g: Mainz; h: Vimose;
$i$ : Krefeld-Gellep; ;j: Furfooz(Namur); k-l: Bonn-Brühl

106

a: Ulbert 1959, Abb. Tafel 66.19

c: Lindenschmitt 1889, Abb. Tafel XXII.25

b: Lindenschmitt 1889, Abb. Tafel XXII.30

d: Lindenschmitt 1870, Abb. aus Bd. II, Heft 4, Tafel 4.3

e: Hübener 1973, Abb. Tafel 12.6

f: Lindenschmitt 1870, Tafel 4.13

g: Lindenschmitt 1870, Tafel 4.4

h: Engelhardt 1869, Abb. Tafel 17.20

i-l: Werner 1968, Abb. Tafel 4.1a,3; Tafel 3.2,1; vgl. hier auch die Messer des 5. bis 7. Jahrhunderts, in der spätrömischen Tradition stehende Einzelmesser und Messerbestecke aus alamannischen, fränkischen und thüringischen Gräbern führender Bevölkerungsgruppen. 
Joachim Werner hat 1968 Messer mit von Gold- und Silberblechhülsen ummantelten Vollgriffen veröffentlicht, deren Verbreitungsgebiet zwischen Seine und Elbe liegt. Die meisten dieser Griffe mit Hülsen, die entweder den gesamten Griff oder das Heft bzw. das Griffende umfassen, stammen aus dem 5. und 6. Jahrhundert. Für die "Goldgriffmesser des merowingischen Westens" stellt Werner als Vorläufer spätrömische Messer des späten 3. und 4. Jahrhunderts aus linksrheinischen Fundorten vor ${ }^{107}$.

Die Verwendung von Messern mit gold- bzw. silberblechummantelten Griffen, die teilweise als reine Tafelbestecke gedient haben, läßt sich im Rheinland kontinuierlich vom Ende des 3. bis in das 6. Jahrhundert nachweisen ${ }^{108}$.

Nördlich der Alpen vergehen mindestens 200 Jahre bis zum nächsten Nachweis eines Messers mit Silberblechummantelung:

1940 veröffentlichte Holger Arbman ein Messer, dessen Griff vollständig von vernieteten Silberblechen umgeben ist. Es wurde in einem Frauengrab nordöstlich der Burg in Birka gefunden ${ }^{109}$.

Ein mittelalterlicher Beleg ist im bearbeiteten Bestand nicht vorhanden, dem Autor ist bisher auch darüber hinaus kein Beispiel bekannt geworden. Erst seit der frühen Neuzeit ist diese Form der Griffverzierung wieder nachweisbar.

Im Museum Boymans-van Beuningen in Rotterdam befindet sich ein Griffangelmesser, dessen Vollgriff von einem in Preßtechnik verzierten, siebeneckigen Kupfer- und Messingblechbeschlag eingefaßt ist ${ }^{110}$. Es wurde in Dordrecht gefunden und wird in das ausgehende 15. Jahrhundert datiert. Ein etwas jüngeres Messer, ebenfalls ohne stratigraphischen Nachweis, wurde in Oslo gefunden, der hölzerne Vollgriff ist von einer Hülse aus Bronzeblech überzogen, die in zwei Zonen unterteilt und mit geometrischen und floralen Mustern verziert worden ist ${ }^{111}$.

Im Barock gehört die Art der Griffgestaltung mittels Edelmetallhülsen, die zumeist mit Kitt gefüllt auf die Angel gesetzt werden, zu einer der gängigen Techniken der Griffproduktion. Als Beispiel wird ein frühes Exemplar aus Amsterdam, stratigraphisch datiert in das zweite bis dritte Viertel des 17. Jahrhunderts, im Katalog vorgestellt ${ }^{112}$.

Eine Verbindung zwischen den merowingerzeitlichen "Goldgriffmessern" und dem "Silbergriffmesser" aus Birka ist nicht nachzuweisen.

Das Messer aus Birka steht isoliert da, lediglich eingebettet in den Kontext eines der wichtigsten wikingerzeitlichen Warenumschlagplätze Nordeuropas.

\footnotetext{
107 Werner 1968, S. 647-660, v.a. S. 655

Pirling 1986, S. 147, Abb. 130

108 Werner 1968, S. 660

109 Vgl. Katalogteil Schweden, Kat. Nr. 2

110 Vgl. Katalogteil Niederlande, Kat. Nr. 39

111 Vgl. Katalogteil Norwegen, Kat. Nr. 30

112 Vgl. Katalogteil Niederlande, Kat. Nr. 92
} 
Für die Beispiele des 17. Jahrhunderts ist sie völlig auszuschließen, in diesem Jahrhundert erlebte die Griffangel mit all ihren Techniken eine regelrechte Wiedergeburt, die sich bis in das 19. Jahrhundert fortsetzte.

Für die Belege des 8./9. Jahrhunderts und des 15. Jahrhunderts fehlen bisher die Möglichkeiten einer Zuordnung. Im 15. Jahrhundert ist allgemein eine rasche $\mathrm{Zu}-$ nahme der Verwendung von Buntmetallen zu beobachten, möglicherweise gelingt es bei der weiteren Vorlage von archäologischem Fundgut, die Lücke zwischen dem 15. und beginnenden 16. und dem 17. Jahrhundert zu schließen und die Beispiele aus Dordrecht und Oslo als frühe Entwicklungsstufen einer seit dem 17. Jahrhundert gängigen Griffform zu kennzeichnen.

Das Beispiel der Mantelbleche am Vollgriff eines Griffangelmessers zeigt jedoch, daß über einen Zeitraum von sechzehnhundert Jahren an einem Gegenstand sowohl Tradition und Kontinuität als auch durch technische Entwicklung bedingte Neuentstehung nachzuweisen sind.

\subsubsection{Griffverzierungen an Griffen aus organischen Materialien vom 9. bis 17. Jahrhundert}

Verzierungen an Griffen aus organischen Materialien, meist Horn, Knochen, Geweih oder Holz, betreffen ausschließlich die Vollgriffe oder Schalen als Träger; Zusatzelemente aus organischen Materialien zu Zierzwecken sind extrem selten und treten in der Regel nur als Einlagen oder in Kombination mit Metallapplikationen auf. Grundsätzlich lassen sich wie bei fast allen Trägern von Ornamenten und Mustern die einzelnen Zierelemente auf Messergriffen aus organischen Materialien in zweidimensionale und dreidimensionale Verzierungen gliedern.

\section{Dreidimensionale Verzierungen}

Dreidimensionale Verzierungen betreffen zumeist Vollgriffe, sie umfassen entweder den gesamten Griff oder Griffteile, vor allem das Griffende.

Neben den üblichen Profilierungen und - bei Bein- und Holzgriffen - gedrechselten Ornamenten treten halb- und vollplastische Gestaltungen auf. Halbplastischer Dekor ist nur sehr selten nachgewiesen worden, in der Regel sind dreidimensional verzierte Griffe vollplastisch ausgeschnitzt worden.

Die inhaltliche Palette reicht von Linear- und Rollprofilierungen und Durchbrechungen der Griffkante über die flächendeckende Ornamentierung der Schauseiten oder der ganzen Griffe mit gedrechselten oder geschnittenen, längs, schräg und hoch verlaufenden Profilrillen, Netzwerk und schuppenartigen Mustern sowie zonal angeordneten Gurtgruppen bis zu plastisch ausgeschnitzten Kronen, Profilwülsten, Ballustern, Hohlkehlen, Spitzkehren und floralen Abschlüssen, Schwalbenschwanz und Flechtknoten, zumeist an den Griffenden. 
Die aufwendigste Art der halb- bzw. vollplastisch verzierten Griffe ist die zoomorphe oder anthropomorphe Gestaltung von Griffen oder Griffenden. Sie läßt sich vom 12. bis 17. Jahrhundert im Bearbeitungsraum nachweisen, von schlichten, stilisiert angedeuteten Tierkopfenden über einfache Büsten und maskenhafte Köpfe bis zu detailliert ausgearbeiteten Tier- und Menschendarstellungen als Vollgriffe oder Griffenden. Unter letzteren verdient eine Gruppe aus dem 13. bis 14. Jahrhundert besondere Beachtung; sie wird im Anschluß an diesen Abschnitt gesondert vorgestellt und umfaßt Darstellungen mittelalterlicher Idealdarstellungen wie Falkner, Ritter und höfische Damen.

\section{Zweidimensionale Verzierungen}

Die zweidimensionalen Griffverzierungen treten bei Vollgriffen und Griffschalen gleichermaßen auf. Sie lassen sich in lineare, geometrische und übertragene Ornamente unterteilen $^{113}$.

Einzelne Ornamente treten innerhalb der Gruppen der verzierten Messergriffe seltener auf, meistens erscheinen sie zu Mustern gereiht und flächendeckend oder zonal angeordnet.

Der lineare Dekor umfaßt Gurte, Gurtpaare und Kreuz- bzw. Schrägschraffur sowie horizontal, schräg oder vertikal verlaufenden, auch s-förmig geschwungenen Kammstrich.

Geometrische Muster bestehen aus Dreiecken, Kreuzen, Schrägkreuzen, Zick-ZackBändern (aneinandergereihte Dreiecke) sowie Zirkelschlag (Punkt- oder Kreisauge). Vor allem das letztere Element tritt in vielschichtiger Funktion auf. Punktaugen mit einfachem oder doppeltem Kreis (einfache Rosette) wurden als Einzelornamente, als zusammengesetzte Ornamente (Kreuze, Würfelaugengruppe, Rosette) sowie als zu Bändern zusammengesetzte Muster beobachtet. Außerdem treten sie auf als Füllwerk in Winkeln von Kreuzschraffuren, Drei- oder Vierecken, in den Tälern von Wellenbändern und in Flechtwerkbögen, sie füllen Freiräume in zonal gegliederten Mustern und sind in selteneren Fällen unregelmäßig flächendeckend angeordnet worden. Auf organischem Material sind sie bei Messergriffen vom 11. bis zum 16. Jahrhundert aufgetreten, ihre Verwendung als Zierelement läßt sich aber zum Beispiel anhand von Knochenkämmen sehr viel weiter zurückverfolgen ${ }^{114}$.

\footnotetext{
113 Übertragene Ornamente: Aus der Natur, der Architektur etc. übernommene und naturalistisch oder stilisiert wiedergegebene Ornamentformen

114 Das einfache Zirkelschlagmotiv (Kreisauge) ist wie der Kammstrich zwar materialimmanent entstanden, aber nicht an ein Material gebunden (vgl. Katalogteil Norwegen, Kat. Nr. 2). Es ist bereits in vorchristlicher Zeit nachweisbar und seit der römischen Kaiserzeit im Untersuchungsgebiet vorhanden. $\mathrm{Da}$ es auf Messergriffen außer auf dem silberblechbeschlagenen Griff aus Birka erst seit dem 11. Jahrhundert auftritt, ist als Zufall zu bewerten, seine Hauptverbreitungszeit auf Messergriffen beginnt aber sicherlich erst nach dem 11. Jahrhundert.

Vgl. u.a.: Die Kelten in Mitteleuropa 1980, S. 83 und S. 169, S. 209, S. 266 und S. 267;

Tempel 1972, S. 57 ff.; $\quad$ Tempel 1969, S. 34 ff.

Nerman 1958, u.a. Abb. S. 123 und 125;

Hilczerowna 1961

Koltschin 1971, S. 16-18 und Abb. 3, S. 17
} 
Die Ornamentierung aus übertragenen Mustern beinhaltet neben Flechtbandmotiven, Schuppenmustern, Wellenbändern und Perlschnüren in zwei Fällen auch Elemente der skandinavischen Tierstile sowie architektonische Entlehnungen als Rahmenwerk einer Tänzergruppendarstellung; derart szenische Darstellungen sind jedoch extrem selten, sie sind ansonsten nur bei plastischen Verzierungsarten aufgetreten $^{115}$.

Eine sehr seltene Art der Griffverzierung besteht in der Verwendung von zusätzlich angebrachten, aus organischem Material bestehenden Elementen. So wurden Griffplättchen aus Knochen, profilierte Heftund Griffendzwingen sowie Einlagen aus Bernstein oder Harz in wenigen Fällen beobachtet ${ }^{116}$.

Zumeist treten die genannten Ornamente und Muster kombiniert auf, auch dreidimensional verzierte Griffe können zusätzlich flächig ornamentiert sein, vor allem, wenn nur das Griffende ausgeschnitzt worden ist.

\section{Katalog der Verzierungsarten und -elemente (Abb. 130)}

Im folgenden werden die Verzierungselemente mit einem oder mehreren Beispielen listenartig vorgestellt. Die Liste enthält neben der Abbildung des Ornaments, Musters oder Griffs den Nachweis des Fundlandes und die jeweilige Katalognummer sowie graphisch den zeitlichen Rahmen des jeweiligen Fundstücks. In Klammern gesetzte Datierungen bezeichnen unsichere Datierungsgrundlagen, gestrichelte Linien markieren eine mögliche Erweiterung des chronologischen Rahmens. Die genauen Datierungshinweise sind dem Katalog zu entnehmen. Die Griffe sind nach folgenden Gesichtspunkten angeordnet worden:

\section{Zweidimensionale Verzierungen}

übertragene Muster; Punkt- oder Kreisaugenmotive; geometrische oder lineare Muster; Zusatzelemente aus organischen Materialien

\section{Dreidimensionale Verzierungen}

Einfache Gestaltung des Griffs, unter anderem gedrechselte Gurt- und Wulstgruppen; Gestaltung von Heft und Griffende zu Wülsten, Ballustern, Flechtknoten, Kronen und Ähnlichem; Einlagen; regionaltypische Griffverzierungen (Zeeuws Vlaanderen); flächendeckende halbplastische Muster; halbplastische und vollplastische Ausschnitzung $\mathrm{zu}$ anthropomorphen und zoomorphen Griffenden und Vollgriffen.

\footnotetext{
115 Vgl. Katalogteil Polen, Kat. Nr. 12, 13 und 128 zu den Messern aus Niestronno und Gniezno vgl.: J. Zak 1963 Zum Ringkettenmuster vgl. Schoknecht 1971, dort auch weiterführende Literatur zur insularen Entstehung des Ringkettenmusters, der Verbreitung im westslawischen Raum und Beispiele (S. 282 ff.)

116 Vgl. Katalogteil Niederlande, Kat. Nr. 35, 121, 125; Schweden, Kat. Nr. 118, 119; Polen, Kat. Nr. 315
} 


\begin{abstract}
Abb. 130
(15 Seiten)

Katalog der Griffverzierungen

an Griffen aus organischem Material

vom 9. bis zum 17. Jahrhundert
\end{abstract}

(Die Länderbezeichnungen in den folgenden Abbildungen beziehen sich auf die bis 1987 benutzte Literatur, die für die Erstellung des Kataloges ausgewertet wurde.

\begin{tabular}{|l|l|l|}
\hline So bezeichnen: & DDR & $\begin{array}{l}\text { Bundesrepublik Deutschland } \\
\text { (neue Bundesländer) }\end{array}$ \\
\hline & VRP & Polen \\
\hline & UdSSR & Staaten der ehemaligen Sowjetunion \\
\hline
\end{tabular}




\section{Verzierungen an Messergriffen}

Zwei- und dreidimensional an organischem Material

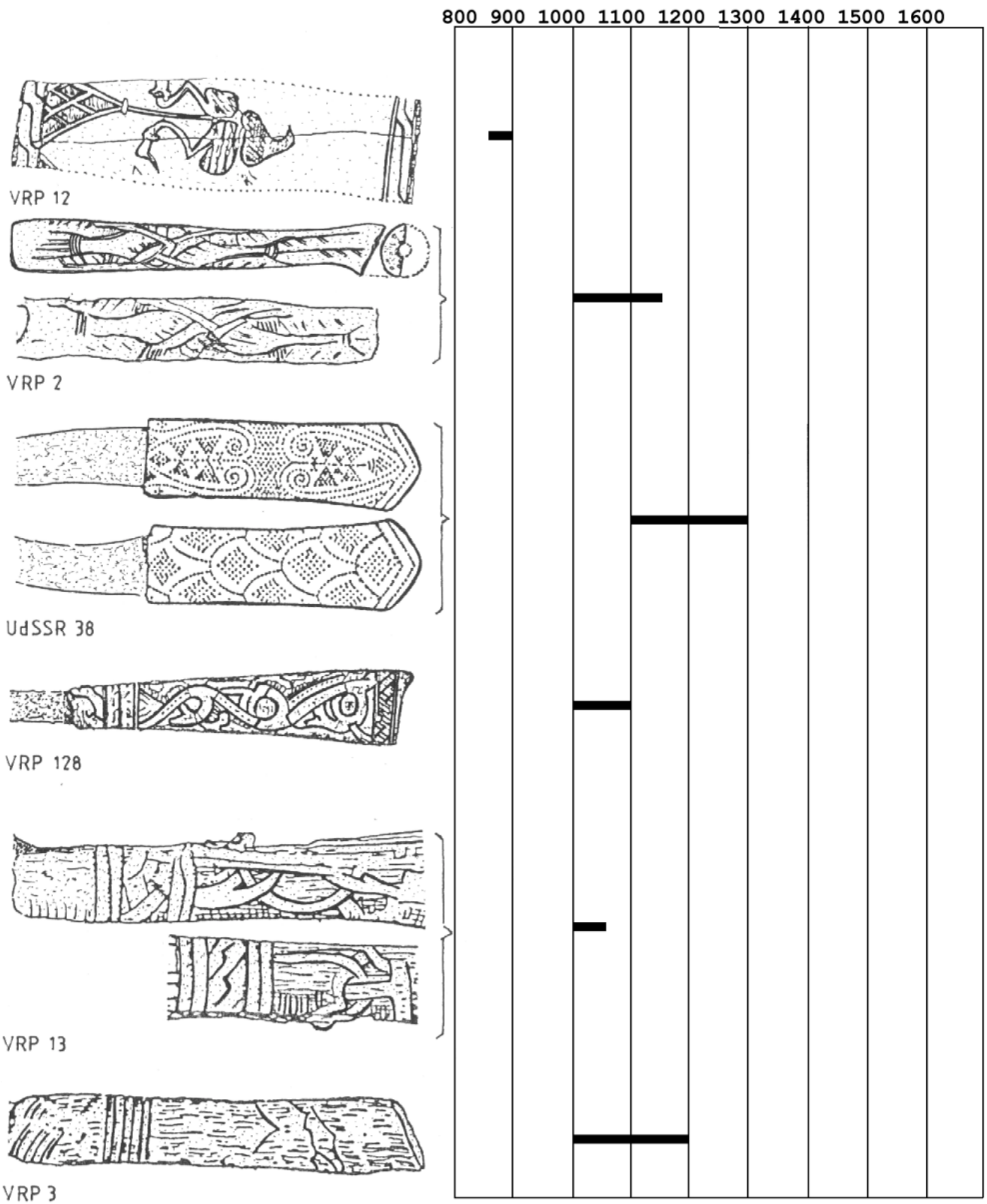




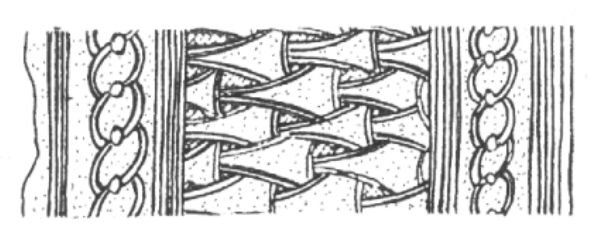

$\begin{array}{lllllllll}800900 & 1000 & 1100 & 1200 & 1300 & 1400 & 1500 & 1600\end{array}$

VRP 330

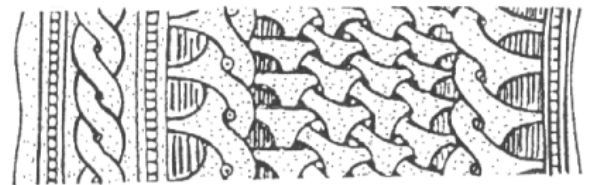

JDR 75

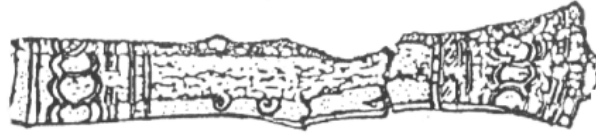

DDR 2

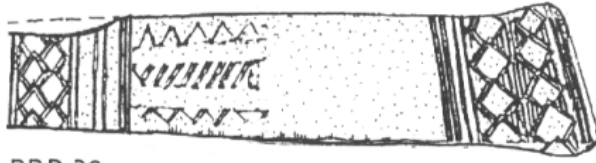

DDR 32

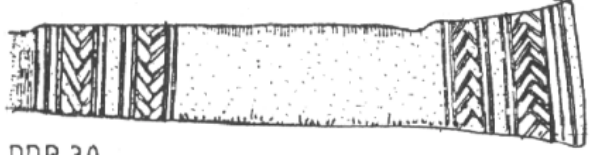

DDR 30

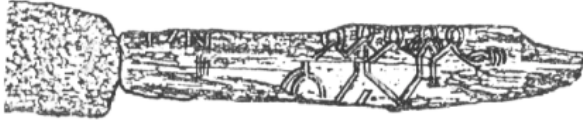

SCHWED 8

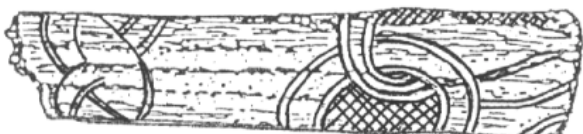

VRP 130

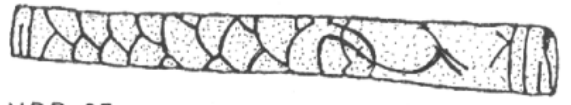

VRP 87

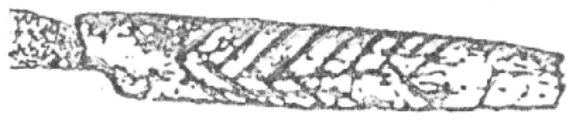

DDR 127

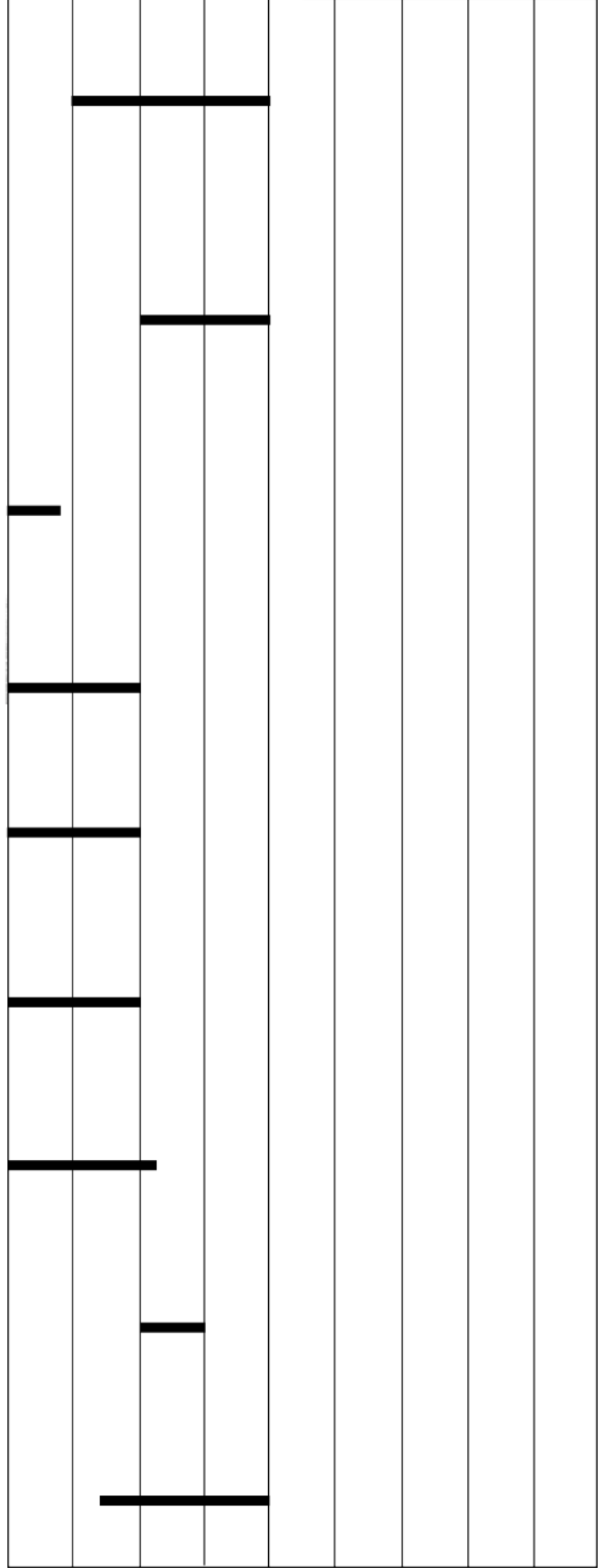


8009001000110012001300140015001600

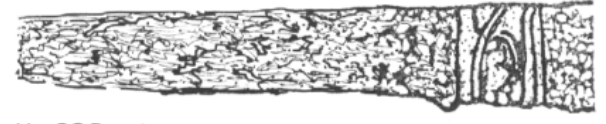

UdSSR 18

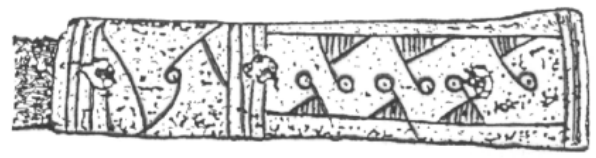

DDR 160

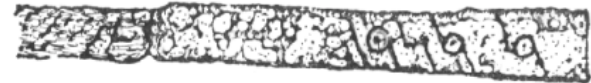
VRP 113
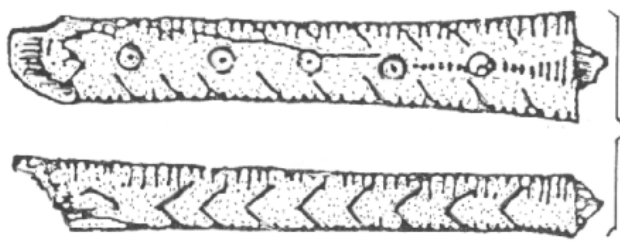
BRD 179
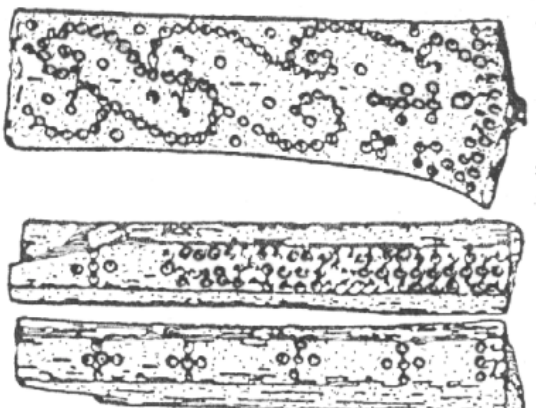

UASSR 39

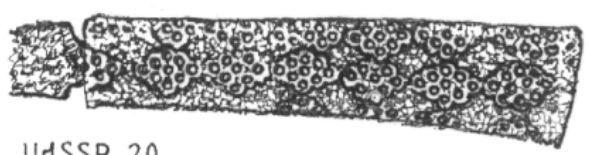

UASSR 20

BRD 150
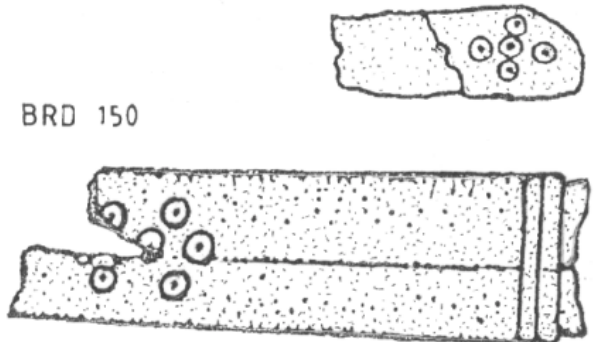

BERL.IN-W. 144

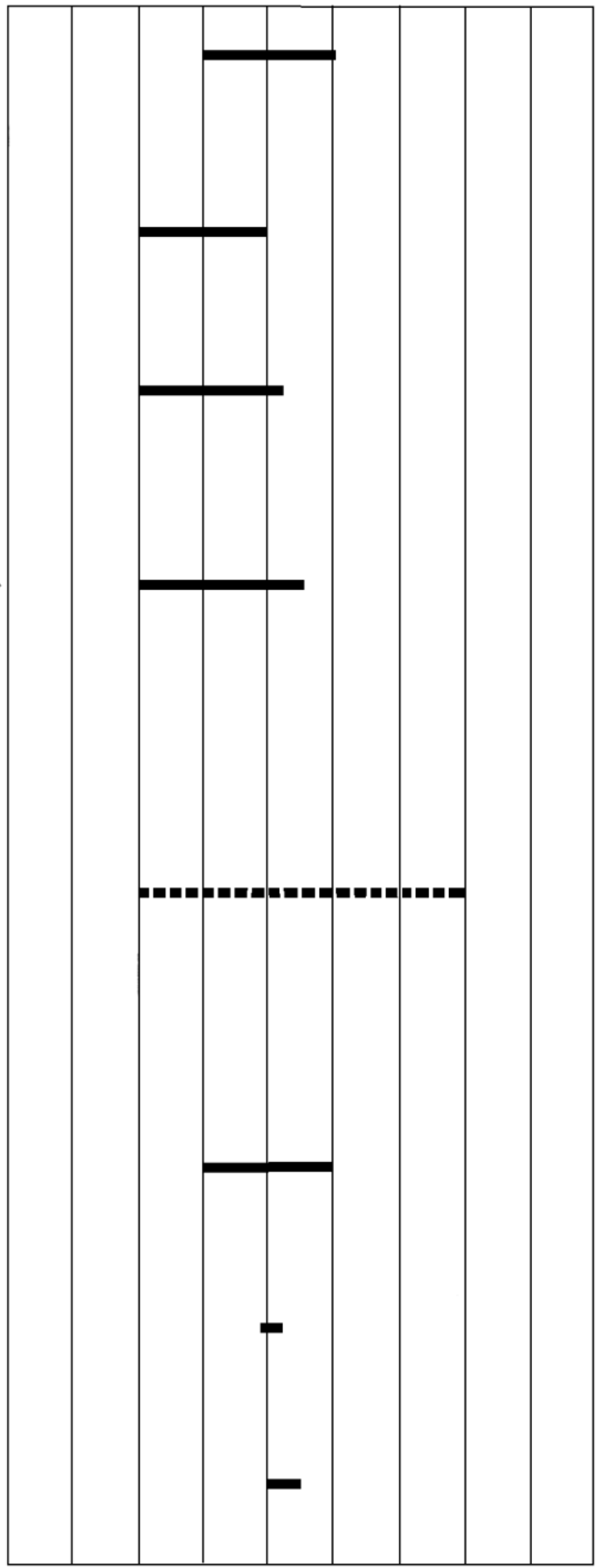




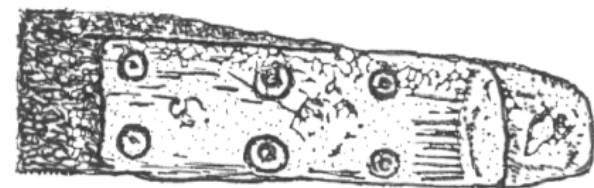

VRP 72

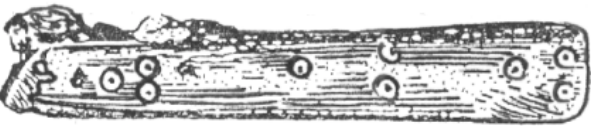

VRP 1600

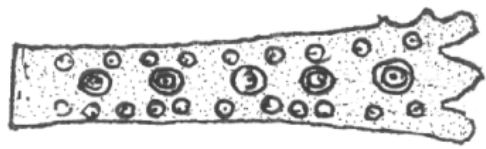

NL 28

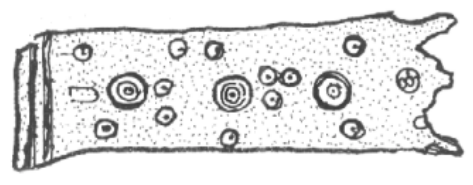

NL 96
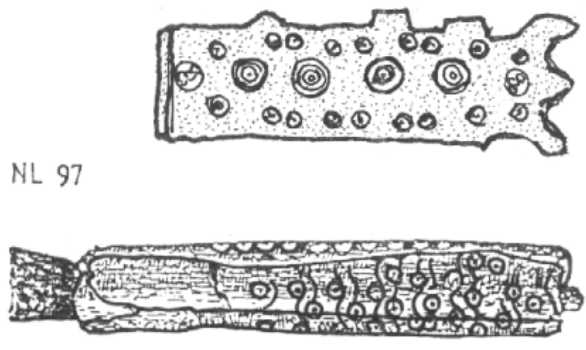

VRP 177

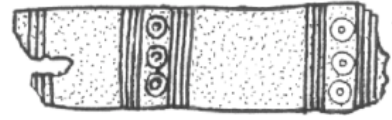

VRP 338

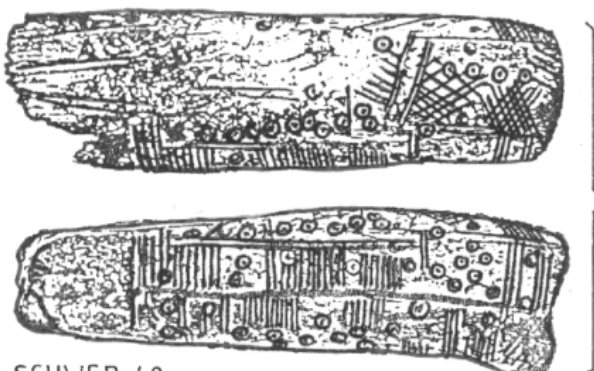

SCHWED. 49

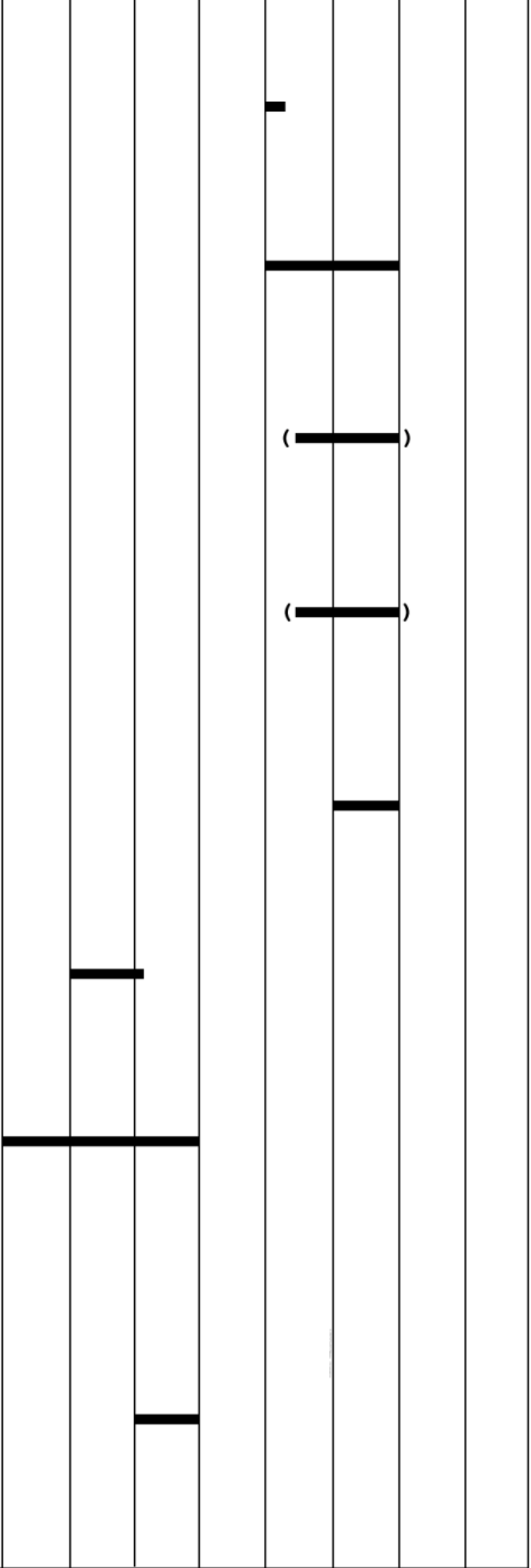


VRP 281

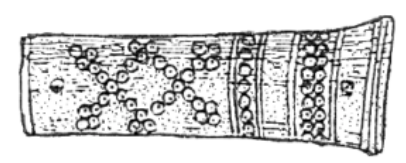

$\begin{array}{llllllll}800900 & 1000 & 1100 & 1200 & 1300 & 1400 & 1500 & 1600\end{array}$

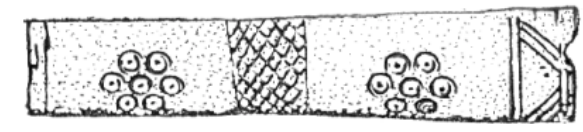

DÄN. 20

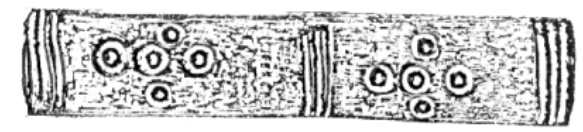

NOR 26

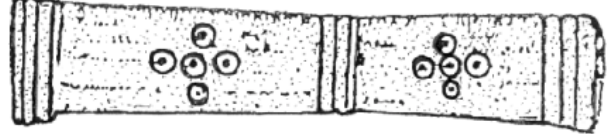

BRD 30

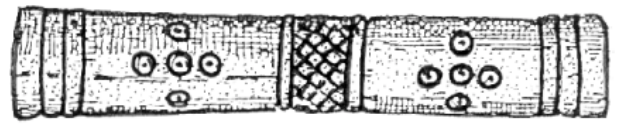

VRP 76

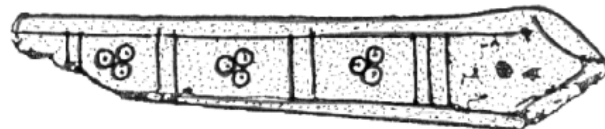

SCHWED 126

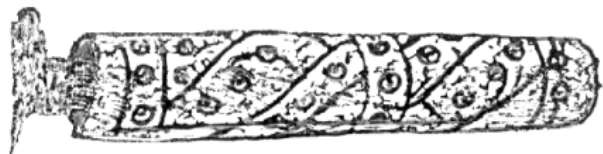

BPD 163

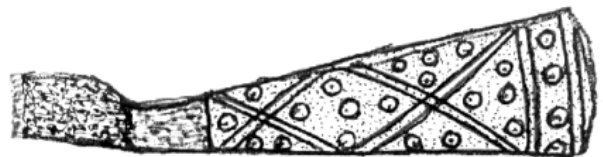

BRD 133
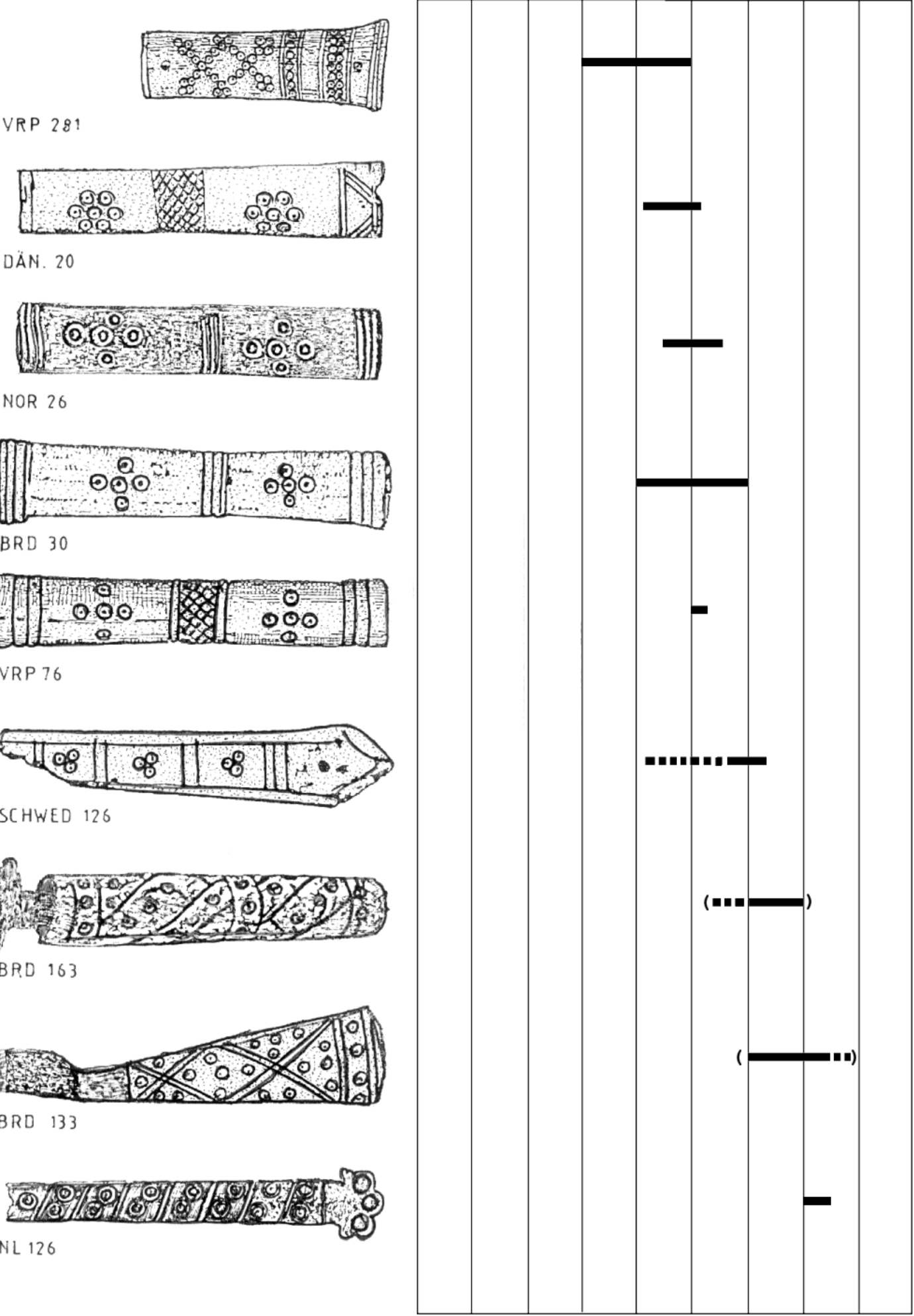


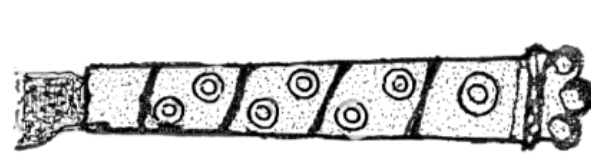

SCHWED. 110

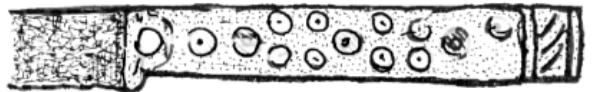

SCHWED. 113

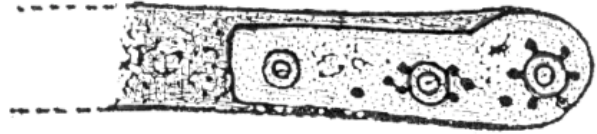

BRD 94

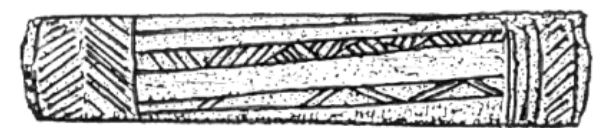

NiL 26

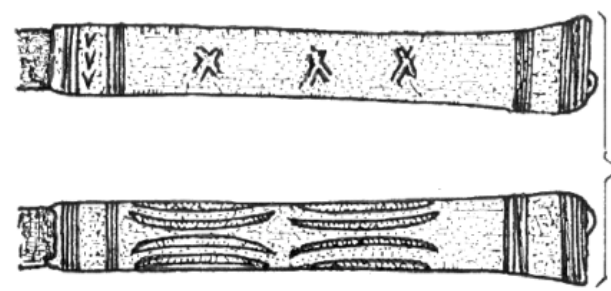

DDR 31

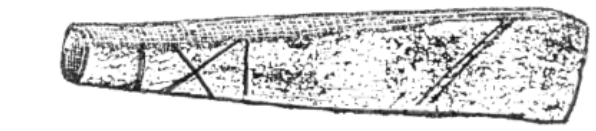

SCHWED 48

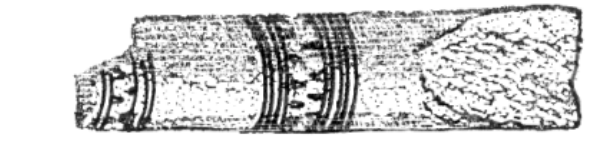

SCHWED 47
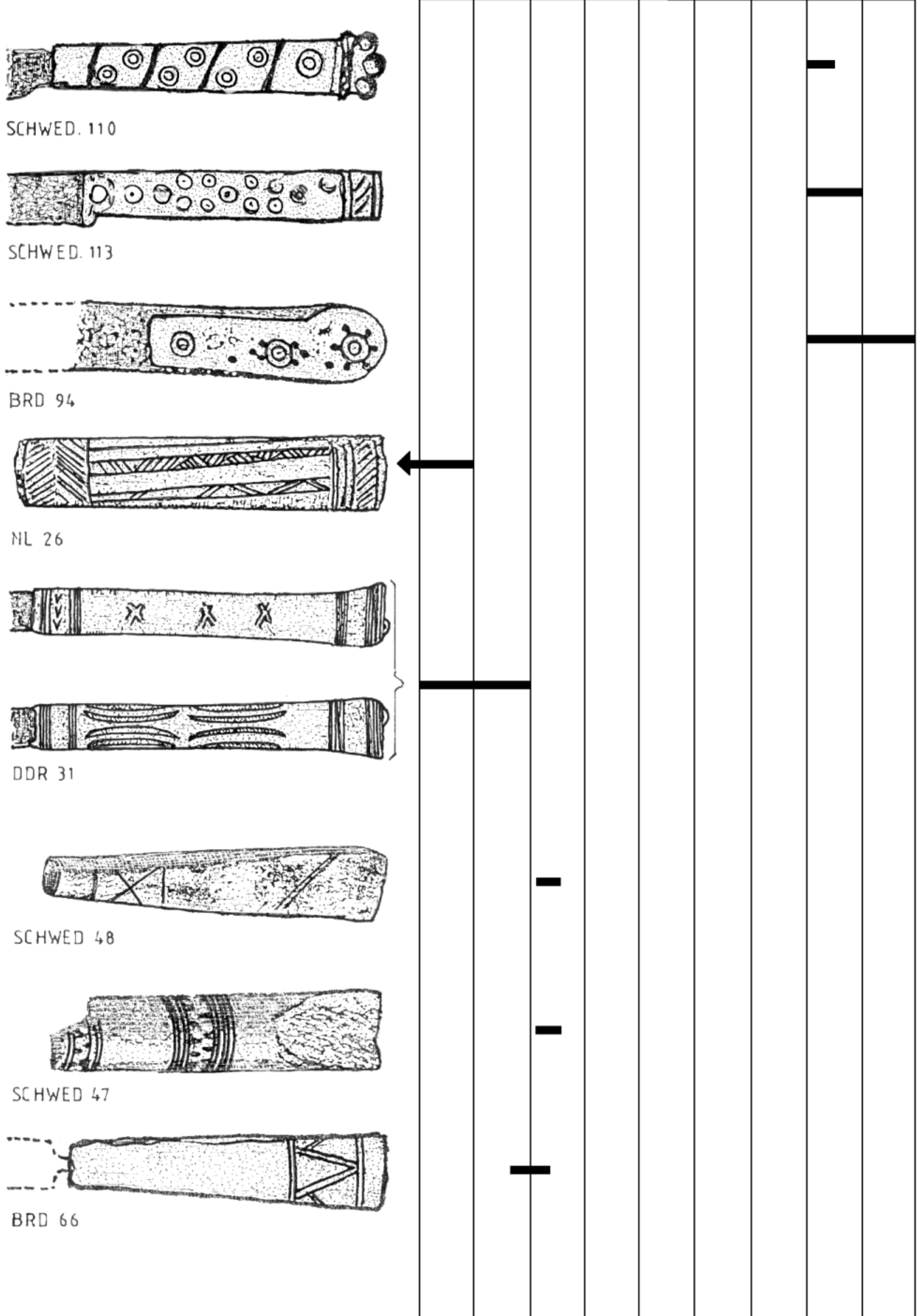


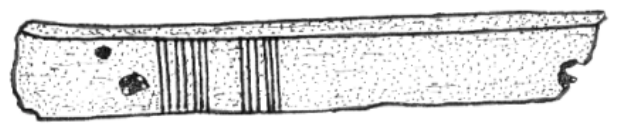

VRP 337

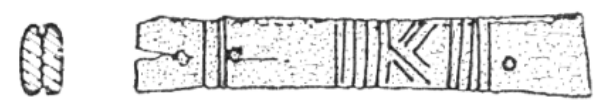
VRP 332

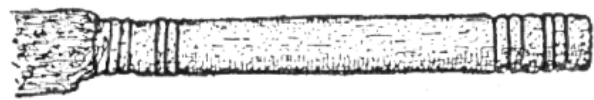
VRP 230

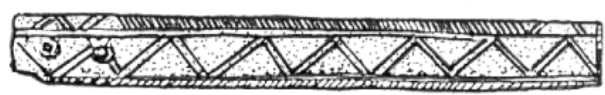
UASSR 82

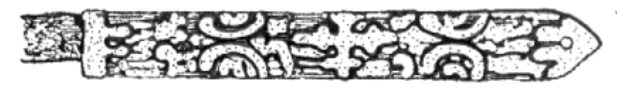

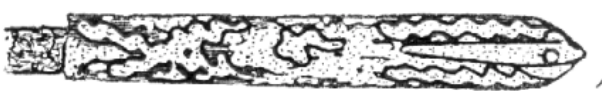
UASSR 40

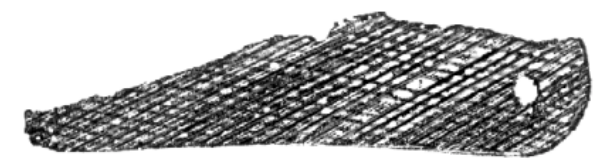

DÄN 34

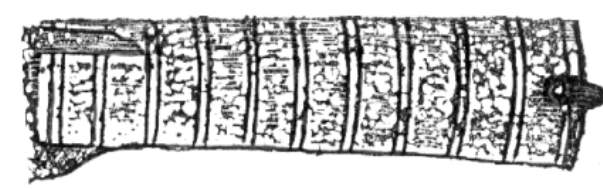

$\mathrm{NL} 38$

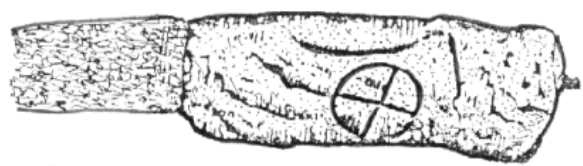

DDR O.Nr.

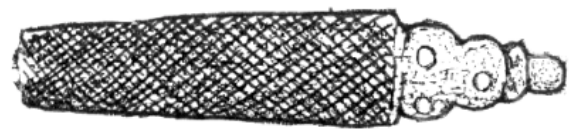

NL 128
$800 \quad 900 \quad 1000 \quad 1100 \quad 1200 \quad 1300 \quad 1400 \quad 1500 \quad 1600$

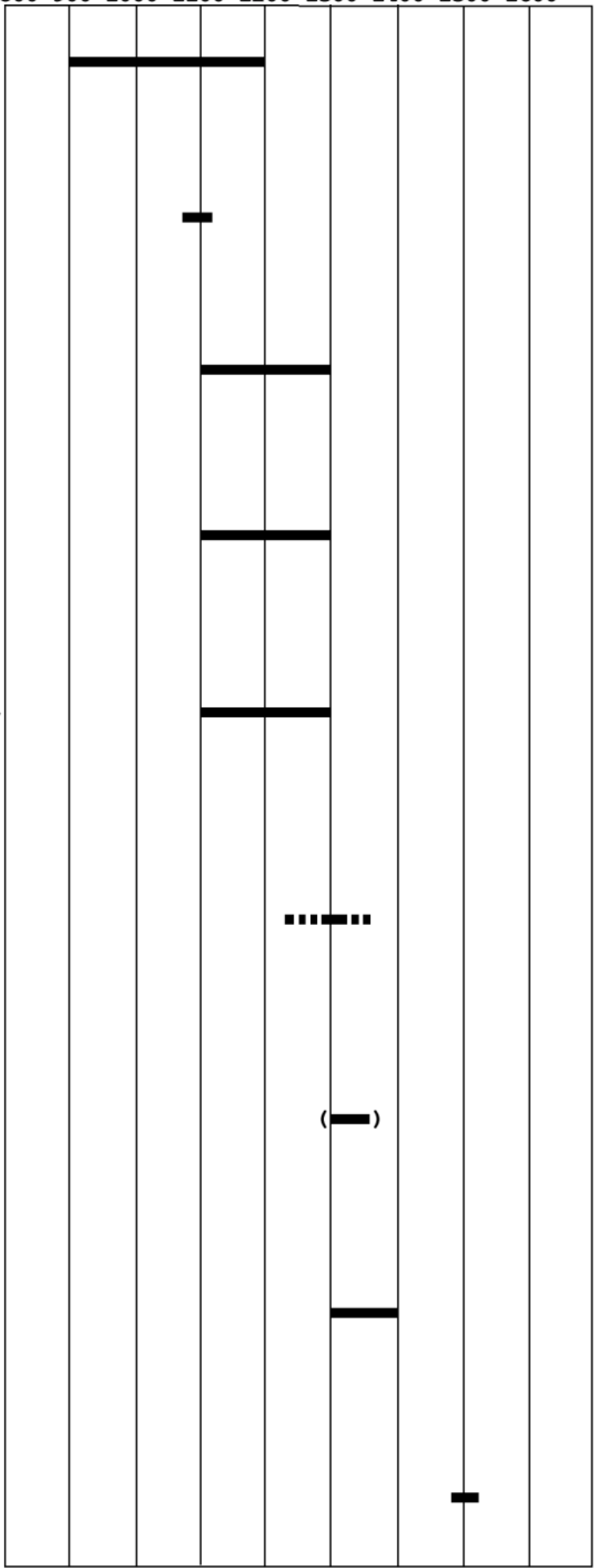




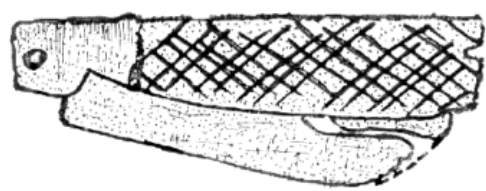

DÄN 58

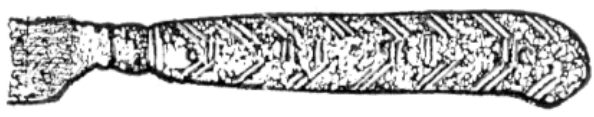

BRD 86

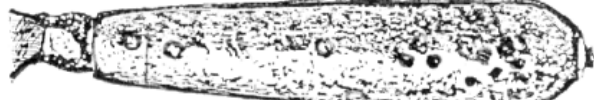

BRD 88

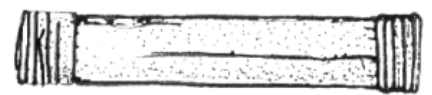

(1)

SCHWED. 118
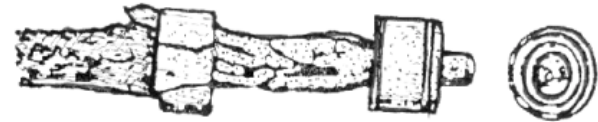

SCHWED. 119

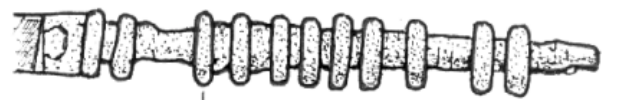

(a)

VRP 315

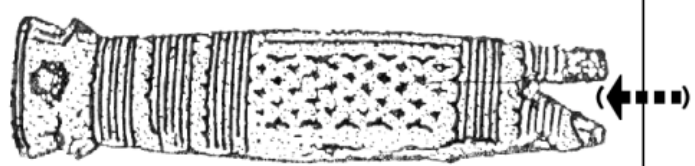

NL 25

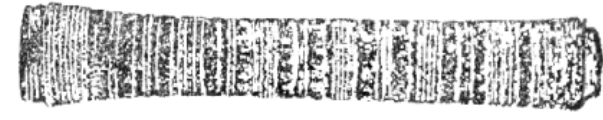

SCHWED. 51

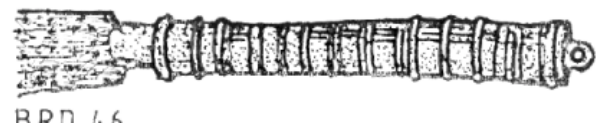

$\begin{array}{lllllll}800 \quad 900 \quad 1000 & 1100 & 1200 & 1300 & 1400 & 1500 & 1600\end{array}$

BRD 46

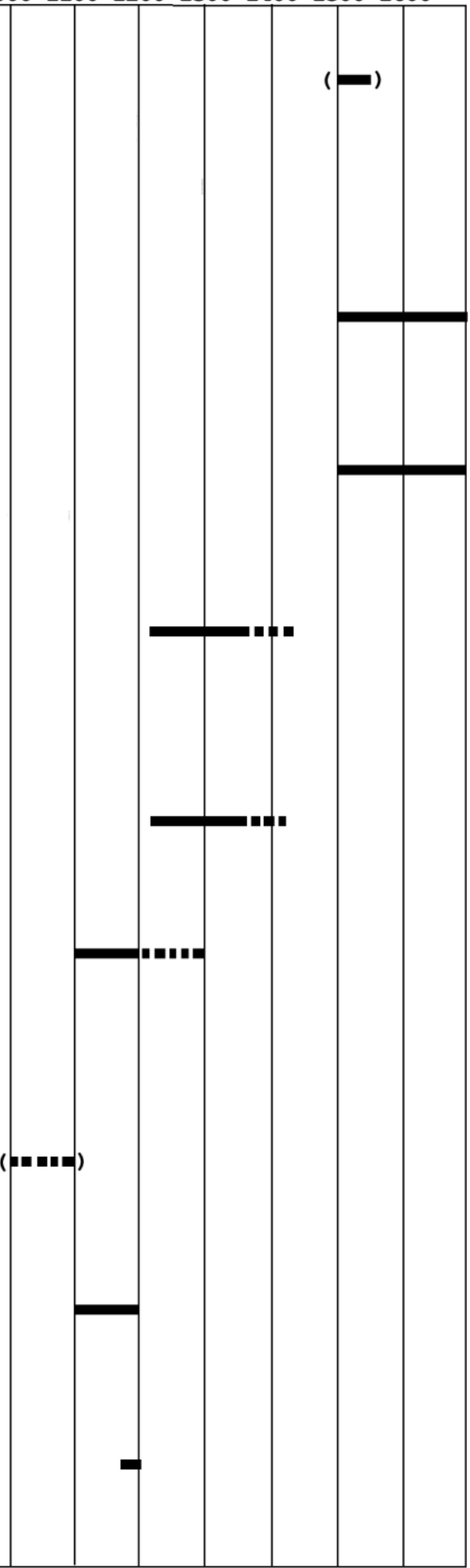




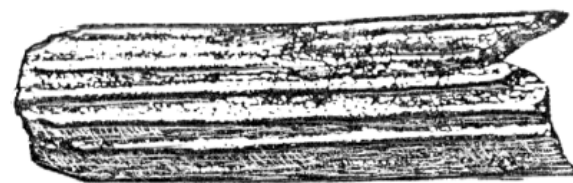

SCHWED. 46

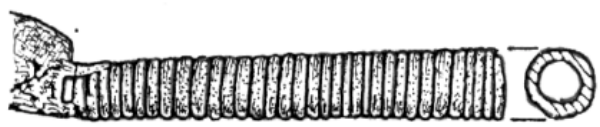
VRP 172

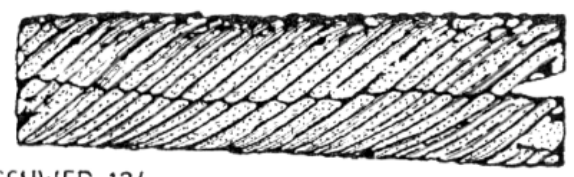

SCHWED. 124

DÄN. 90
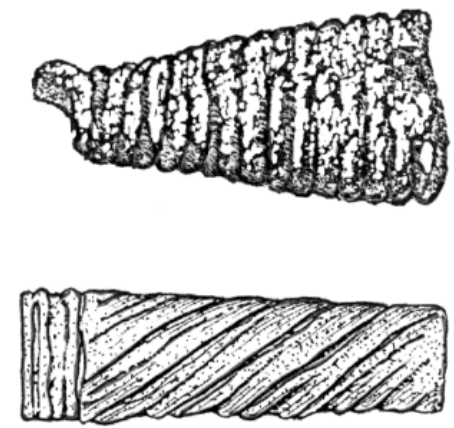

NOR. 36

(0) DDR 98

\section{DDR 99}
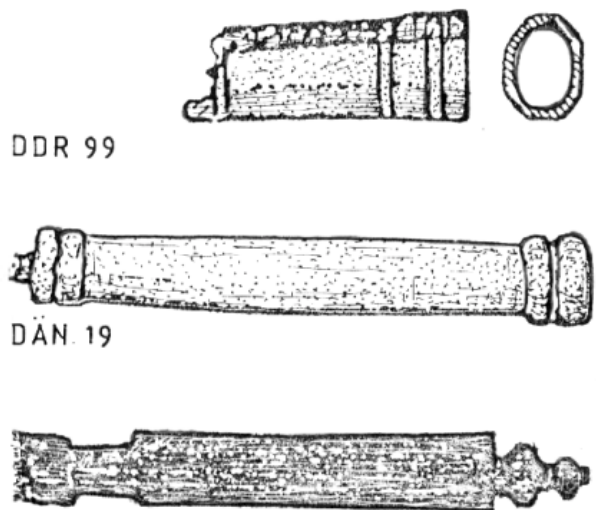

UASSR 10

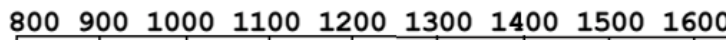

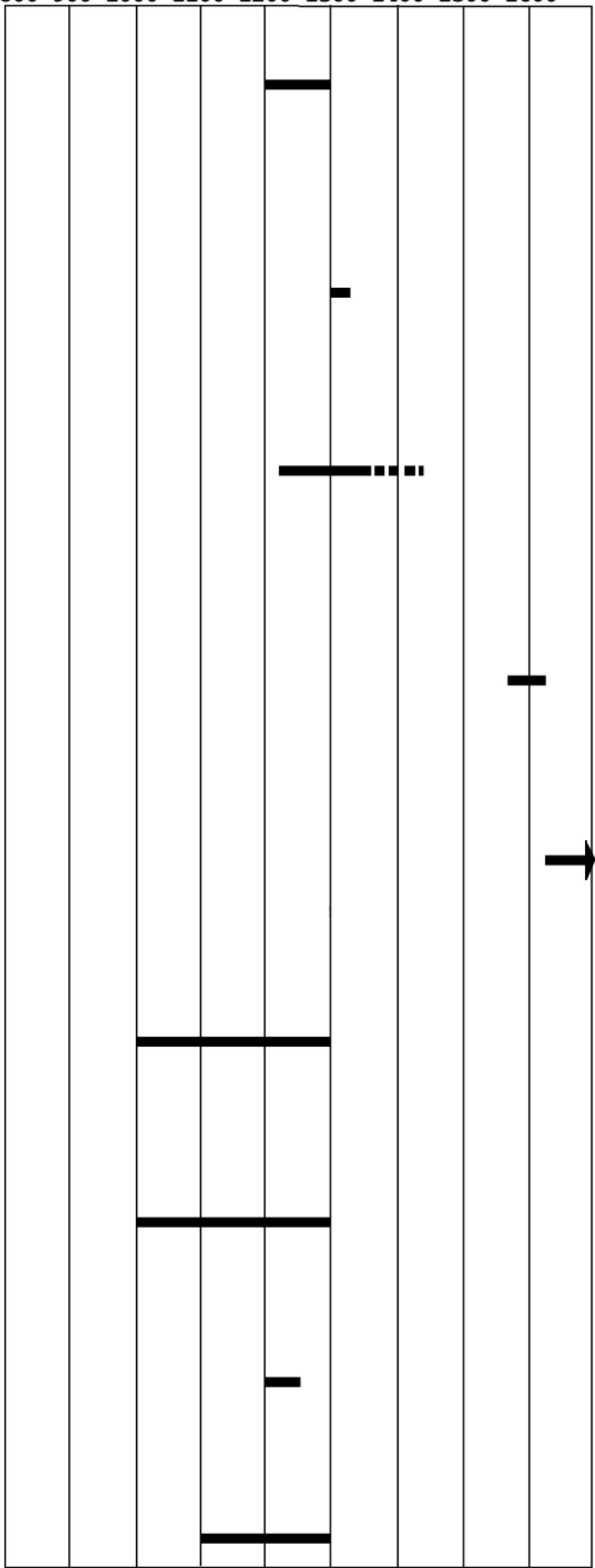




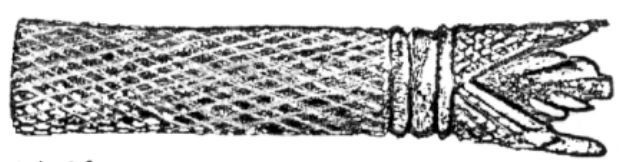

$800900 \quad 1000 \quad 1100 \quad 1200 \quad 1300 \quad 1400 \quad 1500 \quad 1600$
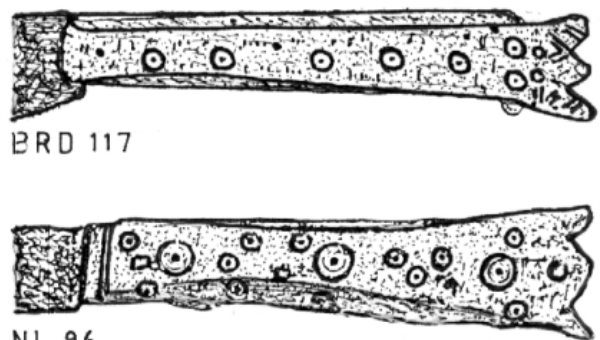

NL 96

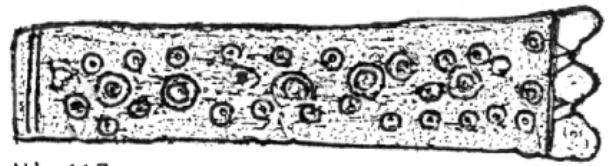

NL 117

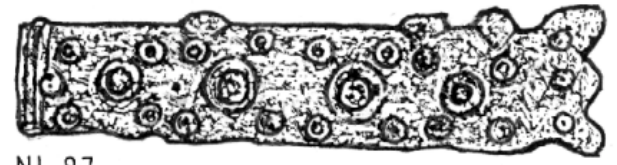

NL 97

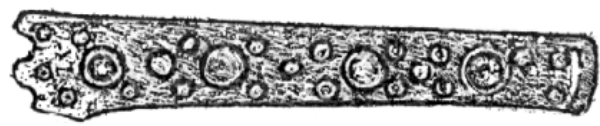

NL 122

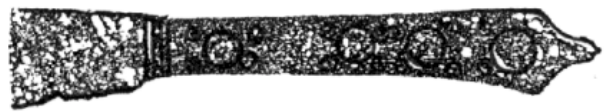

NL 35

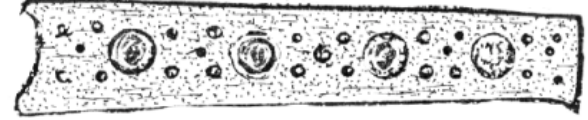

NL 121
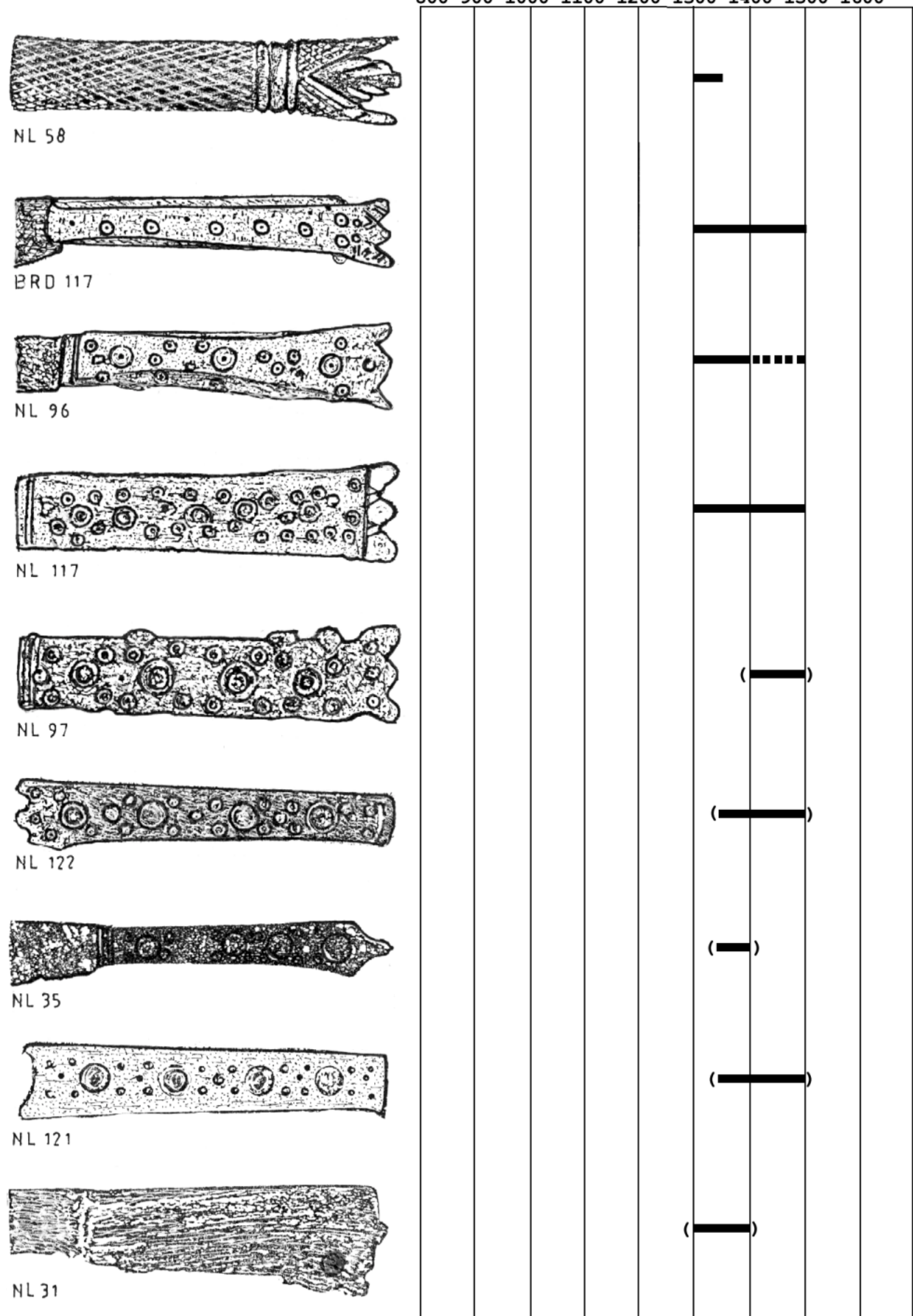


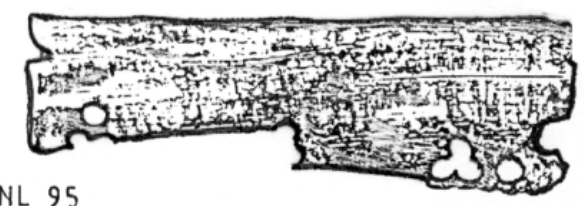

$\begin{array}{llllllll}800900 & 1000 & 1100 & 1200 & 1300 & 1400 & 1500 & 1600\end{array}$

NL 29
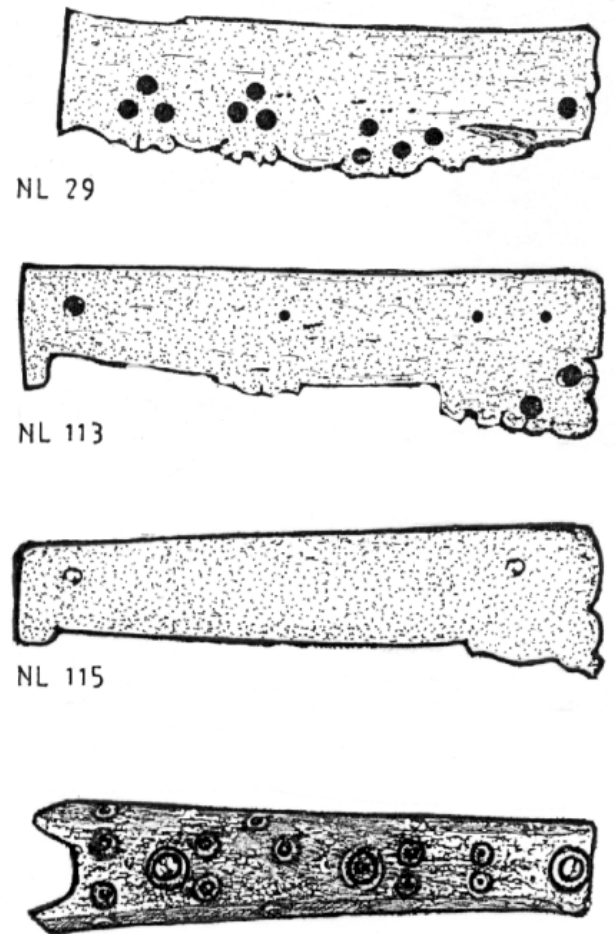

NL 109

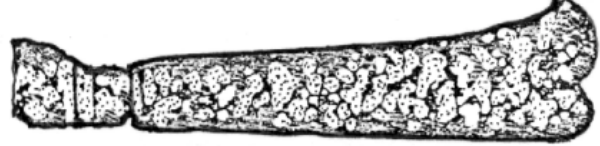

NL 36

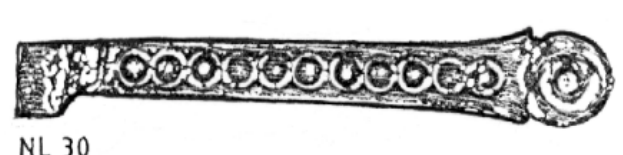

NL 30
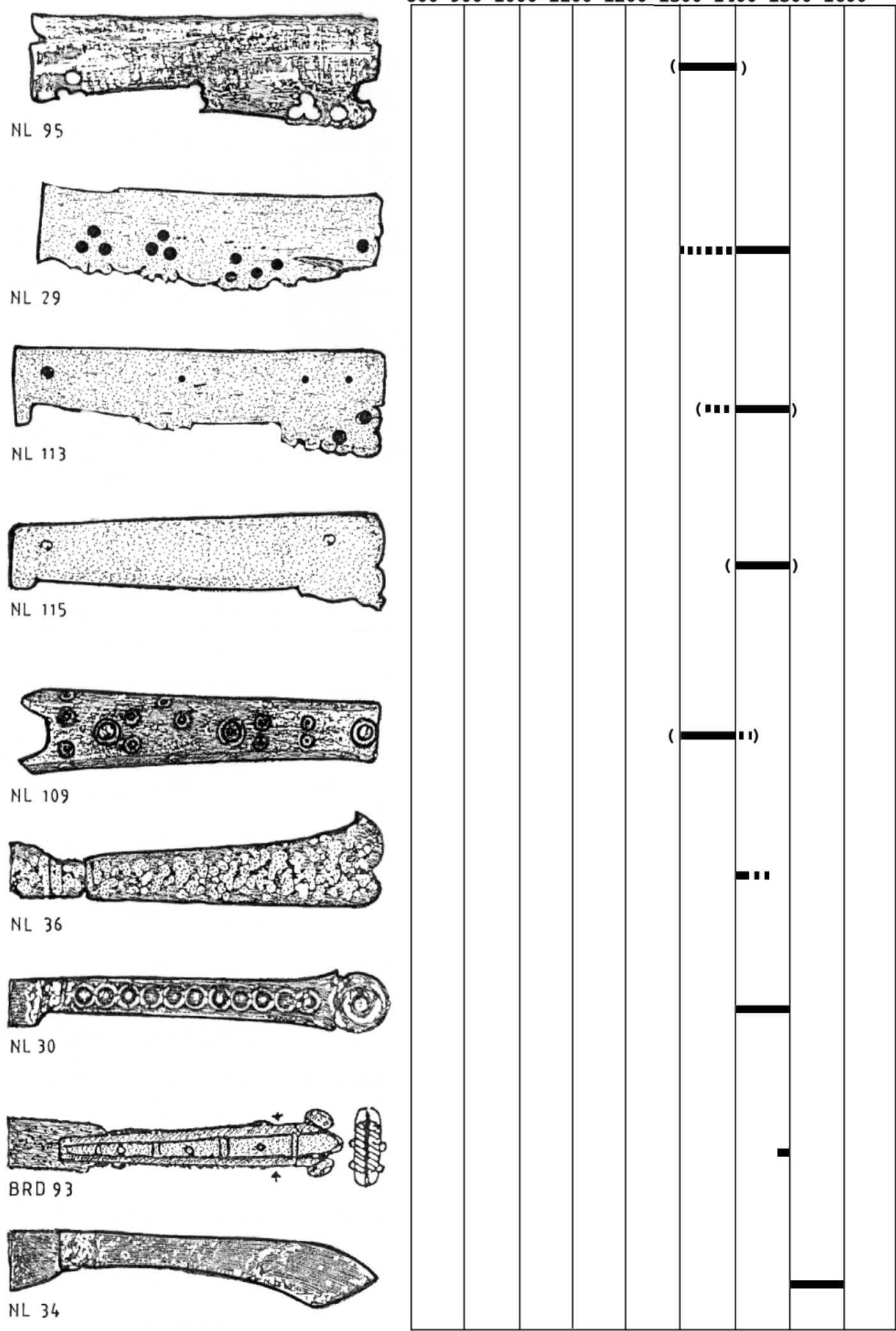


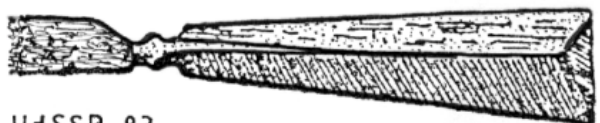

8009001000110012001300140015001600

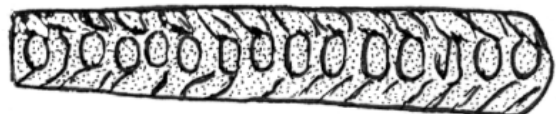

NOR 35
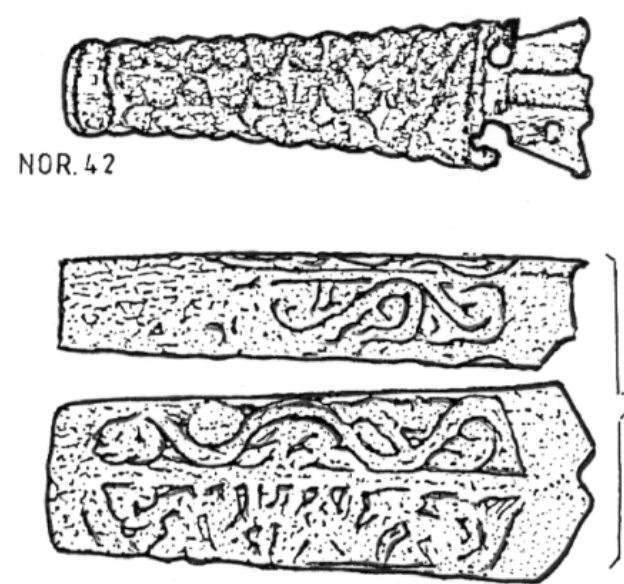

DDR 168

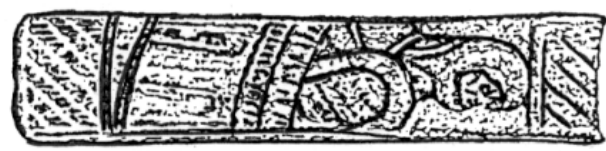

DDR 169

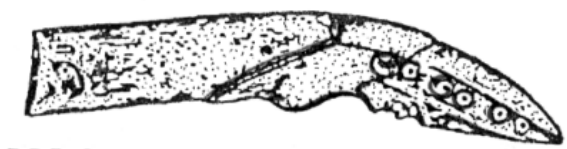

BRD 31

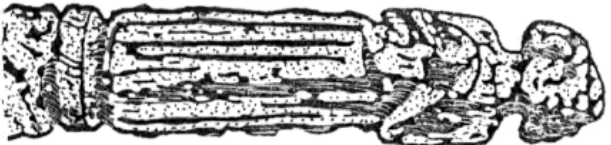

JUSSR 45

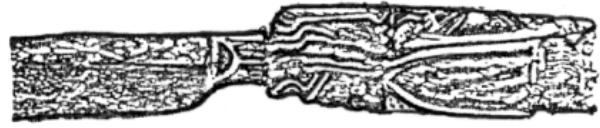

BRD 74

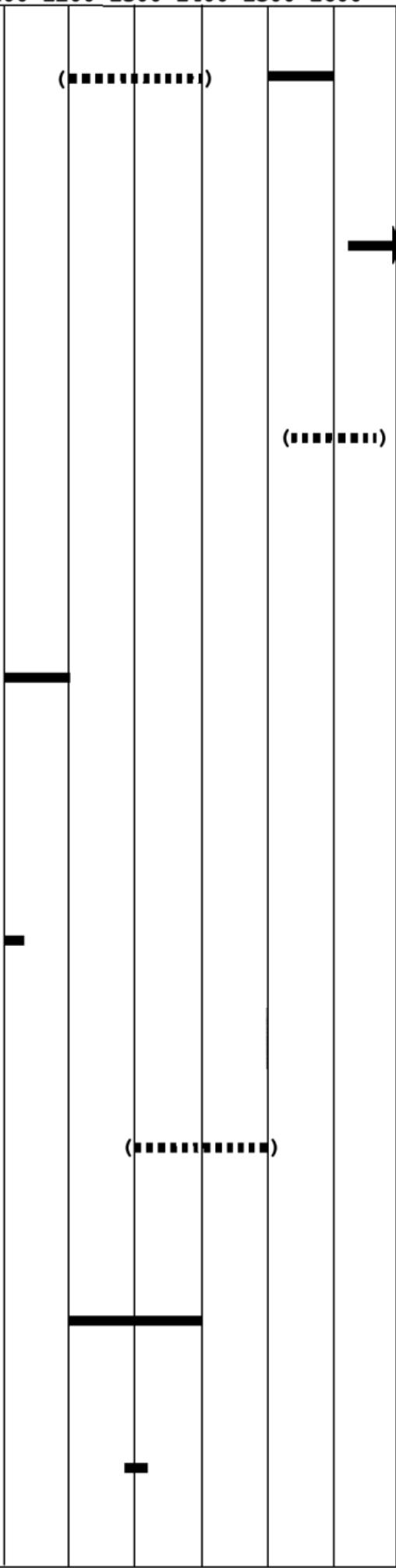




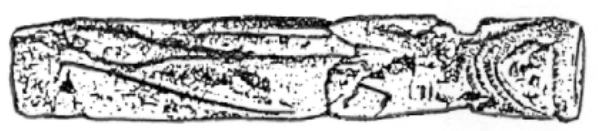

$\begin{array}{llllllll}800900 & 1000 & 1100 & 1200 & 1300 & 1400 & 1500 & 1600\end{array}$

BRD 134

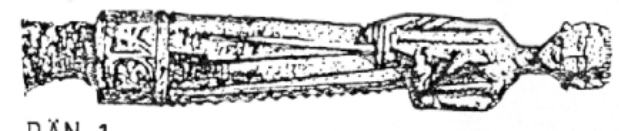
DÄN 1

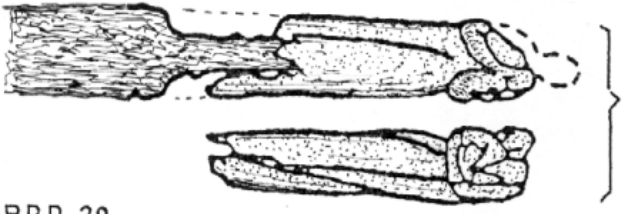

BRD 29

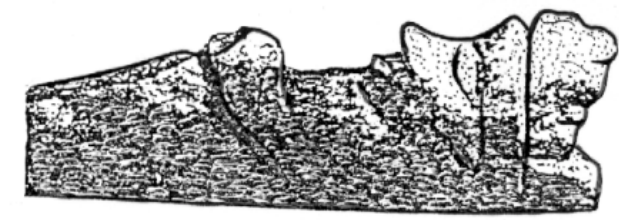

SCHWED.116

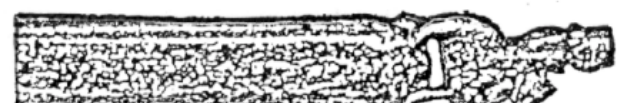

BRD 172

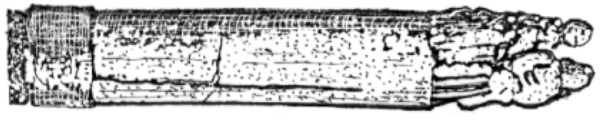

SCHWED. 44

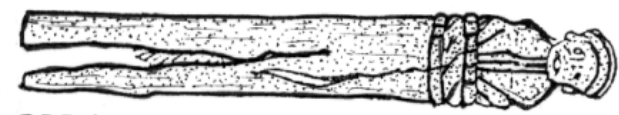

BRD 49

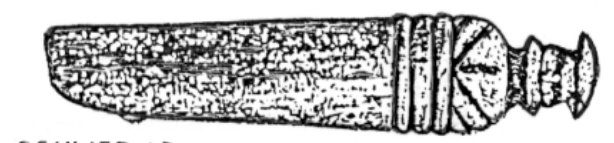

SCHWED. 43

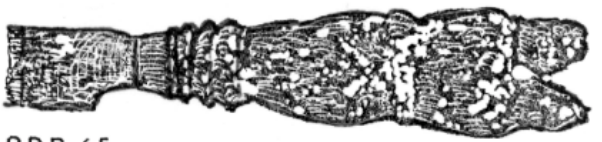

BRD 65

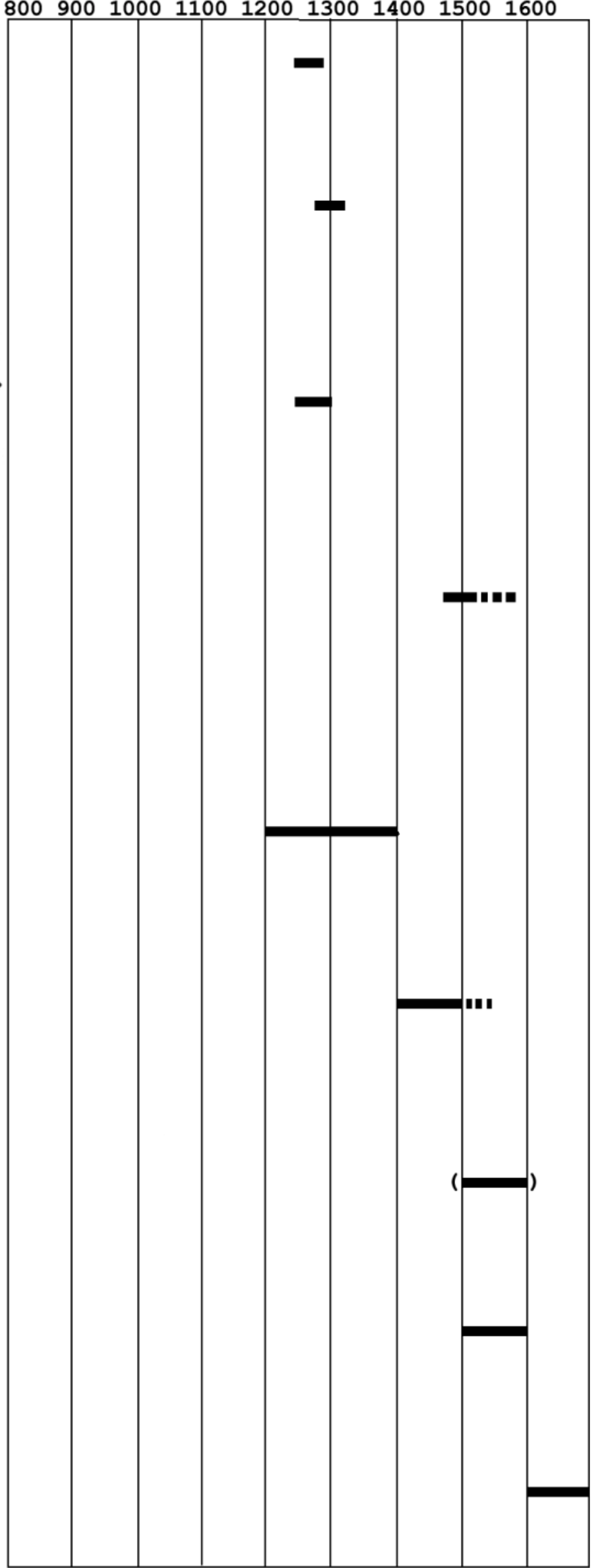




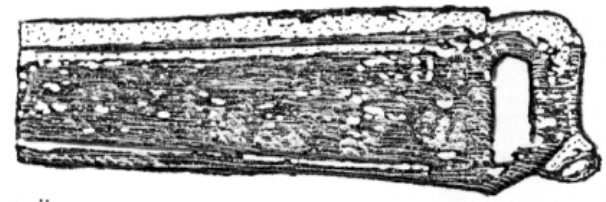

DÄN. 65

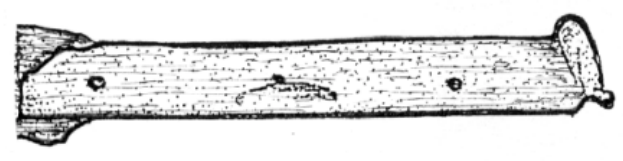

BRD 110

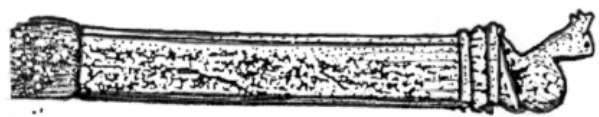
DÄN. 75

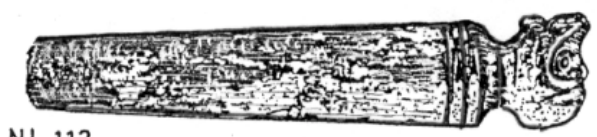

NL 112

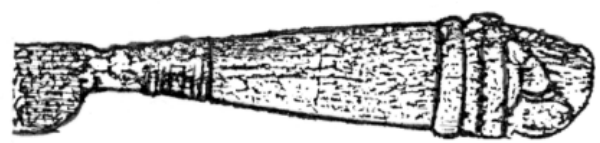

NL 94
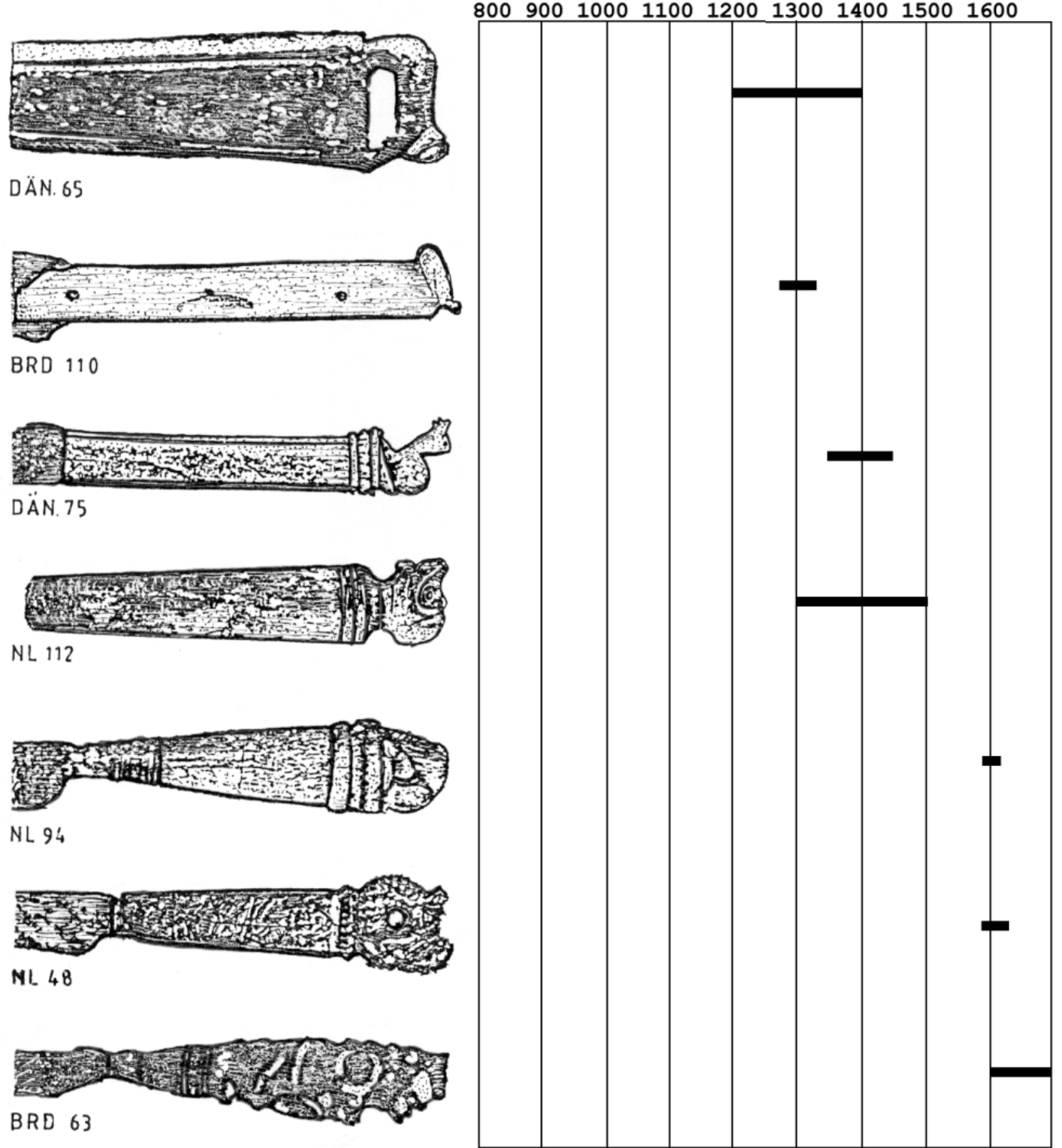

Nachtrag:

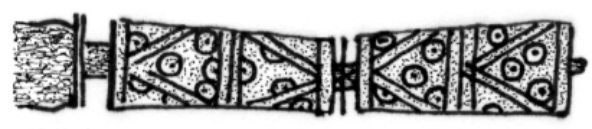
VRP 348

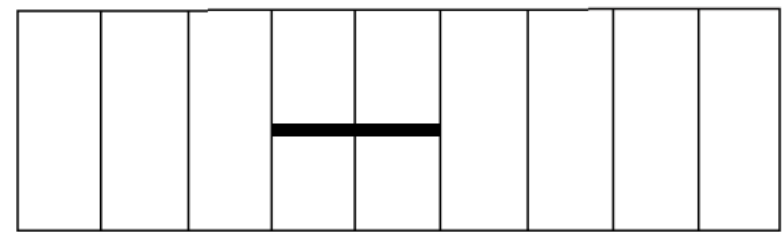




\subsection{Anthropomorph verzierte mittelalterliche Messergriffe - hoch- adelige Topoi in der Sachkultur der mittelalterlichen Tafel}

1974 veröffentliche Lech Leciejewicz einen Aufsatz "Über den Ursprung der Geweihskulptur aus dem Schloßberg von Szczechin", in dem er eine bestimmte Gruppe anthropomorph ausgeschnittener Geweih- und Beingriffe des 13. bis 14 . Jahrhunderts vorstellte ${ }^{117}$. Diese Griffe, neben Stettin auch solche aus Riga, Reval, Rostow, Herlufsholm und Hamburg, stellen in relativ einheitlicher Ausführung den hochmittelalterlichen Topos eines höfischen Falkners dar. Leciejewicz sah in ihnen Beispiele für die Übernahme von Motiven aus dem Bereich monumentaler Architekturplastik in das Umfeld gehobener Sachkultur, deren motivische Herkunft er für Westeuropa annahm, unter Hinweis auf die frühgotischen Plastiken an den Portalen der Kathedralen von Chartre und Le Mans sowie auf die Elfenbeinschnitzereien französischer Reliquienbehälter aus der zweiten Hälfte des 12. und 13. Jahrhunderts. Zur Frage der Einfuhrträgerschaft führte er mit Hinweisen auf skandinavische Parallelen den dänischen Einfluß in Pommern und im Baltikum am Ende des 12. und zu Beginn des 13. Jahrhunderts unter Waldemar II an (Gründung der Stadt Reval 1219) sowie den deutschen Einfluß an (Gründung der Stadt Riga 1201 durch Albert von Buxhövede im Zuge eines Kreuzzugunternehmens an die livländische Küste, Einflußgebiet der Hanse in der zweiten Hälfte des 13. Jahrhunderts).

Ein Jahr später erschien in der Festschrift für H.K. Kristensen ein Aufsatz von Mogens Bencard, in dem er ausgehend von der älteren Bearbeitung eines Falknergriffs aus Ribe durch Kristensen das ihm bekannte Material an Falknergriffen vorstell$\mathrm{te}^{118}$.

Neben den bereits von Leciejewicz publizierten Griffen führte er Beispiele aus Ribe, Roskilde, Skanderborg, Oslo, Kalmar, Schonen, Lund, sowie aus Oxford, Lüttich, Lille, Arras, Scharnegoutum und Friesland an. Weitere Beispiele ohne Fundnachweis stammen aus dem Musée de Cluny in Paris sowie aus der Eremitage in Petersburg. In Anmerkungen werden weitere Beispiele aus Ragnildsholm und aus dem Museum Boymans-van Beuningen in Rotterdam erwähnt.

Zur Herkunft vermutet Bencard, daß das Motiv ursprünglich aus den Elfenbeinwerkstätten in Paris stammt, viele der gröber bearbeiteten Griffe aber wohl lokaler Herstellung entstammen.

Bencard datiert die Gruppe mit einer Ausnahme nach stilistischen, ikonographischen und kleidungsgeschichtlichen Merkmalen in die Zeit von 1250 bis 1350.

\footnotetext{
117 Leciejewicz 1974, S. 177-188

118 Bencard 1975, S. 36-61
} 
Zur sozial- und kulturgeschichtlichen Einordnung führt er sie als Beispiel an für das Motiv des mittelalterlichen Fürsten in Verbindung mit der privilegierten Falkenjagd, welches internationalen Strömungen durch ganz Europa folge ${ }^{119}$.

Neben den von Leciejewicz und Bencard vorgestellten 27 Falknergriffen wurden mit dem dieser Untersuchung zugrunde liegenden Material noch dreizehn weitere vollplastisch anthropomorph ausgeschnitzte Messergriffe sowie zwei halbplastisch bearbeitete Messergriffe und ein stark stilisierter Holzgriff erfaßt. Dreizehn dieser Griffe zeigen Frontaldarstellungen, darunter zwei Falknerfiguren, drei Griffe zeigen Seitenansichten $^{120}$.

Von den insgesamt 45 erfaßten Messergriffen sind vier ohne Fundnachweis. Sie stammen aus dem Musée de Clúny Paris, der Eremitage, dem Kunstgewerbemuseum Budapest und aus der Sammlung d'Allemagne.

\subsection{Beschreibung aller erfaßten Griffe}

\section{Die vollplastische Darstellung}

Von den 41 erfaßten Messergriffen mit Fundnachweis gehören 38 in die von Leciejewicz und Bencard besprochene Gruppe.

Sie zeigen vollplastisch ausgeschnitzte, den ganzen Griff einnehmende anthropomorphe Darstellungen.

Die Motive sind aus zeitgenössischer Dichtung und Bildquellen bekannt:

gekrönte und ungekrönte Adelige, zumeist in Verbindung mit der dem Adel vorbehaltenen Falkenjagd, adelige Damen mit Hund, schildbewehrte Ritter und ein thronender Herrscher.

\footnotetext{
119 Bencard 1975, S. 55-59

Die im Katalog erfaßten Griffe und Messer dieser Gesamtgruppe wurden vom Autor aus anderen Publikationen oder in den jeweiligen Museen aufgenommen, das von Leciejewicz vorgestellte Material wurde dort ebenfalls erfaßt.

Die Publikation von M. Bencard gelangte ihm erst nach Fertigstellung des Katalogs zur Kenntnis, für die er Prof. Leciejewicz sehr zu Dank verpflichtet ist. Weitere Exemplare, die nach Katalogabschluß erfaßt wurden, sind ebenfalls nicht mehr im Katalog enthalten.

An dieser Stelle wird der dem Autor bis jetzt bekannte Bestand vorgestellt und diskutiert, ohne daß er Anspruch auf Vollständigkeit erheben kann.
} 


\section{Frontalansichten}

\section{Westfalen, hansischer Kernraum, Baltikum und Rußland}

In Lübeck wurden in der Hundestraße 9-17 ein Griffangelmesser und ein Messergriff geborgen, welche von den Ausgräbern in die zweite Hälfte des 13. Jahrhunderts datiert wurden ${ }^{121}$. Die beinernen Vollgriffe sind am Heft und am Griffende ausgebrochen. Trotz dieser Beschädigungen sind die Darstellungen einer Frau mit langem Gewand, die einen kleinen Hund auf dem Arm trägt, sowie einer Frau, die einen Gegenstand in ihrer erhobenen rechten Hand hält, noch gut zu erkennen (Abb. 131a-b). Aus dem Vorbericht über die Grabungen in der Hundestraße läßt sich entnehmen, daß das Viertel vor allem im 14. Jahrhundert von "Handwerkern und armen Leuten" bewohnt wurde ${ }^{122}$. Im 13. Jahrhundert wird das Haus Nr. 9 als Privatbesitz erwähnt, welches 1358 von dem Besitzer, dem Sohn des Bürgermeisters Bruno de Warendorpe, in eine Armenstiftung verwandelt wurde ${ }^{123}$.

Seit der ersten Hälfte des 13. Jahrhunderts ist auf den Grundstücken Nr. 13 bis 15 eine Knochenschnitzerwerkstatt belegbar ${ }^{124}$.

Die Messergriffe lassen sich nach diesen Angaben durchaus in einen sozialen Kontext stellen, der vor dem im 14. Jahrhundert einsetzenden Prozeß der Ansiedlung von Armen und mit dem Beginn der differenzierteren Handwerkstätigkeiten anzusetzen ist. Rekonstruierbar erscheint ein Umfeld, welches bereits von Handwerkern und noch von einflußreichen Familien geprägt wurde.

Der Beleg des Knochenschnitzerhandwerks am Fundplatz schließt die Möglichkeit ein, die Griffe könnten dort hergestellt worden sein.

Der von Leciejewicz publizierte Messergriff aus Hamburg wurde zwischen 1880 und 1890 beim Ausbaggern des neuen Zollhafens gefunden. Stilistisch wird er dort in das ausgehende 13. und 14. Jahrhundert datiert ${ }^{125}$. Der Griff aus Walroßzahn ist vollplastisch ausgeschnitzt zu einer stehenden männlichen Figur auf einem Sockel, die einen Falken auf dem linken Arm trägt (Abb. 131c).

Hinweise auf eine soziale und funktionale Einbindung sind der Fundsituation nicht zu entnehmen.

\footnotetext{
121 Vgl. Katalogteil BRD, Nr. 29 und 29a

122 Stephan 1978, S. 78

123 Stephan 1978, S. 78

124 Stephan 1978, S. 78

Mührenberg 1988, S. 98

125 Kartei des Museums für Hamburgische Geschichte, Inv. Nr. 1926-164

Leciejewicz 1974, S. $183 \mathrm{ff}$

Vgl. Katalogteil BRD, Kat. Nr. 48
} 
Daß derartig verzierte Griffe nicht ausschließlich im städtischen Milieu anzutreffen sind, zeigt ein Exemplar im Archäologischen Museum Altenessen ${ }^{126}$. Der beinerne Messergriff zeigt eine vollplastisch ausgeschnitzte Frauenfigur in der typischen Tracht der adeligen Dame des 13. Jahrhunderts mit einem Hund in den sich überkreuzenden Armen (Abb. 131d).

Das Stück wurde vor dem letzten Weltkrieg während einer Grabung des damaligen Ruhrlandmuseums auf der über der Ruhr bei Essen liegenden Ruine der Isenburg "unter dem erhaltenen Fenster des Palas an der Basis des Mauerschuttes" geborgen $^{127}$.

Die Isenburg wurde 1241/42 von Dietrich von Isenberg erbaut, im Zuge der Auseinandersetzungen mit dem Kölner Erzbischof 1244 mit einem Kölner Vogt besetzt und 1288 nach der Schlacht bei Worringen systematisch niedergelegt ${ }^{128}$.

Nach den Berichten der Ausgräber besteht kein Zweifel daran, daß der Messergriff mit zahlreichen Keramikfragmenten und Eisenobjekten des 13. Jahrhunderts in den Funktionsbereich der Burgbewohner zu stellen ist. Das historisch in die Mitte und zweite Hälfte des 13. Jahrhunderts datierte Exemplar von der Isenburg läßt sich aufgrund des noch unpublizierten gesamten Fundinventars ${ }^{129}$ und der historischen Angaben in ein bestimmtes politisches und soziales Umfeld einordnen.

Die Burg wurde von dem Erbauer offenbar nie oder nur sehr kurz bezogen. Die Bewohner rekrutierten sich aus einer Kölner Besatzung unter einem bischöflichen Vogt, ihr historisches Umfeld war von den Auseinandersetzungen zwischen dem Hegemonialanspruch des Kölner Stuhls und der westfälischen Adelsopposition geprägt, deren erster Höhepunkt von dem Gevelsberger Mordanschlag markiert wird $^{130}$. In diesem Spannungsfeld politischer Interessen hatte die Bewohnerschaft der Isenburg unter der Führung eines Amtsadeligen nicht nur die Aufgabe, in einem Grenzgebiet militärische Präsenz zu zeigen. Sie verwaltete darüberhinaus den zur Burg gehörenden Grundbesitz.

Der Besitzer des Messers von der Isenburg ist im Umfeld burgsässigen Amtsadels anzusiedeln.

Ein dem Hamburger Exemplar in der Formgebung des Gewands ähnlicher Griff aus Geweih wurde in Stettin auf dem Schloßberg gefunden (Abb. 131e). Die Fundumstände wurden von Leciejewicz 1974 als "Störung in einer Schicht des 10. Jahrhunderts" beschrieben, er datierte den Griff stilistisch in die ersten Jahrzehnte des 13. Jahrhunderts ${ }^{131}$. Der Kopf der noch $6,9 \mathrm{~cm}$ langen Falknerfigur ist abgebrochen, der Rest der abgebrochenen Angel steckt im Schaft.

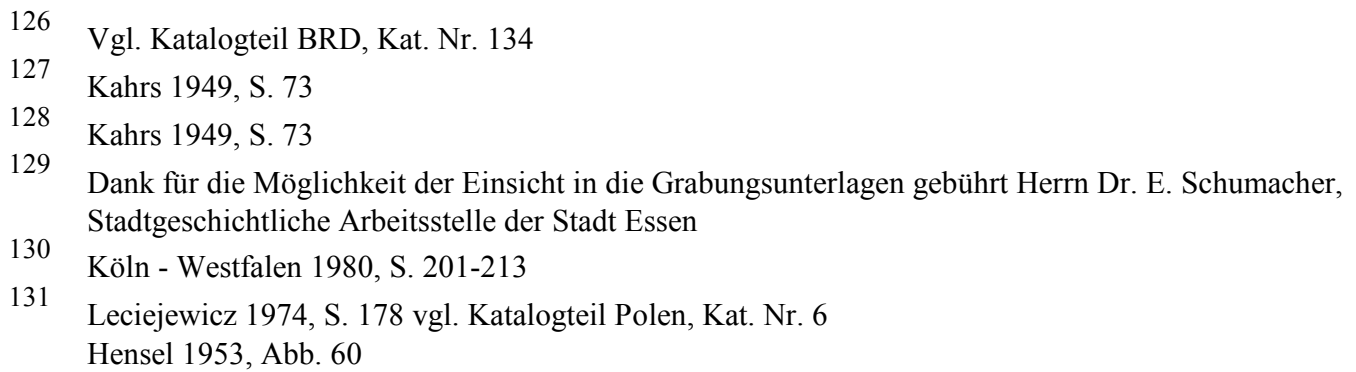


Ob das Messer zu den Bewohnern der Herzogsburg auf dem Schloßberg gehörte oder durch neu ansässige deutsche Kaufleute dorthin gelangte, die seit dem ausgehenden 12., verstärkt seit dem 13. Jahrhundert in die 1237 mit deutschem, 1243 mit Magdeburger Stadtrecht bewidmete Stadt zogen, kann nicht mehr beantwortet wer$\operatorname{den}^{132}$.

Seit Beginn des 13. Jahrhunderts war die Stadt Knotenpunkt zweier Verkehrswege der Hanse, u.a. ein Hauptumschlagplatz auf der Küstenroute von Lübeck nach Riga $^{133}$.

In der Innenstadt von Riga wurden am Albertplatz 1959 ein 8,3 cm langer Falknergriff und 1972 in der Trokēnja-Straße ein fast vollständig erhaltenes Messer mit einem $8 \mathrm{~cm}$ langen Falknergriff in Schichten des 13. bis 14. Jahrhunderts ausgegraben $^{134}$.

Beide Falkner tragen langgezogene Gewänder mit stilisiertem Faltenwurf, ohne die querlaufenden Zierbordüren eines Übergewandes (?) der Beispiele aus Stettin und Hamburg (Abb. 132a-b).

Das Exemplar vom Albertplatz trägt eine Krone, das andere einen Stirnreifen, beide Griffe sind aus Bein gefertigt.

Leciejewicz datierte die Griffe stilistisch unter Hinweis auf Darkewitsch in die erste Hälfte des 13. Jahrhunderts ${ }^{135}$.

Auch hier geben die Fundumstände keine nähere Auskunft über die Träger der beiden Messer.

1201 nach einem Kreuzzugunternehmen durch den Bremer Albert von Buxhövede unter Mitwirkung der Gotländischen Genossenschaft gegründet, wurde Riga Bischofsitz und mit dem Stadtrecht von Visby, nach 1237 mit Hamburger Recht bewidmet.

Bereits bald nach 1200 siedelten sich deutsche Kaufleute an, Riga wurde Stützpunkt des Handels mit russischen Städten über die Flüsse Düna und Dnjepr und war seit der Mitte des 14. Jahrhunderts neben Visby alternierender Hauptort des gotländisch-livländischen Hansedrittels ${ }^{136}$.

Ein von den bisherigen Darstellungen abweichender Falknergriff wurde 1953 in der Altstadt von Reval am Ratusow-Platz ausgegraben und von Leciejewicz mit Hinweis auf Darkewitsch als westeuropäischer Import des 13. Jahrhunderts bezeichnet $^{137}$.

\footnotetext{
132 Leciejewicz 1974, S. 187-188

133 Weczerka 1973, S. 44 ff

Rulewicz 1964, S. 120-124

134 Leciejewicz 1974, S. $180 \mathrm{ff}$

135 Leciejewicz 1974, S. 181, S. 186

136 Grundmann 1975, S. 279, S. 280, S. 293

137

Dollinger 1989, S. 46 ff, S. 129

Leciejewicz 1974, S. 182
} 
Der Griff ist nur $7 \mathrm{~cm}$ lang, er zeigt die Darstellung einer mit Stirnreifen (?) geschmückten Figur in einem langen faltenreichen Gewand, welches schräg gerafft ist. Sie trägt auf dem rechten Arm ein Tier (Hund oder Vogel, Abb. 132c). Der Griff ist kürzer in seinen Proportionen und konturenreicher gearbeitet als die bisher beschriebenen Beispiele.

Reval, der bisher nördlichste Fundort anthropomorph ausgeschnitzter Messergriffe im Baltikum, wurde 1219 im Zuge der dänischen Expansionspolitik unter Waldemar II erobert und blieb bis 1346 dänisch. Sie wurde mit einer Burganlage an der Stelle einer älteren Estenburg befestigt, in deren Schutz sich die von Dänen, Schweden und Deutschen bewohnte Stadt entwickelte.

Mit angeblich 200 deutschen Kaufleuten, die sich 1230 in Reval niederließen, wird der Beginn der deutschen Kaufmannssiedlung angenommen. Die starke Verbindung mit Visby und Lübeck bestand durch die geschäftlichen und familiären Bindungen der die Stadt führenden Kaufmannschaft, die 1259 ihre Verbundenheit mit den Ausgangsstädten in einem Brief an Lübeck dokumentierte. Neben Riga und Dorpat gehörte Reval zu den politisch und wirtschaftlich stärksten der livländischen Städte, sie war wie die beiden genannten von Anfang an Mitglied der Hanse ${ }^{138}$.

In Rostow/Wolga wurde eine aus Knochen geschnitzte bekrönte Figur in der Nähe der Kirche St. Gregor ausgegraben, deren Funktion unsicher zu sein scheint (Abb. 132d). Leciejewicz zitiert Voronin mit der Möglichkeit, sie in einen sakralen Nutzungszusammenhang zu stellen (als Fragment eines Reliquiars), er datiert sie als aus westeuropäischer Provenienz stammend in die zweite Hälfte des 12. bis zu Beginn des 13. Jahrhunderts ${ }^{139}$.

\section{Skandinavien}

Die meisten Griffe wurden in Skandinavien gefunden, von 19 Exemplaren stammen zehn aus Dänemark und Schonen, welches bis zum Frieden von Kalmar 1473 zum dänischen Königreich gehörte, acht aus Schweden und eines aus Norwegen.

1925 wurde bei einer Ausgrabung in der Osloer Altstadt der Rest eines Falknergriffs aus Walroßzahn als Lesefund geborgen ${ }^{140}$. Der noch 5,2 cm lange Griff zeigt einen barhäuptigen Mann mit Schnurrbart, der einen Falken auf der linken Hand trägt (Abb. 133a).

Sowohl das Material als auch die Art und Ausführung der Darstellung lassen vermuten, daß es sich bei diesem Griff um eine örtliche Arbeit handelt, der einheimische Bart- und Haartracht wohl eher als Vorbild diente als französische Monumentalplastik oder kontinentale Buchmalerei.

\footnotetext{
138 Grundmann 1975, S. 293-294 
Ohne verwertbare Fundumstände läßt sich der Träger dieses Messers in der Osloer Kaufmannschaft oder am Hofe der königlichen Burg an der Mündung der Alna in den Oslofjord lediglich vermuten, die von 1286 bis 1350 Residenz der norwegischen Könige war ${ }^{141}$. Mit hoher Wahrscheinlichkeit wurde es als Auftragsarbeit auch am Ort hergestellt ${ }^{142}$, vielleicht unter dem Einfluß deutscher Hansekaufleute, die nach der Mitte des 13. Jahrhunderts dort privilegiert durch die Krone eine führende Rolle im Handel Südnorwegens mit dem Kontinent innehatten und sich seit dem 14. Jahrhundert auch in Oslo niederließen ${ }^{143}$.

In der Silbergasse in Visby auf Gotland wurde 1934 ein Messergriff aus Knochen gefunden, der einen Sackpfeifer darstellt (Abb. 133b). Er ist 9,7 cm lang und am unteren Ende seitlich ausgebrochen.

Die Kopfbedeckung wird in der Kartei des Statens Historiska Museum Stockholm als "volkstümlicher Strohhut des Spätmittelalters" bezeichnet und als vermutlich französische Arbeit um 1400 angesehen ${ }^{144}$. Die Darstellung ist sehr plastisch und weich gearbeitet, sie steht aber in der Gesamtkonzeption und in Details wie dem hinteren Haaransatz klar in der Tradition der älteren Griffe.

Visby, im 13. Jahrhundert neben Lübeck der Hauptstützpunkt der Kaufmannshanse und Stützpunkt der Kriegszüge ins Baltikum, verlor 1293 den Sitz der "Gotländischen Genossenschaft" an Lübeck, blieb aber mit Riga Vorort des gotländischlivländischen Hansedrittels nach der Gründung der Städtehanse. Erst mit der am Ende des 14. Jahrhunderts einsetzenden Seeräuberei wurde der Handel von Visby empfindlich getroffen, nach 1395 setzten sich die Vitalienbrüder in der Stadt fest und wurden erst 1398 durch ein Heer des Deutschen Ordens wieder vertrieben ${ }^{145}$. Im 15. Jahrhundert verlor Visby seine Selbständigkeit als Hansestadt endgültig an die dänische Krone, bis zum Tode der Unionskönigin Margarete 1412 trieben deutsche Kaufleute der Hanse noch in allen skandinavischen Städten privilegierten Han$\mathrm{del}^{146}$.

Sechs weitere Griffe wurden in Kalmar in der schwedischen Provinz Skansen gefunden.

\footnotetext{
141 Molaug 1975, S. 217

142 Bencard 1975, S. 55, S. 59

143 Blom 1973, S. 159, S. 162-163

Weczerka 1973, S. 46

144 Kartei des Statens Historiska Museum, Inv. Nr. 20733, Photo Nr. 1715:56 Dank gebührt Herrn Dr. L. Redin, der dem Autor die Photos und Karteiauszüge der betreffenden Griffe des Statens Historiska Museums Stockholm ermöglichte.

Svahnström 1973, S. 211-217

Grundmann 1975, S. 293-295, 298-299

146 Grundmann 1975, S. 298-299

Dollinger 1989, S. 170
} 
Zwei dieser Griffe zeigen Falknerdarstellungen, einer von ihnen ist beschädigt (Abb. 133g), der Kopf und ein Teil des Sockels ist abgebrochen. Er wird ohne nähere Angaben in die Zeit um 1300 datiert $^{147}$.

Der zweite Griff, $10 \mathrm{~cm}$ lang und aus Bein geschnitten, zeigt einen gekrönten Falkner, der den Falken auf der linken Hand trägt. Während der Körper und das Tier streng stilisierten Linien folgen, sind Gesicht und Krone naturalistischer als die bisherigen Beispiele gestaltet (Abb. 133c). Er wird wohl aus stilistischen Gründen in das ausgehende 13. und beginnende 14. Jahrhundert datiert ${ }^{148}$.

Die drei übrigen Beingriffe aus der Altstadt von Kalmar zeigen männliche Figuren, die jeweils ein mit einem Andreaskreuz versehenes Buch tragen (Abb. 133d-f). Ob es sich bei dem Kreuzgurt um eine um das Buch geschlungene Kordel ${ }^{149}$ oder um einen mit Schrägkreuz versehenen Einband handelt, muß offen bleiben.

Die Griffe sind aus Knochen geschnitzt, die Ausführungsart korrespondiert bis in die Einzelheiten mit den oben beschriebenen Falknergriffen. Zwei der Griffe weisen noch Reste der Klingen auf, das dritte Exemplar ist am unteren Ende ausgebrochen. Ihre Längen betragen 10,4 cm, 9,6 cm und (noch) 7,6 cm. Sie werden stilistisch in das ausgehende 13. und 14. Jahrhundert datiert ${ }^{150}$.

Der letzte aus Kalmar stammende Messergriff wurde in der Schloßbucht gefunden, er ist aus Holz geschnitzt und zeigt eine gekrönte weibliche Figur mit langem geflochten über den Rücken fallendem Haar. Sie ist mit einem faltenreichen Rock und einem über die Schultern fallenden Mantel bekleidet (Abb. 134a). Die 10,3 cm lange Darstellung ist stilisierter als die meisten Beingriffe, dieses und das Material lassen weniger an ein Importstück als an eine einheimische Arbeit denken ${ }^{151}$.

Nähere Angaben zu den Fundumständen fehlen. Kalmar, ein um 1200 mit Wehrturm befestigter Handelsplatz, der bereits eine deutsche Händlerkolonie unter dem Schutz der Svea-Könige enthielt und am Ende des 13. Jahrhunderts mit einer Steinburg befestigt wurde, gehört seit der zweiten Hälfte des 13. Jahrhunderts zu den deutschen Stadtsiedlungen in Skandinavien, die zwar kein Mitglied der Hanse, am

\footnotetext{
147 Bencard 1975, S. 46, Abb. S. 47

151 vgl. D. Selling, Fynd fran Kalmar, in: Statens Historiska Museums Samlingar 5-1948, S. 16. Das Exemplar ist im Statens Historiska Museum unter der Nummer 21144:1169 inventarisiert.
} 
Handel mit der Hanse aber stark beteiligt waren. Vor allem in Kalmar war der Einfluß der deutschen Einwanderung prägend ${ }^{152}$.

Die Tatsache, daß von 19 Griffen aus skandinavischen Fundorten sechs aus Kalmar stammen, zeigt lediglich, wie verbreitet die Griffe möglicherweise waren, denn es ist unzweifelhaft dem Sammeleifer des Dr. Otto Christian Ekmans aus Kalmar zu verdanken, daß sie bewahrt blieben und geschlossen in das Stockholmer Museum gelangten. Erstaunlich ist aber die Vielfalt der Gruppe aus Kalmar, sie weist neben der wahrscheinlich einheimischen Holzskulptur gleich drei Beispiele für die ein Buch tragende Figur auf, die ansonsten im gesamten Untersuchungsgebiet nicht aufgetreten ist.

Lediglich mit der Fundortangabe Småland versehen gelangte ein Elfenbeingriff mit abgebrochenem Kopf in das Statens Historiska Museum Stockholm ${ }^{153}$, der wahrscheinlich derselben Werkstatt entstammt wie der Griff aus Reval (Abb. 134b). Details wie die Gewandraffung, Armhaltung und Sockelgestaltung sowie die Stilistik sind bei beiden Griffen fast identisch. Der am Fuß kauernde Hund findet ikonographisch seine Parallele in dem Griff aus Vysoké Mýto (CSFR), auch die Gesamtkonzeption und Stilistik legen eine weitere Verbindung mit diesem Griff nahe.

Der noch 7,6 cm lange Griff aus Småland darf aufgrund seiner aufwendigen Verarbeitung und seiner stilistischen Vollendung mit hoher Wahrscheinlichkeit als importiertes Luxusobjekt angesehen werden. Seine Herkunft aus dem nordwestfranzösischen Raum ist anzunehmen, da er in seiner Plastizität und stilistischen Einheitlichkeit den von Koechlin publizierten Griffen aus Paris und Nordwestfrankreich (siehe weiter unten) sehr nahe kommt.

Mit ausdrucksstarken Gesichtern und breiter Kopfgestaltung versehen sind zwei von drei Griffen, die in Lund geborgen wurden.

Der wertvollste der drei Griffe zeigt einen Falkner mit Stirnreifen in einem gegürteten Gewand, der in seiner rechten Hand einen Handschuh oder eine Kappe, in seiner linken Hand einen vollplastisch ausgeschnitzten Falken trägt. Haartracht, Gewand und Gesicht sind wie die Attribute sehr fein und in abgestimmteren Proportionen gearbeitet als die gröberen oder stilisierteren bisher vorgestellten Beispiele (Abb. 134c). Der Griff ist 8,1 cm lang und aus Elfenbein geschnitzt.

$\mathrm{Zu}$ den Fundumständen wird lediglich "Lesefund bei einer Ausgrabung" angegeben ${ }^{154}$. Bencard zitiert M. Rydbeck mit der Auffassung, dieser Griff stamme vielleicht aus einer französischen Provinzwerkstatt, sei aber möglicherweise auch eine englische Arbeit nach französischem Vorbild ${ }^{155}$.

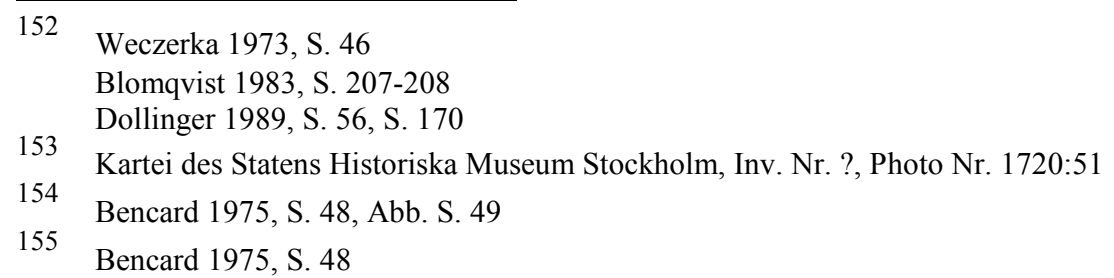


Der zweite in Lund gefundene, 8,9 cm lange Falknergriff aus Bein ist in Proportionen und Ausführung den schlichteren Beispielen aus Hamburg, Stettin und Riga ähnlich, lediglich die breitere Kopf- und Gesichtsgestaltung erinnert an die Griffe aus Lund (1) und Kalmar (1) (Abb. 134f).

Er wurde 1890 als Lesefund bei einer Kloakengrabung geborgen ${ }^{156}$.

In der Kleinen Fischergasse 8 wurde, ebenfalls als Lesefund bei einer Ausgrabung, der letzte der in Lund gefundenen Griffe geborgen ${ }^{157}$. Stark stilisiert und ohne Falken erinnert nur noch die Gesamtgestaltung an die bisher besprochene Gruppe (Abb. 134d).

Der Kopf ist abgebrochen, der Griff aus Bein weist noch eine Länge von $8,6 \mathrm{~cm}$ auf.

Der mit Abstand künstlerisch wie materiell wertvollste Falknergriff, der 1975 Mogens Bencard als Ausgangspunkt seiner Darstellung diente, wurde 1915 in Ribe als Lesefund bei Ausgrabungen in der Baugrube des Hotels Dagmar am Hauptmarkt nordöstlich der Domkirche gefunden ${ }^{158}$.

Er zeigt einen barhäuptigen Falkner in langem Gewand, der auf seiner behandschuhten linken Hand einen z.T. vollplastisch ausgearbeiteten Falken trägt. Dieser sitzt auf einer Stange, deren Ende von der rechten Hand des Trägers gehalten wird.

Der in allen anderen Fällen lediglich abgesetzte Sockel wird hier von einer 8-eckigen Silbermanschette umfaßt, deren Felder mit floralen Mustern verziert sind.

Das Heftfragment der ansonsten vergangenen Klinge weist den Rest einer ausgelegten Marke auf (Abb. 135a).

Der Griff ist aus Elfenbein geschnitzt und 7,5 cm lang.

Nähere Fundumstände sind nicht bekannt, der Fundort Torvet/Overdammen liegt aber an einer so zentralen Stelle der mittelalterlichen Altstadt Ribes, daß Bencard den Träger dieses Messers in "Ribes reicher Bürgerschaft" vermutet ${ }^{159}$. Die dargestellte Männertracht datiert er unter Hinweis auf Hansen von der ersten Hälfte des 13. bis in die Mitte des 14. Jahrhunderts ${ }^{160}$, die Blüte der Elfenbeinschnitzerei mit dem Zentrum Paris von der Mitte des 13. Jahrhunderts bis um $1350^{161}$.

Ribe erlangte 1269 Stadtrecht, die deutschen Kaufleute genossen Hanserecht. Die Stadt wurde zwar nie Mitglied der Hanse, hatte aber eine wikingerzeitliche Vorläufersiedlung östlich der mittelalterlichen Altstadt, einen Königshof vor der Burg des 13. Jahrhunderts und war schon im 12. Jahrhundert einer der führenden dänischen Seeumschlagplätze im Handel mit England und dem Kontinent. Der Dom, in des-

\footnotetext{
156 Bencard 1975, S. 47-48, S. 49

157 Bencard 1975, S. 47, Abb. S. 49

158 Bencard 1975, S. 36 ff, Abb. S. 37 und S. 41, Katalogtext S. 42

159 Bencard 1975, S. 38

160 Bencard 1975, S. 39

161 Bencard 1975, S. 40
} 
sen unmittelbarer Nachbarschaft sich das Messer fand, wurde im 12. Jahrhundert aus rheinischem Tuff und Basalt errichtet ${ }^{162}$.

Auf einem weniger zentralen Grundstück zwischen Grønnegade und Præstegade wurde 1956 in Ribe ein zweiter, am Griffende und am Heft ausgebrochener Falknergriff gefunden. Der Fundort liegt ebenfalls noch innerhalb des mittelalterlichen Stadtkerns. Eine Zuordnung oder Datierung aufgrund archäologischer Befunde liegt nicht vor ${ }^{163}$.

Der noch 8,2 cm lange beinerne Griff ist aufgrund des Materials und der schlichten Ausführung mit den besser erhaltenen Beispielen aus Hamburg, Stettin und Riga zu vergleichen (Abb. 134e).

Bei Ausgrabungen im Bendiktinerinnenkloster Ringkloster bei Skanderborg wurde 1972 ein nur 4,7 cm langer Falknergriff unter einer Brandschicht von 1430 gefun$\operatorname{den}^{164}$.

Die barhäuptige Figur mit Resten eines Metallbeschlags am Heft weist in ihrer Gestaltung eine überproportionale Kopfbetonung auf, ist aber ansonsten von wenigen markanten Strichen im Gewand und der Hervorhebung des Brustbereichs mit dem Falken abgesehen sehr schlicht gehalten (Abb. 135b).

Da das Kloster um 1200 gegründet wurde, liegt mit diesem Griff eine archäologisch begründete Rahmendatierung vor, die aber für weitergehende Fragestellungen zu grob ist.

Stilistisch scheint die Darstellung wegen der weniger starren Gesamthaltung und der leichten Kopfneigung eher in das 14. Jahrhundert zu gehören.

1950 ging im Nationalmuseum Kopenhagen ein 8,2 cm langer Falknergriff aus Bein ein, der als "Lesefund von Herlulfsholm" bezeichnet wurde ${ }^{165}$. In der Besprechung des Gesamtkomplexes führt Bencard als Fundort das Benediktinerkloster Skovkloster (Waldkloster) bei Herlulfsholm an ${ }^{166}$.

Der stark stilisierte, flach und mit geraden Linien geschnittene Griff zeigt einen gekrönten Falkner im langen Gewand (Abb. 135c).

Dieses Exemplar wurde bereits 1974 von L. Leciejewicz publiziert ${ }^{167}$.

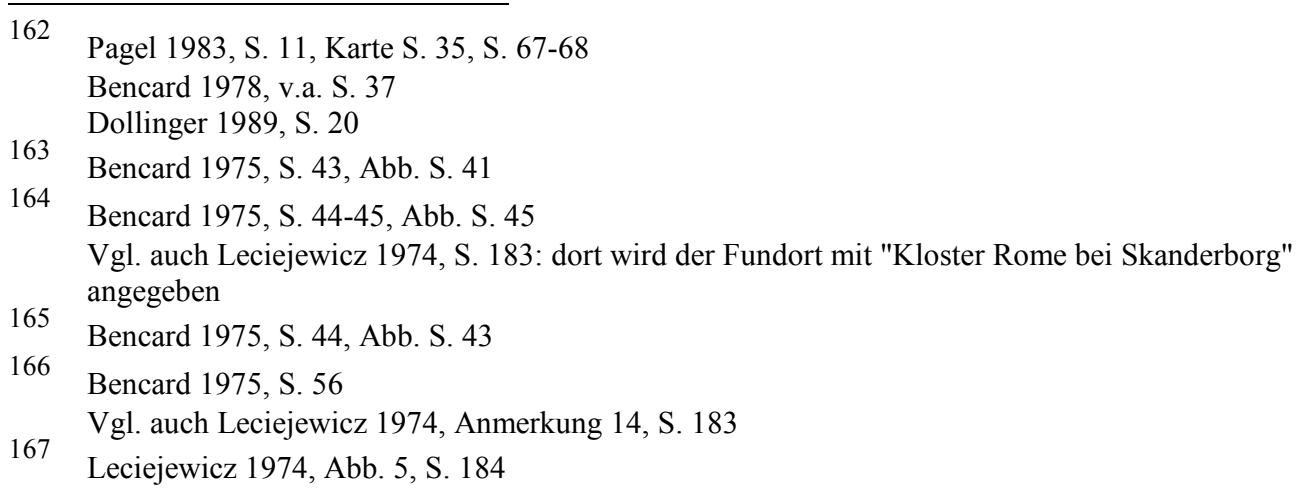


In der vom 10. bis 15. Jahrhundert den dänischen Königen als Residenz dienenden Stadt Roskilde wurde 1875 als Lesefund bei einer Ausgrabung in der Gottorpsvänge ein Messergriff mit einer Doppelfigur gefunden ${ }^{168}$.

Eine gekrönte Falknerfigur in einem langen, faltenreichen Gewand hält mit der linken Hand einen Falken, den sie mit der rechten füttert. Der linke Arm ist um eine zweite Figur gelegt, deren Kopf abgebrochen ist (Abb. 135f).

Der Griff ist aus Elfenbein gearbeitet und $8,8 \mathrm{~cm}$ lang ${ }^{169}$. In dieser Figur vereinen sich das höfische Jagdmotiv mit dem Motiv der Minne, die Darstellung ist mit Ausdruck und leichter Bewegung ausgeführt, wenn auch nicht so naturalistisch wie die Beispiele aus Ribe und Lund. Stilistisch wird er in das 14. Jahrhundert datiert ${ }^{170}$.

An der Fruegade bei der Kirche "Unserer Lieben Frau", an die ein Zisterzienserinnenkloster angeschlossen war $^{171}$, wurde ein weiterer anthropomorph geschnitzter Messergriff in Roskilde gefunden, der das bisherige Motivspektrum erweitert.

Die Darstellung zeigt einen mit Schild bewehrten barhäuptigen Ritter, dessen Kopfgestaltung Ähnlichkeiten mit dem in Oslo gefundenen Falknergriff aufweist (Abb. 135e). Der frontal getragene Schild ist mit einem Wappen versehen, dessen heraldische Zuordnung bisher nicht gelungen ist, möglicherweise handelt es sich wie bei der Figur um eine Idealdarstellung.

Der Griff ist aus "Bein" gefertigt und $9 \mathrm{~cm}$ lang ${ }^{172}$.

Der letzte von Bencard aus Skandinavien veröffentlichte Falknergriff wurde wahrscheinlich in Schonen gefunden, es gibt keine näheren Angaben zum Fundort ${ }^{173}$.

Der Griff zeigt einen Falkner ohne Sockel mit leicht geneigtem Kopf, der eine barettähnliche Bedeckung trägt (Abb. 135d).

Dieses Kleidungsstück bewog M. Rydbeck und M. Bencard, den Griff in das ausgehende 14. bis 15. Jahrhundert bzw. in die Zeit vor oder um 1400 zu datieren ${ }^{174}$.

\section{Westeuropa}

Weitere von Bencard besprochene Beispiele wurden im westlichen Teil des Untersuchungsgebietes gefunden, u.a. in England, Flandern, dem Maasgebiet und Friesland.

\footnotetext{
168 Bencard 1975, S. 43-44, Abb. S. 43

169 Nach der Kartei des Nationalmuseum Kopenhagen, Inv. Nr. D 998, Photo Nr. 21 b 10, 11 Der Autor ist Herrn Dr. N.-K. Liebgott für die Überlassung der Karteiauszüge zu Dank verpflichtet. 
Aus dem mittelalterlichen Stadtgraben von Oxford stammt ein 8,4 cm langer elfenbeinerner Falknergriff, dessen flachere Darstellung an das Exemplar aus Herlulfsholm erinnert ${ }^{175}$. Der gekrönte Falkner trägt den Falken hier auf der linken Hand (Abb. 136a).

Aus den mittelalterlichen Befestigungsanlagen von Arras stammt ein $9 \mathrm{~cm}$ langer Falknergriff aus Elfenbein, der in das beginnende 14. Jahrhundert datiert wird ${ }^{176}$ (Abb. 136b).

Aus dem Fluß Deule in Höhe der ehemaligen Burg Bergneau in Lille wurde ein Griff mit einer gekrönten Falknerfigur von 9,2 cm Länge geborgen, der in das ausgehende 13. Jahrhundert datiert wird ${ }^{177}$.

Möglicherweise aus Lüttich stammt ein barhäuptiger Falknergriff aus Bein mit einer Länge von $8,7 \mathrm{~cm}^{178}$. Die Darstellung ist sehr schlank, das Gewand und der Kopf sind plastisch gestaltet (Abb. 136c).

Ähnlich schlank, aber flacher ausgeprägt ist ein an Kopf und Heft ausgebrochener Falknergriff aus Elfenbein mit einer noch erhaltenen Länge von 7,8 cm, der möglicherweise in Friesland gefunden wurde ${ }^{179}$ (Abb. 136d).

1922 wurde im "Terp" (künstlich aufgeschütteter Grassodenhügel) des Zisterzienserklosters Nijeklooster bei Scharnegoutum in Friesland ein ebenfalls aus Elfenbein geschnitzter, 7,9 cm langer Falknergriff gefunden ${ }^{180}$.

In seiner Gesamtdarstellung erinnert er stark an die Beispiele aus Hamburg, Stettin, Riga und Herlulfsholm. Da der Griff erhebliche Abnutzungsspuren zeigt, läßt sich die Qualität der Ausführung nicht mehr beschreiben, lediglich das Material scheint ihn aus dieser Gruppe herauszuheben (Abb. 136f).

Aus der in Paris entstandenen Sammlung Ren2 d'Allemagne, die später in das $\mathrm{Mu}-$ seum von Rouen gelangte, stammt der Elfenbeingriff eines Messers, dessen nordwestfranzösische Provenienz aufgrund der Entstehungsgeschichte der Sammlung anzunehmen ist ${ }^{181}$ (Abb. 136g).

Er zeigt eine in ein langes, in asymmetrische Falten gegliedertes Gewand gehüllte Figur, die einen Hund auf dem rechten Arm trägt. Nach der haubenartigen Kopfbe-

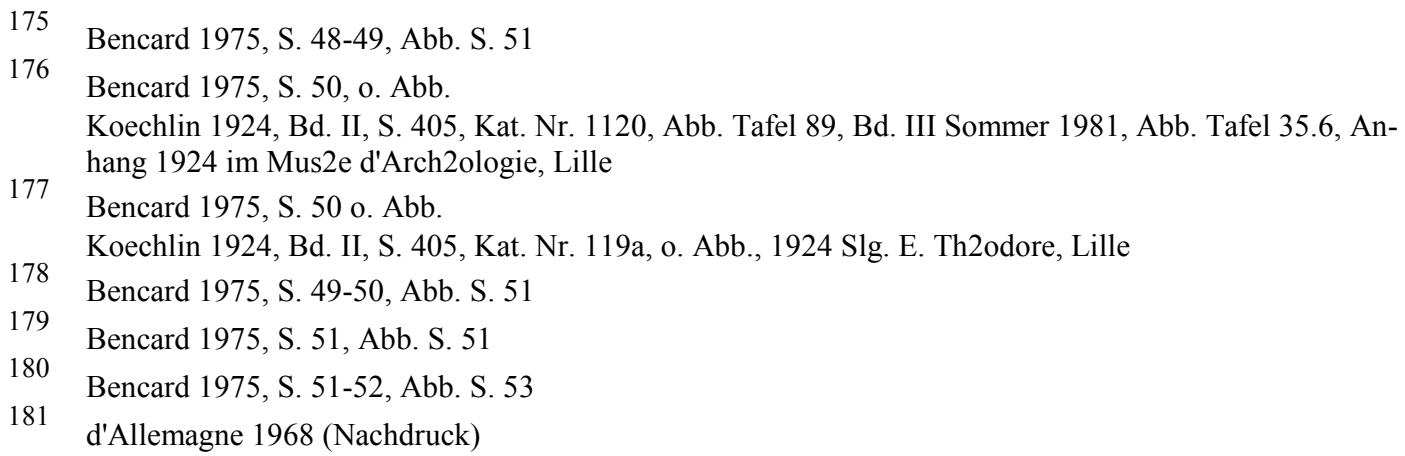


deckung und der Haartracht scheint es sich um eine weibliche Figur zu handeln, die auf einer schmalen Basis steht. Plastizität und Details der Darstellung (Basisgestaltung, Armhaltung, Kopfgestaltung) zeigen Ähnlichkeit mit dem Griff aus Vysoké Mýto (CSFR), die Schrägraffung eines Teils der Gewandung mit denen aus Småland und Reval.

Proportionalität (vor allem der kurze Oberkörper) und das Gesamtbild legen jedoch nahe, in diesem Griff eines der "originalen", das heißt der tatsächlich im stilistischen und ikonographischen Ursprungsraum entstandenen Beispiele $\mathrm{zu}$ sehen, denen die nördlicheren Nachahmungen folgten (Roskilde, Hamburg, Riga etc.).

\section{Osteuropa}

Das einzige dem Autor bekannt gewordene vollständig erhaltene Messer mit einem frontal stehenden, anthropomorph ausgeschnitzten Knochengriff befindet sich im Kunstgewerbemuseum Budapest ${ }^{182}$. Der $8 \mathrm{~cm}$ lange Griff zeigt eine mit Stirnreif versehene männliche Figur auf hohem Sockel, deren Gewand in symmetrisch angelegte und sorgfältig herausgearbeitete Falten fällt. Sie spielt auf einer vor der Brust gehaltenen Laute (Abb. 136e). Angaben zum Fundort wurden nicht gemacht.

Der letzte der hier vorgestellten Falknergriffe, aus einem Rinderknochen geschnitzt, wurde in Vysoké Mýto im Bezirk Wildenschwert (Usti nad Orlici) in Nordostböhmen bei der Ausgrabung einer mit Holzbohlen verschalten Senkgrube gefunden ${ }^{183}$. Keramische Beifunde datieren ihn in das 14. Jahrhundert, nach französischen Analogien wird er in die erste Hälfte des Jahrhunderts datiert ${ }^{184}$.

Der 8,9 cm lange Griff zeigt einen Falkner mit Stirnreifen und in ein bis über die Knie reichendes Gewand gekleidet. Er hält den Falken auf dem rechten Arm, die Hand hält das Obergewand geschlossen. Zu seinen Füßen kauert ein Hund (Abb. 137a).

Die Figur ist in ihren Proportionen am ehesten mit den Exemplaren aus Småland und Reval vergleichbar, wenngleich sich auch Gestaltung und Bildinhalt voneinander unterscheiden, eine allgemeine stilistische Verwandtschaft besteht zu dem Griff aus der Sammlung d'Allemagne.

Sommer stellt die Darstellung in einen allgemeinen, vor allem westeuropäisch geprägten Zusammenhang der Minnesängerkultur ${ }^{185}$.

Vysoké Mýto, im Bezirk Wildenschwert (Usti nad Orlici) an der Wilden Adler, einem Nebenfluß der Oberelbe, liegt an der westlichen Peripherie des im 14. Jahr-

\footnotetext{
182 Benker 1978, S. 47, Abb. 21

183 Sommer 1981, S. 315, Abb. S. 316 und Tafel 34, Anhang

184 Sommer 1981, S. 316

185 Sommer 1981, S. 315
} 
hundert überwiegend von Deutschen bewohnten Siedlungsgebiets Schönhengst im nordöstlichen Böhmen ${ }^{186}$.

Der Ort wurde vor 1265 von deutschen Siedlern gegründet ${ }^{187}$

Ob der exklusive Griff im Zuge der Siedlerbewegung von Westen nach Osten, über das böhmische Kultur- und Handelszentrum Prag oder über Hamburg als Umschlagplatz für Waren, die u.a. von Flandern und den Maasstädten elbeaufwärts über Magdeburg bis nach Böhmen verhandelt wurden ${ }^{188}$, als Luxusimport dorthin gelangte, kann hier nicht geklärt werden.

\section{Seitenansichten}

Die bislang vorgestellten Beispiele waren durchweg Frontalansichten, die seitwärts zur Schneide standen.

Mit dem letzten, vollplastisch anthropomorph ausgeschnitzten Vollgriffmesser, welches aufgrund der besonderen Lagerung vollständig erhalten ist, wird nicht nur die Spanne der Motive und Qualitätsstufen ergänzt, sondern auch eine Seitenansicht vorgestellt.

Im Zuge von Bautätigkeiten im niedersächsischen Heidekloster Wienhausen bei Celle wurde beim Anheben der Fußbodenbohlen im sogenannten Nonnenchor ein vollständig erhaltenes Griffangelmesser geborgen, welches stilistisch in die Zeit um 1300 datiert wurde ${ }^{189}$, wahrscheinlich aber älter ist (siehe weiter unten) (Abb. 138b). Das Messer läßt sich produktionstechnisch in drei Bestandteile gliedern. Die Klinge aus Schmiedeeisen trägt auf der Schauseite eine längs des Rückens verlaufende Silbertauschierung und besitzt im vorderen Klingenbereich eine eingeschlagene Schmiedemarke. Das Heft ist vollständig von einer verzierten Silberblechmanschette umfaßt. Der elfenbeinerne Vollgriff ist ausgeschnitzt zu einer thronenden, bekrönten und mit Schild und Schwert ausgestatteten Königsfigur.

Das Messer besitzt mit der Schmiedemarke eine der frühesten archäologischen Nachweise organisierten Klingenschmiedehandwerks ${ }^{190}$. Die drei erwähnten Produktionsabschnitte lassen auf eine qualitativ hoch anzusetzende und arbeitsteilige Technologie schließen, die in dieser Zeit wohl nur im städtischen Umfeld zu erwarten ist.

Das Messer ging mit zahlreichen weiteren Utensilien aus dem persönlichen Bereich wie Brillen, Nadeln und Amuletten beim Aufenthalt im Nonnenchor verlustig oder wurde dort deponiert.

\footnotetext{
186 Atlas zur Geschichte der deutschen Ostsiedlungen, Velhagen und Klasing 1958, S. 3, 4, 6-7

187 Sommer 1981, S. 317

188 Wecz erka 1973, S. 45

189 Vgl. Katalogteil BRD, Kat. Nr. 74

Appuhn 1973, Text und Abb. S. 9

190 Vgl. Abschnitt 2.6 in diesem Kapitel
} 
Die Bewohnerinnen des Nonnenklosters Wienhausen lassen sich aufgrund historischer Quellen dem Nieder- und Hochadel zuordnen.

Das zwischen 1221 und 1229 von Herzog Heinrich von Braunschweig, einem Sohn Heinrichs des Löwen und seiner Gemahlin Agnes, einer gebürtigen Markgräfin von Meißen, gegründete Nonnenkloster beherbergte in den ersten zwei Jahrhunderten Frauen aus dem niederen Adel. Drei Äbtissinen des 14. Jahrhunderts waren Angehörige des braunschweigisch-lüneburgischen Herrscherhauses ${ }^{191}$.

Das Messer aus Wienhausen findet in seiner künstlerischen wie produktionstechnischen Qualität nur eine Parallele innerhalb der oben beschriebenen Gruppe in dem Exemplar aus Ribe, welches sich ebenfalls durch hohe gestalterische Qualität, einen achtkantigen Silbersockel am Heft und durch Reste einer Silbertauschierung in der Klinge von den übrigen Exemplaren abhebt.

\section{Die halbplastische Darstellung und vollplastische Ausformung des Griffendes}

Daß es im Mittelalter nicht nur vollständig und vollplastisch ausgearbeitete anthropomorphe Griffgestaltung gegeben hat, zeigen die folgenden Beispiele.

Sie umfassen halbplastische und stark stilisierte Darstellungen sowie Griffendverzierungen vom 11. bis 14 . Jahrhundert.

Das älteste im Untersuchungsraum erfaßte Beispiel einer anthropomorphen Griffgestaltung zeigt einen hölzernen Vollgriff, dessen Oberfläche durch Kreisaugen verziert ist und dessen Ende einen stark stilisierten menschlichen Kopf darstellt (Abb. 137b). Der Griff wurde 1926 zusammen mit dem Schlüssel eines Bolzenschlosses im westschwedischen Skara gefunden und in das 11. bis 12. Jahrhundert datiert ${ }^{192}$.

Der Fundplatz an der Südseite des Hauptmarktes in der mittelalterlichen Bischofsstadt Skara ${ }^{193}$ darf unter Berücksichtigung des Beifundes wahrscheinlich als Siedlungsfund einer exponierten Wohnlage gelten, die unsichere Datierung erschwert aber eine Eingrenzung des sozialen Umfeldes.

In Halle an der Saale wurde ein halbplastisch verzierter Messergriff gefunden. Der an den Anfang des 12. Jahrhunderts datierte Griff stellt einen halbplastisch aus einem Röhrenknochen geschnittenen schildbewehrten Ritter dar, der zum Heft und Griffende durch mit Schrägstrichdekor angefüllte Gurtzonen begrenzt ist (Abb. 138a).

Wohl eher wegen der Art der Darstellung wurde er einem dem Fundort benachbarten "ritterlichen Haushalt" zugeordnet ${ }^{194}$.

\footnotetext{
191 Maier 1972, S. 7-8

192 Kartei des Statens Historiska Museum Stockholm, Inv. Nr. 18117:2, Photo Nr. 2002:53

193 Andersson 1983, S. 189

194 Vgl. Katalogteil ehemalige DDR, Kat. Nr. 169

Voigt 1950, S. 176, S. 178, Anmerkung 11
} 
Das Beispiel für die anthropomorphe Griffendgestaltung stammt aus Köln ${ }^{195}$. Der heute im ehemaligen Schnütgen-Museum aufbewahrte, 9,2 cm lange elfenbeinerne Vollgriff wird als Altfund ohne genauere Angaben zum Fundort aufgeführt ${ }^{196}$. Er zeigt einen im Schnitt rechtekkigen Griff, dessen Ende zu einer mit Schild, Waffenrock und Topfhelm bewehrten Reiterfigur ausgeschnitzt wurde (Abb. 137c).

Der als Bodenfund dem Kölner Innenstadtbereich entstammende Griff kann archäologisch lediglich im weitesten Sinne einem nicht näher $\mathrm{zu}$ bestimmenden städtischen Milieu des 13. bis 14. Jahrhunderts zugeordnet werden.

Ein weiterer halbplastisch verzierter Messergriff aus Knochen wurde 1885 bei einer Untersuchung der Ruinen von Burg Falkenberg im schwedischen Falkenberg geborgen. Der 8,6 cm lange Griff zeigt einen gerüsteten Ritter mit hochgeklapptem Visier und vor der Brust gefalteten Händen (Abb. 138c).

Lediglich das Gesicht und die Haartracht erinnern noch an die vollplastischen Falknergriffe. Der Griff, von den Bearbeitern in das 14. Jahrhundert datiert ${ }^{197}$, zeigt an der Griffunterkante, die bei der Führung des Messers von den Fingern umfaßt wurde, eine plastische Konturenführung, während die Oberkante durch einen Grat abgesetzt gerade verläuft. Dadurch ist der Griff hinten breiter als am Heft und erinnert bereits stark an die vor allem im ausgehenden 14. und 15. Jahrhundert entwickelten Griffzungenmesser.

Die im ausgehenden 13. Jahrhundert schriftlich erwähnte Burg Falkenberg wurde 1434 zerstört $^{198}$.

\footnotetext{
195 Vgl. Katalogteil BRD, Kat. Nr. 172

196 Steuer 1982, S. 13

197 Kartei des Statens Historiska Museum Stockholm, Inv. Nr. 7764:174, Photo Nr.1866:37

198 nach Museumskartei: Gustafson, Falkenbergs hus, Kungl. Vitterhets Hist. och Ant. Akademiens månadsblad 1885, S. $198 \mathrm{f}$.
} 

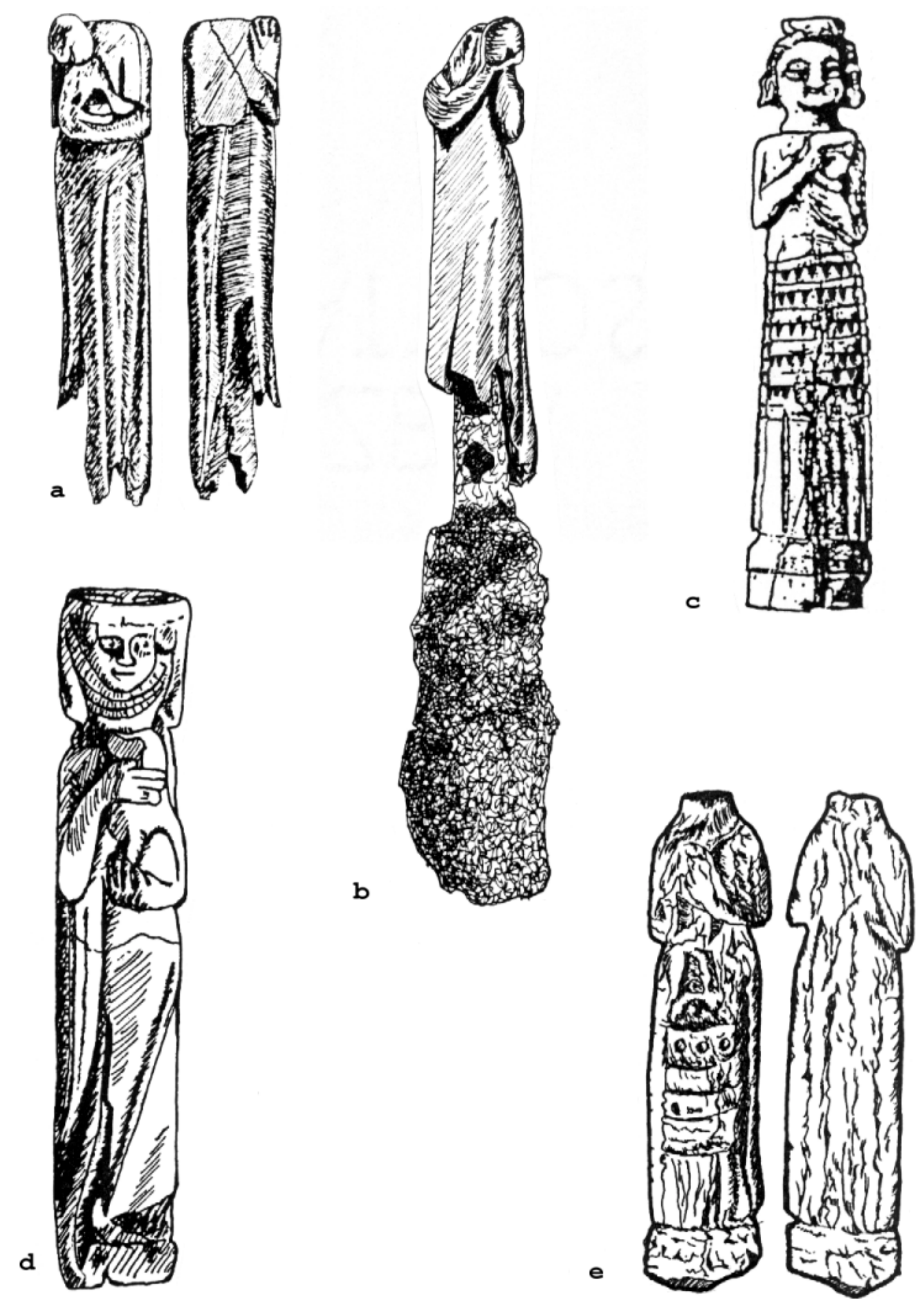

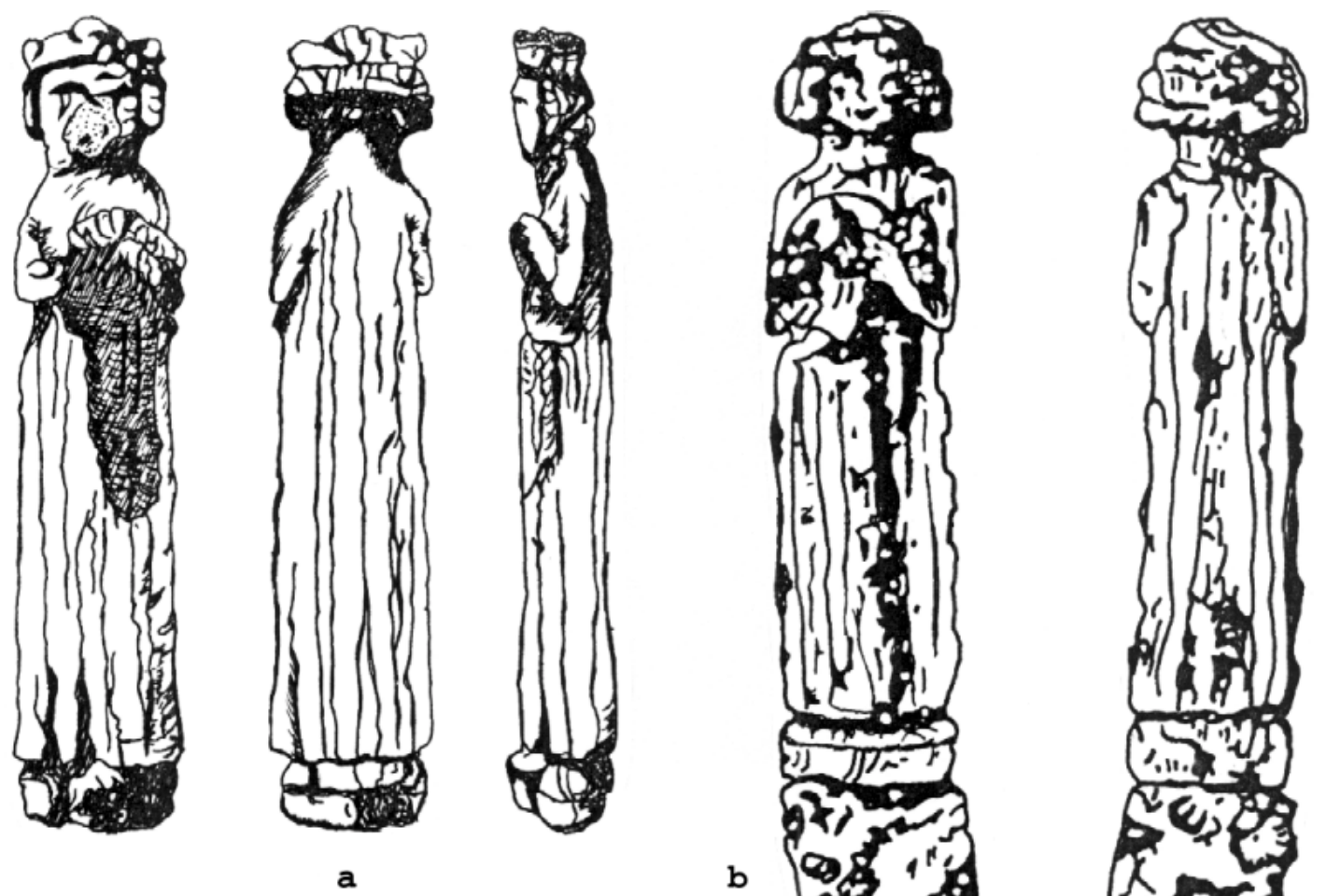

b
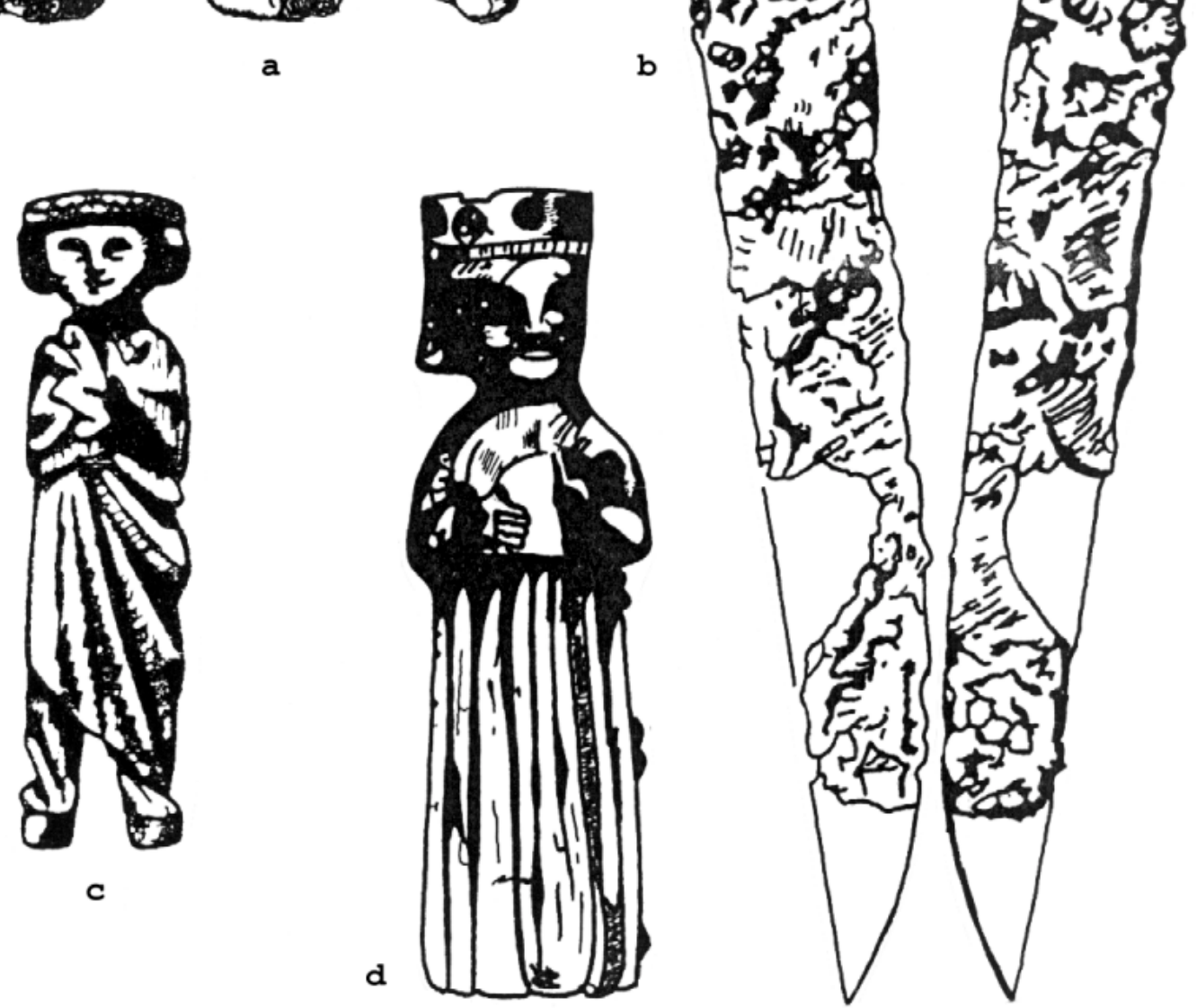


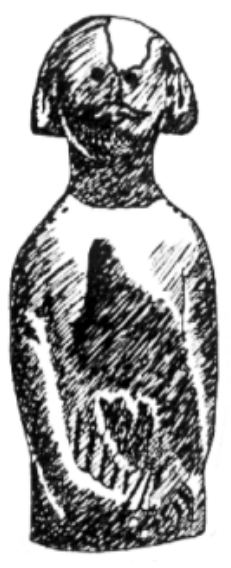

a
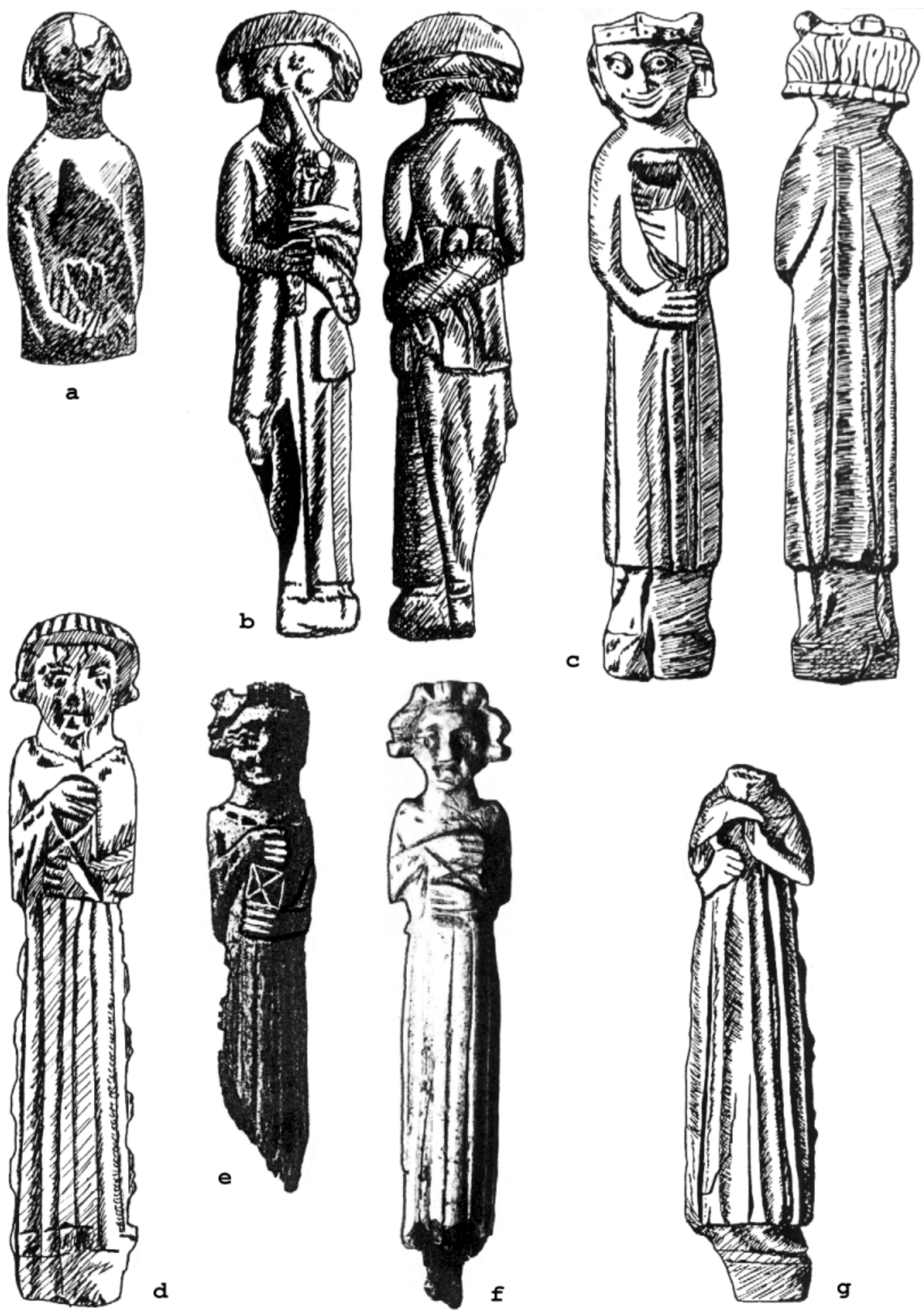

Abb. 133

Anthropomorph gestaltete spätmittelalterliche Messergriffe III a: Oslo, b: Visby; c-g: Kalmar 

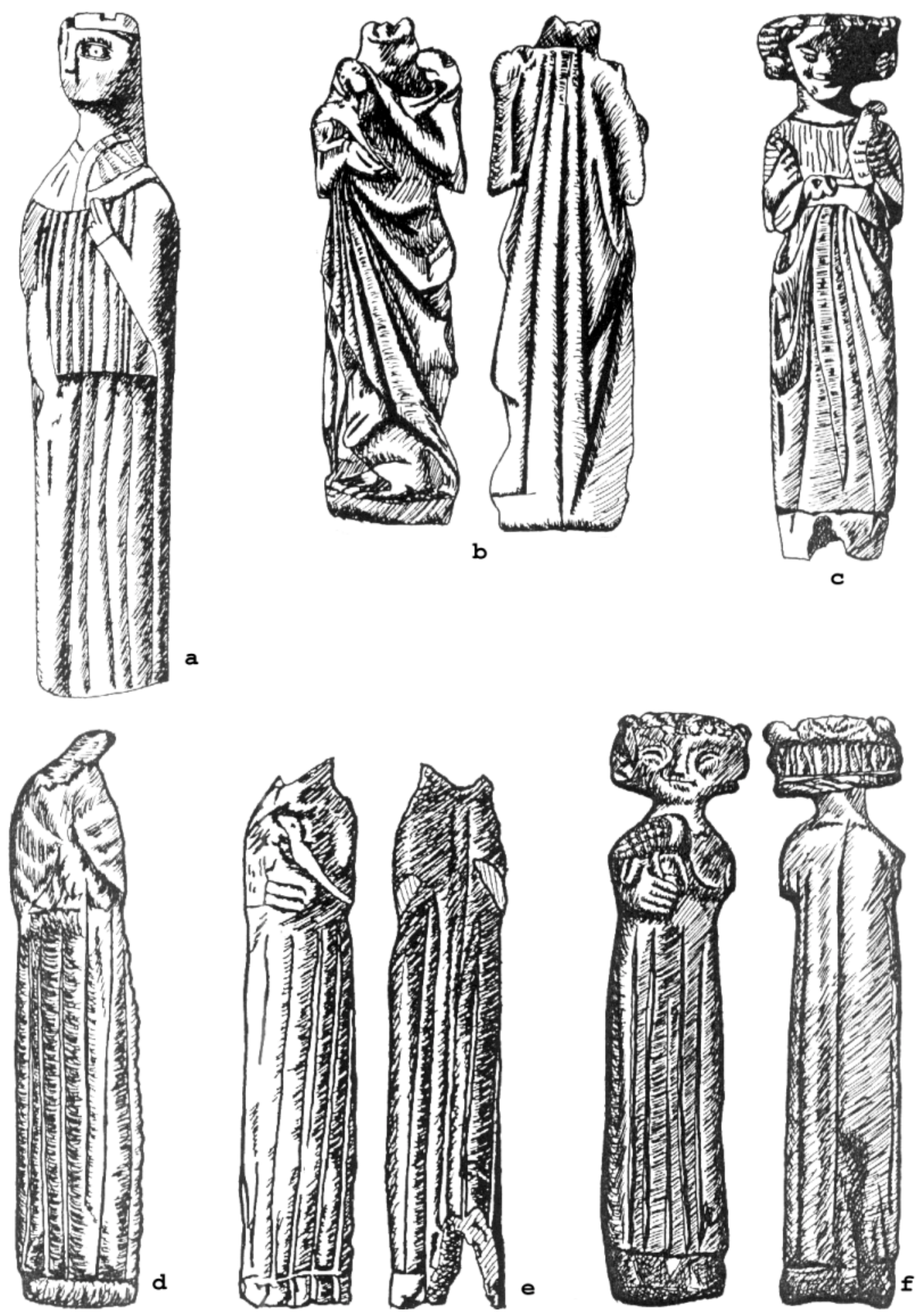

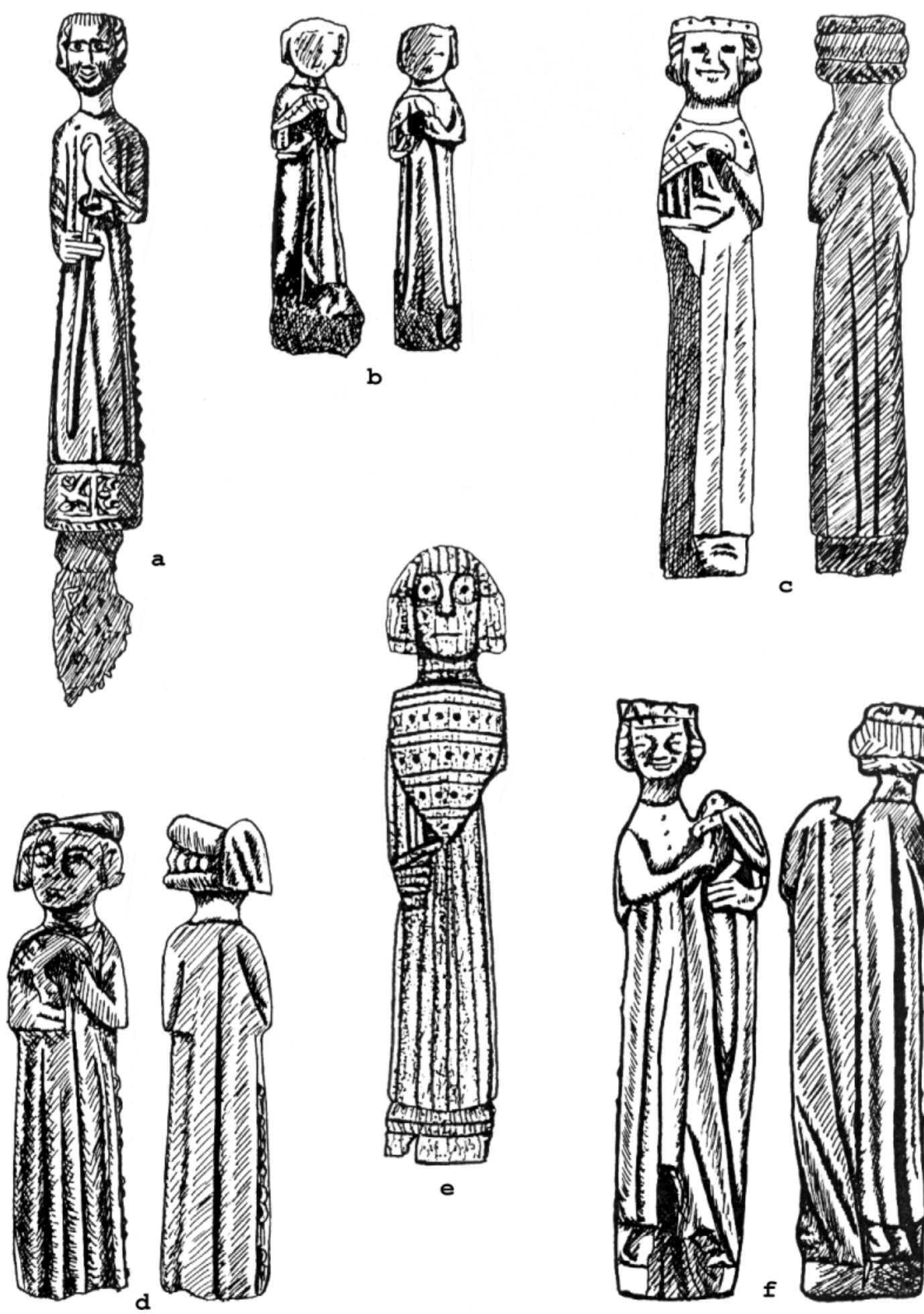

Abb. 135 Anthropomorph gestaltete spätmittelalterliche Messergriffe $V$ $a$ : Ribe; b: Skanderborg; c: Herlufsholm; d: Schonen; e-f: Roskilde 


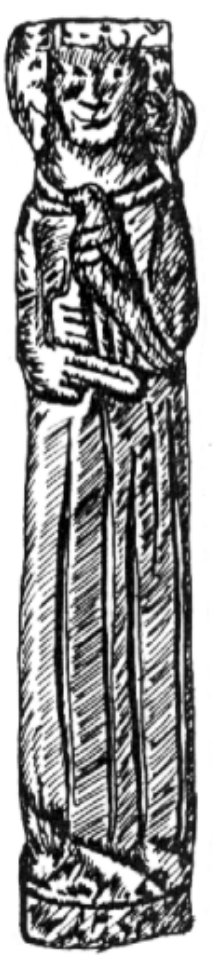

a
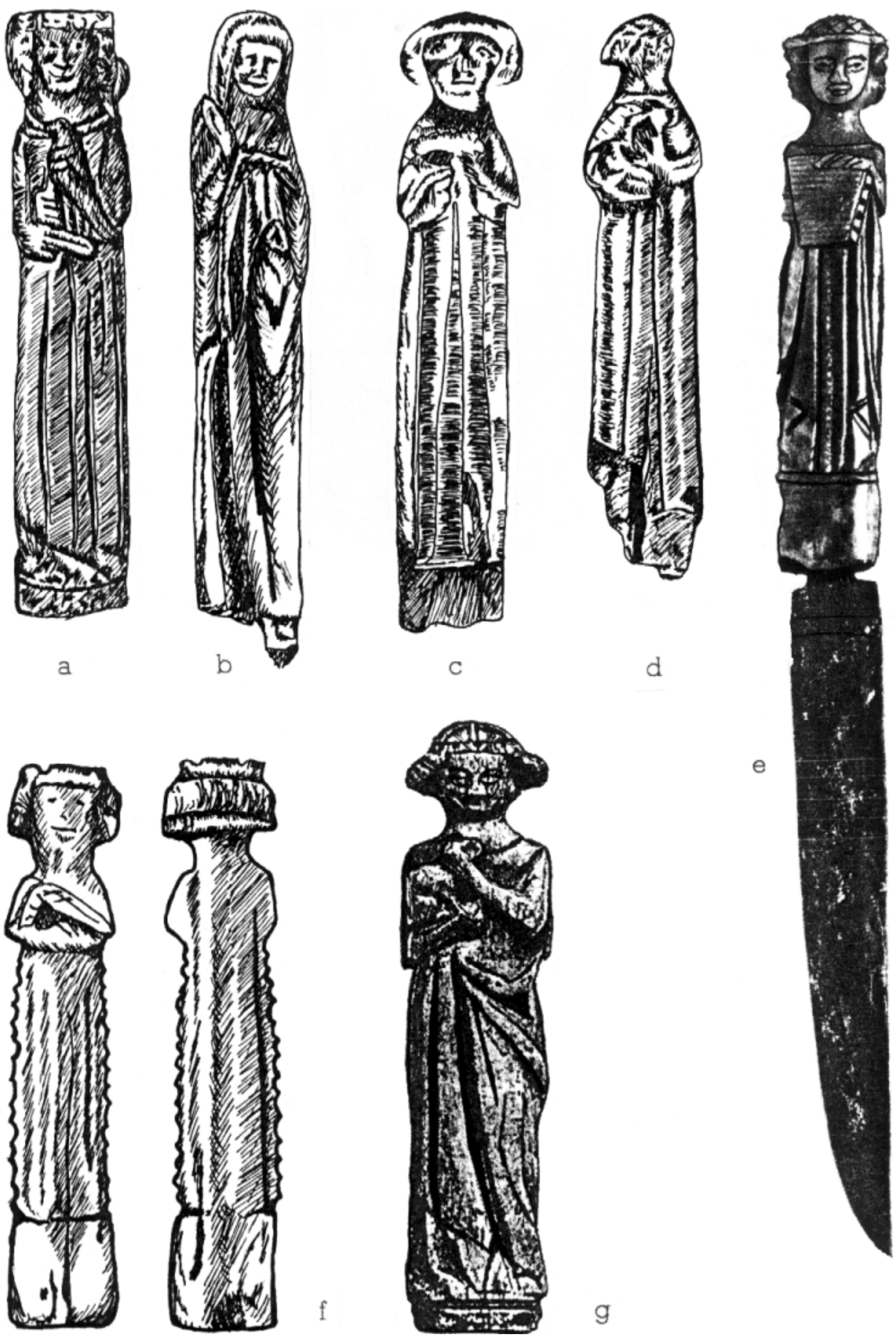

Abb. 136

Anthropomorph gestaltete spätmittelalterliche Messergriffe VI a: Oxford, b. Arras, c: Lüttich; d: Friesland; e. Fundort nicht bekannt; $f$ : Scharnegoutum; g: Fundort nicht bekannt 

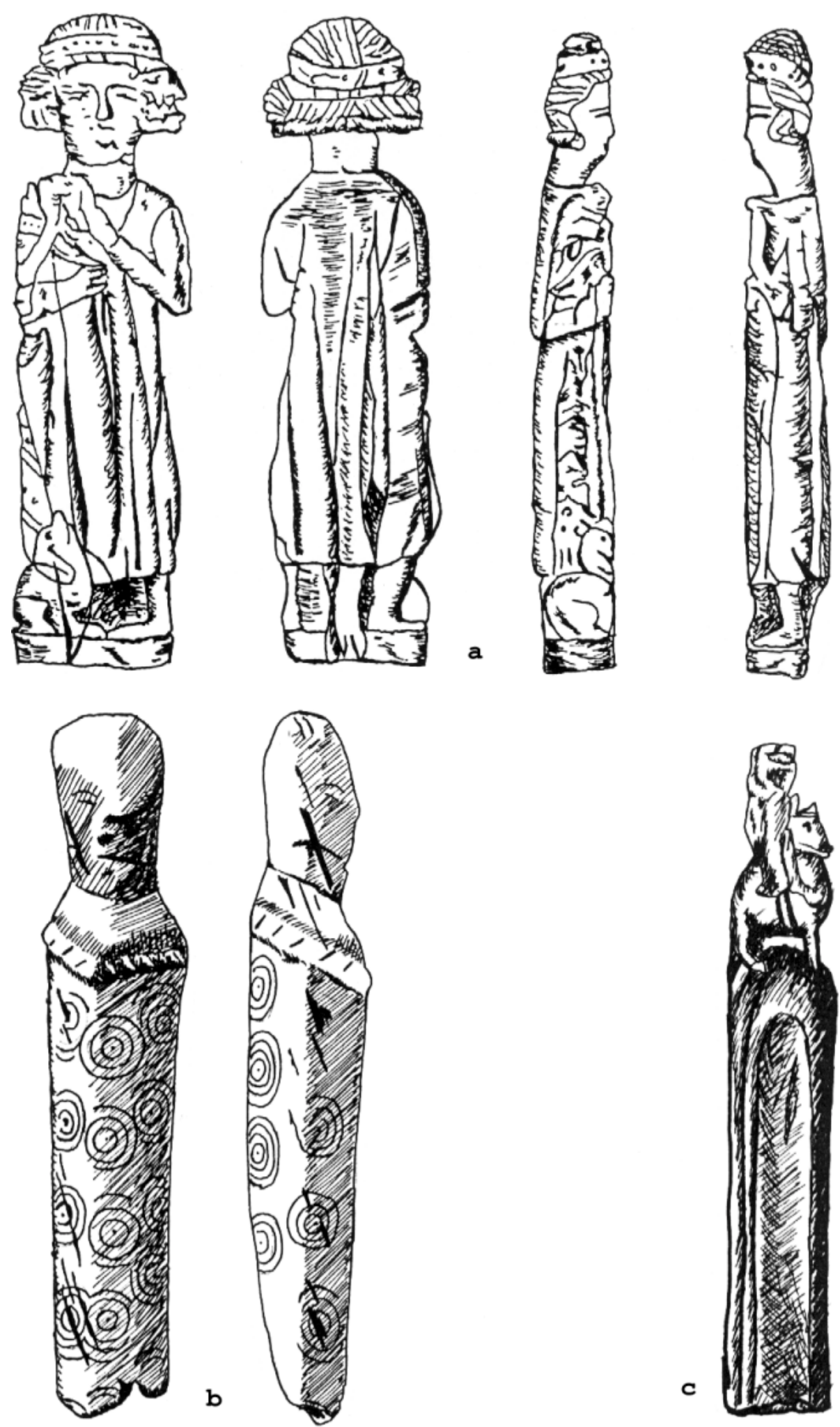

Abb. 137

Anthropomorph gestaltete spätmittelalterliche Messergriffe VII a: Vysoké Mýto, b: Skara, c: Köln 

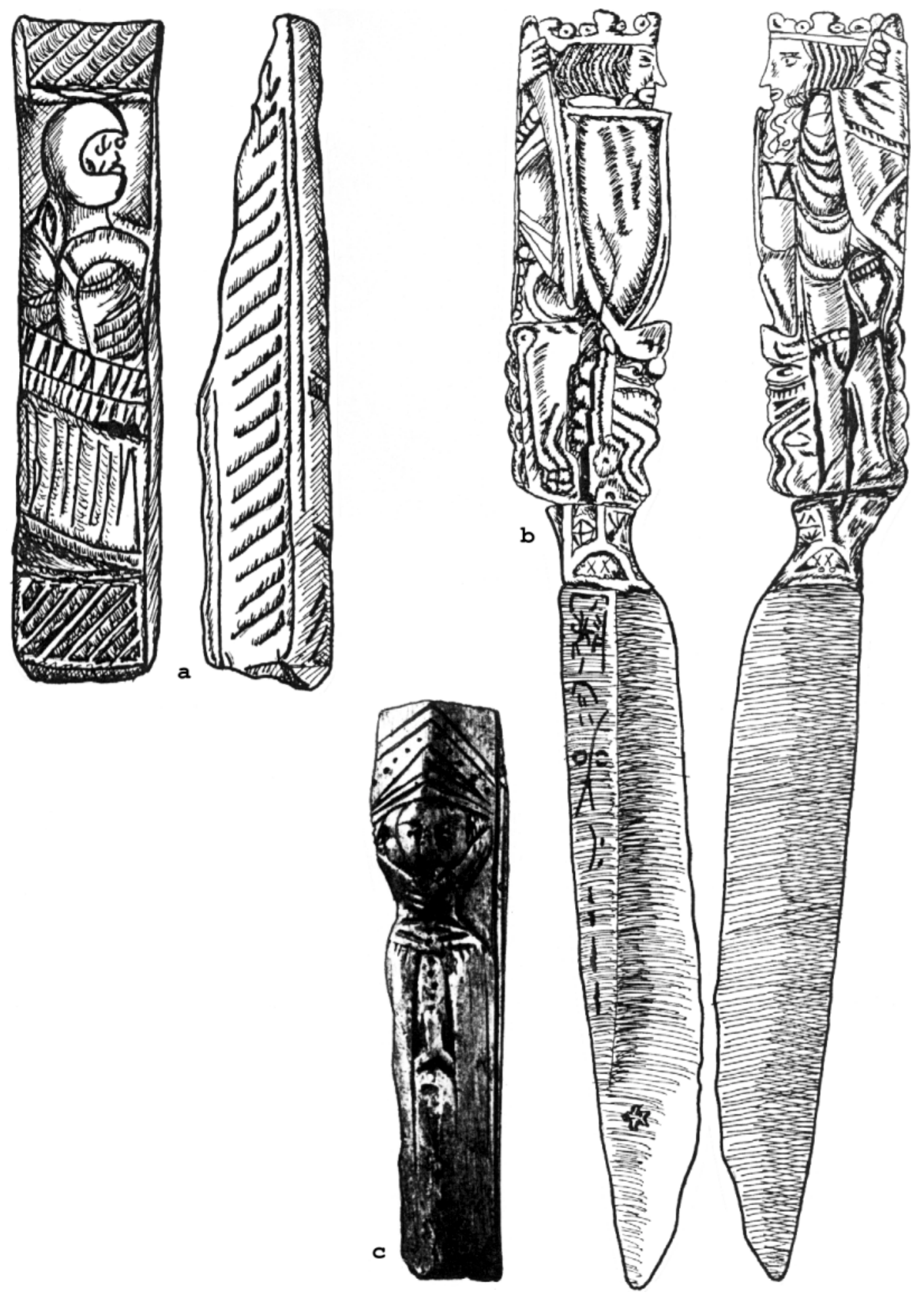

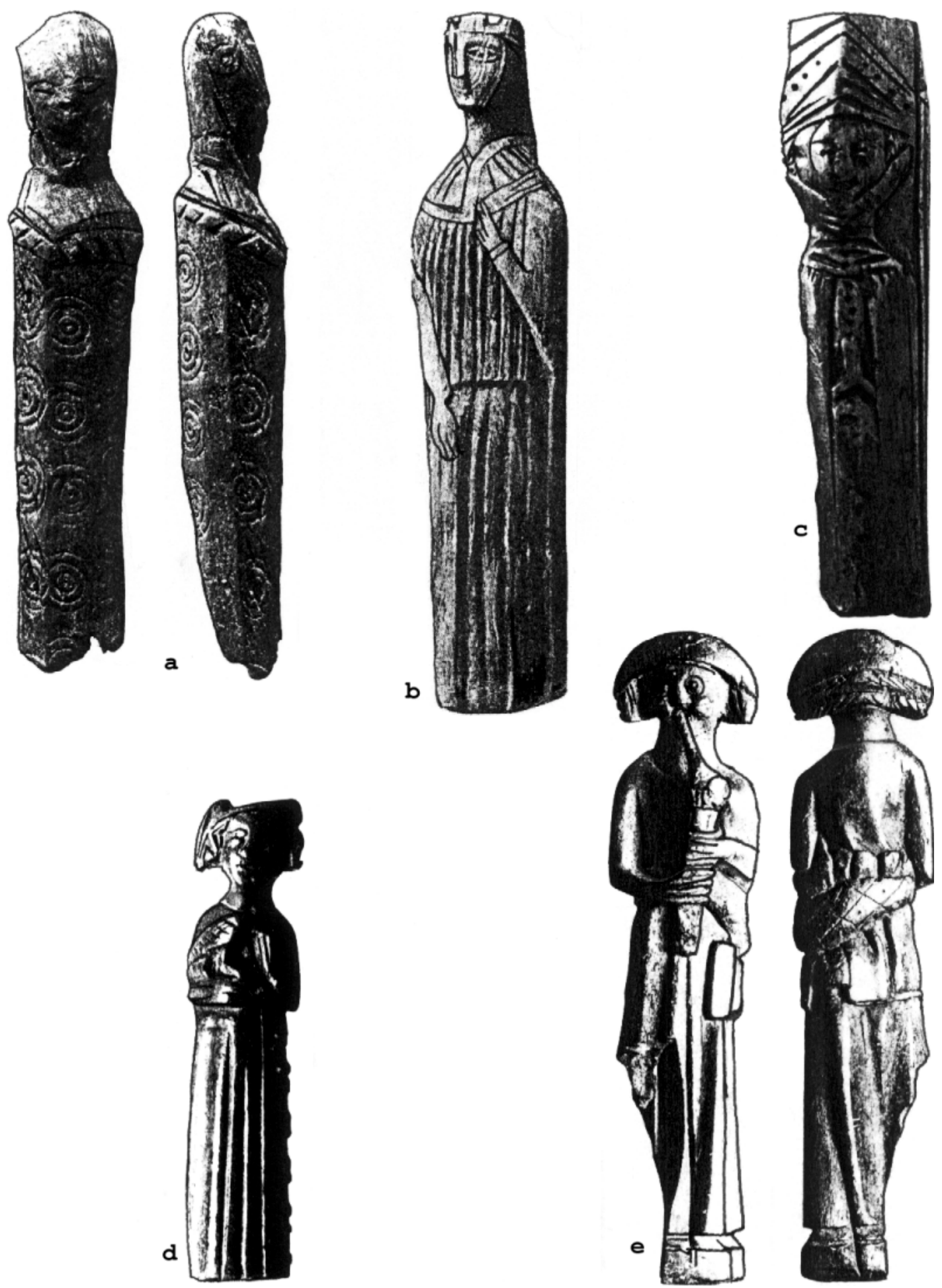

Phototafel I

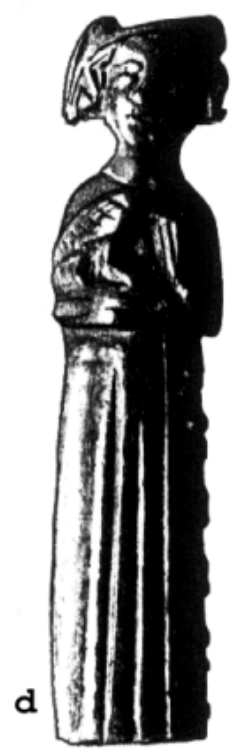

a: Skara; b. Kalmar, c: Falkenberg; d: Schonen, e: Visby 

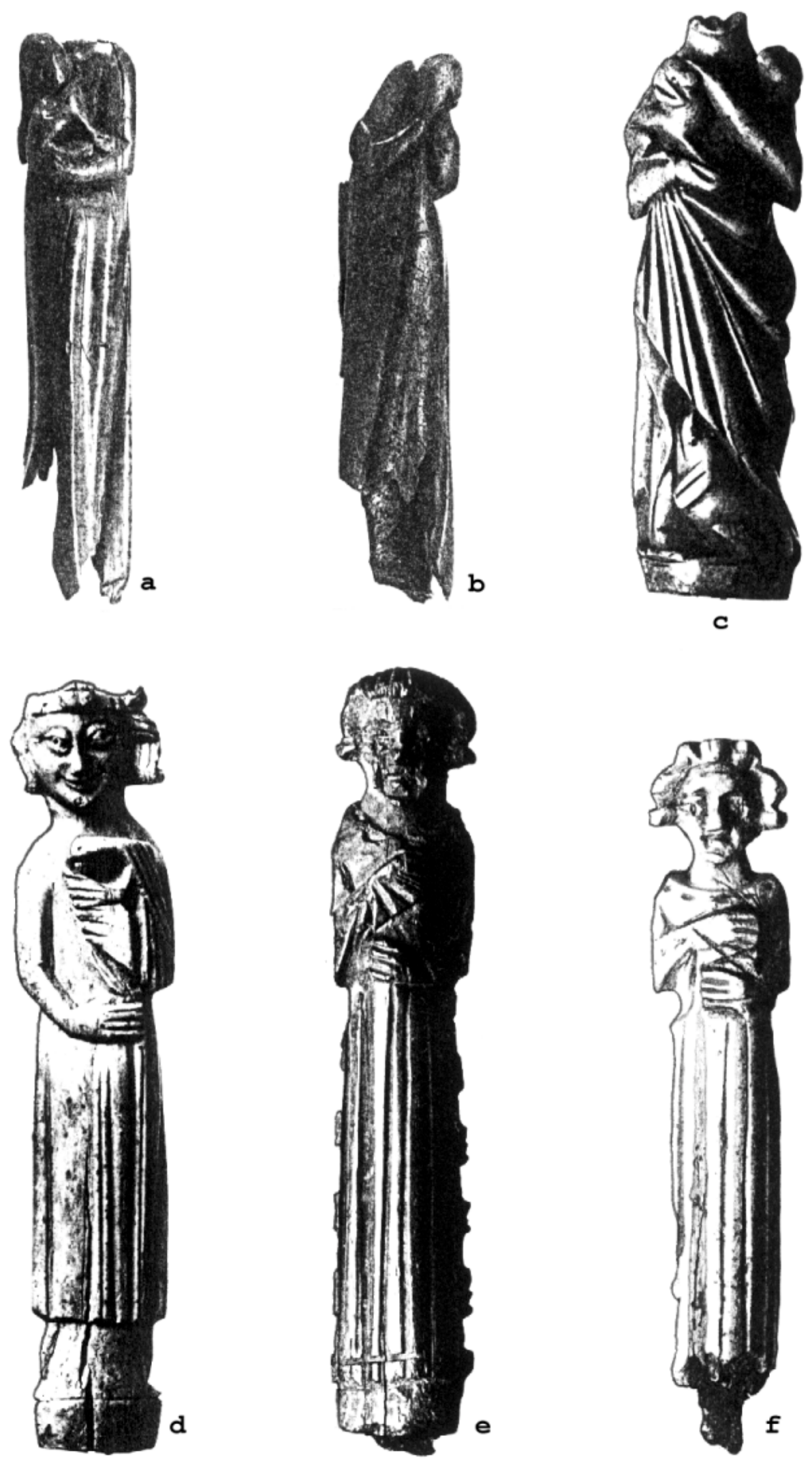

Phototafel II $\quad a-b$ : Lübeck; $c$ : Småland; d-f. Kalmar 

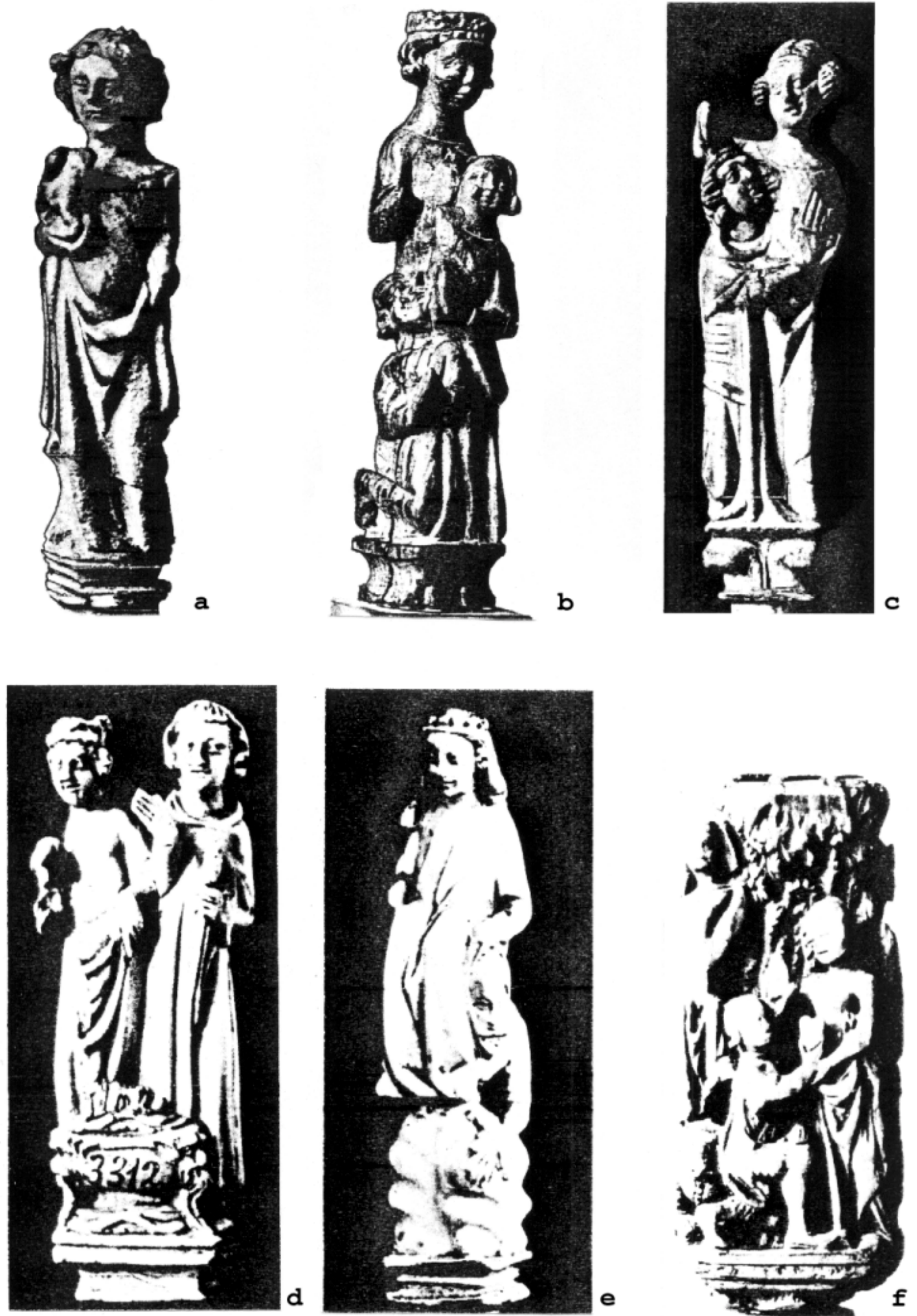

Phototafel III

von R. Koechlin (1924) Pariser Schnitzern zugewiesene Messergriffe des 14. Jh. a: Paris (Seinefund), M. 14.Jh.; b: Frankreich, E. 13.Jh.; c: Frankreich, M. 14.Jh.;

d: Frankreich, 1. H. 14.Jh.; f: Frankreich, 1. H. 14. Jh. 


\subsection{Zur historischen Einordnung der anthropomorph verzierten Messergriffe}

Die bisher geführte Diskussion um die beschriebene Gruppe von Messergriffen geht davon aus, daß die Motive, die als Vorbilder für die dargestellten Personen gedient haben, aus einem allgemeinkulturellen Zusammenhang stammen; das heißt, die Griffe stellen keine Persönlichkeiten, sondern Ideale dar ${ }^{199}$.

Zusammenfassen lassen sich die von Bencard, Leciejewicz und Sommer geäußerten Interpretationen dahingehend, der Ursprung der Griffe sei in Westeuropa mit besonderem Hinweis auf die Elfenbeinschnitzereien in Paris $\mathrm{zu}$ suchen. Ihre Herstellung vermuten sie in französischen oder englischen Provinzwerkstätten unter Pariser Einfluß, z.T. aber auch in lokalen, d.h. im Zusammenhang mit dem Fundort stehenden Werkstätten $^{200}$.

Die Datierung der Griffe erfolgte, abgesehen von dem archäologisch begründeten Zeitrahmen des Fundorts Ringkloster bei Skanderborg durchweg nach kunsthistorischen Gesichtspunkten, wobei die Zeitspanne von 1250 bis 1350, die die Blüte der Pariser Elfenbeinschnitzerei markiert ${ }^{201}$, sowie die aus Bildquellen gewonnene Datierung der dargestellten Kleidung von der ersten Hälfte des 13. Jahrhunderts bis in die Mitte des 14. Jahrhunderts ${ }^{202}$ die plausibelsten sind.

Das größte Hindernis, diese Gruppe von Messergriffen in ihrem ursprünglichen Zusammenhang darzustellen, ergibt sich aus dem weitgehenden Fehlen von Informationen aus Befundzusammenhängen: fast alle Griffe sind quellenkritisch als Einzelfunde zu bezeichnen.

Daher kann eine Einordnung in historische oder soziale Kontexte nur ein Versuch bleiben, die dazu notwendigen Informationen müssen über die dem Archäologen nur zu bekannten Umwege gewonnen werden, wenn sichere Stratigraphien oder Befundzuweisungen fehlen.

\section{Die archäologische Datierung}

Die einzigen Griffe, die sicher in einem engeren Zeitrahmen faßbar sind, wurden auf der historisch datierten Isenburg bei Essen (1244 bis 1288) und in der Hundestraße in Lübeck (stratigraphisch zweite Hälfte des 13. Jahrhunderts) gefunden. Weitere Datierungen auf archäologischer Quellenbasis stammen aus dem Ringkloster bei Skanderborg (1200 bis 1430), aus Riga (13. bis 14. Jahrhundert) und aus Vysoké Mýto (14. Jahrhundert).

\footnotetext{
199 Bencard 1976, S. 40, S. 59-60

200 Bencard 1975, S. 59

Leciejewicz 1974, S. 186 
Faßt man die Gruppe als Ganzes, so ergibt sich ein gesicherter zeitlicher Rahmen vom 13. bis zum 14. Jahrhundert, mit einem Schwerpunkt in der zweiten Hälfte des 13. Jahrhunderts.

Die stilistischen Unterschiede mahnen aber zur Vorsicht, die Messergriffe als eine einheitliche Gruppe zu betrachten.

\section{Die Fundorte}

Die Aussagekraft der Fundorte ist wegen der fehlenden Informationen, die sich aus den Befundkontexten hätten ergeben müssen, eine wichtige Quelle, um Aussagen über Vertrieb und Absatz treffen zu können.

Einige Daten zu den Fundorten wurden bereits in der Beschreibung der Messergriffe erwähnt.

Zunächst läßt sich feststellen, daß die meisten Griffe in Stadtzentren, einige in Burgen und Klöstern gefunden worden sind. Die Mehrzahl der Fundorte wie Lüttich, Köln, Hamburg, Lübeck, Ribe, Roskilde, Visby, Riga und Reval nimmt vor allem im wirtschaftlichen und kulturellen Leben des 13. und 14. Jahrhunderts eine hervorragende Stellung ein.

Die Verbreitungskarte zeigt deutlich die Konzentration auf zwei führende Kulturräume des Spätmittelalters, den Wirtschaftsraum der Hanse von Flandern und dem Niederrhein über den östlichen hansischen Kernraum bis zu wichtigen Stützpunkten an den Küsten der Nord- und Ostsee sowie den politischen Machtbereich des größten Gegenspielers der Hanse, des Königreichs Dänemark (Abb. 139).

Bereits hieraus läßt sich ableiten, daß - wo auch immer die Griffe im einzelnen produziert wurden - sie im Wirtschaftsraum der Hanse transportiert wurden und vor allem städtisches Bürgertum als Abnehmer mit hoher Wahrscheinlichkeit anzunehmen ist.

Die stilistischen und qualitativen Unterschiede der einzelnen Griffe sowie historisch-topographische Informationen über die einzelnen Fundorte lassen aber auch weiterreichende Aussagen zu.

Die beiden herausragenden Beispiele in der Gruppe anthropomorph verzierter Messergriffe sind das Messer aus dem Kloster Wienhausen und das Messerfragment vom Hauptmarkt in Ribe. Beide Exemplare sind bis in die Details vollplastisch aus Elfenbein geschnitzt, ihre künstlerische Gestaltung, die Verwendung von Silberblech am Heft und die Verzierung und Markung der Klingen berechtigen zu der Feststellung, daß sie im Rahmen der gehobenen Sachkultur zu den qualitätvollsten Erzeugnissen in der profanen Kleinkunst des Spätmittelalters zählen.

Im Zuge der Beschreibung des Wienhausener Exemplars ist bereits dargelegt worden, daß das Messer wahrscheinlich älter ist als die Mehrzahl der übrigen Beispiele und die Besitzerin dieses Messers im Kreis der adeligen Bewohnerinnen zu suchen ist. 
Das Messerfragment aus Ribe ist zwar ein Lesefund, dennoch lassen die Fundsituation und das Messer selbst weiterreichende Rückschlüsse zu.

Gefunden wurde es an der Einmündung des Overdammen, der innerstädtischen Verlängerung der mittelalterlichen Salzstraße nach Ribe, auf dem Torvet, dem Hauptmarkt, nordöstlich des Domchores und südöstlich vom alten Rathaus Ribes in der Grønnegade ${ }^{203}$. Das Quartier um den Domplatz und an der Gronnegade wurde im 13. und 14. Jahrhundert bewohnt von stadtsässigem Adel und führenden Kaufmannsfamilien, der früheste nachweisbare Besitzer des steinernen Doppelhauses in unmittelbarer Nähe zum Fundort war Jon Bonde (gest. 1341), Kaufmann und Ratsherr der Stadt, dessen Ehefrau als "Adelige Dame" bezeichnet wurde ${ }^{204}$.

Aus der Serie der Falknerdarstellungen ragt das Exemplar vom Torvet in Ribe durch Proportionalität, Feinheit und Detailreichtum in der Ausführung, durch die verarbeiteten Materialien und die eingelegte Klingenmarke heraus.

Diese Korrespondenz zwischen der topographisch zentralen Lage des Fundplatzes und der herausragenden Qualität des Fundstücks führte bereits Bencard dazu, das Messer in Ribes reicher Bürgerschaft anzu siedeln.

Die Städte, in denen Messergriffe der hier behandelten Gruppe gefunden wurden, werden fast durchweg durch die Anwesenheit deutscher Hansekaufleute gekennzeichnet, zum großen Teil auch durch die Mitgliedschaft in der späteren Städtehanse.

Lübeck und Visby waren vom ausgehenden 12. bis zur Mitte des 14. Jahrhunderts Stützpunkte der Kaufmannshanse, einer Vereinigung von Kaufleuten unterschiedlicher, zu Beginn vor allem nordwestdeutscher Städte, die mit Privilegien ausgestattet an den Küsten der Nord- und Ostsee überregionalen Handel trieben und von Visby aus - gestützt auf den dort bereits vorhandenen gotländischen Fernhandel - schon früh am Rußlandhandel partizipierten ${ }^{205}$.

Im Zuge der Neugründung der Ostseestädte, deren Kaufleute der Kaufmannshanse angehörten, entwickelte sich bereits bis zur Mitte des 13. Jahrhunderts die Hauptachse des hansischen West-Osthandels, die von Groningen/Dortmund bis nach Visby und Riga reichte. Wiebei den meisten Städten in diesem Raum waren deutsche Kaufleute auch bei der Gründung bzw. Neugründung älterer Siedlungsplätze von Reval, Riga, Stettin und Kalmar wesentlich beteiligt ${ }^{206}$.

\footnotetext{
203 Bencard 1975, S. 36

Bencard 1977, S. 60

Bencard 1977, S. 59 
Verbreitungskarte

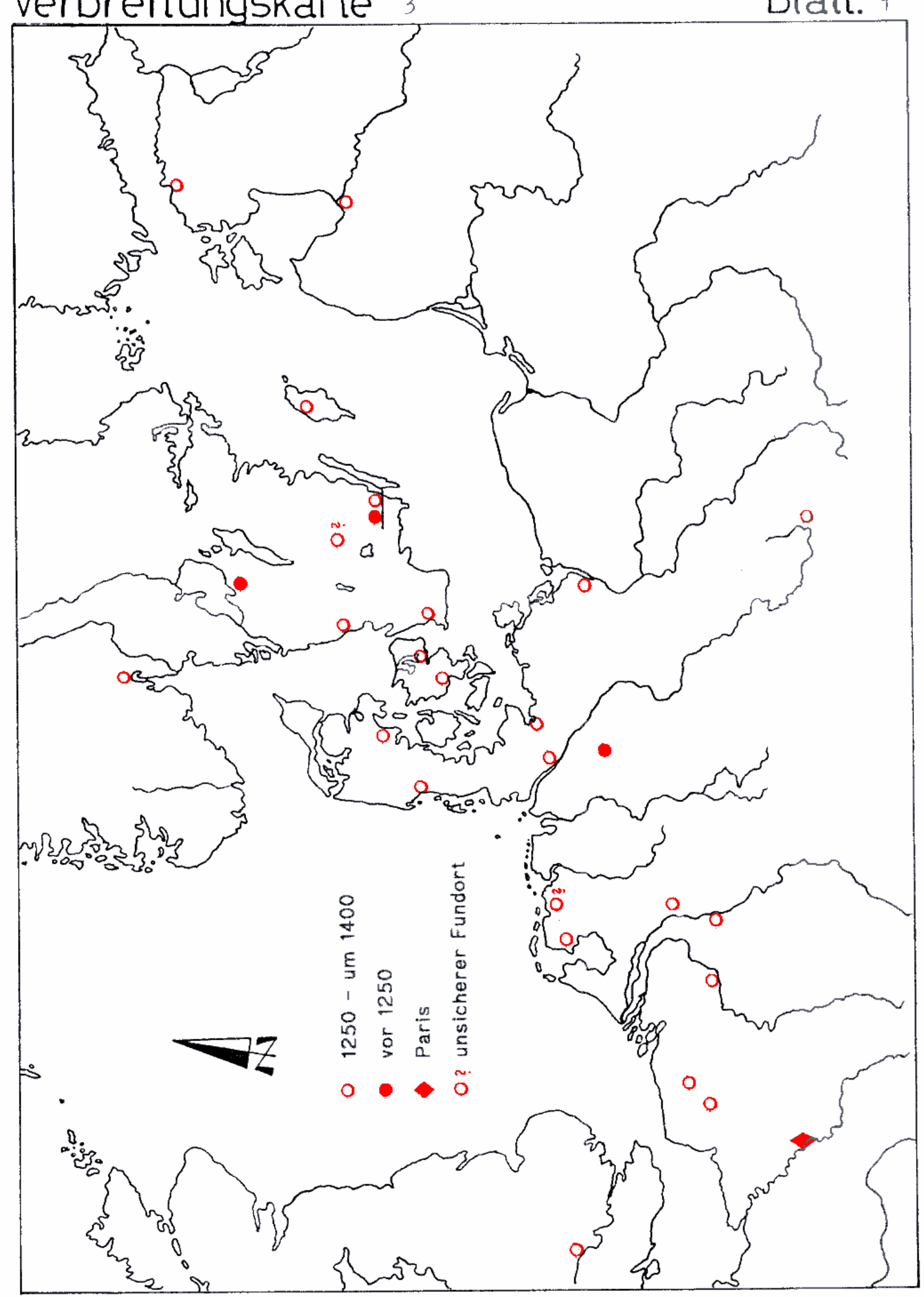

Abb. 139

Die Verbreitung höfischer Topoi auf plastisch gestalteten mittelalterlichen Messergriffen 
In Visby wurden neben der "Gesellschaft der Gotlandfahrer" immer mehr deutsche Kaufleute seßhaft, 1275 wurde die Stadt eine deutsch-gotländische Gemeinde ${ }^{207}$. Das Fehlen von Nachweisen anthropomorph verzierter Messergriffe zwischen Stettin und Riga kann eine Forschungslücke oder Zufall sein. Erstaunlich ist aber dennoch, daß ausgerechnet in den Ländern des Deutschen Ordens, die nicht nur eine hohe Dichte ritterlicher Bevölkerung, sondern auch mit dem Hochmeister des Ordens den einzigen Fürsten in der Organisation der Hanse stellten und wirtschaftlich wie politisch eng mit der Hanse verbunden waren ${ }^{208}$, kein derartiges Beispiel nachzuweisen ist.

In Schweden entwickelte sich seit dem 13. Jahrhundert neben dem Ostseehandel auch der Bergbau unter dem Einfluß deutscher Kauf- und Bergleute. Vor allem Kupfer- und Eisenerz wurden über die Hanse zum Kontinent verschifft. Als Nachfolgerin der Handelsplätze am Mälarsee wurde 1251 Stockholm, danach Kalmar, Söderköping und das finnische Åbo unter starker Beteiligung deutscher Kaufleute als Stadtgemeinden gegründet ${ }^{209}$.

Die in Kalmar gefundenen Griffe sind qualitativ sicher in das obere Drittel der hier vorgestellten Messergriffe einzuordnen. Einen Bezug zum Erzexport herzustellen, wäre hypothetisch. Das Beispiel Kalmar zeigt aber, wie vielfältig die Möglichkeiten sind, die als Grundlage für das Auftauchen oder die Imitation teuren Importgutes im hansischen Wirtschaftsraum in Frage kommen.

Auch in Norwegen war der Einfluß deutscher Kaufleute wirksam. Über die Kontore und Faktoreien in Bergen und Oslo brachten Lübeck, Hamburg und Bremen, später Rostock fast den gesamten norwegischen Ausfuhrhandel unter ihre Kontrolle. In Oslo, Residenzstadt des Herzogs Håkon und seit 1299/1301 königliche Residenz, nahmen norddeutsche Kaufleute eine privilegierte Sonderstellung ein. 1286 von Håkon aufgefordert, nach Oslo zu kommen, genossen sie weitreichende Freiheiten und handelten, anders als in Bergen, selbständig und ohne hansischen Handelshäusern unterstellt zu sein.

Der Handel in Südnorwegen war nie so umfangreich wie in Bergen und betraf vor allem sogenannte Bauernwaren wie Tierhäute, Felle, Schlachtvieh, Teer und Butter im Gegenzug zu Bier, Malz, Getreide und Wein aus Deutschland und den Ostseeländern, die zum Teil mit den Bauern direkt verhandelt wurden ${ }^{210}$.

Es ist vielleicht kein Zufall, daß das Grifffragment aus Oslo stilistisch eine Sonderstellung einnimmt und bereits von Bencard als einheimische Produktion angesehen wurde. Möglicherweise besteht zwischen der Wertigkeit der verhandelten Waren und der geringeren Qualität des Messergriffs ein Zusammenhang.

\footnotetext{
207 Svahnström 1973, S. 216-217

208 Dollinger 1973, S. 28

Pagel 1983, S. 37 ff

209 Weczerka 1973, S. 45-46

Dollinger 1989, S. 56-57

210 Blom 1973, S. 159-162
} 
Die Fundortangabe "gamle byen" bezeichnet das Gebiet zwischen der Königsburg und dem Bischofspalast, die mittelalterliche, in Holzbauweise ausgeführte Stadt ${ }^{211}$. Wie in Bergen, so gab es auch in Oslo eine deutsche Schuhmacherkolonie. Sie stellte in einer vom König zur Verfügung gestellten Hofanlage Schuhe besserer Qualität her als die einheimischen Schuhmacher, was zu Auseinandersetzungen zwischen den beiden Gruppen führte ${ }^{212}$.

Die historisch belegte Anwesenheit privilegierter deutscher Kaufleute und Handwerker legt nahe, fremden Einfluß hinter dem Auffinden eines derartigen Griffs in Oslo zu vermuten. Man könnte ihn entweder als Import (das Ausgangsmaterial Walroßzahn ist für sich noch kein Beleg für eine einheimische Arbeit, da es in großem Umfang als Rohmaterial exportiert wurde) oder als norwegische Fertigung unter fremdem Einfluß betrachten, was aufgrund der eigenwilligen Gestaltung der Gesichtszüge wahrscheinlicher ist.

Eines der wichtigsten von der Hanse aus dem Norden nach ganz Europa verhandelten Güter war gesalzener Hering, konserviert mit Lüneburger Solesalz, bevor das südwesteuropäische Atlantiksalz in großem Umfang im Hansebereich gehandelt wurde. Der wichtigste Umschlagplatz für Hering war Schonen, wo, seit 1201 für Lübeck belegt, auf saisonalen Messen der in den südschwedischen Gewässern gefangene Hering direkt verkauft, eingesalzen und verschifft wurde. Auf den Küstenstreifen in Skanör und Falsterbo entwickelten sich neben dem Heringsvertrieb Messen, auf denen alle Waren des Ostens und Westens ausgetauscht und verkauft wurden, die wichtigsten Städte der Ostseeküste, der Nordseeküste, der Zuiderzee, auch englische, niederländische, norwegische und andere Städte hatten eigene Verkaufs- und Stapelplätze, die sogenannten "Vitten" ${ }^{213}$. Es ist zumindest wahrscheinlich, daß die in Schonen und Lund gefundenen Messergriffe auch vor dem Hintergrund dieser Messen ihre Abnehmer fanden ${ }^{214}$.

Bezüglich der Qualität der Griffe aus Lund und Roskilde läßt sich wiederum ein Bezug zur Bedeutung des Fundortes herstellen.

Roskilde, an der Spitze des gleichnamigen Fjordes in Seeland gelegen, war seit 1020 nach der Verlegung des königlichen Haupthofes durch Knut den Großen Residenz der dänischen Könige und bis 1416 Hauptstadt Dänemarks, seit 1060

\footnotetext{
211 Christie 1966, S. 40 ff.

Molaug 1975, S. 218, 219, $229 \mathrm{ff}$.

212

Blom 1973, S. 160

213 Weczerka 1973, S. 46 ff.

214 Ähnliches gilt vielleicht auch für den Griff aus Ragnildsholm in Bohuslän, der Landschaft nördlich des Göta Älv, an dessen Lauf sich das mittelalterliche Lödöse befand. An der Küste des zu Norwegen gehörenden Bohuslän fand in geringem Umfang ein ähnlicher Handel statt. Vor allem Hamburger, Bremer und später Rostocker Kaufleute fuhren in der Herbstsaison die einheimischen Fischerdörfer an, um Hering aufzunehmen.

siehe Blom 1973, S. 159 und Ekre 1973, S. 170
} 
Bischofssitz und Krönungsort der dänischen Könige ${ }^{215}$. 14 steinerne Pfarrkirchen schreibt Johansen ausländischen Kaufmannssiedlungen innerhalb der Stadtbefestigung zu, deutsche Einwohner sind seit 1133 nachweisbar.

Auch in Lund, der Hauptstadt Schonens und Sitz des nordischen Erzbistums waren zu Beginn des 13. Jahrhunderts bereits 20 Pfarrkirchen vorhanden, deren Errichtung ebenfalls durch ausländische Kaufleute angenommen wird ${ }^{216}$.

Die beiden in Roskilde gefundenen Messergriffe beinhalten die einzigen Beispiele für die Darstellung des schildbewehrten Ritters und die Verbindung der Motive Falkenjagd und Minne in der gesamten Gruppe. Von den drei in Lund geborgenen Exemplaren fällt eines, aus Elfenbein geschnitzt, durch besonders plastische und detailreiche Bearbeitung auf, welche der Qualität des Messers aus Ribe nur wenig nachsteht.

Im Vergleich zu dem Beleg aus Oslo scheint sich zu bestätigen, daß zwischen der potentiellen Käuferschicht sowie der wirtschaftlichen und kulturellen Bedeutung des Ortes und der Qualität der dort gefundenen Messergriffe aus der hier behandelten Gruppe ein Zusammenhang besteht, der, bereits mehrfach behandelt, im übrigen für Luxusimportgüter aller Zeiten Gültigkeit hat ${ }^{217}$.

Die im Ringkloster bei Skanderborg, im Skovkloster in Herlufsholm sowie im Untergrund des Zisterzienserklosters Nijeklooster in Friesland gefundenen Griffe belegen wie das Beispiel aus dem Kloster von Wienhausen, daß nach den zentralen Städten Klöster einen weiteren Abnehmerkreis derartiger Messer darstellten.

Die Fundorte Lille, Arras und Lüttich führen in den Raum, den Sommer, Bencard und Leciejewicz als Ursprungsgebiet der anthropomorph gestalteten Messergriffe annehmen.

Die Funde selbst sagen darüber nichts aus. Weder existieren genauere Aufzeichnungen $\mathrm{zu}$ den Befundumständen noch fallen sie qualitativ besonders auf; die Beispiele aus Lüttich und Arras sind nicht einmal sicher den betreffenden Altstädten zuzuweisen, der Griff aus Lille ist ein Flußfund.

Der Griff, der im Katalog der Sammlung René d'Allemagnes abgebildet ist, zeigt grundsätzliche Ähnlichkeit mit den Beispielen aus Lund, Roskilde und Kalmar; er gelangte in Paris in die Sammlung, eine Fundortzuweisung außer der Wahrscheinlichkeit, daß er nordfranzösischer Provenienz ist, ist daher nicht möglich.

So bleibt als Begründung für die oben erwähnte Ansicht lediglich die hervorragende Bedeutung der Pariser Elfenbeinschnitzerwerkstätten, die Koechlin beschrieben hat $^{218}$.

\footnotetext{
215 Grabois 1981, S. 517

216 Johansen 1978, S. 327-328

217 Es sei an dieser Stelle als ein Beispiel aus dem hier behandelten Zeitraum an die bekannten, über die Hanse verhandelten Dinanderien erinnert, die Römer zu Recht als "Verkaufsschlager der Hanse" bezeichnet, siehe Römer 1973, S. 126/127

Vgl. Abschnitt 2.4.2.1.3 im Anschluß
} 
Der oben beschriebene Griff aus Köln steht stellvertretend für die Möglichkeit weiterer Produktionszentren, die lokalen Herstellern derartiger Gegenstände die Impulse vermittelt haben können ${ }^{219}$.

Der Kontakt hansischer Kaufleute mit Flandern, seit dem ausgehenden 11. bis in das 13. Jahrhundert neben der Lombardei die wichtigste Städte- und Industrielandschaft Europas ${ }^{20}$, und mit den Messen der Champagne in Lagny, Provins, Bar-surAube und Troyes ist sicher schon vor der Mitte des 13. Jahrhunderts anzunehmen, schriftliche Quellen bezeugen Verhandlungen von Vertretern der Gotländischen Genossenschaft 1252 mit Margarete von Flandern und Handelstätigkeiten Kölner (seit 1228), Magdeburger (seit 1239) und Lübecker (1294, 1295, 1302) Kaufleute auf den Champagnemessen ${ }^{221}$. Letztere scheinen von den flämischen Tuchstädten aus direkt auf die Messen weitergezogen zu sein und besaßen offenbar privilegierte Funktionen auch im flandrisch-französischen Handel. So wurde ein königlicher Schutzbrief von 1298 unter Vermittlung eines Lübeckers erwirkt, der sich in Troyes niedergelassen hatte ${ }^{222}$.

Die von hansischen Kaufleuten nach Osten transportierten Waren umfaßten nicht nur billigere Woll- und Webstücke, sondern auch Luxustextilien wie die besonders im Ordensgebiet begehrten feinen leichten Stoffe aus Arras und die Messingprodukte aus den Maasstädten ${ }^{223}$.

Dieser Hintergrund erklärt den stilistischen Zusammenhang zwischen den in Lübeck gefundenen Messergriffen und den Beispielen aus Arras, Lüttich und Friesland $^{224}$. Wenn die Lübecker Griffe in der Werkstatt an der Hundestraße hergestellt worden sind, so geschah dieses wahrscheinlich unter Beeinflussung durch Importe, die bereits im 13. Jahrhundert dorthin gelangten, also noch vor der Verbreitung derartiger Messergriffe im Nord- und Ostseeraum.

Die Anwesenheit privilegierter deutscher Kaufleute im Zentrum des nordwesteuropäischen Warenumschlages und in direkter Nachbarschaft zu Kunstzentren wie Paris erhärtet aber auch die Möglichkeit, daß Messergriffe wie die aus Småland, Reval und Vysoké Mýto, die sich stilistisch möglicherweise noch am ehesten mit den Pariser Elfenbeinarbeiten vergleichen lassen, direkt aus Nordfrankreich nach Osten verhandelt worden sind.

\footnotetext{
219 Fillitz 1977, S. 483-484

220 Römer 1973, S. 123

221 Römer 1973, S. 125

Dollinger 1989, S. 64

Ammann 1978, S. 71

Römer 1973, S. 126, 127 


\subsection{Zur Frage der Herkunft, möglicher Vorbilder und der Bildinhalte}

\section{Einleitende Bemerkungen}

1924 veröffentlichte Raymond Koechlin in Paris sein Werk "Les Ivoires Gothiques Français", in dessen Katalogteil er über 1300 geschnitzte Elfenbeinobjekte des 13. bis 15. Jahrhunderts vorstellte. Diese Arbeit ist bis heute die Basis jeder weiteren wissenschaftlichen Beschäftigung mit gotischen Elfenbeinen geblieben ${ }^{225}$, und es verwundert nicht, wenn nicht nur Kunsthistoriker, sondern auch Archäologen darauf zurückgreifen ${ }^{226}$.

Koechlin hat sein Werk nach Objektgruppen gegliedert. Im ersten Teil beschreibt er neben diesen aber auch die Geschichte der Pariser Elfenbeinschnitzerei unter Anführung zahlreicher schriftlicher Quellen. Hier deutet sich bereits an, was Koechlin selbst quellenkritisch zu seiner Arbeit anmerkt:

Es ist fast unmöglich, neben dem Zentrum Paris andere französische oder außerfranzösische Werkstätten eindeutig nachzuweisen, da offensichtlich der Einfluß der Pariser Werkstätten in ganz Europa vor allem im 13. und 14. Jahrhundert so groß war, daß die Handschrift anderer Werkstätten kaum auszumachen ist ${ }^{227}$.

Die Methode der Gliederung nach Objektgruppen "(Statuetten, Diptychen, Tabernakel, Platten, Kästen, Spiegelkapseln) oder nach Schmucktypen (Diptychen mit Rosetten, mit Arkaturen, mit Arkadenfriesen)"228 hat zunächst nichts mit den tatsächlichen Produktpaletten der jeweiligen Werkstätten zu tun. "Heute besteht die Schwierigkeit darin, das religiöse und profane Oevre dieser Werkstätten neu zu gruppieren"229.

Koechlin hat in einem eigenen Kapitel die Griffe von sogenannten "gravoirs" (Scheitelziehern), Messern und Dolchen behandelt ${ }^{230}$. Bis auf das dort abgebildete Exemplar aus Lille ${ }^{231}$ fallen alle anderen Griffe stilistisch, ikonographisch und kompositorisch aus dem Rahmen der hier behandelten Griffe heraus (Tafel III).

Es ist daher kaum anzunehmen, daß auch nur einer der im Untersuchungsraum ausgegrabenen Messergriffe aus einer Pariser Werkstatt stammt.

Diese einleitenden Anmerkungen zeigen, daß es an dieser Stelle nicht darum gehen kann, mit Hilfe kunsthistorischer Methoden den Herkunftsnachweis archäologischer Funde zu führen. 
Einige historisch und kunsthistorisch ermittelten Ergebnisse zur Erforschung mittelalterlicher Elfenbeinschnitzerei in Europa können aber zur Eingrenzung der Möglichkeiten, die sich aus dem archäologisch erfaßten Material ergeben haben, herangezogen werden.

\section{Zur Elfenbeinschnitzerei im Mittelalter}

Die wichtigsten Veränderungen, die zu stadtsässigen Schnitzergilden und zur Massenproduktion im ausgehenden 13. und 14. Jahrhundert führten, setzten bereits im 12. Jahrhundert ein.

Noch war der Hauptauftraggeber für Elfenbeinobjekte die Kirche, bestand die breite Masse der Produkte aus religiösen Gegenständen wie Reliquienbehältern, Hostienbüchsen, Diptychen, Klappaltären, Buchdeckeln und freistehenden Plasti$\mathrm{ken}^{232}$. Die Zeit der "hervorragenden Einzelleistungen", die von einzelnen Künstlern als Auftragsarbeiten für Kirchen, Klöster und Höfe hergestellt worden waren, in der antike Elfenbeinarbeiten umgearbeitet und vor allem in Kirchenschätzen über Jahrhunderte verwahrt worden waren, wurde jedoch seit dem 12 . Jahrhundert abgelöst durch Veränderungen der beiden wichtigsten Faktoren: Auftraggeber und Produktionsart ${ }^{233}$. Bereits in der Spätromanik konzentrierten sich die Werkstätten, die bisher von unabhängigen, oft mobilen Künstlern betrieben worden waren $^{234}$, in den Städten, in denen jetzt mit ersten Serienfabrikationen begonnen wurde. Fillitz führt zwei Beispiele aus Köln an, die sogenannte "gestichelte Gruppe" $^{235}$, die mehrere sakrale Kunstwerke umfaßt, darunter die Buchdeckel zweier in Köln entstandener Handschriften, sowie die Gruppe der Kölner Kuppelreliquiare, Miniaturnachahmungen byzantinischer Zentralbauten ${ }^{236}$, an denen Reihen von kleinen Elfenbeinstatuetten (v.a. Aposteldarstellungen) befestigt waren. Diese Figuren sowie weitere Elfenbeinteilstücke wurden in den städtischen Werkstätten bereits auf Vorrat hergestellt und variabel verwendet, zum Teil wurde Elfenbein auch schon durch Material wie Walroßzahn ersetzt ${ }^{237}$, welches bereits in der Romanik der Hauptwerkstoff der nordeuropäischen Elfenbeinschnitzerei war $^{238}$. Im Zuge dieser Entwicklung traten starke Qualitätsschwankungen auf, es wurden auf Vorrat produzierte fertige und halbfertige Produkte angeboten ${ }^{239}$.

Elfenbeinplastiken des 12. Jahrhunderts spiegeln im Miniaturformat die zeitgenössische monumentale Kathedralplastik wider, sie entwikkelten im 13. Jahrhundert

\footnotetext{
232 Beigbeder o.J., S. 22-23

233 Fillitz 1977, S. 483-484

234 Gaborit-Chopin 1978, S. 131

235 Fillitz 1977, S. 486

236 Beigbeder o.J., S. 18

Franz 1969, S. 211-212

237 Fillitz 1977, S. 483

238 Williamson 1982, S. 15

239 Fillitz 1977, S. 483
} 
aber eine gewisse Eigenständigkeit ${ }^{240}$, die im 14. Jahrhundert bis zur umgekehrten Beeinflussung führen sollte ${ }^{241}$.

Die Geradlinigkeit und Starrheit der zwischen 1170 und 1180 entstandenen Reliquiarstatuetten, aber auch späterer Großplastiken, z.B. der Kathedralplastiken von Chartres, die zwischen 1200 und 1240 datieren $^{242}$, bewogen Leciejewicz zur Datierung des Stettiner Grifffragments in die erste Hälfte des 13. Jahrhunderts. Die Problematik dieser stilistischen Datierung erhellt aber bereits aus den fließenden Übergängen romanischer und gotischer Elfenbeinschnitzerei, im Verlauf des 13. Jahrhunderts, auch regionale Verschiebungen blieben bei dieser Datierung unberücksichtigt (Abb. 140 und 141).

Im 12. Jahrhundert konzentriert sich die Elfenbeinschnitzerei neben Frankreich auf wenige Gebiete, vor allem auf das Rheinland, das Maasgebiet und Niedersach$\operatorname{sen}^{243}$. Die führende Rolle Frankreichs in der Frühgotik betraf auch die Elfenbeinkunst, erst im 14. Jahrhundert scheinen sich eigenständige Schulen neben den erwähnten Gebieten auch in England, Flandern und Italien voll entwickelt zu haben ${ }^{244}$.

Das Ausgangsmaterial, die Zähne von Elefanten und Flußpferden, stammte überwiegend aus Guinea (Elfenbeinküste) und wurde von den äthiopischen Häfen Aden und Zeila über Alexandria, Acera und Famagusta nach Europa verschifft. Weiterhin wurden die Zähne von Walroß und Narwal vor allem in England und Skandinavien verarbeitet, bereits früher verhandelten skandinavische Wikinger Walroßzahn nach Byzanz $^{245}$, baskische Walfänger belieferten ganz Nordeuropa mit Walbein ${ }^{246}$.

Zwischen 1200 und 1250 konstatiert P. Tudor-Craig einen starken Rückgang in der Herstellung der bisher üblichen Elfenbeinobjekte, die sie nicht nur auf veränderte Gebräuche wie die Zunahme von Leserschichten nichtreligiöser Bücher zurückführt, die zu einem Verschwinden harter Bucheinbände und damit zu einem starken Rückgang von elfenbeinernen Buchdeckelplatten führte, sondern auch auf ein Leerjagen der bisherigen Walroßgründe, also auf Rohstoffmangel; afrikanisches und (seltener) indisches Elfenbein habe aber erst seit der Mitte des 13. Jahrhunderts im nötigen Umfang zur Verfügung gestanden ${ }^{247}$.

\footnotetext{
240 Beigbeder o.J., S. 18-19, 22

241 Madonna von Ste. Chapelle, siehe weiter unten

242 Hürliman u.a. 1954, S. 44, 45, 48, S. 110

243 Fillitz 1977, S. 484

244 Beigbeder o.J., S. 26-27

245 Beigbeder o.J., S. 30-31

246 Williamson 1982, S. 15, 17

247 Williamson 1982, S. 18 Tudor-Craig 1987, S. 94
} 

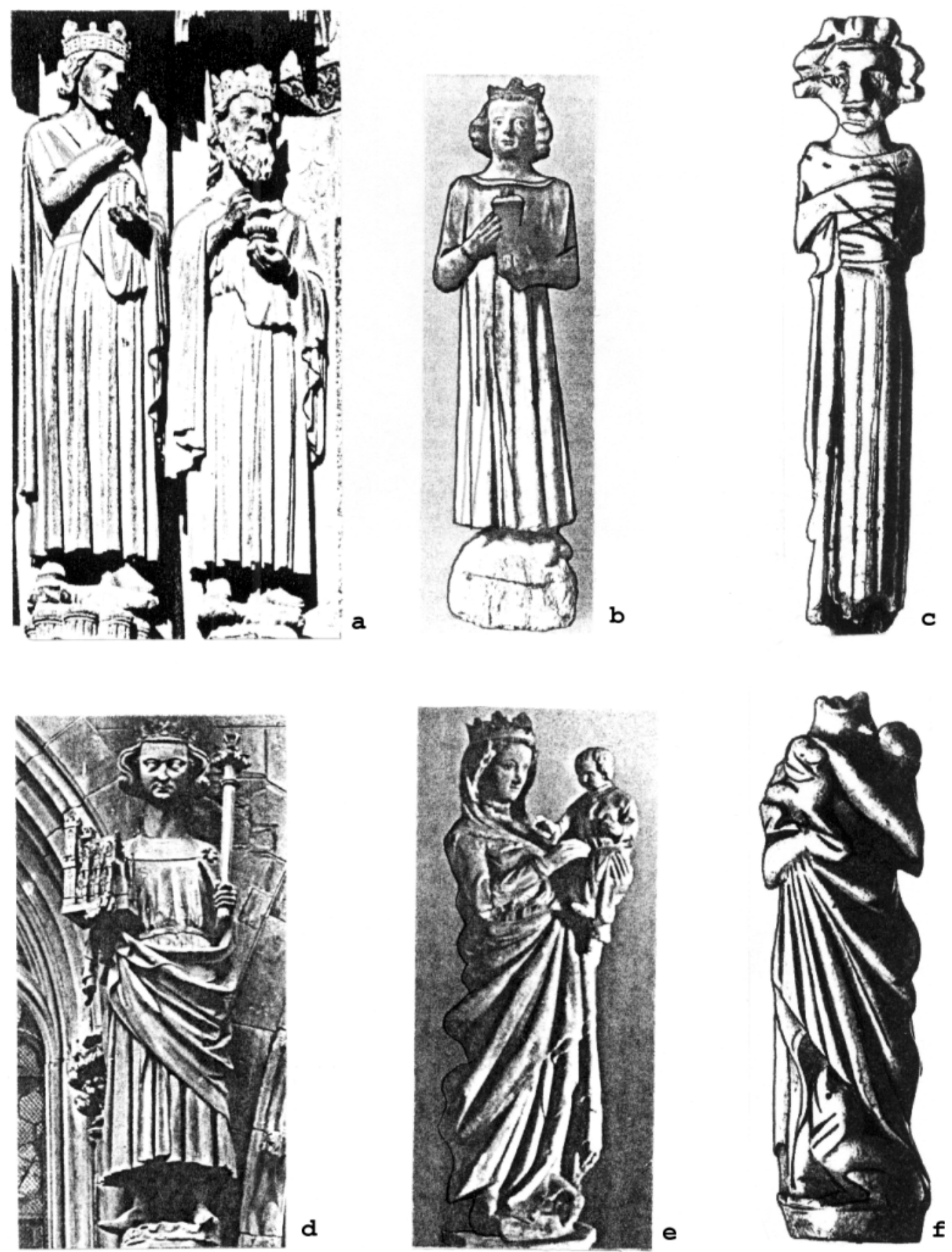

Abb. 140

Messergriffe im Vergleich zur Großplastik des 13. Jh.

a: Amiens, um 1230; b: München/Oberrhein, 1324; c: Kalmar; d: Basel, um 1300; e: Heppenheim, um 1300; f: Småland 

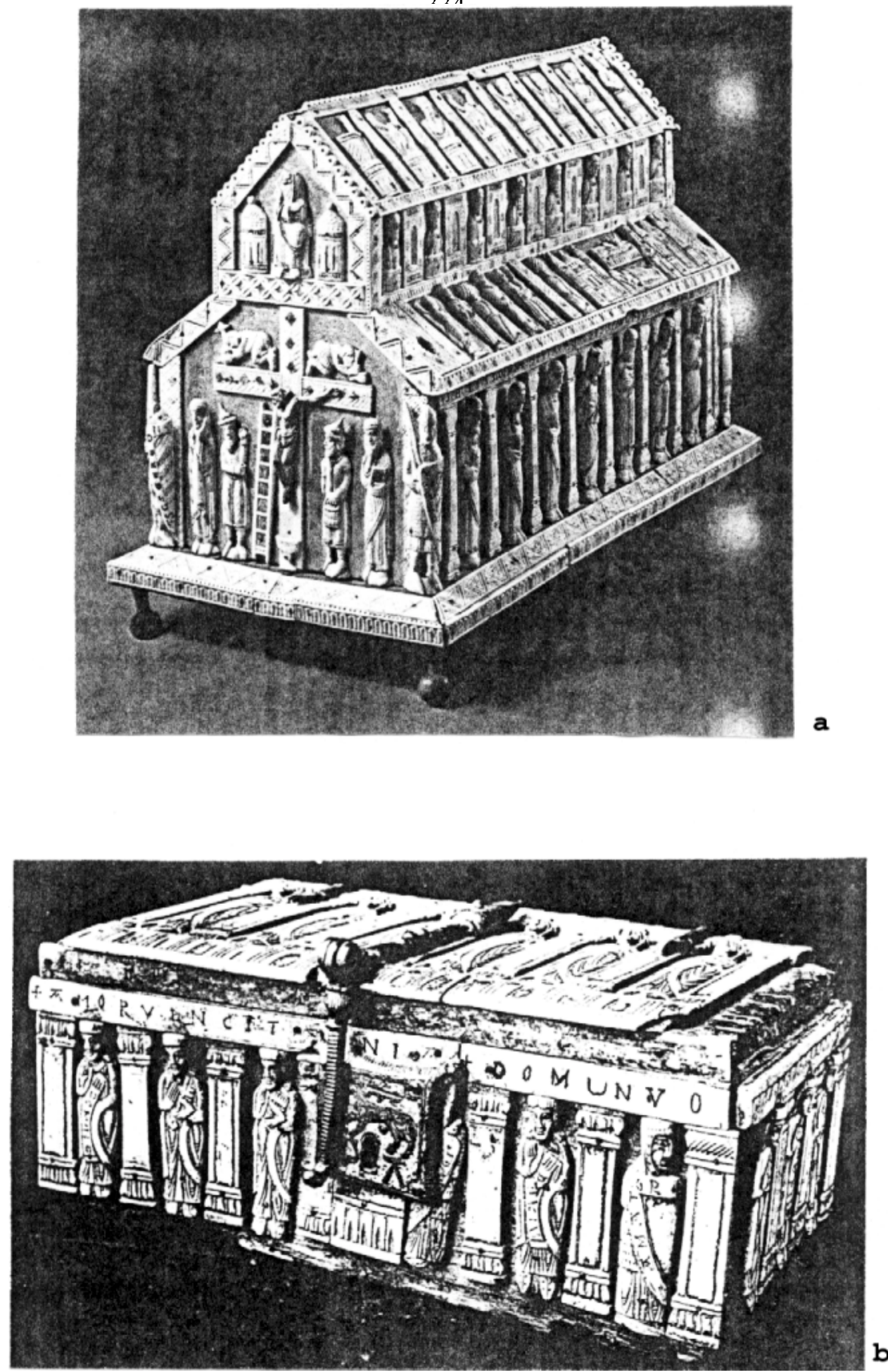

Abb. 141

Statuettenserien des 13. Jahrhunderts

a: Reliquienschrein, Köln, 1.H. 13. Jh., b: Kasten, Köln, 1.H. 13. Jh. 
Seit dieser Zeit wandeln sich sämtliche Faktoren zugunsten einer Serienproduktion elfenbeinerner Objekte. Das Rohmaterial stand in aus reichendem Maße zur Verfügung $^{248}$, die rechtliche und wirtschaftliche Konsolidierung der Städte (so verdoppelte sich z.B die Bevölkerung von Paris zwischen 1214 und $1314{ }^{249}$ ) und neben der wachsenden Umgebung des französischen Königshofes breite neue Käuferschichten durch das wohlhabende Bürgertum ${ }^{250}$ führten zu einer Objektvielfalt auf der einen, zu einer stilistischen Einheitlichkeit auf der anderen Seite ${ }^{251}$.

In Paris etablierte sich ein "System von Boutiquen", welche mit den Zuliefererwerkstätten mehrere Pfarrbezirke umfaßte und nicht nur Aufträge ausführte, sondern auch - wie bereits im ausgehenden 12. Jahrhundert in Köln - Fertigprodukte anbot $^{252}$.

Unsicher scheint der genaue Beginn sowohl der handwerklichen Spezialisierung auf bestimmte Objektgruppen als auch der des Anstiegs profaner Objekte zu sein:

Das "Livre des Métiers", in dem die Pariser Handwerker seit der Mitte des 13. Jahrhunderts aufgeführt sind, führt Messerhersteller schon im 13. Jahrhundert ${ }^{253}$, Elfenbeinschnitzer aber namentlich erst seit dem 14. Jahrhundert $\mathrm{an}^{254}$. D. Gaborit-Chopin erwähnt daher die Möglichkeit, daß die nachweislich im 13. Jahrhundert entstandenen Elfenbeinwerke aus Pariser Werkstätten möglicherweise in Maler-, Bildhauer- oder Goldschmiedewerkstätten entstanden sind ${ }^{255}$, während P. Tudor-Craig bereits seit der Mitte des 13. Jahrhunderts von spezialisierten Elfenbeinschnitzern ausgeht; sie unterscheidet nach Zitat aus derselben Quelle Kamm- und Laternenschnitzer, Maler und Schnitzer von Bildern, Schreibtafelschnitzer, Paternosterhersteller, Kruzifix- und Messergriffschnitzer ${ }^{256}$. Diese Gruppen haben sich offenbar aufgrund des bearbeiteten Rohmaterials ergeben, so wurden für Herstellung von Corpusteilen und Messergriffen die gleichen Teile des Elefantenzahns benötigt.

Die Gleichförmigkeit der zahlreichen Elfenbeinobjekte, die stilistisch in die Zeit zwischen 1250 und 1350 datiert wurden, vor allem der Hausaltäre (Diptychen, Triptychen und Polyptychen), haben sie veranlaßt, auch die Hersteller dieser Objekte trotz fehlender schriftlicher Nachweise vor 1300 anzunehmen ${ }^{257}$.

\footnotetext{
248 Gaborit-Chopin 1978, S. 131

249 Gaborit-Chopin 1978, S. 131

250 Williamson 1982, S. 18

251 Gaborit-Chopin 1978, S. 131

252 Gaborit-Chopin 1978, S. 131

253 Koechlin 1924, Bd. I, S. 420

254 Gaborit-Chopin 1978, S. 131

255 Gaborit-Chopin 1978, S. 131

256 Tudor-Craig 1987, S. 102

257 Tudor-Craig 1987, S. 102
} 
Im Allgemeinen wird seit Koechlin davon ausgegangen, daß in Paris seit ca. 1250 eine Korporation der Elfenbeinschnitzer belegt ist ${ }^{258}$. Die Führung Frankreichs bzw. Paris in der Herstellung und Konzeption elfenbeinerner Objekte religiöser wie profaner Funktion zwischen 1250 und 1350 ist ebenfalls unbestritten, P. Williamson geht davon aus, daß "auch Schnitzer, die in anderen Ländern arbeiteten, in Paris ausgebildet worden waren" 259 .

Erst seit dem beginnenden 14. Jahrhundert wird das verstärkte Auftreten profaner sowie religiöser Objekte für den Privatgebrauch angenommen ${ }^{260}$. Diese Produktion steht in Zusammenhang mit einem Funktionswandel religiöser Bräuche vom öffentlichen Bereich der Kirche in den privaten Bereich eines sehr viel breiteren Abnehmerkreises ${ }^{261}$, der bis zur Bildung privater Prestigeobjekte führte: die Anzahl der Klappaltäre in einem adeligen oder bürgerlichen Haushalt war ein Zeichen besonderen Wohlstandes ${ }^{262}$.

Profane Objekte umfassen neben Behältern wie Schmuckkästchen ("Minnekästchen") und Spiegelkapseln Messer-, Dolch- und "Gravoirs"griffe (Scheitelziehergriffe), Prunksattelapplikationen, Falkenständer (Ständer für Falkenkäppchen), Gürtelschnallen, Würfel, Würfelbecher, Brettspielfiguren, Spielsteine, Griffel, Kämme, Peitschengriffe, Musikinstrumente, Jagdhörner u.a ${ }^{263}$.

Bis auf die bekannten "Olifants" (Jagdhörner), die nordischen Spielfiguren, Kopien antiker Objekte und Kästchen, die stilistisch auf orientalische, romanische und ältere Vorbilder zurückgreifen und bereits seit dem 11. Jahrhundert bekannt sind ${ }^{264}$, werden die gotischen profanen Gegenstände fast durchweg in das 14. Jahrhundert datiert. Bereits hier sei darauf verwiesen, daß die Zeitspanne der hier behandelten Messergriffe archäologisch gesichert bis in das 13. Jahrhundert zurückreicht.

Um die in dieser Arbeit behandelten Messergriffe im Gesamtspektrum mittelalterlicher profaner Elfenbeinarbeiten einordnen zu können, sollen letztere in ihrer Vielfalt und stilistischen Abhängigkeit kurz vorgestellt werden. Die seit Koechlin entwickelten Datierungsmethoden und Datierungsgrundlagen aufgrund stilistischer Vergleiche, dem Herausarbeiten einzelner Werkstattgruppen etc. können hier nicht diskutiert werden; sie sind durchweg der zitierten Literatur entnommen worden.

\footnotetext{
258 Beigbeder o.J., S. 37-38

259 Williamson 1982, S. 18

260 Gaborit-Chopin 1978, S. 131

261 Aubert 1963, S. 114 Busch / Lohse 1962, S. XXII

262 Beigbeder o.J., S. 40-41

263 Gaborit-Chopin 1978, S. 131

Beigbeder o.J., S. 26-27, S. 74

264 Beigbeder o.J., S. 19, 22

Tudor-Craig 1987, S. 105
} 
Ein Vergleich dieser Messergriffe mit den Beispielen im Katalog Koechlins und anderer, französischen Werkstätten zugewiesener Griffe ${ }^{265}$ ergibt zunächst ein klares Bild:

Die aus Paris oder anderen französischen Werkstätten stammenden Griffe sind "naturalistische" Darstellungen, die in ihrer Ausführung und Bewegung wie Kopien der zeitgenössischen Großplastik wirken.

Die einzige Ausnahme bildet der Falknergriff im Archäologischen Museum Lille, welcher in den Befestigungsanlagen von Arras gefunden wurde ${ }^{266}$.

Die hier behandelten, zum größten Teil in nord- und nordosteuropäischen Städten, Klöstern und Burgen ausgegrabenen Griffe sind dagegen durchweg in ihrer starren Haltung und bis auf wenige Ausnahmen (Ribe, Wienhausen, Småland, Vysoké Mýto) stärker stilisierte Darstellungen, die eher der Funktion als Messergriffe genügen.

Nach den Merkmalen, die die kunsthistorische Literatur dem sogenannten "Pariser Stil" zuweist ${ }^{267}$, und nach Vergleich mit wahrscheinlich französischen Griffen sind lediglich die Griffe aus Småland, Reval, Vysoké Mýto und das Exemplar aus der Sammlung d'Allemagne von Paris beeinflußten Werkstätten zuzuordnen.

Die Frage, welche Werkstätten außerhalb von Paris in Frage kommen, berührt, da zwei verschiedene Quellengruppen behandelt werden, zwei unterschiedliche Methoden des Nachweises, die in diesem Fall beide zu keinem erschöpfenden Ergebnis führen.

Der archäologische Nachweis zur Provenienz der aus Elfenbein, Walroßzahn, Knochen und Holz hergestellten Messergriffe ist nicht durch den Fundort zu führen:

"Innerstädtisches Handwerk... ist nur dann als archäologisch nachgewiesen zu betrachten, wenn in der Stadt selbst unzweifelhafte archäologische Befunde die Existenz handwerklicher Produktion am Platz anzeigen." ${ }^{268}$

In einem Beitrag "Zur Lebensweise in der Stadt um 1200"269 führte W. Janssen 1986 zahlreiche Orte an, in denen zu verschiedenen Zeiten im Mittelalter (v.a. 11. bis 14. Jahrhundert) Bein- und Hornverarbeitung archäologisch nachgewiesen werden konnte, darunter die Städte Oslo, Lund, Lübeck und Göttingen.

Die beiden Messergriffe aus Lübeck stammen von einem Gelände an der Hundestraße, auf dem in einem dreischiffigen Hallenhaus innerhalb einer umpflockten Anlage (Grube?) die Abfälle eines Knochenschnitzers oder Kammachers aus der zweiten Hälfte des 13. Jahrhunderts gefunden wurden ${ }^{270}$. Ein direkter Zusammenhang mit dieser Grube ist derzeit noch nicht herstellbar. Da es sich aber um zwei

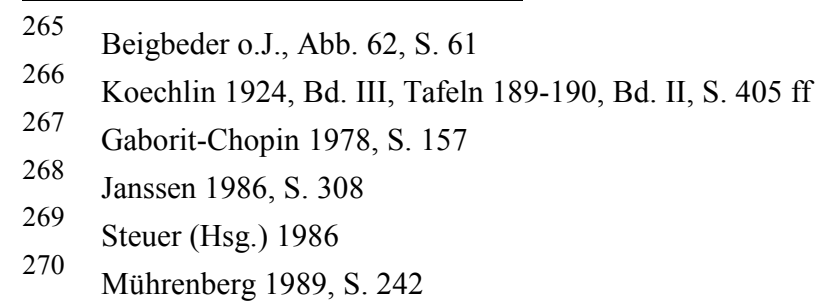


Griffe handelt und ein Benutzer der Messer aus einer der weiter oben beschriebenen städtischen Führungsschichten auf dem Gelände auch historisch nicht nachweisbar ist, ist ihre Herstellung in dieser Werkstatt sehr wahrscheinlich.

Ein derartiger Zusammenhang ist mit den Messergriffen aus Oslo und Lund archäologisch nicht herzustellen. Die Werkstätten produzierten Nadeln und Kämme ${ }^{271}$, die Fundplätze der jeweiligen Messergriffe sind bis auf die kleine Fischergasse 8 in Lund unbekannt, die Werkstätten in Lund wurden südlich der Domkirche ergraben. In Göttingen wurde 1977 in einem "Bürgerhaus" in der Johannisstraße direkt gegenüber der Johanniskirche für die Zeit um 1300 eine Werkstatt ausgegraben, die neben Zeugnissen der Knochenschnitzerei (Kämme, Würfel, Spielzeug, Löffel und Messergriffe) Reste einer Herstellung von Emailarbeiten, Reliquiaren und Tragaltären aufwies ${ }^{272}$.

Ein Messergriff zeigte am Ende die stilisierte Darstellung eines Tierkopfes ${ }^{273}$.

Zwar wurde in dieser Werkstatt kein anthropomorph verzierter Messergriff geborgen, der zoomorph verzierte Griff sowie der Nachweis der Herstellung religiöser Objekte im Schatten der Johanniskirche zeigen aber, daß auch bei anderen Motiven oder weiteren durchaus exklusiven Gegenständen eine Produktion am Ort nachweisbar sein kann.

Archäologisch läßt sich demnach in Lübeck mit einiger Wahrscheinlichkeit die Produktion der hier behandelten Messergriffe am Ort aufgrund der Befundsituation vermuten, in Göttingen läßt sie sich an einem verwandten Motiv mit weiteren $\mathrm{Ob}$ jekten gehobener Sachkultur nachweisen. In Oslo und Lund sind einschlägige Berufe nachweisbar. Diese Folgerungen, gestützt durch die stilistischen und qualitativen Unterschiede der hier behandelten Gruppe zu den möglicherweise in Paris hergestellten Elfenbeingriffen, die Koechlin in seinem Katalog vorstellte, lassen es nahezu unwahrscheinlich werden, die Provenienz von Griffen wie denen aus Hamburg, Stettin, Riga u.a. in Paris oder der Île de France zu suchen.

Von allen übrigen Fundorten sind dem Autor keine archäologischen Nachweise von Werkstätten, die die dort gefundenen Griffe produziert haben könnten, bekannt geworden.

Die Frage, ob und wo genau diese Griffe, die nur in einer Minderzahl aus Elfenbein gefertigt wurden, produziert worden sind, kann hier derzeit nicht beantwortet werden.

$\mathrm{Zu}$ vermuten bleibt aber angesichts der Tatsache, daß knochenverarbeitendes Handwerk in fast jeder größeren Stadtkernuntersuchung auftritt und die publizierten Belege in keiner Relation zum tatsächlichen Aufkommen stehen, die Möglichkeit, eine noch nicht zu umreißende Anzahl dieser Griffe einheimischen Werkstätten zuzuordnen, und dies sicherlich abhängig von der Qualitätstufe, die die jeweiligen Griffe erreicht haben, sowie vom Material. Die in Lübeck gefundenen Griffe gehö-

\footnotetext{
271 Janssen 1986, S. 340, 341

272 Janssen 1986, S. 341

273 Schütte 1984, S. 71, Katalogteil BRD, Kat. Nr. 110
} 
ren zu den weniger qualitätvollen Erzeugnissen dieser Gruppe, obwohl gerade sie nicht ohne Einfluß aus dem französischen Raum entstanden sind (siehe weiter unten).

Elfenbeinverarbeitendes Handwerk ist archäologisch fast nie eindeutig nachgewiesen worden. Der Umweg über die Reidentifizierung von Objekten in Testamenten oder Inventaren, der Nachweis über den historisch abgesicherten Verbleib in Kirchen- oder Klösterschätzen, der stilistische Vergleich sowie der Herkunftsnachweis über den Bildträger wie im Fall der oben erwähnten Kölner Handschriften sind einige Methoden. Die in diesem Zusammenhang seit Koechlin behandelten Pariser Werkstätten, die seit der Mitte des 13. Jahrhunderts aufgeführt und nach 1300 auch namentlich bekannt sind, und ihre zu Hunderten noch vorhandenen profanen Produkte, die eine ganze Epoche geprägt haben, sind als Ausnahme zu bezeichnen.

In der kunsthistorischen Literatur werden aber mehrere Regionen und Orte genannt, in denen die Verarbeitung von Elfenbein im ausgehenden 13. und 14. Jahrhundert angenommen werden kann, obwohl offenbar gerade die Gleichförmigkeit der seit dieser Zeit an mehreren Orten auftretenden Serienproduktion zu den Hauptproblemen bei der Herausarbeitung von Werkstattkreisen und ihren Produkten zählt.

Vor allem im Bereich der Sakralkunst werden neben Paris und der Île de France eine Werkstatt in Franken im Umkreis der Zisterzienser ${ }^{274}$, im Zuge der Diskussion um den elfenbeinernen Kruzifixus in der Kirche zu Herlulfsholm eine Werkstatt in Dänemark genannt, den D. Gaborit-Chopin als ein "Hauptwerk der gotischen Elfenbeinschnitzerei" mit "mehreren verwandten Holzskulpturen aus Dänemark und der Gegend um Lund" bezeichnet ${ }^{275}$. P. Tudor-Craig sieht nach Vergleich mit einer Monumentalplastik an der Kathedrale von Westminster eine enge Verbindung zu demselben Kruzifixus und vermutet um 1250 den Import dieser Skulptur und weiterer Stücke von der Elfenbeinschule in Westminster nach Skandinavien, unter Hinweis auf einen Botenkontakt 1248-49 zwischen Heinrich III. von England und seinem Bruder Haakon IV. von Norwegen ${ }^{276}$. Doch bereits A. Goldschmidt hat die Abhängigkeit dieser Arbeit von älteren Holzskulpturen wie dem Kruzifix aus der Kirche von Fjelstrup belegt und sie wie eine weitere Arbeit aus der Mitte des 13. Jahrhunderts (Abb. 142) einer dänischen Werkstatt zugeschrieben ${ }^{277}$.

\footnotetext{
274 Gaborit-Chopin 1978, S. 132 


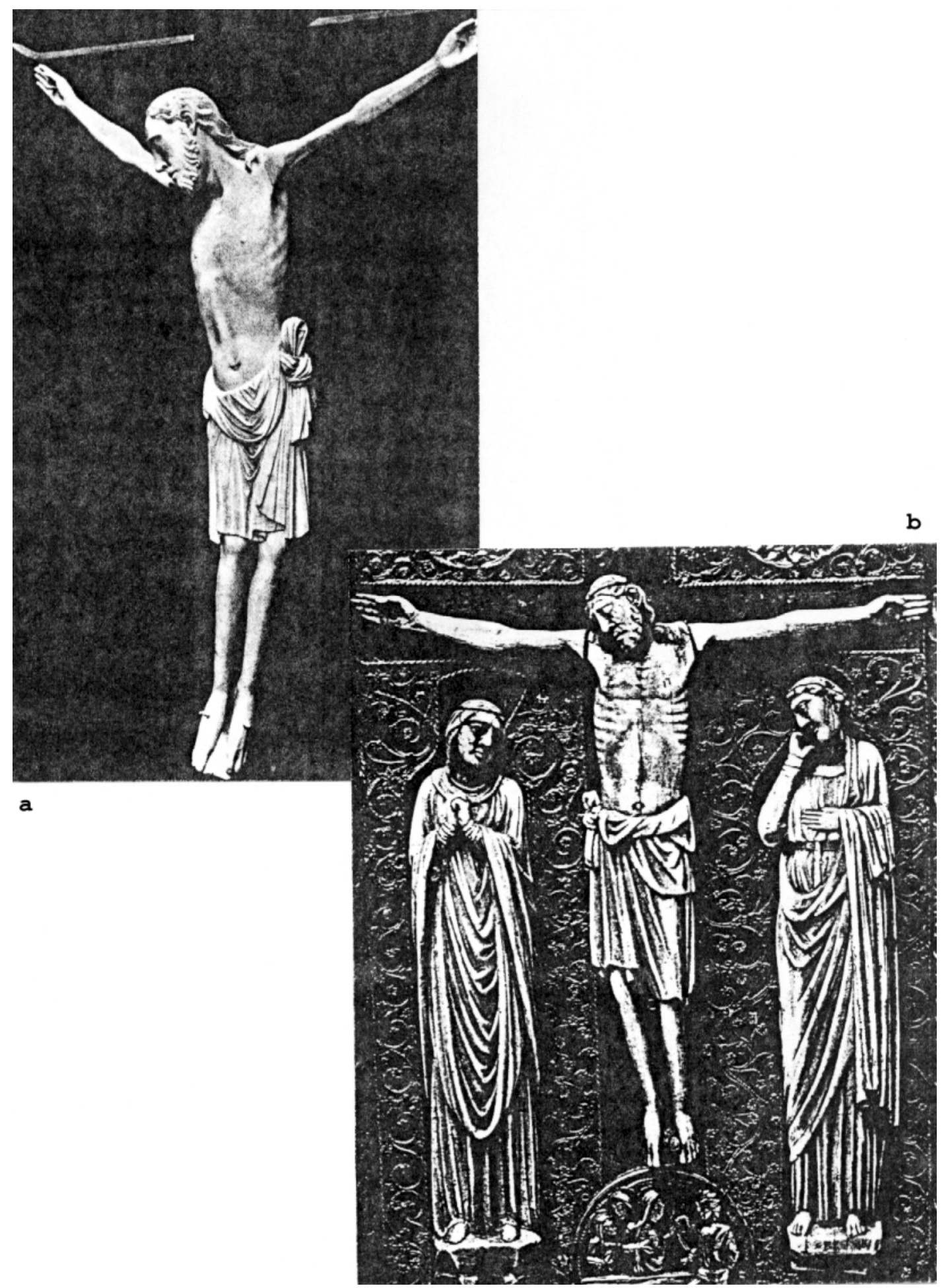


Weitere Werkstätten werden von Nordfrankreich $\left(\right.$ Reims $^{278}$, Noyon ${ }^{279}$ ), von Rou$\mathrm{en}^{280}$, aus dem Rheinland (Köln, 1. Hälfte des 14. Jahrhunderts) ${ }^{281}$, vom Oberrhein

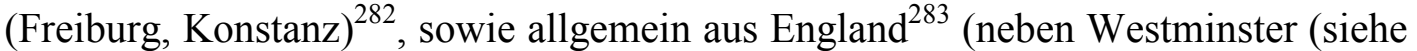
oben) noch Exeter ${ }^{284}$ ) und Italien ${ }^{285}$ (Pisa ${ }^{286}$ ) erwähnt.

Es ist demnach für die Zeit zwischen 1250 und 1350 mit Elfenbeinschnitzerwerkstätten außerhalb Frankreichs im Rheinland, in England und in Skandinavien zu rechnen. Hinzu kommt die Möglichkeit, daß ältere Werkstätten in Niedersachsen und im Maasgebiet (siehe oben) auch in dieser Zeit gearbeitet haben können.

All diese Werkstätten standen in der Hochgotik offenbar unter dem alles beherrschenden Einfluß der Pariser Elfenbeinschnitzer, haben aber alle eine eigenständige Ausprägung entwickelt, wie sie sich auch in den landschaftlichen Sonderformen der Monumentalplastik äußert ${ }^{287}$.

\section{Stilistische und ikonographische Differenzierung der Messergriffe}

Obwohl die hier behandelten Gruppen der Messergriffe stilistisch klar von den wenigen von Koechlin Paris zugeordneten Messer- und Scheitelziehergriffen zu trennen sind, können sie nicht als homogen bezeichnet werden.

\section{Der Griff aus Ribe (1)}

Der Griff aus Ribe ist als qualitätvollste Arbeit der Gruppe der frontal zur Schauseite angebrachten Griffe zu bezeichnen, er steht in der Feinheit der Ausführung, der Proportionalität und im Ausdruck den Pariser Griffen nicht nach, folgt jedoch einem anderen Idealbild und einer anderen Intention in seiner starren und klaren Darstellung.

Seine Herkunft ist ohne eindeutiges Vergleichsmaterial nicht zu klären, der Umschlagplatz und Importhafen Ribe war ausweislich der dort geborgenen Importfunde (Luxusgüter und qualitätvollerer Hausrat) nach England, Friesland und in das Rheinland ausgerichtet.

Der achtkantige, von einem verzierten Silberblech ummantelte Sokkel findet ebenfalls keine Parallele im Bestand der von Koechlin und anderen vorgestellten

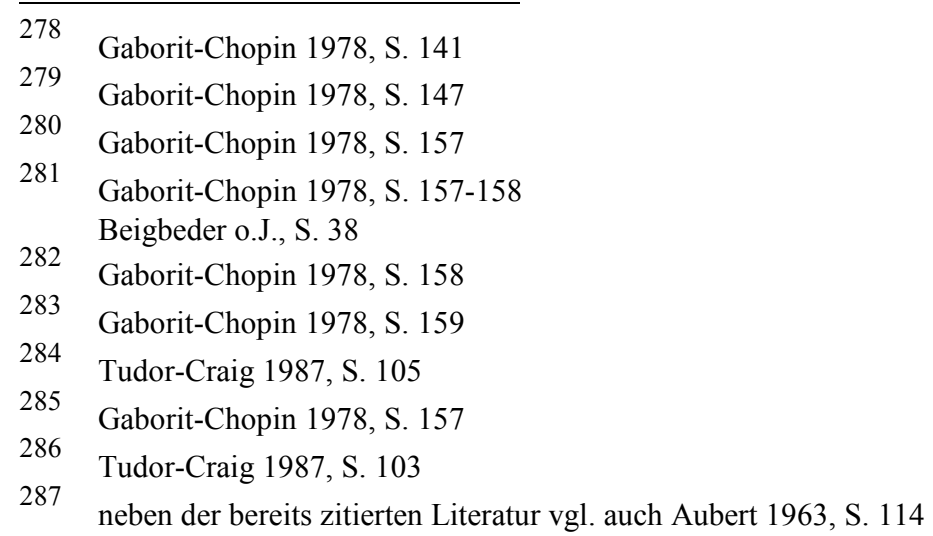


französischen und französisch beeinflußten Messergriffe, deren Sockel durchweg aus dem Ausgangsmaterial herausgeschnitzt wurden. Silberblechummantelungen am Heft sind aber im Bearbeitungsraum von Messern des 13. bis 14. Jahrhunderts aus Store Valby (Dänemark, Kat. Nr. 36, an einem Griff aus Walroßzahn) und aus Anklam (ehemalige DDR, Kat. Nr. 66, an einem Griff aus Wurzelholz) sowie von einem Griff um 1200 (BRD, Kat. Nr. 49a, Elbfund bei Finkenwerder ${ }^{288}$ ) bekannt, sowie an dem weiter oben beschriebenen Elfenbeingriff des Messers aus dem Kloster Wienhausen. Am nächsten kommt ihm das Heft des Messers aus Store Valby. Unter Bezugnahme auf den bereits erwähnten Kruzifixus in der Kirche von Herlulfsholm, der mit einiger Wahrscheinlichkeit dänischer Provenienz, allerdings fast 50 Jahre älter ist, sowie der Heftmanschette des Messers aus Store Valby, besteht die Möglichkeit, daß der Griff in Dänemark hergestellt und auch dort an eine Klinge montiert worden ist.

Der klare Importcharakter des Riber Fundmaterials sowie die Beispiele von Silbermanschetten aus dem hansischen Wirtschaftsraum, vor allem aber die ikonographische Abhängigkeit des Griffs aus Oxford (siehe weiter unten) von dem Riber Griff lassen aber auch an eine englische Herkunft denken. In diesem Fall kann die Montierung an eine Klinge in England oder auf dem Festland erfolgt sein.

\section{Skandinavische Griffe unter englischen Einfluß}

Das Exemplar aus Oxford, flacher und abstrahierter gearbeitet als der Griff aus Ribe, zeigt die gleiche Darstellung, vor allem die Haltung der rechten Hand mit zwei ausgestreckten Fingern läßt auf eine wie auch immer geartete Verbindung schließen (gemeinsames Vorbild, Nachahmung?). Es liegt nahe, aufgrund des Fundortes und der nachweislich vorhandenen Elfenbeinschnitzerei eine englische Provenienz anzunehmen.

Der Knochengriff aus Herlulfsholm zeigt das gleiche Motiv in einer starren, abstrakten Weise, der Kopf ist kantig gearbeitet, der Falke sitzt jetzt auf dem rechten Arm, der aber nicht stark angewinkelt, sondern immer noch rechtwinklig gehalten wird wie am Griff aus Ribe; lediglich die Fütterung des Vogels ist abweichend und weist auf die Ikonographie der Griffe aus Kalmar (2) u.a.

Der Hinweis auf die direkte Verbindung zwischen englischen Elfenbeinschnitzern und dem Ort Herlulfsholm sowie die ikonographische Verbindung lassen die Vermutung zu, den Griff aus Herlulfsholm als einheimische Arbeit eines Knochenschnitzers anzunehmen, dessen elfenbeinerne "Vorbilder" in England hergestellt wurden.

Der Knochengriff aus Ribe (2), dessen Kopf abgebrochen ist, zeigt in der rechtwinkligen Haltung des rechten Arms, in der Stilisierung der Hand, in der Kreuzschraffurfüllung des Falken sowie in der kerbschnitthaften Herausarbeitung 
der Arme auf Vorder- und Rückseite so starke Ähnlichkeit mit dem Beispiel aus Herlulfsholm, daß eine Zuweisung in dieselbe Werkstatt naheliegt.

Die Provenienz des Elfenbeingriffs aus Lund (1) wurde bereits von M. Rydbeck in einer französischen Provinzwerkstatt oder in einer französisch beeinflußten englischen Werkstatt vermutet. Ohne Vergleichsfunde aus englischen Fundorten ist die Werkstatt aber nicht näher einzugrenzen.

Der zweite in Lund geborgene Griff (Lund 2) scheint stilistisch in seiner Abhängigkeit zu stehen, vor allem aufgrund der Kopfgestaltung und dem hoch angesetzten Kopfbereich. Eine Verbindung mit dem Griff aus Herlulfsholm besteht in der Kreuzschraffur des Falken.

Ikonographische und stilistische Ähnlichkeit mit den Griffen Ribe 1 und Lund 1 hat einer der in Kalmar gefundenen Griffe (Kalmar 1): er zeigt mit der mittigen Taille und der Armhaltung, aber auch in der kammstrichartigen Gewandausfüllung Ähnlichkeiten mit den Griffen aus Ribe und Oxford, während die Kopfgestaltung stark an den Elfenbeingriff aus Lund erinnert.

Die Griffe Lund 2 und Kalmar 1 sind aus Knochen gefertigt. Dieses und die stilistischen Ausführungsmerkmale legen eine Herkunft aus einheimischen Werkstätten nahe.

Zusammenfassend läßt sich, ohne daß dafür eindeutige Belege zur Verfügung stehen, folgende Hypothese festhalten:

Den sechs besprochenen Griffen, die alle das Motiv des Falkners verarbeitet haben, scheinen sechs verschiedene Werkstätten zugrunde zu liegen, vermutlich zwei englische Elfenbeinwerkstätten, eine unbekannte Elfenbeinwerkstatt, die möglicherweise französischen Einflüssen unterlag, sowie drei vermutlich skandinavische Knochenschnitzerwerkstätten.

Zwei ikonographisch verschiedene "Vorbilder" wurden in dieser Gruppe verarbeitet, die sich in den Elfenbeingriffen aus Ribe und Lund manifestieren, ohne daß diese deshalb auch an den Anfang zu stellen sind.

\section{Griffe im engeren Einflußbereich der Hanse}

Die umfangreichste Gruppe des hier behandelten Messergriffbestandes umfaßt elf Griffe. Sechs von ihnen zeigen das Motiv des Falkners, die Figur aus Hamburg trägt einen dreieckigen Gegenstand, drei Griffe aus Kalmar stellen Buchträger dar, und ein Griff ist völlig stilisiert. Gemeinsam ist ihnen der hoch angesetzte Brustbereich und eine stereotype Kopfgestaltung, bei den Falknerdarstellungen darüberhinaus eine Gleichförmigkeit der Motivbearbeitung (Fütterung des Vogels).

Stilistik und Ausführung heben einen Knochengriff aus Kalmar (Kalmar 2) heraus, dessen Kopf leider abgebrochen ist. Die Figur steht auf einer profiliert abgesetzten Basis. Das lange Gewand ist in zahlreiche, von der hochsitzenden Taille lang herabfallende Falten gegliedert, beide Arme sind kurz und stark angewinkelt. 
Dieser Darstellungsweise folgen in gröberer Form stilistisch (Basisform) und ikonographisch die Griffe aus Hamburg (Walroßzahn), Stettin (Geweih) und Riga (Knochen). Starke Ähnlichkeiten bis zu Details der Basisgestaltung bestehen zwischen dem Hamburger und dem Stettiner Exemplar sowie zwischen den beiden Rigaer Griffen.

Über die Provenienz des Kalmarer Griffs läßt sich bisher keine begründete Aussage treffen.

Die vier übrigen Griffe stammen vermutlich aus zwei Werkstätten, die verschiedene Materialien bearbeiteten (Walroßzahn und Geweih, Knochen) und auch ikonographisch verschiedene Vorbilder verarbeiteten (verschiedene Attribute sowie die Darstellung eines Übergewandes mit querlaufenden Zierbordüren in Hamburg und Stettin, schlichte Falknerdarstellungen in Riga.

Eine Lokalisierung dieser beiden Werkstätten läßt sich aufgrund der Ausgangsmaterialien sowie der geographischen Fundlage aller fünf Griffe im hansischen Ostseewirtschaftsraum vermuten.

Der Gesamtgestaltung dieser Gruppe sehr ähnlich ist die elfenbeinerne Doppelfigur aus Roskilde (Roskilde 1), sie steht aber auf einer schlichten Basis. Sie ist zwar mit ihren herausgearbeiteten Füßen und zum Teil schräg fallenden Faltenwurf arbeitsaufwendig hergestellt worden und auch aufgrund des Werkstoffs hervorzuheben, reicht aber künstlerisch nicht an die Ausgewogenheit der Darstellung des Kalmarer Griffs heran. Auch für die Elfenbeinschnitzerwerkstatt dieses Griffs wäre ein Lokalisierungsversuch noch Spekulation.

Wesentliche stilistische Merkmale des Roskilder Griffs, darunter die schmale und niedrige Basis, finden sich an vier Knochengriffen, die aber qualitativ starke Unterschiede aufweisen.

Die drei Griffe aus Kalmar mit dem Motiv des Buchträgers (Dichter und/oder Schreiber), vermutlich in zwei verschiedenen Knochenschnitzerwerkstätten entstanden, zeigen noch tief eingeschnittenen Faltenwurf und stark herausgearbeitete Haare und Gesichtszüge, Details wie der Gewandabschluß am Hals lassen vermuten, daß die Griffe Kalmar 3 und 4 aus einer Werkstatt stammen.

Der Knochengriff Lund 3 ist dagegen von minderer Qualität. Der Brustbereich wird nur noch schemenhaft angedeutet und der Faltenwurf zur Längsritzung, die die Basis mit einschließt, stilisiert.

Die fünf Griffe aus Roskilde, Kalmar und Lund stammen demnach aus einer Elfenbeinschnitzerwerkstatt (Roskilde 1), deren Lokalisation unbekannt ist, sowie aus mindestens drei verschiedenen Knochenschnitzerwerkstätten.

Die drei Griffe aus Kalmar sind von zwei verschiedenen Handschriften geprägt. Motivische sowie stilistische Übereinstimmungen lassen in diesem Fall die Vermutung zu, beide Handwerker in einer Stadt, vielleicht sogar in Kalmar selbst, zu suchen, da das Motiv des Buchträgers an keinem weiteren der bearbeiteten Fundorte angetroffen wurde. 
Der Griff Lund 3 ist aufgrund seiner Gestaltung lediglich als Derivat in die Gruppen um die Griffe Kalmar 2 und Roskilde 1 anzusiedeln und wahrscheinlich einheimischer Herkunft.

Keiner der qualitätvolleren Griffe dieser Gruppe (Kalmar 2, Roskilde 1), die theoretisch als Leitformen für die zahlreichen Nachahmungen aus dem hansischen und skandinavischen Raum bezeichnet werden können und mit ihnen wahrscheinlich einer gemeinsamen ikonographischen Tradition folgen, können stilistisch mit französischen Arbeiten in Verbindung gebracht werden, lediglich der Griff aus Lund (1), läßt zumindest teilweise (vor allem das plastisch herausgearbeitete lächelnde und "weiche" Gesicht) französischen Stileinfluß erkennen.

Der Griff des Messers aus dem Budapester Kunstgewerbemuseum zeigt eine männliche Figur, die ein Instrument (Laute?) vor der hoch angesetzten Brust trägt. Das Gewand mit einer Mittelbordüre ist in symmetrisch fallende Falten gegliedert, die Figur steht auf einer hohen Basis. Das Material wird als "Bein" bezeichnet.

Lediglich die Ausgewogenheit der Proportionen, die hohe Ausführungsqualität, der komplizierte Faltenwurf sowie die plastische und weiche Kopfgestaltung geben vielleicht Hinweise auf eine mögliche französische Herkunft dieses Griffs, direkte Parallelen aus diesem oder einem anderen Raum liegen nicht vor.

Der bei Scharnegoutum in Friesland gefundene Elfenbeingriff, eine Falknerdarstellung, zeigt als einziges Beispiel die gleiche hohe Basis. Ansonsten steht sie, mit der Kreuzschraffur des Falken und den ausgeprägt profilierten Seitenbordüren den Funden aus Hamburg, Stettin, Riga und Herlulfsholm nahe.

Der Griff wurde mit hoher Wahrscheinlichkeit im selben Einflußbereich hergestellt wie die angeführten Beispiele. Ob die Werkstatt Elfenbein neben anderen Materialien verarbeitet hat und im Nord- und Ostseeküstenbereich anzusiedeln ist, oder ob sie eine reine Elfenbeinschnitzerwerkstatt war und aufgrund des Fundortes näher am Einflußbereich der nordwestfranzösischen Werkstätten zu vermuten ist, muß offen bleiben.

Der Griff aus der Sammlung René d'Allemagnes in Rouen ist der einzige direkte Hinweis auf eine ursprünglich nordwestfranzösische Provenienz derartiger Griffe, da die Sammlung vor allem aus diesem Raum zusammengetragen wurde. Die mit einer Haube bedeckte und einen Hund auf dem rechten Arm tragende Figur aus Elfenbein zeigt in ausgewogenen Proportionen, mit plastischem Faltenwurf und in qualitätvoller Verarbeitung das Idealbild des Motivs, dem die Griffe aus Hamburg, Stettin, Riga, Scharnegoutum, Roskilde, Kalmar (2) und letztlich auch Ribe (3) folgen.

Als Hypothese mag die Schlußfolgerung gelten, daß zwei nordfranzösische Motivverarbeitungen (Rouen und Budapest, Lund 2) die Impulse für zahlreiche Werkstätten im Nord- und Ostseeraum gaben, die Produkte von unterschiedlichster Qualität aus Elfenbein, Walroßzahn, Knochen und Geweihstangen herstellten. 


\section{Nodwesteuropäische Griffe}

Eine dritte Gruppe von fünf Griffen aus Lüttich (?), Friesland, Arras und Lübeck zeigt eine deutlich andere Verarbeitung der höfischen Motive als die beiden vorangegangenen Gruppen.

In äußerst schmaler Gesamtdarstellung und mit einer starken Zurücknahme der Kopfproportionen zugunsten einer schlanken Griffgestaltung folgen sie einer anderen Stilistik, vor allem die Kopfgestaltung der Griffe aus Lüttich und Arras unterscheidet sich deutlich von der stereotypen Wiederholung des u.a. aus der Manessischen Liederhandschrift bekannten gelockten Idealbildes mit Krone oder Reif, dem in beiden vorangegangenen Gruppen alle Griffe gefolgt sind. Rundstabähnlich gestalteter Faltenwurf und fließende Bewegungen verleihen den Figuren eine hohe Plastizität und ein weiches, gefälligeres Aussehen, mit dem sie den Idealen der Elfenbeinplastik der französischen Hochgotik sehr viel näher stehen als zum Beispiel die Griffe aus Ribe (1), Oxford und Kalmar (2). Lediglich der in Friesland gefundene Elfenbeingriff ist härter und flacher gearbeitet.

Die Griffe aus Lüttich und Arras stammen vermutlich aus einer Knochen- und einer Elfenbeinschnitzerwerkstatt unter direktem französischen Einfluß, der friesische Griff steht mit seiner Gewandgestaltung den Griffen aus Oxford und Herlulfsholm nahe und ist vielleicht unter nordwestfranzösischem Einfluß in England entstanden. Daß dieser Einfluß bis in das Zentrum der Hanse reichte, zeigen die beiden Lübecker Griffe, die wahrscheinlich am Ort hergestellt worden sind. Der einzige möglicherweise archäologisch nachweisbare Knochenschnitzer im NordOstseeraum, der anthropomorph gestaltete Messergriffe herstellte, wäre demnach anderen Einflüssen gefolgt als die zu vermutenden Schnitzer im Einflußbereich der Hanse und in Südskandinavien. Die Datierung der Werkstatt in das 13. Jahrhundert mag ein Hinweis auf die Möglichkeit sein, daß diese Gruppe von Griffen älter sein könnte als die in der Regel um 1300 oder in das 14. Jahrhundert datierten Griffe Ribe 1, Kalmar 2, Budapest und Rouen und ihre zahlreichen Nachahmungen, so auch die Griffe aus Hamburg, Riga und Stettin.

\section{Weitere Griffe aus Skandinavien, dem Rheinland, Niedersachsen und unbe- kannter Herkunft}

Zwei Griffe aus Roskilde (2) und Oslo zeigen deutlich, wie sich auch verschiedenste Einflüsse an einem Beispiel festmachen lassen, und wie problematisch eine regionale Zuweisung aufgrund stilistischer Vergleiche ist.

Die schildbewehrte Ritterfigur des Roskilder Griffs ist im oberen Bereich mit keinem der bisher besprochenen Griffe zu vergleichen. Der plastische Faltenwurf erinnert jedoch stark an den Griff aus Lüttich, dessen Falten ebenfalls gerade fallen, im Gegensatz zu den Griffen aus Arras und Lübeck. Die Basisgestaltung findet ihre 
Entsprechung bei den Beispielen aus Kalmar (2) und der diesem Griff zugeordneten Funde aus Hamburg, Stettin und Riga.

Eine skandinavische Provenienz ist daher eher unwahrscheinlich. Dennoch findet sich zur Kopfgestaltung eine Parallele in dem aus Walroßzahn gefertigten Griffragment aus Oslo, welches aufgrund des Materials und der "individuellen" Gesichtszüge mit Schnurrbart wahrscheinlich am Ort selbst hergestellt wurde.

Die Griffe aus Skanderborg, Schonen und Visby scheinen chronologisch am Ende der anthropomorph gestalteten Messergriffe zu stehen. Das Schlußdatum des Exemplars aus Skanderborg liegt archäologisch vor 1430, die beiden übrigen Griffe wurden aufgrund der Kopfbedeckungen in die Zeit um 1400 datiert.

Die schräge Kopfhaltung und der nahezu identisch gestaltete Brustbereich des Knochengriffs aus Skanderborg und des Walroßzahngriffs aus Schonen machen eine gemeinsame regionale Herkunft wahrscheinlich, ohne daß diese ohne Vergleichsmaterial näher zu lokalisieren ist.

Der Knochengriff aus Visby, ein Sackpfeifer, erinnert in seiner weichen Konturengebung an die Griffe aus Arras und Lübeck, ob er nordwestfranzösischer Herkunft ist, kann lediglich vermutet werden.

Die"Schöne von der Isenburg", ein Knochengriff mit der Darstellung einer Dame mit Hund, ist archäologisch und historisch in die zweite Hälfte des 13. Jahrhunderts, sicher vor 1288 , datiert.

Der Brustbereich, vor allem die Gestaltung und die Haltung der Arme erinnert an die Falknergriffe aus Roskilde (1), Kalmar (2), Hamburg, Stettin und Riga, er ist jedoch nicht so hoch angesetzt.

Das schräg und fließend fallende Gewand ist nicht so plastisch gearbeitet wie bei den Griffen aus Arras und Lübeck, die Gesichtszüge erinnern in ihrer Plastizität an den Griff aus Ribe (1).

Das vollkommene Fehlen von Starrheit bei einer gewissen Flachheit der Körpergestaltung, der detailliert und plastisch gearbeitete Kopf und der kaum erkennbare leichte Schwung in der Gesamtkonzeption lassen französischen Einfluß erkennen, der aber nicht so dominant ist wie bei den Lübecker Griffen. Diese Eigenständigkeit und das hohe Niveau der Arbeit, die sich in der Kopf- und der Gesichtsgestaltung äußert, lassen trotz des Werkstoffes auf eine künstlerisch hochstehende Werkstatt schließen, die die französischen Einflüsse eigenständig verarbeitete.

Diese im benachbarten Köln zu suchen, mit der die Besatzung der Burg durch den Kölner Erzbischof eng verbunden war, liegt nahe, vor allem, da bekannt ist, daß die Kölner Elfenbeinschnitzer auch für sakrale Objekte seit der Spätromanik billigere Ersatzwerkstoffe für Serien von Figuren benutzten.

Die drei Griffe aus Småland, Reval und Vysoké Mýto haben trotz ikonographischer Unterschiede Gemeinsamkeiten (Basisgestaltung, Proportionalität, differenzierter Faltenwurf, Detailreichtum), die auf einen gemeinsamen Entstehungsraum hinwei- 
sen. Vor allem die Griffe aus Småland und Reval scheinen aufgrund ihrer fast identischen Darstellungsweise aus einer Werkstatt zu stammen. Stilistik, Plastizität und die Komplexität des Entwurfs stellen die drei Griffe in die Nähe der von Koechlin publizierten Beispiele, so daß eine Zuweisung nach Nordwestfrankreich wahrscheinlich ist. Nachahmungen minderer Qualität sind nicht bekannt geworden, so daß es sich bei diesen Griffen mit hoher Wahrscheinlichkeit um einzelne, importierte Luxusgüter handelt, die direkt an die Peripherie des wirtschaftlichen und kulturellen Einflußgebietes der hier behandelten Kulturräume gelangten.

Der Griff aus dem Kloster Wienhausen fällt ikonographisch und stilistisch völlig aus dem bisher behandelten Bestand heraus. Der eher an nordische Schachfiguren (Abb. 143a) als an die bisher behandelten schlanken gotischen Idealfiguren erinnernde thronende König steht in seiner kompakten und gedrungen unproportionalen Ausführung, seiner kantigen Linienführung und seinem starren Gesamtbild eher in der Abhängigkeit spätromanischer Formentradition ${ }^{289}$ (Abb. 143d). Die Ausführung ist von hoher Qualität bis in die kleinsten Details, die der verzierten und gemarkten Klinge und der Silbermanschette am Heft entspricht.

Zur Datierung steht im vorliegenden Fall neben dem Griff eine aussagefähige Klinge zur Verfügung, die vielleicht als die älteste bisher bekannte gemarkte Klinge angesehen werden darf.

Um eine Vorstellung von der Zeitstellung der Wienhauser Klinge zu bekommen, wurde sie mit den Klingen der sicher gotischen Messer aus Budapest und Riga sowie mit der sicher romanischen Klinge vom Wall am Dörhai (BRD, Kat. Nr. 81) verglichen.

289 Goldschmidt 1926, Bd. IV, S. 16, S. 47: dort mehrere Beispiele des thronenden Königs in der romanischen Elfenbeinschnitzerei 

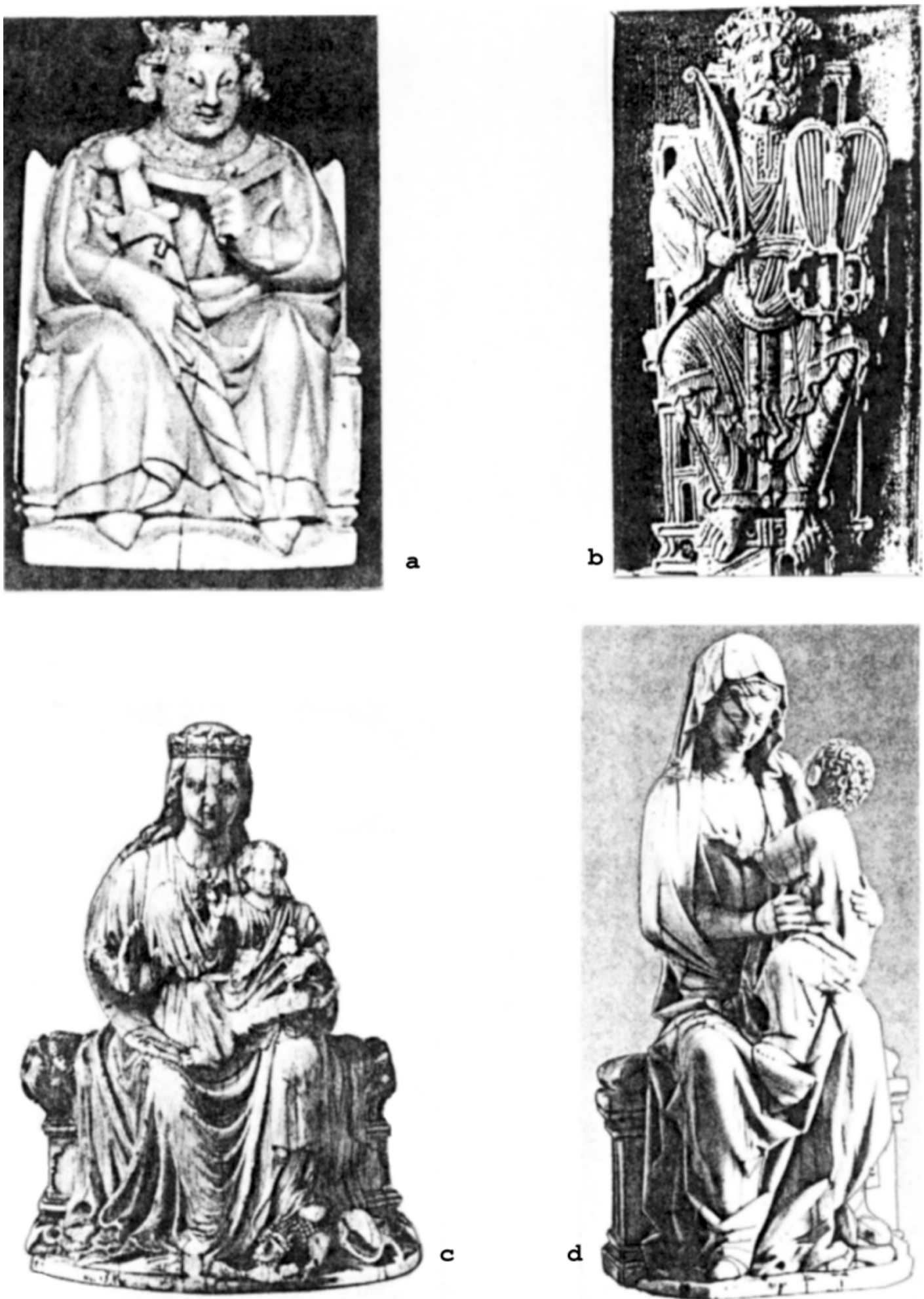

Abb. 143

Zur stilistischen Einordnung des Wienhauser Messergriffs a: Nordisch, 13.Jh., b: Nordfrankreich, 12. Jh.; c. Sachsen oder Rhein-MaasGebiet, um 1220/30; d: Frankreich, um 1270 
Die Verteilung der Klingenform der Messer aus Riga und Budapest (Ib-Ib2) zeigt chronologisch eine allgemein ansteigende Tendenz vom 9. bis zum 14. Jahrhundert, ihre Hauptverbreitungszeit liegt im 13. und 14. Jahrhundert (vgl. Kapitel 6.2.1).

Die Form des Messers vom Dörhai (Vb), dessen verzierte Klinge vergleichbar ist mit der Wienhauser Klinge, ist vom 8. zum 9. und vom 11. bis zum 14. Jahrhundert aufgetreten, ihre Hauptverbreitungszeit im zweiten Abschnitt liegt im 12. Jahrhundert.

Die Klinge aus Wienhausen hat die Form IIIb18.1, welche vor allem vom 8. bis zum 14. Jahrhundert aufgetreten ist, mit regelmäßig abfallender Tendenz. Ihre Hauptverbreitungszeit liegt im 8. bis zum 9. Jahrhundert, vom 11. bis zum 12. Jahrhundert läßt sich ein leichter prozentualer Anstieg beobachten, der im 13. Jahrhundert auf 2,2 \% und im 14. Jahrhundert auf 1,4\% zurückgeht.

Diese chronologischen Verbreitungstendenzen stellen die Klinge zeitlich zwischen die Messer aus Riga und Budapest als Vertreter der gotischen Messergriffe des ausgehenden 13. und beginnenden 14. Jahrhunderts und der Messerklinge vom Dörhai (um 1200), die mit ihrem romanischen Rankenwerk der Verzierung der Wienhauser Klinge näher steht als diese den schlankeren Klingen von Riga und Budapest.

Eine vorläufige Datierung des Wienhauser Messers in die erste Hälfte des 13. Jahrhunderts trägt diesen Überlegungen Rechnung ${ }^{290}$, sie ist dennoch als Vorschlag zu betrachten, denn auch in diesem Fall gilt, was sich bereits für die chronologische und regionale Einordnung der vorangegangenen Messergriffe abzeichnete:

Gerade die herausragenden Stücke qualitätvoller profaner Objektgruppen der Alltagskultur, die zwischen den Gütern sakraler und profaner Kunst und den weniger qualitätvollen Gegenständen des Alltags stehen, sind nur äußerst schwer einem bestimmten historischen Umfeld zuzuordnen.

Sie folgen in der Regel bereits einer überregionalen stereotypen Ikonographie, sind aber oft so selten, daß Vergleichsmöglichkeiten - wie etwa bei der Menge ihrer von unkundigerer Hand und dadurch in individueller Machart gefertigten Nachahmungen - fehlen, die wiederum bei den Objekten ausgebildeter Künstler entweder durch das Oevre eines Meisters oder ganzer Werkstattkreise in höherer Anzahl bekannt und vergleichbar sind.

Daher ist es auch bisher kaum möglich, den Wienhauser Griff einer bestimmten Elfenbeinschnitzerwerkstatt oder ihrem Umfeld zuzuordnen.

290 Zur langen Übergangsphase von der romanischen zur gotischen Elfenbeinschnitzerei vgl. Gaborit-Chopin 1978, S. 132 


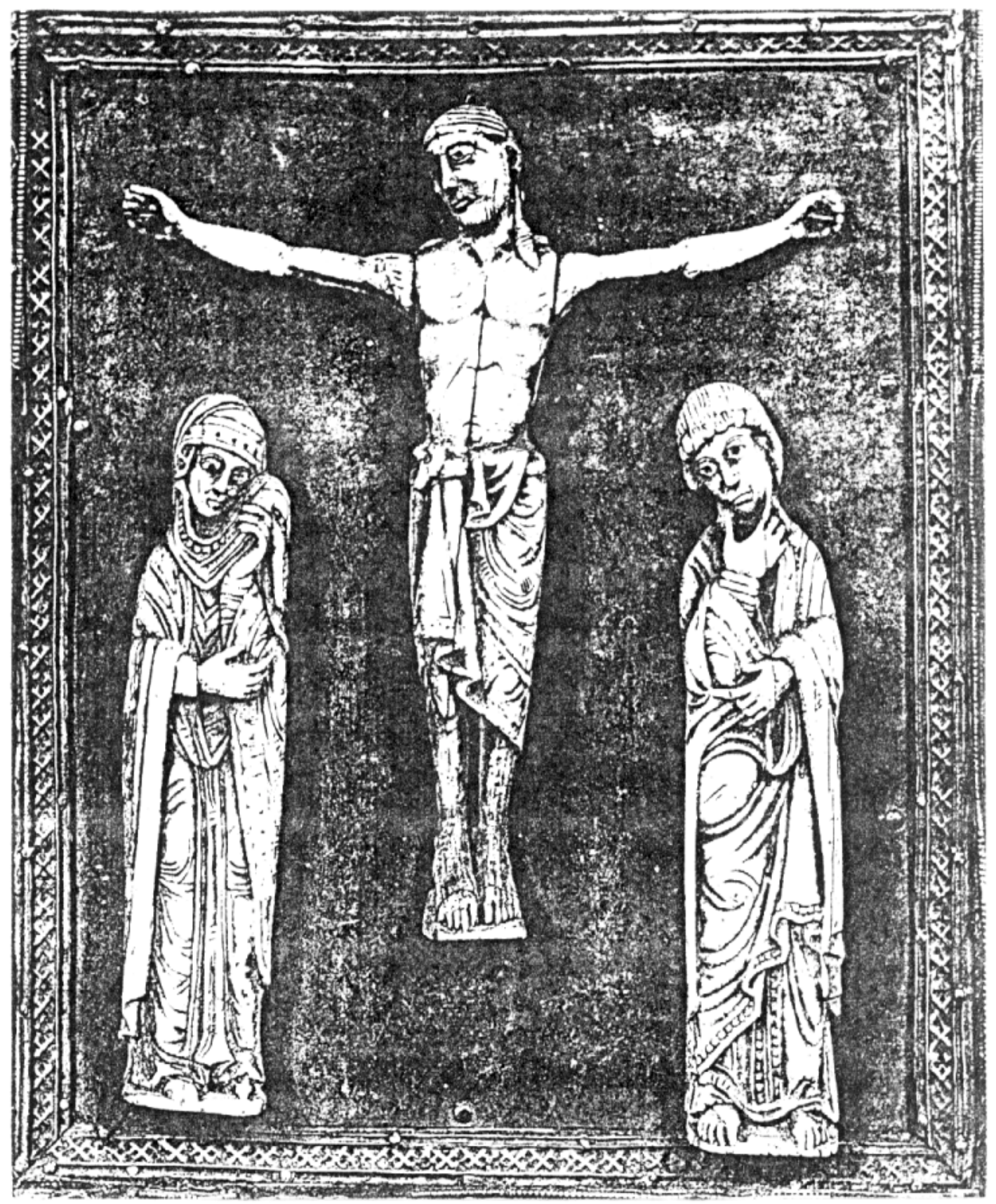

Abb. 144 Kreuzigungsgruppe (Buchdeckelausschnitt) sächsisch (Hildesheim?), E. 12. Jh. 
Es hat in Niedersachsen spätromanische Elfenbeinschnitzerei von hoher Qualität gegeben. Die bekanntesten Schmuckplatten von Tragaltären und Buchdeckeln werden Hildesheim und Helmarshausen zugeschrieben, sie sind in der Regel aus Walroßzahn gearbeitet ${ }^{291}$. Eine ikonographische Verbindung dieser sakralen Traditionsformen folgenden Werke zu dem profanen Messergriff ist jedoch nur schwer herzustellen (Abb. 144).

Die Ähnlichkeit mit dem dem Umfeld der Hildesheimer und welfischen Stiftsministerialität zugeschriebenen Messer vom Dörhai, sowie die eigenwillige, kantige und starre Stilistik des Griffs, die im Umfeld französischer Elfenbeinkunst kaum anzutreffen sein dürfte, macht eine einheimische, das heißt norddeutsche Provenienz der Klinge wahrscheinlich, des Griffs zumindest überlegenswert.

Das Messer aus Wienhausen gehört nicht in die Gruppe der bisher besprochenen Griffe und Messer. Bis weitere Forschungsergebnisse vorliegen, kann es als Einzelstück, das heißt als einzigartige Sonderanfertigung für eine Person aus dem Umfeld der Welfenfamilie oder der Meißener Markgräfin bezeichnet werden, die nach 1229 in das neu gegründete Kloster gelangte oder für eine dort lebende Person angefertigt wurde.

Der Holzgriff aus Kalmar (Slottsfjärden) nimmt ebenfalls eine Sonderstellung ein. Er stellt eine gekrönte Frau dar, die in ein faltenreiches Kleid und einen überwurfartigen Mantel gekleidet ist, ihr Haar fällt zu einem langen Zopf geflochten über den Rücken gerade herunter.

Die Figur steht stilistisch in der Tradition der skandinavischen Romanik und wird von D. Selling in die erste Hälfte des 13. Jahrhunderts datiert. Ihre Bewertung des Griffs als Importgut stützt sich lediglich auf die übrigen in Kalmar gefundenen Knochengriffe $^{292}$.

Das Material, die Verbindung mit der zeitgenössischen einheimischen Großplastik sowie die frühe Zeitstellung lassen aber eher den Schluß zu, daß der Griff in Skandinavien hergestellt wurde.

Gestützt wird diese Annahme durch die Umrißähnlichkeit dieses Griffs mit dem in Skara gefundenen Holzgriff des 11. bis 12. Jahrhunderts, dessen anthropomorpher Charakter nur angedeutet erscheint.

Beide Griffe belegen die Wahrscheinlichkeit, daß es in Skandinavien vor den von kontinentaler Ikonographie beeinflußten gotischen Knochen- und Walroßzahngriffen eine eigenständige Tradition anthropomorph gestalteter Messergriffe aus Holz gegeben hat.

\footnotetext{
291 Kirchliche Kunst des Mittelalters (verschiedene Autoren), in: Stadt im Wandel, Landesausstellung Nie- 


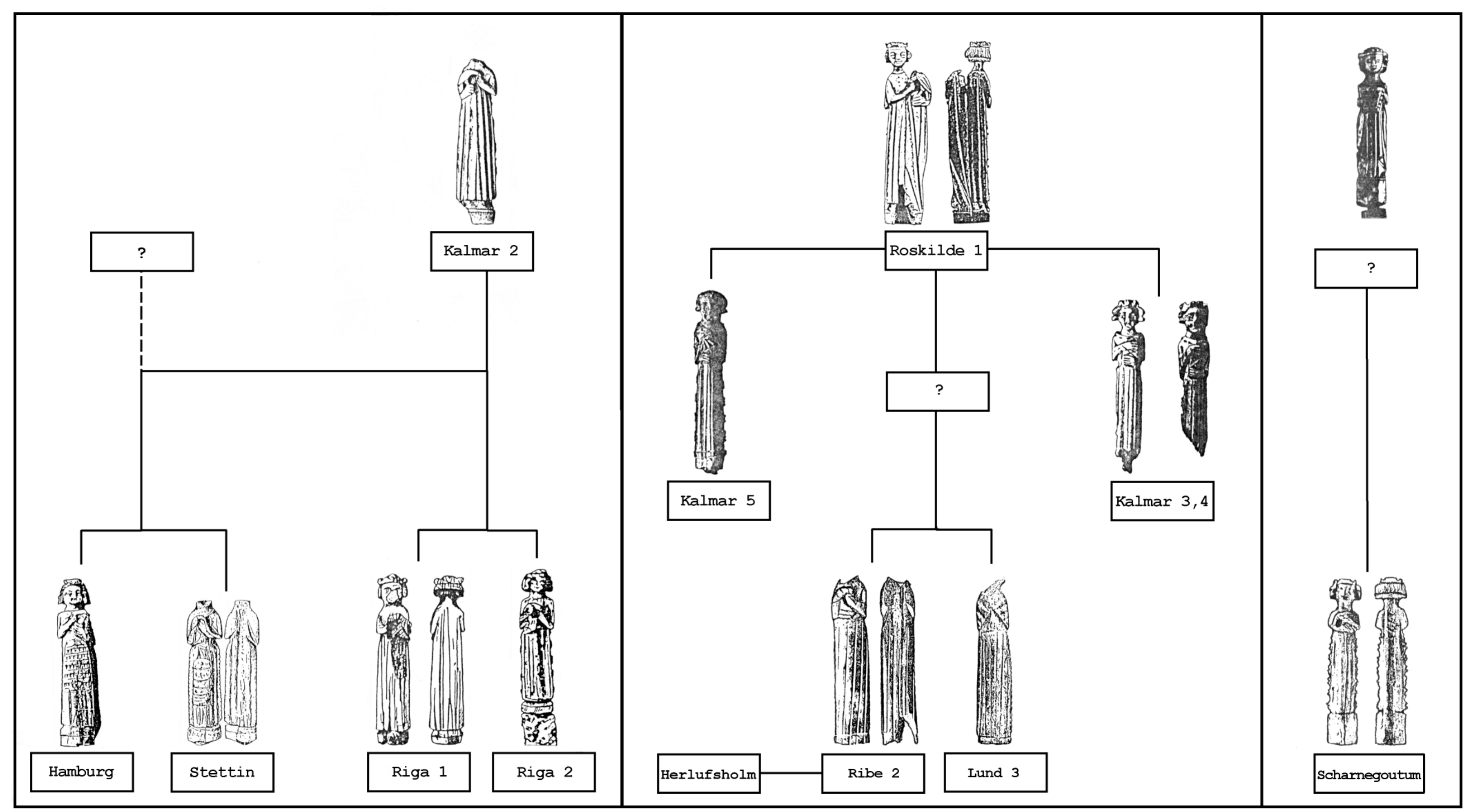

Abb. 145 Formenkreise nach ikonographischen, stilistischen und qualitativen Merkmalen I

Griffe im engeren Einflussbereich der Hanse 


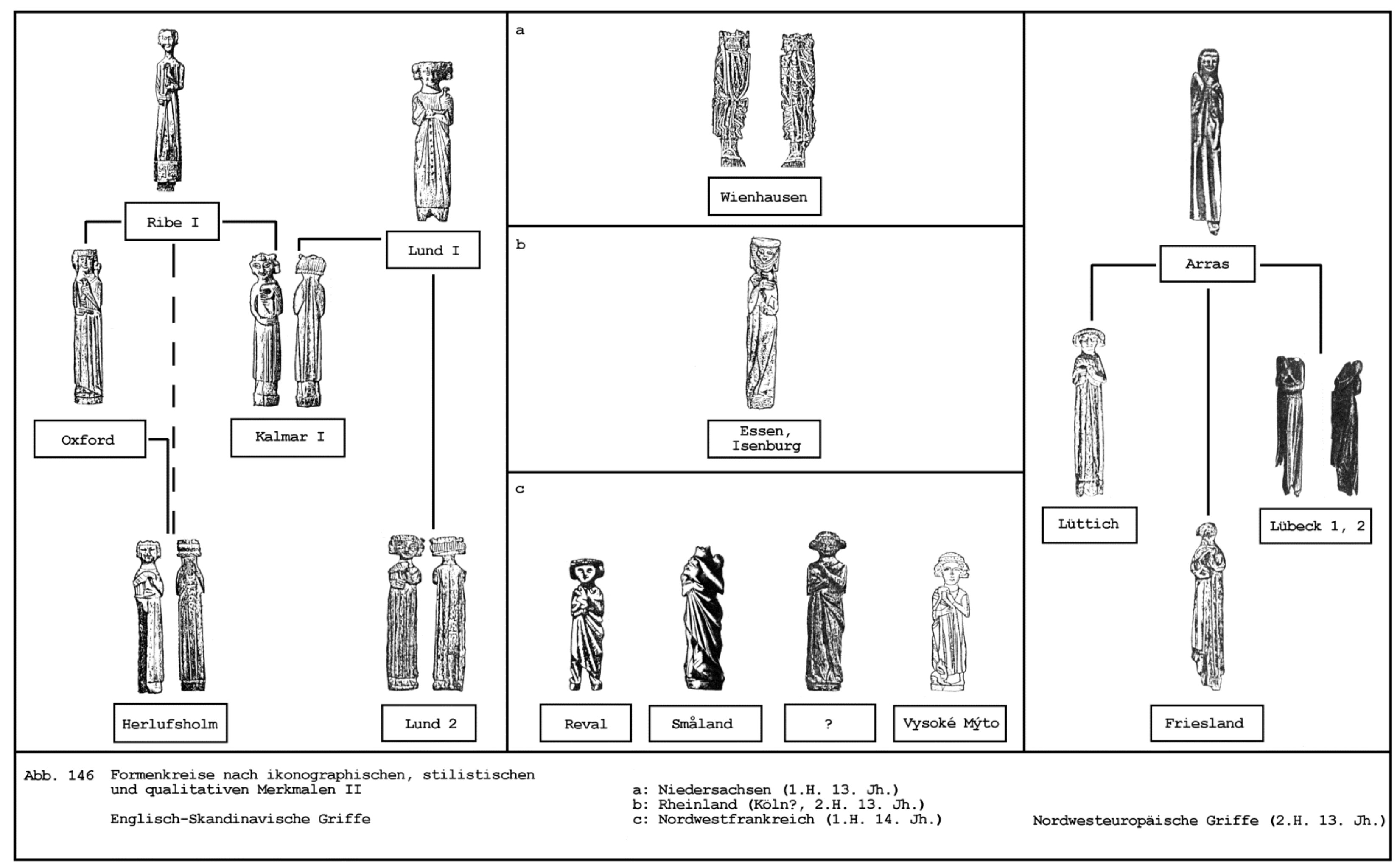




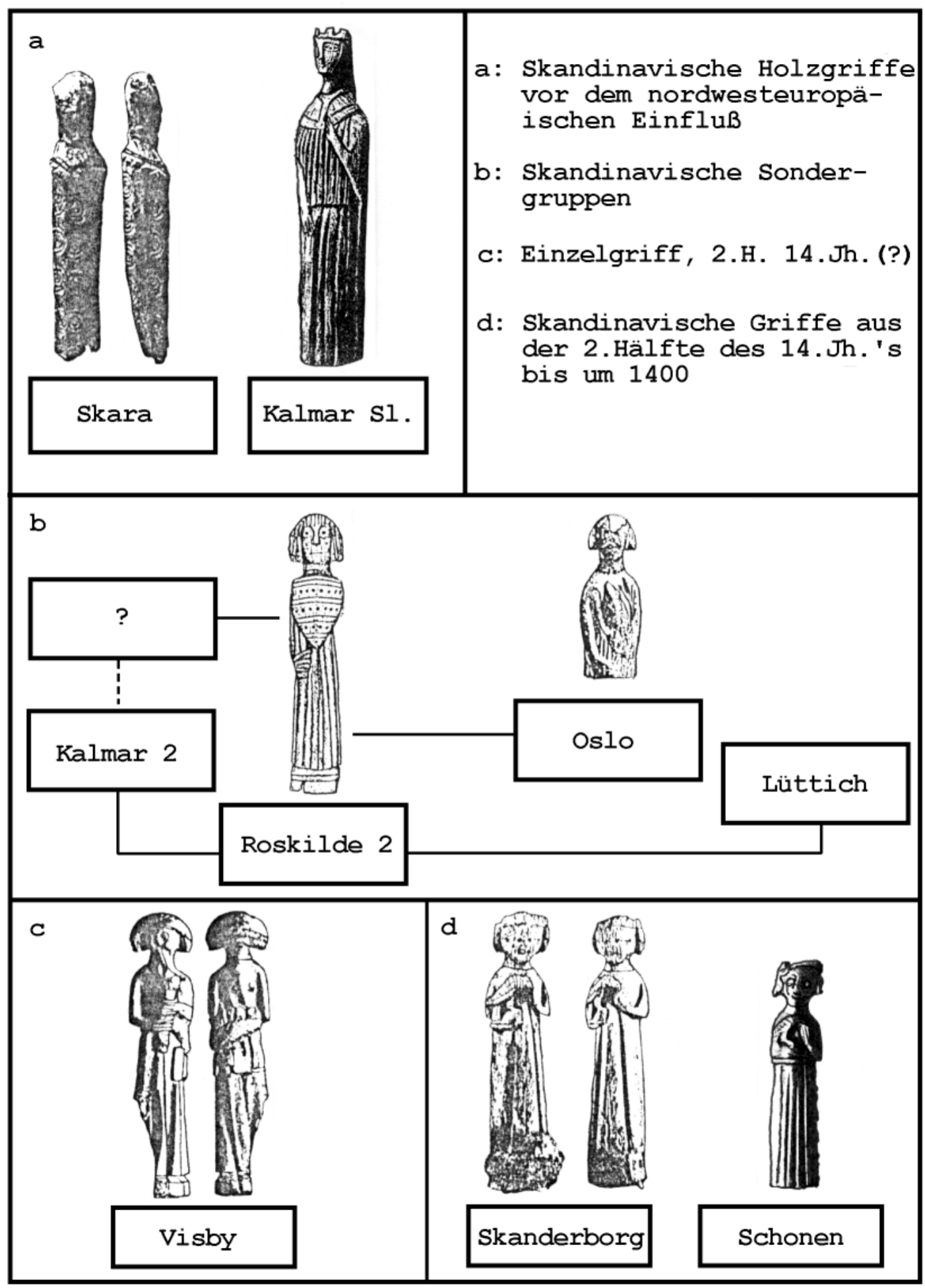




\section{Zum Handel mit Kunst- und Luxusgütern}

Nach der Analyse der Fundorte und der Differenzierung des hier behandelten Fundbestandes kann davon ausgegangen werden, daß ein Teil der Messer oder Messergriffe unabhängig von ihrem Herstellungsort als importiertes Ferngut angesprochen werden kann, wie das Messer aus Ribe (1), die Griffe und Messer aus Småland, Reval und Riga oder der Griff aus Lund (1). Diese Objekte sind sicherlich auf dem See- oder Landweg oder über eine z.T. große Distanz transportiert worden. Andere Funde wie die Griffe aus Scharnegoutum, Hamburg und Stettin haben - je nachdem, wo ihre Herkunft zu lokalisieren ist - kürzere Seewege, vielleicht nur Küstenstrecken überwunden, während Griffe wie die von der Isenburg, aus Friesland und Herlulfsholm auch auf dem Fluß- oder Landweg an ihren Fundort gelangt sein können.

Träger des überregionalen Handels sind, das zeigt die Verbreitungskarte deutlich, mit hoher Wahrscheinlichkeit Fernhandelskaufleute der Hanse, der einzigen Organisation, die all diese Städte und Plätze regelmäßig anfuhr, dort präsent war und nicht nur Handel mit Pelzen, Erzen, Fisch und Salz, sondern auch mit Kunstgegenständen und Luxusgütern trieb und damit zur Verbreitung der Ideale und der Ikonographie dieser Objekte beitrug.

M. Tausch bezeichnet diese Güter als "auf höherer Ebene sich bewegendes Begleitmaterial zu Bernstein, Bier und Butter" ${ }^{293}$. Zeugnisse des hansischen Handels mit sakraler und profaner Kunst reichen von den "hunderten von deutschen Altären, Gemälden und Bildwerken", die "mit hansischen Koggen über die See nach Dänemark, Norwegen, Schweden und Finnland geschafft" wurden ${ }^{294}$, bis zu rheinischem Steinzeug, sie umfassen englische Alabasterfiguren des 14. bis 15. Jahrhunderts, die sogenannten Hanseschalen und Solinger Klingen und Altenaer Kettenpanzer des 13. bis 16. Jahrhunderts, die vor allem über Köln exportiert wurden ${ }^{295}$.

Die daraus entstehenden kulturellen Kontakte führten dazu, daß über die Verkehrsund Handelswege die verschiedenen Kulturzentren untereinander verbunden waren sowie eine weite Außenwirkung erfuhren, so daß verschiedenste Kunstrichtungen und Stilentwicklungen auch über den engeren Wirtschaftsraum der Hanse hinaus verbreitet wurden und in Objekten ihren Niederschlag fanden ${ }^{296}$.

H. Amman verbindet beispielhaft für diese Wirkung mit der Blütezeit der Champagnemessen die "deutsche Fühlungnahme mit der Gotik" und sieht über den

\footnotetext{
293 Tausch 1973, S. 298

294 Tausch 1973, S. 300

295 Leittexte der Ausstellung zum Artikel von Tausch 1973, S. 409-415

296

Tausch 1973, S. 370
} 
Bereich der bildenden Kunst auch Inhalte und Anregungen aus dem "engen Bereich der Messen der Champagne" in die deutsche Dichtung ${ }^{297}$.

Beispielhaft für den Einfluß westeuropäischer Kunst im östlichen Hanseraum, der sich in der Verbreitungskarte der hier behandelten Messergriffe widerspiegelt, steht die Entwicklung der bildenden Kunst in Danzig.

Die ikonographische und stilistische Beeinflussung der in Danzig tätigen Werkstätten erfolgte chronologisch in drei Schüben ${ }^{298}$ :

Im 13. Jahrhundert stand Danzig künstlerisch unter dem Einfluß des deutschen Ordens. In der ersten Hälfte des 14. Jahrhunderts dominierte der Einfluß aus dem Rheinland und Westfalen, für die zweite Hälfte dieses Jahrhunderts konstatiert Wasowicz böhmischen Einfluß, vor allem aus dem Kunstzentrum Prag.

Erst seit dem 15. Jahrhundert finden auch englische, niederländische und weitere norddeutsche Kontakte ihren Niederschlag ${ }^{299}$.

Der Fluß west- und mitteleuropäischer Kultur über die Verkehrswege und Händler der Hanse reichte bis an die äußerste Peripherie:

Nicht nur Importgut wie die Kleinplastik aus Rostow (siehe oben) wurde verhandelt, auch westeuropäische Stilistik im russischen Kunsthandwerk wie die Verwendung gotischer Motive bei Novgoroder Silberarbeiten "kann nur mit der Vermittlung der Hanse erklärt werden" ${ }^{300}$.

Diese Beispiele zeigen, daß unabhängig von der Frage, wieviele der anthropomorph gestalteten Messergriffe tatsächlich als Direktimport aus dem nordwestfranzösisch beeinflußten Initialraum stammen, wieviele als einheimische Nachahmung oder Weiterverarbeitung anzusprechen sind und ob, wie im Fall der Griffe aus Kalmar (Slottsfjärden) und Skara, möglicherweise einheimische Traditionen mit zu berücksichtigen sind, die Hanse als Träger des Objekthandels und als Transporteur der Ideen und mit ihr der Ikonographie, die im Folgenden besprochen werden, die Voraussetzung dieser Verbreitung ist.

\section{Die ikonographischen Vorbilder}

Bereits mehrfach wurde im Zusammenhang mit der europäischen Elfenbeinschnitzerei auf die stilistischen und motivischen Abhängigkeiten hingewiesen, denen die einzelnen Schmuckplatten, Plastiken oder Griffe bei ihrer Entstehung unterlagen.

\footnotetext{
297 Ammann 1978, S. 94-95

298 Wasowicz 1973, S. 254-255

299 Wasowicz 1973, S. 256

300 Angermann 1973, S. 277
} 
Der auch von Leciejewicz angesprochene Vergleich mit der "zeitgenössischen" Monumentalplastik impliziert allerdings einen Zusammenhang, der so direkt nicht bestanden haben wird.

Die Abhängigkeiten sind chronologisch oft verschoben und auch stilistisch verschlungen. Ein direkter Vergleich der Messergriffe mit nordwestfranzösischer Kathedralplastik mag auf den ersten Blick mehr Ähnlichkeiten und stilistische Verwandtschaft zeigen als mit den Pariser Elfenbeingriffen um 1300, doch dieser Eindruck täuscht, da er die tatsächlichen Verhältnisse nicht berücksichtigt (Abb. 140).

Man kann nicht einmal die oft zitierte Abhängigkeit von Kleinplastiken als Miniaturausgabe von monumentaler Steinplastik als Absolutum voraussetzen, wie das Beispiel der Elfenbeinmadonna der Sainte Chapelle in Paris zeigt. Diese Plastik hat in der Folge als individuelle Einzelleistung zahlreiche sakrale Kunstwerke, auch Großplastiken beeinfluß $\mathrm{t}^{301}$, obwohl auch sie nicht ohne Vorbilder entstanden ist.

So bezeichnet D. Gaborit-Chopin die Verbindungen zwischen der Monumentalund der Elfenbeinplastik ausdrücklich als "Zusammenhänge und nicht Abhhängigkeiten" und die Elfenbeinschnitzerei wie die Holzplastik als "Sondergattungen der Großplastik... in häufig eigenständigen Formen" ${ }^{302}$.

Die Kompliziertheit dieser stilistischen Zusammenhänge, die lediglich der Ausdruck einer komplexen Verflechtung von Künstlern und Werkstätten zur Zeit der Entstehung darstellen, führen dazu, daß nicht einmal für die bedeutendsten Werke der Elfenbeinplastik eindeutige Werkstattzuweisungen möglich sind (vgl. zum Beispiel den Kruzifixus von Borgello und seine regionale Einordnung ${ }^{303}$ ).

Vergleiche mit zeitgenössischen Goldschmiedearbeiten, Miniaturen, Holz- und Steinskulpturen ermöglichen den Kunsthistorikern die Herausarbeitung von regionaltypischen Stilistika, deren Kriterien auf allen plastischen Bildern eines Stilkreises wiederkehren, wie "der vergleichsweise schwere und strenge Stil", geprägt von "hartbrechenden Gewandfalten" und "großen, geometrisch bestimmten Formen", der, zum Teil von Paris beeinflußt, um die Mitte des 13. Jahrhunderts nördlich der Île de France angesiedelt wird ${ }^{304}$.

Seit Beginn des 14. Jahrhunderts verändert sich nicht nur der Gesamtstil, der sich, mit der Herrschaft Philips des Schönen (1285-1314) verbunden, im ausgehenden 13. Jahrhundert von Paris aus in fast ganz Europa durchsetzt ${ }^{305}$.

Für die Datierung der hier behandelten Messergriffe fast noch wichtiger ist die Festlegung der Ikonographie.

\footnotetext{
301 Tudor-Craig 1987, S. 99 
Die Hauptschwierigkeit, die jetzt zahlreichen elfenbeinernen Kleinkunstwerke sakraler Nutzung bestimmten Werkstätten zuzuweisen, die seit Beginn des 14. Jahrhunderts in viel größerer Anzahl auch namentlich bekannt sind, liegt in dieser "stereotypen Ikonographie", deren Schemata seit Anfang des 14. Jahrhunderts festliegen und bis zum Beginn des 15. Jahrhunderts "unermüdlich wiederholt" wer$\operatorname{den}^{306}$.

Genau dies trifft auch für die profanen Messergriffe $\mathrm{zu}$, sie folgen bis auf die beiden Exemplare des frühen 13. Jahrhunderts alle einer bestimmten Ikonographie, auch die um 1400 datierten Beispiele aus Skanderborg, Schonen und Visby ${ }^{307}$.

Abgesehen von dem stereotypen Lächeln, welches am deutlichsten an der Falknerfigur des Riber Messers (1) auftritt, stilisiert aber selbst an den Griffen minderer Qualität (Herlulfsholm, Hamburg, Lund 2) noch erkennbar ist, und welches P. Tudor-Craig im Zusammenhang mit der Madonna der Sainte Chapelle als Ausgangspunkt zahlreicher lächelnder Engel, Teufel und Jungfrauen bezeichnet, das Europa ein halbes Jahrhundert lang "überflutete" ${ }^{308}$, lassen sich die Vorbilder der Messergriffe kaum noch im Repertoire der sakralen Ikonographie des 14. Jahrhunderts ausmachen.

Eine nahezu vollständige Aufführung der Motive und ihrer Ikonographie, von denen die Falkner, Damen und andere Motive, die an den Messergriffen begegnen, entlehnt wurden, zeigt die wahrscheinlich kurz nach 1300 von Rüdiger Manesse in Zürich initiierte und bis kurz nach 1330 mit Zusätzen versehene Große Heidelberger Liederhandschrift, dem Codex Manesse, mit dem von dem Züricher Dichter Hadlaub überlieferten Zweck, "das Lob der Frauen im Minnesang zu bewahren und weiterhin zu pflegen"309.

Diese mit 137 Miniaturen versehene Sammlung führt nicht nur die zeitgenössisch relevanten Dichter mit ihren Minneliedern auf, sie liefert ebenso einen Katalog der ikonographischen Topoi, mit denen neben den dargestellten Dichtern auch die Szenerie, die Gestik und die Symbolik der epischen Inhalte bildlich dokumentiert werden.

Die Miniaturen des Codex Manesse (Abb. 148 bis 153) sind nicht als direkte Vorbilder für die Messergriffe zu bewerten.

\footnotetext{
306 Gaborit-Chopin 1978, S. 156

307 Die in diesem Zusammenhang von Gaborit-Chopin erhobene Forderung nach einer "detaillierteren Photo-Dokumentation als jener von Koechlin" berührt direkt ein Problem, welches zusätzlich zur archäologischen Quellenkritik bei der Bearbeitung der anthropomorph gestalteten Messergriffe auftritt. Um die Aussagen über den Bestand auch nur annähernd wissenschaftlicher Wahrscheinlichkeit zuzuführen, müssen alle vorhandenen Griffe dieser Gattung erfaßt werden, auch die in Privatsammlungen befindlichen, selbst wenn ein großer Teil von ihnen ohne Fundnachweis bleiben sollte. Bis jetzt wurden nicht einmal alle im Museumsbesitz befindlichen Griffe publiziert, so daß der hypothetische Charakter der meisten Aussagen unumgänglich bleibt. 
So finden sich Vorbilder für viele dieser Miniaturen unter anderem in den Elfenbeinerzeugnissen des ausgehenden 13. Jahrhunderts wieder, die ihrerseits als Ergebnis der "in Frankreich ausgebildeten Typik der Darstellungen aus dem Ritterleben" betrachtet werden ${ }^{310}$.

Ursprung und Enstehung dieser Bildtradition sind älter. Seit dem ausgehenden 12. Jahrhundert sind Darstellungen von Szenen oder Szenenfolgen ritterlichen Lebens bekannt, das Eingangsbild der Manessehandschrift (Kaiser Heinrich) greift in Abänderung der älteren Weingartener Handschrift auf Kirchenfenster und Siegelbilder des beginnenden 13. Jahrhunderts zurück ${ }^{311}$.

Die ritterlich-höfischen Motive, die in illustrierten Handschriften, an Elfenbeinkästchen ("Minnekästen"), Spiegelkapseln und Griffen begegnen, stammen aus der "Übernahme von Figuren und Kompositionsschemata" aus zeitgenössischer Epik $^{312}$, sind also das Ergebnis einer direkten Herausnahme aus der mittelalterlichen Erzähltradition und Dichtung in die bildliche Darstellungsweise, die sich an der Quelle dieser Translation genauso sinnbildlich äußert wie am Ende der Rezeption an einem Werkzeug der mittelalterlichen Tafel.

Es verwundert daher nicht, wenn die Motive der Messergriffe, die zum Teil archäologisch sicher in die zweite Hälfte des 13. Jahrhunderts datiert werden, in einer Handschrift um 1300, die zudem noch rund 1000 Kilometer weiter südlich entstanden ist, wiederkehren. Die um 1300 festgehaltenen Motive und Szenenfolgen im Zusammenhang mit der Tradition der Minnesängerkultur und der sich gegenständlich äußernden Lebensart ritterlicher Stände konnten variabel gehandhabt werden, je nachdem, was auf welche Weise und für wen dargestellt werden soll$\mathrm{te}^{313}$.

Dabei wurde von Seiten des Buchmalers, der Motivvorbilder suchte, auch auf andere und ältere Bildträger zurückgegriffen. Selbst aus dem Bereich der Sakralkunst wurden Motive aufgegriffen und dem neuen Bildinhalt angepaßt ${ }^{314}$. O. Beigbeder führt dafür mit Belegen profaner gotischer Elfenbeinwerke unter anderem die ikonographische Verbindung zwischen der Marienkrönung aus dem Marienkult und der Krönung einer Dame aus dem Minnezyklus $\mathrm{an}^{315}$.

Die Möglichkeiten der Darstellungsfülle veränderten sich mit der Größe und dem Zweck des Bildträgers. Illustrierte Handschriften weisen ganze Sammlungen von Bilderzyklen auf, textbegleitende Romanillustrationen "erzählen" ähnlich wie die fast textlosen Armenbibeln, auf den Schauseiten der elfenbeinernen oder hölzernen "Minnekästchen" erscheinen noch einzelne Szenenfolgen, auf Spiegelkapseln noch eine einzige Szene mit mehreren Figuren, bis auf Griffen von Messern bestenfalls

\footnotetext{
310 Vetter 1988, S. 276

311 Vetter 1988, S. 276-277, S. 290-291

312 Vetter 1988, S. 284-285

313 Vgl. z.B. die Veränderung der Vorlage des Bildes von Wachsmut von Künzingen, Vetter 1988, S. 280, 281

314 Vetter 1988, S. 188-289

315 Beigbeder o.J., S. 33, 34
} 
eine Situation, in der Regel nur noch eine stereotype Figur als Symbol wiedergegeben wird.

All diese Abbildungen sind letztlich verschiedene Ausdrucksformen einer bestimmten und in all ihrer Symbolik auch bekannten Ideenwelt, Lebensart und eines Selbstverständnisses, das wie die Rezeption ritterlicher Topoi nicht auf Personen des Adels beschränkt war.

So wie in den Miniaturen des Codex Manesse neben dem Kaiser und Vertretern des Hochadels Mitglieder des Amtsadels und des Bürgertums als Schöpfer höfischer Dichtung auftreten, so belegen die Fundorte der Messer und Messergriffe vor allem das städtische Bürgertum als Rezipienten höfischer Idealität. 


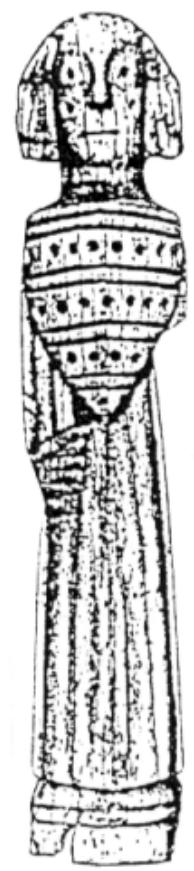

a
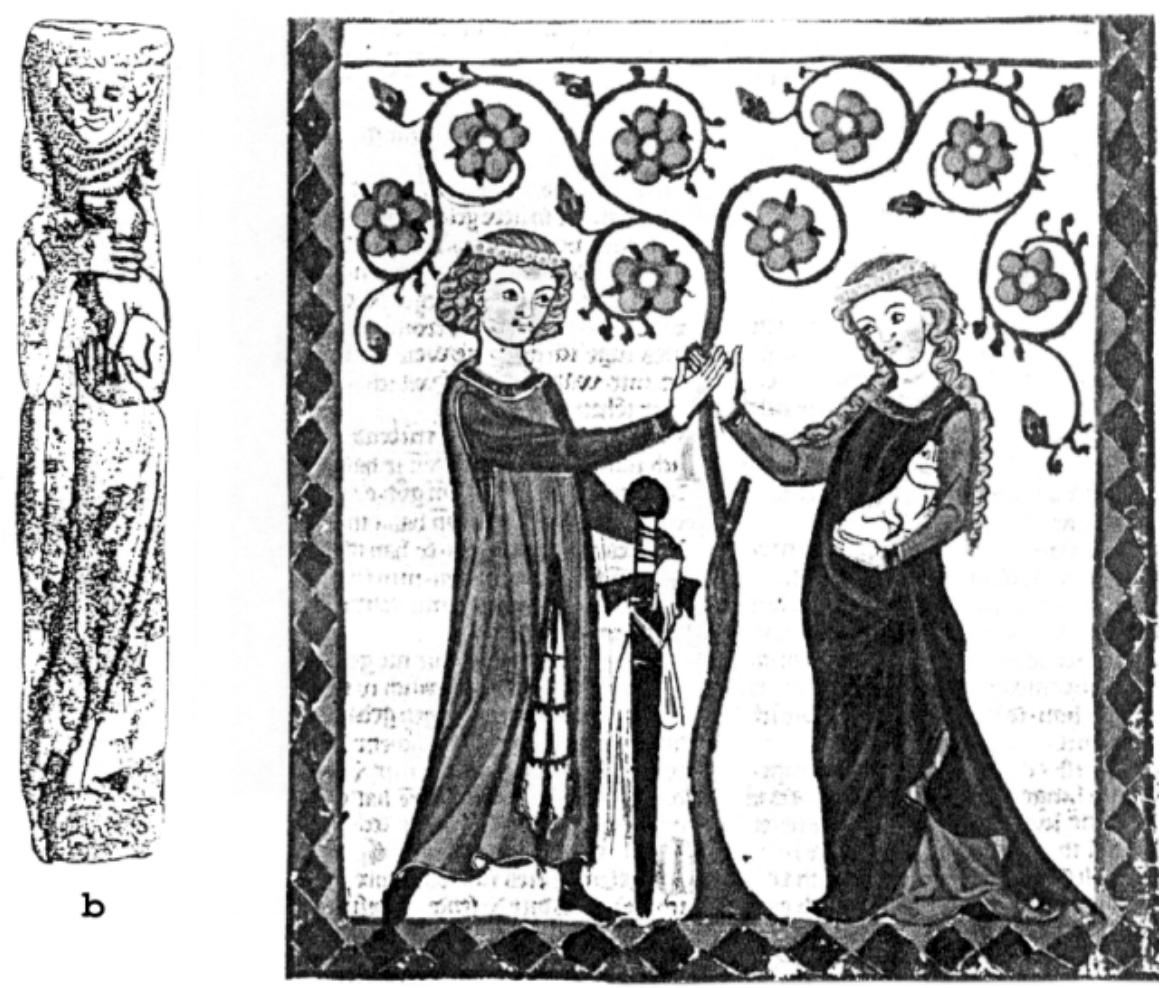

c

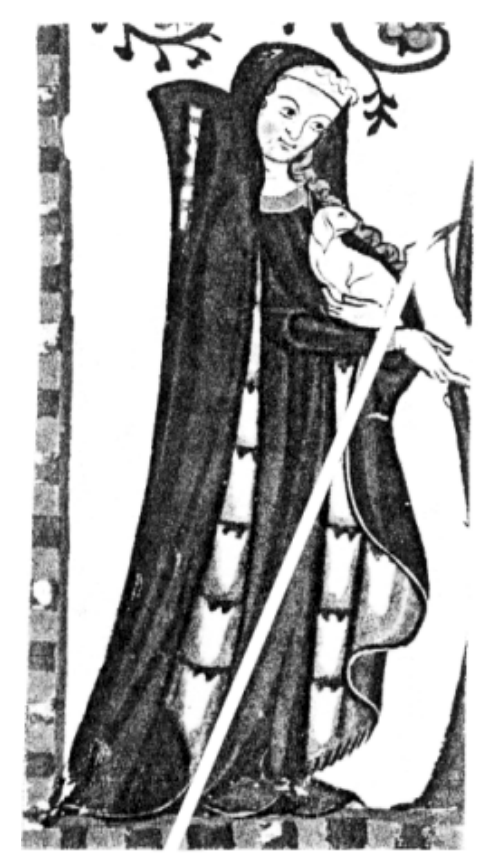

d

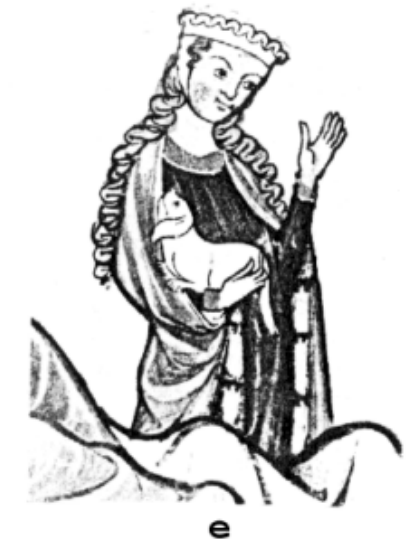

e

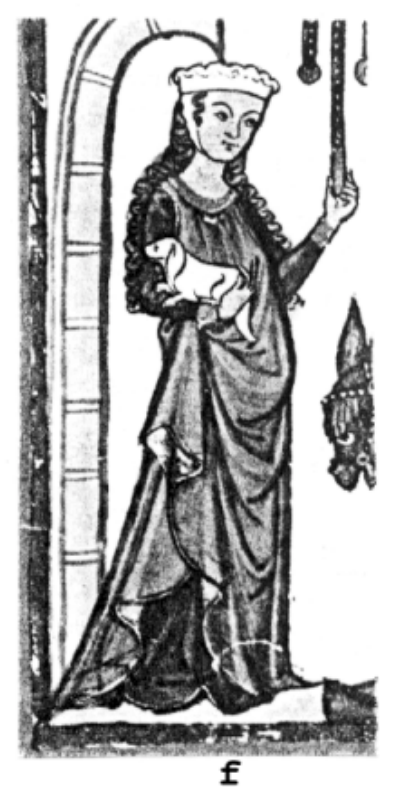

Abb. 148

Die ikonographischen Vorbilder I

Ritter und Dame mit Hund

a: Roskilde; b: Isenburg (Essen); c-f: Codex Manesse, Tafeln 55, II4, 34 u. 27 

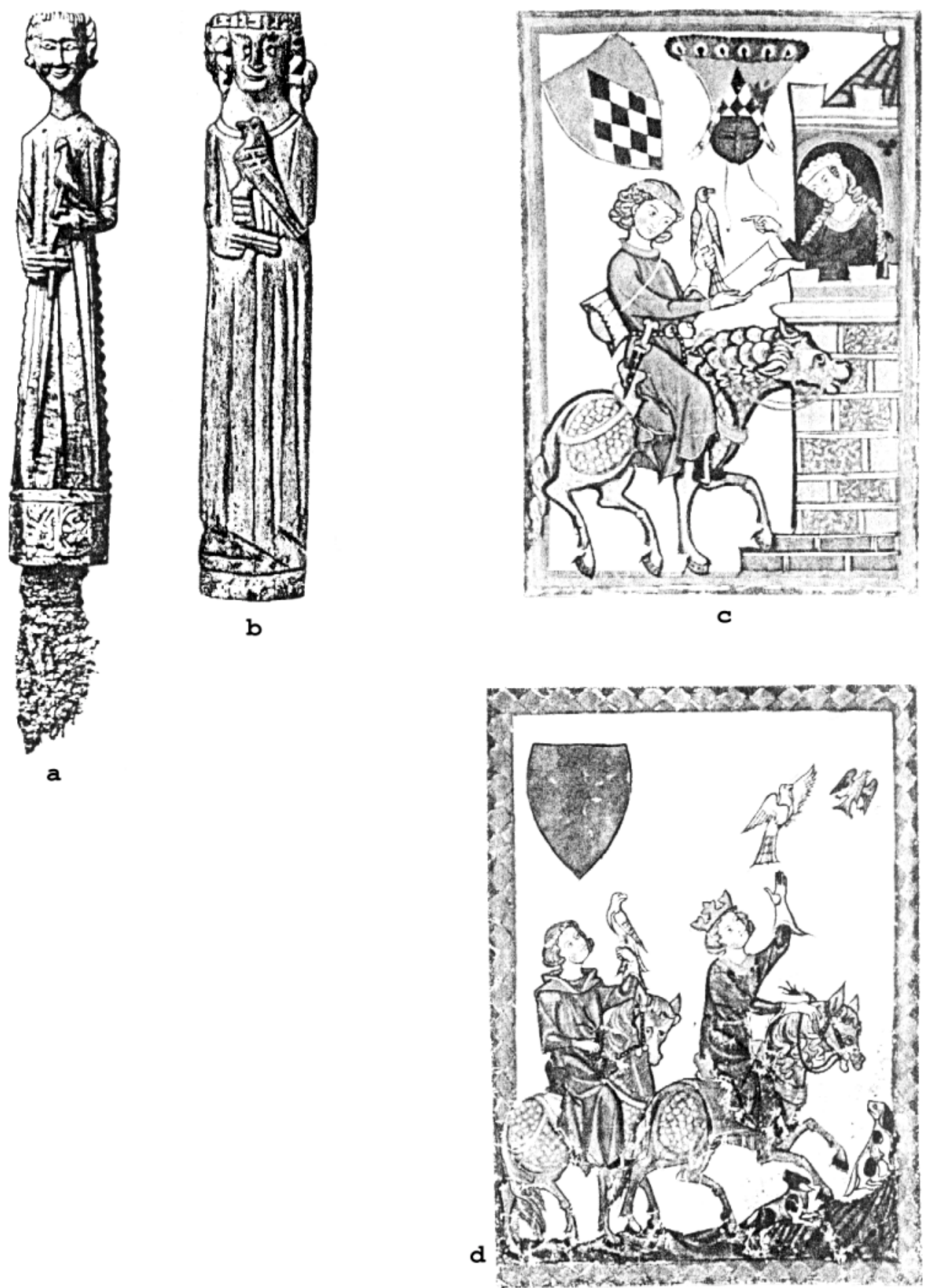

Abb. 149

Die ikonographischen Vorbilder II

Falkner

a: Ribe, b: Oxford; c-d: Codex Manesse, Tafeln 52, 2 


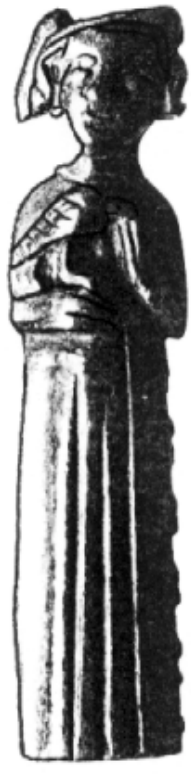

a
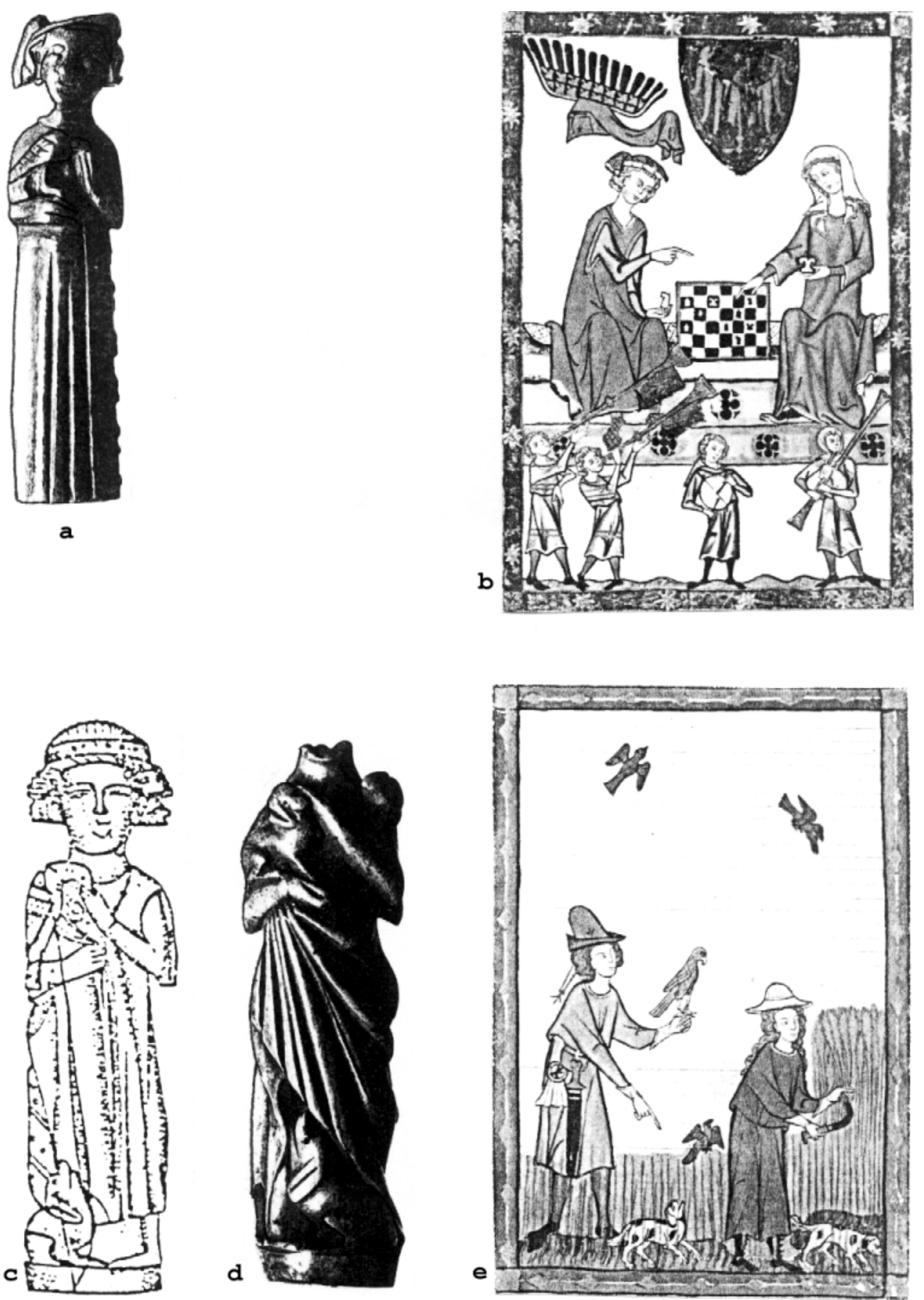

Abb. 150

Die ikonographischen Vorbilder III

Falkner mit barettähnlicher Kopfbedeckung;

Falkner mit Hund / Dame mit zwei Hunden

a: Schonen, c: Vysoké Mýto; d: Småland; b,e: Codex Manesse, Tafeln 6,125 

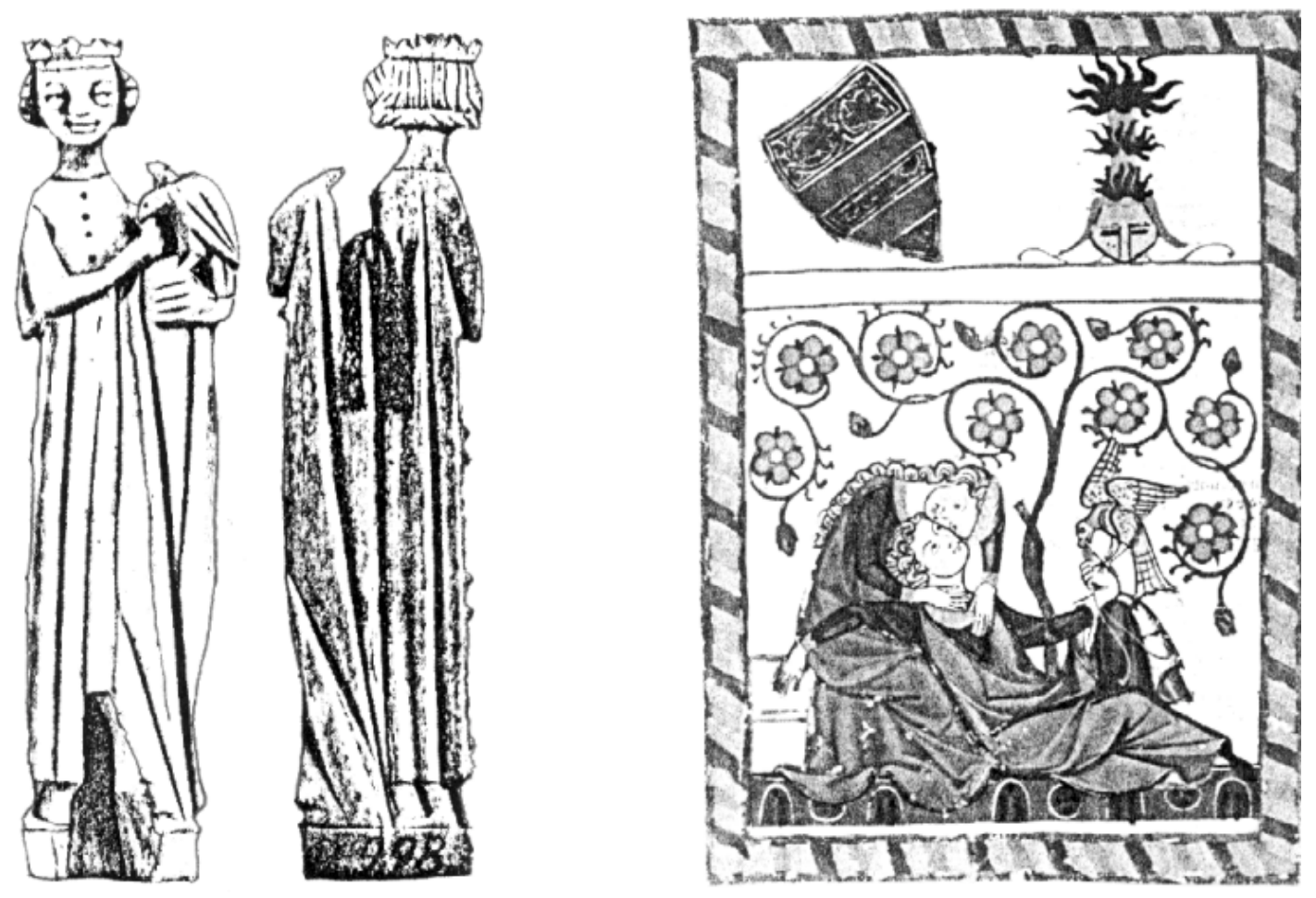

a

$\mathrm{b}$

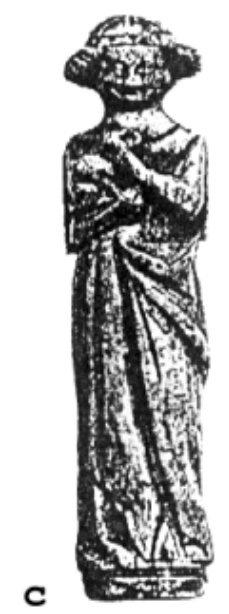

Abb. 151
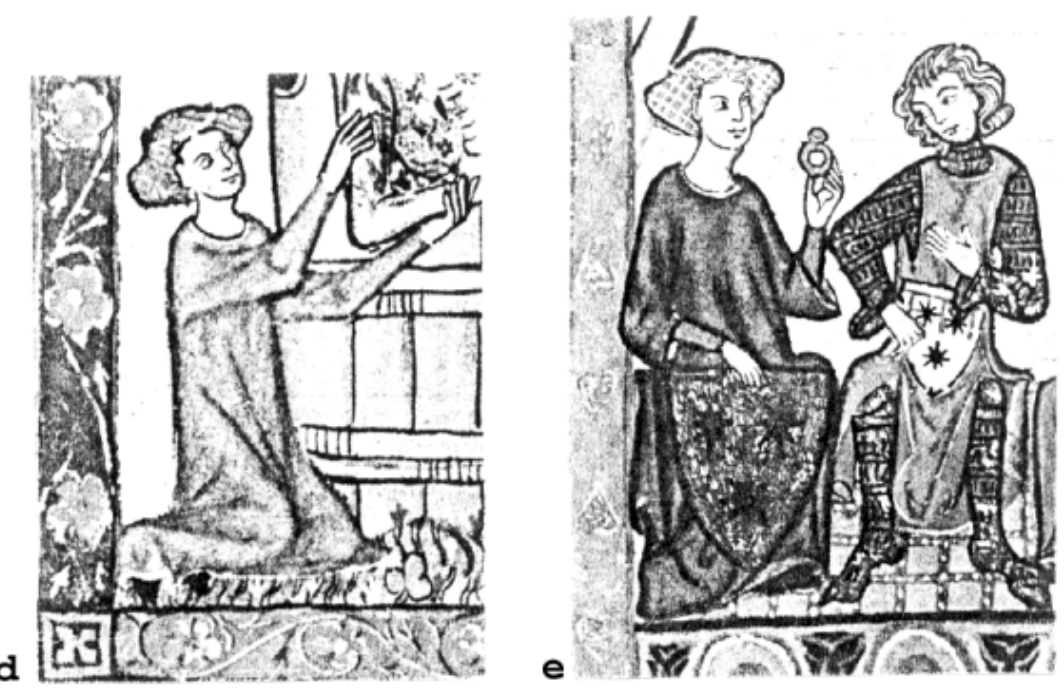

Die ikonographischen Vorbilder IV

Paar mit Falke, Dame (?) mit Haube / Haarnetz

a: Roskilde; c: Nordfrankreich, b, d, e: Codex Manesse, Tafeln 80, 20, 76 

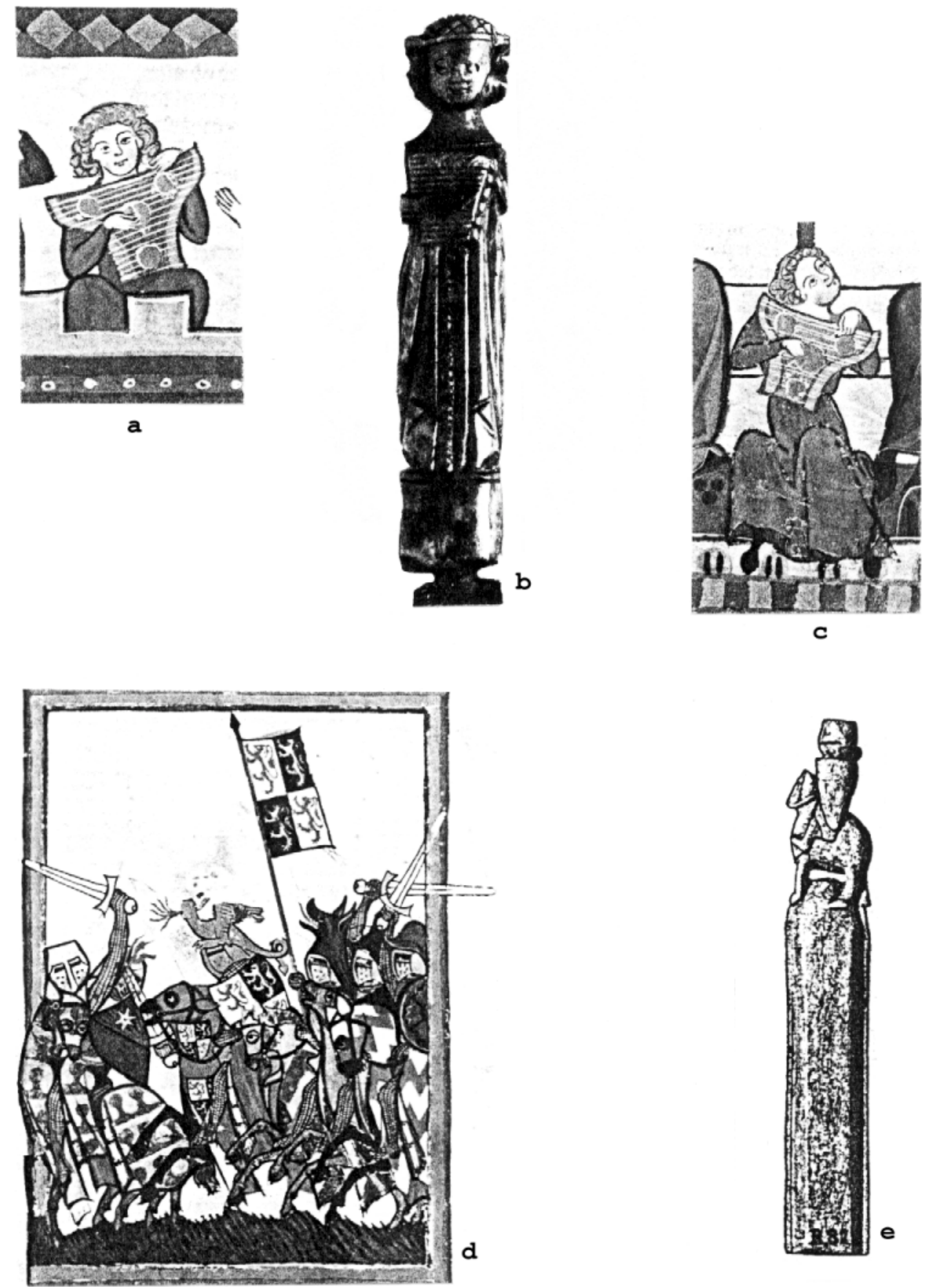

Abb. 152

Die ikonographischen Vorbilder IV

Hackbrett / Sänger; Ritter zu Pferd mit Topfhelm

b: Nordfrankreich (?); e: Köln; a, c, d: Codex Manesse, Tafeln 131, 91, 9 


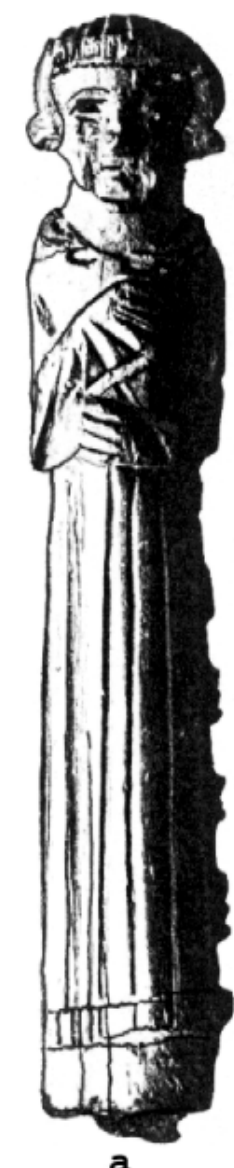

a

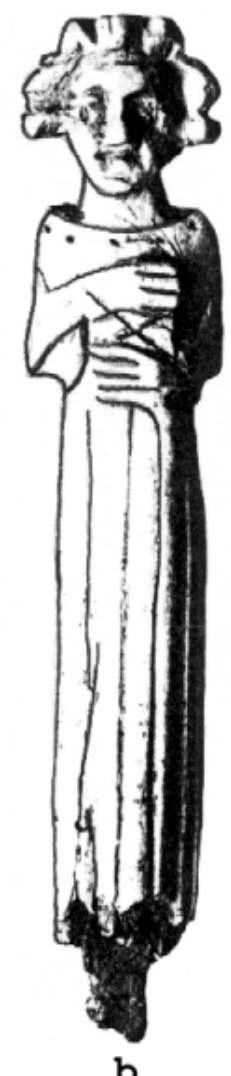

b
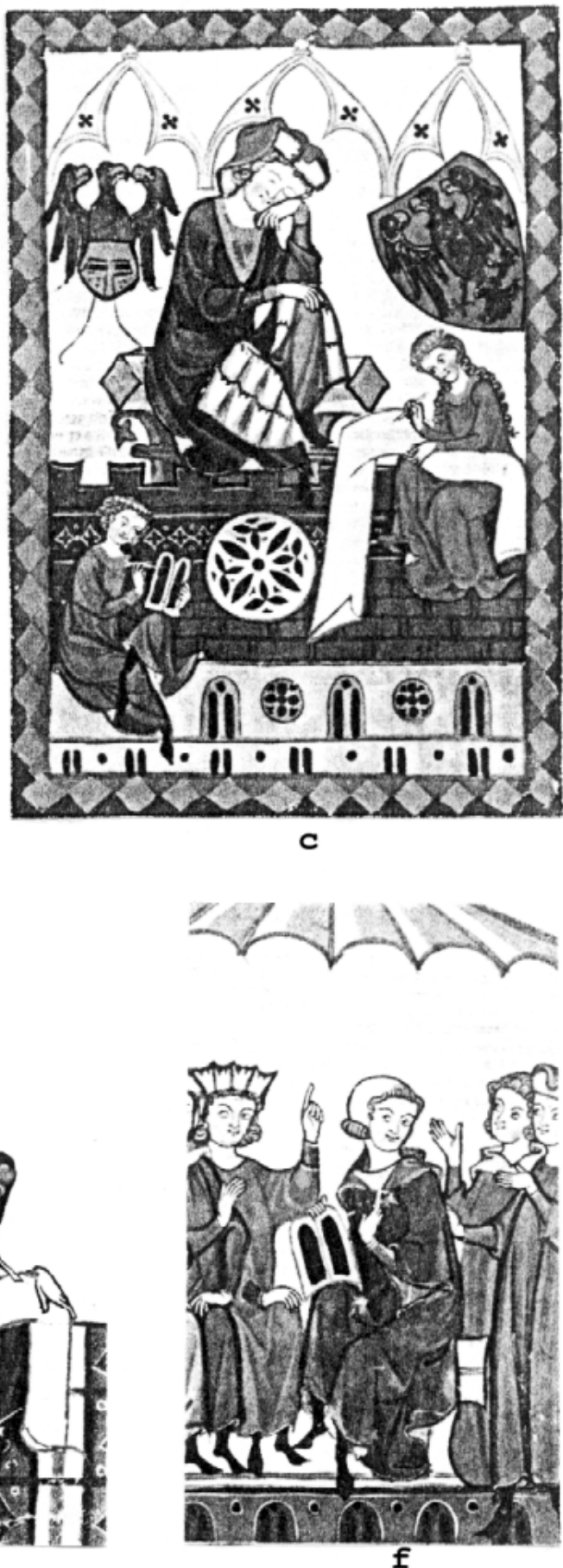

Abb. 153

Die ikonographischen Vorbilder VI

"Buchträger": Dichter / Schreiber (?)

$a, b$ : Kalnar, $c, d$, e, f: Codex Manesse, Tafeln 112, 28, 58, 121 


\section{Bildinhalte und Symbolik}

Die Inhalte der auf den profanen Elfenbeinobjekten wiedergegebenen Szenen und Motive sind eng verknüpft mit den Themen der in Frankreich seit dem 13. Jahrhundert entstandenen Lyrik und höfischer Romane, die in kürzester Zeit auch in England, Flandern, Deutschland, Spanien und Italien Nachahmung fanden ${ }^{316}$.

Ein zentrales Thema ist die "höfische Minne", das Synonym für eine ideale, reine Liebe, reinster Schönheit und Unberührtheit, die vor allem in Frankreich in noch übersteigerter Form auf die Verehrung der Maria übertragen wurde; ein weiteres das Ideal der Ritterlichkeit, der Sieg der göttlichen Gnade und des ritterlichen Geistes über die rohe Gewalt ${ }^{317}$.

Die Inhalte dieser Dichtung haben als Ausdruck eines veränderten Lebensgefühls, einer Verfeinerung der Sitten und einer veränderten Stellung der Frau Eingang gefunden in den profanen Alltag, ob sie nun tatsächlich umgesetzt wurden oder nicht. Die Motive auf Spiegelkapseln und Kästchen tauchen im 14. Jahrhundert zu Hunderten in stetiger Wiederholung auf (Abb. 154 bis 155):

Der Ritt durch den Wald, das Schachspiel und die gegenseitige Bekränzung als Minnemotive; Hirschjagd, Entführung einer Dame und weitere Motive des Parzival- und Artuszyklus aus dem höfischen Roman; Gawain auf dem Bett der Gefahr und der Angriff auf die Burg der Liebe als profane Versionen religiöser Riten (Burg der Liebe - Gralsburg - Heilige Stadt der Apokalypse) $)^{318}$.

Von den zahlreichen Motiven auf Gravoir- und Messergriffen, die Koechlin feststellte (Aristotelesmotiv, Waldritt, Liebeserklärung, "entretien galant", "Offrande du coer", Tristan und Isolde, Liebeskranzszene, Mönch, Dame mit Hund, Vogel oder Falke, Sackpfeifer, Falkner, Dame mit Instrument), tauchen nur einige im hier behandelten Bestand auf. Vorherrschend ist das Motiv des Falkenträgers, auch im Zusammenhang mit dem Minnemotiv des Griffs aus Roskilde (1).

\footnotetext{
316 Aubert 1963, S. 11

317 Aubert 1968, S. 11

318 Beigbeder o.J., S. 45-47
} 

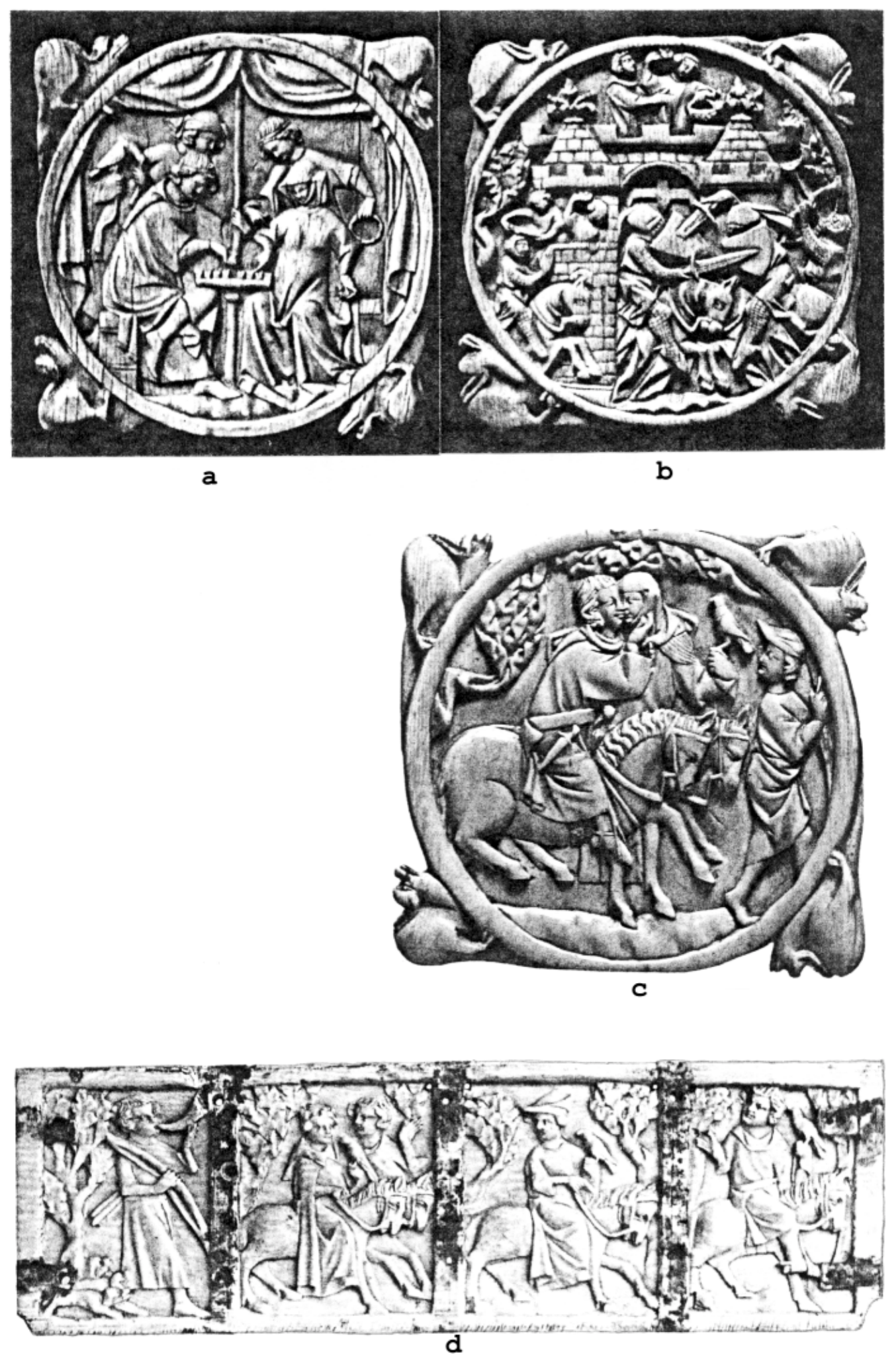

Abb. 154

Französische Elfenbeinobjekte des 14. Jahrhunderts a: Schachspiel, b: Sturm auf der Burg der Liebe; $c$, d: Ritt durch den Wald (Falkenjagd) 


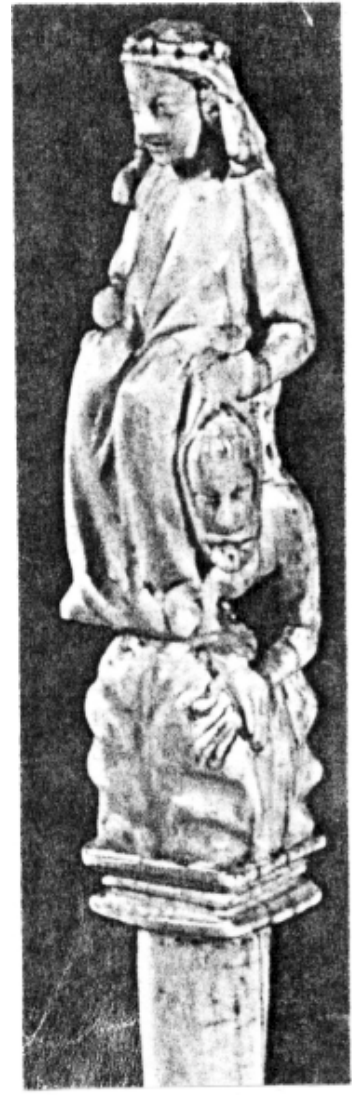

a
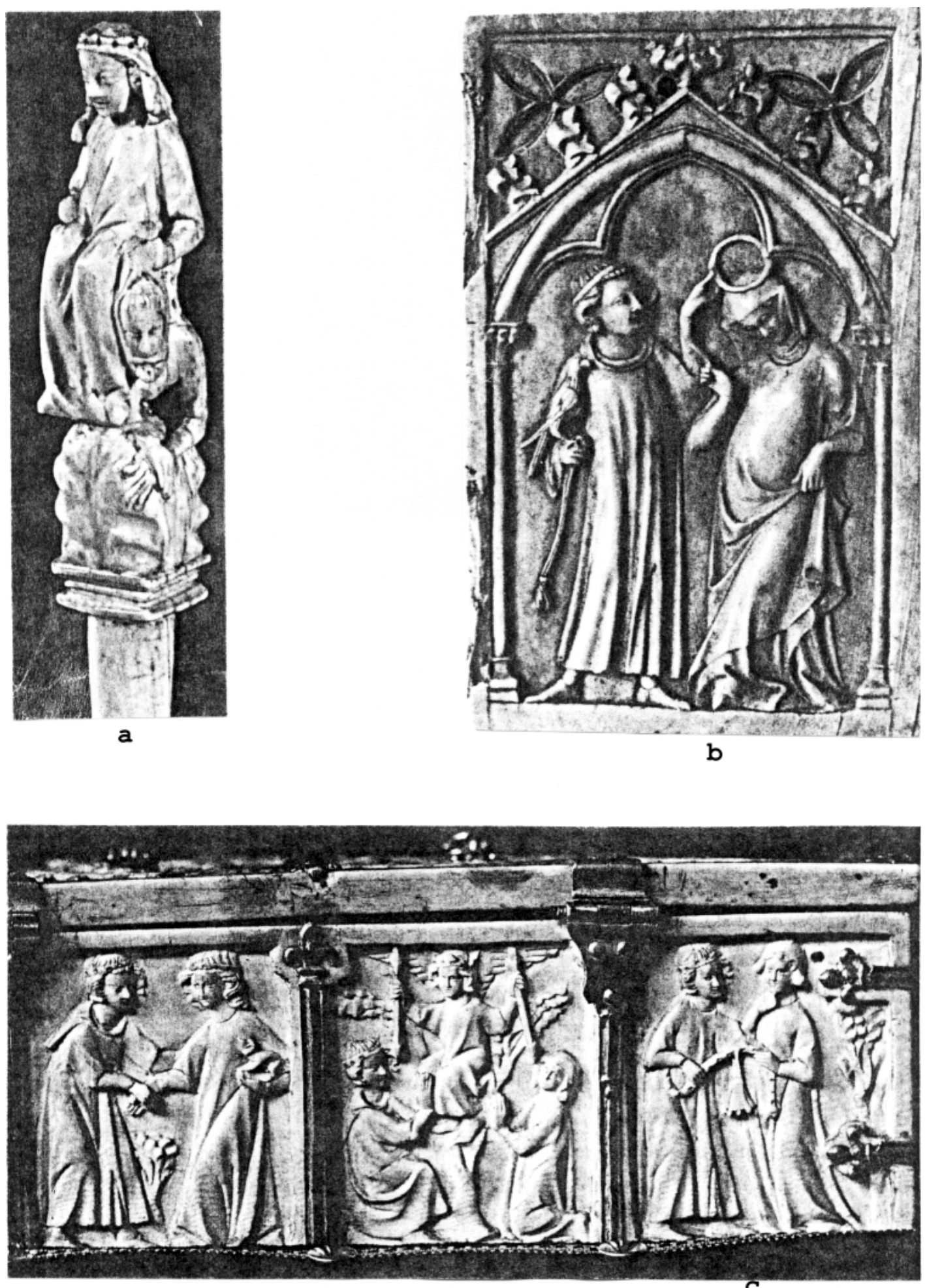


\section{Das Motiv des Falken}

Die Falkenjagd war von allen mittelalterlichen Jagdarten die aufwendigste, teuerste und "edelste" Jagd, sie war keine Ertragsjagdart, sondern eine reine Kunstjagd, der "Lieblingssport der adeligen Gesellschaft" ${ }^{319}$.

Als Einzelgänger nur schwer abzurichten, hat der Falke als Symbol für Kühnheit, "Kraft, Schönheit, Adel und Freiheit" eine zentrale Bedeutung in der Literatur und Kunst des europäischen Mittelalters ${ }^{320}$.

D. Walz führt vier Bereiche an, deren Bedeutungsträger das Motiv des Falken in besonderer Weise war.

Als Symbol und Attribut für Königtum erscheint der Falke noch vor dem römischer Tradition entstammenden Adler auf Reichsiegeln, Evangeliaren, Elfenbeindosen, in Psaltern und illustrierten Handschriften. Als Königssymbol wurde er unter den Staufern wiederbelebt, die das Recht der Falkenjagd ausschließlich der königlichen Jagd vorbehielten ${ }^{321}$.

Auf Fürstensiegeln, auf dem Teppich von Bayeux, als Kathedralskulptur, in Glasfenstern und Kalendaren erscheint der Falke auch als Symbol für Adel und Rittertum, nicht nur für den ausgebildeten und erprobten Ritter, sondern auch für Fürstinnen und Jungherren vor der Schwertleite ${ }^{322}$.

Stellvertretend für das Ritterideal (Parzival) erscheint er in der Literatur. Verbunden mit ritterlichen Tugenden wie Disziplin, Mut, Tapferkeit und Wachsamkeit war der Falke auch Symbol für höfische Gesinnung. D. Walz führt als Beispiel die Vergabe höchster Ämter an bewährte Falkner durch Friedrich II. an, dessen Buch über die Falkenjagd ebenso Ausdruck für die hohe Bedeutung des Falken unter den Staufern ist ${ }^{323}$.

Eine weitere zentrale Bedeutung haftete dem Falken im Bereich der Minne an. In Miniaturen und Texten illustrierter Liederhandschriften erscheint er als Synonym für das Herz der Dame, welches sie zum Beispiel vor dem Werbenden verbirgt, sowie für das Herz des Werbenden, der es an die Angebetete verliert ${ }^{324}$.

Als Symbol für menschliche Freiheit erscheint der Falke in den Liedern der Minnesänger. Das Risiko, daß der freiheitsliebende Falke trotz Abrichtung nicht mehr zurückkehrt, wird verbunden mit der Freiheit des Dichters, zu schreiben und zu lieben, was und wen er will. Die Bedeutung umfaßt im Bereich der Minne also nicht nur die Gefangenschaft des Werbenden, sondern auch die Freiheit des untreuen Liebhabers ${ }^{325}$.

\begin{tabular}{ll}
\hline 319 & Walz 1988, S. 350 \\
320 & Walz 1988, S. 350 \\
321 & Walz 1988, S. 351 \\
322 & Walz 1988, S. 352 \\
323 & Walz 1988, S. 353 \\
324 & Walz 1988, S. 353-354 \\
325 & Walz 1988, S. 355
\end{tabular}


Die Themen, die der Falke als Träger und Vermittler von Bedeutungen berührt, liegen vor allem in den Bereichen Herrschaft, Adel und ritterliche Ideale. Die Freiheit, die abseits von Verpflichtung und Gebundenheit jenseits der gesellschaftlichen Zwänge möglicherweise direkt mit der geistigen Freiheit jenseits von scholastischem Denken verbunden ist, scheint vor allem im engeren Kreis der Minnedichtung verbreitet gewesen zu sein.

$\mathrm{Da}$ die Bedeutung des Falken und der Falkenträger an Messergriffen den Trägern und Benutzern dieser Messer bewußt war, wird wahrscheinlich durch den Funktionszusammenhang mit den Mahlzeiten, die vor allem im Hochadel und beim Klerus mit Bedeutungen behaftet und ritualisiert waren.

Zwei Beispiele mögen als Belege für die Einbindung von Mahlzeiten in das Kirchenjahr oder in einem festgelegten Tagesrhytmus dienen, die sich bis auf das Besteck ausgewirkt haben:

Die Tradition, in mittelalterlichen Klöstern den Mönchen während der Mahlzeiten, des einzigen Genusses, der ihnen gestattet war, mit Bildern oder Lesungen aus der Apokalypse die Unsicherheit ihres Daseins vor Augen zu führen, findet ihren Niederschlag in Darstellungen von Ungeheuern oder Szenen des Jüngsten Gerichts an Griffen von profanen Tranchiermessern, die bis in die Renaissance ikonographisch romanischen Traditionen folgen und gebunden waren an den Teil des Tafelbestecks, der mit der Funktion des Vorschneidens und Servierens eine bevorzugte Stellung einnahm ${ }^{326}$.

Koechlin beschreibt anhand von französischen Quellen aus der Mitte des 14. Jahrhunderts die Bedeutung der verschiedenen Messergriffe zu bestimmten Zeiten des Kirchenjahres an der königlichen Tafel:

In der Fastenzeit bestand der Griff aus Ebenholz, zu gewöhnlichen Zeiten aus Elfenbein, und zu Pfingsten aus beiden Materialien ${ }^{327}$.

\section{Das Motiv des "Buchträgers"}

Unabhängig vom Attribut stehen die Griffe aus Kalmar (3, 4 und 5) ikonographisch und stilistisch in derselben Tradition wie die Griffe aus Hamburg, Riga, Roskilde (1) und letztlich auch Budapest.

Die Suche nach dem Motiv "Buch" blieb jedoch innerhalb der zeitgenössischen profanen Ikonographie erfolglos. Weder im Codex Manesse oder in der Weingartener Liederhandschrift noch in den zahlreichen Bildprogrammen auf Kästchen und Spiegelkapseln ließ sich eine Parallele belegen. So war die zunächst aus diesem Zusammenhang naheliegende Bedeutung als Symbol des Sängers, Schreibers oder Dichters nicht eindeutig zu verifizieren, auch ist in den Codices das Attribut des Dichters/Sängers durchgehend ein Spruchband.

\footnotetext{
326 Beigbeder o.J., S. 86-90

327 Koechlin 1924, Band I, S. 420
} 
In der christlichen Ikonographie hat das Buch als Attribut seinen festen Platz als Symbol des "Heiligen Buches", der Bibel. Es findet sich als Attribut für Heilige, Apostel und Propheten, in den Händen von Christus und Maria, selbst bei Stifterfiguren wieder (Abb. 156 und 157), unabhängig von Stilrichtungen und Schulen.

Spruchband/Schriftrolle und Buch als feste Attribute wurden abhängig von ihrer liturgischen Funktion innerhalb der sakralen Ikonographie vielseitig verwendet, wie das Beispiel des Bildprogramms eines der bedeutendsten romanischen Vortragekreuze (Maasgebiet, 1160-1170) ${ }^{328}$ zeigt:

Die an sechs verschiedenen Orten verstreuten Grubenschmelzplatten zeigen Christus mit Buch und Spruchband, Aaron und Moses mit Buch (Gesetzestafel), die Evangelisten Marcus, Lucas und Matthäus mit Schriftrollen am Schreibpult sowie den Evangelisten Johannes mit Buch am Schreibpult (Abb. 156a-b).

Da das Bildprogramm der hier behandelten Messergriffe sich durchweg an die Ikonographie der höfischen profanen Kunst anlehnt, ist die Deutung der drei Kalmarer Griffe über das Attribut "Buch" als Darstellungen von Aposteln oder Propheten aber ebensowenig möglich.

Zum einen gibt es im gesamten Bestand keinen Beleg für ein aus der älteren oder zeitgenössischen Bildtradition übernommenes sakrales Attribut, zum anderen hätte die Abbildung eines religiösen Themas eher die gesamte Griffgestaltung umfaßt, wie die Beispiele des 15. Jahrhunderts (Mönche, Heilige) aus Paris und Köln, die Koechlin vorgestellt hat ${ }^{329}$, zeigen.

Die Geschlossenheit des ikonographischen Kontextes wurde nicht einmal innerhalb einer sakralen Umgebung durch einen Themenwechsel durchbrochen, auch die Griffe aus den Klöstern folgen dem höfischen Programm. Sie hängt vielleicht ebenso von der profanen Funktion als Messergriff ab wie die Ikonographie innerhalb der christlichen Liturgie.

So bleibt zu vermuten, daß das Motiv des Buchträgers, welches lediglich in Kalmar, dort aber mit drei Exemplaren belegbar ist, trotz fehlender Vorbilder dennoch in das weltliche Programm gehört.

\footnotetext{
328 Die Zeit der Staufer, Katalog der Ausstellung, Bd. 1, Stuttgart 1977,

329 Kat. Nr. 550, S. $414 \mathrm{ff}$

Koechlin 1924, Bd. II (Katalog), Kat. Nr. 1129, 1129a, 1130
} 


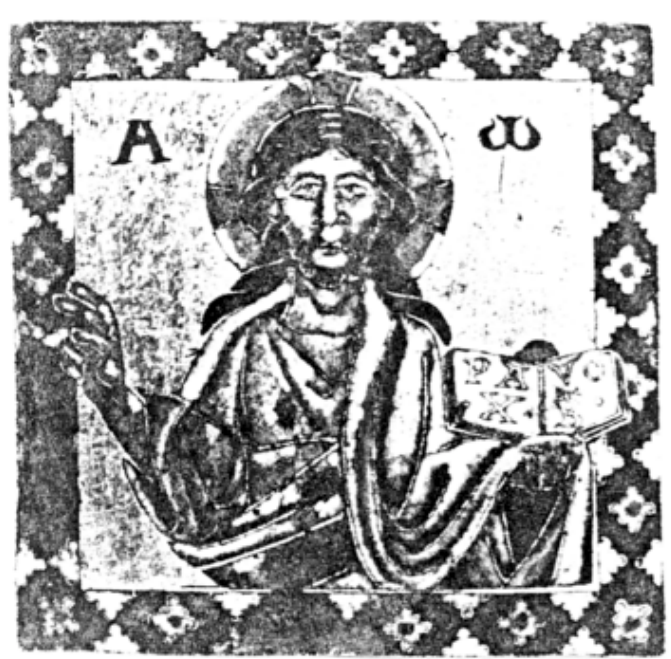

a

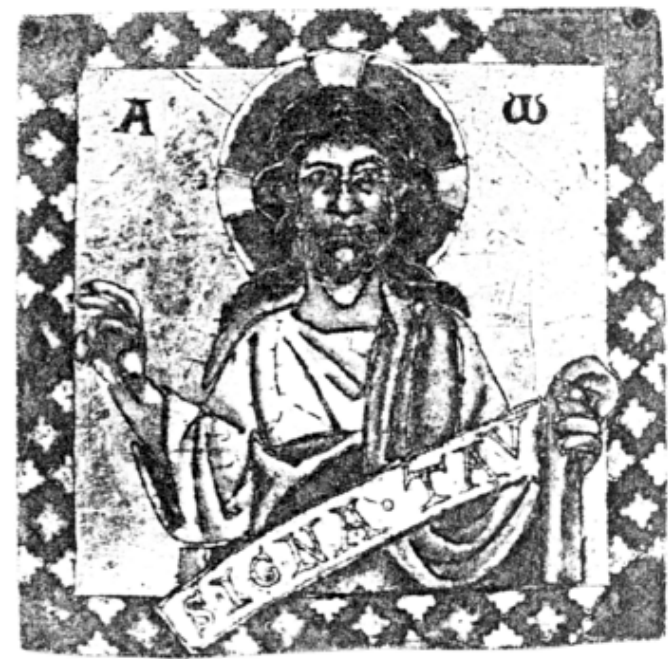

b

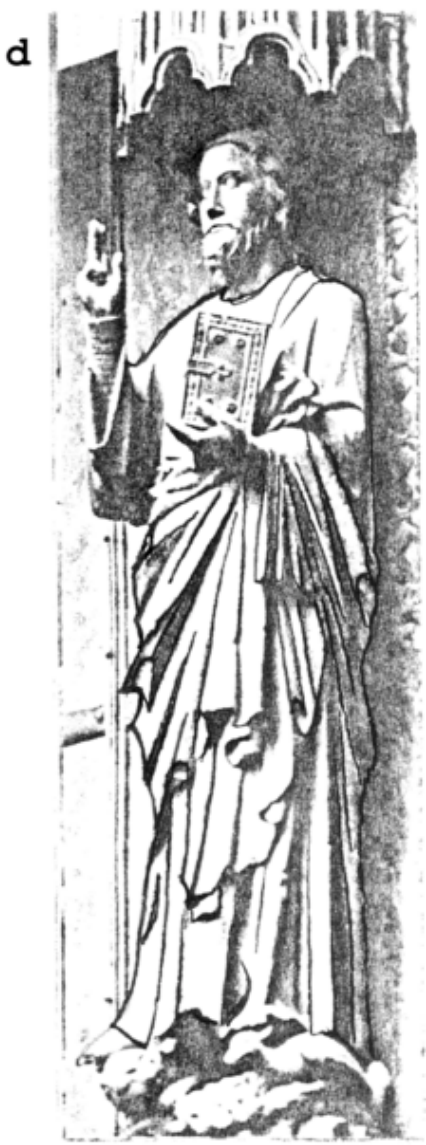

Abb. 156 Die Verwendung der Symbole „Buch“ und „Spruchband“ in der mittelalterlichen Ikonographie I

a, b: Christus, 2.H. 12.Jh., c: Apostel, 1.H. 13. Jh.; d: Christus, um 1230 

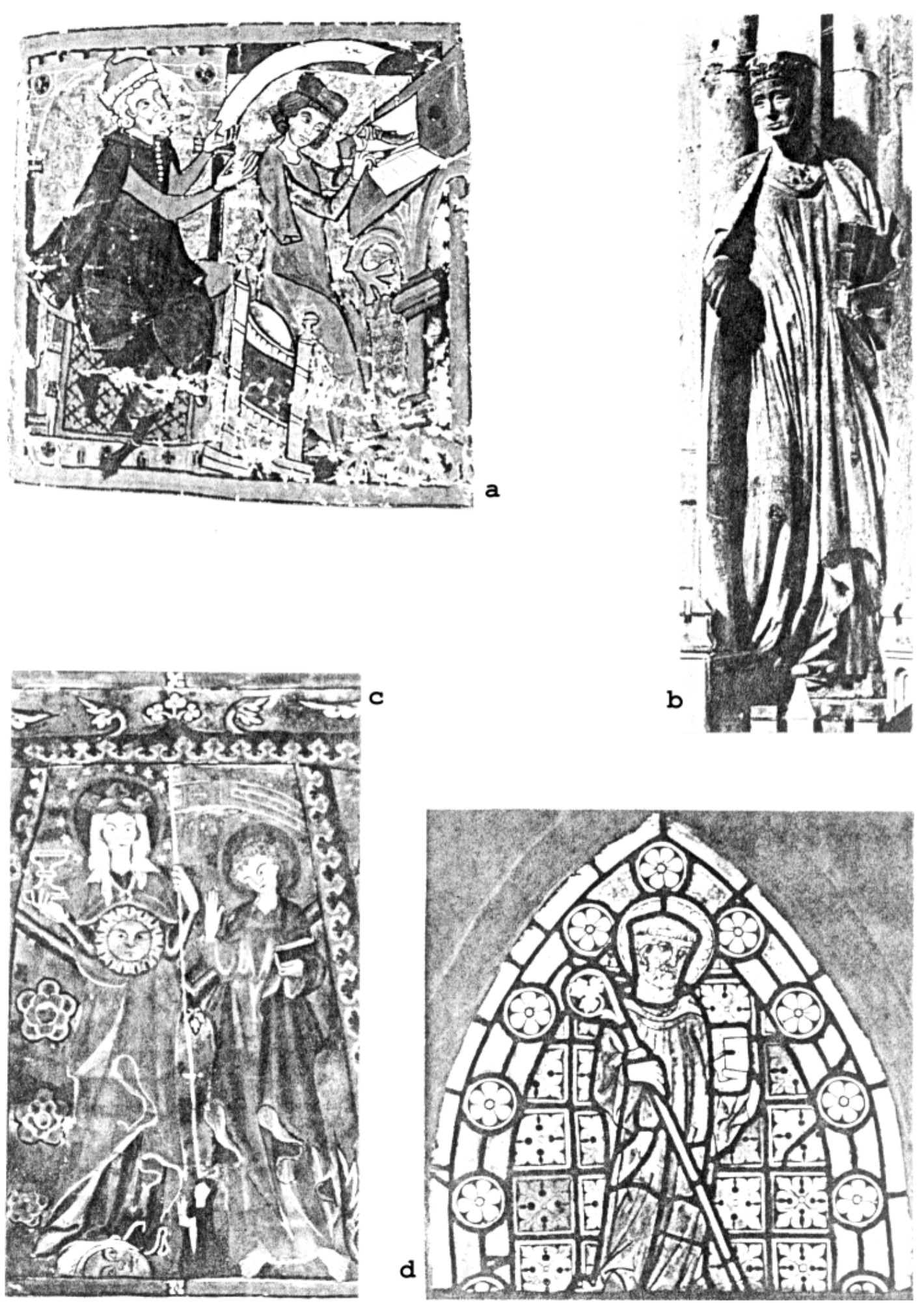

$A b b .157$

Die Verwendung der Symbole „Buch“ und „Spruchband“ in der mittelalterlichen Ikonographie II

a: Dichter und Schreiber, um 1270/80, b: Stifterfigur, um 1250; c: Maria und Johannes, um 1312; d: St. Benedikt von Nursia, um 1330-1340 
Möglicherweise stand dem skandinavischen Knochenschnitzer, der sicherlich im Repertoire eingebunden war in die bekannte und verbreitete Bildfolge gotischer höfischer Idealvorstellungen, als Vorbild das sakrale Attribut "Buch" näher als die Pergamentrolle, die in der Weltchronik des Rudolf von Ems ausdrücklich als Zeichen des Dichters im Gegensatz zum Buch des Schreibers (Abb. 157a), im Codex Manesse aber auch als Attribut des Schreibers begegnet (Abb. 153e), und er verwandte das Symbol entgegen seiner ursprünglichen Bedeutung als ein Zeichen für die Kunst des Dichtens im Kontext der höfischen Minne und ritterlicher Lebensart, so wie die Darstellung des Budapester Griffs im gleichen Zusammenhang den Sänger repräsentiert (Abb. 152a-c).

Wer waren die Käufer und Benutzer dieser Messer des ausgehenden 13. und 14. Jahrhunderts, die mit Attributen und Symbolen des höfischen Adels behaftet waren, ihren inhaltlichen und ikonographischen Ursprung in der nordwestfranzösischen Gotik finden und im gesamten nördlichen Europa verbreitet waren, deren Beliebtheit offensichtlich so weit ging, daß sie als einziges der zahlreichen profanen Objektgruppen der führenden Elfenbeinschnitzereien auf breiter Basis nachgeahmt und formal bis zur Unkenntlichkeit verändert wurden?

\section{Zur Rezeption höfischer Topoi - Ein mittelalterlicher Beleg zur volkskundli- chen Regel vom sinkenden Kulturgut}

Nach Ausweis der Fundorte ist das Gros der Abnehmer anthropomorph gestalteter Messergriffe innerhalb des städtischen Bürgertums zu suchen.

Ohne die Griffe aus Schonen, Friesland, Lüttich, Rotterdam und Ragnildsholm, deren Zuweisung nicht mehr möglich bzw. dem Verfasser unbekannt geblieben ist, stammen 24 Griffe aus städtischem Umfeld, vier aus Klöstern und zwei, vielleicht vier von Burgen (Burg Falkenberg, Isenburg, möglicherweise die Burg in Stettin und Burg Dergneau in Lille).

Die Inhomogenität der Fundgruppe, die von exklusivem Fernimport bis zum einheimischen Surrogat reicht, legt aber nahe, den Kreis der Abnehmer als ähnlich inhomogen zu betrachten.

Die ältesten Belege aus der ersten Hälfte des 13. Jahrhunderts stammen aus Kalmar und dem Kloster Wienhausen.

Der Klostergriff aus der Schloßbucht von Kalmar kann aus quellenkritischen Gründen nur bedingt der Vorläuferanlage der 1280 bis 1290 errichteten königlichen Burg zugeordnet werden. Das soziale und wirtschaftliche Umfeld der entstehenden Stadt beschreibt N. Blomkvist als von ostschwedischen Großgrundbesitzern (unter anderem von Johann Elofson, einem schwedischen Ritter des Deutschen Ordens) und von Klöstern beeinflußt, die hier im Bereich von zahlreichen Umschlagplätzen, Siedlungen und Kronbesitz die Grundlagen legten für die Konzentration von wirt- 
schaftlicher und politischer Macht am Kalmarsund ${ }^{330}$. Eisen als Exportgut wurde bereits genannt ${ }^{331}$, das archäologische Fundgut scheint nicht vor 1200 zu reichen $^{332}$. Die geographische Nähe zum Königshof am Ort der späteren Steinburg, der allgemein am Aufbau der Stadt Kalmar führend beteiligte Personenkreis, die künstlerische Qualität des Griffs sowie die Möglichkeit einer älteren Tradition anthropomorph gestalteter Holzgriffe innerhalb der schwedischen Führungsschicht, belegt durch den Griff aus Skara, sind bis jetzt die einzigen Hinweise, den Besitzer oder Auftraggeber im Bereich führender Adeliger oder Kleriker zu vermuten.

Das Messer aus dem Kloster Wienhausen darf mit einiger Wahrscheinlichkeit im adeligen Umfeld angesiedelt werden, wie bereits weiter oben gezeigt wurde.

Beide Griffe sind älter als die Gruppe der ikonographisch und stilistisch enger zusammhängenden Messergriffe des späten 13. und 14. Jahrhunderts, sie sind aber hier die einzigen Belege für die Vermutung, daß derartige Griffe als Standeszeichen und Prestigeobjekte des Adels auch von führenden Adelsschichten getragen wurden. Unter der Voraussetzung, daß sich diese Vermutung durch weitere Belege erhärten läßt, ist es möglich, die zahlreichen späteren Belege in ein allgemeiner gefaßtes theoretisches Konzept kulturellen Wandels einzubinden.

$\mathrm{Daß}$ die meisten Datierungen lediglich aufgrund stilistischer Merkmale getroffen wurden und dementsprechend weit gefaßt sind, erschwert das Erkennen einer detaillierteren chronologischen Abfolge, die Aussagen über einen Prozeß des Wandels kultureller Phänomene ermöglicht.

Archäologisch sicher vor $1300 \mathrm{zu}$ datieren sind die beiden Griffe aus der Lübecker Hundestraße und der Griff von der Essener Isenburg.

Da die Griffe aus Lübeck mit einiger Wahrscheinlichkeit am Fundplatz hergestellt wurden, geben sie keine direkte Auskunft über ihre Auftraggeber.

Die stilistisch eigenständige, möglicherweise auch insgesamt vor $1300 \mathrm{zu}$ datierende Gruppe um die Lübecker Griffe mit den Griffen aus der Umgebung von Lüttich, Friesland und den Befestigungsanlagen von Arras kann archäologisch kaum einem näher zu umschreibenden Personenkreis zugeordnet werden. Die regionale Diffusität der Fundorte, die größere stilistische Nähe zu französischen Elfenbeinarbeiten, die unterschiedlichen Fundplatzcharakteristika sowie das Fehlen von Nachweisen, daß diese Griffe auch innerhalb eines städtisch-bürgerlichen Kontextes benutzt wurden, sind Faktoren, die im Gegensatz stehen zu der Menge der Griffe um und nach 1300 .

In der zweiten Hälfte des 13. Jahrhunderts sind demnach Mitglieder des Adels als Träger anthropomorph gestalteter Messergriffe, die jetzt den Bildern einer allgemeiner verbreiteten, stereotypen Ikonographie höfischer Thematik folgen, lediglich

\footnotetext{
330 Blomkvist 1983, S. 208

331 Blomkvist 1983, S. 208

332 Andersson 1983, S. 188
} 
auf der Isenburg zu vermuten. Weitere Trägergruppen sind wahrscheinlich, lassen sich aber kaum näher eingrenzen.

Einen Beleg für die Nachahmung derartiger Luxusgüter, im volkskundlichen Kontext hier auch als Prestigeobjekte zu bezeichnen, unter Nutzung eines billigeren Ausgangswerkstoffes (Knochen) in städtischem Umfeld liefert der Befund von der Lübecker Hundestraße bereits für die zweite Hälfte des 13. Jahrhunderts.

Nach allem, was bisher über die Masse profaner Elfenbeinobjekte bekannt geworden ist sowie nach den stilistischen Datierungsvorschlägen zu den Griffen aus Ribe (1), Roskilde (1), Budapest und Vysoké Mýto scheint eine Datierung der bis auf zwei Griffe (Herlulfsholm, Scharnegoutum) aus städtischen Siedlungsschichten stammenden Gruppe (Hamburg, Stettin, Riga; Ribe, Oxford; Ribe (2); Roskilde (1), Lund (2), Lund (1, 3), Kalmar (1, 2), Kalmar (3-5); Roskilde (2), Oslo) in die Zeit um 1300 und in die erste Hälfte des 14. Jahrhunderts vorläufig möglich zu sein.

Diese Gruppe vereint hervorragende Einzelobjekte aus hochstehenden Elfenbeinschnitzerwerkstätten mit mehreren Gruppen von Nachahmungen aus unterschiedlichen Werkstoffen, deren Provenienz im Bereich englischer, skandinavischer, möglicherweise auch kernhansischer Werkstattkreise zu suchen ist.

In einigen Fällen konnte ein Zusammenhang hergestellt werden zwischen der unterschiedlichen Qualität und einer innerstädtisch unterschiedlich zentralen Fundplatzlage (Ribe, Lund) oder einer unterschiedlichen wirtschaftlichen Bedeutung des Fundortes (Oslo), zum Teil waren auch aufgrund der Fundplatzlage indirekte Hinweise auf das soziale Umfeld der möglichen Besitzer zu gewinnen, so in Stettin (Adel?), Roskilde 2 (Kleriker?) und Ribe 1 (führende Kaufleute?).

An Klöstern außerhalb von Städten sind Zisterzienser (Scharnegoutum) und Benediktiner (Herlulfsholm) als Träger zu nennen.

Neben dieser Gruppe stehen drei zeitgleiche Griffe (Småland, Reval, Vysoké Mýto), die wahrscheinlich als Direktimport aus dem nordwestfranzösischen Initialraum an die Peripherie des hansischen Wirtschaftsraums gelangten und als teures Luxusgut ohne nachweisbare Nachahmungen sicherlich erheblichen Prestigewert hatten.

Die Konzentration dieser Griffe um und nach 1300 auf vermögende stadtsässige Personengruppen mit deutlicher qualitativer und sozialer Abstufung (vom luxuriösen Fernimport und qualitätvollen Eigenprodukt oder Nahimport bis zum unkenntlichen Surrogat) läßt sich als deutliche Veränderung gegenüber der Verbreitung der älteren Griffe feststellen.

Stark gewachsenes Imitationsstreben unterschiedlich vermögender Gruppen führte offenbar gleichzeitig zu verstärktem Import von Luxusgütern sowie zur unterschiedlich qualitätvollen einheimischen Produktion. Die Trägerschicht dieser Prestigeobjekte hat sich vom beginnenden 13. zum beginnenden 14. Jahrhundert ebenfalls gewandelt:

Die Griffe aus Wienhausen und Kalmar (Slottsfjärden) lassen zumindest vermuten, dass es sich möglicherweise um Messer handelt, die auf Mitglieder des Adels be- 
schränkt waren, vielleicht auch Symbole ihres Standes und nicht nur Träger höfischer Inhalte waren.

Regional eigenständige Entwicklungen sind $\mathrm{zu}$ vermuten, eine regionale Konzentration des Gebrauchs ist nicht nachweisbar.

In der zweiten Hälfte des 13. Jahrhunderts erscheint das Bild der Trägergruppen diffuser, das Umfeld burgsässigen Adels ist nachweisbar. Die regionale Verbreitung erscheint jetzt konzentrierter, sie ist vor allem an der nördlichen Peripherie der führenden Elfenbeinschnitzerei in Nordwestfrankreich mit stilistischen Abhängigkeiten $\mathrm{zu}$ beobachten. Ikonographische Übereinstimmung und ein Beleg für Surrogatbildung in Lübeck deuten bereits vor 1300 die kommende Entwicklung und Schwerpunktverlagerung in den Wirtschaftsraum der Hanse an, die in diesem Jahrhundert ihre Expansionsphase abschloß und im höchsten Maße wirtschaftlich und politisch innovativ tätig war (Kaufmannshanse, deutsche Stadtgründungen an der Ostseeküste, Ordensstaat, Städtehanse).

Unabhängig von militärischen Auseinandersetzungen sind im 14. Jahrhundert die Konsolidierung und Verdichtung der gewonnenen politischen und wirtschaftlichen Macht die auch kulturell bestimmenden Faktoren im hansischen Einflußbereich. Die erste Hälfte des Jahrhunderts scheint die Hauptverbreitungszeit der anthropomorph gestalteten Griffe gewesen zu sein. Angesiedelt im Bereich mittlerer und führender städtischer Gruppen, ikonographisch einheitlich adelig-höfischen Idealen folgend und von einheimischen Werkstätten nachgeahmt, scheinen sie jetzt von wirtschaftlich stark gewordenen Gruppen als sichtbarer Ausdruck ihres Wohlstandes und Selbstverständnisses.benutzt worden zu sein.

Diese Übernahme eines zunächst vermutlich in höheren Schichten angesiedelten Prestigeobjekts mit Standescharakter durch sozial niedrigere Schichten vor allem in wirtschaftlichen Wachstumsphasen faßt die Volkskunde in der Regel vom sinkenden Kulturgut ${ }^{333}$, wobei in diesem Fall die theoretischen Voraussetzungen für das soziale Absinken materieller Kulturgüter ${ }^{334}$ aufgrund der Quellenlage weiter zu verifizieren wären:

$\mathrm{Zu}$ vermuten ist, daß das Beispiel aus Lund 3, Kleine Fischergasse, ein Hinweis auf die Übernahme gleicher Inhalte bei preiswerterem Ausgangsmaterial (Ersatzstoff) ist, ein reichhaltigeres Angebot ist mit der bekannten Anzahl (Hauptmenge aller Funde um und nach 1300) sicher gegeben. Mit den Griffen aus Småland, Reval und Vysoké Mýto sind auch Direktimporte wahrscheinlich..

Inwieweit die Griffe des frühen 13. Jahrhunderts Belege für eine neue Mode innerhalb des mittelalterlichen Adels sind, das heißt ob sie eine Innovation innerhalb der materiellen Statussymbole adeliger Schichten widerspiegeln, kann derzeit noch nicht beantwortet werden.

\footnotetext{
333 Wiegelmann 1977, S. 51 ff

334 Wiegelmann 1977, S. 56
} 
Der Griff aus Skara weist auf die Möglichkeit eines zumindest regional begrenzten längerfristigen älteren Brauchs hin, der im 14. Jahrhundert eine neue, weitaus größere Verbreitung fand. In dieser Phase war er sicherlich beeinflußt, wurde vielleicht auch neu belebt durch die kulturellen Innovationsschübe der französischen Elfenbeinschnitzerwerkstätten und ihrer englischen und deutschen Pendants. Deren Produkte wurden durch Kaufleute weit nach Norden und Nordosten verhandelt und fanden im Zuge des sozialen Absinkens auf eine kapitalkräftige und weitaus breitere stadtsässige Bevölkerungsschicht genau in dem Großraum ihre größte Verbreitung, in welchem ein Rückgriff auf eine ältere Tradition höherer Schichten möglicherweise angenommen werden kann.

Um das Phänomen des sozialen Absinkens eines einzelnen Gegenstandes in seinen Bezügen zum zeitgenössischen wirtschaftlichen und sozialen Umfeld betrachten zu können, ist es notwendig, die Beziehungen zwischen Adel und Bürgertum und ihre Auswirkungen auf das Selbstverständnis und die Prestigesphäre letzterer kurz anzusprechen.

Im Zuge der weitreichenden Veränderungen, die das Erstarken des städtischen Bürgertums, die Neuorganisierung, Straffung und Absicherung des Fernhandels, die rechtlichen Veränderungen der Stadt als einer neuen Lebensgemeinschaft und viele weitere Faktoren auslösten, blieb die Trennungslinie zwischen Adel und Nichtadeligen nicht unberührt.

Die mittelalterliche Stadtbevölkerung war ungeachtet ihrer regional unterschiedlichen Entwicklung und innerstädtischer Machtverschiebungen stark gegliedert. Die Führungsschicht erlebte vor allem auch in den Hafenstädten der Hanse zu Beginn des 14. Jahrhunderts ihre größte Blüte ${ }^{335}$. Seit dem 13. Jahrhundert wurden Mitglieder des Amtsadels (Ministeriale) überall als Bürger zugelassen und fanden Eingang in das Patriziat ${ }^{336}$.

Die Macht und der politische Einfluß des Patriziats hatten ihre Wurzeln in der wirtschaftlichen Stärke seiner Mitglieder, die vor allem im 13. Jahrhundert im Fernhandel reich geworden waren.

Seit der Anfangsphase der wirtschaftlichen Expansion über die Ostsee, die parallel zur Errichtung deutscher Staatsgebilde verlief, handelten neben den beteiligten Skandinaviern Ordensritter und deutsche Kaufleute Hand in Hand, zum Beispiel bei den Gründungen der Städte Riga, Reval und Dorpat kurz nach $1200^{337}$. Bereits erwähnt wurde, daß der Hochmeister des Ordensstaates als einziger Fürst Mitglied der Hanse war.

Diese frühen Interessenverbindungen blieben nicht ohne strukturelle Konsequenzen. Ähnlich wie im Adel waren die großen Patriziergeschlechter durch verwandtschaftliche Bindungen untereinander verknüpft und schlossen sich zu

\footnotetext{
335 Dollinger 1978, S. 290, 291

336 Dollinger 1978, S. 282

337 Angermann 1973, S. 273
} 
"mächtigen Patriziervereinigungen" zusammen ${ }^{338}$, wie die Kölner Richerzeche oder die Lübecker Zirkelgesellschaft. Patrizier hatten das Recht, Lehen zu empfangen, zahlreiche Mitglieder des städtischen Patriziats stiegen auch persönlich in den Adel auf, seit der Mitte des 14. Jahrhunderts zogen einzelne Patrizierfamilien, dem Vorbild des Adels folgend, aus den Städten auf Landsitze ${ }^{339}$. Weiterhin beschreibt Dollinger den Patrizier als "dominus", in kostbare Gewänder gekleidet, der ein eigenes Wappen führte, silberne und goldene Sporen trug und insgesamt seine Lebensweise der des Adels anglich. "... am Ende des 13. Jahrhunderts... bildete sich über den bürgerlich gebliebenen Geschlechtern ein Stadtadel, bestehend aus eingewanderten Adeligen, Ministerialen und... geadelten Bürgern" ${ }^{340}$.

Die führenden Schichten des städtischen Bürgertums wurden aber bei aller Imitation und Angleichungsbestrebungen als gesellschaftliche Gruppe vom Geburtsadel nie als gleichberechtigt anerkannt ${ }^{341}$. Dieser bezog seine Exklusivität nicht aus Statussymbolen, Prestigeobjekten und Standeszeichen, sondern aus dem erblichen Geburtsrecht. Dieses Recht und das aus ihm resultierende Selbstverständnis war weder durch Reichtum noch durch Handelsprivilegien zu erlangen.

Der aus dieser Unvereinbarkeit entsprungene Bruch zwischen der eigenen Herkunft, einem eigenen Traditionsbewußtsein und dem letztlich unerreichbaren Wunsch nach Zugehörigkeit zur gesellschaftlichen Elite führte Dollinger zu der Bemerkung, das deutsche Bürgertum habe ein ihm wesensfremdes Ideal übernom$\mathrm{men}^{342}$.

Gemessen an der wirtschaftlichen, politischen und militärischen Macht der Hanse, die in der Lage war, mit Dänemark ein ganzes Königreich den eigenen Wirtschaftsinteressen zu unterwerfen, die politische Bündnisse schloß und vermittelte, die mit Boykottmaßnahmen gegen mehrere europäische Staaten die Einhaltung oder Ausweitung einmal erlangter Handelsprivilegien erzwang, erscheint diese Einschätzung als zu weitreichend.

So führte der Ausschluß von ritterlichen Turnieren lediglich dazu, daß die Jugend des zu Pferd kämpfenden Patriziats reicher norddeutscher Städte seit dem 13. Jahrhundert Ersatz schaffte und mit Ringreiten und Rolandstechen ihre eigenen Turniere abhielt. Eine städtische Ritterfigur, belegt seit 1342 (Hamburg) und 1366 (Bremen) als steinerne oder hölzerne Bildsäule mit dem Namen "Roland", wurde zum Sinnbild der autonomen Stadtgerichtsbarkeit und kaiserlicher Privilegien in zahlreichen Hansestädten bis nach Riga (1412).

Die Nachahmung adeliger Bräuche entsprang sicherlich dem Imitationsprozeß einer reich gewordenen Schicht, die Vorbilder in der gesellschaftlichen Elite suchte und

\footnotetext{
338 Dollinger 1978, S. 283

339 Dollinger 1978, S. 284, 285, 290

340 Dollinger 1978, S. 284

341 Dollinger 1978, S. 285

342 Dollinger 1978, S. 286
} 
fand. In seiner Umformung und Anreicherung mit eigenständigen Inhalten spiegelt sich aber eher Unbefangenheit, geistige Freiheit und Unabhängigkeit als Verlust der eigenen Identität.

M. Tausch hat in einem Beitrag zur Frage, ob die Zugehörigkeit zur Hanse als Motivation die Mitglieder zur Schaffung "hansischer Kunst" initiiert habe, Bildzeugnisse als "Selbstdarstellung eines Standes" erst für das 15. und 16. Jahrhundert angeführt, also für eine Zeit, in der die Hanse ihre alte Bedeutung verloren hatte ${ }^{343}$. Dieser Vorgang wird in der Diskussion volkskundlicher Regelund Theoriebildung als Kulturfixierung bezeichnet ${ }^{344}$, die das Festhalten einmal erreichter Standards und die Ausbildung von Traditionsprozessen in Zeiten wirtschaftlichen Niedergangs beschreibt.

Für das 13. und 14. Jahrhundert läßt sich ein umfangreicher Handel mit Kunstobjekten nachweisen, ohne daß dieser zu einem Niederschlag im Sinne einer städtisch geprägten Kunst geführt hat ${ }^{345}$. Unabhängig von der Entwicklung regionaler Sonderstile wie die Verwendung von Backstein im Kirchen- und Klosterbau, an der die Hansestädte maßgeblich beteiligt waren, war der Handel mit Altären, Gemälden, Plastiken und Luxusgütern ein Handel mit Waren, und der Adel bis zu Mitgliedern königlicher Haushaltungen nicht nur Vorbild, sondern vor allem Kunde.

$\mathrm{Zu}$ einer Zeit, in der sich das Patriziat, der Klerus, die Kaufleute und reichen Handwerker in rasch wachsenden und wirtschaftlich florierenden Städten über einen organisierten Fernhandel alles beschaffen konnten, was in den europäischen Kunstzentren hergestellt wurde, und was zu einem großen Prozentsatz auch für andere Bevölkerungsgruppen nur über sie zu bekommen war, liegt es nahe, die aus den empirisch erarbeiteten Theorien und Regeln kulturellen Wandels entwickelten Zwänge und Abhängigkeiten im Verhaltensmuster in dieser Phase weniger bei den Initiatoren dieses Wandels, sondern eher bei der Schicht der alten Elite zu vermuten.

Nach der Regel vom sinkenden Kulturgut zieht eine soziale (und funktionale) Veränderung eines Prestigeobjekts die Aufgabe dieses Gegenstandes (oder Brauchs) in der auf Exklusivität und Abgrenzung nach "unten" bedachten ehemaligen Benutzergruppe nach $\operatorname{sich}^{346}$. Das Auslaufen von Fundplätzen mit direkten Hinweisen auf ein ehemals adeliges Umfeld (Burgen, Klöster mit adeligen Bewohnern) um 1300 kann hier nur als Hinweis auf diese Regel gelten, da die Anzahl der Belege zu gering und die direkte Zuweisung unsicher ist.

Die Veränderung und Vereinheitlichung der Ikonographie der Griffe um und nach 1300 ist, wie auch bei den übrigen sakralen und profanen Gegenständen, wie die Mengenproduktion und das Zielen auf den privaten Gebrauch in den Städten entstanden, in diesem Fall (Elfenbeinobjekte) in Paris und anderen nordfranzösischen Städten. Deren führende Bevölkerungsgruppen, die M. Aubert als "Elite unter den

\footnotetext{
343 Tausch 1973, S. 298

344 Wiegelmann 1977, S. 51 ff, v.a. S. 60 ff

345 Tausch 1973, S. 300

346 Wiegelmann 1978, S. 53, S. 58
} 
Nichtadeligen" bezeichnet ${ }^{347}$, haben nicht nur die politische Entwicklung, sondern auch die Literatur, die Kunst, den Bau von Kathedralen und ihre Ausstattung wesentlich beeinflußt ${ }^{348}$.

So wie in der Sammlung, Abfassung und Fertigstellung der Manessischen Liederhandschrift Züricher Familien primär beteiligt waren ${ }^{349}$, läßt sich die Beliebtheit ritterlich höfischer Ideale in den städtischen Führungsschichten neben Rolandssäulen und Turnieren auch durch die spätere Organisierung preußischer und baltischer Kaufmannsgilden in Artusgesellschaften ${ }^{350}$, in der Pflege "ritterlicher" Dichtung, der Ausschmückung von Innenwänden bürgerlicher Stadthäuser durch ritterliche Motive (Köln, 13. Jahrhundert, Overstolzenhaus ${ }^{351}$ und Zürich, Anfang 14. Jahrhundert, Haus zum Silberschild, Haus zur Hohen Eiche, Haus zum langen Keller $^{352}$ ) bis zur späteren Gestaltung von Mobiliar ${ }^{353}$ (Ende 15. Jahrhundert, Rathausgestühl Reval) belegen.

"Hanse bedeutete nicht nur Warentransport..., sondern auch Verbreitung von Leitbildern, Ideen, literarischen Motiven"354. Der Umgang mit Kunstobjekten und Luxusgütern, die Verbreitung von Kunst und Dichtung hat die Träger dieses Handels nicht unberührt gelassen, sie haben nicht nur Adel und Klerus, sondern auch sich selbst damit versorgt.

Das Ende der Verwendung mittelalterlicher anthropomorph gestalteter Vollgriffe deutet sich mit den Belegen aus dem Ringkloster bei Skanderborg und aus Schonen an, beide Griffe sind vorläufig um 1400 datiert worden. Für die barettähnliche Kopfbedeckung der Falknerfigur aus Schonen, die zu dem späten Datierungsansatz führte, liegen Belege auch aus dem beginnenden 14. Jahrhundert vor (Abb. 150b).

Die Datierung des Griffs aus Visby (Dudelsackpfeifer, um oder kurz nach 1400) gründet sich ebenfalls allein auf die Datierung eines Kleidungsbestandteils (Kopfbedeckung), obwohl auch aus der Manessischen Liederhandschrift ähnliche Hüte bekannt sind. Seine Gesamtgestaltung und Proportionalität stellt ihn eher in die Gruppe der Griffe aus der ersten Hälfte des 14. Jahrhunderts, auf die stilistische Ähnlichkeit mit französischen oder französisch beeinflußten Griffen wurde bereits hingewiesen.

$\mathrm{Ob}$ die späten Datierungsansätze der drei Griffe, archäologisch belegt lediglich durch die Lage des Skanderborger Griffs unterhalb einer Brandschicht von 1430, ausreichend gesichert sind oder nicht, ein Nachweis eines ritterlichen Motivs an

\begin{tabular}{ll}
\hline 347 & Tausch 1973, S. 113 \\
348 & Tausch 1973, S. 113 \\
349 & Werner 1988, S. 1 \\
350 & Tausch 1973, S. 391-392 \\
351 & Steuer 1982, S. 12 \\
352 & Saurma-Jeltsch 1988, S. 323 ff \\
353 & Pagel 1983, S. 199-200 \\
354 & Tausch 1973, S. 391-392 \\
& Tausch 1973, S. 391
\end{tabular}


einem Messergriff aus städtischem Umfeld um 1400 fehlt, obwohl zahlreiche Messerfunde aus Stadtkerngrabungen für diese Zeit vorliegen.

Die Datierung des Messergriffs von der Burg in Falkenberg, zerstört 1434, vor 1350 ist sicherlich einem Irrtum in der Ansprache der Kopfbedeckung, einem Visierhelm, zuzuschreiben ${ }^{355}$, dessen Datierungsansatz eher dem Zerstörungsdatum der Burg entspricht ${ }^{356}$. Auch die Form des Griffs mit der glatten Oberkante und der zum Heft einlaufenden Unterkante setzt frühestens im ausgehenden 14. Jahrhundert ein, so daß dieser Beleg, der die ritterliche Darstellung halbplastisch in die funtkionalere Gesamtform des Griffs eingearbeitet zeigt, eher für eine technischfunktionale Innovation unter Einbeziehung älterer, möglicherweise traditioneller Elemente steht. Im städtisch-bürgerlichen Umfeld sind anthropomorphe Gestaltungen von Messergriffen wieder vereinzelt im 15., regelmäßig erst seit dem 16. Jahrhundert im Zuge allgemeiner neuer Gestaltungsschübe zu beobachten (Katalogteil Schweden, Kat. Nr. 44 und 116).

Von einem Wiederaufgreifen als Standeszeichen des Adels nach der Aufgabe von Messergriffen ritterlich-höfischer Symbolik im städtischen Bürgertum kann aufgrund nur eines Beleges aber nicht ausgegangen werden.

\subsection{Die Griffe aus Reimerswaal und O ud-Krabbendijke als Bei- spiele einer regionaltypischen G ruppe}

An den Stränden der Ooster- und Westerschelde auf der schmalen Landbrücke zwischen Festland und der Halbinsel Zuid-Beveland in der niederländischen Provinz Zeeland werden seit Jahrzehnten Überreste zweier Siedlungen bei den Ortschaften Reimerswaal und Krabbendijke zu Tage gefördert.

Sie werden in der überwiegenden Zahl in das 14. und 15. Jahrhundert datiert und umfassen Teilbereiche einer reichhaltigen Alltagskultur. Ein Teil dieser Funde gelangte über die Privatsammlung van Beuningen-de Friese in das Museum Boymans-van Beuningen in Rotterdam. Unter den Funden befinden sich Messergriffe, die alle aus Knochen geschnitzt und reichhaltig verziert sind. Sie umfassen Vollgriffe und Griffschalen, die plastisch und flächig mit Kronen, rollwerkartigen Profilierungen, einfachen und doppelten Punktaugen (Kreisaugen), durchbrochen

\footnotetext{
355 Kartei des Statens Historiska Museum Stockholm, Inv. Nr. 7764:174, Photo Nr. 1866:37

356 Das Zerstörungsdatum 1434 als terminus post quem weist die Darstellung des geschlossenen Visierhelms mit Halsbrünne als wahrscheinlich frühesten Beleg dieses Helmtyps aus.

Spätgotische Visierhelme sind aus Rüstkammern und Waffensammlungen frühestens seit dem ausgehenden 15. Jahrhundert bekannt. Die Vorläufer der runden, geschlossenen Helme mit hochklappbaren Visieren sind die Hundsgugel mit Absteck- oder Klappvisier sowie die Beckenhaube mit an der Stirnfläche einhakbarem Visier (2. H. 14. Jahrhundert), die in der Frontalansicht aber nicht der Falkenberger Darstellung eines rautenförmigen Visiers entsprechen, ferner die Bicoque, die um 1430 in Westeuropa entwickelt wurde und den frühen Visierhelmen des ausgehenden 15. und beginnenden 16. Jahrhunderts am nächsten kommt. Bidermann 1980, Abb. 73, S. 69; Abb. 171-172, S. 155-156; Abb. 176, S. 160 Müller 1979, S. 18-19; S. 24-25; S. 30-31; S. 34-37 Boeheim 1890, Abb. 21, S. 36; Abb. 29a, S. 42
} 
gearbeiteten Ornamenten wie Dreipässen und mit Einlagen aus Harz oder Bernstein versehen worden sind (Abb. 158). Die genaue Anzahl der bei Reimerswaal und Krabbendijke geborgenen Messergriffe ist aus fundspezifischen Gründen (Lesefunde) nicht bekannt. Die im Katalog erfaßten Griffe stellen nur einen Querschnitt der in Rotterdam befindlichen Exemplare dar.

Außerhalb des kulturellen Bereichs des sogenannten "Zeeuws Vlaanderen" ${ }^{357}$ (Abb. 159) wurden in Dordrecht, Utrecht und im westmünsterländischen Ahaus weitere Beispiele dieser Gruppe gefunden.

Wie weit auch Exemplare einer regional begrenzten Objektgruppe gelangen können, zeigt der Fund einer den zeeländischen Griffen sehr ähnlichen Griffschale im St. Olavskloster in Oslo, vergesellschaftet mit niederländischen Goldgulden des 15. Jahrhunderts $^{358}$. Die Anwesenheit von Niederländern in diesem Dominikanerkloster ist für die gleiche Zeit gesichert ${ }^{359}$; das Messer ist also mit hoher Wahrscheinlichkeit als persönlich mitgeführter Besitz aus den Niederlanden nach Oslo gelangt, als Handelsobjekt kann es kaum angesehen werden (Abb. 161g).

Ob die bei Reimerswaal und Krabbendijke gefundenen Messergriffe dort hergestellt worden sind, kann nur vermutet werden. Ein möglicher Hinweis auf diese These ist die Tatsache, daß vollständige Messer mit Griffen aus diesen Fundorten nach bisheriger Kenntnis nicht aufgetreten sind, obwohl Metallfunde von beiden Fundstellen bekannt sind. Die Messer aus Dordrecht und Ahaus deuten darauf hin, daß die Montierung der Griffe vielleicht an anderen Orten erfolgte.

In jedem Fall handelt es sich bei diesen Griffen um eine südniederländische Formengruppe, deren Verbreitung vornehmlich auf die Provinz Zeeland und angrenzende Gebiete wie Südholland, Utrecht und das östliche Grenzgebiet beschränkt zu sein scheint. Nachweise aus dem südlich angrenzenden Flandern sind in diesem Zusammenhang nicht bekannt geworden.

\footnotetext{
357 Die Information über den Fundort verdankt der Autor Herrn A. Lagerweij und Herrn J. Baart vom Archäologischen Dienst in Amsterdam

358 vgl. Katalogteil Norwegen, Kat. Nr. 30

359 nach Information von Frau Inger H. Vibe Müller vom Museum Oldsaksamlingen der Universität Oslo, vgl. auch Krogstad/Schia 1982, S. 18-20
} 

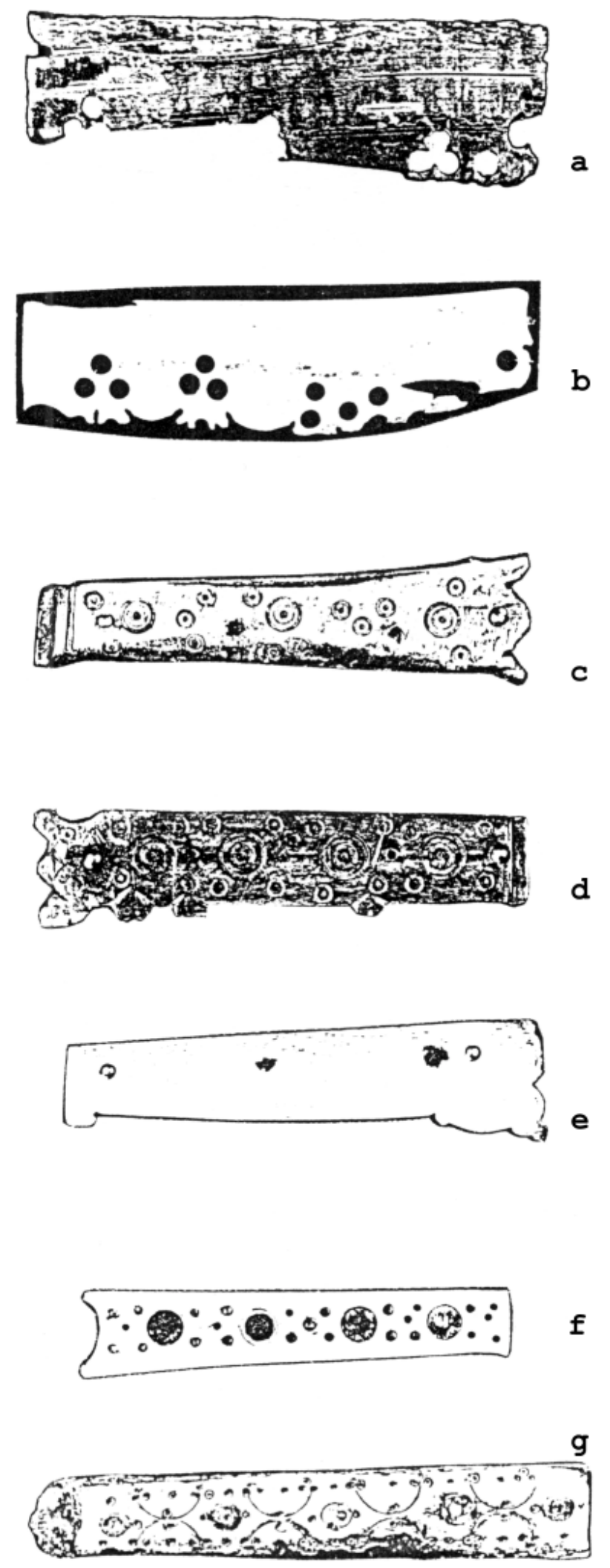

Abb. $158 \quad$ Zeeländische Griffe des 14. bis 15. Jahrhunderts $a-f$ : Niederlande, $g$ : Norwegen 


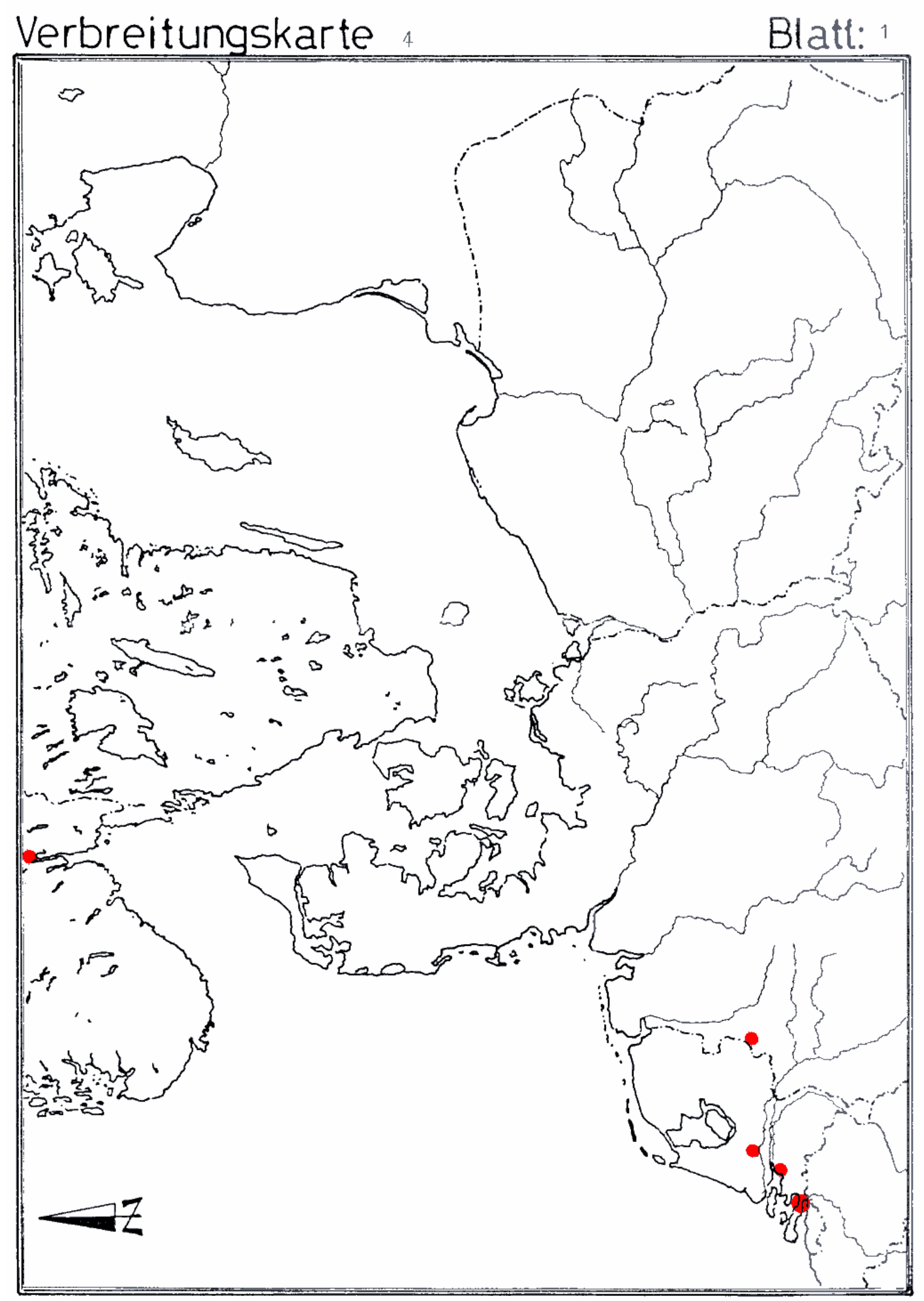

Abb. 159

Die Verbreitung der aus Reimerswaal und Oud-Krabbendijke bekannten Griffformen (14. bis 15. Jahrhundert) 


\subsection{Die Verbreitung von Verzierungselementen - Beispiele}

Neben einfachsten Ornamenten wie Linien, Doppellinien, Kammstrich oder Schraffur sind häufig übertragene Ornamente und das Punkt- oder Kreisauge als Verzierungselemente auf Messergriffen beobachtet worden.

Die Motive der in der Regel zu Mustern angeordneten übertragenen Ornamentik umfassen vor allem Flechtband-, Ringketten- und Wellenbandmuster. Sie sind innerhalb des Untersuchungszeitraums auf das 9. bis 12. Jahrhundert begrenzt (Abb. 109).

\section{Die Verbreitung von Flechtband, Ringkette und Wellenband}

Die kartierte Verbreitung dieser Muster im Untersuchungsraum beinhaltet lediglich die Nachweise an Messergriffen, nicht ihre allgemeine Verbreitung.

Ebenso muß darauf hingewiesen werden, daß die Anzahl publizierter verzierter Griffe aus diesem Zeitraum im östlichen Bereich des Untersuchungsgebietes erheblich größer ist als im Westen, so daß Aussagen in dieser Hinsicht nur tendenziellen Charakter haben können.

Dennoch läßt sich anhand des Verbreitungsbildes feststellen, daß die Übertragung von Flechtband-, Ringketten- und Wellenbandmustern auf Messergriffe vom 9. bis 12. Jahrhundert besonders im westslawischen Bereich verbreitet ist. Außerhalb dieses Kulturraumes erscheinen nur zwei Beispiele mit Flechtbandmotiven in Schweden (Birka) und am Niederrhein ${ }^{360}$ (Abb. 160).

Das Fehlen von Nachweisen nach 1200 sowie die Seltenheit der Motive im westlichen Teil des Untersuchungsgebietes lassen vermuten, daß der kulturelle Einfluß deutscher Siedler und Handwerker, der seit der Mitte des 12. Jahrhunderts mit dem Beginn und der Ausbreitung der Ostkolonisation zu beobachten ist, an der Verbreitung dieser Ornamente unbeteiligt war.

Innerhalb des westslawischen Siedlungsbereichs lassen sich Ringketten- und Flechtbandmuster auf Messergriffen an insgesamt 13 Fundplätzen nachweisen, die ausweislich des Fundmaterials und der beobachteten Siedlungsstruktur bis in das 12. Jahrhundert hauptsächlich von Westslawen bewohnt wurden. Sie sind fast durchweg an Burgwälle oder zentrale Handels- und Verwaltungsorte gebunden ${ }^{361}$. Auf dem Gebiet der ehemaligen DDR konzentriert sich das Aufkommen dieser Ornamente auf Burgwälle im nördlichen Teil, in dem die Kämpfe im Zuge der deutschen Ostexpansion bis in das 12. Jahrhundert andauerten ${ }^{362}$.

\footnotetext{
360 vgl. Katalogteil Schweden, Kat. Nr. 8; BRD, Kat. Nr. 179

361 Zur Verbreitung des Ringkettenmusters auf westslawischem Gebiet vgl. U. Schoknecht 1971, S. 282 ff.

362 vgl. Katalogteil ehemalige DDR, Kat. Nr. 2, 30, 32, 75, 98, 127, 160
} 
In Polen wurden Messergriffe mit den betreffenden Ornamenten in zwei Burgen, einer Vorburgsiedlung, in drei frühstädtischen Siedlungen und in einem Gräberfeld gefunden; sie entstammen mit einer Ausnahme dem 10. bis 12. Jahrhundert ${ }^{363}$ (Abb. 109).

Das Messer aus Birka ${ }^{364}$ weist neben geometrisiertem Flechtmuster Reste eines Flechtbandknotens auf, der Griff vom Husterknupp (Niederrhein) ${ }^{365}$ ein stark stilisiertes Flechtband.

Da aus Skandinavien und aus dem westlichen Teil des Untersuchungsgebietes im bearbeiteten Bestand verzierte Messergriffe vor $1200 \mathrm{zu}$ den Ausnahmen zählen, ist die Verbreitung von Ringketten-, Flechtband- und Wellenbandmustern auf Messergriffen in dieser Auswertung nicht repräsentativ. Weitere Ornamentträger wie Kämme, die häufiger publiziert worden sind, sind als Ergänzung zu dieser Fragestellung hinzugezogen worden.

Die Verbreitung des Ringkettenmusters ist bereits mehrfach beschrieben worden. So gab der Fund eines ringkettenverzierten Messers von der Fischerinsel Ulrich Schoknecht Anlaß zu einer kurzen Darstellung mit Beispielen von der Isle of Man, aus Haithabu sowie von polnischen und tschechischen Fundplätzen ${ }^{366}$.

Die verschiedenen Variationen des Flechtbandmotivs erscheinen vor allem im Kunsthandwerk der Wikinger, wo sie als Verzierungstechnik und als Ornament an Schmuck, Waffen, Bildträgern und Objekten des alltäglichen Lebens begegnen (Abb. 161).

\footnotetext{
363 vgl. Katalogteil Polen, Kat. Nr. 12, 13, 87, 113, 128, 130, 177, 330 


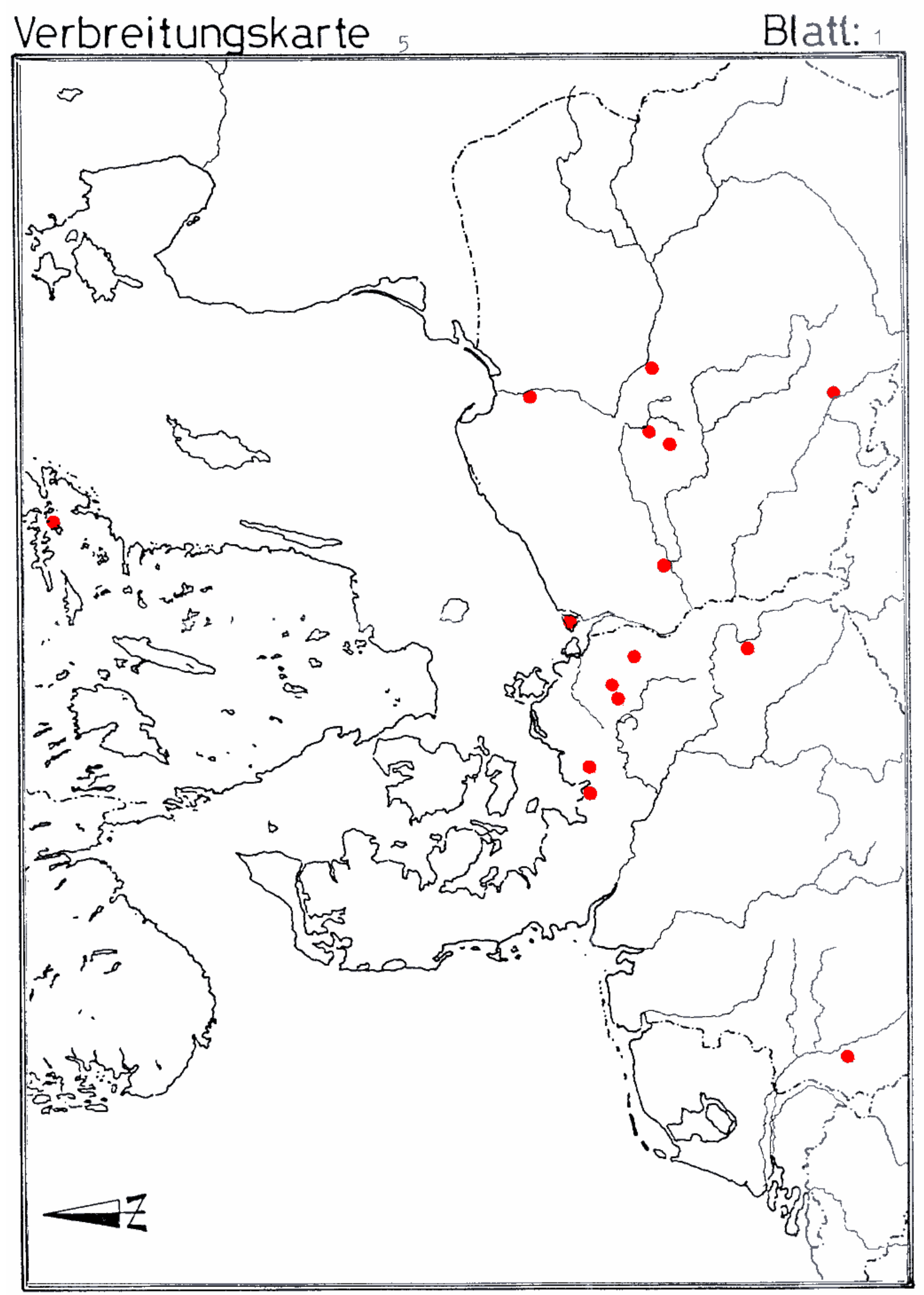

Abb. 160 Die Verbreitung von Flechtband-, Ringketten- und Wellenbandmustern auf Messergriffen des 9 . bis 12. Jahrhunderts 


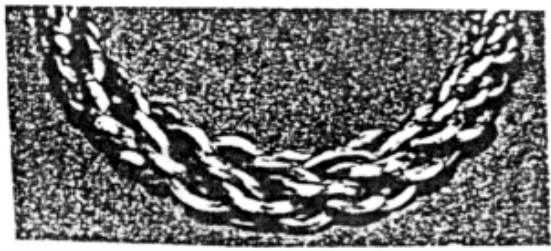

a

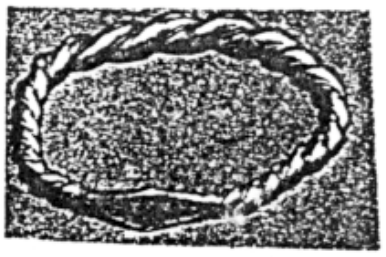

b

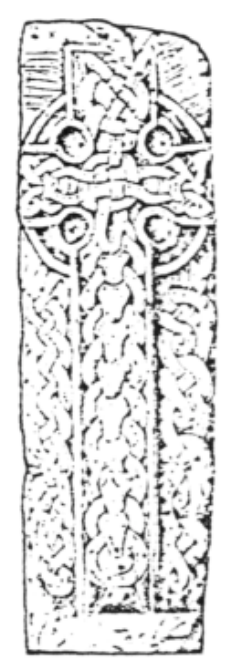

d

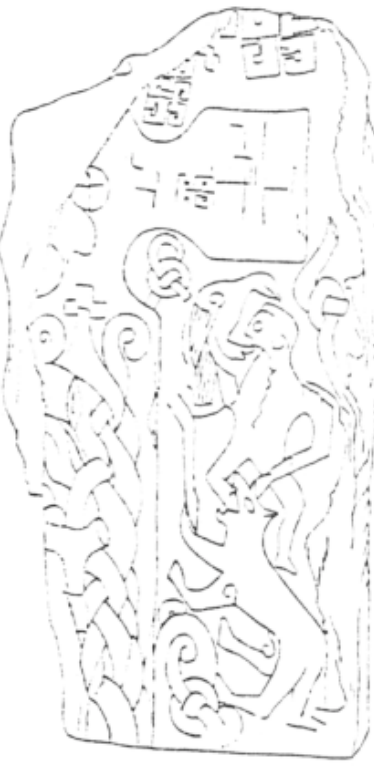

e

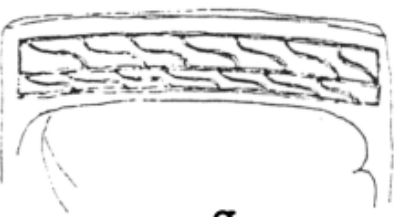

g

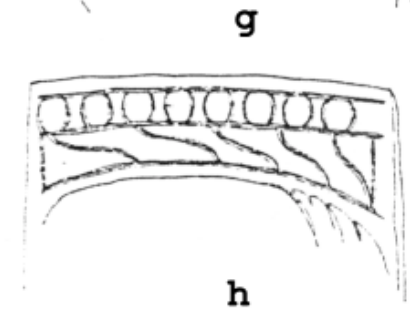

h

$\mathbf{f}$
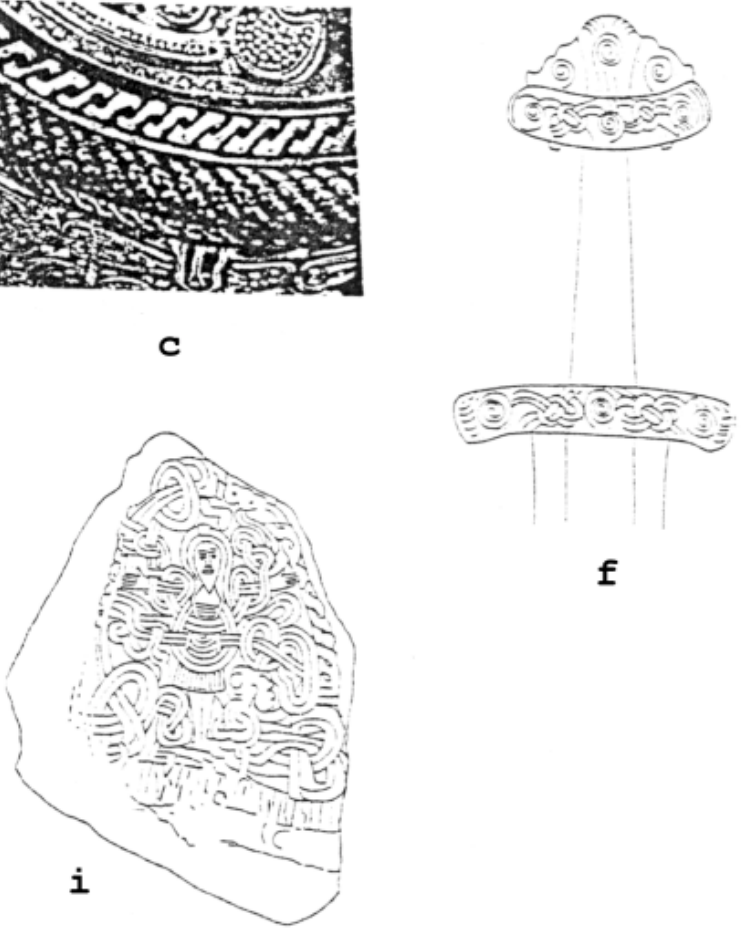

Abb. $161 \quad$ Wikingerzeitliche Flechtband- und Ringkettenmuster ${ }^{367}$ $a-b$ : Schatzfund von Vullum, Norwegen; c: Schultertuchspange (Ausschnitt), Gotland

d: beschnitzte Holzplatte, Isle of Man; e: Thorwaldkreuz, Isle of Man;

$f$ : Schwertgriff aus Södertälje, Schweden;

g-h: Griffleiste aus dem Osebergfund, Norwegen; $i$ : Runenstein von Jellinge, Dänemark

367 a-c, e-i: Almgren u.a. 1975, a-b: Abb. S. 198; c: Abb. S. 221; e: Abb. S. 92; f: Abb. S. 227; g-h: Abb. S. 183; i: Abb. S. 149; d: Graham-Campbell, 1980, Abb. S. 140 
Seine Verbreitung auf Gegenständen des täglichen Gebrauchs ist angesichts der Masse an verzierten Objekten aus Horn, Holz und Knochen geringer als zum Beispiel linearer und geometrischer Dekor, der nicht nur auf Messergriffen über das gesamte Untersuchungsgebiet verbreitet ist. Beispiele von Kämmen und anderen Objekten aus Birka, Arhus, Staraja Ladoga und Haithabu zeigen jedoch Flechtbandmotive verschiedener Ausprägungen auf Gegenständen des täglichen Gebrauchs skandinavischer Provenienz, unter denen der Messergriff aus Birka nur ein Beispiel unter vielen ist (Abb. 162). Somit täuscht der vereinzelte Griff für den skandinavischen Raum eine Leere vor, die sich in der subjektiven Auswahl publizierten und - nicht zuletzt - dem Autor zur Verfügung stehenden Materials begründet. Für den zeitgleichen Bestand der ehemaligen DDR und Polens lassen sich die gleichen Variationen des Flechtbandmusters sowohl auf Messergriffen als auch auf Kämmen und anderen materialidentischen Objekten finden (Abb. 163). Ein Kammfragment aus Danzig zeigt ein aus verschiedenen Motiven völlig aufgelöst komponiertes Flechtband, welches ohne Vergleich mit den weniger abstrahierenden Beispielen kaum noch zu erkennen ist (Abb. 163g).

Somit ist für den skandinavischen und westslawischen Bereich die Verwendung von Ringketten- und Flechtband-, seltener auch von Wellenbandmustern auf Messergriffen, Kämmen und anderen Objekten des gehobenen täglichen Bedarfs aus organischen Materialien gleichermaßen anzunehmen, auch wenn sie sich in dieser Auswertung vor allem in der spätslawischen Kultur manifestiert ${ }^{368}$.

Flechtbandmotive sind im westlichen Teil des Untersuchungsgebietes auf Messergriffen nur einmal nachgewiesen worden ${ }^{369}$. Das Motiv ist zwar stark stilisiert, aber deutlich erkennbar (Abb. 109, BRD-179). In diesem Fall bestätigt der Vergleich mit anderen materialidentischen Objekten das Bild:

Die für den Norden und Osten des Untersuchungsgebietes typische, winklige Ausprägung des Flechtbandmotivs fehlt ganz, im Bestand der Horn- und Knochenobjekte aus dem friesisch-fränkischen Dorestad ist zum Beispiel lediglich die gerundete Ausführung der Darstellung in seltenen Einzelfällen vorhanden (Abb. $119 \mathrm{~h}$ bis 119i), die aus Danzig bekannte Auflösung mit versetzten Punkt- oder Kreisaugen ist zum Wellenband abgewandelt worden (Abb. 119j bis 119k).

\footnotetext{
368 Vgl. zu diesem Thema Herrmann 1982, v.a. S. 165-172 und S. 264-290 zum skandinavisch-slawischen Kulturaustausch

369 Vgl. Katalogteil BRD, Kat. Nr. 179
} 

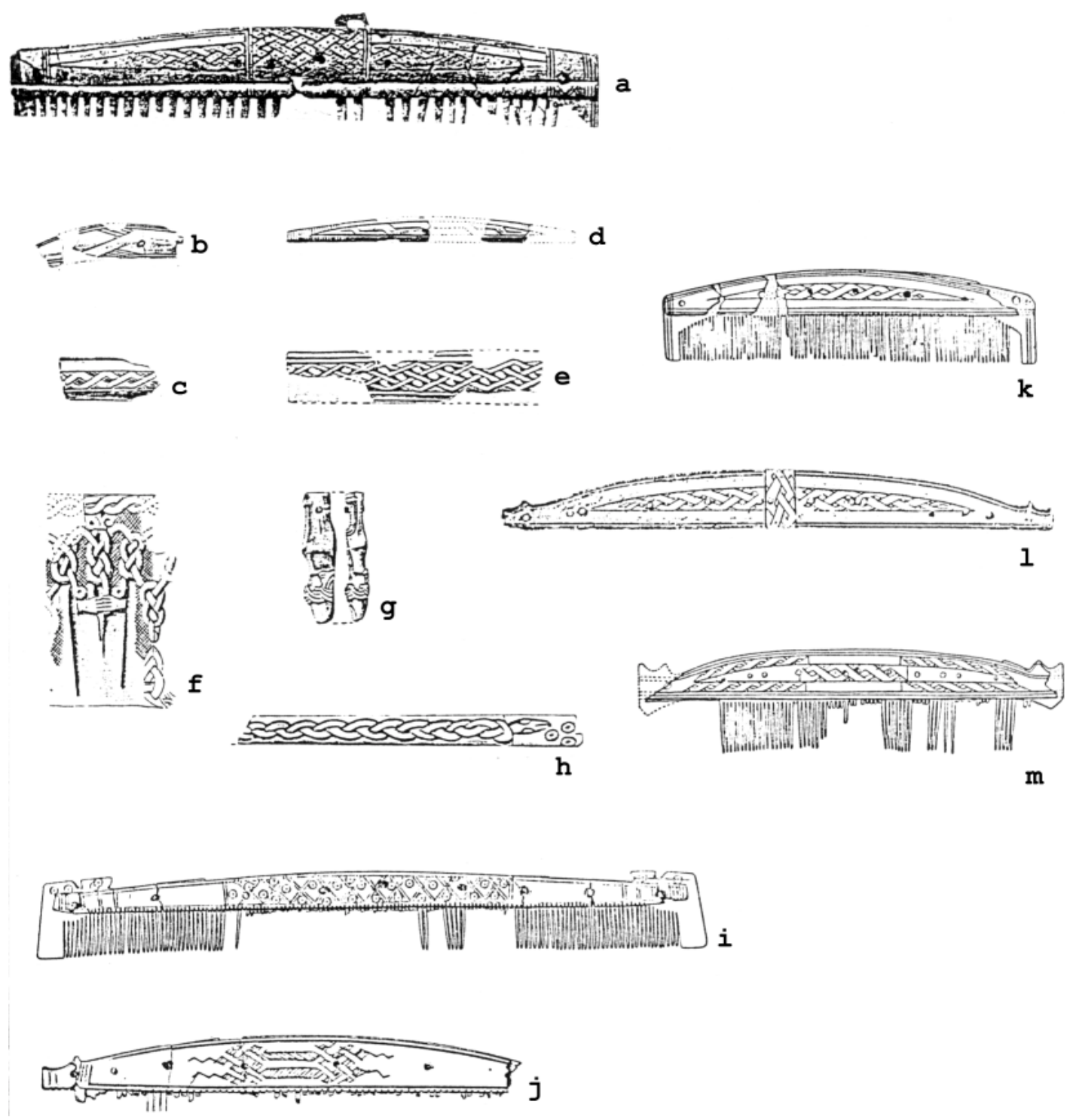

Abb. $162 \quad$ Überragene Muster auf Ornamentträgern aus organischem Material $I^{370}$ a-h: Birka, i-j rhus; $k, m$ : Staraja Ladoga; $l$ : Haithabu

370 a-h: Arbman 1940; a-c: Tafel 159; e, h: Tafel 166; f, g: Tafel 154; i-j: Andersen u.a.

1971, Abb. S. 151 und S. 146; k, m: Davidan 1962, Abb. 2.1 und 2.2, S. 97; 1: Ulbricht 1977, Tafel 16 (Anhang) 


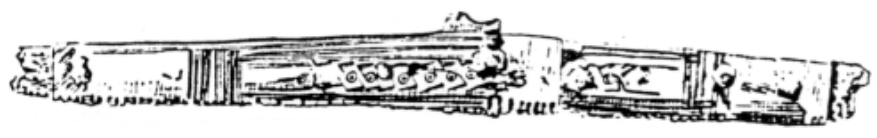

a
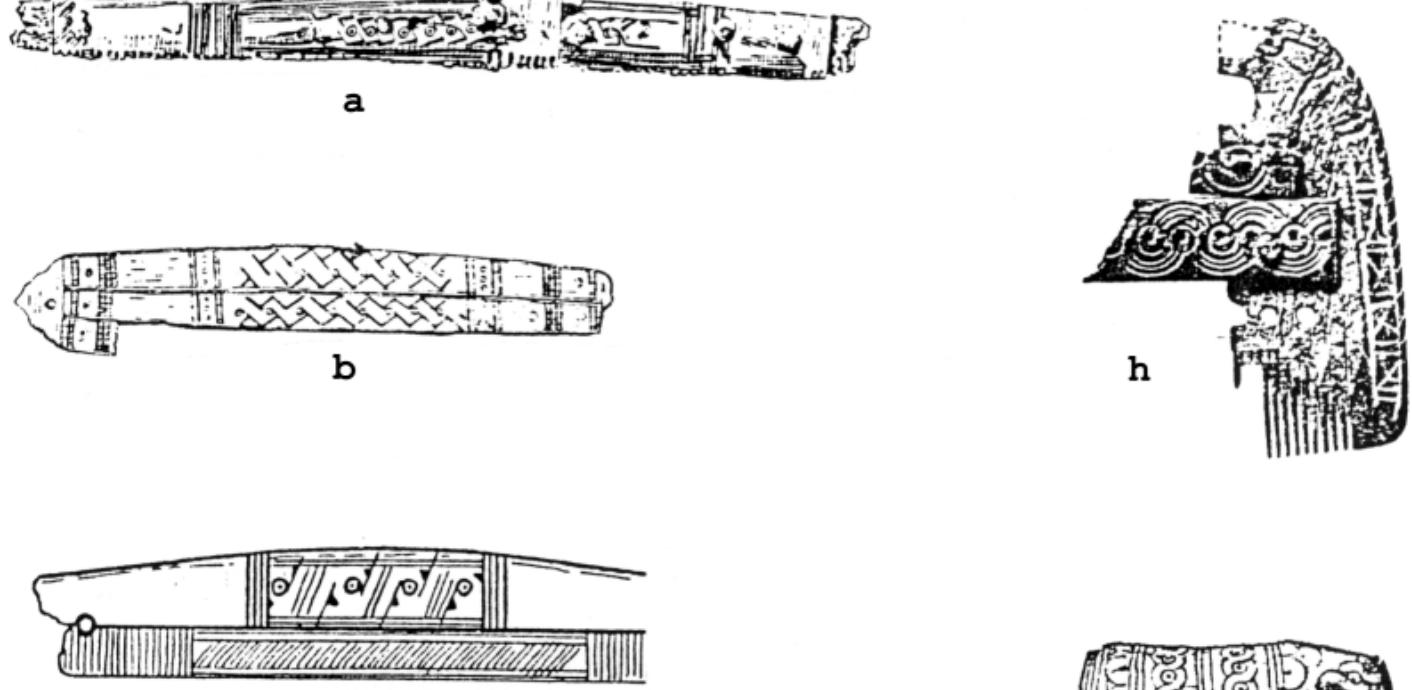

C

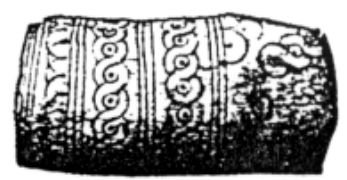

i
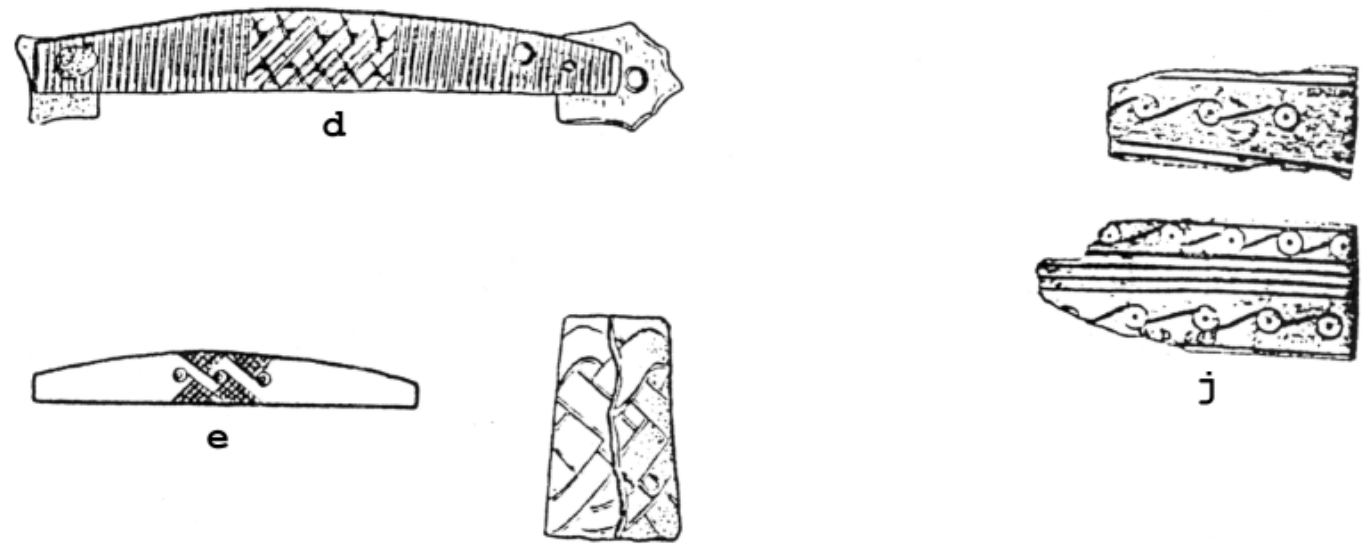

j

e

$\mathbf{f}$

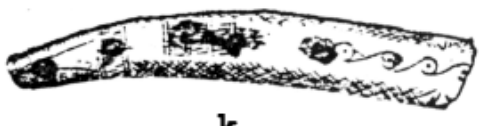

$\mathbf{k}$

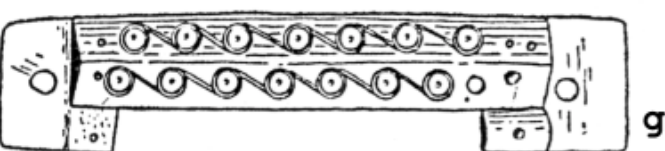

Abb163

Übertragene Muster auf Ornamentträgern aus organischem Material II ${ }^{371}$ $a-b$ : Wolin; c-g: Danzig; h-k: Dorestad

371 a: Cnotliwy 1958, Abb. Tafel III.11

b: Cnotliwy 1956, Abb. Tafel II.6, S. 181

c-g: Hilczerowna 1961; c: Abb. 42, S. 101; d: Abb. 40, S. 99; e: Abb. 43, nach S.102; f: Abb. 31, S. 77; g: Abb. 39, S. 96

h-k: Roes 1965; h: Tafel 29, Abb. 219; i: Tafel 26, Abb. 197; j: Tafel 30, Abb. 225; k: Tafel 29, Abb. 223 
Trotz der quellenkritischen Eingangsbemerkung läßt sich abschliessend festhalten, daß vom 9. bis zum 12. Jahrhundert innerhalb der Grenzen des Untersuchungsgebietes Ringketten- und Flechtbandmotive auf Messergriffen aus organischen Materialien vor allem im westslawischen, auch im skandinavischen Kulturraum anzutreffen sind, korrespondierend mit anderen, materialidentischen Gegenständen des täglichen Gebrauchs.

Im westlichen Teil des Untersuchungsgebietes sind diese Ornamente extrem selten angetroffen worden, obwohl sie sowohl in Haithabu als skandinavischer Import als auch in Dorestad vereinzelt vorhanden sind. Die geringe Anzahl publizierter Funde erlaubt es nicht, weitergehende Schlußfolgerungen zu ziehen.

\section{Die Verbreitung des Punktaugen- (Kreisaugen-) motivs}

Das bereits im keltischen und römischen Kunsthandwerk bekannte Motiv des Zirkelschlags, das Punktauge (Kreisauge, Punktkreis) ${ }^{372}$, ist im Untersuchungsraum vom 11. bis zum 16. Jahrhundert nachweisbar, ältere Beispiele sind von anderen Objekten (v.a. Kämme) verschiedener Materialien bekannt. Wie bei den übertragenen Ornamenten scheint es auch in diesem Fall unterschiedliche Intensität bezüglich der Verwendung derartiger Dekorelemente auf Messergriffen und Kämmen, Spielsteinen etc. gegeben zu haben, die sich sowohl in der Zahl der verzierten Gegenstände als auch im chronologischen Auftreten derselben manifestiert.

Die Verbreitung des Punkt- oder Kreisaugenmotivs auf Messergriffen aus organischen Materialien erstreckt sich auf das gesamte Untersuchungsgebiet (Abb. 164). Vor 1300 ist eine Konzentration vor allem im westslawischen Siedlungsbereich mit sechs Fundplätzen zu beobachten, gegenüber drei Nachweisen aus Lund, vom Husterknupp (Niederrhein) und von der Burg Isenburg (Ruhr).

Im 13. bis 14. Jahrhundert ist eine Ausprägung faßbar, die sich deutlich von den anderen Variationen des Punktaugenmotivs unterscheidet, eine kleine Gruppe anscheinend küstengebundener Vollgriffe, die durch Gurt- und Schraffurbänder in zwei mit kreuz- oder rosettenartig angeordneten Punktaugen angefüllte Zonen gegliedert sind (Abb. 130, Seite 5). Diese Griffverzierungsvariante ist mit vier Exemplaren aus Oslo, Arhus, Lübeck und Danzig bisher nur für küstengebundene Städte aus der Blütezeit der Hanse nachweisbar.

Vom 14. bis 16. Jahrhundert ist im Untersuchungsraum eine Konzentration punktaugenverzierter Messergriffe im westlichen und nördlichen Teil zu beobachten, vor allem im niederländisch-westfälischen Bereich ist für das 14. bis 15. Jahrhundert das Punktauge in Verbindung mit geometrischen und linearen Mustern das gebräuchlichste Verzierungselement auf Messergriffen.

372 Die Bezeichnungen dieses Ornaments sind in der Literatur nicht einheitlich; zu den römischen Beispielen vgl. Abb. 95 und Abb. 108 
Verbreitungskarte

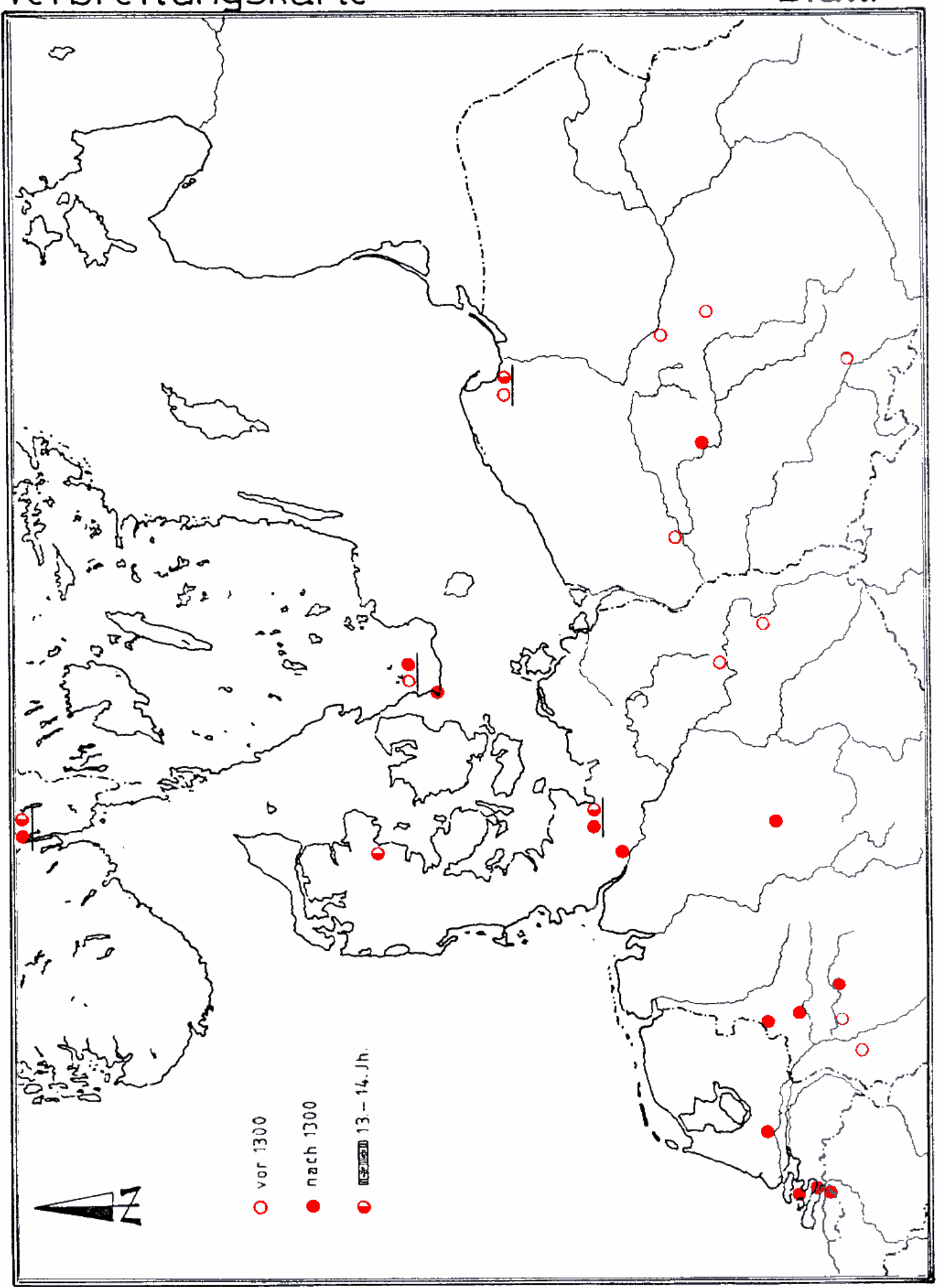

Abb. 164 (Karte) Die Verbreitung des sogenannten Punkt- oder Kreisaugenmotives auf Messergriffen des 11. Bis 16. Jahrhunderts 


\subsubsection{Verzierungen an Griffen aus Metall und die Verwendung von Metallapplikationen vom 9. bis zum 17. Jahrhundert}

Im Gegensatz zu den Griffverzierungen auf organischer Materialgrundlage entspricht das Hauptunterscheidungsmerkmal bei Metallverzierungen an Messergriffen der technologischen Unterscheidung von Griffangel- und Griffzungenmessern.

Ist für die Verzierung von Knochen-, Horn- oder Holzgriffen in der Regel kein besonderer produktionstechnischer Aufwand notwendig, so erfordert die Verwendung von Metallapplikationen aufwendigere Produktionsschritte, die bereits in der Beschaffung und Bearbeitung der Rohmaterialien beginnt.

$\mathrm{Da}$ beide Verzierungsarten nicht nebeneinander, sondern kombiniert angewandt worden sind, zeigen Griffe wie der vom Messer aus Wienhausen ${ }^{373}$ oder ein Griff aus Sluis (Niederlande) aus dem beginnenden 16. Jahrhundert ${ }^{374}$.

Bei den Angelmessern betreffen die Verzierungen entweder den metallenen Griff oder Metallapplikationen.

Die einfachste Art der Verzierung besteht in der Tordierung der Angel; volleiserne, geschmiedete Griffe sind graviert oder tauschiert worden.

Die Verwendung von Applikationen, die als Vollkörper ganze Griffteile ausmachen, betreffen entweder den gesamten Griff oder die hintere Griffhälfte. Eine Variante der volleisernen verzierten Griffe besteht in der Ausformung des Griffendes zu einer mit Schalen belegten Zunge.

Die letzte Art metallener Vollgriffverzierung ist das Ummanteln eines aus organischem Material hergestellten Griffs mit Bunt- oder Edelmetallblechen, später eine gegossene Griffhülse, die mit Kitt gefüllt auf die Angel geschoben wird.

Metallapplikationen, die zusätzlich an Vollgriffen aus organischen Materialien angebracht wurden, umfassen Drahtumwicklungen, verzierte Heftringe und Manschetten, Griffplättchenverzierungen, verzierte Einzelplättchen, Blechbeschläge, gegossene Griffendaufsätze (als Knauf oder Hülse) sowie am Heft befindliche gegossene oder geschmiedete Vollkörper.

Metallverzierungen an Griffzungenmessern betreffen nur sehr selten die Zunge an sich. In zwei Fällen traten durchbrochene Griffzungen auf, deren Hohlräume möglicherweise durch Einlegen von organischen Materialien ausgefüllt worden waren $^{375}$.

Ansonsten sind Griffzungenmesser durch geschmiedete oder gegossene Applikationen verziert worden. Diese können in die aus organischen Materialien bestehenden Griffschalen eingelassen worden sein, wie Zier- oder Blindniete, Silberdraht- oder Messingdrahteinlagen. Sie umfassen weiterhin eiserne oder

\footnotetext{
373 Vgl. Katalogteil BRD, Kat. Nr. 74

374 Vgl. Katalogteil Niederlande, Kat. Nr. 126

375 Vgl. Katalogteil Norwegen, Kat. Nr. 53; Schweden, Kat. Nr. 88
} 
gegossene Buntmetallapplikationen, die vor das Griffende montiert (meistens gelötet) wurden, Beschlagbleche an den Kanten und am Griffende sowie vernietete Buntmetallschalen oder -schalenteilstücke am Heft und am Griffende, die zum Teil bereits in Zierform gegossen und oft zusätzlich mit Gravuren versehen worden sind. Die Formen der Endapplikationen reichen von halbrunden Abschlüssen zu kronenförmig, zoomorph, dreipaß- und palmettenförmig gestalteten Enden bis zu gegenständlichen Abbildungen (zum Beispiel als Hammerkopf) und Scheiben mit Inschriften (Datierungen).

Messergriffe mit gegossenen, montierten Endapplikationen weisen oft noch zusätzliche Verzierungen an den Griffschalen durch Drahtumwicklung, Punktaugen, Zierniete und Drahteinlagen (Tiermasken) auf; die Horn-, Holz- oder Knochenschalen von Griffen mit vernietetem, verziertem Heft und Griffschalenteilstücken sind seltener durch Intarsierung zusätzlich verziert worden.

\section{Katalog der Verzierungsarten und -elemente (Abb. 165)}

Wie die Verzierungselemente auf organischen Materialien werden auch die einzelnen Verzierungen an Metallgriffen listenartig vorgestellt. Sie umfassen ebenfalls die Abbildung des Verzierungselementes anhand von Beispielen aus dem Katalog, das Fundland und die Katalognummer sowie den graphisch dargestellten chronologischen Rahmen.

Tordierte Griffangeln, geschmiedete Eisenvollgriffe und tauschierte Griffe, zungenförmige Griffenden an Eisenvollgriffen, Teile des Griffs umfassende Buntmetallsegmente oder vollständige Bunt- oder Edelmetallgriffe, Mantelbleche und Griffhülsen, Drahtumwicklungen, Heftringe, Griffplättchenverzierung mit und ohne Vollmetallkörper, Zierscheibchen und Beschlagbleche am Griffende, Griffendapplikationen und Heftvollkörper

Eisenapplikationen, durchbrochene Zungen, Blind- und Ziernieten, Drahteinlagen, Beschlagbleche am Griffende, auf die Zunge vernietete, gravierte Buntmetallscheibenstücke (Bänder und Platten) am Griffende, dieselben an Heft und Griffende, vernietete, in Form gegossene oder gravierte und punzierte Buntmetallscheiben, vor das Griffende montierte Buntmetallsegmente 
Abb. 165

(12 Seiten)

Katalog der Griffverzierungen an Griffen aus Metall

und die Verwendung von Metallapplikationen vom 9. bis zum 17. Jahrhundert

(Die Länderbezeichnungen in den folgenden Abbildungen beziehen sich auf die bis 1987 benutzte Literatur, die für die Erstellung des Kataloges ausgewertet wurde.

So bezeichnen:

DDR

Bundesrepublik Deutschland

(neue Bundesländer)

VRP

Polen

UdSSR

Staaten der ehemaligen

Sowjetunion 

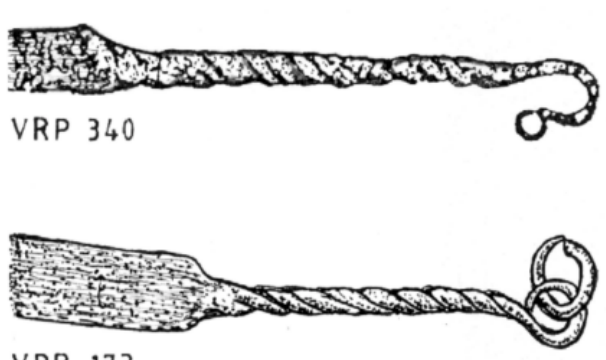

VRP 173

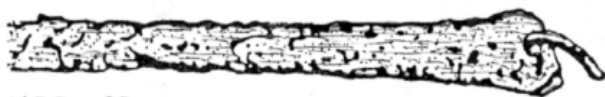
YRP 35
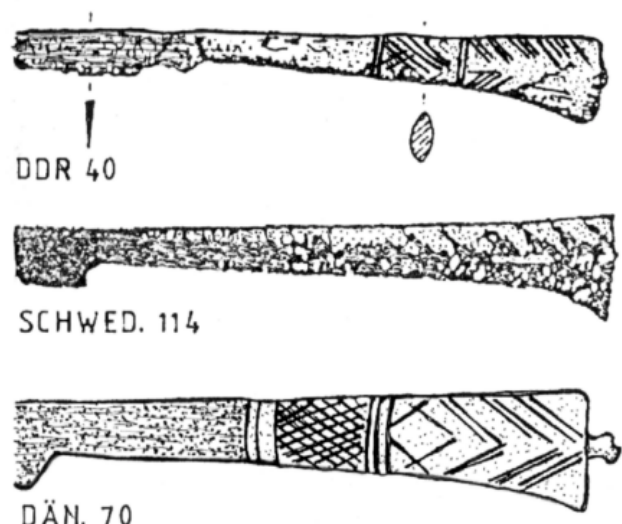

DÄN. 70
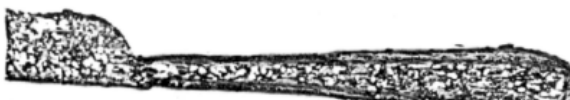

NL 86

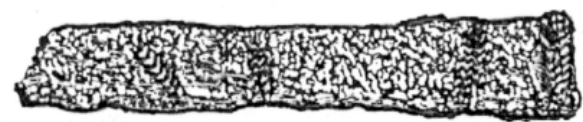

SCHWED. 3

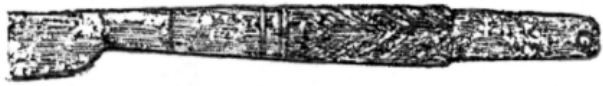

$\mathrm{NL} 78$

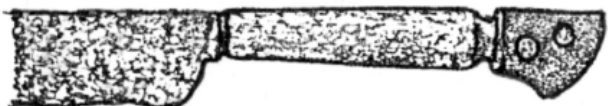

$\begin{array}{ll}\text { NL } & 87\end{array}$

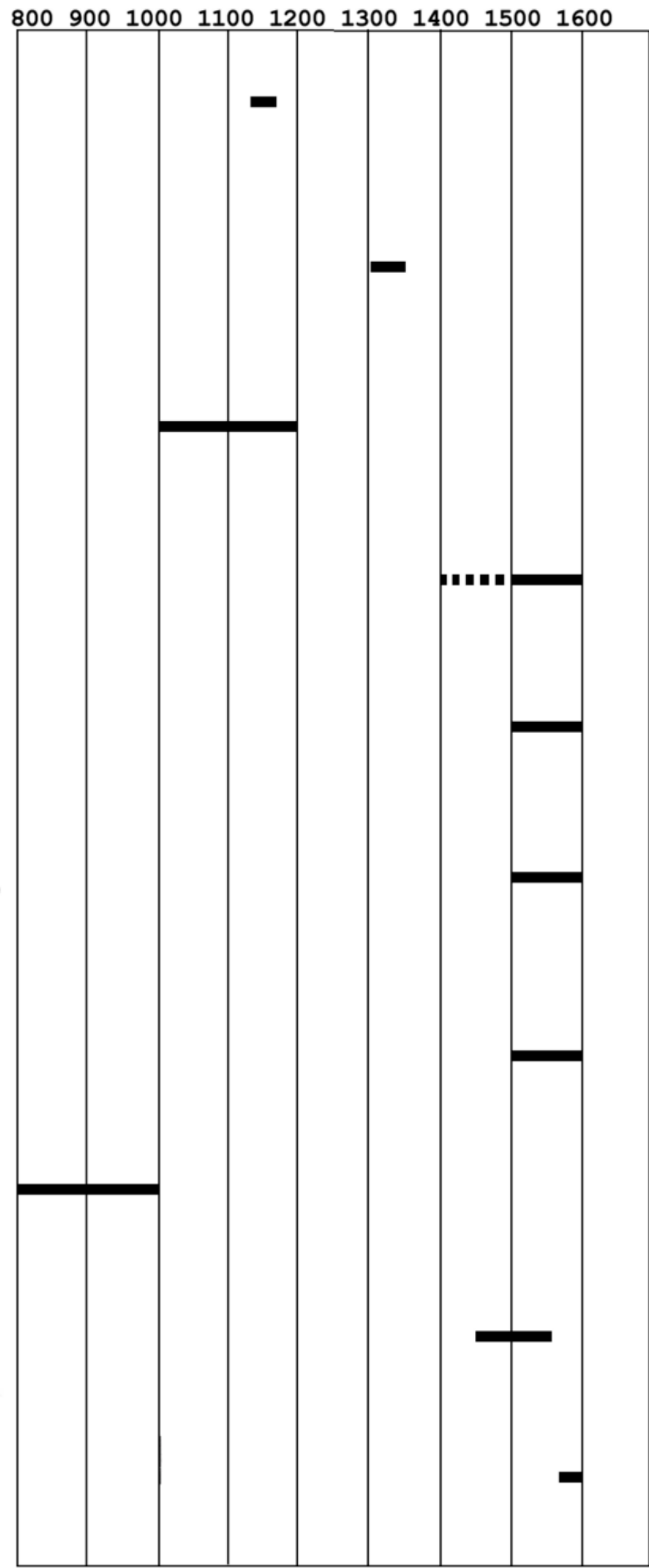




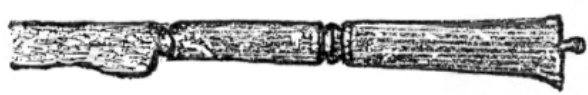
NL 82
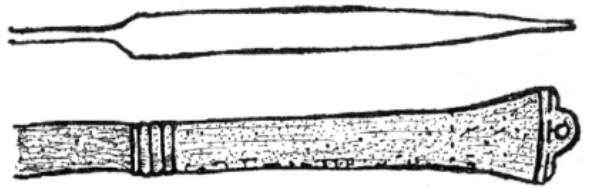
VRP 1
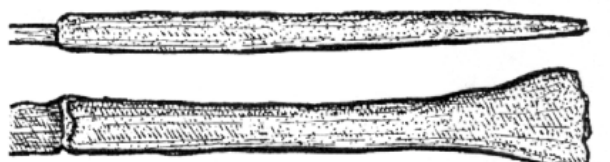
DDR 44

NL 79
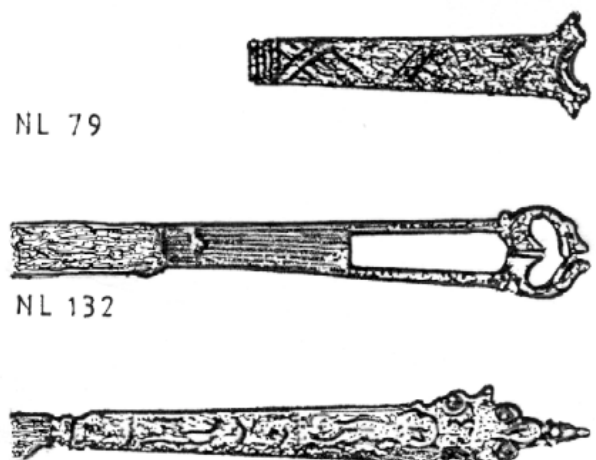
$\mathrm{NL} 47$

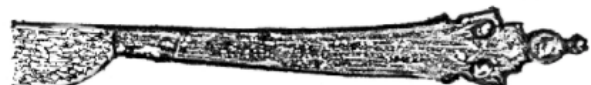
NL 83

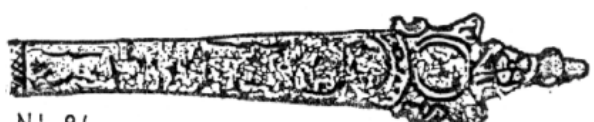

$N L 84$

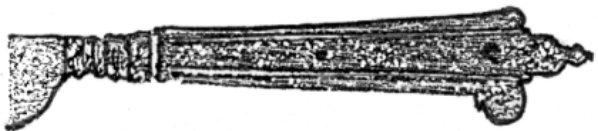

NL 85

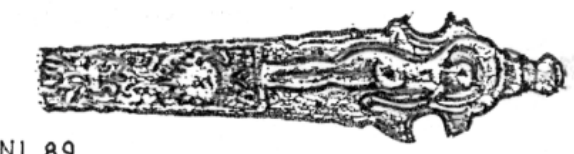
NL 89

$80090010001100120013001400 \quad 1500 \quad 1600$

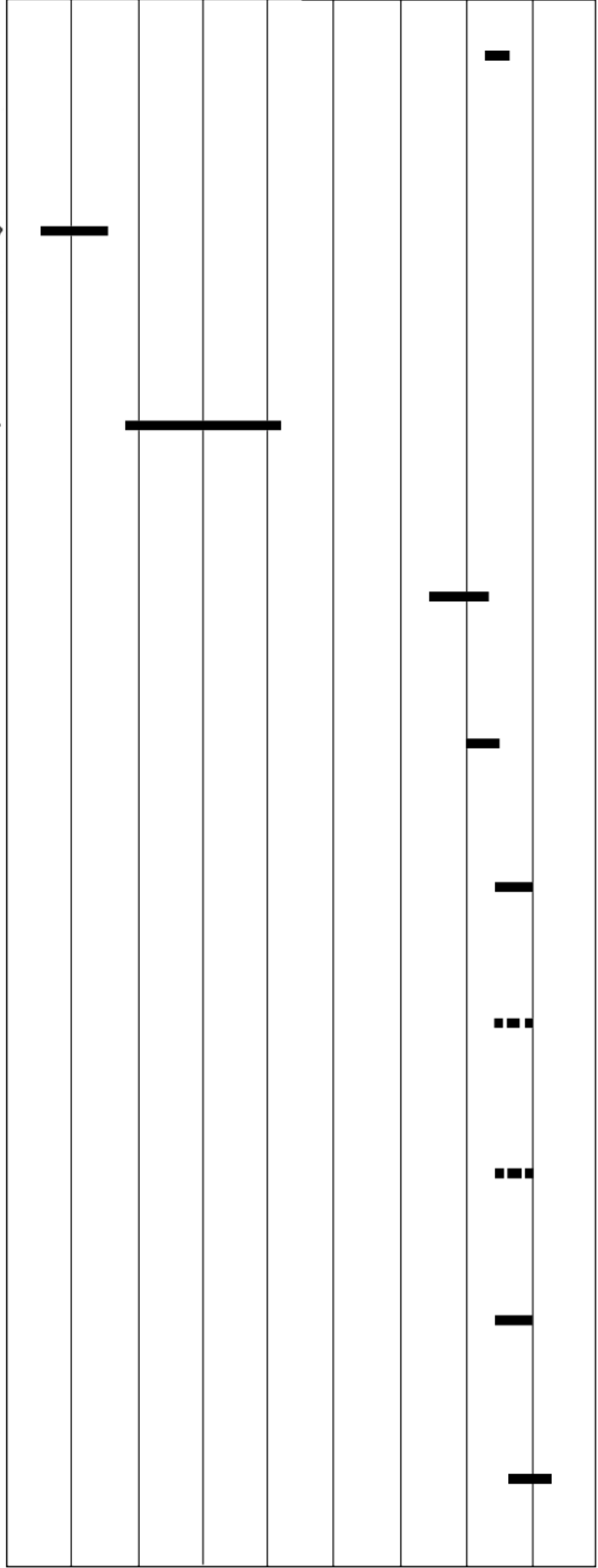




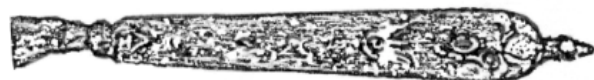

NL 93

NL 90
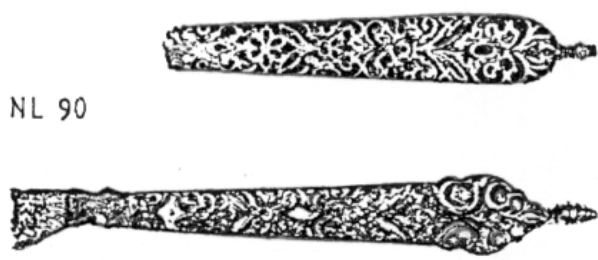

$N L 52$

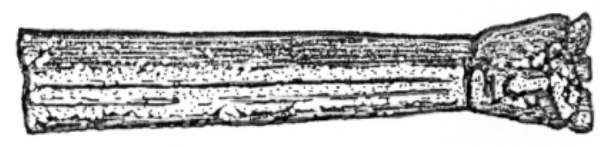

SCHWED. 42

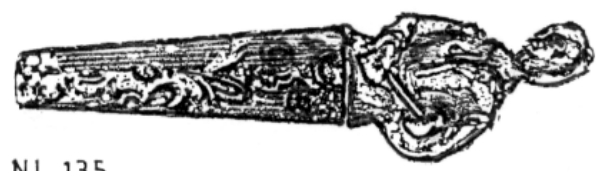

NL 135

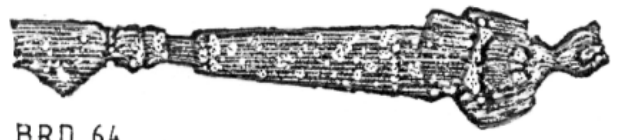

BRD 64

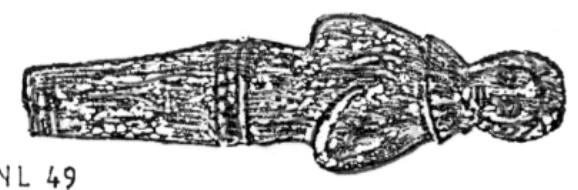

$N L 49$

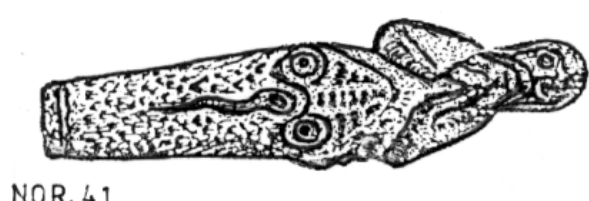

NOR. 41

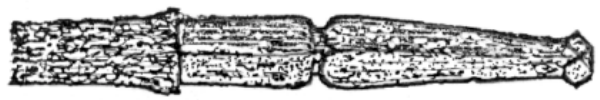

NOR.52

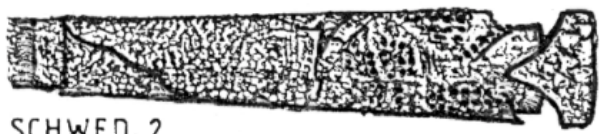

$\begin{array}{lllllll}800900 \quad 1000 & 1100 \quad 1200 & 1300 & 1400 & 1500 & 1600\end{array}$

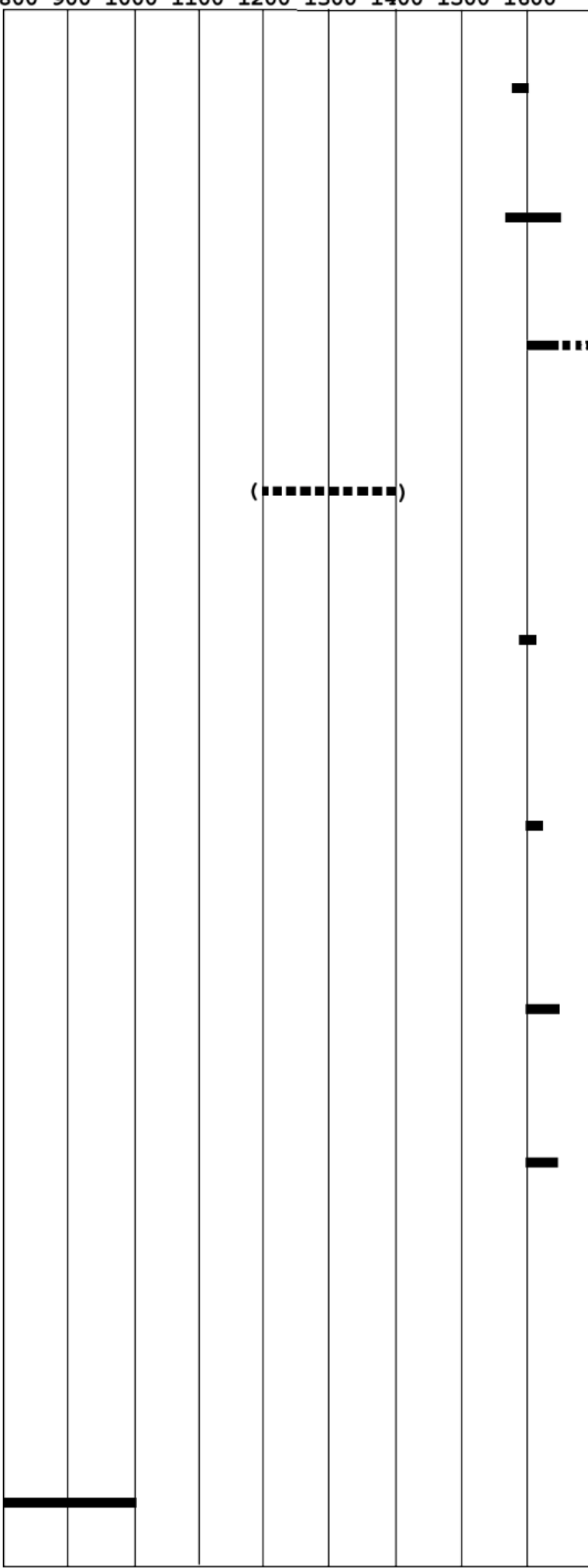




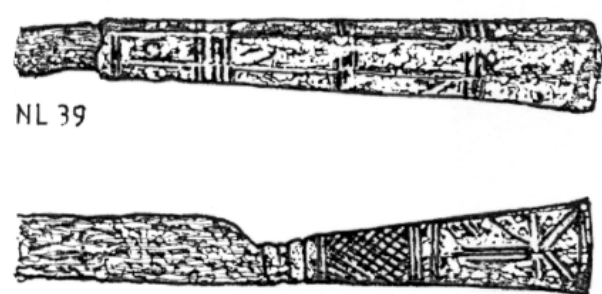

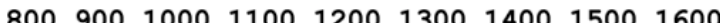

NOR. 32

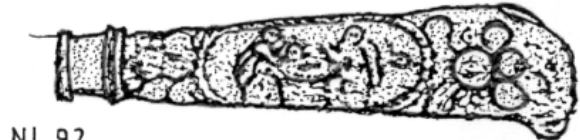

NL 92

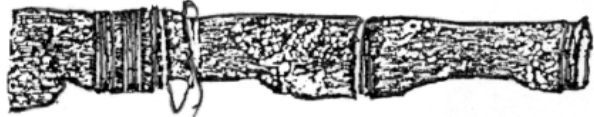

SCHWED. 9

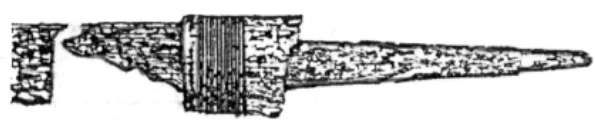

SCHWED. 7

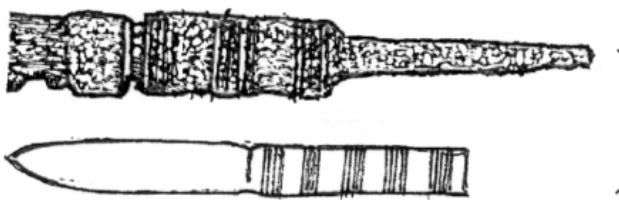

VRP 24

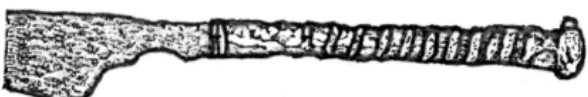

SCHWED. 66

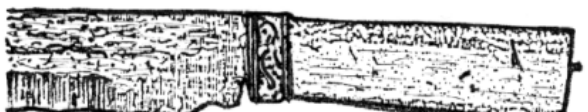

Fos

DDR 66

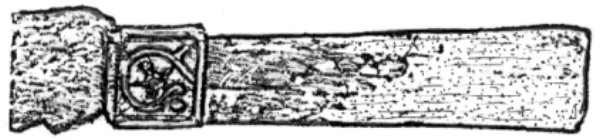

DÄN. 36
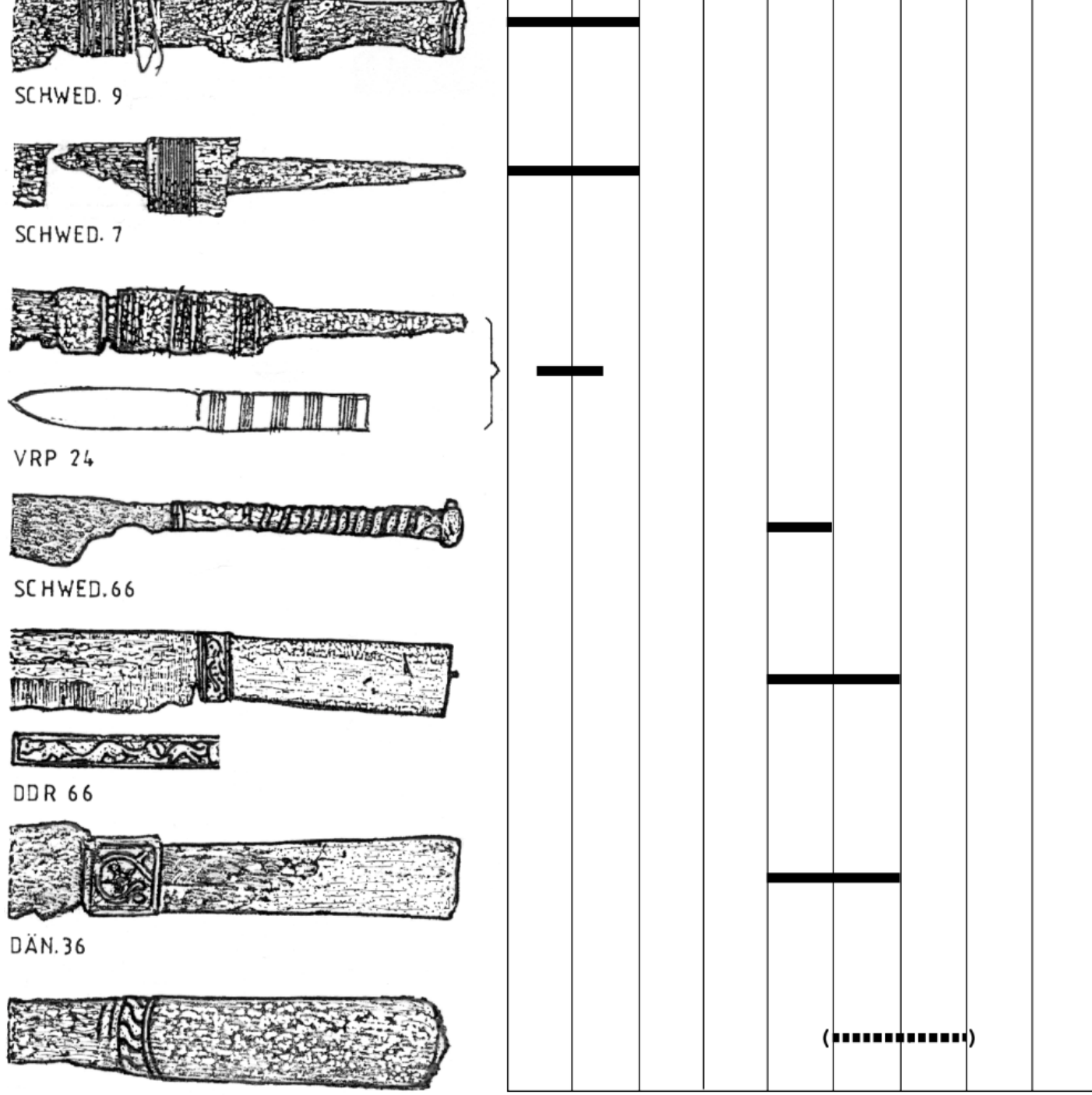

UdSSR 26 


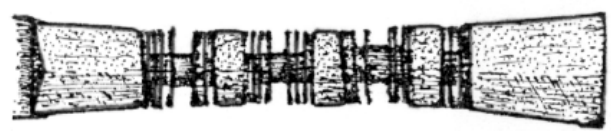

NOR 2

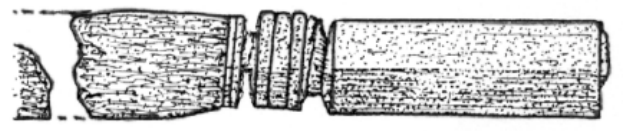
BRD 106

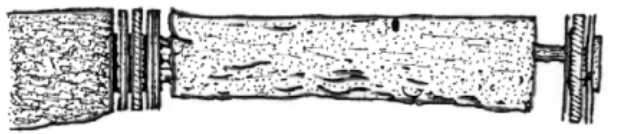
DDR 67

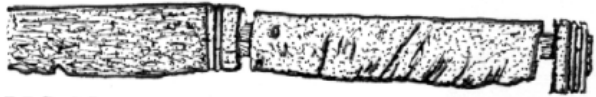
DDR 35

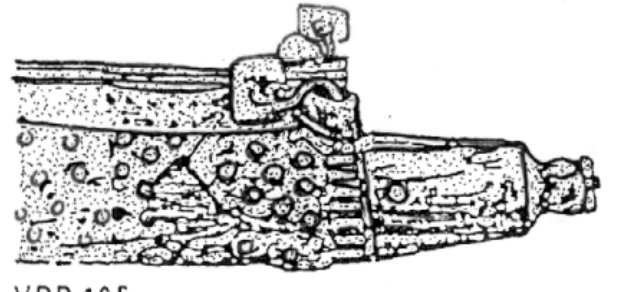

VPP 105
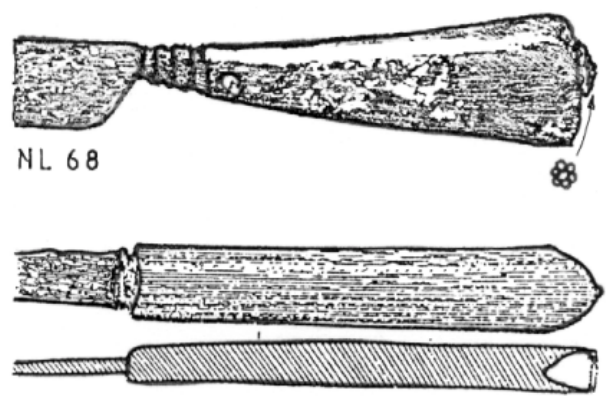

UASSR 74

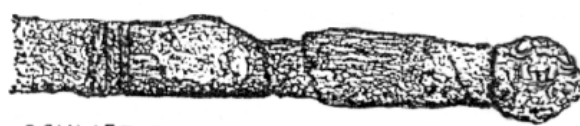

SCHWED. 1

SCHWED.123

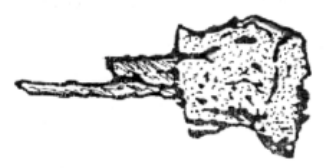

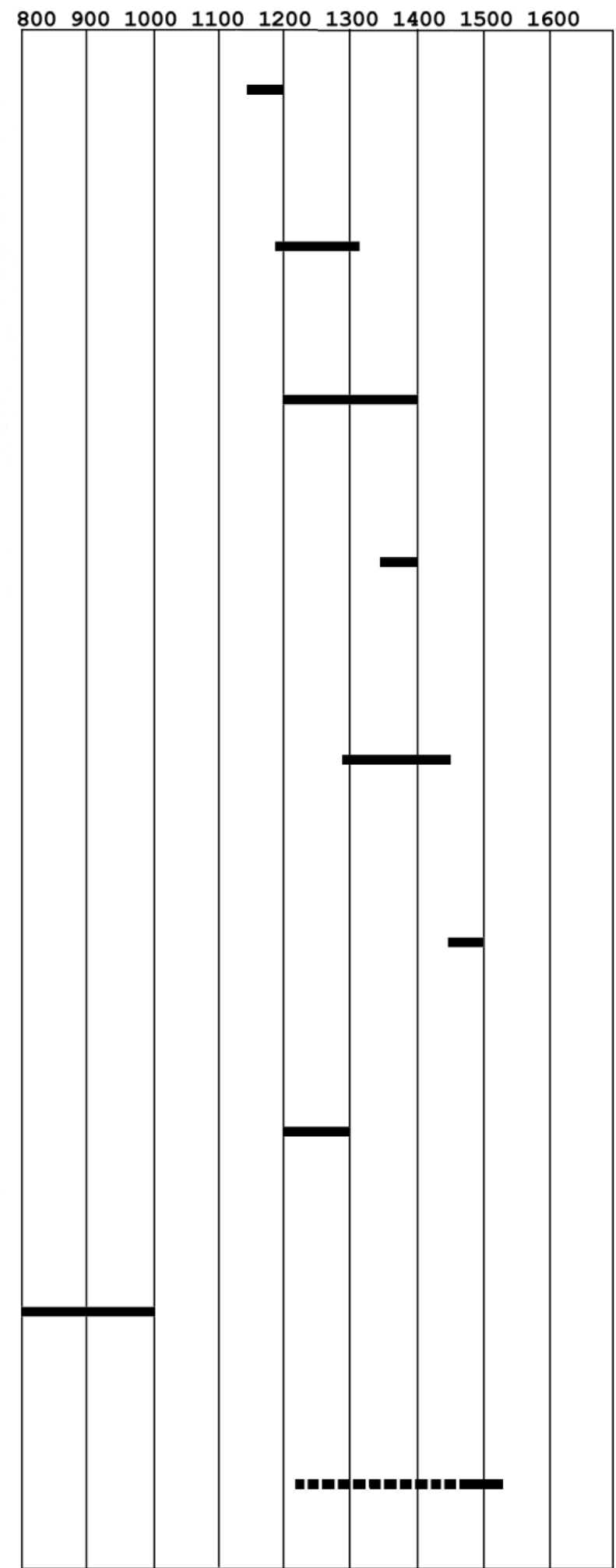




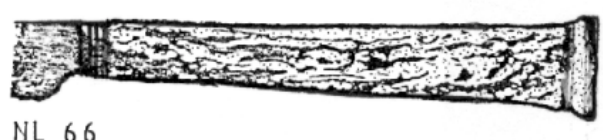

8009001000110012001300140015001600

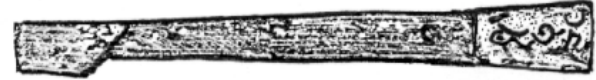
NL 45

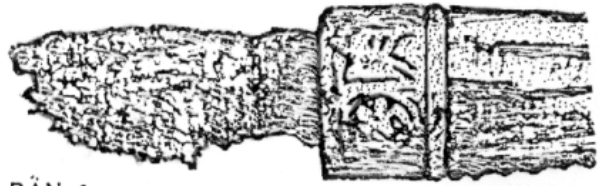

DÄN. 1
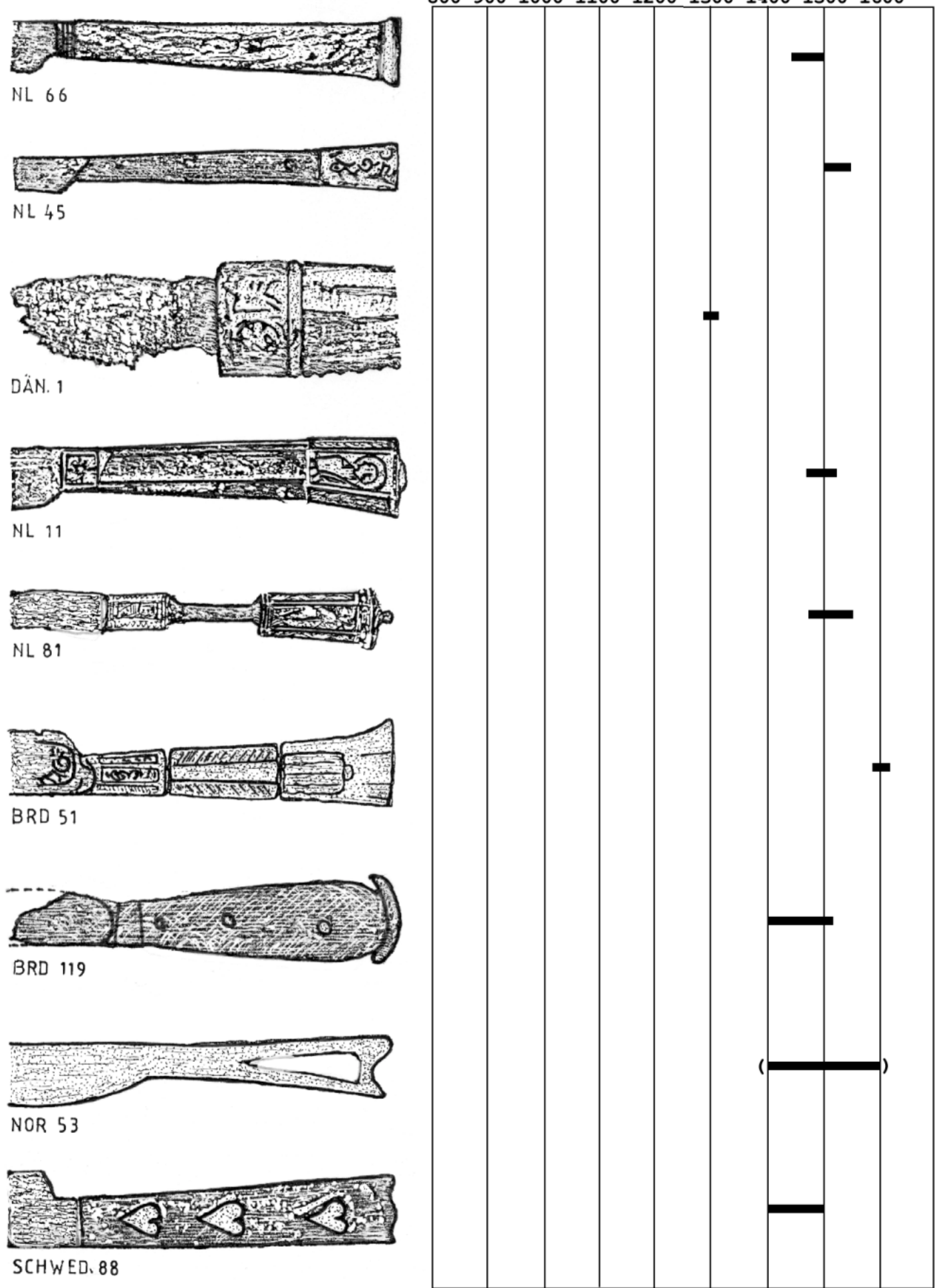

NL 11
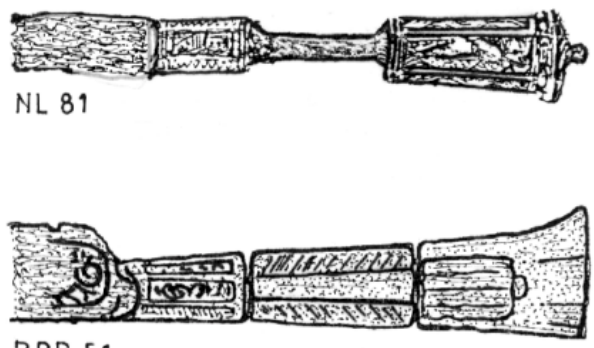

BRD 51

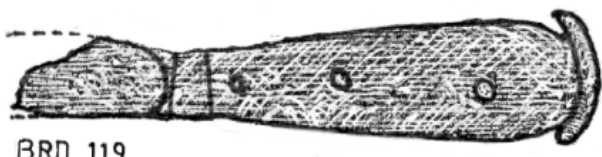

BRD 119

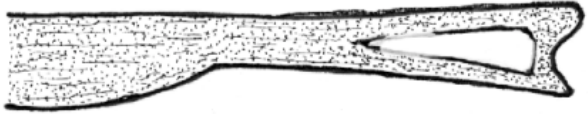

NOR 53

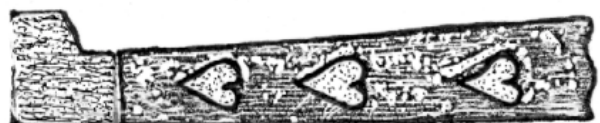

SCHWED、 88 


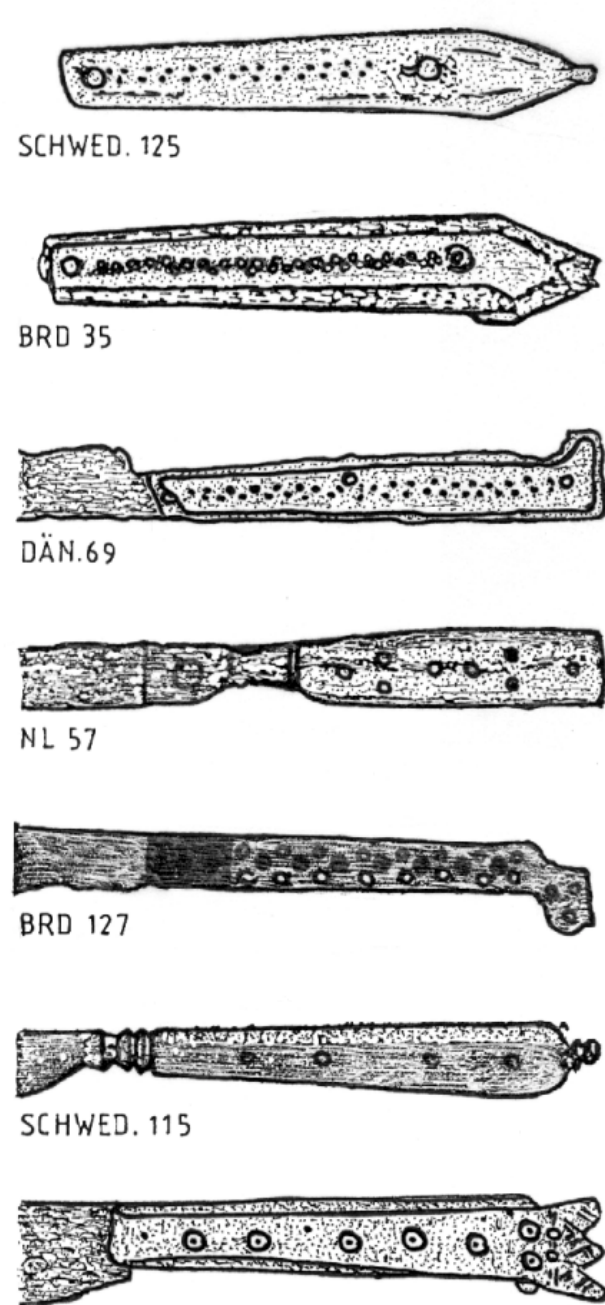

$80090010001100 \quad 12001300140015001600$

BRD 117

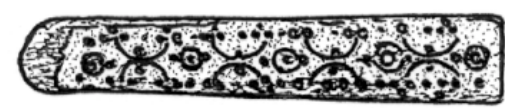

NOR. 30

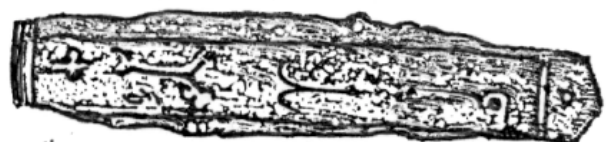

DÄN. 50

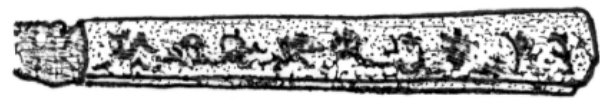

SCHWED. 109

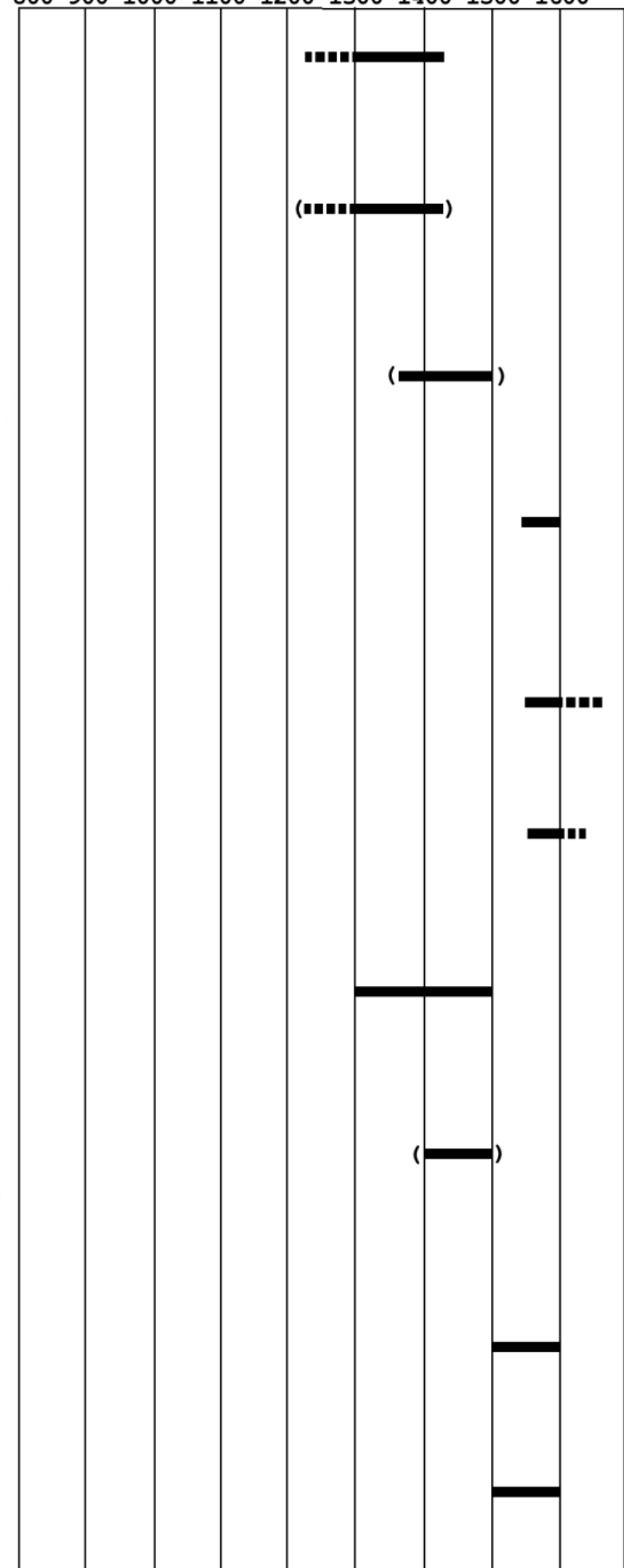




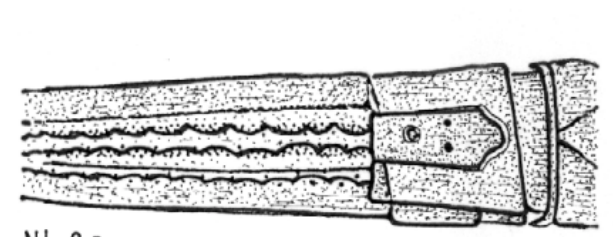

NL Bo

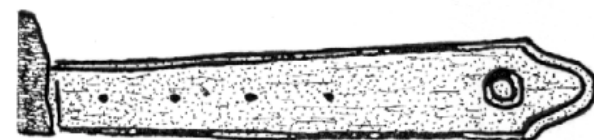

BRD 129

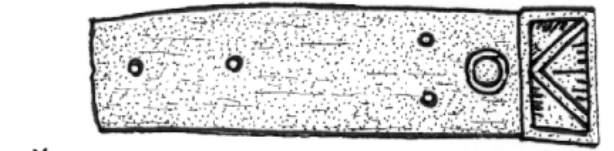
DÄN 86

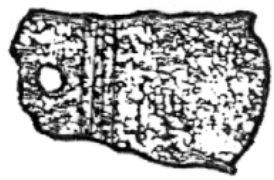

DÄN 83

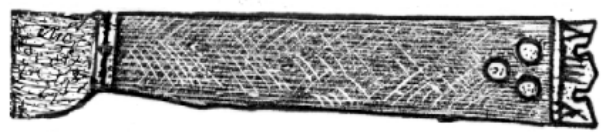

$\triangle \ddot{A N} 3$

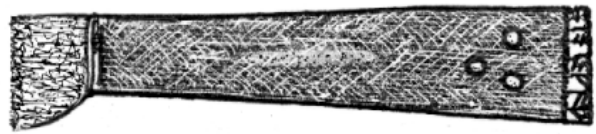

$\triangle \ddot{A N} 2$
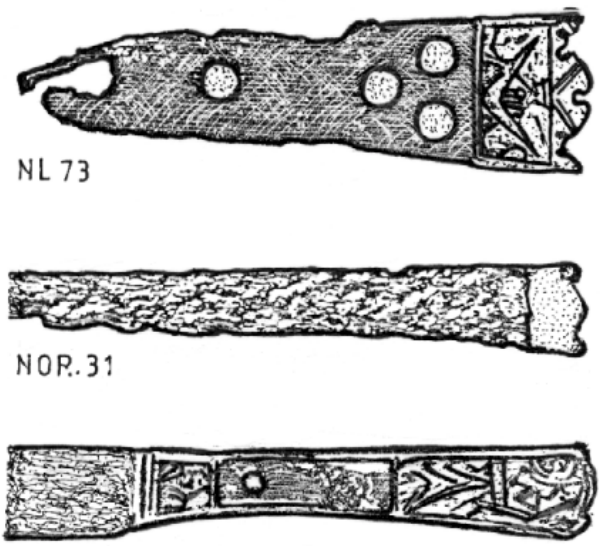

SCHWED. 117

8009001000110012001300140015001600

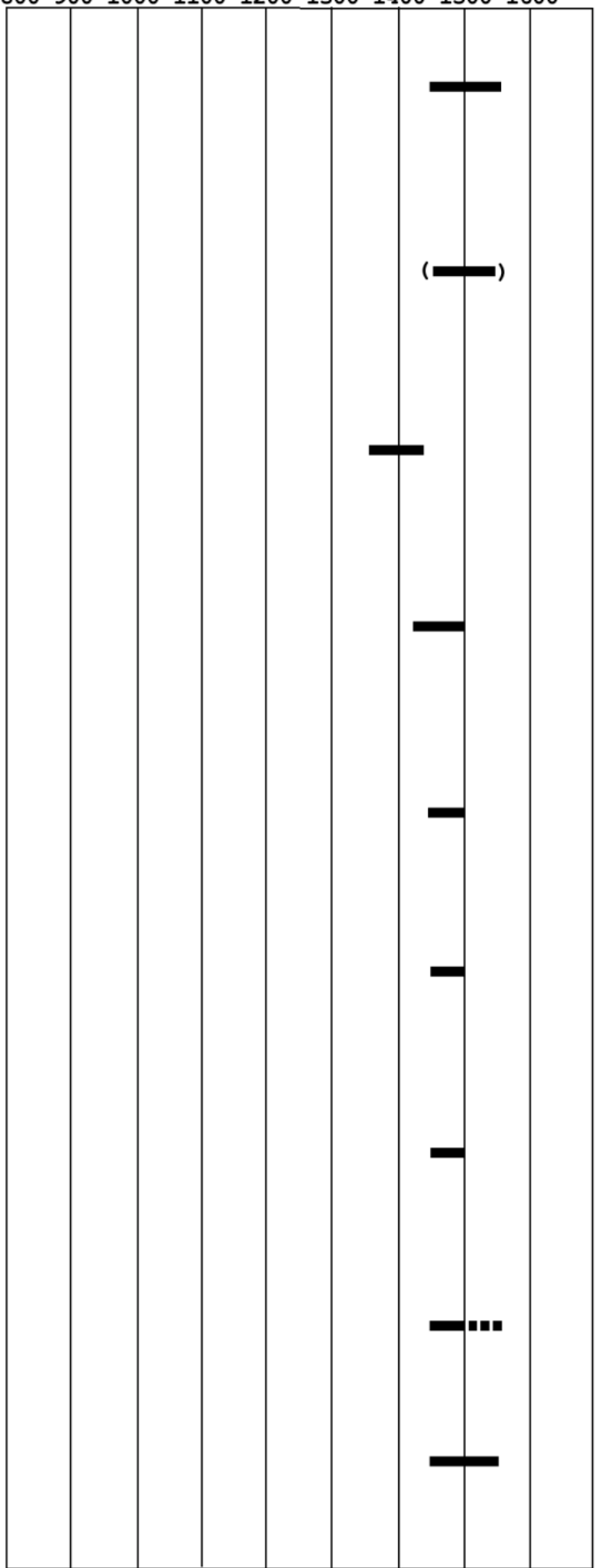




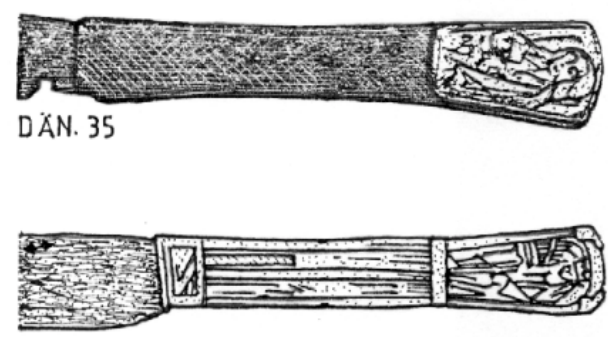

$8009001000 \quad 1100 \quad 1200 \quad 1300 \quad 1400 \quad 1500 \quad 1600$

BRD 34

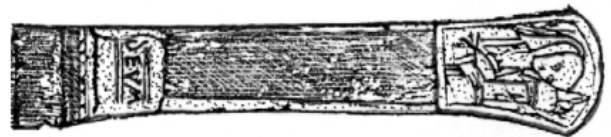

NL 7

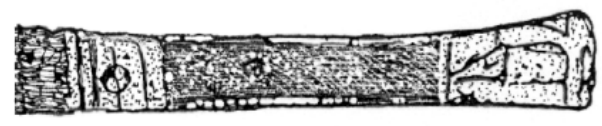

$\mathrm{NL} 80$

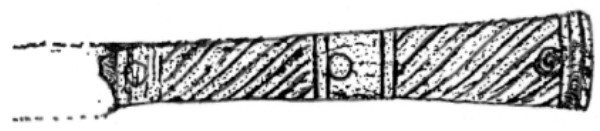

NL. 71

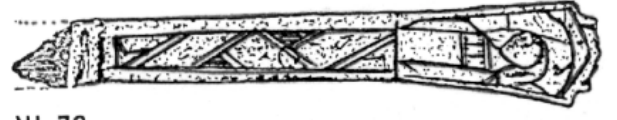

NL 72
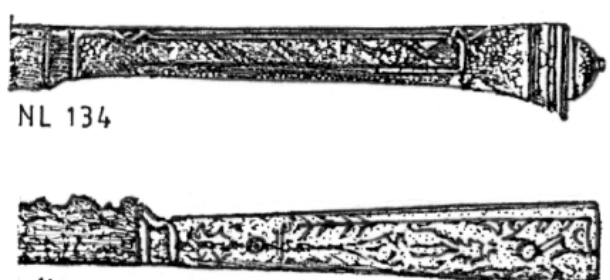

$\square \ddot{A} N 23$

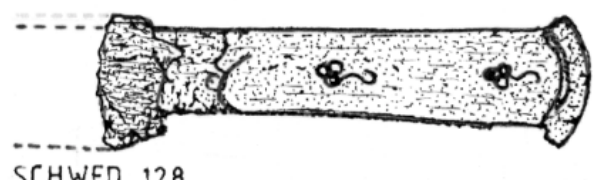

SCHWED. 128

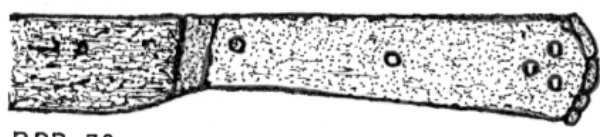

BRD 78

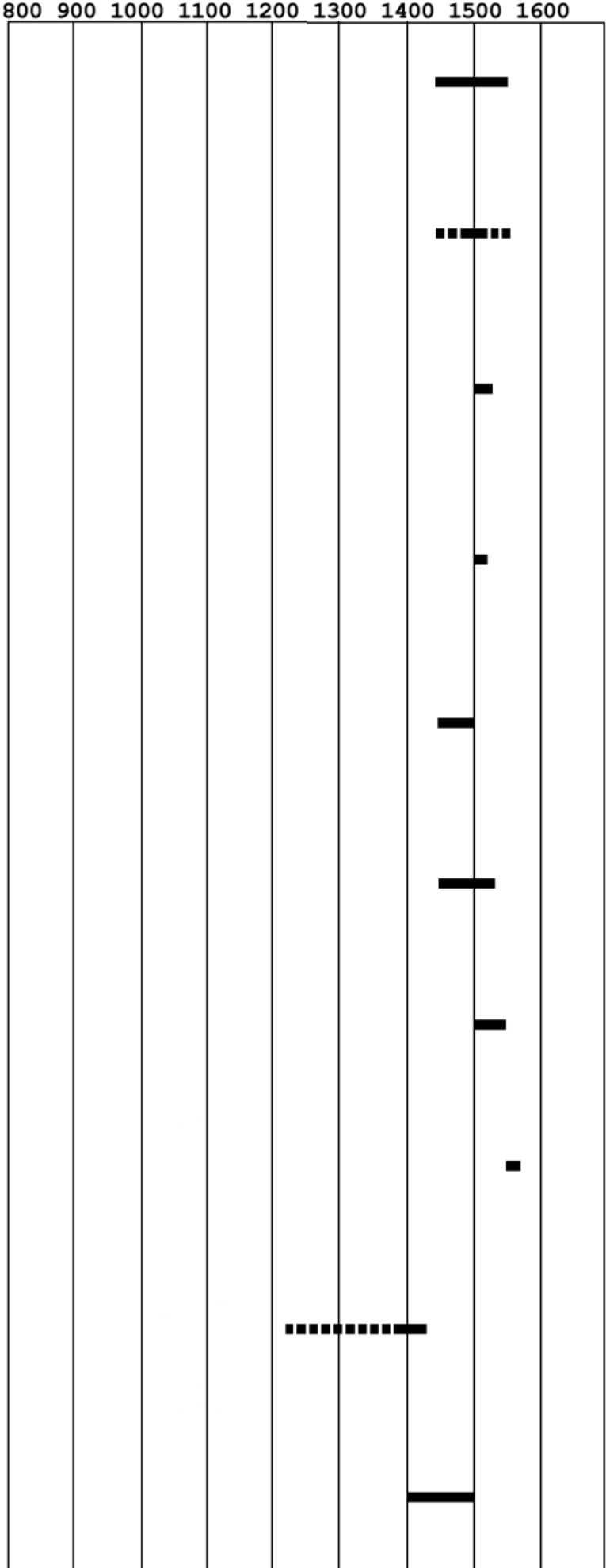




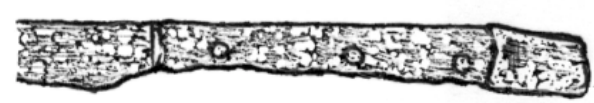

SCHWED. 94

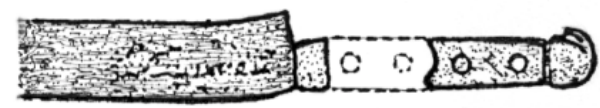

BRD 92

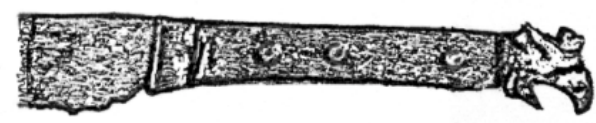

BRD 173

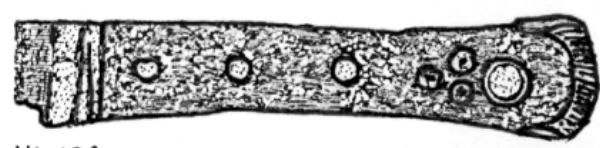

NL 120
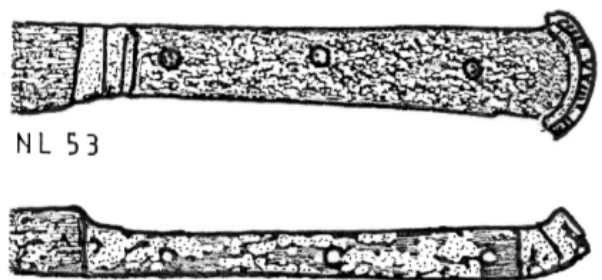

SCHWED. 112

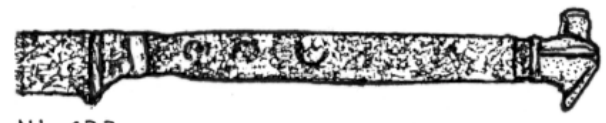

NL 133

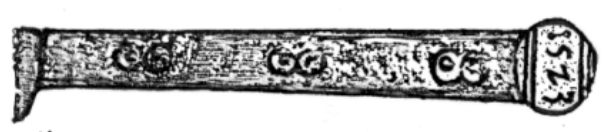

DAN 9 !

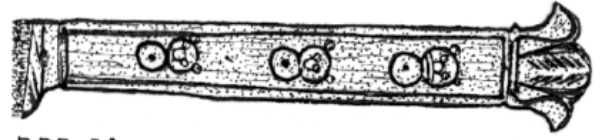

BRD 50
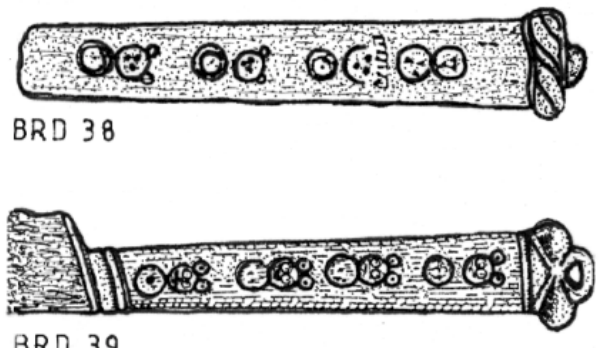

8009001000110012001300140015001600

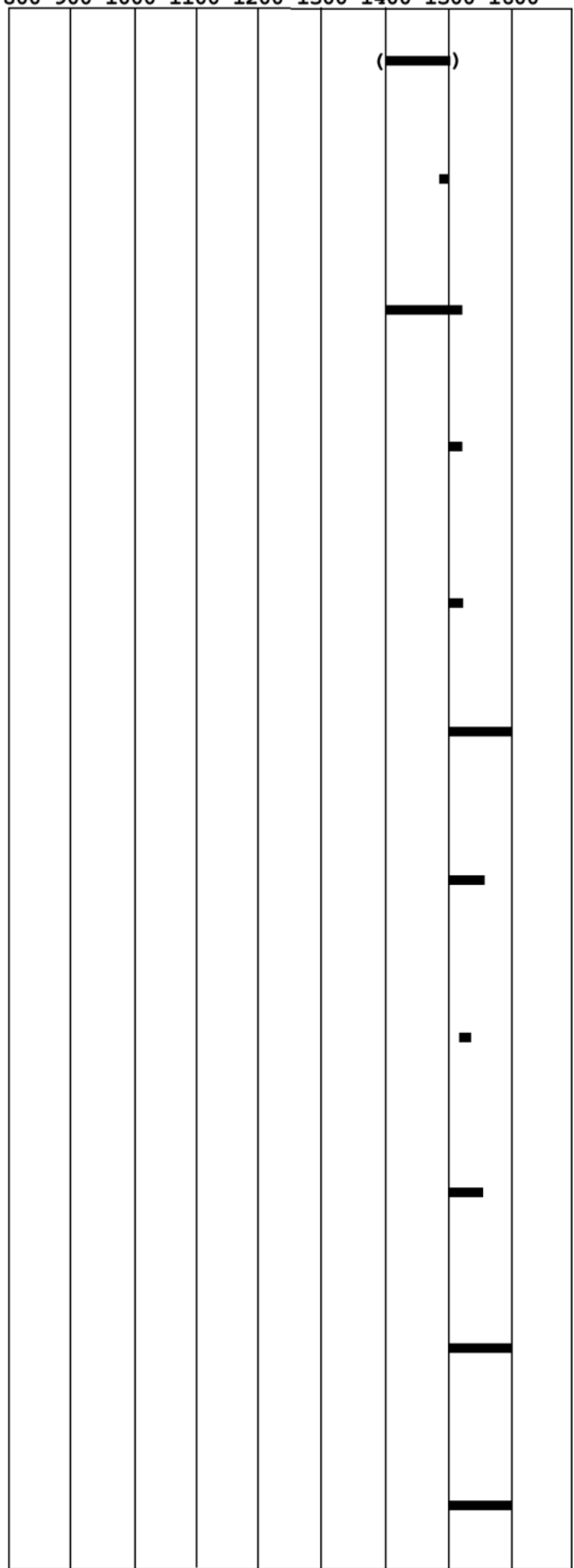




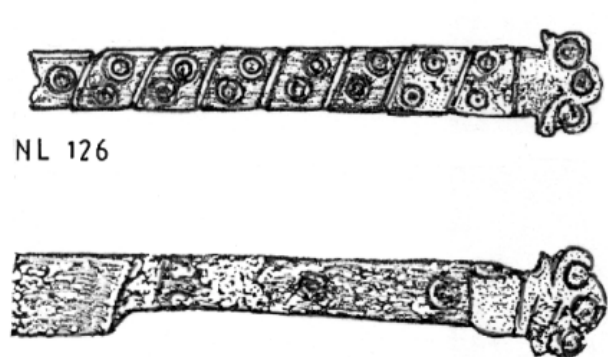

$800 \quad 900 \quad 1000 \quad 1100 \quad 1200 \quad 1300 \quad 1400 \quad 1500 \quad 1600$

NL 51

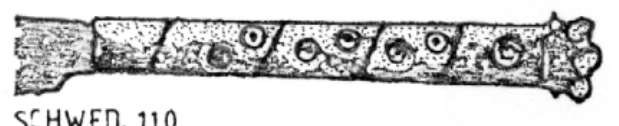

SCHWED. 110

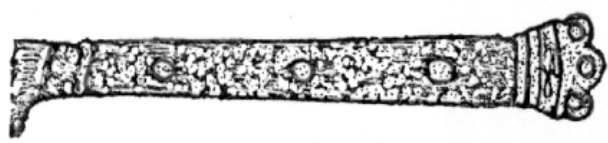

SCHWED. 111

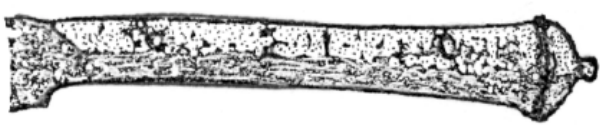

SCHWED. 78

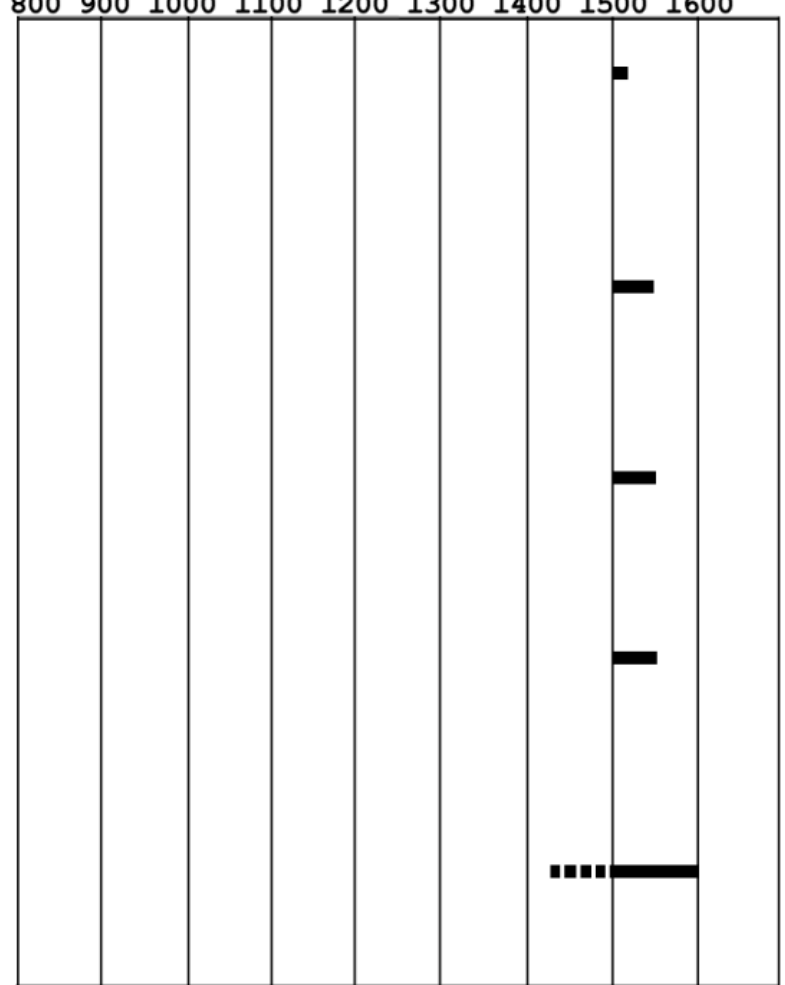

\section{Einzeldarstellungen}

Für alle nach 1400 auftretenden Techniken und Dekorelemente gilt grundsätzlich die Einschränkung, daß von dieser Zeit an in den Publikationen aus der ehemaligen DDR, aus Polen und der ehemaligen nordwestlichen UdSSR keine vergleichbaren Mengen publizierten Materials mehr vorlagen. Die einzige Ausnahme bildeten plättchenverzierte Messergriffe.

Aus diesem Grund wurde bis auf die Griffplättchentechnik auf ausführliche Darstellungen einzelner Metallverzierungsarten verzichtet. Weder waren aus entsprechenden Verbreitungskarten regionale Verteilungen $\mathrm{zu}$ erkennen, noch reichte das erfaßte Material aus, sozial- oder wirtschaftshistorische Fragestellungen zu beantworten.

Die im Folgenden skizzierten Ergebnisse sollen daher auch lediglich den Forschungsstand widergeben, wie er sich aus dem hier erfaßten Fundgut mit allen Einschränkungen ergeben hat. 


\section{Metallene Griffverzierungen an Angelmessern}

Am Beispiel der Verbreitung von Metallgriffen an Angelmessern zeigt sich, daß für die Zeit vor 1400 Eisengriffe, Bunt- bzw. Edelmetallvollgriffe und Mantelbleche auf den nordöstlichen Teil des Untersuchungsgebietes beschränkt sind (Abb. 166 und 167). Tordierte Griffangeln, sowie eiserne und silberne Vollgriffe stammen aus Polen und der ehemaligen DDR, letztere von westslawischen Fundplätzen des 9. bis 12. Jahrhunderts (Burgwall bei "Debia Gora" (Wegry, Sztum), Burgwall BehrenLübchin (Teterow) und frühstädtische Siedlung in Stettin (Schloßberg)) $)^{376}$, erstere aus Hohensalza und Oppeln aus dem beginnenden 14. bzw. 12. Jahrhundert ${ }^{377}$. Mantelbleche sind vor 1400 nur einmal in Birka beobachtet worden, ebenso wie ein tauschierter Eisenvollgriff ${ }^{378}$.

In Lund wurde ein gegossener Vollgriff mit anthropomorpher Griffendgestaltung gefunden, der stilistisch in das Spätmittelalter datiert wird ${ }^{379}$.

Gegossene oder geschmiedete Griffteile vor 1400 sind in Verbindung mit Griffplättchentechnik in Oslo und Höxter als gegossene Buntmetallvollkörper, in Ribe als silbernes Heftvollstück vor einem vollplastisch ausgeschnitzten Falknergriff beobachtet worden ${ }^{380}$.

Geschmiedete Eisengriffe nach 1400 wurden in Lund, Roskilde, Amsterdam und aus der Recknitz (Mecklenburg) geborgen, sie sind alle durch eingeschlagenen linearen Dekor verziert worden. Die sich sehr ähnelnden Griffe werden in das ausgehende 15. und 16. Jahrhundert datiert.

Die Verwendung von Edel- und Buntmetallen (Vollgriffe, Griffhülsen und Applikationen) an Griffangelmessern konzentriert sich auf den niederländischen Raum, die einzigen außerniederländischen Beispiele stammen aus Bremen, Hamburg und Südnorwegen $^{381}$. Sie fallen durchweg in das 16 . bis 17. Jahrhundert.

\section{Metallene Griffverzierungen an Zungenmessern}

Die Verbreitung von nicht vernieteten, sondern vor das Griffende montierten Applikationen aus Buntmetall bei Griffzungenmessern folgt im wesentlichen den publizierten Fundplätzen des 15. bis 16. Jahrhunderts, das heißt, daß Nachweise im östlichen Untersuchungsgebiet fehlen. Im übrigen Gebiet sind sie in Städten wie Amsterdam, Köln, Hamburg, Lübeck, Goslar, Braunschweig und Lund gleichermaßen vertreten, so daß davon ausgegangen werden kann, daß diese Verzierungsart vor allem im 16. Jahrhundert als weit verbreitet angesehen und mit ihrem Erschei-

\footnotetext{
376 Vgl. Katalogteil Polen, Kat. Nr. 1, 85; ehemalige DDR, Kat. Nr. 44

377 Vgl. Katalogteil Polen, Kat. Nr. 173, 340

378 Vgl. Katalogteil Schweden, Kat. Nr. 2, 3

379 Vgl. Katalogteil Schweden, Kat. Nr. 142

380 Vgl. Katalogteil Norwegen, Kat. Nr. 2; BRD, Kat. Nr. 106; Dänemark, Kat. Nr. 1

381 Vgl. u.a. Katalogteil BRD, Kat. Nr. 64, 51; Norwegen, Kat. Nr. 41
} 
nen außerhalb des für diesen Zeitraum faßbaren Gebietes gerechnet werden kann (Abb. 168).

Die Verwendung von gegossenen Platten, die am Heft und am Griffende paarweise vernietet wurden, ist auf das Jahrhundert zwischen 1450 und 1550 beschränkt. In den Fällen, in denen eine stratigraphische Datierungsgrundlage vorlag, verengte sich der Zeitraum auf das ausgehende 15. und beginnende 16. Jahrhundert ${ }^{382}$.

In der Regel sind die Platten aus Buntmetall und mit Gravuren versehen, zum Teil sind sie versilbert worden. Seltener ist die Verwendung von vollständigen, gegossenen Buntmetallschalen. Die frühen Beispiele aus der zweiten Hälfte des 15. Jahrhunderts sind schmale, kronenförmige Bänder oder Platten, die Exemplare um 1500 sind in der Regel halbrund abgeschlossen. Im Verlauf des 16. Jahrhunderts nimmt die Verwendung von Buntmetallschalen stark ab, das einzige Beispiel aus der Mitte des 16. Jahrhunderts aus Randers besitzt bereits einen schlichten, gerade abgeschlossenen Griff, der eher einem Vollgriff ähnelt ${ }^{383}$.

Die Verbreitung dieser Verzierungsart konzentriert sich auf die Niederlande und Südskandinavien, weitere Beispiele wurden in Oslo und in Lübeck gefunden (Abb.169).

Angesichts der Mengen, die sowohl für die Technik der vor das Griffende montierten wie der auf die Zunge vernieteten Buntmetallapplikationen allein in Amsterdam ausgegraben wurden ${ }^{384}$, ist eine weiterführende Interpretation der Verbreitungskarte ohne entsprechendes Vergleichsmaterial unmöglich.

So mag an dieser Stelle der Hinweis genügen, daß angesichts der starken wirtschaftlichen Verbindungen zwischen den niederländischen und skandinavischen Städten im 15. und 16. Jahrhundert das Aufkommen einer zeitlich relativ begrenzten Befestigungs- und Verzierungstechnik in diesem Raum nicht verwundert. Die Handelsverbindungen der Niederlande gingen aber in dieser Zeit erheblich weiter als nur bis Skandinavien, so daß mit Belegen aus weiteren Fundorten durchaus gerechnet werden $\mathrm{mu} \beta^{385}$.

\footnotetext{
382 Vgl. vor allem die in Amsterdam ausgegrabenen Beispiele

383 Vgl. Katalogteil Dänemark, Kat. Nr. 23

384 Die Information und Einsicht in die Bestände verdankt der Autor den Herren J. Baart und A. Lagerweij 385 vom Archäologischen Dienst in Amsterdam

Vgl. u.a. Smolarek 1973 sowie Pagel 1983, S. 235-236
} 
Verbreitungskarte

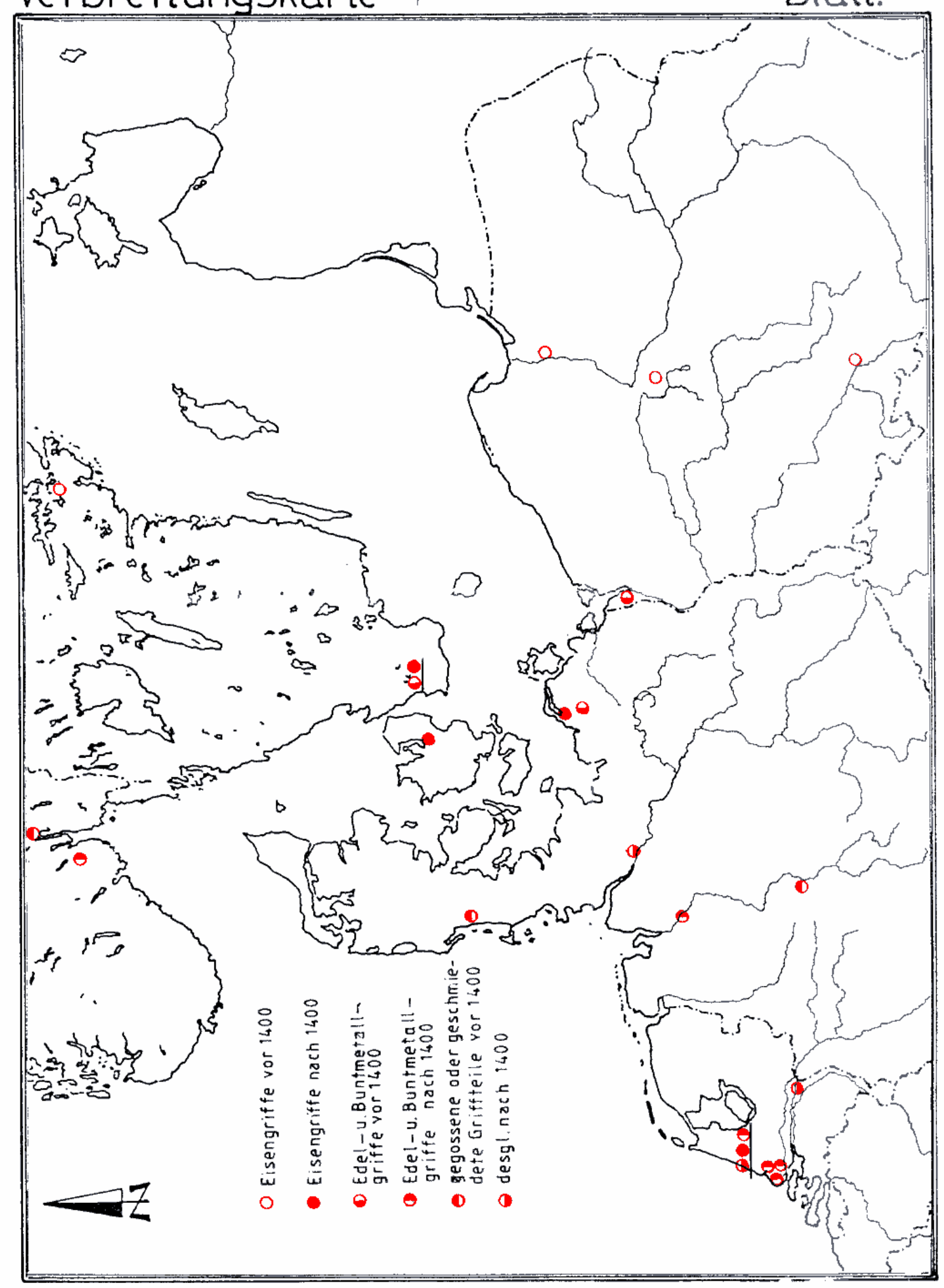

A.bb. 160 Die Verbreitung von Metallgriffen und-griffteilen an Griffangelmessern 


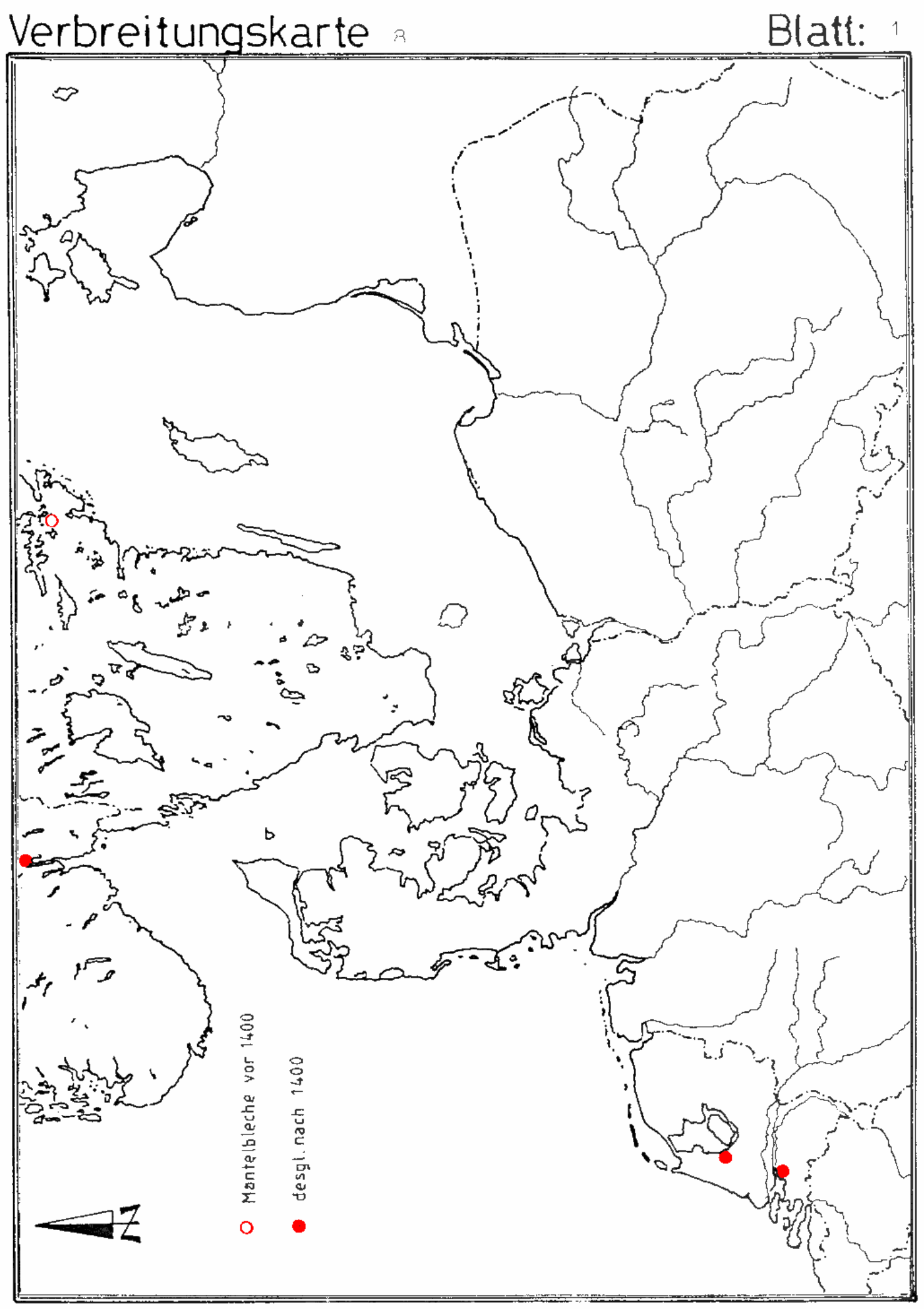

Abb. 107 Die Verbreitung von Mantelblechen an Griffangelmessern 
Verbreitungskarte.

Blatt:

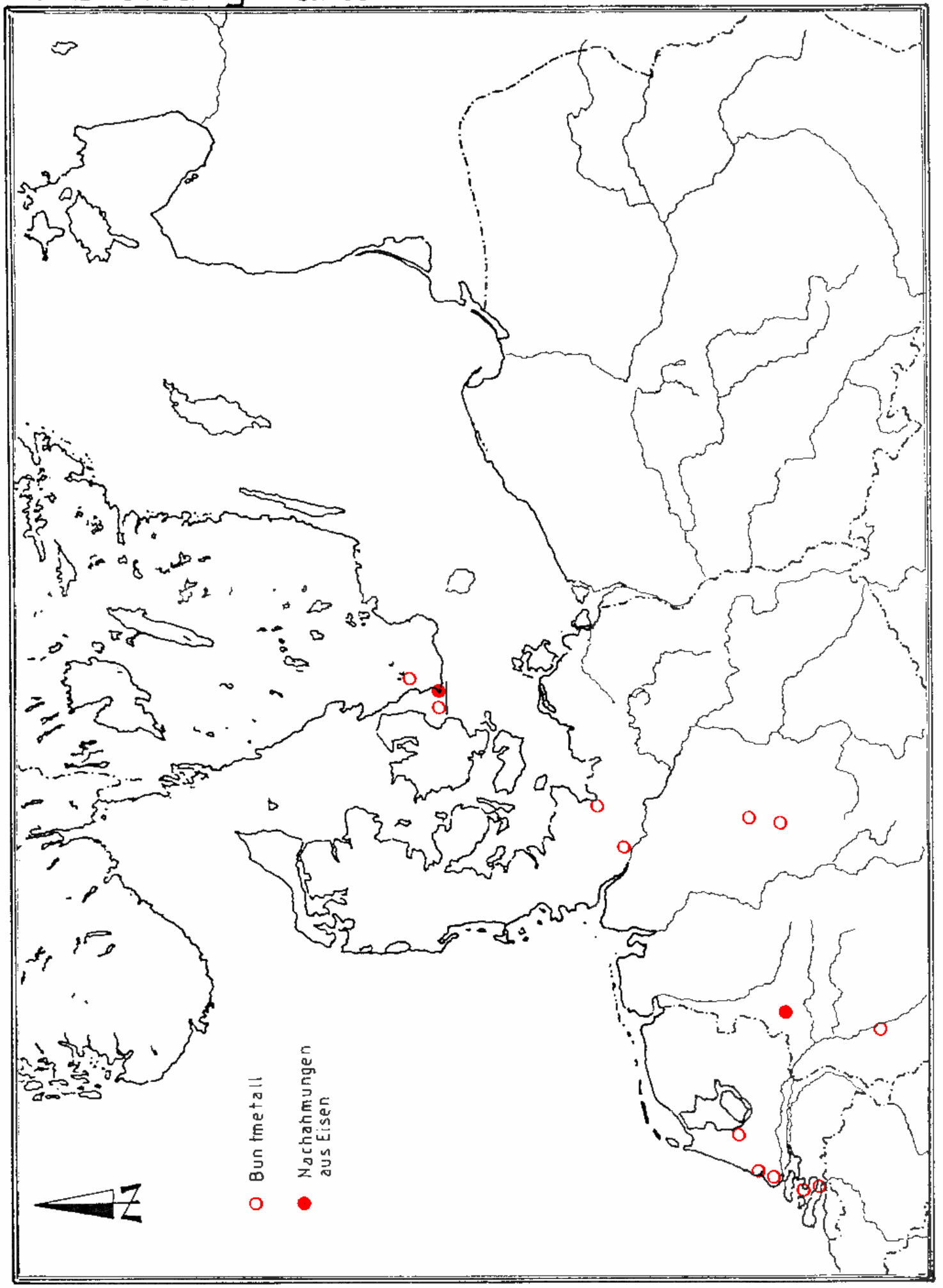

Abb. 168 Die Verbreitung von nicht vernieteten, gegossenen Griffendapplikationen im 15. und 16 . Jahrhundert 


\section{Verbreitungskarte}

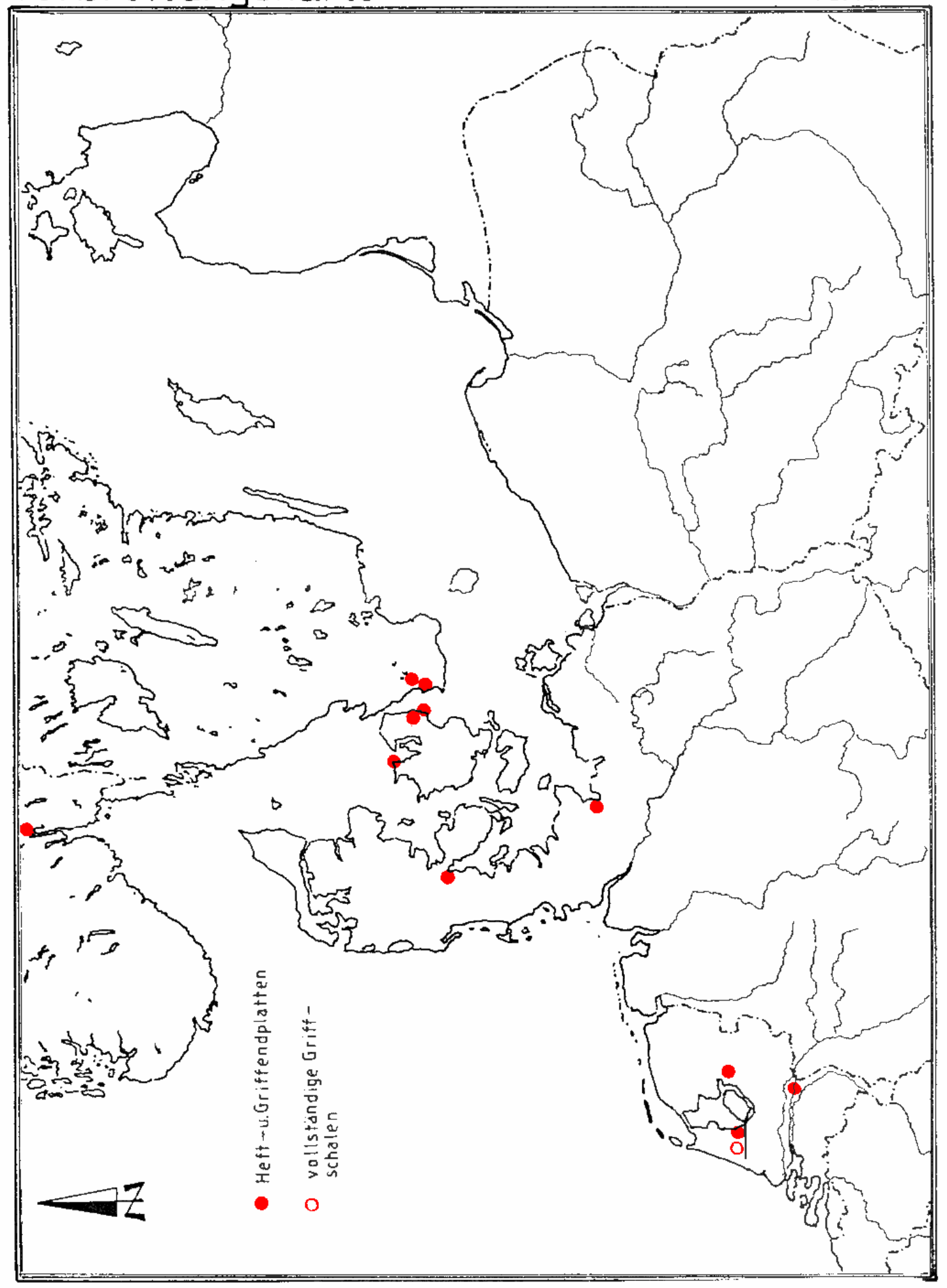

Abb. 169 Die Verbreitung vernieteter und durch Gravuren verzierter Griffplatten und -schalen aus Buntmetall im 15. und 16. Jahrhundert 


\subsection{Die G riffplättchentechnik}

In der Gruppe der Angelmesser, deren Vollgriffe durch Zusatzbefestigungen gesichert worden sind (Form A2), fallen die sogenannten Griffplättchenmesser wegen ihrer aufwendigen Verarbeitung und ihrer optischen Erscheinung besonders auf.

Die zumeist aus organischem Material bestehenden Vollgriffe wurden am Heft und am Griffende mit einzelnen oder mehreren Plättchen aus unterschiedlichen Materialien, in der Regel aus Bronze- oder Messingblech, zwingenartig eingefaßt.

Diese Technik weist an den im Bestand dieser Untersuchung erfaßten Messern aber eine derart große Variationsbreite auf, daß eine detailliertere Betrachtung angebracht erscheint.

Aufgrund ihrer auffälligen Erscheinung ist die Griffplättchentechnik bereits mehrfach in der Literatur behandelt worden. ${ }^{386}$

Vor allem H.A. Knorr hat sie in einem Aufsatz, der auch Stichwaffen mit plättchenverzierten Griffen umfaßt, gesondert behandelt. Er unterscheidet unter anderem plättchenversehene Messer mit geradem und gebogenem Rücken, trennt die Verzierungsabsicht durch mehrere Plättchenlagen von der Befestigungskonstruktion am Heft und am Griffende und stellt mit zahlreichen Beispielen Plättchen verschiedener Formen und Plättchenlagen (-pakete) aus Plättchen verschiedener Materialien (neben Buntmetall Horn, Leder, Blei etc.) vor ${ }^{387}$. Im wesentlichen datiert Knorr die betreffenden Messer in das 14. bis 15. Jahrhundert und skizziert in einer Verbreitungskarte den Raum ihres verstärkten Vorkommens zwischen Elbe und Oder nördlich der Linie Magdeburg-Frankfurt/Oder, weist aber auch auf zwei frühere Beispiele aus Beuthen und Breslau sowie auf skandinavische Beispiele hin.

Innerhalb des hier erfaßten Bestandes konnte an 47 Exemplaren die Verwendung von Griffplättchen nachgewiesen werden. Dazu kommen zwei Messer von der Burg Schartenberg bei Kassel, einzelne Griffplättchen aus der weiteren Umgebung von Kassel und ein von Knorr publizierter Messerrest von der Wiprechtsburg bei Groitzsch, die nicht im Katalog enthalten sind, sowie einzelne Griffplättchen von der Burg Naesholm in Schweden ${ }^{388}$.

Grundsätzlich ist die reine Befestigungstechnik von der Verwendung von Griffplättchen zu Verzierungszwecken zu unterscheiden.

Als Konstruktionselemente sichern Stichplättchen den Vollgriff am Heft und am Griffende.

Bereits hier können mehrere Plättchen aus Messingblech hintereinander angeordnet sein.

\footnotetext{
386 Ullmann 1961

Knorr 1971

387 Knorr 1971, S. 122-126; Abb. 3, S. 127

388 Die Messer und Griffplättchen vom Schartenberg und aus der weiteren Umgebung von Kassel wurden dem Autor vom Finder zur Verfügung gestellt, die Griffplättchen von Naesholm sind im Katalogteil Dänemark unter den Katalognummern 31-33 erfaßt worden.
} 
Griffplättchenverzierte Griffe weisen am Heft und Griffende Pakete von Griffplättchen auf, die ursprünglich auch farbig voneinander abgesetzt sein konnten. Plättchen aus Messing, Zinn/Blei, Knochen, Horn, Leder oder Holz wurden in alternierender Abfolge auf die Angel geschlagen. Bei einigen Griffen traten Griffplättchenpakete auf, die mit Vollgriffsegmenten aus Buntmetall oder organischen Materialien wechselten.

In der Regel sind die hinteren Bereiche der Griffangel abgebrochen und die organischen Bestandteile der Griffe vergangen, so daß die Bandbreite der Griffplättchenverzierung nur fragmentarisch dokumentiert werden kann.

\subsection{Die erfaßten Griffplättchenmesser}

Die Verbreitung griffplättchenversehener Messer umfaßt 37 Fundorte, die sich im Untersuchungsraum auf Burgen der nördlichen deutschen Mittelgebirgszone und auf Städte im östlichen Kernraum der Hanse konzentrieren. Eine Streuung liegt in den Zwischenregionen und in Südskandinavien vor, außerhalb dieses Raumes stammen Einzelbeispiele aus Oslo, Novgorod, Kalisch, Breslau und Beuthen (Abb. 170).

Der zeitliche Rahmen, in dem die Verwendung von Griffplättchen als Technik und Verzierungselement sicher nachzuweisen ist, reicht vom 12. bis zum 15. Jahrhundert.

Die Masse der sicher in das 13. Jahrhundert zu datierenden Messer wurde auf den Burgen Alt-Schieder, Isenburg, Raffenburg, Rücklenburg und Wartenberg gefunden, weitere analoge Beispiele stammen als Lesefunde von den Burgen Altena und Schartenberg (Abb. 172b-172d, 172g, Wartenberg nicht abgebildet ${ }^{389}$, a, e; Abb. 173j; Abb. 174f; Abb. 175g und 175h). Das Messer aus Oslo aus der zweiten Hälfte des 12. Jahrhunderts findet mit seinen Metallsegmenten eine Parallele in einem in Höxter geborgenen Messer des ausgehenden 12. bis beginnenden 14. Jahrhunderts (Abb. 174a, 174b). Weitere Beispiele des 13. Jahrhunderts liegen vom Innenhafen von Duisburg und möglicherweise von der 1256 zerstörten Mecklenburg vor (Abb. 172i, 175f). Schließlich sind ein Flußfund aus der Recknitz bei Plummendorf und ein Moorfund aus der Umgebung von Levetzow-Lübow (Abb. 175c, 172k) zu nennen. Die Datierung der letzten drei Beispiele ist umstritĐans Mecklenburger Messer ist stratigraphisch um $1000 \mathrm{n}$. Chr. datiert, diese Datierung muß aus zwei Gründen hinterfragt werden. Zum einen fällt das Messer (Katalogteil ehemalige DDR, Kat. Nr. 6) aus dem Rahmen der übrigen, durchweg spätslawischen Messer heraus, zum anderen ist die Technik der Griffplättchen bis jetzt nirgendwo vor Mitte bis Ende des 12. Jahrhunderts nachweisbar. Da die Mecklenburg mindestens bis in das 13. Jahrhundert hinein bestanden hat, mögen Zweifel begründet erscheinen.

389 Zu dem griffplättchenversehenen Messer von der Burg Wartenberg in Oberhessen (1225-1265) vgl.: Die Zeit der Staufer 1977, Bd. II (Katalog), Kat. Nr. 263, Abb. 139 
Das Exemplar aus einem mit Waffen des 12. bis 13. Jahrhunderts gefüllten Holzkasten aus einem Moor bei Levetzow-Lübow wurde aufgrund der Datierung eines Sporns in das 15. Jahrhundert aus dem Datierungsrahmen der Waffen herausgenommen $^{390}$, aufgrund der unsicheren Fundumstände (s. Katalogteil ehemalige DDR, Kat. Nr. 16) kann lediglich angeführt werden, daß sowohl die Form des Messers als auch die Technik und Form der Griffplättchen eine Datierung in das 13. Jahrhundert nicht ausschließen.

Das letzte Messer wurde bei Plummendorf aus der Recknitz gebaggert und nach Schoknechts Angaben über den Fund von Levetzow in das 12. bis 13. Jahrhundert datiert $^{391}$. Die Griffkonstruktion ist aus dem 13. Jahrhundert mehrfach belegt, so daß das Messer auch im Vergleich zu den Exemplaren von der Wiprechtsburg und der Burg bei Manebach möglicherweise noch vor das 14. Jahrhundert datiert werden kann (Abb. 175a bis 175c).

Neben die beiden Messer aus Höxter und Oslo tritt ein weiteres Exemplar aus Beuthen (Abb. 173f), welches stratigraphisch in das 12. bis 13. Jahrhundert datiert wurde (Katalogteil Polen, Kat. Nr. 348). Die verwendeten acht Griffplättchen wurden aus dem Blech einer sogenannten Hanseschale geschnitten, was vor dem Hintergrund des frühen Datierungsansatzes den Importcharakter des Messers wahrscheinlich macht. Ebenfalls aus dem 12. bis frühen 13. Jahrhundert stammt ein weiteres Messer aus Breslau (Abb. 174d), welches jedoch keine Buntmetallplättchen aufweist (Katalogteil Polen, Kat. Nr. 315).

Es fällt auf, daß die frühesten Beispiele der Griffplättchentechnik Messer betreffen, die in unterschiedlicher Art diese Technik bereits durch Segmentierung des Griffs als Verzierungselement belegen.

Weitere Beispiele des 13. bis 14. Jahrhunderts stammen von der Wiprechtsburg (vor 1307), der Siedlung in Küsel, Kreis Burg (vor 1308), der Hildagsburg (wohl nach 1289), der Burg bei Manebach (14. Jahrhundert) und aus Minden (um 1300) (Abb. 175b, 175 1; 174g; 172f; 175a; 173 1).

Aus dem nordöstlichen Bereich des Untersuchungsgebietes stammen zwei Messer aus Stargard und eine ganze Serie von griffplättchenverzierten Messern aus Anklam; aus dem östlichen Randbereich begegnet ein Exemplar aus Kalisch.

Leider sind die letzten Beispiele nicht ausreichend eng zu datieren. Das Messer aus Kalisch, in den Vorberichten zu den Stadtkernuntersuchungen in das 11. bis 12. Jahrhundert datiert, ist wahrscheinlich jünger ${ }^{392}$.

Die beiden hier nicht abgebildeten Messer aus Stargard (Katalogteil Polen, Kat. Nr. 8 und 9), in das frühe 14. Jahrhundert datiert, sind nicht eindeutig genug als griffplättchenversehene Stücke zu erkennen.

\footnotetext{
390 Knorr 1971, S. 126 und Anmerkung 12; U. Schoknecht, Ein Fund mittelalterlicher Waffen von Levetzow, Kr. Wismar, in: Bod. Meck.-1967, Rostock 1969, S. 283 ff. 
Die große Anzahl hervorragend erhaltener Messer aus dem Stadtkern von Anklam sind aufgrund keramischer Beifunde in das 13. bis 14. Jahrhundert datiert worden (Abb. 172h, 172j, 1721; 173b, 173c, 173h; 174c).

Die Verzierungen an den Griffen der Messer aus Minden und Anklam betreffen entgegen den früheren Beispielen aus städtischem Umfeld nur noch den Heft- und Griffendbereich, an denen kleine Pakete von alternierend angebrachten Plättchen aus Metall und organischen Materialien den hölzernen oder beinernen Vollgriff einfassen. Wahrscheinlich haben beide Ausführungen nebeneinander bestanden, denn ein Messer des 14. Jahrhunderts aus Lübeck (Abb. 173e) zeigt ebenfalls einen durchgehend segmentierten Griff.

Weitere Beispiele des 13. bis 14. Jahrhunderts stammen aus der Siedlung Glasbach und von der Burg Gommerstedt (Abb. 172m, 172n; 175e) sowie von der Burg Naesholm (ca. 1240 bis 1340; Abb. 173i; 175i, 175k) auf Seeland.

Im Verlauf des 14. und 15. Jahrhunderts scheint der Nachweis griffplättchenversehener Griffe auf das nördliche Konzentrationsgebiet, einem ehemaligen Kernraum der Hanse, beschränkt zu sein. Ist aus der zweiten Hälfte des 14. Jahrhunderts neben Funden aus Kambs, Kreis Schwerin und Winsen an der Luhe noch ein griffplättchenverziertes Messer aus Frankfurt an der Oder nachzuweisen (Abb. 173d, 173k; 174e), so stammen die entsprechenden Messer des 14. bis 15. und 15. Jahrhunderts aus Lübeck, Demmin und von der Burg Wildberg, Kreis Neuruppin (Abb. 173a, 173e, 173g).

Mit diesen letzten drei Messern scheint sich der Zeitraum des beginnenden 15 . Jahrhunderts als die Zeit zu dokumentieren, in der die Modeerscheinung der Griffplättchenverzierung ausläuft.

Daß selbst in dieser Zeit an den alten Randzonen des hansischen Wirtschaftsraumes Messer dieser Art noch vereinzelt auftreten können, belegt das Exemplar aus Novgorod (Abb. 175d).

Die Verwendung von Griffplättchen ist allerdings noch bis in das 16. Jahrhundert vereinzelt nachzuweisen. An Beispielen aus Lund, Lübeck und Hamburg ${ }^{393}$ zeigt sich, daß die Griffplättchen in der Zeit nach der Mitte des 15. Jahrhunderts nur noch Funktionen als Zusatzsicherungen für den Vollgriff an Heft- und Griffende besitzen, beim Messer aus Hamburg (Ende des 16. Jahrhunderts) wurde ein Messingplättchen auf der Abschlußfläche der Griffendhülse aus Eisen verlötet.

Selten sind griffplättchenähnliche Zwingen oder auch Griffplättchen ganz oder zum größten Teil aus organischen Materialien. Neben dem bereits erwähnten Messer aus Breslau (Abb. 174d) aus dem 12. bis frühen 13. Jahrhundert wurden auf der Burg in Skanör (Schonen) neben einem dicken Plättchen aus Blei ein Knochenplättchen sowie ein Messer mit zwei runden Knochenscheiben gefunden (13. bis Anfang 15. Jahrhundert, Abb. 175p, 175r). Knorr erwähnt zu Knochenzwingen auf Griffangelmessern slawische Parallelen ${ }^{394}$.

393 Vgl. Katalogteil Schweden, Kat. Nr. 100 (2. H. 15. Jh.); BRD, Kat. Nr. 36 (2. H. 15. Jh.), 51 (E. 16. Jh.) 


\section{Verbreitungskarte}

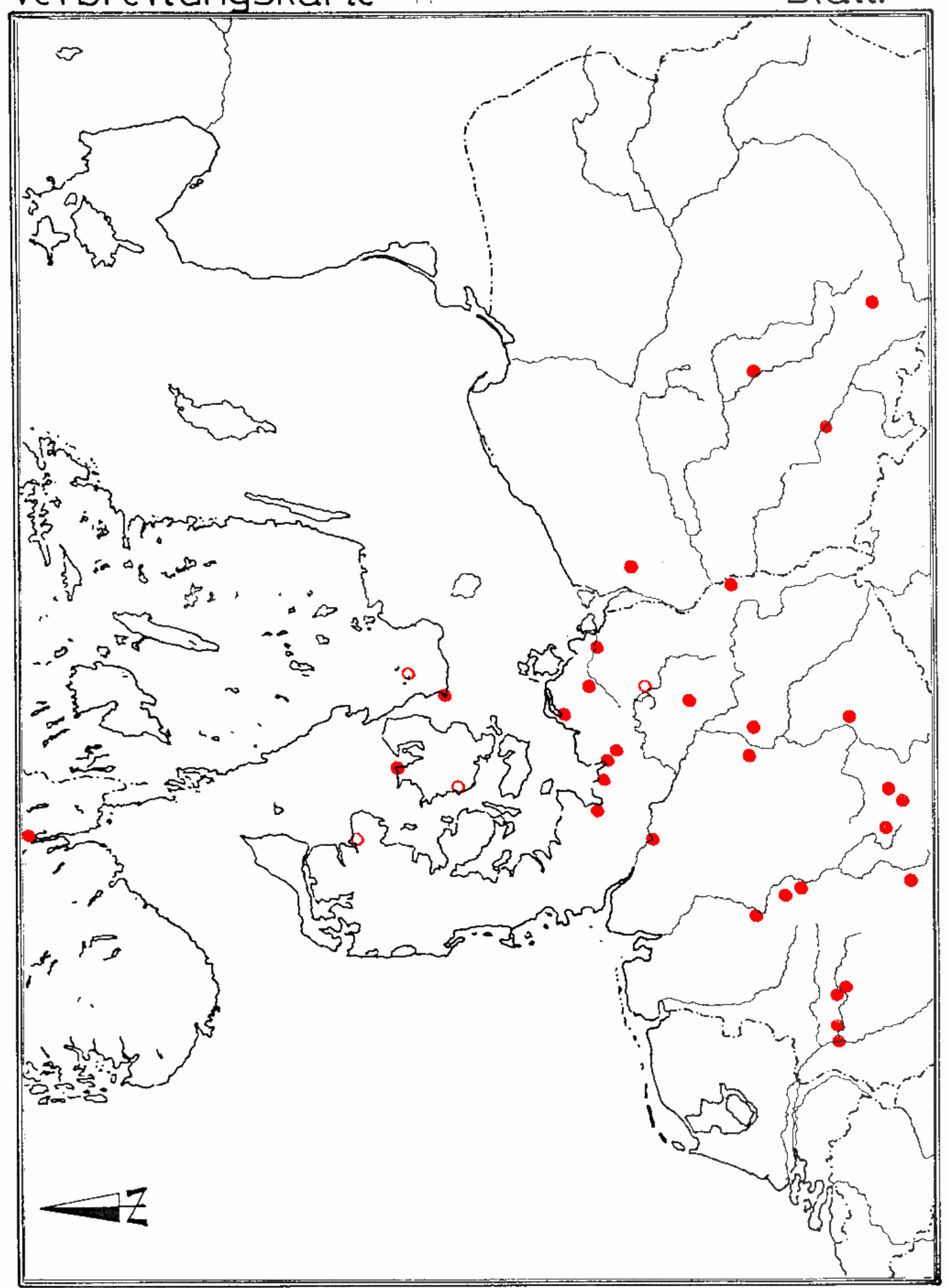

Abb. 170

Die Verbreitung griffplättchenversehener Messer mit den nicht im Katalog enthaltenen funden (offene signatur) aus Allingåbro (Jütland) der Umgebung von Tralleborg (Seeland), Svenstrop (Schonen) und Weisdin (Neustrelitz), Knorr 1971 
Verbreitungskarte

12

Blatt:

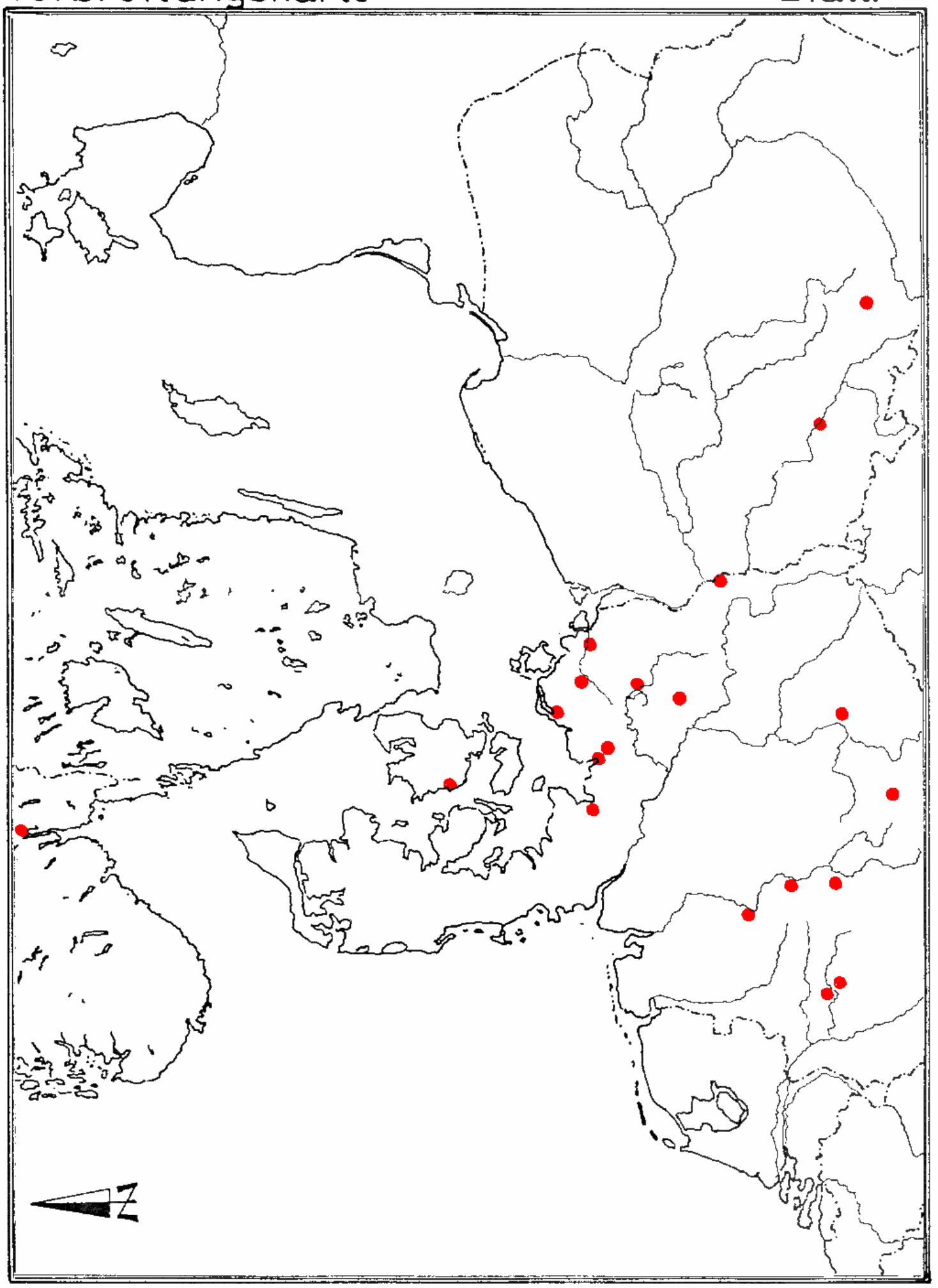

Abb. 171 


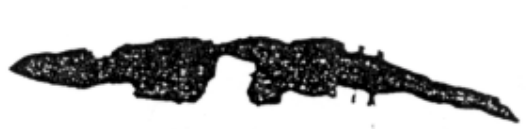

a 可

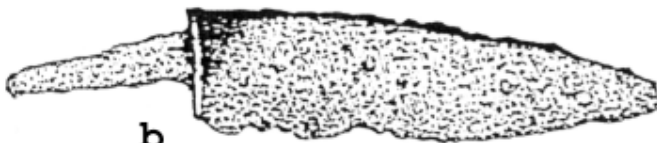

b
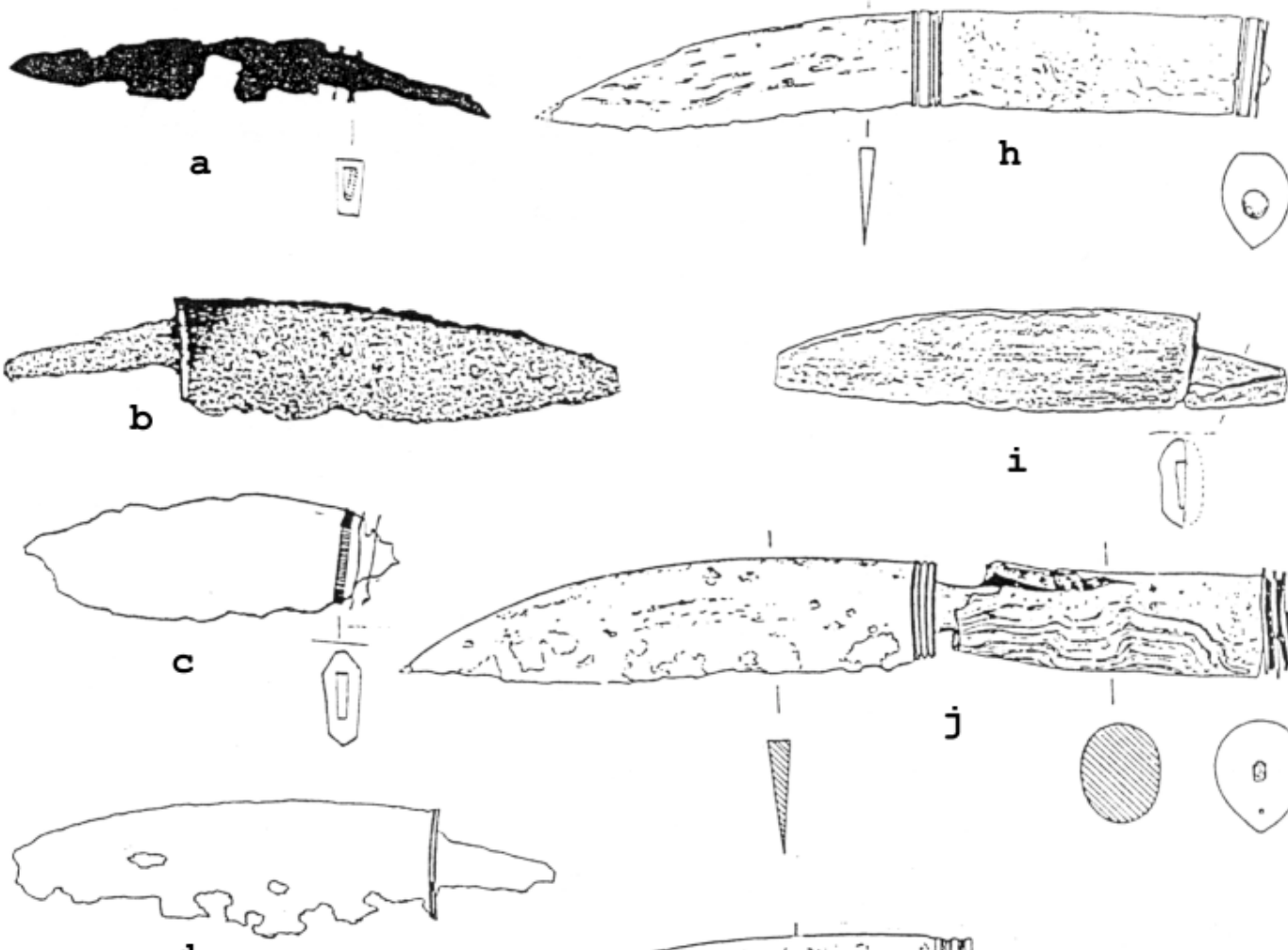

i
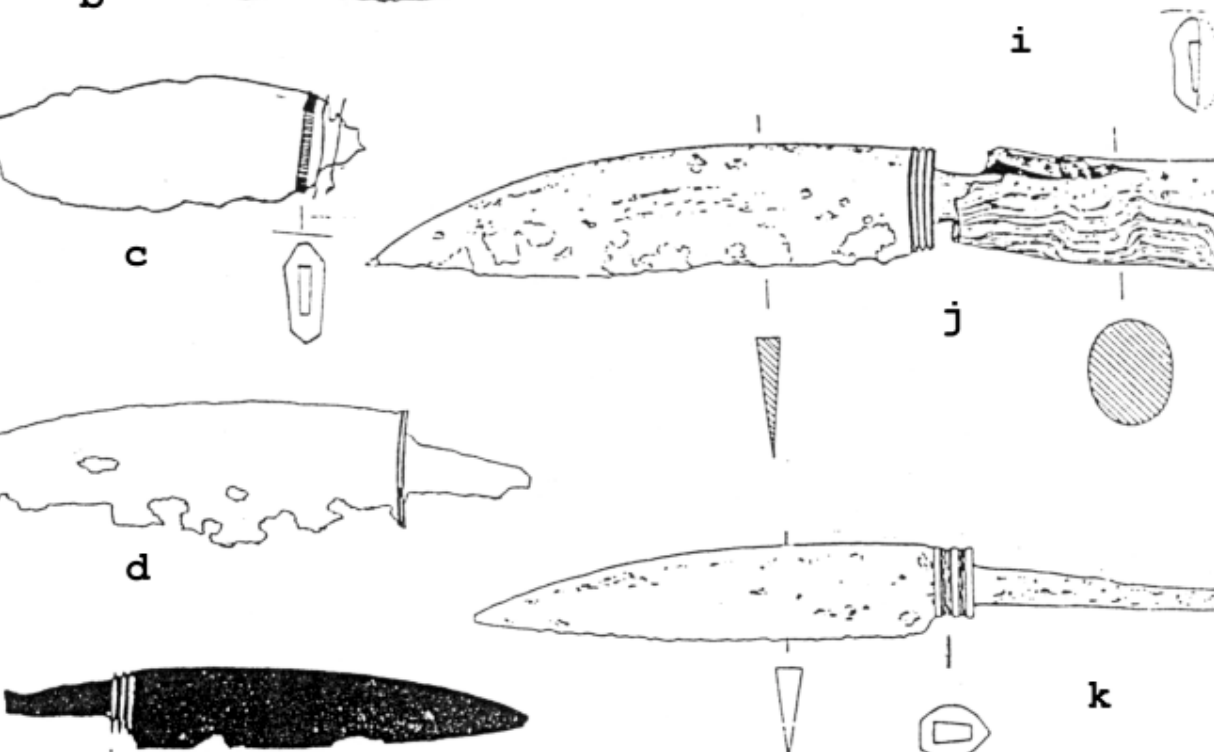

j

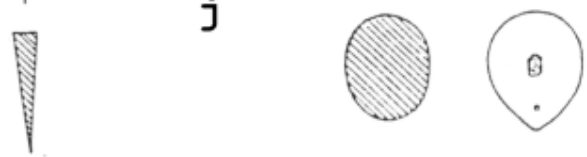

(6) $e$

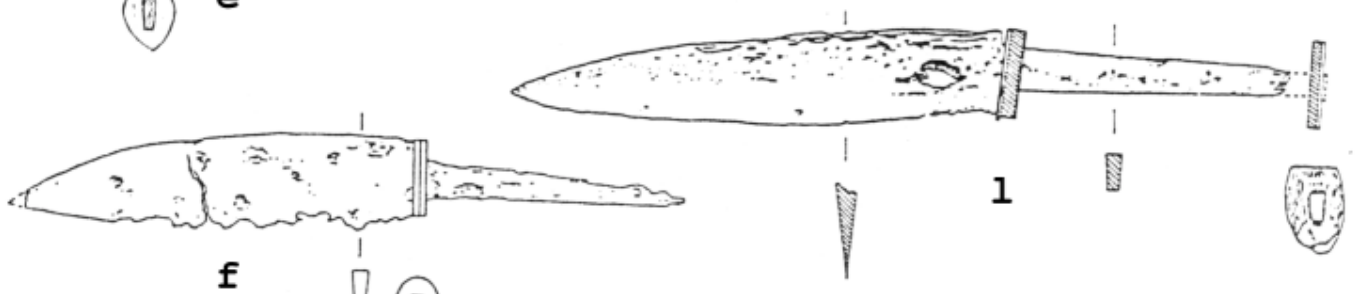

$\mathbf{f}$

(0)

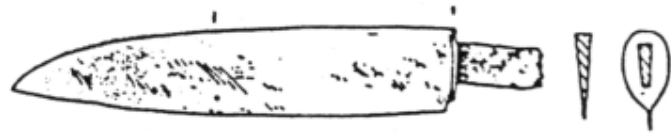

g

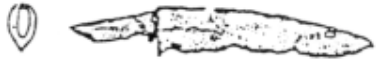

m

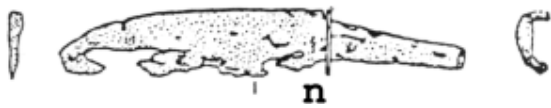

Abb. 172 Griffplättchenversehene Messer mit der Klingenform III b (13. bis 14. Jahrhundert) a: Burg Alrena; b: Rücklenburg; c-d: Raffenburg; e: Burg Schartenberg, $f$ : Hildagsburg; g: Burg in Alt-Schieder; h, j, l: Anklam; $i$ : Duisburg; $k$ : LübowLevetzov; $m, n$ : Glasbach 

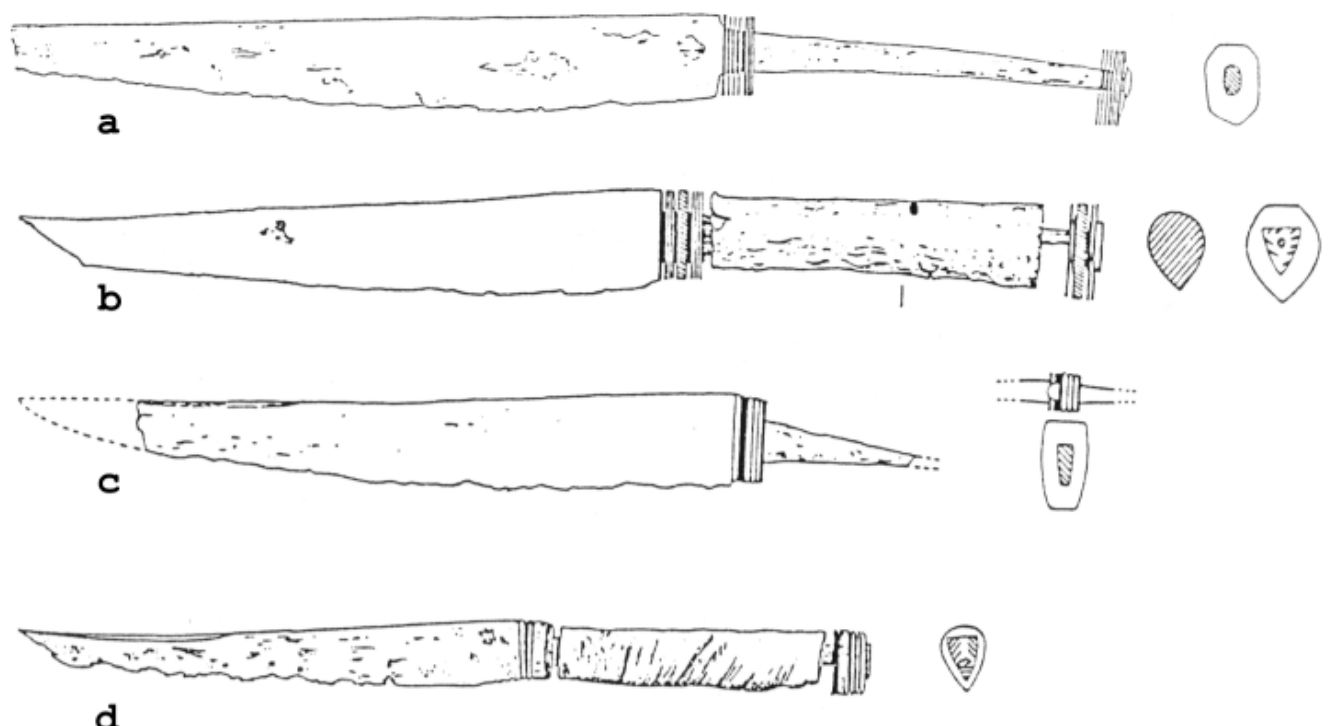

d
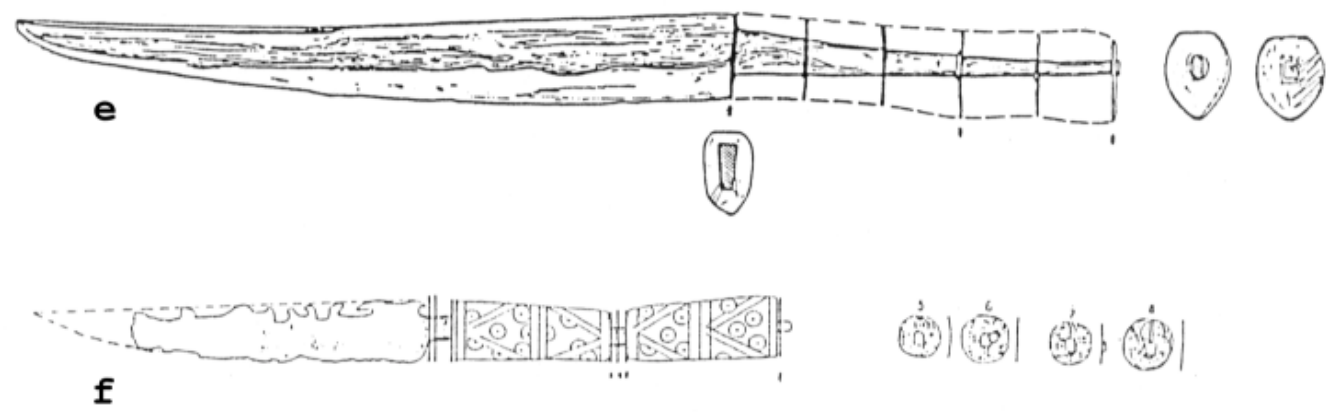

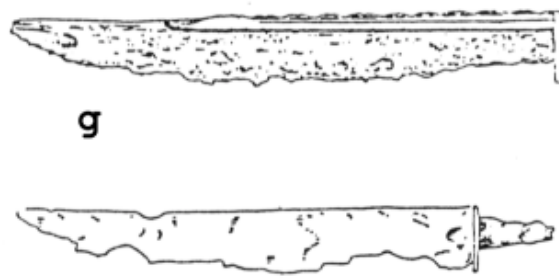

h

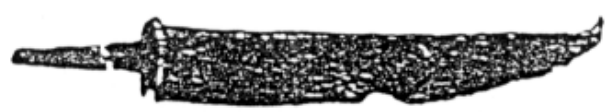

i

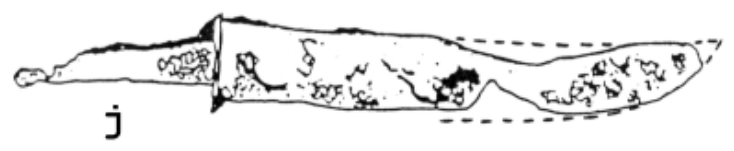

Abb. $173 \quad$ Griffplättchenversehene Messer mit der Klingenform I b, III e, V b und Vc (13. bis 15. Jahrhundert) a: Demmin; b-c: Anklam; d: Kambs; e: Lübeck; $f$ :Beuthen; g: Wildberg; $h$ : Kalisch; $i$ : Naesholm; $j$ : Raffenburg; $k$ : Frankfurt/Oder; $l:$ Minden 

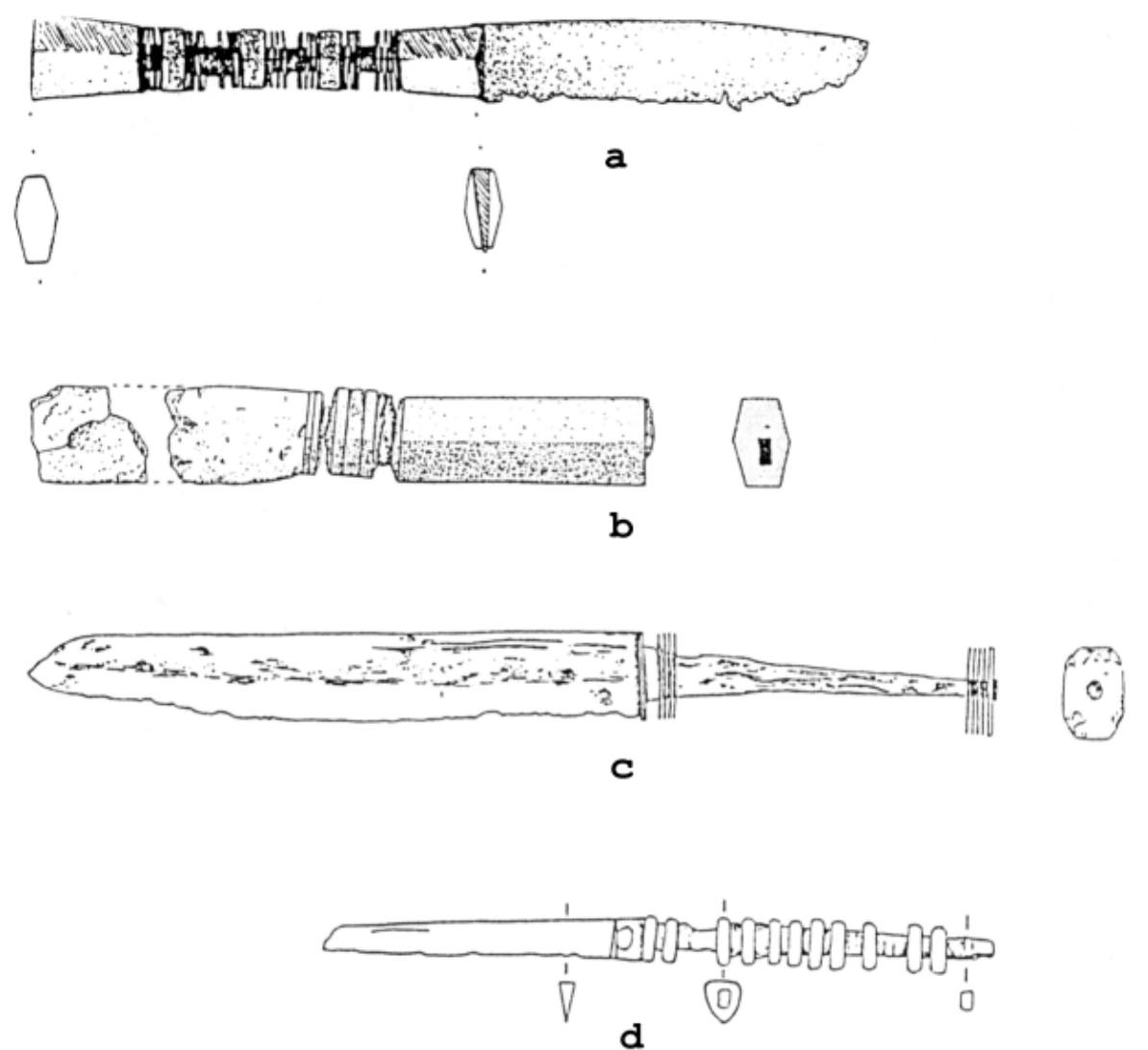

d
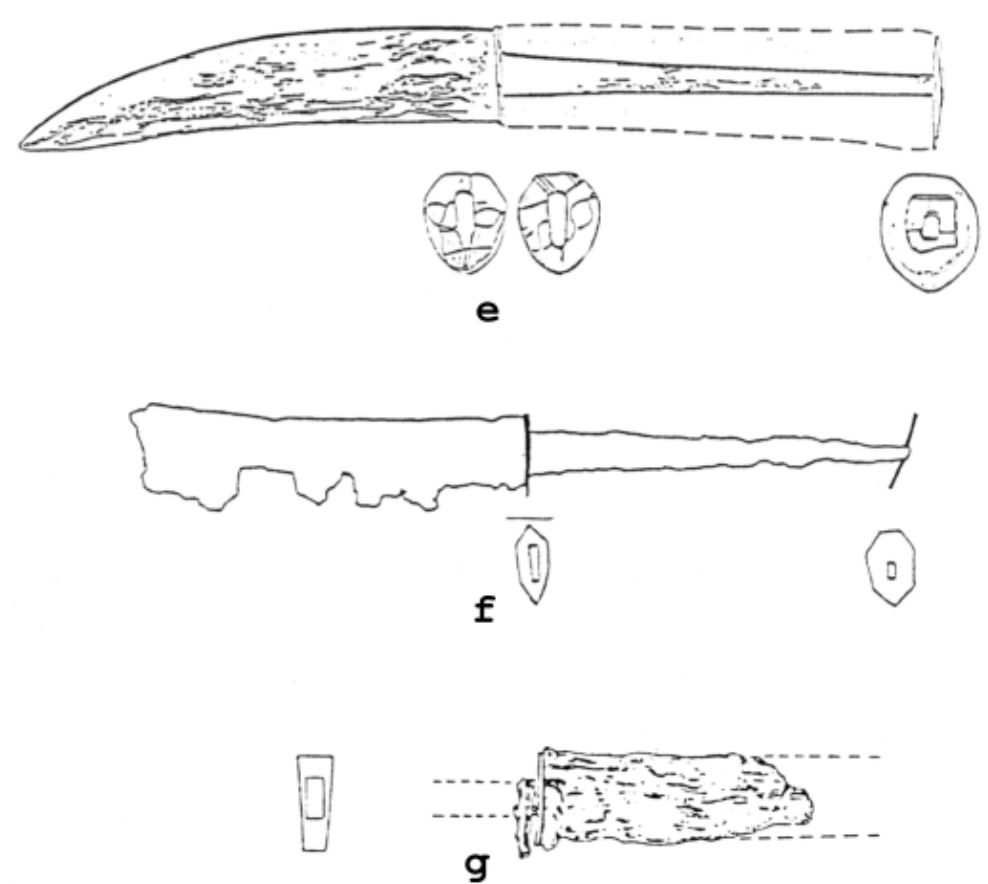

Abb. $174 \quad$ Griffplättchenversehene Messer mit unterschiedlichen Klingenformen (12. bis 14. Jahrhundert)

a: Oslo; b: Höxter; c: Anklam; d: Breslau; e: Winsen/Luhe; f: Raffenburg; g: Wiprechtsburg 


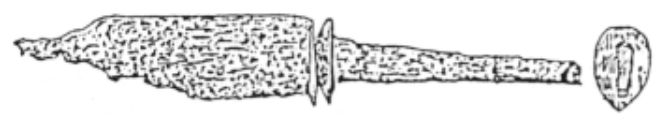

a

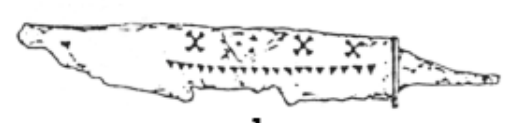

b

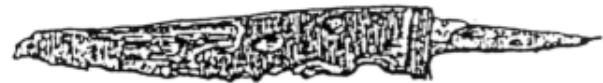

C

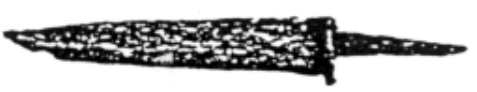

d

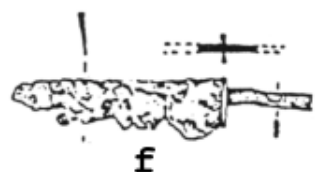

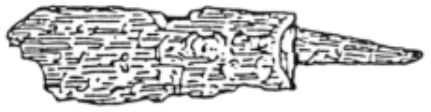

e

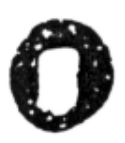

i
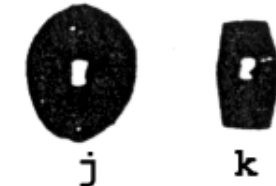

k

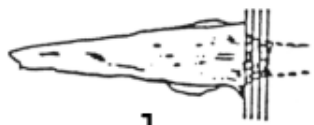

1
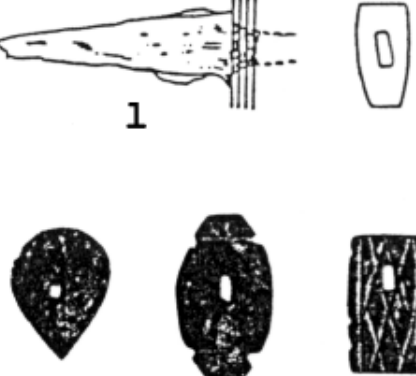

m
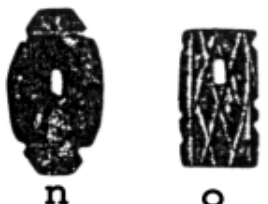

○

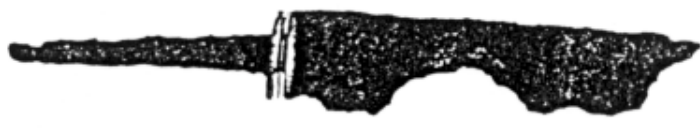

(2.) $\mathrm{g}$

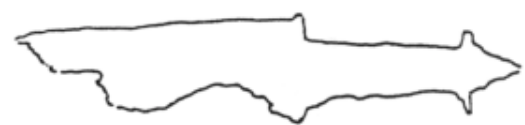

h

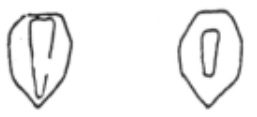

(10)

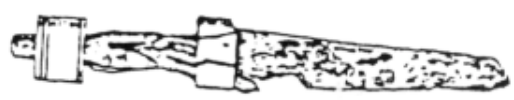

$\mathrm{p}$

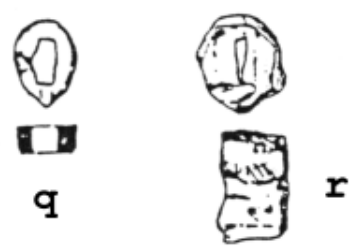

Abb. $175 \quad$ Griffplättchenversehene Messer mit unterschiedlichen Klingenformen (13. bis 15. Jahrhundert)

a: Manebach; b: Wiprechtsburg; c: Recknitzfund; d: Novgorod; e: Gommerstedt; f: Mecklenburg; g: Burg Schartenberg; h: Isenburg; i-k: Naesholm; l: Küsel; m-o: Kassel (Umgebung); p-r: Skanör 


\subsection{Zeitliche und sozialtopographische Verteilung}

Das Bild einer zwischen Technik und Mode stehenden Griffangelkonstruktion, die besonders stark im 13. und 14. Jahrhundert auftritt und über den Kernraum der Hanse hinaus im Bereich des städtischen Bürgertums und des Adels ein bekanntes Attribut, vielleicht sogar, wie Knorr vermutet, Bestandteil einer Tracht gewesen sein mag $^{395}$, läßt sich bei genauerer Analyse der Fundorte weiter spezifizieren.

Von den 37 Fundorten liegen 18 an oder in der Nähe von Flüssen, neun an Meeresküsten (Karte 14).

Besonders auffällig ist im 13. bis 14. Jahrhundert die Häufung an Ruhr und Weser, während vom 12. bis 15. Jahrhundert im nördlichen Teil des Untersuchungsraums von 17 Fundorten zehn küstengebunden sind.

Diese Häufung in Fundorten an schiffbaren Verkehrswegen im gesamten Untersuchungsraum deutet darauf hin, daß das griffplättchenversehene Messer in Verbindung mit Handelstätigkeiten gesehen werden muß, möglicherweise besonders an Fluß- und Seeschiffahrtswegen.

Unterschiede zeigen sich im Vergleich der zeitlichen Verteilung von Griffplättchenmessern in Städten und Burgen.

Nahezu gleichmäßig stark treten in Städten bereits vollentwickelte griffplättchenverzierte Messer im 12. Jahrhundert in Oslo, möglicherweise auch in Kalisch auf, im 12. bis 13. Jahrhundert in Beuthen und Breslau, im 13. Jahrhundert in Duisburg und Höxter (Ende 12. bis Anfang 14. Jahrhundert), im 13. bis 14. Jahrhundert in Anklam und in den Siedlungen Glasbach und Küsel, Kreis Burg, um 1300 in Minden, im 14. Jahrhundert in Frankfurt/Oder, in Winsen an der Luhe und in Stargard, im 14. bis 15. Jahrhundert in Demmin, Lübeck und Novgorod; die letzten Belege für die Verwendung von Griffplättchen als Griffendabsicherung frühneuzeitlicher Messer fanden sich in Lund, Lübeck und Hamburg bis ins 16. Jahrhundert.

Konzentrierungen sind im rheinisch-westfälischen Raum mit den Fundorten Duisburg, Höxter und Minden sowie im Bereich der wendischen Städte mit den Fundorten Winsen/Luhe, Lübeck, Demmin, Anklam und Stargard festzustellen mit einer starken Ausrichtung nach Südosten (Frankfurt/Oder, Kalisch, Breslau und Beuthen).

Fundorte wie der hansische Umschlagplatz Skanör in Schonen und der Eisenverhüttungsplatz und Herbergsort Glasbach an einem mittelalterlichen Fernverkehrsweg legen auch außerhalb städtischer Kontexte eine Verbindung zu überregionalem Verkehr nahe.

Nicht unerwähnt bleiben soll in diesem Zusammenhang das Messer von der Hildagsburg bei Wolmirstedt, die 1129 zerstört wurde. Von 1289 bis in die Mitte des

395 Vgl. hierzu die Ausführungen von H.A. Knorr 1971, S. 129 ff 
16. Jahrhunderts ist der Platz als Wallfahrtsort mit Nicolaikapelle historisch belegt. Da eine Datierung des Messers vor die Mitte des 12. Jahrhunderts zumindest sehr fraglich ist, bleibt als wahrscheinlichere Zuweisung (vor allem aufgrund der großen technischen und formalen Übereinstimmung mit den übrigen Exemplaren des 13. und 14. Jahrhunderts) der Kontext mit letzterem Umfeld bestehen, die Knorr bereits vorgenommen hat.

Ein etwas verändertes Bild ergibt sich bei der zeitlichen Verteilung von griffplättchenversehenen Messern von Burgen. Sie stammen mit hoher Wahrscheinlichkeit fast alle aus dem 13. bis 14. Jahrhundert (von 21 auf Burgen gefundenen Exemplaren wurden 18 vor allem aufgrund historischer Daten in das 13. und 14. Jahrhundert datiert), im nordöstlichen Bereich vielleicht noch aus dem beginnenden 15. Jahrhundert.

Die hohe Anzahl von Messern, die in Burgen an Ruhr und Lenne gefunden wurden, kann ebenso forschungsbedingt sein wie die Menge der in der Altstadt von Anklam geborgenen Messer.

Die zusätzlich beobachtete Konzentrierung von Fundorten des 13. Jahrhunderts im märkischen Sauerland wie der Rücklenburg, der Raffenburg und der Isenburg bei Hattingen ist vielleicht aber auch ein Hinweis auf ein nahegelegenes Produktionszentrum von Messern mit griffplättchenversehenen Griffen, vor allem unter Berücksichtigung des auf Burgen und in den Flußtälern angesiedelten mittelalterlichen Eisengewerbes ${ }^{396}$. Der Fund aus Duisburg, einem hochentwickelten Hafen an der Ruhrmündung im 13. Jahrhundert, könnte auf einen Umschlagplatz verweisen.

Dieses bisher westlichste Gebiet mit nachgewiesenem Aufkommen von Griffplättchenmessern steht aber noch so isoliert da, daß ohne weitere Fundbelege aus eindeutigen Befundsituationen diese Überlegungen spekulativ bleiben müssen.

Die Differenzierung der zeitlichen Verteilung von Griffplättchenmessern in Städten und Burgen, unterschiedliche regionale und zeitliche Konzentrationen und eine genauere Betrachtung der Fundorte lassen Rückschlüsse auf bestimmte Verbraucherschichten zu.

Von der Mitte des 12. bis in das beginnende 15. Jahrhundert sind voll entwickelte Griffplättchenmesser in städtischen Kontexten nachweisbar, ohne daß in diesem Zeitraum Konzentrationsveränderungen feststellbar sind.

Die beobachteten formalen Unterschiede und Konzentrationen einzelner Ausführungsarten können Hinweise auf einen eher modischen Wandel sein, der bisher aber nicht eindeutig belegbar ist.

Der durch die geographische Lage der städtischen Fundorte naheliegende und aufgrund ihrer Verbindung mit der Hanse auch historisch wahrscheinliche

396 Eversberg 1982, S. 20 ff, S. 26 ff, S. 36-37

Sprandel 1968, S. 201-204 
Zusammenhang zwischen dem Verbreitungsbild von Griffplättchenmessern in Städten und überregionalem Handel beinhaltet die Möglichkeit, diese Messer auch als Handelsgut anzusehen. Städte mit einer starken Kaufmannschaft, Orte mit überwiegendem Handelscharakter wie Skanör in Schonen, Wallfahrtsorte und Erzförderstätten als Fundorte legen darüberhinaus nahe, die Besitzer dieser Messer einem mobilen Personenkreis zuzuordnen, wie zum Beispiel Kaufleuten und ihren Begleitern $^{397}$.

Das 13. und 14. Jahrhundert scheint der Zeitraum gewesen zu sein, in dem auf Burgen, vor allem im Bereich der nördlichen deutschen Mittelgebirge, Griffplättchenmesser ebenfalls benutzt wurden. Auch hier legt das Verbreitungsbild eine Verbindung mit überregionalen Verkehrswegen (Flüssen) nahe.

Mit den Funden vom Kambs, Wildberg, Winsen/Luhe, Demmin und Lübeck scheint sich in der zweiten Hälfte des 14. und beginnenden 15. Jahrhunderts der Zeitraum anzudeuten, in dem die Erscheinung des mittelalterlichen Griffplättchenmessers ausläuft.

Der Beginn der Verwendung von Griffplättchen ist dagegen bisher nicht genau zu erkennen. Mit dem Osloer Messer aus der Mitte bis zweiten Hälfte des 12. Jahrhunderts liegt zum frühesten sicher nachweisbaren Zeitpunkt ein bereits technisch und modisch ausgereiftes Exemplar vor.

\subsection{Zur typologischen und regionalen Differenzierung von G riff- plättchenmessern}

Die grundsätzliche Unterscheidung zwischen der Mode der Griffplättchverzierung und der Technik, mit Hilfe von Griffplättchen das Heft und das Griffende zusätzlich abzusichern, kann aufgrund der bisherigen Erkenntnisse kein typologisches Kriterium sein.

Vor- oder Frühstufen fehlen vollkommen, das Griffplättchen taucht sicher datiert im 12. Jahrhundert auf, und zwar bereits in ausgereifter Technik und als Verzierungselement.

Die Messer aus Beuthen und Breslau, in das 12. bis 13. Jahrhundert datiert, sind jeweils völlig unterschiedlich in Technik und Aussehen, besitzen beide verzierte/segmentierte Griffe und sind ebenfalls kaum als Frühstufen einzuordnen.

Auch regional lassen sich keine Hinweise auf Entstehungsräume oder Räume, in denen die Griffplättchentechnik als Verzierungselement vorherrschte, ausmachen.

Der Vergleich zwischen dem Verbreitungsgebiet der Griffplättchenverzierung (Abb. 171) und dem Raum, in dem die allgemeine Verwendung von Griffplättchen bis jetzt nachweisbar ist, zeigt lediglich Unterschiede in der Dichte der Streuung.

397 Dollinger 1989, S. 215-216, S. 224 
$\mathrm{Ob}$ sich mit den beschriebenen Konzentrationsgebieten Produktionsraum und $\mathrm{Ab}$ satzraum zu erkennen geben, ist bisher nur zu vermuten und wird lediglich dadurch gestützt, daß sich im nördlichen Konzentrationsgebiet ein Hauptwirtschaftsraum der Hanse, im südlichen Gebiet Produktionszentren mittelalterlicher Klingenschmiede befinden.

Es fehlt aber bislang jeder exakte Nachweis zu Entstehung und mög lichen Herstellungszentren.

\section{Die verschiedenen Griffplättchenkonstruktionen}

Die in dieser Arbeit erfaßten und ausgewerteten Messer mit griffplättchenversehenen Griffen lassen sich bezüglich der Anzahl und Anordnung der Griffplättchen in zwölf verschiedene Konstruktionsformen gliedern, die zum Teil sehr unterschiedliche Mengen an Einzelbeispielen auf sich vereinigen (Abb. 176).

Bei der Untergliederung der Griffplättchenkonstruktionen ergab sich mit dem häufigen Fehlen der hinteren Griffpartie ein grundsätzliches Problem, welches nur mit Hilfe angenommener Rekonstruktionen auf der Basis der vollständig erhaltenen Beispiele gelöst werden konnte.

Die Abfolge der bis auf eine Form induktiv ermittelten Formen umfaßt zunächst die Verwendung von einem oder zwei Griffplättchen am Heft bzw. am Griffende als Elemente einer Griffsicherung (fünf Formen), sodann die Verwendung von Griffplättchenpaketen und die Segmentierung des Griffs einschließlich der Verwendung von Vollmetallsegmenten als Verzierungselemente (sieben Formen).

Die Verwendung und Anordnung von Griffplättchen aus anderen Materialien, die sich zwischen den einzelnen Plättchen aus Buntmetall befunden haben, wurde nicht berücksichtigt, da sie bei griffplättchenverzierten Griffen in der Regel zwar anzunehmen, oft aber aus Gründen unterschiedlicher Erhaltungsbedingungen nur vereinzelt nachweisbar sind.

Die Ursachen der starken Konzentration auf die einfachste Form der Griffplättchenkonstruktion, die Verwendung eines Stichblattes am Heft, sind wahrscheinlich ebenfalls bedingt durch den schlechten Erhaltungszustand vieler Messer, deren Angeln oft kaum bis zur Griffmitte erhalten sind. Es scheint sich aber anzudeuten, daß die Verwendung von zwei Plättchen am Heft seltener anzunehmen ist als die Stichblattverwendung. Beide Befestigungsarten sind hauptsächlich im 13. und 14. Jahrhundert vertreten, nur ein Messer mit einem Griffend- und zwei Heftplättchen ist für das 15. Jahrhundert belegt.

Die zeitliche Streuung der verschiedenen Griffplättchenverzierungsarten umfaßt neben dem 13. und 14. Jahrhundert als Hauptverbreitungszeit einige Beispiele des 12. bis 13. Jahrhunderts und des 14. bis 15. Jahrhunderts, die vor allem Messer mit segmentierten Griffen umfassen. Die Verwendung von Vollmetallsegmenten scheint auf das 12. bis 13. Jahrhundert beschränkt zu sein, Griffplättchenpakete am 
Heft und am Griffende sind bis auf zwei Exemplare, die möglicherweise bis in das 15. Jahrhundert reichen, vor allem für das 13. bis 14. Jahrhundert belegt.

Die einfachste Form der Segmentierung, die Halbierung des Vollgriffs mit je einem Griffplättchen am Heft, mittig zwischen den Griffsegmenten und am Griffende, ist nur mit einem Messer aus Duisburg belegbar, dessen hintere Griffpartie abgebrochen ist; die Rekonstruktion ist als Hypothese zu betrachten.

Aufgrund der bisherigen Ergebnisse kann nicht davon ausgegangen werden, daß sich die Griffplättchenverzierung aus einer Befestigungstechnik entwickelt hat. Wahrscheinlich haben Verzierungs- und Befestigungsabsichten gleichermaßen eine Rolle gespielt bei der Verwendung von Griffplättchen, und die griffplättchenverzierten Griffe sind möglicherweise als eine zeitgleiche Gruppe innerhalb der Griffplättchentechnik zu betrachten. 

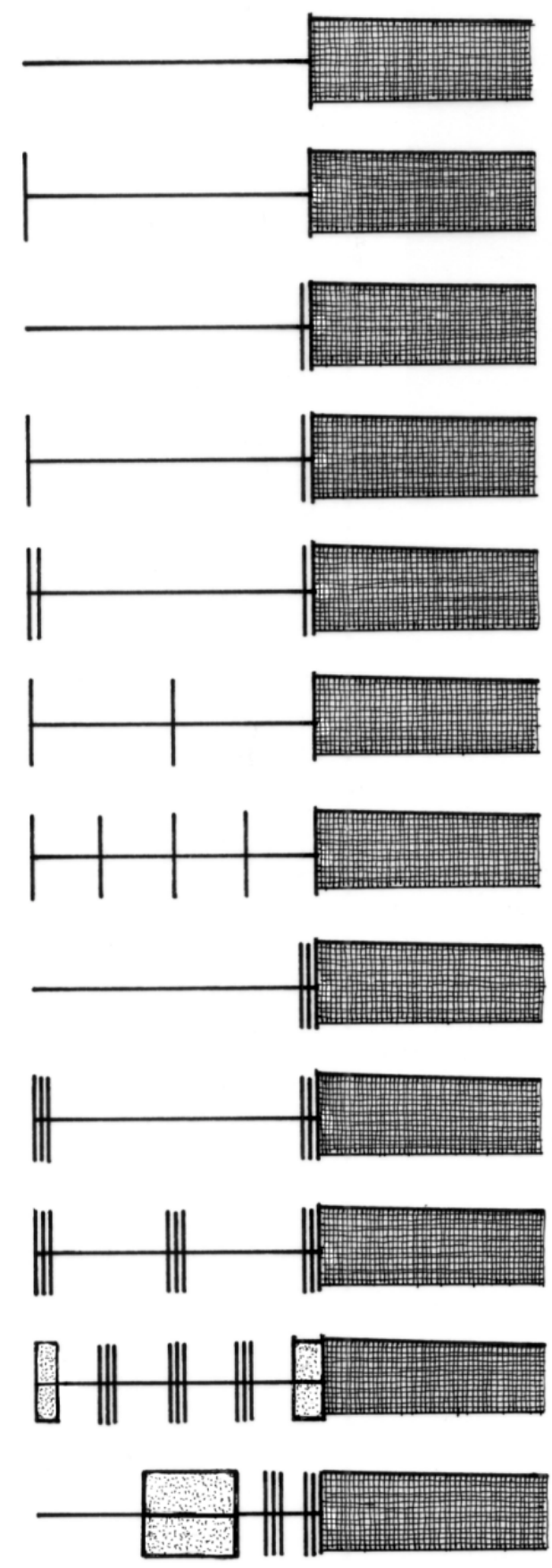

8009001000110012001300140015001600

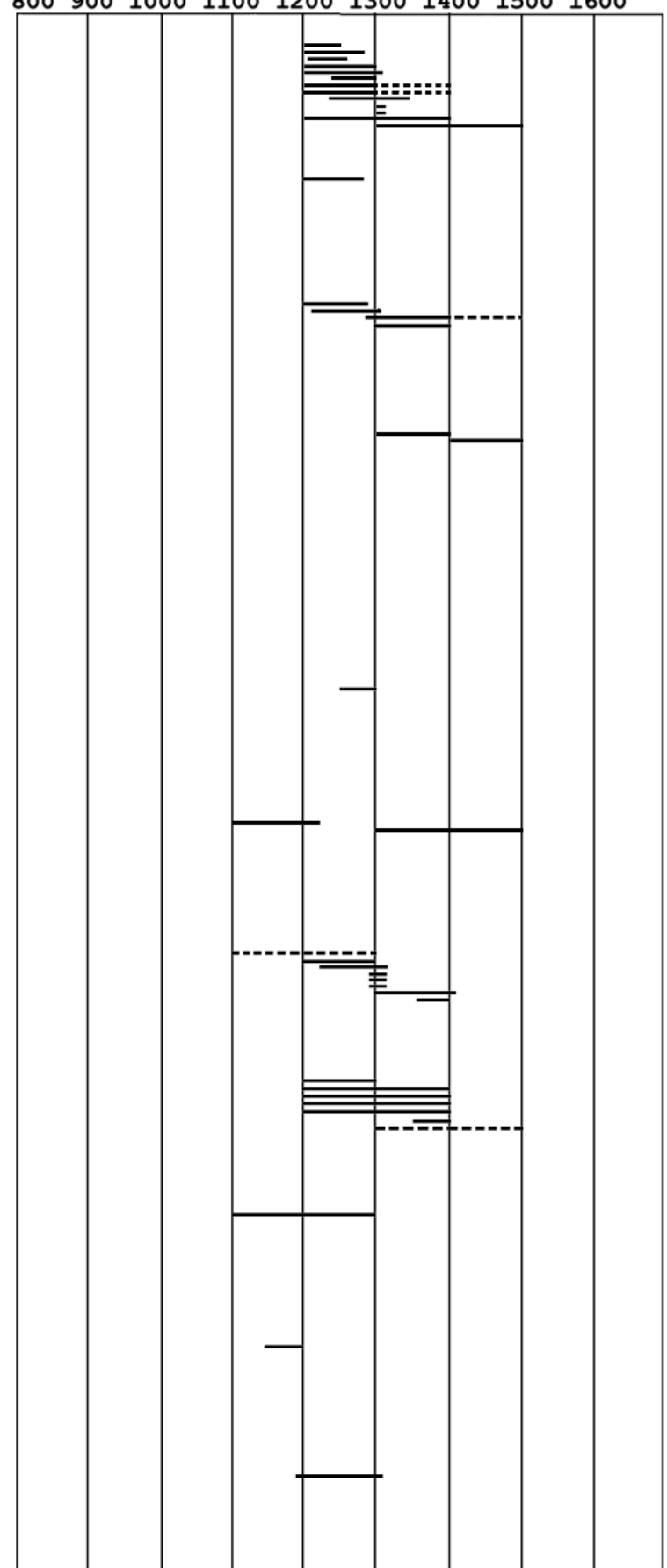

Abb. $176 \quad$ Griffplättchenkonstruktionen - zeitliche Verteilung 


\section{Zur regionalen Verteilung von Klingen- und Griffplättchenformen}

Regionaltypische Gruppen lassen sich -aus dem relativ geringen Bestand nur schwer erkennen ${ }^{398}$.

Den Messern mit Plättchenpaketen aus Anklam, Lübow-Levetzow und Demmin stehen Funde aus Minden, von der Raffenburg und der Burg Schartenberg, der Hildagsburg und der Wiprechtsburg gegenüber, die sich lediglich im Erhaltungszustand voneinander unterscheiden.

Auch die Analyse der an Griffplättchenmessern beobachteten Klingenformen ergibt kaum Anhaltspunkte für eine regionale Gliederung. Am häufigsten tritt in diesem Zusammenhang die Form IIIb auf (Abb. 177), sie scheint im Mittelgebirgsbereich zwischen Rhein und Weser etwas häufiger anzutreffen zu sein als im Bereich der Ostseeküstenstädte; mit hoher Wahrscheinlichkeit ist sie jedoch begrenzt auf das Kerngebiet zwischen Rhein und südwestlicher Ostseeküste (Abb. 178).

Die Formen Ib und IIIe sind zusammen ebenfalls häufig bei griffplättchenversehenen Messern angetroffen worden, ihre Verbreitung erstreckt sich vor allem auf das nördliche Konzentrationsgebiet, seltener anzutreffen sind sie im Mittelgebirgsbereich. Ihre Streuung umfaßt im Gegensatz zu der Form IIIb auch Fundorte außerhalb des Kerngebietes wie Kalisch, Beuthen und die seeländische Burg Naesholm (Abb. 179).

Die übrigen Klingenformen (Va/IIIe4, IIIe3, IIIe14, IIb1/IIIb15) sind zahlenmäßig zu gering vertreten, um Aussagen über ihre Verbreitung zu treffen.

Die bisher erfaßten Griffplättchen umfassen 13 Formen, darunter runde, drei- und mehreckige, ovale und tropfenförmige. Sie wurden aus Buntmetallblechen verschiedener Stärken geschnitten, nachweisbar unter anderem auch aus sekundär verarbeiteten Hanseschalen ${ }^{399}$. Einige Formen, vor allem tropfenförmige und einige mehreckige Plättchen, sind vom ausgehenden 12. bzw. 13. Jahrhundert bis in das 15. Jahrhundert benutzt worden, andere, darunter runde und ovale Plättchen, vor allem im 13. und beginnenden 14. Jahrhundert (Abb. 180).

Auch die räumliche Verteilung der einzelnen Griffplättchenformen (Abb. 181-184) läßt nur bedingt regionale Schwerpunkte erkennen. Die sechseckigen Plättchen des 12. bis 14. Jahrhunderts sind relativ verstreut im gesamten Verbreitungsgebiet aufgetreten (Abb. 181), ebenso wie die tropfenförmigen Plättchen des 13. bis 14. bzw. 15. Jahrhunderts (Abb. 182). Trapezoide und länglich ovale Plättchen des 13. Jahrhunderts scheinen auf den südlichen Teil des Verbreitungsgebietes beschränkt zu sein (Abb. 183). Nach unten spitz zulaufende siebeneckige Plättchen des 13. bis

\footnotetext{
398 Grundsätzlich muß damit gerechnet werden, daß die Räume zwischen den bereits erwähnten Konzentra399 tionsgebieten auf Forschungs- bzw. Publikationsdefizite zurückzuführen sind.

Knorr 1971 und Drescher 1975
} 
14. Jahrhunderts wurden im Bereich der Rhein-Ruhrmündung, in Thüringen und an der mittleren Weser beobachtet, im 14. bis 15. Jahrhundert tritt die Form an einem Messer aus der Peene bei Demmin auf. Die den Proportionen letzterer Form ähnlichen fünf- und sechseckigen Plättchen des 14. bis 15. Jahrhunderts sowie rechteckige Plättchen mit abgeschrägten Ecken scheinen eher an Städte im Küstenbereich gebunden zu sein (Abb. 184).

\section{Zusammenfassung}

Innerhalb des Verbreitungsgebietes der Griffplättchentechnik lassen sich möglicherweise zwei regionale Zentren unterscheiden, die vielleicht auch zeitlich zu differenzieren sind.

Die entsprechenden Messer des 13. Jahrhunderts und der Zeit um 1300 stammen vor allem von Burgen aus dem Bereich der Mittelgebirgszonen vom RheinRuhrmündungsbereich bis Thüringen sowie aus Städten und Siedlungen an der Peripherie dieses Raumes (Duisburg; Minden; Küsel, Kreis Burg). Im 13. bis 14. Jahrhundert lassen sich griffplättchenversehene Messer im gesamten Verbreitungsgebiet nachweisen, welches bis nach Schlesien, Pommern und Skandinavien reicht.

Die frühen Exemplare aus Oslo, Breslau und Beuthen, sowie die Messer von der Mecklenburg, aus dem Moorfund bei Levetzow und aus der Recknitz bei Plummendorf, die alle vor das 14. Jahrhundert datiert worden sind, lassen jedoch erkennen, daß Entstehungsgebiet und -zeit dieser Technik noch nicht klar zu umreißen sind. Möglicherweise sind diese Messer mit deutschen Kaufleuten schon mit der Entstehung und ersten Ausweitung der Hanse an die Peripherie ihres wirtschaftlichen und kulturellen Einflußgebietes gelangt.

Das Fehlen von Beispielen des 13. Jahrhunderts aus dem direkten Einflußbereich der Ostseestädte hat vielleicht aber auch forschungsspezifische Gründe, da für diesen Bereich stratigraphisch oder historisch enger datierte Exemplare auch für das 14. und 15. Jahrhundert selten sind.

Die Messer des 14. bis 15. und 15. Jahrhunderts stammen fast durchweg aus dem Küstenbereich (Lübeck, Cambs, Demmin), welches Knorr mit seiner Verbreitungskarte unter Einbeziehung der südlich angrenzenden Fundorte bereits erfaßt hat ${ }^{400}$. Dieser Raum kann für das ausgehende 14. und 15. Jahrhundert als Rückzugsgebiet der Griffplättchentechnik angesehen werden.

400 Knorr 1971, Abb. 3, S. 127 

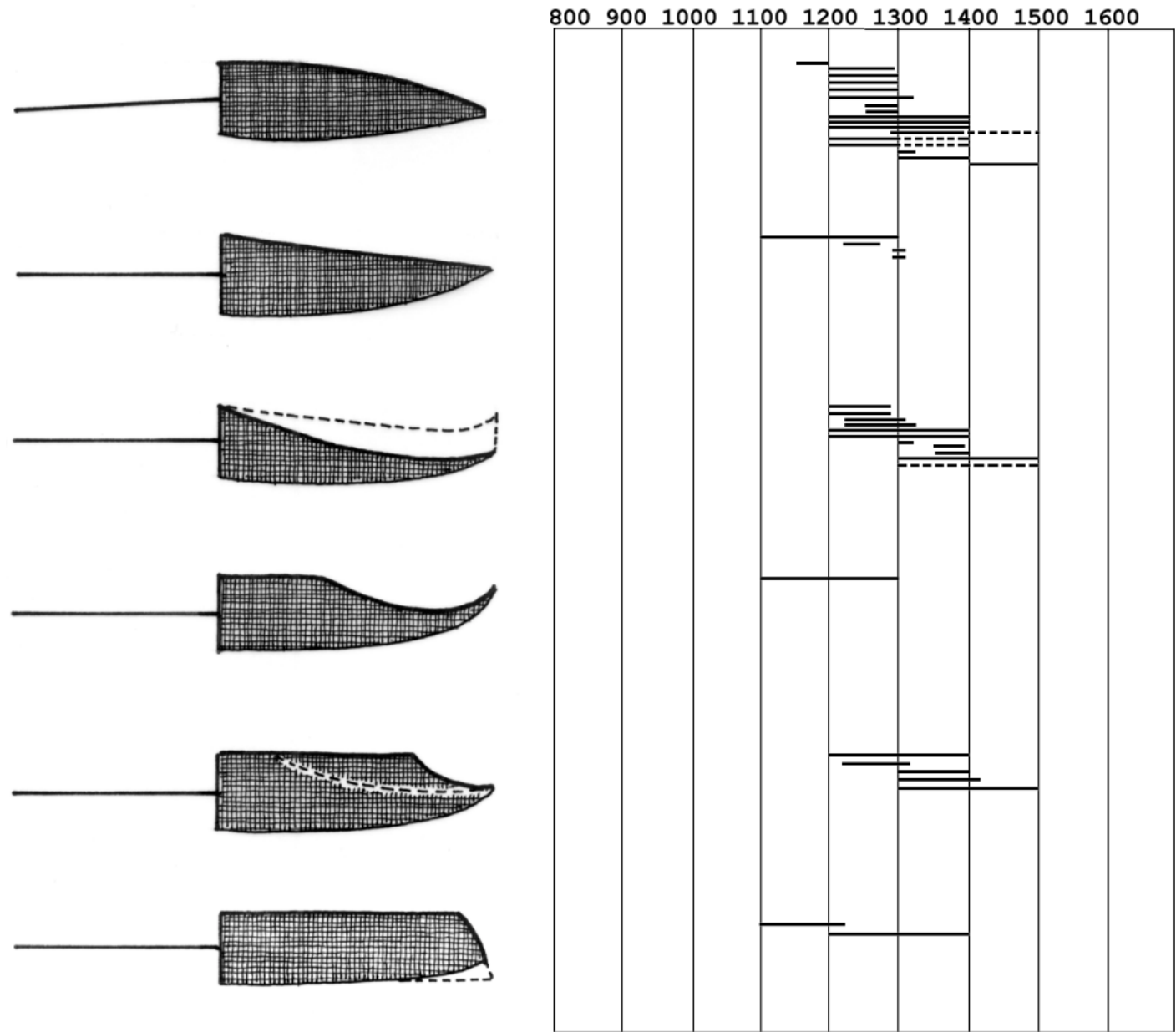

Abb. $177 \quad$ Klingenformen an Griffplättchenmessern - zeitliche Verteilung 


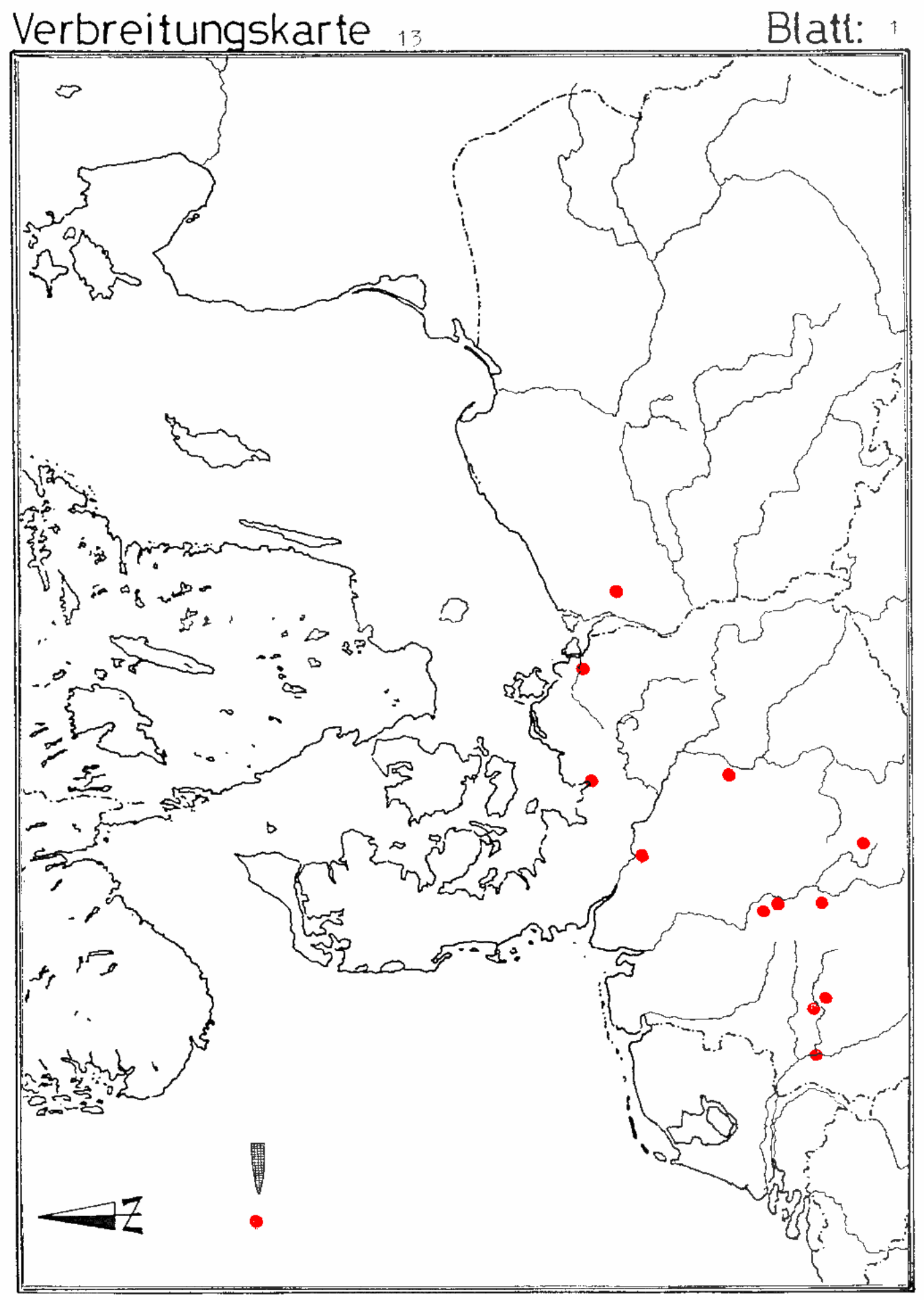

Abb. 178 Die Verbreitung von Griffplättchenmessern mit der Klingenform II b 
Verbreitungskarte

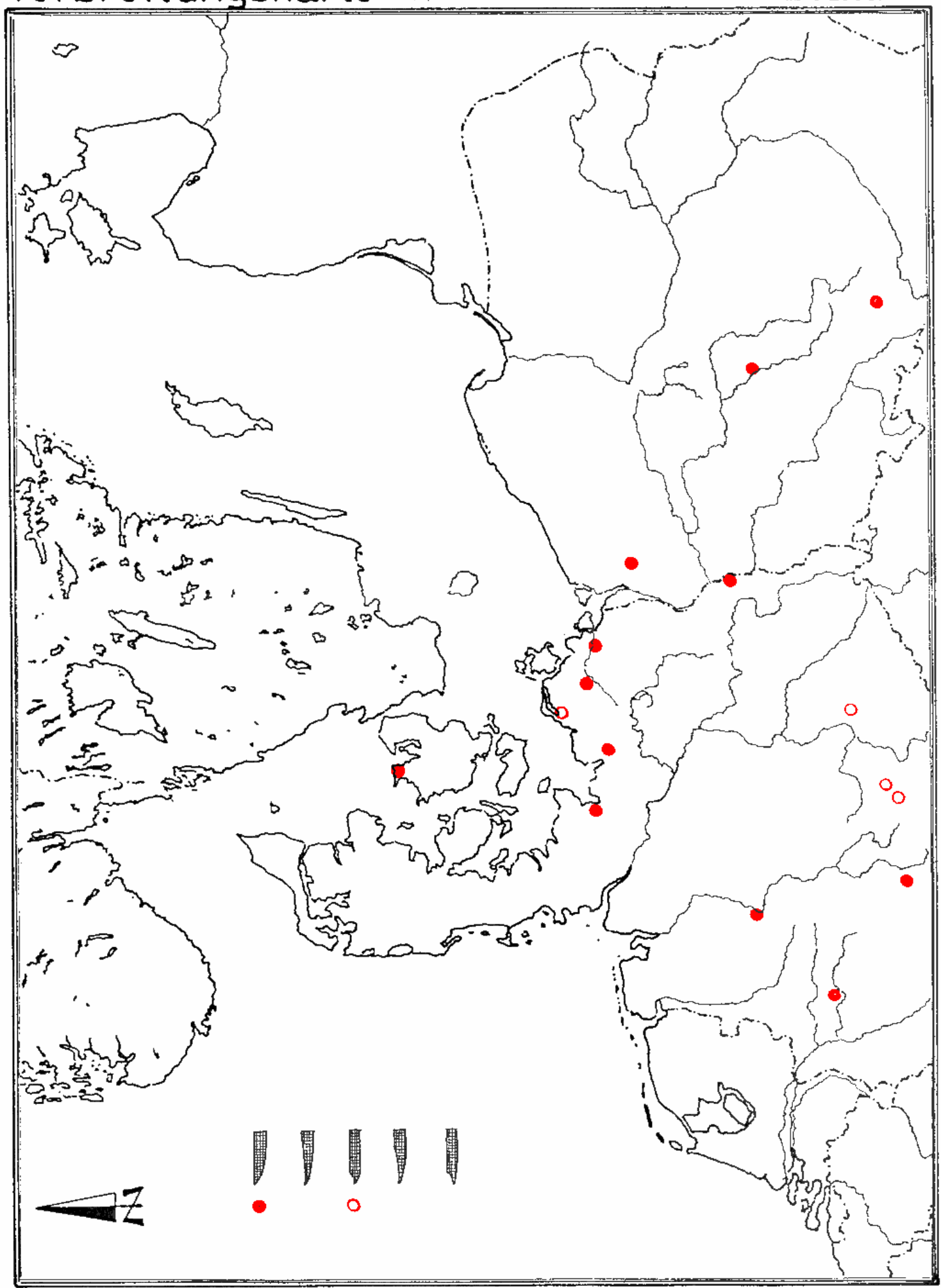

Abb. I79 Die Verbreitung von Griffplättchenmessern mit den Klingenformen I b, JII e. $\mathrm{V}$ b. $\mathrm{Vc} / \mathrm{V}$ a. III e 1 , III e 3 , III e 4 und III e 14 


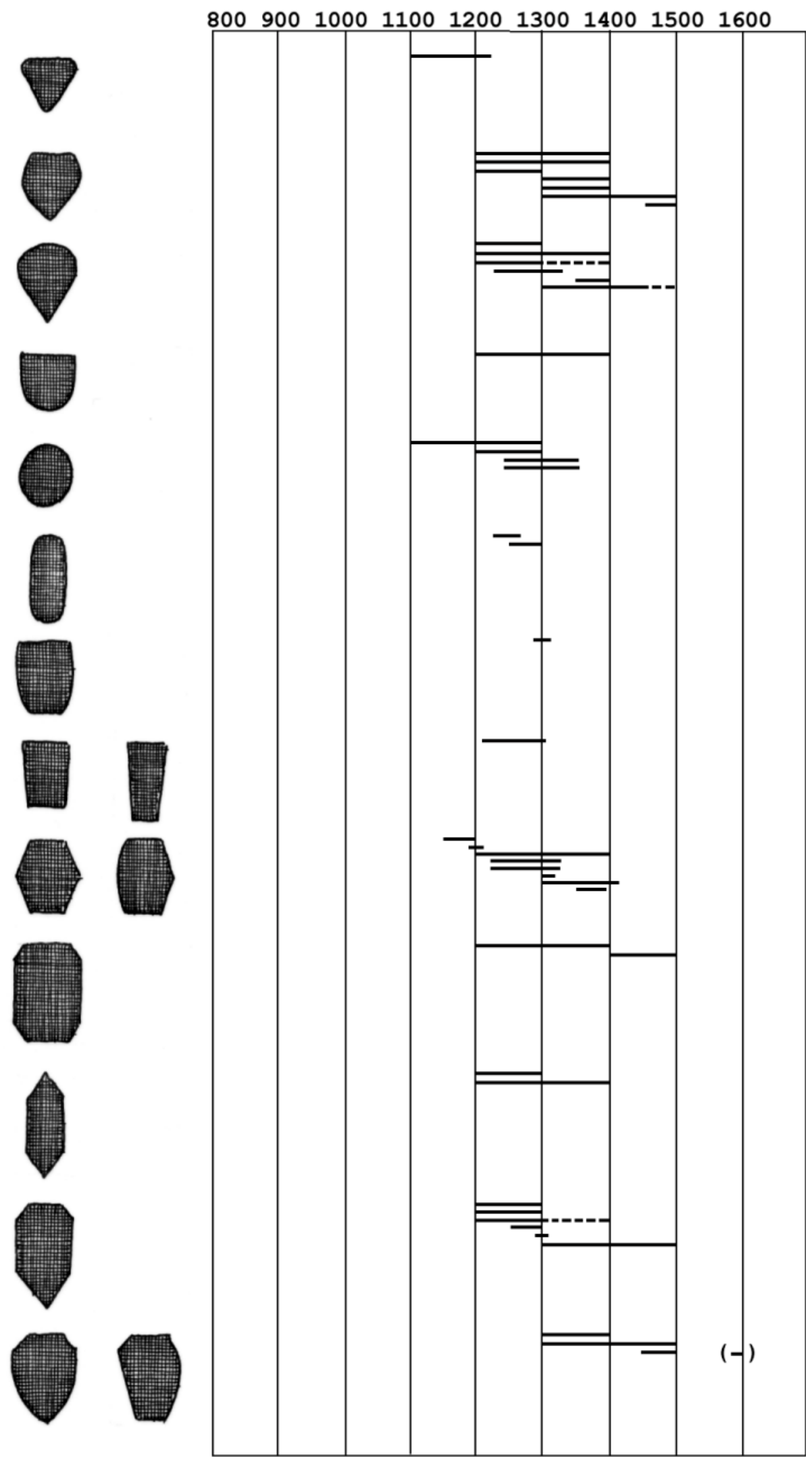

Abb. 180 Griffplättchen - zeitliche Verteilung 


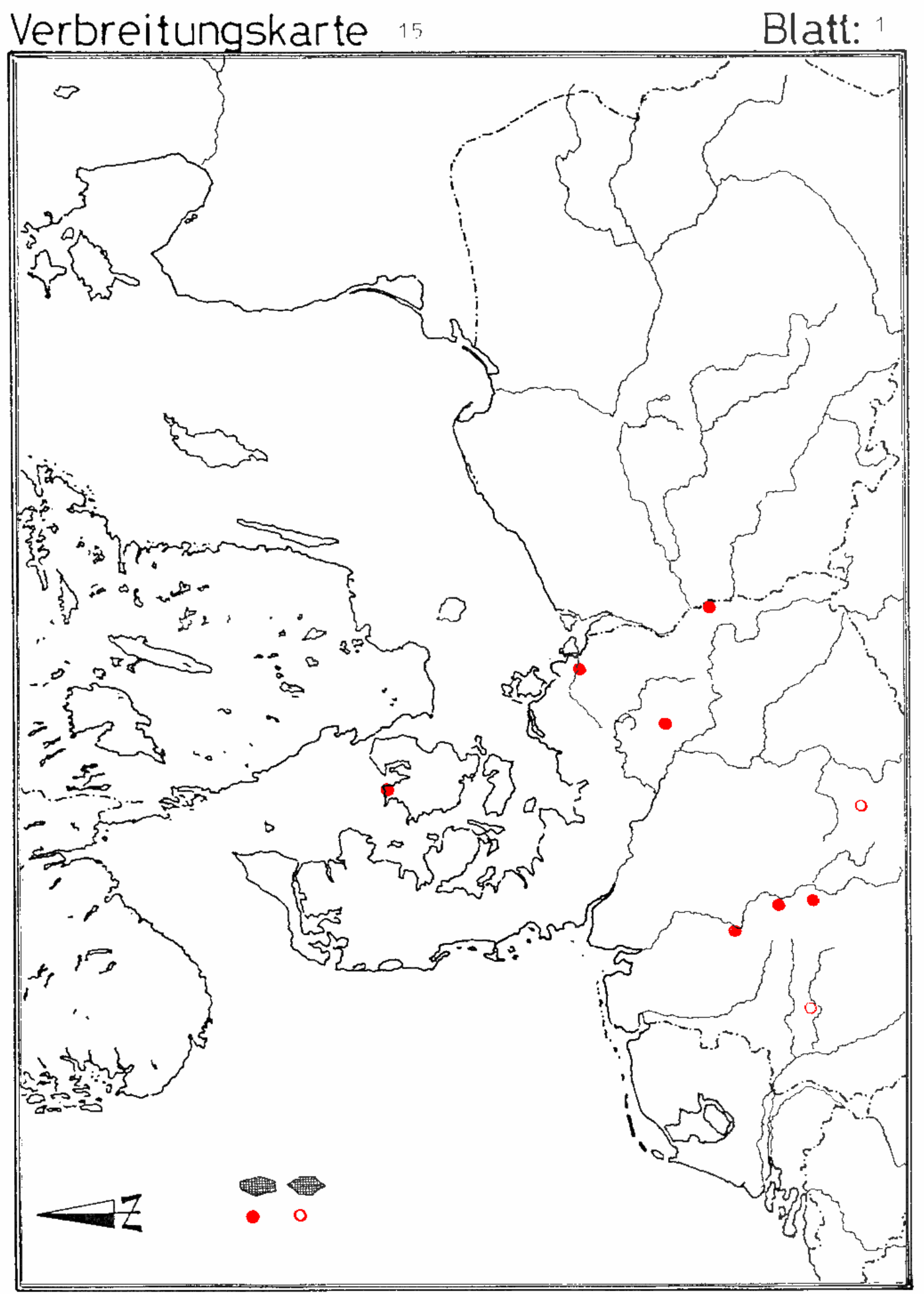

Abb. 181 Die Verbreitung von Griffplättchen I 
Verbreitungskarte

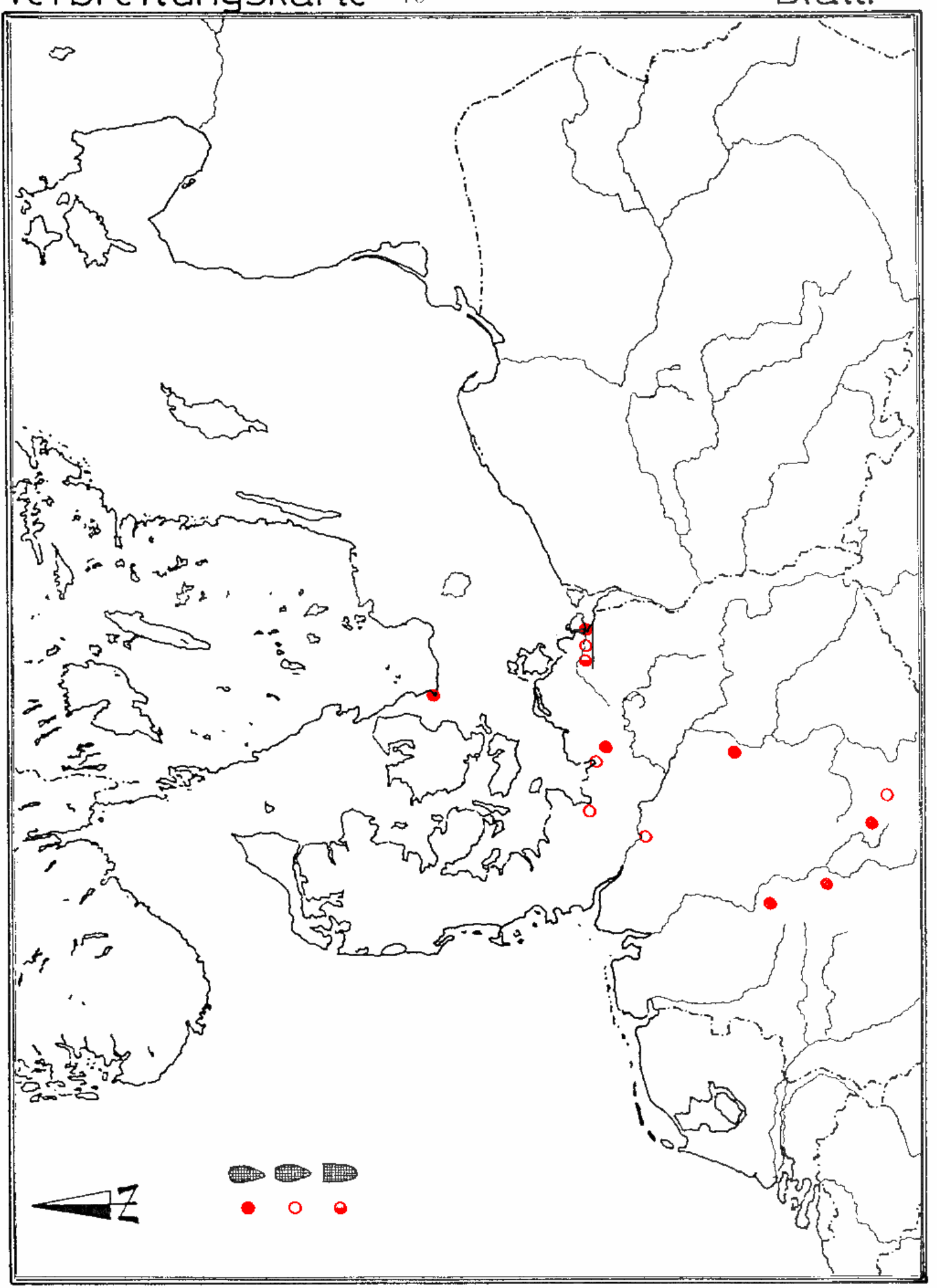

Abb. 182 Die Verbreitung von Griffplättchen II 


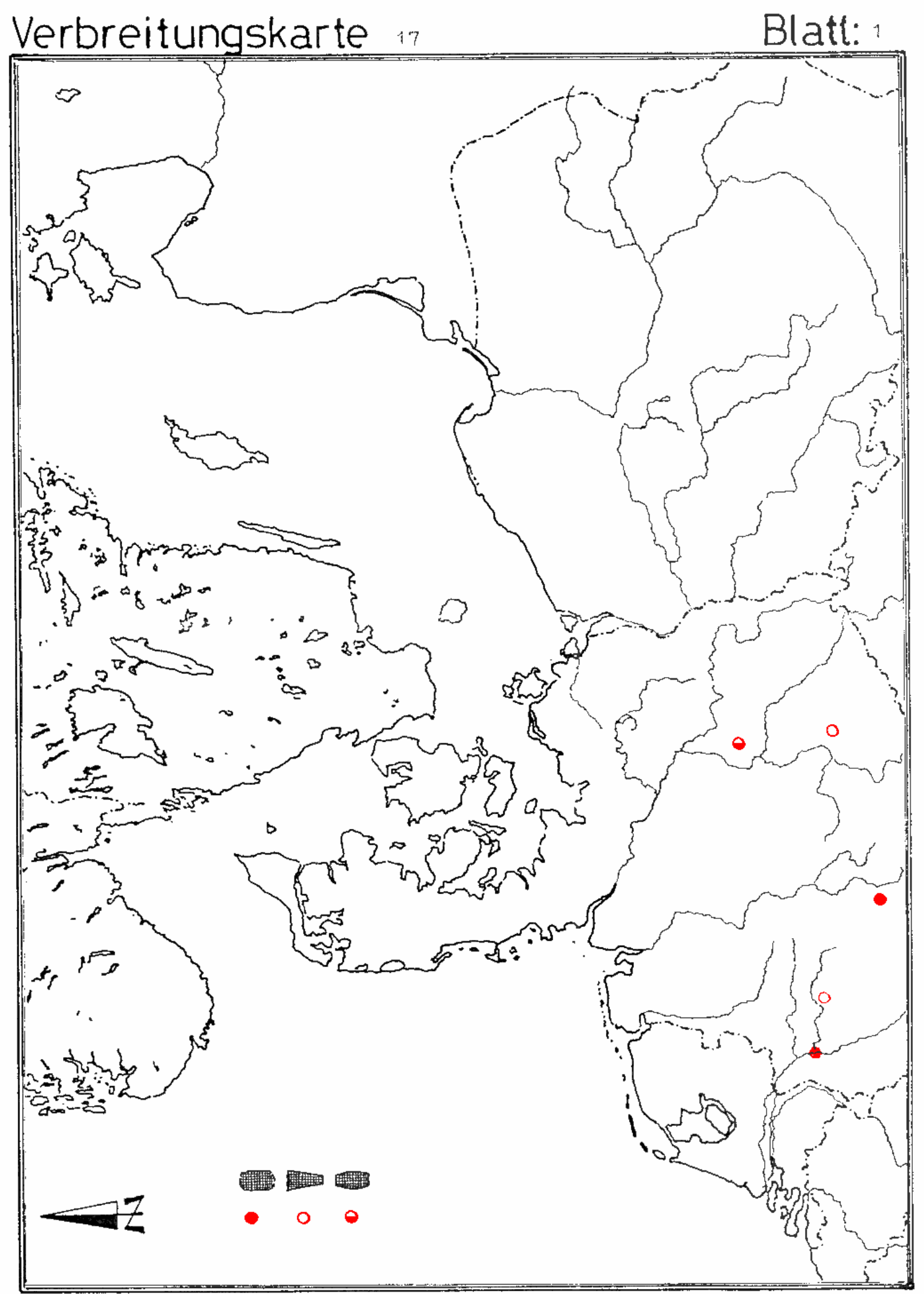

Abb. 183 Die Verbreitung von Griffplättchen III 


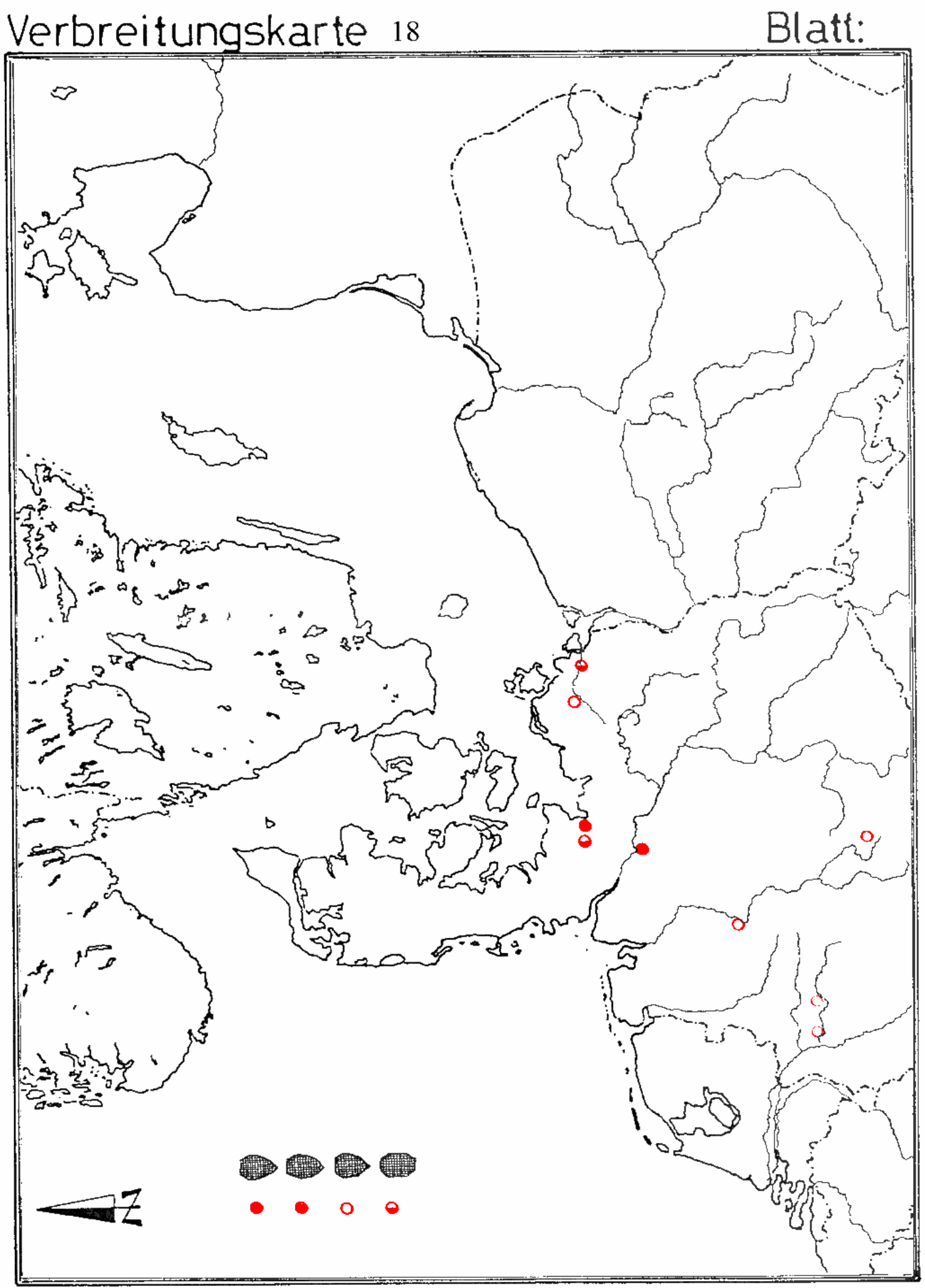

Abb. 184 Die Verbreitung von Griffplättchen IV 


\subsubsection{Klingenverzierungen}

Klingenverzierungen sind im bearbeiteten Fundgut nur selten aufgetreten, dennoch lassen sich bezüglich der Technik und der Verzierungselemente einzelne Gruppen voneinander unterscheiden.

Grundsätzlich können Verzierungen des Klingenrückens und Verzierungen auf der angeschliffenen, der sogenannten Schauseite der Klinge differenziert werden. Beidseitige Klingenverzierungen, wie sie bei Blankwaffen häufiger auftreten, sind auf Messerklingen nur äußerst selten angetroffen worden.

Verzierungen an Messerklingen wurden eingeschlagen (gepunzt), ziseliert, mit Bunt- oder Edelmetallen ausgelegt, in Schienen aufgelegt oder durch Anätzen damasziert oder damastähnlich geschmiedeter Klingen sichtbar gemacht.

Die Motive reichen von einfachen geometrischen Mustern über floralen Dekor bis zu Schriftbändern.

Die frühesten Exemplare verzierter Messerklingen stammen schon aus dem ausgehenden 9. und 10. Jahrhundert, standardisiert und zahlreicher treten Klingenverzierungen aber erst seit dem ausgehenden 16. und 17. Jahrhundert auf.

\subsubsection{Verzierungen des Klingenrückens}

Aus Novogrudok und Posen wurden an drei Messern des 12. bzw. 14. bis 15. Jahrhunderts eingeschlagene Markierungen beobachtet, die bisher nicht eindeutig als Ornament oder Klingenmarke identifiziert worden sind (Abb. 185a bis 185c) ${ }^{401}$. Ein weiteres Exemplar aus Niestronno weist im Rücken der nur noch ansatzweise erhaltenen Klinge eine ähnliche Markierung auf ${ }^{402}$.

Eine eindeutige Verzierung des Klingenrückens liegt mit einem Messer von der Burg Wildberg (Kreis Neuruppin) vor, welches wie das oben erwähnte Messer aus Posen aus dem 14. bis 15. Jahrhundert stammt (Abb. 187c). Dort wurde eine Schiene aus Mesingblech über den Rücken und den oberen Ansatz der Klingenseiten gelötet, die mit einer Reihe rosettenartiger Ornamente verziert worden ist.

\subsubsection{Verzierungen der Klingenseiten}

Häufiger treten Messer auf, die in den Seitenflächen der Klingen verziert worden sind.

\footnotetext{
401 Vgl. u.a. Gurjevitsch 1981, S. 32 mit weiteren Beispielen

402

Vgl. Katalogteil Polen, Kat. Nr. 128
} 
Neben Kupfer- oder Silbertauschierungen stehen einige Beispiele von Klingen mit Damszierungseffekt ${ }^{403}$ (Abb. 185d, 185e). Eingeschlagene (gepunzte) Bänder aus Dreiecken, Rosetten, Kreuzen, Schrägstrichen oder Schlangenlinien liegen vor allem aus Fundorten aus Polen und der ehemaligen DDR vor, sie sind zum Teil mit Silber ausgelegt (tauschiert) worden ${ }^{404}$ (Abb. 185f, 185h, 185i; 186a bis 186f). Die Ornamentzonen befinden sich unterhalb des Rückens, oberhalb der Schneide oder mittig in der Klingenfläche.

Die beidseitige Verzierung wurde nur auf einer Lübecker Klinge beobachtet (Abb. 187b).

Ebenfalls an einem Messer aus Lund sind beide Klingenkanten (sowohl unterhalb des Rückens als auch oberhalb der Schneide) mit Silbertauschierungen verziert worden $^{405}$ (Abb. 185g).

Seltener als geometrische Muster treten zeichenähnliche und übertragene Muster in Messerklingen auf (Abb. 186g bis 186i; 187a, 187b).

Eine Kombination aus geometrischen Ornamenten und einem markenähnlichen Zeichen liegt als Silbertauschierung an einem Messer aus Demmin vor, von dem keine datierenden Fundumstände bekannt sind ${ }^{406}$ (Abb. 186g).

Ein in der Form sehr ähnliches Messer vom Dörhai nahe der Winzenburg (Abb. 186h) mit einer floral anmutenden Kupfertauschierung stammt aus der zweiten Hälfte des 12. Jahrhunderts ${ }^{407}$.

Das hervorragend erhaltene Messer aus dem Nonnenchor des Klosters Wienhausen (Abb. 186i) weist neben einer silbertauschierten Klinge ein mit Silberblech ummanteltes Heft und einen verzierten Griff auf. Weitere Beispiele, wie das Messer von der Wiprechtsburg (Abb. 186b) und das Fragment eines griffplättchenverzierten Messers mit kupfertauschierter Klinge aus Minden (um 1300) ${ }^{408}$ lassen zumindest vermuten, daß in der Regel an einer verzierten Messerklinge ein verzierter oder jedenfalls kostbarer Griff befestigt wurde - der umgekehrte Schluß ist in dieser Form nicht möglich.

\footnotetext{
403 Zur Technik von Klingenverzierungen bzw. des Damaszierungseffekts vgl. u.a. Holubowicz 1956, Abb. 58, S. 154 und S. 156-160 sowie Piaskowski 1960, Abb. 52 und 58, sowie S. 77 


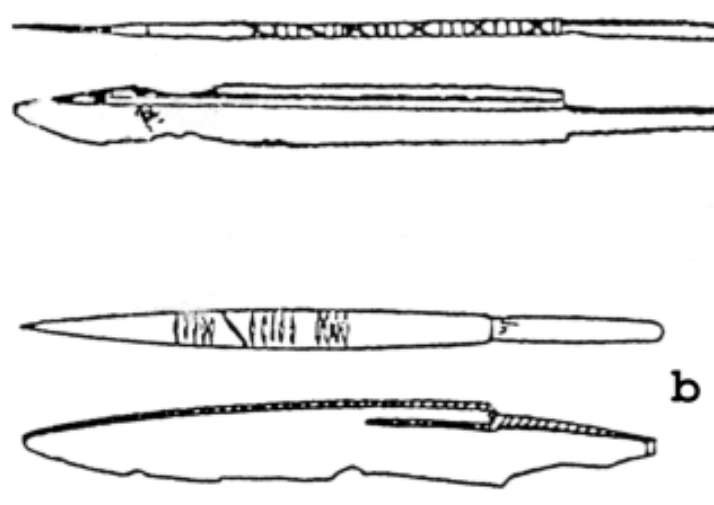

\section{a}

$b$
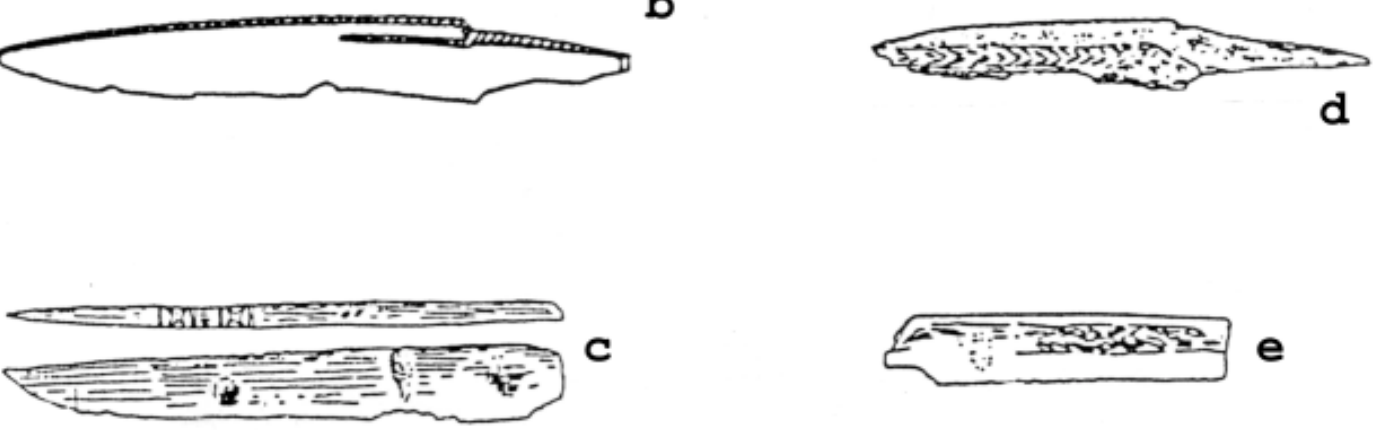

d

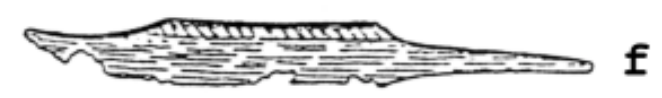

\section{f}
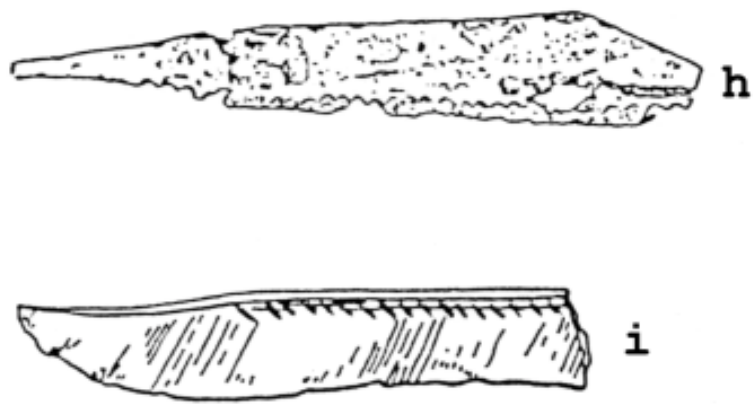

Abb. 185 Klingenverzierungen I

a: Novogrudok, A.-M. 12. Jh.; b: Novogrudok, 2. H. 12. Jh.; c: Posen, 14.-15. Jh.; d-e: Oppeln, 12. Jh.; f: Burgwall in Bardy, 2.H. 9. - 2.H. 10. Jh.; g: Lund, um 1020 -1050 (außer Katalog); h: Oppeln, 12. Jh.; i: Novogrudok, 2.H. 12. Jh. 

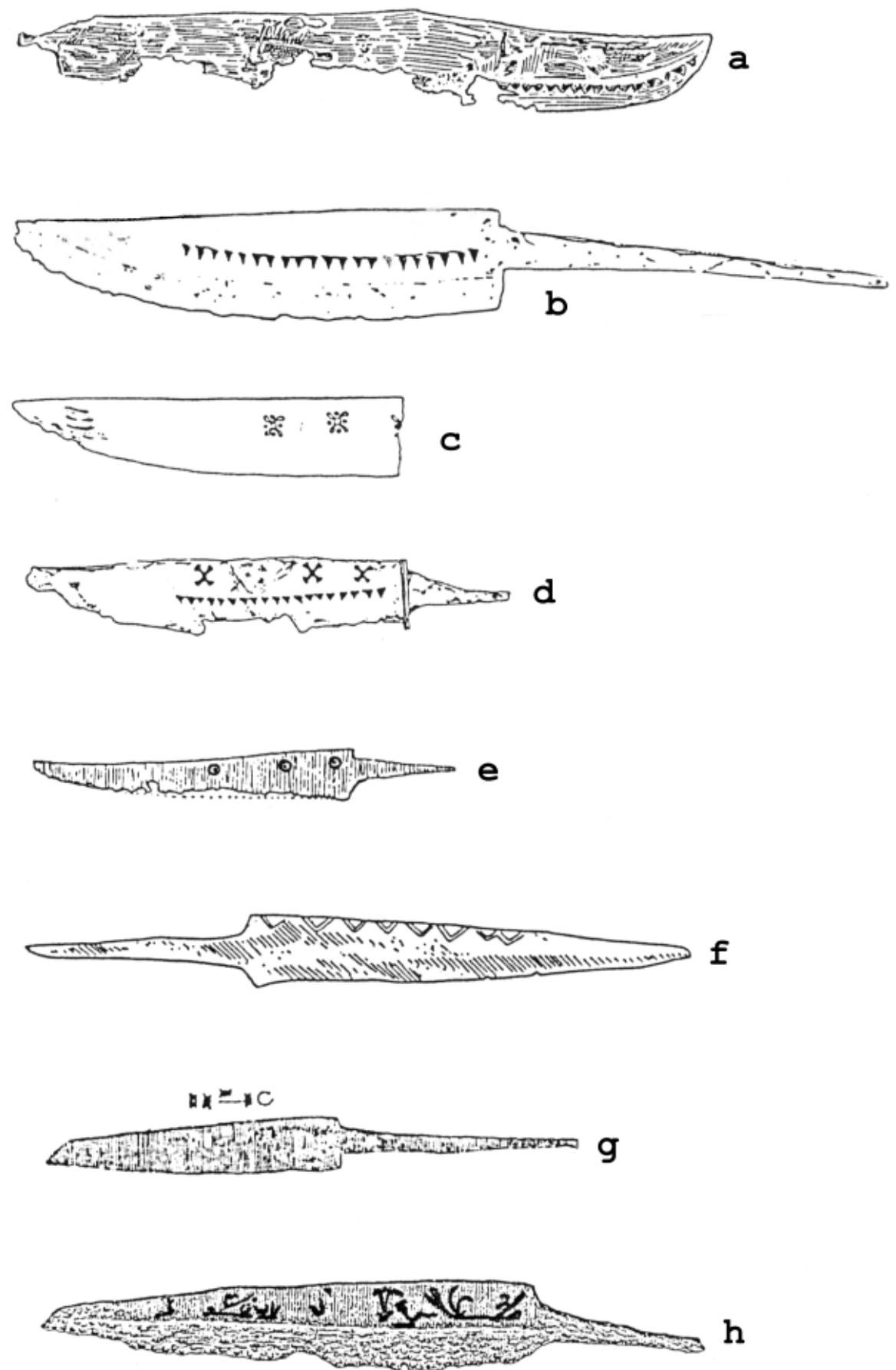

Abb. 186

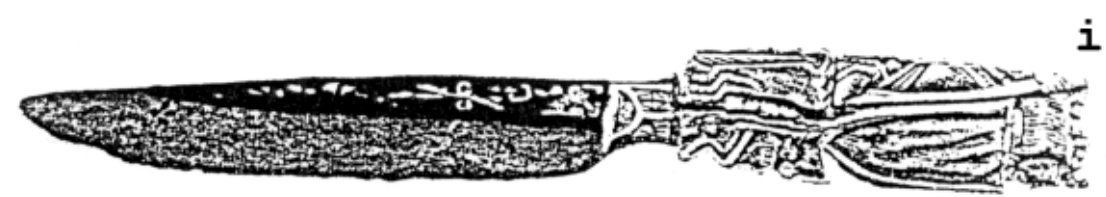

Klingenverzierungen II

a: Gnesen, 10.-13. Jh.; b: Leipzig, 13. Jh.; c: Breslau, 12.-13. Jh.;

d: Wiprechtsburg, 1224-1307; e: Piekáry, 2.H. 13.-1.H. 14.Jh.; f: Grodno, 13.-14.

Jh.; g: Demmin, 12.-13. Jh. (?) (außer Katalog); h: dörhai, E.12. Jh. (?);

$i$ : Kloster Wienhausen, um 1300 (?) 

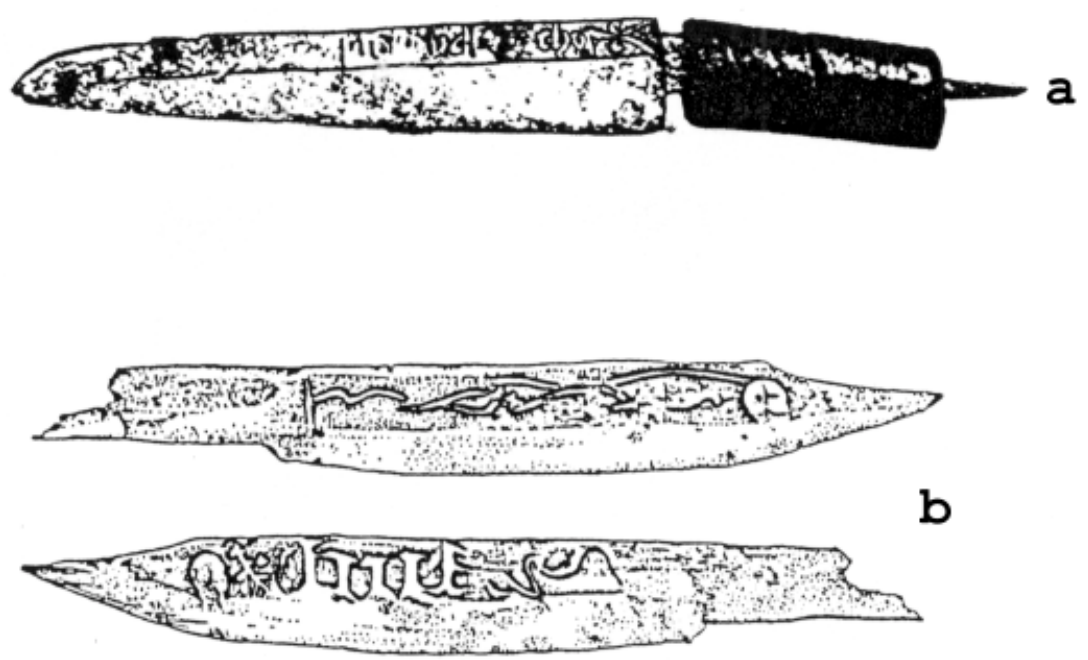

b
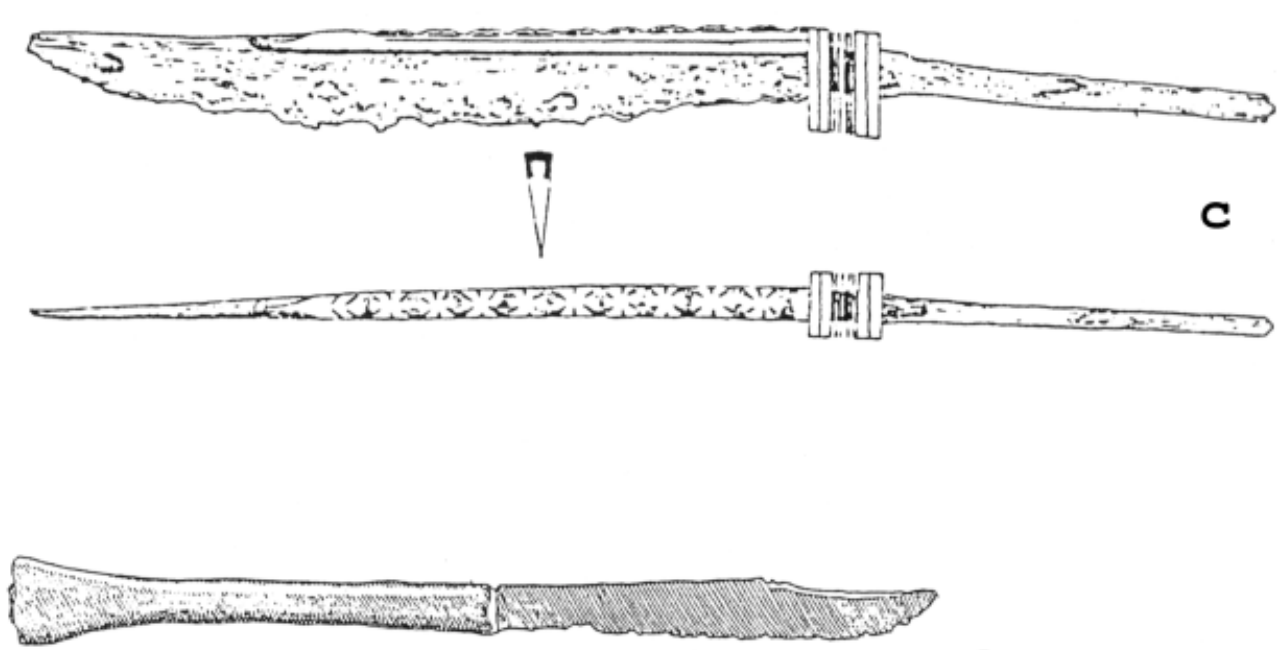

d

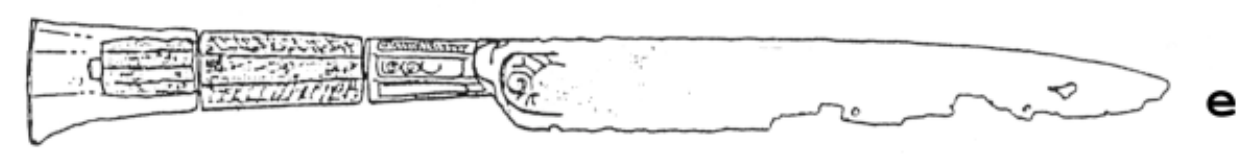

Abb. 187

Klingenverzierungen III

a: Lübeck (außer Katalog, vgl. Anm. 410); Lübeck, 14.-15. Jh. (?);

c: Wildberg, 2.H. 14.-15. Jh.; d. Behren-Lübchin, E. 10.Jh. - um 1200;

e: Hamburg, um 1600 
Messer wie die vom Dörhai, aus dem Kloster Wienhausen, aus der Wildburg und der Wiprechtsburg schränken die Besitzer dieser zum Teil sehr kostbaren Messer auf eine Gruppe ein, die mit hoher Wahrscheinlichkeit im Umkreis vermögender und einflußreicher Bevölkerungsgruppen angesiedelt werden kann.

In selteneren Fällen treten Messer auf, deren Klingen Inschriften aufweisen. Wie bei den tauschierten Klingen mit Mustern übertragener Ornamente sind sie in der Regel in breiten, oberhalb der Schneidenpartie längs des Rückens verlaufenden Zonen angebracht worden.

Als Beispiel seien zwei Messer vorgestellt, die in Lübeck gefunden wurden (Abb. 187a, 187b). Über die Technik (Gravur, Ätzung?) ist dem Autor bisher nichts bekannt geworden ${ }^{409}$.

Auch über die Bedeutung der Inschriften können bestenfalls Vermutungen angestellt werden ${ }^{410}$.

Sie sind aber als Beispiel für eine persönliche Beziehung des Besitzers zu seinem Messer anzusehen, die im Zusammenhang mit der Frage Bedeutung gewinnt, wann das an die Person des Besitzers gebundene Mehrzweck- oder Tafelmesser durch allgemein gebräuchliche, in Form und Herstellung standardisierte Bestecke ersetzt worden ist, die nicht mehr ständig an eine Person gebunden, sondern zum Beispiel vom Gastgeber den Gästen zur Verfügung gestellt wurden. Die Standardisierung in Produktionstechnik, Formgebung und Materialverwendung setzt wahrscheinlich verstärkt erst im 16. Jahrhundert ein ${ }^{411}$, sie betrifft aber in erster Linie Tafelbestecke; persönliche Messer wie die niederländischen "Seemannsmesser" des ausgehenden 16. bis 18. Jahrhunderts belegen die Kontinuität des "privaten" Messers in anderen Funktionsbereichen ebenso wie das norwegische "Tollekniv" oder die bis heute bekannten "Fahrtenmesser"412.

An dieser Stelle sei ein Messer aus Svendborg erwähnt, welches sich im Dänischen Nationalmuseum Kopenhagen befindet. Auf dem Geweihgriff des Angelmessers befindet sich eine Runeninschrift folgenden Inhalts: "Karl schlug das Zeichen in das Heft, Are stellte den Schaft her" ${ }^{413}$. Das Messer wird in das 13. Jahrhundert datiert. Inschriften dieser Art, die auf den Hersteller verweisen und zum Beispiel auf Schwertern vorkommen, sind auf Messerklingen dem Autor bisher nicht bekannt geworden ${ }^{414}$.

\footnotetext{
410 Zur Bedeutung des Wortes "hilf" als Weiheinschrift vgl. Kapitel 9.1.3

411 Vgl. die entsprechenden Auswertungen der Griffkonstruktionen u.a. am Beispiel von Messern aus niederländischen Fundorten in diesem Kapitel

412 "Tollekniv" = Allfunktionales (Mehrzweck-) Messer, vgl. die entsprechenden Ausführungen in Kapitel 6 zu der Frage der Kontinuität des privaten Mehrzweckmessers, vgl. z.B. Katalogteil Niederlande, Kat. Nr. 94 wird in der ständigen Ausstellung der mittelalterlichen Abteilung gezeigt, Inv. Nr. D-8728 
Auf einer bei Southwark aus der Themse geborgenen Messerklinge ist in ähnlicher Art wie bei den Messern vom Dörhai und aus dem Kloster Wienhausen die unterhalb des Rückens verlaufende Klingenpartie beidseitig mit einem silbertauschierten Band aus verschiedenen übertragenen Mustern besetzt ${ }^{415}$. Das Messer wird als "ostbaltischer Import" angesehen, mit finnischen Parallelfunden verglichen und in die Zeit zwischen 1100 und 1250/1300 datiert ${ }^{416}$.

Die bisher vorgestellten Klingenverzierungen fallen vor allem in die Zeit des 10 . bis 14. Jahrhunderts. Eindeutig datierte Messer mit verzierten Klingen aus dem 15. und beginnenden 16. Jahrhundert ließen sich im bearbeiteten Bestand nicht nachweisen.

Erst im Verlauf und am Ende des 16. Jahrhunderts treten verzierte Messerklingen wieder häufiger auf, im archäologischen Fundgut sind sie jedoch nur selten vertreten (Abb. 187e, sowie ein Messer aus Randers) ${ }^{417}$.

Anhand obertägig verwahrter Messer des 16. bis 17. Jahrhunderts aus der Sammlung d'Allemagne (Rouen) seien zum Schluß abweichend der bisherigen Beschränkung auf archäologisches Fundgut die zumeist den Heftbereich der Klingen umfassenden Verzierungen dieser Zeit am Beispiel gut erhaltener Tafelmesser illustriert (Abb. 188) ${ }^{418}$.

Da aus der Literatur oft nicht zu entnehmen war, ob und mit welchen Metallen die eingeschlagenen Muster in den Klingen ausgelegt worden waren, können keine Angaben über die Häufigkeit von gepunzten oder tauschierten Klingenverzierungen gemacht werden. Möglicherweise haben beide Techniken vom 9./10. bis 13./14. Jahrhundert nebeneinander existiert.

Angaben über regionale Verteilungen können ebenfalls nicht vorgenommen werden. Der zahlenmäßige Überhang von verzierten Klingen aus Fundorten der ehemaligen DDR, Polens und der ehemaligen UdSSR vom 9. bis 13. Jahrhundert findet seine Erklärung in der Selbstverständlichkeit, mit der in diesen Ländern seit über 30 Jahren metallurgische Untersuchungen an Metallfunden vorgenommen werden.

Viele Klingenverzierungen sind wegen der schlechten Erhaltung der Bodenfunde erst durch Röntgenaufnahmen oder chemische Behandlung der Klingen sichtbar zu machen, so daß sich das Verteilungsbild verzierter Messerklingen sicherlich noch korrigieren läßt.

\footnotetext{
415 Vgl. Cowen 1971, S. 281-285

416 Cowen 1971, S. 285

417 Vgl. Katalogteil Dänemark, Kat. Nr. 22

418 Die Abbildungen wurden folgender Publikation entnommen: d'Allemagne 1968
} 

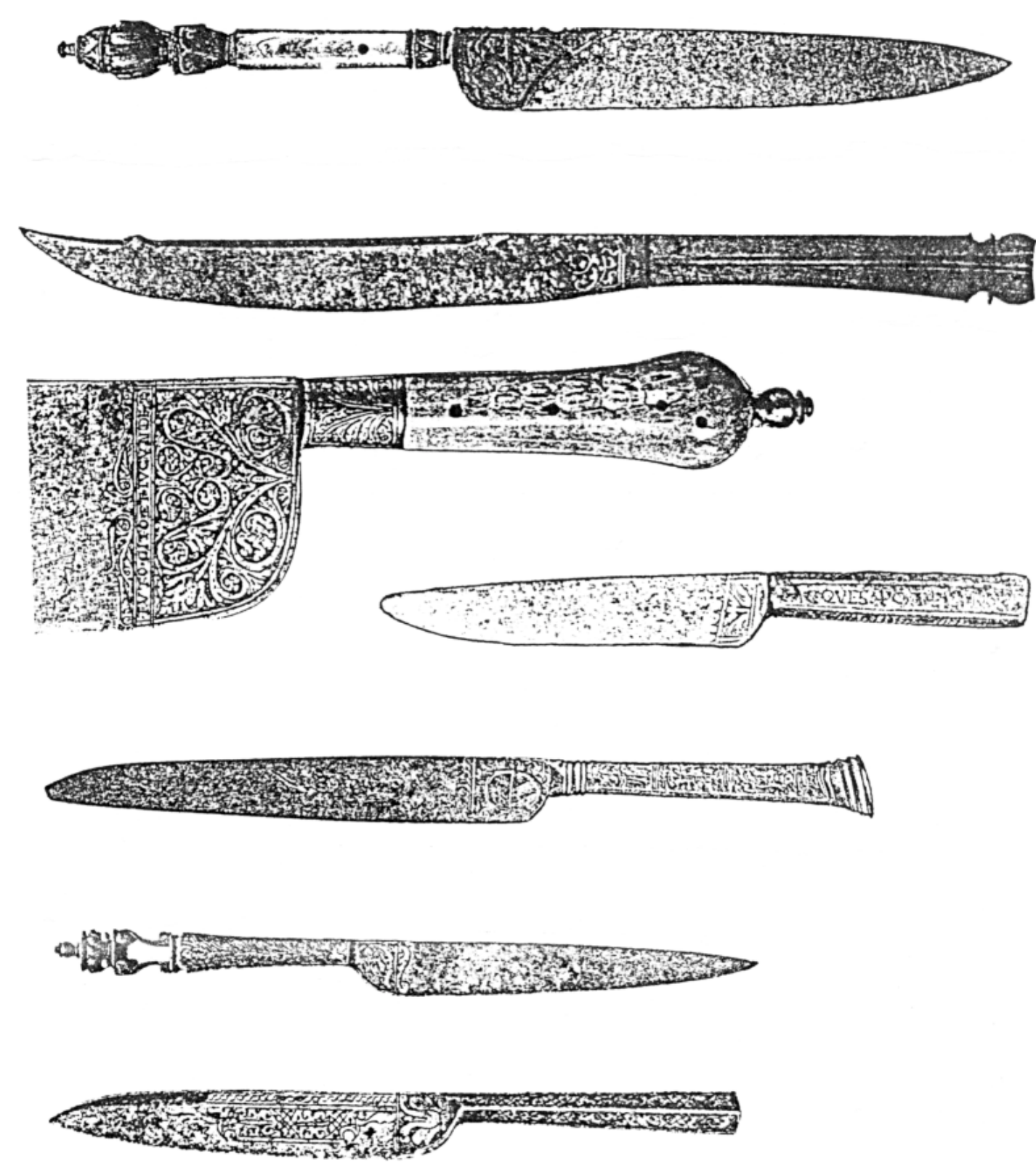

Abb. $188 \quad$ Klingenverzierungen IV

Beispiele verzierter Messerklingen des 16. Jahrhunderts (aus der Sammlung René d'Allemagne, Rouen) 


\subsubsection{Die Klingenmarken}

Die auf insgesamt 54 Messern nachweisbaren Klingenmarken werden in der historischen Literatur als Gütezeichen der Produzenten und - soweit es sich um zwei, seltener um mehrere Marken handelt - des die Produktion überwachenden Prüfers angesehen $^{419}$. Danach gaben sie nicht nur Auskunft über den Produktionsort sowie über Vertrieb und Absatz der gemarkten Ware, sondern wären im Fundgut auch als Indiz für ein bereits bestehendes organisiertes und spezialisiertes Handwerk anzusehen, welches aus historischen Quellen seit dem 14. Jahrhundert bekannt ist.

Die Marken befinden sich in der Regel auf der sogenannten Schauseite der Messerklinge, der Seite des Schneidenanschliffs, sie sind in die Klinge eingeschlagen und zum Teil mit Bunt oder Edelmetall ausgelegt worden ${ }^{420}$.

Die ältesten Beispiele gemarkter Messerklingen stammen aus dem 13. Jahrhundert und aus der Zeit um 1300 (Wienhausen, Ribe) ${ }^{421}$, ältere, ähnlich wertvolle Messer des 12. Jahrhunderts mit Klingenverzierungen (Dörhai-Winzenburg) ${ }^{422}$ weisen noch keine Marken auf.

Schriftliche Erwähnungen von Messerschmieden als Mitglieder eines eigenständigen, von anderen eisenverarbeitenden Produktionsgruppen unterschiedenen Berufszweiges setzen 1285 mit einem Nürnberger Messerschmied ein, dort wird bereits 1290 zwischen Klingen- und Messerschmieden unterschieden ${ }^{423}$.

Im 13. Jahrhundert wird in Braunschweig der Eigenname "metzetwerchte (cultellifex)" erwähnt. Der Messerschmied als von den Schmieden unterschiedener Berufszweig wird dort 1388 genannt. Das Gründungsdatum der zunftmäßig organisierten Messerschmiede in Braunschweig ist möglicherweise das Jahr $1341^{424}$.

Ebenfalls kurz nach 1300 tauchen auf den in der Londoner Innenstadt ausgegrabenen Messern in bereits erheblichem Umfang Klingenmarken auf. Die älteste Marke

\footnotetext{
419 Bracker und Fahl 1983, S. 46

Glaub o.J., S. 10

Helferich-Dörner 1959, S. 37

Timpel 1982, S. 72/74 und Anmerkung 19, S. 96

Wernet 1960, S. 465-468

420

Fuhse 1935, S. 155

Baart u.a. 1977, S. 325-326; Cowgill u.a. 1987, S. 20

Von 61 erfaßten Marken (davon 54 von im Katalog enthaltenen Messern) waren neun Marken nachweis-

421 lich mit Buntmetall ausgelegt.

421 Vgl. Katalogteil BRD, Kat. Nr. 74; Dänemark, Kat. Nr. 1, sowie Kapitel 6.2.4.2.1.3 (Wienhausen)

422 Vgl. Katalogteil BRD, Kat. Nr. 79

423 Wernet 1960, S. 465-466

Glaub o.J., S. 5

Helferich-Dörner 1959, S. 31

Fuhse 1930, S. 36

Fuhse 1935, S. 154
} 
befindet sich dort auf einer Messerklinge des 13. Jahrhunderts. Die Marken beinhalten eine bedeutende Anzahl von verschiedenen Buchstaben, Ornamenten, Zeichen und Symbolen, die zum Teil mit den in dieser Arbeit behandelten Marken korrespondieren. Von den in London gefundenen 110 gemarkten Messerklingen weisen 19 Doppelmarken auf; fast die Hälfte aller Marken ist mit Buntmetall ausgelegt worden.

Über zwei Drittel der Marken waren zunächst nicht sichtbar; sie wurden erst nach einer radiographischen Behandlung der Klingen nachgewiesen. Damit erklärt sich auch die geringe Anzahl von Klingenmarken in dieser Arbeit, die in keinem Verhältnis zur bearbeiteten Menge an Messern auch nach 1300 steht $^{425}$.

In Amsterdam setzen gemarkte Messerklingen in der ersten Hälfte des 14. Jahrhunderts ein ${ }^{426}$.

Abbildungen von Messerschmieden sind unter anderem aus den Hausbüchern der Mendelschen und Landauerschen Zwölf-Brüderstiftungen bekannt, die älteste wird um 1410 datiert, weitere Darstellungen folgen 1446, 1476, 1523, 1542 etc. $^{427}$ (Abb. $191 \mathrm{a}-\mathrm{c})^{428}$. Auch im 1568 in Frankfurt/Main gedruckten sogenannten Ständebuch, deren von Jost Amman gefertigte Holzschnitte mit Versen des Schuhmachermeisters Hans Sachs unterlegt sind (Abb. 191d), ist die Darstellung eines "Messerschmidts" enthalten mit einem Hinweis auf zweierlei Qualitätsstufen innerhalb der Produktion, die auch ihren Niederschlag im Preis fanden, sowie mit der Bemerkung:

"Wer dieser meiner arbeit darff/

Der find mein Zeichen grecht und scharff."

Parallel zu den im 15. und 16. Jahrhundert häufiger werdenden schriftlichen und bildlichen Erwähnungen von Vertretern der Messerschmiede verteilt sich der Anteil der gemarkten Klingen aus dem bearbeiteten Fundgut auf die einzelnen Jahrhunderte (Abb. 189, 190).

Nach den beiden erwähnten Klingen um 1300 sind im 14. Jahrhundert fünf, im 14. bis 15. Jahrhundert drei, im 15. Jahrhundert acht, im 15. bis 16. Jahrhundert sechs, im 16. Jahrhundert 13, im 16. bis 17. und frühen17. Jahrhundert 24 gemarkte Messerklingen nachgewiesen worden ${ }^{430}$.

\footnotetext{
425 Cowgill u.a. 1987, S. VIII f., S. 8 und S. 17-24

426 Baart u.a. 1977, S. $326 \mathrm{ff}$

427 Helferich-Dörner 1959, S. 24

Glaub o.J., S. 9

428 a-c: Helferich-Dörner 1959, Abb. S. 32 und 33

d: Amman 1975, Abb. S. 63

429 Amman 1975, S. 63

430 Vgl. Abb. 138-139, die Messer Polen Nr. 128 und 160 sowie ehemalige UdSSR Nr. 67 und 71 (Rückenmarkierungen) wurden nicht mitgezählt
} 
Dänemark

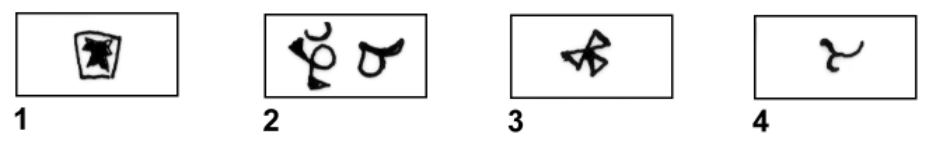

Schweden

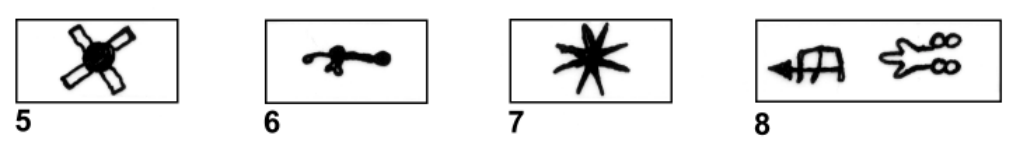

Niederlande
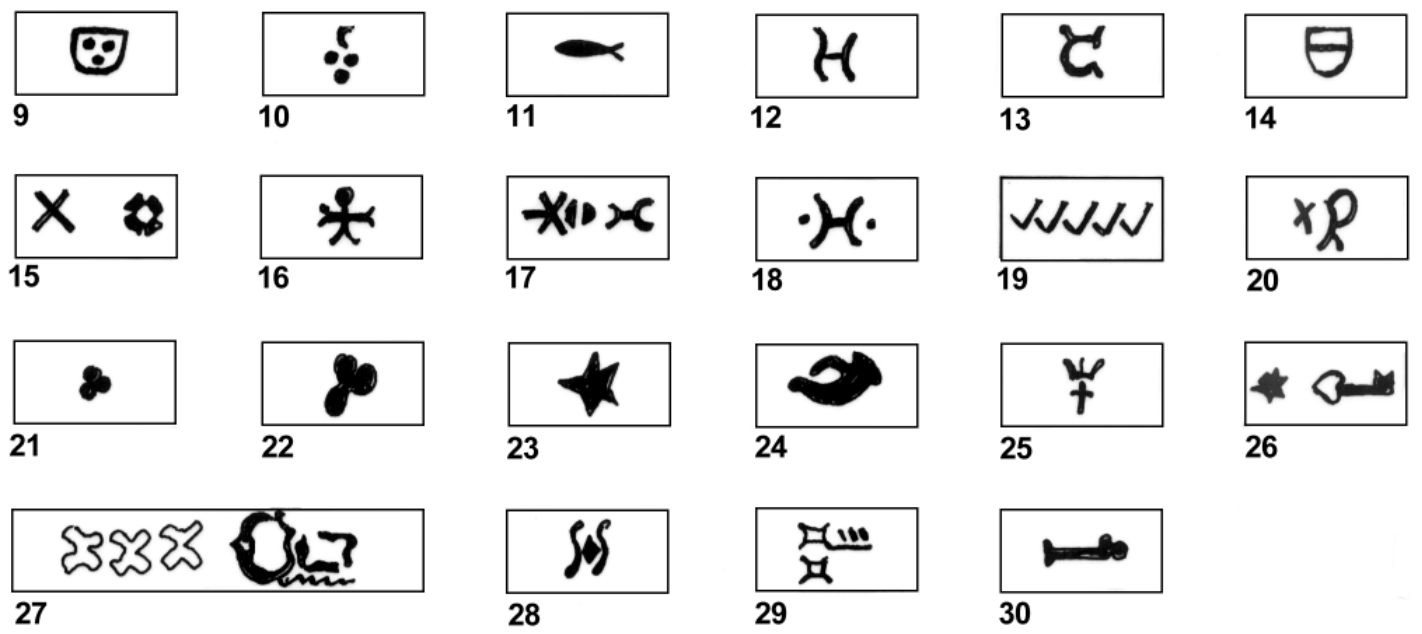

\section{Bundesrepublik}
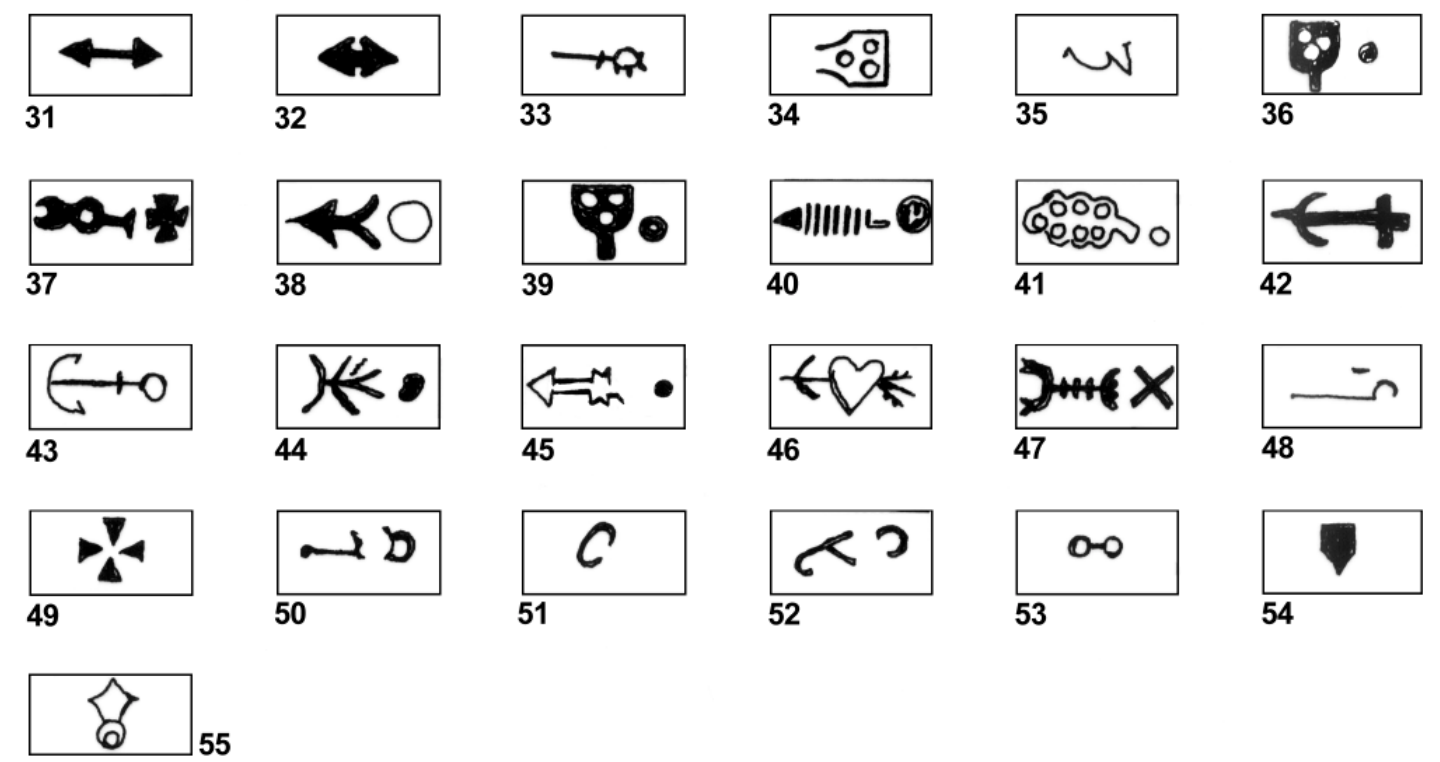

Abb. 189 
ehemalige DDR

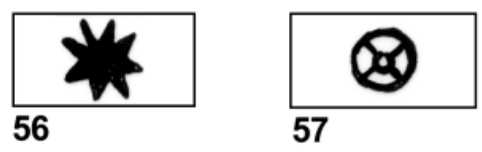

Polen

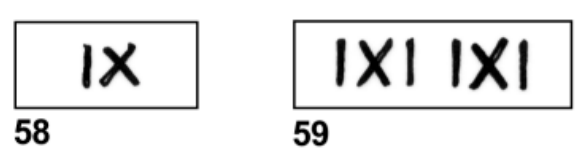

ehemalige UdSSR

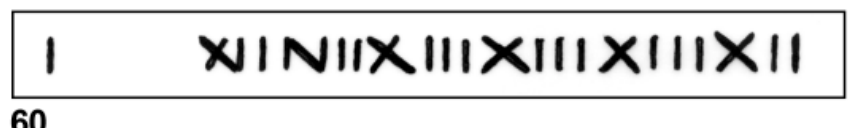

60

Abb. 190 

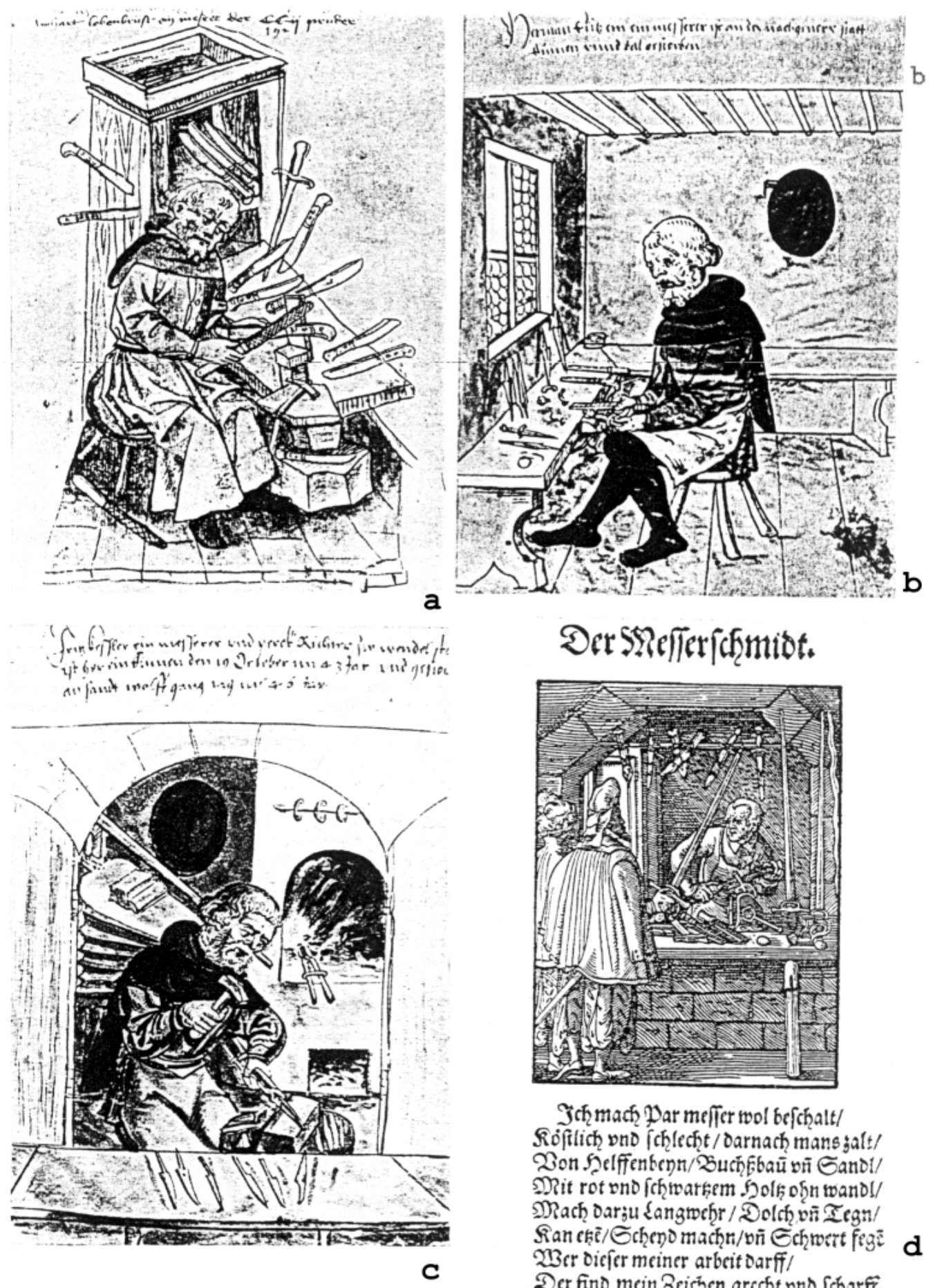

Ichrmact Par mefier wol befchalt/ Röftlich vno icḩlectht / Darnach mans galt/

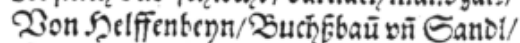
Nit rot vnd ichinathem Sols ofn twand//

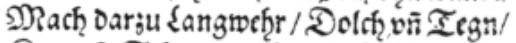
Sancểe/ Echerno machn/vñ Echwert fege W3er oicjer meiner arbeit darff/

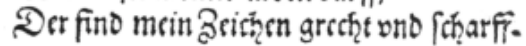

Abb. 191

Die Herstellung von Messern in zeitgenössischen Darstellungen ${ }^{431}$ a: Hausbuch der Mendelschen Zwölf-Brüderstiftung, 1476; b-c: Hausbuch der Landauerschen Zwölf-Brüderstiftung, um 1542 und 1543-1546; d: Jost Amman, Ständebuch, 1568

431 a-c: Helferich-Dörner 1959, Abb. S. 32 und 33 d: Amman 1975, Abb. S. 63 
Um einen Eindruck von der Abhängigkeit dieser Zahlen von der Relativität des bearbeiteten Fundgutes im Vergleich zu den tatsächlichen Verhältnissen vor allem des letzten Zeitabschnitts zu vermitteln, sei darauf verwiesen, daß von den 24 Marken des 16. bis 17. und frühen 17. Jahrhunderts 20 Marken auf Klingen des Hamburger Wrackfundes entfallen ${ }^{432}$.

Aus dem Ammanschen Ständebuch ist bereits zitiert worden, daß zum Beispiel um 1560/70 der Nürnberger Messerschmied Messer unterschiedlicher Güte herstellte. Bereits für Messer des 11. bis 13. Jahrhunderts aus $\operatorname{Posen}^{433}$, des 10. bis 12. Jahrhunderts aus Oppeln ${ }^{434}$ und des 10. bis 14. Jahrhunderts aus Danzig ${ }^{435}$ sind aufgrund eingehender metallurgischer Untersuchungen ebenfalls erhebliche Qualitätsunterschiede bezüglich der Klingenproduktion nachweisbar, die nicht nur über einen längeren Zeitraum durch veränderte Produktionsverhältnisse und Absatzbedingungen verursachte Prozesse dokumentieren. Da sie auch an zeitgleichen Beständen auftreten, sind auch zeitgleich verschiedene Käufergruppen anzunehmen. Ähnliche Unterschiede sind bei einer Untersuchung von Messerklingen des 9. bis 10. Jahrhunderts aus Haithabu nachgewiesen worden ${ }^{436}$.

Bereits im 11. bis 12. Jahrhundert treten in Polen und im ehemaligen Litauen Messer auf, die in den Rücken der Klinge eingeschlagene Muster zeichenhaften Charakters aufweisen ${ }^{437}$. Eine Verbindung zwischen den Qualitätsstufen der Klingen und diesen Mustern ist allerdings bisher kaum herzustellen, da es neben den wenigen derart behandelten Klingen zahlreiche ungemarkte Klingen gibt, die mit großem produktionstechnischen Aufwand hergestellt worden sind ${ }^{438}$.

Mit der Vorlage des Fundmaterials der Burg und Siedlung Gommerstedt (Thüringen) liegen aus einem zeitlich begrenzten Siedlungskomplex 92 Messer vor, die zum Teil am Ort produziert, zum Teil importiert worden sein sollen. Unter den letzteren befinden sich zwei gemarkte Klingen des 13. bis 14. Jahrhunderts, die der Bearbeiter mit einer 1424 erwähnten "weit entwickelten Zunft der Messerer" in Schmalkalden in eine mögliche Verbindung bringt ${ }^{439}$.

Angesichts der historisch oft zitierten Auffassung über Kontrollen, der die Endprodukte vor dem Absatz unterlagen, sowie über die Verpflichtung eines jeden der

\footnotetext{
432 Vgl. Katalogteil BRD, Kat. Nr. 52, 54-57, 59, sowie Abb. 139. Die Zeichnungen der Marken des Hamburger Wrackfundes a-h wurden folgender Publikation entnommen: Bracker und Fahl 1983, Abb. Anlage 1, S. 49 
Zunft angehörigen Meisters zur Markierung seines Produkts ${ }^{440}$ läßt das Verhältnis von zwei gemarkten zu neunzig ungemarkten Messern im 13. bis 14. Jahrhundert auch dann Vermutungen über die tatsächlichen Verhältnisse entstehen, wenn man berücksichtigt, daß es sich bei diesem Zeitabschnitt um den Beginn des nachweislich faßbaren, spezialisierten Messerschmiedehandwerks in großen Städten wie Braunschweig und Nürnberg handelt.

Die Folgerung, daß die Bewohner von Gommerstedt einen Teil ihrer eisernen Bedarfsgüter importiert haben ${ }^{441}$, ist nur schlüssig, wenn man davon ausgeht, daß jedes gemarkte Objekt aus organisierter städtischer Produktion, jedes ungemarkte Objekt aus nicht organisierten, möglicherweise dörflichen oder Burgschmieden stammt ${ }^{442}$.

Diese neben den stadtgebundenen, teiweise spezialisierten Messerschmieden existierenden Dorf- und Burgschmiede haben, das zeigt das Fundmaterial von Gommerstedt, wahrscheinlich keine Marken verwendet, allem Anschein nach aber das Gros des "täglichen Bedarfs" der Umgebung an Eisengerät wie neben Messern auch Hufeisen, Nägel, Beschlagteile, zum Teil auch Waffen (Bolzen, Pfeilspitzen u.ä.) geliefert ${ }^{443}$.

Spezialisierte Schmiedehandwerke sind ausweislich der archäologischen Quellen innerhalb des Bearbeitungszeitraums gebunden an Siedlungen frühstädtischen Charakters mit Handwerk, Handel und zum größten Teil auch mit zentralen Verwaltungsfunktionen und vom 10./11. bis 14. Jahrhundert zunächst anhand aufwendiger und differenzierter Produktionstechniken nachweisbar, die am Produkt selbst feststellbar sind ${ }^{44}$. Aufwendig hergestellte und/oder verzierte Messerklingen sind selbst im 14. Jahrhundert nur selten gemarkt worden.

$\mathrm{Da} ß$ es in nachmittelalterlichen Großstädten tatsächlich Kontrollen gegeben hat, das heißt, daß hinter den Marken ein gewisser Anspruch stand, der aber inhaltlich noch immer weitgehend offen ist, zeigt ein Messerfund des 16. Jahrhunderts, der 1969 in London aus der Themse geborgen wurde. Es handelt sich dabei um 60 Messer mit verbogenen Klingen, die als von der Londoner Messerschmiedezunft verworfene Ware interpretiert wurde ${ }^{445}$.

\footnotetext{
440 Timpel 1982, Anmerkung 19, S. 96 Helferich-Dörner 1959, S. 37 Wernet 1960, S. 466 Timpel 1982, S. 72 und 74; diese Annahme wurde unter der Voraussetzung getroffen, daß die von den städtischen Messerschmieden produzierte Ware, die den Gütebestimmungen der Zunft entsprach, in jedem Fall mit Marken versehen wurde.

442 Vgl. die Diskussion in Kapitel 9, Abschnitt 1.3

443 Timpel 1982, Anmerkung 16, S. 96 Eversberg 1982, S. 22 und S. 37

444 Vgl. Kapitel 9, Abschnitt 1 (Produktionstechniken)

445 Bund 1973, S. 402-403
} 
Die 1987 vorgelegte Bearbeitung der Messerfunde aus dem mittelalterlichen Londoner Stadtkern hat für das späte 13. Jahrhundert die erste Marke auf einer Messerklinge erbracht, einen Halbmond ${ }^{446}$. Der zeitgleiche Bestand enthält daneben eine an Rücken und Schauseite tauschierte Messerklinge, die ungemarkt ist ${ }^{447}$.

Im frühen und hohen 14. Jahrhundert sind Klingen etwas regelmäßiger gemarkt worden, aber seit dem späten 14. und frühen 15. Jahrhundert sind ungemarkte Klingen die Ausnahme ${ }^{448}$.

Die bisher zitierte Auffassung, das Messerschmiedehandwerk sei seit ca. 1300 ein spezialisiertes, zum Teil eigenständiges oder den Schmiedezünften angeschlossenes Handwerk, dessen Bedeutung bereits im 14. Jahrhundert erheblich, dessen Blüte aber im 15. bis 17. Jahrhundert liege ${ }^{449}$, muß differenzierter betrachtet werden.

Unter Berücksichtigung regionaler Besonderheiten wie Rohstoffunabhängigkeit und Verbindungen zu älterem oder gleichzeitig bestehendem Eisengewerbe (z.B. Schmalkalden, Märkisches Sauerland) ${ }^{450}$ sind möglicherweise auch außerhalb des organisierten städtischen Handwerks Qualitätserzeugnisse auf dem Land hergestellt worden. Dabei ist generell zu beachten, daß die meisten historischen Quellen, die bezüglich des spezialisierten städtischen Handwerks zitiert werden, erst im 15. Jahrhundert einsetzen.

Serielle Analysen der Klingen, das zeigt das Beispiel Novgorod, bringen eher Erkenntnisse über die tatsächlichen Verhältnisse, deren regionale Unterschiede aufgrund verschiedenartiger historischer Gegebenheiten sich bereits abzeichnet ${ }^{451}$.

Zur weiteren Arbeitsteilung innerhalb der Messerherstellung sei am Schluß auf die Anmerkung von Mummenhoff verwiesen, nach der die Nürnberger Messerschmiede das Horn für die Griffschalen u.a. aus den Abnehmerorten ihrer Ware bezogen und es vor der Montage von den Kammachern zuschneiden lassen mußten ${ }^{452}$.

\footnotetext{
446 Cowgill u.a. 1987, S. 81

447 Cowgill u.a. 1987, S. 81

448 Cowgill u.a. 1987, S. $84 \mathrm{ff}$

449 Helferich-Dörner 1959, S. 31, 34, 39

Glaub o.J., S. 6

$\mathrm{Da} ß$ die schriftlichen Erwähnungen wie auch die gemarkten Klingen mit dem 15. Jahrhundert stark zu-

nehmen, dokumentiert eher die Normierung der Ware als eine Zunahme von Produktion und Absatz.

Eversberg 1982, S. $11 \mathrm{ff}$

Sprandel 1974, S. S. 7-17

451 Vgl. Kapitel 9, Abschnitt 1.1

452 Mummenhoff 1924, S. 109-110, ohne zeitliche Angaben
} 


\subsubsection{Analyse fundortspezifischer Unterschiede von Mes- serklingen}

Die folgende Untersuchung versucht, das Aufkommen von Klingenformen in Städten, Burgen und ländlichen Siedlungen miteinander zu vergleichen, um Hinweise $\mathrm{zu}$ erhalten, ob sich regionale, wirtschaftliche oder soziale Unterschiede im Formenspektrum des untersuchten Fundguts manifestieren.

Eine Analyse der Zusammensetzung vom Messerklingenformen aus spezifischen Fundortgruppen, deren Funktionen weitgehend unbekannt und deren Benutzer nur im weitesten Sinne sozial eingrenzbar sind, kann nur feststellen, ob sich die Klingenformenbestände aus Städten oder Burgen in einem vergleichbaren Zeitraum generell voneinander unterscheiden, oder ob zeitgleiche Bestände in Städten unterschiedlicher Regionen, d.h. im überregionalen Vergleich, einander in etwa entsprechen.

Jede weitergehende Fragestellung wie etwa Unterschiede innerhalb eines städtischen Sozialgefüges muß an den quellenkritischen Einschränkungen, denen das gesamte hier bearbeitete Fundgut unterliegt, scheitern. Eine vergleichbare Ausgangsbasis liegt weder für eine bestimmte Fundortgattung noch für einen eingrenzbaren Kulturraum vor.

Die unterschiedlichen prozentualen Anteile der einzelnen Klingenformen innerhalb der Fundorte bzw. Fundortgruppen lassen sich nicht direkt funktionalen, regionalen oder sozialen Unterschieden zuweisen. Funktionsgebundene Formen lassen sich nur im Vergleich mit Quellen anderer Disziplinen, z.B. mit bildlichen Darstellungen, herausarbeiten, und eine soziale Differenzierung wäre neben der Berücksichtigung der Fundortgattung nur mit Wissen um die Funktionen der betreffenden Messer zu treffen.

Es kann hier nur um die Frage gehen, ob sich Unterschiede im alltäglichen Leben und Arbeiten zwischen Stadt-, Landbevölkerung und Burgbewohnern überhaupt an den von ihnen im grundsätzlich gleichen Zusammenhang benutzten Messern bemerkbar machen. Dabei soll nicht nach den bekannten Wohlstandsindikatoren wie Qualität des Materials, Verzierungsgrad oder Verarbeitungsaufwand gefragt werden, sondern nach Unterschieden in der Form und nach formaler Vielfalt. Diese Indikatoren lassen Rückschlüsse auf ähnliche oder verschiedenartige Funktionen oder Funktionsspannen zu. Dabei ist zu berücksichtigen, daß die Ergebnisse nur die Gegenstände, nicht die Personen oder Lebensumstände direkt betreffen. So soll nur untersucht werden, ob sich im Funktionsrahmen von Messern Unterschiede in den Beständen von Burgen, ländlichen Siedlungen und Städten zeigen. Eine inhaltliche Beschreibung derartiger Unterschiede kann allein aufgrund des vorliegenden Materials nicht vorgenommen werden. 
Als Indikatoren für derartige Unterschiede wurden Verschiebungen in der Verteilung der Klingenformen vom 11./12. bis 14. Jahrhundert benutzt, die in dieser Zeit neben den Klingenlängen das einzige der untersuchten Kriterien zu sein scheint, an dem sich unterschiedliche Funktionen oder ein Wandel von Funktionen abzeichnen $^{453}$.

An zwei Beispielen wurden Mikroanalysen durchgeführt. Sie betreffen das slawische Siedlungskonglomerat "Lieps" im Norden und die deutsche Burg und zugehörige Siedlung Gommerstedt im Süden der ehemaligen DDR.

Außerdem wurden die Bestände westslawischer Siedlungen und Burgwälle des 11. bis 13. Jahrhunderts, deutscher Burgen und Siedlungen (meist Rodungsdörfer) des 12. bis 14. Jahrhunderts aus dem Bereich der nördlichen deutschen Mittelgebirge sowie die Bestände von ausgewählten Burgen und Städten des 12. bis 14. Jahrhunderts aus dem gesamten Untersuchungsraum analysiert.

Die Bandbreite des Formenspektrums, welche den einzelnen Analysen zugrunde liegt, entspricht dem Maximalaufkommen von Klingenformen innerhalb des untersuchten Siedlungsgebietes (z.B. nordwestslawischer Siedlungsraum) oder der untersuchten Fundortgattung (z.B. Städte des 13. bis 14. Jahrhunderts).

\section{Der Bestand von Messerklingenformen in der westslawischen Sied- lungskammer "Lieps" (Neubrandenburg, 11. bis 13. Jahrhundert)}

Aus den zahlreichen Siedlungeinheiten der Lieps wurden zwei Siedlungsplätze mit erheblichem Formenaufkommen an Messern untersucht, die Markt- und Handwerkersiedlung auf der Fischerinsel und die befestigte Siedlung auf dem Hanfwerder $^{454}$.

Die Verteilung der einzelnen Klingenformen ergab für den Gesamtbestand beider Siedlungen eine Dominanz der Formen IIIb und IIIe mit 40,9\% und 25\%, gefolgt von der Form Ib mit 18,2\% (Abb. 192). Die Formen Ilb, IVf und Va waren nur gering, die Form $\mathrm{Vb}$, von anderen slawischen Siedlungen bekannt, gar nicht vertreten.

Auf der Fischerinsel, einer Handwerker- und Marktsiedlung mit nachweislichem Anschluß an den Fernhandel, die auf das Herrschaftszentrum auf dem Hanfwerder ausgerichtet war, ergeben sich folgende Abweichungen:

Sonderform IVf kommt hier nicht vor, die Form IIb ist aber mit 4,8\% gegenüber dem allgemeinen Anteil von 2,3\% stärker vertreten (Abb. 193).

\footnotetext{
453 Die Auswahl wurde nach den Erkenntnissen der vorangegangenen Untersuchungen bezüglich der Messerklingen getroffen. Sie betrifft den Zeitraum, in dem stärkere Unterschiede der Standardabweichungen zu den Mittelwerten der Klingenlängen und das verstärkte Auftreten von Klingen der Formen IV und V im gesamten Untersuchungsraum Hinweise auf eine möglicherweise breitere Palette von funktionsgebundenen Messern geben als in der Zeit der Mehzweckmesser vor und in der Zeit der standardisierten und funktional gebundenen Massenware nach diesem Zeitraum.

Vgl. die Anmerkungen im Katalogteil ehemalige DDR unter den Katalognummern 75 und 98
} 
Der Bestand auf dem Hanfwerder, dem befestigten Hauptsiedlungsplatz in der Lieps, auf dem der Sitz einer adeligen Führungsschicht sowie ein überregionales slawisches Kultzentrum angenommen wird ${ }^{455}$,zeigt ebenfalls Unterschiede zum Gesamtbild. Der dominierende Anteil der Formen IIIb und IIIe sowie der Form Ib ist im Kern vorhanden, die anteilige Differenz zwischen IIIb und IIIe allerdings geringer (Abb. 194). Die Form IIb fehlt hier ganz, die Form IVf ist mit 4,4\% vorhanden; die hauptsächlich im Siedlungsbereich der Westslawen nachgewiesene Form Va ist mit 8,9\% gegenüber 4,8\% auf der Fischerinsel erheblich stärker vertreten.

Es hat sich gezeigt, daß auf zwei westslawischen Siedlungsplätzen mit unterschiedlichen Funktionen (Markt- und Handwerkersiedlung, politisches Zentrum) der Grundbestand an Klingenformen in etwa gleich ist, daß sich aber durchaus erhebliche Unterschiede in den Details feststellen lassen.

Im Vergleich zu dieser Analyse wurden die Bestände von anderen westslawischen Burgen und Siedlungen ${ }^{456}$ des 11. bis 13. Jahrhunderts untersucht. Sie betreffen Messer von der Mecklenburg sowie von den Burgwällen in Behren-Lübchin, Sanzkow, Fergitz, Berlin-Spandau und Berlin-Köpenick, weiterhin die Vorburgsiedlung der Mecklenburg sowie von Siedlungen im Trenntsee, in Jatzke (Vorburgsiedlung), Pasewalk, Tornow-16-Eichen und Cottbus ${ }^{457}$.

Der gesamte untersuchte Bestand weist in seiner anteiligen Verteilung der Klingenformen ein dem Gesamtbestand der Lieps ähnliches Bild auf (Abb. 195). Der Hauptbestandteil wird von den Formen IIIb, IIIe und Ib abgedeckt. Die Form Va wurde nicht mehr, die Form Vb im Gegensatz dazu nachgewiesen.

Aufschlußreich erscheint die Gegenüberstellung der Anteilsverteilungen der Messerformen von den betreffenden Burgen und den Siedlungen. Im Gegensatz zu den materiell und kulturell als herausragend zu bezeichnenden Siedlungen auf der Fischerinsel und dem Hanfwerder, die beide exponierte Funktionen innehatten, stehen sich nun Bestände von Siedlungsplätzen mit erheblich schlichteren Lebensumständen und alltäglicheren Funktionen gegenüber. Es zeigt sich hier, daß die Formenvielfalt in offenen und Vorburgsiedlungen größer ist als auf den zeitgleichen Burgen (Abb. 196, 197):

Die Formen IIb, IVf und Vb treten innerhalb der untersuchten Fundplätze nur noch in Siedlungen auf, der Bestand der innerhalb der Burgwälle wohnenden Menschen umfaßt lediglich den überall nachweisbaren Grundbestand mit den Formen Ib, IIIb

\footnotetext{
455 Spuren von handwerklicher Tätigkeit wurden nicht nachgewiesen, vgl. Katalogteil ehemalige DDR, Kat. 
und IIIe, wobei die Form Ib auf den Burgen erheblich stärker vertreten ist als in den offenen Siedlungen.

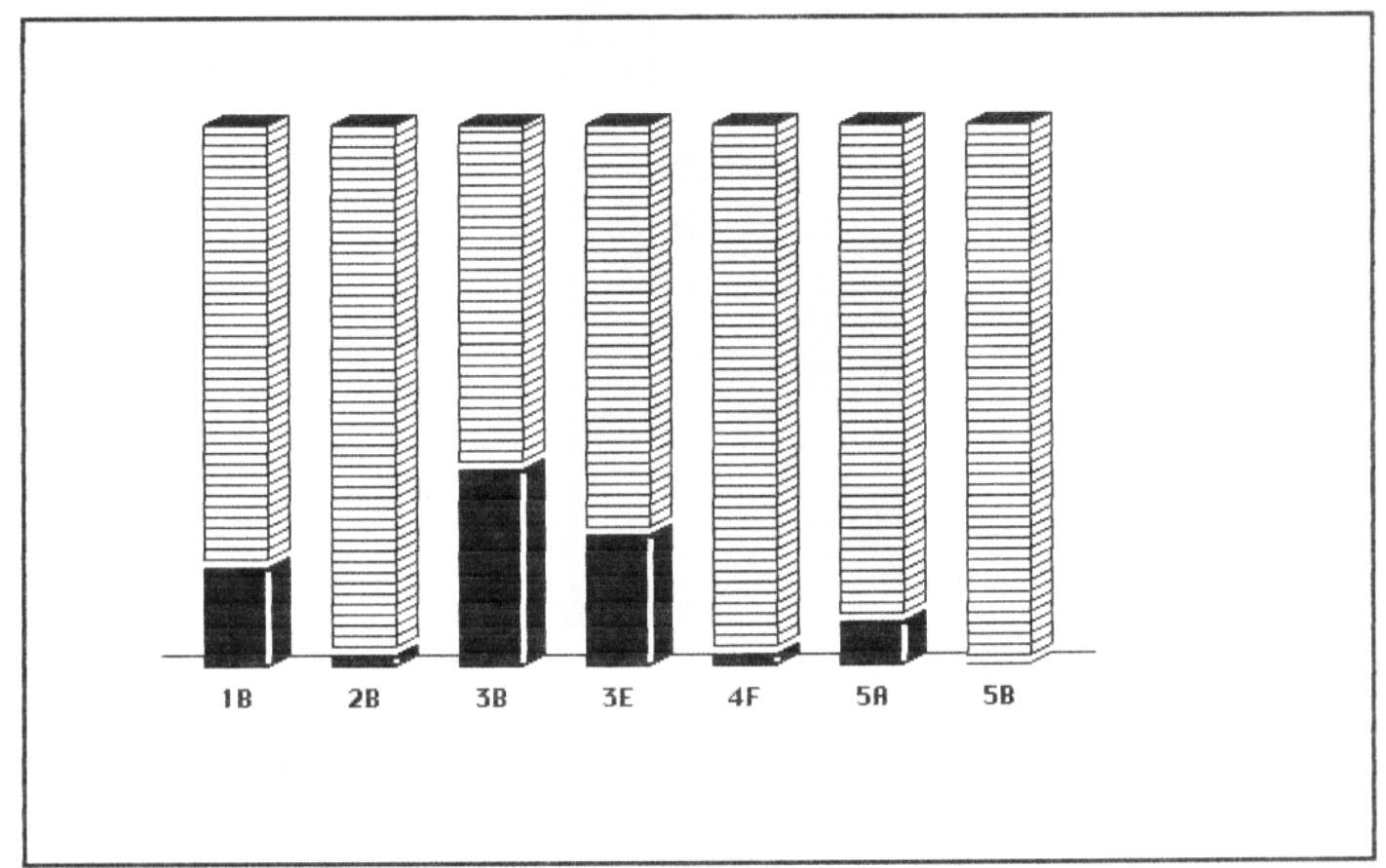

Abb. 192 Die Verteilung der Klingenformen in der spätslawischen Siedlungskammer "Lieps" im 11.. 12. und beginnendem 13. Jahrhundert 


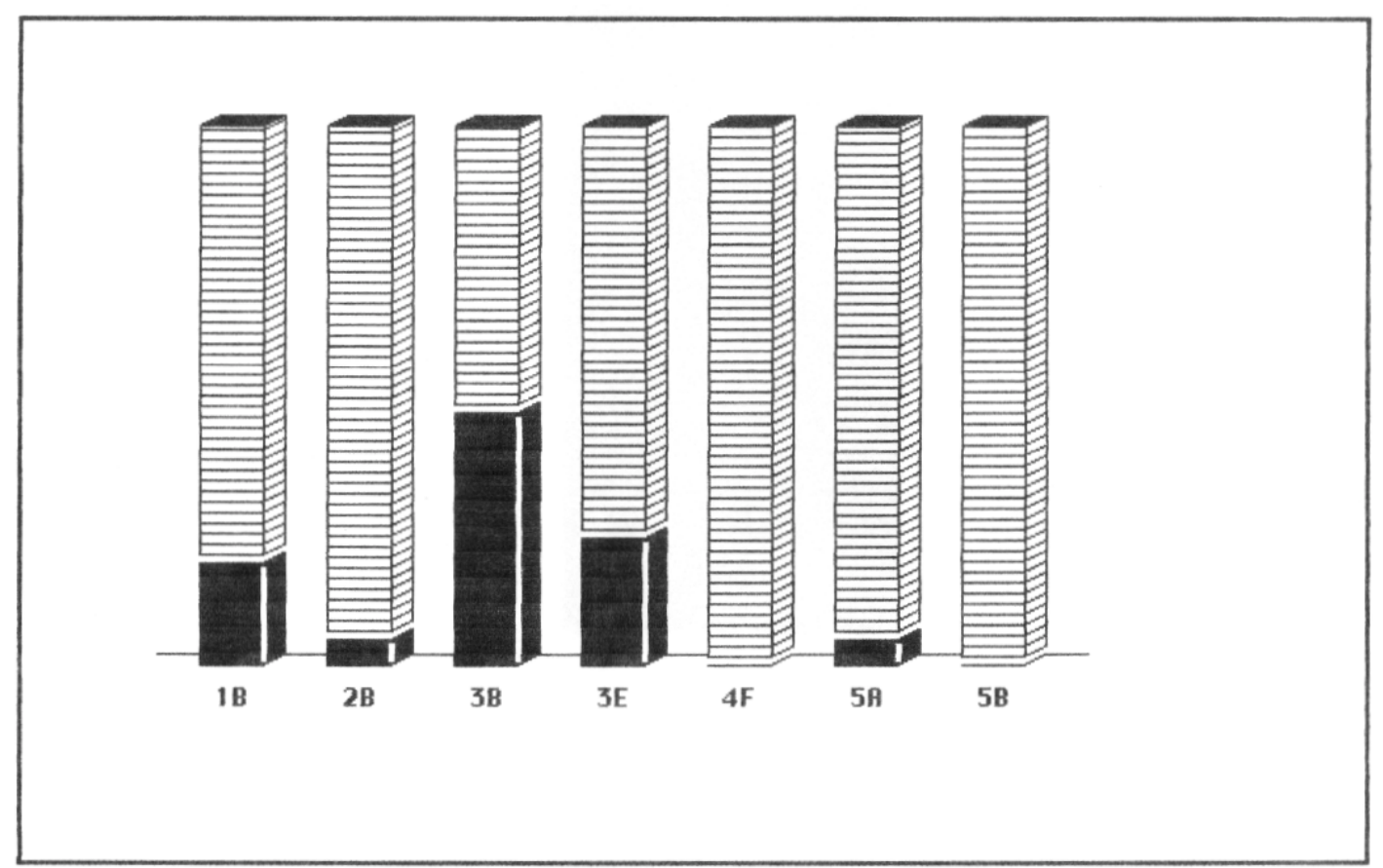

Abb. 193 Die Verteilung der Klingenformen auf der Fischerinsel im Tollense-See vom frühen 11. Jahrhundert bis um 1200

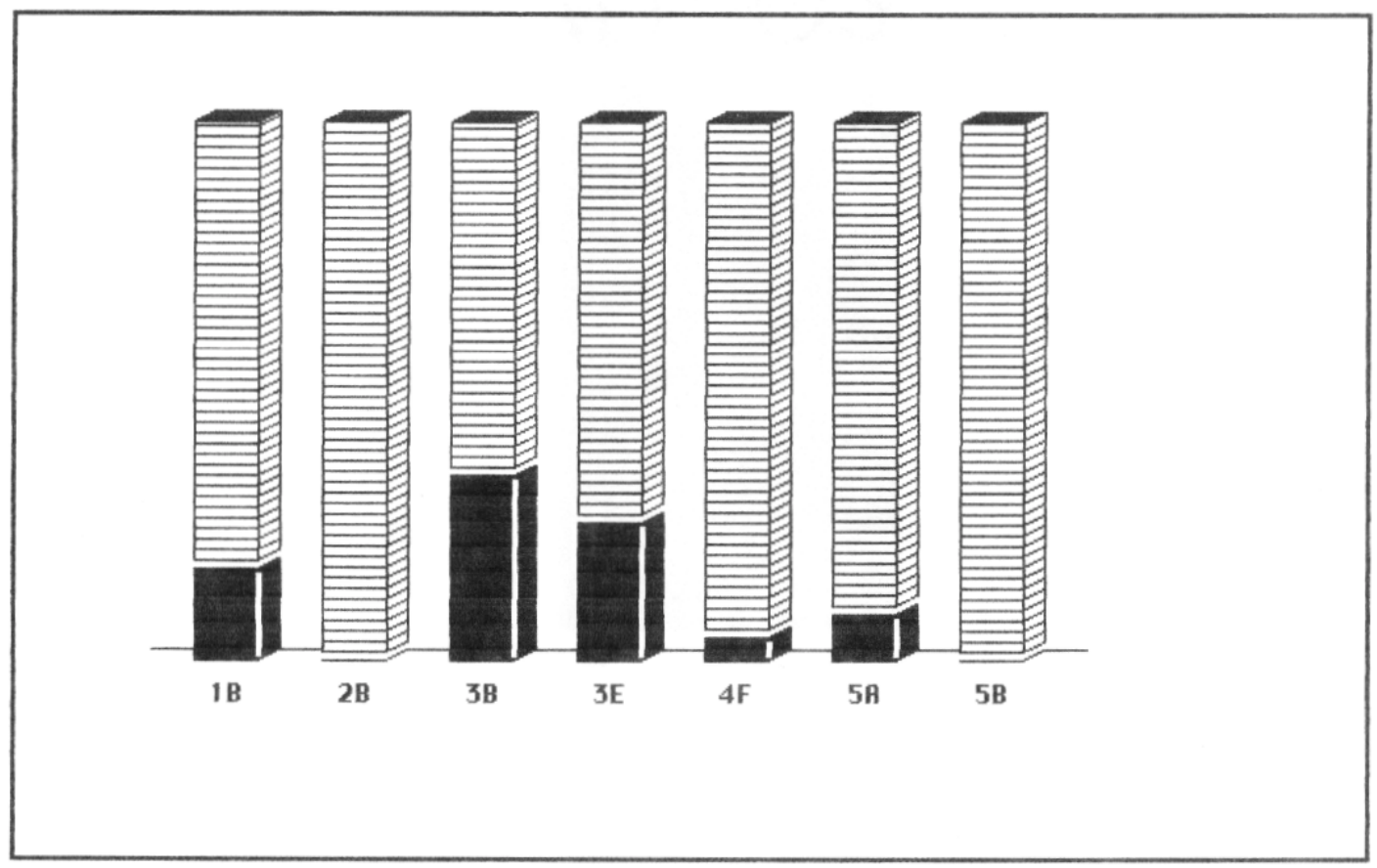

Abb. 194

Die Verteilung der Klingenformen auf dem Hanfwerder in der Lieps vom 11. Jahrhundert bis zum 13. Jahrhundert 


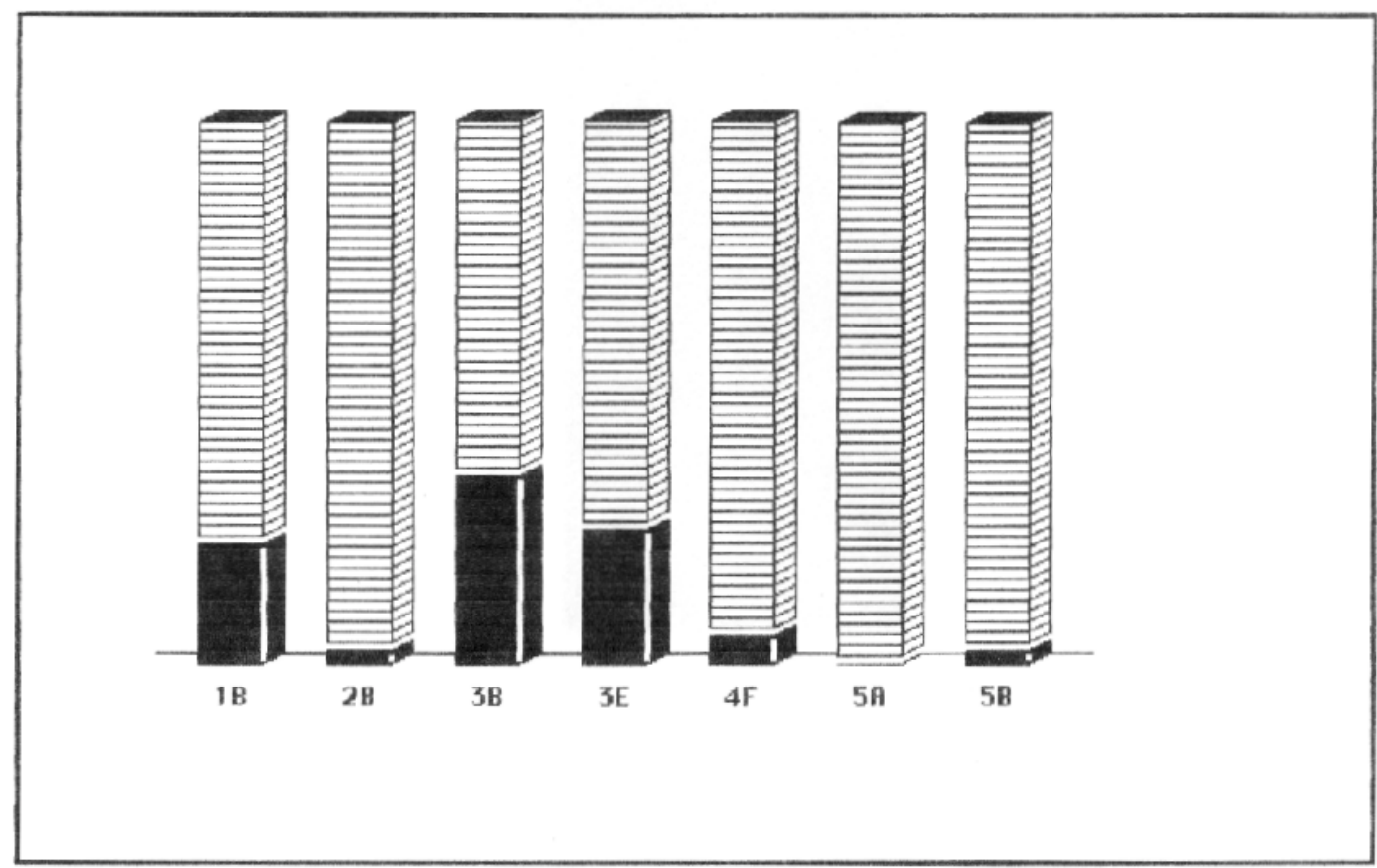

Abb. $195 \quad$ Die Verteilung der Klingenformen in spätslawischen Burgen und Siedlungen auf dem Gebiet der ehemaligen nördlichen DDR vom 11. bis zur ersten Hälfte des 13. Jahrhunderts

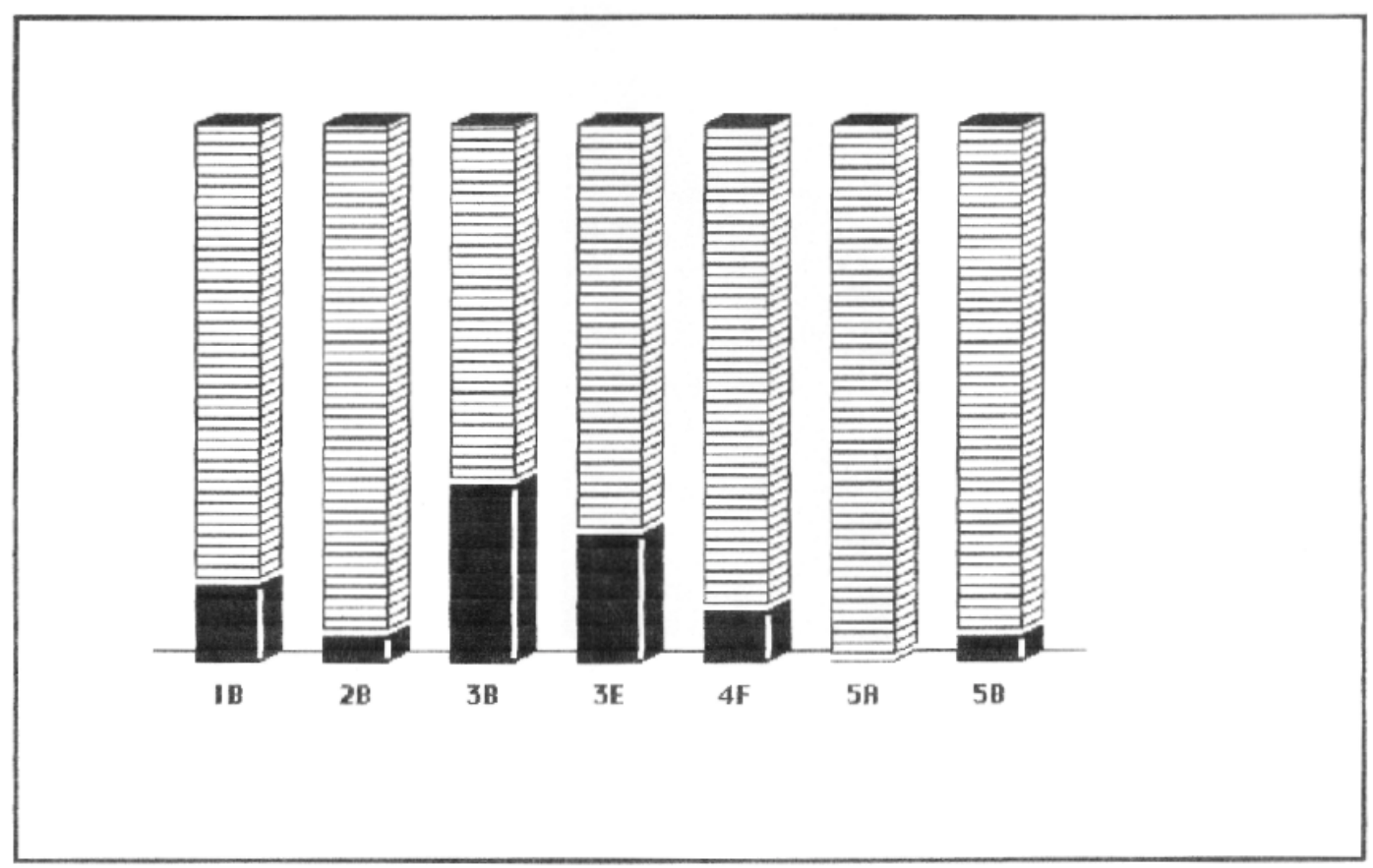

Abb 196

Die Verteilung der Klingenformen in spätslawischen Siedlungen auf dem Gebiet der ehemaligen nördlichen DDR 


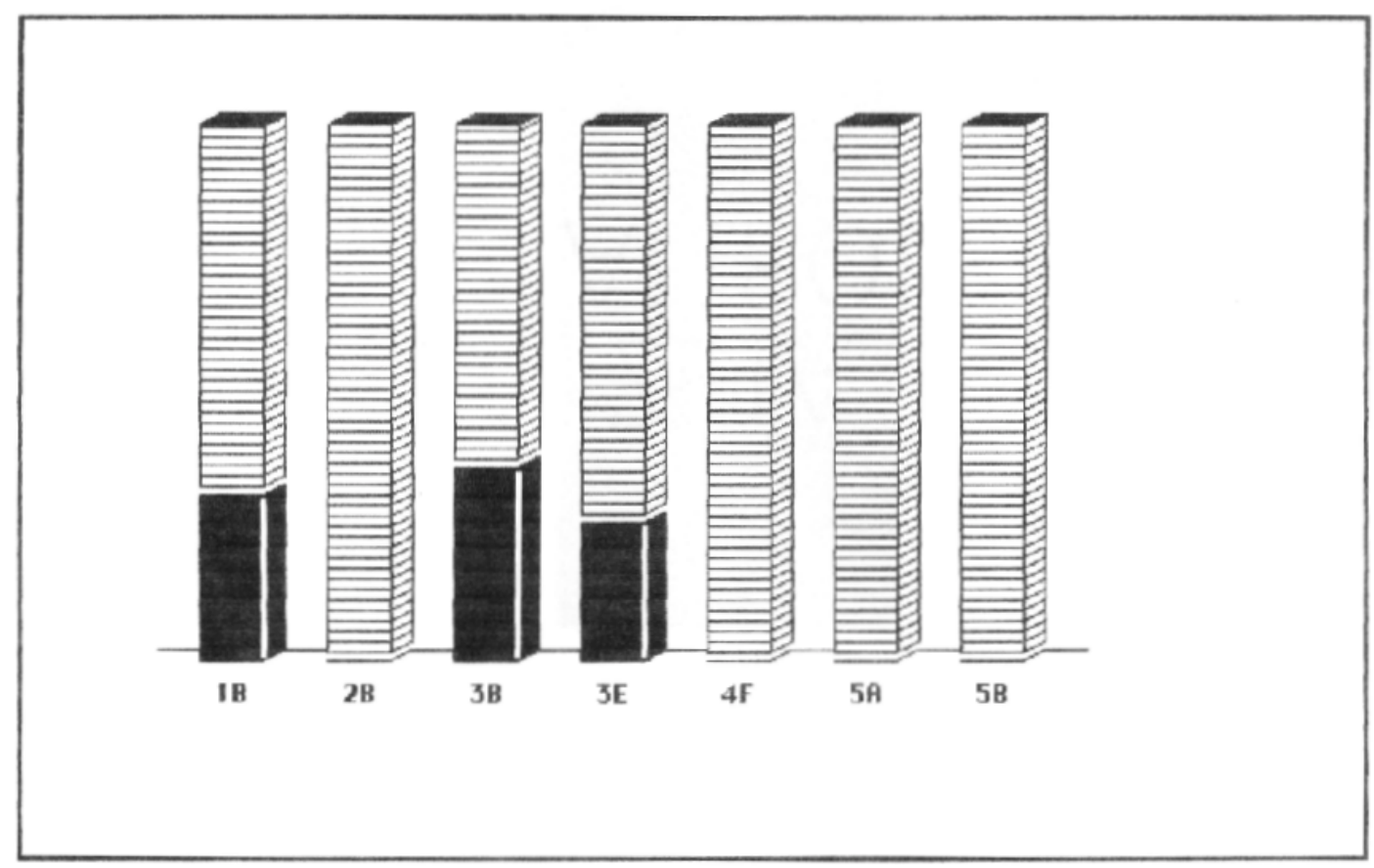

Abb. 197

Die Verteilung der Klingenformen in spätslawischen Burgwällen auf dem Gebiet der ehemaligen DDR

Als Hinweis kann festgehalten werden, daß sich innerhalb des untersuchten nordwestslawischen Siedlungsgebietes ein Grundbestand an Formen nachweisen läßt, der offenbar als Allgemeingut angesehen werden kann.

Neben diesem Grundbestand stehen jedoch Unterschiede in der prozentualen Verteilung dieser Formen und das Aufkommen oder Fehlen weiterer Formen, die möglicherweise zurückgehen auf unterschiedliche Funktionen und Lebensumstände auf Siedlungsplätzen mit sozial und wirtschaftlich unterschiedlichen Grundlagen.

\section{Der Bestand an Messerklingenformen in der Burg und Siedlung Gom- merstedt, Thüringen (13. bis 14. Jahrhundert)}

Der Siedlungskomplex Gommerstedt umfaßt neben einem kleinen Adelssitz eine aus wenigen Höfen, einer Zehntscheune und einer Kapelle bestehende, zur Burg gehörende Siedlung auf vorwiegend landwirtschaftlicher Grundlage, in welcher die Produktion einfacher Eisengegenstände angenommen wird ${ }^{458}$. Die Funde, vor allem die Metallfunde, gehören zum größten Teil dem 13. bis 14. Jahrhundert an.

Verglichen wurde die anteilige Verteilung der Klingenformen von 13 Messern aus der Siedlung mit 20 Messern aus der Burg.

Die Palette an Klingenformen in Gommerstedt ist erheblich, vor allem Formen wie IIc und IIa sind gegenüber den Beständen anderer zeitgleicher Fundplätze unver- 
hältnismäßig stark vertreten. Die Ursache dafür ist zunächst nicht auszumachen, es steht aber zu vermuten, daß sie in der Vollständigkeit der durchgeführten und vorgelegten Materialaufnahme zu suchen ist.

Die Herkunft der Messer ist nur schwer einzugrenzen. Einige Metallanalysen sowie der Nachweis einer am Ort befindlichen Eisenverarbeitung lassen vermuten, daß zumindest ein Teil am Ort selbst hergestellt worden ist ${ }^{459}$.

Der Bestand innerhalb der Burg zeigt neben starken Anteilen der Formen IIIb und IIIe mit je 15\% einen Anteil der Form IVa von 10\% (Abb. 198). Dazu treten die Sonderformen IVf, IVg und IVh mit je 5\%. Die Formen IVa und IVh sind aus Bildquellen als Tafelmesser bekannt ${ }^{460}$,die Form IVa findet sich auch auf anderen zeitgleichen Burgen $^{461}$.

Die Formen Ib, Ilb, IIc und Va, zum Teil möglicherweise als Mehrzweckmesser anzusprechen, fehlen auf der Burg ganz.

In absoluten Zahlen stehen auf der Burg Gommerstedt sechs Messern der Formen IIIb und IIIe fünf Messer mit Sonderformen gegenüber, von denen die meisten dem Bereich der Tafel zugeordnet werden dürfen.

In der Siedlung verteilen sich die Klingen auf die Formen IIIb und IIIe mit 23,1\% und 15,4\%, die auf der Burg nicht nachgewiesenen Formen Ib und IIb sind mit je $7,7 \%$ vertreten (Abb. 199). Die ebenfalls nur in der Siedlung auftretende Form Va, die möglicherweise noch vor das 13. Jahrhundert datiert werden $\operatorname{kann}^{462}$, ist mit zwei Exemplaren (15,4\%) nachweisbar. Die in der Burg vorhandenen Formen IVf, IVg und IVh fehlen ganz.

Erstaunlicherweise fanden sich aber in der Siedlung drei Messer der Form IVa und eines der Form IIc, welches mit seinem fast stumpfen vorderen Abschluß der Form IVa sehr nahe kommt. Das Auftreten dieser Formen außerhalb der Burg mag ein Hinweis auf den möglichen Produktionsort sein, es kann aber ebensogut ein Indiz für die Annahme sein, daß die innerhalb des bearbeiteten Bestandes in der Regel in Burgen und Klöstern auftretende Form IVa/IIc (Klingen mit senkrechtem oder stumpf konkav verlaufendem vorderen Abschluß) nicht ausschließlich an höhere soziale Schichten gebunden war.

Trotz unterschiedlicher kultureller Voraussetzungen bestätigt sich aber insgesamt das bereits für den westslawischen Kulturbereich in Ansätzen angetroffene Bild:

Die Palette der Grundformen, d.h. möglicherweise die Bandbreite unterschiedlicher Funktionen, scheint in offenen Siedlungen größer zu sein als auf Burgen.

\footnotetext{
459 Timpel 1982, S. 72

460 Vgl. Kapitel 9, Abschnitt 2

461 Vgl. u.a. die Bestände der Raffen- und der Rücklenburg, Katalogteil BRD, Kat. Nr. 151-161, sowie der Burg Wartenberg, Kat. Nr. 197-205

462

Vgl. Katalogteil ehemalige DDR, Kat. Nr. 207 und 208
} 
So fanden sich im Weiler Gommerstedt sieben verschiedene Grundformen von Klingen; davon tauchen nur drei Formen auf der Burg auf.

Im Gegensatz zu den vorangegangenen Untersuchungen begegnet man auf der mittelalterlichen Burg Gommerstedt mindestens drei Sonderformen, die in der Siedlung nicht vorkommen. Das Messer der Form IVh ist nach seiner Größe und Machart sicherlich als Teil der adeligen Tafel zu betrachten. Es kann möglicherweise als Beispiel für eine an die Burg gebundene Funktion genannt werden, die im Zusammenhang mit den differenzierten Tätigkeiten an der mittelalterlichen Tafel des Adels steht ${ }^{463}$.

Das Fehlen von Formen mit Mehrzweckcharakter, die größere absolute Anzahl von Messerfunden und der höhere Anteil von Sonderformen auf der Burg legen den Schluß nahe, daß dort die Bandbreite der Funktionen von Messern kleiner, die Spezialisierung innerhalb eines engeren Funktionsrahmens und vielleicht auch der vorhandene Bestand an Messern dagegen größer war als in der zugehörigen Siedlung.

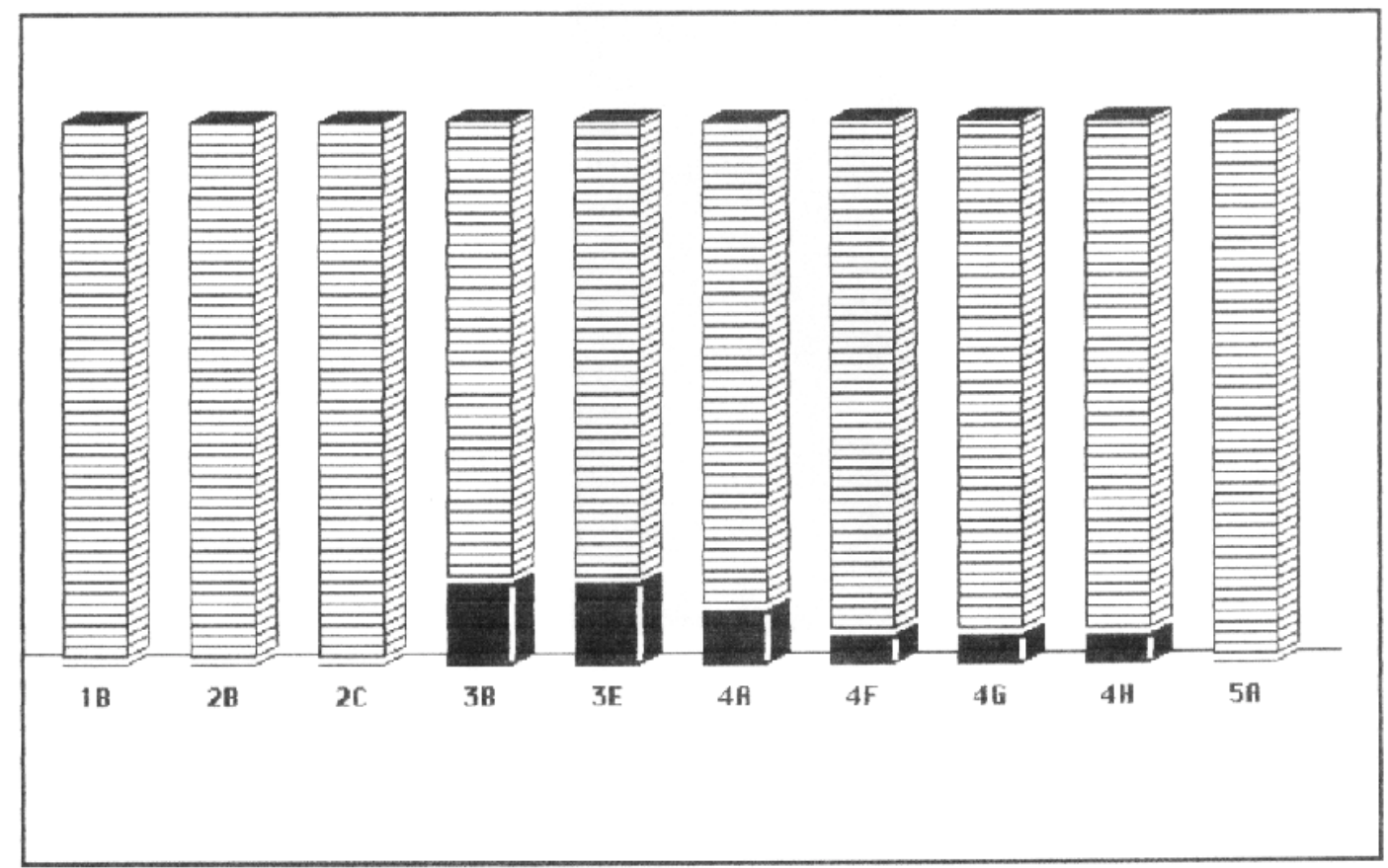

Abb. 198

Die Verteilung der Klingenformen in der Burg Gommerstedt im 13. und 14. Jahrhundert 


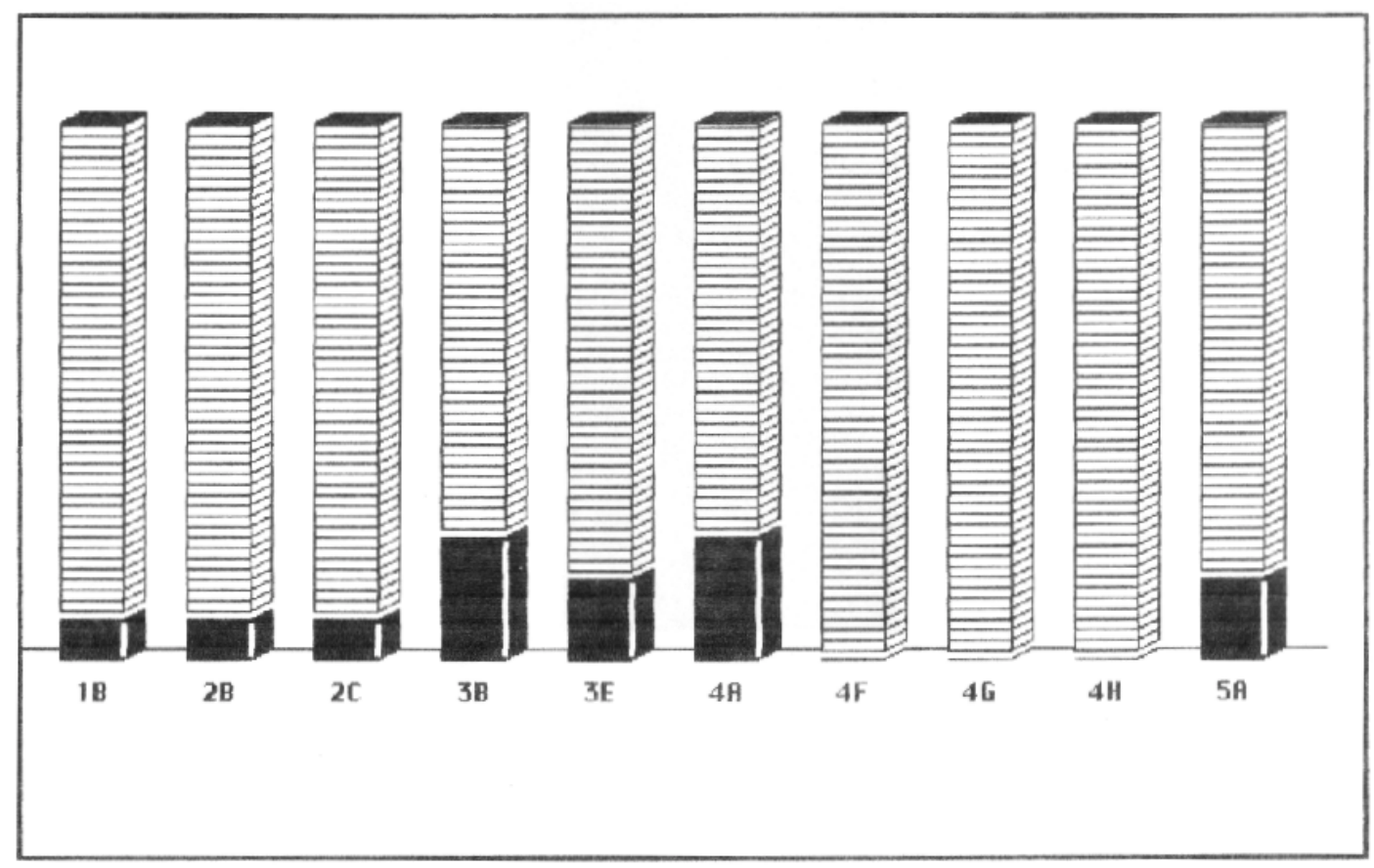

Abb. 199

Die Verteilung der Klingenformen in der Siedlung Gommerstedt im 13. und 14. Jahrhundert

\section{Messerklingenformen in weiteren deutschen Burgen und Siedlungen des 12. bis 14. Jahrhunderts}

Für die folgenden Analysen von Burgen des 13. Jahrhunderts und Siedlungen des

12. bis 14. Jahrhunderts aus den deutschen Mittelgebirgen wurden die Bestände der Isenburg (Essen), der Burg Isenberg (Hattingen), der Raffenburg, der Rücklenburg, der Burg Altena und der Burg Wartenberg sowie die Bestände der mittelalterlichen Siedlungen Schulenrode, Bengerode, Hohenrode und Glasbach miteinander verglichen ${ }^{464}$.

Die Aussagefähigkeit eines solchen Vergleichs ist begrenzt, da für beide Fundortgruppen kein stratigraphisch ausreichend vergleichbares Material zur Verfügung stand. Die Menge reicht aber aus, um grundsätzlich nach Unterschieden zwischen burgsässigem Adel und Landbevölkerung zu fragen.

Der durchweg in das 13. Jahrhundert zu datierende Bestand der erwähnten Burgen zeigt neben dem bekannten Grundbestand der Formen Ib, IIIb und IIIe (mit deutlicher Dominanz der beiden letzteren Formen) einen prozentual der Form Ib entsprechenden Anteil der Form IVa sowie prozentual der Form IIb entsprechende Anteile der Sonderformen IVh, IVi und Vc (Abb. 200). Die Form IVf ist nur sehr gering vertreten.

464 Vgl. Katalogteile BRD und ehemalige DDR 
Der Bestand der wüsten Rodungsdörfer Schulenrode (12. bis 13. Jahrhundert), Bengerode (12. bis 14. Jahrhundert), Hohenrode (Mitte 12. bis 14. Jahrhundert) und Glasbach (13. bis 14. Jahrhundert) weist einen prozentualen Anteil von über 50\% für die Form IIIb auf, gefolgt von der Form Ilb, deren Anteil mehr als doppelt so hoch ist wie im Bestand der oben angegebenen Burgen (Abb. 201). Die in etwa gleichen Anteile der Formen Ib und IIIe liegen etwas unter denen der betreffenden Anteile in den Burgen. Die auf den Burgen nachgewiesenen Formen IVh, IVi und Vc sowie die in Gommerstedt auch in der Siedlung auftretende Form IVa fehlen ganz.

Das Ergebnis dieses Vergleichs zeigt zunächst einen erheblich größeren Anteil verschiedener Klingenformen auf den untersuchten Burgen als in den offenen Siedlungen, während in Gommerstedt das Verhältnis umgekehrt war.

Ähnlich wie in Gommerstedt treten Sonderformen wie IVa und IVh nur in den Burgbeständen auf. Der gegenüber der Burg erhöhte Anteil der Formen IIIb und IIb in der offenen Siedlung tritt hier noch deutlicher hervor. Im Detail lassen sich aber noch weitere Unterschiede zu den Ergebnissen aus Gommerstedt erkennen.

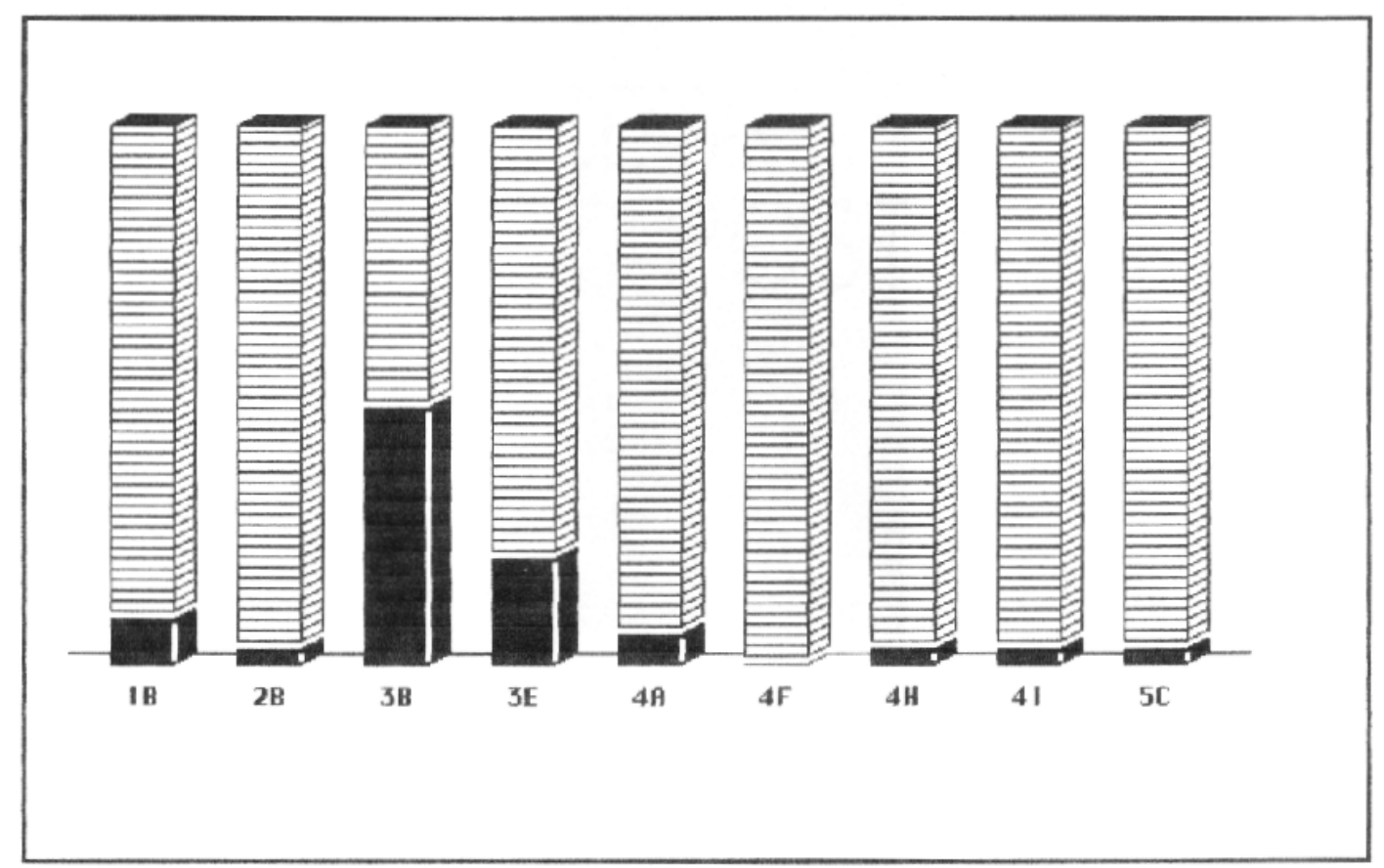

Abb. 200

Die Verteilung der Klingenformen in deutschen Burgen des 13. Jahrhunderts 


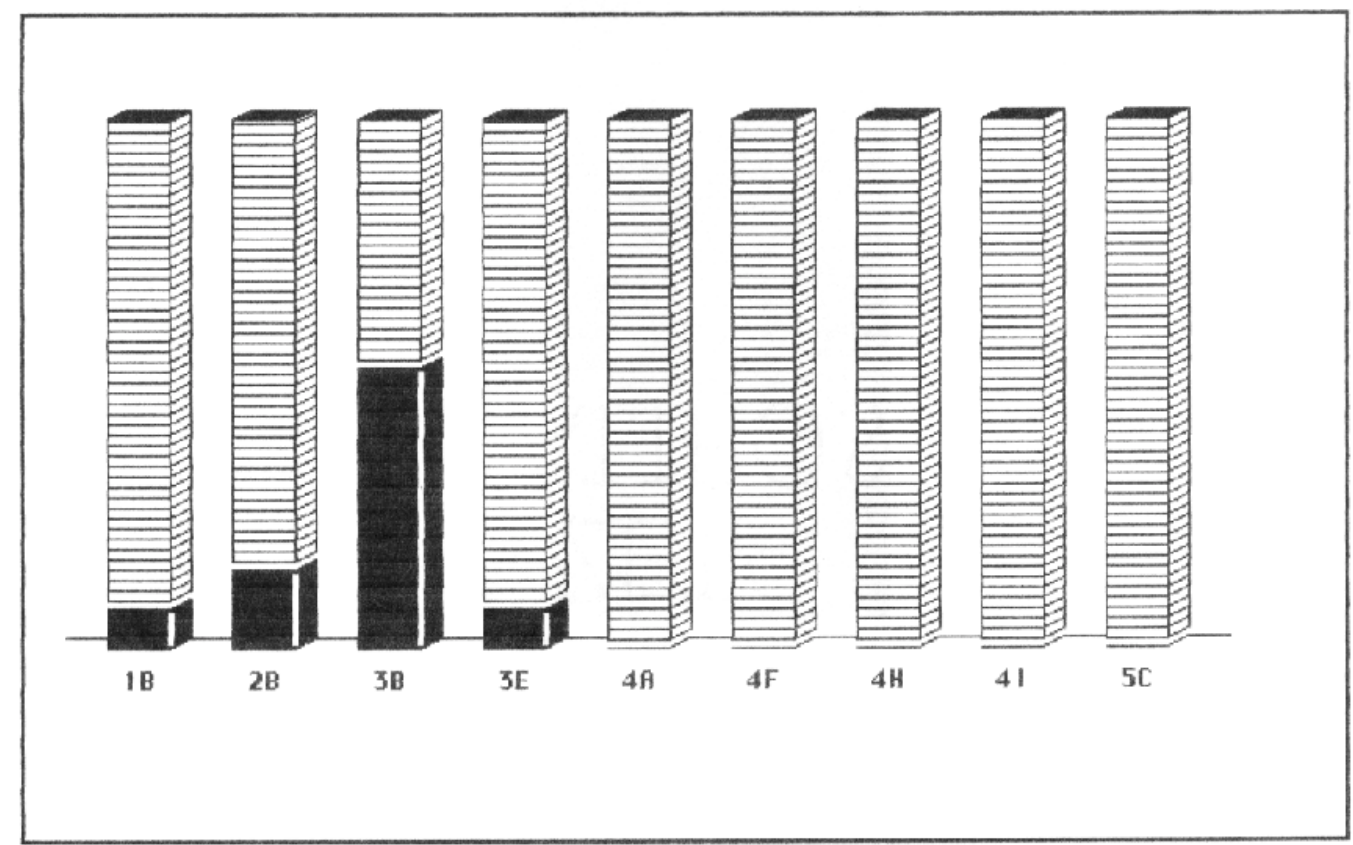

Abb. 201

Die Verteilung der Klingenformen din deutschen Siedlungen am Beispiel von vier Rodungsdörfern des 12. bis 14. Jahrhunderts

In Gommerstedt ist der beobachtete Bestand in der Siedlung um vier Grundformen reicher als auf der Burg, drei Sonderformen treten nur auf der Burg auf.

In den übrigen untersuchten offenen Siedlungen ist nur noch eine Grundform mehr beobachtet worden als auf den Burgen. Dagegen finden sich vier Sonderformen nur in den Burgbeständen, wobei die Sonderform IVf nur in Gommerstedt auf der Burg vorkommt, auf den untersuchten Höhenburgen und in den offenen Siedlungen aber fehlt $^{465}$.

Zur Interpretation dieser Verschiebungen läßt sich anführen, daß es sich in Gommerstedt um ein Dorf mit kleinem Herrensitz handelt und selbst ähnliche Strukturen hier möglicherweise anders ausgeprägt waren als in den untersuchten offenen Rodungsdörfern.

Neben ihren Funktionen, die zumindest Teilbereiche der dörflichen Arbeit bestimmte, haben die Lebensformen auf der Burg in Gommerstedt vielleicht auch Auswirkungen auf die privaten Bereiche der Dorfbewohner gehabt, wie zum Beispiel beim Essen und Trinken. Dieser mögliche Einfluß fehlte in den Rodungsdörfern, in denen der Anteil der Formen IIb und IIIb, die eher als Mehrzweckmesser dienlich waren, höher liegt als im Weiler Gommerstedt, während die

465 Die Form IVf, die in sehr unterschiedlichen Proportionen erscheint, ist in der Regel in Siedlungen häufiger anzutreffen als in Burgen (vgl. BRD, Kat. Nr. 98 und 100, wobei das Exemplar von der Burg Gommerstedt durchaus andere Proportionen besitzt). In den untersuchten Städten ist sie von allen Formen der Gruppen IV und V am stärksten vertreten (Abb. 204). 
zum Teil ausweislich zeitgenössischer Bildquellen auf der gehobenen Tafel verwendeten Sonderformen IVa, IVh und IVi dort gar nicht auftreten.

Fraglich ist, ob das Ergebnis von Gommerstedt typisch für die Siedlungsform Dorf oder Weiler mit kleinem Herrensitz ist, oder ob es andere Faktoren wirtschaftlicher oder regionaltypischer Art gibt, die dieses Verteilungsbild beeinflußt haben.

Hier werden die Grenzen der Interpretationsmöglichkeit allein auf archäologischer Quellenbasis sichtbar:

$\mathrm{Da}$ es Unterschiede im Bestand von Klingenformen auf Burgen und in ländlichen Siedlungen gibt, wurde deutlich.

$\mathrm{Da}$ es offenbar nicht gleichgültig ist, ob eine offene Siedlung an eine Burg angeschlossen oder als eigenständige Siedlung organisiert war, wurde ebenfalls deutlich. Welche Rolle dabei aber die Art und Funktion der jeweiligen Burgen spielt, welche Bedeutung der Lebensgrundlage der offenen Siedlungen - hier Rodungsdörfer, die sicherlich auch gewerbliche Produktion (Glasbach) betrieben haben - zukommt, all dies sind Fragen, die grundsätzlich nur mit Hilfe anderer historischer Quellen beantwortet werden können.

Aber auch im Bereich der archäologischen Quellen werden Defizite sichtbar:

Das Fundmaterial reicht weder in der publizierten Menge noch in seiner unterschiedlichen Interpretationsqualität aus, um genauere Analysen durchzuführen. Nach wie vor stehen zu viele Streufunde, zu viele "befundlose" Fundobjekte aus Grabungen zu wenig aus interpretationsfähigen Befunden stammendem Material gegenüber, welches sozial und funktional eingrenzbar ist.

\section{Der Bestand an Messerklingenformen in mittel- und nordeuropäischen Städ- ten des 13. bis 14. Jahrhunderts im Vergleich}

Der überwiegende Anteil der bei Stadtkernuntersuchungen geborgenen Messer dieser Untersuchung wurde von den Ausgräbern in das 15. bis 17. Jahrhundert datiert. Um für den Zeitraum des 13. bis 14. Jahrhunderts die Verteilung der einzelnen Klingenformen mit der zeitgleicher Bestände von Burgen und ländlichen Siedlungen $\mathrm{zu}$ vergleichen, wurden 76 Messer aus dem gesamten Untersuchungsgebiet ausgewählt und analysiert, die in der Regel aus stratigraphisch gesicherten Fundumständen stammen. Sie wurden in Oslo, Tönsberg, Arhus, Roskilde, Lund, Danzig, Lübeck, Anklam, Hamburg, Winsen/Luhe, Stargard, Amsterdam, Minden, Posen, Coesfeld, Dordrecht, Höxter, Göttingen, Duisburg und Krakau gefunden ${ }^{466}$. Regionale Faktoren fallen auf der Grundlage dieser Auswahl als Daten zur Interpretation weg. Die Ergebnisse spiegeln dementsprechend nur allgemeine Tendenzen wider, die sich in kleinräumigeren Untersuchungen sicherlich differenzieren lassen.

466 Die für diese Untersuchung ausgewählten Messer des 13. und 14. Jahrhunderts sind unter den angegebenen Fundorten im Katalog beschrieben worden. 
Das Ergebnis (Abb. 202) zeigt eine ähnliche Verteilung wie bei der Analyse der Klingenformenanteile auf ausgewählten deutschen Burgen des 13. Jahrhunderts (Abb. 200), vor allem die Dominanz der Formen Ib, IIIb und IIIe ist evident.

Zum Vergleich wurden die Bestände von Burgen des 12. bis 14. Jahrhunderts aus dem gesamten Untersuchungsgebiet sowie von polnischen Burgen herangezogen. Beise Analysen (Abb. 203-204) zeigen, daß sich am Bild der anteiligen Formenverteilung nichts wesentliches ändert:

Im 12. bis 14. Jahrhundert herrschen die Klingenformen IIIb, IIIe und Ib in dieser Abfolge auf den Burgen, in der Abfolge IIIb, Ib und IIIe in den Städten vor, die Form IIIe scheint demnach auf Burgen, die Form Ib in den Städten etwas mehr verbreitet gewesen zu sein. Die letzte Grundform, die Form IIb, tritt auf Burgen und in den Städten seltener auf, sie wurde nur bei der Analyse der Messerfunde aus den vier ausgewählten deutschen Rodungsdörfern stärker beobachtet (Abb. 201). Auch hier bot sich ansonsten das gleiche Bild in der Abfolge IIIb - Ib - IIIe.

Damit zeigt sich der Grundbestand an Klingenformen in relativ festen anteiligen Mengen vom 12. bis 14. Jahrhundert als eine konstante Größe, die in Burgen, ländlichen Siedlungen und Städten gleichermaßen auftritt, mit einem Spektrum, welches die Grundformen IIIb, IIIe und Ib umfaßt.

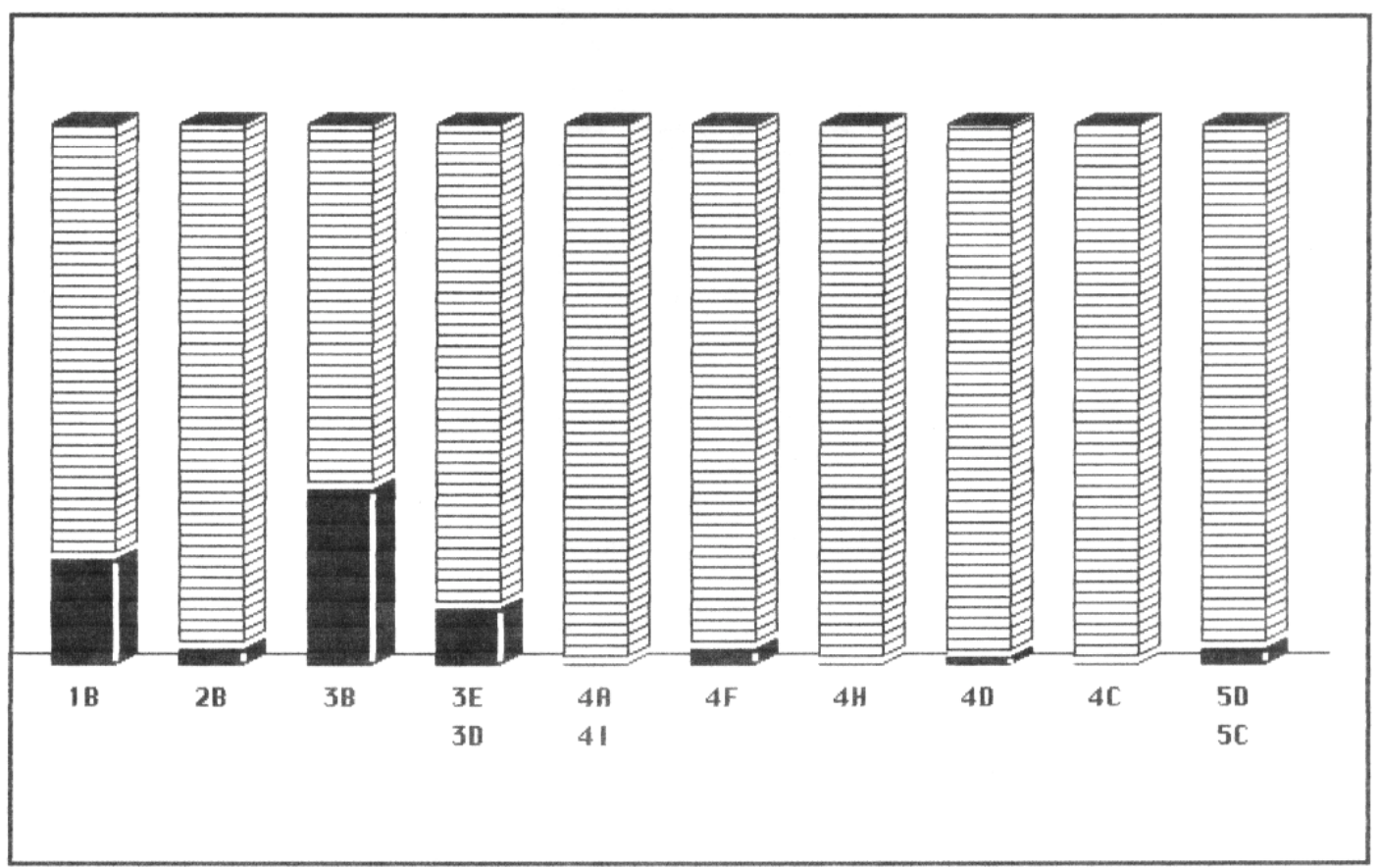

Abb. 202

Die Verteilung der Klingenformen in nord- und mitteleuropäischen Städten des 13. bis 15. Jahrhunderts 


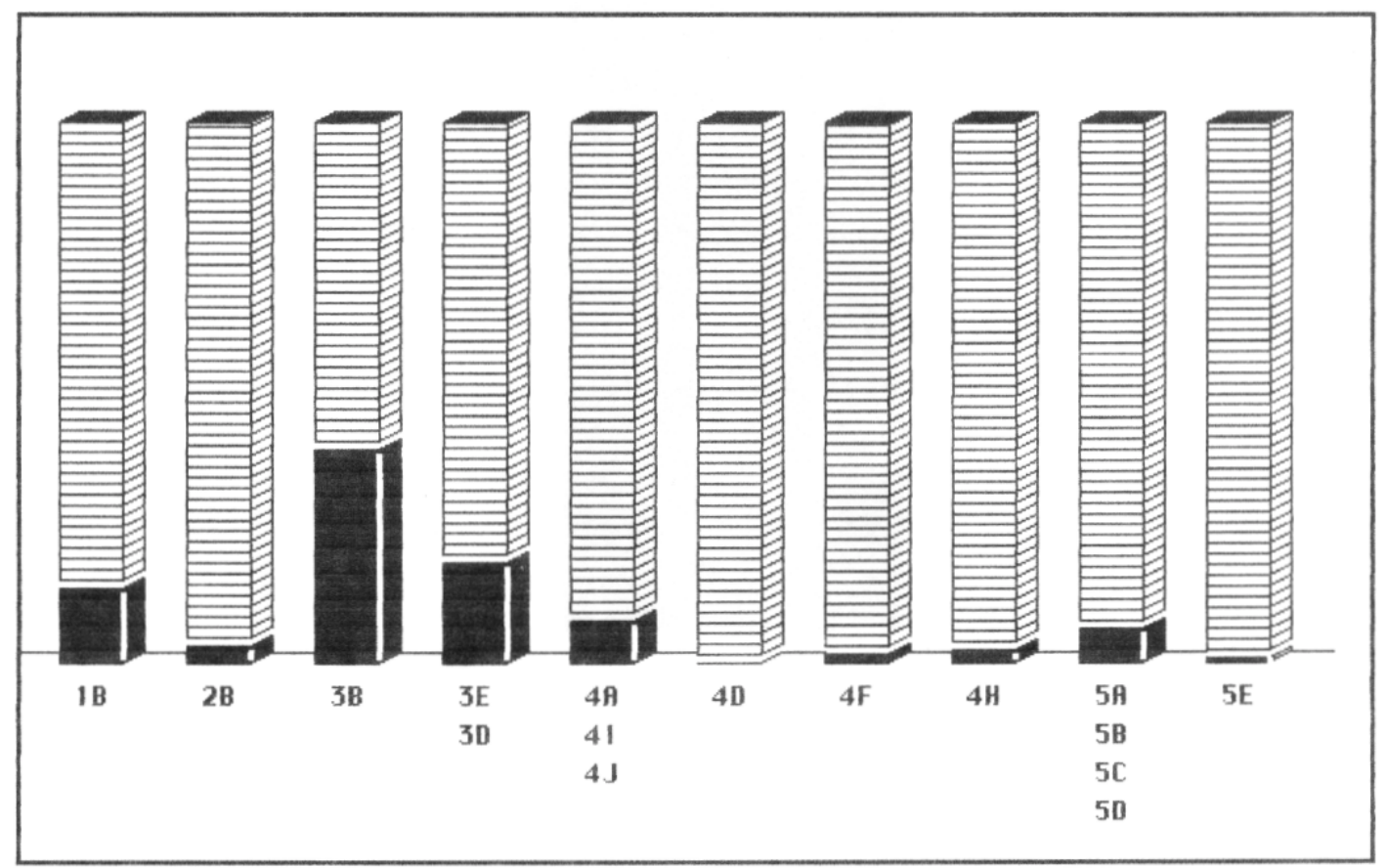

Abb. 203 Die Verteilung der Klingenformen in nord-und mitteleuropäischen Burgen des 12. bis 13. Jahrhunderts

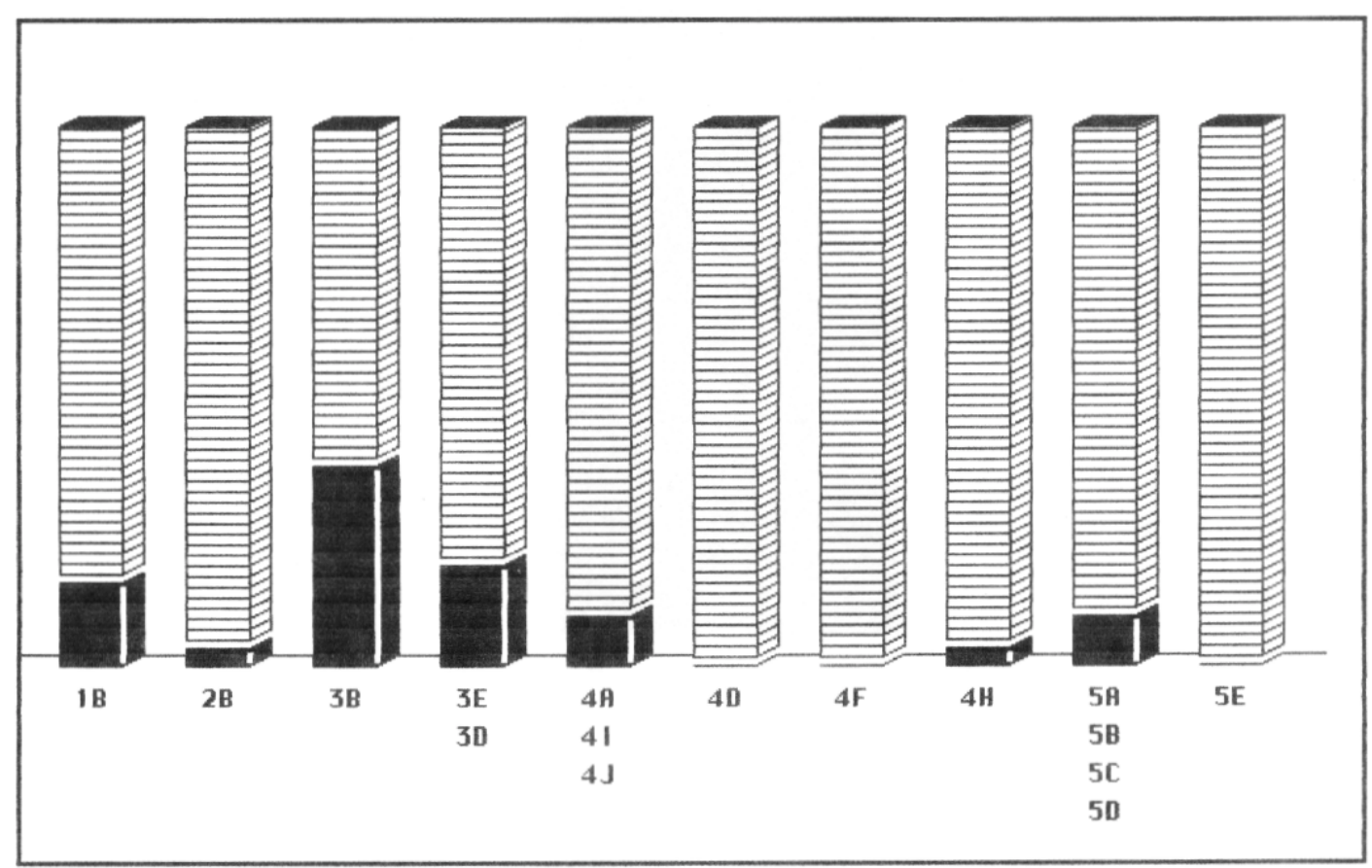

Abb. 204

Die Verteilung der Klingenformen in polnischen Burgen des 12. bis 14. Jahrhunderts 
Alle anderen Formen variieren zum Teil stark: so sind die Klingen der Formen IVa, IVi und IVh, die in den untersuchten Burgen mit erkennbaren Anteilen vorhanden sind, in den untersuchten Städten nicht aufgetreten. Unterschiede in den untersuchten Messerbeständen sind sowohl zwischen Burgen und Städten als auch zwischen Burgen und ländlichen Siedlungen erkennbar, ohne daß diese auf archäologischer Quellenbasis näher zu interpretieren sind.

Trotz der einseitigen Quellenlage und unter Vorbehalt der oben angeführten Quellenkritik wurde im Fall der Messerfunde aus Stadtkerngrabungen versucht, eine historische Dimension in die Analysen miteinzubeziehen.

Der Zeitraum des 13. bis 14. Jahrhunderts wurde nicht nur in Anlehnung an die vorangegangenen Analysen gewählt. Er kennzeichnet die Blütezeit der Stadtentwicklung, in diesem Zeitraum haben sich die wichtigsten Städtelandschaften im Untersuchungsgebiet herausgebildet, vor allem der Städtebund der Hanse, des wichtigsten überregionalen Trägers von Handel und Kulturaustausch im Untersuchungsraum.

Verglichen wurden für das 13. und 14. Jahrhundert die Messerbestände aus skandinavischen Städten, aus niederländisch-westfälischen Städten, aus Städten im weiteren Bereich der südlichen Ostseeküste, des Weser-Werra-Raums und aus östlichen Hansestädten im heutigen Polen.

Die Auswahl dieser Kulturräume, die sowohl in ihrer regionalen Ausbreitung als auch in ihrer kulturräumlichen und wirtschaftlichen Bedeutung zu differenzieren sind und auch nach außen verschieden wirksam waren, mithin sicherlich keine idealen historischen Vergleichslandschaften bilden, erfolgte auf der Basis des vorhandenen Fundmaterials und der Dichte der Fundstreuung innerhalb dieser Räume. So fielen z.B. die Kölner Bucht und das Rhein-Ruhr-Mündungsgebiet heraus, da nur aus Duisburg einige Messerklingen zur Verfügung standen.

Bei drei Analysen (Skandinavien, niederländisch-westfälischer Raum, südliche Ostseeküste) zeigte sich das gleiche Bild wie bei der Auswertung des Gesamtbestandes an Klingen aus Stadtkerngrabungen: Dominierend war die Grundform IIIb, im südlichen Ostseeraum gefolgt von der Grundform IIIe, in den übrigen Untersuchungsräumen von der Grundform Ib, die Grundform IIb war nur gering oder gar nicht vertreten. Die Verteilung der übrigen Formen, vielleicht auch der Sonderformen der Gruppen IV und V, war jeweils unterschiedlich (Abb. 205 bis 207).

Ein anderes Bild ergab sich aus der vergleichenden Analyse der Messerfunde aus Städten des Weser-Werra-Raums und des osteuropäischen Hanseeinflußgebiets (Abb. 208 und 209).

Entgegen allen anderen überregionalen Untersuchungen, in denen sich vor allem Unterschiede und Verschiebungen im Detail zeigten, erscheint hier in beiden Fällen als dominante Form die Grundform Ib, gefolgt von den Formen IIIb, IIIe und IVa. 
Der einzige Unterschied in den ansonsten fast identischen Verteilungsbildern besteht in der Grundform IIb, die im Weser-Werra-Raum nicht aufgetreten ist. Die geringe Anzahl der aus diesem Raum aufgenommenen Messer darf dafür wahrscheinlich als Ursache gelten.

Nach dem einheitlichen Bild, welches sich bei den anderen Städtelandschaften auf ähnlich niedriger Quellenbasis dennoch ergeben hat, scheidet der Zufall und die geringe Zahl der untersuchten Messerklingen als Erklärung für diese Abweichung aus. Eine Kontrollanalyse, bei der die Klingenformen von 72 Messern aus den Städten Stargard, Danzig, Posen, Breslau, Kalisch, Lublin, Oppeln und Krakau vom 12. bis 14. Jahrhundert ausgewertet wurden, ergab die gleiche Abfolge der dominanten Formen Ib, IIIb, IIIe und IIa. Ein Vergleich mit der Auswertung von 172 Messern aus 24 Städten des gesamten Untersuchungsraumes vom 12. bis 14. Jahrhundert bestätigte, daß dieses Erscheinungsbild auf die Städte des Weser-Werra-Raums und die Städte mit hohem deutschen Bevölkerungsanteil östlich der Oder beschränkt bleibt.

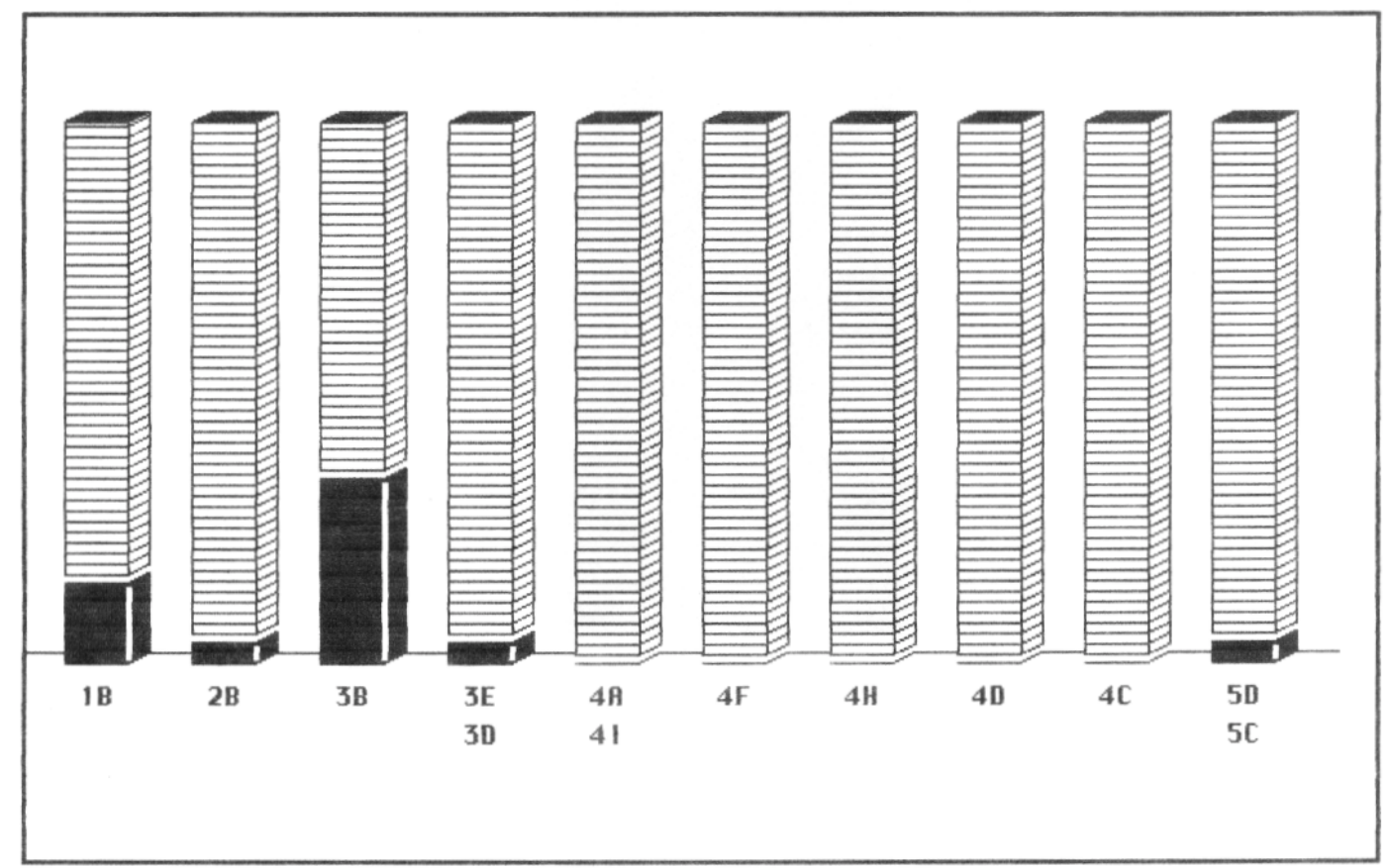

Abb. 205

Die Verteilung der Klingenformen in skandinavischen Städten des 13. und 14. Jahrhunderts 


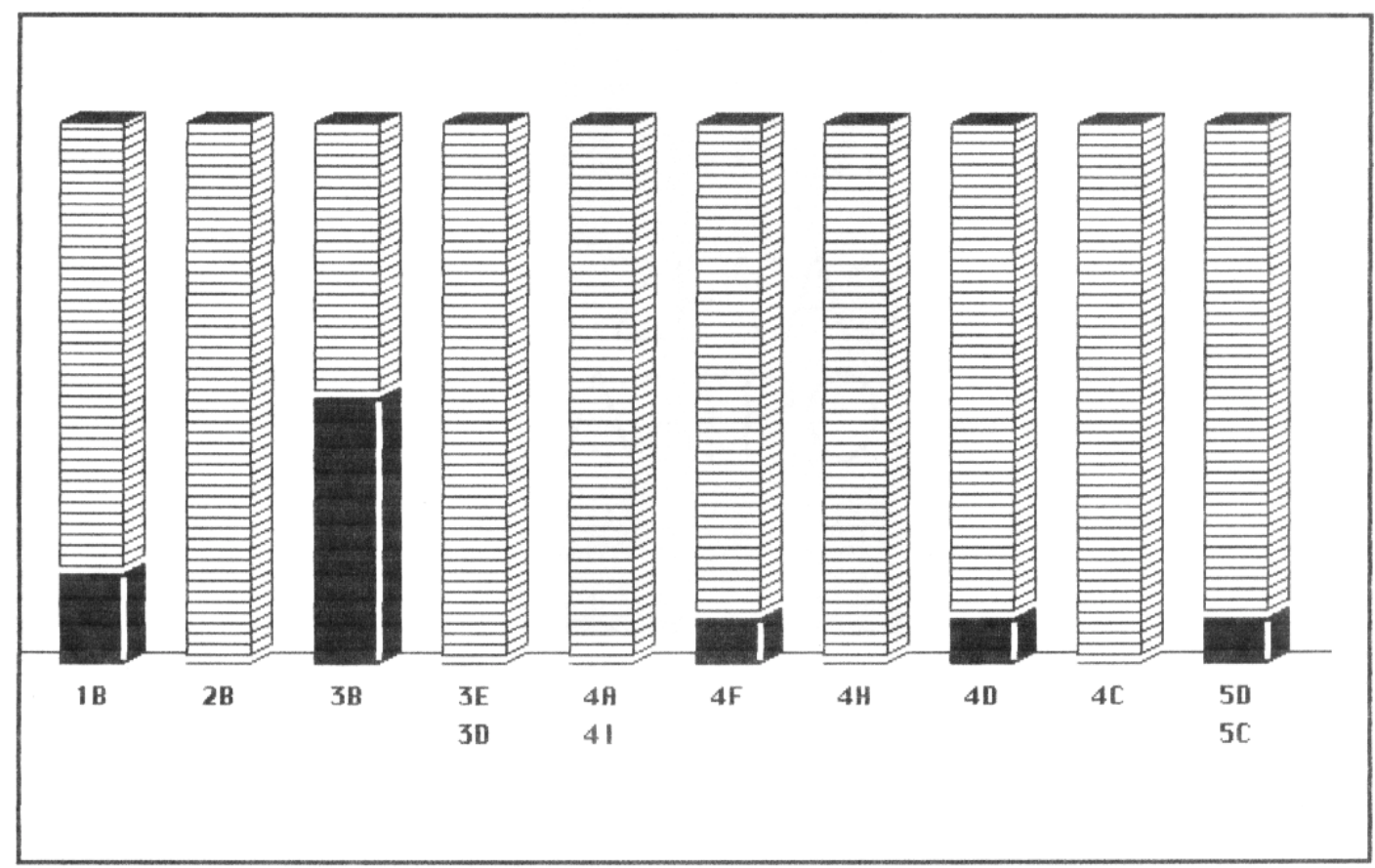

Abb. 206 Die Verteilung der Klingenformen in Städten aus den Niederlanden und dem niederländisch-westfälischen Grenzraum im 1.3 und 14. Jahrhundert

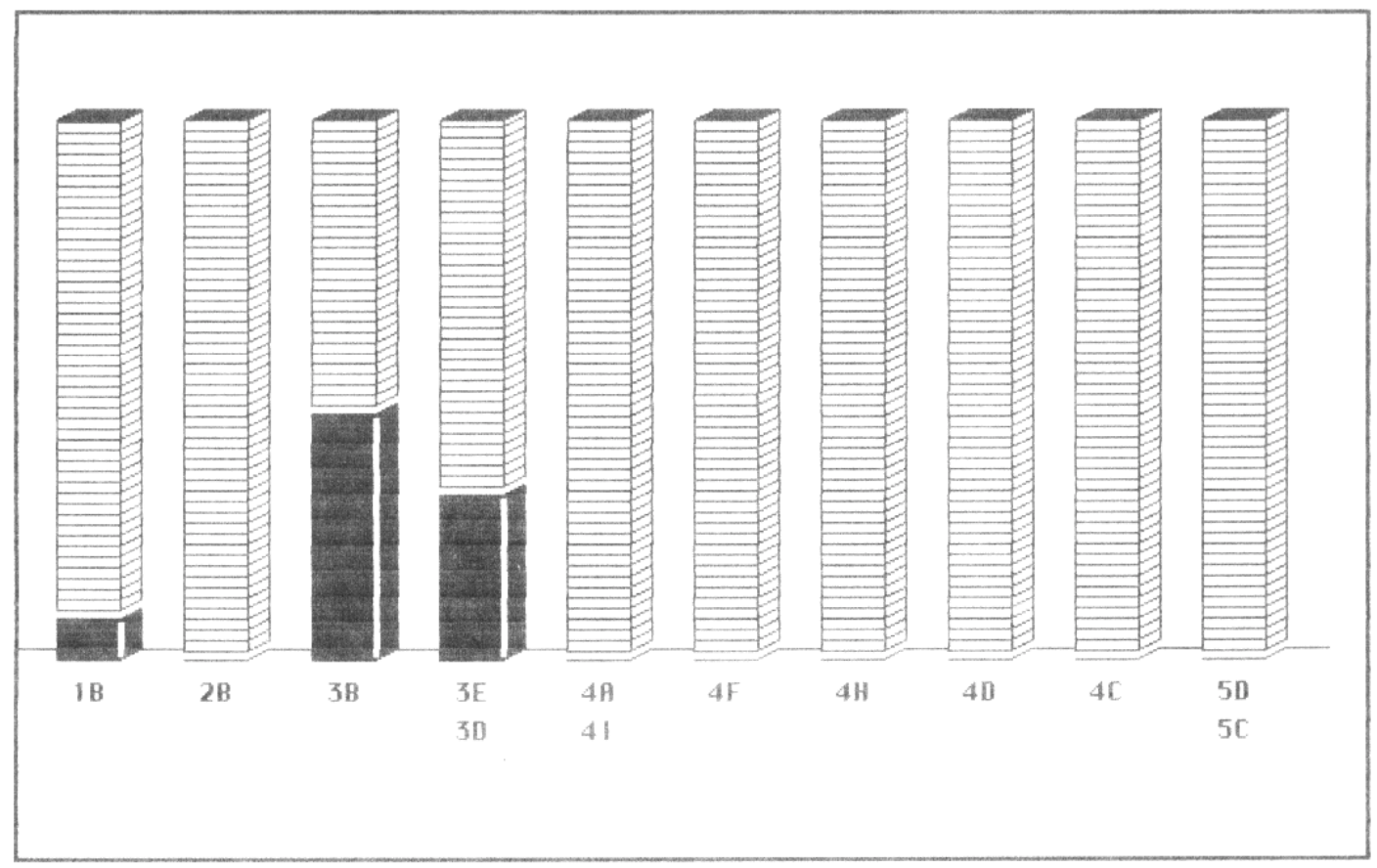

Abb. 207

Die Verteilung der Klingenformen in Städten aus dem weiten Bereich der südlichen Ostseeküste im 13 und 14. Jahrhundert 


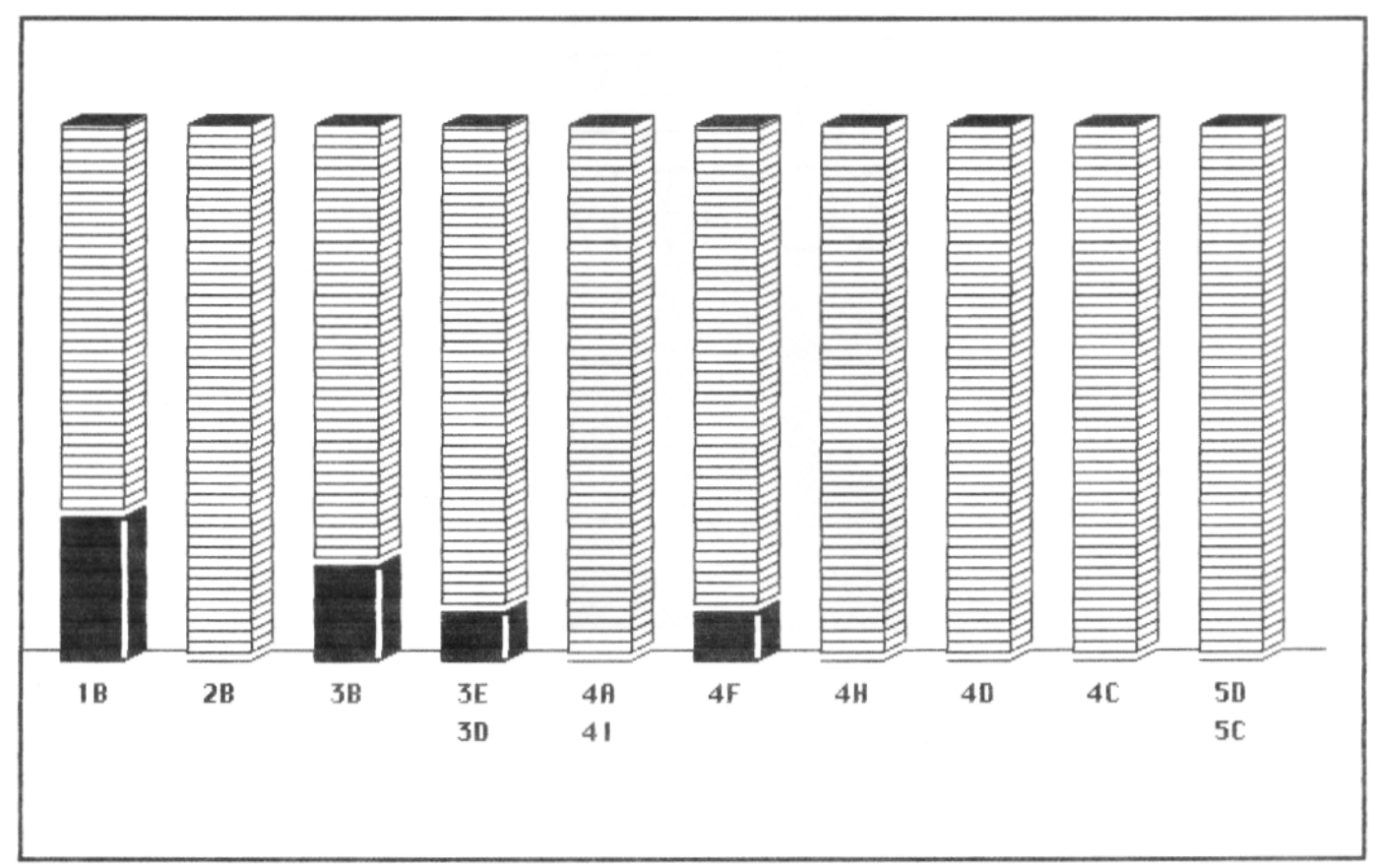

Abb. 208 Die Verteilung der Klingenformen in Städten des Weser-Werra-Raumes im 13 und 14. Jahrhundert

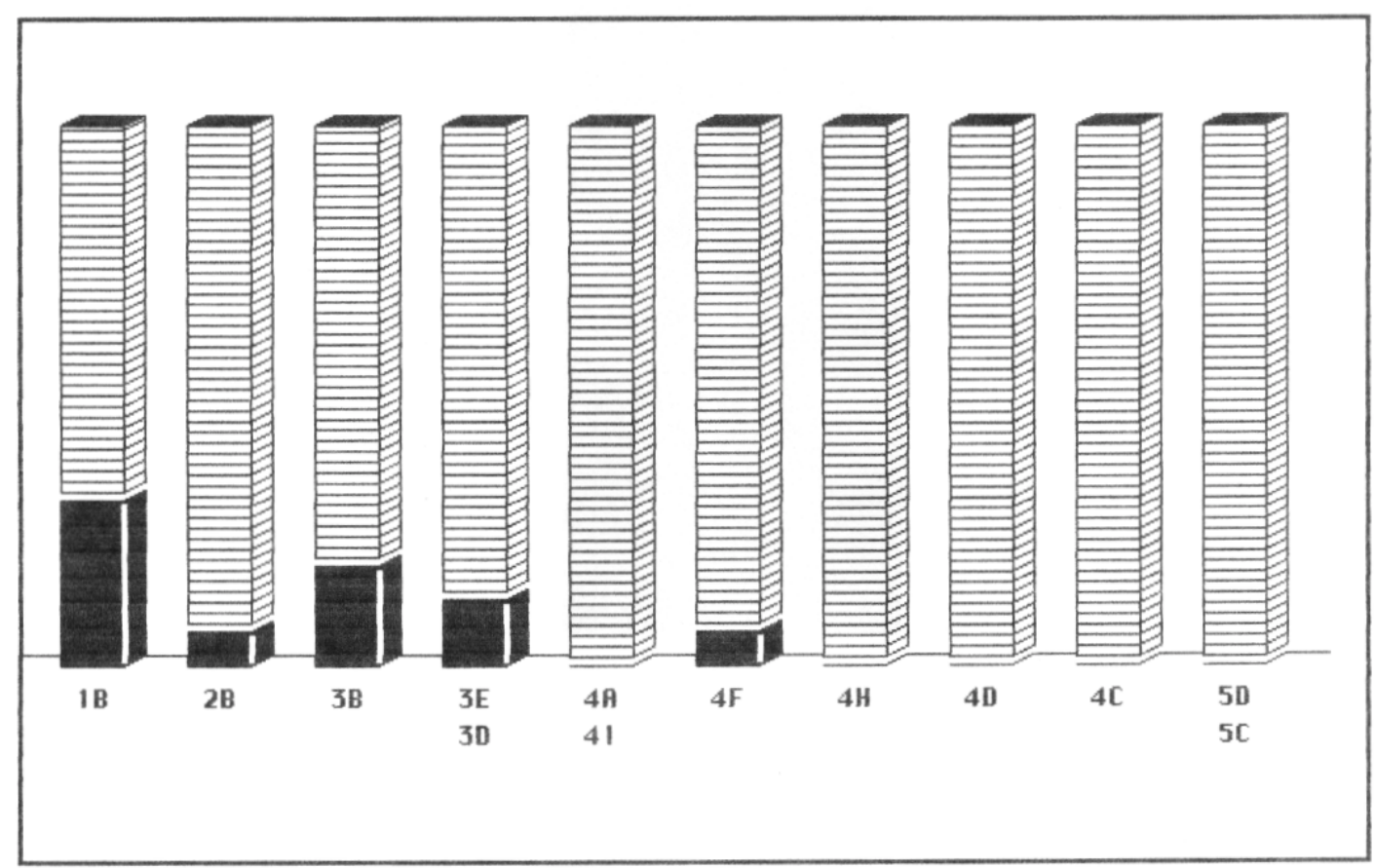

Abb. 209 Die Verteilung der Klingenformen in polnischen Städten des 13. und 14. Jahrhunderts 
In fast allen hier bisher behandelten Fragestellungen zur Verbreitung von Einzelphänomenen waren deutsche Kaufleute am Aufbau und an der Entwicklung der in Frage kommenden Fundorte entscheidend beteiligt. Eine derart deutliche Übereinstimmung von fast $90 \%$ der Formen eines wichtigen Kriteriums wie der Klingenform, welches sich ansonsten im gesamten Untersuchungsraum mehr oder weniger ähnlich darstellt, ist abseits dieser beiden Kulturräume jedoch nicht mehr beobachtet worden.

Die nächstliegende Erklärung für dieses Erscheinungsbild findet sich in der systematischen Ansiedlung deutscher Bürger und Bauern im 13. und 14. Jahrhundert östlich der Oder.

Die in dieser Analyse als Fundorte auftretenden östlichen Städte waren durchweg von den polnischen Fürsten privilegierte deutschrechtliche Städte mit einem hohen Anteil deutscher Bevölkerung, dazu kam der Zuzug deutscher Siedler über Lokatoren, dessen Höhepunkt von der Mitte des 13. bis in das ausgehende 14. Jahrhundert reichte und vor allem Schlesien, Pommerellen und die westlichen Teile Großpolens umfaßte $^{467}$. Für die Regierungszeit Kasimirs III. (gest. 1370) stellt Rhode sehr allgemein in den Städten ein überwiegend deutsches Bürgertum fest ${ }^{468}$, welches er dahingehend spezifiziert, daß vor allem im 13. Jahrhundert das Bürgertum in seinen oberen Schichten deutsch blieb, während der polnische Bevölkerungsanteil in den Städten seit dem 14. Jahrhundert wuchs ${ }^{469}$.

Blaszczyk stellt als Städtelandschaften, die seit der ersten Hälfte des 13. Jahrhunderts vor allem deutsche Bewohner aufweisen, Niederschlesien, Westpommern und Preußen heraus, im einzelnen besonders Krakau, Breslau, Posen, Danzig und Stettin; die Blüte dieser deutschrechtlichen Städte sieht er im 14. Jahrhundert ${ }^{470}$.

Die hier in Frage kommenden Fundorte werden bis auf Stargard unter anderem von Dollinger behandelt ${ }^{471}$.

Krakau und Posen wiesen bereits seit Otto von Bamberg deutsche Kaufmannsviertel auf. Das Krakauer Gründungsprivileg durch Herzog Boleslav 1257 galt nur für deutsche Siedler, bis 1316 konnten Polen kein Bürgerrecht erwirken. Erst 1387 als Hansestadt bezeichnet, war Krakau schon früh in den hansischen Wirtschaftsraum eingebunden, vor allem durch den Handel mit slowakischem Kupfer ${ }^{472}$.

In Danzig liegt der Beginn einer deutschen Ansiedlung im ausgehenden 12. Jahrhundert. 1238 wurde sie von Herzog Svantopolk zur Stadt erhoben, eine weitere Gründung erfolgte im 14. Jahrhundert. Noch im letzten Drittel dieses Jahr-

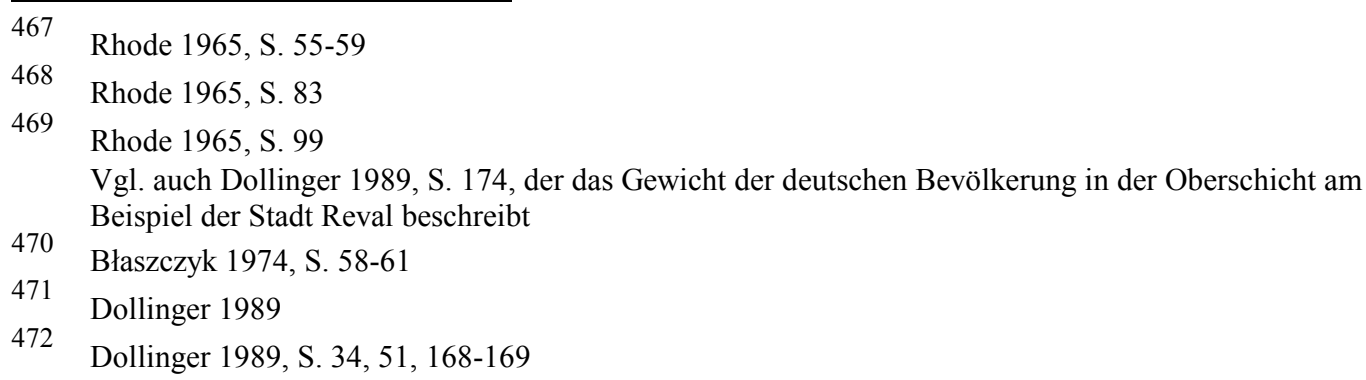

472 Dollinger 1989, S. 34, 51, 168-169 
hunderts betrug der Anteil der Zuwanderer aus Gebieten westlich der Elbe an der Gesamtbevölkerung $25 \%{ }^{473}$.

Die Herkunft der deutschen Bevölkerungsteile in den Städten und Dörfern östlich der Oder liegt vor allem in den überbevölkerten und städtereichen Landschaften Westfalens, Niedersachsens und dem Niederrhein, neben geringeren Anteilen aus den Niederlanden, Flandern und anderen westeuropäischen Gebieten ${ }^{474}$. Dementsprechend bezeichnet zum Beispiel Wasowicz den westfälisch-rheinländischen Einfluß als prägend für die Entwicklung der angewandten Kunst in Danzig zwischen 1300 und $1350^{475}$.

Als Beispiel für den Einfluß westfälischer Kaufmannsfamilien in der Städtelandschaft östlich der Oder führt Rothert das Dortmunder Geschlecht der Sudermann an, die seit 1257 in Krakau ansässig waren und später zu den "einflußreichsten Krakauer Ratsfamilien" zählte ${ }^{476}$, sowie den westfälischen Zuzug nach Danzig in der zweiten Hälfte des 14. Jahrhunderts ${ }^{477}$.

$\mathrm{Da}$ sich dieser Einfluß auch auf alltägliche Gebrauchsgüter erstreckt, zeigen die Funde der Stadtkerngrabungen in Stettin, Posen, Gnesen, Danzig und vielen weiteren polnischen Städten. Da es sich bei den meisten Holzgefäßen, Irdenwaren und Trachtbestandteilen nicht um Importgut, sondern um einheimische Produkte handelt, zeichnet sich der Herstellungs- und Formenwandel im lokalen Alltagsgut mit dem verstärkten Einsetzen deutscher Besiedlung deutlich ab.

Bisher liegen jedoch noch keine überregionalen Untersuchungen vor, die über das Fundgut aus Siedlungen und Städten östlich der Oder regionale Eingrenzungen in den westlichen Ursprungsgebieten zuließen. Bei vielen Alltagsobjekten wäre dieser Ansatz auch wenig sinnvoll, da sie sich auch in den in Frage kommenden nördlichen deutschen Landschaften kaum voneinander unterscheiden lassen, wie zum Beispiel Gürtelschnallen, Daubenschalen, Kugeltopfformen oder Holzlöffel.

Die untersuchten Messer aus Städten des Weser-Werra-Raums geben aber einen Hinweis auf die Möglichkeit, daß sich mit dem ihrer Formenverteilung identischen Erscheinungsbild in den östlichen Städten auch die Herkunft der Neusiedler dokumentiert, da sie sich auch von den übrigen untersuchten Räumen sowie vom Gesamtraum unterscheidet.

Das hier benutzte Fundgut aus den Städten Minden, Höxter und Göttingen kann allerdings kaum stellvertretend für Hauptorte der Auswanderung wie Dortmund oder Soest stehen.

Dollinger 1989, S. 52, 173

Dollinger 1989, S. 172

Weczerka 1973, S. $44 \mathrm{f}$

Schoppmeyer 1990, S. 139-142

Wasowicz 1973, S. 254-255

476

Rothert (I) 1981, S. 255

Rothert (I) 1981, S. 422
} 
So wäre diese Annahme durch eine entsprechende Untersuchung von Messern des 13. und 14. Jahrhunderts aus westfälischen und niedersächsischen Städten zu verifizieren, die in ausreichender Menge bisher nicht vorgelegt worden sind.

Faßt man die Ergebnisse zusammen, so zeigt sich in der überregionalen Analyse der gleiche Kernbestand mit Beispielen der Leitformen IIIb, IIIe und Ib wie auf zeitgleichen Burgen, lediglich die prozentuale Abfolge variiert.

Die Untersuchung anteiliger Formenverteilungen in verschiedenen historischen Städtelandschaften hat zunächst dieselben Leitformen ergeben, im Norden und Westen des Untersuchungsraums auch dieselbe prozentuale Abfolge wie in der überregionalen Auswertung.

Die fast identischen Verteilungsbilder der Bestände aus Städten des Weser-WerraGebietes und aus östlichen Hansestädten unterscheiden sich von allen übrigen Auswertungen. Sie stehen vielleicht in Zusammenhang mit dem hohen Anteil aus Westfalen und Niedersachsen stammender Bürger in östlichen Hansestädten. 


\section{Messerformen vor 800 n. Chr.}

Die für das 11. bis 14. Jahrhundert belegte Formenvielfalt an Messerklingen hat sich in der darauffolgenden Zeit, in der sich ein Wandel der Klingenformen fast ausschließlich in Proportionsveränderungen manifestiert, nicht fortgesetzt ${ }^{478}$. Die Herstellung von Messern wird einheitlicher, dementsprechend auch die Form von Klingen und Griffen ${ }^{479}$.

Die Frage nach den Hintergründen der mittelalterlichen Formenvielfalt konnte aufgrund der archäologischen Quellenbasis nur angeschnitten werden, in einigen Fällen können Bildquellen Hinweise geben ${ }^{480}$.

Die wenigen Beispiele aus der Zeit des 9. und 10. Jahrhunderts lassen erkennen, daß in jedem Fall bereits die Leitformen des Formenmodells nachweisbar sind, Klingen mit geraden und gebogenen Rücken und Schneiden waren in den Hauptvarianten der Leitformen Ib, IIb, IIIb und IIIe bereits vorhanden ${ }^{481}$. Der Anzahl an Klingen mit weiter differenzierten Formen oder mit den aus dem Kernmodell herausfallenden Sonderformen des 11. bis 14. Jahrhunderts stehen für das 9. bis 10 . Jahrhundert keine statistisch vergleichbaren Mengen gegenüber, so daß der Anschein, die Bandbreite an Klingenformen sei in dieser Zeit geringer gewesen als im Hoch- und Spätmittelalter, nur bedingt verifizierbar ist.

Für den Vergleich dieser Bestände mit Messern des 5. bis 8. Jahrhunderts konnte aus ähnlichen Gründen nur die Frage von Belang sein, ob sich die Leitformen des Modells bereits vor und während der Karolingerzeit nachweisen ließen oder nicht. Es soll an dieser Stelle nicht versucht werden, die ganze Bandbreite völkerwanderungszeitlicher und frühkarolingischer Messerformen zu umreißen; auch die Frage nach der Herkunft dieser Formen und ihrer Abhängigkeit von der Entwicklung der gleichzeitigen Waffenformen (vor allem der Entwicklung der Saxklingen) würde an dieser Stelle den Rahmen der Untersuchung sprengen ${ }^{482}$.

Die Formen Ib, IIb und IIIb lassen sich anhand einiger ausgewählter Beispiele aus den Moorfunden von Kragehul und Nydam in Dänemark bereits für das 4. bis 5 . Jahrhundert belegen, die Griffangeln sind durchläufig und die Vollgriffe (Hilzen) bereits mit Zwingen oder Griffendknäufen befestigt ${ }^{483}$ (Abb. 210a bis 210d). Diese bereits voll ausgeprägten Angelmesser mit zusätzlich gesicherten Griffen sind aufgrund ihrer Form nicht zu datieren, sie sind bis in das 14. Jahrhundert nachweisbar.

\footnotetext{
$478 \quad$ Vgl. Kapitel 6, Abschnit2.1.

479 Vgl. Kapitel 6, Abschnitt 2.3. bis 2.4. und Abschnitt 2.6.

480 Vgl. Kapitel 9, Abschnitt 2

481 Vgl. Kapitel 6, Abschnitt 2.1.

482 Vgl. Hundt 1953, S. 104 ff.

483 Engelhardt 1867 und 1865
} 
Erst mit dem Auftreten des Absatzes zwischen Griff und Schneide im 14. bis 15. Jahrhundert verändert sich ihre Gesamtform.

Ähnliches gilt für die drei Beispiele aus Ihre (Hellvi) und Hallvede (Eke) auf Gotland (Abb. 210f bis 210h). Abgesehen von den Bronzebeschlägen der Griffe ist vor allem das Messer aus Hallvede als typisches skandinavisches Mehrzweckmesser anzusehen, das sich in Form und Proportionen bis auf den heutigen Tag nicht verändert hat ${ }^{484}$. Die drei Beispiele stammen aus der zweiten Hälfte des 7. Jahrhunderts.

Vier Messer aus der skandinavischen Siedlung Grobin-Seeburg (West-Kurland) mit den Klingenformen Ib und IIIb sind als Beispiele für einen Aspekt ausgewählt worden, der die Frage nach den Längen der Klingen berührt. Drei der vier Messer stammen aus der zweiten Hälfte des 7. Jahrhunderts (Abb. 211a bis 211c), ihre Klingenlängen liegen zwischen 6 und $13 \mathrm{~cm}$. Auch bei Berücksichtigung des verschliffenen Zustandes der ersten beiden Messer ist aufgrund des unterschiedlichen Einfallwinkels der Messerrücken von erheblichen Unterschieden bei den Klingenlängen auszugehen, die möglicherweise Hinweise auf unterschiedliche Funktionen geben $^{485}$. Das vierte Messer aus Grobin-Seeburg (Abb. 211d) mit der Klingenform IIIb stammt aus einem Männergrab der zweiten Hälfte des 8. Jahrhunderts ${ }^{486}$.

Weitere Formen der Gruppen Ib und IIIb sowie Klingen, deren Rückenverlauf abgeknickt ist, sind am Beispiel der befestigten Anlage des 7. bis 8. Jahrhunderts von der Sternberger Burg ${ }^{487}$ (Abb. $211 \mathrm{i}$ bis 211n) sowie des friesischen Gräberfeldes von Dunum aus dem 8 . bis 9 . Jahrhundert ${ }^{488}$ (Abb. 211e bis 211h) aufgeführt worden.

\footnotetext{
484 Nerman 1958, S. 119; der Autor konnte 1980 in Schweden ein neues Messer erstehen, welches mit dem Exemplar aus Hallvede nahezu identisch ist, allerdings wird die Scheide mittlerweile aus Hartplastik produziert. 

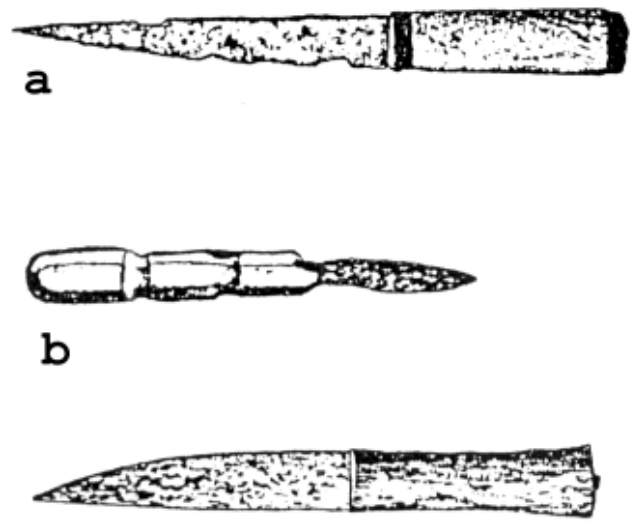

C
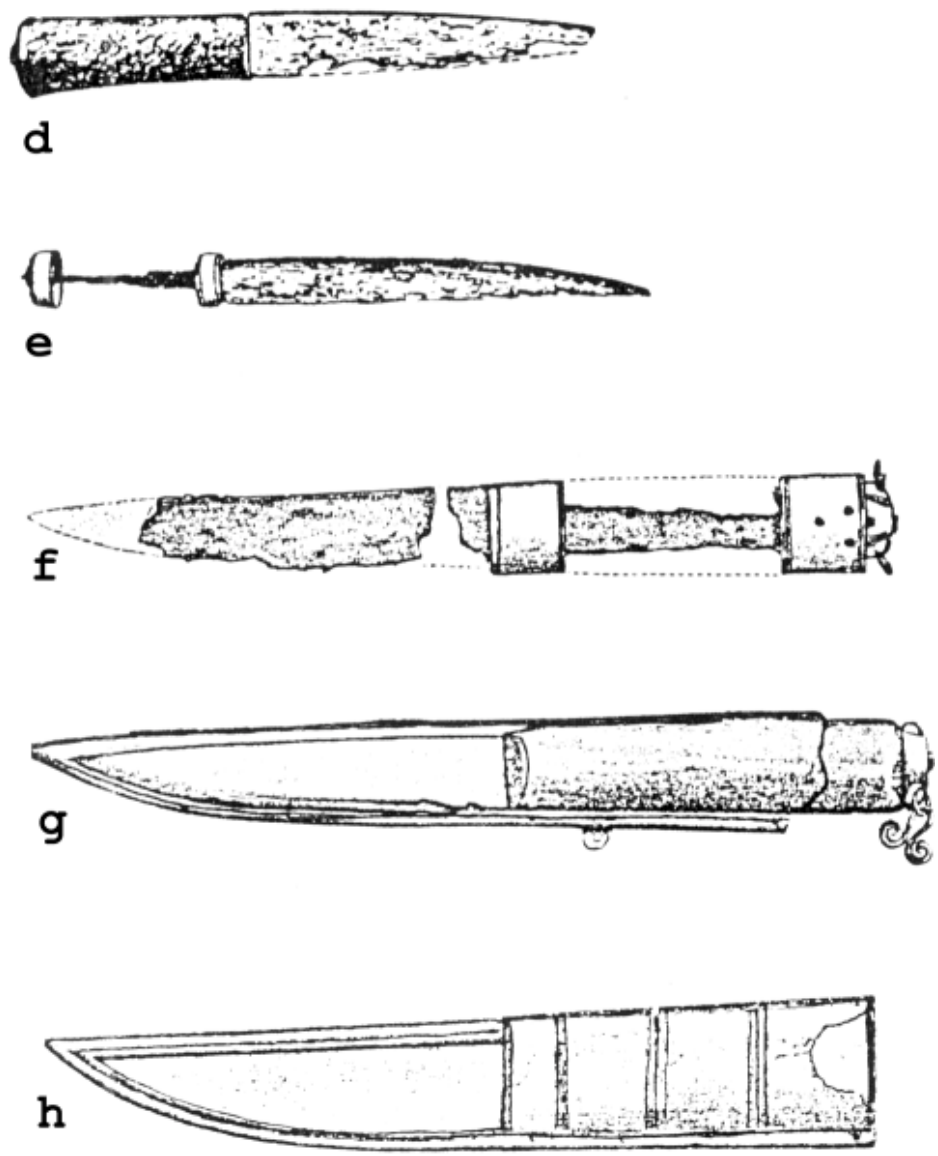

Abb. $210 \quad$ Messerformen vor 800 n.Chr. $I^{489}$

$a-b, e:$ Kragehul, 4.-5. Jahrhundert; $c$-d: Nydam, 4.-5. Jahrhundert; f, h: Ihre (Hellvi), 2. Hälfte 7. Jahrhundert; g: Hallvede (Eke) 2. Hälfte 7.Jahrhundert

489 a-b, e: Engelhardt 1867, Tafel 6.1-3

c-d: ders. 1865, Tafel 15.2, 7

f-h: Nerman 1958, Textfiguren 167-168, S. 119 

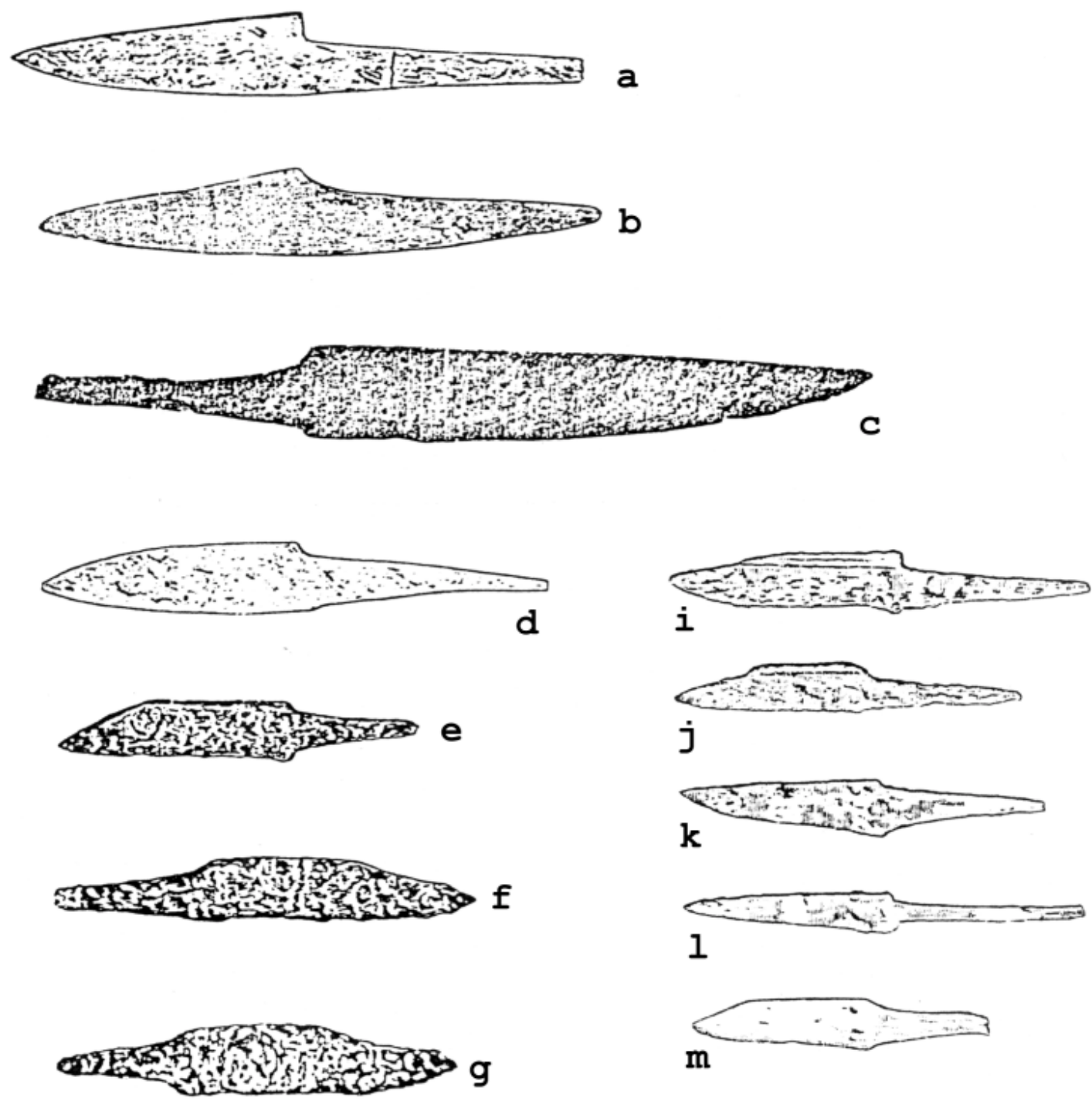

1

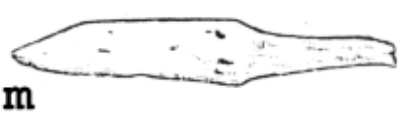

n

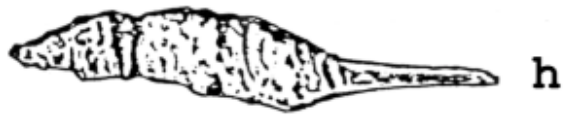

Abb. $211 \quad$ Messerformen vor 800 n.Chr. II ${ }^{490}$

a-d: Grobin-Seeburg, 2. Häfte 7. - 1. Hälfte 9. Jahrhundert;

e-h: Dunum, 8. - 9. Jahrhundert; i-n: Sternberger Burg, 7. - 8. Jahrhundert

490 a-d: Nerman 1958, Tafeln 53.375, 28.159, 24.132 und 36.212

e-h: Schmidt 1970, Abb. 2, S. 44; Abb. 3, S. 45; Abb. 5, S. 54

i-n: vgl. Katalogteil ehemalige DDR, Kat. Nr. 22-29 

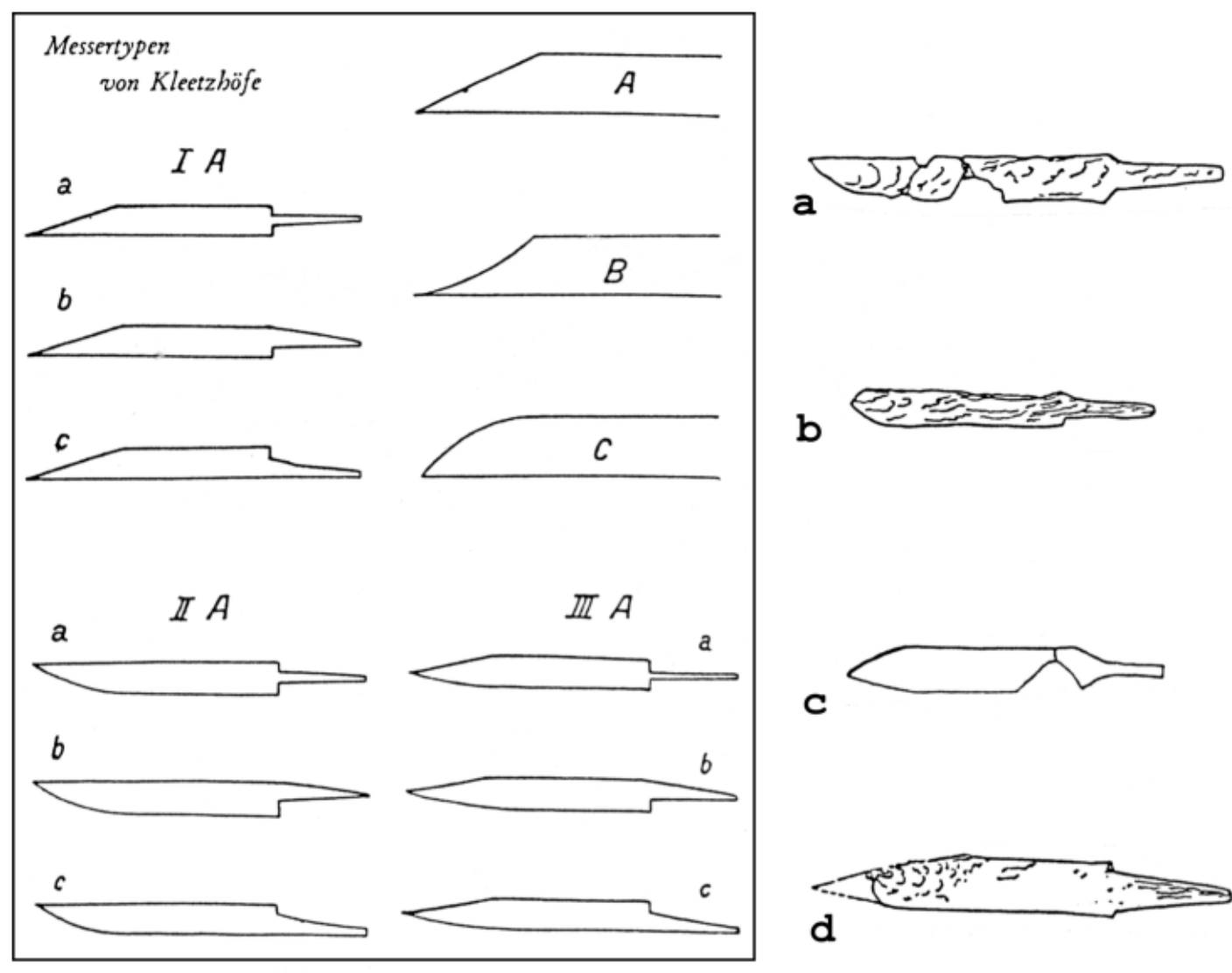

b

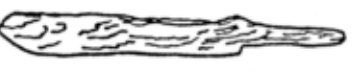

c

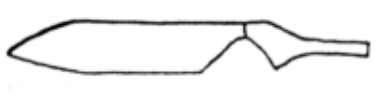

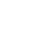

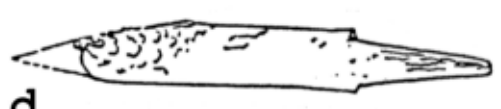

d

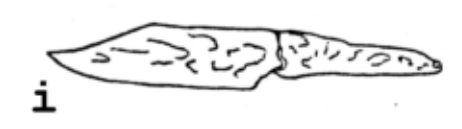

e

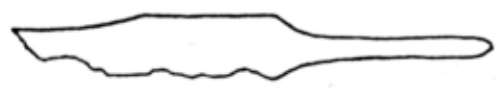

f

HF=

g

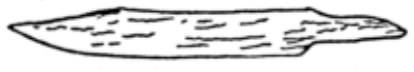

i
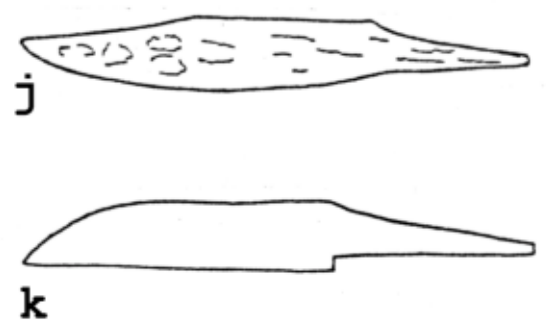

\section{0}

Abb. 212 Messerformen eines karolingerzeitlichen Reihengräberfeldes am Beispiel von Feldendorf-Kleetzhöfe, Kulmbach ${ }^{491}$

links oben die von Max Hundt anhand des Kleetzhöfer Messermaterials entworfene Formentypologie

a-b: Ib1; c-d: IIIb 1/2; e-j: Beispiele unterder Leitform III e; $k$-l: IIb 1

491 Hundt 1953, Abb. S. 53

a-1: Abb. S. 56-57 
Auffallend ist der bereits erwähnte frühe Ansatz der Form IVf ${ }^{492}$. Eine genauere Form- und Herstellungsanalyse von Messern des 7. bis 8. Jahrhunderts liegt bisher nicht vor, es darf an dieser Stelle aber die Vermutung geäußert werden, daß es sich bei der Form IVf in dieser Ausprägung möglicherweise um eine produktionstechnisch bedingte Form handelt. Der Absatz im Rücken (Abb. 211f, 211g, 211i, 211j, 211n) scheint mit den im hinteren Klingenabschnitt unterhalb des Rückens verlaufenden Rillen in Zusammenhang $\mathrm{zu}$ stehen, da diese nicht bis zur Spitze durchlaufen. Ob die frühe Ausprägung des Typs IVf als Vorläufer der Formen IIIe1-4 oder als regional auf die friesischen und westslawischen Küstenregionen begrenzbare Form anzusehen ist, muß dahingestellt bleiben. Schuldt stellte jedoch bei der Vorlage des Materials von der Sternberger Burg fest, daß "unter den Messern aus der... Siedlung Groß-Raden, die um die Mitte des 9. Jahrhunderts gegründet wurde, die Form mit dem zur Spitze hin gekehlten Rücken eine Seltenheit ist, während sie hier überwiegt" ${ }^{493}$. Den Unterschied zwischen der frühen Ausprägung der Form IVf und den späteren, nun wirklich "gekehlten" Klingenformen IIIe1-4 hat Schuldt nicht erwähnt.

Die Formen der Gruppe IIIe, deren vordere Rückenpartie einen gleichmäßig zur Spitze einfallenden, konkav gebogenen Verlauf nimmt, sind seit dem 9. Jahrhundert im bearbeiteten Bestand nachweisbar ${ }^{494}$.

1953 veröffentlichte Max Hundt das karolinigische Reihengräberfeld von Felkendorf-Kleetzhöfe, Landkreis Kulmbach ${ }^{495}$. Aufgrund einer Formanalyse des Messerbestandes dieses Gräberfeldes stellte er Messertypen zusammen, die bereits wesentliche Formkriterien zur Erfassung von Klingen und Griffangelkonstruktionen enthielten (Abb. 212) ${ }^{496}$.

Der Bestand der Messerbeigaben des Gräberfeldes von Kleetzhöfe enthält die wichtigsten Klingenformen, die bis in das ausgehende Mittelalter vorkommen, Formen der Typen Ib, IIb, IIIb und IIIe (Abb. 212a bis 212 1). Bereits hier stehen Klingen unterschiedlicher Formen und gleicher Klingenlängen formengleiche Klingen unterschiedlicher Längen gegenüber (Abb. 212e, 212h).

\footnotetext{
492 Vgl. Kapitel 6, Abschnitt 2.1.

493 Schuldt 1982, Berlin 1983, S. 140

494 Vgl. Kapitel 6, Abschnitt 2.1.

495 Hundt 1952

496 Diese Übersicht bewog den Verfasser, das im ersten Kapitel dieser Untersuchung vorgelegte Klassifizierungsmodell der Klingen- und Grifformen (Formenmodell) zu entwickeln. Die Kombination der von Hundt vorgestellten Klingen- und Grifftypen ergeben einen Ausschnitt des vom Verfasser entwickelten Modells; die Form c betrifft im wesentlichen ein bestimmtes Verschliffstadium. Die in Kleetzhöfe vorhandene Möglichkeit, den Rückeneinfall zur Schneide vor oder auf der Klingenmitte beginnen zu lassen, ist in der Übersicht nicht vorhanden.
} 


\section{Zusammenfassung}

Die vorgestellten Beispiele haben gezeigt, daß der Bestand hoch- und spätmittelalterlicher Klingenformen und Angelkonstruktionen zumindest seit dem 8. bis 9 . Jahrhundert in seinen grundsätzlichen Anlagen vollständig vorhanden ist. Die Formen Ib, IIb und IIIb sowie Griffangelkonstruktionen mit Zusatzbefestigungen sind bereits für das 5. bis 7. Jahrhundert nachweisbar.

Das Beispiel Kleetzhöfe zeigt auch, daß es bereits für das 8. bis 9. Jahrhundert schwer sein wird, den einzelnen Formen bestimmte Funktionen zuzuweisen, die sich in bedeutenden Unterschieden der Klingenlängen zu erkennen geben.

Die Vermutung, daß sich funktionale und produktionstechnische Innovationen weniger an Formen als an Herstellungsverfahren, Maßen und Konstruktionselementen festmachen lassen, scheint sich auf die Jahrhunderte vor dem Bearbeitungszeitraum ausdehnen zu lassen ${ }^{497}$. 


\section{Klappmesser}

Klappmesser (Einschlag-, Faltmesser) sind über den gesamten Bearbeitungszeitraum verstreut nachgewiesen worden.

Der bearbeitete Bestand an Klappmessern läßt sich in zwei Gruppen unterteilen:

Klappmesser mit einer Klinge, die sich über eine Scharniervorrichtung am Griffende in den Griff versenken läßt, sind bereits aus provinzialrömischen Fundplätzen bekannt (Abb. 213i, 213j) und innerhalb des Bearbeitungszeitraums vom 9. bis zum 17. Jahrhundert nachweisbar.

Klappmesser mit einer Doppelklinge, bei denen wie bei Messern mit feststehenden Klingen immer eine Klinge offen liegt, sind vom 8. bis 12. Jahrhundert beobachtet worden (Abb. 214).

Die einfachste Form des Klappmessers mit einer Klinge weist hinter dem Scharnierloch an der Klinge einen schräg nach oben weisenden Dorn auf, der die stehende Klinge stabilisiert und ein weiteres Umklappen der Klinge verhindert (Abb. 213e bis 213h). Bei Messern, deren Klinge vom Griff vollständig aufgenommen wird, ist dieser Dorn stärker ausgebildet, um über ihn die Klinge hebelartig aufzuklappen. Bei einigen Klappmessern des 13. Jahrhunderts aus Novgorod ist der Dorn als Öse ausgestaltet worden, die gleichzeitig als Haltevorrichtung diente (Abb. 213c bis 213e).

Bei Klappmessern, deren Klingen im geschlossenen Zustand nur teilweise vom Griff umfaßt wurden, war ein Klapphebel dieser Art nicht erforderlich (Abb. 213a).

Im 16. bis 17. Jahrhundert wird die Scharniervorrichtung vom Griff getrennt; sie wird in ein heftartig abgesetztes Vollmetallstück eingefügt, das durch eine Stoßkante vom Griff (in der Regel Griffschalen) getrennt wird (Abb. 215e bis 215g). Im aufgeklappten Zustand unterscheidet sich ein Klappmesser in dieser Zeit nicht mehr von Messern mit feststehenden Klingen.

In Berlin-Spandau wurde ein ähnliches Messer geborgen, welches in das 14. Jahrhundert datiert worden ist (Abb. 215a). Das Beispiel aus der Burg Kakesbeck, welches aufgrund der Fundumstände und Begleitfunde kaum vor das 16. Jahrhundert datiert werden $\mathrm{kann}^{498}$, zeigt zwar schon das vom eigentlichen Griff getrennte Scharnier, aber noch keinen Absatz (Abb. 215d). Vergleichbare Messer werden ausschließlich in das ausgehende 16. bzw. in die erste Hälfte des 17. Jahrhunderts datiert ${ }^{499}$, so daß die Datierung des Spandauer Exemplars unzutreffend erscheint.

\footnotetext{
498 Vgl. Katalogteil BRD, Kat. Nr. 126

499 Vgl. neben den drei Beispielen aus Coesfeld (Katalogteil BRD) Katalogteil Dänemark, Kat. Nr. 54 sowie Katalogteil BRD, Kat. Nr. 95
} 
Klappmesser mit Doppelklingen sind aus Novgorod, Dorestad und einem lettischen Gräberfeld für das 8. bis 12. Jahrhundert belegt (Abb. 214a bis 214d, 214f).

Ein Klappmesser aus Birka besitzt an jedem Griffende je eine einklappbare Klinge, deren Spitze jeweils nach außen hochgebogen worden ist. Die Spitze der Klinge diente möglicherweise zum Aufklappen (Abb. 214e).

Angesichts der Menge an Klappbestecken, die zumindest aus dem 16. und 17. Jahrhundert aus zahlreichen Kunstgewerbesammlungen bekannt geworden ist $^{500}$, kann damit gerechnet werden, daß das archäologisch erfaßte Material nur einen Ausschnitt aus der Fülle derartiger Messer zu bieten vermag.

An dier Stelle sei noch einmal auf das Problem der Zuweisung der Form A6 $6^{501}$ verwiesen. Klingen mit ausgeprägten Griffangeln und Nietlöchern im Heftbereich sind an erhaltenen Klappmessern innerhalb des bearbeiteten Bestandes nicht bekannt geworden. Klingen mit dornartigen, nach oben weisenden Spitzen und gelochtem Heft sind dagegen eindeutig als Klappmesser zu identifizieren ${ }^{502}$.

500 Vgl. d'Allemagne 1968 (Neudruck), Abb. 363, 384, 387, 388

501 Vgl. Kapitel 6, Abschnitt 2.3.

502 Vgl. den Bestand an Messern von der Isenburg (Hattingen), Katalogteil BRD, vor allem die Katalognummern 145, 146, 149 und im Gegensatz zu diesen die Nr. 148 

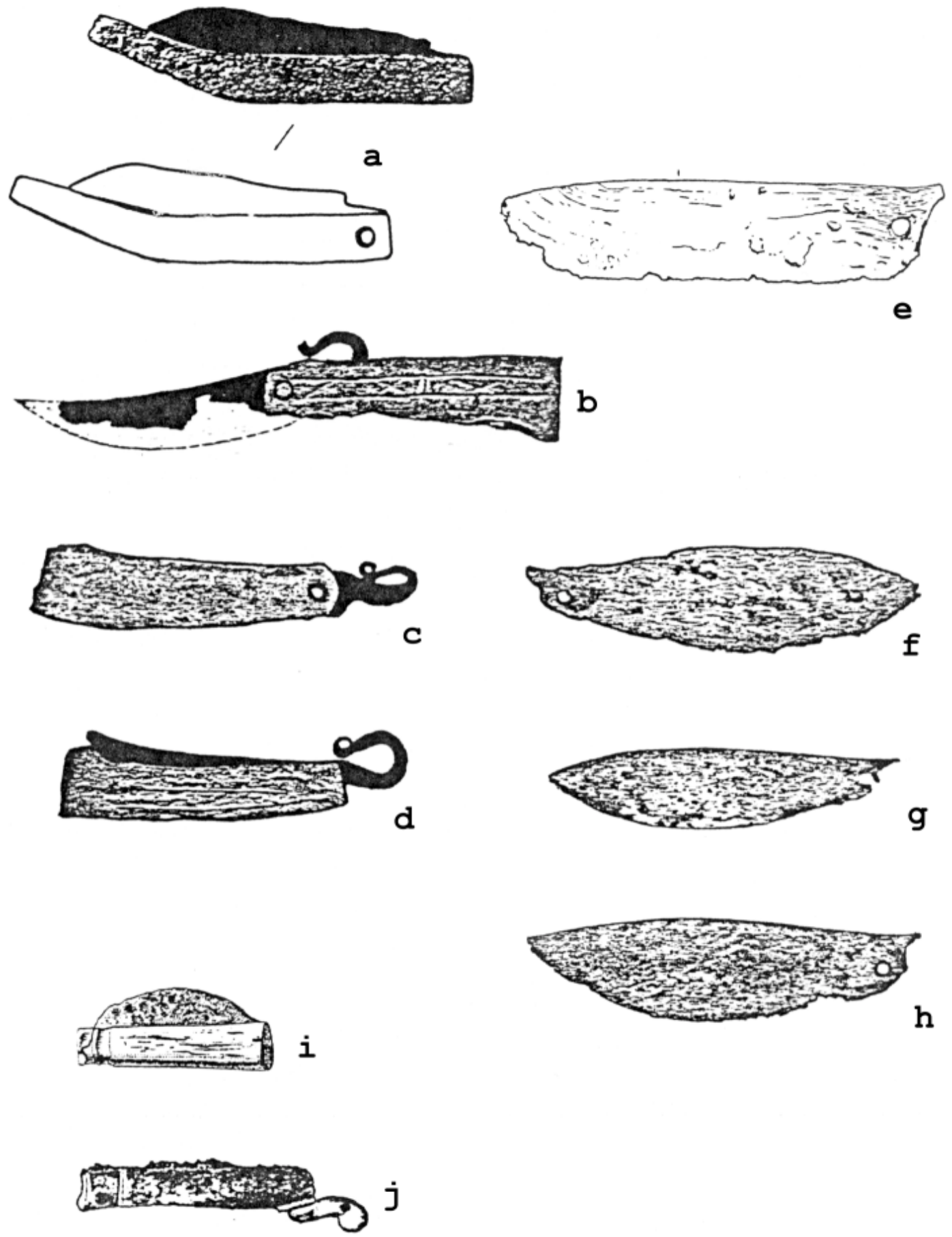

Abb. 213

Klappmesser (Falt-, einschlagmesser) mit einer Klinge (RKZ und 9.-14. Jh.) ${ }^{503}$ a: Birka, 9.-10- jh., b-d: Novgorod, 13. Jh.; e: Århus, A.13.-A.14.Jh., f-h: Novgorod, 13. Jh., i: Heddernheim, röm. Kaiserzeit; j: Mainz, röm. Kaiserzeit

503 a: Arbman 1940, Tafel 184.3

b-d, f-h: Arzichovskij/Koltschin 1959, Abb. 45.1-3 und 4-6, S. 58

e: Andersen/Macken 1971, Abb. S. 160 

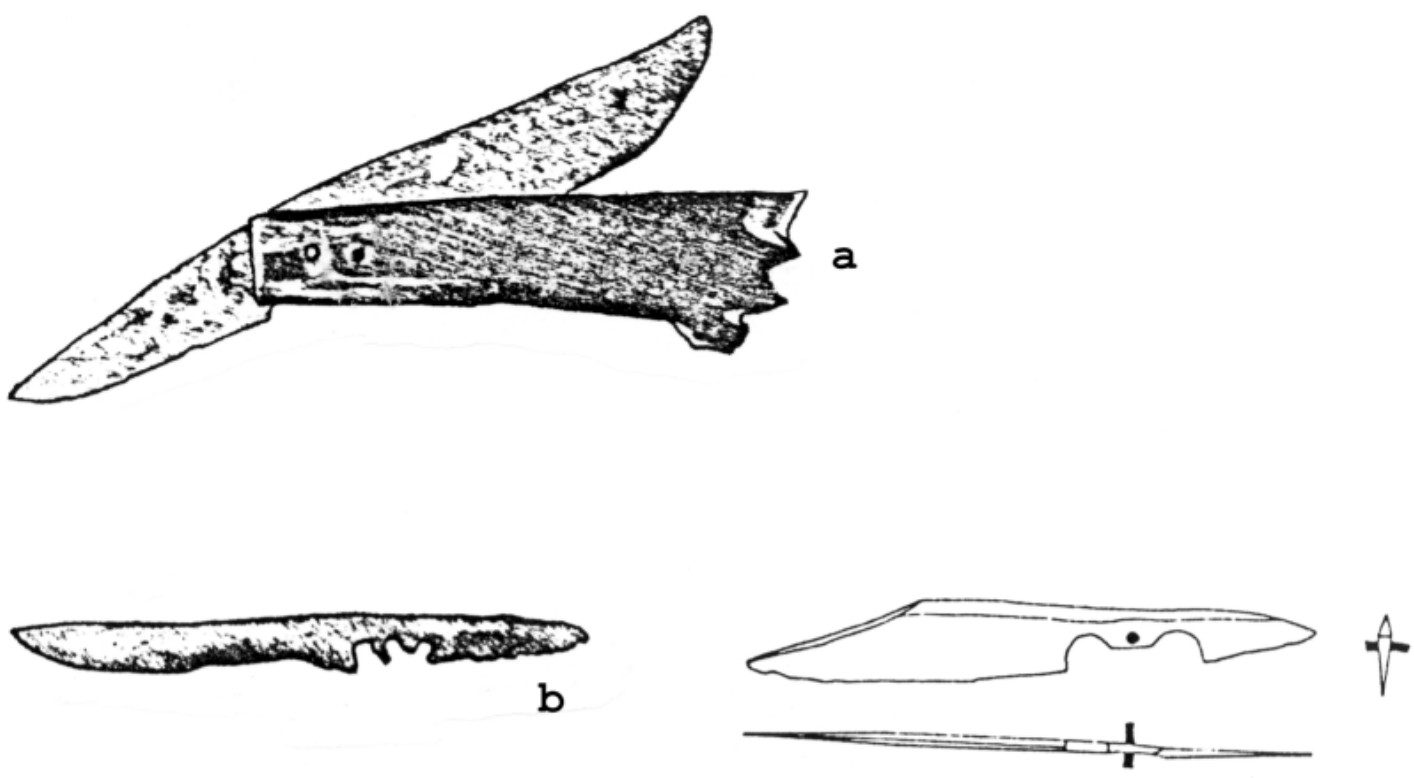

C

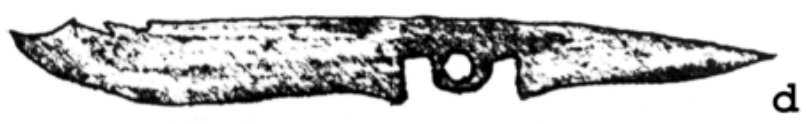

d
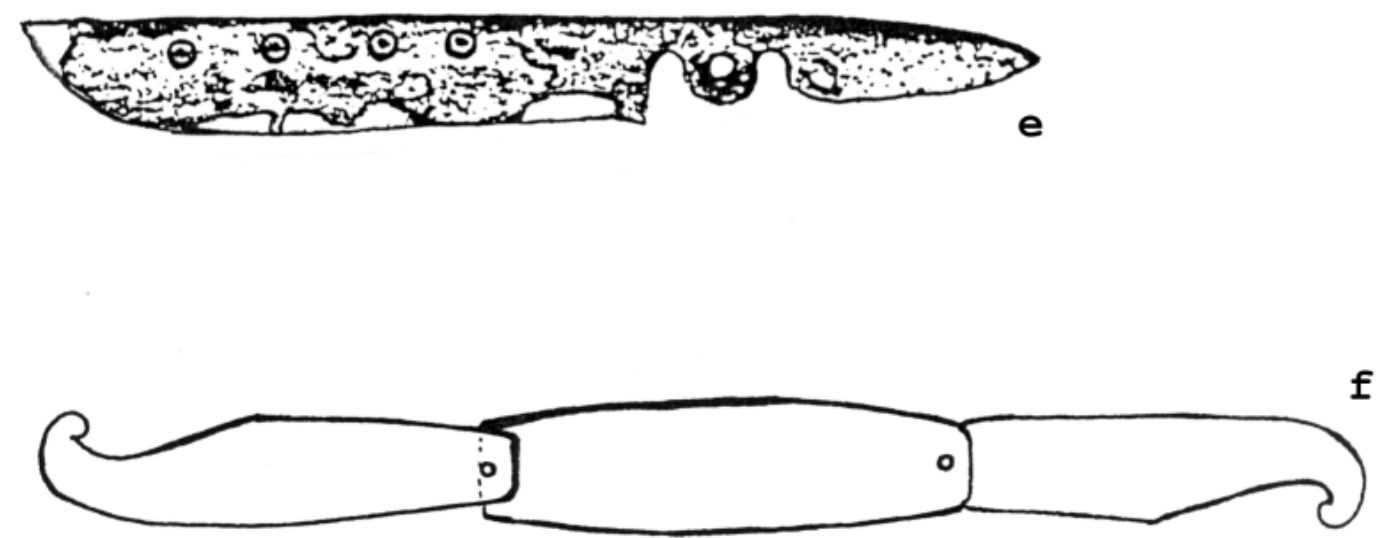

Abb. $214^{504}$

Klappmesser (Falt-, Einschlagmesser) mit Doppelklinge (8.-12. Jahrhundert) a-c: Novgorod, 11.-12. Jh.; d: Mardi, Viljandi (Lettisches Gräberfeld), 10. A.13.Jh.; e: Birka, 9.-10. Jh.; f: Dorestad, 8.-9. Jh.

504 a-c: Arzichovskij/Koltschin 1959, Abb. 44.1-3, S. 57

d: Selirand 1981, Abb. 4.5, S. 213

e: Arbman 1940, Tafel 184.4

f: van Es/Verwers 1980, Abb. 137.11, S. 185 

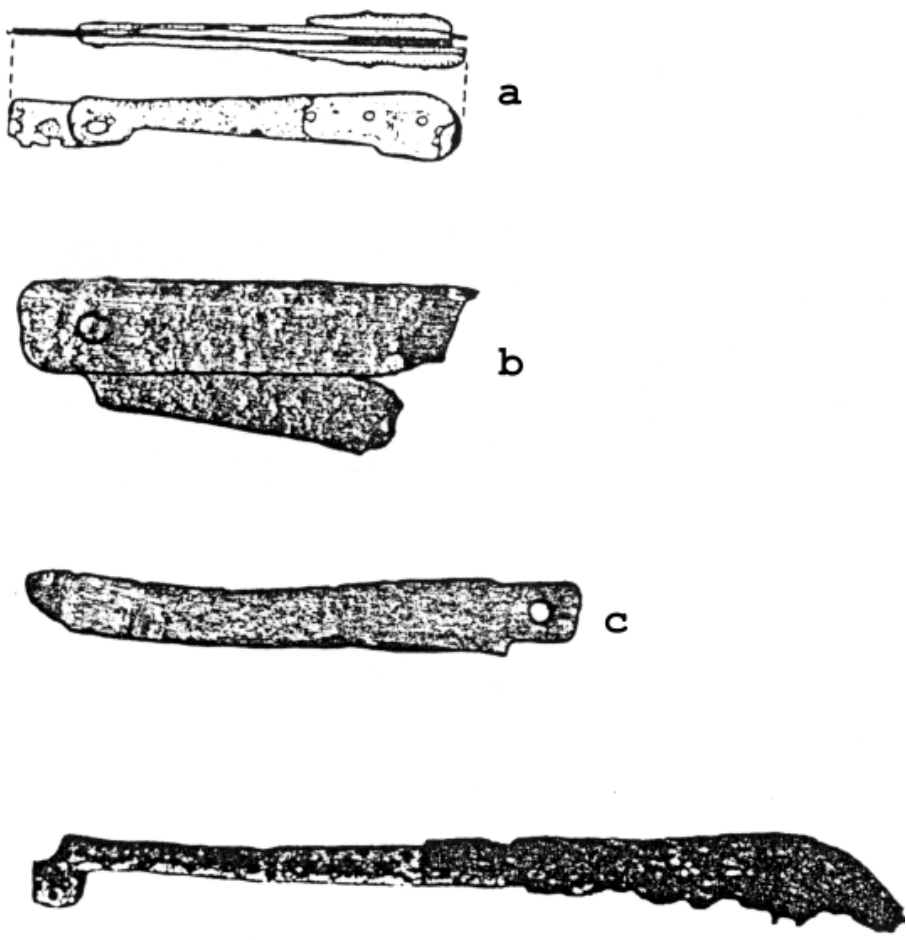

d
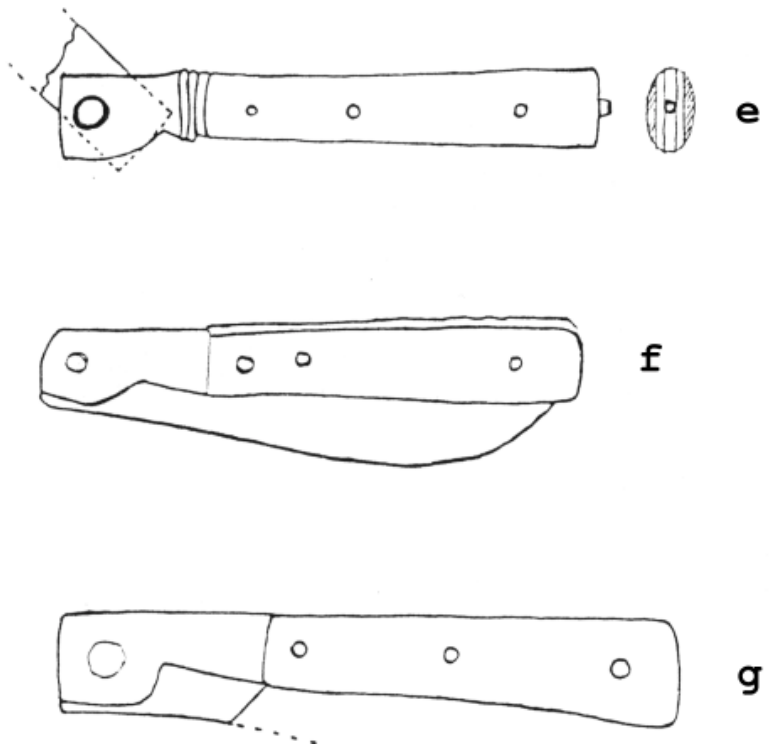

g

Abb. $215^{505}$

Klappmesser (Falt-, Einschlagmesser) 14.-A. 17. Jahrhundert) a: Berlin-Spandau 14. Jahrhundert (?); b-c: Store Valby, o.A.;

d: Burg Kakesbeck, 2. H. 16. - 1. H. 17. Jahrhundert; e-g: Coesfeld, E. 16. - 1. H. 17. Jahrhundert

505 a: Katalogteil ehemalige DDR, Kat. Nr. 143

b-c: Steensberg/Ostergard/Christensen 1974, Tafel 174.3-4, S. 369

d: Katalogteil BRD, Kat. Nr. 127

e-g: Katalogteil BRD, Kat. Nr. 220-222 


\section{Produktion und Gebrauch}

\subsection{Zur Herstellung von Messerklingen}

\subsubsection{Anmerkungen zu Herstellungsverfahren von Mes- serklingen}

Metallurgische Untersuchungen zur Feststellung der wichtigsten Produktionstechniken von Messerklingen betreffen bis auf wenige Ausnahmen Fundbestände des 9. bis 14. Jahrhunderts aus Osteuropa ${ }^{506}$. Die Verfahrenstechniken dieser Untersuchungen sollen an dieser Stelle nicht behandelt werden, sie sind aber in der Regel in den jeweiligen Untersuchungsberichten detailliert aufgeführt worden $^{507}$.

Die bisher an Messerklingen beobachteten Produktionstechniken betreffen in der Regel zwei Eigenschaften, die ein auf Dauer belastbares Messer besitzen muß: Bruchsicherheit der Klinge und Härte der Schneide ${ }^{508}$.

Das den Werkzeugschmieden im Früh-, Hoch- und Spätmittelalter zur Verfügung stehende Ausgangsmaterial an ungereinigtem, zum Teil erheblich verschlacktem Eisen mußte mit oft erheblichem Aufwand bearbeitet werden, um es zunächst von aus dem Verhüttungsprozeß herrührenden Einschlüssen zu säubern und dem zu bearbeitenden Werkstück dann die gewünschten Eigenschaften zu verleihen ${ }^{509}$.

\footnotetext{
506 Pleiner 1983, S. 85; vgl. dort auch das Literaturverzeichnis mit der wichtigsten Literatur zu metallurgi- 
Die an Messerklingen beobachteten Technologien bestehen aus dem Verarbeiten verschiedener Materialien, vor allem dem Bündeln und Verschweißen von Eisenund Stahlbändern, sowie aus Härtungsverfahren wie dem Aufkohlen und thermischen Behandlungen. Dazu kommen Ätzverfahren an Klingen aus alternierend verschweißten Eisen- und Stahlbändern zu Verzierungszwecken (Damast- und Damaszierungseffekt $)^{510}$.

Die aufwendigste Art, eine Messerklinge aus weichem Schweiß- oder Schmiedeeisen zu härten, um vorzeitigem Verschliff vorzubeugen, besteht in der Aufkohlung (Vermehrung des Kohlenstoffanteils), die die Klinge zwar härter, aber auch starrer und damit bruchanfälliger macht. Um der Bruchgefährdung vorzubeugen, kann die Klinge anschließend thermisch behandelt werden. Sie wird je nach Anforderung so weit erhitzt, daß der Kohlenstoff in bestimmten Partien der Klinge, in der Regel in den äußeren Schichten, wieder verbrennt. Dieser Prozeß wird durch abschließendes schockartiges Löschen der Klinge beendet. Dadurch entsteht eine Klinge, die zwar insgesamt aus Schmiedeeisen besteht, in der äußeren bzw. in der inneren Partie aber verschiedene Gitterstrukturen aufweist:

die innere Partie ist starr und hart (sie wird durch Anschliff an der Schneide freigelegt), die äußeren Partien sind weicher, aber auch geschmeidiger und schützen die Klinge vor Bruch ${ }^{511}$.

Die Anwendung dieser Methode ist durch veränderte Behandlung variabel in ihren Auswirkungen (z.B.: Aufkohlung der äußeren Schichten bei weichem Kern, Aufkohlung der Schneidenpartie bei weicher Rückenpartie etc.). Neben der verschiedenartigen Bearbeitung eines Ausgangswerkstoffs steht die Verbindung verschiedener Werkstoffe. Bei Messern, Scheren, Feuerstählen und anderen Eisenobjekten ist die Verwendung von Stahl und Eisen nachgewiesen worden ${ }^{512}$.

Die Verbindung von Stahl- und Eisenbändern nutzt die unterschiedlichen Eigenschaften des kohlenstoffreicheren, harten Stahls und des weicheren Schmiedeeisens, wobei der technisch langwierigere Prozeß des Aufkohlens durch das Einschweißen von Stahlpartien ersetzt wird.

Dieses Prinzip ist bei vertikal oder horizontal angelegten Mehrschichtenklingen zu beobachten, wobei die Stahlpartie nach dem Anschliff der Klinge die Schneide bildet.

demie der Wissenschaften, Bd. 25), S. 423-436, dort auch weiterführendes Literaturverzeichnis S. 437

511 Piaskowski 1960, S. 45 ff. Pleiner 1983, S. 67, S. 84

Cowgill u.a. 1987, S. 8

512 Vgl. die zusammenfassende Darstellung mit schematischen Graphiken der drei wichtigsten Mehrschichtenklingen bei Pleiner 1983, S. 84 ff. sowie Cowgill u.a. 1987, S. 8-11 
Am Beispiel metallurgisch untersuchter Messer aus Haithabu, Oppeln, Posen, Krakau-Debniki, Danzig, Novgorod und London werden im folgenden die Methoden aufgrund der publizierten Untersuchungsergebnisse vorgestellt.

Aus dem Fundmaterial von Haithabu wurden fünf Messer des 9. und drei Messer des 10. Jahrhunderts untersucht ${ }^{513}$. Festgestellt wurde eine breite Palette an Produktionstechniken und Qualitätsstufen, die von mangelhafter Ware bis zum Spitzenprodukt reicht ${ }^{514}$.

Für das 9. Jahrhundert wurden ein Messer aus weichem Schmiedeeisen sowie ein aufgekohltes Eisenmesser mit Eisenschneide festgestellt. Am ersteren Exemplar waren Aufkohlungsspuren ohne nennenswerten Erfolg zu beobachten, das zweite Messer wird von Pleiner als "Fehlerzeugnis" bewertet, da trotz thermischer Behandlung das Resultat der Aufkohlung der Schneide fehlt ${ }^{515}$.

Ein weiteres Messer des 9. Jahrhunderts ist wahrscheinlich ein Beispiel für eine erfolgreiche Aufkohlung der Klingenseiten und der Schneide mit anschließender Härtung der Schneide in Wasser, der Produktionsprozeß selbst wird allerdings von Pleiner als zu aufwendig und zu wenig kontrollierbar bezeichnet, um für eine Serienproduktion geeignet $\mathrm{zu} \operatorname{sein}^{516}$.

In einem Fall konnte ein Messer mit einer Klinge aus ungehärtetem Stahl nachgewiesen werden, deren Schneide einen stärkeren Kohlenstoffgehalt aufwies als die übrigen Klingenteile ${ }^{517}$.

Das letzte untersuchte Messer des 9. Jahrhunderts besitzt eine Dreischichtenklinge. Ein hartes Stahlband wurde zwischen zwei weicheren Eisenbändern verschweißt und angeschliffen, so daß die Stahlschneide freigelegt wurde ${ }^{518}$. Anschließend wurde die Klinge ganz durchgehärtet und erneut erhitzt, um der Bruchgefahr vorzubeu gen $^{519}$.

Die drei untersuchten Messer des 10. Jahrhunderts sind ebenfalls von sehr unterschiedlicher Qualität.

Das erste Beispiel ist wiederum aus weichem Schmiedeeisen produziert worden, Härtungsversuche wurden nicht festgestellt ${ }^{520}$. Im zweiten Fall handelt es sich um eine Klinge aus zwei mehrlagig verschweißten Eisenbändern, die im unteren Bereich eine Stahleinlage umschließen; diese bildete bei Anschliff der Klinge die Schneide ${ }^{521}$.

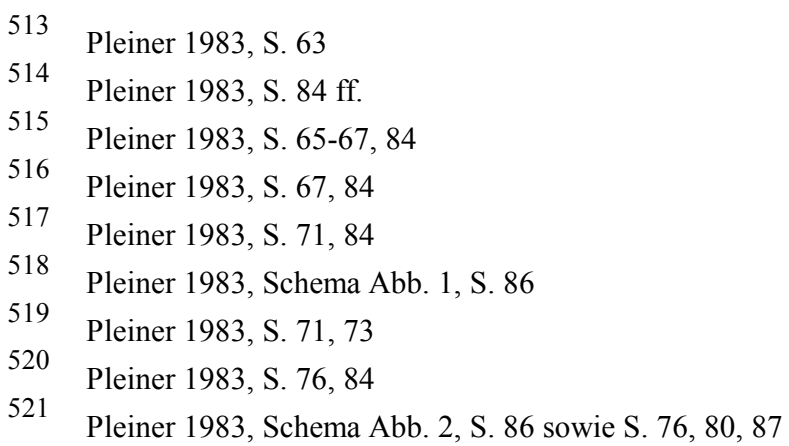


Im Gegensatz zur oben erwähnten Dreischichtenklinge läuft die Stahleinlage nicht bis zum Rücken, sondern nur bis kurz vor die Klingenmitte durch ${ }^{522}$. War im Fall der Dreischichtenklinge die Härte der Schneide bis zum totalen Verschliff garantiert, so reichte sie im letzteren Fall nur bis zur Hälfte der Klinge ${ }^{523}$.

Das letzte Beispiel aus Haithabu betrifft eine Klinge, die aus zwei Ausgangsstoffen, Eisen im Rückenbereich und Stahl im unteren Bereich, stumpf zusammengeschweißt worden ist ${ }^{524}$. Die weichere Rükkenpartie besteht aus drei horizontal verschweißten Eisenbändern mit homogenem Gefüge ${ }^{525}$, die untere Partie aus drei vertikal verschweißten Stahlbändern mit unterschiedlichen Gefügen ${ }^{526}$. Die Schneide ist wahrscheinlich im Anschluß an die Fertigstellung durch Tauchen in Fett

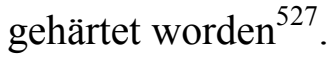

Aus der Innenstadt von Oppeln wurden Messer des 10. bis 12. Jahrhunderts metallurgisch untersucht ${ }^{528}$.

50\% aller in Oppeln geborgenen Eisenwerkzeuge bestanden aus Messern, von diesen war ebenfalls die Hälfte unter Verwendung von Stahlpartien produziert worden. Seit dem 10. Jahrhundert sind Mehrschichtenklingen nachweisbar ${ }^{529}$, wobei der Anteil der Messer mit eingeschweißten Stahlschneiden in den älteren Schichten größer ist als in den jüngeren Horizonten ${ }^{530}$. Neben Klingen aus Eisen- und Stahlbändern wurden auch zahlreiche Messer aus weichem Schmiedeeisen geborgen ${ }^{531}$.

Zwei Messer aus Oppeln verdienen besondere Beachtung, ihnen wird von den Bearbeitern aufgrund ihrer aufwendigen Produktionstechnik Waffencharakter zugeschrieben $^{532}$.

Das erste Messer aus der Schicht C1 besteht aus vier verschweißten Partien. Der Rücken wurde aus einem leicht aufgekohlten Eisenband gefertigt, im mittleren Teil der Klinge sind zwei Lagen zu unterscheiden, eine aus je einem Eisen- und Stahldraht zu einer gemusterten Stange verdrillten und ausgeschmiedeten oberen Lage und eine untere Lage aus einem weichen Eisenband, in dessen untere Schweißkante Kerben eingeschlagen worden waren. In dieser gekerbten Fläche wurde die Schneidenpartie aus Stahl verschweißt, aus den gekerbten Schweißflächen entstand nach dem Schweißvorgang eine längs über der Schneide verlaufende Wellenlinie, die 
neben ihrem technologischen Ursprung auch eine ästhetische Wirkung erzielt, korrespondierend mit dem Mustergürtel der zweiten Lage unterhalb des Rückens ${ }^{533}$.

Im Fall des zweiten Messers aus der Schicht B3 wurden fünf oder sieben verschiedene Partien zu einer Klinge verbunden. Das Prinzip ähnelt dem des ersten Beispiels. Die mittlere Klingenpartie besteht jedoch aus einer Lage, die aus zwei jeweils verdrillten Stangen (vier alternierend verdrillte Eisen- und Stahldrähte) zu einem Band verschmiedet wurden, so daß in der Fläche der fertigen Klinge ein Fischgrätenmuster entstand ${ }^{534}$. Für diesen Produktionsprozeß waren Temperaturen von $1275{ }^{\circ} \mathrm{C}$ bis $1400{ }^{\circ} \mathrm{C}$ erforderlich ${ }^{535}$.

Von den in der Innenstadt von Posen geborgenen Metallgegenständen des 11. bis 13. Jahrhunderts wurden ebenfalls mehrere Messer metallurgisch untersucht ${ }^{536}$.

Neben zwei Messern des 11. bis 12. Jahrhunderts aus aufgekohltem und thermisch gehärteten Schmiedeeisen ${ }^{537}$, die neben harten Schneiden auch nahezu schlackefreie Ausgangswerkstoffe aufwiesen, stand mit einem Exemplar ein Messer minderer Qualität, welches ein weiches Schmiedeeisen aufwies, dessen starke Verunreinigung durch Schlakkereste trotz versuchter Durchschmiedung nicht beseitigt worden waren ${ }^{538}$. Die Belastbarkeit der Schneide wird mit $682 \mathrm{~kg} / \mathrm{mm}^{2}$ angegeben $^{539}$.

An zwei Messern wurde die Verwendung von Eisen- und Stahlbändern nachgewiesen.

Das erste Messer aus der zweiten Hälfte des 11. Jahrhunderts wies eine klassische Dreischichtenklinge mit durchläufiger, vertikal verschweißter Mittellage aus Stahl auf $^{540}$; das Messer wurde nach der Fertigstellung in rotglühendem Zustand in Öl oder Blut gelöscht (thermische Härtung).

Meit dem zweiten Messer aus der zweiten Hälfte des 13. Jahrhunderts (Schicht III) lag eine horizontal angelegte Zweischichtenklinge vor, deren weiche und elastische Rückenpartie aus mehreren verschweißten Eisenblechen besteht ${ }^{541}$. Die Schneidenpartie aus sorgfältig geschmiedetem, hochgekohlten Stahl wies an der Schneide eine Belastbarkeit von $572 \mathrm{~kg} / \mathrm{mm}^{2}$ auf, die Belastbarkeit der übrigen Klingenpartien liegt zwischen $233 \mathrm{~kg} / \mathrm{mm}^{2}$ und $420 \mathrm{~kg} / \mathrm{mm}^{2}{ }^{542}$. In die Klingenfläche war nach der Fertigstellung ein Wellenband eingestichelt worden ${ }^{543}$. Aufgrund des auf-

\footnotetext{
533 Holubowicz 1956, Abb. 60 und 61, S. 157 
wendigen Produktionsprozesses und der Form der Klinge wird das Messer als Rasiermesser bezeichnet ${ }^{544}$.

In Krakau-Debniki wurden innerhalb einer slawischen Siedlung des 9. bis 12. Jahrhunderts mit lokaler Schmiedetätigkeit auch zahlreiche Messer geborgen, von denen acht metallurgisch untersucht wurden ${ }^{545}$.

Drei Messer bestanden aus weichem Schmiedeeisen, welches in einem einfachen Rennfeuerofen gewonnen worden war. Zusätzliche Härtungsmaßnahmen wurden an ihnen nicht festgestellt.

An zwei weiteren Messern wurden aufgekohlte Partien nachgewiesen, eine Klinge war ganz aus Stahl hergestellt worden.

Zwei Messer des 9. bis 11. Jahrhunderts wiesen horizontal angelegte Zweischichtenklingen auf; die Rückenpartien waren aus weicherem Eisen, die Schneidenpartien aus gehärtetem Stahl produziert worden ${ }^{546}$.

Im Ramen der Reihe "Gdańsk Wczesnośredniowieczny" (Das frühmittelalterliche Danzig), die die wichtigsten Ausgrabungsergebnisse aus der Innenstadt von Danzig in Monographien vorstellt, ist der Eisenverarbeitung und der Produktionstechnik der einzelnen Gegenstände aus Eisen vom 10. bis 14. Jahrhundert 1960 ein eigener Band gewidmet worden $^{547}$.

Die lokale Verarbeitung von Eisenerzen zu schmiedbarem Eisen geht möglicherweise zurück bis in die Mitte des 11. Jahrhunderts, seit der Mitte des 12. Jahrhunderts ist die Produktion von relativ hartem Stahl $(0,5$ bis $0,8 \%$ C) am Ort gesichert $^{548}$.

Von 44 untersuchten schneidenden Eisengeräten (darunter viele Messer) waren neun Gegenstände $(20,4 \%)$ durch Aufkohlungsprozesse gehärtet worden, fünf von ihnen wiesen neben aufgekohlten Schneiden auch Aufkohlungsspuren im Rückenbereich auf; von den 37 Messern war nur eines ganz aus Stahl produziert worden ${ }^{549}$. $66,1 \%$ der untersuchten Gegenstände sind in der Mehrschichtentechnik hergestellt worden, im Danziger Fundmaterial nachweisbar seit der Mitte des 11. Jahrhunderts. Das Verhältnis von Mehrschichtenklingen zu Objekten mit durch Aufkohlung gehärteten Partien wuchs von 1,67:1 im 11. Jahrhundert auf 5:1 im 13. Jahrhundert, die Stahlpartien betreffen in der Mehrzahl der untersuchten Schneidegeräte die Schneide ${ }^{550}$.

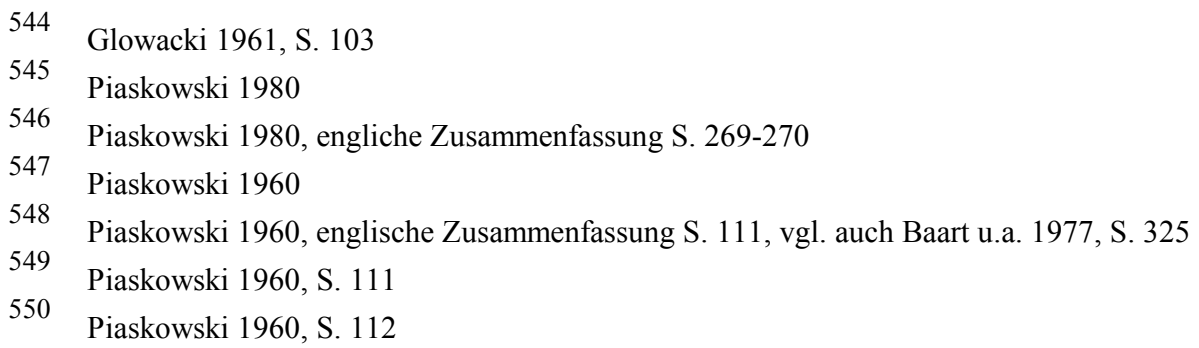


Thermische Behandlung zur nachträglichen Härtung wurde bei acht aufgekohlten, zwei stählernen und an allen mehrschichtigen Objekten nachgewiesen ${ }^{551}$.

Die Masse der analysierten Gegenstände korrespondiert in technologischer Hinsicht mit Schmiedeerzeugnissen von anderen polnischen Fundorten; von 37 untersuchten Messern wiesen 78,4\% Produktionstechniken auf, die auch an anderen Orten wie Kolberg und Leczyca nachweisbar sind ${ }^{552}$.

Die am meisten verbreitete Technik war die horizontal angelegte Zweischichtenklinge, die seit circa 1020 auftritt und um 1090 als gebräuchliche Produktionsart anzusehen ist. Um 1200 übertrifft die Zahl von Zweischichtenklingen alle übrigen am Ort nachgewiesenen Herstellungsverfahren von Messerklingen ${ }^{553}$.

Besonders hervorzuheben sind Beispiele mit "damaszierten" Klingen, einer Technik, die zu den qualitätvollsten Erzeugnissen mittelalterlicher Schmiedetechnik zählt ${ }^{554}$. Bei diesen Messern wurde zwischen der Rücken- und der Schneidenpartie eine Lage eingeschweißt, die aus alternierend verschweißten Stahl- und Eisenstreifen oder -bündeln bestand. Die unterschiedliche Färbung der helleren Eisen- und dunkleren Stahlbänder wurde durch Anätzung der Klingenoberfläche in der Abfolge der Schweißnähte sichtbar gemacht, so daß Muster ähnlich denen der Damaszenerklingen entstanden (Streifendamast, "wurmbunter Mittelbarren") ${ }^{555}$. Ob diese Messer am Ort produziert oder importiert worden sind, ist nicht sicher zu entscheiden. ${ }^{556}$

Vom 10. bis 13. Jahrhundert ließen sich zwei unterschiedliche Produktionsphasen nachweisen, die eine Entwicklung zu vermehrter Herstellung bei gleichzeitiger Vereinfachung und Standardisierung der verschiedenen Fertigungstechniken beinhalten ${ }^{557}$.

Insgesamt umfaßten die an den Danziger Messern beobachteten Techniken neben einfacher Verarbeitung von Eisen das Aufkohlen, thermische Härtungsverfahren, die Verarbeitung von Stahl, das Verschweißen von Eisen und Stahl sowie das Bündeln und Verschweißen von Eisen- und Stahlbändern. ${ }^{558}$

In Novgorod ist eine innerstädtische Erzaufbereitung und Eisenproduktion nicht nachgewiesen worden, das Rohmaterial wurde von den ländlichen Verhüttungsplätzen in die Stadt importiert und dort weiterverarbeitet. ${ }^{559}$

\footnotetext{
551 Piaskowski 1960, S. 112

552 Piaskowski 1960, S. 112

553 Piaskowski 1960, S. 112

554 Piaskowski 1960, S. 113

555 Pleiner 1983, S. 88-89

556 Piaskowski 1960, S. 113

557 Piaskowski 1960, S. 113

558 Piaskowski 1960, S. 113

559 Thompson 1967, S. 73; Baart u.a. 1977, S. 325

Arzichowski und Koltschin 1959
} 
Am Beispiel der 304 metallurgisch untersuchten Messer aus Novgorod läßt sich der Beginn der Massenproduktion, der für das 12. Jahrhundert konstatiert worden ist ${ }^{560}$, mit einem deutlichen Qualitätsrückgang der Klingengüte in Verbindung setzen ${ }^{561}$. Die Klingen des ausgehenden 10., 11. und beginnenden 12. Jahrhunderts bestehen zum größten Teil aus vertikal angelegten Drei- oder Mehrschichtpaketen unter der Verwendung von Stahl- und Eisenbändern (Messer der Kulturhorizonte 28-20) ${ }^{562}$. Mit Beginn der Massenproduktion, die mehr und mehr auch auf das weitere Umland zielte, welches bis dahin in ländlichen Einzelschmieden sich selbst versorgt hatte $^{563}$, setzt im Verlauf des 12. Jahrhunderts die horizontal angelegte Zweischichtenklinge mit stumpf verschweißten eisernen Rücken- und stählernen Schneidepartien ein (Messer der Kulturhorizonte 18-12), wobei die Stahlpartien in der Regel ein Drittel bis die Hälfte der Klinge ausmachten; bis weit in das 12. Jahrhundert hinein ist auch das Einschweißen einer vertikal angelegten, nicht mehr bis zum Rücken durchläufigen Stahllamelle in mehrere Eisenschalen zu beobachten, daneben treten reine Eisenklingen auf ${ }^{564}$. Seit der Mitte des 13. Jahrhunderts ist eine Standardisierung der Produktionstechnik festzustellen, die sich mit einer Ausnahme (eine Ganzstahlklinge um 1400) in Klingen mit angeschweißten, zum Teil äußerst dünnen Stahlschneiden äußert ${ }^{565}$. Diese Ware war für Käufer bestimmt, die viele und billige Messer benötigten ${ }^{566}$, sie ist qualitativ kaum besser als Eisenklingen mit aufgekohlten Schneiden (Messer der Kulturhorizonte 11-5, ausgehendes 13. bis beginnendes 15. Jahrhundert). Auch die Qualität des Stahls, die im 11. Jahrhundert ihren Höhepunkt erreichte, nahm im Verlauf dieser Entwicklung stetig ab ${ }^{567}$.

Aus der Masse der in der Londoner Innenstadt geborgenen, zumeist stratigraphisch eingeordneten Messer wurden zehn Messerklingen metallurgisch untersucht, sie umfassen den Zeitraum vom späten 12. bis zum 15. Jahrhundert ${ }^{568}$.

Neben zwei Klingen aus einfachem Schmiedeeisen (Ende 13. Jahrhundert, spätes 14. Jahrhundert) wurde ein Beispiel einer horizontal angelegten Zweischichtenklinge mit stumpf verschweißten Partien für das ausgehende 13. Jahrhundert nachgewiesen $^{569}$. Die Methoden der schräg angesetzten und der von zwei eisernen Schalen eingefaßten, nicht durchläufigen Stahlschneide begegnen am Beispiel zweier Klingen aus der Mitte des 13. bzw. aus dem späten 12. Jahrhundert ${ }^{570}$.

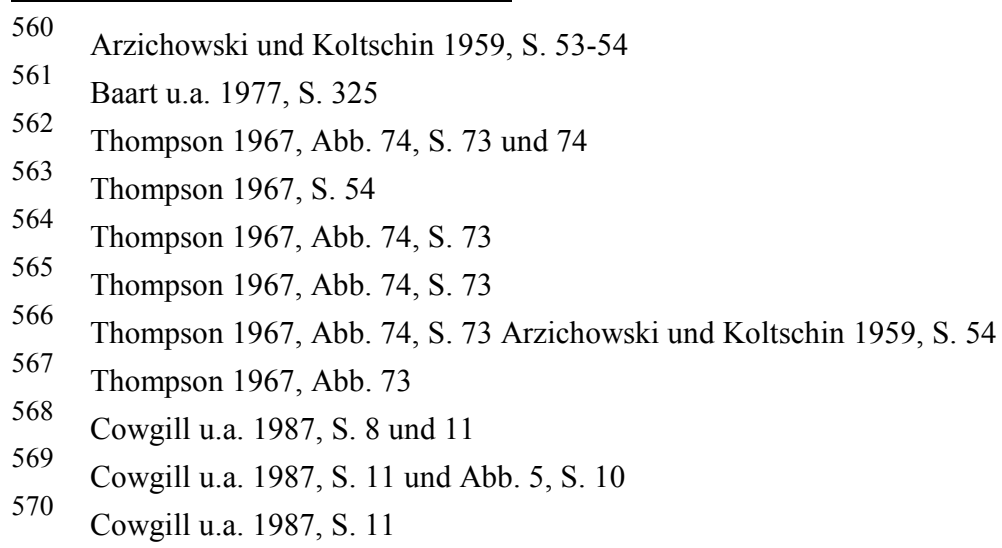


Die in London geborgenen Klingen des 12. bis 13. Jahrhunderts umfassen damit die auch von anderen Fundorten bekannten Produktionstechniken und Qualitätsspannen. Lediglich die bisher aus dem 9. bis 12. Jahrhundert bekannten, in Haithabu, Skandinavien und mit circa 150 Exemplaren aus Nordosteuropa bekannten durchläufigen Dreischichtenklingen, die ihre Blütezeit im 10. und 11. Jahrhundert hatten $^{571}$, wurden hier nicht nachgewiesen.

Die in London analysierten Messerklingen des 14. und 15 Jahrhunderts bestanden in drei Fällen aus einem Eisenstück, um das an der Schneide beidseitig ein Stahlmantel geschweißt worden war ${ }^{572}$. Die Methode, für die Schneide ein härteres Material zu verwenden als für den Rest der Klinge, wurde im Prinzip bis in das 15. Jahrhundert fortgeführt; die Stahllamelle erscheint im Gegensatz zu den früheren Klingen jetzt direkt an der Außenhaut der Klinge.

Auch die Qualitätsunterschiede sind zu Beginn der Neuzeit weiterhin bedeutend. So erscheinen im späten 14. Jahrhundert neben den Klingen mit Stahlschneiden noch eine reine Eisenklinge im gleichen Zeitraum sowie in der Mitte des 15. Jahrhunderts je ein Beispiel einer Klinge, die aus mehreren verschweißten Eisenbändern besteht ${ }^{573}$.

Daß die Qualität einer Klinge nicht unbedingt allein von der Produktionstechnik abhängen muß, zeigen zwei Messer aus der Mitte des 14. Jahrhunderts, die beide mit außen angeschweißten Stahlmänteln zur Schneidenbildung versehen sind ${ }^{574}$. Die Qualitätsstufen der beiden Klingen (Härte und Dauerhaftigkeit der Schneide und Bruchsicherheit der Klinge $)^{575}$ werden bei dem einen Messer mit "gering", im anderen Fall mit "hervorragend" angegeben ${ }^{576}$.

Zur Übersicht wurden die wichtigsten Ergebnisse der metallurgischen Untersuchungen an Messerklingen anhand der oben beschriebenen Beispiele in einer vergleichenden Tabelle zusammengefaßt (Abb. 216). Sie enthält lediglich die grundlegenden Produktionstechniken wie Eisen-, Stahl- und Mehrschichtenklingen; Feinunterscheidungen nach Güte oder Variationen der bekannten Techniken wurden nicht gesondert aufgelistet. Eingetragen wurden nur explizit besprochene Beispiele oder - wie im Fall Novgorod - die zeitliche Verteilung ganzer Gruppen. Die gestrichelten Linien betreffen in den Publikationen erwähnte Techniken mit zeitlichen Angaben, die nicht mit Beispielen belegt worden waren.

Die wenigen hier aufgeführten Beispiele haben gezeigt, daß metallurgische Serienuntersuchungen Ergebnisse liefern können, die das Messer sowohl als äußerst

\footnotetext{
571 Pleiner 1983, S. 84-87

572 Cowgill u.a. 1987, S. 10 und Abb. 5, S. 11

573 Cowgill u.a. 1987, S. 11

574 Cowgill u.a. 1987, S. 11

575 Cowgill u.a. 1987, S. 62

576 Cowgill u.a. 1987, S. 11
} 
differenziert in seiner Herstellungsart als auch als wirtschaftlichen Entwicklungen unterworfenes Handelsgut charakterisieren.

Die große Spanne der Produktionstechniken läßt Rückschlüsse auf verschiedene Funktionen, aber auch auf unterschiedliche Käuferschichten zu.

Die Stabilisierung städtischer Strukturen und des damit verbundenen breiteren Wohlstands dokumentiert sich in Danzig, wo im Verlauf von 180 Jahren die Entwicklung der Klingentechnologie korrespondiert mit dem auch tatsächlich umgesetzten Qualitätsanstieg von Messerklingen als Massenware.

In die entgegengesetzte Richtung weisen die Ergebnisse des Novgoroder Fundmaterials. Dort konnte nachgewiesen werden, daß im Verlauf der Monopolisierung der Stadt Novgorod gegenüber ihrem ländlich geprägten Umland die Qualität der Klingen, die für den Export in ehemals relativ autarke Siedlungen produziert wurden, mit steigender Nachfrage sank. 


\section{Produktion von Messerklingen 9. - 15. Jahrhundert}<smiles>C1CC1</smiles>

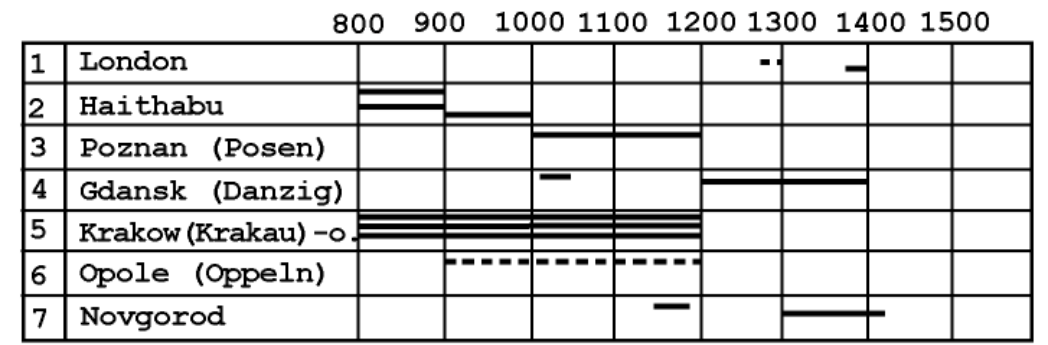

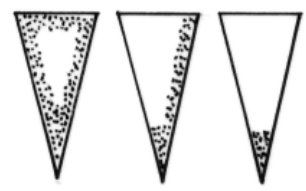

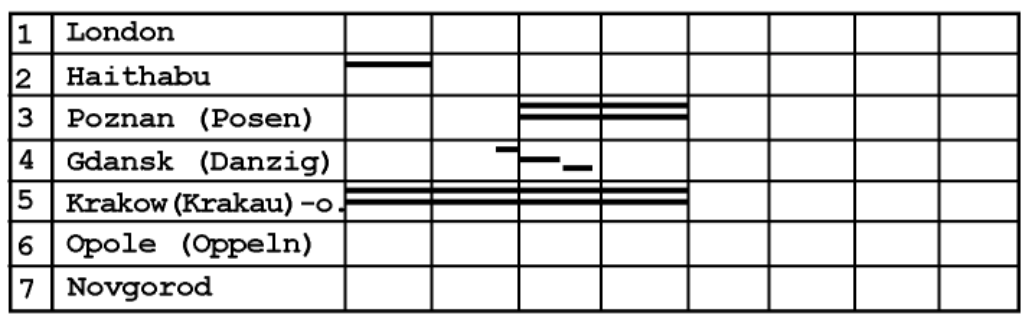

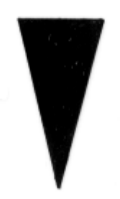

\begin{tabular}{|l|l|l|l|l|l|l|l|l|l|}
\hline 1 & London & & & & & & & & \\
\hline 2 & Haithabu & & & & & & & & \\
\hline 3 & Poznan (Posen) & & & & & & & & \\
\hline 4 & Gdansk (Danzig) & & & & & & & & \\
\hline 5 & Krakow (Krakau) -o & & & & & & & \\
\hline 6 & Opole (Oppeln) & & & & & & & & \\
\hline 7 & Novgorod & & & & & & & & \\
\hline
\end{tabular}

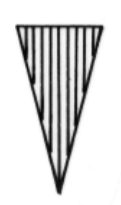

\begin{tabular}{|l|l|l|l|l|l|l|l|l|l|}
\hline 1 & London & & & & & & & - & \\
\hline 2 & Haithabu & & & & & & & & \\
\hline 3 & Poznan (Posen) & & & & & & & & \\
\hline 4 & Gdansk (Danzig) & & & & & & & & \\
\hline 5 & Krakow (Krakau)-o. & & & & & & & \\
\hline 6 & Opole (Oppeln) & & & & & & & & \\
\hline 7 & Novgorod & & & & & & & & \\
\hline
\end{tabular}

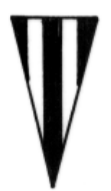

\begin{tabular}{|l|l|l|l|l|l|l|l|l|l|}
\hline 1 & London & & & & & & & & \\
\hline 2 & Haithabu & & & & & & & & \\
\hline 3 & Poznan (Posen) & & & & & & & & \\
\hline 4 & Gdansk (Danzig) & & & & & & & & \\
\hline 5 & Krakow (Krakau) -o. & & & & & & & \\
\hline 6 & Opole (Oppeln) & & & & & & & & \\
\hline 7 & Novgorod & & & & & & & \\
\hline
\end{tabular}

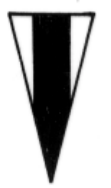

\begin{tabular}{|l|l|l|l|l|l|l|l|l|l|}
\hline 1 & London & & & & & & & & \\
\hline 2 & Haithabu & & & & & & & & \\
\hline 3 & Poznan (Posen) & & & - & & & & & \\
\hline 4 & Gdansk (Danzig) & & & & & & & & \\
\hline 5 & Krakow (Krakau) -o. & & & & & & & \\
\hline 6 & Opole (Oppeln) & & & & & & & & \\
\hline 7 & Novgorod & & & & & & & \\
\hline
\end{tabular}

Abb. 216 (Blatt 1) 


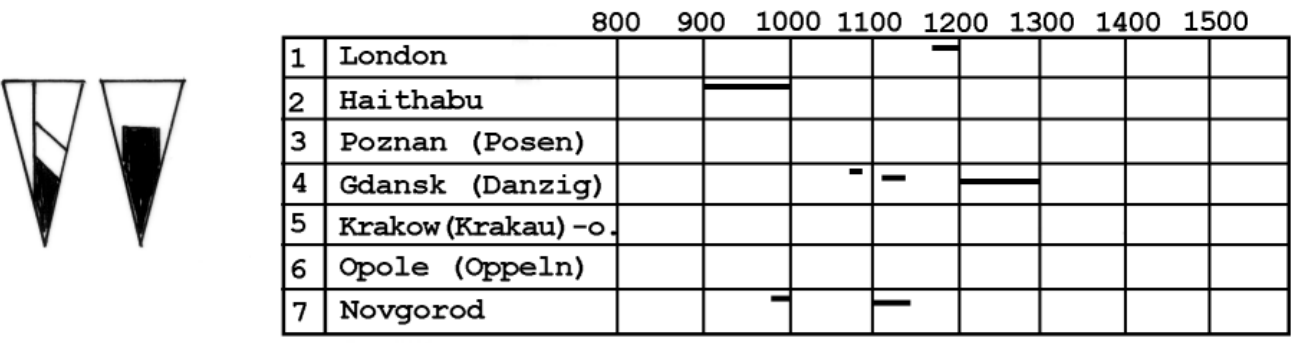

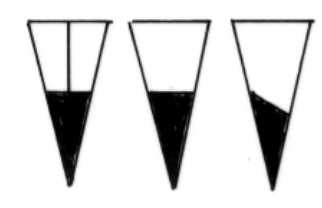

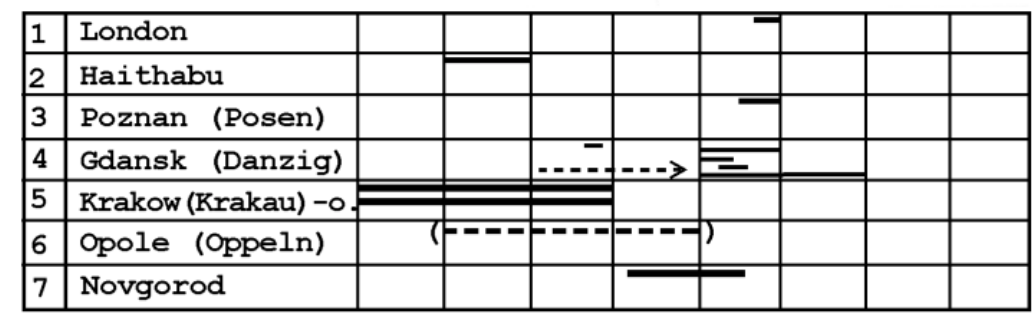

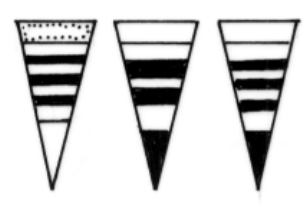

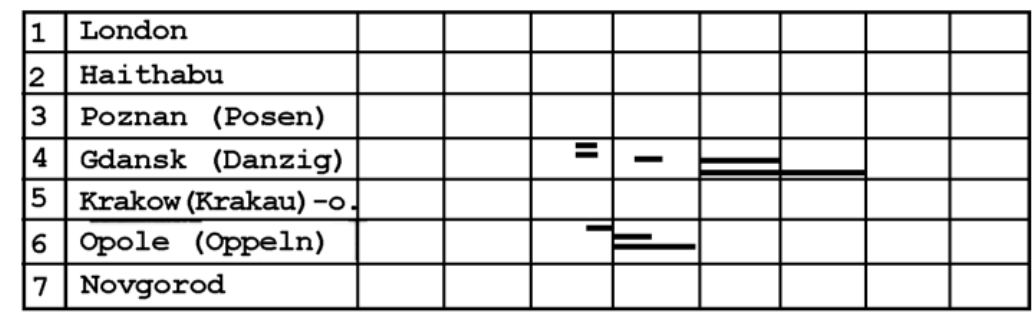

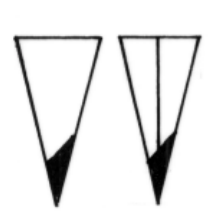

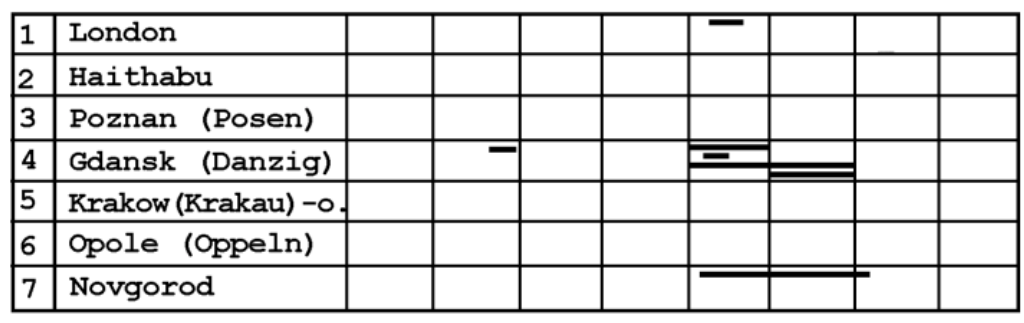

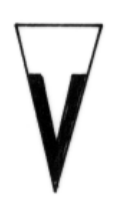

\begin{tabular}{|l|l|l|l|l|l|l|l|l|l|}
\hline 1 & London & & & & & & I & - & \\
\hline 2 & Haithabu & & & & & & & & \\
\hline 3 & Poznan (Posen) & & & & & & & & \\
\hline 4 & Gdansk (Danzig) & & & & & & & & \\
\hline 5 & Krakow (Krakau) -o. & & & & & & & \\
\hline 6 & Opole (Oppeln) & & & & & & & & \\
\hline 7 & Novgorod & & & & & & & & \\
\hline
\end{tabular}

\section{Legende}

$\square$ Eisen

aufgekohlte Eisempartie

Stahl

zwei Eisenlagen

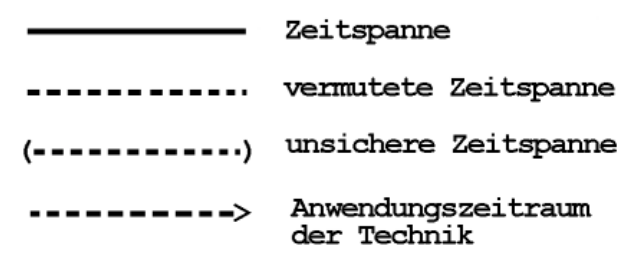

Abb. 216 (Seite 2) 


\subsubsection{Eisenerzförderung und Eisenhandel}

Die im vorangegangenen Abschnitt behandelten Verfahren zur Herstellung von Messern betrafen ausschließlich Techniken, die mit Hilfe naturwissenschaftlicher Methoden am Objekt selbst feststellbar waren. Schriftliche Quellen zu früh- und hochmittelalterlichen Produktionstechniken stehen nur derart vereinzelt zur Verfügung, daß sie lediglich zu Vergleichszwecken herangezogen werden können.

Die Sage von Wieland dem Schmied ist wohl die bekannteste Quelle. Sie dient aufgrund der ausführlichen Schilderung der Qualitätsverbesserung einer Schwertklinge in der Regel als Beleg für die oft vertretene Auffassung, daß Erfahrung und Tradition mittelalterlicher Klingenschmiede $\mathrm{zu}$ einem hohen Kenntnisstand über chemische Prozesse bei der Eisenbearbeitung geführt haben ${ }^{577}$. Die Ergebnisse der metallurgischen Untersuchungen an Klingen scheinen dieses zu bestätigen.

Die Voraussetzungen für die serienmäßige Herstellung von mittel- bis hochwertigen Klingen, welche im Untersuchungsraum durch die Anzahl der Funde und ihrer technischen Analysen seit dem 11. Jahrhundert belegt ist, waren Förderung und Handel von Eisenerz in ausreichender Menge und in der benötigten Qualität.

Für die früh- und hochmittelalterliche Produktion von Eisen standen Erze zweier verschiedener Arten von Lagerstätten zur Verfügung, die in Niederungsgebieten anstehenden Raseneisenerze (Rasen-, See-, Sumpf- und Wiesenerze ${ }^{578}$ ) und Bergerze.

Da eine in die Karolingerzeit oder weiter in das Mittelalter reichende Kontinuität des römischen Erzbergbaus nur in einem Fall (lombardische Alpentäler) wahrscheinlich ist ${ }^{579}$, darf die Bedeutung des Raseneisenerzes trotz der von Sprandel wegen seines Phosphatgehaltes erhobenen Bedenken ${ }^{580}$ nicht unterschätzt werden.

Von den zahlreichen Beiträgen zur regionalen Eisenproduktion aus in Feuchtgebieten ausgefällten und abgelagerten Eisenerzen seien hier nur beispielhaft die Arbeiten von Hingst ${ }^{581}$, Thieme ${ }^{582}$ und Winkelmann ${ }^{583}$ genannt, die für den Zeitraum von der römischen Eisenzeit bis zum Hochmittelalter Belege für einen ausgedehnten Abbau und die Verhüttung dieser Erze in Norddeutschland nördlich der Mittelgebirge liefern.

\footnotetext{
577 Als Beispiel der älteren wissenschaftlichen Forschung vgl. Johannsen 1924, S. 23, zum heutigen Stellenwert dieses Werks vgl. Sprandel 1968, S. 16 
Für den Zeitraum des Beginns dieser Untersuchung belegen beispielhaft die Befunde im Staatsforst Flensburg ${ }^{584}$ und aus der sächsischen Siedlung bei Warendorf in Westfalen ${ }^{585}$ den systematischen Erzabbau, die Verhüttung durch Batterien freistehender Rennfeueröfen und möglicherweise die Weiterverarbeitung des gewonnenen Eisens für die Zeit von um 800 bis um 1200 nach Christus. Mit diesem Eisen scheint der Großteil des Eigenbedarfs an Eisengeräten hergestellt worden zu sein. $\mathrm{Ob}$ Teile der Ausbeute wie während der vorrömischen Eisenzeit und der römischen Kaiserzeit auch verhandelt worden sind, ist den zitierten Arbeiten nicht zu entnehmen.

Bis auf vereinzelte große Lagerstätten in Südschweden, England, Südpolen, im Saarland, in Lothringen, in der Steiermark, in Mähren und in der nördlichen Lombardei, in denen der spätrömische Erzbergbau schon im Frühmittelalter, zum Teil noch früher in unterschiedlichem Umfang wieder aufgenommen wurde ${ }^{586}$, setzt ein in großem Umfang betriebener Bergbau nach Eisenerzen mit den zugehörigen Verhüttungsplätzen und der Kohleproduktion erst im 12. Jahrhundert wieder ein. Zwar lassen mehrere karolingerzeitliche Quellen erkennen, daß in größerem Maße als vorher Eisen regional abgebaut wurde, Erzgruben und Hüttenplätze als Einnahmequellen von Grundherrschaften erscheinen und Eisen als Abgaben an Grundherren genannt werden ${ }^{587}$, zur Eisenproduktion im Karolingerreich stellt Sprandel jedoch ausdrücklich fest, daß es sich außerhalb einiger unter direkter königlicher Verwaltung stehender Produktionsgebiete um die Deckung des "unmittelbaren örtlichen Bedarfs" ${ }^{588}$ gehandelt hat. Die Knappheit des Rohmaterials Eisen war offenbar so groß, daß eiserne Gegenstände importiert und zum Beispiel Waffenexporte verboten wurden ${ }^{589}$.

Vor allem im 12. Jahrhundert setzten die Entwicklungen ein, die zu der blühenden spätmittelalterlichen Eisenproduktion führten. Mit dem ständig steigenden Bedarf an Eisen, der Erschließung neuer Siedlungsgebiete und dem intensiven Suchen nach neuen Erzquellen ging in unterschiedlicher Form die rechtliche Loslösung der Eisenproduktion aus der direkten Abhängigkeit und Bedarfsdeckung von Grundherrschaften (z.B. die Entstehung der kommunalen Berghoheit) einher, die einen unabhängigeren, an überregionalen wirtschaftlichen Bedürfnissen orientierten $\mathrm{Ab}$ bau ermöglichte und über den Fernhandel unter Einbindung neuer Unternehmergruppen auf geldwirtschaftlicher Basis auch den Vertrieb des gewonnenen Eisens sicherstellte ${ }^{590}$.

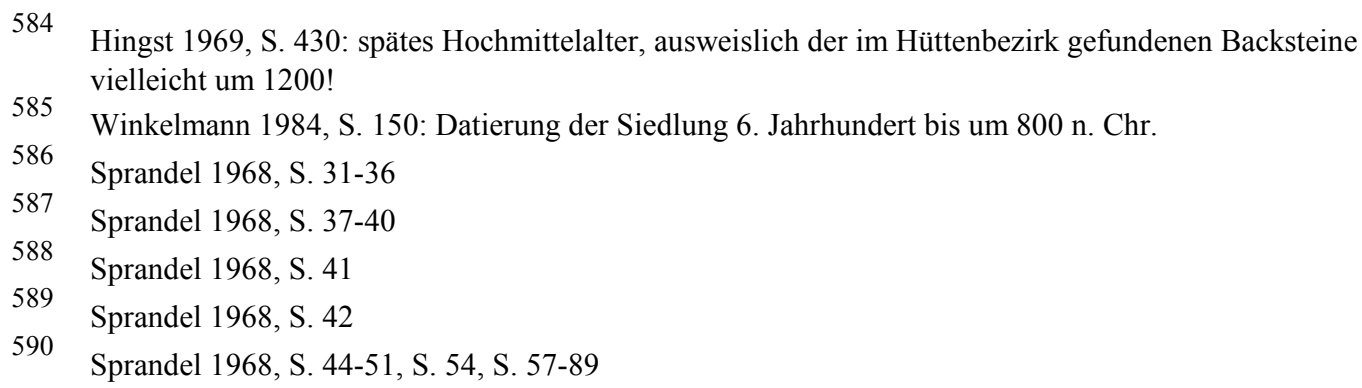


Insgesamt gilt für die wichtigen europäischen Eisenproduktionsgebiete im 13. bis 15. Jahrhundert eine Konzentration auf wenige, aber effektiv arbeitende Großräume. So fehlen zum Beispiel in Bayern und Österreich kleinere Zentren, die in Frankreich noch lange vorhanden waren, abseits der innerösterreichischen und oberpfälzischen Produktionszentren fast ganz, von den vier bekannt gewordenen Orten liegen die Zeugnisse der Eisenproduktion bei dreien vor 1200. In der Regel wird mit dem Produktionsanstieg in den Großräumen die verstreute Kleinproduktion zur lokalen Eisenversorgung im Verlauf des Spätmittelalters schrittweise aufgegeben $^{591}$.

Ausgehend von Sprandels umfassender Arbeit über die mittelalterliche Eisenproduktion in Europa werden im folgenden die wichtigsten Produktionsgebiete kurz vorgestellt.

\section{Oberitalien}

An den Südhängen der italienischen Alpen, vor allem in den Territorien der Herzöge von Savoyen und der Städte Mailand, Brescia und Venedig, befand sich im Mittelalter eines der bedeutendsten Erzabbau- und Verhüttungsgebiete Europas.

Kaiserliche Urkunden aus der zweiten Hälfte des 12. Jahrhunderts belegen eine zentrale Berghoheit, die in einigen Tälern zu früher Selbstverwaltung der in geschlossenen Bezirken zusammengefaßten Bergbausiedlungen führte. Andere eisenproduzierende Täler unterstanden Grundherrschaften oder wurden von Städten verwaltet, die zum Teil selbst über große Territorien verfügten.

Nach der Eroberung Brescias durch Mailand 1335 gelangte das zentrale Gebiet der oberitalienischen Eisenproduktion in den Machtbereich der Viscontis, die eine planmäßige Förderung und Verarbeitung der Erze sowie einen zentral geregelten Handel mit Eisen (und Waffen) über Mailand betrieben.

Nach der Eroberung Friauls (1420) sowie Bergamos und Brescias (1426) durch Venedig polarisierte sich die oberitalienische Eisenproduktion. Der Wirtschaftskampf zwischen Mailand und Venedig führte zur Teilung in zwei verschiedene Rechtsbezirke.

Unter dem venezianischen Konkurrenzdruck intensivierte Mailand die Produktion, konzessionierte seit der Mitte des 15. Jahrhunderts immer mehr kapitalkräftige Unternehmer in den nun getrennt und gezielt geförderten Bereichen Erzabbau und Verhüttung sowie in der Mailänder Rüstungsproduktion. Mit der Gründung von Lohnunternehmen ging die ältere kommunale Berghoheit zurück.

Im venezianischen Einflußbereich gab es mehr beteiligte Grundherrschaften, die wie die kommunalen Rechtsträger ihre Autonomie weitgehend bewahren konn$\operatorname{ten}^{592}$.

591 Sprandel 1968, S. 158

592 Sprandel 1968, S. 110-118 


\section{Spanien}

In Spanien befand sich neben kleineren erzfördernden Landschaften (u.a. das Baskenland) eine Konzentration von Bergbauhütten im Nordwesten, die die gebrochenen Eisenerze in küstengebundenen Verhüttungsplätzen veredeln ließen und über die Seehäfen direkt nach England, Frankreich und in die Niederlande verhandelten. Nach Frankreich und Italien gelangte vor allem baskisches Eisen. Starke königliche Initiativen und eine ausgeprägte kommunale Bewegung (Selbstverwaltung und eigene Rechtssprechung der eisenproduzierenden Siedlungen und Gebiete) führten zu Erzförder- und Verhüttungslandschaften, die weitgehend von grundherrschaftlichen Elementen befreit waren ${ }^{593}$.

\section{Elba}

Im 13. Jahrhundert wurde die Erzförderung auf Elba fast vollständig von der Stadt Pisa kontrolliert und getragen, die Verhüttung wurde vom Abbau getrennt auf dem italienischen Festland vorgenommen (1185 erster Hüttenbeleg bei Pisa). 1282 begann der Städtekrieg zwischen Pisa und Genua, in dessen Folge Pisa die Förderung, Verarbeitung und den Vertrieb des Eisens mehr und mehr geldwirtschaftlich betrieb. Bereits 1283 ist der erste konzessionierte Kaufmann belegt, der auf eigene Rechnung Elbaeisen kaufte und verhandelte. Große Erzhändler folgten, bis private Bergwerksbesitzer über Lohnarbeiter (ab 1305 belegt) Eingang fanden, darunter auch Pisaner Bürger.

1342 übernahm ein Konsortium von Pisaner und Genueser Kaufleuten den Erzvertrieb von Elba, die Verhüttung fand im 14. Jahrhundert vor allem an der italienischen Küste südlich von Genua über Lohnarbeiter oder im Rahmen von Grundherrschaften statt, die die Verhüttung in Verlagsverhältnissen Hüttenmeistern übertrugen. Eine weitere Konzentration von Hüttenbezirken bildete sich in der Toskana.

1399 wurde das Pisaner Monopol endgültig beendet, im 15. Jahrhundert wurde die Erzausbeute Elbas unter den Herzögen der Appianos für Jahre im voraus an Kaufmannsgesellschaften verkauft. Seit 1477 waren auch die Medicis beteiligt, die Erze wurden jetzt direkt an grundherrliche oder private Hüttenwerke und an städtische Handwerker verkauft.

Elba versorgte nur einen Teil Italiens mit Erzen, der Rest importierte Eisen und Erze aus Spanien und der Lombardei ${ }^{594}$.

$\begin{array}{ll}593 & \text { Sprandel 1968, S. 94-101 } \\ 594 & \text { Sprandel 1968, S. 101-108 }\end{array}$ 


\section{Kleinere nordwesteuropäische und alpine Regionen}

Schwerpunkte mit geringer Fördermenge bildeten sich im Dauphiné, in Lothringen, Wallonien und der Eifel, in der oberen Normandie sowie in der Schweiz, die aber alle nicht die Bedeutung der südeuropäischen Zentren erlangten. Frankreich blieb trotz dieser und weiterer kleiner Förderbezirke vom Import von Eisen abhängig ${ }^{595}$. In der Eifel trugen Landes- und kleinere Grundherren (Malberg, Manderscheid, Blankenheim und Schleiden) die Eisenproduktion. Letztere verliehen die Ausbeute und Verhüttung abgabenpflichtig an ländliche Handwerker, die Herzöge von Jülich, die Grafen von Virneburg und die Trierer Erzbischöfe beteiligten städtische Unternehmerkreise an der Verhüttung ${ }^{596}$.

Die schweizer Eisenproduktion erfolgte vor allem im grundherrschaftlichen Rahmen, im nordwestlichen Konzentrationsgebiet um die Fricktaler Gruben und die Hammerwerke bei Lauffenburg entstanden freie Bergwerksgemeinden und die Beteiligung der Stadtgemeinde Lauffenburg ${ }^{597}$.

\section{Innerösterreich}

Bereits vor dem Beginn der deutschen Ostsiedlung bestand im Inneren Österreichs eine slawische Eisenproduktion, die in den großräumigen slawischen Fürstentümern betrieben wurde. Der grundherrschaftliche Rahmen änderte sich auch im 12 . Jahrhundert unter deutschen Landesherrschaften und Grundherrschaften nicht. Durch die Rodungstätigkeit deutscher Siedler erfolgte eine Ausweitung der Produktionsgebiete.

Bereits im 13. Jahrhundert sind Beteiligungen von Kommunen und Fernhandel am Steierischen Erzberg belegt, dessen Produktion zentral gefördert wurde. In der zweiten Hälfte des Jahrhunderts erfolgte die Trennung der einzelnen Produktionsschritte und eine Ausweitung der jeweiligen Kapazitäten: selbständige Hammerwerke und größere Schmelzhütten wurden in einem weiteren Bezug um den Erzberg dezentral angesiedelt. Die Eisenproduktion in Kärnten und Krain ist historisch erst im 14. Jahrhundert belegt (1330 gelangt Hüttenberger Eisen nach München), sie wird jedoch ebenfalls - mit einer slawischen Vorläuferproduktion bereits für das 13. Jahrhundert angenommen.

Im 14. Jahrhundert setzen die Quellen über den Bergbau in diesen drei Gebieten verstärkt ein.

Das steierische Produktionsgebiet gehörte seit dem 14. Jahrhundert zum Herrschaftsbereich der Habsburger, die den Erzberg zu Lasten älterer Hütten (Klosterhütten Admont und St. Lambrecht bereits im 12. Jahrhundert) förderten. An die Stelle einer dezentralen Flächenausdehnung trat nun eine konsequente Intensivierung und Arbeitsteilung der Erzförderung, Verhüttung und S95 Spandel 1968, S. 19-136

596 Sprandel 1968, S. 133

597 Sprandel 1968, S. 136-141 
vierung und Arbeitsteilung der Erzförderung, Verhüttung und Verschmiedung am Erzberg mit seinem Vorort Steyr, die Produktion anderer Förderorte wurde eingeschränkt, das Bergregal blieb bei den habsburgischen Kontrollinstanzen.

Erst in der ersten Hälfte des 15. Jahrhundert begann neben der ständigen Vergrößerung der Werke am Erzberg wieder eine räumliche Expansion, 1406 ist steierischer Erzexport nach Venedig belegt.

Die zentralen Förderorte in Kärnten und Krain lagen auf Territorien des Erzbistums Salzburg und des Bistums Bamberg. Die Produktion von Hüttenberg wurde im 14. Jahrhundert durch den Erzbischof gefördert, 1381 erhielt Althofen das Monopol zur Ausfuhr Hüttenberger Eisens.

Die Hammerwerke im Kanaltal, die ihr Erz vom Hüttenberg bezogen, unterstanden wie die Bergwerke bei Waldenstein dem Bamberger Bistum.

Zahlreiche weitere Erzförderplätze und Verhüttungsbezirke (darunter Eisnern, Jesenice, Lavamünd und das Katschtal) lagen in Herrschaftsbereichen verschiedener Grundherrschaften, die im 14. Jahrhundert fast alle ihre Berghoheit behielten, so daß an Stelle einer zentralen Intensivierung zahllose kleinere Produktionsstätten entstanden.

Erst als in der zweiten Hälfte des 15. Jahrhunderts die Habsburger ihr Bergrecht auf die Brixener, Salzburger und Bamberger Bezirke ausdehnten, erhielt Hüttenberg die habsburgische Bergordnung und wurde an habsburgische Handelsorte angebunden, in Friesach durfte das dort geförderte und verhüttete Eisen nur regional begrenzt verkauft werden, das Krainer Gebiet wurde nach dem Aussterben der ehemaligen Grundherrschaft an eine Privatperson verpachtet und im Katschtal die Produktion von 1492 bis 1517 ganz verboten.

Unter der Oberherrschaft großräumiger Grundherrschaften (zumeist Landesherren), vor allem unter der zentraldirigistischen, wirtschaftlichen Gesichtspunkten unterliegenden habsburger Herrschaft entfalteten sich kommunale Verbände und Unternehmerkräfte. Arbeitsteilung, Verlagswesen und zentral geförderte Beteiligung von Orten und Städten an den Produktionsorten führten zur wirtschaftlichen Verflechtung von Unternehmergruppen, Handelsgesellschaften und einzelnen Kapitaleignern, deren Konkurrenz vor allem im 15. Jahrhundert durch die Zentralmacht der Habsburger zugunsten des steierischen Erzbergs reguliert wurde. Vor allem dort entfalteten sich neben dem Unternehmertum kommunale Kräfte wie Hammerbrüderschaften, die städtische Eisenhändlergemeinde Leoben und die Berggemeinde am Erzberg ${ }^{598}$.

\section{Oberpfalz}

In Österreich und Bayern gelangte im Mittelalter neben den oben genannten nur noch eine eisenproduzierende Region zu überregionaler Bedeutung, die Oberpfalz mit den zentralen Bergwerkstädten Sulzbach und Amberg. Seit dem 14. Jahrhundert

598 Sprandel 1968, S. 141-158 
war vor allem die pfalzgräflich privilegierte Kaufmannschaft Ambergs aktiv an der Ausweitung des oberpfälzer Eisengewerbes beteiligt. An den Flüssen Pegnitz, Vils und ihren Nebenflüssen zogen sich zahlreiche Hammerwerke entlang bis vor die Tore Nürnbergs, deren Bürger sich seit Ende des 14. Jahrhunderts vornehmlich durch Kauf oder Leihe von Hämmern an der Produktion beteiligten. Bereits seit der Mitte des Jahrhunderts waren sie in den Fernhandel mit oberpfälzischem Eisen eingeschaltet.

Neben dem Nürnberger Eisen- und Waffengewerbe, welches auch durch Eisen aus stadteigenen Hammerwerken versorgt wurde, spezialisierten sich die beteiligten Nürnberger Kaufleute vor allem auf die seit der zweiten Hälfte des 14. Jahrhunderts aufkommende Blechherstellung, die bis zum 15. Jahrhundert in Europa eine Monopolstellung innehatte ${ }^{599}$.

\section{Die ostdeutschen und osteuropäischen Gebirgszüge}

Südlich der im Spätmittelalter zentralen Produktionsgebiete, die sich vom Erzgebirge und Riesengebirge über die Sudeten und Oberschlesien bis zur Lysa Gora erstreckten, bestand in Böhmen, Mähren und Südpolen in zahlreichen slawischen Fürstentümern bereits vor der deutschen Ostsiedlung eine rege Eisenproduktion, die grundherrschaftlich organisiert von Dorf- und Hofbewohnern nebenberuflich betrieben wurde.

Die meisten dieser Förder- und Verhüttungsplätze blieben auch nach der Eingliederung in deutsche und deutsch beeinflußte Adels- und Kirchenherrschaften in Funktion.

Mit der Luxemburger Herrschaft, die im Verlauf des 13. Jahrhunderts auch Schlesien, die östlichen Sudeten, das Görlitzer Land, die Niederlausitz und das Herzogtum Schweidnitz-Jauer mit der Stadt Hirschberg betraf, wurde die Zahl der Bergbau- und Verhüttungsplätze erhöht und die Produktion nach Norden ausgedehnt. Das Zentrum der Eisenproduktion lag jetzt in Oberschlesien, hinzu kamen das Erzgebirge, die Niederlausitz, der Fläming, die Sudeten und das Katzengebirge nördlich von Breslau.

Bis in das 13. Jahrhundert fand die Produktion in ähnlichen grundherrschaftlich gebundenen Verhältnissen statt wie unter den slawischen Fürsten. Vor dem Beginn der Hussitenkriege (1420) ist die Trennung von Bergbau und Verhüttung nicht belegt, Eisenproduktionsstätten als von der eigentlichen Grundherrschaft getrennte Objekte von Leiheverträgen oder Abgabenbemessungen werden ebenfalls erst im 14. Jahrhundert genannt.

Erst mit dem steigenden Einfluß deutscher Neusiedler und der zunehmenden Verstädterung der nördlich der Gebirgszüge anschließenden Regionen nahmen auch die Aktivitäten von Unternehmern und Handwerkern sowie die rechtliche und wirt-

599 Sprandel 1968, S. 158-174, Zur Herstellung des verzinnten Weißblechs, welches bereits 1372 als Import in Paris erwähnt wird, vgl. S. 167 
schaftliche Verbindung mit Bergstädten $\mathrm{zu}$, das hier produzierte Eisen fand als Handelsgut über Danzig Eingang in den hansischen Ostseehandel.

Nach einer Phase der Stagnation während der Hussitenkriege entstanden in der zweiten Hälfte des 15. Jahrhunderts Konzentrationsgebiete mit intensiver Produktion $^{600}$.

\section{Ungarn}

Neben Eisenproduktionsstätten in Kroatien, Siebenbürgen, Bosnien und im Burgenland wurde vor allem in den ungarischen Karpaten Erz gefördert und verhüttet, belegt an sechs Orten zwischen 1370 und dem Anfang des 15. Jahrhunderts. Das hier produzierte Eisen gelangte über Danzig und Thorn auch in den nordeuropäischen Fernhandel ${ }^{601}$.

\section{Mittelgebirge zwischen Elbe und Rhein}

Die Eisenproduktionslandschaft in den mittel- und westdeutschen Mittelgebirgszügen unterlag im Mittelalter keiner großräumigen Bergordnung, zahlreiche kleinere Erzförderstellen und Hüttenbezirke entstanden in den einzelnen Grundherrschaften und größeren Territorien, die zwar gelegentlich Konzentrationen und kommunale Organisation durch Gewerbestädte förderten, aber auch ältere Förderformen wie die sogenannten Waldschmiede schützten ${ }^{602}$.

Im Wesergebirge werden Produktionsstätten bereits im 12. bis 13. Jahrhundert vermutet, schriftliche Belege setzen in der zweiten Hälfte des 14. Jahrhunderts ein. Die Förderung und Verhüttung erfolgte ohne Unternehmerbeteiligung in verschiedenen Grundherrschaften, das Eisen wurde zum Teil über Bremen und Hamburg verhandelt.

Die Harzer Eisenproduktion geht mindestens bis in das 13. Jahrhundert zurück. Sie wurde von Territorial- und Landesfürsten gefördert, die städtische Bürger beteiligten. So entstanden in Wernigerode und Stolberg Vereinigungen von Stahlschmieden, die auch Bergwerke und einen Verhüttungsplatz (Blasofen) betrieben. Daneben förderten auch klostereigene Werke ${ }^{603}$.

Vom Thüringer Wald erstreckte sich eine Kette kleinerer, aber bedeutender Produktionslandschaften bis an die obere Ruhr. Die thüringische Eisenproduktion ist bereits in der Karolingerzeit belegt, wurde aber erst seit dem 14. Jahrhundert intensiviert und ausgebaut. Ihre Zentren lagen in den Grafschaften Henneberg und

\footnotetext{
600 Sprandel 1968, S. 177-190

601 Sprandel 1968, S. 188-189

602 Sprandel 1968, S. 204

603 Sprandel 1968, S. 191-193
} 
Schwarzburg sowie bei Schmalkalden und Coburg mit bedeutenden Hammerschmieden.

Ab 1408 ist in Schmalkalden städtisches Stahlgewerbe belegt, welches zum Teil auch Bergwerke auf Lehensbasis betrieb. Die bekannten schmalkaldischen Schwert- und Klingenschmiede bezogen ihr Eisen hauptsächlich aus den Hammerschmieden der Grafschaft Henneberg ${ }^{604}$.

Eine Sonderstellung nimmt der hessische Waldschmiedebezirk ein. Im nördlichen Spessart, am Vogelsberg, im östlichen Rothaargebirge, im Westerwald, Taunus und im nördlichen Odenwald wurde die Eisenproduktion durch Waldschmiede vorgenommen $^{605}$, die auf zugewiesenen Stellen in den Bergwäldern Erz förderten, verhütteten und das gewonnene Eisen dann direkt an den Eisenhandel vertrieben. Sie wohnten abseits der Dörfer im Wald an ihren Arbeitsplätzen. Diese Arbeitsform erinnert an die Anfänge mittelalterlicher Eisenproduktion durch die zisterziensische Kolonisation $^{606}$, sie ist aber schriftlich erst im letzten Drittel des 14. Jahrhunderts bis um 1490 belegt. Der Begriff wird als Rechtsbegriff interpretiert, der die Waldschmiede, die keiner Siedlungsgemeinschaft angehörten, vor Diskriminierung schützte ${ }^{607}$.

Die auch archäologisch faßbaren Waldschmiede, die zunächst im "wilden Wald", dem nicht grundherrschaftlich erfaßten Wald arbeiteten, zum Teil neben den rechtlich anders gestellten klösterlichen Waldhütten, wurden mit der grundherrschaftlichen Durchdringung der Wälder in der Regel von Lehensverträgen mit Grundherren ausgenommen und verblieben so in ihrer "archaischen" Lebensform $^{608}$.

Im Vergleich zum hessischen Waldschmiedebezirk wurde die Eisenproduktion im Siegerland und im Dillkreis in einem festgefügten, landesherrlich kontrollierten System betrieben.

Erste schriftliche Quellen erwähnen 1298 Siegen und 1313 das Bergwerk von Müsen. Möglicherweise gelangte bereits hier schon Siegener Eisen in den Kölner Fernhandel. Die in großem Umfang betriebene Produktion setzte aber erst im 15. Jahrhundert ein, vor allem an den Höhen über der Sieg bei Siegen, am Berg von Müsen und am Bieberstein im Dillkreis.

Neben Waldschmieden entstanden organisierte Bergwerke mit getrennten Hüttenplätzen und Hämmern, die Förderung erfolgte durch organisierte und an der Förderung beteiligte Bergknappen, die unter der Aufsicht landesherrlicher Bergmeister oder der jeweiligen Grundherren standen. Seit dem ausgehenden 15.

\footnotetext{
604 Sprandel 1968, S. 193-196

Sprandel 1974, S. 11: dort werden 45 einzelne Werke von der Mitte des 14. bis zum Ende des 15. Jahrhunderts genannt 
Jahrhundert gaben die Grafen von Nassau über ihre Bergbauverwaltung der Produktion eine umfassende Bergordnung.

Neben diesen Herrschaften beteiligte sich die Stadt Siegen mit ihrem Gewerbe (Stahlschmiedezunft ${ }^{609}$ ) und ihrer Kaufmannschaft an der Produktion und dem Vertrieb des Eisens, das vor allem nach Köln und an den Niederrhein verhandelt wurde ${ }^{610}$.

Die mittelalterliche Eisenproduktion des Sauerlandes ist vom 11. bis 14. Jahrhundert fast ausschließlich durch archäologische Befunde nachweisbar ${ }^{611}$. Für die Umgebung von Lüdenscheid wies Sönnecken 181 Rennfeuerverhüttungsplätze nach, für das westliche Sauerland errechnete er 1961500 Plätze $^{612}$, bis 1969 stieg die Zahl der nachgewiesenen Verhüttungsplätze auf $1116 \mathrm{an}^{613}$.

Die sauerländische Eisenproduktion des 14. und 15. Jahrhunderts verteilte sich hauptsächlich auf die Territorien Kurkölns, der Grafschaft Mark und des Herzogtums Berg. Schriftliche Quellen liegen erst seit der Mitte des 14. Jahrhunderts vor (1348 aus dem Einkünfteverzeichnis der Grafschaft Arnsberg). Weitere Belege betreffen im 15. Jahrhundert ein klösterliches Hammerwerk im südlichen Sauerland, Bergwerke bei Engelskirchen und Remscheid, Lüdenscheid und Meinerzhagen. 1480 wird Lenneper Eisen in Osnabrück genannt, 1491 eine Stahlschmiede in Radevormwald.

Impulse zum Erzbergbau gingen vor allem von den märkischen Eisengewerbestädten Breckerfelde, Altena und Iserlohn aus. Bereits 1391 wurde einem Altenaer Bürger ein "Steinberg" und eine Hütte verliehen, auch der bekannte mittelalterliche Eisendraht aus Altena und Iserlohn wurde aus Eisen der näheren Umgebung hergestellt. Wie in Siegen entstand auch in Breckerfeld ein organisiertes Stahlgewerbe. Aufgrund der schriftlichen Belege des 15. Jahrhunderts, die vor allem Neugründungen von Bergwerken, Hüttenplätzen und Hämmern sowie eine erneut einsetzende Erzsuche betreffen, vermutet Sprandel eine Unterbrechung und einen strukturellen Wandel der spätmittelalterlichen sauerländischen Eisenproduk$\operatorname{tion}^{614}$.

Die Gründe für diesen Wandel beschreibt Scheler in seinem Beitrag zur mittelalterlichen Eisenproduktion und -verarbeitung im niederbergisch-märkischen Hügelland südlich des heutigen Ruhrgebiets ${ }^{615}$. Zunächst stehen die von Sönnecken 1958 und 1961 vorgestellten Hüttenplätze des 11. bis 14. Jahrhunderts nicht mehr so isoliert da, wie es Sprandel 1968 erscheinen mochte.

\footnotetext{
609 Sprandel 1974, S. 15

610 Sprandel 1968, S. 198-204

611 Sönnecken 1958, 1961

612 Sprandel 1968, S. 202

613 Sönnecken 1986

Sonnenschein 1987, S. 27

614 Sprandel 1968, S. 201-204

615 Scheler 1990, S. 111-117
} 
Vor allem durch die Ausgrabungen auf der Burg Isenberg bei Hattingen (11941225) ist die mittelalterliche Verhüttung von Eisen im grundherrschaftlichen Rahmen belegt ${ }^{616}$. Weiterhin zitiert Scheler Sönnecken mit der Auffassung, daß sich bei Radevormwald die Territorialgrenze zwischen der Grafschaft Mark und dem Herzogtum Berg an der Begrenzung des archäologisch nachgewiesenen Eisenhüttenbezirks "In der Mark" orientierte ${ }^{617}$. Nach der Befundbeschreibung von Eversberg war auch der aus einem Wohnturm, zwei Rennfeueröfen und einem unterkellerten Gebäude bestehende Hüttenkomplex auf der Unterburg der Burg Isenberg ein getrennter Bezirk mit eigenem Wohnbereich, der wahrscheinlich die umfangreiche Produktion der Burgschmiede auf der Oberburg mit Eisen versorgte ${ }^{618}$.

Die zahlreichen archäologischen Nachweise aus dem märkischen Sauerland ${ }^{619}$ sowie die oben zitierten Beispiele aus Radevormwald und Hattingen belegen nicht nur den Umfang der mittelalterlichen Eisenproduktion, sondern auch ihre zumindest partielle Einbindung in grund- und landesherrschaftliche Interessen.

Den Rückgang der Eisenproduktion bringt Scheler in Verbindung mit der seit dem 13. Jahrhundert einsetzenden Nutzung von Wasserkraft für die Blasebälge der Schmelzhütten, die zunehmend in die Bach- und Flußtäler verlegt wurden ${ }^{620}$. Diese Entwicklung und die Qualitätszunahme des Siegerländer Eisens hat nach seiner Ansicht zu einer Verlagerung der Eisenproduktion nach Süden und zur Spezialisierung auf die Herstellung von Eisenwaren im Norden zwischen Iserlohn und Schwerte bis Solingen und Ratingen geführt ${ }^{621}$.

Die von Sprandel zitierten Belege für einen Neubeginn der Erzsuche und Eisenproduktion im 15. Jahrhundert müssen vor dem Hintergrund der starken Konkurrenz im Stahl- und Eisenwarengewerbe gesehen werden. Nach dem Ausfall des Dortmunder Stahlhandels am Ende des 14. Jahrhunderts hielt Köln die Führungsrolle im Handel mit Eisen und Stahl aus dem Siegerland und dem märkischen Sauerland. Differenzierte Schmiedegewerbe in Dortmund und Köln, Essen, Ratingen und weiteren früher mit der Eisenproduktion beschäftigten Städten schufen im 15. Jahrhundert ein Klima, daß von Konkurrenz und Monopolisierungsbestrebungen geprägt war $^{622}$ und sicherlich mit dazu beitrug, alle

\footnotetext{
616 Eversberg 1982 
erreichbaren und mit erweiterten und verbesserten Herstellungsmethoden auch verwertbaren Eisenressourcen auszuschöpfen.

\section{Mittelschweden}

Die ältere schwedische Eisenproduktion hatte ihre Blütezeit vor 1100 in Südschweden, wo vor allem See- und Sumpferze ausgebeutet wurden. In den zum Teil dänischen Regionen Südschwedens reichen die schriftlichen Belege bis zum 13. Jahrhundert (Kloster Sorö in Halland am Anfang des Jahrhunderts, 1284 wird Eisen aus Blekinge in Flensburg erwähnt, das über Kalmar verschifft wurde).

Unter dem Einfluß der Hanse verlagerte sich die schwedische Eisenproduktion nach Mittelschweden, wo seit Beginn des 14. Jahrhunderts (1303) Bergerze gefördert wurden. 1368 wird in Lübeck erstmalig der Import mittelschwedischen Eisens genannt.

In Bergwerksdistrikte eingeteilt, unterstanden die Hütten königlichem Recht und genossen eine gewisse Bergfreiheit, 1434 wurden die Bergleute von Grundzinsen und grundherrschaftlichen Abgaben befreit ${ }^{623}$.

\section{England}

Die zentrale mittelalterliche Eisenproduktion Englands lag mit 70 Eisenhütten (1282) im nördlich von Gloucester gelegenen Forest of Dean. Sie wurde von Wanderschmieden und kleineren Bergwerken, darunter die "Große Schmiede" des Königs, unter königlicher Verwaltung betrieben. Der Einfluß einzelner Grundherrschaften blieb unbedeutend, städtische Unternehmer sind nicht bekannt.

Von den zwischen 1250 und 1300 nachweisbaren 150 Eisenhütten in ganz England, Wales und Schottland wurden in Mittel- und Nordengland zahlreiche Werke von Zisterziensern betrieben.

Die Pest von 1348 brachte einen Einbruch, der spätestens 1354 zu einem Eisenausfuhrverbot und seit dem ausgehenden 14. Jahrhundert zu einer starken Ausweitung der englischen Eisenimporte führte.

Im Forest of Dean bestanden 1436 noch 33 Eisenhütten, bereits ab 1375 ist die Intensivierung der Produktion durch Arbeitsteilung (Trennung von Berg- und Hüttenwerken) belegt. Auch in Yorkshire setzte nach dem Pesteinbruch eine rege Produktionstätigkeit ein, die bis in die Mitte des 15. Jahrhunderts reichte.

Insgesamt schrumpfte die einheimische Eisenherstellung jedoch nach 1350 fortschreitend, in Nordwestengland enden die schriftlichen Quellen mit dem ausgehenden 14. Jahrhundert.

Eine Sonderstellung nahm die Abtei Durham ein, deren Produktion von 1212 bis 1433 kontinuierlich auf das Sechsfache anstieg.

623 Sprandel 1968, S. 206

zum Handel mit schwedischem Eisen über Lübeck vgl. Dollinger 1989, S. 311-312 
Ein weiterer Versuch, die einheimische Eisenproduktion zu beleben, wird durch die auf königliche Initiative zurückgehende Neugründung von Berg- und Hüttenwerken im Weald (Südostengland) am Ende des 15. Jahrhunderts markiert ${ }^{624}$.

\subsubsection{Produktion und Handel mit Eisen- und Stahlwaren}

Diese kurze Übersicht über die wichtigsten Standorte der mittelalterlichen und frühneuzeitlichen Eisenproduktion in Europa zeigt, wie unterschiedlich sich im einzelnen Abbau, Verhüttung, Verarbeitung und Vertrieb entwickelt haben. Von den unterschiedlichen Bedingungen hingen nicht nur die Intensität und das Volumen der Handelsgüter Stahl und Eisen ab, sondern auch die des eisenverarbeitenden Gewerbes und des Handels mit Eisenwaren.

Bis heute liegt eine der Arbeit Sprandels vergleichbare Untersuchung zur Herstellung und zum Vertrieb mittelalterlicher Eisen- und Stahlwaren nicht vor.

Die oft listenartig zusammengetragenen oder zu verallgemeinernden Darstellungen ausgewählten Informationen in zahlreichen Regional- und Landesgeschichten lassen zwar den Umfang und die wirtschaftliche Bedeutung der gewerblichen Produktion von Eisenwaren in Städten wie Braunschweig ${ }^{625}$ oder Regionen wie das südliche Westfalen ${ }^{626}$ erkennen, ein Bezug zu den archäologisch erfaßten Funden aus Eisen ist daraus aber nicht herzustellen.

In der Diskussion um die Marken auf Messerklingen wurde bereits deutlich, daß eine Verbindung zwischen ihnen und historisch faßbaren organisierten Schmiedevereinigungen - wie etwa bei den Blankwaffen des 16. und 17. Jahrhunderts bisher nicht nachweisbar ist.

Ausweislich der historischen Ersterwähnungen von organisierten Schmieden in größeren Städten um 1300 und dem gleichzeitigen Auftreten von Klingenmarken ${ }^{627}$ kann davon ausgegangen werden, daß ein allgemeiner Zusammenhang zwischen beiden Ereignissen besteht, der aber bisher nicht näher einzugrenzen ist.

Die in Rats-, Zunft- oder Ämterverordnungen des 16. und 17. Jahrhunderts beschriebenen Strukturen, darunter auch die Markierung der Ware, können nicht auf mittelalterliche Zustände angewandt werden. Das kontinuierliche Ansteigen gemarkter Messerklingen im 14. und 15. Jahrhundert, vor allem in Beständen wie denen aus London und Amsterdam, lassen vermuten, daß sich diese Strukturen langsam, aber kontinuierlich entwickelt haben, und daß die Überprüfbarkeit der Ware und die Entstehung von Markenzeichen ähnliche Ursprünge haben mögen wie die fortschreitende Kontrolle und Organisation des Gewerbes und Handels.

\footnotetext{
624 Sprandel 1968, S. 212-215

625 Fuhse 1930, 1935

626 Rothert 1981

627

Vgl. Kapitel 6.2.6
} 
Fuhse beschreibt die historische Entwicklung des Schmiedehandwerks in Braunschweig ähnlich ${ }^{628}$. Für die Zeit vor dem 16. Jahrhundert standen ihm nur vereinzelte Quellen zur Verfügung, die Schmiede als Gesamtkorporation werden 1293 erstmalig genannt ${ }^{629}$, die Messerschmiede tauchen als Eigenname ebenfalls im 13. Jahrhundert, als Ortsbezeichnung 1357, als Berufsgruppe 1388 auf, das Gründungsdatum der Messerschmiedezunft 1341 ist ein Rückschluß ${ }^{630}$.

In Magdeburg wird 1431 zum ersten Mal die Gruppe der stadtsässigen Schmiede differenziert und unter anderem die der Messerschmiede erwähnt ${ }^{631}$, städteübergreifende Organisationsbemühungen wie die der Schlosser, Sporen- und Büchsenmacher von Braunschweig, Magdeburg, Hildesheim, Hannover, Goslar, Einbeck, Göttingen, Celle, Hameln, Northeim und Osterwieck 1571 sind ebenfalls erst nach dem Mittelalter nachweisbar ${ }^{632}$.

Unabhängig davon, daß eine Spezialisierung innerhalb des eisenverarbeitenden Gewerbes zum Teil schon seit der vorrömischen Eisenzeit anzunehmen ist (z.B. Waffenschmiede) ${ }^{633}$, steht das späte Einsetzen von ausreichend vergleichbaren Quellen zur Handwerksorganisation und zum Vertrieb der Waren im Gegensatz zur Quellensituation des eisenproduzierenden Gewerbes.

Die steigende Nachfrage nach Eisen und Stahl, die bereits aus dem 14. Jahrhundert bekannten Wirtschaftskämpfe um Monopole und Absatzmärkte sowie die zahlreichen Funde von Eisengegenständen belegen einen stetig wachsenden Verbrauch, der von bäuerlichen und Dorfschmieden in grundherrschaftlicher Abhängigkeit, von Kloster- und Burgschmieden und von städtischen, schon früh spezialisierten und seit dem 14. Jahrhundert in ersten Ansätzen organisierten Schmiedegruppen beliefert wurde. In welchem Rahmen sich die seit dem ausgehenden 14. Jahrhundert aus Stadtkerngrabungen bekannte Massenproduktion entwickelte, wie der Vertrieb in regionale und überregionale Märkte organisiert wurde, in welchem Umfang und zu welchen Zwecken Messer, Scheren, Armbrustbolzen, Beile, Äxte und andere Werkzeuge markiert wurden, welche Rolle die städtischen Schmiedeorganisationen in großen Städten wie Köln, Braunschweig oder Magdeburg oder die Monostruktur von eisenproduzierenden und -verarbeitenden Städten wie Breckerfeld in der Verbreitung ihrer Organisations- und Produktionsstrukturen in mittleren und Kleinstädten oder auf dem Land gespielt haben, läßt sich bisher, von Einzeluntersuchungen abgesehen, für die Eisenverarbeitung nur vermuten ${ }^{634}$.

\footnotetext{
628 Fuhse 1930

629 Fuhse 1930, S. 10

630 Fuhse 1930, S. 36

631 Fuhse 1930, S. 10

632 Fuhse 1930, S. 11

633 Jankuhn 1969, S. 86, S. 89 ff, S. 164 f

634 Die folgenden Anmerkungen haben keinen Anspruch auf den Charakter einer Darstellung, die wenigen zitierten Beispiele ersetzen nicht die Zusammentragung der Ergebnisse aus den zahlreichen regionalen Untersuchungen landesgeschichtlicher Aufsätze, deren Durcharbeitung den Rahmen dieser Untersuchung gesprengt hätte.
} 
Sprandel belegt für die Eisenproduktion und zum Teil auch für die Weiterverarbeitung in der Karolingerzeit das Prinzip der Selbstversorgung ${ }^{635}$. Lediglich für einige Gebiete, die der königlichen Verwaltung direkt unterstanden, nimmt er eine über den Eigenbedarf hinausgehende Produktion an, die jedoch nicht dem Reichsbedarf genügte und auch nicht als Handelsgut angesehen wurde ${ }^{636}$. Exportverbote und Fremdimporte (wie Messer aus englischen Klosterschmieden) bestätigen das Bild einer vor allem agrarisch ausgerichteten Gesellschaft, in der ein überregionaler Handel mit Eisen und Eisengeräten zur Ausnahme zählte ${ }^{637}$.

Im Hochmittelalter läßt sich der Handel mit Eisengeräten, Halbwaren und Roheisen in ganz Europa belegen, wobei die Quellen zum Gerätehandel (Waffenhandel) eher einsetzen $^{638}$. Die von Sprandel zitierten Belege vom 10. bis 13. Jahrhundert zum europäischen Eisenhandel beinhalten mehrfach Eisen und Waffen (Exportverbote in den Orient und nach Rußland) ${ }^{639}$, so daß davon ausgegangen werden kann, daß die von ihm erarbeitete Verbreitungskarte zur Entwicklung des Eisenhandels ${ }^{640}$ wahrscheinlich auch einen Teil des überregionalen Gerätehandels miteinschließt. Als wesentliche Voraussetzung für die Intensivierung und Ausweitung des europäischen Eisenhandels nennt Sprandel die Entstehung von Eisenhüttenlandschaften und Produktionszentren, die weit über den Bedarf der umliegenden Gebiete hinaus produzierten $^{641}$. Daß ähnliches auch für den überregionalen Handel mit Eisen- und Stahlwaren im Hoch- und Spätmittelalter gilt, zeigen Schmiedekonzentrationen wie die in Nürnberg, im Sauerland und in Schmalkalden, die in unmittelbarer Nähe von Verhüttungslandschaften entstanden, sowie in Venedig und Passau, die ihr Eisen aus den subalpinen Tälern bzw. aus der Steiermark bezogen. Zentren der Stahlproduktion zogen dementsprechend die Herstellung von Waffen und anderen Stahlerzeugnissen an, wie zum Beispiel in Breckerfeld, wo im 14. Jahrhundert eine spezielle "Messer-"produktion entstand ${ }^{642}$.

Diese Voraussetzungen erfüllten zwei Hüttenlandschaften, die beispielhaft die verschiedenen Entwicklungsmöglichkeiten $\mathrm{zu}$ einer Eisenwarenexportlandschaft belegen: die Oberpfalz mit Nürnberg und das märkische Sauerland.

Die bereits beschriebene Eisenproduktion in der Oberpfalz führte nicht nur zu einem Eisenhandel, der vor allem über Nürnberg lief, sondern auch zur Entstehung eines spezialisierten Eisen- und Stahlwarengewerbes in dieser Stadt, die mit ihren Produkten vom ausgehenden 13. bis in das 15. Jahrhundert einen ausgeprägten Fernhandel betrieb.

\footnotetext{
635 Sprandel 1968, S. 41

636 Sprandel 1968, S. 42

637 Sprandel 1968, S. 42

638 Sprandel 1968, S. 83-84, S. 86; dort werden 971 venezianischer und 1192 nordwestspanischer Waffenhandel gennant. Sprandel 1968, S. 86-87

640 Sprandel 1968, S. 85

641 Sprandel 1968, S. 89

642 Scheler 1990, S. 112
} 
Die vor allem auf Veredelung und Export ausgerichtete Wirtschaftsstruktur führte in Nürnberg zu einer in Deutschland einzigartigen städtischen Organisation, die bereits im 13. Jahrhundert jede Form handwerklicher Eigenorganisation verbot und stattdessen die gesamte Produktion in der Stadt unter die direkte Aufsicht des Rates stellte ${ }^{643}$.

Diese frühe Form einer zentralen Gewerbeaufsicht, die auch der Aufstand der städtischen Handwerker 1348/1349 nicht beseitigen konnte, verbunden mit einer frühen Übernahme italienischer Finanzwirtschaft und Buchführung und der konsequenten Durchsetzung des Verlagssystems führte zu hochspezialisierten Gewerben vor allem im Bereich der Eisenwaren, die eine ansonsten fast nirgendwo erreichte Menge an Produkten gleichbleibender Qualität für den Fernhandel lieferte ${ }^{644}$. Dieser lag in den Händen reicher Kaufmannsgeschlechter, die in Köln und Lübeck Zollfreiheit genossen und somit über Köln die Märkte in Flandern, Brabant und England, über Lübeck Skandinavien und das Baltikum, weiterhin Polen und Ungarn, zwischen 1378 und 1422 auch Österreich, Italien und Spanien relativ ungehindert erreichten. Verbote wie die der Lübecker Krämerzunft bezüglich des Kleinhandels mit Nürnberger Messern 1353 belegen die Intensität des Handels ${ }^{645}$.

Zusätzliche Aufgeschlossenheit gegenüber technischen Neuerungen führte zum Beispiel zu Anfang des 15. Jahrhunderts dazu, daß Nürnberger Draht und Drahtwaren 1428 die Brabanter Messen und kurz darauf den gesamten nördlichen Markt beherrschten, so daß das bis dahin führende sauerländische Drahtgewerbe sich nur mit Kettenhemden und Tuchkratzen behaupten konnte ${ }^{646}$.

Als Beispiel für die übergreifenden Handelstätigkeiten Nürnberger Familien sei die Mendelsche Handelsgesellschaft genannt, deren Mitglieder von 1305 bis 1449 im regional begrenzteren Nürnberger Raum Hammerwerke betrieb und die Eisenwaren als Verleger neben zahlreichen anderen Produkten und Geldgeschäften von Venedig über Nürnberg nach Frankfurt, Köln, Antwerpen und Brügge vertrieben. In Köln (seit 1350) und Venedig (seit 1364) besaßen sie feste Niederlassungen ${ }^{647}$.

Die von Sprandel und Scheler beschriebene Verschiebung der Eisenproduktion aus dem nördlichen Sauerland nach Süden und die Entstehung einer eisenwarenprozierenden Landschaft im märkischen Sauerland und im nördlichen Bergischen Land führte in zahlreichen Orten zu einer Spezialisierung auf die Massenherstellung einzelner Stahl- und Eisenwaren, die sich möglicherweise zu frühen Markenartikeln im überregionalen Handel entwickelten.

Die in der Literatur regelmäßig zitierten Schwertklingen des Kölner Fernhandels vor dem 13. Jahrhundert nach England, deren Provenienz in Köln, Solingen oder in

\footnotetext{
643 Mummenhoff 1924, S. 28-29, S. 42-44, S. 134

644 Stromer 1973, S. 334-337

645 Stromer 1973, S. 334-337

646 Stromer 1973, S. 337

647 Stromer 1973, S. 338
} 
Siegen vermutet wird ${ }^{648}$, belegt 1303 eine englische Zollveranlagung (ein Schiff mit sechs Sendungen von Schwertern und Helmen (?), die auf Rechnung Kölner Kaufleute verhandelt wurden ${ }^{649}$ ).

Etwas regelmäßigere und genauere Nachrichten zu den einzelnen Produkten und ihren Herstellungsorten setzen mit dem 14. Jahrhundert ein ${ }^{650}$. Neben den bereits erwähnten Breckerfelder "Messern", die nach Scheler als "Breckerfelder" in den Handel gelangten, ist vor allem die Drahtproduktion der märkischen Städte Altena, Iserlohn und Lüdenscheid zu nennen, darunter die bekannten Altenaer und Lüdenscheider Kettenhemden (letztere seit 1351 belegt) ${ }^{651}$.

Nachrichten wie die Übersiedlung von Medebacher und Attendorner Bürgern nach Köln um die Mitte des 12. Jahrhunderts, der Direkthandel eines Iserlohners in Ypern 1284, westfälischer Stahl 1340 in Kampen ${ }^{652}$ sowie der mögliche Direktvertrieb von Eisenwaren aus Attendorn nach England im 14. Jahrhundert über die westfälische Kaufmannsvereinigung ${ }^{653}$ belegen lediglich weitere Möglichkeiten des Handels mit Stahl- und Eisenprodukten, ohne diese jedoch spezifizieren zu können. Auch der Beginn der Produktion Solinger Messerklingen ${ }^{654}$, Schwerter, Harnische $^{655}$, Marsberger Ringpanzer ${ }^{656}$, Breckerfelder Sporen und Dolche ${ }^{657}$ sowie der Produkte aus Altenbeken und dem lippischen Kohlstädt (Eisenverarbeitung seit 1365 belegt $)^{658}$ im 13. oder 14. Jahrhundert ist ohne genauen Nachweis lediglich eine begründete Vermutung, da erst seit der Wende zum 15. Jahrhundert Belege für eine bestehende Fernhandelsproduktion vorhanden sind.

Einen Eindruck von dieser Produktion vermittelt die Güteraufstellung, die unter anderem Henning 1980 für die Zeit um 1400 vorgestellt hat ${ }^{659}$ :

Auf dem Kölner Stahl- und Eisenwarenmarkt waren neben den Stahllieferungen Iserlohn, Balve, Altena, Lüdenscheid und Plettenberg mit Draht und Drahtproduk-

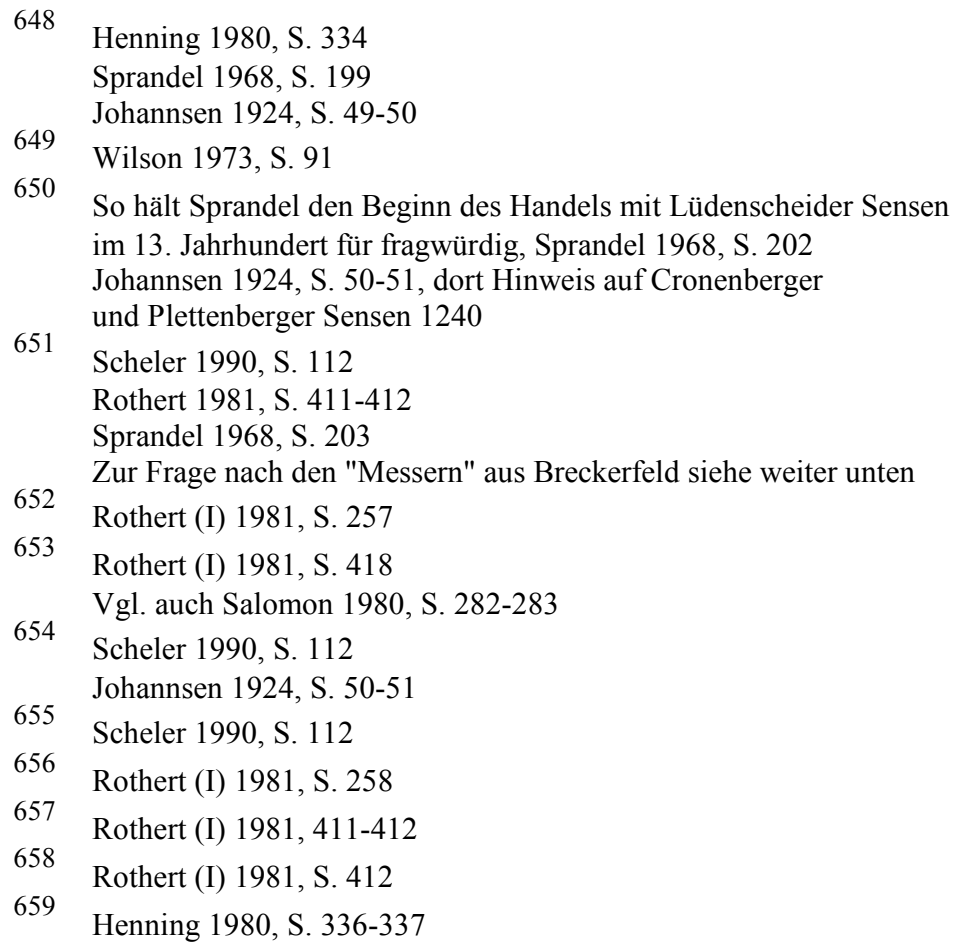


ten, Plettenberg mit Scheren und Schnallen, Plettenberg und Meinerzhagen mit Nägeln, Engstfeld mit Schellen, Kreuzberg, Drolshagen und Olpe mit Kesseln, Pfannenscheiben, Pfannen und (Drolshagen) mit Öfen vertreten.

Eine wesentliche Veränderung brachte das 15. Jahrhundert in der Waffenproduktion.

Neben älteren Produkten wie Kettenhemden, die noch 1437 von Iserlohn nach Livland geliefert werden ${ }^{660}$, entstanden im 15. Jahrhundert Büchsen- und Geschützproduktionen vor allem in Essen (1470) und Dortmund, die wie andere Handwerke teilweise von älteren Standorten übernommen worden waren ${ }^{661}$. So scheint Essen neben seiner Tuchscherenproduktion, die 1439 über die Anwerbung von Ratinger Scherenschmieden angesiedelt wurde, auch Ratinger Büchsenmacher geworben zu haben ${ }^{662} .1448$ werden in Münster sauerländische Geschütze genannt $^{663}$, Dortmund lieferte seine Geschütze bis nach Rußland ${ }^{664}$.

Wenn Messer in der Kölner Aufstellung um 1400 fehlen, so mag das für diesen Zeitpunkt Zufall sein, auch war Köln nicht die einzige Stadt, die märkische Stahlwaren in den Fernhandel brachte. Vor 1400 war Dortmund der Exportmarkt für das märkische Sauerland, während Köln vor allem die Produkte des Siegerlandes verhandelte ${ }^{665}$.

Nach der Dortmunder Finanzkrise (Fehde 1388/89) brachte Köln den märkischen Stahlhandel an sich, was möglicherweise aber nicht vollständig gelang und nicht unbedingt den Handel mit Stahlwaren nach sich zog.

Noch zwischen 1489 und 1492 versuchten zwei Kölner Stahlhandelsgesellschaften, sich das Monopol über die gesamte Produktion der über 200 Breckerfelder Schmiede für den Englandhandel zu sichern ${ }^{666}$.

Neben möglichen Zentren der Messerherstellung wie Breckerfeld, Solingen und Siegen hatten die Messerschmiede der größeren Handelsstädte eine möglicherweise noch größere Rolle in der Klingenproduktion für den Fernhandel inne.

Die Kölner und Dortmunder Schmiedegewerbe waren im 14. und 15. Jahrhundert spezialisiert und breit gefächert ${ }^{667}$. Während das wirtschaftliche Hinterland neben den erwähnten Eisen- und Stahlwaren vor allem Rohmaterial und Halbzeug her-

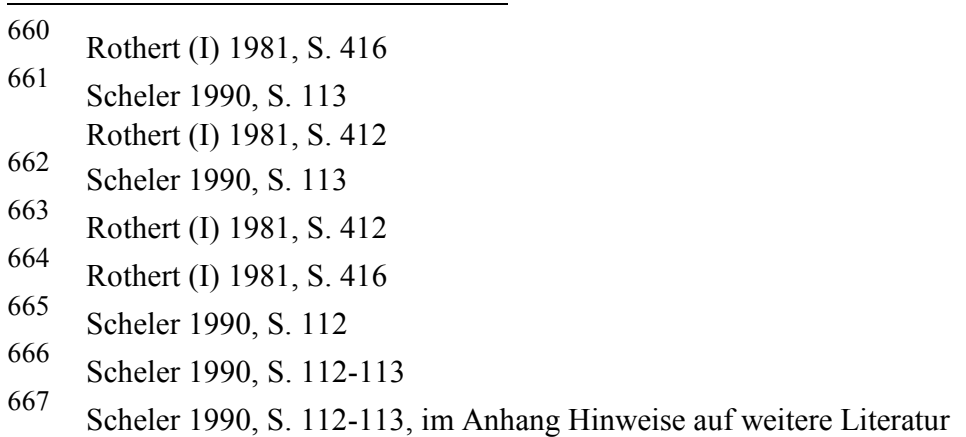


stellte, konzentrierte sich das städtische Handwerk auf die Weiterverarbeitung und Veredelung ${ }^{668}$.

So verhandelte Köln auch nach Paris vor allem Metallwaren, Waffen und Werkzeuge, 1471 wird dort ein Lager von 600 Kölner Sicheln genannt ${ }^{669}$.

Scheler nimmt den Beginn einer umfangreicheren Konkurrenzproduktion zu den Kölner und Dortmunder Eisen- und Stahlwaren im 16. Jahrhundert an, die zitierten Quellen des 15. Jahrhunderts, vor allem bezüglich der Monopolisierungsbestrebungen und Abwerbungen lassen ihn zu dem Schluß kommen, daß es im gesamten Raum des Siegerlands, des märkischen Sauerlands und der größeren Städte "qualifiziertes Eisen- und Stahlwarenhandwerk" bereits im 14. und 15. Jahrhundert gab $^{670}$.

Im 16. Jahrhundert nahmen die Standardisierung in der Messerproduktion und ihr Vertrieb erheblich zu. Nicht nur die Mengenzunahme in den Fundbeständen von zahlreichen Stadtkerngrabungen mit vergleichbaren Erhaltungsbedingungen und in den Sammlungen einschlägiger Museen, sondern auch die Ergebnisse dieser Untersuchung wie das Aufkommen nahezu identischer Messer in den Niederlanden und Skandinavien lassen vermuten, daß auch in der Produktion eine zunehmende Konzentration stattgefunden hat.

Johannsen beschrieb bereits 1924 die Rolle des Überseexports für die Messerproduktion. Dieser führte in Solingen zu einer Trennung zwischen sogenanntem Messengut, welches auf die Frankfurter, Leipziger und Braunschweiger Messen, aber auch nach Italien, Frankreich und in die Niederlande geliefert wurde, und sogenanntem Seegut, welches unter anderem über Amsterdam in den Überseehandel gelangte $^{671}$.

Der bereits im Zusammenhang mit den Klingenmarken erwähnte Elbfund von Wittenbergen belegt mit etwa 150 Messern dieses Seegut für die Zeit um $1600^{672}$. Die gemarkten Klingen aus Eisen mit Rücken und Schneiden aus Stahl sind relativ einheitlich in der Form. Vor allem die Griffverarbeitung und die Holzschalen sind von einer für diese Zeit beispiellos schlechten Qualität, so daß ein Absatz in Europa nur schwer vorstellbar ist.

Die Diskussion um die Herkunft der Messer hat London als möglichen Produktionsort genannt, Solingen aufgrund fehlender Nachweise in der dortigen Schmiedemarkenrolle ausgeschlossen ${ }^{673}$, so daß der Eindruck entsteht, das Schiff

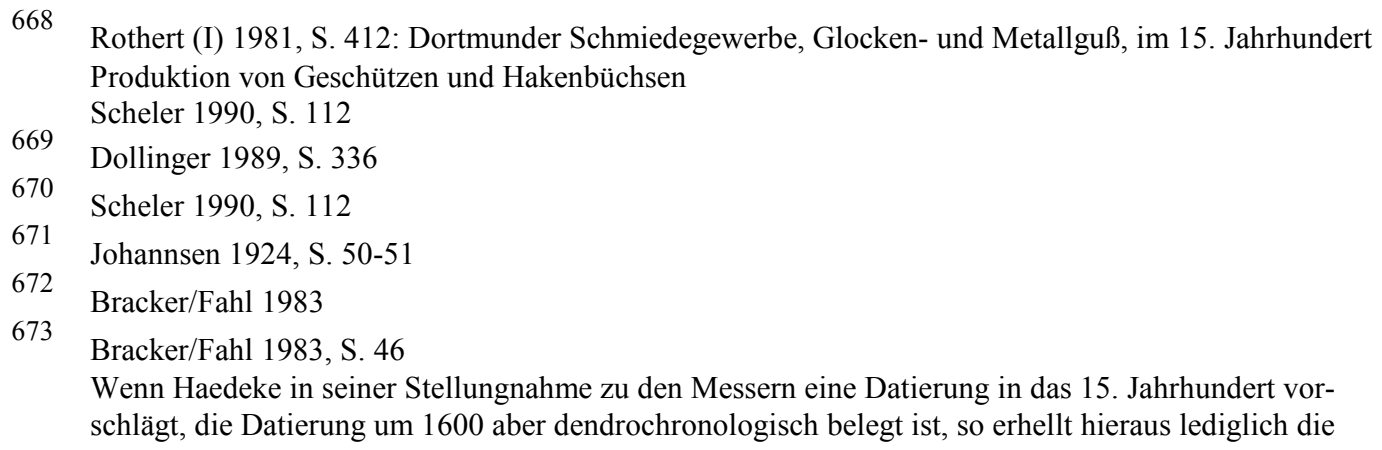


sei beim Anlaufen Hamburgs als Bestimmungshafen der Ladung gesunken. Da es aber zu der Wittenberger Messerladung im hier behandelten Bestand an Messern, aber auch in weiteren Beständen wie aus London, Amsterdam und Lübeck für das 15. bis 17. Jahrhundert keine einzige Parallele gibt, die am angetroffenen Fundort benutzt wurde und demnach für den europäischen Gebrauch bestimmt war, ist eine Charakterisierung als Ware für den Überseexport mehr als wahrscheinlich.

Seit dem ausgehenden 16. Jahrhundert lassen sich Schmiedemarken auf Werkzeugen und Messern über die eingetragenen Markenrollen an Produktionsorten wie Nürnberg und Solingen in größerem Umfang zurückverfolgen, in der zweiten Hälfte dieses Jahrhunderts setzen auch Stadtwappen als Marken ein ${ }^{674}$.

Der ebenfalls bereits erwähnte Messerfund aus der Themse bei Blackfriars (London) zeigt für das beginnende 16. Jahrhundert die Messer, die als englisches Importgut vom Kontinent dem Solinger Messengut entsprechen mögen. Über 60 zum größten Teil verbogene oder zerbrochene Dolche und Messer wurden von den Bearbeitern als archäologischer Beleg zu dem aus historischen Quellen bekannten Recht der Londoner Messerschmiedezunft gewertet, ausländische Ware auf ihre Qualität hin zu überprüfen ${ }^{675}$.

Die Messer entsprechen vollkommen den auch an anderen Orten wie Amsterdam, Lübeck und Lund ausgegrabenen Beispielen um 1500, so ein Griffzungenmesser mit Messingplatten am Heft, hölzernen Griffschalen und einer gegossenen Griffendapplik in Form eines Pferdehufes, dessen Klinge verbogen war $^{676}$.

Mit der Oberpfalz und dem siegerländischen und sauerländischen Bergland wurden Produktionslandschaften beschrieben, die über auf Export ausgerichtete mittelalterliche Städte wie Nürnberg, Köln und Dortmund Eisen- und Stahlwaren in großem Umfang in den Handel brachten.

Die folgenden Beispiele stehen stellvertretend für weitere Versorgungsmöglichkeiten und chronologische Fragestellungen zum Einsetzen der bisher nachprüfbaren Belege eines überregionalen Handels mit Eisen- und Stahlwaren.

Die gängige Vorstellung vom Versorgungsprinzip städtereicher Exportlandschaften, die mit ihrer Überschußproduktion vor allem den Norden und Osten Europas mit Luxusartikeln und veredelten Bedarfsgütern belieferten, läßt sich gerade im Bereich der Eisen- und Stahlgeräte differenzieren.

Am Beispiel der Städte Lödöse und Danzig zeigt sich, daß auch in überwiegend auf den Export- und Transithandel ausgerichteten Städten einige Warengruppen der Selbstversorgung vorbehalten blieben.

Unmöglichkeit, für diese Waren entsprechende kontinentaleuropäische oder insulare Parallelen des 16. 
Im schwedischen Lödöse, welches durch die Göta Älv mit dem Kattegat und der Küste Bohusläns verbunden ist, sind durch umfangreiche Grabungen detaillierte Einblicke in das Handelsgut der mittelalterlichen Stadt möglich, deren wirtschaftliche Blüte von den Eckdaten einer eigenen Münzprägung (ca. 1170 bis ca. 1365) markiert wird.

Als Warenumschlagsplatz und Transithandelsort zwischen Mittelschweden und dem Nordseehandel führte die Stadt vor allem Butter, Felle, Häute, Fleisch, Holz, lebende Pferde und Eisen aus den mittelschwedischen Bergwerken aus, ergänzende schriftliche Quellen belegen diesen Handel zwischen 1306 und um 1400.

Eingeführt wurden Salz, Bier, Wein, Hering, Tuche und "Kolonialwaren", historisch bis in das 15. Jahrhundert belegt, dazu Keramik, Glas und Textilien sowie Trockenfisch, Rengeweihe und Walknochen, belegt durch die Grabungsergebnisse vor allem im 13. und 14. Jahrhundert.

Der etwas befremdlich wirkende Begriff "Kolonialwaren" beinhaltet geringerwertige alltägliche Bedarfsgüter, zu denen auch Messer, Scheren, Nadeln u.ä. zählen konnten.

Bei den Grabungen wurden aber am Randbezirk der Stadt Belege einer eigenen Eisen- und Geräteversorgung gefunden. Die Menge und Qualität des in Hüttenplätzen aufbereiteten und in benachbarten Schmieden verarbeiteten Mooreisens reichte nicht aus, um die dortige Produktion in den Fernhandel zu bringen. Sie diente nach der Befundinterpretation der Versorgung der Stadt, möglicherweise auch der umliegenden Regionen ${ }^{677}$.

Eine ähnliche Situation scheint in Danzig vorzuliegen. Das durch Grabungsergebnisse nachgewiesene hochentwickelte Eisengewerbe der Stadt vor der Einnahme der Burg 1308 durch den Deutschen Orden, dessen Produkte einen hohen Qualitätsstandard erreichten ${ }^{678}$, belieferte wahrscheinlich die einheimischen Handwerker und die nähere Umgebung. Eisenwaren wie Messer werden in den historischen Quellen zu den aus oder über Danzig verhandelten Gütern (wie Holz, Pelze, Leder und Bernstein) nicht genannt. Ein Vertrieb der Messer nach Süden wird zwar vermutet, ist aber ebensowenig belegbar ${ }^{679}$.

Städte wie Lödöse, Danzig oder Novgorod, das ebenfalls über eine umfangreiche Messerproduktion verfügte und, wie bereits erwähnt, nicht nur sich selbst, sondern auch sein umliegendes Territorium versorgte, kamen als Absatzorte damit für die mitteleuropäischen Produktionslandschaften weniger in Frage.

\section{Warenkennzeichnungen}

Über Schmiedemarken reidentifizierbare Waren des Hoch- und Spätmittelalters sind fast ausschließlich Waffen, vor allem Schwerter. Vom 9./10. bis in das

\footnotetext{
677 Ekre 1973, S. 186-187 
14. Jahrhundert treten vor allem im niederdeutschen Raum Schwertklingen auf, die die Namen ihrer Hersteller tragen. Der bekannteste so namentlich genannte Schwertfeger/Klingenschmied ist sicherlich Ulfberht, der vermutlich im 9./10. Jahrhundert lebte und seinen Klingen mit seinem Namenszug auch ein Gütezeichen verlieh. Die hervorragende Qualität seiner Produkte wurde offensichtlich auch nach seinem Tod, möglicherweise auch in derselben Werkstatt durch die Beibehaltung dieser namentlichen Kennzeichnung unterstrichen, so daß Ulfberht-Schwerter im niederdeutschen und im Ostseeraum noch in Kontexten des 12. Jahrhunderts auftauchen $^{680}$.

Neben weiteren Namenszügen wie Ingelred und Benno sind vor allem die Schwertklingen des Gicelin zu erwähnen, die im 13. und 14. Jahrhundert ebenfalls in Niederdeutschland auftreten. Auch hier hat die Benutzung des Namens die Arbeitsspanne des Schmieds überlebt, in diesem Fall auch belegt durch typologische Hinweise wie Buchstabenumkehrungen und Namensverstümmelungen ${ }^{681}$.

Kombinationen aus Buchstaben wie NED oder DIC gehören zu der Gruppe der Weiheinschriften (nomine eterni dei etc.) ${ }^{682}$, weiterhin sind Namens- und Inschriftenimitationen, zum Teil aus buchstabenähnlichen Zeichen bestehend, und frühe Schmiedemarken (Rosetten) bekannt geworden ${ }^{683}$.

Für die Diskussion um die Funktionen von Marken ist vor allem festzuhalten, daß die Kennzeichnung der Ware als Gütezeichen für Qualität vom 9./10. bis 14. Jahrhundert bereits losgelöst vom tatsächlichen Werk des ursprünglichen Namensträgers erscheint und zwei wichtige Komponenten einer standardisierten, überregional verhandelten Qualitätsware enthält: Die Führung einer Kennzeichnung als Gütesiegel über einen langen Zeitraum und die Fälschung dieser Kennzeichnung zur Vortäuschung einer bekannten und geschätzten Herkunft, die eine Teilnahme am Handel mit dieser Qualität ermöglicht.

Das bereits erwähnte dänische Messer aus Svendborg (13. Jahrhundert), welches am Griff Angaben zum Klingenschmied und Messerbereiter enthält ${ }^{684}$, ist als Einzelerscheinung zu bewerten.

Mit dem Passauer Wolfszeichen liegt im 13. Jahrhundert nördlich der Alpen ${ }^{685}$ der erste Beleg für eine Schmiedemarke vor, die als städtische Herkunftsmarke und

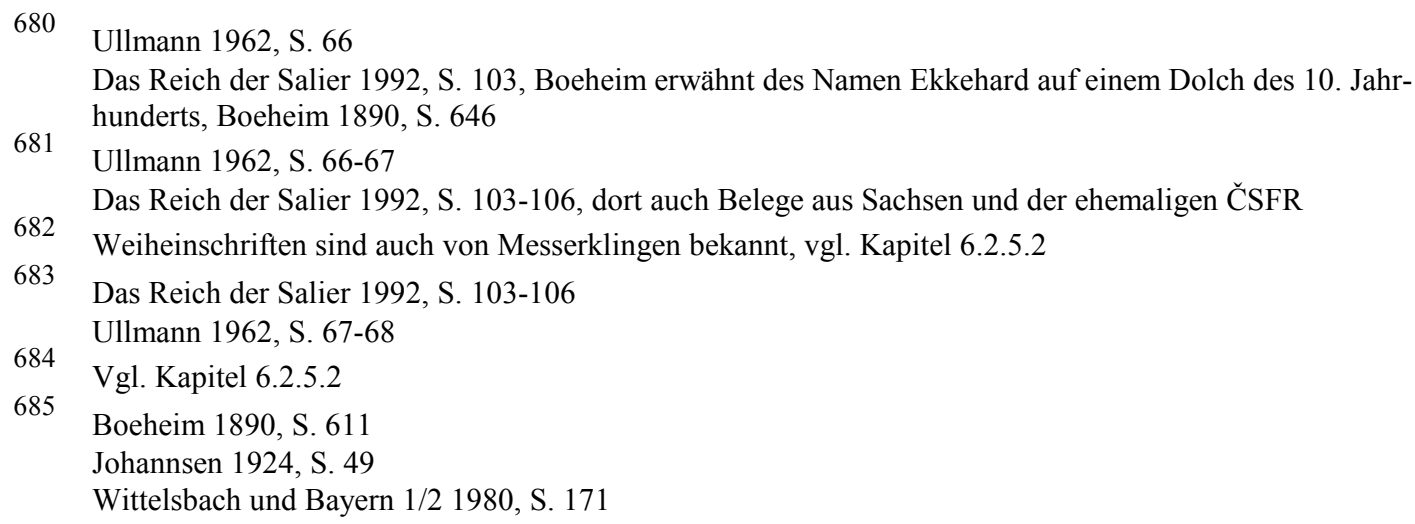


Gütezeichen eine Garantie für die Qualität der Schwertklinge darstellt ${ }^{686}$. Daß mit dieser Einführung ${ }^{687}$ eine für den Exporthandel bestimmte Serienproduktion geschützt wurde, belegen die zahllosen Nachahmungen (u.a. durch Solingen und im 15. Jahrhundert in Spanien) auch dieses Zeichens ${ }^{688}$.

Weitere Schmiedemarken des ausgehenden 13. Jahrhunderts wie der gekrönte böhmische Löwe auf einem Zeremonienschwert Ottokars II. von Böhmen sind möglicherweise eher als Kennzeichnung eines besonderen Auftrages zu bewerten $^{689}$.

Ältere Marken wie das Kreuz im Kreis auf sarazenischen Klingen des 12. und 13. Jahrhunderts ${ }^{690}$ gelten als die ersten Gütemarken im europäischen Waffenhandel, die von italienischen Werkstätten bis in das 14. Jahrhundert verwandt wurden. In dieser Zeit treten auch in Deutschland weitere zeichen- oder monogrammähnliche Schmiedemarken auf ${ }^{691}$, die aber im Gegensatz zu italienischen Marken ${ }^{692}$ keiner Stadt oder Region zuzuordnen sind.

Für die hoch- und spätmittelalterlichen Kennzeichnungen (Namenszüge, Marken und Kombinationen aus beiden) kann davon ausgegangen werden, daß sie als Qualitätsgarantie aus bekannten Werkstätten für den europäischen Fernhandel gedacht waren und als solche auch akzeptiert wurden. Von Anfang an wurden sie von anderen Werkstätten, die bis dahin keine Marken führten, aufgegriffen und nachgeahmt, um Wettbewerbsvorteile auszugleichen. Dieses gilt bis in das 14. Jahrhundert vor allem für Waffen und kann, wie weiter unten begründet wird, nicht ohne weitere Untersuchungen auf die seit dem ausgehenden 13. Jahrhundert langsam einsetzenden Marken auf Messerklingen übertragen werden.

Im 15. Jahrhundert belegen schriftliche Quellen und gemarkte Waffen eine Zunahme, für die meisten bekannten Produktionsorte den Beginn namentlich bekannter Plattner und Schwertfeger oder ihrer Herkunft:

Boeheim führt für das ausgehende 15. Jahrhundert monogrammartige Marken von Plattnern und Klingenschmieden (Schwertfegern) aus Mailand, Frankreich, aus Burgund (Arbois), Wien, Augsburg, Landshut und den Niederlanden an, erst im 16. Jahrhundert folgen Belege für Spanien (Valencia, Toledo), Brescia, Venedig, Lyon

\footnotetext{
686 Frühere Marken wie der Dreipaß auf einer Schwertklinge aus Zuilen (Niederlande) aus dem 11. Jahrhundert sind keinem Ort oder Produzenten zuweisbar, Das Reich der Salier 1992, S. 103/105 Die offizielle Vergabe der Wolfsmarke 1349 durch den bayerischen Herzog Albrecht ist umstritten, Boeheim 1890, S. 611-612 Johannsen 1924, S. 49 Beleg für die Ausrichtung des Handels mit dem christlichen Europa an, Boeheim 1890, S. 674 
und Nürnberg, darunter auch Beschaumarken der jeweiligen Ämter, obwohl die Klingenproduktion in fast allen aufgeführten Orten und Ländern älter ist ${ }^{693}$.

Namentlich bekannte Meister ${ }^{694}$ (Plattner, Klingenschmiede, Messerer und Armbrustmacher) und ein Teil der von ihnen verwandten Marken setzen bereits im 14. Jahrhundert ein (Brüssel um 1304, vor 1314; Passau Mitte bis 2. Hälfte des 14. Jahrhunderts, Bordeaux um 1375; Paris um 1380; Mailand um 1390; Wien um 1392), darunter befinden sich zwei Messerer (Passau, Paris).

Im 15. Jahrhundert überwiegen Benennungen aus Burgund (sieben Meister in Brüssel und Brügge vor 1450, 13 Meister in Brüssel, Brügge und Arbois nach 1450), gefolgt von Frankreich (drei Meister in Paris, Lyon, Aix vor 1450, fünf Meister in Paris, Lyon, Angiers und Tours nach 1450), Deutschland (insgesamt vier Meister aus Nürnberg und Augsburg sowie vier aus Innsbruck) und Italien (insgesamt fünf Meister aus Mailand, Venedig, Florenz und Rom). Aus Spanien werden zwei Meister aus Tortosa und Toledo genannt, darunter ein maurischer Klingenschmied, der außer in Toledo noch in Granada, Saragossa und Sevilla arbeitete und u.a. das Passauer Wolfszeichen führte.

Im 16. Jahrhundert nehmen namentliche Nennungen in Deutschland, Österreich, Italien und Spanien/Portugal stark zu, in Burgund und Frankreich gehen sie zurück: Für Deutschland und Österreich führt Boeheim 50 Namen an, die in Solingen (15), Dresden (6), Annaberg (2), Nürnberg (11), Augsburg (10), München (2), Landshut (2), Innsbruck (3), Wien (1) und Graz (1) arbeiteten. Der Solinger Klingenschmied Klemens Horn (Anfang 16. Jahrhundert) benutzte neben seiner eigenen Marke den Passauer Wolf und die Marke Brescias, ebenso der um 1595 in Solingen tätige Klingenschmied Peter Munsten.

In Frankreich sind zwischen 1528 und 1550 noch fünf Meister bekannt, drei von ihnen in Paris, einer in Lyon und einer in Tours. Ebenfalls fünf Namen werden aus Brüssel, Mecheln, Brügge, Gent und Flandern genannt.

Die italienischen Plattner und Klingenschmiede hatten ihre Werkstätten vor allem in Mailand (12), gefolgt von Brescia (4), Neapel (3), Belluno (2), Florenz (2), Pisa (1), Padua (1), Mantua (1) und Asolo (1, Armbrustmacher).

Die 24 genannten spanischen Meister, zumeist Klingenschmiede, arbeiteten alle in Toledo, acht von ihnen darüberhinaus in San Clemente, Valencia, Sevilla, Cordoba und Madrid. Für Madrid, Mouzon und Saragossa werden vier Armbrustmacher erwähnt.

\footnotetext{
693 Boeheim 1890, S. 674-680

694 Für die folgenden Angaben wurde das Namens- und Markenregister aus dem Anhang von Boeheim 1890 ausgewertet, die Geschützgießer, Büchsenmacher etc. wurden nicht berücksichtigt, ebensowenig Graveure, Ätzer und Treibarbeiter.
} 
Die von Boeheim aufgelisteten Namen und Marken, die er 1890 nach Durchsicht von Archiven und unter Berücksichtigung der wichtigsten europäischen Rüstkammern und Sammlungen abschloß, repräsentieren die Elite der europäischen Waffenhersteller, ein großer Teil von ihnen belieferte im ausgehenden 15. und 16. Jahrhundert die wichtigsten europäischen Fürstenhöfe ${ }^{695}$.

Viele der von ihnen benutzten Marken wurden über mindestens zwei Generationen geführt und entwickelten sich zu frühen Firmenmarken, die dementsprechend auch von nicht autorisierten Werkstätten benutzt wurden ${ }^{696}$.

Die frühen Erwähnungen von Messerern in Passau und Paris können nicht als Beleg dienen, vergleichbare Verhältnisse in der Messerproduktion anzunehmen. Es ist zwar davon auszugehen, daß einige der hier genannten Werkstätten auch Messer herstellten (wie in Passau durch die Bezeichnung Messerer und Klingenschmied belegt) ${ }^{697}$, auch werden die seit dem 16. Jahrhundert verbreiteter auftretenden Kurzwehren mit beigefügten Bestecksätzen von denselben Werkstätten hergestellt worden sein (Abb. 217).

Dennoch muß zwischen der technisch und qualitativ hervorragenden Produktion dieser in Europa führenden Werkstätten, der Durchschnittsproduktion der zahlreichen Waffenhersteller, die bereits von Boeheim nicht mehr erfaßt wurden, und schließlich der Messerproduktion für den alltäglichen Gebrauch unterschieden werden.

In einer Abhandlung zur Geschichte des rheinisch-westfälischen Waffenhandwerks spricht Ullmann die grundsätzlichen Probleme, die auch hier zu berücksichtigen sind, bereits an ${ }^{698}$.

Der in den historischen Quellen oft benutzte Begriff Messer ("meste") bezeichnet häufig keine Werkzeug- oder Speisemesser, sondern Dolch- und Stechmesser ${ }^{699}$. Die von Ullmann, Knorr und Schoknecht ${ }^{700}$ übereinstimmend als Trachtbestandteil und Standeszeichen vor allem bürgerlicher Schichten bezeichneten Kurzwaffen des 14. bis 16. Jahrhunderts, deren Tragen in den Städten oft reglementiert oder verboten wurde ${ }^{701}$, lassen sich mit den vor allem aus Grabungen bekannten Nierendolchen und -dolchmessern verbinden, die in den zeitgenössischen Verordnungen als "stekemes" und "pook", aber auch als "Hildesheimer", "Breckerfelder"

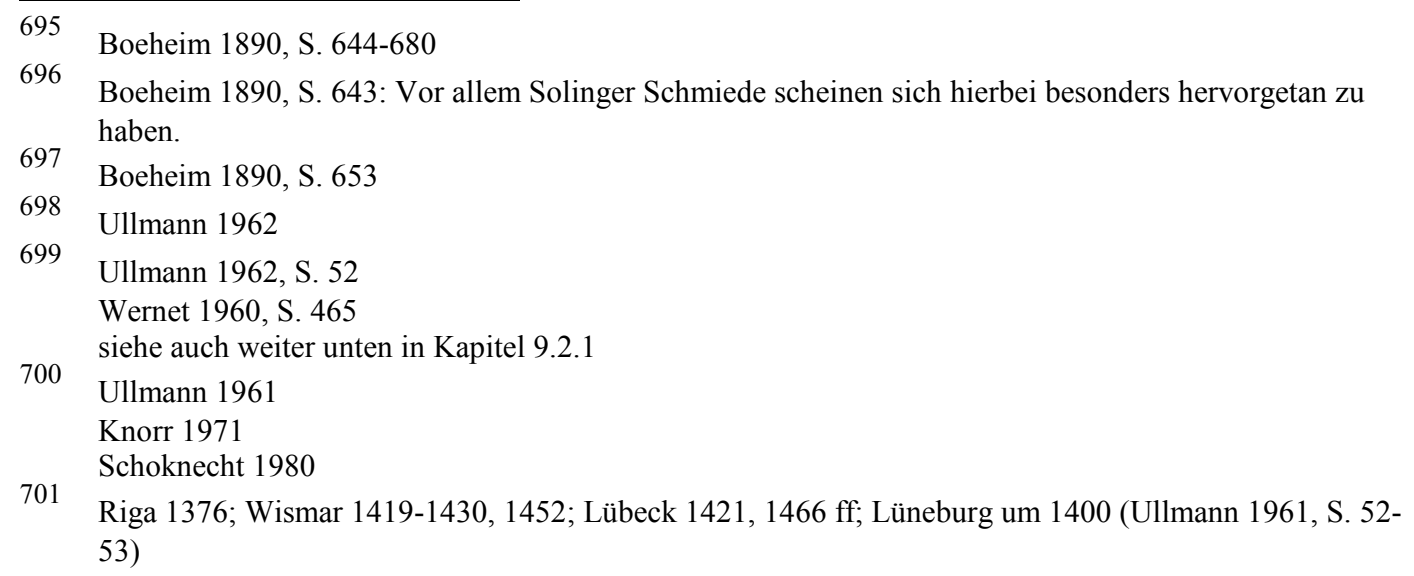


oder "Kölnische Messer" (die letzteren beiden als Handelsgut) bezeichnet wur$\operatorname{den}^{702}$. Ullmann unterscheidet das Stechmesser als eher bürgerlich-bäuerliche, den pook (auch als Meisterstück genannt) als eher oberschichtliche Waffe, ordnet sie aber beide als Waffe zwischen Messer und Dolch stehend in die Gruppe der Nierendolche ein ${ }^{703}$.

Wenn in der allgemeineren Literatur zu Fernhandelsgütern des Hoch- und Spätmittelalters von Messern als Exportgut gesprochen wird, so ist zum Beispiel bei den oben erwähnten Kölnischen Waren und den ebenfalls bereits zitierten Breckerfelder Messern im einzelnen zu prüfen, ob eine Differenzierung zwischen Messern und Dolchmessern überhaupt möglich ist. Ullmann erwähnt die Ausfuhr Breckerfelder Dolchmesser in das nordöstliche Hansegebiet, "Kölnische Messer" nach Lübeck und in das mecklenburgische Küstengebiet, aber auch pooke mit vermutlich Lübecker Marken in den westfälischen Raum ${ }^{704}$.

Ein weiterer wichtiger Aspekt ist die Frage nach der Herstellung und der Möglichkeit einer Herkunftsbestimmung, die Ullmann für die angesprochene Gruppe von Kurzwaffen ebenfalls anspricht.

Grundsätzlich ist innerhalb der spätmittelalterlichen städtischen Messerproduktion zwischen den Messerschmieden (Klingenproduktion), den Messerbereitern (Endmontage) und den Eisenkrämern (Vertrieb) zu unterscheiden ${ }^{705}$.

Wenn in Darstellungen zum Messerschmiedehandwerk davon gesprochen wird, daß Zunftbeschränkungen und Kontrollen in der Regel der innerstädtischen Produktion eine Monopolstellung verschafft hätten und die Ware der angeschlossenen Schmiede zur Qualitätssicherung mit einer oder zwei Marken (Schmiede- und Beschaumarke) versehen werden mußte ${ }^{706}$, so kann dieses nicht einmal für den nachmittelalterlichen Zeitraum verallgemeinert werden, aus dem die wenigen historischen Quellen stammen. Das gilt auch für die Auffassung, den Werkstätten sei der Handel mit fremden oder bereits einmal verkauften Messern verboten gewesen ${ }^{707}$.

Der wirtschaftliche Hintergrund erhellt eher aus den von Ullmann zitierten Beispielen zu Auseinandersetzungen in der Produktion und im Handel mit Messern, die, das zeigt die bereits erwähnte Lübecker Verordnung von 1353, im Gegensatz zu langen Blankwaffen und Dolchen vor allem im Kleinhandel der Krämer anzutreffen sind.

Die Trennung zwischen Messerklingenschmieden und Messerbereitern ist in Lübeck 1479 durch einen Streit belegt, in dem die Messerbereiter unter Verweis auf älteres Recht auch das Schmieden der Klingen ausüben wollen. Der Vergleich ver-

\footnotetext{
702 Ullmann 1962, S. 53

703 Ullmann 1962, S. 53, 62

704 Ullmann 1962, S. 53

705 Ullmann 1962, S. 53-54

706 Wernet 1960, S. 466

707 Wernet 1960, S. 466
} 
ordnet den Schmieden daraufhin, nur die von ihnen hergestellten Messer mit Griffen zu versehen und die Klingen zur Kennzeichnung dieser Abgrenzung mit Maken $\mathrm{zu}$ versehen, die nicht verändert werden dürften ${ }^{708}$. Zum Ausgleich für das Verbot, Klingen zu schmieden, erhalten die Messerbereiter das Recht, neben Lübecker auch außerhalb von Lübeck geschmiedete Messerklingen aufzukaufen und zu verkaufsfertiger Ware zu verarbeiten ${ }^{709}$.

Bereits 1512 erfolgt eine neue Regelung, nach der die Lübecker Klingenschmiede auch fremde Klingen aufkaufen dürfen, in einem späteren Zusatz wird den Amtsmeistern des Schmiedeamtes verboten, lübische Klingen und fertige Dolchmesser aufzukaufen, in einem nach 1512 verfaßten Dokument erhalten die Messerschmiede das Recht, ihre Kurzwaffen und Messer verkaufsfertig zu produzieren, wobei unsicher ist, ob dieses Recht die Verarbeitung von Holz und Messing zu Griffteilen miteinschließt $\mathrm{t}^{710}$.

Dem hier geschilderten Streit liegt in erster Linie der Gewinn zugrunde, der mit fertigen Waren zu erzielen war, wobei die Auseinandersetzung nicht nur das Recht der beiden an der Produktion beteiligten Handwerke zur Endmontage berührt, sondern auch den Verkauf der Ware zwischen dem Schmiedeamt, dem die Klingenschmiede in Lübeck zu dieser Zeit angehörten, den Messerbereitern, die diesem Amt nicht angehörten, und den Eisenkrämern, die dem Krämeramt angeschlossen waren ${ }^{711}$.

Die erwähnte Markenverordnung zeigt, daß es zumindest auch Schmiedemarken gegeben hat, die nichts mit einem Gütesiegel oder einer amtlichen Kontrolle über die Warenqualität zu tun hatten, sondern eine bestimmte Gruppe von Halbfertigprodukten innerhalb der am Produktionsprozeß beteiligten Handwerke zum Zweck der Kompetenzabgrenzung kenntlich machen sollten.

Die erwähnten Auseinandersetzungen bezüglich des Handels mit den Fertigprodukten, die in dem oben zitierten Streit ebenfalls zum Ausdruck kommen, belegt Ullmann durch weitere Beispiele.

Die Regel, das heißt allgemein verbreitete Rechtsauffassung ${ }^{712}$, scheint die Aufteilung nach Objektgruppen gewesen zu sein:

Die Schwertfeger (Endbereiter von Blankwaffen) hielten den Handel mit langen Blank- und Feuerwaffen, die Eisenkrämer den Handel mit Messern, Stechmessern und Dolchmessern ${ }^{713}$.

\footnotetext{
708 Ullmann 1962, S. 54

709 Ullmann 1962, S. 55

710 Ullmann 1962, S. 55-56: Zum Beispiel ist unsicher, ob die Holz-, Knochen- oder Elfenbeingriffe von den Messerbereitern, die das Rohmaterial oft noch von anderen Handwerkern wie den Kammachern vorarbeiten lassen mußten, oder von den Messerschmieden hergestellt wurden.

712 So wurde bei innerstädtischen Streitigkeiten in anderen Städten nach den dortigen Regelungen nachgefragt, zum Beispiel innerhalb des Schwertfegerkreises der sechs wendischen Städte, vgl. Fuhse 1930, S. 32-33; Ullmann 1962, S. 59-61

Ullmann 1962, S. 57, S. 60-61
} 
Aber auch hier gehen Anspruch und Wirklichkeit auseinander, wobei häufig nicht nur die Warengattung, sondern auch die Herkunft der Ware ein Streitpunkt war.

Der innerstädtische Verkauf bzw. Handel von importierten Messern und Waffen, belegt 1353 in Lübeck, scheint seit dem 15. Jahrhundert als Konkurrenz zur einheimisch produzierten Ware üblich gewesen $\mathrm{zu} \operatorname{sein}^{714}$. 1566 werden in einem Nachlaßinventar Waren eines Lübecker Krämers genannt, die dieser in ländlichen Gemeinden und kleineren Städten zwischen Lübeck und Rostock verkaufte: "iseren poke", der "fin pok" und "Kollensche Messe", letzteres möglicherweise Erzeugnisse des bergischen oder märkischen Eisengewerbes, welche über Köln verhandelt wurden $^{715}$.

In Münster beanspruchen die Krämer 1584 den Vertrieb von Messern "woher auch immer", extra aufgeführt wird Nürnberger, Schmalkalder, Braunschweiger, Kölner und Antwerpener Ware, die nicht "mit Ellen gemessen" und "mit Schalen gewogen" werden kann ${ }^{716}$.

1560 versucht das Hamburger Schmiedeamt, durch eine Verordnung den Import von Messern, Dolchen, Blank- und Feuerwaffen zu drosseln, um den innerstädtischen Produktabsatz zu fördern und fügt erklärend hinzu, es wolle damit dem einheimischen Bürger die Möglichkeit geben, preisgünstig seine Bewaffnung zu ergänzen ${ }^{717}$,.

Der innerstädtische Streit um fremde wie eigene Ware findet seinen Niederschlag in zahlreichen Auseinandersetzungen:

In Hamburg verpflichten sich 1598 die Krämer, keine Feuer- und Blankwaffen zu verkaufen, welches Recht den Schwertfegern zustehe ${ }^{718}$.

Im gleichen Jahr klagt das Schmiedeamt gegen die Eisenkrämer, sie griffen mit der Montage und dem Vertrieb kleiner Dolche in das hier dem Amt unterstehende Schwertfegergewerbe ein ${ }^{719}$, wobei zu bemerken ist, daß der Handel mit pook und stekemes eher den Krämern als den Schwertfegern zustand. Der Vergleich dieses Streits berührt auch gar nicht diese Waren, sondern verbietet den Krämern den Handel mit den unbereiteten und bereiteten Waffen und deren Gefäßen (Griffpartien), die die Schwertfeger bearbeiteten ${ }^{720}$.

Schon 1491 wird ebenfalls in Hamburg ein Ausgleich zwischen dem Schmiedeund dem Krämeramt geschaffen, nachdem ersteres versucht hatte, sich in den Kleineisenhandel einzuschalten ${ }^{721}$.

In Braunschweig klagen die Schmiede 1488 gegen Schwertfeger und Krämer, weil diese Messer und Dolche verkaufen ${ }^{722}$. Ob die Krämer in Braunschweig dieses 
Recht besaßen, erhellt nicht. Die Schwertfeger hielten es jedoch "seit alters her" für ihr Recht, Dolche, Jagdmesser, Messer und Pfrieme als vorgefertigte Ware zu kaufen, aufzubereiten und zu verkaufen ${ }^{723}$. Möglicherweise ist dies ein Hinweis darauf, daß es Unterschiede zwischen Bestecksätzen an Jagd- und Kampfwaffen und einzeln verkauften Messern und Kurzwehren gab. In Münster gehörte 1619 zu den Meisterstücken des Messerschmieds neben einem Speisemesser ein "Hauer mit kleinen messern, pren und specknadeln" ${ }^{724}$.

Der Kampf der Schwertfeger um das alleinige Recht, die von ihnen montierten und aufbereiteten Waffen ohne Einschaltung der Krämer direkt zu verhandeln, wird bereits 1473 in Lübeck durch den Versuch deutlich, dieses Recht in der Zunftrolle festzuschreiben $^{725}$.

Um die einzelnen Warengattungen, die von den Krämern und Schwertfegern in beiderseitiger Überschneidung beansprucht wurden, voneinander zu trennen, legte Lübeck im 16. Jahrhundert die Maße für Schwerter und kleine Waffen fest:

Die von den Schwertfegern bearbeiteten und verhandelten Blankwaffen durften nicht unter $50 \mathrm{~cm}$, die von den Messerbereitern bearbeiteten und von den Krämern verhandelten Kurzwehren nicht über $50 \mathrm{~cm}$ lang $\operatorname{sein}^{726}$. Diesen Maßen folgten die Ordnungen in Hamburg, Stade, Lüneburg, Salzwedel und Hildesheim, während in Dresden und Augsburg 1575 die Schwertfeger kurze und lange Waffen kaufen, bereiten und verkaufen durften ${ }^{727}$.

$\mathrm{Da}$ es ähnliche Abgrenzungen, möglicherweise auch zwischen Kurzwehren und Messern gegeben hat, belegt die Gegenüberstellung der Klingenlängen von Messern und Nierendolchen ${ }^{728}$, wobei die Gründe in diesem Fall eher in der Trennung zwischen Waffe und Werkzeug zu suchen sind, da beide Artikel von derselben Berufsgruppe verhandelt wurden.

Trotz aller Konkurrenzstreitigkeiten hatten Klingenschmiede, Schwertfeger und Krämer neben ihrem Rückhalt in gleichen oder verschiedenen Ämtern ausreichende

\footnotetext{
714 Ullmann 1962, S. 57

715 Ullmann 1962, S. 57

716 Ullmann 1962, S. 57

717 Ullmann 1962, S. 59

718 Ullmann 1962, S. 57

719 Ullmann 1962, S. 60

720 Ullmann 1962, S. 61

721 Ullmann 1962, S. 57

722 Ullmann 1962, S. 58

723 Fuhse 1930, S. 34

724 Ullmann 1962, S. 59

725 Ullmann 1962, S. 60-61

726 Ullmann 1962, S. 58

Fuhse 1930, S. 33 (Anmerkung 58) 
eigene Produktreserven, die sich nicht überschnitten. Dieses trifft nicht für die Messerbereiter $\mathrm{zu}$, die letztlich lediglich einen geringen Marktanteil zwischen Vorprodukt und Endverkauf besaßen, in ihren Rechten oft eingeengt und von allen Seiten regelmäßig beschnitten wurden. Die Stellung dieser Berufsgruppe in den Niederlanden wird deutlich in einer Erklärung der niederländischen Klingenschmiede und Schwertfeger, die es 1571 ablehnen, die "Messerschmiede" als gleichberechtigtes Handwerk anzuerkennen: "wir halten es für ein bloß umlaufendes Handwerk", ähnlich etwa dem der Scherenschleifer ${ }^{729}$.

Die hier zitierten Quellen belegen im 15. und 16. Jahrhundert verschiedene Ansätze zu einer kontrollierten Produktion der beteiligten Handwerke, die alle um ihren Marktanteil kämpfen.

Nürnberg stand in dieser Entwicklung insofern günstiger da, als es durch zentraldirigistische Maßnahmen derartige Auseinandersetzungen in der Regel gar nicht erst aufkommen ließ und dadurch die gesamte Messerproduktion, dessen Rohstoff Stahl es aus eigenen und von ihm kontrollierten Hütten bezog, ohne den innerstädtischen Zwischenhandel direkt in den Markt bringen konnte, zu Lasten der an der Produktion beteiligten Handwerke.

In der Regel waren bis zum Verkauf des Endprodukts Messer aber mehrere Zwischenhandelsstationen beteiligt:

Die Ausgangsrohstoffe Eisen und Stahl, oft bereits über mehrere Stationen verhandelte Handelsprodukte, standen - gemessen an der Gesamtzahl an Klingenproduzenten in den zahlreichen mittelalterlichen und frühneuzeitlichen Städten - nur den wenigsten Städten direkt und in relativ unbegrenzter Menge zur Verfügung, wie etwa Köln, Nürnberg, Schmalkalden oder Freiberg. Selbst Passau bezog seinen Stahl aus steirischen Hütten, wenn auch landesherrlich privilegiert. Die historischen Quellen scheinen bis in das 16. Jahrhundert einen unbeständigen Zustand zu beschreiben, in dem jeder Bezieher und Weiterverarbeiter von Stahl versuchte, die gesamte Gewinnspanne bis zum Verkauf des Endprodukts auszuschöpfen, und die zitierten Streitigkeiten zeigen, daß dieser Zustand um 1600 noch nicht abgeschlossen war.

Es ist unwahrscheinlich, daß diese Entwicklung von einem bereits spezialisierten, regulierten und einheitlich festgelegten Produktions- und Vertriebssystem ausging, auch wenn in Einzelfällen wie in Nürnberg bereits im 14. Jahrhundert ein kontrolliertes Verlagssystem den überregionalen Vertrieb von Kleineisen- und Stahlwaren übernommen hatte oder in Köln nach dem vorübergehenden Niedergang der Dortmunder Konkurrenz seit ca. 1400 zentralistische und monopolistische Initiativen zum Tragen kamen.

Ullmann und Fuhse beschreiben die zitierten innerstädtischen Auseinandersetzungen des 16. Jahrhunderts als Ergebnis eines technischen 
Spezialisierungsprozesses im Schmiedehandwerk, der bei den Schwertfegern vermutlich im 14., bei den Messerschmieden wohl erst im 15. Jahrhundert einsetzte ${ }^{730}$. Die erwähnte Vorschrift von 1479 bezüglich einer Markierung von Klingen betraf lediglich einen Ausschnitt aus der in Lübeck hergestellten und von dort in und auBerhalb der Stadt verkauften Messer, und zwar zur Abgrenzung gegenüber den Messern der Messerbereiter in derselben Stadt.

$\mathrm{Zu}$ den Klingenmarken an Messern des 14. und beginnenden 15. Jahrhunderts sagt diese Quelle nichts aus, lediglich der Zweck der Verordnung und der Zeitpunkt - möglicherweise nur wenige Jahrzehnte nach der Trennung der Messerbereiter und der Messerklingenschmiede, als der ältere Zustand noch erinnerlich war und zur Begründung eines Anspruchs ausreichend erschien - lassen auch für den um 1300 einsetzenden Brauch der Markierung ähnliche wirtschaftliche Beweggründe wahrscheinlich werden.

Eine gewollte Identitätsverleihung zum Zweck der Qualitätsgarantie wie bei den Blankwaffen ist dagegen eher unwahrscheinlich. So sind die meisten Marken vor dem 17. Jahrhundert eher zeichenhaft und weit verbreitet, im Gegensatz zu den monogrammartigen oder beschreibenden Marken an Waffen, die in einigen Fällen im 16. Jahrhundert bereits den Namen oder die Anfangsbuchstaben der Werkstätten tragen.

Cowgill führt für die Motivwahl der auf 110 in London ausgegrabenen Messern beobachteten Marken mehrere mögliche Gründe auf, darunter auch den Namen des Schmieds bezüglich einer kleinen Gruppe von Buchstabenmarken ${ }^{731}$.

Eine Marke in Dolchform führt er auf eine Bestimmung von 1606 zurück, nach der diese Marke für die der Zunft unterstellten Produzenten bindend war, wahrscheinlich seit $1594^{732}$. Zur Verbreitung der meisten Marken in ganz Europa wird auf eine Marke hingewiesen, die ihre Entsprechung auf einem Vorschneidemesser mit dem Wappen des burgundischen Kanzlers Nicholas Rolin (15. Jahrhundert) findet ${ }^{733}$.

Dennoch finden sich im Vergleich der von Cowgill vorgestellten Marken auf in London ergrabenen Messern zu den hier erfaßten Marken nur wenige Überschneidungen, vor allem zu Messern aus niederländischen Städten. Auch die Marken der Messer aus dem Schiffswrack von Wittenbergen sind trotz ihrer Vielfalt eine deutlich zu unterscheidende Einzelgruppe innerhalb der beiden anderen Zusammenstellungen.

Keine Berücksichtigung haben in dieser Diskussion die Messer erfahren, die im Zuge der Selbstversorgung in den in grundherrschaftlicher Abhängigkeit stehenden Hof-, Dorf- oder Burgschmieden produziert worden sind. So naheliegend es auch erscheint, diese aus den Messerkontingenten des überregionalen Handels auszu-

\footnotetext{
730 Fuhse 1930, S. 32

Ullmann 1962, S. 54

731 Cowgill (u.a.) 1987, S. 24

732 Cowgill (u.a.) 1987, S. 20

733 Cowgill (u.a.) 1987, S. 20
} 
klammern, eine regionale Bedeutung vor allem als Konkurrenz zur Ware der Eisenkrämer und innerstädtischer Produzenten aus in der Nähe liegenden Städten kann ihnen unbegründet nicht abgesprochen werden.

Über ihre Produktion ist abgesehen von Grabungsergebnissen nichts bekannt, und diese können in der Regel das eiserne Fundmaterial nicht nach ihrer Herkunft differenzieren ${ }^{734}$. Daß aber auch in diesem Rahmen eine über den direkten Bedarf hinausgehende Produktion denkbar ist, zeigt die bereits erwähnte Eisenverhüttung und -verarbeitung auf der Hattinger Isenburg, die zumindest über alle Faktoren einer rohstoffunabhängigen Waffen- und Werkzeugproduktion verfügte ${ }^{735}$.

\section{Ausblick}

Die vorgenannten Ausführungen zeigen, mit welchen Problemen eine Untersuchung über die Produktions- und Vertriebsformen von Messern und anderen Kleineisenwaren zu rechnen hat. Herkunft, Verkehrswege und Absatzorte der Endprodukte sind, auch wenn die unterschiedlichen Möglichkeiten einzugrenzen sind, noch weitgehend unbekannt.

Eine möglichst vollständige Vorlage der in städtischen Zunftrollen enthaltenen Schmiedemarken könnte im Vergleich mit den ebenfalls noch weitgehend unbeobachteten und unpublizierten Marken an eisernen Fundobjekten zumindest den Prozentsatz an Eisen- und Stahlgeräten bestimmen helfen, der in möglicherweise regional begrenzten Bezirken aus reidentifizierbaren Werkstätten stammt, ohne daß damit ein derartiger historischer Anspruch verbunden wird.

Da ein nicht unerheblicher Teil der Klingen mehrfach gemarkt wurde, läßt sich vielleicht auch ein Teil der von Cowgill beschriebenen, europaweit benutzten Kleinzeichen ${ }^{736}$ regional oder objektbezogen begrenzen.

Die Intentionen, die hinter den Marken standen, werden wahrscheinlich eher im Bereich der Kontingentbegrenzung vor dem Hintergrund von Konkurrenz- und Absatzregulierungen $\mathrm{zu}$ suchen sein; archäologische Quellen tragen $\mathrm{zu}$ dieser Fragestellung nur in günstigen Ausnahmefällen bei.

Naturwissenschaftliche Methoden zur Herkunftsbestimmung des verwandten Eisens gibt es bisher nicht.

Im Gegensatz zu Keramikgefäßen und Buntmetallobjekten, bei denen die Ton- und Erzlagerstätten über eine Analyse der Fundobjekte vielfach zu ermitteln sind, stehen der Reidentifizierung von Eisen grundsätzliche Probleme entgegen.

\footnotetext{
734 Vgl. die Diskussion um die Messer aus der Siedlung Gommerstedt, Kapitel 6, Abschnitt 2.6 (Die Klingenmarken) 
Die für eine Ermittlung der Erzlagerstätten notwendigen Begleitelemente Phosphor, Arsen, Nickel, Kohlenstoff, seltener auch Silicium, Mangan, Titan und Vanadium sind wohl in den Schlacken des Verhüttungsprozesses im Rennfeuerverfahren vorhanden, im ausgehämmerten Roheisen werden günstigstenfalls noch 2 bis $5 \%$ mechanisch eingeschlossene Schlackenreste angetroffen. Im aufbereiteten und $\mathrm{zu}$ Stahl veredelten Endwerkstoff, wie er in fertiggestellten Eisen- und Stahlwaren begegnet, fehlen diese Elemente.

Im indirekten Verfahren (Hochofenverfahren) gewonnenes Roheisen, welches seit ca. 1000 nach Christus belegt ist und seit dem 14. Jahrhundert stärker einsetzt, sind Silicium und Mangan enthalten. Beide Elemente sind aber zu allgemein verbreitet, um allein über sie ein Ausschlußverfahren von Lagerstätten durchzuführen.

Ähnliche Faktoren (wie der zu geringe Anteil von Blei) verhindern die Herkunftsermittlung über eine Isotopenbestimmung.

Zusätzliche Probleme liegen in der grundsätzlichen Art der Beweisführung. Auch im günstigsten Fall wie auf der Hattinger Isenburg, wo bereits die Befundsituation einen Zusammenhang zwischen dem anstehenden Spateisenstein, den Verhüttungsanlagen und der Schmiede nahe legt, ließe sich über die Schlackenreste lediglich ein hoher Wahrscheinlichkeitsgrad naturwissenschaftlich ermitteln, da die Methode lediglich die weniger wahrscheinlichen Lagerstätten ausschliessen kann, eine Beweissicherung kann sie nicht liefern.

Darüber hinaus sind die chemischen Zusammensetzungen der Erze in den in Frage kommenden mittelalterlichen Eisenerzlagerstätten nicht ausreichend bekannt, um derartige Ausschlußverfahren durchzuführen ${ }^{737}$.

Für die zukünftige archäologische Feldforschung bedeutet dieser bisher nahezu vollständige Ausschluß naturwissenschaftlicher Identifikationsmöglichkeiten, Befunden von Eisenerzverhüttung besondere Aufmerksamkeit zu widmen.

Das notwendige Vergleichsmaterial an verhütteten Bergerzen ist regional weitgehend begrenzt auf die von Sprandel beschriebenen Lagerstätten, die in der Regel die Verhüttungsplätze in ihre unmittelbare Nähe oder in einem begrenzten Umfeld an sich zogen.

Abschließend sei nocheinmal darauf hingewiesen, daß sich, abseits der Bergbauregionen an zahlreichen Stellen Abbau, Verhüttung und Weiterverarbeitung von in Feuchtgebieten ausgefällten Eisenerzen auch archäologisch nachweisen lassen. Die Untersuchungen zu den Verhüttungs- und Schmiedetechniken vom 10. bis 14. Jahrhundert in Danzig, dessen Ausgangsrohstoff wahrscheinlich Marscherze des Überschwemmungsgebiets der Mottlau waren ${ }^{738}$, belegen die verbleibenden Möglichkeiten naturwissenschaftlicher und archäologischer Forschung in eindrucksvoller Weise.

737 Der hier zusammengefaßte Überblick zum bisherigen Stand archäometallurgischer Forschung wurde dem Autor dankenswerterweise von Herrn Dr. Rehrend vom Institut für Archäometallurgie (Zollern-Institut) 


\subsection{Zur Funktion von Messern}

Bei dem Versuch, die Funktionen der Messer näher zu bestimmen, stößt man an die Grenzen der Aussagefähigkeit des archäologischen Quellenmaterials.

Der Gebrauch dieses im gesamten Untersuchungszeitraum eminent wichtigen Gegenstandes manifestiert sich eher in zahlreichen schriftlichen und bildlichen Quellen, die das breite Spektrum vom einfachen Mehrzweckwerkzeug bis zum von Tabus erfaßten und von Verboten beladenen Statussymbol umfassen.

Die Frage nach dem sozialen und funktionalen Umfeld des Messers zeigt das Ende dieser Untersuchung an und eröffnet ein neues Thema mit neuen Fragestellungen und neuen Quellen.

Wie bereits zu Anfang erwähnt, ist das Erarbeiten von breit angelegten Materialgrundlagen auf archäologischer Quellenbasis im Bereich der historischen Wissenschaften nicht unumstritten, da die direkten Ergebnisse oft im Unverhältnis zu dem benötigten Aufwand zu stehen scheinen. Dennoch sind sie angesichts der in Millionenhöhe anfallenden Funde, angesichts der zahlreichen offenen Fragen im Kontext alltäglichen Lebens mittelalterlicher Menschen notwendig. Sie dienen dem engeren Zusammenschluß von Materialbasis und Theoriebildung, von Arbeitsgrundlage und wissenschaftlichem Ergebnis.

Die wesentlichen Daten zur Funktionsbestimmung eines ausgegrabenen Messers, die aus archäologischer Sicht relevant sind, können aus den angetroffenen Begleitumständen, den Befunden, gewonnen werden, oder - zumindest teilweise - dem Fundobjekt selbst entnommen werden. Leider wurden bei zahlreichen Messern die näheren Fundumstände nicht oder nur unzureichend registriert, vor allem bei den älteren Funden.

Ein Messer, welches zum Beispiel mit Mahlzeitresten und weiteren Objekten wie Tellern, Gläsern etc. in einem Einzelbefund innerhalb einer Kloake angetroffen wird, kann mit hoher Wahrscheinlichkeit mit diesen Relikten in Zusammenhang gebracht werden, es erfüllt in etwa die Kriterien eines "geschlossenen Fundes", wie etwa ein Grabfund mit Messern, Nahrungsmitteln und Geschirr.

Derartige Befundsituationen sind aber nicht die Regel. Der überwiegende Teil der hier bearbeiteten Messer ist als Verlust oder nach Beschädigung bzw. Verschliff in den Boden gelangt, lösgelöst von seinem ursprünglichen Funktionsumfeld. Die Fundumstände geben demnach nur selten Auskunft über die Art und Dauer der Benutzung.

Die Form eines Messers kann Hinweise geben auf eine mögliche funktionale Einengung, wie die Formen der Gruppe IV. Sie gibt zwar keine Auskunft über die Art dieser Einengung, die Vielfalt der beobachteten Formen vor allem vom 11. bis 
14. Jahrhundert läßt jedoch Rückschlüsse auf eine relativ hohe Anzahl unterschiedlicher Funktionen zu.

Im Vergleich hat die Analyse der Klingenlängen und Klingenformen ergeben, daß sowohl unterschiedliche Klingenlängen einer Klingenform als auch verschiedene Klingenformen mit gleicher Länge auftreten.

Ebenfalls beobachtet wurde vom 11. bis zum 15. Jahrhundert eine größere Spannweite der Klingenlängen inclusive der Extremwerte, während im 16. und 17. Jahrhundert ein deutlicher Rückgang der Extremwerte und eine Standardisierung der Klingenlängen zu verzeichnen ist.

Auch diese Daten geben klare Hinweise darauf, daß mit einer starken Differenzierung innerhalb des Rahmens, in dem Messer benutzt worden sein können, zu rechnen ist, ebenfalls darauf, daß vom 15. zum 16. Jahrhundert ein allgemeiner Funktionswandel stattgefunden hat, der sich auch im Rückgang einiger Grundformen dokumentiert, und auf eine allgemeine Einengung des funktionalen Rahmens der in dieser Untersuchung bearbeiteten Messer hinweist.

Der lange Zeitraum und die damit enthaltenen Quellenlücken machen eine Rückschlußinterpretation, wie sie in der Volkskunde bei sehr viel kleineren Zeiträumen und besserer Belegbarkeit (z.B. noch lebende Gewährspersonen) angewandt wird, unmöglich, selbst dann, wenn man feststellt, daß die wesentlichen Grundformen von Messern auch heute noch üblich sind, und man aus guten Gründen wie den Substanzunterschieden von Fleich und Brot gemeinhin Messer mit gebogenen Rücken und geraden Schneiden zum Brotschneiden, Messer mit gebogenen Schneiden und geraden Rücken zum Fleischschneiden benutzt.

Die Archäologie kann derartige Fragen nur in günstigsten Einzelfällen beantworten. Die vorliegende Untersuchung erhebt daher nicht den Anspruch auf Vollständigkeit, sondern ist in vielen Bereichen als Arbeitshypothese zu verstehen.

Im Zusammenhang mit der Frage nach der Funktion eines mittelalterlichen oder frühneuzeitlichen Gegenstandes kommt zeitgenössischen Darstellungen eine große Bedeutung zu.

Der Umgang mit Bildquellen ist in der Behandlung mittelalterlicher wie neuzeitlicher Themen längst üblich geworden. Die Flut von Abhandlungen über das Leben in mittelalterlichen Dörfern, Burgen und Städten ist oft mit zahlreichen "zeitgenössischen" Darstellungen versehen worden, die in der Regel den Eindruck vermitteln, die Autoren griffen bei ihrer Auswahl auf eine breit angelegte, durchstrukturierte und thematisch aufgearbeitete Quellenbasis zurück ${ }^{739}$.

739 Vgl. Übersichtsarbeiten und populärwissenschaftliche Werke wie W. Meyer und E. Widmer, Das große Burgenbuch der Schweiz, Zürich $1978^{(2)}$; ders. und E. Lessing, Deutsche Ritter, Deutsche Burgen, Sonderausgabe des Bertelsmann-Verlages, München 1984; Beispiele gründlicherer Quellenbearbeitung wie A. Wolf-Graaf, Die verborgene Geschichte der Frauenarbeit, Weinheim und Basel 1983; H. Kühnel (Hsg.), Alltag im Spätmittelalter, Graz-Wien-Köln 1984; H. Boockmann, Stadt im Spätmittelalter, Auf- 
Einrichtungen wie das österreichische Institut für mittelalterliche Realienkunde und Bildarchive in großen Kulturgeschichtsmuseen können jedoch nicht die noch immer weitgehend ausstehende Aufarbeitung dieser Quellengattung ersetzen. Es mangelt an Arbeiten, die die einzelnen Gegenstände in ihren Funktionen und ihrem Funktionswandel, ihrer Stellung innerhalb des alltäglichen Lebens aufgrund der zahlreich vorhandenen Bildquellen darstellen. Die Aufarbeitung dieser Quellengattung wäre ein weiterer Schritt, und erst die Verbindung fachspezifischer Ergebnisse, die auf regionale Untersuchungen und auf überregional angelegte Materialvorlagen zurückgreifen, wird ein sicheres Fundament für die weitergehende Erforschung mittelalterlicher und neuzeitlicher Lebensumstände sein können.

Wenn am Schluß dieser Arbeit die Frage nach den Funktionen von Messern ins Blickfeld rückt und mit ihr die Quellengattung der zeitgenössischen bildlichen Darstellungen, so mag dies als Anregung zu einer neuen Untersuchung dienen, die lediglich den Zweck hat, einige wenige Aspekte dieses Themas zu behandeln und in Beispielen einige der Probleme zu berühren, die möglicherweise zu bewältigen sein werden.

sätze wie D.B. Harden, Table-glass in the Middle Ages, in: Rott. Pap. II-1975; J.A. Brongers und H.F. Wijnman, Chronological Classification of roemers with the help of $17^{\text {th }}$ century paintings in the Low Countries, in: Rott. Pap. I-1968; W.A. Forbes, Ontwikkelingsfasen in het Middeleeuws bestek, in: Rott. Pap. II-1975, deren Aussagekraft je nach Intensität der Quellenbearbeitung sehr unterschiedlich ist. 


\subsubsection{Funktion und Funktionswandel aus der Sicht der his- torischen Forschung - Beispiele}

Die gängige Meinung vom Wandel, dem das Messer in seinen Funktionsspektren vom frühen Mittelalter bis in die Neuzeit unterworfen war, unterteilt die Entwicklung grob in das frühgeschichtliche Messer mit Waffencharakter, das mittelalterliche Mehrzweckmesser und das frühneuzeitliche Tafelmesser ${ }^{740}$. Differenzierungen betreffen nahezu jede Epoche, so daß sich ein zum Teil widersprüchliches Bild ergibt:

Das vorgeschichtliche Messer als persönlich am Gürtel getragenes Universalwerkzeug wird zum Teil als dem auch "zur Speise gebrauchten kurzen Schwert" verwandt oder diesem nachfolgend beschrieben, die Begründung erfolgt in der Regel etymologisch ${ }^{741}$. Die Übernahme derartiger Überlegungen in mittelalterliche Lebensverhältnisse führt zu unterschiedlichen Ergebnissen. So steht auf der einen Seite die Annahme, das vorgeschichtliche Universalmesser sei im Mittelalter an der Tafel nicht benutzt worden, man habe mit den Händen gegessen, und die zwei oder drei Messer am Tisch seien von allen benutzte Tranchiermesser gewesen; erst allmählich habe wieder jeder sein Messer selber mitgebracht ${ }^{742}$. An anderer Stelle wird das persönliche Mehrzweckmesser als das Speisemesser des Mittelalters angesehen, welches erst im 15. Jahrhundert vom regelrechten Tafelmesser abgelöst werde $^{743}$, andere begrenzen das mittelalterliche Mehrzweckmesser am Tisch auf die unteren Bevölkerungsschichten und sehen unter anderem in den aus Bildquellen bekannten Sonderformen IVe und IVj (zum Teil kombiniert) die Tafelmesser der mittelalterlichen höfischen Tafel ${ }^{744}$.

Zwei der beliebtesten Themen, die das Messer des ausgehenden Mittelalters und der frühen Neuzeit betreffen, sind die ritualisierte Bedienung von Fürsten und Königen am Tisch und die aus schriftlichen Quellen bekannten Verhaltensmaßregeln an der Tafel.

Das bekannte Buch von Schiedlausky über mittelalterliche Tischsitten ${ }^{745}$ betrifft vor allem nachmittelalterliche Zustände. Die im 13. Jahrhundert auftauchenden

\footnotetext{
740 Hundt 1953, S. 109-110

Wühr 1961, S. 6

Mat og Drikke i middelalderen, Bryggens Museum (Beiheft) 1980, S. 26. Helferich-Dörner 1959,

S. $12 \mathrm{ff}$.

Wühr 1961, S. 6

Helferich-Dörner 1959, S. 12

742 Wühr 1961, S. 22, 24

743 Mat og Drikke 1980, S. 26

744 Helferich-Dörner 1959, S. 24, 25

Forbes 1975, S. 17 ff.

745 Schiedlausky 1956
} 
Tischzuchten ${ }^{746}$, die seit dem ausgehenden 15. Jahrhundert in gedruckter Form weitere Verbreitung finden ${ }^{747}$, beschreiben nicht die Zustände, die zu dieser Zeit an den Höfen und im höheren Bürgertum herrschten, sondern eher das Gegenteil. Sie sind keine Chroniken, sondern Erziehungsmittel in einer Zeit, die Elias als Epoche beginnender Affektkontrollen und vorrückender Scham- und Peinlichkeitsschwellen beschreibt $^{748}$. Elias selbst sagt von seinen zitierten Beispielen (unter anderen die Tisch- und Hofzucht des Tannhäuser aus dem 13. Jahrhundert), sie "repräsentieren ziemlich rein das Verhalten der jeweiligen Oberschicht" ${ }^{749}$. Quer durch die Vorschriften des Mittelalters wiederholen sich die gleichen Gebote, bis Erasmus von Rotterdam 1530 definitiv von der Beherrschung der Affekte spricht ${ }^{750}$, ein wohl deutliches Zeichen für die tatsächlichen "mittelalterlichen Umgangsformen"751. Schiedlausky zitiert Einhard, der den "würdevollen Ablauf" der Mahlzeiten Karls des Großen beschreibt ${ }^{752}$. Ob auf den zugigen und feuchten früh- und hochmittelalterlichen Burgen selbst der adeligen Führungsschicht in erwiesenermaßen spartanischen Lebensverhältnissen die "Zuocht und Maze"753 oder das Ritual der Bedienung des jeweils Ranghöchsten durch die ihm direkt Untergebenen auch so stattgefunden haben, muß dahingestellt bleiben. Die Ausstattung der Tafel des Adels ist selbst im 16. Jahrhundert zum Teil noch äußerst schlicht ${ }^{754}$. Auch die zahlreichen Tischzuchten späterer Jahrhunderte, vor allem des 15. bis 16. Jahrhunderts, müssen mit ihren Vorschriften zum Gebrauch des in dieser Zeit sicher als Teil des Tafelbestecks zu bezeichnenden Messers ${ }^{755}$ nicht notwendigerweise aufgrund des verstärkten Auftretens auch Rückschlüsse auf immer zivilisierteres Verhalten und eingeschränkteren Gebrauch des Messers zulassen; als ein Beispiel zentraldirigistischer Maßnahmen zur Befriedung menschlicher Verhaltensweisen sind sie sicherlich nicht völlig erfolglos gewesen. Darauf weist auch die anhand der hier bearbeiteten Messer sichtbar gewordene Einengung des funktionalen Rahmens und der Rückgang der Klingenlängen seit dem 14. bis 15. Jahrhundert hin. Der Grad ihres Erfolgs - und damit ein tatsächlicher Prozeß der funktionalen Einschränkung des Messergebrauchs - ist aus einer Quellengattung allein nicht festzustellen.

So ist erst seit dem 15. Jahrhundert damit zu rechnen, daß an den mitteleuropäischen Höfen unter dem Eindruck der burgundischen Kultur der Ausbildung und Pflege der "Kunst des Tranchierens" mit verschiedenen Messern

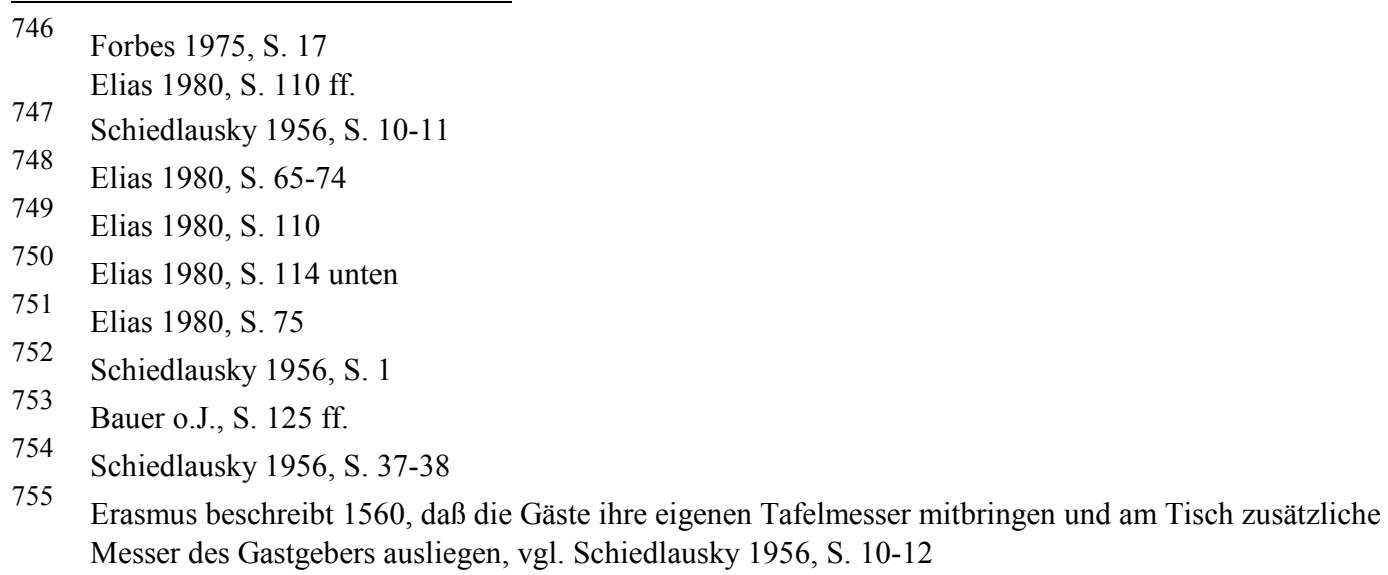


ein breiter Raum gewidmet war ${ }^{756}$. Die aus dem Mittelalter nur aus allerhöchsten Anlässen, dann aber mit großem Aufwand betriebene Form des Servierens, Vorschneidens und Vorkostens mit Spezialmessern und anderem Gerät, die neben dem geschilderten Ablauf der Mahlzeiten Karls des Großen und in einer Beschreibung eines Gastmahls des französischen Königs Ludwig IX. $1227^{757}$ auch in Bildquellen erscheint (zum Beispiel das Festmahl des Erzbischofs Balduin in Trier $1308^{758}$, bei dem zu Pferd serviert wurde), wurde zu Beginn der Neuzeit sicherlich von einer breiten Schicht des Adels und des vermögenden Bürgertums getragen ${ }^{759}$. Darauf deuten auch die relativ zahlreich erhaltenen Tranchierbestecke und -messer des 15. bis 16. Jahrhunderts hin.

Aber auch in dieser Zeit betreffen die beschriebenen Zustände nur einen geringen Teil der Bevölkerung. Die Schilderung des Erasmus von Rotterdam, in städtischen Gasthöfen befinde sich nur das notwendigste an Geschirr, Messer habe der Gast selbst mitzuführen ${ }^{760}$, bezeugt das Vorhandensein von persönlich getragenen Messern noch im 16. Jahrhundert; und die diese Messer betreffenden noch zu erwähnenden Tabus und Verbote legen den Schluß nahe, daß es sich dabei nicht nur um zierliche Tafelmesser gehandelt hat.

Der Zeitraum des Aufkommens reiner Tafelmesser, oft unberechtigterweise gleichgesetzt mit dem Niedergang des Mehrzweckmessers, ist in der Literatur keineswegs einheitlich festgelegt. Haedeke setzt ihn an das Ende des 16. Jahrhunderts, er unterscheidet seit dieser Zeit zwischen "vornehmen Häusern", in denen jedem Gast ein Messer an der Tafel zur Verfügung gestellt wurde, und "einfachen Leuten", die ihr Messer selber mitbrachten ${ }^{761}$. Wühr sieht die Verbreitung des Tafelmessers bereits im 15. Jahrhundert, nach seiner Ansicht wurde es zusammen mit einem größeren Messer zum Zerlegen in einer Scheide getragen und von jedem Gast bei sich geführt ${ }^{762}$.

Die Form der Tafelmesser wird übereinstimmend beschrieben. Es handelt sich um schlanke, oft spitze Messer, die sich durch geringere Klingenlänge und feinere Griffgestaltung dokumentieren $^{763}$.

Genau diese Messer mit schlanken Klingen tauchen im bearbeiteten Fundgut aber schon im 14. Jahrhundert auf. Sie besitzen in der Regel seit dem ausgehenden

\footnotetext{
756 Schiedlausky 1956, S. 23-24, 26

Bauer o.J., S. 117-118

Helferich-Dörner 1959, S. 74 ff.

Haedeke 1981, S. 22

Helferich-Dörner 1959, S. 74

Schiedlausky 1956, Abb. 5, Anhang

Helferich-Dörner 1959, S. 76-77

Schiedlausky 1959, S. 40

Wühr 1961, S. 119

Schiedlausky 1956, S. 38

Haedeke 1981, S. 5

762 Wühr 1961, S. 32

763 Wühr 1961, S. 32

Haedeke 1981, S. 34
} 
14. Jahrhundert Griffzungenkonstruktionen, an ihnen zeigt sich der Rückgang der Klingenformen der Gruppe IIIe und das Aufkommen von vernieteten Zusatzbefestigungen aus Buntmetallapplikationen. Ihre Hauptverbreitungszeit ist das 15. bis 17 . Jahrhundert, im ausgehenden 16. und 17. Jahrhundert werden ihre Griffe wieder mehr und mehr durch Angelkonstruktionen mit oft verzierten Vollgriffen aus Buntund Edelmetallen gebildet ${ }^{764}$.

Ein ähnliches Ergebnis hat die Auswertung der in London ergrabenen Messerbestände erbracht ${ }^{765}$. Dort wurde festgestellt, daß sich vor 1350 die Masse der in Scheiden befindlichen Messer und insgesamt mehr als doppelt so viele Scheiden wie Messer fanden; seit der Mitte des 14. Jahrhunderts nahm die Anzahl der in Scheiden befindlichen Messer rapide ab, und im ausgehenden 14. Jahrhundert kommen auf elf Scheiden 173 Messer $^{766}$. Nach 1400 kommt auf 28 Messer nur noch eine Scheide. Vor 1300 wurden ausschließlich Angelmesser beobachtet, nach 1360 besaßen bereits über die Hälfte der geborgenen Messer Zungenkonstruktionen. Ebenfalls seit 1300 wurden schlankere Klingen und seit der Mitte des 14 . Jahrhunderts verstärkt Metallapplikationen festgestellt ${ }^{767}$. Die Ergebnisse dieser lokal angelegten Auswertung werden von den Bearbeitern dahingehend interpretiert, daß sich am Rückgang der Scheiden als Indikator für das persönlich mitgeführte Messer die Trennung von Mehrzweck- und Tafelmesser im 14. Jahrhundert festmachen lasse ${ }^{768}$. Da die Messer dort aufbewahrt worden seien, wo sie benutzt wurden, sei davon auszugehen, daß seit dem ausgehenden 14. Jahrhundert mit dem rapiden Rückgang der Scheiden im Fundgut die Gäste in der Regel ihr Messer nicht mehr selbst mit zum Essen gebracht hätten ${ }^{769}$.

Dieses Ergebnis ist zunächst auf die Lebensumstände in spätmittelalterlichen Städten zu begrenzen, denn auch im vom Verfasser bearbeiteten Fundgut betraf diese Entwicklung Funde aus Städten wie Dordrecht, Rotterdam und Amsterdam.

Für die Zeit vor dem 14. Jahrhundert nehmen Cowgill (und andere) an, daß die Gäste einer Mahlzeit ihr eigenes Messer mitbrachten; die auf mittelalterlichen Darstellungen zu beobachtende geringe Anzahl von Messern werten sie als Ausdruck oder Brauch des Vertrauens der Gäste, sich ein Messer zu teilen ${ }^{770}$. Diese persönlich geführten Messer stehen nach ihren Ausführungen als Mehrzweckmesser individuell bestimmter Funktionen vor der Trennung zwischen Messer zur Nahrungszubereitung/-aufnahme und spezialisierten Werkzeugmessern, die das Mehrzweckmesser im Verlauf des 14. Jahrhunderts überflüssig gemacht habe ${ }^{771}$.

\footnotetext{
764 Vgl. als Auswahl Niederlande, Kat. Nr. 33, 35, 41, 43, 44-49, 60, 63-65, 82, 88, 91

765 Cowgill u.a. 1987

766 Cowgill u.a. 1987, S. VIII

767 Cowgill u.a. 1987, S. VIII-IX

768 Cowgill u.a. 1987, S. 51

769 Cowgill u.a. 1987, S. 61

770 Cowgill u.a. 1987, S. 55

771 Cowgill u.a. 1987, S. 52
} 
Koltschin geht in seiner Analyse des Fundguts von Novgorod noch weiter. Er definiert griffunterständige Klingen in Unterscheidung zu gleichständigen Klingen als Messer der Holzbearbeitung und unterscheidet bereits für das 12. bis 13. Jahrhundert Haushalts- und Küchenmesser, Tafelmesser und Messer holzverarbeitender Spezialhandwerker $^{772}$. Für den Zeitraum bis zum 14. Jahrhundert fügt er noch Schuster- und Chirurgenmesser an ${ }^{773}$.

Derartige Zuweisungen sind nach wie vor stark hypothetisch. Es ist nicht bekannt, ob sich selbst in Städten wie London, Amsterdam, Rotterdam und Dordrecht das Mehrzweckmesser neben dem Aufkommen des Tafelmessers im 14. Jahrhundert weiter gehalten hat. Die Auswertungen dieser Untersuchung haben immer wieder gezeigt, daß die Proportionalitätsverschiebungen von breiten zu schlanken Klingen, der Rückgang der Klingenlängen und das Auslaufen von Formen der Leitform IIIe im ausgehenden 14. und 15. Jahrhundert als Indizien für das Aufkommen des frühneuzeitlichen Tafelmessers vor allem den Teil des bearbeiteten Fundguts betrifft, der bei Stadtkerngrabungen geborgen wurde.

Die Formen der oben beschriebenen Tafelmesser mit frühen Zungenkonstruktionen wurden allerdings auch auf Burgen des 13. bis 14. Jahrhunderts (beispielsweise Aranaes, Gommerstedt) gefunden, sie fehlen auf Burgen des 13. Jahrhunderts (zum Beispiel der Isenburg, Raffenburg). Es erscheint durchaus möglich, daß die in den Städten beobachteten Tafelmesser des ausgehenden 14. und 15. Jahrhunderts auch auf zeitgleichen Burgen gefunden werden, so wie es für das 16. und 17. Jahrhundert nachweisbar ist (zum Beispiel Kakesbeck, Barntrup).

Tafelmesser mit schlanken und breiter proportionierten Klingen, versehen mit zum Teil aufwendigen Angelkonstruktionen, sind jedoch auch aus dem 12. und 13. Jahrhundert bekannt, ebenso wie in Lederscheiden geführte Messersätze mit und ohne Waffen aus dem 15. und 16. Jahrhundert, die in ihren Proportionen und Formen den in London ausgegrabenen Tafelmessern sehr nahe kommen ${ }^{774}$ (Abb. 218).

An dieser Stelle sei noch einmal auf die plättchenverzierten Messer verwiesen. Knorr hat vor allem die Exemplare des 14. bis 15. Jahrhunderts als persönlich geführte Messer von Frauen und Männern aus bürgerlichem und adeligem Umfeld gekennzeichnet, als Kleidungsbestandteile und - was die Messer mit längeren Klingen betrifft - als Dolchmesser mit Doppelfunktion, zum Teil mit dem Charakter eines Standeszeichens ${ }^{775}$. Die von ihm zitierten schriftlichen Quellen, die vor allem die soziale Differenzierung bezüglich des Tragens von Dolchmessern und Dolchen in der Stadt betreffen, weisen bis in das ausgehende 15. Jahrhundert ${ }^{776}$. Dort wird im 15. Jahrhundert im Zusammenhang mit dem Pook, einem Stechmesser, welches

\footnotetext{
772 Arzichowskij, Koltschin 1959, S. 54-56

773 Arzichowski, Koltschin 1959, Abb. 43, S. 55

774 Vgl. Katalogteil BRD, Kat. Nr. 73, 129; Niederlande, Kat. Nr. 9; vgl. auch Wühr 1961, S. 31

775 Knorr 1971, S. 129-132

776 Knorr 1971, S. 132-135
} 
Knorr als "kleines spitzes Gebrauchsmesser in Art eines Fahrtenmessers" charakterisiert, "das locker in der Tasche des Volkes und der Junker saß..."777, vom "spisenpook"778 gesprochen, allem Anschein nach also von einem an der Seite getragenen Messer, das auch zum Essen benutzt wurde.

Es muß damit gerechnet werden, daß trotz nachweisbaren Auftretens von Tafelmessern, die möglicherweise schon im Verlauf des 14. Jahrhunderts im Haus verwahrt und nur an der Tafel benutzt wurden, das persönlich in einer Scheide bei sich geführte Messer entweder in Form eines Bestecks mit und ohne Waffe oder als Mehrzweckmesser bis in das 15. Jahrhundert anzutreffen ist, bevor es zur endgültigen Trennung von Messern und verschiedenartigsten Waffen kam. In den Jagdbestecksätzen des Adels, die seit der zweiten Hälfte des 16. Jahrhunderts erhalten sind, hat es sich bis in das 17. und 18. Jahrhundert gehalten ${ }^{779}$.

Der Begriff "Mehrzweckmesser" ist ein fiktiver Arbeitsbegriff, denn konkrete Anhaltspunkte für den funktionalen Rahmen der Messer, die weder reine Tafelmesser noch spezielle Werkzeugmesser oder Kurzwehren waren, liegen noch kaum vor. Möglicherweise richtete sich die Funktion des mittelalterlichen an der Seite getragenen Mehrzweckmessers nach der sozialen Stellung und den naturräumlichen Voraussetzungen, nach Wohnort, Geschlecht und Alter der Trägerin oder des Trägers. Daß sich diese Unterschiede nur selten - wie im Fall der kleineren, zum Teil zierlichen Ausprägung der Form Va - in Form und regionaler Verbreitung ausdrückt, hat die Untersuchung über die Verbreitung der einzelnen Klingenformen gezeigt. Wie stark aber das Führen des eigenen Messers in der Bevölkerung verankert war, zeigen nicht nur die zahlreichen Tabus und Verbote vor allem des 14. und 15. Jahrhunderts, sondern auch Beispiele von Kinderspielzeug, wie das Beispiel aus Danzig für das ausgehende 11. Jahrhundert zeigt (Abb. 217).

\footnotetext{
777 Knorr 1971, S. 138

778 Knorr 1971, S. 135

779 Haedeke 1981, S. 28

Vgl. in diesem Zusammenhang die Kontinuität des norwegischen "Tollekniv", einem persönlichen Mehrzweckmesser, welches Christensen in Gegensatz zur englischen und kontinentalen Entwicklung stellt, in der das Mehrzweckmesser nach ca. $1000 \mathrm{n}$. Chr. durch Spezialmesser für Küche, Tafel und Werkstatt ersetzt worden sei und nur im Klappmesser weitergelebt habe.

In Norwegen ist die Benutzung des "Tollekniv" durchläufig bis heute, mit standardisierten Klingenmaßen und Funktionen wie dem Zerlegen, Aufschneiden und Filtrieren von Elchen und Fischen, dem Schnitzen von Holzgegenständen, dem Essen u.s.w. Für das Mittelalter wird eine lokale Klingenproduktion angenommen, die zusätzliche Griffbefestigung beginnt möglicherweise in der Renaissance. Es wurden nur Angelkonstruktionen beobachtet, die Benutzung des "Tollekniv" reicht von ca. $200 \mathrm{n}$. Chr. bis heute. (Die Information erhielt der Autor von A.E. Christensen vom Museum Oldsaksamlingen der Universität Oslo. Vgl. Christensen 1983 sowie Breivik o.J.)
} 


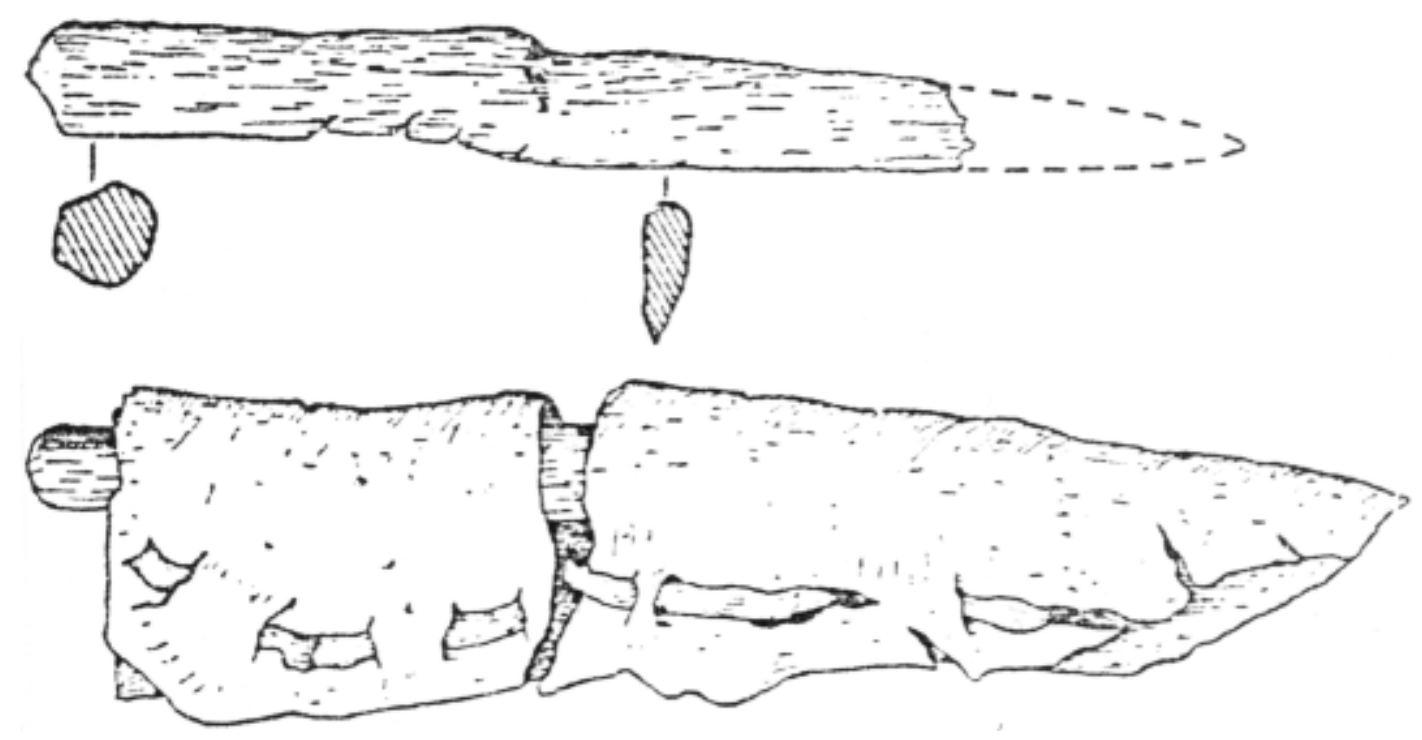

Abb. 217

Hölzernes Spielzeugmesser in Lederscheide Danzig, um 1065-1080

Die bereits mehrfach angesprochene Grauzone zwischen dem Messer als Gerät und Werkzeug und dem Stechmesser, einer Waffe, ist ebenfalls aus archäologischem Quellenmaterial allein nicht einzugrenzen. Abgesehen davon, daß jedes beliebige Messer gegebenenfalls zu einer Waffe werden kann, fällt es vor allem dann schwer, diese Zone zu umreißen, wenn die Griffapplikationen (Vollgriff oder Hilze, Abschluß- oder Heftelemente) fehlen. In diesem Fall können die Dicke des Rückens (Nackens), die Länge (beispielsweise ab 18 bis $20 \mathrm{~cm}$ ) und die Form der Klinge (gleichseitiges, spitzes Dreieck) Aufschluß über den Waffencharakter des Objekts geben. Knorr und Schoknecht haben sich mit der Frage eingehend beschäftigt und die Verbindungen zwischen langen Messern, Dolchmessern und Dolchen (Nierendolchen) beschrieben ${ }^{781}$. 


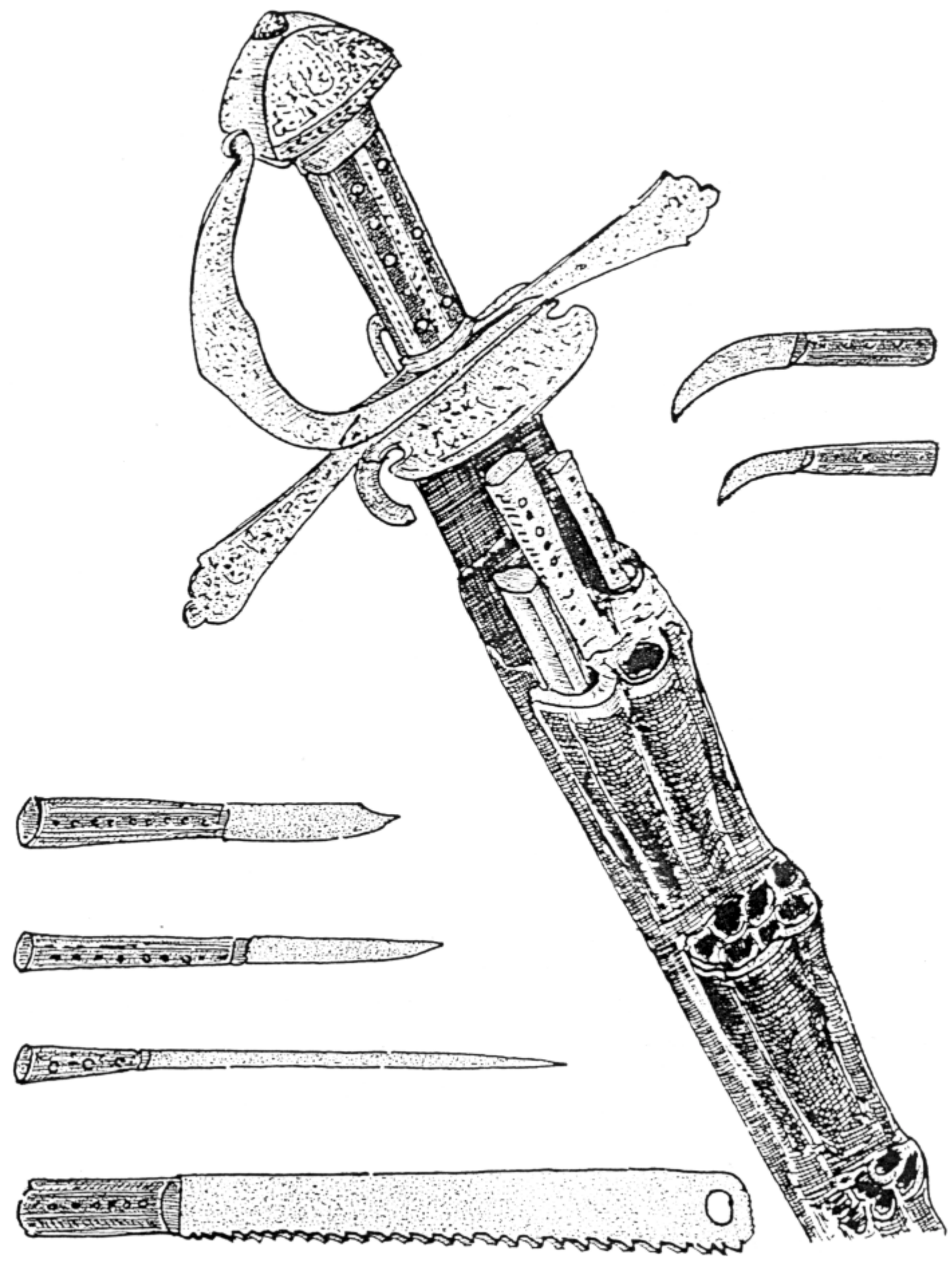

Abb. 218

Jagdbesteck, Deutschland, Mitte 16. Jahrhundert ${ }^{782}$

Waffe mit 22 Besteckteilen

782 Zeichnung nach: Deutsche Geschichte 500-1789, Führer des Museums für deutsche Geschichte Berlin, Berlin o.J., Abb. S. 54 
Die Klingenform allein kann aber ebensowenig wie die Länge der Klinge Werte für die Trennung zwischen Messer und Waffe liefern. So gibt es bei den Klingenlängen zwischen kurzen Nierendolchen und in dieser Untersuchung bearbeiteten längeren Messern ebenfalls eine Zone von mindestens $4 \mathrm{~cm}$ (etwa zwischen 16 und $20 \mathrm{~cm}$ ), die beide Funktionen umfaßt, auch wenn die üblichen mittleren Werte weit darüber bzw. darunter liegen (bei den Dolchen 24,6 und 35,7 cm, bei dem zeitlich weiter gefaßten Bestand von Messern dieser Untersuchung 9,56 cm $)^{783}$. Der Durchschnitt der Klingenlängen kleiner Nierendolche fällt mit $18,4 \mathrm{~cm}$ jedoch genau in diese Zone ${ }^{784}$.

Knorr nennt neben der gleichseitigen Dolchklinge mit beidseitiger Schneide als weitere Beispiele für Stichwaffen die Pook-Klinge mit geradem Rücken (gleichseitige, einseitig schneidende Klinge) und die Dolchmesserklinge (gerader oder gebogener Rücken, gebogene Schneide). Bei den beiden letzteren ist die Spitze im Schnitt halbkreisförmig verdickt, die Dolchmesserklinge mißt nur eine Länge von $12,7 \mathrm{~cm}^{785}$.

Bei Klingen mit erhaltenen Griffen wird die Unterscheidung zwischen Messer und Waffe in der Regel davon abhängig gemacht, ob am Heft ein Pariersockel oder eine Parierstange vorliegt. Bei den von Knorr und Schoknecht vorgelegten Beispielen handelt es sich um Nierendolche, die entweder am Heft einen zumeist eisernen Parierbalken mit über den Klingenansatz gezogenen Backen aufweisen, auf dem ein Pariersockel aus Plättchenpaketen oder die nierenförmige Verdickung des Vollgriffs (der Hilze) aufsitzt, oder Nierendolche, bei denen die Stoßkante nur durch einen am Heft verdickten Vollgriff gebildet wird ${ }^{786}$.

Fehlen diese Griffteile, bleibt ein Restbestand von einseitig geschärften Messerklingen übrig, der Längenmaße von 12 bis $20 \mathrm{~cm}$ umfassen kann, in der Regel zwischen 16 und $20 \mathrm{~cm}$ liegt und stabile, meist schlanke und spitze Klingen mit relativ starken Rücken (Nacken) aufweist. Die Formen der Klingen umfassen die Leitformen IIIb und IIIe sowie die Einzelform Ib0.1. Nicht enthalten in dieser Gruppe sind Klingen, die sich durch ihre Gestaltung (zum Beispiel im Schnitt dreikantige Klingen oder Klingenpartien) auch ohne Pariervorrichtungen am Heft als Waffen zu erkennen geben ${ }^{787}$.

Dieser funktional derzeit kaum faßbare Bestand enthält wahrscheinlich einen großen Teil der "langen Messer", "Stekemessen" etc., die im Zuge der von Elias beschriebenen Pazifizierung immer breiterer Bevölkerungsschichten auch unter die zahlreichen Waffenverbote fallen ${ }^{788}$.

\footnotetext{
783 Schoknecht 1980, S. 225, 227 sowie Kapitel 6, Abschnitt 2.2. (Klingenlängen)

784 Schoknecht 1980, S. 227

785 Knorr 1971, S. 138, Abb. 11, S. 137

786 Knorr 1971, Abb. 8, S. 136; Abb. 9-10, S. 137

Schoknecht 1980, Abb.1 S. 212; Abb. 2, S. 215; Abb. 4, S. 219

787

vgl. Katalogteil ehemalige DDR, Kat. Nr. 130

Elias 1980, Bd. I, S. 263-283
} 
Diese umfassen seit dem 13. Jahrhundert nicht nur regelrechte Waffen (um 1150 wird den Bauern in Bayern und Österreich bereits das Tragen von Schwertern verboten $)^{789}$, sondern auch bereits Dolch- und Stechmesser ${ }^{790}$.

Dabei wurde das heimliche Tragen derartiger Messer vor allem in den Städten ganz verboten, das auf bestimmte Bevölkerungsschichten beschränkte offene Tragen durch Begrenzung der Messerlängen eingeschränkt (Mühlhausen, erste Hälfte des 14. Jahrhunderts) ${ }^{791}$.

Differenzierungen betrafen zum Beispiel Bauern des Stadtgerichtsbezirks, die überhaupt keine Messer oder Waffen innerhalb der Mauern führen durften, und Ratsmitglieder, die vor allem auf Ratssitzungen das Schwert und Messer tragen mußten $^{792}$. Die Verbote richteten sich in der Regel an niedere oder lohnabhängige Schichten; im 14. und 15. Jahrhundert gehört das Stechmesser, zu dem Knorr auch die Nierendolche mittlerer Größe zählt, zur Kleidung des städtischen Bürgers; daneben erwähnt er noch Fremde wie Seeleute, Kaufleute und deren Bedienstete, die ebenfalls Messer und Waffen trugen ${ }^{793}$.

Die schweren Strafen, die vor allem dem Mißbrauch dieser zwischen Mehrzweckutensil und Waffe stehenden "langen Messer" betrafen, werfen ein Licht auf ihre Verbreitung trotz der Verbote ${ }^{794}$. Die Hintergründe von Familienfehden, Raubüberfällen und spontan entstehenden Wirtshausstechereien innerhalb der Stadtmauern korrespondieren durchaus mit den zum Teil unsicheren Zuständen außerhalb der Städte, so daß die aus Schriftquellen bekannten Verbote eher im negativen Sinn Rückschlüsse zulassen und wohl erst in kleinen Schritten und langen Zeiträumen wirksam wurden ${ }^{795}$ (Abb. 219).

\footnotetext{
$789 \quad$ Epperlein 1975, S. 89

790 Knorr 1971, S. 132

791 Knorr 1971, S. 133 und Anmerkung 39

792 Knorr 1971, S. 133

793 Knorr 1971, S. 133

794 Handbook of Bryggens Museum (to the cultural history of the Middle Ages), Bergen 1978, S. 84

795 Elias 1980, Bd. I, S. 266, 268-271, 273-276; Bd. II, S. 407-408

zu Einschränkungen in Wirtshäusern vgl. K. Hoyer 1910, dort wird auf die Schwierigkeiten bei der Durchsetzung des Verbots von Waffen in Wirtshäusern verwiesen, S. 59-63
} 


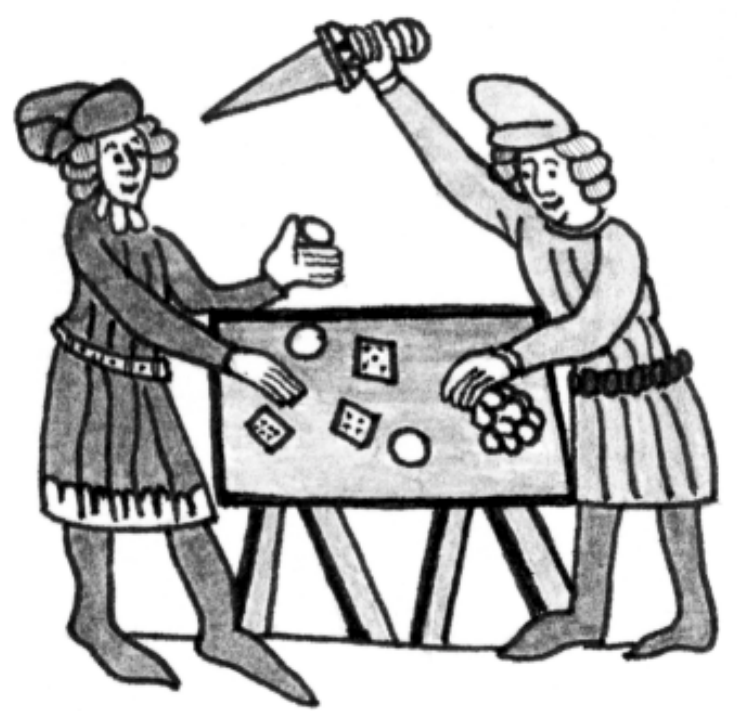

Abb. 219

Stecherei beim Würfelspiel ${ }^{796}$

In der offiziellen Bewaffnung der Bürgerschaft spielen Messer und Dolch nur eine untergeordnete Rolle: in einer Liste des Stadtarchivs Deventer werden in 23 Eintragungen von 1455 bis 1495 Angriffs- und Verteidigungswaffen im Besitz städtischer Bürger aufgezählt. Neben durchläufig erwähnten Utensilien wie Brustharnisch, Helm und Kettenhemd tauchen außer acht Schwertern nur fünf Messer und Dolche auf, davon zwei im Besitz eines Kaufmanns ${ }^{797}$. Daraus kann nicht abgeleitet werden, daß diese Waffen in der zweiten Hälfte des 15. Jahrhunderts zahlenmäßig geringer vertreten gewesen seien. Ihr Stellenwert ist wahrscheinlich eher als der eines Kleidungsbestandteils oder Standeszeichens anzusehen, die für die Schicht der waffenbesitzenden Bürger u.a. mit dem Nierendolch in Verbindung gebracht wird $^{798}$.

\subsubsection{Beispiele von Messern in Bildquellen}

Zum Schluß sei an einigen Beispielen der Rahmen von Speisemesserformen umrissen, die sich als solche durch Bildquellen belegen lassen.

Auf zehn Tafeln werden aus bildlichen Darstellungen vergrößerte und umgezeichnete Messer in etwa zeitgleichen und in der Form ähnlichen Fundstücken vom 11. bis 17. Jahrhundert gegenübergestellt. Alle Messer, die aus Bildquellen umgezeichnet worden sind, entstammen Darstellungen aus dem unmittelbaren Bereich der Tafel und wurden hier der Übersicht halber in Rahmen gesetzt.

\footnotetext{
796 Kjersgaard 1978, Abb. 71, S. 95 
Die dem Teppich von Bayeux entnommenen zwei Messer von der mit sechs Gästen besetzten Tafel Wilhelms des Eroberers kurz vor seiner Abfahrt nach England zeigen eine stark hochgebogene Klinge sowie eine etwas stabilere und kürzere Klinge mit einem Einsprung im vorderen Klingenbereich (Abb. 220a). Auf der Tafel liegen Fisch und Brot.

Aus der ersten Hälfte des 11. Jahrhunderts stammen drei weitere zum Essen benutzte Messer, von denen zwei neben dem Einsprung im vorderen Rückenabschnitt einen stumpfen vorderen Abschluß mit leicht hochgezogener Spitze aufweisen (Abb. 221b). Auch hier besteht das Essen unter anderem aus Fisch und Brot.

Die drei Exemplare aus Lund aus der gleichen Zeit sowie das Messer aus Oslo aus dem 12. Jahrhundert besitzen modifizierte, in den Grundformen ähnliche Klingen, mit Klingenlängen von $10,4 \mathrm{~cm}, 12,5 \mathrm{~cm}, 11,2 \mathrm{~cm}$ und $8 \mathrm{~cm}$ (Abb. 220c bis 220f).

Im 12. und 13. Jahrhundert tauchen Messer mit hoch- oder auf die Schneideebene gezogenen Spitzen auf (Abb. 221a, 221b), die ebenfalls zur Nahrungsaufnahme dienen. Im Beispiel aus dem Hortus Deliciarum der Herrad von Landsberg (um 1170) wird mit ihnen Fisch verzehrt (Abb. 221a, unten), während die beiden Messer mit geradem Rücken bzw. mit abgeknicktem Rücken (Abb. 221a, oben) auf dem benachbarten Anrichtetisch mit Brot und Wildschwein liegen. Es muß offen bleiben, ob im folgenden Gang mit dem Fleisch auch das Besteck wechselte.

Die Form der umgebogenen, nach unten weisenden Spitze ist im Fundgut nicht sicher vertreten. Das einzige mögliche Exemplar, gefunden auf der Burg Wartenberg, kann auch eine ausgebrochene Klinge der Form IVa sein (Abb. 221e).

Die besonders auffälligen Fischmesser aus dem Speyerer Evangeliar (12. Jahrhundert) zeigen Klingen mit stark geschwungenen Rücken, Einkerbungen in der Schneide und stumpfen vorderen Abschlüssen mit spitz ausgezogenen Ecken (Abb. 221b). Direkte Beispiele für diese Formen sind nicht aufgetreten, entfernt ähnliche Klingen stammen aus dem 13. Jahrhundert (Abb. 221f, 221g).

Andere Sonderformen lassen sich dagegen im Fundgut besser dokumentieren. Die Form IVa, abgebildet auf einer spätromanischen Abendmahlsdarstellung aus Dänemark (Abb. 222a), findet ihre Entsprechung vor allem in Funden aus Burgen des 13. und 13. bis 14. Jahrhunderts, und zwar mit Angel- und Zungenkonstruktionen (Abb. 222c bis 222e).

Auch die drei Messer des 13. Jahrhunderts mit mehr und weniger stark ausgeprägten Einsprüngen im Rücken und, in einem Fall, hochgezogener Spitze, lassen sich mit ergrabenen Beispielen des 12. bis 13. und des 14. Jahrhunderts belegen (Abb. 222b, 222f bis 222h).

$\mathrm{Zu}$ den Messern, die auf der großen Tafel des Erzbischofs Balduin von Trier (1308) liegen, finden sich identische und ähnliche Formen vom 12. bis 14. Jahrhundert aus Burgen und mittelalterlichen Stadtkernen, die auf eine möglicherweise starke Differenzierung an der Tafel führender Bevölkerungsschichten hinweisen (Abb. 223). An der Tafel des Erzbischofs wurde zu Pferde serviert, man aß unter anderem Geflügelgerichte. Für die zehn abgebildeten Gäste liegen acht Messer bereit. 
Angesichts der speziellen Formen, die zumindest auf den Tischen des hohen Adels und der hohen Geistlichkeit vom 11. bis 14. Jahrhundert vorhanden sind und sich zum Teil auch im Fundgut widerspiegeln, läßt sich die Vermutung äußern, daß die Differenzierung der Speisefolge und der aus den späteren Tischzuchten bekannten Tafelsitten mit einer bereits ebenso differenzierten Existenz von Spezialmessern am Tisch korrespondiert. Messer wie die aus dem Speyerer Evangeliar sind sicherlich kaum ständig mitgeführte Mehrzweckmesser, sondern eher als vielleicht bei besonderen Anlässen und möglicherweise ausschließlich zu Fischgerichten benutzte Spezialtafelmesser anzusehen.

Auch die drei Messer aus einer Kölner Abendmahlszene um 1360 lassen sich im Fundgut mit Beispielen von Burgen belegen, ein weiteres Mal mit verschiedenen Griffkonstruktionen (Abb. 224a, 224d bis 224f). Die in Funktion dargestellten Tranchierbestecke aus dem ausgehenden 14. und beginnenden 15. Jahrhundert sind kaum direkt nachweisbar, da sich die oft kostbaren Stücke nur selten im Fundgut finden (Abb. 224b, 225a). Ein in der bäuerlichen Siedlung Store Valby gefundenes, als Geschenk eines Klosters angesehenes Vorschneidemesser vermittelt einen Eindruck von der Art der frühen Großmesser am Tisch, zeigt aber auch deutlich, wie fließend die soziale Abgrenzung der Benutzer sein kann (Abb. 224i).

Eine Auswahl skandinavischer Messerformen aus Abendmahlszenen des 15. Jahrhunderts zeigt neben dem bekannten Einsprung in der vorderen Klingenpartie auch Klingen mit hochgezogenen Spitzen, die zwei möglicherweise etwas älteren Funden von der Burg Naesholm und aus der Siedlung Store Valby entsprechen (Abb. 225b, 225d, 225e).

Auf einem Basler Bildteppich des 15. Jahrhunderts, auf dem unter anderem eine Liebesgartenszene dargestellt wird, schneidet ein Mann mit einem zierlichen Messer eine Weintraube von der Ranke, am Gürtel trägt er ein kurzes Schwert, dessen Scheide zwei weitere Messer enthält. Ein viertes Messer liegt auf dem Tisch vor einem Paar (Abb. 226b, 226c). Die Zuordnung des Griffzungenmessers aus Lübeck (Abb. 226a) zu dieser Szene ist - wie auch in den vorangegangenen Fällen - als Vorschlag zu verstehen. Geht man jedoch davon aus, daß der funktionale Rahmen des hier zum Abschneiden einer Traube benutzten Messers sicherlich nicht auf diesen Vorgang beschränkt war, daß der Mann dasselbe Messer auch zum Zerschneiden von Obst, zum Essen an der Tafel wie auch im Freien hat verwenden können, dann liegt mit derartigen Messern ein Bestand ähnlich dem des sicherlich als persönlicher Besitz anzusehenden Lübecker Exemplars vor, der noch in der zweiten Hälfte des 15. Jahrhunderts zur individuellen Benutzung des Besitzers in Scheiden an der Seite getragen wurde. Dieser Bestand ähnelt den Londoner Beispielen in Formen und Proportionen sehr, die dort offensichtlich als im Haus verwahrte Tafelmesser anzusprechen sind. Im Fall des Basler Bildteppichs werden die Messer zusammen mit einem Kurzschwert getragen, ein Beispiel aus Celle zeigt ein Nierendolchbesteck mit Messer, ähnlich dem Dolchbesteck eines niederländi- 
schen Schiffswracks bei Lelystad ${ }^{799}$. Das in einer Scheide getragene Messerbesteck aus der Burg Kakesbeck zeigt, daß das Tragen von reinen Schneidemessern, die man - isoliert aufgefunden - jederzeit als Tafelmesser bezeichnen würde, bis weit in das 15. Jahrhundert, vermutlich noch bis in das 16. Jahrhundert hinein üblich war $^{800}$. Diese Messer unterscheiden sich von den mittelalterlichen Mehrzweckmessern dadurch, daß sie weniger stabil, in ihrem funktionalen Rahmen also tatsächlich eingeschränkt waren und offensichtlich nicht mehr allein, sondern als Besteck mit oder ohne Waffen getragen wurden. Trug im Mittelalter in der Regel jede Frau und jeder Mann ein derartiges Mehrzweckutensil bei sich, das in den beschriebenen Konstellationen auch als Waffe verwendet werden konnte ${ }^{801}$, so werden sie im 15. und beginnenden 16. Jahrhundert neben oder zusammen mit Waffen von Mitgliedern des Adels oder privilegierter Bürgerschichten getragen. Nicht nur die sozialen Verhältnisse haben sich geändert, auch im Nutzungsrahmen ist ein Wandel festzustellen. War das Mehrzweckmesser des Mittelalters vermutlich eines der wichtigsten Werkzeuge, vielfach genutzt ${ }^{802}$, ein Gerät, das seinen Sinn und auch seine gesellschaftliche Wertigkeit in der Nutzbarkeit und zum Teil auch in der Wehrhaftigkeit im Alltag erfüllte, so scheint das Messer an Schwert oder Dolch oder als reines Messerbesteck eher ein Attribut des Standes ${ }^{803}$ und ein Utensil für zum Teil modische, zum anderen rein private Verrichtungen zu sein.

Es hat sich gezeigt, daß eine chronologische Abfolge vom persönlichen Mehrzweckmesser zum speziellen Tafelmesser zu hinterfragen sein wird, da im Mittelalter offensichtlich an der Seite getragene Messer des vielfältigen, vom Träger abhängigen alltäglichen Gebrauchs neben spezielleren Tafelmessern (wie Fischmessern) gleichzeitig auftreten. Hier läuft die Trennung mit hoher Wahrscheinlichkeit parallel zu den gesellschaftlichen Trennungslinien.

Vom ausgehenden Mittelalter bis weit in die frühe Neuzeit sind wiederum reine Tafelmesser neben an der Seite getragenen Messern und Messerbestecken zu beobachten. Diese scheinen ihre Trägerschaft vertauscht zu haben, die Trennungslinie ist noch nicht genau zu umreißen. Die Messer in den Bestecken haben ihre auch auf Arbeit und eine gewisse Wehrhaftigkeit ausgerichtete Stabilität verloren, ohne daß sie deshalb als reine Tafelmesser anzusprechen sind.

In weiteren Bildquellen werden Messerformen des 15. Jahrhunderts gezeigt, die sich in der Regel auch im bearbeiteten Fundgut nachweisen lassen (Abb. 226d bis 226f, $227 \mathrm{f}$ bis $227 \mathrm{j}$ ). Es fällt allerdings auf, daß die Formen der Leitform IIIe in den

\footnotetext{
799 Vgl. Katalogteil Niederlande, Kat. Nr. 8a; BRD, Kat. Nr. 73

Diese generalisierte, in der Literatur oft zitierte Auffassung berücksichtigt nicht mögliche soziale Differenzierungen, die aber noch nicht näher zu benennen sind. gerlich-ständischen Selbstbewußtseins verkörpert und weniger als Waffe gedacht ist" (Knorr 1971, S. $133,135)$
} 
Bildquellen bis in das 16. Jahrhundert hinein erscheinen (Abb. 227c, 227d, 227f, $229 \mathrm{~g}$ ), während sie im bearbeiteten Fundgut bereits im 15. Jahrhundert fast vollständig zurückgehen (Abb. 227g).

Die im Fundgut vom 13. bis 15. Jahrhundert in fünf sicheren Fällen nachgewiesenen Formen IVf und IVh (Abb. 228b-f), die ähnlich wie die Klingen mit heraufoder herabgebogenen Spitzen (Abb. 221a, 221b, 222b) möglicherweise als Tafelmesser anzusehen sind, finden in den Bildquellen kaum Parallelen. Ein entfernt ähnliches Exemplar des ausgehenden 15. Jahrhunderts weist in die gleiche Richtung, es ist jedoch nicht in direktem Funktionszusammenhang dargestellt, sondern liegt neben einem Gericht aus Brot und Fleisch auf einem Tischchen in einer Darstellung der Geburt Mariä um 1470 (Abb. 228a).

Messerdarstellungen des 16. bis 17, Jahrhunderts (Abb. 229) entsprechen in ihren Klingenformen, Griffkonstruktionen und der Verwendung von Heft- und Griffendapplikationen oder der Griffgestaltung bis in die Einzelheiten dem vorliegenden Fundmaterial. Daß Formen wie die von zwei Messern der tafelnden Amsterdamer Schützengilde von 1533 im Fundgut nicht repräsentiert sind, liegt neben dem enormen Gestaltungsschub in der Sachkultur der Renaissance wahrscheinlich auch in dem Wert begründet, den derartige Messer darstellten (Abb. 229g, oben). 

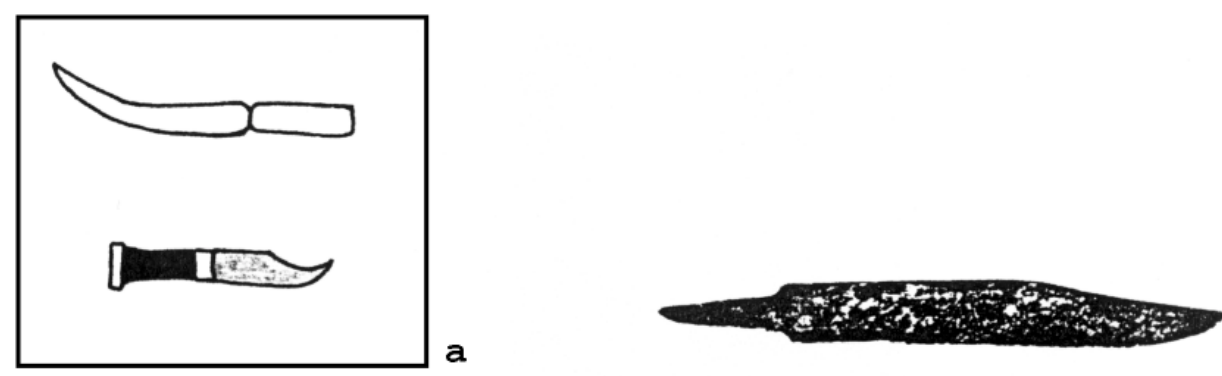

C: Lund (72)

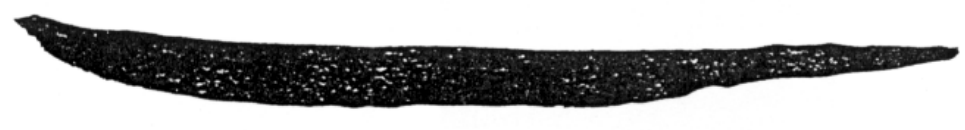

d: Lund (53)

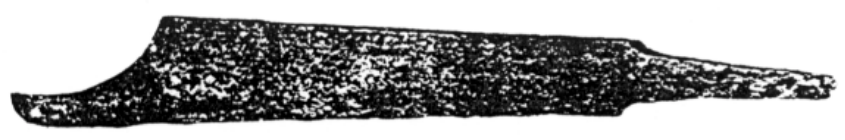

e: Lund (54)

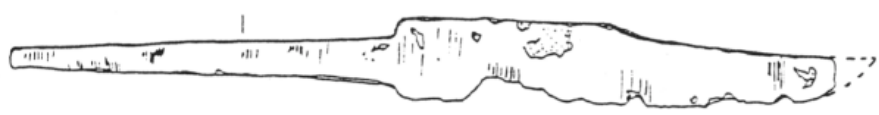

f. Oslo (7)

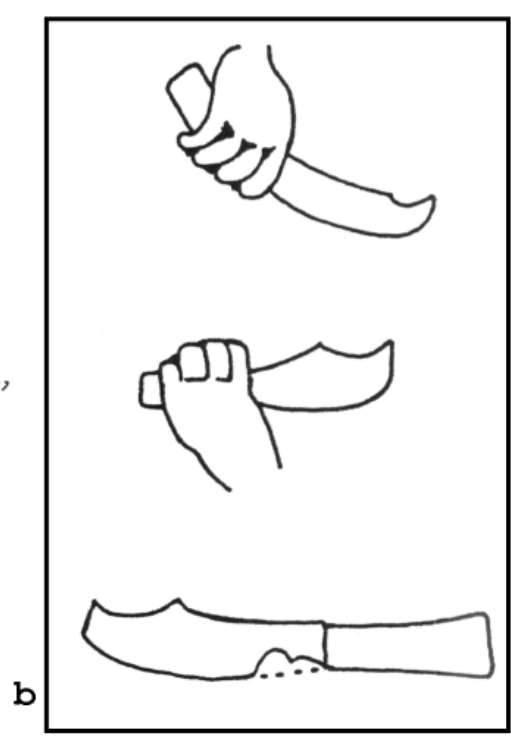

Abb. 220

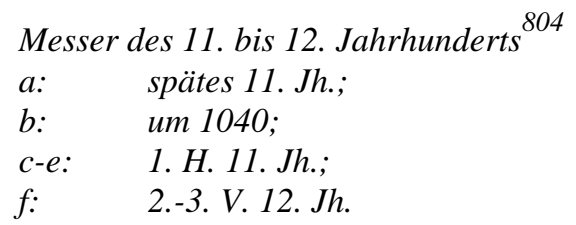

804 a: Teppich von Bayeux, spätes 11. Jahrhundert (Abschlußmahlzeit vor der Überfahrt, Ausschnitt) nach einer Postkarte der Collection Ville de Bayeux Nr. 43

b: Echternacher Codex, um 1040 (Gastmahl des Ahasver) in: Schiedlausky 1956, Abb. 1, Anhang 

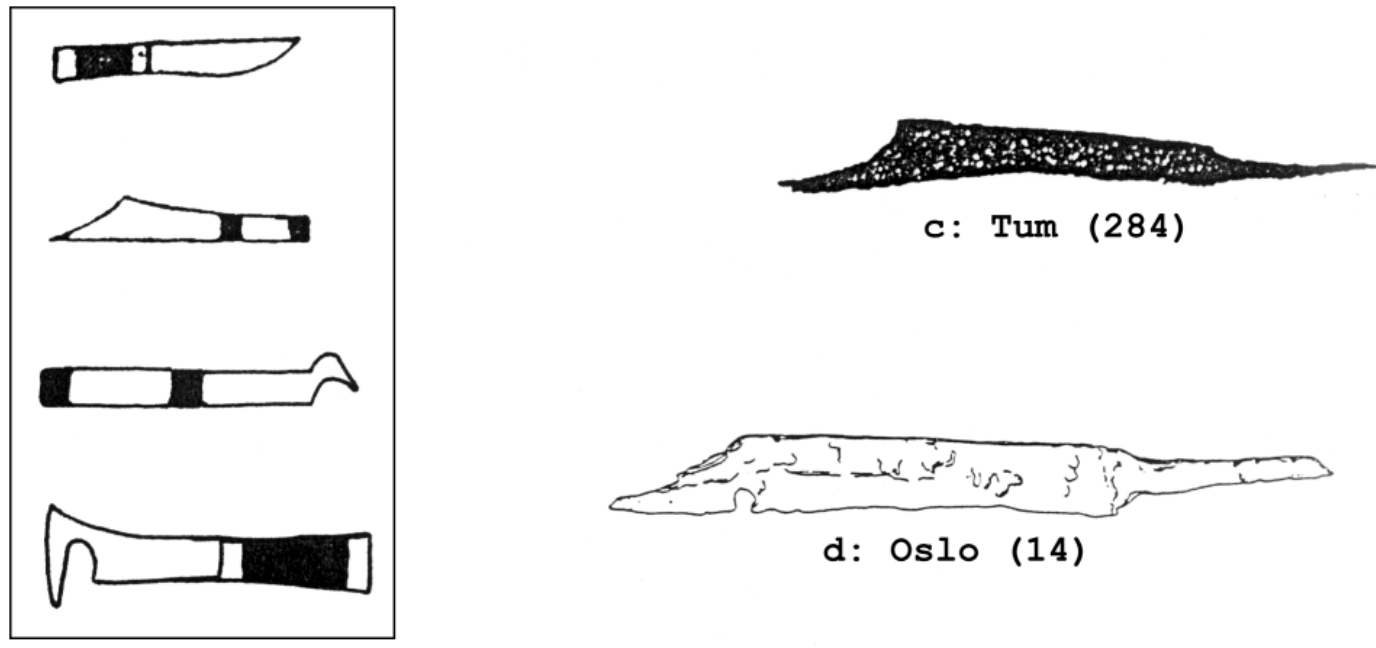

a

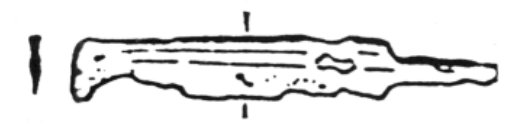

e: Wartenberg (204)
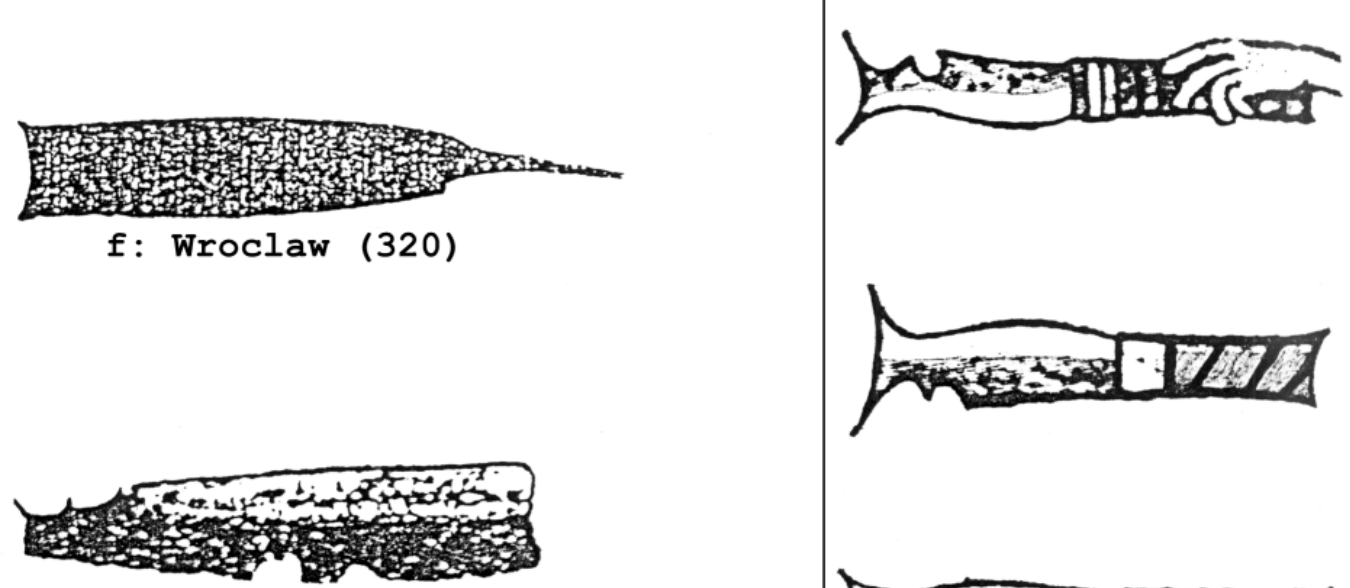

g: Wroclaw (322)

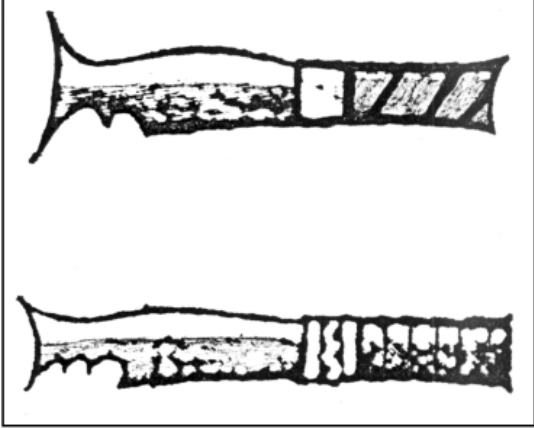

Abb. 221

$$
\begin{array}{ll}
\text { Messer des 12. bis 13. Jahrhunderts } & 805 \\
a: & \text { um 1170; } \\
b: & \text { 12. Jh.; } \\
c: & \text { 12. - 13. Jh.; } \\
d: & \text { 2. H. I2. Jh.; } \\
e-g: & \text { 13. Jh. }
\end{array}
$$

805 a: Hortus Deliciarum der Herrad von Landsberg, um 1170, in: Wühr 1961, Abb. 8 und Abb. 2, Anhang b: Speyerer Evangeliar, 12. Jh. in: Helferich-Dörner 1959, Abb. S. 25 


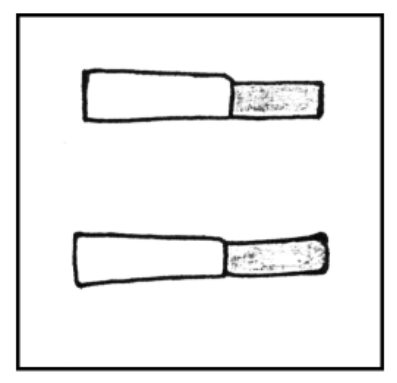

a
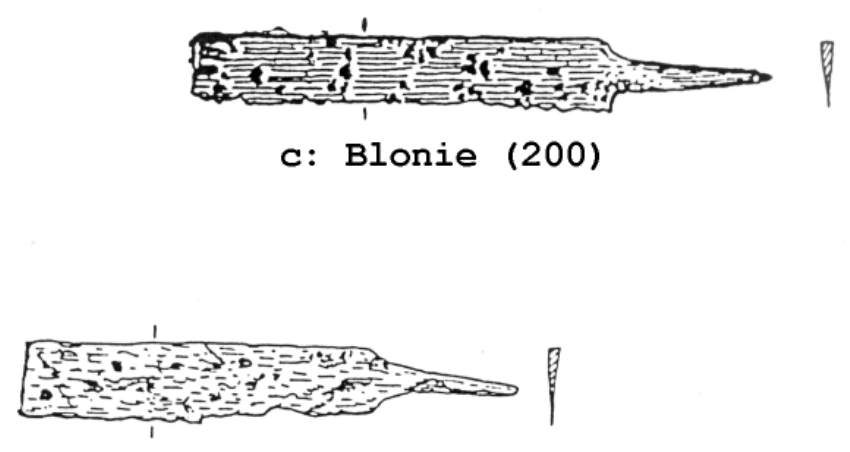

d: Gommerstedt (195)

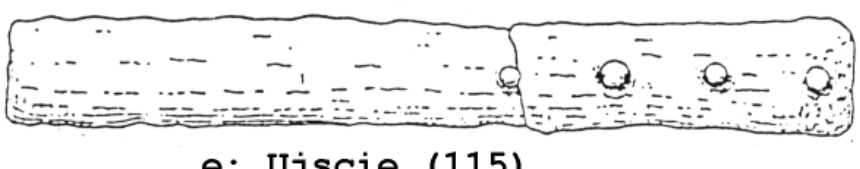

e: Ujscie (115)

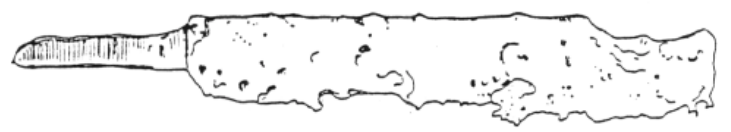

f: Oslo (17)

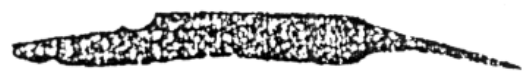

g: Wrocław (316)

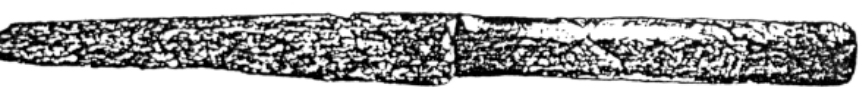

h: Amsterdam (60)

b

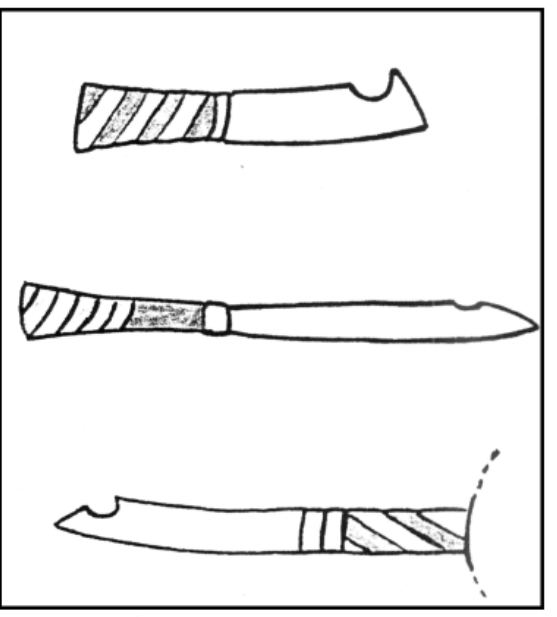

Abb. 222

Messer des 12. bis 14. Jahrhunderts ${ }^{806}$

a: 12. - 13. Jh. (?); b-c: 13. Jh.; d: 13. - 14. Jh.;

e: 14. Jh.; f-g: 12. - 13. Jh.; h: 14. Jh.

806 a: spätromanisches Fresko in der Kirche von Estvad (Skive), Dänemark, in: E. Kjersgaard 1978, Abb. 99 , S. 130

b: Miniatur aus einem Psalmenkommentar, 13. Jh., in: H. Bauer o.J., Abb. S. 97 (vgl. auch Schiedlausky 1956, Abb. S.7) 

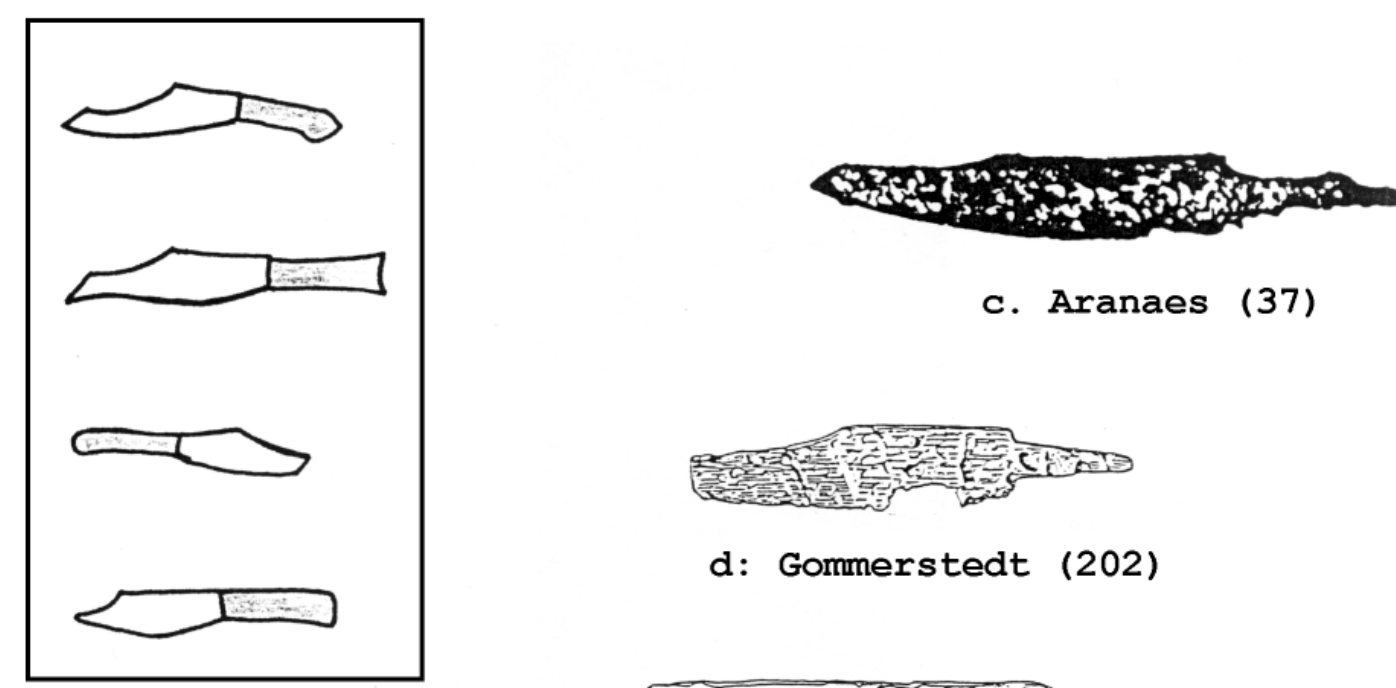

c. Aranaes (37)

a

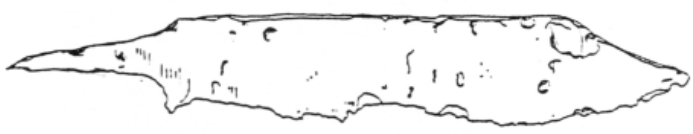

e: Oslo (16)

\section{d: Gommerstedt (202)}

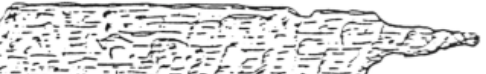

f: Gommerstedt (216)
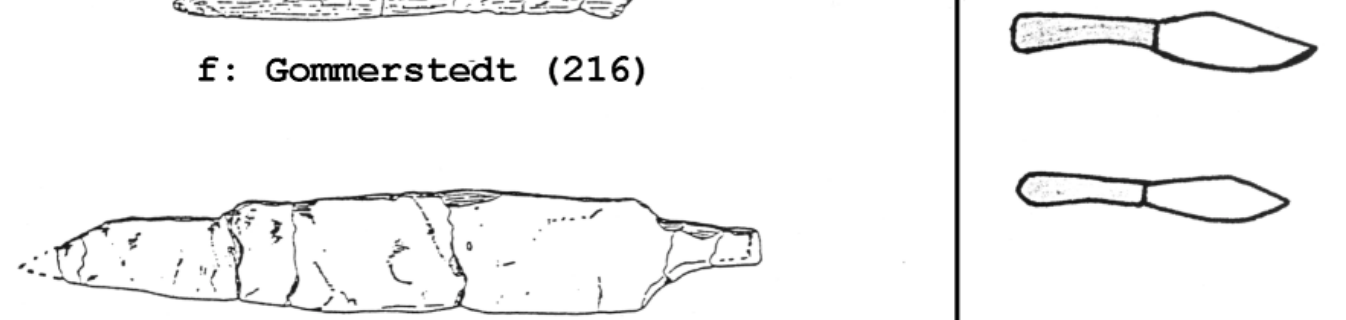

g: Oslo (14)
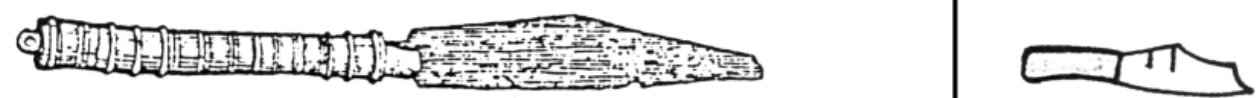

\section{h: Hamburg (46)}

Abb. 223

$$
\begin{aligned}
& \text { Messer des 12. bis 14. Jahrhunderts } \\
& \text { a-b: 1. H. 14. Jh.; c: 12. - 14. Jh.; d, f: 13. - 14. Jh.; } \\
& \text { e: 4. V. 12. - 1. V. 13. Jh; g: 12. Jh.; h: 4. V. 12. Jh. }
\end{aligned}
$$

807 a-b: Codex Balduineus (aus dem Gastmahl des Erzbischofs Balduin von Trier 1308), 1. H. 14. Jh., in: Schiedlausky 1956, Abb. 5, Anhang 

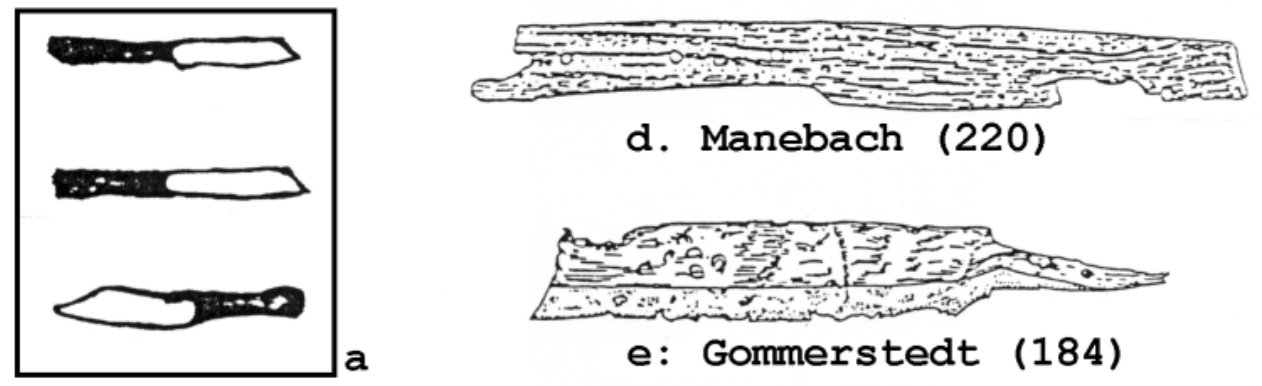

d. Manebach (220)
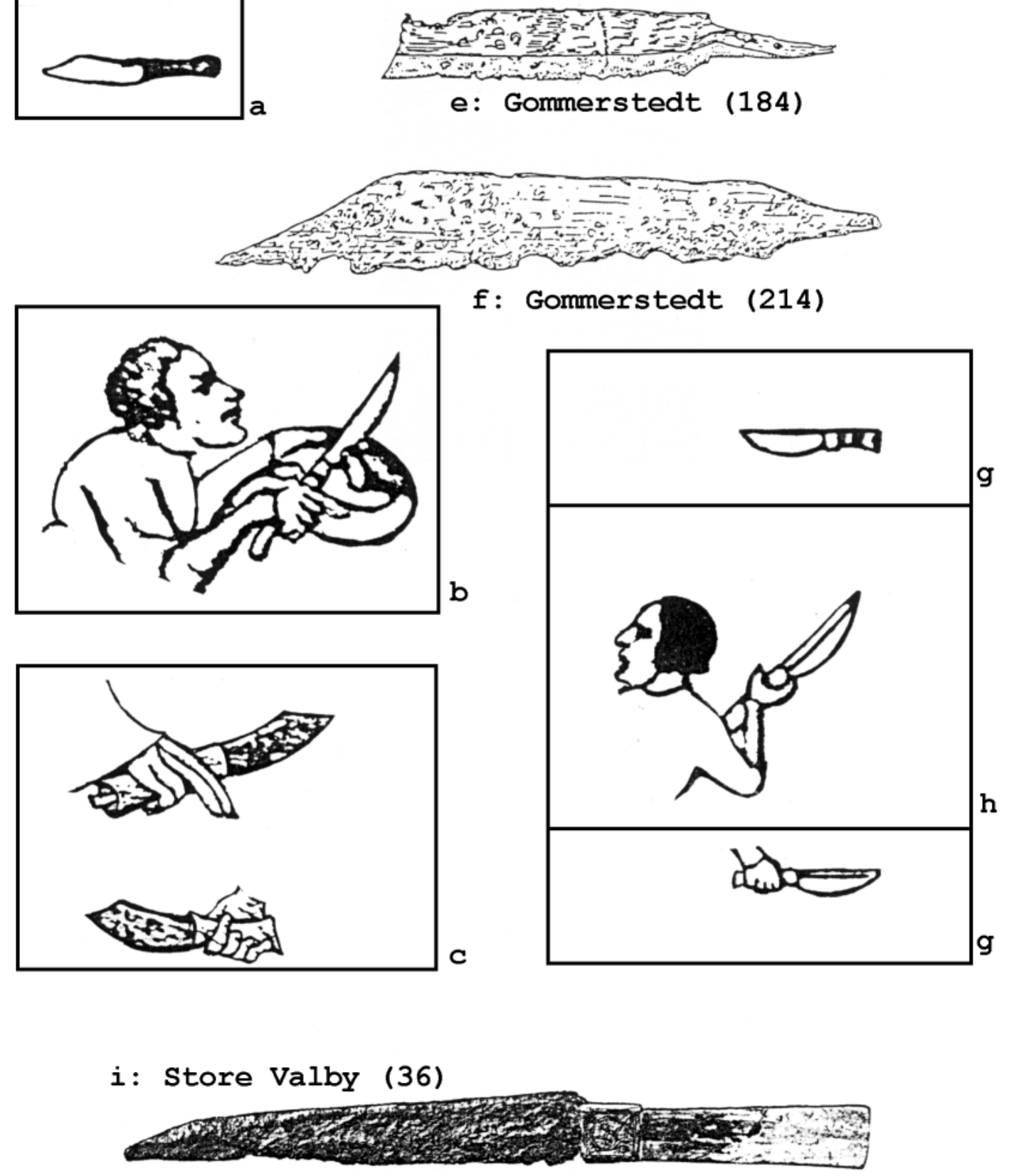

Abb. 224

Messer des 13. bis 14. Jahrhunderts 808
a: um 1360; b: um 1375-1380; c: E. 14. Jh.; d: 14. Jh.;
e-f: 13. - 14. Jh.; g-h: E. 14. Jh.; i: 13. - 14. Jh.

808 a: Das letzte Abendmahl, Köln um 1360 (Wallraf-Richartz-Museum, Köln), in: A. Helferich-Dörner 1959, Abb. S. 26

b: Grandes Chroniques de France, 1375-1380 (aus dem Bankett König Karls V 1377 in Paris), in: Schiedlausky 1956, Abb. 10, Anhang

c: Meister Bertram von Minden, Abendmahl, E. 14. Jh., in: T. Dexel, Gebrauchsgerätetypen I, Braunschweig 1980, Tafel I 

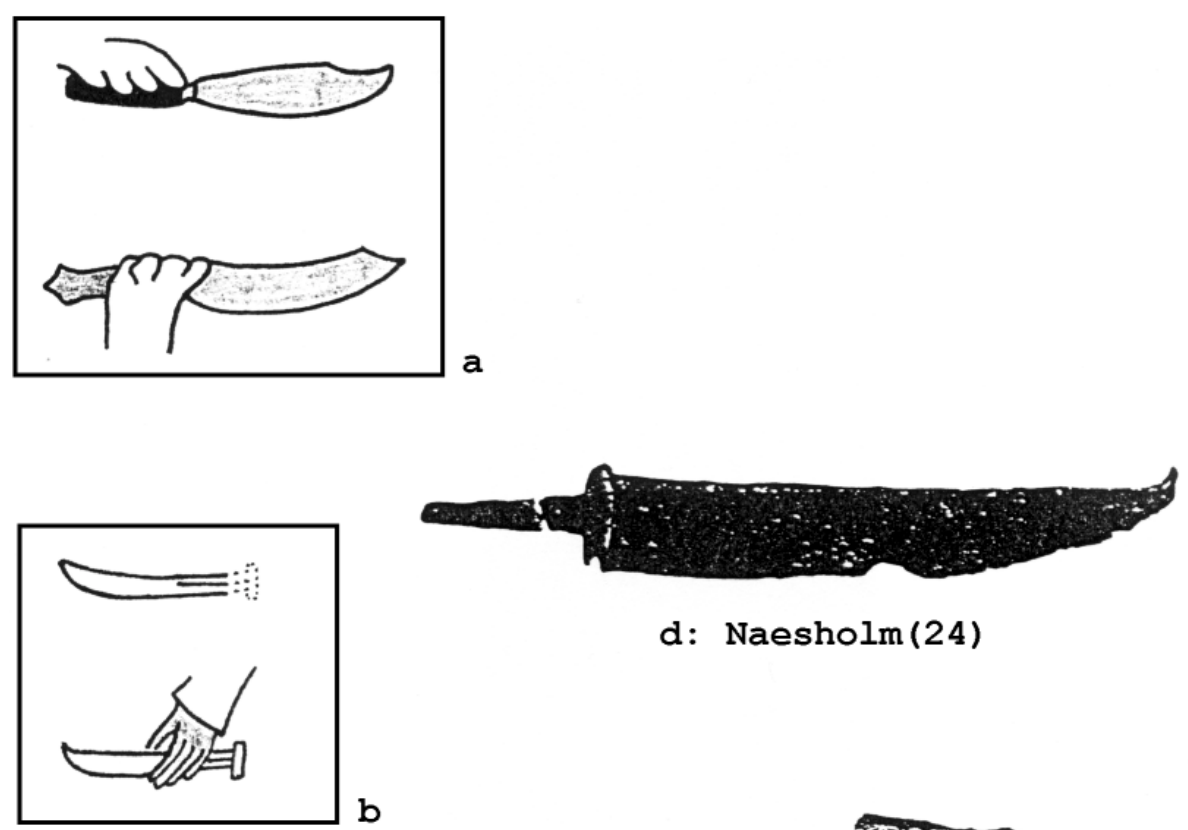

d: Naesholm(24) b
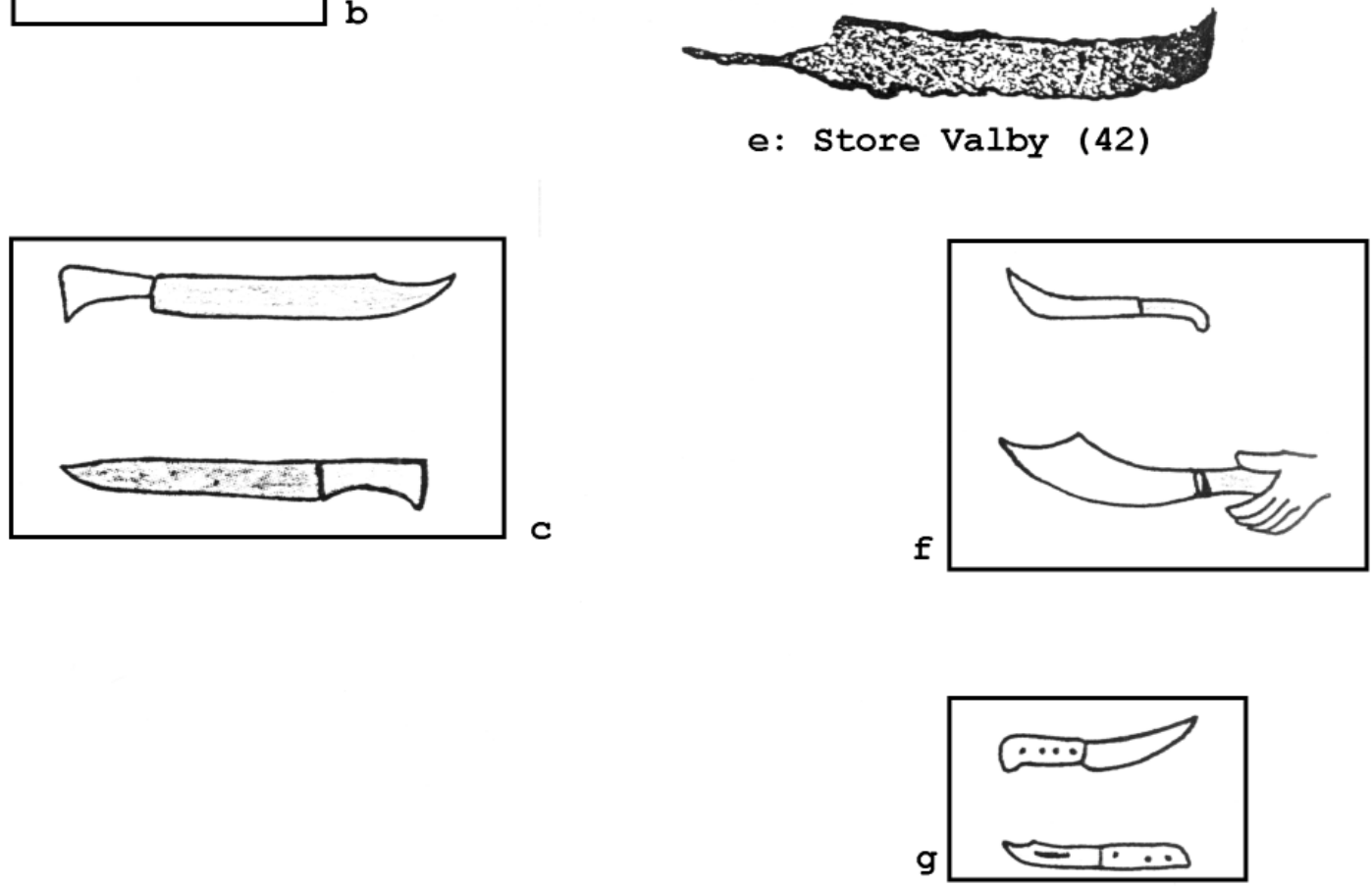

Abb. 225

Messer des 14. bis 15. Jahrhunderts 809

a: A. 15. Jh.; b: um 1425; c: um 1430; d: 1. H. 14. Jh. (?);

e: mittelalterlich (?); $f:$ um 1450; g:1496

809 a: "Les Belles Heures" des Jean Duc de Berry, vor 1416 (aus dem Neujahrgastmahl des Herzogs),

in: Schiedlausky 1956, Abb. 21, Anhang

b: Fresko in der Kirche von Gerlev (Slagelse), Dänemark, um 1425 (Das letzte Abendmahl),

in: Kjersgaard 1978, Abb. 1, S. 10

c: Fresko in der Kirche von Nordrup (Slagelse), Dänemark, um 1430 (Das letzte Abendmahl),

in: Kjersgaard 1978, Abb. 60, S. 83

f: Fresko in der Kirche von Morkov (Tudse), Dänemark, um 1450 (Das letzte Abendmahl),

in: Kjersgaard 1978), Abb. 6, S. 15

g: Fresko in der Kirche von Bellinge (Fyn), Dänemark, 1496 (Das letzte Abendmahl),

Kjeersgaard 1978, Abb. 144, S. 165 


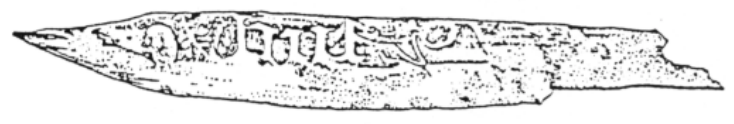

a: Lübeck (37)
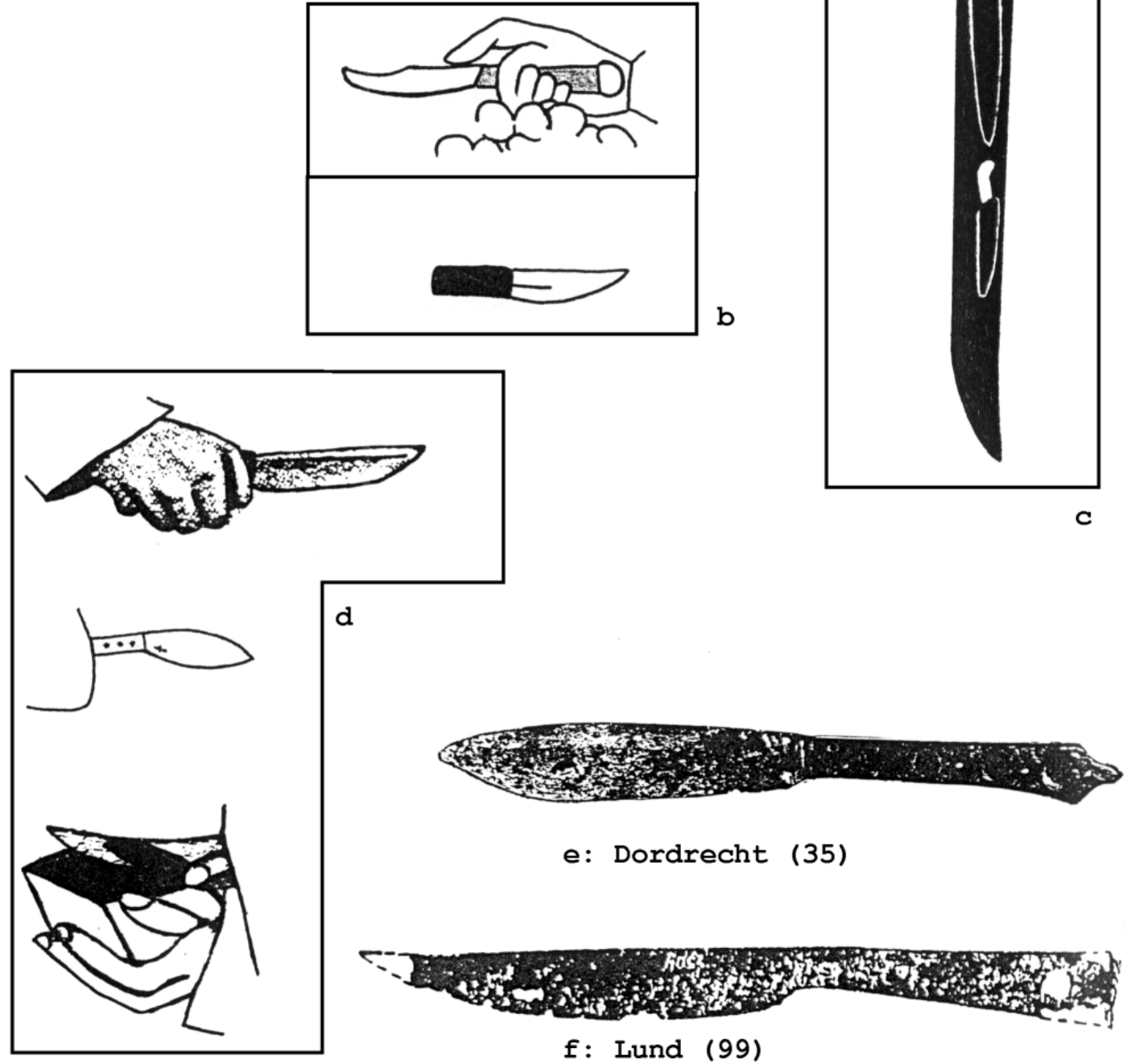

Abb. 226

Messer des 14. bis 15. Jahrhunderts 810

a: 2. H. 14. Jh. (?); b-c: um 1465; d: 2. H. 15. Jh.;

e: 2. H. 14. Jh. (?); f: 15. Jh.

810 b-c: Baseler Bildteppich, um 1465 (Szene im Liebesgarten), in: A. Helferich-Dörner 1959, Abb. S. 35 d: Meister der Lyversberger Passion, 2. H. 15. Jh., (Das letzte Abendmahl), nach der Funk-Postkarte Nr. 1355 

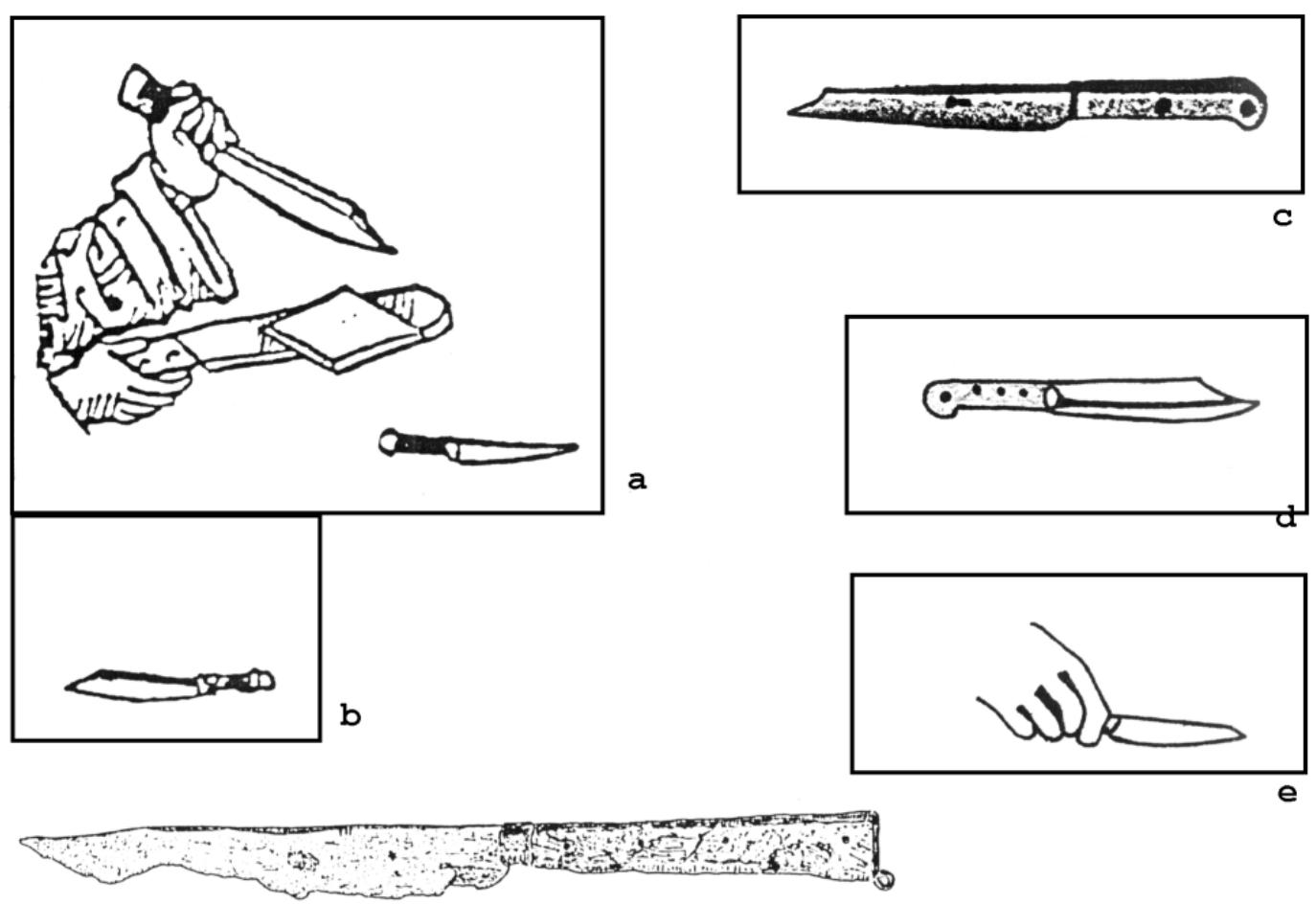

g: Amsterdam (65)

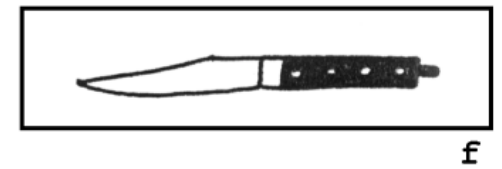

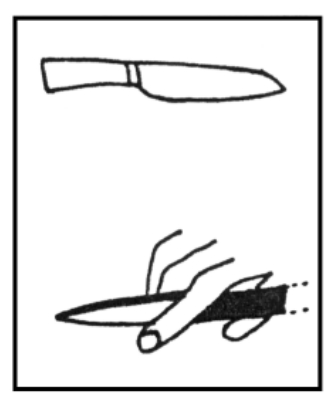

$\mathrm{h}$

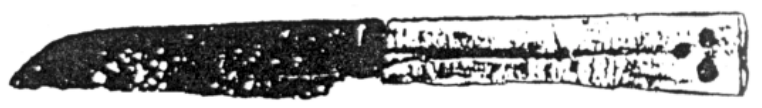

i: Lund (93)

$A b b .227$ Messer des 15. Jahrhunderts ${ }^{811} a-b: 1491 ; c: 3$. V. 15. Jh.; d: E. 15. Jh.; e: 3. V. 14. Jh.; f: E. 15. Jh.; g: E. 14. - A. 15. Jh.; h: um 1460; i: 15. Jh.; j: 2. H. 15. Jh.

811 a-b: Holzschnitt von Michael Wolgemut, Nürnberg 1491 (Das Bankett des Königs), a: Tranchierbesteck, b: Messer des Königs, in: Schiedlausky 1956, Abb. S. 13

c: Dirk Bouts, 1464-1467, Abendmahlsaltar, Löwen (Peterskirche), in: W. Rotzler, Glas im Spiegel der Kunst, in: du-438 (Zürich, August 1977)

d: Corvinus Graduale, Folio 127, E. 15. Jh., in: E. Soltesz, Das Corvinus Graduale, Budapest 1980, dt. Übersetzung F. Gottschlig, Hanau 1982, Abb. S. 119

e: Dirk Bouts, Die Feier des Passahmahls, 1464/67, in: Heidrich 1924, Abb. 52

f: Breviario Grimani (Miniatur), Niederlande E. 15. Jh. (Der Fürst an der Tafel), in:Schiedlausky 1956, Abb. 14, Anhang

h: Dirk Bouts, Christus im Hause des Pharisäers Simon, um 1460, in: E. Heidrich, Alt-Niederländische Malerei, Jena 1924, Abb. 64 

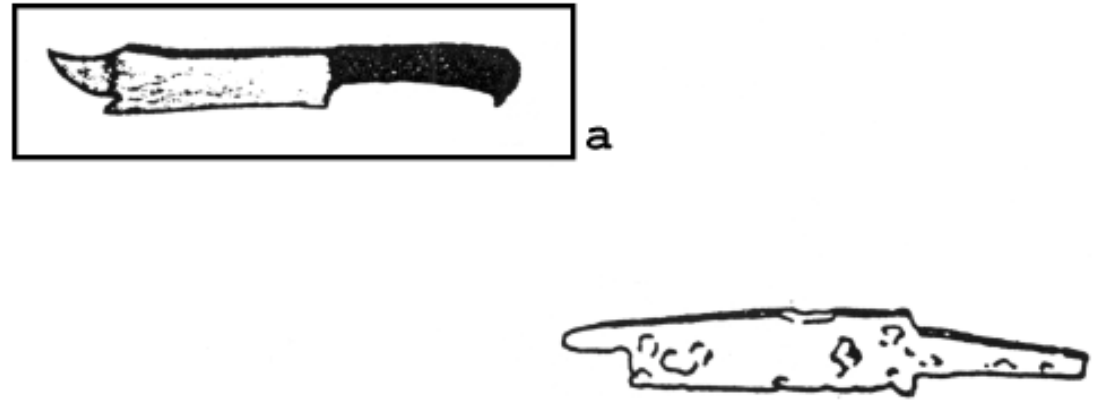

b: Wartenberg (202)

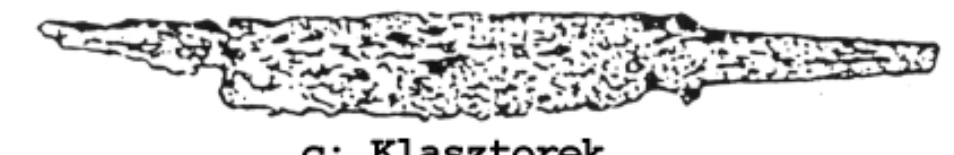

\section{c: Klasztorek}

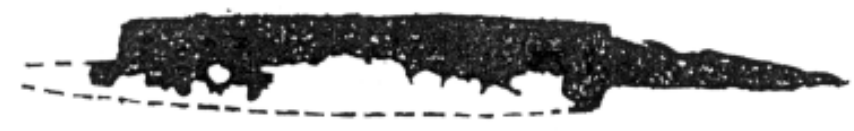

d: Lund (75)

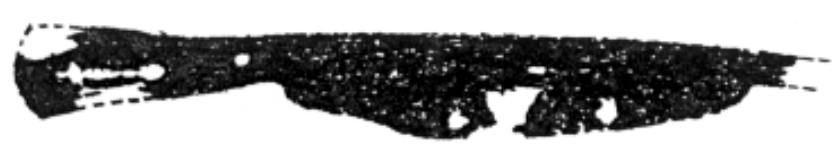

e. Lund (103)

f: Gommerstedt (196)

Abb. 228

Klingenformen IV f und IV $h^{812}$

a: um 1470; b: 13. Jh.; c: 13. - 14. Jh. (?); e: 15. Jh.; f: 13. - 14. Jh. 


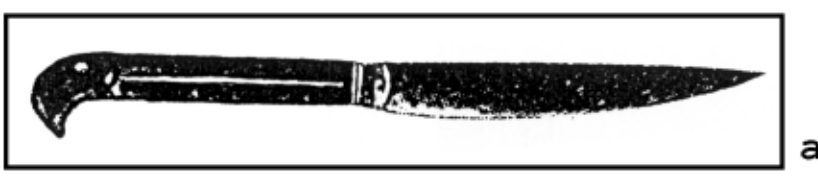
a

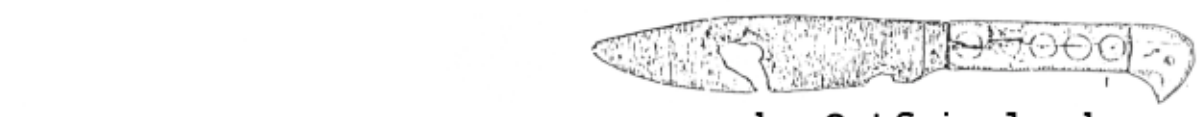

b: Ostfriesland
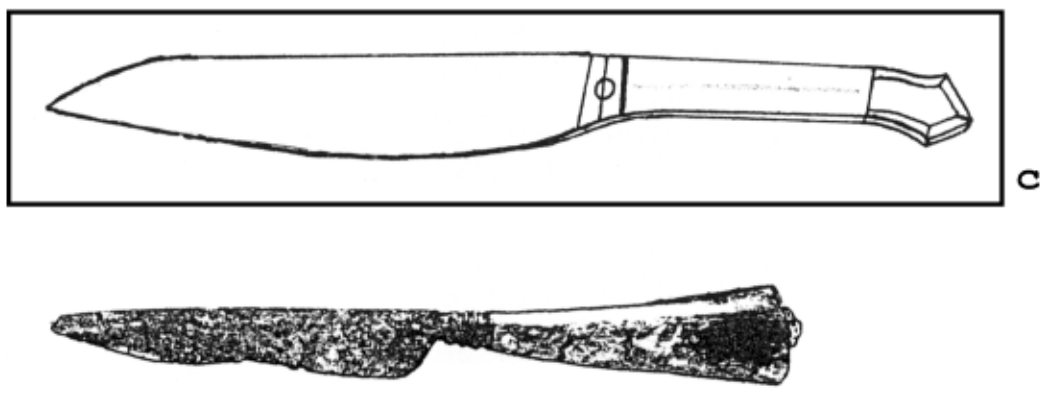

d: Amsterdam
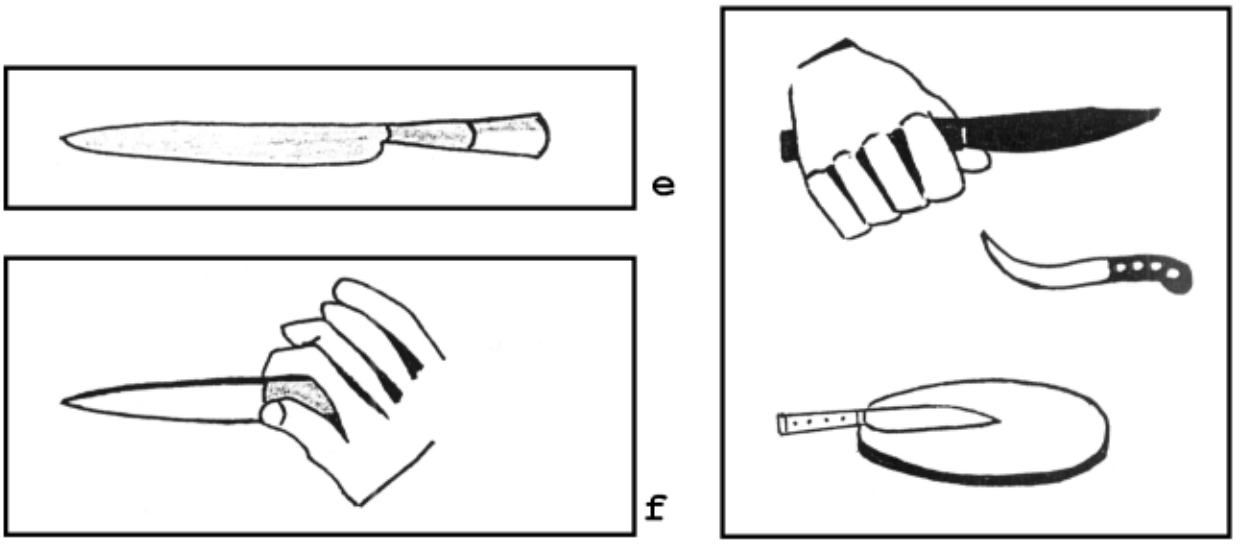

g

\section{$M$}

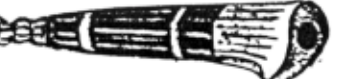

$\mathrm{h}$

Abb. 229

Messer des 15. bis 17. Jahrhunderts 813

a: A. 16. Jh.; b: 15. Jh. (?); c: um 1540; d: 2. H. 15. Jh.; e: 1559;

$f:$ um 1503; g: 1533; $h: 1635 ; i: 1608$

813 a: Joos van Cleve (?), Die Heilige Familie, frühes 16. Jh. in: Heidrich 1924, Abb. 129

b: Ostfriesland, Bodenfund (Das Messer wurde dem Autor freundlicherweise von Herrn Dr. E. Schumacher, Essen, zur Verfügung gestellt. Es ist im Archäologischen Museum Altenessen unter der Nummer RE 65/88 A inventarisiert. Die Zeichnung wurde von Herrn A. Zeischka, Oberhausen, angefertigt.)

c: Maerten van Heemskerk (?), Familienbildnis, um 1540, in Heidrich, Alt-Niederländische Malerei, Jena 1924, Abb. 157

d: Niederlande, Kat. Nr. 68

e: Hans August Kluber, Bildnis der Familie des Goldschmieds Hans Rudolf Faesch, Basel 1559,

in: Schiedlausky 1956, , Anhang Abb. 41 
f: Gerard David, Die Hochzeit zu Kanaa, um 1503, in: Heidrich, Alt-Niederländische Malerei, Jena 1924, Abb. 84

g: Cornelis Anthonisz, Bankett der Schützengilde, Amsterdam 1533, nach einer Postkarte des historischen Museums Amsterdam, a.h.m. 1975

h: Georg Flegel, Stilleben mit Hirschhornkäfer, 1635, nach einer Postkarte des ehem. Wallraf-RichartzMuseums, Bestell Nr. WRM 25

i: Osias Beert, Stilleben mit Kirschen und Erdbeeren, 1608, in: Rotzler 1977, Abb. S. 46 


\section{Schluß}

Die aufgeführten Beispiele haben gezeigt, daß erst im Vergleich archäologischer, historischer und kunsthistorischer Quellen fundiertere Aussagen über Fragen der Sachkultur und des Alltagslebens im Mittelalter und in der frühen Neuzeit möglich sind. Für eine umfassende und hinreichend problembezogene Darstellung reicht es nicht aus, archäologisch gewonnene Erkenntnisse mit einigen Quellen anderer Gattungen zu konfrontieren.

Die Frage, ob zunächst neben den archäologischen die Schrift- und Bildquellen intensiv und zunächst isoliert nach den ihren Disziplinen eigenen Methoden aufgearbeitet werden, oder ob die Bearbeitung von Teilbereichen mittelalterlicher und neuzeitlicher Lebensformen von Anfang an alle zur Verfügung stehende historischen Quellen berücksichtigen sollte, ist nicht zuletzt eine Frage der derzeitigen Realisierbarkeit.

Es wurde bereits mehrfach darauf verwiesen, daß selbst innerhalb der archäologischen Fachrichtungen in der Regel nicht alle Möglichkeiten der Quellenerfassung und -bearbeitung ausgeschöpft werden, so daß wissenschaftliche Untersuchungen zumeist lokal oder regional beschränkt bleiben und nur in seltenen Fällen Grabungsmonographien einen repräsentativen Querschnitt des Fundmaterials liefern. Viele Publikationsvorhaben kommen über den Status eines Vorberichts nicht hinaus.

Nur an wenigen Stellen in der Bundesrepublik wird - regional begrenzt - historische Forschung interdisziplinär betrieben, werden die archäologisch erfaßten Befunde mit den vorhandenen historischen Quellen konfrontiert und gemeinsam interpretiert. An den Ergebnissen sind durchweg Gruppen von Wissenschaftlern und Mitarbeitern beteiligt, die im Rahmen langjähriger Projekte durch zusätzliche Finanzierungsprogramme arbeiten können.

Außerhalb dieser Projekte ist es lediglich dem Forschungseinsatz einzelner Wissenschaftler zu verdanken, daß an anderen Orten und Instituten interdisziplinäre Arbeit im engsten Rahmen überhaupt stattfindet. Die Mehrzahl dieser Bemühungen ist in den letzten Jahren durch finanzielle Einschnitte reduziert worden, die amtliche Bodendenkmalpflege ist aus den gleichen Gründen kaum in der Lage, ihrer Arbeit vor Ort nachzukommen.

In dieser Situation bleibt vielen Mittelalter- und Neuzeitarchäologen nur die Möglichkeit, sich selbst um die zur Interpretation notwendigen Quellen der Nachbardisziplinen zu bemühen. Im Rahmen lokal oder regional begrenzter Forschung ist dieses auch aufgrund des interdisziplinären Ansatzes der Fachrichtung und Ausbildung durchführbar.

Jede Ausweitung dieses engen Rahmens stößt jedoch an die bekannten Grenzen, die durch sachgerechte und interpretationsfähige Quellenanalyse in allen beteiligten Disziplinen gesetzt werden. Wenn die herangezogenen Quellen des jeweiligen wissenschaftlichen Nachbarn nicht schmückendes Beiwerk bleiben sollen, ist eine 
intensive und langfristige Zusammenarbeit in regionaler und überregionaler Kulturgeschichtsforschung unumgänglich.

Wo diese strukturell von Anfang an möglich ist, können sich Quellenanalyse und Methoden auch von Anfang an ergänzen.

Wo diese aber nur bedingt oder gar nicht möglich ist, bleibt jenseits von "Mikroanalysen" nur die Möglichkeit, wie bisher in der Ur- und Frühgeschichte üblich, einzelne, oft regional eingeschränkte Epochen, größere Grabungskomplexe oder Objekte und Objektgruppen überregional darzustellen. Allein die zeitlichen Vorgaben und die zur Verfügung stehenden finanziellen Mittel machen weitergehende Anforderungen, die sich im Fall der Mittelalter- und Neuzeitarchäologie aufgrund der besonderen Quellensituation ergeben, zur Zeit unrealistisch.

$\mathrm{Da} ß$ vor allem überregional angelegte Studien verschiedener Disziplinen sich oft nicht gegenseitig ergänzen, sondern beziehungslos nebeneinander stehen, mag diejenigen nachdenklich stimmen, die für Ausbildungsbestimmungen und Prüfungsordnungen, aber auch für Stellenpläne, Wissenschafts- und Kulturfinanzierung Verantwortung tragen. 


\section{Nachweis der Abbildungen ${ }^{814}$}

Tafel I

S. 397
a - Statens Historika Museum Stockholm, Photo Nr. 2002:53
b - Statens Historika Museum Stockholm, Photo Nr. 2433:11
c - Statens Historika Museum Stockholm, Photo Nr. 1715:56
d - Statens Historika Museum Stockholm, Photo Nr. 1720:51c
e - Statens Historika Museum Stockholm, Photo Nr. 7764:174 (1866:37c)

Tafel II S. 398 a - Amt für Vor- und Frühgeschichte Lübeck, Photo Nr. B 3086/3087

b - Amt für Vor- und Frühgeschichte Lübeck, Photo Nr. B 3085

c - Statens Historika Museum Stockholm, Photo Nr. 916:8a

d - Statens Historika Museum Stockholm, Photo Nr. 1720:51a

e - Statens Historika Museum Stockholm, Photo Nr. 1720:51d

f - Statens Historika Museum Stockholm, Photo Nr. 1720:51b
Tafel III S. 399 a - Koechlin 1924, Band 2 (Katalog), Tafel 189, Kat. Nr. 1122
b - Koechlin 1924, Band 2 (Katalog), Tafel 189, Kat. Nr. 1143
c - Koechlin 1924, Band 2 (Katalog), Tafel 189, Kat. Nr. 1136
d - Koechlin 1924, Band 2 (Katalog), Tafel 190, Kat. Nr. 1137

\footnotetext{
814 Die Nachweise zu den im Katalog und im laufenden Text benutzten Photographien, Zeichnungen und abbildungen sind in der Regel im Kataloganhang nach jedem Bearbeitungsland , im kaufenden Text in anmerkungen geführt worden. Hier erscheinen nur die dort nicht aufgeführten Nachweise.
} 
e - Koechlin 1924, Band 2 (Katalog), Tafel 190, Kat. Nr. 1138

f - Koechlin 1924, Band 2 (Katalog), Tafel 190, Kat. Nr. 1140

Abb. 140 S. 416 a - Amiens (Kathedrale), um 1230, in Hürliman u.a. 1954, Abb. S. 110

b - München (Lorenzkirche), Oberrhein/Süddeutschland 1324, In Wittelsbach und Bayern 1/2 1980, Abb. 381, S. 250

d - Basel (Münster), um 1300, in Deuchler 1976, Abb. 102

e - Heppenheimer Madonna, um 1300, in Deuchler 1976, abb. 104

Abb. $141 \quad$ S. 417

a - Die Zeit der Staufer II 1977 (Katalog), Kat. Nr. 633, Abb. 138

b - Goldschmidt III 1923, Tafel 25, Abb. 72

Abb. 142 S. 440 a - Beigbeder o.J., Abb. 23, S. 27 vgl. auch Goldschmidt III 1923, Tafel 45, Abb. 126

b - Goldschmidt III 1923, Tafel 46, Abb. 127

Abb. 143 S. 440 a - Beigbeder o.J., Abb. 82, S. 82

b - Goldschmidt IV 1926, Tafel II, Abb. 38

c - Handbuch des Museums für Kunst und Gewerbe Hamburg, München 1980, Abb. 106. S. 58

d - Handbuch des Museums für Kunst und Gewerbe Hamburg, München 1980, Abb. 107, S. 58

Abb. 144 S. 443

Goldschmidt III 1923, Tafel 18, Abb. 56

Abb. 148 S. 456 c - Codex Manesse 1988, Tafel 55

d - Codex Manesse 1988, Tafel 114

e - Codex Manesse 1988, Tafel 34

F - $\quad$ Codex Manesse 1988, Tafel 27 
Abb. 149 S. 457 c - Codex Manesse 1988, Tafel 52

d - Codex Manesse 1988, Tafel 2

Abb. 150 S. 150 b - Codex Manesse 1988, Tafel 6

e - Codex Manesse 1988, Tafel 125

Abb. 151 S. 151 b - Codex Manesse 1988, Tafel 80

d - Codex Manesse 1988, Tafel 20

e - Codex Manesse 1988, Tafel 76

Abb. 152 S. 460 a - Codex Manesse 1988, Tafel 131

c - Codex Manesse 1988, Tafel 91

d - Codex Manesse 1988, Tafel 9

Abb. 153 S. 461 c - Codex Manesse 1988, Tafel 112

d - Codex Manesse 1988, Tafel 28

e - Codex Manesse 1988, Tafel 58

f - Codex Manesse 1988, Tafel 121

Abb. 154 S. 464 a - Paris, um 1320-1330, in: Deuchler 1976 abb. 113, S. 94

b - Frankreich, um 1330, in Deuchler 1976, Abb. 114, S. 94

c - Frankreich, 1.H. 14. Jahrhundert, in: Mittler/ Werner 1988, Kat. Nr. K 26 Abb. S. 653

d - Frankreich, 1.H. 14. Jahrhundert, in: Mittler/ Werner 1988, Kat. Nr. K 24 Abb. S. 653

Abb. 155 S. 465 a - Scheitelziehergriff, Frankreich, 14. Jahrhundert, in: Beigbeder o.J., Abb. 62, S. 61

b - Schreibtafel, Frankreich, 14. Jahrhundert, in: Beigbeder o.J., Abb. 28, S. 31

c - Minnekästchen, Frankreich, frühes 14. Jahrhundert (St. Ursula, Köln), in: Mittler / Werner 198, Abb. 11, S. 283 
Abb. 156 S. $470 \quad a-b$ Mittelplatten eines vortragekreuzes, Maasgebiet 1160 - 1170, in: Die Zeit der Staufer II, 1977 , Kat. Nr. 550, Abb. 343

c - Turmförmiges Reliquiar, Köln, 1.H. 13. Jahrhundert, in: Die Zeit der Staufer II 1977, Kat. Nr. 634, Abb. 439

d - Amiens (Kathedrale, Westfasade), um 1230, in: Deuchler 1976, Abb. 79, s. 69

Abb. 157 S. 471 a - Rudolf von Ems, Weltchronik, Blatt 1, Straßburg (?), um 1270-1280, in Mittler / Werner 1988, Kat. Nr. H 15 Abb. S. 608

b - Naumburg (Dom, Sifterfigur im Westchor), um 1250, in: Die Zeit der Staufer II 1977, Kat. Nr. 452, Abb. 256

c - Graduale aus St. Katharinental (Initiale a, Blatt 258), Katharinental (ß) um 1312, in: Mittler / werner 1988, Kat. Nr. J 16, Abb. S. 637

d - Kloster Wienhausen, Chorgangfenster, um 1330 1340, in: U.-D. Korn, Kloster Wienhausen Band V Die Glasmalereien, Kloster Wienhausen 1975, Abb. S. 24 


\section{Abkürzungsverzeichnis für benutzte Zeitschriften und Periodika}

\section{NIEDERLANDE}

B.R.O.B.

Berichten van het Rijksdienst voor Opgravingen en Bodemonderzoek

R.O.B. Rijksdienst voor Opgravingen en Bodemonderzoek, Amersfoort

BUNDESREPUBLILK DEUTSCHLAND (alte Bundesländer)

Lüb. Schriften

Lübecker Schriften zur Archäologie und Kulturgeschichte

Arch. Korrbl.

Archologisches Korrespondenzblatt

Z.A.M.

Zeitschrift für Archäologie des Mittelalters

N.N.U.

Nachrichten aus Niedersachsens Urgeschichte

N.A.F.N.

Neue Ausgrabungen und Forschungen in Niedersachsen

BUNDESREPUBLIK DEUTSCHLAND (neue Bundesländer)

Bod. Meck.

A.u.F.

POLEN

Mat.W.

Sl. Ant.

Spr. Arch.

P.i.M.

P.A.

St. Arch. Po.

Mat. Zach.

St.W.

Prz.Arch.

F.A.P.
Bodendenkmalpflege in Mecklenburg (Jahrbücher)

Ausgrabungen und Funde

Materiały Wczesnośredniowieczne

Slavia Antiqua

Sprawozdania Archeologiczne

Prace i Materiały

Prace Archeologiczne

Studia Archeologica Poneranica

Materiały Zachodnio-Pomorskie

Studia Wczesnośredniowieczne, Wrocław

Przeglad Archeologiczny, Poznań Wrocław

Fontes Archaeologici Posnanienses 


\section{Literaturverzeichnis}

A. Abramowicz, Przedmioty Ozdobne Z Grodziska Łęczyckiego, in: A. Nadolski, Z Badań Nad Wczesnośredniowieczn Łęczycą, in: St. W. 3, Wrocław 1955

H.R. d'Allemagne, Decorated Antique Ironwork _ A Pictorial Treasory, New York 1968, Nachdruck der Tafeln des 1924 in Paris erschienenen Katalogs "Ferronnerie ancienne" des Museums "Le Secq des Tournelles" in Rouen. Bearbeitet von Vera K. Ostoia, Metropolitan Museum of Art New York

B. Almgren u.a., Die Wikinger, Essen 1975

H. Amman, Deutschland und die Messen der Champagne, in: H. Stoob

(Hsg.), Altständisches Bürgertum Bd. II, Darmstadt 1978, S. 51-95

J. Amman, Das Ständebuch, Insel-Verlag, Leipzig 1975

H. Andersson, Veränderungen oder Kontinuität im mittelalterlichen schwedischen Städtewesen des 12. und 13. Jahrhunderts unter besonderer Berücksichtigung der Entwicklung im Ostseegebiet, in: Lübecker Schriften zur Archäologie und Kulturgeschichte 7-1983, S. 185-194

H. Hellmuth Andersen, Die Burg in Itzehoe, Neumünster 1980

H. Hellmuth Andersen / H.J. Macken, Århus Søndervold, København 1971

N. Angermann, Die Hanse und Rußland, in: Hanse in Europa, Katalog und Begleitband zur gleichnamigen Ausstellung in der Kunsthalle Köln, Köln 1973, S. 273-280

H. Appuhn, Der Fund vom Nonnenchor, Kloster Wienhausen Bd. 4-1973

H. Arbman, Birka I - Die Gräber, Uppsala 1940

Archäologie des Mittelalters und der Neuzeit (H.G. Peters, red.), Eine Ausstellung des niedersächsischen Ministers für Wissenschaft und Kunst, Hannover 1978

A.V. Arzichovskij / B.A. Koltschin, Trudy Novgorodskoj Archeologičeskoj Ekspeditii, Tom I, Mockwa 1956 (Tom II, Mockwa 1959)

M. Aubert, Hochgotik (in der Reihe "Kunst der Welt"), Baden-Baden 1963

Ausgrabungen in Minden, Ausstellungskatalog der gleichnamigen Ausstellung des Westfälischen Museums für Archäologie Münster, Münster 1987

J. Baart u.a., Opgravingen in Amsterdam - 20 jaar stadskernonderzoek, Amsterdam 1977

J.M. Baart, Mittelalterliche Holzfunde aus Amsterdam, in: ZAM 10/1982 W. Barner, Ein kupfertauschiertes Messer aus dem Wall am Dörhai, Krs. Alfeld, in: NNU 351966

W. Barner, Stand und Aufgabe der Forschung im Burgenbereich der hohen Schanze, in: NNU 39-1970

R. Barnycz-Gupieniec, Wyniki Nowych Prac Archeologicznych Na Stanowisku 4, in: Gdańsk Wczesnośredniowieczny VI, Gdańsk 1967

H.-J. Barthel, Der Große Herrmannstein bei Manebach, in: Alt-Thüringen 10-1968/69

H.-J. Barthel u.a., Eine mittelalterliche Produktionsstätte für Knochenspielwürfel, in: AltThüringen 16-1979 
J. Bartys, Wczesnohistoryczne Cmentarżysko Skieletowe We Wsi Samborzec, Pow. Sandomierz, in: Prz. Arch. Tom V, Rok 1933-1936, Poznań1936

H. Bauer, Tisch und Tafel in alten Zeiten, Leipzig o.J.

W. Bauer, Die Funde, in: K. Maurer, Burg Wartenberg bei Angersbach, in: Prähistorische Zeitschrift 39-1961

G. Bechthold, Zur Geschichte der Stadt Essen, in: Führer zu vor- und frühgeschichtlichen Denkmälern Band 15, Mainz 1969

D. Becker, Die slawische Inselsiedlung im Trenntsee, Krs. Sternberg, in: Bod. Meck. Jb. 1980, Berlin 1981

O. Beigbeder, Elfenbein, Frankfurt/Main o.J.

M. Bencard, Om et middelalderligt knivskaft fra Ribe, in: Festschrift H.K. Kristensen, Ribe Amt 1975

M. Bencard, Om Ribes Rådhus, in: hikuin 3-1977, S. 59-80

M. Bencard, Ribe durch tausend Jahre, Esbjerg 1978

G. Benker, Alte Bestecke, München 1978

R. Berge, Norsk Bondesylv, Oslo 1975

F. Białęcka u.a., Badania Archeologiczne Na Zawodziu W Kaliszu, in: Spr. Arch. XIII1961

F. Białęcka, Sprawozdanie Z Badań Archeologicznych W Koscielnej Wsi, Pow. Kalisz, in: Spr. Arch. XIII-1961

G.H. Bidermann, Burg Hornberg - Rüstzeugschau 1980, Schwäbisch-Hall 1980

J. Billberg, Kniv med Figurer, in: Malmöya Nr. 2, Malmö 1979

W. Błaszczyk, Wyniki Badań Arkeologicznych W Stretie Osady Šw. Gotarda Na Starym Miešcie W Poznaniu, in: F.A.P. XXII-1971, Poznań 1972

W. Błaszczyk, Die Anfänge der polnischen Städte im Lichte der Bodenforschung, Begleitheft zur gleichnamigen Ausstellung im Archäologischen Museum Poznań, Poznań 1974

W. Bleicher, Die Burg auf dem Raffenberg, in: Museumsführer Hohenlimburg, Raffenberg-Piepenbrink, Heft 1-1979

W. Bleicher, Die Abteilung Ur- und Frühgeschichte, in: Museum Hohenlimburg, Museumsführer und Auswahlkatalog, Hagen 1980

W. Bleicher, Fundbericht Nr. 34 der Stadt Hagen, Hagen-Holthausen, in: Ausgrabungen und Funde in Westfalen-Lippe 1-1983, S. 149-150

W. Bleicher, Neufunde von der Raffenburg, in: Hohenlimburger Heimatblätter 7-1984

G.A. Blom, Die Hanseaten in Oslo und Tønsberg, in: Hanse in Europa, Katalog und Begleitband zur gleichnamigen Ausstellung in der Kunsthalle Köln, Köln 1973

N. Blomqvist, Der marktökonomische Durchbruch und der frühe Urbanisierungsprozeß: Das Kalmarsundgebiet als peripheres Beispiel, in: Lübecker Schriften zur Archäologie und Kulturgeschichte 7-1983, S. 203-211

R. Blomqvist / A.W. Mårtensson, Thulegrävningen 1961, Lund 1963 
W. Boeheim, Handbuch der Waffenkunde, Leipzig 1890

M. Bogucka, Das alte Polen, Leipzig-Jena-Berlin 1983

A. Bolta u.a., Polsko-Jugosławiañskie Badania Archeologiczne W Powiecie Kaliszkim, in: Spr. Arch. XV-1963

H.-J. Brachmann, Die Wallburg "Der Kessel" von Kretzschau-Groitzschen, Krs. Zeitz, in: Siedlung, Burg und Stadt, Festschrift P. Grimm, Berlin 1969

J. Bracker und A. Fahl, Vorbericht zu den Schiffsfunden in Wittenbergen, in: Beiträge zur deutschen Volks- und Altertumskunde Bd. 21, Hamburg 1983

A. Brackmann / W. Unverzagt, Zantoch, eine Burg im deutschen Osten I, Leipzig 1936

T. Breivik, Tollekniven, Oslo o.J.

J.A. Brongers / H.F. Wijnman, Chronological Classification of Roemers with the Help of $17^{\text {th }}$ Century Paintings in the Low Countries, in: Rott. Pap. I-1968

O. Brunner, Europäisches und Russisches Bürgertum, in: H. Stoob (Hsg.), Altständisches Bürgertum I, Darmstadt 1978

V. Bulkin u.a., Novgorod, Leningrad 1984

K. Bund, Ausstellungsübersicht im Katalog der gleichnamigen Ausstellung "Hanse in Europa" in der Kunsthalle Köln, Köln 1973

H. Busch, B. Lohse (Hsg.), Gotische Plastik in Europa, Frankfurt/Main 1962, darin: H. Weigert, Elfenbeine (S. XXII ff)

R. Busch, Skelettgräberfeld Woltwiesche, Krs. Peine, in: Frühgeschichtliche Funde aus dem Braunschweiger Land, Veröffentlichungen des Braunschweiger Landesmuseums 6, Göttingen 1976

M. Cabalsku, Tuligłowy, in: Recherches Archeologiques de 1979, Kraków 1980

W. Campe, Baggerfunde aus dem Unterlauf der Recknitz, in: Bod. Meck. Jb. 1973, Berlin 1974

T. Capelle, Die frühgeschichtlichen Metallfunde von Domburg auf Walcheren, in: Nederlandse Oudheden 5, ROB Amersfoort o.J.

U. Cappe, Ruine Neideck in Arnstadt, in: Alt-Thüringen 15-1978

E.M. Carus-Wilson, Die Hanse und England, in: Hanse in Europa, Katalog und Begleitband zur gleichnamigen Ausstellung in der Kunsthalle Köln, Köln 1973, S. 87106

J. Cherry, Medieval Metal Finds from Lübeck, in: Lübecker Schriften zur Archäologie und Kulturgeschichte 3-1980

A. Chmielowska u.a., Sieradz Wśredniowieczu, in: P.i.M. 7, Łodż 1962 (darin v.a. über Stanowisku 1: J. Kamińska)

A. Chmielowska, Z Badań Nad Wczesnośredniowiecznym Kompleksem Osadniczym W Rozprzy, Pow. Piotrków Tribunalski, in: P.i.M. 13-1966

A.F. Christensen, Tollekniven gjennom Tidene, Handzettel des Bryggens Museum Bergen, Bergen 1983

H. Christie, Old Oslo, in: Medieval Archaeology 10-1966, London 1967, S. 45-58 
E. Cnotliwy, Z Badań Nad Rzemioslem, Zajmujacym Sie Obrobka Rogu I Kosci Na Pomorzu Zachodnim, in: Mat. Zach. Pom. II-1956

E. Cnotliwy, Wczesnośredniowieczne Przedmioty Z Rogu I Kosci Z Wolina, in: Mat.Zach. Pom. IV-1958

E. Cnotliwy, Wyniki Badań Archeologiznych Na Osadzie Wczesnośredniowiecznej W Gardżu, Pow. Kamień Pom., in: Mat. Zach. XII-1966

E. Cnotliwy, R. Rogosz, Badania Archeologiczne Na Wzgórzu Zamkowym W Szczecine W Latach 1967-68, in: Spr. Arch. XXII-1970

W. Coblenz, Ausgrabungen auf dem Burgberg Meißen - Vorbericht 1959, in: A.u.F. 5-1960

W. Coblenz, Die slawische Sumpfschanze von Brohna, Berlin 1969

Codex Manesse, Die Miniaturen der großen Heidelberger Liederhandschrift, Frankfurt/Main 1988 (siehe: I.F. Walther)

A. Cofta, Wyniki Badań Na Grodzisku Wczesnośredniowiecznym W Błoniu, in: Mat. W. III-1951

A. Cofta-Broniewska, Przeszłośč Kruszwicy W Šwietle Badań, in: Spr. Arch. XIV-1962

A. Cofta-Broniewska, Zaplecze Gospodarcze Konwentu Oo. Franciszkanów W Inowrocławiu, Poznań 1979

W. La-Cour, Näsholm, København 1961

J. Cowgill, M. de Neergaard, N. Griffiths, Knives and scabbards, Medieval finds from excarvations in London I, London 1987

J.D. Cowen, The Southwark Knife Reconsidered, in: The Antiquaries Journal LI-1971

I./K. Dąbrowscy / R. Kozłowska, Badania Archeologiczne Na Zawodziu W Kaliszu, in: Spr. Arch. XVI-1964

E. Dąbrowska, Osadnictwo Wczesnośredniowieczne Na Terenie Powiatu Krakowskiego, in: P.A. 4-1962

E. Dąbrowska, Sprawozdania Z Badań Sondazowych Przeprowadzonych Na Grodzisku Wczesnośredniowiecznym W Demblinie, Pow. Dąbrowa Tarnowska, in: Spr. Arch. XVII-1965

I. Dąbrowska u.a., Materiały Wczesnośredniowieczne Z Kalisza I Piwonic, in: Mat. W. V1960

Das Reich der Salier, Katalog zur rheinland-pfälzischen Landesausstellung in Speyer 1992, Sigmaringen 1992, dort S. 81 ff: B. Theune-Großkopf, Bewaffnung und Reiterzubehör (Katalogtexte zu den ausgestellten Schwertern)

O.I. Davidan, Grebni Staroj Ladogi, in: Sbornik-4, Leningrad 1962

K. Dębska-Luty, Badania Wykopaliskowe W Poznaniu Na Posesij Przy Ul. Szewskiej 6, W Roku 1960, in: F.A.P. XXII-1971, Poznań 1972

F. Deuchler, Gotik, Stuttgart 1976

Die Kelten in Mitteleuropa, Katalog der Salzburger Landesausstellung 1980 in Hallein, Salzburg 1980 
L. Diemer, Baggerfunde aus Peene, Tollense und Treben, in: Bod. Meck. Jb. 1955, Rostock 1957

Die Zeit der Staufer, Katalog der Ausstellung im Württembergischen Landesmuseum Stuttgart Bd. 2, Stuttgart 1977

Dokumentation zur Archäologie Niedersachsens in Denkmalpflege und Forschung (H.G. Peters, red.), Hannover 1975

$\mathrm{Ph}$. Dollinger, Die Hanse, in: Hanse in Europa, Katalog und Begleitband zur gleichnamigen Ausstellung, Köln 1973, S. 19-38

Ph. Dollinger, Die deutschen Städte im Mittelalter, in: H. Stoob (Hsg.), Altständisches Bürgertum Bd. II, Darmstadt 1978, S. 269-300

Ph. Dollinger, Die Hanse ${ }^{(4)}$, Stuttgart 1989

A. Dorgeloh, Het Oude Bischopshof te Deventer, in: BROB 7-1956

P. Donat, Die Mecklenburg, in: Schriften zur Ur- und Frühgeschichte 37, Berlin 1984

E. Donnert, Das Kiewer Rußland, Leipzig 1983

H. Drescher, Die mittelalterlichen Funde von den Kirchplätzen in Hitfeld, HamburgSinstorf und Hamburg-Wilstorf, in: Harburger Jahrbücher 1968/72, HamburgHarburg 1973

H. Drescher, Messerbeschläge aus Hanseschalenblech, in: ZAM 3-1975

A. Dymaczewski, Badania Wykopaliskowe W Ogrodzie Przy Ul. Wieżowej 2-4 W Poznaniu W Latach 1939, 1950-53, in: Poznań We Wczesnym Šredniowieczu Tom III-1961, Wrocław/Warszawa 1961

W. Dzieduszycki / J. Fogel, Gród Wczesnośredniowieczny W Šriemie, in: Sl. Ant. XXVI1979

R. Ekre, Lödöse - Stadt der Faktoreien, in: Hanse in Europa, Katalog und Begleitband zur gleichnamigen Ausstellung in der Kunsthalle Köln, Köln 1973

N. Elias, Über den Prozeß der Zivilisation, 2 Bände, Frankfurt/Main) $1980^{(7)}$

D. Ellger (Hsg.), Denkmalpflege und Forschung in Westfalen 2, Beiträge zur archäologischen Burgenforschung und zur Keramik des Mittelalters in Westfalen, Bonn 1979

W. Elling, Siedlungsfunde im Kreis Borken 800-1900, Vreden 1978

C. Engelhardt, Nydam Mosefund, København 1865

C. Engelhardt, Kragehul Mosefund, København 1867

C. Engelhardt, Vimose Fundet, København 1869

S. Epperlein, Der Bauer im Bild des Mittelalters, Berlin 1975

J. Ericsson, Futterkamp Bd. I, Neumünster 1981

W.A. van Es / W.J.H. Verwers, Excavations at Dorestad 1_ The Harbour: Hoogstraat 1, in: Nederlandse Oudheden 9, ROB Amersfoort $19 \overline{80}$

H. Eversberg, Eisenverhüttung und Eisenverarbeitung in der Burg Isenberg in Hattingen a.d. Ruhr, Hattingen 1982 
A. Falk, Metallfunde aus der Lübecker Innenstadt, in: Archäologie in Lübeck, Hefte zur Kunst- und Kulturgeschichte der Hansestadt Lübeck 3-1980

A. Falk, Knochengeräte des späten Mittelalters und der frühen Neuzeit, in: Zeitschrift des Vereins für Lübecker Geschichte und Altertumskunde 63-1983

A. Falk, Mittelalterlicher Hausrat, in: Hausbau in Lübeck, Jahrbuch für Hausforschung 351986

G. Fehring, Einführung in die Archäologie des Mittelalters, Darmstadt 1978

R. Feustel u.a., Die wüste Kapelle Glasbach am Rennsteig, in: Alt-Thüringen 5-1961

W. Filipowiak, Port Wczesnośredniowiecznego Wolina, in: Mat. Zach. II-1956

W. Filipowiak, Kamień Wczesnodziejowy, Szczecin 1959

W. Filipowiak, Der Götzentempel von Wolin, Kult und Magie, in: Beiträge zur Ur- und Frühgeschichte II (Festschrift W. Coblenz), Berlin 1982

H. Fillitz, Elfenbein, in: Die Zeit der Staufer, Katalog der Ausstellung im Württembergischen Landesmuseum Stuttgart Bd. 1, Stuttgart 1977, S. 483-494

W.A. Forbes, Ontwikkelingsfasen in het Middeleeuws bestek, in: Rott. Pap. II-1975

H.G. Franz, Spätromanik und Frühgotik, Baden-Baden 1969

F. Fuhse, Schmiede und verwandte Gewerbe in der Stadt Braunschweig, Leipzig 1930

F. Fuhse, Handwerksaltertümer, Braunschweig 1935

D. Gaborit-Chopin, Elfenbeinkunst im Mittelalter, Fribourg 1978

L. Gajewski, / J. Gurba, Wczesnośredniowieczny Zespół Osadniczy Na Terenach Wsi Spiczyni I Kijany, Pow. Lubartów, in: Spr. Arch. XI-1960

J. Gąssowski, Cmentarzysko W Koñskich, in: Mat. W. II-1950

W. Gebers, Ausgrabungen in der Siedlungskammer Bosau, in: Archäologisches Korrespondenzblatt 4-1974

B. Gediga, Badania Wykopaliskowe Na Ostrówku Opolu, in: Spr. Arch. XVIII-1966

B. Gediga, Wyniki Badań Na Ostrówku W Opolu W Latach 1964 I 1965, in: Spr. Arch. XIX-1968

B. Gediga, Początki I Roźwój Wczesnośredniowiecznego Ośrodka Miejskiego Na Ostrówsku W Opolu, in: Sl. Ant. XVI-1969

B. Gediga, Wyniki Archeologicznych Badań Wczesnośredniowiecznego Opola W 1966 Roku, in: Spr. Arch. XX-1969

B. Gedl, Zbrojewsko, district of Kłobuck, site 1 (A Medieval Motte), in: Recherches Archéologiques De 1974, Kraków 1976

B. Gedl, Zbrojewsko, district of Kłobuck, in: Recherches Archéologiques De 1974, Kraków 1976

R. Gensen u.a., Der Burgwall "Hünenkeller" bei Korbach-Lengenfeld, Krs. Waldeck, in: Fundberichte aus Hessen 13-1973

B. Glaub, Das Messer, Besteckmuseum Bodo Glaub, Köln o.J.

P. Glazema, Oudheidkundige Opgravingen te Tiel, in: BROB 1-3, 1950/51, Jg. I/II 
Z. Glowacki, Uwagi Na Temat Technologii Wykonania Zabytkow Metalowych Z XI-XIII W.Z. Ostrowa Tumskiego W Poznaniu, in: Poznań We Wczwsnym Šredniowiecu III-1961

A. Goldschmidt, Die Elfenbeinskulpturen aus der romanischen Zeit, Band 3 und 4, Berlin 1923,1926

J. Górska u.a., Grodziska Mazowza I Podlasia, Wrocław-Warszawa-Kraków-Gdańsk 1976

A. Grabois, Illustrierte Enzyklopädie des Mittelalters, Athenäum 1981

J. Graham-Campbell, Das Leben der Wikinger, Berlin/Hamburg 1980

R. Grenz, Die slawischen Funde aus dem hannoverschen Wendtland, in: Göttinger Schriften zur Vor- und Frühgeschichte 2-1961

S. Grieg, Middelalderske Byfund fra Bergen og Oslo, Oslo 1933

H.G. Griep, Ausgrabungen und Bodenfunde im Stadtgebiet Goslar, in: Harz-Zeitschrift 24/25-1972/73

P. Grimm, Hohenrode, eine mittelalterliche Siedlung im Südharz, Halle/Saale 1939

P.Grimm, Beiträge zu Handwerk und Handel in der Vorburg der Pfalz Tilleda, in: Zeitschrift für Archäologie 6-1972, S. 104 ff

P. Grimm, Die untere Vorburg der Pfalz Tilleda, in: Zeitschrift für Archäologie 10-1976, S. $261 \mathrm{ff}$

P. Grimm, Tilleda - Eine Königspfalz am Kyffhäuser Teil 2 Die Vorburg und Zusammenfassung, Berlin 1990

G. Groedilov, Raskopki Drewnego Pskova, in: Sbornik 4-1962, Leningrad 1962

E. Grohne, Bremische Boden- und Baggerfunde, Jahresschrift des Fokke-Museums, Bremen 1929

K. Grote, Bengerode, Ein spätmittelalterlicher Töpferort bei Fredersloh im südlichen Niedersachsen, in: NNU 45-1976

H. Grundmann, Wahlkönigtum, Territorialpolitik und Ostbewegung im 13. und 14. Jahrhundert, Gebhard, Handbuch der deutschen Geschichte ${ }^{(9)}$, Band 5, Stuttgart 1975

F.D. Gurjevitsch, Drewnije Novogrudok, Leningrad 1981

R. Haarberg, Bericht über die Funde bei der Grabung der Burg Rödersen, Krs. Wolfhagen, in: Fundberichte aus Hessen 4-1964

W. Haarnagel, Emden - Handelssiedlung, in: Friesisches Jahrbuch 30-1955

H.-U. Haedeke, Bestecke (Deutsches Klingenmuseum Solingen), Köln-Bonn 1981

K.-D. Hahn, Grabung Königstraße 59-63, Lübeck, Katalog der Kleinfunde, in: Lübecker Schriften 1-1978

D.P. Hallewas, Een Gat in de Breestraat te Leiden, in: Bodemonderzoek in Leiden 4-1981

(82), erschienen als ROB Publicatie 310

Handbook of Bryggens Museum (to the cultural history of the Middle Ages), Bergen 1978

D.B. Harden, Table Glass in the Middle Ages, in: Rott. Pap. II-1975

E. Heidrich, Alt-Niederländische Malerei, Jena 1924 
A. Helferich-Dörner, Messer, Löffel, Gabel - Zur Kulturgeschichte des Bestecks, Schwäbisch-Hall 1959

F.-W. Henning, Mittelalterliche Handelsgüter, in: Köln-Westfalen 1180-1980, Landesgeschichte zwischen Rhein und Weser, Bd. I des Ausstellungskatalogs der gleichnamigen Ausstellung in Münster und Köln, Münster 1980, S. 329-337

W. Hensel, Les investigations sur l'origine de l'Etat polonais (résumé), in: Sl. Ant. IV-1953

W. Hensel, Słowiaciszczyzna Wczesnośredniowieczna, Warszawa 1956

W. Hensel, Najdawniejsze Stolice Polski, Warszawa 1960

W. Hensel, Archeologica O Poczatkach Miast Słowiañskich, Wrocław/Warszawa/Kraków 1963

W. Hensel, Anfänge der Städte bei den Ost- und Westslawen, Bautzen 1967

W. Hensel, Ur- und Frühgeschichte Polens, Bd. II, Berlin 1974

Z. Hensel, Badania Metaloznawcze Wyróbow Źelaznych Z IX-XII Wieku Z Wolina, in: Mat. Zach. XXI-1975

J. Herrmann, Die Ausgrabungen in der Altstadt von Köpenick, in: Ausgrabungen und Funde 3-1958, Heft 3

J. Herrmann, Ergebnisse der archäologischen Stadtkernuntersuchungen in Berlin, Bd. 15: Köpenick, Berlin 1962

J. Herrmann, Feldberg, Rethra und das Problem der Wilzischen Höhenburgen, in: Sl. Ant. XVI-1969

J. Herrmann, Zwischen Hradschin und Vineta, Leipzig 1971

J. Herrmann (Hsg.), Die Slawen in Deutschland, Berlin 1974

J. Herrmann, Wikinger und Slawen, Neumünster 1982

H. Herrnbrodt, Der Husterknupp, Köln/Graz 1958

Z. Hilczerowna, Rogownictwo Gdańskie W X-XIV Wieku, in: Gdańsk Wczesnośredniowieczny IV, Gdańsk 1961

H. Hingst, Vor- und frügeschichtliche Eisenverhüttung in SchleswigHolstein, in: W. Krämer (Hsg.), Neue Ausgrabungen in Deutschland, Berlin 1958, S. 258-267

H. Hingst, Ein Eisenverhüttungsrevier im Staatsforst Flensburg, in: K. H. Otto und J. Herrmann (Hsg.), Siedlung, Burg und Stadt (Band 25 der Schriften der Deutschen Akademie der Wissenschaften Berlin, Sektion für Vor- und Frühgeschichte), Berlin 1969, S. 423-437

H. Hingst, Haneburg, eine mittelalterliche Burg in Westerohrstedt, in: Offa 30-1973

H. Hinz, Die Ausgrabungen auf dem Kirchberg in Morken, Krs. Bergheim (Erft), in: Rheinische Ausgrabungen 7, Düsseldorf 1969

H. Hinz, Archäologische Beobachtungen in der Altstadt von Kiel, in: Offa 28-1971

S. Hoczyk-Sikowa, Sprawozdania Z Badań Lublina Przedlokaczyjnego, in: Spr. Arch. XXVI-1974

F. Hohenschwert, Ur- und frühgeschichtliche Befestigungen in Lippe, Münster 1978 
F. Hohenschwert, (Fundmeldungen aus Barntrup): Brunnenfund in der Stadt Barntrup, in: Ausgrabungen und Funde in Westfalen-Lippe Jg. 2-1984

A. Hollnagel, Burg und Wik von Marlow, Krs. Ribnitz-Damgarten, in: Bod. Meck. 1973, Berlin 1974

W. Holtmann, Die Münzen, Marken, Plomben und Gewichte vom Coesfelder Markt, in: Geschichtsblätter des Kreises Coesfeld 1980, Heft 1/2

H.C. Holubowiczowa, Slowiansko-Wareskie Cmentarzysko Kuranowe Kolo Porzecza W Pow. Dziśnieńskim, in: Prz. Arch. Tom VI, Lata 1937-1939, Poznań 1939

W. Holubowicz, Prace Wykopaliskowe Na Ostrówku W Opolu W 1954 Roku, in: Spr. Arch. I-1955

W. Holubowicz, Opole W Wiekach X-XII, Wydawnictwo "Šląsk", Katowice 1956

W. Holubowicz, Z Badań Na Ostrówku W Opolu W 1957 Roku, in: Spr. Arch. IX-1960

K. Hoyer, Das ländliche Gastwirtsgewerbe im deutschen Mittelalter, Oldenburg 1910

W. Hübener, Die römischen Metallfunde von Augsburg-Oberhausen, Kallmünz/Opf. 1973

Hürliman/Clemen/Meyer, Gotische Kathedralen in Frankreich, Zürich 1954

M. Hundt, Das karolingische Reihengräberfeld von Felkendorf-Kleetzhöfe, in: Die Plassenburg 6-1953

G. Isenberg, Ausgrabungen auf dem Coesfelder Marktplatz - Grabungsbefund, in: Jahrbuch des Kreises Coesfeld 1981

R. Jamka u.a., Badania Wykopaliskowe W Piekarach W Fowiecie Krakowski, Kraków 1939

H. Jankuhn, Deutsche Agrargeschichte (I), Stuttgart 1969

S. Janosz, Wczesnośredniowieczna Półziemianka W Górze, Pow. Poznański, in: Prz. Arch. XII, Rocznik 34-1959, Wrocław 1960

W. Janssen, Handwerksbetriebe und Werkstätten in der Stadt um 1200, in: H. Steuer (Hsg.), Zur Lebensweise in der Stadt um 1200, ZAM Beiheft 4-1986, S. 301378

W. Janssen / H. Müller-Wille, Das Fundmaterial der Grabungen der Niederungsburg bei Haus Meer, in: Rheinische Ausgrabungen I, Köln 1968

D. Jaskanis, Materiały Z Badań Wczesnośredniowiecznego Cmentarzyska W Miejscowości Podroś Koło Wołkowyska W BSRR, in: Rocznik Białostocki Tom IV-1963

K. Jażdżwewski, Cmentarzysko Wczesnośredniowieczne W Lutomiersku Pod Łodzią W Świetle Badań, in: Mat. W. I-1949

K. Jazdżewski / W. Chmielewski, Gdańsk Wczesnośredniowieczny W Swietle Badań Wykopaliskowych Z Lat 1948/49, in: St. W. 1-1952

K. Jazdżewski, Wczesnośredniowieczne Osadnictwo Miasta Włocławka I Jego Najblizszej Okoliaj, in: Mat. W. IV-1956

A. Jodłowski, Wyniki Badań Archeologicznych Przeprowadzonych Na Terenie Wieliczki W 1965 Roku, in: Spr. Arch. XIX-1968 
A. Jodłowski, Wczesnośredniowieczny Gród W Łapczycy, Pow. Bochnia, W Swietle Badań Lat 1965-67, in: Spr. Arch. XX-1969

O. Johannsen, Geschichte des Eisens, Düsseldorf 1924

P. Johannsen, Die Kaufmannskirche im Ostseegebiet, in: H. Stoob (Hsg.), Altständisches Bürgertum II, Darmstadt 1978, S. 301-335

E. Kahrs, Aus Essens Vor- und Frühgeschichte, Essen 1949

J. Kamińska, Grodziska Stożkowate Šladem Posiadłości Rycerskich XIII-XIV Wieku, in: P.i.M. 13-1966

J. Każmierczyk, Sprawozdania Z Badań Wykopaliskowe Na Terenie Prawobrzeżnego Opola W Latach 1952, 53 I 55, in: Spr. Arch. VI-1959

J. Każmierczyk, Z Badań Lewobrzeżnego Wrocławia, in: Spr. Arch. XIII-1961

J. Każmierczyk, / J. Lodowski, Z Badań W Regionie Placu Nowy Targ We Wrocławiu W Latach 1960-61, in: Spr. Arch. XV-1963

J. Każmierczyk, Z Badań Niemczy Šląskiej, in: Spr. Arch. XVII-1965

H. Keiling, Kurze Fundberichte 1980, Bezirk Schwerin, in: Bod. Meck. Jb. 1981, Berlin 1982

E. Kjersgaard, Mad og Ø1 i Danmarks Middelalder, København 1978

J. Klein, Alte Siedlungsspuren im Ortskern von Senden, in: Geschichtsblätter des Kreises Coesfeld 6-1981, Heft 1/2

Kloster Tom Roden, Ausstellungskatalog des Westfälischen Museumsamtes und Westfälischen Museums für Archäologie, Münster1982

H.A. Knorr, Messer und Dolch, in: Veröffentlichungen für Ur- und Frühgeschichte, Potsdam 6-1971

W. Kóčka / E. Ostrowska, Prace Wykopaliskowe We Wrocławiu W Latach 1949-51, in: St. W. 3-1955

W. Kóčka / E. Ostrowska, Wyniki Prac Wykopaliskowich We Wrocławiu Na Ostrowie Tumskim W Latach 1954, in: Spr. Arch. II-1956

R. Koechlin, Les Ivoires Gothiques Francais, 3 Bände, Paris 1924

Köln-Westfalen 1180-1980, Bd. 2 des Ausstellungskatalogs der gleichnamigen Ausstellung in Münster und Köln, Münster 1980

B.A. Koltschin, Cernaja Metallurgija I Metalloobrabotka V Drewnej Rusj, in: Materiali I Issledovanija Po Archeologii SSSR-32 (Domongolskij Perjod), Mockwa 1953

B.A. Koltschin, Gelesoobrabatiwajuschtsche Remeslo Novgoroda Welnikogo, in: Arzichowskij/Koltschin (II) 1959

B.A. Koltschin, Novgorodskie Drewnosti, Teil 2, Mockwa 1971

J. Kostrzewski (red.), Gród Prasłowiański W Biskupinie, Poznań 1938 J. Kostrzewski, Pradzieje Polski, Wrocław 1965

J. Kostrzewski, Kultura Prapolska, Warschau 1962

M. Krogstad / E. Schia, Vandring i Gamlebyen, Oslo 1982

H. Kühnel (Hsg.), Alltag im Spätmittelalter, Graz-Wien-Köln 1984 
L. Kunicka-Okuliczowa, Wczesnośredniowieczne Zabawki I Gry Z Gdańska, in: Gdańsk Wczesnośredniowieczny I, Gdańsk 1959

E. Kuszewska, Wczesnośredniowieczne Cmentarzysko W Kałdusie, Pow. Chełmno, in: P.i.M. 5-1960

E. Kuszewska, Gorzędziej Wczesnośredniowieczny W Šwietle Wstepnych Prac Archeologicznych, in: Gdańsk Wczesnośredniowieczny IV, Gdańsk 1961

E. Kuszewska, Tymczasowie Sprawozdanie Z Badań Przeprowadzonych W 1965 Roku Na Grodzisku Sredniowiecznym W Klasztorku, Pow. Kwidzyn, in: Spr. Arch. XIX-1968

E. Kuszewska, Sprawozdania Z Badań Wykopaliskowych Na Wczesnośredniowiecznym Grodzisku W Szczaworyżn, Pow. Busko, in: Spr. Arch. XX-1969

E. Kuszewska, Wyniki Badań Na Grodzisku W Klasztorku W 1966 Roku, in: Spr. Arch. XX-1969

W. Lampe, Baggerfunde aus dem Unterlauf der Recknitz, in: Bod. Meck. 1973, Berlin 1974

G.S. Lebedev, Der slawische Burgwall Gorodec bei Luga (UdSSR), in: Beiträge zur Urund Frühgeschichte II (Festschrift W. Coblenz), Berlin 1982

L. Leciejewicz, O Pochodzeniu Rogowej Rzeźby Ze Wzgórza Zamkowego W Szczecinie, in: St. Arch. Pom. 12-1974

T. Lenkiewicz, Wyniki Observacji Wykopów Przypadkowych I Archeologicznych Badań Sondażowych Prowadzonych Na Terenie Klasztoru Norbertanek Na Zwierzyńcu W Krakowie, in: P.A. 4-1962

B. Lepówna, Gdańsk im 10. bis 13. Jahrhundert, in: Hanse in Europa, Katalog und Begleitband der gleichnamigen Ausstellung in der Kunsthalle Köln, Köln 1973

N.-K. Liebgott, En Gruppe Nyredolke fra Randers-Egnen, in: hikuin 3-1977

N.-K. Liebgott, Stakhavn, Arkäologiske Undersøgelser i senmiddelalderens Dragør, København 1979

A.D. Linde, Vondsten uit de Voorst, in: J.G.N. Renaud u.a., Het Kasteel Voorst, Zwolle 1983

L. Lindenschmitt, Die Alterthümer unserer heidnischen Vorzeit, Mainz 1870

L. Lindenschmitt (Hsg.), Das Römisch-Germanische Central-Museum, Mainz 1889

J. Lindt / J. Brendalsmo, Funn fra en Utgravning (Tønsberg), Akadem. Forlag 1983

U. Lobbedey, Die Geschichte der Pfarrkirche zu Albersloh, in: Westfalen 50-1972

U. Lobbedey, Funde von der Burg Isenberg in Hattingen, in: Westfalen 61-1983, I

G. Loewe, Archäologische Funde und Denkmäler des Rheinlandes III, Krs. KempenKrefeld, Düsseldorf 1971

C.D. Long, Excavations in the Medieval City of Trondheim, Norway, in: Medieval Archeology 19, London 1975

W. Łosiński, Wczesnośredniowieczne Cmentarzysko W Cewlinie, Pow. Koszalin, in: Mat. Zach. IV-1958 
W. Łosiński, E. Tabaczyńska, Z Badań Nad Rzemiosłem We Wczesnośredniowiecznu Kołobrzegu, Poznań 1959

W. Łosiński, Sprawozdanie Z Badań Archeologicznych Ekspedycji Wykopaliskowej IHKM Pan W Šwielubiu I Bardach, Pow. Kołobrzeg, in: Spr. Arch. XVI-1964

R. Maczijewski, Spandauer Altstadtgrabung Lindenufer, in: Ausgrabungen in Berlin 31972, Berlin 1973

K. Maier, Kloster Wienhausen Bd. 1, Wienhausen 1972

M. Malinowska, Badańia Na Stanowisku Ostrów Tumski 17 W Poznaniu W Latach 195354, in: Poznań We Wczesnym Sredniowieczu Tom III-1961, Wrocław/Warszawa 1961

M. Malinowska, Sprawozdanie Z Archeologcznych Prac Wykopaliskowych Na Placu Katedralnym Na Ostrowie Tumskim W Poznaniu, in: Spr. Arch XIII-1961

G. Mangelsdorf, Untersuchungen auf der Wüstung Göritz bei Rädel, Krs. Brandenburg, in: A.u.F. 28-1983, Heft 2

J. Marciniak, Tymczasowe Wyniki Badań Przeprowadzonych Na Cmentarzysku Wczesnohistorycznem W Strzemieszycach Wielkich, in: Prz. Arch. Tom IV Rok 1928/32, Poznań 1933

J. Marciniak, Wczesnośredniowieczne Zespół Osadniczy Z Miejscowośki Bazar Nowy Pow. Maków Masowiecki, in: Mat. W. V-1960

A. Martensson / C. Wahlöö, Lyndafund, Karlshamn 1970 (ersch. als Bd. IV der Reihe Archäologica Lundensia)

A.W. Martensson, Uppgrävt Förflutet för PK-Banken i Lund, Malmö 1976 (ersch. als Bd. VII der Reihe Archaeologica Lundensia)

Masterpieces of Cutlery and the Art of Eating, Ausstellungskatalog der gleichnamigen Ausstellung im Victoria \& Albert Museum, London 1979

Mat og Drikke i middelalderen, Bryggens Museum (Beiheft) 1980

P.W. Meister (u.a), Messer, Löffel, Gabel, Museum für Kunsthandwerk Frankfurt, Frankfurt/Main 1965

W. Meyer und E. Widmer, Das große Burgenbuch der Schweiz, Zürich 1978

W. Meyer und E. Lessing, Deutsche Ritter, Deutsche Burgen, Sonderausgabe des Bertelsmann-Verlages, München 1984

E. Mittler / W. Werner (Hsg.), Codex Manesse, Katalog zur Ausstellung, Heidelberg 1988

H. Młynarczyk, Badania Wczesnośredniowiecznej Osady Warszawa-Wilanów, Stan. 13 "Pasieka", in: Spr. Arch. XXXII-1980

P.B. Molaug, Oslo im Mittelalter, in: Zeitschrift für Archäologie des Mittelalters 3-1975, S.217-260

D. Mührenberg, Archäologische Untersuchungen in der Hundestraße zu Lübeck, in: Lübecker Schriften zur Archäologie und Kulturgeschichte 17, Bonn 1988, S. 98-101

D. Mührenberg, Archäologische und Baugeschichtliche Untersuchungen im Handwerkerviertel zu Lübeck, in: Lübecker Schriften zur Archäologie und Kulturgeschichte 16, Bonn 1989, S. 233-290

H. Müller, Alte Helme, Katalog des Museums für Deutsche Geschichte Berlin, Berlin 1979 
M. Müller-Wille, Eisengeräte aus Haithabu, Neumünster 1973

E. Mummenhoff, Der Handwerker, Band 8 der Reihe "Die deutschen Stände in Einzeldarstellungen", Jena 1924

A. Nadolski, Prace Wykopaliskowe Na Grodzisku W Tumie Pod Łęczyca, in: St. W. 1, Wrocław 1952

A. Nadolski, Z Badań Nad Wczesnośredniowieczną Łęczycą, in: St. W. 3, Wrocław 1955

E. Naumowicz, Sprawozdanie Z Badań Archeologicznych W Ujściu, Pow. Chodziez, in: Spr. Arch. XV-1963

B. Nerman, Grobin-Seeburg, Uppsala 1958

W. Neugebauer u.a., Alt-Lübeck - ein Forschungsbericht, in: Offa 21-22/1964-65

G. Neumann, Burg Camburg an der Saale historisch und archäologisch, in: Siedlung, Burg und Stadt, Festschrift P. Grimm, Berlin 1969

A. Niesiotowska / M. Perzyńska / J. Żak, Badania Na Posesji Ostrów Tumski 13 W Latach 1950-53, in: Poznań We Wczesnym Šredniowieczu Tom II, Wrocław/Warszawa 1960

T. Nilsson, Nagot om hushallet och des inventarium, in: Uppgrävt förflutet för PK-banken i Lund (red. A.W. Mårtensson), Lund (Malmö) 1976

R. Odoj, Sprawozdanie Z Prac Wykopaliskowych Przeprowadzonych Równinie Dolnej, Pow. Kętrzyn, in: Rocznik Olsztyński I, Olsztyn 1958

R.J. Ooyevaar, Een Proef-Opgraving in het Gein, gem. Nieuwegein, in: Westerheem 26-2, 1977

E. Ostrowska, Odcrycia Archeologiczne Na Ostrowie Tumskim We Wrocławiu W 1957 Roku, in: Spr. Arch. IX-1960

E. Ostrowska, Wykopaliska Na Wyspie Tumskiej We Wrocławiu, in: Spr. Arch. XIII-1961

K. Pagel, Die Hanse, Neubearbeitung von F. Naab, Braunschweig 1983

J. Piaskowski, Technika Gdańskiego Hutnietwa I Kowalstwa żelaznego X-XII Wieku, in: Gdańsk Wczesnośredniowieczny II, Gdańsk 1960

J. Piaskowski, Mateloznawcze Badania Przedmiotow Z Krakowa-Debnik, in: Spr. Arch. XXXII-1980

R. Pirling, Die Gräberfelder von Krefeld-Gellep, in: Ausgrabungen in Deutschland Teil 2, Mainz 1975, S. 165-180

R. Pirling, Römer und Franken am Niederrhein, Mainz 1986

Ch. und F. Plate, Ein mittelalterlicher Hausgrundriss aus Dagow, Neuglobsow, Krs. Gransee, in: A.u.F. 25-1980, Heft 2

Ch. und F. Plate, Die slawische Siedlung von Tornow-Sechzehneichen, Krs. Kyritz, in: Veröffentlichungen des Museums für Ur- und Frühgeschichte Potsdam 17-1983

R. Pleiner, Zur Technik von Messerklingen aus Haithabu, in: Berichte über die Ausgrabungen in Haithabu-18, Neumünster 1983

E. Plümer, Die Wüstung Oldendorp bei Einbeck, Einbeck 1978

R. Pohl-Weber (Hsg.), Aus dem Alltag der mittelalterlichen Stadt, Handbuch zur gleichnamigen Ausstellung des Bremer Landesmuseums, Bremen 1982 
Z. Pokuta / L. Wojda, Wczesnośredniowieczne Cmentarzysko We Wsi Dębina woj. Sieradzkie, in: P.i.M. 26-1980

Z.A. Rajewski, Wielkopolskie Cmentarzyska Rzędowe Okresu Wczesnodziejowego, in: Prz. Arch. VI, Poznań 1939

M. Rauschert, Ein Fundplatz neben den slawischen Brücken im Ober-Ückersee, in: A.u.F. 22-1977, Heft 3

L. Redin, Skanör, Falsterbo und die Hanse, in: Hanse in Europa, Katalog und Begleitband zur gleichnamigen Ausstellung in der Kunsthalle Köln, Köln 1973, S. 193-208

E. Reinbacher, Beiträge zur Frügeschichte Spandaus, in: Prähistorische Zeitschrift 38-1960

H.R. Reinders u.a., Drie Schepen uit de late Middeleeuwen, Ministerie van Verkeer en Waterstaat (Hsg.), Lelystad o.J.

G. Rhode, Kleine Geschichte Polens, Darmstadt 1965

H. von Rimscha, Geschichte Rußlands, Darmstadt 1970

A. Roes, Vondsten van Dorestad, Archaeologica Traiectina VII, Groningen 1965

C. Römer, Die Hanse und die niederländische Städtewelt, in: Hanse in Europa, Katalog und Begleitband zur gleichnamigen Ausstellung in der Kunsthalle Köln, Köln 1973

R. Rogosz, Wczesnośredniowieczne Cmentarzysko Szkieletowe W Kunowie pow. Stargard Szczeciński, in: Mat. Zach X-1964

R. Rogosz, Dotychczasowe Badania Archeologiczne Nad Wczesnośredniowiecznym Stargardem, in: Mat. Zach. XII-1966

R. Rogosz, Początki Stargardu W Swietle Dotychczasowych Wyników Badań Archeologicznych, in: Mat. Zach. XIX-1973

R. Rogozińska-Gosczczyńska, Sprawozdania Z Badań Cmentarzyska Wczesnośredniowiecznego W Miejscowości Pałecznica, Pow. Proszowice, in: Spr. Arch. XVIII-1966

H. Rothert, Westfälische Geschichte (2 Bände), Nachdruck Gütersloh 1981

Rotzler, Glas im Spiegel der Kunst, in: du-438, Zürich, August 1977

M. Rulewicz u.a., Z Przesłošci Szczecina, in: Popularnonaukowa Biblioteka Archeologiczna $12-1964$

M. Rulewicz u.a., Tymczasowe Wyniki Badań Archeologicznych Na Podzamczu W Szczecinie 1975-78, in: Spr. Arch. XXXVI-1984

O. Rydbeck, Den Medeltida Borgen i Skanör, Lund 1935

H. Sarfatij, Dordrecht - deel 2, in: Spiegel Historiael 7-1972, Nr. 12

M.Sauerlandt, Werkformen deutscher Kunst, Leipzig 1926

L.E. Saurma-Jeltsch, Das stilistische Umfeld der Miniaturen, in: E. Mittler / W. Werner (Hsg.), Codex Manesse, Katalog zur Ausstellung, Heidelberg 1988, S. 302-349

D. Scheler, Kohle und Eisen im mittelalterlichen "Ruhrgebiet", in: Vergessene Zeiten Mittelalter im Ruhrgebiet, Band 2 des Katalogs der gleichnamigen Ausstellung im Ruhrlandmuseum Essen, Essen 1990, S. 111-117

E. Schia (red.), Fra Christianias Bygrunn, Riksantikvarens Skrifter 4, Oslo 1981 
G. Schiedlausky, Essen und Trinken, Tafelsitten bis zum Ausgang des Mittelalters, München 1956

R. Schindler, Die Ausgrabungen in der Hamburger Altstadt i.J. 1948, in: Hammaburg 11948/49

P. Schmidt, Das frühmittelalterliche Gräberfeld von Dunum, Krs. Wittmund, in: NAFN-5, 1970

V. Schmidt, Untersuchungen am slawischen Burgwall von Jatzke, Kreis Neubrandenburg, in: Bod. Meck. Jb. 1981, Berlin 1982

V. Schmidt, Lieps, Eine Siedlungskammer am Südende des Tollensesees, Berlin 1984

B. Schnittger / H. Rydh, Aranaes, en 1100-tals Borg i Västergötland, Stockholm 1927

U. Schoknecht, Ein Fund mittelalterlicher Waffen von Levetzow, Krs. Wismar, in: Bod. Meck. Jb. 1967, Schwerin 1969

U. Schoknecht, Bemerkenswerte mittelalterliche Neufunde aus dem Bezirk Neubrandenburg, in: Bod. Meck. Jb. 1970, Schwerin 1971

U. Schoknecht, Mecklenburgische Nierendolche und andere mittelalterliche Funde, in: Bod. Meck. Jb. 1979, Berlin 1980

U. Schoknecht, Kurze Fundberichte 1980, Bezirk Neubrandenburg, in: Bod. Meck. Jb. 1981, Berlin 1982

U. Schoknecht, Nierendolche in Mecklenburg (Teil II), in: Bod. Meck. Jb. 1982, Berlin 1983

H. Schoppmeyer, Die Hanse zwischen Ruhr und Lippe, in: Vergessene Zeiten - Mittelalter im Ruhrgebiet, Band 2 des Katalogs der gleichnamigen Ausstellung im Ruhrlandmuseum Essen, Essen 1990

S. Schütte, Funde und Befunde des Mittelalters und der frühen Neuzeit vom Markt 4 in Göttingen, in: NAFN 12-1978

S. Schütte, 5 Jahre Stadtkernarchäologie - Das neue Bild des alten Göttingen, Göttingen 1984

E. Schuldt, Altslawisches Handwerk, Ausstellung zur 800-Jahr-Feier der Stadt Schwerin Museum für Ur- und Frühgeschichte Schwerin, Schwerin 1960

E. Schuldt, Behren-Lübchin, Berlin 1965

E. Schuldt, Burg und Siedlung von Groß-Raden, Schwerin 1978

E. Schuldt, Handwerk und Gewerbe des 8. bis 12. Jahrhunderts in Mecklenburg, Schwerin 1980

E. Schuldt, Die frühslawische Befestigung von der Sternberger Burg, Krs. Sternberg, in: Bod. Meck. Jb. 1982, Berlin 1983

W. Schwabenicky, Grabung an der Wasserburg in Beerwalde, in: A.u.F. 14-1969, Heft 2

J. Selirand, Über das frühmittelalterliche Schmiedehandwerk in Estland, in: Offa 37-1980 (Festschrift H. Hinz), Neumünster 1981

K. Siuchniński, Sprawozdanie Z Badań Wykopaliskowych Na Wczesesnośredniowiecznym Grodzisku W Radaczu Pow. Szczecinek, in: Mat. Zach. VIII-1962 
Slawen und Deutsche, Begleitheft zur gleichnamigen Ausstellung im Museum für Vor- und Frühgeschichte - Staatliche Museen Preußischer Kulturbesitz in Berlin, Berlin 1983

P. Smolarek, Gdańsk, sein Handel und seine Schiffahrt vom 14. bis 17. Jahrhundert, in: Hanse in Europa, Katalog und Begleitband zur gleichnamigen Ausstellung in der Kunsthalle Köln, Köln 1973, S. 235-250

P. Sommer, Strenka ve tvaru sokolnika z Vysokého Mýta, in: Festschrift J. Filip, Praehistorica VIII - Varia Archaeologica 2, Prag 1981, S. 315-318

H. Sonnenschein u.a., Westfälisches Freilichtmuseum Technischer Kulturdenkmale Hagen (Museumsführer), Hagen 1987

R. Sprandel, Das Eisengewerbe im Mittelalter, 1968

R. Sprandel, Die Betriebsformen der Eisenproduktion in Westdeutschland in vorindustrieller Zeit, in: Vortragsreihe der Gesellschaft für westfälische Wirtschaftsgeschichte e.V., Heft 18, Dortmund 1974

E. Stahl, Die Bunkenburg, ein mittelalterlicher Ringwall bei Ahlden, Krs. SoltauFallingbostel, in: NNU 47-1978

A. Steensberg / J.L. Østergaard Christensen, Store Valby, København 1974

H.G. Steffens, Die Ausgrabungen in der Großen Reichenstraße zu Hamburg, in: Hammaburg 4-1953/54

H.-G. Stephan, Archäologische Grabungen im Handwerkerviertel der Hansestadt Lübeck, in: Lübecker Schriften zur Archäologie und Kulturgeschichte 1-1978

H. Steuer, Zum Lebensstandard in der mittelalterlichen Stadt, in: Aus dem Alltag der mittelalterlichen Stadt, Handbuch der gleichnamigen Sonderausstellung im Bremer Landesmuseum (Fokke-Museum), Bremen 1982

H. Steuer, Spiegel des täglichen Lebens - Archäologische Funde des Mittelalters aus Köln, Katalog des Kölner Stadtmuseums, Köln 1983

W. von Stromer, Konkurrenten der Hanse: Die Oberdeutschen, in: Hanse in Europa, Kata$\log$ und Begleitband zur gleichnamigen Ausstellung in der Kunsthalle Köln, Köln 1973, S. 331-340

B. Stürup, Transtraedegravningen, in: hikuin 3-1977

A. Stubavs, Kentes Pilskalns Un Apmetne, Ryga 1976

G. Svahnström, Das mittelalterliche Visby, in: Hanse in Europa, Katalog und Begleitband zur gleichnamigen Ausstellung in der Kunsthalle Köln, Köln 1973, S. 211-217

W./Z. Szafrańscy, Z Badań Nad Wczesnośredniowiecznym Osadnictwem Wiejskim W Biskupinie, Wrocław/Warszawa 1961

J. Szydłowski, Bytom Pradziejei Początki Miasta, Bytom 1966

M. Tausch, Hanse und Kunst, in: Hanse in Europa, Katalog und Begleitband zur gleichnamigen Ausstellung in der Kunsthalle Köln, Köln 1973, S. 297-300

W.D. Tempel, Die Kämme aus Haithabu, in: Berichte über die Ausgrabungen in Haithabu 4, Neumünster 1969 
W.D. Tempel, Unterschiede zwischen den Formen der Dreilagenkämme in Skandinavien und auf den friesischen Wurten vom 8.-10. Jahrhundert, in: Archäologisches Korrespondenzblatt 2-1972

W. Thieme, Eisenverhüttung in Norddeutschland, Blatt 41 des Helms-Museums (Hamburgisches Museum für Vor- und Frühgeschichte), Hamburg 1979

M.W. Thompson, Novgorod The Great, New York / Washington 1967

Thuis in de late Middeleeuwen, Ausstellungskatalog des Provinciaal Overijssels Museum, Zwolle 1980

W. Timpel, Gommerstedt, ein hochmittelalterlicher Herrensitz in Thüringen, Weimar 1982

A. Tode, Ausgrabungen im mittelalterlichen Schulenrode bei Harzburg, in: Harz-Zeitschrift $2-1950$

T. Trębaczkiewicz, Cmentarzysko Wczesnośredniowieczne We Wsi Psary, Pow. Piotrków Tribunalski, in: P.i.M. 9-1963

P. Tudor-Craig, (Ivory) Europe: Late Middle Ages, in: (Fiona St. Aubyn, Hsg.) Ivory, London 1987

G. Ulbert, Die römischen Donau-Kastelle Aislingen und Burghöfe, Berlin 1959

G. Ulbert, Das römische Donaukastell Rißtissen, Stuttgart 1970

J. Ulbricht, Einige Aspekte der Geweihverarbeitung in Haithabu, in: Archäologisches Korrespondenzblatt 7-1977

K. Ullmann, Dolchmesser, Dolche und Kurzwehren des 15. und 16. Jahrhunderts im Kernraum der Hanse, in: Waffen- und Kostümkunde 1-2 1961

K. Ullmann, Das Werk der Waffenschmiede, Schriften zur Kulturgeschichte des Rheinisch-Westfälischen Industriegebietes, Heft 11, Essen 1962

M. Untermann, Die Grabungen auf der Burg Berge-Altenberge, in: Beiträge zur Archäologie des Mittelalters III, Sonderdruck 1984

J. Vellev, Arkæologiske Undersøgelser i Randers, in: hikuin 3-1977

E.M. Vetter, Bildmotive - Vorbilder und Parallelen, in: E. Mittler / W. Werner (Hsg.), Codex Manesse, Katalog zur Ausstellung, Heidelberg 1988, S. 275-301

H.J. Vogt, Die Ausgrabungen auf der Wiprechtsburg in Groitzsch, Wurzen 1965

T. Voigt, Zwei Zeugnisse "romanischer" Kleinkunst aus Halle/Saale, in: Jahresschrift für mitteldeutsche Vorgeschichte Bd. 34, Halle/Saale 1950

N.N. Voronin, Drevnee Grodno, in: Materialy I Issledovanija Po Archeologii Drevnerysskich Gorodov Tom III, Mockwa 1954

I.F. Walther (Hsg.), Codex Manesse, Die Miniaturen der Großen Heidelberger Liederhandschrift, Frankfurt/Main 1988

D. Walz, Falkenjagd-Falkensymbolik, in: E. Mittler und W. Werner (Hsg.), Codex Manesse, Katalog zur Ausstellung, Heidelberg 1988, 350-371

N. Wand, Die Büraburg bei Fritzlar, in: Kasseler Beiträge zur Ur- und Frühgeschichte 4-1974

A. Wapińska, Wyniki Badań Archeologicznych Na Stanowisku 4 W Gdańsku, in: Gdańsk Wczesnośredniowieczny VI-1967 
J.H. Wasowicz, Die Kunst in Gdańsk vom 10. bis 17. Jahrhundert, in: Hanse in Europa, Katalog und Begleitband zur gleichnamigen Ausstellung in der Kunsthalle Köln, Köln 1973, S. 253-269

H. Weczerka, Verkehrsnetz und Handelsgüter der Hanse, in: Hanse in Europa, Katalog und Begleitband zur gleichnamigen Ausstellung in der Kunsthalle Köln, Köln 1973, S. 41-56

H. Weigert, Elfenbeine, in: H. Busch / B. Lohse (Hsg.), Gotische Plastik in Europa, Frankfurt/Main 1962, S. XXII ff

J. Werner, Das Messerpaar aus Basel-Kleinhüningen Grab 126, in: Festschrift für R. LaurBelart, Basel 1968

W. Werner, Schicksale der Handschrift, in: E. Mittler / W. Werner (Hsg.), Codex Manesse, Katalog zur Ausstellung, Heidelberg 1988, S. 1-21

K.F. Wernet, Der Messerschmied, in: H. Focke (Hsg.), Geschichte der Handwerksberufe Bd. 2, Waldshut/Baden 1960

G. Wetzel, Funde aus der Altstadt von Cottbus, in: A.u.F. 15-1970, Heft 3

B. Wiącek, Sprawozdanie Z Badań Wykopaliskowych Na Osadzie Z Okresów Rzymskiego I Wczesnośredniowiecznego W W grach, Pow. Szlum, in: Spr. Arch. XVIII1966

B. Wiącek, Sprawozdanie Z Badań Osady Z Póznego Okresu Rzymskiego I Grodziska Wczesnośredniowiecznego Na Dębiej Górze W Węgrach, Pow. Szłum, in: Spr. Arch. XX-1969

T. Wieczorowski, Sprawozdanie Z Prac Wykopaliskowych W Szczecinie W 1954 Roku, in: Spr. Arch. I-1955

G. Wiegelmann, Theorien und Methoden, in: G. Wiegelmann, M. Zender, G. Heilfürth, Volkskunde - Eine Einführung (Grundlagen der Germanistik, Bd. 12), Berlin 1977, S. 39-86

H. Wiklak, Cmentarzysko Z XII I XIII Wieku W Poddębicach, in: P.i.M. 5-1960

H. Wiklak, Osada Wczesnośredniowieczna W Stobnicy-Trzymorgach, Woj. Piotrków Trybunalski, Stan. 2, in: Spr. Arch. XXXV-1983

L. Wilhelmsson, Nördet "nappade" på Fiskaregatan, in: Malmöya Nr. 1, Malmö 1979

P. Williamson, Elfenbeinschnitzereien (Victoria u. Albert Museum, London), Bern/Stuttgart 1982

W. Winkelmann, Archäologische Zeugnisse zum frühmittelalterlichen Handwerk in Westfalen, in: Beiträge zur Frühgeschichte Westfalens, Münster 1984, S. 150-167

Wittelsbach und Bayern - Die Zeit der frühen Herzöge, Band I/II des Katalogs zur Ausstellung Wittelsbach und Bayern auf der Burg Trausnitz in Landshut, München/Zürich 1980, dort S. 166 ff: M. Junkelmann u.a., Rittertum und Kriegsführung (Katalogtexte zu den ausgestellten Schwertern)

J. Wojtasik, Przetmioty Drewniane Znalezione Na Stanowisku 4 W Wolinie, in: Mat. Zach. IX-1963

A. Wolf-Graaf, Die verborgene Geschichte der Frauenarbeit, Weinheim und Basel 1983

H. Wühr, Altes Eßgerät, Darmstadt 1961 
J. Żak, Badania W Ogrodzie Przy Ul. Ostrow Tumski 10 W 1946, in: Poznań We Wczesnym Średniowieczu, Tom I, Wrocław-Warszawa 1959

J. Żak, "Importy" Skandynawskie Na Ziemiach Zachodniosłowiańskychod IX Do XI Wieku, Poznań 1963

J. Żak, Die Frage nach dem Ursprung des Ringkettenmusters auf westslawischem Gebiet, in: Studien zur Europäischen Vor- und Frühgeschichte, Neumünster 1968

A. Żaki, Badania Nad Przedlokacyjnym Krakowem (Seria X), in: Spr. Arch. XVII-1965

A. Zbierski, Struktura Zawodowa, Spoleczna i Etniczna Ludnosci Gdańska w IX-XIII Wieku, in: P.i.M. 25-1979

P. Zbiorowa, Sprawozdanie Z Badań Na Grodzisku W Gielzu W Pow. Šredzkim Za Rok. 1949, in: H./W. Hołubowicz, Z Badań Na Šlęży W 1949 Roku, in: St. W. 11952

A. Zeischka, Grabungsbericht Burg Kakesbeck 1971/72, Teil B - Fundkatalog, Eigenverlag o.J.

K. Źurowski, Sprawozdanie Z Badań Wykopaliskowych Na Górze Lecha W Gnieźnie, in: St. W. II-1953 


\section{1}

Verbreitung der

Fundorte

im Bearbeitungsgebiet

Das Messer im nördlichen Europa

Legende:

-
0 offent

Burg
Kloster

Kloster
$* \quad$ Streufund u. Fund aus Schiffsyrach
$+\quad$ Gräberfeld

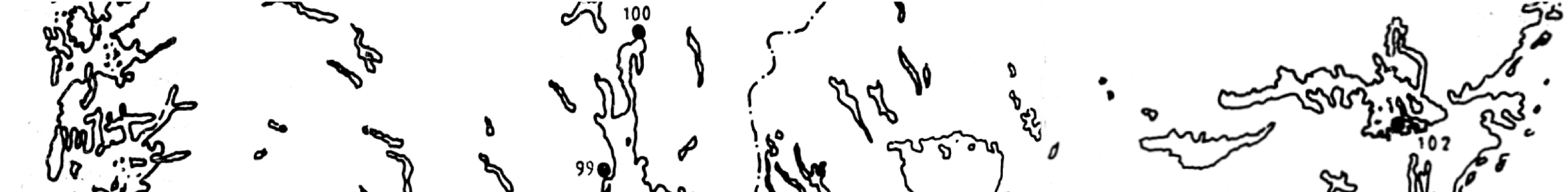

in

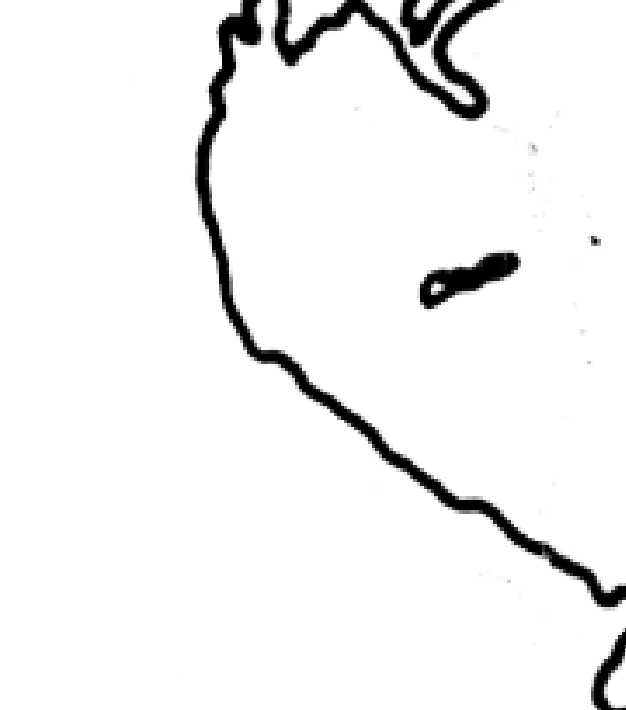

cose

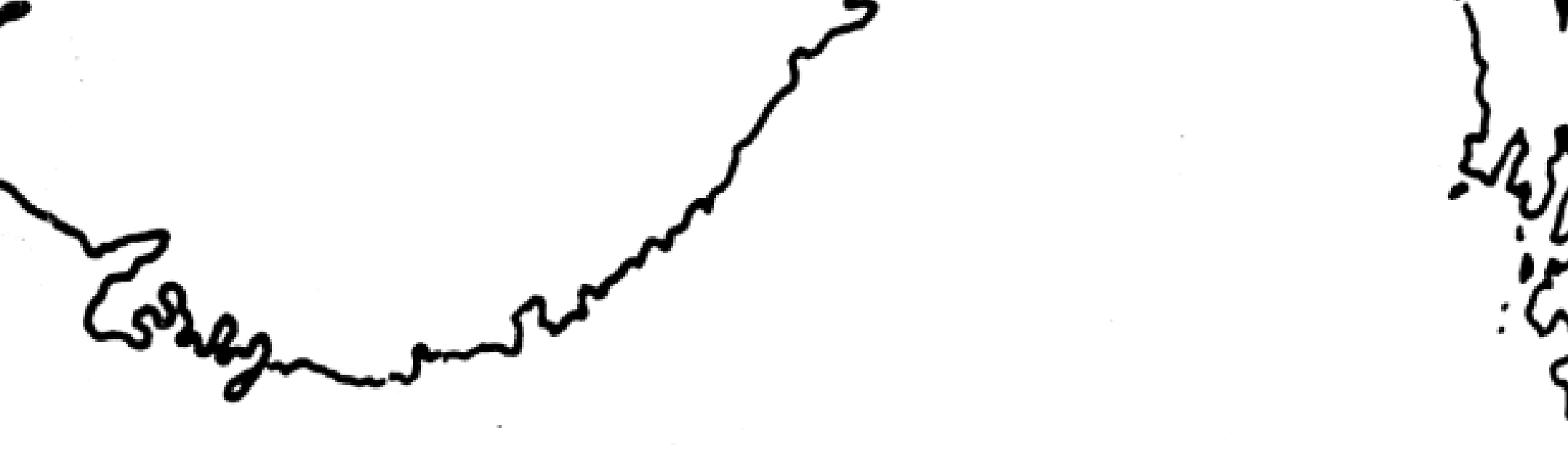

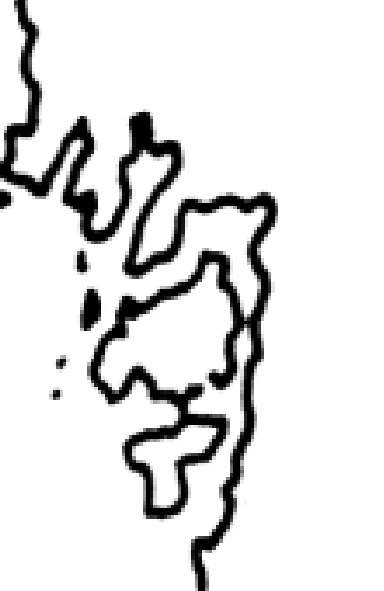

$20^{5}$
0.0

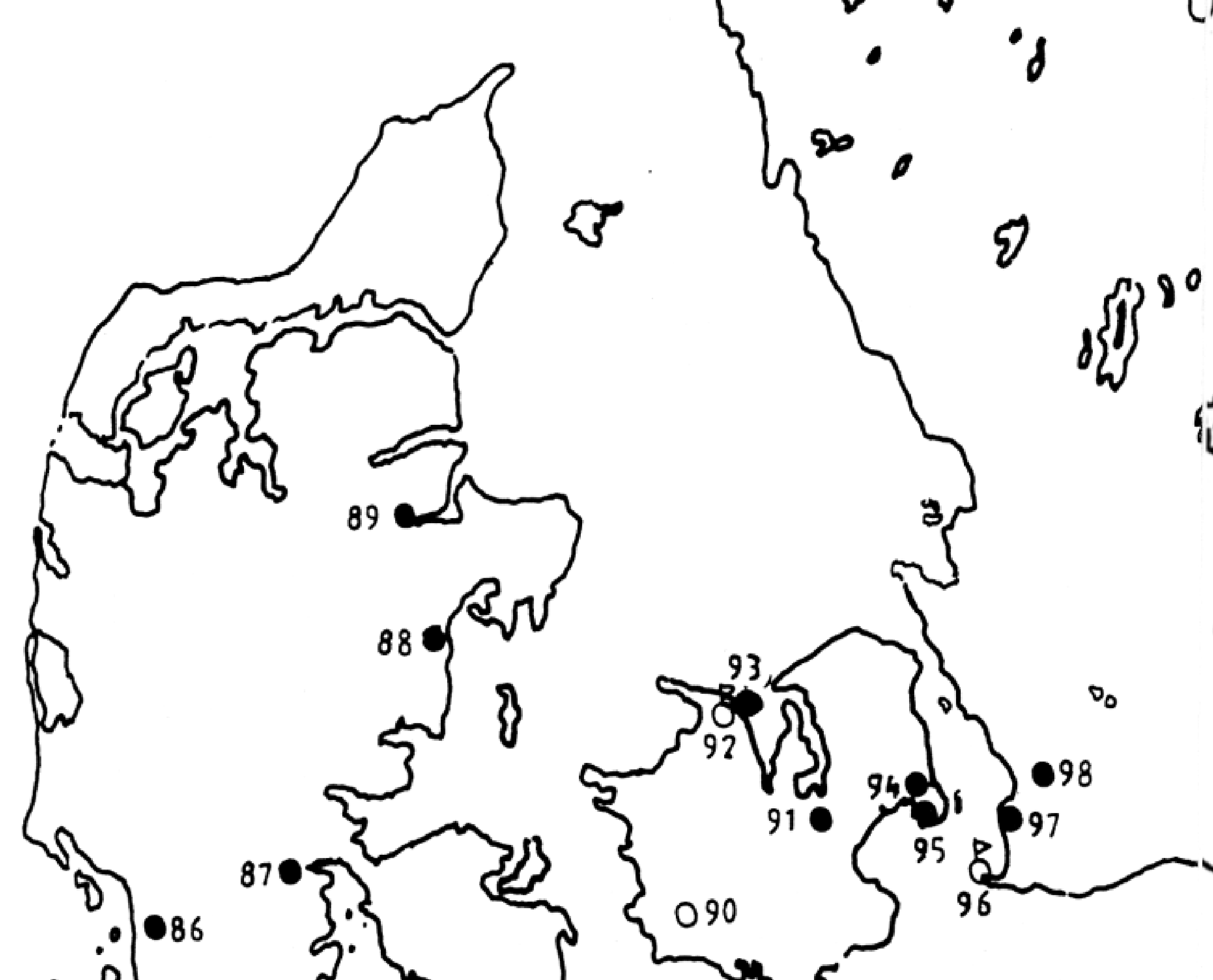

का

$\begin{array}{lll}\infty & 0 \\ \infty & 0\end{array}$ .

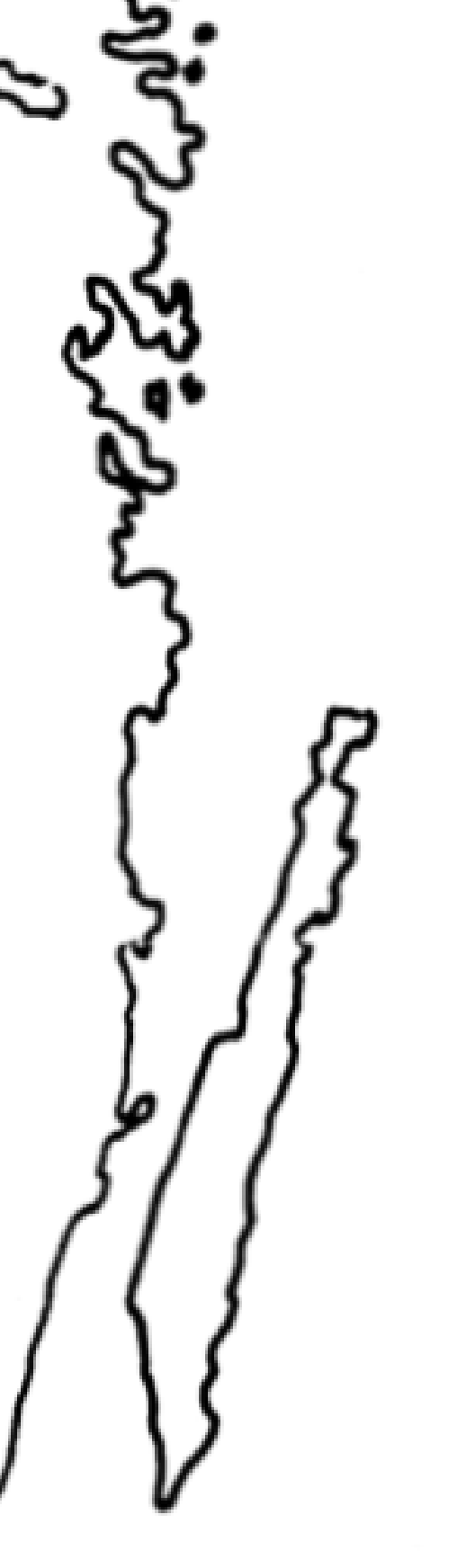

\{\}$^{3}$

$\vartheta$

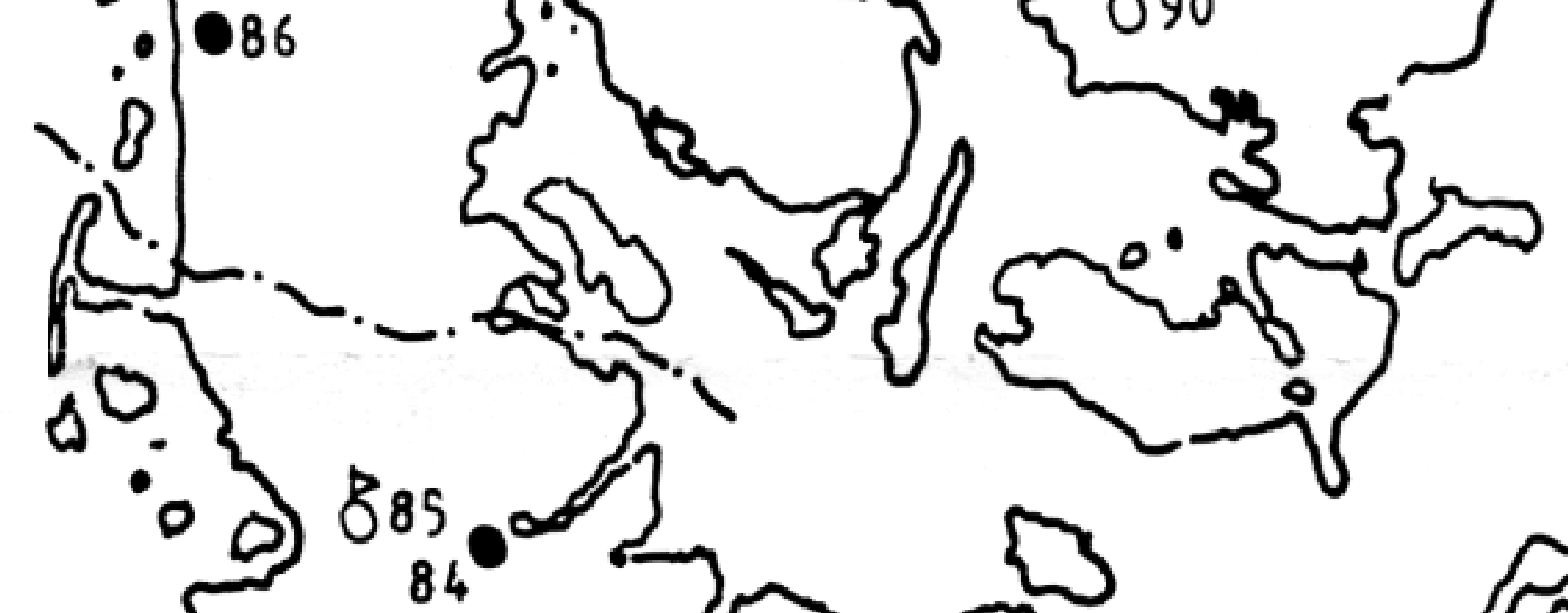

(
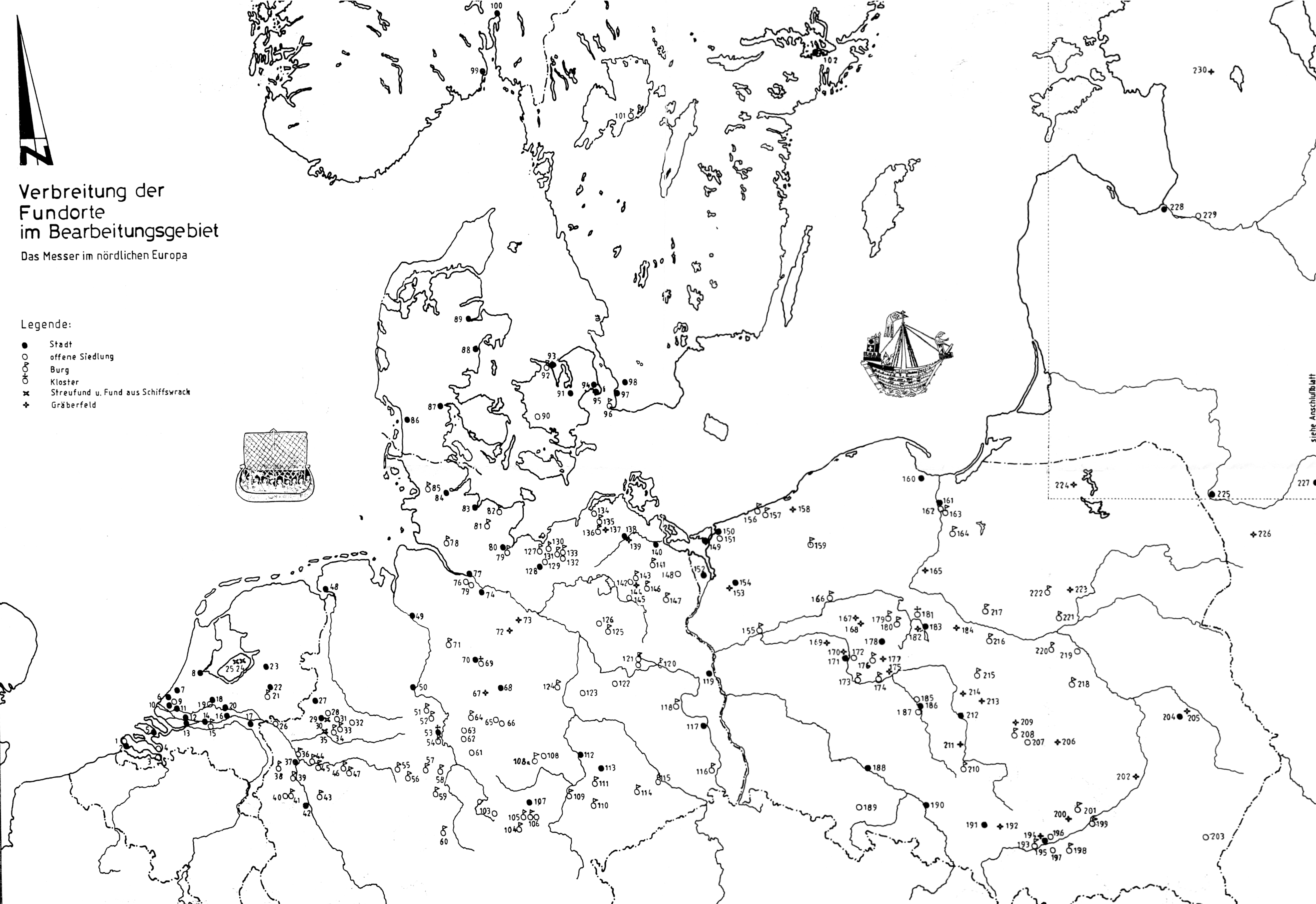

$230+\vartheta$ 
